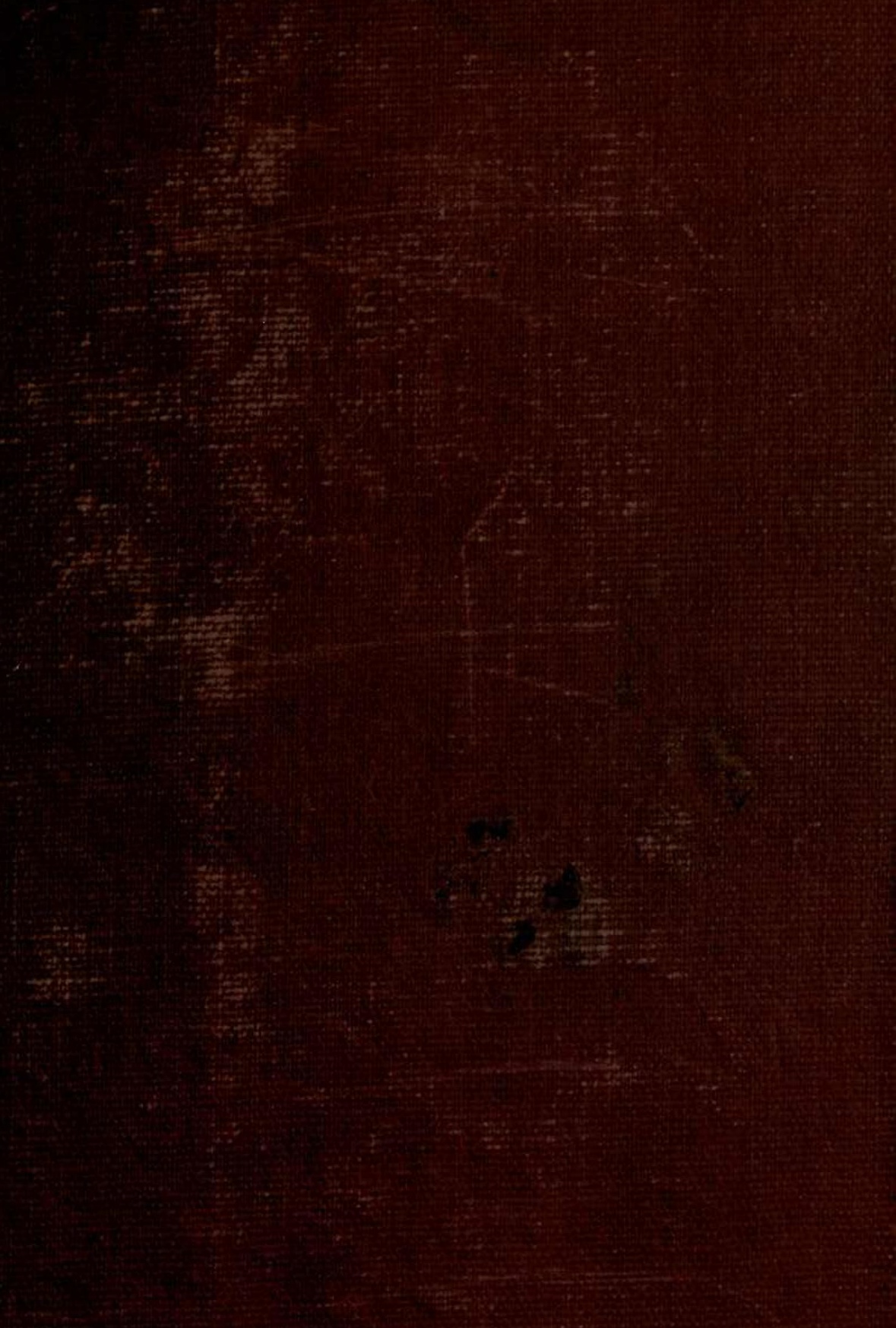




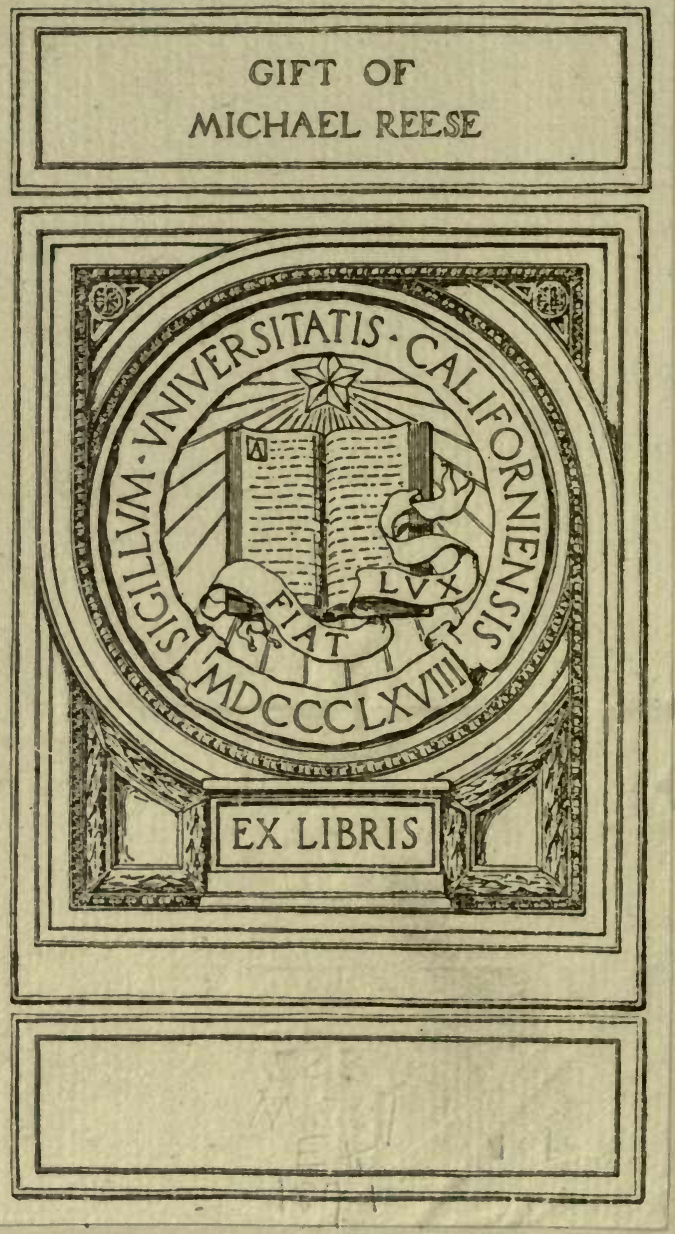






\section{.}





\section{PRINCIPLES OF CHEMISTRY}

VOL, I. 
PRATTED BX

SPOTTISWOODE AND CO., NEW-STRERT SQUARE LONDOX 


\title{
PRINCIPLES OF CHEMISTRY
}

BY

\author{
D. MENDELÉEFF
}

TRANSLATED FROM THE RUSSIAN (FIFTH EDITION) BY

GEORGE KAMENSKY, A.R.S.M.

OF THE IMPERIAL MINT, ST PETERSBURG

EDITED BY

A. J. GREENAWAY, F.I.C.

SCB-EDTTOR OF THE JOURNAL OF THE CHEMICAL SOCIETY

IN TWO VOLUMES

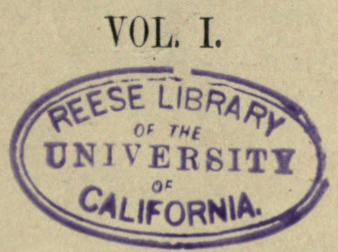

LONDON

LONGMANS, GREEN, AND CO. AND NEW YORK: 15 EAST $16^{\text {th }}$ STREET 1891 


$$
\therefore \quad p^{30}
$$

48.630 


\section{CALIFORNIA.}

\section{PREFACE}

TO THE

\section{ENGLISH TRANSLATION}

Is presenting to the scientific world an English translation of the text-book of Chemistry written by the great master of the Periodic Law, we feel that no apology is necessary, for it was in preparing the first edition of this book that the author was led to those considerations which resulted in the discovery of that law, and, moreover, the book is quite unique in its treatment of its subject.

In order to convey as nearly and clearly as possible the exact meaning of the author, it has been our endeavour to give, as far as the genius of the two languages permits, a literal rendering of the original work. Some exception may no doubt be taken to some of the sentences, but it was felt on the whole that it would be better to have some inelegance of language rather than to risk the loss of the exact shade of meaning that the author had intended to convey.

We have not considered ourselves at liberty to make any alterations in the matter of the work, save the omission of two notes referring to the meaning of Russian words, and of some details referring to the waters of the streams near St. Petersburg, which required local knowledge to be of any utility. It has, however, been necessary to make a considerable change in the 
illustrations, as electro-types of the figures in the original could not be obtained.

Since the publication of the Russian fifth edition, Professor Mendeléeff has issued some appendices to the work, which will be found printed at the end of Volume II. We have to express our thanks to the Managers of the Royal Institution for permission to reprint the lecture delivered at the Royal Institution by Professor Mendeléeff (Appendix I.), and to the Council of the Chemical Society for permission to reprint the Faraday lecture which forms Appendix II.

In conclusion, we would express our gratitude to Professor Kinch for the aid so kindly given in revising the sheets for the press.

G. K.

A. J. G.

October, 1891. 


\section{AUTHOR'S PREFACE}

To

\section{THE FIFTH EDITION}

TuIs work was written during the years 1868-1870, its object being to acquaint the student not only with the methods of observation, the experimental facts, and the laws of chemistry, but also with the aspect of this science towards the invariable substance of varying matter. If the facts themselves include the person who observes them, then how much more inevitable is the reflection of personality in giving an account of methods and of philosophical speculations? For the same reason there will inevitably be much that is subjective in every objective exposition of science. And as an individual production is only significant in virtue of that which has preceded and which surrounds it, so it essentially resembles a mirror which in reflecting exaggerates the size and clearness of neighbouring objects, and causes a person near it to see reflected most plainly those objects which are on the side to which it is directed. Although I have endeavoured to make my book a true mirror directed towards the domains of chemical transformations, yet involuntarily those influences near to me have been the most clearly reflected, the most brightly illuminated, and have tinted the entire work with their colouring. In this way the chief peculiarity of the book has been determined. Experimental and practical data occupy their place, but the philo- 
sophical principles of our science form the chief theme of the work. In former times sciences, like bridges, could only be built up by supporting them on a few deep abutments and long girders. In addition to the exposition of the principles of chemistry, it has been my desire to show how science has now been built up like a suspension bridge, supported by the united strength of a number of slender, but firmly-fixed, threads, which individually are of little strength, and has thus been carried over difficulties which before appeared impassable. In comparing the science of the past, the present, and the future, in placing the particulars of its restricted experiments side by side with its aspirations for unbounded and infinite truth, and in restraining myself from yielding to a bias towards following the most attractive representation, I have endeavoured to incite in the reader a spirit of inquiry, which, unsatisfied with speculative reasonings alone, should subject every idea to experiment, excite the habit of stubborn work, necessitate a knowledge of the past, and a search for fresh threads to complete the bridge over the bottomless unknown. Experience proves that it is possible by this means to avoid two equally pernicious extremes, the Utopian - a visionary contemplation which proceeds from a current of thought only-and the realistic stagnation which is content with bare facts. In sciences like chemistry, which treat of ideas as well as of the substances of nature, experience demonstrates at every step that the work of the past has availed much, and that without it it would be impossible to advance "into the ocean of the unknown.' We are compelled to value their history, to cast aside classical illusions, and to engage in a work which not only gives mental satisfaction but is also practically useful. ${ }^{1}$

I Chemistry, like every other science, is at once a means and an end. It is a means of attaining certain practicable aspirations. Thus, by its assistance, the obtaining of matter in its various forms is facilitated; it shows new possibilities of availing ourselves of the forces of nature, indicates the methods of preparing many substances, points out their properties, etc. In this sense chemistry is closely connected with the work of the manufacturer and the artisan, its sphere is active, and is a means of promoting general welfare. Besides this honourable vocation, ehemistry has another. With it, as with every other elaborated science, there are many lofty aspirations, the contemplation of which serves to inspire its workers and partisans. This eontemplation comprises not only the principal data 


\section{Thus the desire to direct those thirsting for truth to the pure} source of the science of the forces acting throughout nature forms

of the science, but also the generally-accepted deductions, and also hypotheses, which refer to phenomena as yet but imperfectly known. In this latter sense scientific contemplation varies much with times and persons, it bears the stamp of creative power, and comprehends the highest branch of scientific progress. In that pure enjoyment experienced on approaching to the ideal, in that eagerness to draw aside the veil from the hidden truth, and even in that discord which exists between the various workers, we ought to see the surest pledges of further scientific success. Science thus advances, discovering new truths, and at the same time obtaining practical results. The edifice of science not only requires material but also a plan, and necessitates the work of preparing the materials, putting them together, working out the plans and the symmetrical proportions of the various parts. To conceive, understand, and grasp the whole symmetry of the scientific edifice, including its unfinished portions, is equivalent to tasting that enjoyment only conveyed by the highest forms of beauty and truth. Without the material, the plan alone is but a castle in the air, a mere possibility, whilst the material without a plan is but useless matter; all depends on the concordance of the materials with the plan and execution, and the general harmony thereby attained, In the work of science, the artisan, architect, and creator are very often one and the same individual, but sometimes, as in other walks of life, there is a difference between them; sometimes the plan is preconceived, sometimes it follows the preparation and accumulation of the raw material. Free access to the edifice of science is not only allowed to those who devised the plan, worked out the detailed drawings, prepared the materials, or piled up the brickwork, but also to all those who are desirous of making a close acquaintance with the plan, and wish to avoid dwelling in the vaults or in the garrets where the useless lumber is stored.

Knowing how contented, free, and joyful is life in the realms of science, one fervently wishes that many would enter their portals. On this account many pages of this treatise are unwittingly stamped with the earnest desire that the habits of chemical contemplation which I have endeavoured to instil into the minds of my readers will incite them to the further study of science. Science will then flourish in them and by them, on a fuller acquaintance not only with that little which is enclosed within the narrow limits of my work, but with the further learning which they must imbibe in order to make themselves masters of our science and partakers in its further advancement.

Those who enlist in the cause of science have no reason to fear when they remember the urgent need for practical workers in the spheres of agriculture, arts, and manufacture. By summoning aciherents to the work of theoretical chemistry, I am confident that I call them to a most useful labour, to the habit of dealing correctly with nature and its laws, and to the possibility of becoming truly practical men. In order to become actual chemists, it is necessary for beginners to be well and closely acquainted with three important branches of chemistry-analytical, organic, and theoretical. That part of chemistry which is dealt with in this treatise is only the groundwork of the edifice. For the learning and development of chemistry in its truest and fullest sense, beginners ought, in the first place, to turn their attention to the practical work of analytical chemistry; in the second place, to practical and theoretical acquaint. 
the first and most important aim of this book. The time has arrived when a knowledge of physics and chemistry forms as important a part of education as that of the classics did two centuries ago. In those days the nations which excelled in classical learning stood foremost, just as now the most advanced are those which are superior in the knowledge of the natural sciences. I also wished to show in an elementary treatise on chemistry the palpable advantages gained by the application of the periodic law, which I first saw in its entirety in the year 1869 when I was engaged in writing the first edition of this book, in which, indeed, the law was first enunciated. Then, however, this law was not established so firmly as now, when so many of its consequences have been verified by the researches of numerous chemists, and especially by Roscoe, Lecoq de Boisbaudran, Nilson, Brauner, Thorpe, Carnelley, Laurie, Winkler, and others. As the entire scheme of this work ${ }^{2}$ is subjected to the law of periodicity, which may be illustrated in a

ance with some special chemical question, studying the original treatises of the investigators of the subject (at first, under the direction of experienced teachers), because in working out particular facts the faculty of judgment and of correct criticism becomes sharpened; in the third place, to a knowledge of current scientific questions through the special chemical journals and papers, and by intercourse with other chemists. The time has come to turn aside from visionary contemplation, from platonic aspirations, and from classical verbosity, and to enter the regions of actual labour for the common weal, and to prove that the study of science is not only an excellent education for youth, but that it instils the virtues of labour and truth, and creates solid national wealth, material and mental, which without it would be unattainable. Science, which deals with the infinite, is itself without bounds.

2 I recommend those who are commencing to study chemistry with my book to first learn only what is printed in the large type, because in that part I have endeavoured to concentrate all the fundamental, indispensable knowledge required for the study of chemistry. In the footnotes, printed in small type (which I advise being read only after the large text has been mastered), certain details are discussed; they are either further examples, or debatable questions on existing ideas which I thought indispensable to lay before those entering into the sphere of science, or certain historical and technical details which might be withdrawn from the fundamental portion of the book. Without intending to attain in my treatise to the completeness of a work of reference, I have still endeavoured to express the principal developments of science as they concern the chemical elements viewed in that aspect in which they appeared to me after long continued study of the subject and participation in the contemporary advance of knowledge. 
tabular form by placing the elements in series, groups, and periods, two such tables are given at the end of this preface.

In this fifth edition I have not altered any essential feature of the original work, but have enlarged it in two directions. First, the doctrine of chemical equilibria, originally introduced by Berthollet and Henri Sainte-Claire Deville, is discussed more fully and minutely than in the earlier editions, as it has during recent years been established on a much firmer footing; and, second, the descriptive data referring to the elements have been increased by many new facts.

D. MENDELEEFF. 


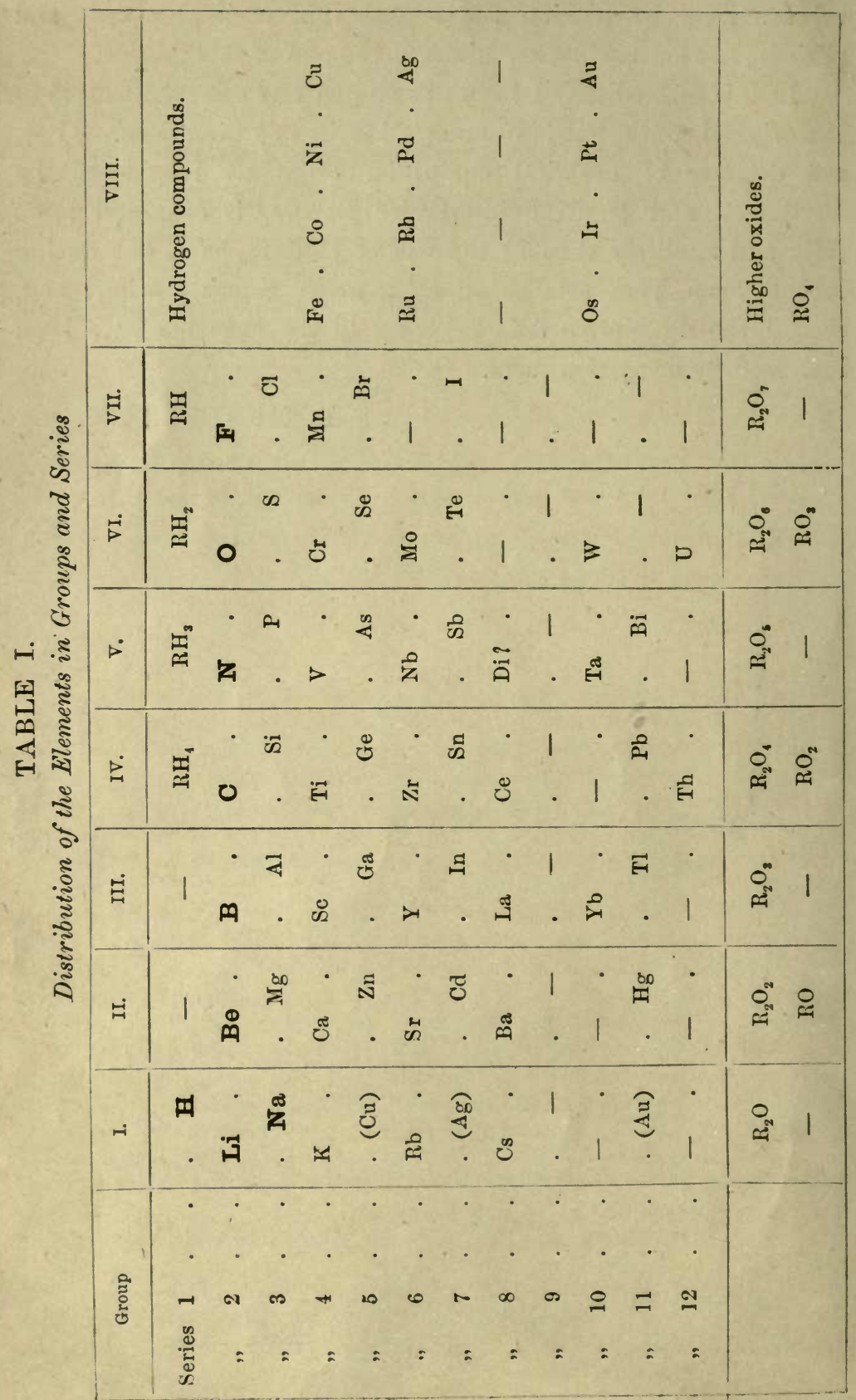




\section{xiii}

\section{TABLE II.}

The Atomic Weights of the Elements

Distribution of the Elements in Periods

\begin{tabular}{|c|c|c|c|c|c|c|c|c|}
\hline \multirow{2}{*}{ Groups } & \multirow{2}{*}{$\begin{array}{c}\text { Higher } \\
\text { Salt- } \\
\text { forming } \\
\text { Oxides }\end{array}$} & \multirow{2}{*}{\multicolumn{2}{|c|}{$\begin{array}{c}\text { Typical or } \\
\text { 1st small } \\
\text { Period }\end{array}$}} & \multicolumn{5}{|c|}{ Large Periods } \\
\hline & & & & 1st & $2 n d$ & $3 r d$ & 4th & 5th \\
\hline I. & $\mathrm{R}_{2} \mathrm{O}$ & $\mathbf{L i}$ & $=7$ & K 39 & $\mathrm{Rb} 85$ & Cs 133 & - & - \\
\hline II. & Ro & $\mathrm{Be}$ & $=9$ & Ca 40 & S 87 & Ва 137 & - & - \\
\hline III. & $\mathrm{R}_{2} \mathrm{O}_{3}$ & $B$ & $=11$ & Sc 44 & $\begin{array}{l}Y \\
\end{array}$ & La 138 & Yb 173 & - \\
\hline IV. & $\mathrm{RO}_{2}$ & $\mathrm{C}$ & $=12$ & $\mathrm{Ti} 48$ & $\mathrm{Zr} 90$ & Ce 140 & - & Th 232 \\
\hline v. & $\mathrm{R}_{2} \mathrm{O}_{3}$ & $\mathrm{~N}$ & $=14$ & V 51 & $\mathrm{Nb} 94$ & - & Ta 182 & - \\
\hline VI. & $\mathrm{RO}_{3}$ & o & $=16$ & Cr 52 & Mo 96 & - & W 184 & $\mathrm{Ur} 240$ \\
\hline VII. & $\mathrm{R}_{2} \mathrm{O}_{7}$ & $\mathrm{~F}$ & $=19$ & Mn 55 & - & - & - & - \\
\hline & & & & Fe 56 & $\mathrm{Ru} 103$ & - & Os 191 & - \\
\hline vili. & & & & Co $58 \cdot 5$ & Rh 104 & - & Ir 193 & - \\
\hline & & & & $\mathrm{Ni} 59$ & Pd 106 & - & Pt 196 & - \\
\hline I. & $\mathrm{R}_{2} \mathrm{O}$ & $\mathrm{H}=1 . \mathrm{Na}$ & $=23$ & $\mathrm{Cu} 63$ & Ag 108 & - & Au 198 & - \\
\hline II. & no & $\mathrm{Mg}$ & $=2 \star 2$ & $\mathrm{Zn} 65$ & $\mathrm{Cd} 112$ & - & $\mathrm{Hg} 200$ & - \\
\hline III. & $\mathrm{R}_{2} \mathrm{O}_{3}$ & $\mathrm{Al}$ & $=27$ & Ga 70 & In 113 & - & Tl 204 & - \\
\hline IV. & $\mathrm{RO}_{2}$ & $\mathrm{Si}$ & $=28$ & Ge 72 & Sn 118 & - & $\mathrm{Pb} 206$ & - \\
\hline v. & $\mathrm{R}_{2} \mathrm{O}_{5}$ & $\mathbf{P}$ & $=31$ & As 75 . & $\mathrm{Sb} 120$ & - & Bi 208 & - \\
\hline VI. & $\mathrm{RO}_{3}$ & $\mathbf{S}$ & $=32$ & Se 79 & Te 125 & - & - & - \\
\hline VII. & $\mathrm{R}_{2} \mathrm{O}_{7}$ & Cl & $=35.5$ & $\mathrm{Br} 80$ & I $\quad 127$ & - & - & - \\
\hline & & $\underset{\mathrm{Pe}}{2 \text { nd }}$ & $\begin{array}{l}\text { small } \\
\text { riod }\end{array}$ & 1st & 2nd & $3 \mathrm{rd}$ & 4th & 5 th \\
\hline & & & & & & Per & & \\
\hline
\end{tabular}



CHAPTKR

X. SODIUM CHLORIDE. BERTHOLLET'S LAWS. HYDROCHLORIC ACID .

1. THE HALOGENS: CHLORINE, BROMINE, IODINE, AND FLUORINE • 45 XII. SODIUM

XIII. POTASSIUM, RUBIDIUM, CESIUM, AND LITHIUM. SPECTRUM ANALYSIS.

Xiv. THE VALENCY AND SPECIFIC heat OF the MEtals. MagNesium, CALCIUM, STRONTICM, BARIUM, AND BERYLLIUM

\section{Erratum.}

Page 91, line 4 from foot, for Prinsep read Pierson. 


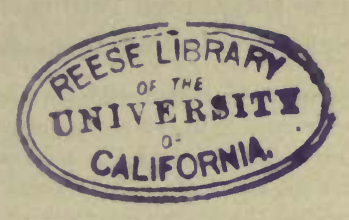

\section{PRINCIPLES OF CHEMISTRY}

\section{INTRODUCTION}

\section{Chemistry is concerned with the study ${ }^{1}$ of the homogeneous substances}

1 The investigation of a substance or phenomenon of nature consists $(a)$ in determining the relation of the thing under investigation to that which is already known, either from former studies, or from experiment, or from the consciousness of the common surroundings of life-that is, in determining and expressing the quality of the unknown by the aid of that which is known; $(b)$ in measuring all that which can be subjected to measurement, and thereby denoting the quantitative relation of that under investigation to that already known and its relation to the categories of time, space, temperature, mass, \&c.; $(c)$ in determining the position held by the thing under investigation in the system of the things known, guided by both qualitative and quantitative data; $(d)$ in finding, from the quantities which have been measured, the empirical (visible) dependence (function, or 'law,' as it is sometimes termed) of variable factors-for instance, the dependence of the composition of the substance on its properties, of temperature on time, of time on locality, \&c.; $(e)$ in framing hypotheses or propositions as to the actual cause and true nature of the relation between that studied (measured or observed) and that which is known or the categories of time, space, \&c.; $(f)$ in verifying the logical consequences of the hypotheses by experiment; and $(g)$ in advancing a theory which shall account for the nature of the properties of that studied in its relations with things already known and with those conditions or eategories among which it exists. It is certain that it is only possible to thus study, when we have taken as a basis some incontestable fact which is self-evident to our understanding; as, for instance, number, time, space, movement, or mass. The determination of such primary or fundamental conceptions (categories), although not excluded from the possibility of investigation, frequently does not subject itself to our present mode of scientific generalisation. Hence it follows in the investigation of anything, there always remains something which is recognised without investigation, or admitted as a known factor. The axioms of geometry may be taken as an example. Thus in the science of biology it is necessary to admit the faculty of organisms for multiplying themselves, as a conception whose meaning is yet unknown. Thus in the study of chemistry the notion of elements must be recognised without hardly any further analysis. However, by first investigating that which is visible and subject to direct observation by the organs of the senses, we may hope that, first, hypotheses will be arrived at, and afterwards theories of that which has now to be placed at the basis of our investigations. The minds of the ancients strove to at once seize the very fundamental categories of investigation, whilst all the successes of recent knowledge are based on the above-cited method of investigation without the determination of ' the beginning of all beginnings.' By following this inductive method, the exact sciences

VOL. I. 


\section{or material ${ }^{2}$ of which all the objects of the universe are made up, with} the transformations of these substances into each other, and with the phenomena ${ }^{3}$ which accompany such transformations. Every chemical

have already succeeded in becoming acquainted with certainty with much of the invisible world, which directly is imperceptible to the organs of sense (for example, the molecular movement of all bodies, the composition of the heavenly luminaries, the paths of their movement, the necessity for the existence of substances which cannot be subjected to experiment, \&c.), and have verified the knowledge thus obtained, and employed it for increasing the interests of human life; and therefore it may be safely said that the inductive method of investigation is a more perfect mode of acquiring knowledge than the deductive method alone (starting from a little of the unknown accepted as incontestable to arrive at the much which is visible and observable) by which the ancients strove to embrace the universe. By investigating the universe by an inductive method (endeavouring from the much which is observable to arrive at a little which may be verified and is indubitable) the new science refuses to recognise dogma as truth, but through reason, by a slow and laborious method of investigation, strives for and attains to true deductions.

${ }^{2}$ A substance or material is that which occupies space and has weight. That is, which presents a mass which is attracted by the earth and by other masses of material, and of which the objects of nature are composed, and through which the movements and phenomena of nature are accomplished. It is easy to find out by examining and investigating, by various methods, the objects met with in nature and in the arts, that some of them are homogeneous, whilst others are composed of a mixture of several homogeneous substances. This is most clearly seen in solid substances. The metals nsed in the arts (for example, gold, iron, copper) should be distinguished for their homogeneity, otherwise they are brittle and unfit for many uses. Homogeneous matter exhibits similar properties in all its parts. By breaking up a homogeneous substance we obtain parts which, although different in form, resemble each other in their properties. Glass, the best qualities of sugar, marble, \&c., are examples of homogeneous substances. But examples of non-homogeneous substances are much more frequent in nature and the arts. Thus the majority of the rocks are not homogeneous. In porphyries bright pieces of a mineral called 'orthoclase' are often seen strewn amongst the dark mass of the rock. In ordinary red granite it is easy to distinguish large pieces of orthoclase mixed with dark semi-transparent quartz and flexible laminæ of mica. Nor are plants and animals homogeneous. Thus leaves are composed of a skin, fibre, pulp, sap, and a green colouring matter. This is clearly seen by examining under a microscope a thin slice cut off a leaf. As an example of those non-homogeneous substances which are produced artificially, gunpowder may be cited, which is prepared by mixing together known proportions of sulphur, nitre, and charcoal. Many liquids, also, are not homogeneous, as may be observed by the aid of the microscope, when drops of blood are seen to consist of a colourless liquid in which red corpuscules, invisible to the naked eye owing to their small size, are floating about. It is these corpuscules which give blood its peculiar colour. Milk is also a transparent liquid, in which microscopical drops of fat are floating, and which rise to the top when milk is left at rest, forming cream. When the fat is beaten up (churned) the separate drops collect into one mass. It is possible to extract from every nonhomogeneous substance those homogeneous substances of which it is made up. Thus orthoclase may be separated from porphyry by breaking it off. So also gold is extracted from gold-bearing sand by washing away the mixture of clay and sand. Chemistry deals only with the homogeneous substances met with in nature, or extracted from natural or artificial non-homogeneous substance. The various mixtures found in nature form the subjects of other natural sciences-as geognosy, botany, zoology, anatomy, \&c.

3 All those events which are accomplished by substances in time, are termed 'phenomena.' Phenomena in themselves form the fundamental subject of the study of physics. Movement is the primary and most generally understood form of phenomenon, and thereforewe endeavour to reason about other phenomena as clearly as when dealing with move- 
change or reaction, ${ }^{4}$ as it is called, can only take place under a condition of most intimate and close contact of the reacting substances, ${ }^{5}$ and is determined by the forces proper to the smallest invisible particles (molecules) of matter. We must distinguish three chief classes of chemical transformations.

1. Combination is a reaction in which the union of two substances yields a new one, or in general terms, from a given number of substances a lesser number is produced. Thus, by heating a mixture of iron and sulphur ${ }^{6}$ a single new substance is produced, iron sulphide, in which the constituent substances cannot be distinguished even by the highest magnifying power. Before the reaction, the iron could be separated from the mixture by a magnet, and the sulphur by dissolving it in certain oily liquids $;^{7}$ in general, before combination they might be mechanically separated from each other, but after combination both substances penetrate into each other, and are then neither mechanically separable nor individually distinguishable. As a rule, reactions of direct combination are accompanied by an evolution of heat, and the common case of combustion, evolving heat, consists in the combination of combustible substances with a portion (oxygen) of the atmosphere,

ment. Therefore, mechanics, which treats of movement, forms the fundamental science of natural philosophy, and all other sciences endeavour to reduce the phenomena with which they are concerned to mechanical principles. Astronomy was the first to take to this path of reasoning, and succeeded in many cases in reducing astronomical to purely mechancal phenomena. Chemistry and physics, physiology and biology are proceeding in the same direction.

4 The verb 'to react' means to act or change chemically.

3 If a phenomenon proceeds at visible or measurable distances (as, for instance, magnetic attraction or gravity) it cannot be ascribed to chemical phenomena, which are only accomplished at distances immeasurably small and undistinguishable to the eye or the microscope ; that is to say, which belong to the number of purely molecular phenomena. When a change of material is accomplished within a substance without visible motion or the interference of foreign matters (for instance, when new wine 'ages' by keeping, and acquires a peculiar aroma), it may be classed as a chemical phenomenon; but the ordinary eases of chemical reaction are accomplished by the mutual action of different substances which, previously free, on reaction mutually permeate each other.

${ }^{6}$ For this purpose a piece of iron may be made red hot in a smith's furnace, and then placed in contact with a lump of sulphur, when iron sulphide will be obtained as a molten liquid, the combination being accompanied by a visible increase in the glow of the iron. Or else iron filings are mixed with powdered sulphur in the proportion of 5 parts of iron to 3 parts of sulphur, and the mixture placed in a glass tube, which is then partially heated. Combination does not commence without the aid of external heat, but when once started in any portion of the mixture it extends throughout the entire mass, because the portion first heated evolves sufficient heat in forming iron sulphide to raise the adjacent parts of the mixture to the temperature required for starting the reaction. The rise in temperature thus obtained is so high as to soften the glass tube.

7 Sulphur is slightly soluble in many thin oils; it is very soluble in carbon bisulphide and in some other liquids. Iron is insoluble in carbon bisulphide, and therefore the sulphur can be dissolved away from the iron. 
the gases and vapours contained in the smoke being the products of combination.

2. Reactions of decomposition are cases the reverse to those of combination, that is, in which one substance gives two-or, in general, a given number of substances a greater number. Thus, by heating wood (and also coal and many animal or vegetable substances) without access to air, a combustible gas, a watery liquid, tar, and carbon are obtained. It is in this way that tar, lighting gas, and charcoal are prepared on a large scale. ${ }^{8}$ All limestones, for example, flagstones, chalk, or marble, are decomposed by heating to redness into lime and a peculiar gas called carbonic anhydride. A similar decomposition, taking place, however, at a much lower temperature, proceeds with the green copper carbonate which enters into the composition of malachite. This example will be studied more in detail presently. Whilst heat is evolved in the ordinary reactions of combination, it is, on the contrary, consumed in the reactions of decomposition.

3. The third class of chemical reactions - where the number of acting substances is equal to the number of substances formed-consists, as it were, of an association of decomposition and combination. If, for instance, two compounds $\mathrm{A}$ and $\mathrm{B}$ are taken and they react on each other to form the substances $\mathrm{C}$ and $\mathrm{D}$, then supposing that $\mathrm{A}$ is decomposed into $\mathrm{D}$ and $\mathrm{E}$, and that $\mathrm{E}$ combines with $\mathrm{B}$ to form $\mathrm{C}$, we have a reaction in which two substances $A$, or $D E$, and $B$ were taken and two others $\mathrm{C}$, or $\mathrm{E} \mathrm{B}$, and $\mathrm{D}$ were produced. Such reactions ought to be placed under the general term of reactions of 'rearrangement,' and the particular case where two substances give two fresh ones, reactions of 'substitution.' 9 Thus, if a piece of iron be immersed in a solution of blue vitriol (copper sulphate), copper is formed-or, rather,

8 Decomposition of this kind is termed 'dry distillation' because, as in distillation, the substance is heated and vapours are given off which, on cooling, condense into liquids. In general, decomposition, in absorbing heat, presents much in common to a physical change of state-such as, for example, that of a liquid into a gas: Deville likened complete decomposition to boiling, and compared partial decomposition, when a portion of a substance is not decomposed in the presence of its products of decomposition (or dissociation), to evaporation.

9 A reaction of rearrangement may in certain cases take place with one substance only; that is to say, a substance may by itself change into a new isomeric form. Thus, for example, if hard yellow sulphur be heated to a temperature of $250^{\circ}$ and then poured into cold water it gives, on cooling, a soft, brown variety. Ordinary phosphorus, which is transparent, poisonous, and phosphorescent in the dark (in air), gives, after being heated at $270^{\circ}$ (in an atmosphere incapable of supporting combustion, such as steam), an opaque, red, and non-poisonous isomeric variety, which is not phosphorescent. Cases of isomerism point out the possibility of an internal rearrangement in a substance, and are the result of an alteration in the grouping of the same elements, just as a certain number of balls may be grouped in figures and forms of different shapes and of various properties. 
separated out, and green vitriol (iron sulphate, which only differs from the blue vitriol in that the iron has replaced the copper) is obtained in solution. In this manner iron may be coated with copper, so also copper with silver; such reactions are frequently made use of in practice.

The majority of the chemical changes accomplished in nature and the arts are very complicated, as they consist of an association of many separate and simultaneous combinations, decompositions, and replacements. In this natural complexity of chemical phenomena is discovered the chief reason why for so many centuries chemistry did not exist as an exact science; that is to say, that although many chemical changes were known and made use of, ${ }^{10}$ yet their real nature was unknown, nor could they be foreseen or directed at will. Another reason for the tardy progress of chemical knowledge is the participation of gaseous substances, especially air, in many reactions. The true comprehension of air as a ponderable substance, and of gases in general as peculiar elastic and dispersive states of matter, was only arrived at in the sixteenth and seventeenth centuries, and it was only after this that the transformations of substances could form a science. Up to that time, without understanding the invisible and yet ponderable gaseous and vaporous states of substances, it was impossible to form any fundamental chemical evidence, because gases escaped from notice between the acting and resultant substances. It is easy from the impression conveyed to us by the phenomena we observe to form the opinion that matter is created and destroyed : a whole mass of trees burn, and there only remains a little charcoal and ash, whilst from one small seed there grows little by little a majestic tree. In one case matter seems to be destroyed, and in the other to be created. This conclusion is arrived at because the formation or consumption of gases, being under the circumstances invisible to the eye, is not noted. When wood burns it undergoes a chemical change into gaseous products, which escape as smoke. A very simple experiment will prove this. By collecting the smoke it may be observed that it contains gases which differ entirely from air, being incapable of supporting combustion or respiration. These gases may be weighed, and it will then be seen that their weight exceeds that of the wood taken. This increase in weight arises from the fact that, in burning, the component parts of the wood combine with a portion of the air ; in like manner iron increases in weight by rusting. In burning gunpowder its substance is not destroyed, but only converted into gases and smoke. So also in the growth of a tree; the seed does not

10 Thus the ancients knew how taconvert the juice of grapes containing the saccharine principle (glucose) into wine or vinegar, or how to extract metals from the ores which are found in the earth's crust, und how to prepare glass from earthy substances. 
increase in mass of itself and from itself, but it grows because it absorbs gases from the atmosphere and sucks water and substances dissolved therein from the earth through its roots. The sap and solid substances which give plants their form are produced from these absorbed gases and liquids by complicated chemical processes. The gases and liquids are converted into solid substances by the plants themselves. Plants not only do not increase in size, but die, in a gas which does not contain the constituents of air. When moist substances dry they decrease in weight; when water evaporates we know that it does not disappear, but will return from the atmosphere as rain, dew, and snow. When water is absorbed by the earth, it does not disappear there for ever, but accumulates somewhere underground, from whence it afterwards flows forth as a spring. Thus matter does not disappear and is not created, but only undergoes various physical and chemical transformations - that is to say, changes its locality and form. Matter remains on the earth in the same quantity as before ; in a word it is, as far as we are concerned, everlasting. It was difficult to submit this simple and primary truth of chemistry to investigation, but when once made clear it rapidly spread, and now seems as natural and simple as many truths which have been acknowledged for ages. Mariotte and other savants of the seventeenth century already suspected the existence of the law of the indestructibility of matter, but they made no efforts to express it or to apply it to the ends of science. The experiments by means of which this simple law was arrived at were made during the latter half of the last century by the founder of contemporary chemistry, LAvoisier, the French Academician and mayor. The numerous experiments of this savant were conducted with the aid of the balance, which is the only means of directly and accurately determining the quantity of matter.

Lavoisier found, by weighing all the substances, and even the apparatus, used in every experiment, and then weighing the substances obtained after the chemical change, that the sum of the weights of the substances formed was always equal to the sum of the weights of the substances taken; or, in other words: MATTER Is NoT CREATED AND DOES NOT DISAPPEAR, or that, matter is everlasting. This expression naturally includes a hypothesis, but our only aim in using it is to concisely express the following lengthy period-That in all experiments, and in all the investigated phenomena of nature, it has never been observed that the weight of the substances formed was less or greater (as far as accuracy of weighing permits) than the weight of the sub-. stances originally taken, and as weight is proportional to mass ${ }^{11}$ or

11 The idea of the mass of matter was first shaped into an exact form by Galileo (died 1642), and more especially by Newton (born 1643, died 1727), in the glorious epoch of the 
quantity of matter, it follows that no one has ever succeeded in observing a disappearance of matter or its appearance in fresh quantities. The law of the indestructibility of matter endows all chemical investigations with exactitude, as, on its basis, an equation may be formed for every chemical reaction. If in any reaction the weights of the substances taken be designated by the letters A, B, C, \&c., and the weights of the substances formed by the letters $\mathrm{M}, \mathrm{N}, \mathrm{O}$, \&c., then

$$
\mathrm{A}+\mathrm{B}+\mathrm{C}+\ldots \ldots \ldots=\mathrm{M}+\mathrm{N}+\mathrm{O}+\ldots \ldots \ldots
$$

Therefore, should the weight of one of the acting or resultant substances be unknown, it may be determined by solving the equation. The chemist, in applying the law of the indestructibility of matter, must never lose sight of any one of the acting or resultant substances. Should such an oversight be made, it will at once be remarked from the sum of the weights of the substances taken being unequal to the sum of the weights of the substances formed. All the progress made by chemistry during the end of the last, and in the present, century is entirely and immovably founded on the law of the indestructibility of matter. It is absolutely necessary in beginning the study of chemistry to become familiar with the simple truth which is expressed by this law, and for this purpose several examples elucidating its application will now be cited.

1. It is well known that iron rusts in damp air, ${ }^{12}$ and that when heated to redness in air it becomes coated with scoria (oxide), having, like rust, the appearance of an earthy substance resembling some of the iron ores from which metallic iron is extracted. If the iron is weighed before and after the formation of the scoria or rust, it will be found that the metal has increased in weight during the operation. ${ }^{13}$ It

developinent of the principles of inductive reasoning enunciated by Bacon and Descartes in their philosophical treatises. Shortly after the death of Newton, Lavoisier, whose fame in natural philosophy should rank with that of Galileo and Newton, was born on August 26, 1743. The death of Lavoisier occurred during the Reign of Terror of the French Revolution, when he, together with twenty-six other chief farmers of the revenue, was guillotined on May 8, 1794, at Paris, but his works and thoughts have made him immortal.

12 By covering iron with an enamel, or varnish, or with unrustable metals (such as nickel), or a coating of paraffin, or other similar substances, it is protected from the air and moisture, and so kept from rusting.

${ }^{13}$ Such an experiment may easily be made by taking the finest (unrusted) iron filings (ordinary filings nust be first washed in ether, dried, and passed through a very fine sieve). The filings thus obtained are capable of burning directly in air (by oxidising or forming rust), especially when they hang (are attracted) on a magnet. A compact piece of iron does not burn in air, but spongy iron glows and smoulders like tinder. In making the experiment, a horse-shoe magnet is fixed, with the poles downwards, on one arm of a rather sensitive balance, and the iron filings are applied to the magnet (on a 
can easily be proved that this increase in weight and formation of earthy substances from the metal is accomplished at the expense of the atmosphere, and mainly, as Lavoisier proved, at the expense of that portion which is called oxygen, and, as will afterwards be explained, supports combustion. In fact, in a vacuum, or in gases which do not contain oxygen, for instance, in hydrogen or nitrogen, the iron neither rusts nor becomes coated with scoria. Had the iron not been weighed, the participation of the oxygen of the atmosphere in its transformation into an earthy substance might have easily passed unnoticed, as was formerly the case, when phenomena like the above were, for this reason, misunderstood. It is evident from the law of the indestructibility of matter that as the iron increases in weight in its conversion into rust, the latter must be a more complex substance than the iron itself, and its formation is due to a reaction of combination. Were not this chemical change studied in regard to mass, and did we not know of the ponderability of air, and of its capacity to take part in the phenomena of combustion, we might form an entirely wrong opinion about it, and might, for instance, consider rust to be a simpler substance than iron, and explain the formation of rust as the removal of something from the iron. Such, indeed, was the general opinion prior to Lavoisier, when it was held that iron contained a certain unknown substance called 'phlogiston,' and that rust was iron deprived of this supposed substance.

2. Copper carbonate (in the form of a powder, or as the well-known green mineral called 'malachite,' which is used for making ornaments, or as an ore for the extraction of copper) changes into a black substance called 'copper oxide' when heated to redness. ${ }^{14}$ This black sheet of paper) so as to form a beard about the poles. The balance pan should be exactly under the filings on the magnet, in order that any which might fall from it should not alter the weight. The filings, having been weighed, are set light to by applying the flame of a candle; they easily take fire, and go on burning by themselves, forming rust. When the combustion is ended, it will be clear that the iron has increased in weight; from $5 \frac{1}{2}$ parts by weight of iron filings taken, there are obtained, by complete combustion, $7 \frac{1}{2}$ parts by weight of rust. Consequently, if about 5 grams of filings be applied to the magnet, the increase in weight will be clearly seen by the weights that are required to restore equilibrium. This experiment proceeds so easily and quickly that it may be conveniently demonstrated, as a proof of the increase of weight at the expense of air and of its transformation into the solid iron-rust.

14 For the purpose of experiment, it is most convenient to take copper carbonate, prepared by the experimenter himself, by adding a solution of sodium carbonate to a solution of copper sulphate. The precipitate (deposit) so formed is collected on a filter, washed, and dried. The decomposition of copper carbonate into copper oxide is effected by so moderate a heat that it may be accompished in a glass vessel heated by a lamp. For this purpose a thin glass tube, closed at one end, and called a 'test tube,' may be employed, or else a vessel called a 'retort.' The experiment is carried on, as described in the third example above, by collecting the carbonic anhydride over a water bath, as will be afterwards explained. 
substance is also obtained by heating copper to redness in air-that is, it is the scoria or oxidation product of copper. The weight of the black oxide of copper left is less than that of the copper carbonate originally taken, and therefore we consider the reaction which occurred to have been one of decomposition, and that by it something was separated from the green copper carbonate, and in fact by closing the orifice of the vessel in which the copper carbonate is heated with a wellfitting cork, through which a gas delivery tube ${ }^{15}$ passes whose end is immersed under water, it will be observed that on heating, a gas is formed which bubbles through the water. This gas can be easily collected, as will presently be described, and it will be found to essentially differ from air in many respects; for instance, a burning taper is extinguished in it as if it had been plunged into water. If weighing had not proved to us that some substance had been separated, the formation of the gas might easily have escaped our notice, for it is colourless and transparent like air, and is therefore evolved without any striking feature. The carbonic acid gas evolved may be weighed ${ }^{16}$ and it will be seen that the sum of the weights of the black copper

15 Gas delivery tubes are usually made of glass tubing as prepared at glass works. It is made of various diameters and thicknesses. If of small diameter and thickness, a glass tube is easily bent by heating in a gas jet or the flame of a spirit lamp, and may also be easily divided at a given point by making a deep scratch with a file and then breaking the tube at this point with a sharp jerk. These properties, together with their impermeability, transparency, hardness, and regularity of bore, makes glass tubes most useful in experiments with gases. Naturally they might be replaced by straws, india-rubber, metallic, or other tubes, but these are more difficult to fix on to a vessel, and are not entirely impervious to gases. A glass gas delivery tube may be hermetically fixed into a vessel by fitting it into a perforated cork, which should be soft and free from flaws, and fixing the cork into the orifice of the vessel. Sometimes the cork is previously soaked in paraffin, or it is replaced by an india-rubber cork.

16 Gases, like all other substances, may be weighed, but, owing to their extreme lightness and the difficulty of dealing with them in large masses, they can only be weighed by very sensitive balances; that is, in such as, with a considerable load, indicate a very small difference in weight-for example, a centigram or milligram with a load of 1,000 grams. In order to weigh a gas, a glass globe furnished with a stop-cock (which must not leak in any part, and therefore must be kept well lubricated) is first of all exhausted of air by an air-pump (a Sprengel pump is the best). The stop-cock is then closed, and the exhausted globe weighed. As the pressure of the atmosphere acts on the walls of the globes, they should be thick. Glass is found to bear the strain of the inequality of the exterior and interior pressures best. If the gas to be weighed is then let into the globe, its weight can be determined from the increase in the weight of the globe. It is necessary, how. ever, that the temperature and pressure of the air about the balance should remain constant for both weighings, as the weight of the globe in air will (according to the laws of hydrostatics) vary with its density. The volume of the air displaced, and its weight, must therefore be determined by observing the temperature, density, and moisture of the atmosphere during the time of experiment. This will be partly explained later, but may be studied more in detail by physies. Owing to the complexity of all these operations, the mass of a gas is usually determined from its volume and density, or the weight of one volume. 
oxide and carbonic acid gas is equal to the weight of the copper carbonate $^{17}$ originally taken, and thus by carefully following out the various stages of all chemical reactions we arrive at a confirmation of the law of the indestructibility of matter.

3. Red mercury oxide (which is formed as mercury scoria by heating mercury in air) is decomposed like copper carbonate (only by heating more slowly and at a somewhat higher temperature), with the formation of the peculiar gas, oxygen. For this purpose the mercury oxide is placed in a glass tube or retort, ${ }^{18}$ to which, by means of a cork, a gas delivery tube is attached. This tube is bent downwards, as shown

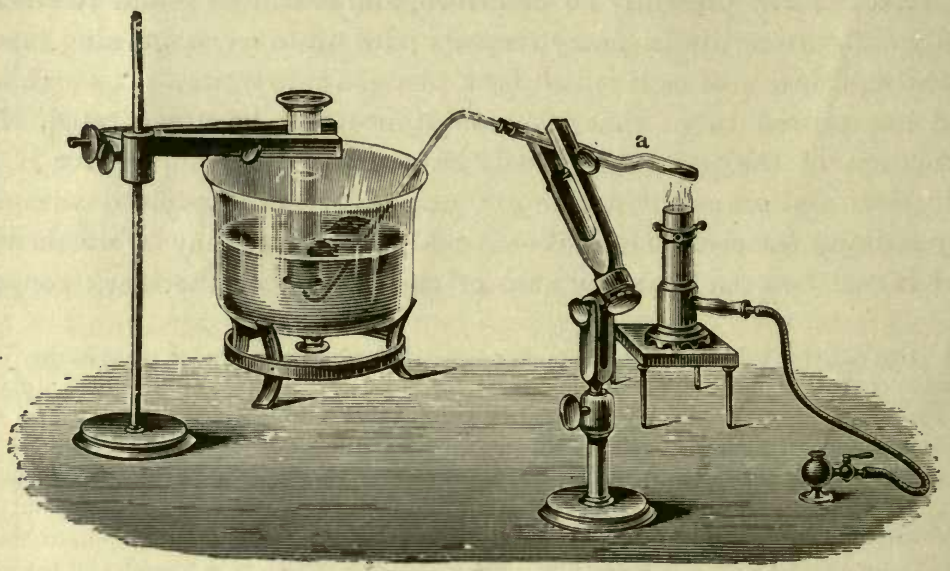

Fig. 1.-Apparatus for the decomposition of red mercury oxide.

in the drawing (Fig. 1). The open end of the gas delivery tube is immersed in a vessel filled with water, called a pneumatic trough. ${ }^{19}$ When

17 The copper carbonate should be dried before weighing, as otherwise-besides copper oxide, and carbonic anhydride-water will be obtained in the decomposition. Water forms a part of the composition of malachite, and has therefore to be taken into consideration. The water produced in the decomposition may be all collected by absorbing it in sulphuric acid or calcium chloride, as will be described further on. In order to dry a salt it must be heated at about $100^{\circ}$ until its weight remains constant, or be placed under an air pump over sulphuric acid, as will also be presently described. As water is met with almost everywhere, and as it is absorbed by many substances, the possibility of its presence should never be lost sight of.

18 As the decomposition of red oxide of mercury requires so high a temperature, near redness, as to soften ordinary glass, it is necessary for the experiment to take a retort (or test tube) made of infusible (German) glass, which is able to stand high temperatures without softening. For the same reason, the lamp used must give a strong heat and a large flame, capable of embracing the whole bottom of the retort, which should be as small as possible for the convenience of the experiment.

19 The pneumatic trough may naturally be made of any material (china, earthenware, or metal, \&c.), but usually a glass one, as shown in the drawing, is used, as it allows the progress of experiment being better observed. For this reason, as well as the ease with which they are kept clean, and from the fact also that glass is not acted on by many sub- 
the gas begins to be evolved in the retort it is obliged, having no other outlet, to escape through the gas delivery tube into the water in the pneumatic trough, and therefore its evolution will be rendered visible by the bubbles coming from this tube. In heating the retort containing the mercury oxide, the air contained in the apparatus is first partly expelled, owing to its expansion by heat, and then the peculiar gas called 'oxygen' is evolved, and may be easily collected as it comes off. For this purpose a vessel (an ordinary cylinder, as in the drawing) is filled quite full with water and its mouth closed; it is then inverted and placed in this position under the water in the trough; the mouth is then opened. The cylinder will remain full of waterthat is, the water will remain at a higher level in it than in the sur-

stances which affect other materials (for instance, metals), glass vessels of all kindssuch as retorts, test tubes, cylinders, beakers, Hlasks, globes, \&c.-are preferred to any other for chemical experiments. Glass vessels may be heated without any danger if the following precautions be observed: 1st, they should be made of thin glass, as otherwise they are liable to crack from the bad heat-conducting power of glass; 2nd, they should be surrounded by a liquid or with sand (Fig. 2), or sand bath as it is called; or else should

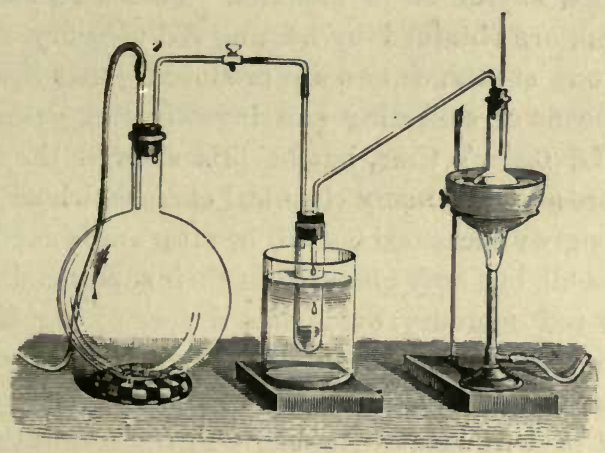

Fig. 2.-Apparatus for distilling under a diminished pressure l:quids which decompose at their boiling points under the ordinary pressure. The apparatus in which the liquili is distilled is connected with a large globe from which the air is pumped out; the liquid is lieated, and the receiver cooled.

stand in a current of hot gases without touching the fuel from which they proceed, or in the flame of a smokeless lamp. A common candle or lamp forms a deposit of soot on a cold object placed in their flames. The soot interferes with the transmission of heat, and so a glass vessel when covered with soot often cracks. And for this reason spirit lamps, which burn with a smokeless flame, or gas burners of a peculiar construction, are used. In the Bunsen burner the gas is mixed with air, and burns with a non-luminous and smokeless flame. On the other hand, if an ordinary lamp (petroleum or benzine) does not smoke it may be used for heating a glass vessel without danger, provided the glass is placed well above the flame in the current of hot gases. In all cases, the heating should be begun very carefully by raising the temperature by degrees, and not all at once, or the glass will break. 
rounding vessel, owing to the atmospheric pressure. The atmosphere presses on the surface of the water in the trough, and prevents the water from flowing out of the cylinder. The mouth of the cylinder is placed over the end of the gas delivery tube, ${ }^{20}$ and the bubbles issuing from it will rise into the cylinder and displace the water contained in it. Gases are generally collected in this manner. When a sufficient quantity of gas has accumulated in the cylinder it can be clearly shown that it is not air, but another gas which is distinguished by its capacity for vigorously supporting combustion. In order to show this, the cylinder is closed, under water, and removed from the bath; its mouth is then turned upwards, and a smouldering taper plunged into it. As is well known, a smouldering taper will be extinguished in air, but in the gas which is given off from red mercury oxide it burns clearly and vigorously, showing the capacity this gas has for vigorously supporting combustion, and thus enabling it to be distinguished from air. It may be observed in this experiment that, besides the formation of oxygen, metallic mercury is formed, and, being volatilised at the high temperature required for the reaction, condenses on the cooler parts of the retort as a mirror or in globules. Thus two substances, mercury and oxygen, are obtained by heating red mercury oxide. In this reaction, from one substance two are produced - that is, decomposition ensues. The means of collecting and investigating gases were already known before Lavoisier's time, but he first showed the real part they played in the processes of many chemical changes which before his era were either wrongly understood (as will be afterwards explained) or were not explained at all, but only observed in their superficial aspects. This experiment on red mercury oxide has a special significance in the history of chemistry contemporary with Lavoisier, because the oxygen gas which is here evolved is contained in the atmosphere, and plays a most important part in nature, especially in the respiration of animals, in combustion in air, and in the formation of rusts or scoria (earths, as they were then called) from metals - that is, of earthy substances, like the ores from which metals are extracted. The law of the indestructibility of matter could not be discovered or confirmed by the balance until the part played by the atmosphere as regards the participation of its oxygen in the numerous chemical phenomena, known either from the everyday experiences of life (combustion, respiration) or from the researches of

20 In order to avoid the necessity of holding the cylinder, its open end is widened (and also ground so that it may be closely covered with a ground-glass plate when needful), and placed on a stand below the level of the water in the bath. This stand is called ' the bridge.' It has several circular openings cut through it, and the gas delivery tube is placed under one of these, and the cylinder for collecting the gas over it. 
previous observers (the transformations of the metals into their earths or oxides), had been explained.

4. In order to illustrate by experiment one more example of chemical change and the application of the law of the indestructibility of matter, we will take some common table salt and lunar caustic, which is well known from its use in cauterising wounds. By taking a clear solution of each and mixing them together, it will at once be remarked that a solid white substance is formed, which settles to the bottom of the vessel, and is insoluble in water. This substance may be separated from the solution by filtering; it is then found to be an entirely different substance from either of those taken originally in the solutions. This is evident from the fact that it does not dissolve in water. On evaporating the liquid which passed through the filter, it will be found to contain a new substance unlike either table salt or lunar caustic, but, like them, soluble in water. Thus table salt and lunar caustic, two substances soluble in water, being taken, by their mutual chemical action produced two new substances, one insoluble in water, and the other remaining in solution. Here, from two substances two others are obtained, consequently there occurred a reaction of substitution. The water served only to convert the acting substances into a liquid and mobile state. If the lunar caustic and salt be dried ${ }^{21}$ and weighed, and if about $58 \frac{1}{2}$ parts by weight-for instance, grams ${ }^{22}$ - of salt and 170 grams of lunar caustic be taken, then $143 \frac{1}{2}$ grams of insoluble silver chloride and 85 grams of sodium nitrate will be obtained. The sum of the weights of the acting and resultant substances are seen to be similar and equal to $228 \frac{1}{2}$ grams, as necessarily follows from the law of the inclestructibility of matter.

21 Drying is necessary in order to remove any water which may be held in the salts (see Note 17). If the original and resultant substances be dried, then the water employed for solution, and which is removed in drying, may be taken in indefinite quantities.

22. The exact weights of the acting and resulting substances are determined with the greatest difficulty, not only from the possible inexactitude of the balance (every weighing is only correct within the limits of the sensitiveness of the balance) and weights used in weighing, not only from the difficulty in making corrections for the weight of air displaced by the vessels holding the substances weighed and by the weights themselves, but also from the hygroscopic nature of many substances (and vessels) causing absorption of moisture from the atmosphere, and from the difficulty in not losing any of the substance to be weighed in the many operations (filtering, evaporating, and drying, \&c.) which have to be gone through before arriving at a final result. All these circumstances have to be taken into consideration in exact researches, and their elimination requires very many special precautions which are impracticable in preliminary experiments; these arrive within only a certain comparatively rough proximity to those weights (expressed by chemical formulæ) which (all with a certain, definite, and inevitable error) correspond with reality. 
Having accepted the truth of the above law, the question involuntarily arises whether there is any limit to the various chemical transformations, or are they unrestricted in number - that is to say, is it possible from a given substance to obtain an equivalent quantity of all other substances? In other words, does there exist a perpetual and infinite change of one kind of material into all other kinds, or is the cycle of these transformations limited? This is the second essential problem of Chemistry, a question of quality of matter, and one, it is evident, which is more complicated than the question of quantity. It cannot be resolved by a mere superficial glance at the subject. Indeed, on seeing how all the varied forms and colours of plants are built up from air and the elements of the soil, and how metallic iron can be transformed into dyes, such as inks and Prussian blue, we might be led to think that there is no end to the qualitative changes to which matter is susceptible. But, on the other hand, the everyday experiences of life compel us to acknowledge that food cannot be made out of a stone, or gold out of copper. Thus a definite answer can only be looked for in a close and diligent study of the subject, and the problem has been resolved in different ways at different times. In ancient times the opinion most generally held was that everything visible was composed of four elements-Air, Water, Earth, and Fire. The origin of this doctrine can be traced far back into the confines of Asia, whence it was handed down to the Greeks, and most fully expounded by Empedocles, who lived before 460 B.c. By accepting so small a number of elements it was easy to arrive at the conclusion that the cycle of chemical changes was, if not infinite, at all events most extensive. This doctrine was not arrived at by the results of exact research, but was only founded on the speculations of philosophers. It apparently owes its origin to the clear division of bodies into gases (like air), liquids (like water), and solids (like the earth). It seems that the Arabs were the first who tried to solve the question by means of experiment, and they introduced, through Spain, the taste for the study of similar problems into Europe, where from that time there appear many adepts in chemistry, which was considered as an unholy art, and called 'alchemy.' As the alchemists were ignorant of any exact or strict law which could guide them in their researches, they resolved the question of the transformation of substances in a most varied manner. Their chief service to chemistry was that they made a number of experiments, and discovered many new chemical transformations; but it is well known how they solved the fundamental problem of chemistry. Their view may be taken as a positive acknowledgment of the infinite transmutability of matter, for they aimed at 
discovering the Philosopher's Stone, capable of converting everything into gold and diamonds, and of making the old young again. This solution of the question was afterwards most decidedly refuted, but it must not, for this reason, be thought that the hopes held by the alchemists were only the fruit of their imaginations. On the contrary, the first chemical experiments might well lead them to their conclusion. They took, for instance, the bright metallic mineral galena, and they extracted metallic lead from it. Thus they saw that from a metallic substance which is unfitted for use they could obtain another metallic substance which is ductile and valuable for many uses in the arts. Furthermore, they took this lead and obtained silver, a still more valuable metal, from it. Thus they might easily conclude that it was possible to ennoble metals by means of a whole series of transmutations - that is to say, to obtain from them those which are more and more precious. Having got silver from lead, they only aimed at getting gold from silver. The mistake they made was that they never weighed or measured the substances used or produced in their experiments. Had they done so, they would have learnt that the weight of the lead was much less than that of the galena from which it was obtained, and the weight of the silver infinitesimal compared with that of the lead. Had they looked more closely into the process of the extraction of the silver from lead (and now silver is chiefly obtained from the lead ores) they would have seen that the lead does not change into silver, but that it only contains a certain small quantity of it, and this amount having once been separated from the lead it cannot by any further operation give more. The silver which the alchemists extracted from the lead was in the lead, and was not obtained by a chemical change of the lead itself. This is now well known from experiment, but the first view of the nature of the process was very likely to be erroneous. ${ }^{23}$ The methods of research adopted by the alchemists could not but give little

25 Besides which, in the majority of cases, the first judgment on most subjects which do not repeat themselves in everyday experience under various aspects, but always in one form, or only at intervals and infrequently, is usually untrue. Thus the daily evidence of the rising of the sun and stars evokes the erroneous idea that the heavens move and the earth stands still. This apparent truth is far from being the real truth, and is even contradictory to it. Similarly, an ordinary mind and everyday experience concludes that iron is incombustible, whereas it burns not only as filings, but even as wire, as we shall afterwards see. With the progress of knowledge very many primitive prejudices have been obliged to give way to true ideas which have been verified by experiment. In ordinary life we often reason at first sight with perfect truth, only because we are taught a right judgment by our daily experience. It is a necessary consequence of the nature of our minds to reach the attainment of truth through elementary and often erroneous reasoning and through experiment, and it would be very wrong to expect a knowledge of truth from a simple mental effort. Naturally, experiment itself cannot give truth, but it gives the means of destroying erroneous representations whilst confirming those which are true in all their consequences. 
success, for they groped in the dark, making all kinds of mixtures and experiments, without setting themselves clear and simple questions whose answers would aid them to make further progress. Thus they did not form one exact law, but, nevertheless, they left numerous and useful experimental data as an inheritance to chemistry; they studied, in particular, the transformations proper to metals, and for this reason chemistry was for long afterwards entirely confined to the study of metallic substances.

In their researches, the alchemists frequently made use of two chemical processes which are now termed 'reduction ' and 'oxidation.' The rusting of metals, and in general their conversion from a metallic into an earthy form, is called 'oxidation,' whilst the extraction of a metal from an earthy substance is called ' reduction.' A large number of metals - for instance, iron, lead, and tin-are oxidised by heating in air alone, and may be again reduced by heatirng with carbon. Such oxi. dised metals are found in the earth, and form the majority of metallic ores. The metals, such as tin, iron, and copper, may be extracted from these ores by heating them together with carbon. All these processes were well studied by the alchemists. It was afterwards shown that all earths and minerals are formed of similar metallic rusts or oxides, or of their combinations. Thus the alchemists knew of two forms of chemical changes: the oxidation of metals and the reduction of the oxides so formed into metals. The explanation of the nature of these two classes of chemical phenomena was the means for the discovery of the most important chemical laws. The first hypothesis on their nature is due to Becker, and more particularly to Stahl, a surgeon to the King of Prussia. Stahl writes in his 'Fundamenta Chymix,' 1723 , that all substances consist of an imponderable fiery substance called 'phlogiston' (materia aut principium ignis non ipse ignis) and of another element having particular properties for each substance. The greater the capacity of a body for oxidation, or the more combustible it is, the richer it is in phlogiston. Carbon contains it in great abundance. In oxidation or combustion phlogiston is emitted, and in reduction it is consumed or enters into combination. Carbon reduces earthy substances because it is rich in phlogiston, and gives up a portion of its phlogiston to the substance reduced. Thus Stahl supposed metals to be compound substances consisting of phlogiston and an earthy substance or oxide. This hypothesis is distinguished for its very great simplicity, and for this and other reasons it acquired many supporters. ${ }^{24}$

24 It is true that Stall was acquainted with a fact which directly disproved his hypothesis. It was already known (from the experiments of Geber, and more especially of Ray, in 1630) that metals increase in weight by oxidation, whilst, according to Stahl's 
Lavoisier proved by means of the balance that every case of rusting of metals or oxidation, or of combustion, is accompanied by an increase in weight at the expense of the atmosphere. He formed, therefore, the natural opinion that the heavier substance is more complex than the lighter one. ${ }^{25}$. The following remarkable experiment was made by Lavoisier in 1774, and gave indubitable support to his opinion, which was in many respects contradictory to Stahl's doctrine. Lavoisier

hypothesis, they should decrease in weight, because phlogiston is separated by oxidation. Strhl speaks on this point as follows:- 'I know well that metals, in their transformation into earths, increase in weight. But not only does this fact not disprove my theory, but, on the contrary, confirms it, for phlogiston is lighter than air, and, in combining with substances, strives to lift them, and so decreases their weight; consequently, a substance which has lost phlogiston must be heavier.' This argument, it will be seen, is founded on an improper understanding of the properties of gases, regarding them as laving no weight and as not being attracted by the earth, or else on a confused idea of phlogiston itself, as it was first defined as imponderable. The conception of imponderable phlogiston tallies well with the habit and methods of the last century, when recourse was often had to imponderable fluids for explaining a large number of phenomena. Heat, light, magnetism, and electricity were explained as being peculiar imponderable fluids. In this sense the doctrine of Stahl corresponds entirely with the spirit of his age. If heat be now regarded as movement or energy, then phlogiston also should be considered in this light. In fact, in combustion, of coals, for instance, heat and energy are evolved, and not combined in the coal, although the oxygen and coal do combine. Consequently, the doctrine of Stahl contains the essence of a true representation of the evolution of energy, but naturally this evolution is only a consequence of the combination going on between the coal and oxygen. As regards the history of chemistry prior to Lavoisier, besides Stahl's work (to which reference has been made above), Priestley's Experiments and Observations on Different Kinds of Air, London, 1790, and also Scheele's Opuscula Chimica et Physica, Lips., 1788-89, 2 vols., must be recommended as the two leading works of the English and Scandinavian chemists showing the condition of chemical learning before the propagation of Lavoisier's views. A most interesting memoir on the history of phlogiston is that of Rodwell, in the Philosophical Magazine, 1868, in which it is shown that the idea of phlogiston dates very far back, that Basil Valentine (13941415), in the Cursus Triumphalis Antimonii Paracelsus (1493-1541), in his work, De Rerum Natura, Glauber(1604-1668), and especially John Joachim Becher (1625-1682), in his Physica Subterranea, all referred to phlogiston, but under different names.

${ }^{25}$ An Englishman, named Mayow, who lived a whole century before Lavoisier (in 1666), nnclerstood certain phenomena of oxidation in their true aspect, but was not able to develop his views with cleamess, or make his doctrine a universal inheritance, or express it by instructive experiments; he, therefore, cannot be considered, like Lavoisier, as the founder of contemporary chemical learning. Science is a universal heritage, and therefore it is only just to give the highest honour in science, not to those who first enunciate a certain truth, but to those who are first able to convince others of its authenticity and establish it for the general welfare. It should be observed, with reference to scientific discoveries, that they are rarely made all at once, but, as a rule, the first teachers do not succeed in convincing others of the truth they have discovered; with time, however, the store of materials for its demonstration increases, and other teachers come forward, possessing every means for making the truth apparent to all. They are rightly considered as the founders; but it must not be forgotten they are entirely indebted to the labours and mass of data accumulated by many others. Such was Lavoisier, and such are all the great founders of science. They are the enunciators of all past and present learning, and their names will always be revered by posterity.

VOL. I. 
poured four ounces of pure mercury into a glass retort (fig. 3), whose neck was bent as shown in the drawing and dipped into the ressel $\mathrm{R} s$, also full of mercury. The projecting end of the neck was covered with a glass bell jar P. The weight of all the mercury taken, and the volume of air remaining in the apparatus, namely, that in the upper portion of the retort, and under the bell-jar, were determined before beginning the experiment. In this experiment it was most important to know the volume of air in order to learn what part it played in the oxidation of the mercury, because, according to Stahl, phlogiston is emitted into the air, whilst, according to Lavoisier, the mercury in

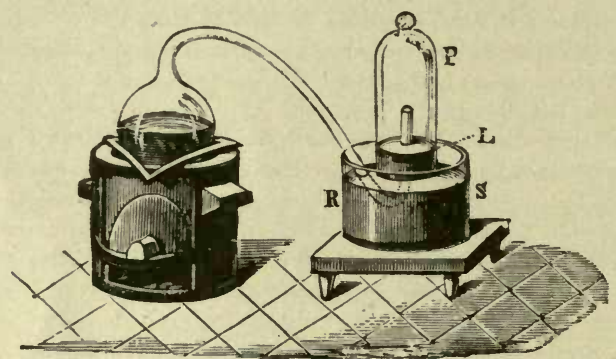

FIG. 3.-Lavoisier's apparatus for determining the composition of air and the reason of metals increasing in weight when they are calcined in air.

oxidising absorbs a portion of the air ; and consequently it was absolutely necessary to determine whether the amount of air increased or decreased in the oxidation of the metal. It was, therefore, most important to measure the volume of the air in the apparatus both before and after the experiment. For this purpose it was necessary to know the total capacity of the retort, the volume of the mercury poured into it, the volume of the bell-jar above the level of the mercury, and also the temperature and pressure of the air at the time of its measurement. The volume of air held in the apparatus and isolated from the surrounding atmosphere could be determined from these data. Having arranged his apparatus in this manner, Lavoisier heated the retort holding the mercury for a period of twelve days at a temperature near the boiling point of mercury. The mercury became covered with a quantity of small red scales; that is, it was oxidised or converted into an earth. This substance is the same mercury oxide which has already been mentioned (example 3). After the lapse of twelve days the apparatus was cooled, and it was then seen that the volume of the air in the apparatus had diminished during the time of the experiment. This result was in exact contradiction to Stahl's hypothesis. Out of 50 cubic inches of air originally taken, there only remained 42 . 
Lavoisier's experiment led to other no less important results. The weight of the air taken decreased by as much as the weight of the mercury increased in oxidising ; that is, the portion of the air was not destroyed, but only combined with mercury. This portion of the air may be again separated from the mercury oxide, and has, as we saw (example 3), properties different from those of air. That portion of the air which remained in the apparatus and did not combine with the mercury does not oxidise metals, and cannot support either combustion or respiration, so that a lighted taper is immediately extinguished if it be dipped into the gas which remains in the bell-jar. 'It is extinguished in the remaining gas as if it had been plunged into water,' writes Lavoisier in his memoirs. This gas is called 'nitrogen.' Thus air is not a simple substance, but consists of two gases, oxygen and nitrogen, and therefore the opinion that air is an elementary substance is erroneous. The oxygen of the air is absorbed in combustion and the oxidation of metals, and the earths produced by the oxidation of metals are substances composed of oxygen and a metal. By mixing the oxygen with the nitrogen the same air as was originally taken is re-formed. The existence of compound substances was incontestably proved by these experiments. It has also been shown by direct experiment that on reducing an oxide with carbon, the oxygen contained in the oxide is transferred to the carbon, and gives the same gas as is obtained by the combustion of carbon in air. Therefore this gas is a compound of carbon and oxygen, just as the earthy oxides are composed of metals and oxygen.

The many examples of the formation and decomposition of substances which are met with convince us that the majority of substances with which we have to deal are compounds made up of several other substances. By heating chalk (or else copper carbonate, as in the second example) we obtain lime and the same carbonic acid gas which is produced by the combustion of carbon. On bringing lime into contact with this gas and water, at the ordinary temperature, we again obtain the compound carbonate of lime, or chalk. Therefore chalk is a compound. So also are those substances from which it may be built up. Carbonic anhydride is formed by the combination of carbon and oxygen ; and lime is produced by the oxidation of a certain metal called 'calcium.' By breaking up substances in this manner into their component parts, we arrive at last at such as are indivisible into two or more substances by any means whatever, and which cannot be formed from other substances. All we can do is to make such substances combine together or act on other substances. Substances which cannot be formed from or decomposed into others are termed simple substances (elements). Thus 
all homogeneous substances may be classified into simple and compound substances. This view was introduced and established as a scientific fact during the lifetime of Lavoisier. The number of these elements is very small in comparison with the number of compound substances which are formed by them. At the present time, only seventy elements are known with certainty to exist. Some of them are very rarely met with in nature, or are found in very small quantities, whilst others are yet doubtful. The number of elements with whose compounds we commonly deal in everyday life is very small. Elements cannot be transmuted into one another - at least up to now not a single case of such a transformation has been met with; it may therefore be said that, as yet, it is impossible to transmute one metal into another. And as yet, notwithstanding the number of assays which have been made in this direction, no fact has been discovered which could in any way support the idea of the complexity of those indubitably-known elements ${ }^{26}$ - such as oxygen, iron, sulphur, dc. Therefore, from its conception, an element is not susceptible to reactions of decomposition. ${ }^{27}$

26 Many ancient philosophers admitted the existence of one elementary form of matter. This idea still appears in our times, in the constant efforts which are made to reduce the number of the elements; to prove, for instance, that bromine contains chlorine or that chlorine contains oxygen. Many methods, founded both on experiment and theory, have been tried to prove the compound nature of the elements. All labour in this direction has as yet been in vain, and the assurance that elementary matter is not so homogeneous (single) as the mind would desire in its first transport of rapid generalisation is strengthened from year to year. At all events, there are as yet no experimental or theoretical evidences of the compound nature of our elements. With the methods and evidence now at our disposal it is impossible to. even imagine the possibility of a method by which the different elements conld be formed from one elementary material. Cases of isomerism and of polymerism of compound substances certainly show the possibility of the formation, from one and the same elements, of substances with different properties, but every change of this kind is completely levelled and nullified by a certain rise in temperature by which every isomeride and polymeride is converted into one variety and changes its original properties. All our knowledge shows that iron and other elements remain, even at such a high temperature as there exists in the sun, as different substances, and are not converted into one common material. Admitting, even mentally, the possibility of one elementary form of matter, a method must be imagined by which it could give rise to the various elements, as also the modus operandi of their formation from one material. If it be said that this diversitude only takes place at low temperatures, as is observed with isomerides, then there would be reason to expect, if not the transition of the various elements into one particular and more stable form, at least the mutual transformation of some into others. But nothing of the kind has yet been observed, and the alchemist's hope to manufacture (as Berthollet puts it) elements has no foundation of fact or theory.

27 The weakest point in the idea of elements is the negative character of the determinative signs given them by Lavoisier, and from that time ruling in chemistry. They do not:decompose, they do not change into one another. But it must be remarked that elements form the limiting horizon of our knowledge of matter, and it is always difficult to determine a positive side on the borderland of what is known. But all the same, if not for all, at all events for the majority, of those having the properties of metals, there is a series of positive common signs (they possess a particular appearance and lustre, 
The quantity, therefore, of each element remains constant in all chemical changes; which fact may be deduced as a consequence of the law of the indestructibility of matter, and of the conception of elements themselves. Thus the equation expressing the law of the indestructibility of matter acquires a new and still more important signification. If we know the quantities of the elements which occur in the acting, it may be compound, substances, and if from these substances there proceed, by means of chemical changes, a series of new compound substances, then the latter will together contain the same quantity of each of the elements as there originally existed in the reacting substances. The essence of chemical change is embraced in the study of how, and with what substances, each element is combined before and after change.

In order to be able to express various chemical changes by equations, it has been agreed to represent each element by the first or some two letters of its (Latin) name. Thus, for example, oxygen is represented by the letter $\mathrm{O}$; nitrogen by $\mathrm{N}$; mercury (hydrargyrum) by $\mathrm{Hg}$; iron (ferrum) by $\mathrm{Fe}$; and so on for all the elements, as is seen in the tables on page 24. A compound substance is represented by placing the symbols representing the elements of which it is made up side by side. For example, red mercury oxide is represented by $\mathrm{HgO}$, which shows that it is composed of oxygen and mercury. Besides this, the symbol of every element corresponds with a certain relative quantity of it by weight, called its 'combining' weight, or the weight of an atom; so that the chemical formula of a compound substance not only designates the nature of the elements of which it is composed, but also their quantitative proportion. Every chemical process may be expressed by an equation composed of the formulæ corresponding with those substances which take part in it and are produced by it. The amount by weight of the elements in every chemical equation must be equal on both sides of the equation, because no element is either formed or destroyed in a chemical change.

On pages 24,25 , and 26 a list of the elements, with their symbols and combining or atomic weights, is given, and we shall see afterwards on what basis the atomic weights of elements are determined. At present we will only point out that a compound containing the elements $\mathrm{A}$ and $\mathrm{B}$ is designated by the formula $\mathbf{A}^{n} \mathbf{B}^{m}$, where $m$ and $n$ are the coefficients or multiples in which the combining weights of the

they conduct an electric current without decomposing) which allow them to be distinguished at a glance from other kinds of matter. Besides, there is no doubt (from the results of spectrum analysis) that the elements are distributed as far as the most distant stars, and that they support the highest attainable temperatures without decomposing. 
elements enter into the composition of the substance. If we represent the combining weight of the substance $A$ by $a$ and that of the substance $\mathbf{B}$ by $b$, then the composition of the substance $\mathbf{A}^{n} \mathbf{B}^{m}$ will be expressed thus : it contains na parts by weight of the substance $A$ and $m b$ parts by weight of the substance $\mathrm{B}$, and corsequently in 100 parts of our compound there is contained $\frac{n a 100}{n a+m b}$ percentage parts by weight of the substance $\mathrm{A}$ and $\frac{m b 100}{n a+m b}$ of the substance $\mathrm{B}$. It is evident that as a formula shows the relative amounts of all the elements contained in a compound, the actual weights of the elements contained in a given weight of a compound may be calculated from its formula. For example, the formula $\mathrm{NaCl}$ of table salt shows (as $\mathrm{Na}=23$ and $\mathrm{Cl}=35.5$ ), that 58.5 lbs. of salt contain $23 \mathrm{lbs}$. of sodium and $35.5 \mathrm{lbs}$. of chlorine, and that 100 parts of it contain $39 \cdot 3$ per cent. of sodium and $60 \cdot 7$ per cent. of chlorine.

What has been said above clearly limits the province of chemical changes, because from substances of a given kind there can be obtained only such as contain the same elements. But, notwithstanding this primary limitation, the number of possible combinations is infinitely great. Only a comparatively small number of compounds have yet been described or subjected to research, and any one working in this direction may easily discover new compounds which had not before been obtained. It often happens, however, that such newly-discovered compounds were foreseen by chemistry, whose object is the apprehension of that uniformity which rules over the multitude of compound substances, and whose aim is the comprehension of those laws which govern their formation and properties. When once the conception of elements had been established, the most intinate object of chemistry was the determination of the properties of compound substances on the basis of the determination of the quantity and kind of elements of which they are composed; the investigation of the elements themselves; the determination of what compound substances can be formed from each element and the properties which these compounds show ; and the apprehension of the nature of the connection between the elements in different compounds. An element thus serves as the starting point, and is taken as the primary conception under which all other bodies are embraced.

When we state that a certain element enters into the composition of a given compound (when we say, for instance, that mercury oxide contains oxygen) we do not mean that it contains oxygen as a gaseous substance, but only desire to express those transformations which mercury oxide is capable of making ; that is, we wish to say that it is 
possible to obtain oxygen from mercury oxide, and that it can give up oxygen to various other substances; in a word, we desire only to express those transformations of which mercury oxide is capable. Or, more concisely, it may be said that the composition of a compound is the expression of those transformations of which it is capable. It is useful in this sense to make a clear distinction between the conception of an element as a separate homogeneous substance, and as a material but invisible part of a compound. Mercury oxide does not contain two simple bodies, a gas and a metal, but two elements, mercury and oxygen, which, when free, are a gas and a metal. Neither mercury as a metal nor oxygen as a gas is contained in mercury oxide ; it only contains the substance of these elements, just as steam only contains the substance of ice, but not ice itself, or as corn contains the substance of the seed but not the seed itself. The existence of an element may be recogr nised without knowing it in the uncombined state, but only from an investigation of its combinations, and from the knowledge that it gives, under all possible conditions, substances which are unlike other known combinations of substances. Fluorine is an example of this kind. It was for a long time unknown in a free state, and was, nevertheless, recognised as an element because its combinations with other elements were known, and their difference from all other similar compound substances was determined. In order to grasp the difference between the conception of the visible form of an element as we know it in the free state, and of the intrinsic element (or 'radicle,' as Lavoisier called it) contained in the visible form, it should be remarked that compound substances also combine together forming yet more complex compounds, and that they evolve heat in the process of combination. The original compound may often be extracted from these new compounds by exactly the same methods as elements are extracted from their corresponding combinations. Besides, many elements exist under various visible forms whilst the intrinsic element contained in these various forms is something which is not subject to change. Thus carbon appears as charcoal, graphite, and diamond, but yet the element carbon alone contained in each is one and the same. Carbonic anhydride contains carbon, and not charcoal, or graphite, or the diamond.

Elements alone, although not all of them, have the peculiar lustre, opacity, malleability, and the great heat and electrical conductivity which are proper to metals and their mutual combinations. But elements are far from all being metals. Those which do not possess the physical properties of metals are called non-metals (or metalloids). It is, however, impossible to draw a strict line of demarcation between metals and non-metals, there being many intermediary substances. 
Thus graphite, from which pencils are manufactured, is an element with the lustre and other properties of a metal ; but charcoal and the diamond, which are composed of the same substance as graphite, do not show any metallic properties. Both classes of elements are clearly distinguished in definite examples, but in particular cases the distinction is not clear and cannot serve as a basis for the exact division of the elements into two groups.

At all events, the conception of elements forms the basis of chemical knowledge, and if we give a list of them at the very beginning of our work, it is that we wish to symbolise the condition of the contemporary information on the subject. Altogether about seventy elements are now authentically known, but many of them are so rarely met with in nature, and have been obtained in such small quantities, that we possess but a very insufficient knowledge of them. The substances most widely distributed in nature contain a very small number of elements. These elements have been more completely studied than the others because a greater number of investigators have been able to carry on experiments and observations on them. The elements most widely distributed in nature are :-

Hydrogen, $\mathbf{H}=1$.

Carbon, $\quad \mathrm{C}=12$. In organisms, coal, limestones.

Nitrogen, $\quad \mathrm{N}=14$. In air and in organisms.

Oxygen, $\mathrm{O}=16$. In air, water, earth. It forms the greater part of the mass of the earth.

Sodium, $\quad \mathrm{Na}=23$. In common salt and in many minerals.

Magnesium, $\mathrm{Mg}=24$. In sea-water and in many minerals.

Aluminium, $\mathrm{Al}=27$. In minerals and clay.

Silicon, $\quad \mathrm{Si}=28$. In sand, minerals, and clay.

Phosphorus, $\mathrm{P}=31$. In bones, ashes of plants, and soil.

Sulphur, $\mathrm{S}=32$. In pyrites, gypsum, and in sea-water.

Chlorine, $\quad \mathrm{Cl}=35.5$. In common salt, and in the salts of seawater.

Potassium, $\quad K=39$. In minerals, ashes of plants, and in nitre.

Calcium, $\quad \mathrm{Ca}_{\mathbf{a}}=40$. In limestones, gypsum, and in organisms.

Iron, $\quad \mathrm{Fe}=56$. In the earth, iron ores, and in organisms.

Beside these, the following elements, although not very largely distributed in nature, are all more or less well known from their applications to the requirements of everyday life or the arts, either in a free state or in their compounds :-

Lithium, $\mathrm{Li}=7$. In medicine $\left(\mathrm{Li}_{2} \mathrm{CO}_{3}\right)$, and in photography $(\mathrm{LiBr})$.

Boron, $\mathrm{B}=11$. As Borax, $\mathrm{B}_{4} \mathrm{Na}_{2} \mathrm{O}_{7}$, and as boric anhydride, $\mathrm{B}_{2} \mathrm{O}_{3}$. 
Fluorine, $\quad \mathbf{F}=19$. As fluor spar, $\mathrm{CaF}_{2}$, and as hydrofluoric acid, $\mathrm{HF}$.

Chromium, $\mathrm{Cr}=52$. As chromic anhydride, $\mathrm{CrO}_{3}$, and potassium dichromate, $\mathrm{K}_{2} \mathrm{Cr}_{2} \mathrm{O}_{7}$.

Manganese, $\mathrm{Mn}=55$. As manganese peroxide, $\mathrm{MnO}_{2}$, and potassium permanganate, $\mathrm{MnKO}_{4}$.

Cobalt, $\quad$ Co $=59$. In smalt and blue glass.

Nickel, $\quad \mathrm{Ni}=59$. For electro-plating other metals.

Copper, $\quad \mathrm{Cu}=63$. The well-known red metal.

Zinc, $\quad Z n=65$. Used for the plates of batteries, roofing, \&c.

Arsenic, $\quad \mathrm{As}=75$. White arsenic, $\mathrm{As}_{2} \mathrm{O}_{3}$.

Bromine, $\mathrm{Br}=80$. A brown volatile liquid; sodium bromide, $\mathrm{NaBr}$.

Strontium, $\mathrm{Sr}=87$. In coloured fires $\left(\mathrm{SrN}_{2} \mathrm{O}_{6}\right)$.

Silver,

Cadmium, $\quad \mathbf{C d}=112$. In alloys. Yellow paint (CdS).

Tin, $\quad S_{n}=118$. The well-known metal.

Antimony, $\mathrm{Sb}=122$. In alloys such as type metal.

Iodine, $\quad I=12 \pi$. In medicine and photography; free, and as KI.

Barium, $\quad \mathrm{Ba}=137$. "Permanent white," and as an adulterant in white lead, and in heary spar, $\mathrm{BaSO}_{4}$.

Platinum, $\quad \mathrm{Pt}=196$.

Gold, $\quad \mathrm{Au}=197$.

Mercury, $\quad \mathrm{Hg}_{\mathrm{g}}=200$. Well-known metals.

Lead, $\quad \mathrm{Pb}=207$.)

Bismuth, $\quad \mathrm{Bi}=208$. In medicine and fusible alloys.

Uranium, $\quad \mathrm{U}=240$. In green fluorescent glass.

The compounds of the following metals and semi-metals have fewer applications, but are well known, and are somewhat frequently met with in nature, although in small quantities:-

$\begin{array}{lll}\text { Beryllium, } & \mathrm{Be}=9 . & \text { Palladium, } \mathrm{Pd}=106 . \\ \text { Titanium, } & \mathrm{Ti}=48 . & \text { Cerium, } \mathrm{Ce}=140 . \\ \text { Vanadium, } \mathrm{V}=51 . & \text { Tungsten, } \mathrm{W}=184 . \\ \text { Selenium, } \mathrm{Se}=78 . & \text { Osmium, } \mathrm{Os}=193 . \\ \text { Zirconium, } \mathrm{Zr}=90 . & \text { Iridium, } \mathrm{Ir}=195 . \\ \text { Molybdenum, } \mathrm{M}=96 . & \text { Thallium, } \mathrm{Tl}=204 .\end{array}$

The following rare metals are still more seldom met with in nature and are not yet applied to the arts, but have been studied somewhat fully :- 


$\begin{array}{lll}\text { Scandium, } \mathrm{Sc}=44 . & \text { Indium, } & \mathrm{In}=113 . \\ \text { Gallium, } \mathrm{Ga}=68 . & \text { Tellurium, } \mathrm{Te}=125 . \\ \text { Germanium, } \mathrm{Ge}=72 . & \text { Cresium, } \mathrm{Cs}=132 . \\ \text { Rubidium, } \mathrm{Rb}=85 . & \text { Lanthanum, } \mathrm{La}=138 . \\ \text { Yttrium, } \mathrm{Y}=89 . & \text { Didymium, } \mathrm{Di}=143 . \\ \text { Niobium, } \mathrm{Nb}=94 . & \text { Ytterbium, } \mathrm{Yb}=173 . \\ \text { Ruthenium, } \mathrm{Ru}=104 . & \text { Tantalum, } \mathrm{Ta}=182 . \\ \text { Rhodium, } \mathrm{Rh}=104 . & \text { Thorium, } \mathrm{Th}=234 .\end{array}$

Besides these 66 elements there have been discovered :-Erbium, Terbium, Samarium, Thullium, Holmium, Mosandrium, Phillipium, Vesbium, Actinium, and several others. But their properties and combinations, owing to their extreme rarity, are very little known, and even their existence as independent substances ${ }^{28}$ is doubtful.

It has been incontestably proved from observations on the spectra of the heavenly bodies that many of the most common elements (such as $\mathrm{H}, \mathrm{Na}, \mathrm{Mg}, \mathrm{Fe}$ ) occur on the far distant stars. This fact confirms the belief that those forms of matter which appear on the earth as elements are widely distributed over the entire universe. But why, in nature, the mass of some elements should be greater than that of others we do not yet know.

The capacity of each element to combine with one or another element, and to form compounds with them which are in a greater or less degree prone to give new and yet more complex substances, forms the fundamental character of each element. Thus sulphur easily combines with the metals, oxygen, chlorine, or carbon, forming stable substances, whilst gold and silver enter into combinations with difficulty, and form unstable compounds, which are easily decomposed by heat. Compounds, and also elements, may be divided into two classes-those which easily enter into many different chemical changes, and those which enter into but few combinations, which are characterised by their small capacity for the direct formation of new, more complex substances. The cause or force which induces substances to enter into chemical change must be considered, as also the cause which holds different substances in combination-that is, which endues the substances formed with their particular degree of stability. This cause or force is called affinity (affinitus, affinité, verwandtschaft), or chemical affinity. ${ }^{29}$

28 It may be that some of them are compounds of other already-known elements. Pure and incontestably independent compounds of these substances are unknown, and some of them have not even been separated but are only supposed to exist from the results of spectroscopic researches. There can be no mention of such contestable and doubtful elements in a short general handbook of chemistry.

${ }_{29}$ This word, first introduced, if I mistake not, into chemistry by Glauber, is based on the idea of the ancient philosophers that combination can only take place when the sub- 
As this force must be regarded as exclusively an attractive force, like gravity, many writers (for instance, Bergmann at the end of the last, and Berthollet at the beginning of this, century) supposed affinity to be essentially similar to the universal force of gravity, from which it only differs in that the latter acts at observable distances whilst aftinity only evinces itself at the smallest possible distances. But chemical affinity cannot be entirely identified with the universal attraction of gravity, which acts at observable distances and which is dependent only on mass and distance, and not on the quality of the material on which it acts, whilst it is by the quality of matter that affinity is most forcibly influenced. Neither can it be entirely identified with cohesion, which gives to homogeneous solid substances their crystalline form, elasticity, hardness, ductility, and other properties, and to liquids their surface, drop formation, capillarity, and other properties, because affinity acts between the component parts of a substance and cohesion on a substance in its homogeneity, although both act at imperceptible distances (by contact) and have much in common. Chemical force, which makes one substance penetrate into another, cannot be entirely identified with even those attracting forces which make different substances adhere to each other, or hold together (as when two plane-polished surfaces of solid substances are brought into close contact), or which cause liquids to soak into solids, or adhere to their surfaces, or gases and vapours to condense on the surfaces of solids. These forces must not be confounded with chemical forces, which cause one substance to penetrate into the substance of another and to form a new substance, which is not the case with cohesion. But it is evident that the forces which determine cohesion form a connecting-link between mechanical and chemical forces, because they only act by intimate contact and between different kinds of matter. For a long time, and especially during the first half of this century, chemical attraction and chemical forces were identified with electrical forces. There is certainly an intimate relation between them, for electricity is evolved in chemical reactions, and it, in its turn, has a powerful influence on chemical processes-for instance, compounds are decomposed by the action of an electrical current. But the exactly similar relation which exists between chemical phenomena and the phenomena of heat (heat being developed by chemical phenomena, and heat being able to decompose compounds) only proves the unity of the forces of nature, the capability of one force to produce and to be trans-

stances combining have something in common-a medium. As is generally the case, another idea evolved itself in antiquity, and has lived until now, side by side with the first, to which it is exactly contradictory; this considers union as dependent on contrast, on polar difference, on an effort to fill up a want. 
formed into others. Therefore the identification of chcmical force with electricity will not bear experimental proof. ${ }^{30}$ As of all the (molecular) phenomena of nature which act on substances at immeasurably small distances, the phenomena of heat are at present the best (comparatively) known, having been reduced to the simplest fundamental principles of mechanies (of energy, equilibrium, and movement), which, since Newton, have been subjected to strict mathematical analysis, it is quite natural that an effort, which has been particularly pronounced during recent years, should have been made to bring chemical phenomena into strict correlation with, and under the theory founded on, the already investigated phenomena of heat, without, however, aiming at any identification of chemical with heat phenomena. The true nature of chemical force is still a secret to us, just as is the nature of the universal force of gravity, and yet without knowing what gravity realiy is, by applying mechanical conceptions, astronomical phenomena have been subjected not only to exact generalisation but to the detailed prediction of a number of particular facts ; and so, also, although the true nature of chemical affinity may be unknown, there is reason to hope for considerable progress in chemical science by applying the laws of mechanics to chemical phenomena by means of the mechanical theory of heat. But as yet this portion of chemistry has been but little worked at, and therefore, while forming a current problem of the science, it is treated more fully in that particular

30 Especially conclusive are those cases of so-called metalepsis (Dumas, Laurent). Chlorine, in combining with hydrogen, forms a very stable substance, called ' hydrochloric acid,' which is split up by the action of an electrical current into chlorine and hydrogen, the chlorine appearing at the positive and the hydrogen at the negative pole. From this electro-chemists considered hydrogen to be an electro-positive and chlorine an electronegative element, and that they are held together in virtue of their opposite electric charges. It appears, however, from metalepsis, that chlorine can replace hydrogen (and reversely hydrogen replaces chlorine) in its compounds without in any way changing the grouping of the other elements, or altering their chief chemical properties. Thus the capacity of acetic acid to form salts is not altered by replacing its hydrogen by chlorine. Here an electro-positive element is replaced by an electro-negative element, which is quite contrary to the electrical theory of the origin of chemical attraction, which has thus been entirely overthrown by the facts of metalepsis. We must remark, whilst considering this subject, that the explanation suggesting electricity as the origin of chemical phenomena is unsound in that it strives to explain one class of plenomena whose nature is almost unknown by another class which is no better known. It is most instructive to remark that together with the electrical theory of chemical attraction there arose and survives a view which explains the galvanic current as being a transference of chemical action through the circuit-i.e., regards the origin of electricity as being a chemical one. It is evident that the connection is very intimate, but both kinds of phenomena are independent and represent different forms of molecular (atomic) movement, whose real nature is not yet understood. Nevertheless, the connection between the phenomena of both categories is not only in itself very instructive, but it extends the applicability of the general idea of the unity of the forces of nature, conviction of the truth of which has held so important a place in the science of the last ten years. 
province which is termed either 'theoretical' or 'physical' chemistry, or, better still, chemical mechanics. As this province of chemistry requires a knowlerlge not only of the various homogeneous substances which have yet been obtained and of the chemical transformations which they undergo, but also of the phenomena (of heat and other kinds) by which these transformations are accompanied, it is only possible to enter on the study of chemical mechanics after an acquaintance with the fundamental chemical conceptions and substances which form the subject of this book. ${ }^{31}$

31 I consider that in an elementary textbook of chemistry, like the present, it is only possible and advisable to mention, in reference to chemical mechanics, a few general ideas and some particular examples referring more especially to gases, whose mechanical theory must be regarded as the most complete. The molecular mechanics of liquids and solids is as yet in embryo, and contains much that is disputable; for this reason, chemical mechanics has made less progress in relation to these substances. It may not be superfluous to here remark, with respect to the conception of chemical affinity, that up to the present time gravity, electricity, and heat have been respectively applied to its elucidation. Efforts have also been made to introduce the luminiferous ether into theoretical chemistry, and should that connection between the phenomena of light and electricity which was established by Maxwell be worked out more in detail, doubtless these efforts to elucidate all or a great deal by the aid of luminiferous ether will yet again appear in theoretical chemistry. An independent chemical mechanics of the material particles of matter, and of their internal (atomic) changes, would, in my opinion, arise as the result of these efforts. Just as the progress made in chemistry in the time of Lavoisier was reflected over all natural science, so there is reason to think that an independent chemical mechanics would shed a new light on all molecular mechanics, which must be considered as the fundamental problem of the exact sciences in our times. Two hundred years ago Newton laid the foundation of a truly scientific theoretical mechanics of extemal visible movement, and erected the edifice of celestial mechanics on this foundation. One hundred years ago Lavoisier arrived at the first fundamental law of the internal mechanics of invisible particles of matter. This subject is far from having been developed into a harmonious whole, because it is much more difficult, and, although many details have been completely investigated, it does not possess any starting points. Newton was possible only after Copernicus and Kepler, who had discovered the exterior empirical simplicity of celestial phenomena. Lavoisier and Dalton may, in respect to the chemical mecinanics of the molecular world, be compared to Copernicus and Kepler. But a Newton has not yet appeared in the molecular world; when he does, I think that he will find the fundamental laws of the mechanics of the invisible movements of matter more easily and more quickly in the chemical structure of matter than in physical phenomena (of electricity, heat, and light), for these latter are accomplished by already-disposed particles of matter, whilst it is now clear that the problem of chemical mechanics mainly lies in the apprehension of those movements which are invisibly accomplished by the smallest atoms of matter. The general laws of mechanics, established by Newton, will probably serve as starting points for molecular mechanics, but the independence of its range becomes more evident when chemical molecules are compared with the celestial systems, such as the solar system. Chemical atoms may be regarded as separate members of such systems (as, for instance, the sun, planets, comets, and other heavenly bodies), whilst the ether of light may be likened to the cosmic dust which without doubt is distributed throughout space. The present condition of molecular mechanies is, to a certain extent, copied from celestial mechanics, but there is nothing to prove the entire similarity of both worlds, although it appears to the mind that, starting from the primary elements of the unity of creation, such a representation is the most likely. 
As the chemical changes to which substances are liable proceed from internal forces proper to these substances, as chemical phenomena certainly consist of movements of material parts (from the laws of the indestructibility of matter and of elements), and as the investigation of mechanical and physical phenomena proves the law of the indestructibility of forces, or the conservation of energy - that is, the possibility of the transformation of one kind of movement into another (of visible or mechanical into invisible or physical) - we are inevitably obliged to acknowledge the presence in substances (and especially in the elements of which all others are composed) of a store of chemical energy or invisible movement inducing them to enter into combinations. If heat be evolved in a reaction, it means that a portion of chemical energy is transformed into heat $;^{32}$ if heat be absorbed in a reaction, ${ }^{33}$ that it is

32 The theory of heat gave the idea of a store of internal movement or energy, and therefore with it, it became necessary to acknowledge chemical energy, but there is no foundation whatever for identifying heat energy with chemical energy. It may be supposed, but not positively affirmed, that heat movement is proper to molecules and chemical movements to atoms, but that as molecules are made up of atoms, the movement of the one passes to the other, and that for this reason heat strongly influences reaction and appears or disappears (is absorbed) in reactions. These relations, which are. apparent and hardly subject to doubt on general lines, still present much that is doubtful in detail, because all forms of molecular and atomic movement are able to pass into each other. On broad general lines it must be acknowledged that as mechanical energy can entirely pass into heat energy (but the reverse transition is accomplished only partially, according to the second law of heat), so also heat energy may pass into chemical energy, but it is doubtful, and even unlikely, that chemical energy passes altogether into heat energy. Therefore, the heat evolved in chemical reactions cannot serve as the total measure of chemical energy, more especially as there are a number of reactions of combination in which heat is absorbed; for instance, the combination of charcoal with sulphur is accompanied by an absorption of heat-probably because the molecules of charcoal are complex, and those of carbon bisulphide less so, and the breaking up of the complex molecules of charcoal requires a large absorption of heat (whose measure we do not know) -and whilst the combination of charcoal with sulphur is accompanied by an evolution of heat, yet we only observe the difference of these two heat effects.

53 The reactions which take place (at the ordinary or at a high temperature) directly between substances may be clearly divided into exothermal, which are accompanied by an evolution of heat, and endothermal, which are accompanied by an absorption of heat. It is evident that the latter require a source of heat. They are determined either by the directly surrounding medium (as in the formation of carbon bisulplide from charcoal and sulphur, or in decompositions which take place at high temperatures), or else by a simultaneously proceeding secondary reaction. So, for instance, hydrogen sulphide is decomposed by iodine in the presence of water at the expense of the heat which is evolved by the solution in water of the hydrogen iodide produced. This is the reason why this reaction, as exothermal, only takes place in the presence of water; otherwise it would be accompanied by a cooling effect. As in the combination of dissimilar substances, the bonds existing between the molecules and atoms of the homogeneous substances have to be broken asunder, whilst in reactions of rearrangement the formation of any one substance proceeds parallel with the formation of another, and, as in reactions, a series of physical and mechanical changes take place, it is impossible to separate the heat directly depending on a given reaction from the total sum of the observed heat effect. For this 
partly transformed (rendered latent) into chemical energy. The store of force or energy going to the formation of new compounds may, after several combinations, accomplished with an absorption of heat, at last diminish to such a degree that indifferent compounds will be obtained, although these sometimes, by combining with energetic elements or compounds, give more complex compounds, which may be capable of entering into chemical combination. Among elements gold, platinum, and nitrogen have but little energy, whilst potassium, oxygen, and chlorine have a very marked degree of energy. When dissimilar substances enter into combination they often form substances of diminished energy. Thus sulphur and potassium when heated easily burn in air, but when combined together their compound is neither inflammable nor burns in air like its component parts. Part of the energy of the potassium and of the sulphur was evolved in their combination in the form of heat. Just as in the passage of substances from one physical state into another a portion of their store of heat is absorbed or evolved, so in combinations or decompositions and in every chemical process, there occurs a change in the store of chemical energy, and at the same time an evolution or absorption of heat. ${ }^{34}$

For the comprehension of chemical phenomena in a mechanical sense - i.e., in the study of the modus operandi of chemical phenomenait is at the present time most important to consider : (1) the facts gathered from stoïchiometry, or that part of chemistry which treats of the quantitative relation, by weight or volume, of the. reacting substances ; $(2)$ the distinction between the different forms and classes of chemical reactions ; (3) the study of the changes in properties produced by alteration in composition ; (4) the study of the phenomena which accompany chemical transformation; (5) a generalisation of the conditions under which reactions occur. As regards stoïchiometry, this branch of chemistry has been worked out most thoroughly, and embraces laws (of Dalton, Avogadro-Gerhardt, and others) which bear so deeply on all parts of chemistry that its entire contemporary standing may be

reason, thermo-chemical data are very complex, and cannot by themselves give the key to many chemical problems, as it was at first supposed they might. They ought to form a part of chemical mechanics, but alone they do not constitute it.

34 As chemical reactions are effected by heating, so the heat absorbed by substances before decomposition or change of state, and called 'specific heat,' goes in many cases to the preparation, if it may be so expressed, of reaction, even when the limit of the temperature of reaction is not attained. The molecules of a substance $\mathbf{A}$, which is able to react on a substance $\mathrm{B}$ below a temperature $t$ by being heated from a somewhat lower temperature to $t$, undergoes that change which had to be arrived at for the formation of $\mathrm{A} B$. This idea is often extended; for instance, it is supposed that a given substance in its passage from a liquid to a gaseous state gives chemically or materially new, lighter, and simpler molecules (is depolymerised, according to De Haen). 
characterised as the epoch of their circumstantial application to particular cases. The expression of the quantitative (volumetric or gravimetric) composition of substances now forms the most important problem of chemical research, and therefore the entire further exposition of the subject is subordinate to stoïchiometrical laws. All other branches of chemistry are clearly subordinate to this most important portion of chemical knowledge. Even the very signification of reactions of combination, decomposition, and rearrangement, acquired, as we shall see, a particular and new character under the influence of the progress of exact ideas concerning the quantitative relations of substances entering into chemical changes. Furthermore, in this sense there arose a new-and, up to then, unknown - division of compound substances into definite and indefinite compounds. Even at the beginning of this century, Berthollet had not made this distinction. But Prout showed that a number of compounds contain the substances of which they are composed and into which they break up, in exact definite proportions by weight, which are unalterable under any conditions. Thus, for example, red mercury oxide contains sixteen parts by weight of oxygen for every 200 parts by weight of mercury, which is expressed by the formula $\mathrm{HgO}$. But in an alloy of copper and silver one or the other metal may be added at will, and in an aqueous solution of sugar, the relative proportion of the sugar and water may be altered and nevertheless a homogeneous whole with the sum of the independent properties will be obtained-i.e., in these cases there was indefinite chemical combination. Although in nature and chemical practice the formation of indefinite compounds (such as alloys and solutions) plays as essential a part as the formation of definite chemical compounds, yet, as the stoïchiometrical laws at present apply chiefly to the latter, all facts concerning indefinite compounds suffer from inexactitude, and it is only during recent years that the attention of chemists has been directed to this province of chemistry.

In chemical mechanics it is, from a qualitative point of view, very important to clearly distinguish at the very beginning between reversible and non-reversible reactions. One or several substances capable of reacting on each other at a certain temperature produce substances which at the same temperature either can or cannot give back the original substances. For example, salt dissolves in water at the ordinary temperature, and the solution so obtained is capable of breaking up at the same temperature, leaving salt and separating the water by evaporation. Carbon bisulphide is formed from sulphur and carbon at the same temperature at which it can be resolved into sulphur and carbon. Iron, at a certain temperature, separates hydrogen from water, forming iron oxide, which, 
in contact with hydrogen at the same temperature, is able to produce iron and water. It is evident that if two substances, $\mathrm{A}$ and $\mathrm{B}$, give two others $\mathrm{C}$ and $\mathrm{D}$, and the reaction be reversible, then $\mathrm{C}$ and $\mathrm{D}$ will form $\mathrm{A}$ and $\mathrm{B}$, and, consequently, by taking a definite mass of $\mathrm{A}$ and $\mathrm{B}$, or a corresponding mass of $\mathrm{C}$ and $\mathrm{D}$, we shall obtain, in each case, all four substances - that is to say, there will be a state of chemical equilibrium between the reacting substances. By increasing the mass of one of the substances we obtain a new condition of equilibrium, so that reversible reactions present a means of studying the influence of. mass on the modus operandi of chemical changes. Many of those reactions which occur with very complicated compounds or mixtures may serve as examples of non-reversible reactions. Thus many of the compound substances of animal and vegetable organisms are broken up by heat, but cannot be re-formed from their products of decomposition at any temperature. Gunpowder, as a mixture of sulphur, nitre, and carbon, on burning, forms gases from which the original substances cannot be re-formed at any temperature. In order to obtain them, recourse must be had to an indirect method of combination at the moment of separation. If $\mathrm{A}$ does not under any circumstances combine directly with $\mathrm{B}$, it does not imply that it cannot give a compound A B. For $A$ can often combine with $\mathrm{C}$ and $\mathrm{B}$ with $\mathrm{D}$, and if $\mathrm{C}$ has a great affinity for $\mathrm{D}$, then the reaction of $\mathrm{A} \mathrm{C}$ on $\mathrm{B} \mathrm{D}$ produces not only $\mathrm{C} \mathrm{D}$, but also $\mathrm{A} \mathrm{B}$. As on the formation of $\mathrm{C} \mathrm{D}$, the substances $\mathrm{A}$ and $\mathrm{B}$ (previously in A C and B D) are left in a peculiar state of separation, it is supposed that their mutual combination occurs because they meet together in this nascent state at the moment of separation (in statu nascendi). Thus chlorine does not directly combine with charcoal, graphite, or the diamond, nevertheless there are compounds of chlorine with carbon and many of them are distinguished by their stability. They are obtained during the action of chlorine on hydrocarbons, as the separation products from the direct action of chlorine on hydrogen. Chlorine takes up the hydrogen, and the freed carbon at the moment of its separation enters into combination with another portion of the chlorine, so that in the end the chlorine is combined with both the hydrogen and the carbon. ${ }^{35}$

35 It is possible to imagine that the cause of a great many of such reactions is, that substances taken in a separate state, for instance, charcoal, present a complex molecule composed of separate atoms of carbon which are fastened together (united, as is usually said) by a considerably affinity; for atoms of the same kind, just like atoms of different kinds, possess a mutual affinity. The affinity of chlorine for carbon, although unable to break this bond asunder, may be sufficient to form a stable compound with already separate atoms of carbon. Such a view of the subject presents a hypothesis which, although dominant at present, is without sufficiently firm foundation. Were the matter

VoL. I. 
As regards those phenomena which accompany chemical action, the most important circumstance in reference to chemical mechanics is that not only do chemical processes produce a mechanical displacement (a visible disturbance), heat, light, electrical potential and current; but that all these agents are themselves capable of changing and governing chemical transformations. This reciprocity or reversibility naturally depends on the fact that all the phenomena of nature are only different kinds and forms of visible and invisible (molecular) movement. First sound, and then light, was shown to consist of vibratory movements, as the laws of physics have proved and developed beyond a doubt. Then, the connection between heat and mechanical motion and work has ceased to be a supposition, but has become a known fact, and the mechanical equivalent of heat (424 kilogrammetres of mechanical work correspond with one kilogram unit of heat or Calorie) gives a mecha: nical measure for heat phenomena. Although the mechanical theory of electrical phenomena cannot be considered so fully developed as the theory of heat, nevertheless there can be no doubt but that the electrical state of substances, and electric or galvanic currents, represent a peculiar form of motion; more especially as both statical and dynamical electricity are produced by mechanical means (in common electrical machines or in Gramme or other dynamos), and, as conversely, a current (in electric motors) can produce mechanical motion, as heat produces motion in heat (steam, gas, or air) engines. Thus by passing a current through the poles of a Gramme dynamo it may be made to revolve, and, conversely, by revolving it an electrical current is produced, which demonstrates the reversibility of electricity into mechanical motion. Therefore, chemical mechanics must look for the fundamental lines of its advancement in the correlation of chemical with physical and mechanical phenomena. But this subject, owing to its complexity and comparative novelty, has not yet been subjected to a harmonious theory, or even to a satisfactory hypothesis, and therefore we shall avoid lingering over it.

A chemical change in a certain direction is accomplished not only

as simple as it appears to be, according to this hypothesis, one would expect, for instance, that the compounds of carbon with chlorine would be easily decomposable by reason of the supposed considerable affinity of the separate atoms of carbon, which should therefore tend to mutual combination and the formation of charcoal. It is evident, however, that not only does reaction itself consist of movements, but that in the compound formed (in the molecules) the elements (atoms) forming it are in harmonious stable movement (like the planets in the solar system), and this movement will affect the stability and capacity for reaction, and therefore these depend not only on the affinity of the participating substances, but also on the conditions of reaction which change the state of movement of the elements in the molecules, as well as on the nature, form, and inten. sity of those movements which the elements have in their given state. In a word, the mechanical side of chemical action nust be exceedingly complex. 
by reason of the difference of masses, the composition of the substances concerned, the distribution of their parts, and their affinity or chemical energy, but also by reason of the conditions under which the substances occur, and these conditions differ for every particular reaction. In order that a certain chemical reaction may take place between substances which are capable of reacting on each other, it is often necessary to have recourse to conditions which are sometimes very different from those in which the substances usually occur in nature. For example, not only is the presence of air (oxygen) necessary for the combustion of charcoal, but the latter must also be heated to redness. The red-hot portion of the charcoal burns-i.e., combines with the oxygen of the atmosphere-and in doing so evolves heat, which heats the adjacent parts of charcoal, which are thus able to burn. Just as the combustion of charcoal is dependent on its being heated to redness, so also every chemical reaction only takes place under certain physical, mechanical, or other conditions. The following are the chief conditions which exert an influence on the progress of chemical reactions.

(a) Temperature.-Chemical reactions of combination only take place within certain definite limits of temperature, and cannot be accomplished outside these limits. As examples we may cite, not only that the combustion of charcoal begins at a red heat, but also that chlorine and salt only combine with water at a temperature below $0^{\circ}$. These compounds cannot be formed at a higher temperature, for they are then wholly or partially broken up into their component parts. A certain rise in temperature is necessary to start combustion. In certain cases the effect of this rise may be explained as causing one of the reacting bodies to change from a solid into a liquid or gaseous form. The transference into a fluid form facilitates the progress of the reaction, because it aids the intimate contact of the particles acting on each other. Another reason, to which must be ascribed the chief influence of heat in exciting chemical action, is that the physical cohesion, or the internal chemical union, of homogeneous particles is thereby weakened, and therefore the separation of the particles of the substances taken, and their transference into new compounds, is rendered easier. When a reaction absorbs heat - as in decomposition, where the heat is transformed into latent chemical energy - the reason why heat is necessary is self-evident.

It is most important to observe the effect of an elevation of temperature on all compounds, as there is reason to believe that they are all decomposed at a more or less high temperature. We have already seen examples of this in describing the decomposition of mercury oxide 
into mercury and oxygen, and the decomposition of wood under the influence of heat. Many substances are decomposed at a very moderate temperature ; for instance, the fulminating salt which is employed in cartridges is decomposed at a little above $120^{\circ}$. The majority of those compounds which make up the mass of animal and vegetable matters are decomposed at $250^{\circ}$. On the other hand, there is reason to think that at a very low temperature no reaction whatever can take place. Thus plants cease to carry on their chemical processes during the winter. Every chemical reaction requires certain limits of temperature for its accomplishment, and, doubtless, many of the chemical changes observed by us cannot take place in the sun, where the temperature is very high, or on the moon, where it is very low.

The influence of heat on reversible reactions is particularly instructive. If, for instance, a compound which is capable of being reproduced from its products of decomposition be heated up to the temperature at which decomposition begins, the decomposition of a mass of the substance contained in a definite volume is not immediately completed. Only a certain fraction of the substance is decomposed, the other portion remaining unchanged, and if the temperature be raised, the quantity of the substance decomposed increases; furthermore, for a given volume the ratio between the part decomposed and the part unaltered corresponds with each definite rise in temperature until it reaches that at which the compound is entirely decomposed. This partial decomposition under the influence of heat is called dissociation. It is possible to distinguish between the temperatures at which dissociation begins and ends. . Should dissociation proceed at a certain temperature, yet should the product or products of decomposition not remain in contact with the still undecomposed portion of the compound, then decomposition will go on to the end. Thus limestone is decomposed in a limekiln into lime and carbonic anhydride, because the latter is carried off by the draught of the furnace. But if a certain mass of limestone be enclosed in a definite volume-for instance, in a gun barrel-which is then sealed up, and heated to redness, then, as the carbonic anhydride cannot escape, a certain proportion only of the limestone will be decomposed for every increment of heat (rise in temperature) higher than that at which dissociation begins. Decomposition will cease when the carbonic anhydride evolved presents a maximum dissociation pressure corresponding with each rise in temperature. If the pressure be increased by increasing the quantity of gas, then combination begins afresh; if the pressure be diminished decomposition will recommence. Decomposition in this case is exactly similar to evaporation; if the steam given off by evaporation cannot escape, its 
pressure will reach a maximum corresponding with the given temperature, and then evaporation will cease. Should steam be added it will be condensed in the liquid; if its quantity be diminished-i.e., if the pressure be lessened, the temperature being constant - then evaporation will go on. We shall afterwards discuss more fully these phenomena of dissociation, which were first discovered by Henri St. Claire Deville. We will only remark that the products of decomposition re-combine with greater facility the nearer their temperature is to that at which dissociation begins, or, in other words, that the initial temperature of dissociation is near to the initial temperature of combination.

(b) The influence of an electric current, and of electricity in general, on the progress of chemical transformations is very similar to the influence of heat. The majority of compounds which conduct electricity are decomposed by the action of a galvanic current, and there being great similarity in the conditions under which decomposition and combination proceed, combination often proceeds under the influence of electricity. Electricity, like heat, must be regarded as a peculiar form of molecular motion, and all that which refers to the influence of heat also refers to the phenomena produced by the action of an electrical current, only with this difference, that a substance can be separated into its component parts with much greater ease by electricity, as the process goes on at the ordinary temperature. The most stable compounds may be decomposed by this means, and a most important fact is then observed - namely, that the component parts appear at the different poles or electrodes by which the current passes through the substance. Those substances which appear at the positive pole (anode) are called 'electro-negative,' and those which appear at the negative pole (cathode, that in connection with the zinc of an ordinary galvanic battery) are called 'electro-positive.' The majority of non-metallic elements, such as chlorine, oxygen, \&c., and also acids and substances anaiogous to them, belong to the first group, whilst the metals, hydrogen, and analogous products of decomposition appear at the negative pole. Chemistry is indebted to the decomposition of compounds by the electric current for many most important discoveries. Many elements have been discovered by this method, the most important being potassium and sodium. Lavoisier and the chemists of his time were not able to decompose the oxygen compounds of these metals, but Davy showed that they might be decomposed by an electric current, the metals sodium and potassium appearing at the negative pole.

(c) Certain unstable compounds are also decomposed by the action of light. Photography is based on this property in certain substances (for instance, in the salts of silver). The mechanical energy of those vibra- 
tions which determine the phenomena' of light is very small, and therefore only certain, and these generally unstable, compounds can be decomposed by light-at least under ordinary circumstances. But there is one class of chemical phenomena dependent on the action of light which forms as yet an unsolved problem in chemistry - these are the processes accomplished in plants under the influence of light. Here there take place most unexpected decompositions and combinations, which are often unattainable by artificial means. For instance, carbonic anhydride, which is so stable under the influence of heat and electricity, is decomposed, and evolves oxygen in plants under the influence of light. In other cases, light decomposes unstable compounds, such as are usually easily decomposed by heat and other agents. Chlorine combines with hydrogen under the influence of light, which shows that combination, as well as decomposition, can be determined by its action, as was likewise the case with heat and electricity.

(d) Mechanical effects exert, like the foregoing agents, an action both on the process of chemical combination and of decomposition. Many substances are decomposed by friction or by a blow-as, for example, the compound called iodide of nitrogen (which is composed of iodine, nitrogen, and hydrogen), and silver fulminate. Mechanical friction causes sulphur to burn at the expense of the oxygen contained in potassium chlorate.

(e) Besides the various conditions which have been enumerated above, the progress of chemical reactions is accelerated or retarded by the condition of contact in which the reacting bodies occur. Other conditions remaining constant, the rate of progress of a chemical reaction is accelerated by increasing the number of points of contact. It will be enough to point out the fact that sulphuric acid does not absorb ethylene under ordinary conditions of contact, but only after continued shaking, by which means the number of points of contact is greatly increased. To ensure full action between solids, it is necessary to reduce them to very fine powder and to mix them as thoroughly as possible, as by this means their reaction is greatly accelerated. M. Spring, the Belgian chemist, has shown that finely-powdered solids which do not react on each other at the ordinary temperature may undergo reaction under an increased pressure. Thus, under a pressure of 6,000 atmospheres, sulphur combines with many metals at the ordinary temperature, and the powders of many metals form alloys. It is evident that an increase in the number of points or surfaces must be regarded as the chief cause producing reaction, which is doubtless accomplished in solids, as in liquids and gases, in virtue of an internal movement or mobility of the particles, which movement, although in different degrees 
and forms, must exist in all the states of matter. It is very important to direct attention to the fact that the internal movement or condition of the parts of the particles of matter must be different on the surface of a substance from what it is inside ; because in the interior of a substance similar particles are acting on all sides of every particle, whilst at the surface they only act on one side. Therefore, the condition of a substance at its surfaces of contact with other substances must be more or less modified by them-it may be in a manner similar to that caused by an elevation of temperature. These considerations throw some light on the action in the large class of contact reactions; that is, such as seem to proceed from the mere presence (contact) of certain special substances. Porous or powdery substances are very prone to act in this way, especially spongy platinum and charcoal. For example, sulphurous anhydride does not combine directly with oxygen, but this reaction takes place in the presence of spongy platinum. ${ }^{36}$

The above general and introductory chemical conceptions cannot be thoroughly grasped in their true sense without a knowledge of the particular facts of chemistry to which we shall now turn our attention. It was, however, absolutely necessary to become acquainted on the very threshold with such fundamental principles as the laws of the indestructibility of matter and of the conservation of energy, as it is only by their acceptance, and under their direction and influence, that the examination of particular facts can give practical and fruitful results.

36 Contact phenomena are separately considered in detail in the work of Professor Konovaloff (1884). In my opinion, one must consider that the state of the internal movements of the atoms in molecules is modified at the points of contact of substances, and this state determines chemical reactions, and therefore, that reactions of combination, decomposition, and rearrangement are accomplished by contact. Professor Konovaloff showed that a number of substances under certain conditions of their surfaces act by contact; for instance, powdery silica (from the hydrate) acts just like platinum, decomposing certain compound ethers. As reactions are only accomplished under close contact, it is probable that those modifications in the distribution of the atoms in molecules which come about by contact phenomena prepare the way for them. By this the rôle of contact phenomena is considerably extended. By such phenomena the fact should be explained why a mixture of hydrogen and oxygen yields water (explodes) at different temperatures according to the kind of heated substance which transmits this temperature. In chemical mechanics, phenomena of this kind have great importance, but as yet they have been but little studied. 


\section{CHAPTER I}

\section{ON WATER AND ITS COMPOUNDS}

WATER is found almost everywhere in nature, and in all three physical states. As vapour, water occurs in the atmosphere, and in this form it is distributed over the entire surface of the earth. The vapour of water in condensing, by cooling, forms snow, rain, hail, dew, and fog. One cubic metre (or 1,000,000 cubic centimetres, or 1,000 litres, or 35.316 cubic feet) of air can contain at $0^{\circ}$ only 4.8 grams of water, at $20^{\circ}$ about 17.0 grams, at $40^{\circ}$ about 50.7 grams ; but ordinary air only contains about 60 per cent. of the possible moisture. Air containing less than 40 per cent. of the possible moisture is felt to be dry, and air which contains more than 80 per cent. of the possible moisture is considered as already damp. ${ }^{1}$ Water in the liquid state, in falling as rain

1 In practice, the chemist has to continually deal with gases, and gases are often collected over water; in which case a certain amount of water passes into vapour, and this vapour mingles with the gases. It is therefore most important that he should be able to calculate the amount of water or of moisture in air and other gases. Let us consider the relations in volume and weight which exist in this case. Let us imagine a cylinder standing in a mercury bath, and filled with a dry gas whose volume equals $v$, temperature $t^{\circ}$, and pressure or tension $h \mathrm{~mm}$. ( $h$ millimetres of the column of mercury at $\left.0^{\circ}\right)$. We will introduce water into the cylinder in such a quantity that a small part remains in the liquid state, and consequently that the gas will be saturated with aqueous vapour; the volume of the gas will then increase (if a larger quantity of water be taken some of the gas will be dissolved in it, and the volume may therefore be diminished). We will further suppose that the temperature remains constant after the addition of the water; then the pressure (as the volume increases the mercury in the cylinder falls, consequently the pressure is increased) and the volume is increased. In order to investigate the phenomenon we will artificially increase the pressure, and reduce the volume to the original volume $v$. Then the pressure or tension will prove greater than $h$, namely $h+f$, which means that by the introduction of aqueous vapour the tension of the gas is increased. The researches of Dalton, Gay-Lussac, and Regnault showed that this increase is equal to the maximum pressure which is proper to the aqueous vapour at the temperature at which the observation is made. The maximum pressure for all temperatures may be found in the tables made from observations on the tension of aqueous rapour. The quantity $f$ will be equal to this maximum pressure of aqueous vapour. This may be expressed thus: the maximum tension of aqueous vapour (and of all other vapours) saturating a space in a vacuum or in any gas is the same. This rule is known as Dalton's law. Thus we have a volume of dry gas $v$, under a pressure $h$, and a volume of moist gas, saturated with vapour, under a pressure $h+f$. The volume $v$ of the dry gas under a pressure $h+f$ occupies, according to the law of Mariotte, a 
and snow, soaks into the soil and collects together into springs, lakes, rivers, seas, and oceans. It is absorbed from the soil by the roots of

volume $\frac{v h}{h+f}$; consequently the volume occupied by the aqueous vapour under the pressure $h+f$ equals $v-\frac{v h}{h+f}$, or $\frac{v f}{h+f}$. Thus the volumes of the dry gas and of the moisture which occurs in it, at a pressure $h+f$, are in the ratio $f: h$. And, therefore, if the aqueous vapour saturates a space at a pressure $n$, the volumes of the dry air and of the moisture which is contained in it are in the ratio $n-f: f$, where $f$ is the pressure of the vapour according to the tables of vapour tension. Thus, if a volume $\mathrm{N}$ of a gas saturated with moisture be measured at a pressure $H$, then the volume of the gas, when dry, will be equal to $\mathrm{N} \frac{\mathrm{H}-f}{\mathrm{H}}$, because the volume $\mathrm{N}$ requires to be divided into parts which are in the ratio $\mathrm{H}-f: f$. In fact, the entire volume $\mathrm{N}$ must be to the volume of dry gas $x$ as $\mathrm{H}$ is to $\mathrm{H}-f$; therefore, $\mathrm{N}: x=\mathrm{H}: \mathrm{H}-f$, from which $x=\mathrm{N} \frac{\mathrm{H}-f}{\mathrm{H}}$. Under any other pressure-for instance, $760 \mathrm{~mm}$.- the volume of dry gas will be $\frac{x \mathrm{H}}{760}$, or $\frac{\mathrm{H}-f}{760}$ and thus we obtain the following practical rule: If a volume of a gas saturated with aqueous vapour be measured at a pressure $\mathrm{H} \mathrm{mm}$., then the volume of dry gas contained in it will be obtained by finding the volume corresponding with the pressure $H$, less the pressure due to the aqueous vapour at the temperature of observation. For example, 37.5 cubic centimetres of air saturated with aqueous vapour was measured at a temperature of $15 \cdot 3^{\circ}$, and under a pressure of $747.3 \mathrm{~mm}$. of mercury $\left(\right.$ at $0^{\circ}$ ). What will be the volume of dry gas at $0^{\circ}$ and $760 \mathrm{~mm}$.? The pressure of aqueous vapour corresponding with $15.3^{\circ}$ is equal to $12.9 \mathrm{~mm}$, and therefore the volume of dry gas at $15.3^{\circ}$ and $747.3 \mathrm{~mm}$. is equal to $37.5 \times \frac{747 \cdot 3-12 \cdot 9}{747 \cdot 3}$; at $760 \mathrm{~mm}$. it will be equal to $37.5 \times \frac{734.4}{760}$; and at $0^{\circ}$ the volume of dry gas will be $37 \cdot 5 \times \frac{734 \cdot 4}{760} \times \frac{273}{273-15 \cdot 3}=34 \cdot 31$ c.c.

From this rule may also be calculated what fraction of a volume of gas is occupied by moisture under the ordinary pressure at different temperatures; for instance, at $30^{\circ} \mathrm{C}$ $f=31 \cdot 5$, consequently 100 volumes of a moist gas or air, at $760 \mathrm{~mm}$., cortain a volume of aqueous vapour $100 \times \frac{31.5}{760}$, or 4.110 ; also it is found that at $0^{\circ}$ there is contained 0.61 p.c. by volume, at $10^{\circ} 1.21$ p.c., at $20^{\circ} 2 \cdot 29$ p.c., and at $50^{\circ}$ up to $12 \cdot 11$ p.c. From this it may be judged how great an error might be made in the volumetric determination of gases were the moisture not taken into consideration. From this it is also evident how great are the variations in volume of the atmosphere when it loses or gains aqueous vapour, which again explains a number of atmospheric phenomena (winds, variation of pressure, precipitations, storms, \&c.).

If aqueous vapour does not saturate a gas, then it is indispensable that the degree of moisture should be known in order to determine the volume of dry gas from the volume of moist gas. The preceding ratio gives the maximum quantity of water which can be held in a gas, and the degree of moisture shows what fraction of this maximum quantity occurs in a given case, when the vapour does not saturate the space occupied by the gas. Consequently, if the degree of moisture equals 50 p.c.-that is, half the maximum - then the volume of dry gas at $760 \mathrm{~mm}$. is equal to the volume of dry gas at $760 \mathrm{~mm}$. multiplied by $\frac{h-0.5 f}{760}$, or, in general, by $\frac{h-r f}{760}$, where $r$ is the degree of mois-

ture. If, therefore, it is required to measure the volume of a moist gas, it must either be entirely dried or quite saturated with moisture, or else the degree of moisture determined. The first and last methods are inconvenient, and therefore recourse is usually had to the second. For this purpose water is introduced into the cylinder holding the gas to be measured; it is left for a certain time so that the gas may become saturated, 


\section{plants, which, when fresh, contain from 40 to 80 per cent. of water by weight. Animals contain about the same amount of water. In a}

the precaution being taken that a portion of the water remains in a liquid state; then the volume of the moist gas is determined, from which that of the dry gas may be calculated. In order to find the weight of the aqueous vapour in a gas it is necessary to know the weight of a cubic measure at $0^{\circ}$ and $760 \mathrm{~mm}$. Knowing that one cubic centimetre of air under these circumstances weighs $0.001293 \mathrm{gram}$, and that the density of aqueous vapour is 0.62 , we find that one cubic centimetre of aqueous vapour at $0^{\circ}$ and $760 \mathrm{~mm}$. weighs 0.0008 gram, and at a temperature $t^{\circ}$ and pressure $h$ the weight of one cubic centimetre will be $0.0008 \times \frac{h}{760} \times \frac{273}{273+t}$. We already know that $v$ volumes of a gas at a temperature $t^{\circ}$ pressure $h$ contain $v \times \frac{f}{h}$ volumes of aqueous vapour which satu. rate it, therefore the weight of the aqueous vapour held in $v$ volumes of a gas will be $\frac{v \times f}{h} \times 0.0008 \times \frac{h}{760} \times \frac{273}{273+t^{\circ}}$, or $v \times 0.0008 \times \frac{f}{760} \times \frac{273}{273+t}$.

Consequently, the weight of the water which is held in one volume of a gas is only dependent on the temperature and not on the pressure. This also signifies that evaporation proceeds to an equal extent in air as in a vacuum, or, in general terms (this is Dalton's law), vapours and gases diffuse into each other as if into a vacuum. In a given space there enters, at a given temperature, a constant quantity of vapour whatever be the pressure of the gas filling that space. If the degree of moisture equals $r$ then the weight of the vapour in $v$ cubic centimetres will be $p=v \times 0.0008 \times \frac{f r}{760} \times \frac{273}{273+t}$ grams.

From this it is clear that if the weight of the vapour held in a given volume of a gas be known, it is easy to determine the degree of moisture $r=\frac{p}{v \times 0^{*} 0008} \times \frac{760}{t} \times \frac{273+t}{273}$ On this is founded the very exact determination of the degree of moisture of air by the weight of water contained in a given volume. It is easy to calculate from the preceding formula the number of grams of water contained at all pressures in one cubic metre or million centimetres of air saturated with vapour at various temperatures; for example, at $30^{\circ} f=31 \cdot 5$, therefore $p=1000000 \times 0.0008 \times \frac{31.5}{760} \times \frac{273}{273+30}$ or 29.84 grams.

The laws of Mariotte, Dalton, and Gay-Lussac, which are here applied to gases and vapours, are not entirely exact, but are approximately true. Were they quite exact, a mixture of several liquids, having a certain vapour pressure, would be able to give vapours of a very great pressure, which is not the case. In fact the pressure of aqueous vapour is slightly less in a gas than in a vacuum, and the weight of aqueous vapour held in a gas is slightly less than it should be according to Dalton's law, as was shown by the experiments of Regnault and others. This means that the tension of the vapour is less in air than in a vacuum, which also is the reason why the weight of vapour is less than the theoretical weight. The difference between the pressure of rapours in air and in a vacunm does not, however, exceed $\frac{1}{20}$ of the total pressure of the vapours, and therefore in practice the application of Dalton's law may be followed. This decrement in vapour tension which occurs in the intermixture of vapours and gases, although small, indicates that there is then already, so to speak, a beginning of chemical change. The essence of the matter is that in this case there occurs as on contact (see preceding footnote) an alteration in the movements of the atoms in the molecules, and therefore also a change in the movement of the molecules themselves.:

In the uniform intermixture of air and other gases with aqueous vapour, and in the capacity of water to pass into vapour and form a uniform mixture with air, we may perceive an instance of a physical phenomenon which is analogous to chemical phenomena, forming indeed a transition from one class of phenomena to the other. Between water and dry air there exists a kind of affinity which obliges the water to saturate the 
solid state water appears as snow, ice, or in an intermediate form between these two, which is seen on mountains covered with perpetual snow. The water of rivers, ${ }^{2}$ springs, oceans and seas, lakes, and wells

air. But such a lomogeneous mixture is formed (almost) independently of the nature of the gas in which evaporation takes place; even in a vacuum the phenomenon occurs in exactly the same way as in a gas, and therefore it is not the property of the gas, nor its relation to water, but the property of the water itself, which obliges it to evaporate, and therefore in this case chemical affinity is not yet acting-at least its action is not clearly pronounced. That it does, however, play a certain part is seen from the deviation from Dalton's law.

In falling through the atmosphere, water dissolves the gases of the atmosphere, nitric acid, ammonia, organic compounds, salts of sodium, magnesium, and calcium, and mechanically washes out a mixture of dust and microbes which are suspended in the atmosphere. The amount of these and certain other constituents is very variable. Even in the beginning and end of the same rainfall, a variation which is often very considerable may be remarked. Thus, for example, Bunsen found that rain collected at the beginning of a shower contained 3.7 grams of ammonia per cubic metre, whilst that collected at the end of the same shower contained only 0.64 gram. The water of the entire shower contained an average of $\mathbf{1 . 4 7}$ grams of ammonia per cubic metre. In the course of a year rain supplies an acre of ground with up to $5 \frac{1}{2}$ kilos of nitrogen in a combined form. Marchand found in one cubic metre of snow water 15.63 , and in one cubic metre of rain water 10.07, grams of sodium sulphate. Angus Smith showed that after a thirtyhours' fall at Manchester the rain still contained $34 \cdot 3$ grams of salts per cubic metre. A considerable amount of organic matter, namely 25 grams per cubic metre, has been found in rain water. The total amount of solid matter in rain water reaches 50 grams per cubic metre. Rain water contains generally very little carbonic acid, whilst stream water contains a considerable quantity of it. In considering the nourishment of plants, it is necessary to keep in view the substances which are carried into the soil by raín.

River water, which is accumulated from springs and sources fed by atmospheric water, contains from 50 to 1,600 parts by weight of salts in 1,000,000 parts. The amount of solid matter, per $1,000,000$ parts by weight, contained in the chief rivers is as follows:- the Don 124, the Loire 135, the St. Lawrence 170, the Rhone 182, the Dnieper 187, the Danube from 117 to 234 , the Rhine from 158 to 317 , the Seine from 190 to 432 , the Thames at London from 400 to 450 , in its upper parts 387 , and in its lower parts up to 1,617 , the Nile 1,580, the Jordan 1,052. The Neva is characterised by the remarkably small amount of solid matter it contains. From the investigations of Prof. G. K. Trapp, a cubic metre of Neva water contains 32 grams of incombustible and 23 grams of organic matter, or altogether about 55 grams. This is one of the purest waters which is known in rivers. The large amount of impurities in river water, and especially of organic impurity produced by pollution with putrid matter, makes the water of many rivere unfit for use.

The chief part of the soluble substances in river water consists of the calcium salts. 100 parts of the solid residues contain the following amounts of calcium carbonatefrom the water of the Loire 53, from the Thames about 50, the Elbe 55, the Vistula 65, the Danube 65, the Rhine from 55 to 75 , the Seine 75 , the Rhone from 82 to 94 . The Neva contains 40 parts of calcium carbonate per 100 parts of saline matter. The considerable amount of calcium carbonate held by stream water is very easily explained from the fact that water which contains carbonic acid in solution easily dissolves calcium carbonate, which occurs all orer the earth. Besides calcium carbonate and sulphate, river water contains magnesium, silica, chlorine, sodium, potassium, aluminium, nitric acid, and manganese. The presence of salts of phosphoric acid has not yet been determined with exactitude for all rivers, but the presence of nitrates has been proved with certainty in almost all kinds of well-investigated river water. The quantity of calcium phosphate does not exceed 0.4 gram in the river of the Dnieper, and the Don does not contain more 
contains various substances in solution, mostly salts - that is, sub-
stances resembling common table salt in their physical properties and

than 5 grams. The water of the Seine contains about 15 grams of nitrates, and the Rhone about 8 grams. The amount of ammonia is much less; thus in the water of the Rhine about 0.5 gram in June, and 0.2 gram in October; the water of the Seine contains the same amount. This is less than in rain water. Notwithstanding this insignificant quantity, the water of the Rhine alone, which is not so yery large a river, carries 16,245 kilograms of ammonia into the ocean every day. The difference between the amount of ammonia in rain and river water depends on the fact that the soil through which the rain water passes is able to withhold the ammonia. (Soil can also absorb many other substances, such as phosphoric acid, potassium salts, \&c.)

The water of springs, rivers, wells, and in general of those localities from which it is taken for drinking purposes, may be very injurious to the health if it contains much organic pollution-all the more, as in such water the lower organisins (bacteria) may rapidly develop, and these organisms often serve as the carriers or causes of infectious diseases. Thanks to the work of Pasteur, Koch, and many others, this province of researcl has made considerable progress during the past ten years, and has shown the possi bility of investigating even the number and properties of the germs held by water because those pathogenic bacteria which produce sickness, such as typhoid fever, Siberiar plague, \&c., have been distinguished. In bacteriological researches, a gelatinou: medium, enabling the germs to develop and multiply, is prepared with gelatin and water which has previously been heated several times, at intervals, to $100^{\circ}$ (it is thus renderec sterile-that is to say, all the germs in it are killed). The water to be investigatec is added to this prepared medium in a definite and small quantity (it is sometimes diluted with sterilised water to facilitate the calculation of the number of germs), it is protected from dust (which contains germs), and is left at rest until whole families o lower organisms are developed from each germ. These families (colonies) are visible to the naked eye (as spots), they may be counted, and by examining them under the microscope and observing the number of organisms they produce, their significance mas be determined. The majority of bacteria are harmless, but there decidedly are patho genic bacteria whose presence is one of the causes of malady, and of the spreading 0 certain diseases. The number of bacteria in one cubic centimetre of water sometime attains the immense figures of hundreds of thousands and millions. Certain well, spring and river waters contain very few bacteria, and are free from disease-producing bacteri under ordinary circumstances. By boiling water, the bacteria in it are killed, but th organic matter necessary for their nourishment remains in the water. The best kind of water for drinking purposes do not contain more than 300 bacteria in a cubi centinetre.

The presence in water of every residue of destroyed organisms may be partly judge from the amount of combined nitrogen, as all organisms contain nitrogen compound It is most essential to distinguish and determine nitrogen in the form of organic matter and in the form of oxides (nitric acid). The former is not separated, on heating, fron water by the action of reducing agents, such as sulphurous anyhdride, whilst th nitrogen which occurs as oxide is evolved by this means. Thus on adding hydrochlori acid and ferrous chloride to water, the nitrogen of the nitric acid gives oxide of nitrogen which may be determined. The presence of nitric acid indicates that the organi matter in water has already been oxidised. Water which contains more than 1 par of nitrogen (in this form) in a million parts is considered as injurious, and should no be used. Frankland found about 1.8 parts of nitrogen in an oxidised form, and fron 0.22 to 0.5 part in organic combinations in the water of the Thames at London.

The amount of gases dissolved in river water is much more constant than that of it solid constituents. One litre, or 1,000 c.c., of water contains 40 to 55 c.c. of ga measured at normal temperature and pressure. In winter the amount of gas is greate than in summer or autumn. Allowing that a litre contains 50 e.c. of gases, it may b admitted that these consist, on the average, of 20 vols. of nitrogen, 20 vols. of carboni 
chief chemical transformations. Further, the quantity and nature of these salts differ in different waters. ${ }^{3}$ Everybody knows that there

anlydride (proceeding in all likelihood from the soil and not from the atmosphere), and of 10 rols. of oxygen. If the total amount of gases be less, the constituent gases are still in about the same proportion; in many cases, however, carbonic anhydride predominates. The water of many deep and rapid rivers contains less carbonic anhydride, which shows their rapid formation from atmospheric water and that they have not succeeded, during a long and slow course, in absorbing a greater quantity of carbonic anlyydride. Thus, for instance, the water of the Rhine, near Strasburg, according to Deville, contains 8 c.c. of carbonic anhydride, 16 c.c. of nitrogen, and 7 c.c. of oxygen per litre. From the researches of Prof. M. R. Kapoustin and his pupils, it appears that in determining the quality of a water for drinking purposes, it is most important to investigate the composition of the dissolved gases.

3 Spring water is formed from rain water percolating through the soil. Naturally a part of the rain water is evaporated straightway from the surface of the earth and from the vegetation on it. It has been shown that out of 100 parts of water falling on the earth only 36 parts flow to the ocean; the remaining 64 are evaporated, or percolate far underground. The collection of water by means of ponds, common wells, or artesian wells is dependent on the presence of subterranean water. After flowing underground along some impervious strata, water comes out at the surface in many places as springs, whose temperature is determined by the depth from which the water has flowed. Springs penetrating to a great depth may become considerably heated, and this is why hot mineral springs, with a temperature of up to $30^{\circ}$ and higher, are often met with. For instance, there is one Caucasian spring whose temperature is $90^{\circ}$. Most likely in this case the water is heated owing to its penetrating near a rock formation which is heated by volcanic action. The composition of spring water is most varied. When a spring water contains substances which endow it with a peculiar taste, and especially if these substances are such as are only found in minute quantities or not at all in river and other flowing waters, then the spring water is termed a mineral water. Many such waters are employed for medicinal purposes. Mineral waters are classed according to their composition into-(a) saline waters, which often contain a large amount of common salt; (b) alkaline waters, which contain sodium carbonate; $(c)$ bitter waters, which contain magnesia; $(d)$ chalybeate waters, which hold iron carbonate in solution; $(e)$ aërated waters, which are rich in carbonic anhydride; $(f)$ sulphuretted waters, which contain hydrogen sulphide. Sulphuretted waters may be recognised by their smell of rotten eggs, and by their giving a black precipitate with lead salts, and also by their tarnishing silver objects. Aërated waters, which contain an excess of carbonic anhydride, effervesce in the air, have a sharp taste, and redden litmus paper. Saline waters leave a large residue of soluble solid matter on evaporation, and have a salt taste. Chalybeate

\begin{tabular}{|c|c|c|c|c|c|c|c|c|c|c|c|c|}
\hline - & 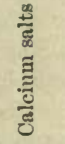 & 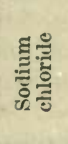 & 点苞 & 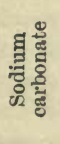 & 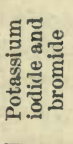 & 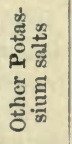 & 률 & 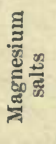 & 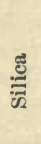 & 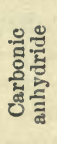 & 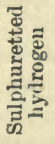 & 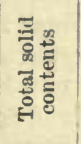 \\
\hline I. & 1,928 & - & 152 & - & - & 24 & - & 448 & 152 & 1,300 & 80 & 2,609 \\
\hline II. & 816 & 386 & 1,239 & 26 & - & 43 & 9 & 257 & 46 & 1,485 & - & 2,812 \\
\hline III. & 1,085 & 1,430 & 1,105 & - & 4 & 90 & - & 187 & 65 & 1,326 & 11 & 3,950 \\
\hline IV. & 343 & 3,783 & 16 & $\mathbf{3 , 4 3 1}$ & - & 14 & - & 251 & 112 & 2,883 & - & 7,950 \\
\hline V. & 3,406 & 15,049 & - & - & 2 & - & 17 & 1,587 & 229 & - & 76 & 20,290 \\
\hline VI. & 352 & 3,145 & - & 85 & 35 & 50 & 1 & 260 & 11 & 20 & - & 3,970 \\
\hline VII. & 308 & 1,036 & 2,583 & 1,261 & - & - & 4 & 178 & 75 & - & - & 5,451 \\
\hline VIII. & 1,726 & 9,480 & - & - & 40 & 120 & 26 & 208 & 40 & - & - & 11,790 \\
\hline IX. & 551 & 2,040 & 1,150 & 999 & - & 1 & 30 & 209 & 50 & 2,740 & - & 4,070 \\
\hline $\mathrm{x}$. & 285 & 558 & 279 & 3,813 & - & - & 7 & 45 & 45 & 2,268 & - & 5,031 \\
\hline XI. & 340 & 910 & Iron an & alumi & $\mathrm{m}$ sul & hates & $\left\{\begin{array}{l}1,020 \\
1,660\end{array}\right.$ & 940 & 190 & $\begin{array}{r}2,550 \\
330\end{array}$ & \multicolumn{2}{|c|}{$\begin{array}{l}\text { Sulphuric } \\
\text { and hydro- } \\
\text { chloricacids }\end{array}$} \\
\hline
\end{tabular}


are salt, fresh, iron, and other waters. The presence of about $3 \frac{1}{2}$ per cent. of salts renders sea-water ${ }^{4}$ heavy and bitter to the taste. Fresh water also contains salts, only in a comparatively small quantity. Their presence may be easily proved by simply evaporating water in a vessel. By evaporation the water passes away as vapour, whilst the salts are left behind. This is why a crust (incrustation), consisting of salts, previously in solution, is deposited on the insides of kettles or boilers, and other ressels in which water is boiled. Running water (rivers, \&c.) is charged with salts, owing to its being formed from the collection of rain water percolating through the soil. While percolating the water dissolves certain parts of the soil. Thus water which filters or passes through saline or calcareous soils becomes charged with salts or contains calcium carbonate (chalk). Rain water and snow are much purer than river or spring water. This is because snow and rain are only condensed aqueous vapour, and salts do not pass into the vapour.

waters have an inky taste, and are colsured black by an infusion of galls; on being exposed to the air they usually give a brown precipitate. Generally, the character of mineral waters is mixed. In the table on page 45 are given the analysis of certain mineral springs which are known for their medicinal properties. The quantity of the substances is expressed in millionths by weight-that is, in grams per cub. metre or milligrams per litre.

I. Sergieffsky, a sulphur water, Gov. of Samara (temp. $8^{\circ}$ C.), analysis by Clause. II. Geléznovodskya water source No. 10, near Patigorsk, Caucasus (temp. 22.5), analysis by Fritzsche. III. Aleksandroffsky, alkaline-sulphur source, Patigorsk (temp. 46.5 ${ }^{\circ}$, a verage of analyses by Herman Zinin and Fritzsche. IV. Bougountouksky, alkaline source, No. 17, Essentoukah, Caucasus (temp. $21^{\circ}{ }^{\circ}$ ), analysis by Fritzsche. V. Saline water, Staro-Russi, Gov. of Novgorod, analysis by Nelubin. VI. Water from artesian well at the factory of state papers, St. Petersburg, analysis by Struve. VII. Spriidel, Carlsbad (temp. $83^{\circ} 7^{\circ}$, analysis by Berzelius. VIII. Kriitznach spring (Elisenquelle), Prussia (temp. $8^{\circ} 8^{\circ}$, analysis by Bauer. IX. Eau de Seltz, Nassau, analysis by Henry. X. Vichy water, France, analysis by Berthier and Puvy. XI. Paramo de Ruiz, New Granada, analysis by Levy; it is distinguished by the amount of free acids.

- Sea-water contains more non-volatile saline constituents than the usual kinds of fresh water. This is explained by the fact that the waters flowing into the sea supply it with salts, and whilst a large quantity of vapour is given off from the surface of the sea, the salts remain behind. Even the specific gravity of sea-water differs considerably from that of pure water. It is generally about 1.02, but in this and also in respect to the amount of salts contained, samples of sea-water from different localities and from different depths offer rather remarkable variations. It will be sufficient to point out that one cubic metre of water from the undermentioned localities contains the following quantity in grams of solid constituents:-Gulf of Venice 19,122, Legliorn Harbour 24,312, Mediterranean, near Cetta, 37,655, the Atlantic Ocean from 32,585 to 35,695 , the Pacific Ocean from 35,233 to 34,708 . In closed seas which do not communicate, or are in very distant communication, with the ocean, the difference is often still greater. Thus the Caspian Sea contains 6,300 grams; the Black Sea and Baltic 17,700. Common salt forms the chief constituent of the saline matter of sea- or ocean-water; thus in one cubic metre of sea-water there are $25,000-31,000$ grams of common salt, 2,6006,000 grams of magnesium chloride, $1,200-7,000$ grams of magnesium sulphate, $1,500-6,000$ grams of calcium sulphate, and $10-700$ grams of potassium chloride. The small amount of organic matter and of the salts of phosphoric acid in sea-water is very remarkable. 
Nevertheless, in passing through the atmosphere, rain and snow succeed in catching the dust held in it, and dissolve air, which is found in every water. The dissolved gases of the atmosphere are partly disengaged, as bubbles from water on heating, and water after long boiling is quite freed from them.

In general terms water is called pure when it is clear and free from insoluble particles held in suspension and visible to the naked eye, from which it may be freed by filtration through charcoal, sand, or porous (natural or artificial) stones, and when it possesses a clean fresh taste. It depends on the absence of any tastable, decomposing organic matter, on the quantity of air ${ }^{5}$ and atmospheric gases in solution, and on the presence of mineral substances to the amount of about 300 grams per ton (or cubic metre, or, what is the same, 300 milligrams to a kilogram or litre of water), and of not more than 100 grams of organic matter. ${ }^{6} \quad$ Such water is suitable for drinking and every practical

3 The taste of water is greatly dependent on the quantity of dissolved gases it contains. On boiling, these gases are given off, and it is well known that, even when cooled, boiled water has, until it has succeeded in absorbing gaseous substances from the atmosphere, quite a different taste from fresh water containing a considerable amount of gas. The dissolved gases, especially oxygen and carbonic anhydride, have an important influence on the health. The following instance is very instructive in this respect. The Grenelle artesian well at Paris, at the first period of its opening, supplied a water which had an injurious effect on animals and people. It appeared that this water did not contain oxygen, and in general was very poor in gases. As soon as it was made to fall in a cascade, by which it absorbed air, it proved entirely fit for consumption. In long sea voyages by steamer sometimes fresh water is not taken or only taken in a small quantity because it spoils by keeping, and becomes putrid from the organic matter it contains undergoing decomposition. Fresh water may be obtained directly from sea-water by distillation. The distilled water no longer contrins sea salts, and is therefore fit for consumption, but it is very tasteless and has the properties of boiled water. In order to render it palatable certain salts, which are usually held in fresh water, are added to it, and it is made to flow in thin streams exposed to the air in order that it may become saturated with the component parts of the atmosphere-that is, absorb gases.

B Hard water is such as contains much mineral matter, and especially a large proportion of calcium salts. Such water, owing to the amount of lime it contains, does not form a lather with soap, prevents vegetables boiled in it from softening properly, and forms a great deal of incrustation on vessels in which it is boiled. Owing to its high degree of hardness, it is injurious for drinking purposes, which is evident from the fact that in many large cities the death-rate decreased after introducing a soft water in the place of a hard water. Putrid water contains a considerable quantity of decomposing organic matter, chiefly vegetable, but in populated districts, especially in towns, chiefly animal remains. Such water acquires an unpleasant smell and taste, by which stagnant bog water and the water of certain wells in inhabited districts are particularly characterised. Such water is especially harmful at a period of epidemic. It may be partially purified by passing through charcoal, which retains the putrid and certain organic substances, and also certain mineral substances. Turbid water may be purified to a certain extent by the addition of alum, which aids, after standing some time, the formation of a sediment. Condy's fluid (potassium permanganate) is another means for purifying putrid water. A solution of this substance, even if very diluted, is of a red colour; on adding it to a putrid water, the permanganate oxidises and destroys the organic matter. When added to water in such a quantity as to impart to it an almost imperceptible rose 
application, but evidently it is not pure in a chemical sense. A chemically pure water is necessary not only for scientific purposes, as. an independent substance having constant and definite properties, and as the chief component of all forms of water which play such an important part in nature, but also for many practical purposes-for instance, in photography and in the preparation of medicines-because many properties of substances in solution are changed by the impurities of natural waters. Water is usually purified by distillation, because the solid substances in solution are not transformed into vapours in this process. Such distilled water is prepared by chemists and in laboratories by boiling water in closed metallic boilers or stills, and causing the steam produced to pass into a condenser-that is, through tubes (which should be made of tin, or, at all events, tinned, as water and its impurities do not act on tin) surrounded by cold water, and in which the steam, being cooled, condenses into water which is collected $^{7}$ in a colour it destroys much of the organic substances it contains. It is especially salutary to add a small quantity of Condy's fluid to impure water in times of epidemic.

The presence in water of one gram per litre, or 1,000 grams per cubic metre, of any substance whatsoever renders it unfit and even injurious for consumption by animals, and this whether organic or mineral matter predominate. The presence of 1 p.c. of chlorides makes water quite salt, and produces thirst instead of assuaging it. The presence of magnesium salts is most unpleasant; they have a disagreeable bitter taste, and in fact impart to sea water its peculiar taste. A large amount of nitrates is only found in impure water, and is usually injurious, as they may indicate the presence of decomposing organic matter.

7 Distilled water may be prepared, or distillation in general carried on, either in a

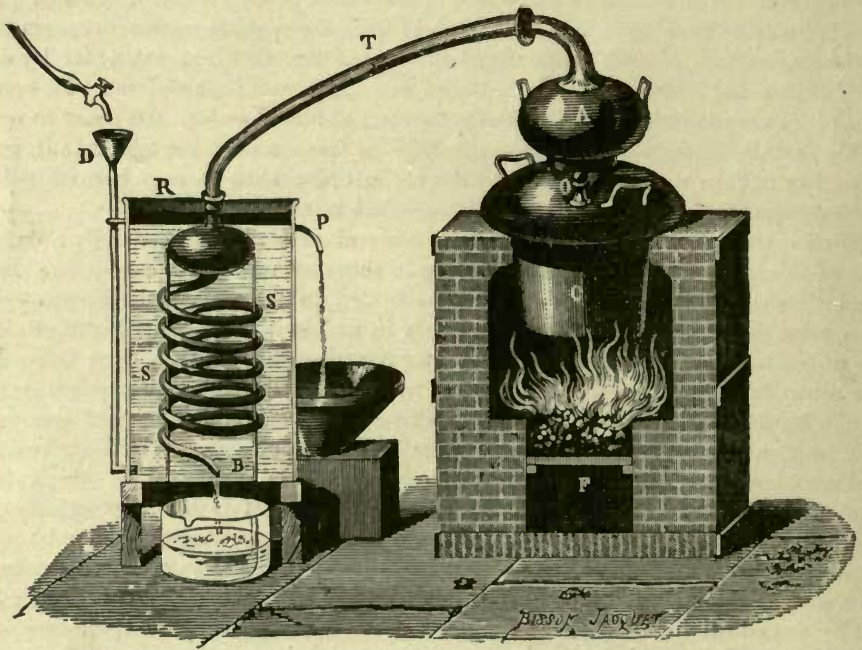

FIG. 4.-Distillation by means of a metallic still. The liquid in $\mathrm{C}$ is heated by the fire F. The vapours rise through the head $A$ and pass by the tube $T$ to the worm $S$ placed in a vesscl $R$, through which a current of cold water flows by means of the tubes D and P. 
receiver. By standing exposed to the atmosphere, however, the water in time absorbs air, and dust carried in the air, and ceases to be entirely pure. However, the amount of impurities in distilled water is so small that they have hardly any effect on the properties of the water, and it is fit for many purposes. Nevertheless, in distillation, water retains, besides air, a certain quantity of volatile impurities (especially organic) and the walls of the distillation apparatus are partly corroded by the water, and a portion, although small, of their substance renders the water not entirely pure, thus a sediment is obtained on evaporation: ${ }^{8}$

Still, for certain pliysical and chemical researches it is necessary to have completely pure water. To obtain it a solution of potassium permanganate is added to distilled water until it all becomes tinted light rose colour. By this means the organic matter in the water is destroyed (converted into gases or non-volatile substances). An excess

metal still with worm condenser (fig. 4), or on a small scale in the laboratory in a glass retort (fig. 5) heated by a lamp (see footnote 19, Introduction). Fig. 5 illustrates the main parts of the usual glass laboratory apparatus used for distillation. The steam

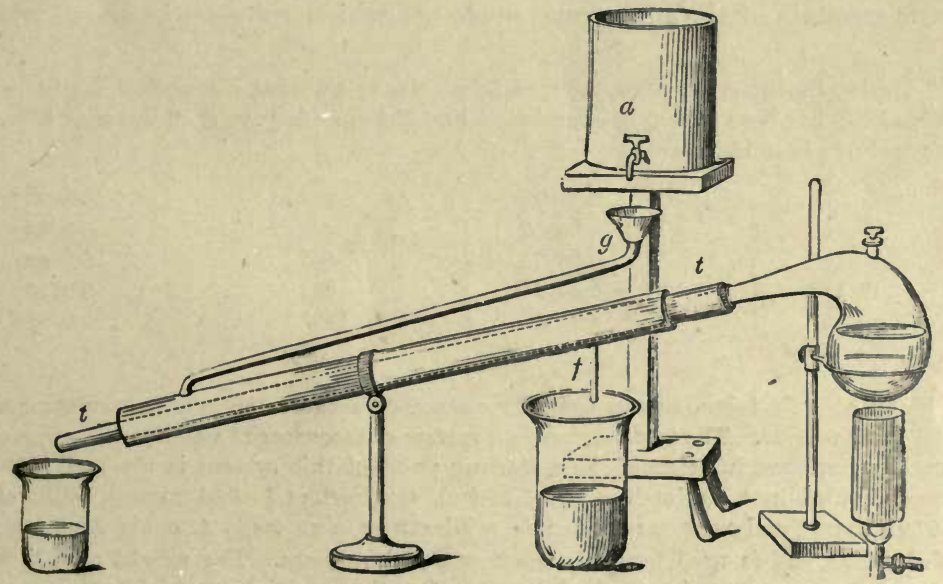

FIG. 5,-Distillation from a glass retort. The nesk of the retort fits into the inner tube of the Liebig's condenser. The sp.ce between the inmer and outer tube of the condenser is filled with cold water, which enters by the tube $g$ and flows out at $f$.

issuing from the retort (on the right-hand side) passes through a glass tube surrounded by a larger tube, through which a stream of cold water passes, by which the steam is condensed and trickles into a receiver (on the left-hand side).

8 One of Lavoisier's first memoirs (1770) referred to this question. He investigated the formation of the earthy residues in the distillation of water in order to prove whether it was possible, as was affirmed, to convert water into earth, and he found that the residue was produced by the action of water on the walls of the vessel holding it, and not from the water itself. He proved this to be the case by direct weighing.

VOL. I. 
of potassium permanganate does no harm, because in the next distillation it is left behind in the distillation apparatus. The next distillation should then be from a platinum retort with a platinum receirer. Platinum is a metal which is not in any way changed either by air or water, and therefore nothing passes from it into the water. The water obtained in the receiver still contains air. It must then be boiled for a long time, and afterwards cooled in a vacuum under the receiver of an air pump. Pure water on evaporation does not give any sediment, does not in the least change, however long it be kept, and if air have no access to it does not putrefy like water only once distilled or impure; and it does not give bubbles of gas on heating, nor does it change the colour of a solution of potassium permanganate. These are a few signs by which the complete purity of water may be recognised.

Water, purified as above described, has constant physical and chemical properties. For instance, it is of such water only that one cubic centimetre weighs one gram at $4^{\circ}$ C. - i.e., it is only such pure water whose specific gravity equals 1 at $4^{\circ} \mathrm{C} .^{9} \quad$ Water in a solid state forms crystals of the hexagonal system ${ }^{10}$ which are seen in snow, which

9 Taking the generally-accepted specific gravity of water at its greatest density-i.e. at $4^{\circ}$ as 1 -it has been shown by experiment that the specific gravity of water at different temperatures is as follows:-

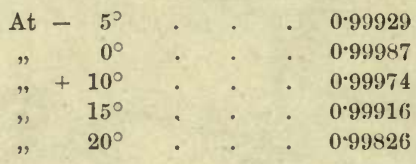

$\begin{array}{rrrrrr}\text { At } & 30^{\circ} & . & . & . & 0.99577 \\ " & 40^{\circ} & . & . & . & 0.99236 \\ " & 50^{\circ} & . & . & . & 0.98817 \\ " & 80^{\circ} & . & . & . & 0.97192 \\ " & 100^{\circ} & . & . & . & 0.95854\end{array}$

Water at $4^{\circ}$ is taken as the basis for reducing measures of length to measures of weight and volume. The metric, decimal, system of measures of weights and volumes is generally employed in science. The starting point of this system is the metre $(39.37$ inches) divided into decimetres $(=0.1$ metre), centimeties $(=0.01$ metre), millimetres $(=0.001$ metre), and micrometres ( $=$ one millionth of a metre). A cubic decimetre is called a litre, and is used for the measurement of volumes. The weight of a litre of water at $4^{\circ}$ in a vacuum is called a kilogram. One thousandth part of a kilogram, or one cubic centimetre, of water weighs one gram. It is divided into decigrams, centigrams, and milligrams $(=0.001 \mathrm{gram})$. An English pound equals 453.59 grams. The great advantage of this system is that it is a decimal one, and that it is universally adopted in science and in most international relations. All the measures cited in this work are metrical. The units most often used in science are:-Of length, the centimetre; of weight, the gram; of time, the second; of temperature, the degree Celsius or Centigrade.

10 As solid substances appear in independent, regular, crystalline forms which are dependent, judging from their cleavage or lamination (in virtue of which mica breaks up into laminae and Iceland spar, \&c., into pieces bounded by faces inclined to each other at angles which are definite for each substance), on an inequality of attraction (coinesion hardness) in different" directions which intersect at definite angles; therefore, the determination of crystalline forms offers one of the most important external marks 
grenerally consists of star-like clusters of several crystals, and also in the half-melted scattered ice floating on rivers in spring time. At characterising separate, definite chemical compounds. The elements of crystallography which comprise a special science, should therefore be familiar to all who desire to work

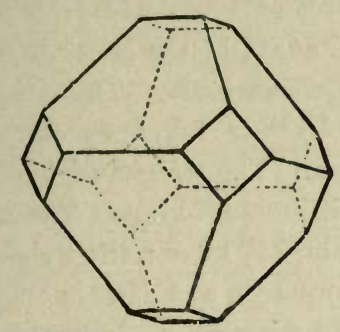

Fur. 6.-Example of the form belonging to the regular system. Combination of an octahedron and a cube. The former predominates. Alum, fluor spar, suboxide of copper, and others.

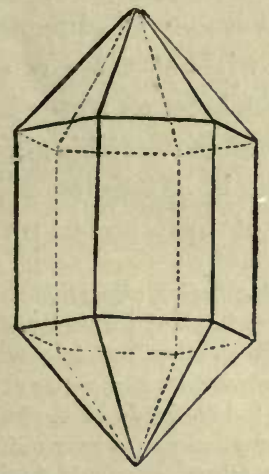

FIG. ४.-Hexagonal prism terminated by liexagonal pyramids. Quaitz, de.

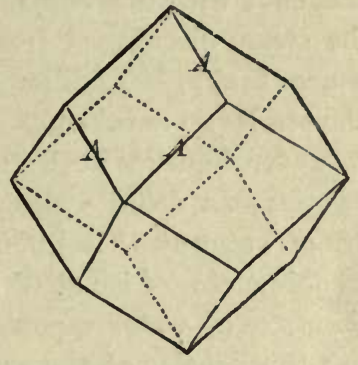

Fig. 7.-Rhombic Dodecahedron of the resular system. Garnet.

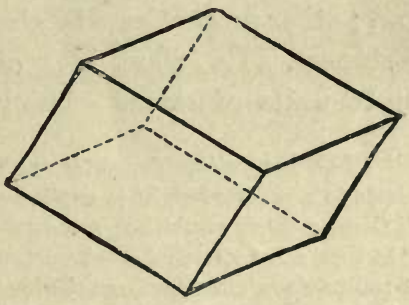

Fi木. 9.-Rhomboledron. Ca!c spar, \&c.

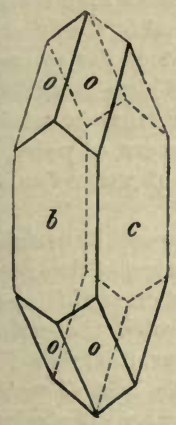

FIG. 10.-Rhombic system. Desmine.

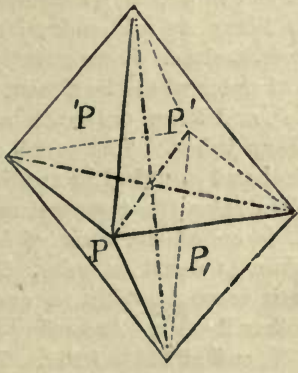

FIg. 11.-Triclinic pyramid.

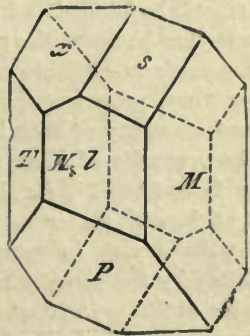

FIG. 12.-Triclinic system. Albite, \&c.

in scientific chemistry. In this work we shall only have occasion to speak of a few crystalline forms, some of which are shown in Figs. 6 to 12. 
this time of the year the ice splits up into spars or prisms, bounded by angles proper to substances crystallising in the hexagonal system. The temperatures at which water passes from one state to another are taken as fixed points on the thermometer scale; namely, the zero corresponds with the temperature of melting ice, and the temperature of the steam disengaged from water boiling at the normal barometer pressure (that is 760 millimetres measured at $0^{\circ}$, at the latitude of $45^{\circ}$, at the sea level) is taken as $100^{\circ}$ of the Celsius scale. Thus, the fact that water liquefies at $0^{\circ}$ and boils at $100^{\circ}$ is taken as one of its properties as a definite chemical compound. The weight of one cubic metre of water at $4^{\circ}$ is 1,000 kilos, at $0^{\circ}$ it is 999.8 kilos. The weight of a cubic metre of ice at $0^{\circ}$ is less-namely, 917 kilos ; the weight of a cubic metre of water vapour at $760 \mathrm{~mm}$. pressure and $100^{\circ}$ is only 0.60 kilos; the density of the vapour compared with air $=0.62$, and compared with hydrogen $=9$.

These data briefly enumerate the physical properties of water as a separate substance. As a supplement to this it may be added that water is a mobile liquid, colourless, transparent, without taste or smell, drc. It is unnecessary to dwell on these properties here, as water is familiar to all ; other properties will also be pointed out in describing less known substances. Its latent heat of vaporisation is 534 units, of liquefaction 79 units of heat. ${ }^{11}$ The large amount of heat stored up in water

11 Of all known liquids, water exhibits the greatest cohesion of particles. Indeed, it ascends to a greater height in capillary tubes than other liquids; for instance, two and a half times as high as alcohol, nearly three times as high as ether, and to a much greater height than oil of vitriol, \&c. In a tube of two millimetres diameter, water at $0^{\circ}$ ascends 15.3 millimetres, counting from the level of the liquid to two-thirds of the height of the meniscus, and at $100^{\circ}$ it rises 12.5 millimetres. The cohesion varies very uniformly with the temperature; thus at $50^{\circ}$ the height of the capillary column equals 13.9 millimetresthat is, the mean between the columns at $0^{\circ}$ and $100^{\circ}$. This uniformity is not destroyed even on approaching the freezing point, and gives reason to think that at high temperatures cohesion will vary as uniformly as at ordinary temperatures; that is, the difference between the columns at $0^{\circ}$ and $100^{\circ}$ being 2.8 millimetres, the height of the column at $500^{\circ}$ should be $15 \cdot 2-(5 \times 2.8)=1 \cdot 2$ millimetres. Consequently, at these high temperatures the cohesion between the particles of water would be almost nil. Only certain solutions (sal ammoniac c.-il lithium chloride), and these only with a great excess of water, rise higher than pure water in capillary tubes. The great colnesion of water doubtless determines many of both its physical and chemical properties.

The quantity of heat required to raise the temperature of one part by weight of water from $0^{\circ}$ to $1^{\circ}$, i.e., by $1^{\circ} \mathrm{C}$, is called the unit of heat or calorie; the specific heat of liquid water at $0^{\circ}$ is taken as equal to anity. The variation of this specific heat with a rise in temperature is inconsiderable in comparison with the variation exhibited by the specific heats of other liquids. According to Ettinger, the specific heat of water at $20^{\circ}=1 \cdot 016$, at $50^{\circ}=1.039$, and at $100^{\circ}=1 \cdot 073$. The specific heat of water is greater than that of all other known liquids; for example, the specific heat of alcoliol at $0^{\circ}$ is $0.5475-i . e$., the quantity of heat which raises 55 parts of water $1^{\circ}$ raises 100 parts of alcohol $1^{\circ}$. The specific heat of oil of turpentine at $0^{\circ}$ is 0.4106 , of ether 0.529 , of acetic acid 0.5274 , of mercury 0.033 . This means that water is the best condenser or 
vapour and also in liquid water (for its specific heat is greater than that of other liquids) renders it available in both forms for heating

absorber of heat. This property of water has an important significance in practice and in nature. Water impedes rapid cooling or heating; it tempers cold and heat. The specific heats of ice and aqueous vapour are much less than that of water; namely that of ice is 0.504 , and of steam 0.48 .

With an increase in pressure equal to one atmosphere, the compressibility of water is 0.000047 , of mercury 0.00000352 , of ether 0.00012 at $0^{\circ}$, of alcohol at $13^{\circ} 0.000095$. The addition of various substances to water generally simultaneously decreases its compressibility and colhesion. The compressibility of other liquids increases with a rise of temperature, but for water it decreases up to $53^{\circ}$ and then increases like other liquids.

The expansion of water by heat (Note 9) also exhibits many peculiarities which are not found in other liquids. The expansion of water at low temperatures is very small compared with other liquids; at $4^{\circ}$ it reaches even 0 , and at $100^{\circ}$ it is equal to 0.0008 ; below $4^{\circ}$ it is negative-i.e., water on cooling then expands, and does not decrease in volume. In passing into a solid state, the specific gravity of water decreases; at $0^{\circ}$ one c.c. of water weighs 0.999888 gram, and one c.c. of ice at the same temperature weighs only 0.9175 gram. The ice formed, however, contracts on cooling like the majority of other substances. Thus 100 volumes of ice are produced from 92 volumes of water-that is, water expands considerably on freezing, which fact determines a number of natural phenomena. The freezing point of water falls with an increase in pressure $\left(0.007^{\circ}\right.$ per atmosphere), because in freezing water expands (Thomson), whilst with substances which contract in solidifying the melting point rises with an increase in pressure; thus, for paraffin it is at one atmosphere $46^{\circ}$ and at 100 atmospheres $49^{\circ}$.

When liquid water passes into vapour, the cohesion of its particles must be destroyed, as the particles are removed to such a distance from each other that their mutual attraction no longer exhibits any influence. As the cohesion of aqueous particles varies at different temperatures, the quantity of heat which is expended in overcoming this cohesion-or the latent heat of evaporation - for this reason alone will be different at different temperatures. The quantity of heat which is consumed in the transformation of one part by weight of water, at different temperatures, into vapour was determined by Regnault with great accuracy. His researches showed that one part by weight of water taken at $0^{\circ}$, in passing into vapour having a temperature $t^{\circ}$, consumes $606.5+0.305 t$ units of heat, at $50^{\circ} 621 \cdot 7$, at $100^{\circ} 637^{\circ} 0$, at $150652 \cdot 2$, and at $200^{\circ} 667 \cdot 5$. But this quantity includes also the quantity of heat required for heating the water from $0^{\circ}$ to $t^{\circ}-$ i.e., besides the latent heat of evaporation, also that heat which is used in heating the water in a liquid state to a temperature $t^{\circ}$. On deducting this amount of heat, we obtain the latent of evaporation of water as 606.5 at $0^{\circ}, 571$ at $50^{\circ}, 534$ at $100^{\circ}, 494$ at $150^{\circ}$, and only 453 at $200^{\circ}$, which shows that the conversion of water at different temperatures into vapour at a constant temperature requires very different quantities of heat. This is chiefly dependent on the difference of the cohesion of water at different temperatures; the cohesion is greater at low than at high temperatures, and therefore at low temperatures a greater quantity of heat is required to overcome the cohesion. On comparing these quantities of heat, it will be observed that they decrease rather uniformly, namely their difference between $0^{\circ}$ and $100^{\circ}$ is 72 , and between $100^{\circ}$ and $200^{\circ}$ is 81 units of heat. From this we may conclude that-this variation will be approximately the same for high temperatures also, and therefore that no heat would be required for the conversion of water into vapour at a temperature of about $400^{\circ}-600^{2}$. At this temperature, water passes into vapour whatever be the pressure (see chap. II. The absolute boiling point of water, according to Dewar, is $370^{\circ}$, the critical pressure 196 atmospheres). It must here be remarked that water, in presenting a greater cohesion, requires a larger quantity of heat for its conversion into vapour than other liquids. Thus alcohol consumes 208 , ether 90 , turpentine 70 , units of heat in their conversion into vapour.

The whole amount of heat which is consumed in the conversion of water into vapour is not used in surmounting the colesion-that is, in internal work accomplished in the 
purposes. The chemical reactions which water undergoes, and by means of which it is formed, are so numerous, and so closely allied to

liquid. A part of this heat is employed in moving the aqueous particles; in fact, aqueous vapour at $100^{\circ}$ occupies a volume 1,650 times greater than that of water (at the ordinary pressure), consequently a portion of the heat or work is employed in lifting the aqueous particles, in overcoming pressure, or in external work, which may be usefully employed and which is so employed in steam engines. In order to determine this work we will first separately consider all the factors necessary for this calculation, and we will then make a deduction from the comparison of these factors.

The maximum pressure or tension of aqueous vapour at different temperatures has been determined with great exactitude by many observers. The observations of Regnault in this respect, as on those preceding, deserve special attention from their comprehensiveness and accuracy. The pressure or tension of aqueous vapour at various temperatures is given in the adjoining table, and is expressed in millimetres of the barometric column having a temperature of $0^{\circ}$.

\begin{tabular}{|c|c|c|c|}
\hline Temperature & Tension & Temperature & Tension \\
\hline$-20^{\circ}$ & $0: 9$ & $70^{\circ}$ & $233 \cdot 3$ \\
$-10^{\circ}$ & $2 \cdot 1$ & $90^{\circ}$ & $525 \cdot 4$ \\
$0^{\circ}$ & $4 \cdot 6$ & $100^{\circ}$ & $760 \cdot 0$ \\
$+10^{\circ}$ & $9 \cdot 1$ & $105^{\circ}$ & $906 \cdot 4$ \\
$15^{\circ}$ & $12 \cdot 7$ & $110^{\circ}$ & $1075 \cdot 4$ \\
$20^{\circ}$ & $17 \cdot 4$ & $115^{\circ}$ & $1269 \cdot 4$ \\
$25^{\circ}$ & $23 \cdot 5$ & $120^{\circ}$ & $1491 \cdot 3$ \\
$30^{\circ}$ & $31 \cdot 5$ & $150^{\circ}$ & $3581 \cdot 0$ \\
$50^{\circ}$ & $92 \cdot 0$ & $200^{\circ}$ & $11689 \cdot 0$ \\
\hline
\end{tabular}

The table shows the boiling points of water at different pressures. Thus on the summit of Mont Blanc, where the average pressure is about $424 \mathrm{~mm}$., water boils at $84^{\circ} 4^{\circ}$. In a rarefied atmosphere water boils at even the ordinary temperature, but in evaporating it absorbs heat from the neighbouring parts, and therefore it becomes cold and may even freeze if the pressure does not exceed $4.6 \mathrm{~mm}$., and especially if the vapour be rapidly absorbed as it is formed. Oil of vitriol, which absorbs the aqueous vapour, is used for this purpose. Thus ice may be obtained artificially at the ordinary tenperature with the aid of an air-pump. This table of the tension of aqueous vapour also shows the temperature of water contained in a closed boiler if the pressure of the steam formed be known. Thus at a pressure of five atmospheres (a pressure of five times the ordinary atmospheric pressure-i.e., $5 \times 760=3,800 \mathrm{~mm}$.) the temperature of the water would be 152 '. The table also shows the pressure produced on a given surface by steam on issuing from a boiler. Thus steam having a temperature of $152^{\circ}$ exerts a pressure of $517 \mathrm{kilos}$. on a piston whose surface equals 100 sq. c.m., for the pressure of one atmosphere on one sq. c.m. equals 1,033 kilos., and steam at $152^{\circ}$ has a pressure of five atmospheres. As a column of mercury $1 \mathrm{~mm}$. high exerts a pressure of 1.35959 grams on a surface of 1 sq. c.m., therefore the pressure of aqueous vapour at $0^{\circ}$ corresponds with a pressure of 6.25 grams per square centimetre. The pressures for all temperatures may be calculated in a similar way, and it will be found that at $100^{\circ}$ it is equal to $1,033.28$ grams. This means that if a cylinder be taken whose sectional area equals 1 sq. c.m., and if water be poured into it and it be closed by a piston weighing 1,033 grams, then on heating it in a vacuum to $100^{\circ}$ no steam will be formed, because the steam cannot orercome the pressure of the piston; and if at $100^{\circ} 534$ units of heat be transmitted to each unit of weight of water, then the whole of the water will be converted into vapour having the same temperature; and so also for every other temperature. The question now arises, To what height does the piston rise under these circumstances; that is, in other words, What is the volume occupied by the steam under a known pressure? For this we must know 
the reactions of many other substances, that it is impossible to describe the majority of them at this early stage of chemical exposition. Afterwards we shall become acquainted with many of them, but at present we shall only cite certain compounds formed by water. In order to see clearly the nature of the various kinds of compounds formed by

the weight of a cubic centimetre of steam at various temperatures. It has been shown by experiment that the density of steam, which does not saturate a space, varies very inconsiderably at all possible pressures, and is nine times the density of hydrogen under similar conditions. Steam which saturates a space varies in density at different temperatures, but this difference is very small, and its arerage density with reference to air is 0.64 . We will employ this figure in our calculation, and will calculate what volume the steam occupies at $100^{\circ}$. One cubic centimetre of air at $0^{\circ}$ and $760 \mathrm{~mm}$. weighs $0.001293 \mathrm{gram}$, at $100^{\circ}$ and under the same pressure it will weigh $\frac{0.001293}{1 \cdot 368}$ or about

0.000946 gram, and consequently one cubic centimetre of steam whose density is 0.64 will weigh 0.000605 gram at $100^{\circ}$, and therefore one gram of aqueous vapour will occupy a volume of about 1,653 c.c. Consequently, the piston in the cylinder of 1 sq. c.m. sectional area, and in which the water occupied a height of 1 c.m., will be raised $1,653 \mathrm{c} . \mathrm{m}$. on the conversion of this water into steam. This piston, as has been mentioned, weighs 1,033 grams, therefore the external work of the steam-that is, that work which the water does in its conversion into steam at $100^{\circ}$-is equal to lifting a piston weighing 1,033 grams to a height of $1,653 \mathrm{c.m}$., or $17 \cdot 07$ kilogram-metres of work-i.e., is capable of lifting 17 kilograms 1 metre, or 1 kilogram 17 metres. One gram of water requires for its conversion into steam 534 gram units of heat or 0.534 kilogram units of heat - i.e., the quantity of heat absorbed in the evaporation of one gram of water is equal to the quantity of heat which is capable of heating 1 kilogram of water $0.534^{\circ}$. Each unit of heat, as has been shown by accurate experiment, is capable of doing 424 kilogrammetres of work. Therefore, in evaporating, one gram of water expends $424 \times 0.534=$ (almost) 226 kilogram-metres of work. The external work was found to be only 17 kilogram-metres, therefore 209 kilogram-metres are expended in overcoming the internal colesion of the aqueous particles, and consequently about 92 p.c. of the heat or work consumed goes in overcoming the internal cohesion. The following figures are thus calculated approximately :-

$\begin{array}{cc}\text { Temperature } & \begin{array}{c}\text { Total work of } \\ \text { evaporation in } \\ \text { Kilogram-metres }\end{array} \\ 0^{\circ} & 255 \\ 50^{\circ} & 242 \\ 100^{\circ} & 226 \\ 150^{\circ} & 209 \\ 200^{\circ} & 192\end{array}$
Exterual work of vapour in Kilograml-metres

$\begin{array}{ll}13 & 242 \\ 15 & 227 \\ 17 & 209 \\ 19 & 190 \\ 20 & 172\end{array}$

Internal work of vapour

Thus it will be remarked from this table that the work necessary for overcoming the internal colresion of water in its passage into vapour decreases with the rise in temperature; this is in connection with the decrease of cohesion with a rise in temperature, and, in fact, the variations which take place in this case are very similar to those which are observed in the heights to which water rises in capillary tubes at different timperatures. It is evident, therefore, that the amount of externul-or, as it is termed, useful-work which water can supply by its evaporation is very small compared with the amount which it expends in its conversion into vapour.

In considering certain physico-mechanical properties of water, I had in view not only their inportance for theory and practice, but also their purely chemical significance, for it is evident from the above considerations that in even a physical change of state the greatest part of the work accomplished goes in overcoming cohesion, and that chemical cohesion, or affinity, is an enormous internal energy. 
water we will begin with the most feeble, which are determined by purely mechanical superficial properties of the reacting substances. ${ }^{12}$

Water is mechanically attracted by many substances; it adheres to their surfaces just as dust adheres to objects, and one polished glass adheres to another. Such attraction is termed ' moistening,' 'soaking,' or' 'absorption of water.' Thus water moistens clean glass and adheres to its surface, is absorbed by the soil, sand, and clay, and does not flow away from them but lodges itself between their particles. Similarly, water soaks into a sponge, cloth, hair, or paper, dcc., but fat and greasy substances in general are not moistened. Attraction of this kind does not alter the physical or chemical properties of water. For instance, under these circumstances water, as is known from everyday experience, may be expelled from objects by drying. Water which is in any way held mechanically may be dislodged by mechanical means, by friction, pressure, centrifugal force, \&c. Thus water is squeezed from wet cloth by pressure or centrifugal machines. But objects which in practice are called dry (because they do not wet people's hands) often still contain moisture, as may be proved by heating the object in a glass tube closed at one end. By placing a piece of paper, dry earth, or any similar object (especially porous substances) in such a glass tube, and heating that part of the tube where the object is situated, it will be remarked that water condenses on the cooler portions of the tube. The presence of such absorbed, or, as it is termed, 'hygroscopic,' water is generally best recognised in non-volatile substances by drying at $100^{\circ}$,

12 When it is necessary to heat a considerable mass of liquid in different vessels, it would be very uneconomical to make use of metallic vessels and to construct a separate fire grate under each one; such cases are continually met with in practice. A considerable mass of water, for instance, may have to be heated for making solutions, or it may be required to expel volatile liquids from different vessels at intermittent periods; as, for instance, alcohol from partially fermented liquors, \&c. In such cases one boiler or vessel containing water is made use of. Steam from this boiler is introduced into the liquid, or, in general, into the ressel which it is required to heat. The steam, in condensing and passing into a liquid state, parts with its latent heat, and as this is very considerable a small quantity of steam will produce a considerable heating effect. If it be required, for instance, to heat 1,000 kilos. of water from $20^{\circ}$ to $50^{\circ}$, which requires approximately 30,000 units of heat, steam heated to $100^{\circ}$ is passed into the water from a boiler. Each kilogram of water at $50^{\circ}$ contains about 50 units of heat, and each kilogram of steam at $100^{\circ}$ contains 637 units of heat; therefore, each kilogram of steam in cooling to $50^{\circ}$ gives up 587 units of heat, and consequently 52 kilos of steam are capable of accomplishing the required heating of 1,000 kilos. of water from $20^{\circ}$ to $50^{\circ}$. Water is very often applied for heating in chemical practice. For this purpose metallic vessels or pans, called 'water-baths,' are made use of. They are closed by a cover formed of concentric rings lying on each other. The objects-such as beakers, evaporating basins, retorts, \&c.-containing liquids are placed on these rings, and the water in the bath is heated. The steam given off heats the bottom of the ressels to be heated, and thus accomplishes the evaporation or distillation or other required process. A water-bath may also be used for heating a vessel directly inmersed in the water. 
or under the receiver of an air-pump and over substances which attract water chemically. By weighing a substance before and after drying, it is easy to determine the amount of hygroscopic water from the loss in weight. ${ }^{13}$ Only in this case the amount of water must be judged with

13 In order to dry any substance at about $100^{\circ}$ - that is, at the boiling point of water (hygroscopic water passes off at this temperature) - an apparatus called a 'drying-oven' is employed. It consists of a double copper box; water is poured into the space between the internal and external boxes, and the oven is then heated over a stove or by any other means, or else steam from a boiler is passed between the walls of the two boxes. When the water boils, the temperature inside the inner box will be approximately $100^{\circ} \mathrm{C}$. The substance to be dried is placed inside the oven, and the door is closed. Several holes are cut in the door to allow the free passage of air, which carries off the aqueous vapour by the chimney on the top of the oven. Often, however, desiccation is carried on in copper ovens heated directly over a lamp fig. 13). In this case any desired

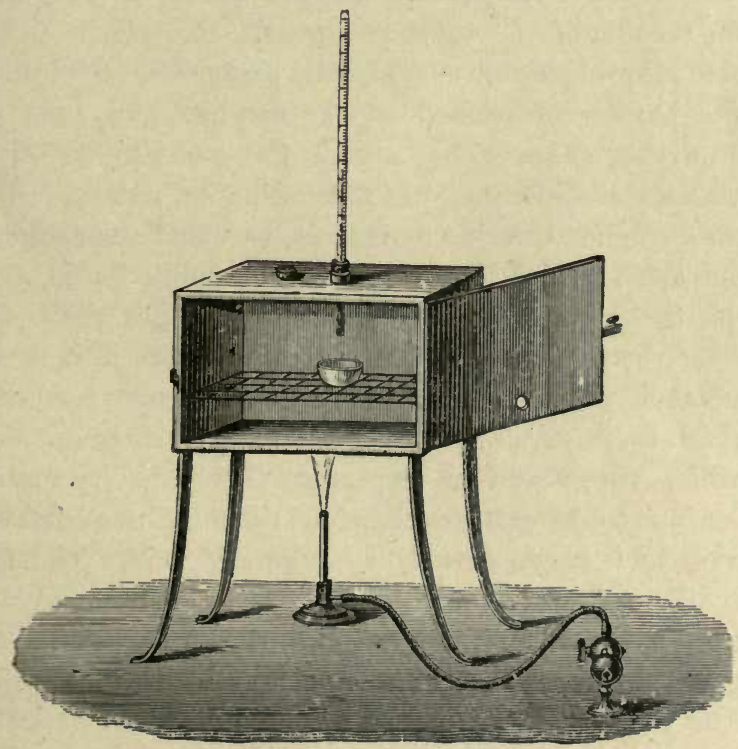

Fig. 13.-Drying ovell, composed of brazed copper. It is heated by a lamp. The object to be dried is placel on the gauze inside the oven. The thermometer indicates the temperature.

temperature may be obtained, which is determined by a thermometer fixed in a special orifice. There are substances which only part with their water at a much higher temperature than $100^{\circ}$, and then such air baths are very useful. In order to directly determine the amount of water in a substance which does not part with anything except water at a red heat, the substance is placed in a bulb tube. By first weighing the tube empty and then with the substance to be dried in it, the weight of the substance taken may be found. The tube is then connected on one side with a gas-helder full of air, which, on opening a stop-cock, passes first through a flask containing sulphuric acid, and then into a vessel containing lumps of pumice stone moistened with sulphuric acid. In passing through these vessels the air is thoroughly dried, having given up all its moisture to the sulphuric acid. Thus dry air will pass into the bulb tube, and as hygroscopic water is entirely given up from a substance in dry air at even the ordinary temperature, and still 
care, because the loss in weight may sometimes proceed from the decomposition of the substance itself, with disengagement of gases or vapour. In making exact weighings the hygroseopic capacity of substances - that is, their capacity to absorb moisture - must be continually kept in view, as otherwise the weight will be untrue from the presence of moisture. The quantity of moisture absorbed depends on the degree of moisture of the atmosphere (that is, on the tension of the aqueous vapour in it) in which a substance is situated. In an entirely dry atmosphere, or in a vacuum, the hygrosiopic water is expelled, being converted into vapour; therefore, if we have the means of drying gases (or a vacuum) - that is, of removing the aqueous vapour from themobjects impregnated with water may be entirely dried by placing them in such a desiccated atmosphere. The process is aided by heat, as it increases the tension of the aqueous vapour. Phosphoric anhydride (a white powder), liquid sulphuric acid, solid and porous calcium chloride, or the white powder of ignited copper sulphate are most generally employed in drying gases. They absorb the moisture contained in air and all gases to a considerable, but not unlimited, extent. Phosphoric anhydride and calcium chloride deliquesce, become damp, sulphuric acid changes from an oily thick liquid into a more mobile liquid, and ignited copper sulphate becomes blue; after which changes these substances partly lose their capacity of holding water, and can, if it be in excess, even give up their water to the atmosphere. We may remark that the order in which these substances are placed above corresponds with the order in which they stand in respect to their capacity for absorbing moisture. Air dried by calcium chloride still contains a certain amount of moisture, which it can give up to sulphuric acid. The most complete desiccation takes place with phosphoric anhydride. Water is also removed from many substances by placing them in a basin over a vessel containing a substance absorbing water under a glass bell. ${ }^{14}$ The bell, like the receiver of an air pump, should be hermetically closed.

more rapidly on heating, the moisture given up by the substance in the tube will be carriet off by the air passing through it. This damp air then passes through a $U$-shaped tulue full of pieces of pumice stone moistened with sulphuric acid, which absorbs all the moisture given off from the substance in the bulb tube. Thus all the water expelled from the substance will collect in the $U$ tube, and so, if this be weighed before and after, the difference will show the quantity of water expelled from the substance. If only water (and not any gases) come over, the increase of the weight of the $U$ tube will be equal to the decrease in the weight of the bulb tube.

14 Instead of under a glass bell, drying over sulphuric acid is often carried on in a desiccator composed of a wide-mouthed low glass vessel, closed by a well-fitting groundglass stopper. Sulphuric acid is poured over the bottom of the desiccator, and the substance to be dried is placed on a glass stand above the acid. A lateral glass tube with a stop-cock is often fused into the desiccator in order to connect it with an air pump, and so allow drying under a diminished pressure, when the moisture evaporates more rapidly. 
In this case desiccation takes place ; because sulphuric acid, for instance, first dries the air in the bell by absorbing its moisture, the substance to be dried then parts with its moisture to the dry air, from which it is again absorbed by the sulphuric acid, dc. Desiccation proceeds still better under the receiver of an air pump, for then the aqueous vapour is formed more quickly than in a bell full of air.

From what has been said above, it is evident that the transference of moisture to gases and the absorption of hygroscopic moisture present great resemblance to, but still are not, chemical combinations with water. Water, when combined as hygroscopic water, does not lose its properties and does not form new substances. ${ }^{15}$

The attraction of water for substances which dissolve in it is of a different character. In the solution of substances in water there proceeds a peculiar kind of indefinite combination ; there is formed a new homogeneous substance from the two substances taken. But here also the bond connecting the substances is very unstable. Water containing different substances in solution boils at a temperature near to its usual boiling point, and acquires properties which are closely allied to the properties of water itself and of the substances dissolved in it. Thus, from the solution of substances which are lighter than water itself, there are obtained solutions of a less density than water-as, for example, in the solution of alcohol in water; whilst a heavier substance in dissolving in water gives it a higher specific gravity. Thus salt water is heavier than fresh. ${ }^{16}$

We will consider aqueous solutions somewhat fully, because, among other reasons, solutions are constantly being formed on the earth and in the waters of the earth, in plants and in animals, in chemical practice and in the arts, and these solutions play an important part in the chemical transformations which are everywhere taking place, not only because water is everywhere met with, but chiefly because a substance in solution presents the most propitious conditions for the process of chemical changes, which require a mobility of parts and an intimate

15 Chapuy, however, determined that in wetting 1 gram of charcoal with water 7 units of heat are evolved, and on pouring carbon bisulphide over 1 gram of charcoal as much as 24 units of heat are evolved. Alumina (1 gram), when moistened with water, evolves $2 \frac{1}{2}$ calories. This indicates that even in respect to evolution of heat moistening already presents a transition towards exothermal combinations (those evolving heat in their formation), like solutions.

${ }_{16}$ Strong acetic acid $\left(\mathrm{C}_{2} \mathrm{H}_{4} \mathrm{O}_{2}\right)$, whose specific gravity at $15^{\circ}$ is 1.055 , does not become lighter on the addition of water (a lighter substance, sp. gr. $=0.999$ ), but heavier, so that a solution of 80 parts of acetic acid and 20 parts of water has a specific gravity of 1.074, and even a solution of equal parts of acetic acid and water ( 50 p.c.) has a sp. gr. of 1.065 , which is still greater than that of acetic acid itself. This shows the high degree of contraction which takes place on solution. In fact, solutions-and, in general, liquids-on mixing with water, decrease in volume. 
contact. In dissolving, a solid substance acquires a mobility of parts, and a gas loses its elasticity, and therefore reactions often take place in solutions which do not proceed in the undissolved substances. Further, a substance, clistributed in water, evidently breaks up (or 'disintegrates ') - that is, becomes more like a gas and acquires a greater mobility of parts. All these considerations require that in describing

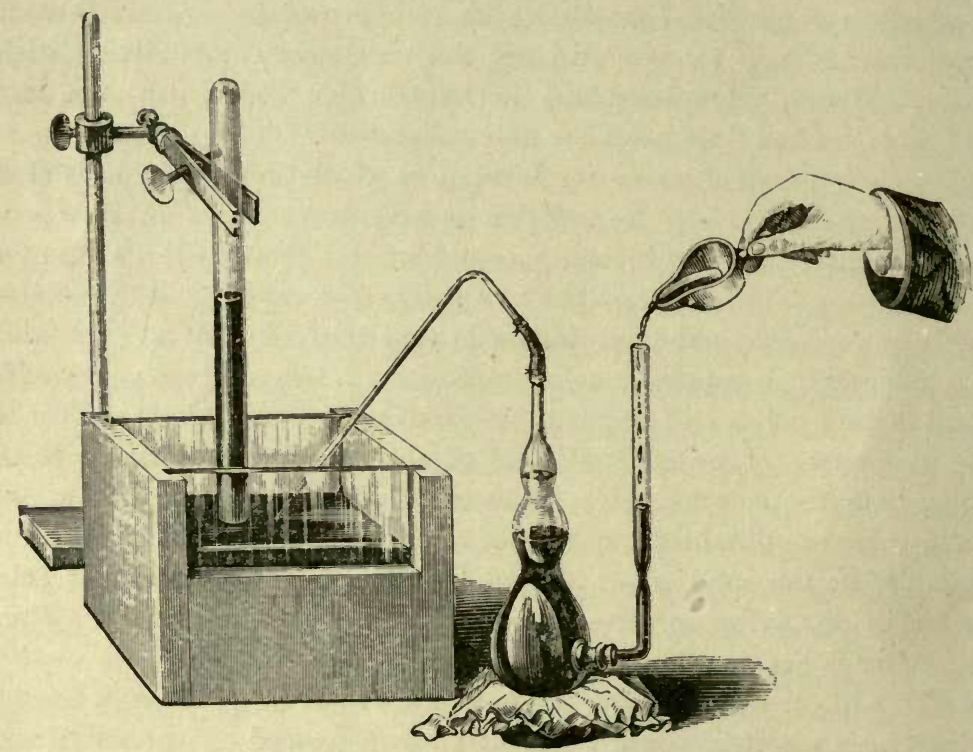

FIt. 14.-Metlod of transfermng a gas into a cylinder filled with mercury and whose open end is inmersel under the mercury in a bath having two glass silles. The apparatus containing the gas is represented on the right. Its upper extremity is furnished with a tube extending under the cylinder. The lower part of the vessel communicates with a vertical tube. If mercury bc poured into this tube, the pressure of the gas in the apnaratus is increasel, and it passes through the gasconducting tube into the cylinder, where it displaces the mercury, and can be measuret or subjectel to the action of absorbing agents, such as water.

the properties of substances, particular attention should be paid to their relation to water as a solvent.

Everybody knows that water dissolves many substances. Salt, sugar, alcohol, and a number of other substances, by dissolving in water form with it homogeneous liquids. To clearly show the solubility of gases in water a gas should be taken which has a high co-efficient of solubility-for instance, ammonia. This is introduced into a bell (or cylinder, as in fig. 14), which is previously filled with mercury and stands in a mercury bath. If water be then introduced into the cylinder, the mercury will rise, owing to the water dissolving the ammonia gas. If the column of mercury be less than the barometric 
column, and if there be sufficient water to dissolve the gas, all the ammonia will be absorbed by the water. The water is introduced into the cylinder by a glass pipette, with a bent end. Its bent end is put into water, and the air is sucked out from the upper end. When full of water, its upper end is closed with the finger, and the bent end placed in the mercury bath under the orifice of the cylinder. The water will then be forced from the pipette by the atmospheric pressure, and will rise to the surface of the mercury in the cyiinder owing to its lightness. The solubility of a gas like ammonia may be demonstrated by taking a flask full of the gas, and closed by a cork with a tube passing through it. On placing the tube under water, the water will rise into the flask (this may be accelerated by heating the flask), and begin to play like a fountain inside it. Both the rising of the inercury and the fountain clearly show the considerable affinity of water for ammonia gas, and the force acting in this dissolution is rendered evident. For both the homogeneous intermixture of gases (diffusion) and the process of solution a certain period of time is required, which depends, not only on the surface of the participating substances, but also on their nature. This is seen from experiment. Prepared solutions of different substances heavier than water, such as salt or sugar, are poured into tall jars. Pure water is then most carefully poured into these jars (through a funnel) on to the top of the solutions, so as not to disturb the lower stratum, and the jars are then left undisturbed. The line of demarcation between the solution and the pure water will be visible, owing to their different co-efficients of refraction. Notwithstanding that the solutions taken are heavier than water, after some time complete intermixture will ensue. Gay-Lussac convinced himself of this fact by this particular experiment, which he conducted in the cellars under the Paris Astronomical Observatory. These cellars are well known as the locality where numerous interesting researches have been conducted, because, owing to their depth under ground, they have a uniform ternperature during the whole year; the temperature does not change during the day, and this was indispensable for the experiments on the diffusion of solutions, in order that no doubt in their results should arise from a daily change of temperature (the experiment lasted several months), which would set up currents in the liquids and intermix their strata. Notwithstanding the uniformity of the temperature, the substance in solution in time ascended into the water and distributed itself uniformly through it, proving that there exists between water and a substance dissolved in it a particular kind of attraction or striving for mutual interpenetration in opposition to the force of gravity. Further, this effort, or rate of diffusion, is different for salt or sugar or for 
various other substances. Consequently, in solution there acts a peculiar force, as in actual chemical combinations, and solution is determined by a peculiar kind of movement (by the chemical energy of a substance) which is proper to the substance dissolved and to the solvent.

Graham made a series of experiments similar to those above described, and he showed that the rate of diffusion ${ }^{17}$ in water is very variable-that is, a uniform distribution (under perfest rest, and with such an arrangement of the strata of the solutions that uniformity takes place in opposition to gravity) of a substance in the water dissolving it is attained in different periods of time with different solutions. Graham compared diffusive capacity with volatility. There are substances which diffuse easily, and there are others which diffuse with difficulty, just as there are more or less volatile substances. Seven hundred cubic centimetres of water was poured into a jar, and by means of a syphon (or a pipette) 100 cub. centimetres of a solution containing 10 grams of a substance was cautiously poured in so as to occupy the lower portion of the jar. After the lapse of several days, successive layers of 50 cubic centimetres were taken from the top downwards, and the quantity of substance dissolved in the different layers determined. Thus, common table salt, after fourteen days, gave the following amounts (in milligrams) in the respective layers, beginning from the top : 104. 120, $126,198,267,340,429,535,654,766,881,991,1,090,1,187$, and 2,266 in the remainder; whilst albumin in the same time gave, in the first seven layers, a very small amount, and beginning from the eighth layer, $10,15,47,113,343,855,1,892$, and in the remainder 6,725 milligrams. Thus, the diffusive power of a solution depends on time and on the nature of the substance dissolved, which fact may serve, not only for the explanation of the process of solution, but also in distinguishing one substance from another. Graham showed that substances which rapidly diffuse through liquids are able to rapidly pass through membranes and crystallise, whilst substances which diffuse slowly and do not crystallise are colloids, that is, resemble glue, and penetrate through

17 The researches of Graham, Fick, Nernst, and others showed that the quantity of a dissolved substance which is transmitted (rises) from one stratym of liquid to another in a vertical cylindrical vessel is not only proportional to the time and to the sectional area of the cylinder, but also to the amount and nature of the substance dissolred in a stratum of liquid, so that each substance has its corresponding co-efficient of diffusion. The cause of the diffusion of solutions must be considered as essentially the same as the cause of the diffusion of gases-that is, as dependent on movements which are proper to their molecules; but here most probably those purely chemical, although feebly-developed, forces, which incline the substances dissolved to the formation of definite compounds, also play their part. 
a nembrane slowly, and form jellies; that is, occur in insoluble forms. ${ }^{18}$

13 The rate of diffusion-like the rate of transmission - through membranes, or dialysis (which plays an important part in the vital processes of organisms and also in technical work), presents, according to the researches of Graham, a sharply-defined change in passing from such crystallisable substances as the majority of salts and acids to substances which are capable of giving jellies (gum, gelatin, \&c.). The former diffuse into solutions and pass through membranes much more rapidly than the latter, and Graham therefore distinguishes between crystalloids, which diffuse rapidly, and colloids, which diffuse slowly. On breaking solid colloids into pieces, a total absence of cleavage is remarked. The fracture of such substances is like that of glue or glass. It is termed a 'conchoidal' fracture. Almost all the substances of which animal and vegetable bodies consist are colloids, and this is, at all events, partly the reason why animals and plants hare such varied forms, which have no resemblance to the crystalline forms of the majority of mineral substances. The colloid solid substances in organisms-that is, in animals and plants-are usually soaked with water, and take most peculiar forms, of networks, of granules, of hairs, of mucous, shapeless masses, \&c., which are quite different from the forms taken by crystalline substances. When colloids separate out from solutions, or from a molten state, they present a form which is similar to that of the liquid from which they were formed. Glass may be taken as the best example of this. Colloids are distinguishable from crystalloids, not only. by the absence of crystalline form, but by many other properties which admit of clearly distinguishing both these classes of solids, as was shown by the above-mentioned English scientific man, Graham. Nearly all colloids are capable of passing, under certain circumstances, from a soluble into an insoluble state. The best example is shown by white of eggs (albumin) in the raw and soluble form, and in the hard-boiled and insoluble form. The majority of colloids, on passing into an insoluble form in the presence of water, give substances having a gelatinous appearance, which is familiar to every one in starch, solidified glue, jelly, \&c. Thus gelatin, or common carpenter's glue, when soaked in water, swells up into an insoluble jelly. If this jelly be heated, it melts, and is then soluble in water, but on cooling it again forms a jelly which is insoluble in water. One of the properties which distinguish. colloids Fif. 15.-Dialrser. Apparatus for tl e separation of subfrom crystalloids is that the former stances which pass through a membrane from those pass very slowly through a mem-

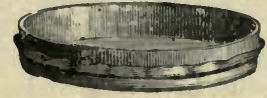
brane, whilst the latter penetrate very rapidly. This may be shown by taking a cylinder, open at both ends, and by covering its lower end with a bladder or with vegetable parch. ment (unsized paper immersed for two or three minutes in a mixture of sulphuric acid and half its volume of water, and then washed), or any other membranous substance (all such substances are themselves colloids in an insoluble form). The membrane must be firmly tied to the cylinder, so as not to leave any opening. Such an apparatus is called a dialyser (fig. 15), and the process of separation of crystalloids from colloids by means of such a membrane is termed dialysis. An aqueous solution of a crystalloid or colloid, or a mixture of both, is poured into the dialyser, which is then placed in a vessel containing water, so that the bottom of the membrane is corered with water. Then, after a certain period of time, the crystalloid passes through the membrane, whilst the colloid, if it does pass through at all, does so at an incomparably slower rate. The crystalloid 
If it be desired to increase the rate of solution, recourse must be had to stirring, shaking, or some such mechanical movement, obliging the solution formed round the given substance to rise upwards if the solution be heavier than water. But if once a uniform solution is formed, it will remain uniform if the temperature be uniform, no matter how heavy the dissolved substance is, or how long the solution be left at rest, which fact again shows the presence of a force holding together the particles of the body dissolved and of the solvent. ${ }^{19}$

naturally passes through into the water until the solution attains the same strength on both sides of the membrane. By replacing the outside water with fresh water, a fresh quantity of the crystalloid may be separated from the dialyser. While a crystalloid is passing through the membrane, a colloid remains almost entirely in the dialyser, and therefore a mixed solution of these two kinds of substances may be separated from each other by a dialyser. The study of the properties of colloids, and of the phenomena of their passage through membranes, should elucidate much respecting the phenomena which are accomplished in organisms.

19 The formation of solutions may be considered in two aspects, from a physical and from a chemical point of view, and it is more evident in solutions than in any other department of chemistry that these provinces of natural science are allied together in a most intimate manner. On one hand solutions form a particular aspect of a physico-mechanical interpenetration of homogeneous substances, and a juxtaposition of the molecules of the substance dissolved and of the solvent, similar to the juxtaposition which is exlibited in homogeneous substances. From this point of view this diffusion of solutions is exactly similar to the diffusion of gases, with only this difference, that the nature and store of energy is different in gases from what it is in liquids, and that in liquids there is considerable friction whilst in gases there is comparatively little. The penetration of a dissolved substance into water is likened to evaporation, and solution to the formation of vapour. This resemblance was clearly expressed even by Graham. In recent years the Dutch chemist, Van't Hoff, has developed this view of solutions in great detail, having shown (in a memoir in the Transactions of the Swedish Academy of Science, Part 21, No. 17, 'Lois de l'équilibre chimique dans l'état dilué, gazeux au dissous,' 1886), that for dilute solutions the osmotic pressure follows the same laws (of Boyle, Mariotte, Gay-Lussac, and Avogadro-Gerhardt) as for gases. The osmotic pressure of a substance dissolved in water is determined by means of membranes which allow the passage of water, but not of a substance dissolved in it, through them. This property is found in animal protoplasmic membranes and in porous substances covered with an amorphous precipitate such as is obtained by the action of copper sulphate on potassium ferrocyanide (Pffeifer Traube). If, for instance, a one p.c. solution of sugar be placed in such a vessel, which is then closed and placed in water, then the water passes through the walls of the vessel and increases the pressure by $50 \mathrm{~mm}$. of the barometric column. If the pressure be artificially increased inside the vessel, then the water will be expelled through the walls. The osmotic pressure of dilute solutions determined in this mamer (from observations made by Pffeifer and De Vries) was shown to follow the same laws as those of the pressure of gases; for instance, by doubling or increasing the quantity of a salt (in a given volume) $n$ times, the pressure is doubled or increases $n$ times. One of the extreme consequences of the resemblance of osmotic pressure to gaseous pressure is that the concentration of a uniform solution varies in parts which are heated or cooled. Soret (1881) indeed observed that a solution of copper sulphate containing 17 parts of the salt at $20^{3}$ only contained 14 parts after heating the upper portion of the tube to $80^{\circ}$ for a long period of time. This aspect of solution, which is now being very carefully and fully worked out, may be called the physical side. Its other aspect is purely chemical, for solution does not take place between any two substances, but requires a 
In the consideration of the process of solution, besides the conception of diffusion, another fundamental conception is necessary, namely, that of the saturation of solutions.

special and particular attraction or affinity between them. A vapour or gas permeates into any other rapour or gas, but a salt which dissolves in water may not be in the least soluble in alcohol, and is quite insoluble in mercury. In considering solution as a manifestation of chemical forces (and of chemical energy), it must be acknowledged that they are here developed to so feeble an extent that the definite compounds (that is, those formed according to the law of nultiple proportions) which are formed between water and a soluble substance dissociate at even the ordinary temperature, forming a homogeneous system-that is, one where both the compound and the products into which it decomposes (water and the aqueous compound) occur in a liquid state. The chief difficulty in the comprehension of solutions depends on the fact that the mechanical theory of the structure of liquids has not yet been so fully developed as the theory of gases, and solutions are liquids. The conception of solutions as liquid dissociated definite chemical compounds is based on the following considerations: (1) that there exist certain undoubtedly definite chemical crystalline compounds (such as $\mathrm{H}_{2} \mathrm{SO}_{4}, \mathrm{H}_{2} \mathrm{O}$; or $\mathrm{NaCl}, 10 \mathrm{H}_{2} \mathrm{O}$; or $\mathrm{CaCl}_{2}, 6 \mathrm{H}_{2} \mathrm{O}$; \&c.) which melt on a certain rise of temperature, and then form real solutions; (2) that metallic alloys in a molten condition are real solutions, but on cooling they often give entirely distinct and definite crystalline compounds, which are recognised by the properties of alloys; (3) that between the solvent and the substance dissolved there are formed, in a number of cases, many undoubtedly definite compounds, such as compounds with water of crystallisation; (4) that the physical properties of solutions, and especially their specific gravities (a property which is very accurately observable), vary with a cliange in composition, and in such a manuer as the formation of one or several definite but dissociating compounds would require. Thus, for example, on adding water to fuming sulphuric acid its density is observed to decrease until it attains the definite composition $\mathrm{H}_{2} \mathrm{SO}_{4}$, or $\mathrm{SO}_{3}+\mathrm{H}_{2} \mathrm{O}$, when the specific gravity increases, although on further diluting with water it again falls. Further (Mendeléeff, The Investigation of Aqueous Solutions from their Specific Gravities, 1887), the increase in specific gravity $(d s)$, with the augmentation $(d p)$ of the percentage amount of a substance dissolved, varies in all well-known solutions with the percentage amount of the substance dissolved, so that a rectilinear dependence is obtained (i.e., $\frac{d s}{d p}=\mathrm{A}+\mathrm{B} p$ ) between the limits of definite compounds which must be acknowledged to exist in solutions; this would be expected to be the case from the dissociation hypothesis. So, for instance, from $\mathrm{H}_{2} \mathrm{SO}_{4}$ to $\mathrm{H}_{2} \mathrm{SO}_{4}+\mathrm{H}_{2} \mathrm{O}$ (both these substances exist as definite compounds in a free state), the fraction $\frac{d s}{d p}=0.0729-0.000749 p$ (where $p$ is the percentage amount of $\mathrm{H}_{2} \mathrm{SO}_{4}$ ). For alcohol $\mathrm{C}_{2} \mathrm{H}_{6} \mathrm{O}$, whose aqueous solutions have been more accurately investigated than all others, three definite compounds must be acknowledged in its solutions, $\mathrm{C}_{2} \mathrm{H}_{6} \mathrm{O}+12 \mathrm{H}_{2} \mathrm{O}$, $\mathrm{C}_{2} \mathrm{H}_{6} \mathrm{O}+3 \mathrm{H}_{2} \mathrm{O}$, and $3 \mathrm{C}_{2} \mathrm{H}_{6} \mathrm{O}+\mathrm{H}_{2} \mathrm{O}$.

The two aspects of solution above mentioned, and the hypotheses which have as yet been applied to the examination of solutions, although they have partially different starting points, yet will doubtless in time lead to a general theory of solutions, because the same common laws govern both physical and chemical phenomena, inasmuch as the properties and movements of molecules, which determine physical properties, are dependent on the movements and properties of atoms, which determine chemical mutual actions. For details of the questions dealing with the theories of solution recourse must now be had to special memoirs and to works on theoretical (physical) chemistry; for this subject forms one of special interest at the present epoch of the development of our science. In working out chiefly the chemical side of solutions I consider it to be necessary to reconcile the two aspects of the question; this seems to me.to be all the more possible, as the physical side is limited to dilute solutions only, whilst the chemical side deals mainly with strong solutions.

VOL. I. 
Just as damp air may be added to any quantity of dry air it be desired, so also a solvent liquid may be taken in an indefinitely large quantity and yet a uniform solution will be obtained. But more than a definite quantity of aqueous vapour cannot be introduced into a certain volume of air at a certain temperature. The excess above the point of saturation will remain in the liquid form. ${ }^{20}$ The relation between water and substances dissolved in it is similar. More than a definite quantity of a substance canıot, at a certain temperature, dissolve in a given quantity of water; the excess does not unite with the water. Just as air or a gas becomes saturated with vapour, so water becomes saturated with a substance dissolved in it. If an excess of a

¿o A juxtaposition of (chemically or physically) reacting substances taken in various states-for instance, some solid, others liquid or gaseous-is termed a heterogeneous system. Up to now it is only systems of this kind which can be subjected to detailed examination in the sense of the mechanical theory of heat. Solutions present liquid homogeneous systems, which as yet are subjected to investigation with difficulty.

In the case of limited solution of liquids in liquids, the difference between the solvent and the substance dissolved is clearly seen. The former (that is, the solvent) may be added in an unlimited quantity, and yet the solution obtained will always be uniform, whilst of the substance dissolved there can only be taken a definite saturating proportion. We will take water and common (sulphuric) ether. On shaking the ether with the water it will be remarked that a portion of it dissolves in the water, forming a solution. If the ether be taken in such a quantity that it saturates the water and a portion of it remains undissolved, then this remaining portion will act as a solvent, and water will diffuse through it and also form a saturated solution of water in the ether taken. Thus two saturated solutions will be obtained. One solution will contain ether dissolved in water, and the other solution will contain water dissolved in ether. These two solutions will arrange themselves in two layers, according to their density; the ethereal solution of water will be on the top, as the lightest, and the aqueous solution of ether at the bottom, as the heaviest. If the upper ethereal solution be poured off from the aqueous solution, any quantity of ether may be added to it; this shows that the dissolving substance is ether. If water be added to it, it is no longer dissolved in it; this shows that water saturates the ether-here water is the substance dissolved. If we act in the same manner with the lower layer, we shall find that water is the solvent and ether the substance dissolved. By taking different amounts of ether and water, the degrce of solubility of ether in water, and of water in ether, may be easily determined. Thus, for example, in the above case it is found that water approximately dissolves $\frac{1}{10}$ of its volume of ether, and ether dissolves a very small quantity of water. Let us imagine that the liquid poured in dissolves a considerable amount of water, and that water dissolves a considerable amount of the liquid. For instance, let us imagine that the saturation of 100 parts of water require 80 parts of the liquid, and that 100 parts of the liquid would require 125 parts of water for its saturation. What would then take place if the liquid be poured in water? Two layers could not be formed, because the saturated solutions would resemble each other, and therefore they would intermix in all proportions. Indeed, in the saturated aqueous solution there. would be 0.8 parts of the liquid taken to 1 part of water, and in the solution of water in the liquid taken there would be on saturation 1 part of water to 0.8 parts of the liquid. There would be noline of demarcation between the layers of the liquids, or, in other words, they would intermix in all proportions. This is, consequently, a case of a phenomena where two liquids present considerable co-efficients of solubility in each other, but where it is impossible to say what these co-efficients are, because it is impossible to obtain a saturated solution. 
substance be added to water which is already saturated with it, it will remain in its original state, and will not spread through the water. The quantity of a substance (either by volume with gases, or by weight with solids and liquids) which is capable of saturating 100 parts of water is called the co-efficient of solubility or the solubility. In 100 grams of water at $15^{\circ}$, there can be dissolved not more than 35.86 grams of common salt. Consequently, its solubility at $15^{\circ}$ is equal to $35 \cdot 86 .{ }^{21}$ It is most

21 The solubility, or co-efficient of solubility, of a substance is determined by various methods. Either a solution is expressly prepared with a clear excess of the soluble substance and saturated at a given temperature, and the quantity of water and of the substance dissolved in it determined by evaporation, desiccation, or other means; or else, as is done with gases, known quantities of water and of the soluble substance are taken, and the amount remaining undissolved is determined.

The solubility of a gas in water is determined by means of an apparatus called an absorptiometer (fig. 16). It consists of an iron stand $f$, on which an india-rubber ring rests. A wide glass tube is placed on this ring, and is pressed down on it by the ring $h$ and the screws $i i$. The tube is thus firmly fixed on the stand. A cock $r$, communicating with a funnel $r$, passes into the lower part of the stand. Mercury can be poured into the wide tube through this funnel, which is therefore made of steel, as copper would be affected by the mercury. The upper ring $h$ is furnished with a

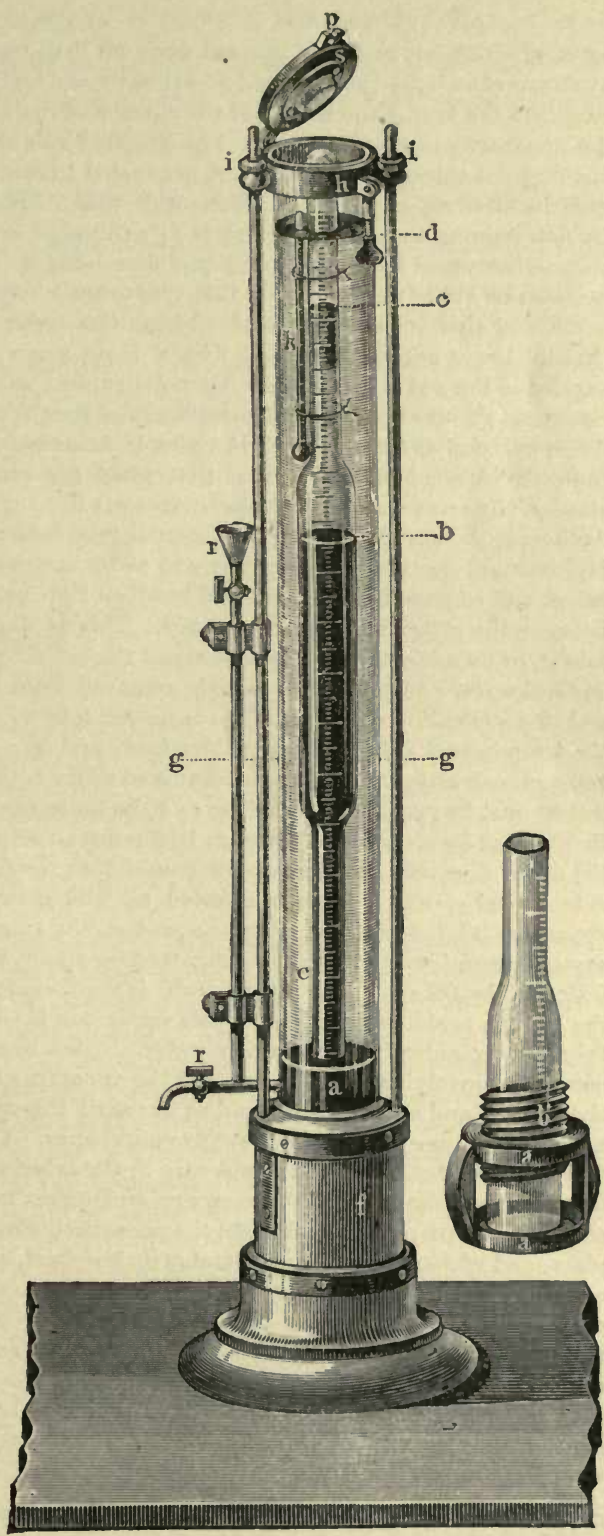

Frg.16.-Bunsen's absorptiometer. Apparatus for determining the solubility of gases in liquids. 
important to turn attention to the existence of the solid insoluble substances of nature, because on them depends the shape of the

cover $p$, which can be firmly pressed down on to the wide tube, and hermetically closes it by means of an india-rubber ring. The tube $r r$ can be raised at will, and so by pouring mercury into the funnel the height of the column of mercury, which produces pressure inside the apparatus, can be increased. The pressure can also be diminished at will, by letting mercury out through the cock $r$. A graduated tube $e$, containing the gas and liquid to be experimented on, is placed inside the wide tube. This tube is graduated in millimetres for determining the pressure, and it is calibrated for volumes, so that the number of volumes occupied by the gas and liquid dissolving it can be easily calculated. This tube can also be easily removed from the apparatus. To the right of the figure, the lower portion of this tube when removed from the apparatus is shown. It will be observed that its lower end is furnished with a male screw $b$, fitting in a nut $a$. The lower surface of the nut $a$ is covered with india-rubber, sn that on screwing up the tube its lower end presses upon the india-rubber, and thus hermetically closes the whole tube, for its upper end is fused up. The nut $a$ is furnished with arms $c c$, and in the stand $f^{\prime}$ there are corresponding spaces, so that when the screwed-up internal tube is fixed into stand $f$, the arms $c c$ fix into these spaces cut in $f$. This enables the internal tube to be fixed on to the stand $f$. When the internal tube is fixed in the stand, the wide tube is put into its right position, and mercury and water are poured into the space between the two tubes, and communication is opened between the inside of the tube $e$ and the mercury between the interior and exterior tubes. This is done by either revolving the interior tube $e$, or by a key turning the nut about the bottom part of $f$. The tube $e$ is filled with gas and water as follows: the tube is removed from the apparatus, filled with mercury, and the gas to be experimented on is passed into it. The volume of the gas is measured, the temperature and pressure determined, and the volume it would occupy at $0^{\circ}$ and $760 \mathrm{~mm}$. calculated. A known volume of water is then introduced into the tube. The water must be previously boiled, so as to be quite freed from air in solntion. The tube is then closed by screwing it down on to the india-rubber on the nut. It is then fixed on to the stand $f$, mercury and water are poured into the intervening space between it and the exterior tube, which is then screwed up and closed by the cover $p$, and the whole apparatus is left at rest for some time, so that the tube $e$, and the gas in it, may attain the same temperature as that of the surrounding water, which is marked by a thermometer $k$ tied to the tube $e$. The interior tube is then again closed by revolving it in the nut, the cover $p$ again shut, and the whole apparatus is shaken in order that the gas in the tube $e$ may entirely saturate the water. After several shakings, the tube $e$ is again opened by revolving it in the nut, and the apparatus is left at rest for a certain time; it is then closed and again shaken, and so on until the volume of gas does not diminish after a fresh shaking - that is, until saturation ensues. Observations are then made of the temperature, the height of the mercury in the interior tube, and the level of the water in it, and also of the level of the mercury and water in the exterior tube. All these data are necessary in order to calculate the pressure under which the solution of the gas takes place, and what volume of gas remains undissolved, and also the quantity of water which serves as the solvent. By varying the temperature of the surrounding water, the amount of gas dissolved at various temperatures may be determined. Bunsen, Carius, and many others determined the solution of various gases in water, alcohol, and certain other liquids, by means of this apparatus. If in a determination of this kind it is found that $n$ cubic centimetres of water at a pressure $h$ dissolve $m$ cubic centimetres of a given gas, measured at $0^{\circ}$ and $760 \mathrm{~mm}$., when the temperature under which solution took place was $t^{\circ}$ and pressure $h \mathrm{~mm}$., then it follows that at the temperature $t$ the co-efficient of solubility of the gas in 1 volume of the liquid will be equal to $\frac{m}{n} \times \frac{760 \text {. }}{h}$

This formula is very clearly understood from the fact that the co-efficient of solubility of gases is that quantity measured at $0^{\circ}$ and $760 \mathrm{~mm}$., which is absorbed at a pressure 
substance of the earth's surface, and of plants and animals. There is so much water on the earth's surface, that were the surface of substances formed of soluble matters it would constantly change, and however substantial their forms might be, mountains, river banks and sea shores, plants and animals, or the habitations and coverings of men, could not exist for any length of time. ${ }^{22}$

of $760 \mathrm{~mm}$. by one volume of a liquid. If $n$ cubic centimetres of water absorb $m$ cubic centimetres of a gas, then one cubic centimetre absorbs $\frac{m}{n}$. If $\frac{m}{n}$ c.c. of a gas are absorbed under a pressure of $h \mathrm{~mm}$., then, according to the law of the variation of solubility of a gas with the pressure, there would be dissolved, under a pressure of $760 \mathrm{~mm}$., a quantity varying in the same ratio to $\frac{m}{n}$ as $760: h$. In determining the residual volume of gas its moisture (note 1) must be taken into consideration.

Below are given the number of grams of several substances saturating 100 grams of water-that is, their co-efficients of solubility by weight at three different temperatures:-

\begin{tabular}{|c|c|c|c|c|c|c|}
\hline & - & & & At $0^{\circ}$ & At $20^{\circ}$ & At $100^{\circ}$ \\
\hline $\begin{array}{l}\text { Gases } \\
\text { Liquids }\end{array}$ & $\left\{\begin{array}{l}\text { Oxygen, } \mathrm{O}_{2} \\
\text { Carbonic anhydride, } \mathrm{CO}_{2} \\
\text { Ammonia, } \mathrm{NH}_{3} \\
\text { Phenol, } \mathrm{C}_{6} \mathrm{H}_{6} \mathrm{O} \\
\text { Amyl alcohol, } \mathrm{C}_{5} \mathrm{H}_{12} \mathrm{O} \\
\text { Sulphuric acid, } \mathrm{H}_{2} \mathrm{SO}_{4} \\
\text { Gypsum, } \mathrm{CaSO}_{4}, 2 \mathrm{H}_{2} \mathrm{O} \\
\text { Alum, AlKS } \mathrm{O}_{2}, 12 \mathrm{O}_{2} \mathrm{O} \\
\text { Anhydrous sodium sulphate, } \\
\text { Common } \mathrm{Salt}_{\mathrm{NaCl}} \mathrm{N} \\
\text { Nitre, } \mathrm{KNO}_{3}\end{array}\right.$ & $\begin{array}{l}: \\
\vdots: \\
\vdots: \\
\mathrm{Na}_{2} \mathrm{SO}_{4} \\
:\end{array}$ & $\begin{array}{l}: \\
\vdots \\
\vdots \\
:\end{array}$ & $\begin{array}{r}\frac{6}{1000} \\
\frac{35}{100} \\
90 \cdot 0 \\
4 \cdot 9 \\
4 \cdot 4 \\
\infty \\
\frac{1}{5} \\
3 \cdot 3 \\
4 \cdot 5 \\
35 \cdot 7 \\
13 \cdot 3\end{array}$ & $\begin{array}{l}\frac{4}{1000} \\
\frac{18}{100} \\
51 \cdot 8 \\
5 \cdot 2 \\
2 \cdot 9 \\
\infty \\
\frac{1}{4} \\
15 \cdot 4 \\
20 \\
36 \cdot 0 \\
31 \cdot 7\end{array}$ & $\begin{array}{c}\overline{-} \\
7 \cdot 3 \\
\infty \\
-\infty \\
\infty \\
\frac{1}{5} \\
43 \cdot 5 \\
39 \cdot 7 \\
246 \cdot 0\end{array}$ \\
\hline
\end{tabular}

Sometimes a substance is so slightly soluble that it may be considered as insoluble. Many such substances are met with both in solids and liquids, and such a gas as oxygen, although it does dissolve, does so in so small a proportion by weight that it might be considered as zero did not the solubility of even so little oxygen play an important part in nature (as in the respiration of fishes) and were not an infinitesimal quantity of a gas by weight so easily measured by volume. The sign $\infty$, which stands on a line with sulphuric acid in the above table, indicates that it intermixes with water in all proportions. There are many such cases among liquids, and everybody knows, for instance, that spirit (absolute alcohol) can be mixed in any proportion with water. Common corn spirit (vodky) is a mixture of about fifty parts by weight of pure spirit to 100 parts by weight of water.

22 Just as the existence must be admitted of substances which are completely undecomposable (chemically) at the ordinary temperature-for there are substances which are entirely non-volatile at such a temperature (as wond and gold), although capable of decomposing (wood) or volatilising (gold) at a higher temperature-so also the existence must be admitted of substances which are totally insoluble in water without some degree of change in their state. Although mercury is partially volatile at the ordinary temperature, there is no reason to think that it and other metals are soluble in water, alcohol, or other similar liquids. However, mercury forms solutions, as it dissolves other metals. On the other hand, there are many substances found in nature which are so very slightly soluble in water, that in ordinary practice they may be considered as insoluble (for example, barium sulphate). For the comprehension of that general plan according to which a change of state of substances (combined or dissolved, solid, liquid, or gaseous) 
Substances which are easily soluble in water bear a certain resemblance to it. Thus sugar and salt in many of their superficial features remind one of ice. Metals, which are not soluble in water, have no points in common with it, whilst on the other hand they dissolve each other in a molten state, forming alloys, just as oily substances dissolve each other ; for example, tallow is soluble in petroleum and in olive oil, although they are all insoluble in water. From this it is evident that the analogy of substances forming a solution plays an important part, and as aqueous and all other solutions are liquids, there is good reason to believe that in the process of solution solid and gaseous substances change in a physical sense, passing into a liquid state. These considerations elucidate many points of solution-as, for instance, the variation of the co-efficient of solubility with the temperature and the evolution or absorption of heat in the formation of solutions.

The solubility - that is, the quantity of a substance necessary for saturation-varies with the temperature, and, further, with an increase in temperature the solubility of solid substances generally increases, and that of gases decreases ; this might be expected, as solid substances by heating, and gases by cooling, approach to a liquid or dissolved state. ${ }^{23}$ A graphic method is often employed to express the variation of solubility with temperature. On the axes of abscissie or on a horizontal line, temperatures are marked out and perpendiculars are raised corresponding with each temperature, whose length is determined by the solubility of the salt at that temperature - expressing, for instance, one part by weight of a salt in 100 parts of water by one unit of length, such as a millimetre. By joining the summits of the perpendiculars, a curve is obtained which expresses the degree of solubility at different. temperatures. For solids, the curve is generally an ascending one-i.e., recedes from the horizontal line with the rise in temperature. These curves clearly show by their inclination the degree of rapidity of increase in solubility with the temperature. Having determined several points

takes place, it is very important to make a distinction at this boundary line (on approaching zero of decomposition, volatility, or solubility) between an insignificant amount and zero, but the present methods of research and the data at our disposal at the present time do not yet touch such questions. It must be remarked, besides, that water in a number of cases does not dissolve a substance as such, but acts on it chemically and forms a soluble substance. Thus glass and many rocks, especially if taken as powder, are chemically changed by water, but are not directly soluble in it.

${ }_{23}$ Beilby (1883) experimented on paraffin, and found that one cubic decimetre of solid paraffin at $21^{\circ}$ weighed 874 grams, and when liquid, at its melting-point $38^{\circ}, 783$ grams, at $49^{\circ}, 775$ grams, and at $60^{\circ}, 767$ grams, from which the weight of a litre of liquefied paraffin would be $795^{\circ} 4$ grams at $21^{\circ}$ if it could remain liquid at that temperature. By dissolving solid paraffin in lubricating oil at $21^{\circ}$ Beilby found that 795.6 grams occupy one cubic decimetre, from which he concluded that the solution contained liquefied paraffin. 
of a curve - that is, having made a determination of the solubility for several temperatures - the solubility at intermediary temperatures may be determined from the sinuosity and form of the curve so formed; in this way the empirical law of solubility may be followed. ${ }^{24}$ The results of research have shown that the solubility of certain salts-as, for example, common table salt - varies comparatively little with the temperature ; whilst for other substances the solubility increases by equal amounts for equal increments of temperature. So, for example, for the saturation of

24 Gay-Lussac was the first to have recourse to such a graphic method of expressing solubility, and he considered, in accordance with the general opinion, that by joining up the summits of the ordinates in one harmonious curve it is possible to express the entire change of solubility with the temperature. Now, there are many reasons for doubting the accuracy of such an admission, for there undoubtedly are critical points in curves of solubility (for example, of sodium sulphate, as shown further on), and it may be that definite compounds of dissolved substances with water, in decomposing within known limits of temperature, give critical points more often than would be imagined; it may even be, indeed, that instead of a continuous curve, solubility should be expressed-if not always, then not unfrequently-by straight or broken lines. According to Ditte, the solubility of sodium nitrate, $\mathrm{NaNO}_{5}$, is expressed by the following figures per 100 parts of water:-

$\begin{array}{ccccccccc}0^{\circ} & 4^{\circ} & 10^{\circ} & 15^{\circ} & 21^{\circ} & 29^{\circ} & 36^{\circ} & 51^{\circ} & 68^{\circ} \\ 66 . & 71.0 & 76.3 & 80.6 & 85^{\circ} \cdot & 92 \cdot 9 & 99 \cdot 4 & 13 \cdot 6 & 125 \cdot 1\end{array}$

According to my opinion (1881), these data should be expressed with exactitude by a straight line, $67 \cdot 5+0.87 t$, which entirely agrees with the results of experiment. According to this the figure expressing the solubility of the salt at $0^{\circ}$ exactly coincides with the composition of a definite chemical compound- $-\mathrm{NaNO}_{3}, 7 \mathrm{H}_{2} \mathrm{O}$. The experiments made by Ditte showed that all saturated solutions between $0^{\circ}$ and $-15 \cdot 7^{\circ}$ have such a composition, and that at the latter temperature the solution completely solidifies into one homogeneous whole. Ditte slows, in the first place, that the solubility of sodium nitrate is expressed by a broken straight line, and, in the second place, confirms the idea, which I had already traced, that in solutions we have definite chemical compounds in a state of dissociation. In recent times (1888) Etard discovered a similar phenomenon in many of the sulphates. Brandes, in 1830, shows a diminution in solubility below $100^{\circ}$ for manganese sulphate. The percentage by weight (i.e., per 100 parts of the solution, and not of water) of saturation for ferrous sulphate, $\mathrm{FeSO}_{4}$, from $-2^{\circ}$ to $+65^{\circ}=13 \cdot 5+0 \cdot 3784 t$ that is, the solubility of the salt increases. The solubility remains constant from $65^{\circ}$ to $98^{\circ}$ (according to Brandes the solubility then increases; this divergence of opinion requires proof), and froin $98^{\circ}$ to $150^{\circ}$ it falls as $=104.35-0.6685 t$. Hence, at about $+156^{\circ}$ the solubility should $=0$, and this has been confirmed by experiment. I observe, on my side, that Etard's formula gives $38^{\circ} 1$ p.c. of salt at $65^{\circ}$ and $38^{\circ} 8$ p.c. at $92^{\circ}$, and this maximum amount of salt in the solution very nearly corresponds with the composition $\mathrm{FeSO}_{4}, 14 \mathrm{H}_{2} \mathrm{O}$, which requires $37 \cdot 6$ p.c. Thus, in this case, as in that of sodium nitrate, the formation of a definite solution may be presupposed. From what has been said, it is evident that the data concerning solubility require a new method of investigation, which, in the first place, should have in view the entire scale of solubility-from the formation of completely solidified solutions (cryolnydrates, which we sliall speak of presently) to the separation of salts from their solutions, if this is accomplished at a higher temperature (for manganese and cadmium sulphates there is an entire separation, according to Étard), or to the formation of a constant solubility (for potassium sulphate the solubility, according to Etard, remains constant from $163^{\circ}$ to $220^{\circ}$ and equals 24.9 p.c.); and, in the second place, should endeavour to apply the conception of definite compounds existing in solutions to constant and critical solutions, corresponding with a maximum of solubility or of its limits. From these aspects solution should present a new and particular interest. 
100 parts of water by potassium chloride there is required at $0^{\circ}, 29 \cdot 2$ parts, at $20^{\circ}, 34 \cdot 7$, at $40^{\circ}, 40 \cdot 2$, at $60^{\circ}, 45 \cdot 7$; and so on, for every $10^{\circ}$ the solubility increases by 2.75 parts by weight of the salt. Therefore the solubility of potassium chloride in water may be expressed by a direct equation : $\alpha=29 \cdot 2+0 \cdot 275 t$, where $\alpha$ represents the solubility at $t^{\circ}$. For other salts, more complicated equations are required. For example, for nitre: $a=13 \cdot 3+0 \cdot 574 t+0 \cdot 01717 t^{2}+0 \cdot 0000036 t^{3}$, which shows that when $t=0^{\circ} \quad \alpha=13 \cdot 3$, when $t=10^{\circ} \quad \alpha=20 \cdot 8$, and when $t=100^{\circ}$ $\alpha=246 \cdot 0$.

Curves of solubility give the means of judging with accuracy the amount of a salt separated by the cooling to a known extent of a solution saturated at a given temperature. For instance, if 200 parts of a solution of potassium chloride in water saturated at a temperature of $60^{\circ}$ be taken, and it be asked how much of the salt will be separated by cooling the solution to $0^{\circ}$, if its solubility at $60^{\circ}=45 \cdot 7$ and at $0^{\circ}=29 \cdot 2$ ? The answer is obtained in the following manner : At $60^{\circ}$ a saturated solution contains $45 \cdot 7$ parts of potassium chloride per 100 parts by weight of water, consequently $145 \cdot 7$ parts by weight of the solution contains $45 \cdot 7$ parts, or, by proportion, 200 parts by weight of the solution contains $62 \cdot 7$ parts of the salt. The amount of salt remaining in solution at $0^{\circ}$ is calculated as follows: In 200 grams taken there will be $137 \cdot 3$ grams of water ; consequently, this amount of water is capable of holding only $40^{\circ} 1$ grams of the salt, and therefore in lowering the temperature from $60^{\circ}$ to $0^{\circ}$ there should separate from the solution $62 \cdot 7-40 \cdot 1=22 \cdot 6$ grams of the dissolved salt.

The difference in the solubility of salts, \&c., with a rise or fall of temperature is often taken advantage of, especially in technical work, for the separation of salts in intermixture from each other. Thus a mixture of potassium and sodium chlorides (this mixture is met with in nature at Stassfürt) is separated from a saturated solution by subjecting it alternately to boiling (evaporation) and cooling. The sodium chloride separates out in proportion to the amount of water expelled from the solution by boiling, and is removed, whilst the potassium chloride separates out on cooling, as the solubility of this salt rapidly decreases with a lowering in temperature. Nitre, sugar, and many other soluble substances are purified (refined) in a similar manner.

Although in the majority of cases the solubility of solids increases with the temperature, yet just as there are substances whose volume diminishes with a rise in temperature (for example, water from $0^{\circ}$ to $4^{\circ}$ ), so there are not a few solid substances whose solubilities fall on heating. Glauber's salt, or sodium sulphate, historically forms a particu- 
larly instructive example of the case in question. If this salt be taken in an ignited state (deprived of its water of crystallisation), then its solubility in 100 parts of water varies with the temperature in the following manner : at $0^{\circ}, 5$ parts of the salt form a saturated solution ; at $20^{\circ}, 20$ parts of the salt, at $33^{\circ}$ more than 50 parts. As will be seen, the solubility increases with the temperature, as is the case with nearly all salts; but starting from $33^{\circ}$ it suddenly diminishes, and at a temperature of $40^{\circ}$, there dissolves less than 50 parts of the salt, at $60^{\circ}$ only 45 parts of the salt, and at $100^{\circ}$ about 43 parts of the salt in 100 parts of water. This phenomenon may be traced to the following facts: Firstly, that this salt forms various compounds with water, as will be afterwards explained; secondly, that at $33^{\circ}$ the compound $\mathrm{Na}_{2} \mathrm{SO}_{4}+10 \mathrm{H}_{2} \mathrm{O}$ formed from the solution at lower temperatures, melts; and thirdly, that on evaporation at a temperature above $33^{\circ}$ there separates out an anhydrous salt, $\mathrm{Na}_{2} \mathrm{SO}_{4}$. It will be seen from this example how complicated such a seemingly simple phenomenon as solution really is ; and all data concerning solutions lead to the same conclusion. This complexity becomes eviclent in investigating the heat of solution. If solution consisted of a physical change only, then in the solution of gases there would be evolved-and in the solution of solids, there would be absorbed-so much heat as answers to the change of state; but in reality a large amount of heat is always evolved in solution, depending on the fact that in the process of solution there is accomplished an act of chemical combination, accompanied by an evolution of heat. Seventeen grams of ammonia (this weight corresponds with its formula $\mathrm{NH}_{3}$ ), in passing from a gaseous into a liquid state, evolve 4,400 units of heat (latent heat); that is, the quantity of heat necessary to raise the temperature of 4,400 grams of water $1^{\circ}$. The same quantity of ammonia, in dissolving in an excess of water, evolves twice as much heat-namely 8,800 units - showing that the combination with water is accompanied by the evolution of 4,400 units of heat. Further, the chief part of this heat is separated in dissolving in small quantities of water, so that 17 grams of ammonia, in dissolving in 18 grams of water (this weight corresponds with its composition $\mathrm{H}_{2} \mathrm{O}$ ), evolve 7,535 units of heat, and therefore the formation of the solution $\mathrm{NH}_{3}+\mathrm{H}_{2} \mathrm{O}$ evolves 3,135 units of heat beyond that due to the change of state. As in the solution of gases, the heat of liquefaction (of physical change of state) and of chemical combination with water are both positive $(+)$, therefore in the solution of gases in water a heat effect is always observed. This phenomenon is different in the solution of solid substances, because their passage from a solid to a liquid state is accompanied by an absorption 
of heat (negative, - heat), whilst their chemical combination with water is accompanied by an evolution of heat (+heat); consequently, their sum may either be a cooling effect, when the positive (chemical) portion of heat is less than the negative (physical), or it may be, on the contrary, a heating effect. This is actually the case. 124 grams of sodium thiosulphate (employed in photography) $\mathrm{Na}_{2} \mathrm{~S}_{2} \mathrm{O}_{3}, 5 \mathrm{H}_{2} \mathrm{O}$ in melting (at $48^{\circ}$ ) absorbs 9,700 units of heat, but in dissolving in a large quantity of water at the ordinary temperature it absorbs 5,700 units of heat, which shows the evolution of heat (about $+4,000$ units), notwithstanding the cooling effect observed in the process of solution, in the act of the chemical combination of the salt with water. ${ }^{25}$ But in

25 The latent heat of fusion is determined at the temperature of fusion, whilst solution takes place at the ordinary temperature, and one must think that at this temperature the latent heat would be different, just as the latent heat of evaporation varies with the temperature (see note 11, p. 52). Besides which, in solution there occurs a disunion (disintegration) of the particles of both the solvent and the substance dissolved, which in its mechanical aspect resembles evaporation, and which therefore must consume much heat. The heat emitted during the solution of a solid must be therefore considered (Personne) as composed of three factors-(1) positive, the effect of combination; (2). negative, the effect of transference into a liquid state ; and (3) negative, the effect of disintegration. In the solution of a liquid by a liquid the second factor is removed; and therefore if the heat evolved in combination is greater than that absorbed in disintegration a heating effect is observed, and in the reverse case a cooling effect; and, indeed, sulphuric acid, alcohol, and many liquids evolve heat in dissolving in each other. But the solution of chloroform in carbon bisulphide (Bussy and Binget), or of phenol (or aniline): in water (Alexéeff), produces cold. In the solution of a small quantity of water in acetic acid (Abasheff), or hydrocyanic acid (Bussy and Binget), or amyl alcohol (Alexéeff), cold is produced, whilst in the solution of these substances in an excess of water heat is evolved.

The fullest information concerning the solution of liquids in liquids has been gathered by W. 'T. Alexéeff (1883-1885), still these data are far from being sufficient to. resolve the mass of problems respecting this subject. He showed that two liquids which dissolve in each other, intermix together in all proportions at a certain temperature. Thus the solubility of phenol, $\mathrm{C}_{6} \mathrm{H}_{6} \mathrm{O}$, in water, and the converse, is linited up to $70^{\circ}$, whilst above this temperature they intermix in all proportions. This is seen from the following figures, where $p$ is the percentage amount of phenol and $t$ the temperature at which the solution becomes cloudy-that is, that at which it is saturated:-

$\begin{array}{cllllllll}p=7 \cdot 12 & 10^{\circ} 20 & 15 \cdot 31 & 26 \cdot 15 & 28.55 & 36.70 & 48.86 & 61 \cdot 15 & 71 \cdot 97 \\ t=1^{\circ} & 45^{\circ} & 60^{\circ} & 67^{\circ} & 67^{\circ} & 67^{\circ} & 65^{\circ} & 53^{\circ} & 20^{\circ}\end{array}$

It is exactly the same in the solution of benzene, aniline, and other substances in molten sulphur. Alexéeff discovered a sinilar complete intermixture for solutions of secondary butyl alcohol in water at about $107^{\circ}$; at lower temperatures the solubility is not only limited, but between $50^{\circ}$ and $70^{\circ}$ it is at its minimum, both for solutions of the alcohol in water and for water in the alcohol; and at a temperature of $5^{\circ}$ both solutions exhibit a fresh change in their scale of solubility, so that a solution of the alcohol in water which is saturated between $5^{\circ}$ and $40^{\circ}$ will become cloudy when heated to $60^{\circ}$. In the solution of liquids in liquids, Alexéeff observed a lowering in temperature (an absorption of heat) and an absence of change in specific heat (calculated for the mixture) much more frequently than had been done by previous observers. As regards his affir- 
most cases solid substances in dissolving in water evolve heat, notwithstanding the passage into a liquid state, which indicates so considerable an evolution of $(+)$ heat in the act of cumbination with water that it exceeds the absorption of $(-)$ heat dependent on the passage into a liquid state. Thus, for instance, calcium chloride, $\mathrm{CaCl}_{2}$, magnesium sulphate, $\mathrm{MgSO}_{4}$, and many other salts in dissolving evolve heat; for example, 60 grams of magnesium sulphate evolves about 10,000 units of heat. Therefore, in the solution of solid bodies there is produced either a cooling ${ }^{26}$ or a heating ${ }^{27}$ effect, according to the difference of the reacting affinities. When they are considerable-that is, when water is with difficulty separated from the resultant solution, and only with a rise of temperature (such substances absorb water) -then much heat is evolved in the process of solution, just as in many reactions of direct combination, and therefore a considerable heating of the solution is observed. Of such a kind, for instance, is the solution

mation (in the sense of a mechanical and not a chemical representation of solutions) that substances in solutions preserve their physical states (as gases, liquids, or solids), it is very doubtful, for it would necessitate admitting the presence of ice in water or its vapour. His theory starts from an unsupported hypothesis-which is, however, held by many - that the sizes (weights) of the molecules of one and the same substance are very different in different physical states. At present the weight of gaseous molecules is determined from the freezing of solutions (see later), and therefore it must either be admitted that solutions contain gaseous molecules or else that the weight of liquid molecules is the same as that of gaseous molecules, which is far simpler and more probable.

From what has been said above, it will be clear that even in so very simple a case as solution, it is impossible to calculate the heat emitted by chemical action alone, and that the chemical process cannot be separated from the physical and mechanical.

26 The cooling effect produced in the solution of solids (and also in the expansion of gases and in evaporation) is applied to the production of low temperatures. Ammonium nitrate is very often used for this purpose; in dissolving in water it absorbs 77 units of heat per each part by weight. On evaporating the solution thus formed, the solid salt is re-obtained. The application of the various freezing mixtures is based on the same principle. Snow or broken ice frequently enters into the composition of these mixtures, advantage being taken of its latent heat of fusion in order to obtain the lowest possible temperature (without altering the pressure or employing heat, as in other methods of obtaining a low temperature). For laboratory work recourse is most often had to a mixture of three parts of snow and one part of common salt, which causes the temperature to fall from $0^{\circ}$ to $-21^{\circ} \mathrm{C}$. Potassium thiocyanate, $\mathrm{KCNS}$, mixed with water ( $\frac{3}{4}$ by weight of the salt) gives a still lower temperature. By mixing ten parts of crystalline calcium chloride, $\mathrm{CaCl}_{2}, 6 \mathrm{H}_{2} \mathrm{O}$, with seven parts of water, the temperature may even fall from $0^{\circ}$ to $-55^{\circ}$.

27 The heat which is evolved in solution, or even i. the dilution of solutions, is also sometimes made use of in practice. Thus caustic soda $(\mathrm{NaHO})$, in dissolving or on the addition of water to a strong solution of it, evolves so much heat that it can replace fuel. In a steam boiler, which has been previously heated to the boiling point, another boiler is placed containing caustic soda, and the exhaust steam is made to pass through the latter; the formation of steam then goes on for a somewhat long period of time without any other heating. Norton makes use of this for smokeless street boilers. 


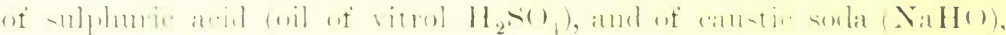

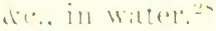

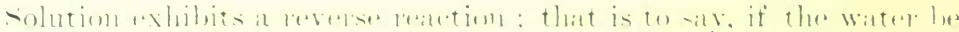

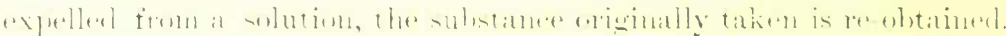

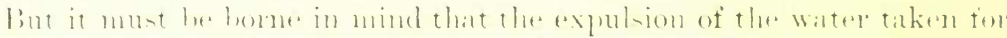

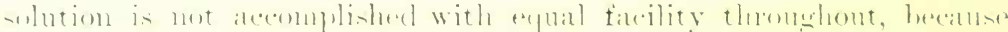

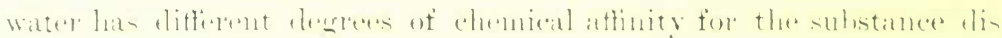

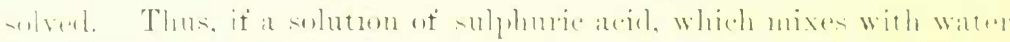

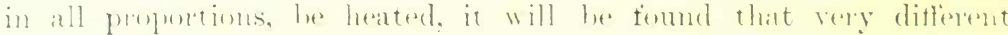

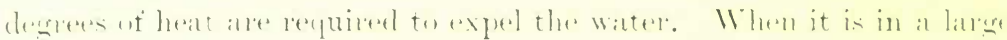

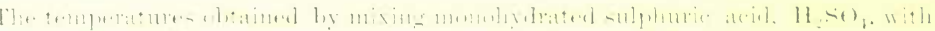

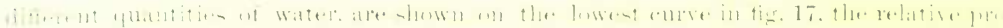

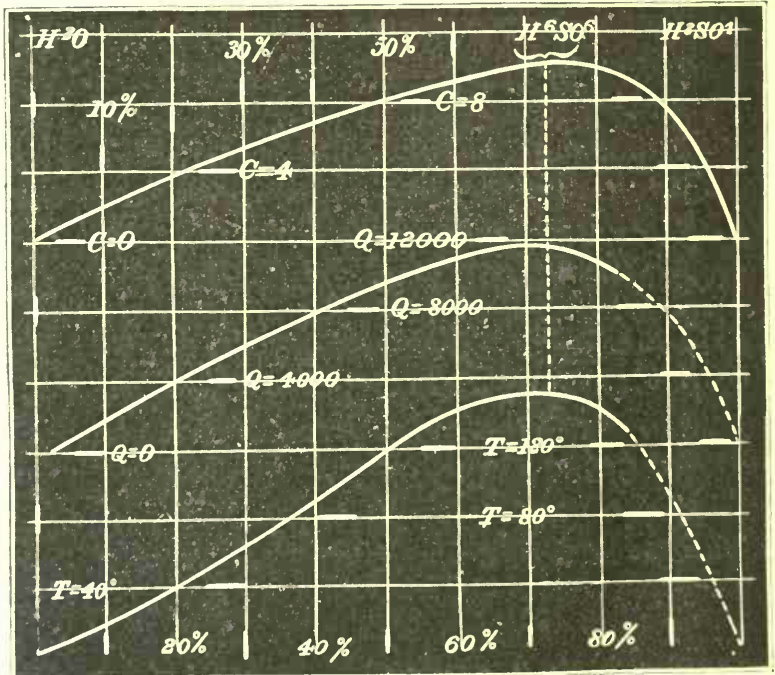

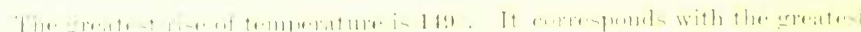

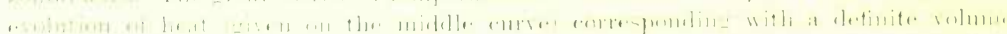

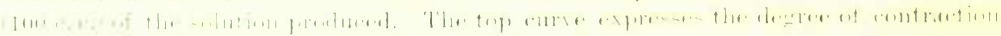

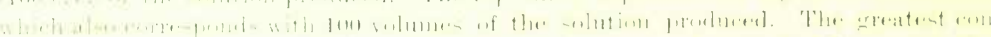

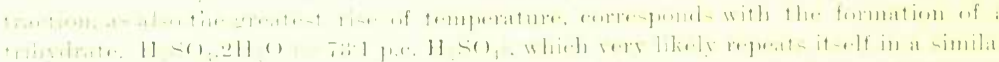

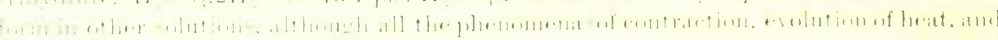

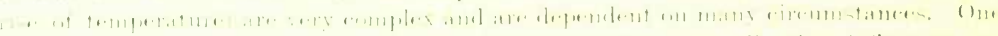

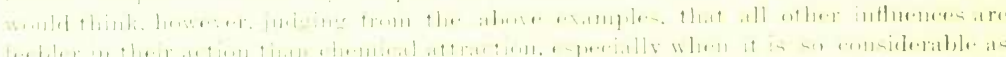

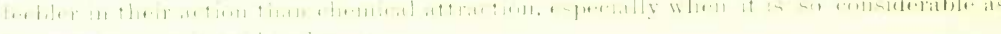


excess, water already begins to come off at a temperature slightly above $100^{\circ}$, but if it be in but a small proportion there is such a relation between it and the sulphuric acid that at $120^{\circ}, 150^{\circ}, 200^{\circ}$, and even at $300^{\circ}$, water is still held by the sulphuric acid. The bond between the remaining quantity of water and the sulphuric acid is evidently stronger than the bond between the sulphuric acid and the excess of water. The force acting in solutions is consequently of different intensity, starting from so feeble an attraction that the properties of water-as, for instance, its power of evaporation - are but very little changed, and ending with cases of strong attraction between the water and the substance dissolved in or chemically combined with it. In consideration of the very important signification of the phenomena, and of the cases of the breaking up of solutions with separation of water or of the substance dissolved from them, we shall further discuss them separately, after having acquainted ourselves with certain peculiarities of the solution of gases and of solid bodies.

The solubility of gases, which is usually measured by the volume of $\operatorname{gas}^{29}$ (at $0^{\circ}$ and $760 \mathrm{~mm}$. pressure) per 100 volumes of water, varies not only with the nature of the gas (and also of the solvent), and with the temperature, but also with the pressure, because gases themselves change their volumes considerably with the pressure. As might be expected, (1) gases which are easily liquefied (by pressure and cold) are more soluble than those which are liquefied with difficulty. Thus, in 100 volumes of water there dissolve at $0^{\circ}$ and $760 \mathrm{~mm}$. only two volumes of hydrogen, three volumes of carbonic oxide, four volumes of oxygen, \&c., for these are gases which are liquefied with difficulty; whilst

29. If a volume of gas $v$ be measured under a pressure of $h \mathrm{~mm}$. of mercury (at $0^{\circ}$ ) and at a temperature $t^{\circ}$ Centigrade, then, according to the laws of Boyle, Mariotte, and of Gay-Lussac combined, its volume at $0^{\circ}$ and $760 \mathrm{~mm}$. will equal the product of $v$ into 760 divided by the product of $h$ into $1+a t^{\circ}$, where $a$ is the co-efficient of expansion of gases, which is equal to 0.00367 . The weight of the gas will be equal to its volume at $0^{\circ}$ and $760 \mathrm{~mm}$. multiplied by its density referred to air and by the weight of one volume of air at $0^{\circ}$ and $760 \mathrm{~mm}$. The weight of one litre of air under these conditions being= 1.293 grams. If the density of the gas be given in relation to hydrogen this must be divided by 14.4 to bring it in relation to air. If the gas be measured when saturated with aqueous vapour, then it must be reduced to the volume and weight of the gas when dry, according to the rules given in Note 1 . If the pressure be determined by a column of mercury having a temperature $t$, then by dividing the height of the column by $1+0.00018 t$ the corresponding height at $0^{\circ}$ is obtained. If the gas be enclosed in a tube in which a liquid stands above the level of the mercury, the height of the column of the liquid being $=\mathrm{H}$ and its density $=\mathrm{D}$, then the gas will be under a pressure which is equal to the barometric pressure less $\begin{gathered}\mathrm{HD} \\ 13.59\end{gathered}$, where 13.59 is the density of mercury. $\mathrm{By}$ these methods the quantity of a gas is determined, and its observed volume reduced to normal conditions or to parts by weight. The physical data concerning vapours and gases must be continually kept in sight in dealing with and measuring gases. The student nust become perfectly familiar with the calculations relating to gases. 
there dissolve 180 volumes of carbonic anhydride, 130 of nitrous oxide, and $43 \pi$ of sulphurous anhydride, for these are gases which are rather easily liquefied. (2) The solubility of a gas is diminished by heating, which is easy to understand from what has been said previously - that the elasticity of a gas becomes greater as it is further removed from a liquid state. Thus 100 volumes of water at $0^{\circ}$ rissolve $2 \cdot 5$ volumes of air, and at $20^{\circ}$ only 1.7 volumes. For this reason cold water, when brought into a warm room, parts with a portion of the gas dissolved in it. ${ }^{30}$ (3) The quantity of the gas dissolved varies directly with the pressure. This rule is called the lime of Henry and Dalton, and is applicable to those gases which are little soluble in water. Therefore a gas is separated from its solution in water in a vacuum, and water saturated with a gas under great pressure parts with it if the pressure be diminished. Thus many mineral springs are saturated underground with carbonic anhydricle under the great pressure of the column of water above them. On coming to the surface, the water of these springs boils and foams in giving up the excess of dissolved gas. Sparkling wines and aerated waters are saturated under pressure with the same gas. They hold the gas so long as they are in a well-corked vessel. When the cork is removed and the liquid comes in contact with air at a less pressure, part of the gas, unable to remain in solution at a lesser pressure, is separated as foam with the hissing sound familiar to all. It must be reniarked that the law of Henry and Dalton belongs to the class of approximate lares, like the laws of gases (Gay-Lussac's and Mariotte's) and many others - that is, it expresses only a portion of a complex phenomenon, the limit towards which the phenomenon aims. The matter is rendered complicated from the influence of the degree of solubility and of affinity of the dissolved gas for water. Gases which are little soluble - for instance, hydrogen, oxygen, and nitrogen - follow the law of Henry and Dalton the most closely. Carbonic anhydride exhibits a decided deviation from the law, as is seen from the determinations of Wroblewski (1882). He showed that at $0^{\circ}$ a cubic centimetre of water absorbs 1.8 cubic centimetres of the gas under a pressure of one atmosphere ; under 10 atmospheres, 16 cubic centimetres (and not 18 , as it should be according to the law) ; under 20 atmospheres,

so According to Bunsen, 100 volumes of water under a pressure of one atmosphere absorb the following volumes of gas (measured at $0^{\circ}$ and $760 \mathrm{~mm}$.) :-

$\begin{array}{rcccccccccc} & 1 & 2 & 3 & 4 & 5 & 6 & 7 & 8 & 9 & 10 \\ 0^{\circ} & 4 \cdot 11 & 2 \cdot 03 & 1 \cdot 93 & 179 \cdot 7 & 3 \cdot 3 & 130 \cdot 5 & 437 \cdot 1 & 68 \times \cdot 6 & 5 \cdot 4 & 104960 \\ 11^{\circ} & 3 \cdot 25 & 1 \cdot 61 & 1 \cdot 93 & 118 \cdot 5 & 2 \cdot 6 & 92 \cdot 0 & 35 \times \cdot 6 & 513 \cdot 8 & 4 \cdot 4 & 81280 \\ 210^{\circ} & 2 \cdot 84 & 1 \cdot 40 & 1 \cdot 93 & 90 \cdot 1 & 2 \cdot 3 & 67 \cdot 0 & 290 \cdot 5 & 362 \cdot 2 & 3 \cdot 5 & 65400\end{array}$

1, oxygen ; 2, nitrogen : 3 , lyylrogen : 4, earbonic anltydrile ; 5 , carbonic oxide ; 6 , nitrous oxide ; 7 , bydrogen sulphide; 8,5 liphurous aulyydrile ; 9 , marsh gas ; 10 , ammonia. 
26.6 cubic centimetres (instead of 36 ) ; and under 30 atmospheres, $33 \cdot 7$ cubic centimetres. ${ }^{31}$ However, as the researches of Sechenoff show, the absorption of carbonic anhydride within certain limits of change of pressure, and at the ordinary temperature, by water -and even by solutions of salts which are not chemically changed by it, or do not form compounds with it--very closely follows the law of Henry and Dalton, so that the chemical bond between this gas and water is so feeble that the breaking up of the solution with separation of the gas is accomplished by a decrease of pressure alone. ${ }^{32}$ The case is different if a considerable affinity exists between the dissolved gas and water. Then it might even be expected that the gas would not be entirely separated from water in a vacuum, as should be the case with gases according to the law of Henry and Dalton. Such gases-and, in general, all which are very soluble - exhibit a distinct deviation from the law of Henry and Dalton. As examples, ammonia and hydrochloric acid gas may be taken. The former is separated by boiling and decrease of pressure, while the latter is not, but they both deviate distinctly from the law.

\begin{tabular}{|c|c|c|}
\hline $\begin{array}{c}\text { Pressure in mm. } \\
\text { of mercury }\end{array}$ & $\begin{array}{c}\text { Ammonia dissolved } \\
\text { in } 100 \text { grams of } \\
\text { water at } 0^{\circ}\end{array}$ & $\begin{array}{c}\text { Hydrochlorie acid } \\
\text { gas dissolved in 100 } \\
\text { grans of water at } 0^{\circ}\end{array}$ \\
\hline 100 & Grams & Grams \\
500 & $28 \cdot 0$ & $65 \cdot 7$ \\
1,000 & $69 \cdot 2$ & $78 \cdot 2$ \\
1,500 & $112 \cdot 6$ & $85 \cdot 6$ \\
\hline
\end{tabular}

It will be remarked, for instance, from this table that whilst the pres-

31 These figures show that the co-efficient of solubility decreases with an increase of pressure, notwithstanding that the carbonic anhydride approaches a liquid state. And, indeed, liquefied carbonic anhydride does not intermix with water, and does not exhibit a rapid increase in solubility at its temperature of liquefaction. This indicates, in the first place, that solution does not consist in liquefaction, and in the second place that the solubility of a substance is determined by a peculiar attraction of water for the substance dissolving. Wroblewski even considers it possible to admit that a dissolved gas retains its properties as a gas. This he deduces from his experiments, which showed that the rate of diffusion of gases in a solvent is, for gases of different densities, inversely proportional to the square roots of their densities, just as the velocities of movement of gaseous molecules (see Note 34 on p. 80 ). Wroblewski showed the affinity of water, $\mathrm{H}_{2} \mathrm{O}$, for carbonic anhydride, $\mathrm{CO}_{2}$, from the fact that on expanding moist compressed carbonic anlyydride (compressed at $0^{\circ}$ under a pressure of 10 atmospheres) he obtained (a fall in temperature takes place from the expansion) a very unstable definite crystalline compound, $\mathrm{CO}_{2}+8 \mathrm{H}_{2} \mathrm{O}$.

$32 \mathrm{As}$, according to the researches of Roscoe and his collaborators, ammonia exhibits a considerable deviation at low temperatures from the law of Henry and Dalton, whilst at $100^{\circ}$ the deviation is small, it would appear that the dissociating influence of temperature tells on all gaseous solutions; that is, at high temperatures, the solutions of all gases will follow the law, and at lower temperatures there will in all cases be a deviation from it. 
sure increased 10 times, the solubility of ammonia only increased $4 \frac{1}{2}$ times.

A number of examples of such cases of the absorption of gases by liquids might be cited which do not in any way, even approximately, agree with the laws of solubility. Thus, for instance, carbonic anhydride is absorbed by a solution of caustic potash in water, and if there be sufficient caustic potash it is not separated from the solution by a decrease of pressure. This is a case of more intimate chemical combination. A less completely studied, but similar and clearly chemical, correlation appears in certain cases of the solution of gases by water, and we shall afterwards take an example of this in the solution of hydrogen iodide ; but first we will stop to consider a remarkable application of the law of Henry and Dalton ${ }^{33}$ in the case of the solution of a mixture of two gases, and this we must do all the more because the phenomena which then take place cannot be foreseen without a clear theoretical representation of the nature of gases. ${ }^{34}$

35 The ratio between the pressure and the amount of gas dissolved was discovered by Henry in 1805 , and Dalton in 1807 pointed out the adaptability of this law to cases of gaseous mixtures, introducing the conception of partial pressures which is absolutely necessary for a right comprehension of Dalton's law. The conception of partial pressures essentially enters into that of the diffusion of vapours in gases (fontnote 1); for the pressure of damp air is equal to the sum of the pressures of dry air and of the aqueous vapour in it, and it is admitted as a sequence to Dalton's law that evaporation in dry air takes place as in a vacuum. It is, however, necessary to remark that the volume of a mixture of two gases (or vapours) is only approximately equal to the sum of the volumes of its constituents (the same, naturally, also refers to their pressures)-that is to say, in mixing gases a change of volume occurs, which, although small, is quite apparent when carefully measured. For instance, in 1888 Brown showed that on mixing various volumes of sulphurous anhydride $\left(\mathrm{SO}_{2}\right)$ with carbonic anhydride (at equal pressures of $760 \mathrm{~mm}$. and equal temperatures) a decrease of pressure of 3.9 millimetres of mercury was observed The possibility of a chemical action in similar mixtures is evident from the fact that equal volumes of sulphurous and carbonic anhydrides at $-19^{\circ}$ form, according to Pictet's researches in 1888 , a liquid having the signs of a chemical compound, or a solution similar to that given when sulphurous anhydride and water combine into an unstable chemical whole.

31 The origin of the now generally-accepted kinetic theory of gases, according to which they are animated by a rapid progressive movement, is very ancient (Bernoulli and others in the last century had already developed a similar representation), but it was only generally accepted after the mechanical theory of heat had been established, and after the work of Krönig (1855), and especially after its mathematical side had been worked out by Clausius and Maxwell. The pressure, elasticity, diffusion, and internal friction of gases, the laws of Boyle, Mariotte, and of Gay-Lussac and Avogadro-Gerhardt are not only explained (deduced) by the kinetic theory of gases, but also expressed with perfect exactitude; thus, for example, the magnitude of the internal friction of different gases was foretold with exactitude by Maxwell, by applying the theory of probabilities to the concussion of gaseous particles. The kinetic theory of gases must therefore be considered as one of the most brilliant acquisitions of the latter half of the present century. The velocity of the progressive movement of the gaseous particles of a gas, one cubic centimetre of which weighs $d$ grams, is found, according to the theory, to be equal to the square root of the product of $3 p \Gamma g$ divided by $d$, where $p$ is the pressure under which 
The law of partial pressures is as follows:-The solubility of gases in intermixture with each other does not depend on the influence of the total pressure acting on the mixture, but on the influence of that portion of the total pressure which is due to the volume of each given gas in the mixture. Thus, for instance, if oxygen and carbonic anhydride were mixed in equal volumes and exerted a pressure of 760 millimetres,

$d$ is determined expressed in centimetres of the mercury column, $D$ the weight of a cubic centimetre of mercury in grams $(D=13 \cdot 59, p=76$, consequently the normal pressure $=$ 1,033 grams on a sq. c. m.), and $g$ the acceleration of gravity in centimetres $(g=980.5$, at the sea level and long. $45^{\circ},=981.92$ at St. Petersburg; in general it varies with the longitude and altitude of the locality). Therefore, at $0^{\circ}$ the velocity of hydrogen is 1,843 , and of oxygen 461, metres per second. This is the average velocity, and (according to Maxwell and others) it is probable that the velocities of individual particles are different, that is, they occur in, as it were, different conditions of temperature, which is very important to take into consideration in the investigation of many phenomena proper to matter. It is evident from the above determination of the velocity of gases, that different gases at the same temperature and pressure have average velocities, which are inversely proportional to the square roots of their densities; this is also shown by direct experiment on the flow of gases through a fine orifice, or through a porous wall. This dissimilar velocity of flow for different gases is frequently taken advantage of in chemical researches (see Chap. II. and also Chap. VII. on the law of Avogadro-Gerhardt) in order to separate two gases having different densities and velocities. The difference of the velocity of flow of gases also determines the phenomenon cited in the following footnote for demonstrating the existence of an internal movement in gases.

If for a certain mass of a gas which fully and exactly follows the laws of Mariotte and Gay-Lussac the temperature $t$ and the pressure $p$ be simultaneously changed, then the entire change would be expressed by the equation $p r=C(1+a t)$, or, what is the same, $p v=R T$, where $T=t+273$ and $C$ and $R$ are constants which vary not only with the units of measurement but with the nature of the gas and its mass. But as there are discrepancies from both the fundamental laws of gases (which will be spoken of in the following chapter), and as, on the one hand, a certain attraction between the gaseous molecules must be admitted, and on the other hand it must be acknowledged that the gaseous molecules themselves occupy a portion of a space, therefore for ordinary gases, within any considerable variation of pressure and temperature, recourse should be had to Van der Waal's formula-

$$
\left(p+\frac{a}{v_{2}}\right)(v-p)=R(1-a t)
$$

where $a$ is the true co-efficient of expansion of gases. As the actual co-efficient of expansion of air at the atmospheric pressure and between temperatures of $0^{\circ}$ and $100^{\circ}=$ 0.00367 , when determined from the change of pressure (according to Regnault's data) and when determined from the change of volume $=0.00368$ (according to Mendeléeff and Kayander), and for other gases there is a discrepancy, although not a large one (see the following chapter), which is considerable at high pressures and for great densities, therefore that co-efficient of expansion should be taken which all gases have at low pressures. This quantity is approximately 0.00367.

The formula of Van der Waal has an especially important significance in the case of the passage of a gas into a liquid state, because the fundamental properties of both gases and liquids are equally well expressed, although only in their general features, by it.

The further development of the questions referring to the subjects here touched on, which are of especial interest for the theories of solutions, must be looked for in special memoirs and works on theoretical and physical chemistry. A small part of this subject will be partially considered in the footnotes of the following chapter.

VOL. I. 
then water would dissolve so much of each of these gases as would be dissolved if each separately exerted a pressure of half an atmosphere, and in this case, at $0^{\circ}$ one cubic centimetre of water would dissolve 0.02 cubic centimetre of oxygen and 0.90 cubic centimetre of carbonic anhydride. If the pressure of a gaseous mixture equals $h$, and in $n$ volumes of the mixture there be $a$ volumes of a given gas, then its solution will proceed as though this gas were dissolved under a pressure $\frac{h \times a}{n}$. That portion of the pressure under influence of which the solution proceeds is termed the 'partial' pressure.

In order to represent to oneself the cause of the law of partial pressures, an explanation must be given of the fundamental properties of gases. Gases are elastic and disperse in all directions. All that is known of gases obliges one to think that these fundamental properties of gases are due to a rapid progressive movement, in all directions, which is proper to their smallest particles (molecules). ${ }^{35}$ These molecules in impinging against an obstacle produce a pressure. The greater the number of molecules impinging against an obstacle in a given time, the greater the pressure. The pressure of a separate gas or of a gaseous mixture depends on the sum of the pressures of all the molecules, on the number of blows in a unit of time on a unit of surface, and on the mass and velocity (or the vis viva) of the impinging molecules. To the obstacle all molecules (although different in nature) are alike; it is submitted to a pressure due to the sum of their vis viva. But, in a chemical action such as the solution of gases, on the contrary, the

55 Although the actual movement of gaseous molecules, which is acknowledged by the kinetic theory of gases, cannot be seen, yet its existence may be rendered evident by taking advantage of the difference in the velocities which undoubtedly belongs to different gases which are of different densities under equal pressures. The molecules of a light gas must move more rapidly than the molecules of a heavier gas in order to produce the same pressure. Let us take, therefore, two gases-hydrogen and air ; the former is 14.4 times lighter than the latter, and hence the molecules of hydrogen must move almost four times more quickly than air (more exactly $3 \cdot 8$, according to the formula given in the preceding footnote). Consequently, if air occurs inside a porous cylinder and hydrogen outside, then in a given time the volume of hydrogen which succeeds in entering the cylinder will be greater than the volume of air leaving the cylinder, and therefore the pressure inside the cylinder will rise until the gaseous mixture (of air and hydrogen) attains an equal density both inside and outside the cylinder. If now the experiment be reversed and air surround the cylinder, and hydrogen be inside the cylinder, then more gas will leave the cylinder than enters it, and hence the pressure inside the cylinder will be diminished. In these considerations we irave replaced the idea of the number of molecules by the idea of volumes. We shall learn afterwards that equal volumes of different gases contain an equal number of molecules (the law of Avogadro-Grerhardt), and therefore instead of speaking of the number of molecules we can speak of the number of volumes. If the cylinder be partially immersed in water the rise and fall of the pressure can be observed, and consequently the experiment can be rendered selfevident. 
nature of the impinging molecules plays the most important part. In impinging against a liquid, a portion of the gas enters into the liquid itself, and is held by it so long as other gaseous molecules impinge against the liquid - exert a pressure on it. As regards the solubility of a given gas, for the number of blows it makes on the surface of a liquid, it is immaterial whether other molecules of gases impinge side by side with it or not. Therefore, the solubility of a given gas will be proportional, not to the total pressure of a gaseous mixture, but to that portion of it which is due to the given gas separately. Further, the saturation of a liquid by a gas depends on the fact that the molecules of gases that have entered into a liquid do not remain at rest in it, although they enter in a harmonious kind of movement with the molecules of the liquid, and therefore they throw themselves off from the surface of the liquid (just like its vapour if the liquid be volatile). If in a unit of time an equal number of molecules penetrate into (leap into) a liquid and leave (or leap out of) a liquid, it is saturated. It is a case of mobile equilibrium, and not of rest. Therefore, if the pressure be diminished, the number of molecules departing from the liquid will exceed the number of molecules entering into the liquid, and a fresh state of mobile equilibrium only takes place under a fresh equality of the number of molecules departing from and entering into the liquid. Thus are explained the main features of the solution, and furthernore of that special (chemical) attraction (penetration and harmonious movement) of a gas for a liquid, which determines both the measure of solubility and the degree of stability of the solutions produced.

The consequences of the law of partial pressures are exceedingly numerous and important. All liquids in nature are in contact with the atmosphere. The atmosphere, as we shall afterwards see more fully, consists of an intermixture of gases, chiefly four in number - oxygen, nitrogen, carbonic anhydride, and aqueous vapour. 100 volumes of air contain, approximately, 78 volumes of nitrogen, and about 21 volumes of oxygen; the quantity of carbonic anhydride, by volume, does not exceed 0.05 . Under ordinary circumstances, the quantity of aqueous vapour is much greater, but it varies with the moisture of the atmosphere. Consequently, the solution of nitrogen in a liquid in contact with the atmosphere will proceed under a partial pressure equal to $\frac{78}{100} \times 760 \mathrm{~mm}$. if the atmospheric pressure equal $760 \mathrm{~mm}$.; consequently, under a pressure of $600 \mathrm{~mm}$. of mercury, the solution of oxygen will proceed under a partial pressure of about $160 \mathrm{~mm}$., and the solution of carbonic anhydride only under the very small pressure of $0.4 \mathrm{~mm}$. Therefore, although the amount of nitrogen in air is 
large, yet, as the solubility of oxygen in water is twice that of the nitrogen in water, the proportion of oxygen dissolved in water will be greater than its proportion in air. It is easy to calculate what quantity of each of the gases will be contained in water, and we will take the most simple case, and calculate what quantity of oxygen, nitrogen, and carbonic anhydride will be dissolved from air having the above composition at $0^{\circ}$ and $760 \mathrm{~mm}$. pressure. Under a pressure of $760 \mathrm{~mm} .1$ cubic centimetre of water dissolves 0.0203 cubic centimetre of nitrogen, or under the partial pressure of $600 \mathrm{~mm}$. it will dissolve $0.0203 \times \frac{60}{7} \frac{0}{0}$, or 0.0160 cubic centimetre ; of oxygen $0.0411 \times \frac{16}{7} \frac{6}{6}$, or 0.0086 cubic centimetre ; of carbonic anhydride $1.8 \times \frac{0.4}{760}$ or 0.00095 cubic centimetre; consequently, 100 cubic centimetres of water will contain at $0^{\circ}$ altogether 2.55 cubic centimetres of atmospheric gases, and 100 volumes of air dissolved in water will contain about 62 p.c. of nitrogen, 34 p.c. of oxygen, and 4 p.c. of carbonic anhydride. The water of rivers, wells, \&c., usually contains more carbonic anhydride. This proceeds from the oxidation of organic substances falling in the water. The amount of oxygen, however, dissolved in water appears to be actually about $\frac{1}{3}$ the dissolved gases, whilst air contains only $\frac{1}{5}$ of it by volume.

According to the law of partial pressures, whatever gas be dissolved in water will be expelled from the solution in an atmosphere of another gas. This depends on the fact that gases dissolved in water escape from it in a vacuum, because the pressure is nil. An atmosphere of another gas acts like a vacuum on a gas dissolved in water. Separation then proceeds, because the molecules of the dissolved gas no longer impinge upon the liquid, are not dissolved in it, and those previously held in solution depart from the liquid in virtue of their elasticity. ${ }^{36}$ For the same

36 Here there may be, properly speaking, two cases: either the atmosphere surrounding the solution may be limited, or it may be proportionally so vast as to be unlimited, like the earth's atmosphere. If a gaseous solution be brought into an atmosphere of another gas which is limited-for instance, as in a closed vessel-then a portion of the gas held in solution will be expelled, and thus pass over into the atmosphere surrounding the solution, and will evince its partial pressure. Let us imagine that water saturated with carbonic anhydride at $0^{\circ}$ and under the ordinary pressure be brought into an atmosphere of a gas which is not absorbed by water; for instance, that 10 c.c. of an aqueous solution of carbonic anhydride be introduced into a vessel holding 10 c.c of such a gas. The solution will contain $18 \mathrm{c.c}$ of carbonic anhydride. The expulsion of this gas goes on until a state of equilibrium is arrived at. The liquid will then contain a certain amount of carbonic anhydride, which is retained under the partial pressure of that gas which has been expelled. Now, how much gas will remain in the liquid and how much will pass over into the surrounding atmosphere? In order to solve this problem, let us suppose that $x$ cubic centimetres of carbonic anhydride are retained in the solution. It is evident that the amount of carbonic anlyydride which passed over into the surrounding atmosphere will be $18-x$, and the total volume of gas will be $10+18-x$ or $28-x$ cubic centimetres. The partial pressure under 
reason a gas may be entirely expelled from a gaseous solution by boiling - at least, in many cases when it does not form particularly stable compounds with water. In fact the surface of the boiling liquid will be occupied by aqueous vapour, and therefore all the pressure acting on the gas will belong to the aqueous vapour. Consequently, the partial pressure of the dissolved gas will be very inconsiderable. For this, and for no other reason, a gas separates from a solution on boiling the liquid holding it. At the boiling point of water the solubility of gases in water is still sufficiently great for a considerable quantity of a gas to remain in solution. The gas dissolved in the liquid is carried away, together with the aqueous vapour; if boiling be continued for a long time, then in the end all the gas will be separated. ${ }^{37}$

which the carbonic anhydride is then dissolved will be (supposing that the common pressure remains constant the whole time) equal to $\frac{18-x}{28-x}$, consequently there is not in solution 18 c.c of carbonic anhydride (as would be the case were the partial pressure equal to the atmospheric pressure), but only $18 \frac{18-x}{28-x}$, which is equal to $x$, and consequently we obtain the equation $18 \frac{18-x}{28-x}=x$, hence $x=8.69$. Again, where the atmosphere into which the gaseous solution is introduced is not only that of another gas but also unlimited, then the gas dissolved will, on passing over from the solution, diffuse itself through this atmosphere, and from its limitedness produce an infinitely small pressure in the unlimited atmosphere. Consequently, no gas can be retained in solution under this infinitely small pressure, and it will be entirely expelled from the solution. For this reason water saturated with a gas which is not contained in air, will be entirely deprived of the dissolved gas if left exposed to air. Water also passes off from a solution into the atmosphere, and it is evident that there might be such a case as a constant proportion between the quantity of water vaporised and the quantity of a gas expelled from a solution, so that not the gas alone, but the entire gaseous solution, would pass off. A similar case is exhibited in solutions which are not decomposed by heat (such as those of hydrogen chloride and iodide), as will afterwards be considered.

57 However, in those cases when the variation of the co-efficient of solubility with the temperature is not sufficiently great, and when a known quantity of aqueous vapour and of the gas passes off from a solution at the boiling point, an atmosphere may be obtained having the same composition as the liquid itself. In this case the amount of gas passing over into such an atmosphere will not be greater than that held by the liquid, and therefore such a gaseous solution will distil over without change, and without altering its composition during the whole period of boiling or distillation. The solution will then represent, like a solution of hydriodic acid in water, a liquid which is not changed by distillation, while the pressure under which this distillation takes place remains constant. Thus in all its aspects solution presents gradations from the most feeble affinities to examples of intimate chemical combination. The amount of heat evolved in the solution of equal volumes of different gases is in distinct relation with these variations of stability and solubility of different gases. 22.3 litres of the following gases (at 760 $\mathrm{mm}$. pressure) evolve the following number of (gram) units of heat in dissolving in a large mass of water; carbonic anhydride 5,600, sulphurous anhydride 7,700, ammonia 8,800 , hydrochloric acid 17,400 , and hydriodic acid 19,400. The two last-named gases, which are not expelled from their solution by boiling, evolve approximately twice as much heat as such gases as ammonia, which are separated from their solutions by boiling, whilst gases which are only slightly soluble evolve less heat than the latter gases. 
It is evident that the conception of the partial pressures of gases should not only be applied to the formation of solutions, but also to all cases of chemical action of gases. Especially numerous are its applications to the physiology of respiration, for in these cases it is only the oxygen of the atmosphere that acts. ${ }^{38}$

The solution of solids, whilst depending only in a small measure on the pressure under which solution takes place (because solids and liquids are almost incompressible), is very clearly dependent on the temperature. In the great majority of cases the solubility of solids in water increases with the temperature; and further, the rapidity of solution increases also. The latter is determined by the rapidity of diffusion of the solution formed into the remainder of the water. The solution of a solid in water, although it is as with gases, a physical passage into a liquid state, is determined, however, by its chemical affinity for water; which is particularly clear from the fact that in solution there occurs a diminution in volume, a change in the boiling point of water, a change in the tension of its vapour, in the freezing point, and in many similar properties. Were solution a physical, and not a chemical, phenomenon, it would naturally be accompanied by an increase and not by a diminution of volume, because generally in melting a solid increases in volume (its density diminishes). Contraction is the usual phenomenon accompanying solution, and takes place even in the addition of solutions to water, ${ }^{39}$ and in the solution

38 Among the numerous researches concerning this subject, certain results obtrined by Paul Bert are cited in Chapter III., and here we will point out that Prof. Sechenoff, in his researches on the absorption of gases by liquids, very fully investigated the phenomena of the solution of carbonic anhydride in solutions of various salts, and arrived at many important results, which showed that, on the one hand, in the solution of carbonic anhydride in solutions of salts on which it is capable of acting chemically (for example, sodium carbonate, borax, ordinary sodium phosphate), there is not only an increase of solubility, but also a distinct deviation from the law of Henry and Dalton; and, on the other hand, that solutions of salts which are not acted on by carbonic anhydride (for example, the chlorides, nitrates, and sulphates) absorb less of it, by reason of the competition of the already dissolved salt, and follow the law of Henry and Dalton, but all the same show undoubted signs of a chemical action between the salt, water, and carbonic anhydride. Sulphuric acid (whose co-efficient of absorption is 92 vols. per 100), when diluted with water, absorbs less and less carbonic anhydride, until the hydrate $\mathrm{H}_{2} \mathrm{SO}_{4}, \mathrm{H}_{2} \mathrm{O}$ (co-eff. of absorption then equals 66 vols.) is formed; then on further addition of water the solubility again rises until a solution of 100 p.c. of water is obtained.

39 Kremers made this observation in the following simple form :-He took a narrownecked flask, with a mark on the narrow part (like that on a litre flask which is used for accurately measuring liquids), poured water into it, and then inserted a funnel, having a fine tube which reached to the bottom of the flask. Through this funnel he carefully poured a solution of any salt, and (having removed the funnel) allowed the liquid to attain a definite temperature (in a water bath); he then filled the flask up to the mark with water. In this manner two layers of liquid were obtained, the heavy saline solution 
of liquids in water, ${ }^{40}$ just as happens in the combination of substances when evidently new substances are produced. ${ }^{41}$ The contraction which takes place in solution is, however, very small, a fact which depends on the small compressibility of solids and liquids, and on the insignificance of the compressing force acting in solution. ${ }^{42}$ The change of volume which takes place in the solution of solids and liquids, or the alteration in specific gravity ${ }^{43}$ corresponding with it, depends on peculiarities of the dissolving substances, and of water, and, in the majority of cases, is not proportional to the quantity of the substance dis-

below and water above. The flask was then shaken in order to accelerate diffusion, and it was observed that the volume became less if the temperature remained constant. This can be proved by calculation, if the specific gravity of the solutions and water be known. Thus at $15^{\circ}$ one c.c. of a 20 p.c. solution of common salt weighs $1 \cdot 1500$ grams, hence 100 grams occupy a volume of 86.96 c.c. As the sp.gr. of water at $15^{\circ}=0.99916$, therefore 100 grams of water occupy a volume of 100.08 c.c. The sum of the volumes is 187.04 c.c. On mixing, 200 grams of a 10 p.c. solution are obtained. Its specific gravity is 1.0725 (at $15^{\circ}$ and referred to water at its maximum density), hence the 200 grams will occupy a volume of 186.48 c.c. The contraction is consequently equal to 0.56 c.c.

40 The contractions produced in the case of the solution of sulphuric acid in water are shown in the diagram Fig. 17 (page 76). Their maximum is $10^{\circ} 1$ c.c. per 100 c.c. of the solution formed. A maximum contraction of 4.15 at $0^{\circ}, 3.78$ at $15^{\circ}$, and 3.50 at $30^{\circ}$, takes place in the solution of 46 parts by weight of anhydrous alcohol in 54 parts of water. This signifies that if, at $0^{\circ}, 46$ parts by weight of alcohol be taken per 54 parts by weight of water, then the sum of their separate volumes will be $104 \cdot 15$, and after mixing their total volume will be 100 .

41 This subject will be considered later in this work, and we shall then see that the contraction produced in reactions of combination (of solids or liquids) is very variable in its amount, and that there are, although very rare, reactions of combination in which contraction does not take place, or when an increase of volume is produced.

42 The compressibility of solutions of common salt is less, according to Grassi, than that of water. At $18^{\circ}$ the compression of water per million volumes $=48$ vols. for a pressure of one atmosphere; for a 15 p.c. solution of common salt it is 32 , and for a 24 p.c. solution 26 vols. Similar determinations were made by Brown (1887) for saturated solutions of sal ammoniac (38 vols.), alum ( 46 vols.), common salt $(27$ vols.), and sodium sulphate at $+1^{\circ}$, when the compressibility of water $=47$ per million volumes. This investigator also showed that substances which dissolve with an evolution of heat and with an increase in volume (as, for instance, sal-ammoniac) are partially separated from their saturated solutions by an increase of pressure (this experiment was especially convincing in the case of sal-ammoniac), whilst the solubility of substances which dissolve with an absorption of heat or diminution in volume increases, although very slightly, with an increase of pressure. Sorby observed the same phenomenon with common salt (1863).

43 The most trustworthy data relating to the variation of the specific gravity of solutions with a change of their composition and temperature, are collected and discussed in my work cited in footnote 19. The practical (for the amount of a substance in solution is determined by the aid of the specific gravities of solutions, both in works and in laboratory practice) and the theoretical (for specific gravity can be more accurately observed than other properties, and because a variation in specific gravity gorerns the variation of many other properties) interest of this subject, besides the strict rules and laws to which it is liable, make one wish that this province of data concerning solutions may soon be enriched by further observations of as accurate a nature as possible. Their collection does not present any great difficulty, although requiring much time and attention. 
solved, ${ }^{14}$ showing the existence of a chemical action between the solvent and the sulstance dissolved which is of the same nature as in all otlee forms of chenical relation. ${ }^{4 .}$

Althugh an alteration of the external pressure does not usually decompose solutions of solirls, nevertheless the terble development of

th hatsmuch ats the degree of ehamge exhihited in many properties on the formation of

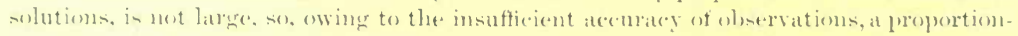

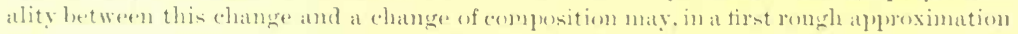

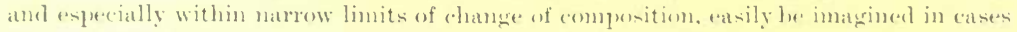
where it does not eren exist. The conclusion of Michel and hivat is particularly instruc-

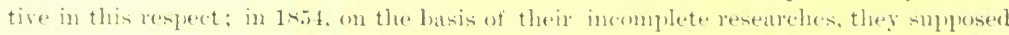
the increment of the speceifice gravity of solutions to be proportional to the increment of at salt in a giren volume of a solution, which is only true for deteminations of specific gratrity which are exact to the second decimal plater-an accuracy insufficient even for tochuical determinations. Acenrate measurements do not comfirm a proportionality

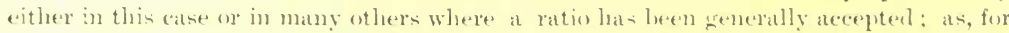
example, for the rotatory power (with respect to the plane of polarisatson of solutions, and for their calpillarity, dic. Vevertheless, such a mether is not only still marle une of, hut even hats its adrantages when applied to solutioms within a linited seoper-ats, for instance, very weak solutions, and for a first aceprantance with the flhenomena acompanying solution, and also as a means for facilitating the alplication of mathematical analysis to the investigation of the plenomenom of solution. Judging by the results oldatined in my researches on the specitic gravity of solutions, I think that in many catses it would be nearer the truth to talie the ehange of properties as proprertional, not to the amount of a substance dissolved, but, to the product of this quantity and the amomut of water in which it is dissolved; all the more so as many chemical relations vary in proportion to the reacting masises, and a similar ratio has been established for many phememena of attraction studied by mechanies. This product is teasily arived at when the gnantity of water in the solutions to be compured is constant, as is shown in investigating the fall of temperature in the formation of ice (sre fortuote $49, \mathrm{p}$. 911 .

1.) All the different forms of (liemical reaction mat he satid to take place in the process of solutions. (1) Combrinations between the solvent and the snbatance dissolved, which are more or less stallele (more or less dissociated). This form of reatetion in the most prebal,le, and is that now often olserved. (2) Reactions of sulstitution of of double fecompresition leetweren the molecules. Thus it may les mpleserd that in the solntion of

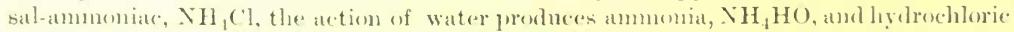
ascid, HC'I, which atre dissolved in the water and simultaneonsly attract wath other. As these solutions and mamy other's do indeed exhibit signs which are sometimes indispu-

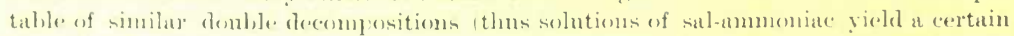
amount of anmonial, it is probiblue that this form of reation is more often met with that is generally theught. (:3) Reatetions of isomeresum or eplacement are also probably met with in solution, all the more as here nolereules of different kinds ronse into intimate (contatet, and it is very likely that the configuration of the atoms in the mestecules under these influeseres is somewhat different from what it was in its original and isolated

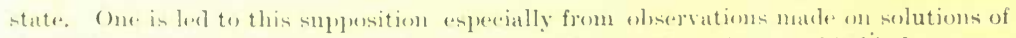

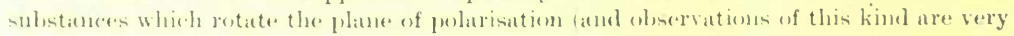

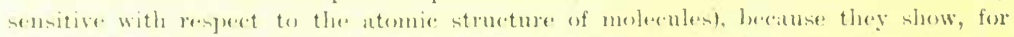

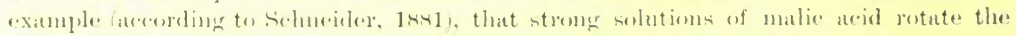

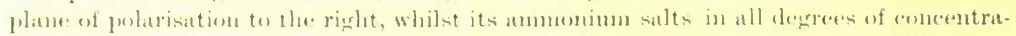
tion rotate the plane of probrisation to the left. (t) Reactions of decompersition under

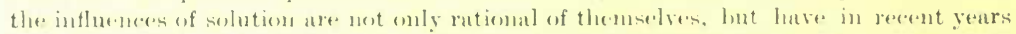

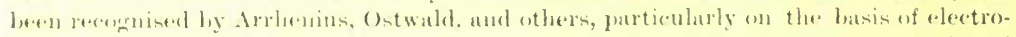
Jytical determinations. If a fertion of the molecules of a selution oxenr in a condition of

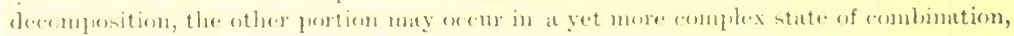


the chemical affinities acting in solutions of solids becomes evident from those multifarious methods by which their solutions are decomposed, whether they be saturated or not. On heating (absorption of heat), on cooling, and by internal forces alone, aqueous solutions in many cases separate into their components or their definite compounds. The water contained in solutions is removed from them as vapour, or, by freezing, in the form of ice, ${ }^{46}$ but the tension of the vapour of water ${ }^{47}$ held in solution is less than that of water in a free

just as the velocity of the movement of different gaseous molecules may be far from being the same (see Note $34, \mathrm{p} .80$ ).

It is, therefore, very probable that the reactions taking place in solution vary both quantitatively and qualitatively with the mass of water in the solution, and the great difficulty in arriving at a lasting decision on the question as to the nature of the chemical relations which take place in the process of solution will be understood, and if besides this the existence of a physical process, like the sliding between and interpenetration of two homogeneous liquids, be also recognised in solution, then the complexity of the problem as to the actual nature of solutions, which is now to the fore, appears in its true light. However, the efforts which are now being applied to the solution of this problem are so numerous and of such varied aspect that they will offer the coming investigators a vast mass of material towards the construction of a complete theory of solution.

For my part, I think that the study of the physical properties of solutions (and especially of weak ones) which now reigns, cannot give any fundamental and complete solution of the problem whatever (although it should add much to both the provinces of physics and chemistry), but that, parallel with it, should be undertaken the study of the influence of temperature, and especially of low temperatures, the application to solutions of the mechanical theory of heat, and the comparative study of the chemical properties of solutions. The beginning of all this is already established, but it is impossible to consider in so short an exposition of chemistry the further efforts of this kind which have been made up to the present date.

46 If solutions are regarded as being in a state of dissociation (see footnote 19, p. 64) it would be expected that they would contain free molecules of water, which form one of the products of the decomposition of those definite compounds whose formation is the cause of solution. In separating as ice or vapour, water makes, with a solution, a heterogeneous system (made up of substances in different physical states) similar, for instance, to the formation of a precipitate or volatile substance in reactions of double decomposition.

47 If the substance dissolved is non-volatile (like salt or sugar), or only slightly volatile, then the whole of the tension of the vapour given off belongs to the water, but if a solution of a volatile substance-for instance, a gas or a volatile liquid-evaporates, then only a proportion of the pressure belongs to the water, and the whole pressure observed consists of the sum of the pressures of the vapours of the water and of the substance dissolved. The majority of researches bear on the first case, which will be spoken of presently, and the observations of D. P. Konovoloff (1881) refer to the second case. He showed that in the case of two volatile liquids, mutually soluble in each other, forming two layers of saturated solutions (for example, ether and water, note 20, p. 66), both solutions have an equal vapour tension (in the case in point the tension of both is equal to $431 \mathrm{~mm}$. of mercury at $\left.19 \cdot 8^{\circ}\right)$. Further, he found that for solutions which are formed in all proportions, the tension is either greater (solutions of alcohol and water) or less (solutions of formic acid) than that which answers to the rectilinear change (proportional to the composition) from the tension of water to the tension of the substance dissolved; thus the tension, for example, of a 70 p.c. solution of formic acid is less, at all 


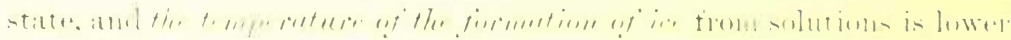

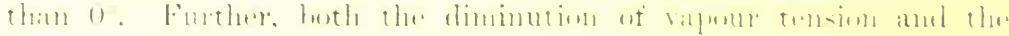

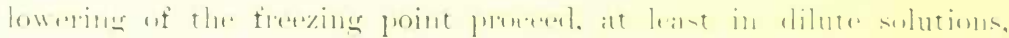

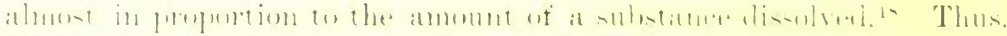

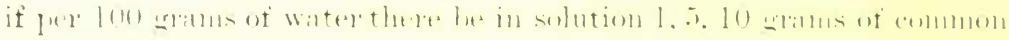

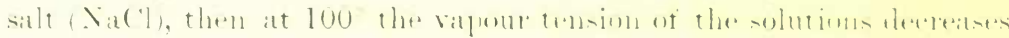

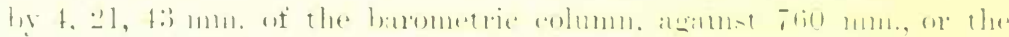

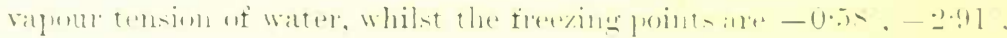

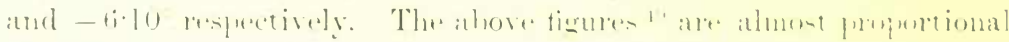

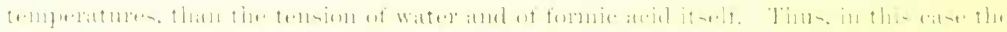

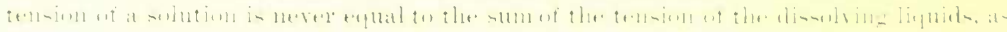

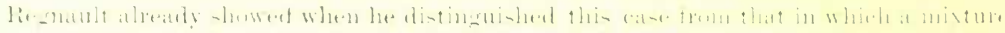

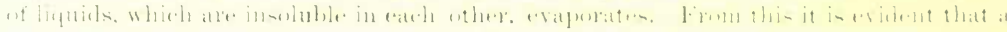

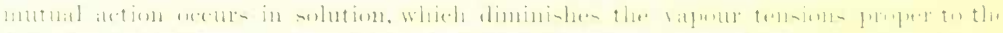

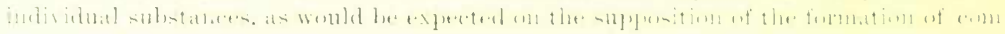

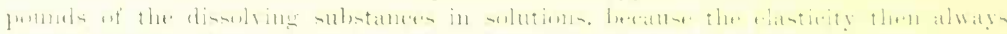
himinian-lit..

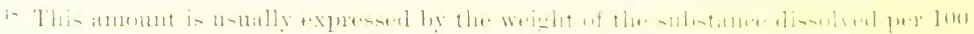

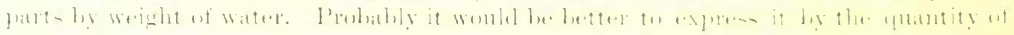

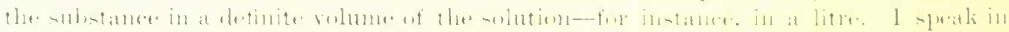

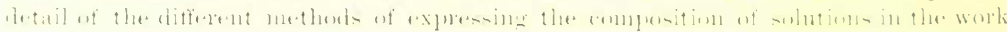
me-ntimed in mit. 1!). 1. tit.

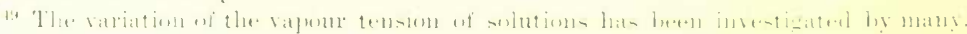

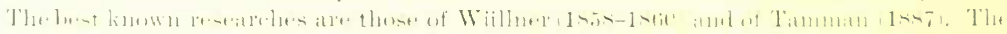

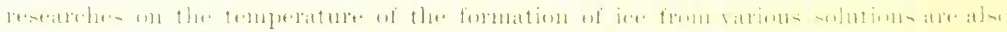

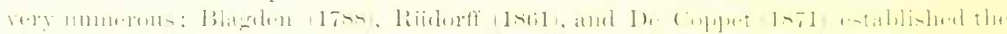

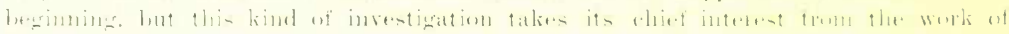

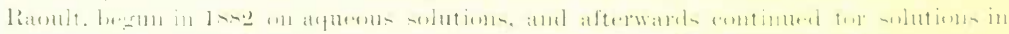

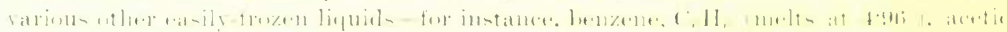

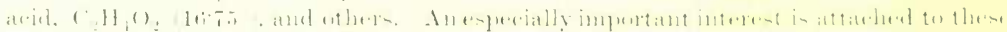

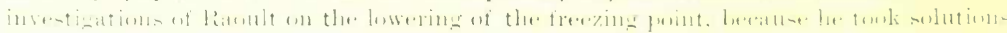

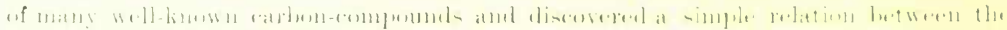

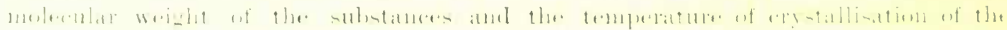

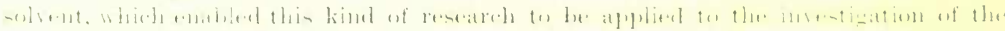

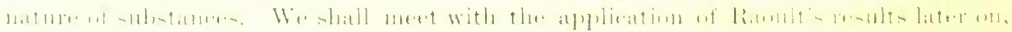

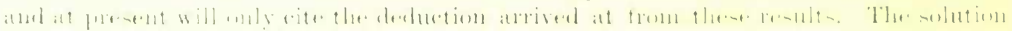

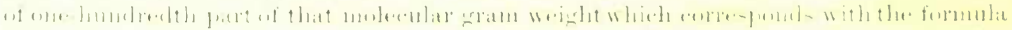

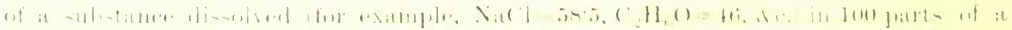

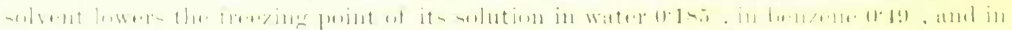

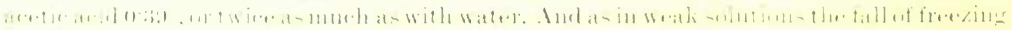

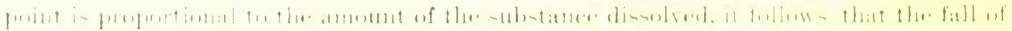

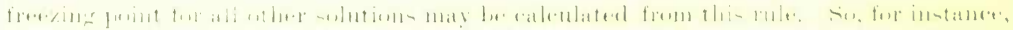

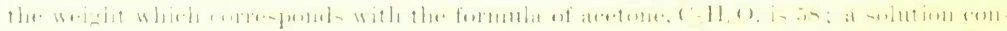

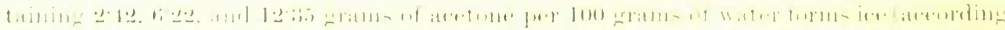

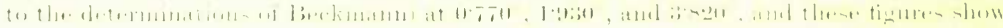

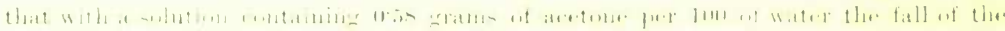

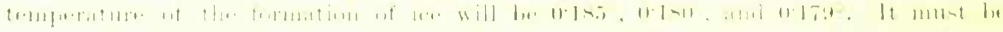

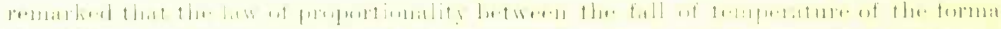

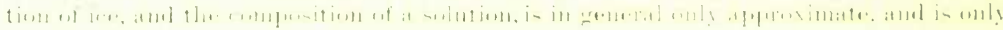

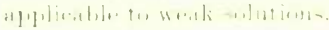

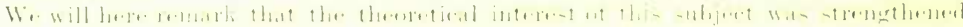

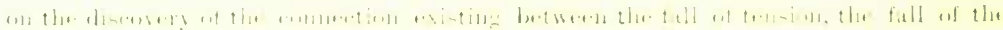


to the amounts of salt in solution ( 1,5 , and 10 per 100 of water). Furthermore, it has been shown by experiment that the ratio of the diminution of vapour tension to the vapour tension of water at different temperatures in a given solution is an almost constant quantity, ${ }^{50}$ and

temperature of the formation of ice, of osmotic pressure (Van't Hoff, note 19), and of the electrical conductivity of solutions, and we will therefore supplement what we have already said on the subject by some short remarks on the method of investigating the phenomenon, and on its theretical results.

In order to determine the temperature of the formation of ice (or of crystallisation of other solvents), a known solution is prepared and poured into a cylindrical vessel surrounded by a second similar vessel, leaving a layer of air between the two, which, being a bad conductor, prevents any rapid change of temperature. The bulb of a sensitive and corrected thermometer is immersed in the solution, and also a bent platinum wire for stirring the solution; the whole is then cooled (by immersing the apparatus in a freezing mixture), and the temperature at which ice begins to separate observed. If the temperature at first falls slightly lower, nevertheless, it becomes constant when ice begins to form. By then allowing the liquid to get just warm, and then again observing the temperature of the formation of ice, an exact determination may be arrived at. If there be a large mass of solution, the formation of the first crystals may be accelerated by dropping a small lump of ice into the solution already partially over-cooled. This only imperceptibly changes the composition of the solution. The observation should be made at the point of formation of only a very small amount of crystals, as otherwise the composition of the solution will become altered from their separation. Every precaution must be taken to prevent the access of moisture to the interior of the apparatus, which might also alter the composition of the solution or properties of the solvent (for instance, when using acetic acid).

The very great theoretical interest of these observations on the fall of the temperature of the formation of ice, which are essentially very simple, dates from the time when Van't Hoff (note 19) showed that their consequences are in complete accord with those derived from observations on osmotic pressure. These latter showed that a molecular (expressed by formulae) quantity of a substance evinces an osmotic pressure in a solution, which is equal to the atmospheric pressure (when $i=1$ ), or which is greater than it by $i$ times. The magnitude $i$, determined from osmotic observations on aqueous solutions, is also obtained from observations on the fall of the temperature of the formation of ice, if the fall corresponding with a solution containing 1 gram of a substance per 100 parts water be multiplied by the molecular weight (according to the formula of the substance, and expressing the weight of a molecule) of the substance dissolved, and divided by 18.5. Thus from the above data for acetone, it is seen that with a solution containing $1 \mathrm{gram}$, the fall of temperature of the formation of ice equals $0.318^{\circ}$, and after multiplying by the molecular weight (58), and dividing by $18 \cdot 5$, we have $i=1$. With sugar and many other substances (among salts, magnesium sulphate, for instance), with carbonic anhydride, \&c., both methods give a figure which is nearly unity. For potassium and sodium chlorides, potassium iodide, nitre, and others, $i$ is greater than 1 but less than 2 ; for sulphuric and hydrochoric acids, sodium and calcium nitrates, and others, $i$ is nearly 2; for solutions of barium and magnesium chlorides, potassium carbonate and dichromate, $i$, according to both methods, is greater than 2 but less than 3 . The further investigation of this subject should show whether these conclusions are entirely general, and would probably explain better than they do now those remarkable correlations which are arrived at with the present data.

50 This fact, which was established by Gay-Lussac, Prinsep, and v. Babo, is confirmed by the latest observations, and enables us to express not only the fall of tension $\left(p-p^{\prime}\right)$ itself, but its ratio to the tension of water $\left(\frac{p-p^{\prime}}{p}\right)$. It is to be remarked that in the absence of any chemical action. the fall of tension is either very small, or does not 


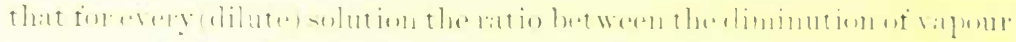

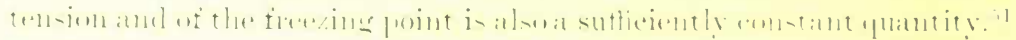

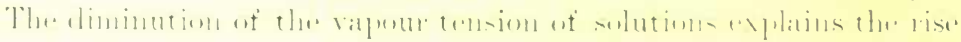

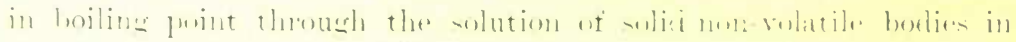

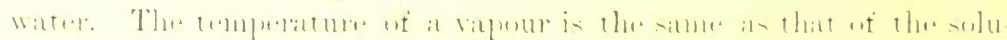

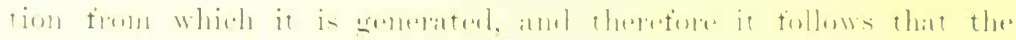

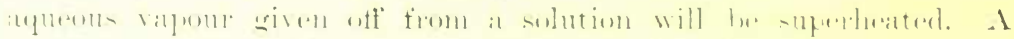

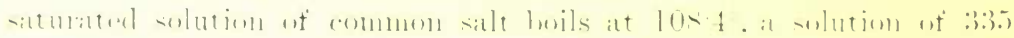

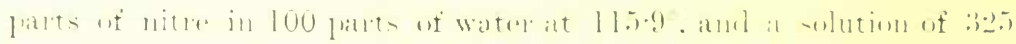

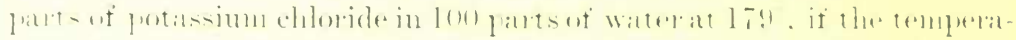

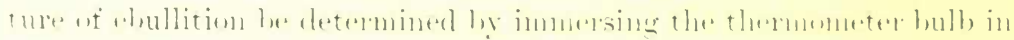

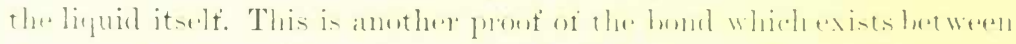

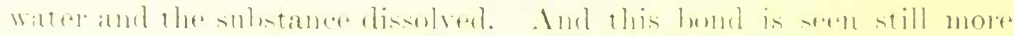

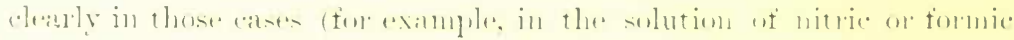

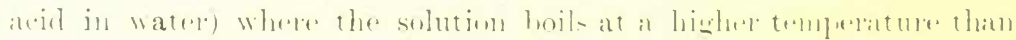

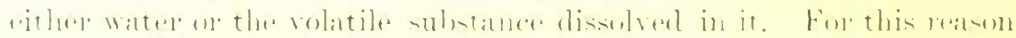

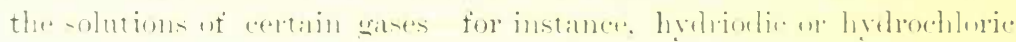
acirl bril almose 100 .

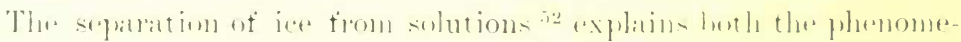

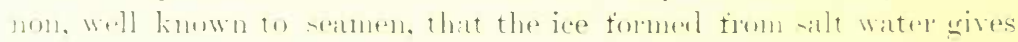

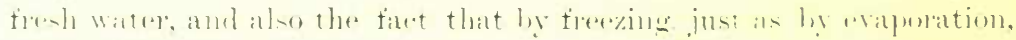

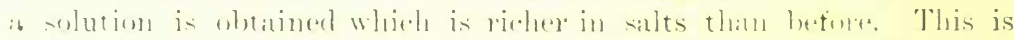

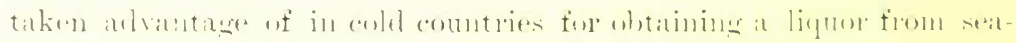

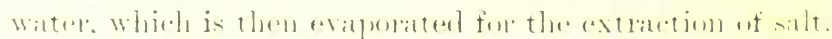

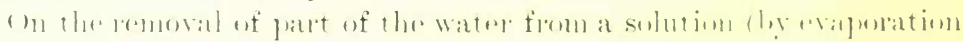

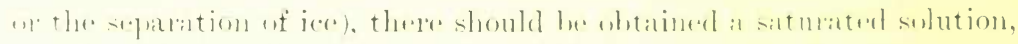

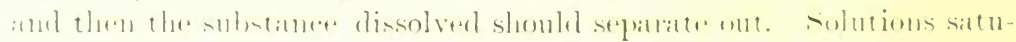

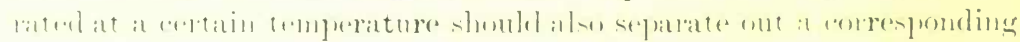

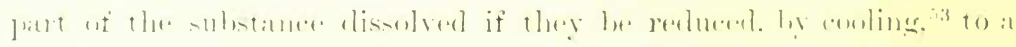

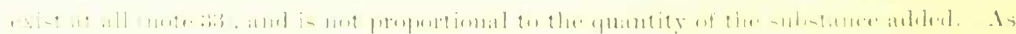

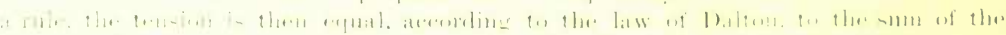

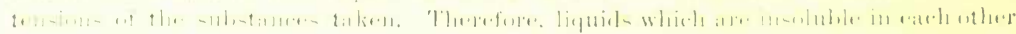

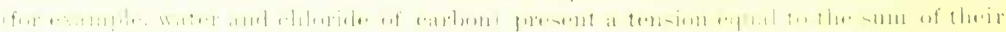

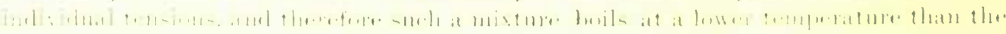

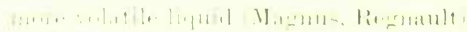

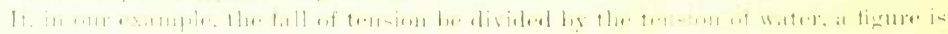

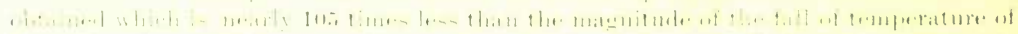

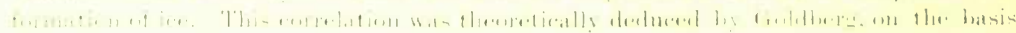

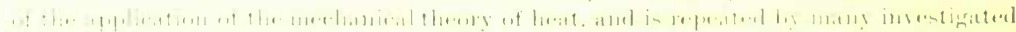
sillin'init.

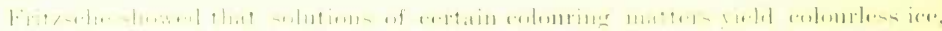

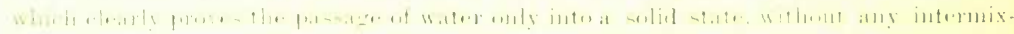

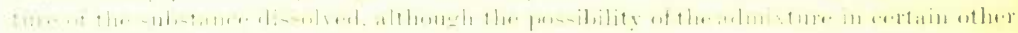

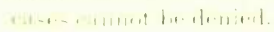

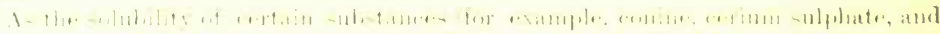

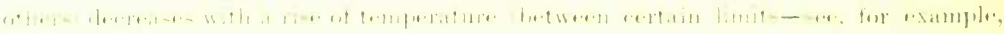


temperature at which the water can no longer hold the former quantity of the substance in solution. If this separation, by cooling a saturated solution or by evaporation, take place slowly, crystals of the substance dissolved are in many cases formed; and this is the method by which crystals of soluble salts are usually obtained. Certain solids rery easily separate out from their solutions in perfectly-formed crystals, which may attain very large dimensions. Such are nickel sulphate, alum, sodium carbonate, chrome-alum, copper sulphate, potassium ferricyanide, and a whole series of other salts. The most remarkable circumstance in this is that many solids in separating out from an aqueous solution retain a portion of water, forming crystallised solid substances which contain water. A portion of the water previously in the solution remains in the separated crystals. The water which is thus retained is called the water of crystallisation. Alum, copper sulphate, Glauber's salt, and magnesium sulphate contain such water, but neither salammoniac, nor table salt, nor nitre, nor potassium chlorate, nor silver nitrate, nor sugar, contains any water of crystallisation. One and the same substance may separate out from a solution with or without water of crystallisation, according to the temperature at which the crystals are formed. Thus common salt in erystallising from its solution in water at the ordinary or a higher temperature does not contain water of crystallisation. But if its separation from the solution takes place at a low temperature, namely below $-5^{\circ}$, then the crystals contain 38 parts of water in 100 parts. Crystals of the same substance which separate out at different temperatures may contain different amounts of water of crystallisation. This proves to us that a solid dissolved in water may form various compounds with it, differing in their properties and composition, and capable of appearing in a solid separate form like many ordinary definite compounds. This is indicated by the numerous properties and phenomena connected with solutions, and gives reason for thinking that there exist in solutions themselves such compounds of

note 24), so these substances do not separate from their saturated solutions on cooling but on heating. Thus a solution of manganese sulphate, saturated at $70^{\circ}$, becomes cloudy on further heating. The point at which a substance separates from its solution with a change of temperature gives an easy means of determining the co-efficient of solubility, and this was taken advantage of by Prof. Alexéeff for determining the solubility of many substances. The phenomenon and method of observation is here essentially the same as in the determination of the temperature of formation of ice. If a solution of a substance which separates out on heating be taken (for example, the sulphate of calcium or manganese), then at a certain fall of temperature ice will separate out from it, and at a certain rise of temperature the salt will separate out. From this example, and from general considerations, it is clear that the separation of a substance dissolved from a solution should present a certain analogy to the separation of ice from a solution. In both cases, a heterogeneous system of a solid and a liquid is formed from a homogeneous (liquid) system. 


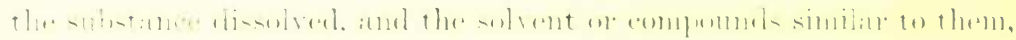

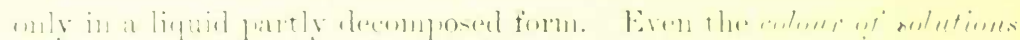

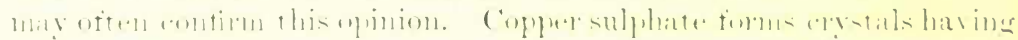

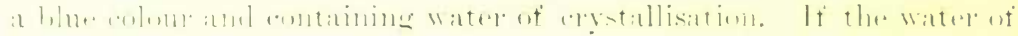

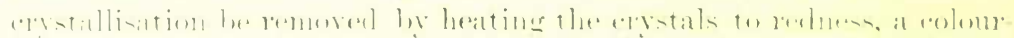

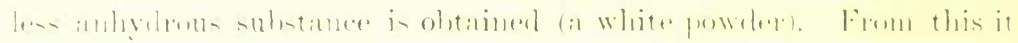

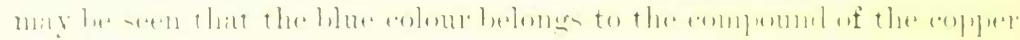

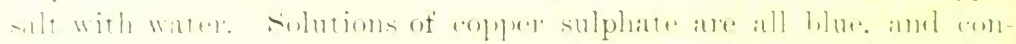

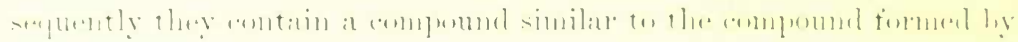

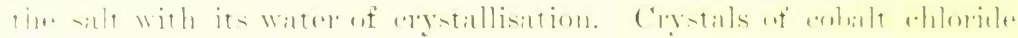

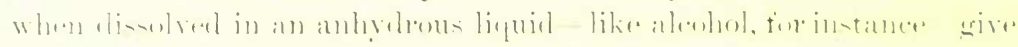

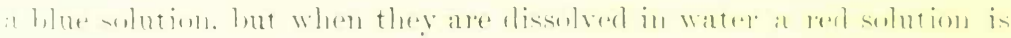

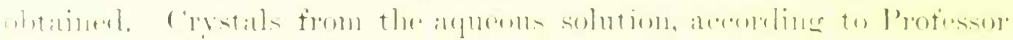

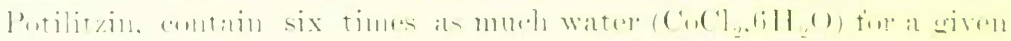

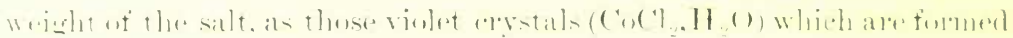

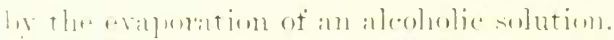

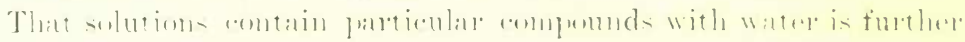

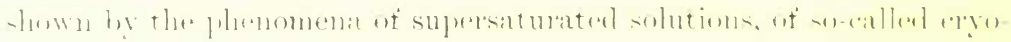

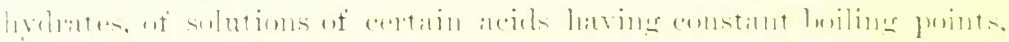

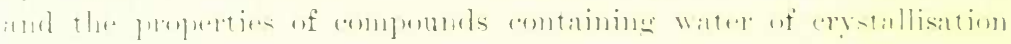

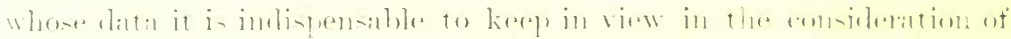
$-1,11+\cdots, 1$, .

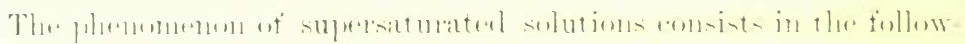

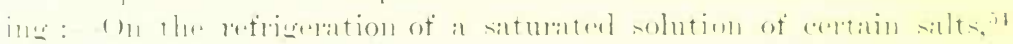

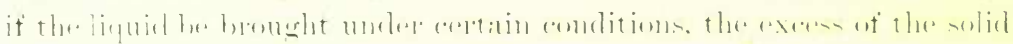

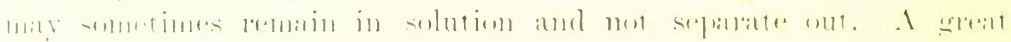

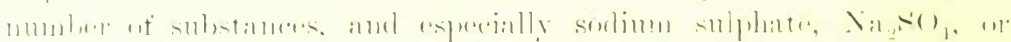

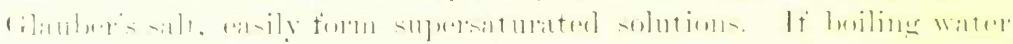

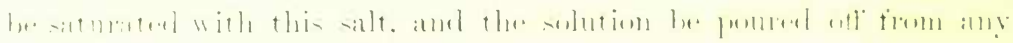

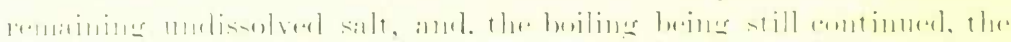

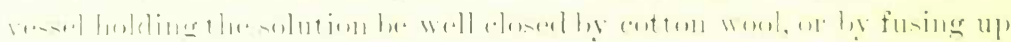

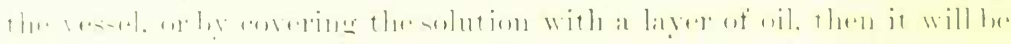

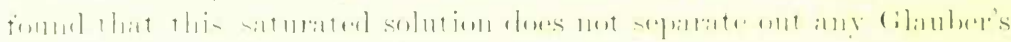

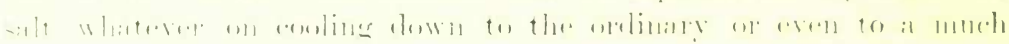

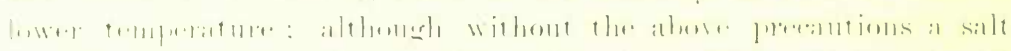

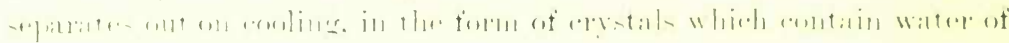

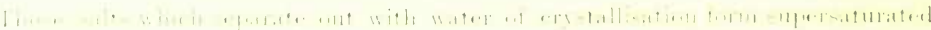

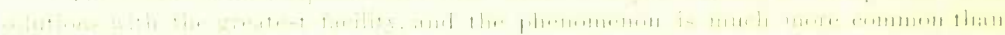

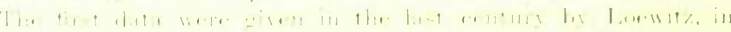

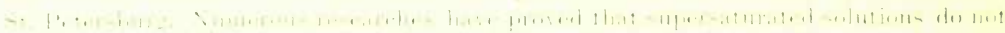

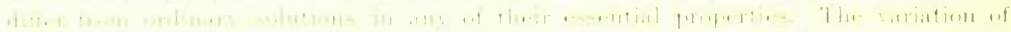

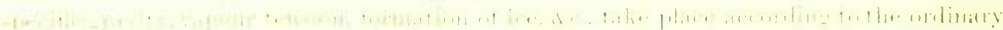


crystallisation to the amount of $\mathrm{Na}_{2} \mathrm{SO}_{4}, 10 \mathrm{H}_{2} \mathrm{O}$-that is, 180 parts of water for 142 parts of anhydrous salt. The supersaturated solution may be moved about or shaken inside the vessel holding it, and no crystallisation will take place; the salt remains in the solution in as large an amount as at a higher temperature. If the vessel holding the supersaturated solution be opened and crystals of Glauber's salt be thrown in, crystallisation suddenly takes place. ${ }^{55}$ A considerable rise in temperature is noticed during this rapid separation of crystals, which is explained by the salt, previously in a liquid state, passing into a solid state, by which, as is known, latent heat is evolved. This somewhat resembles the fact that water may be cooled below $0^{\circ}\left(\right.$ even to $\left.-10^{\circ}\right)$ if it be left at rest, under certain circumstances, and evolves heat in suddenly crystallising. Although from this point of view there is a resemblance, yet in reality the phenomenon of supersaturated solutions is much more complicated. Thus, on cooling, a saturated solution of Glauber's salt deposits crystals containing $\mathrm{Na}_{2} \mathrm{SO}_{4}, 7 \mathrm{H}_{2} \mathrm{O},{ }^{56}$ or 126 parts

55 Inasmuch as air, as has been shown by direct experiment, contains, although in very small quantities, minute crystals of salts, and among them of sodium sulphate, air can bring about the crystallisation of a saturated solution of sodium sulphate in an open vessel, but it has no effect on saturated solutions of certain other salts; for example, lead acetate. According to the observations of De Boisbaudran, Gernez, and others, isomorphous salts (analogous in composition) are capable of evoking crystallisation. Thus, a supersaturated solution of nickel sulphate crystallises by contact with crystals of sulphates of other metals analogous to it, such as those of magnesium, cobalt, copper, and manganese. The crystallisation of a supersaturated solution, brought about by the contact of a minute crystal, starts from it in rays with a definite velocity, and it is evident that the crystals as they form propagate the crystallisation in definite directions. This phenomenon recalls the evolution of organisms from germs. An attraction of similar molecules ensues, and they dispose themselves in definite similar forms.

56 In these days a view is very generally accepted, which regards supersaturated solutions as homogeneous systems, which pass into heterogeneous systems (composed of a liquid and a solid substance), in all respects exactly resembling the passage of water cooled below its freezing point into ice and water, or the passage of crystals of rhombic sulphur into monoclinic crystals, and of the monoclinic crystals into rhombic. Although many phenomena of supersaturation are thus clearly understood, yet the spontaneous formation of the unstable hepta-hydrated salt (with $7 \mathrm{H}_{2} \mathrm{O}$ ), in the place of the more stable deca-hydrated salt (with mol. $10 \mathrm{H}_{2} \mathrm{O}$ ), indicates a property of a saturated solution of sodium sulphate which obliges one to admit that it has a different structure form an ordinary solution. Stcherbacheff affirms, on the basis of his researches, that a solution of the deca-hydrated salt gives, on evaporation, without the aid of heat, the deca-hydrated salt, whilst after heating above $33^{\circ}$ it forms a supersaturated solution and the hepta-hydrated salt, which gives reason for thinking that the state of salts in supersaturated solutions is different from that in ordinary solutions. But in order that this view should be accepted, some signs must be discovered distinguishing solutions (which are, according to this view, isomeric) containing the hepta-hydrated salt from those containing the decahydrated salt, and all efforts made in this direction (the study of the properties of the solutions) have given negative results. Further, according to this view, one would expect that all supersaturated solutions would contain particular forms of erystallohydrates, and, although this is possible, yet up to now nothing of the kind has been observed, 
of water per 142 parts of anhydrous salt, and not 180 parts of water, as in the above-mentioned salt. Further, the crystals containing $7 \mathrm{H}_{2} \mathrm{O}$ are distinguished for their instability ; if they stand in contact not only with crystals of $\mathrm{Na}_{2} \mathrm{SO}_{4}, 10 \mathrm{H}_{2} \mathrm{O}$, but with many other substances, they immediately become opaque, forming a mixture of anhydrous and decahydrated salts. It is evident that between water and a soluble substance there may be established different kinds of greater or less stable equilibrium, of which solutions form one aspect. ${ }^{57}$

and one must think that the connection with the fusibility of the deca-hydrated salt (and of all salts which easily give supersaturated solutions and are capable of forming several crystallohydrates), and with that decomposition (formation of the anlyydrous salt) which the deca-hydrated salt suffers on melting-plays its part here. As some crystallohydrates of salts (alums, sugar of lead, calcium chloride) melt without decomposing, whilst others (like $\mathrm{Na}_{2} \mathrm{SO}_{4}, \mathrm{H}_{2} \mathrm{O}$ ) are decomposed, then it may be that the latter are only in a state of equilibrium at a higher temperature than their melting point. Did experiment show that the hepta-hydrated salt began to crystallise below $33^{\circ}$, and that then only the crystals grow, then all the data concerning supersaturated solutions of sodium sulphate cuuld be explained exclusively in the sense of a super-cooling effect. At present, however, these questions, notwithstanding the mass of research to which they have been subjected, cannot be considered as fully resolved. It may liere be observed that in melting crystals of the deca-hydrated salt, there is formed, besides the solid anhydrous salt, a saturated solution giving the hepta-hydrated salt, so that this passage from the deca- to the hepta-hydrated salt, and the reverse, takes place with the formation of the anhydrous (or it may be, mono-hydrated) salt.

The researches of Pickering (1887) on the amount of heat which is evolved in the solution of hydrous and anhydrous salts at different temperatures, give reason to think that at a certain temperature no heat will be evolved in the combination with water; that is, that probably such a combination will not take place. Thus 106 grams (the molecular weight in grams) of anhydrous sodium carbonate, $\mathrm{Na}_{2} \mathrm{CO}_{3}$, in dissolving in 7,200 grams $\left(=400 \mathrm{H}_{2} \mathrm{O}\right)$ of water, evolve 4,300 calories at $4^{\circ}, 5,300$ at $16^{\circ}$, and 5,850 calories at $25^{\circ}$ (in other cases the heat evolved in solution also increases with a rise of temperature). If, however, the crystallo. hydrate, $\mathrm{Na}_{2} \mathrm{CO}_{3}, 10 \mathrm{H}_{2} \mathrm{O}$, be taken, then (for the same quantity of anhydrous salt) an absorption of heat is observed; at $4^{\circ}-16,250$, at $16^{\circ}-16,150$, and at $25^{\circ}-16,300$ calories. As in this case a portion of the heat absorbed is due to the fact that the water of crystallisation taken in a solid state appears in a liquid state, Pickering subtracts the latent heat of liquefaction of ice, and obtains in the given case at $4^{\circ}-1,700$, at $16^{\circ}-600$, and at $28^{\circ}-0$ calories. From this, the heat of the formation of the crystallohydrate, or the heat evolved by the combination of $\mathrm{Na}_{2} \mathrm{CO}_{3}$ with $10 \mathrm{H}_{2} \mathrm{O}$, may be calculated (by subtracting the former quantities from the first). At $4^{\circ}$ it is equal to $+6,000$, at $16^{\circ}+5,900$, at $25^{\circ}+5,850$ calories; that is, it distinctly decreases, although but slightly, with the rise of temperature. It may be that for $\mathrm{Na}_{2} \mathrm{SO}_{4}$ at $33^{\circ}$ the heats of the formation of $+10 \mathrm{H}_{2} \mathrm{O}$ and $7 \mathrm{H}_{2} \mathrm{O}$ differ but very slightly.

57 Emulsions, like milk, are composed of a solution of glutinous or like substances, or of oily liquids suspended in a liquid in the form of drops, which are clearly visible under a microscope, and form an example of a mechanical formation which resembles solutions. But the difference from solutions is here evident. There are, however, solutions which approach very near to emulsions in the facility with which the substance dissolved separates from them. It has long been known, for example, that a particular kind of Prussian blue, $\mathrm{KFe}_{2}(\mathrm{CN})_{6}$, dissolves in pure water, but, on the addition of the smallest quantity of either of a number of salts, it curdles and becomes quite insoluble. If copper sulphide $(\mathrm{CuS})$, cadmium sulphide (CdS), arsenic sulphide $\left(\mathrm{As}_{2} \mathrm{~S}_{3}\right)$, and many other metallic sulphides, be obtained by a method of double decomposition (by precipi- 
Solutions of salts on refrigeration below $0^{\circ}$ deposit ice or crystals (which then usually contain water of crystallisation) of the salt dissolved, and on arriving by this means at a certain degree of concentration they soliclify in their entire mass. These soliditied masses are termed cryolyydrates. My researches on solutions of common salt (1868) showed that its solution solidifies when it reaches a composition $\mathrm{NaCl}+10 \mathrm{H}_{2} \mathrm{O}(180$ parts of water per 58.5 parts of salt), which takes place at about $-23^{\circ}$. The solidified solution melts at the same temperature, and both the portion melted and the remainder preserve the above composition. Guthrie (1874-1876) obtained the cryohydrates of many salts, and he showed that certain of them are formed at comparatively low temperatures, whilst others (for instance, corrosive sublimate, alums, potassium chlorate, and various colloids) are formed on a slight cooling, to $-2^{\circ}$ or even before, and that these contain a very large amount of water. One can easily imagine that these two series of cryohydrates differ considerably from each other, but the insufficiency of the existing data ${ }^{58}$ does not permit of a true judgment being formed. Nevertheless, in the case of common salt, the cryo-

tating salts of these metals by hydrogen sulphide), and be then carefully washed (by allowing the precipitate to settle, pouring off the liquid, and again adding sulphuretted lydrogen water), then, as was shown by Schulze, Spring, Prost, and others, the previously insoluble sulphides pass into transparent (for mercury, lead, and silver, reddish brown ; for copper and iron, greenish brown; for cadmium and indium, yellow; and for zinc, colourless) solutions, which may be preserved (the weaker they are the longer they keep) and even boiled, but which, nevertheless, in time become curdled-that is, settle in an insoluble form, and then sometimes become crystalline and quite incapable of re-dissolving. Graham and others observed the power shown by colloids (see note 18) of forming similar hydrosols or solutions of gelatinous colloids, and, in describing alumina and silica, we shall have occasion to speak of such solutions once more.

In the existing state of our knowledge concerning solution, such solutions may be looked on as a transition between emulsion and ordinary solutions, but no fundamental judgment can be formed about them until a study has been made of their relations to ordinary solutions (the solutions of even soluble colloids freeze immediately on cooling below $0^{\circ}$, and, according to Guthrie, do not form cryohydrates), and to supersaturated solutions, with which they have certain points in common.

38 Offer (1880) concludes, from his researches on cryohydrates, that they are simple mixtures of ice and salts, having a constant melting point, just as there are alloys having a constant point of fusion, and solutions of liquids with a constant boiling point (see note 60 ). This does not, however, explain in what form a salt is contained, for instance, in the cryohydrate, $\mathrm{NaCl}+10 \mathrm{H}_{2} \mathrm{O}$. At temperatures above $-10^{\circ}$ common salt separates out in anhydrous crystals, and at temperatures near $-10^{\circ}$, in combination with water of crystallisation, $\mathrm{NaCl}+2 \mathrm{H}_{2} \mathrm{O}$, and, therefore, it is very improbable that at still lower temperatures it would separate without water. If the possibility of the solidified cryohydrate containing $\mathrm{NaCl}+2 \mathrm{H}_{2} \mathrm{O}$ and ice be admitted, then it is not clear why one of these substances does not melt before the other. If alcohol does not extract water from the solid mass, leaving the salt behind, this does not prove the presence of ice, because alcohol also takes up water from the crystals of many lydrated substances (for instance, from $\mathrm{NaCl}+2 \mathrm{H}_{2} \mathrm{O}$ ) at about their melting-points. Besides which, a simple observation on the cryohydrate, $\mathrm{NaCl}+10 \mathrm{H}_{2} \mathrm{O}$, shows that with the most careful cooling it does not

VOL. I. 
hydrate with 10 molecules of water, and in the case of sodium nitrate, the cryohydrate ${ }^{59}$ with 7 molecules of water (i.e., 126 parts of water per 85 of salt) should be accepted as established substances, capable of passing from a solid to a liquid state and conversely; and therefore it may be thought that in cryohydrates we have solutions which are not only undecomposable by cold, but also have a definite composition which would present a fresh case of definite equilibrium between the solvent and the substance dissolved.

The formation of definite but unstable compounds in the process of solution becomes evident from the phenomena of a marked decrease of vapour tension, or from the rise of the temperature of ebullition which occurs in the solution of certain volatile liquids and gases in water. As an example, we will take hydriodic acid, HT, a gas which liquefies on a very considerable reduction of temperature, giving a liquid which boils at $-20^{\circ}$. A solution of it containing 57 p.c. of hydriodic acid is distinguished by its great stability. If it be evaporated by heating, the hydriodic acid volatilises together with the water in the same proportions as they occur in the solution, so that the gas passes off together with the aqueous vapour, and therefore such a solution may be distilled unchanged, for the distillate will contain the same proportion of hydriodic acid and water as was originally taken. The solution boils at a higher temperature than water. The physical properties of the gas and water in this case already disappear; there is formed a stable compound between water and the gas, a new substance which has its definite boiling point. To put it more correctly, this is not the temperature of ebullition, but the temperature at which the compound formed decomposes, forming the vapours of the products of dissociation, which, on cooling, re-combine. The above-described aqueous solution boils at $127^{\circ}$. Should a less amount of hydriodic acid be dissolved in water than the above, then, on heating such a solution, water only will at first be distilled over, until the solution attains the abovementioned composition; it will then distil over unaltered. If more hydriodic acid be passed into such a solution a fresh quantity of the gas will dissolve, which, however, may be very easily removed. It must not, however, he thought that those forces which determine the

on the addition of ice deposit ice, which would occur if ice in intermixture with the salt were formed on solidification.

I may add with regard to cryohydrates that, in investigating aqueous solutions of alcohol (note 19), I concluded, on the basis of the specific gravity, that a compound, $\mathrm{C}_{2} \mathrm{H}_{6} \mathrm{O}+12 \mathrm{H}_{2} \mathrm{O}$, existed, and a solution of this composition completely solidifies on cooling to $-20^{\circ}$, forming well-formed crystals, which melt at about $-18^{\circ}$, as was shown by observations made by W. E. Tischenko and myself. This definite compound reminds one of cryohydrates in many respects.

59 See note 24. 
formation of ordinary gaseous solutions play no part whatever in the formation of a solution having a definite boiling point; that they do act is shown from the fact that such constant gaseous solutions vary in their composition under different pressures. ${ }^{60}$ Therefore, it is not at

60 For this reason (the want of entire constancy of the composition of constant boiling solutions with a change of pressure) many deny the existence of definite hydrates formed by volatile substances-for instance, by hydrochloric acid and water. They generally argue as follows: If there did exist a constancy of composition, then it would not be altered by a change of pressure. But the distillation of constant boiling hydrates is undoubtedly accompanied (judging by the vapour densities determined by Bineau), like the distillation of sal-ammoniac, sulphuric acid, \&c., by an entire decomposition of the previous compound-that is, these substances do not exist in a state of vapour, but their products of decomposition (hydrochloric acid and water) are gases at the temperature of volatilisation, which dissolve in the volatilised and condensed liquids; but the solubility of gases in liquids depends on the pressure, and, therefore, the composition of constant boiling solutions may, and even ought to, vary with a change of pressure, and, further, the smaller the pressure and the lower the temperature of volatilisation, the more likely is a true compound to be obtained. According to the researches of Roscoe and Dittmar (1859), the constant boiling solution of hydrochloric acid proved to contain 18 p.c. of hydrochloric acid at a pressure of 3 atmospheres, 20 p.c. at 1 atmosphere, and 23 p.c. at $\frac{1}{10}$ of an atmosphere. On passing air through the solution until its composition became constant (i.e., forcing the excess of aqueous vapour or of hydrochloric acid to pass away with the air), then acid was obtained containing about 20 p.c. at $100^{\circ}$, about 23 p.c. at $50^{\circ}$, and about 25 p.e. at $0^{\circ}$. From this it is seen that by decreasing the pressure and lowering the temperature of evaporation one arrives at the same limit, where the composition should be taken as $\mathrm{HCl}+6 \mathrm{H}_{2} \mathrm{O}$, which requires $25 \cdot 26$ p.c. of hydrochloric acid. Fuming hydrochloric acid contains more than this.

The most important fact in evidence of the existence of definite compounds in acids boiling at a constant temperature is the fall of tension. The gas loses its tension, does not follow the law of Henry and Dalton with a diminution of pressure; its solution only parts with water; the vapour tension of a volatile liquid in solution is less than its own or that of the water combined with it. This loss of tension is a loss of movement brought about by the action of the attraction existing between the water and the substance dissolved. In the case already considered, as in the case of formic acid in the researches of D. P. Konovaloff (note 47), the constant boiling solution corresponds with a minimum tensionthat is, with a boiling point higher than that of either of the component elements. But there is another case of constant boiling solutions similar to the case of the solution of propyl alcohol, $\mathrm{C}_{3} \mathrm{H}_{8} \mathrm{O}$, when a solution, undecomposed by distillation, boils at a lower point than that of the more volatile liquid. However, in this case also, if there be solution, the possibility cannot be denied of the formation of a definite compound in the form $\mathrm{C}_{3} \mathrm{H}_{8} \mathrm{O}+\mathrm{H}_{2} \mathrm{O}$, and the tension of the solution is not equal to the sum of tensions of the components. There are possible cases of constant boiling mixtures even when there is no solution nor any loss of tension, and consequently no chemical action, because the amount of liquids that are volatilised is determined by the product of the vapour densities into their vapour tensions (Wanklyn), in consequence of which liquids whose boiling point is above $100^{\circ}$-for instance, turpentine and ethereal oils in general-when distilled with aqueous vapour, pass over at a temperature below $100^{\circ}$. Consequently, it is not in the constancy of composition and boiling point (temperature of decomposition) that the signs of a clear chemical action should be seen in the above-described solntions of acids, but in the great loss of tension, which completely resembles the loss of tension observed, for instance, in the perfectly-definite combinations of substances with water of crystallisation (see later, note 65). Sulphuric acid, $\mathrm{H}_{2} \mathrm{SO}_{4}$, as we shall learn later, is also decomposed by distillation, like $\mathrm{HCl}+6 \mathrm{H}_{2} \mathrm{O}$, and exhibits, moreover, all the signs of a 
every, but only at the ordinary, atmospheric pressure that a constant boiling solution of hydriodic acid will contain 57 p.c. of the gas. At another pressure the proportion of water and hydriodic acid will be different. It varies, however, judging from observations made by Roscoe, very little for considerable variations of pressure. This variation in composition directly indicates that pressure exerts an influence on the formation of unstable chemical compounds which are easily dissociated (with formation of a gas), just as it influences the solution of gases, only the latter is influenced to a more considerable degree than the former. ${ }^{61}$ Hydrochloric, nitric, and other acids form solutions having definite boiling points, like that of hydriodic acid. They show further the common property, if containing but a small proportion of water, that they fume in air. Strong solutions of nitric, hydrochloric, hydriodic, and other gases are even termed 'fuming acids.' The fuming liquids contain a definite compound, whose temperature of ebullition (decomposition) is higher than $100^{\circ}$, and contain also an excess of the volatile substance dissolved, which (the substance) exhibits a capacity to combine with water and form a hydrate, whose vapour tension is less than that of aqueous vapour. On evaporating in air, this dissolved substance meets the atmospheric moisture and forms a visible vapour (fumes) with it, which consists of the above-mentioned compound. The attraction or affinity which binds, for instance, hydriodic acid with water is evinced not only in the evolution of heat and the diminution of vapour tension (rise of boiling point), but also in many purely chemical relations. Thus hydriodic acid is produced from iodine and hydrogen sulphide in the presence of water, but unless water is present this reaction does not take place. ${ }^{62}$

definite chemical compound. The study of the variation of the specific gravities of solutions as dependent on their composition (see note 19) shows that phenomena of a similar kind, although of different dimensions, take place in the formation of both $\mathrm{H}_{2} \mathrm{SO}_{4}$ from $\mathrm{H}_{2} \mathrm{O}$ and $\mathrm{SO}_{3}$, and of $\mathrm{HCl}+6 \mathrm{H}_{2} \mathrm{O}$ (or of aqueous solutions analogous to it) from $\mathrm{HCl}$ and $\mathrm{H}_{2} \mathrm{O}$.

61 The essence of the matter may be thus represented. A substance $A$, either gaseous or easily volatile, forms with a certain quantity of water, $n \mathrm{H}_{2} \mathrm{O}$, a definite complex compound $A n \mathrm{H}_{2} \mathrm{O}$, which is stable up to a temperature $t^{\circ}$ higher than $100^{\circ}$. At this temperature it is decomposed into two substances, $A+\mathrm{H}_{2} \mathrm{O}$. Both boil below $t^{\circ}$ at the ordinary pressure, and therefore at $t^{\circ}$ they distil over and re-combine in the receiver. But if a part of the substance $\mathrm{An} \mathrm{H}_{2} \mathrm{O}$ is decomposed or volatilised, there still remains a portion of undecomposed liquid in the vessel, which can partially dissolve one of the products of decomposition, and that in quantity varying with the pressure and temperature, and therefore the solution at a constant boiling point will have a slightly-different composition at different pressures.

62 For solutions of hydrochloric acid in water there are still greater differences in reactions. For instance, strong solutions decompose antimony sulphide (forming hydrogen sulphide, $\mathrm{H}_{2} \mathrm{~S}$ ), and precipitate common salt from its solutions whilst weak solutions do not act thus. 
Many compounds containing water of crystallisation are solid substances (when melted they are already solutions-i.e., liquids); furthermore, they are capable of being formed from solutions, as is ice or aqueous vapour. I propose calling them crystallo-hydrates. Inasmuch as the direct presence of ice or aqueous vapour cannot be admitted in solutions (for these are liquids), although the presence of water may be, so also there is no basis for acknowledging the presence in solutions of substances in an already-existing state of combination with water of crystallisation, although they are obtained from solutions as such. ${ }^{63}$ It is evident that such substances present one of the many forms of equilibrium between water and a substance dissolved in it. This form, however, reminds one, in all respects, of solutions-that is, aqueous compounds which are more or less easily decomposed, with separation of water and the formation of a less aqueous or an anhydrous compound. In fact, there are not a few crystals containing water which lose a part of their water at the ordinary temperature. Of such a kind, for instance, are the crystals of soda, or sodium carbonate, which, when separated from an aqueous solution at the ordinary temperature, are quite transparent; but when left exposed to air, lose a portion of their water, becoming opaque, and, in the process, lose their crystalline appearance, although preserving their original form. This process of the separation of water at the ordinary temperature is termed the efflorescence of crystals. Efflorescence takes place more rapidly under the receiver of an air pump, and especially at a gentle heat. This breaking up of a crystal is dissociation at the ordinary temperature. Solutions are decomposed in exactly the same manner. ${ }^{64}$ The tension of the aqueous vapour, which is given off from

63 Supersaturated solutions give an excellent proof in this respect. Thus a solution of copper sulphate generaliy crystallises in penta-hydrated crystals, $\mathrm{CuSO}_{4}+5 \mathrm{H}_{2} \mathrm{O}$, and its saturated solution gives such crystals if it be brought into contact with the minutest possible crystal of the same kind. But, according to the observations of Lecoq de Boisbaudran, if a crystal of ferrous sulphate (an isomorphous salt, see note 55), $\mathrm{FeSO}_{4}+7 \mathrm{H}_{2} \mathrm{O}$, be placed in a saturated solution of copper sulphate, then crystals of hepta-hydrated salt, $\mathrm{CuSO}_{4}+7 \mathrm{H}_{2} \mathrm{O}$, are obtained. It is evident that neither the penta- nor the hepta-hydrated salt is contained as such in the solution. The solution presents its own particular liquid form of equilibrium.

64 Efflorescence, like every evaporation, proceeds from the surface. Inside crystals which have effloresced there is usually found a non-effloresced mass, so that the majority of effloresced crystals of washing soda show, in their fracture, a transparent nucleus coated by an effloresced, opaque, powdery mass. It is a remarkable circumstance in this respect that efflorescence proceeds in a completely regular and uniform manner, so that the angles and planes of similar crystallographic character effloresce simultaneously', and in this respect the crystalline form determines those parts of crystals where efflorescence starts, and the order in which it continues. In solutions evaporation also proceeds from the surface, and the first crystals which appear on. its reaching the required degree of saturation are also formed at the surface. After falling to the bottom the crystals naturally continue to grow (see Chap. X.). 
crystallo-hydrates is naturally, as with solutions, less than the vapour tension of water itself ${ }^{65}$ at the same temperature, and therefore many anhydrous salts which are capable of combining with water absorb aqueous vapour from moist air ; that is, they act like a cold body on which water is deposited from steam. It is on this that the desiccation of gases is based, and it must further be remaried in this respect that certain substances - for instance, potassium carbonate $\left(\mathrm{K}_{3} \mathrm{CO}_{3}\right)$ and calcium chloride $\left(\mathrm{CaCl}_{2}\right)$-not only absorb the water necessary for the formation of a solid crystalline compound, but also give solutions, or deliquesce, as it is termed, in moist air. Many crystals do not effloresce in the least at the ordinary temperature ; for example, copper sulphate, which may be preserved for an indefinite length of time without efflorescing, but when placed under the receiver of an air pump, if efflorescence be once started, it goes on at the ordinary temperature. The temperature at which the entire separation of water from crystals takes place varies considerably, not only for different substances but also for different portions of the contained water. Very often the temperature at which dissociation begins is very much higher than the boiling point of water. So, for example, copper sulphate, which contains 36 p.c. of water, gives up 28.8 p.c. at $100^{\circ}$, and the remaining quantity, namely $7 \cdot 2$ p.c., only at $240^{\circ}$. Alum, out of the 45.5 p.c. of water which it contains, gives up 18.9 p.c. at $100^{\circ}, 17 \cdot 7$ p.c. at $120^{\circ}, 7 \cdot 7$ p.c. at $180^{\circ}$, and 1 p.c. at $280^{\circ}$; it only loses the last quantity (1 p.c.) at their temperature of decomposition. These examples clearly show that the annexation of water of crystallisation is accompanied by a rather profound, although, in comparison with instances which we shall consider later, still incon-

65 According to Lescoeur (1883), at $100^{\circ}$ a thick solution of barium hydrcxide, $\mathrm{BaH}_{2} \mathrm{O}_{2}$, on first depositing crystals (with $+\mathrm{H}_{2} \mathrm{O}$ ) has a tension of about $630 \mathrm{~mm}$. (instead of $760 \mathrm{~mm}$., the tension of water), which decreases (because the solution evaporates) to $45 \mathrm{~mm}$., when all the water is expelled from the crystals, $\mathrm{BaH}_{2} \mathrm{O}_{2}+\mathrm{H}_{2} \mathrm{O}$, which are formed, but they also lose water (dissociate, effloresce at $100^{\circ}$ ), leaving the hydroxide, $\mathrm{BaH}_{2} \mathrm{O}_{2}$, which is perfectly undecomposable at $100^{\circ}$-that is, does not part with water. At $73^{\circ}$ (the tension of water is then $265 \mathrm{~mm}$.) a solution, containing $.33 \mathrm{H}_{2} \mathrm{O}$, on crystallising has a tension of $230 \mathrm{~mm}$. ; the crystals $\mathrm{BaH}_{2} \mathrm{O}+8 \mathrm{H}_{2} \mathrm{O}$, which separate out, have a tension of $160 \mathrm{~mm}$.; on losing water they give $\mathrm{BaH}_{2} \mathrm{O}_{2}+\mathrm{H}_{2} \mathrm{O}$. This substance does not decompose at $73^{\circ}$, and therefore its tension $=0$. Miiller-Erzbach (1884) determines the tension (with reference to liquid water) by placing similar long tubes with water and the substances experimented with in a desiccator, the rate of loss of water giving the relative tension. Thus, at the ordinary temperature, crystals of sodium phosphate, $\mathrm{Na}_{2} \mathrm{HPO}_{4}+12 \mathrm{H}_{2} \mathrm{O}$, present a tension of 0.7 compared with water, until they lose $5 \mathrm{H}_{2} \mathrm{O}$, then 0.4 until they lose $5 \mathrm{H}_{3} \mathrm{O}$ more, and on losing the last equivalent of water the tension falls to 0.04 compared with water. It is clear that the different molecules of water are held by an unequal force. Out of the five molecules of water in copper sulphate the two first are comparatively easily separated, even at the ordinary temperature (but only after several days in a desiccator, according to Latchinoff); the next two are more difficultly separated, and the last equivalent is held firmly, even at $100^{\circ}$. 
siderable, change of its properties. In certain cases the water of crystallisation is only given off when the solid form of the substance is destroyed: when the crystals melt on heating. The crystals are then said to melt in their water of crystallisation. Further, after the separation of the water, a solid substance remains behind, so that by further heating it acquires a solid form. This is seen most clearly in crystals of sugar of lead or lead acetate, which melt in their water of crystallisation at a temperature of $56 \cdot 25^{\circ}$, and in so doing begin to lose water. On reaching a temperature of $100^{\circ}$ the sugar of lead solidifies, having lost all its water; and then at a temperature of $280^{\circ}$ the anhydrous and solidified salt again melts. Sodium acetate $\left(\mathrm{C}_{2} \mathrm{H}_{3} \mathrm{NaO}_{2}, 3 \mathrm{H}_{2} \mathrm{O}\right)$ melts at $58^{\circ}$ (but resolidifies only on contact with a crystal, otherwise it may remain liquid even at $0^{\circ}$; as the temperature does not change during solidification, the melted salt can be used for obtaining a constant temperature of $\left.58^{\circ}\right)$. According to Jeannel, the latent heat of fusion is about 28 calories, and, according to Pickering, the heat of solution is 35 calories. When melted, this salt boils at $123^{\circ}$ - that is, the tension of the aqueous vipour given off then equals the atmospheric pressure.

It is most important to recognise in respect to the water of crystallisation that its ratio to the quantity of the substance with which it is combined is always a constant quantity. However often we may prepare copper sulphate, we shall always find $36 \cdot 14$ p.c. of water in its crystals, and these crystals always lose four-fifths of their water at $100^{\circ}$, and one-fifth of the whole amount of the water contained remains in the crystals at $100^{\circ}$, and is only expelled from them at a temperature of about $240^{\circ}$. The determination of the amount of water of crystallisation is easily made if a weighed quantity of crystals is dried in an air or other bath. What has been said about crystals of copper sulphate refers also to crystals of every other substance which contain water of crystallisation. It is impossible to here increase either the relative proportion of the salt or of the water, without changing the homogeneity of the substance. If once a portion of the water be lost-for instance, if once efflorescence takes place - a mixture is obtained, and not a homogeneous substance, namely a mixture of a substance deprived of water with a substance which has not yet lost water-i.e., decomposition has already commenced. This constant ratio is an example of the fact that in chemical compounds the quantity of the component parts is quite definite; that is, it is an example of the so-called definite chemical compounds. They may be distinguished from solutions, and from all other so-called indefinite chemical compounds, in that at least one, and sometimes both, of the component parts may be added in a large quantity to an indefinite chemical compound without destroying 
its homogeneity, as in solutions, whilst it is impossible to add any one of the component parts to a definite chemical compound without destroying the homogeneity of the entire mass. Definite chemical compounds only decompose at a certain rise in temperature; on a lowering in temperature they do not, at least with very few exceptions, yield their components like solutions which form ice or compounds with water of crystallisation. This obliges one to consider that solutions contain water as water, ${ }^{66}$ although it may sometimes be in a very small quantity. Therefore solutions which are capable of entirely solidifying (for instance, cryohydrates and crystallo-hydrates - i.e., compounds with water of crystallisation which are capable of melting - or the compound of $84 \frac{1}{2}$ parts of sulphuric acid, $\mathrm{H}_{2} \mathrm{SO}_{4}$, with $15 \frac{1}{2}$ parts of water, $\mathrm{H}_{2} \mathrm{O}$, or $\mathrm{H}_{2} \mathrm{SO}_{4}, \mathrm{H}_{2} \mathrm{O}$, or $\mathrm{H}_{4} \mathrm{SO}_{3}$ ) appear as true definite chemical compounds. If, then, we imagine such a definite compound in a liquid state, and admit that it partially decomposes in this state, separating waternot as ice or vapour (for then the system would be heterogeneous, including substances in different physical states), but in a liquid form, when the system will be homogeneous - then we shall form an idea of a solution as an unstable, decomposing fluid equilibrium between water and the substance dissolved. Just as the component elements may be added to a gaseous mixture without destroying its homogeneity, so both the solvent may be added to a solution (the solution will then be obtained diluted, and no longer presenting a definite composition), and also the substance dissolved may be added (with a solid and a saturated solution a supersaturated solution will be obtained), which may, however, owing to the force of the cohesion of its parts, separate out from the solution in a crystallised form. In adding the solvent, or the substance dissolved, without destruction of the homogeneity of the whole, we altered their relative quantity (the proportion of the acting masses), by which there will be an alteration, both in the quantity of the water, forming one of the products of dissociation, and also of the relative quantity of one or many of the definite compounds between the water and the substance dissolved. Owing to this change, there occurs an alteration in the properties of a solution (contraction, change of vapour tension, dc.) ; not in the sense of a purely mechanical change in the proportion of the components (as in the intermixture of non reacting

66 Such a phenomenon frequently presents itself in purely chemical action. For instance, let a liquid substance $A$ give, with another liquid substance $B$, under the conditions of an experiment, a mere minute quantity of a solid or gaseous substance $C$. This small quantity will separate out (pass away from the sphere of action, as Berthollet expressed it), and the remaining masses of $A$ and $B$ will again give $C$; consequently, under these conditions, action will go on to the end. Such, it seems to me, is the action in solutions when they yield ice or vapour indicating the presence of water. 
gases), but in the sense of an alteration in the quantity of those definite liquid chemical compounds which are determined by the chemical attraction between water and the substance dissolved in it, and by their eapacity for forming with it diverse compounds, ${ }^{67}$ which is seen in the capacity of one substance to form with water many various crystallohydrates, or compounds with water of crystallisation, showing diverse and independent properties. From these considerations, solutions ${ }^{68}$ may be regarded as fuid, unstable, definite chemical compounds in a state of dissociation. ${ }^{69}$

67 Certain substances are capable of forming only one compound, others several, and these of the most varied degrees of stability. The compounds of water are instances of this kind. In solutions of sulphuric acids (see note 19), for example, the existence must be acknowledged of several different definite compounds. Many of these have not yet been obtained in a free state, and it may be that they cannot be obtained in any other but a liquid form-that is, dissolved; just as there are many undoubted definite compounds which only exist in one physical state. Among the hydrates such instances occur. The compound $\mathrm{CO}_{2}+8 \mathrm{H}_{2} \mathrm{O}$ (see note 31 ), according to Wroblewski, only occurs in a solid form. Hydrates like $\mathrm{H}_{2} \mathrm{~S}+12 \mathrm{H}_{2} \mathrm{O}$ (De Forcrand and Villard), $\mathrm{HBr}+\mathrm{H}_{2} \mathrm{O}$ (Roozeboom), can only be accepted on the basis of a decrease of tension, but present themselves as very transient substances, incapable of existing in a stable free state. Even sulphuric acid, $\mathrm{H}_{2} \mathrm{SO}_{4}$, itself, which undoubtedly is a definite compound, fumes in a liquid form, evolving the anhydride, $\mathrm{SO}_{3}$-that is, exhibits a very unstable equilibrium. The crystallohydrates of chlorine, $\mathrm{Cl}_{2}+8 \mathrm{H}_{2} \mathrm{O}$, of hydrogen sulphide, $\mathrm{H}_{2} \mathrm{~S}+12 \mathrm{H}_{2} \mathrm{O}$ (it is formed at $0^{\circ}$, and is completely decomposed at $+1^{\circ}$, as then 1 vol. of water only dissolves 4 vols. of hydrogen sulphide, while at $0 \cdot 1^{\circ}$ it dissolves about 100 vols.), and of many other gases, are instances of hydrates which are very unstable.

38 Of such a kind are also other indefinite chemical compounds; for example, metallic alloys. These are solid substances or solidified solutions of metals. They also contain definite compounds, and may contain an excess of one of the metals. According to the experiments of Laurie (1888), the alloys of zinc with copper in respect to the electromotive force in galvanic batteries behave just like zinc if the proportion of copper in the alloy does not exceed a certain percentage-that is, until a definite compound is attained - for then there are yet particles of free zinc; but if a copper surface be taken, and it be covered by only one-thousandth part of its area of zinc, then only the zinc will act in a galvanic battery.

69 According to the above supposition, the condition of solutions in the sense of the kinetic hypothesis of matter (that is, on the supposition of an internal movement of molecules and atoms) may be represented in the following form:-In a homogeneous liquid-for instance, water-the molecules occur in a certain state of, although mobile, still stable, equilibrium. When a substance $A$ dissolves in water, its molecules form with several molecules of water, systems $A n \mathrm{H}_{2} \mathrm{O}$, which are so unstable that when surrounded by molecules of water they decompose and re-form, so that $A$ passes from one mass of molecules of water to another, and the molecules of water which were at this moment in harmonious movement with $A$ in the form of the system $A n \mathrm{H}_{2} \mathrm{O}$, in the next instant may have already succeeded in getting free. The addition of water or of molecules of $A$ may either only alter the number of free molecules, which in their turn enter into systems $A n \mathrm{H}_{2} \mathrm{O}$, or they may introduce conditions for the possibility of building up new systems $A m \mathrm{H}_{2} \mathrm{O}$, where $m$ is either greater or less than $n$. If in the solution the relation of the molecules be the same as in the system $\mathrm{AmH}_{2} \mathrm{O}$, then the addition of fresh molecules of water or of $A$ would be followed by the formation of new molecules $A n \mathrm{H}_{2} \mathrm{O}$. The relative quantity, stability, and composition of these systems or definite compounds will vary in one or another solution. Such a view of solutions came to rne from a most intimate study of the variation of their specific gravities, to which my book, cited in note 19 , is 
In regarding solutions from this point of view they come under the head of those definite compounds which chemistry mainly treats of. ${ }^{70}$ For this reason we will direct our particular attention to one side of the subject under consideration, which touches on the essential property

devoted. Definite compounds, $A n_{1} \mathrm{H}_{2} \mathrm{O}$ and $A m_{1} \mathrm{H}_{2} \mathrm{O}$, existing in a free-for instance, solid-form, may in certain cases be held in solutions in a dissociated state (although but partially); they are similar in their structure to those definite substances which are formed in solutions, but nothing obliges one to think that it is such systems as, for instance, $\mathrm{Na}_{2} \mathrm{SO}_{4}+10 \mathrm{H}_{2} \mathrm{O}$, or $\mathrm{Na}_{2} \mathrm{SO}_{4}+7 \mathrm{H}_{2} \mathrm{O}$, or $\mathrm{Na}_{2} \mathrm{SO}_{4}$, that are contained in solutions. The comparatively more stable systems $A n_{1} \mathrm{H}_{2} \mathrm{O}$ which exist in a free state and change their physical state must present, although within certain limits of temperature, an entirely harmonious kind of movement of $A$ with $n_{1} \mathrm{H}_{2} \mathrm{O}$; the property also and state of systems $A n \mathrm{H}_{2} \mathrm{O}$ and $A m \mathrm{H}_{2} \mathrm{O}$, occurring in solutions, is that they are in a liquid form, although partially dissociated. Substances $A_{1}$, which give solutions, are distinguished by the fact that they can form such unstable systems $A n \mathrm{H}_{2} \mathrm{O}$, but besides them they can give other much more stable systems $A n_{1} \mathrm{H}_{2} \mathrm{O}$. Thus ethylene, $\mathrm{C}_{2} \mathrm{H}_{4}$, in dissolving in water, probably forms a system $\mathrm{C}_{2} \mathrm{H}_{4} n \mathrm{H}_{2} \mathrm{O}$, which easily splits up into $\mathrm{C}_{2} \mathrm{H}_{4}$ and $\mathrm{H}_{2} \mathrm{O}$, but it also gives the system of alcohol, $\mathrm{C}_{2} \mathrm{H}_{4}, \mathrm{H}_{2} \mathrm{O}$ or $\mathrm{C}_{2} \mathrm{H}_{6} \mathrm{O}$, which is comparatively stable. Thus oxygen can dissolve in water, and it can combine with it, forming peroxide of hydrogen. Turpentine, $\mathrm{C}_{10} \mathrm{H}_{16}$, does not dissolve in water, but it combines with it in a comparatively stable hydrate. In other words, the chemieal structure of hydrates, or of the definite compounds which are contained in solutions, is distinguished not only by its original peculiarities but also by a diversity of stability. A similar structure to hydrates must be acknowledged in crystallo-hydrates. On melting they give actual (real) solutions. As substances which give crystallo-hydrates, like salts, are capable of forming a number of diverse hydrates, and as the greater the number of molecules of water $(n)$ they $\left(\mathrm{AnH}_{2} \mathrm{O}\right)$ contain the lower is the temperature of their formation, and as the more easily they decompose the more water they hold, therefore, in the first place, the isolation of hydrates holding much water existing in aqueous solutions may be soonest looked for at low temperatures (although, perhaps, in certain cases they cannot exist in the solid state); and secondly, the stability also of such higher lydrates will be at a minimum under the ordinary circumstances of the occurrence of liquid water. Hence a further more detailed investigation of cryohydrates (note $\bar{j} 8$ ) may help to the elucidation of the nature of solutions. But it may be foreseen that certain cryohydrates will, like metallic alloys, present solidified mixtures of ice with the salts themselves and their more stable hydrates, and others will be definite compounds.

70 The above representation of solutions, \&c., considering them as a particular state of definite compounds, excludes the independent existence of indefinite compounds; by this means that unity of chemical conception is obtained which cannot be arrived at by admitting the physico-mechanical conception of indefinite compounds. The gradual transition from typical solutions (as of gases in water, and of weak saline solutions) to sulphuric acid, and from it and its definite, but yet unstable and liquid, compounds, to clearly definite compounds, such as salts and their crystallo-hydrates, is so imperceptible, that by denying that solutions pertain to the number of definite but dissociating compounds, we risk denying the definiteness of the atomic composition of such substances as sulphuric acid or of molten crystallo-hydrates. I repeat, however, that for the present the theory of solutions cannot be considered as firmly established. The above opinion about them is nothing more than a hypothesis which endeavours to satisfy those comparatively limited data which we have for the present about solutions, and of those cases of their transition into definite compounds. By submitting solutions to the Daltonic conception of atomism, I hope that we may not only attain to a general harmonious chemical doctrine, but also that new notives for investigation and research will appear in the problem of solutions, which must either confirm the proposed theory or replace it by another fuller and truer one. 
of definite compounds as a class to whose number solutions should (or at least, may) be referred.

We saw above that copper sulphate loses four-fifths of its water at $100^{\circ}$ and the remainder at $240^{\circ}$. This means that there are two definite compounds of water with the anhydrous salt. Washing soda or carbonate of sodium, $\mathrm{Na}_{2} \mathrm{CO}_{3}$, separates out as crystals, $\mathrm{Na}_{2} \mathrm{CO}_{3}, 1 \mathrm{CH}_{2} \mathrm{O}$, containing 62.9 p.c. of water by weight, from its solutions at the ordinary temperature. When a solution of the same salt deposits crystals at a low temperature, about $-20^{\circ}$, then these crystals contain 71.8 parts of water per 28.2 parts of anhydrous salt. Further, the crystals are obtained together with ice, and are left behind when it melts. If ordinary soda, with 62.9 p.c. of water, be cautiously melted in its own water of crystallisation, there remains a salt, in a solicl state, containing only 14.5 p.c. of water, and a liquid is obtained which contains the solution of a salt which separates out crystals at $34^{\circ}$, which contain 46 p.c. of water and do not effloresce in air. Lastly, if a supersaturated solution of soda be prepared, then at temperatures below $8^{\circ}$ it deposits crystals containing $54 \cdot 3$ p.c. of water. Thus there are known as many as five compounds of anhydrous sola with water; and they are dissimilar in their properties and crystalline form, and even in their solubility. We will mention that the greatest amount of water in the crystals corresponds with a temperature of $20^{\circ}$, and the smallest to the highest temperature. There is apparently no relation between the above quantities of water and the salts, but this is only because in each case the amount of water and anhydrous salt was given in percentages, but if it be calculated for one and the same quantity of anhydrous salt, or of water, a great regularity will be observed in the amounts of the component parts in all these compounds. It appears that for 106 parts of anhydrous salt in the crystals separated out at $-20^{\circ}$ there are 270 parts of water ; in the crystals obtained at $15^{\circ}$ there are 180 parts of water ; in the crystals obtained from a supersaturated solution 126 parts, in the crystals which separate out at $34^{\circ}, 90$ parts, and the crystals with the smallest amount of water, 18 parts. On comparing these quantities of water it may be easily seen that they are in simple proportion to each other, for they are all divisible by 18 , and are in the ratio $15: 10: 7: 5: 1$. Naturally, direct experiment, however carefully it be conducted, is hampered with errors, but taking these inevitable errors into consideration, it will be seen that for a given quantity of an anhydrous substance there occur, in several of its compounds with water, quantities of water which are in very simple multiple proportion. This is observed in, and is common to, all definite chemical compounds. This rule is called the law of multiple proportions. It was discovered 
by Dalton, and will be evolved in detail in the further exposition in this work. For the present we will only state that the law of definite composition enables the composition of substances to be expressed by formulae, and the law of multiple proportions permits the application of co-efficients in a weight of whole numbers, in formulae. Thus the formula, $\mathrm{Na}_{2} \mathrm{CO}_{3}, 10 \mathrm{H}_{2} \mathrm{O}$, directly shows that in this crystallo-hyclrate there are 180 parts of water to 106 parts by weight of the anhyclrous salt, because the formula of soda, $\mathrm{Na}_{2} \mathrm{CO}_{3}$, directly answers to a weight of 106 , and the formula of water to 18 parts, by weight, which are here taken 10 times.

In the above examples of the combinations of water, we saw the gradually-increasing intensity of the bond between water and a substance with which it forms a homogeneous compound. There is a series of such compounds with water, in which the water is held with very great force, and is only given up at a very high temperature, and sometimes cannot be separated by any clegree of heat without the entire decomposition of the substance. In these compounds there is generally no outward sign whatever of their containing water. A perfectly new substance is formed from an anhydrous substance and water, in which sometimes the properties of neither one nor the other substance are observable. In the majority of cases, a considerable amount of heat is evolved in the formation of such compounds with water. Sometimes the heat evolved is so intense that a red heat is produced and light is emitted. It is hardly to be wondered at, after this, that stable compounds are formed by such a combination. Their decomposition requires great heat; a large amount of work is necessary to separate them into their component parts. All such compounds are definite, and, generally, completely and clearly definite. The number of such definite compounds with water or hydrates, in the narrow sense of the word, is generally inconsiderable for each anhydrous substance; in the greater number of cases, there is formed only one such combination of a substance with water, one hydrate, having so great a stability. The water contained in these compounds is often called water of constitution -i.e., water which enters into the structure or composition of the given substance. By this it is desired to express, that in other cases the molecules of water are as it were separate from the inolecules of that substance with which it is combined. It is supposed that in the formation of hydrates this water, even in the smallest particles, forms one complete whole with the anhydrous substance. Many examples of the formation of such hydrates might be cited. The most familiar example in practice is the hydrate of lime, or so-called 'slaked' lime. Lime is prepared by burning limestone, by which the carbonic anhydride 
is expelled from it, and there remains a white stony mass, which is dense, compact, and rather tenacious. Lime is usually sold in this form, and bears the name of 'quick' or 'unslaked' lime. If water be poured over such lime, a great rise in temperature is remarked either directly, or after a certain time. The whole mass becomes hot, part of the water is evaporated, the stony mass in absorbing water crumbles into powder, and if the water be taken in sufficient quantity and the lime be pure and well burnt, not a particle of the original stony mass is leftit all crumbles into powder. If the water be in excess, then naturally a portion of it remains and forms a solution. This process is called 'slaking' lime. Slaked lime is used in practice in intermixture with sand as mortar. Slaked lime is a definite hydrate of lime. If it is dried at $100^{\circ}$ it retains $24 \cdot 3$ p.c. of water. This water can only be expelled at a temperature above $400^{\circ}$, and then quicklime is re-obtained. The heat evolved in the combination of lime with water is so intense that it can set fire to wood, sulphur, gunpowder, \&c. Even on mixing lime with ice the temperature rises to $100^{\circ}$. If lime be melted with a small quantity of water in the dark, a luminous effect is observed. But, nevertheless, water may still be separated from this hydrate. ${ }^{71}$ If phosphorus be burnt in dry air, a white substance called 'phosphoric anhydricle' is obtained. It combines with water with such energy, that the experiment must be conducted with great caution. A red heat is produced in the formation of the compound, and it is impossible to separate the water from the resultant hydrate at any temperature. The hydrate formed by phosphoric anhydride is a substance which is totally undecomposable into its original component parts by this action of heat. Almost as energetic a combination occurs when sulphuric anhydride, $\mathrm{SO}_{3}$, combines with water, forming its hydrate, sulphuric acid, $\mathrm{H}_{2} \mathrm{SO}_{3}$. In both cases definite compounds are produced, but the latter substance, as a liquid, and capable of decomposition by heat, giving off the vapour of its volatile anhydride even at the ordinary temperature, forms an evident link with solutions, and, with an excess of water, it gives, as a soluble substance, a true solution. If 80 parts of sulphuric anhydride retain 18 parts of water, this water cannot be separated from the anhydride, even at a temperature of $300^{\circ}$. It is only by the addition of phosphoric anhydride, or by a series of chemical transformations, that this water can be separated from its compound with sulphuric anhydride. Oil of vitriol,

71 In combining with water one part by weight of lime evolves 245 units of heat. A high temperature is obtained, because the specific heat of the resulting product is small. Sodium oxide, $\mathrm{Na}_{2} \mathrm{O}$, in reacting on water, $\mathrm{H}_{2} \mathrm{O}$, and forming caustic soda (sodium hydroxide), $\mathrm{NaHO}$, evolves 552 units of heat for each part by weight of sodium oxide. 
or sulphuric acid, is such a compound. If a larger proportion of water be taken, it will combine with the $\mathrm{H}_{2} \mathrm{SO}_{4}$; for instance, if 36 parts of water per 80 parts of sulphuric anhydride be taken, a compound is formed which crystallises in the cold, and melts at $+8^{\circ}$, whilst oil of vitriol does not solidify at even $-30^{\circ}$. If still more water be taken, the oil of vitriol will dissolve in the remaining quantity of water. An evolution of heat takes place, not only on the addition of the water of constitution, but in a less degree on further additions of water. ${ }^{72}$ And therefore there is no distinct boundary, but only a gradual transition, between those clremical phenomena which are expressed in the formation of solutions and those which take place in the formation of the most stable hydrates. ${ }^{73}$

72 The diagram given in note 28 shows the evolution of heat on the mixture of sulphuric acid, or mono-hydrate $\left(\mathrm{H}_{2} \mathrm{SO}_{4}\right.$, i.e. $\left.\mathrm{SO}_{3}+\mathrm{H}_{2} \mathrm{O}\right)$, with different quantities of water per 100 vols. of the resultant solution. Per 98 grams of sulphuric acid $\left(\mathrm{H}_{2} \mathrm{SO}_{4}\right)$ there are evolved, on the addition of 18 grams of water, 6,379 units of heat; with double or three times the quantity of water 9,418 and 11,137 units of heat, and with an infinitely large quantity of water 17,860 units of heat, according to the determinations of Thomsen. $\mathrm{He}$ also showed that when $\mathrm{H}_{2} \mathrm{SO}_{4}$ is formed from $\mathrm{SO}_{3}(=80)$ and $\mathrm{H}_{2} \mathrm{O}(=18), 21,308$ units of heat are evolved per 98 parts by weight of the resultant sulphuric acid.

75 Thus, for different hydrates the stability with which they hold water is very dissimilar. Certain hydrates hold water very loosely, and in combining with it evolve little heat. From other hydrates the water cannot be separated by any degree of heat, even if they are formed from anhydrides (i.e., anhydrous substances) and water with little evolution of heat; for instance, acetic anhydride in combining with water evolves an inconsiderable amount of heat, but the water cannot then be expelled from it. If the hydrate (acetic acid) formed by this combination be strongly leated it either volatilises without change, or decomposes into new substances, but it does not again yield the original substances-i.e., the anhydride and water. Here is an instance which gives the reason for calling the water entering into the composition of the hydrate, water of constitution. Such, for example, is the water entering into the so-called caustic soda or sodium hydroxide (see note 71 ). But there are hydrates which easily part with their water; yet this water cannot be considered as water of crystallisation, not only because sometimes such hydrates have no crystalline form, but also because, in perfectly analogous cases, very stable hydrates are formed, which are capable of particular kinds of chemical reactions, as we shall learn afterwards. In a word, there is not a distinct boundary either between the water of hydrates and of erystallisation, or between solution and hydration.

It must be observed that in separating from an aqueous solution, many substances, without having a crystalline form, hold water in the same unstable state as in crystals; only this water cannot be termed 'water of crystallisation' if the substance which separates out has no crystalline form. The hydrates of alumina and silica are examples of such unstable hydrates. If these substances are separated from an aqueous solution by a chemical process, then they always contain water, and when dried at a definite temperature, so that the hvgrosconic water may pass off, these substances hold water in a definite proportion. The formation of a new chemical compound containing water is here particularly evident, for alumina and silica in an anhydrous stats have properties differing from those they show when combined with water, and do not combine directly with it. The entire series of colloids on separating from water form similar compounds with it, which have the aspect of solid substances generally, without crystalline structure. Besides which, colloids retain water in other different states (see notes 57 
We have thus considered many aspects and clegrees of combination of various substances with water, or instances of the compounds of water, when it and other substances form new homogeneous substances, which in this case will evidently be complex--i.e., made up of different substances - and although they are homogeneous, yet it must be admitted that in them there exist those component parts which entered into their composition, inasmuch as these parts may be re-obtained from them. It must not be imagined that water really exists in hydrate of lime, any more than that ice or steam exists in water. When we say that water occurs in the composition of a certain hydrate, we only wish to point out that there are chemical transformations in which it is possible to obtain that hydrate by means of water, and other transformations in which this water may be separated out from the hydrate. This is all sinuply expressed by the words, that water enters into the composition of this hydrate. If a hydrate be formed by feeble bonds, and be decomposed at even the ordinary temperature, then the water appears as one of the products of dissociation, which in all likelihood is the case in solutions, and forms the fundamental distinction between them and other hydrates in which the water is combined with greater stability and forms a solid substance.

and 18), and most often form gelatinous masses. Water is held in a considerable quantity in solidified glue or boiled albumin. It cannot be expelled from them by pressure; hence, in this case there has ensued some kind of combination of the substance with water, This water, however, is easily separated by drying; but not the whole of it, a portion being retained, and this portion belongs, as they say, to the hydrate, although in this case it is very difficult, if possible, to obtain definite compounds. The absence of any distinct boundary lines between solutions, crystallo-hydrates, and ordinary hydrates above referred to, is very clearly seen in such examples. 


\section{CHAPTER II}

THE COMPOSITION OF WATER, HYDROGEN

THE question now arises, Is not water itself a compound substance? Cannot it be formed by the mutual combination of some component parts? Cannot it be broken up into its component parts? There cannot be the least doubt that if it does split up, and if it is a compound, then it is a definite one characterised by the stability of the union between those component parts from which it is formed. From the fact alone that water passes into all physical states as a homogeneous whole, without in the least varying in its properties and without split. ting up into its component parts (neither solutions nor many hydrates can be distilled - they are split up), we must already conclude, from this fact alone, that if water is a compound then it is a stable and definite chemical compound. Like many other great discoveries in the province of chemistry, it is to the end of the last century that we are indebted for the important discovery that water is not a simple substance, that it is composed of two substances like a number of other compound substances. This was proved by two of the methods by which the compound nature of bodies may be determined as self-evident; by analysis and by synthesis - that is, by a method of the decomposition of water into, and of the formation of water from, its component parts. In 1781 Cavendish first obtained water by burning hydrogen in oxygen, both of which gases were already known to him. He concluded from this that water was composed of two substances. But he did not make more accurate experiments, which would have shown the relative quantities of the component parts in water, and which would have determined its complex nature with certainty. Although his experiments were the first, and although the conclusion he drew from them was true, yet such novel ideas as the complex nature of water are not easily recognised so long as there is no series of researches which entirely and indubitably proves the truth of such a conclusion. The fundamental experiments which proved the complexity of water by the method of synthesis, and of its formation from other substances, were made in 1789 by Monge, 
Lavoisier, Fourcroy, and Vauquelin. They obtained four ounces of water by burning hydrogen, and found that water consists of 15 parts of hydrogen and 85 parts of oxygen. It was also proved that the weight of water formed was equal to the sum of the weights of the component parts entering into its composition ; consequently, water contains all the matter entering into oxygen and hydrogen. The com. plexity of water was proved in this manner by a method of synthesis. But we will turn to its analysis - i.e., to its decomposition into its component parts. The analysis may be more or less complete. Either both component parts may be obtained in a separate state, or else only one is separated and the other is converted into a new compound in which its amount may be determined by weighing. This will be a reaction of substitution, such as is often taken advantage of for analysis. The first analysis of water was thus conducted in 1784 by Lavoisier and Meusnier. The apparatus they arranged consisted of a glass retort containing water, naturally purified, and whose weight had been previously determined. The neck of the retort was inserted into a porcelain tube, placed inside an oven, and heated to a red heat by charcoal. Iron filings, which decompose water at a red heat, were placed inside this tube. The end of the tube was connected with a worm, for condensing any water which might pass through the tube undecomposed. This condensed water was collected in a separate flask. The gas formed by the decomposition was collected over a water bath in a bell jar. The aqueous vapour in passing over the red-hot iron was decomposed, and a gas was formed from it whose weight could be determined from its volume, its density being known. Besides the water which passed through the tube unaltered, a certain quantity of water disappeared in the experiment, and this quantity, in the experiments of Lavoisier and Meusnier, was equal to the weight of the gas which was collected in the bell jar plus the increase in weight of the iron filings. Hence the water was decomposed into a gas, which was collected in the bell jar, and a substance, which combined with the iron ; consequently, it is composed of these two component parts. This was the first analysis of water ever made ; but here only one (and not both) of the gaseous component parts of water was collected separately. Both the component parts of water can, however, be simultaneously obtained in a free state. For this purpose the decomposition is brought about by a galvanic current or by heat, as we shall learn directly. ${ }^{1}$

1 The first experiments of the synthesis and decomposition of water did not afford, however, an entirely convincing proof that water was composed of hydrogen and oxygen only. Davy, who investigated the decomposition of water by the galvanic current, thought for a long time that, besides the gases, an acid and alkali were also obtained.

VOL. I. 
Water is a bad conductor of electricity - that is, pure water does not transmit a feeble current; but if any salt or acid be dissolved in it, then its conductivity increases, and on the passage of a current through acidified water it is decomposed into its component parts. Some sulphuric acid is generally added to the water. By immersing platinum plates (electrodes) in this water (platinum is chosen because it is not acted on by acids, whilst many other metals are chemically acted on by acids), and connecting them with a galvanic battery, it will be observed that bubbles of gas appear on these plates. The gas which separates is called detonating gas, ${ }^{2}$ because, on approaching a light, it very easily explodes. ${ }^{3}$ What takes place is as follows :- First, the water, by the action of the current, is decomposed into two gases. The mixture of these gases forms detonating gas. When detonating gas is brought into contact with an incandescent substance-for instance, a lighted taper - the gases re-combine, forming water, the combination being accompanied by a great evolution of heat, and therefore the vapour of the water formed expands considerably, which it does very rapidly, and as a consequence of which an explosion takes place - that is, sound and increase of pressure, and atmospheric commotion, as in the explosion of gunpowder.

In order to discover what gases are obtained by the decomposition of water, the gases which separate at each electrode must be collected separately. For this purpose a V-shaped tube is taken ; one of its ends is open, and the other fused up. A platinum wire, terminating inside the tube in a plate, is fused into the closed end ;

He was only convinced of the fact that water contains nothing but hydrogen and oxygen by a long series of researches, which showed him that the appearance of an acid and alkali in the decomposition of water proceeds from the presence of impurities (especially from the presence of ammonium nitrate) in water. A final understanding of the composition of water is obtained from the determination of the quantities of the component parts which enter into its composition. It will be seen from this how many data are necessary for proving the composition of water--that is, of the transformations of which it is capable. What has been said of water refers to all other compounds; the investigation of each one, the entire proof of its composition, can only be obtained by the juxtaposition of a large mass of data referring to it.

2 This gas is collected in a voltameter.

3 In order to observe this explosion without the slighest danger, it is best to proceed in the following manner. Some soapy water is prepared, so that it easily forms soap bubbles, and it is poured into an iron trough. In this water, the end of a gas-conducting tube is immersed. This tube is connected with any suitable apparatus, in which detonating gas is evolved. Soap bubbles, full of this gas, are then formed. If the apparatus in which the gas is produced be then removed (otherwise the explosion might travel into the interior of this apparatus), and a lighted taper be brought to the soap bubbles, a very sharp explosion takes place. The bubbles should be small to avoid any danger; ten, each about the size of a pea, suffice to give a sharp report, like a pistol shot. 
the closed end is entirely filled with water ${ }^{4}$ acidified with sulphuric acid, and another platinum wire, terminating in a plate, is immersed in the open end. If a current from a galvanic battery be now passed through the wires an evolution of gases will be observed, and the gas which is obtained in the open branch mixes with the air, while that in the closed branch accumulates above the water. As this gas accumulates it displaces the water, which continues to descend in the closed and ascend into the open branch of the tubes. When the water, in this way, reaches the top of the open end, the passage of the current is stopped, and the gas which was evolved from one of the electrodes only is obtained in the apparatus. By this means it is easy to prove that a particular gas appears at each electrode. If the closed end be connected with the negative pole-i.e., with that joined to the zinc - then the gas collected in the apparatus is capable of burning. This may be demonstrated by the following experiment:- The bent tube is taken off the stand, and its open end stopped up with the thumb and inclined in such a manner that the gas passes from the closed to the open end. It will then be found, on applying a lighted lamp or taper, that the gas burns. This combustible gas is hydrogen. If the same experiment be carried on with a current passing in the opposite direction-that is, if the closed end be joined up with the positive pole (i.e., with the carbon, copper, or platinum), then the gas which is evolved from it does not burn of itself, but it supports combustion very vigorously, so that in it a smouldering taper immediately bursts into flame. This gas, which is collected on the anode or positive pole, is oxygen, which is obtained, as we saw before (in the Introduction), from mercury oxide and is contained in air.

Thus in the decomposition of water oxygen appears at the positive pole and hydrogen at the negative pole, so that detonating gas will be a mixture of them both. Hydrogen burns in air from the fact that in doing so it re-forms water, with the oxygen of the air. Detonating gas explodes from the fact that the hydrogen burns in the oxygen mixed with it. It is very easy to measure the relative quantities of one and the other gas which are evolved in the decomposition of water. For this purpose a funnel is taken, whose orifice is closed by a cork through which two platinum wires pass. These wires are connected with a battery. Acidified water is poured into the funnel, and a glass cylinder full of water is placed over the end of each wire (fig. 18). On passing a current, hydrogen and oxygen collect in these cylinders,

4 In order to fill the tube with water, it is turned up, so that the closed end points downwards and the open end upwards, and water acidified with sulphuric acid is poured into it. 
and it will easily be seen that two volumes of hydrogen are evolved for every one volume of oxygen. This signifies that, in clecomposing, water gives two volumes of hydrogen and one volume

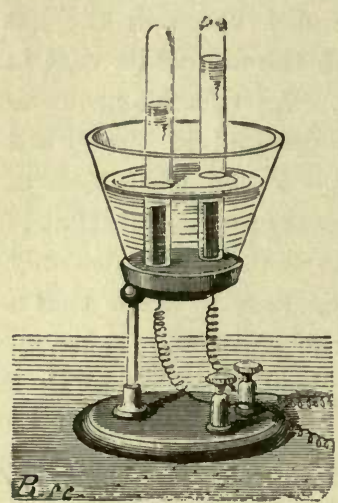

FJG. 18.-Decomposition of water by the galvanic current, for determining the relation between the volumes of hydrogen and oxygen. of oxygen.

Water is also decomposed into its component parts by the action of heat. At the melting point of silver $\left(960^{\circ}\right)$, and in its presence, water is decomposed and the oxygen absorbed by the molten silver, which dissolves it so long as it is liquid. But directly the silver solidifies the oxygen is expelled from it. However, this experiment is not entirely convincing ; it might be thought that in this case the decomposition of the water did not proceed from the action of heat, but from the action of the silver on water-that silver decomposes water, taking up the oxygen. It is impossible to directly show the decomposition of water by the action of heat, because the component parts of water, if they remain together, re-combine with a fall of temperature, and give water back again. For instance, if steam be passed through a red-hot tube, whose internal temperature attains $1,000^{\circ}$, then a portion ${ }^{5}$ of the water decomposes into its component parts, forming detonating gas. But on passing into the cooler portions of the apparatus this detonating gas again reunites and forms water. The hydrogen and oxygen obtained combine together at a lower temperature. ${ }^{6}$ Apparently the problem-

5 As water is formed by the combination of oxygen and hydrogen, the reaction evolving much heat, and as it can also be decomposed, therefore this reaction is a reversible one (see Introduction), and consequently at a high temperature the decomposition of water cannot be complete-it is limited by the opposite reaction. Strictly speaking, it is not known how much water is decomposed at a given temperature, although many efforts (Bunsen, and others) have been made in various directions to solve this question. Not knowing the coefficient of expansion, and the specific heat of gases at such high temperatures, renders all calculations (from observations of the pressure on explosion) doubtful.

6 Grove, about 1840 , observed that a platinum wire fused in the flame of detonating gas-that is, having acquired the temperature of the formation of water-and having formed a molten drop at its end which fell into water, evolved detonating gas-that is, decomposed water. It therefore follows that water already decomposes at the temperature of its formation. At that time, this formed a scientific paradox; this we shall unravel only with the development of the conceptions of dissociation, introduced into science by Henri Sainte-Claire Deville, about 1850. These conceptions form an important epoch in science, and their development is one of the problems of contemporary chemistry. The essence of the matter is that, at high temperatures, water exists but also decomposes, just as a volatile liquid, at a certain temperature, exists both as a liquid and 
to show the decomposability of water at high temperatures-is unattainable. It was considered as such before Henri Sainte-Claire Deville (in the fifties) introduced the conception of dissociation into chemistry, as of a change of chemical state resembling evaporation, if decomposition be likened to boiling, and before he had demonstrated the decomposability of water by the action of heat in an experiment which will presently be described. In order to demonstrate clearly the dissociation of water, or its decomposability by heat, at a temperature approaching that at which it is formed (as a volatile liquid, at a given temperature, can be either in a liquid or vaporous condition) it was necessary to separate the hydrogen from the oxygen at a high temperature, without allowing the mixture to cool. Deville took advantage of the difference between the densities of hydrogen and oxygen.

A wide porcelain tube $\mathbf{P}$ (fig. 19) is placed in a furnace giving a

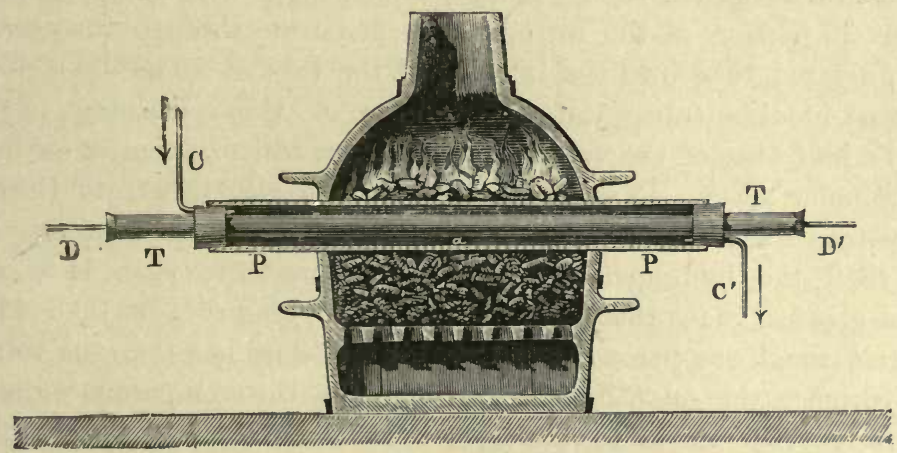

FiG. 19.-Decomposition of water by the action of heat, and the separation of the hydrogen formed by its permeating through a porous tube.

strong heat (it should be heated with small pieces of good coke). In this tube there is inserted a second tube $\mathrm{T}$, of less diameter, and made of unglazed earthenware and therefore porous. The ends of the tube are luted to the wide tube, and two tubes, $\mathrm{c}$ and $\mathrm{c}^{\prime}$, are inserted into the ends, as shown in the drawing. With this arrangement it is possible for a gas to pass into the annular space between the walls of the two tubes, from whence it can be collected. Steam from

as a vapour. Similarly as a volatile liquid saturates a space, attaining its maximum tension, so also the products of dissociation have their maximum tension, and once that is attained decomposition ceases, just as evaporation ceases. Under like conditions, if the vapour be allowed to escape (and therefore its partial pressure be diminished), evaporation recommences, so also if the products of decomposition be removed, decomposition again continues. These simple conceptions of dissociation introduce infinitely varied consequences into the mechanism of chemical reactions, and therefore we shall have occasion to return to them very often. 
a retort or flask is passed through the tube $\mathrm{D}$, into the internal porous tube т. This steam on entering the red hot space is decomposed into hydrogen and oxygen. The densities of these gases are very different, hydrogen being sixteen times lighter than oxygen. Light gases, as we saw above, penetrate through porous surfaces very much more rapidly than denser gases, and therefore the hydrogen passes through the pores of the tube into the annular space very much more rapidly than the oxygen. The hydrogen which separates out into the annular space can only be collected when this space does not contain any oxygen. If any air remains in this space, then the hydrogen which separates out will combine with its oxygen and form water. For this reason a gas incapable of supporting combustion - for instance, nitrogen-is previously passed in the annular space. Thus the nitrogen is passed through the tube c, and the hydrogen, separated from the steam, is collected through the tube $\mathbf{C}^{\prime}$, and will be partly mixed with nitrogen. A certain portion of the nitrogen will penetrate through the pores of the unglazed tube into the interior of the tube T. The oxygen will remain in this tube, and the volume of the remaining oxygen will be half that of the volume of hydrogen which separates out from the annular space. Part of the oxygen will also penetrate through the pores of the tube; but, as was said before, a much smaller quantity than the hydrogen, and as the density of oxygen is sixteen times greater than that of hydrogen, the volume of oxygen which passes through the porous walls will be four times less than the volume of hydrogen (the quantities of gases passing through porous walls are inversely proportional to the square roots of their densities). The oxygen which separates out into the annular space will combine, at a certain fall of temperature, with the hydrogen ; but as each volume of oxygen only requires two volumes of hydrogen, whilst at least four volumes of hydrogen will pass through the porous walls for every volume of oxygen that passes, therefore, part of the hydrogen will remain free, and can be collected from the annular space. A corresponding quantity of oxygen remaining from the decomposition of the water can be collected from the internal tube.

The decomposition of water is produced much more easily by a method of substitution, taking advantage of the affinity of substances for the oxygen or the hydrogen of water. If a substance be added to water, which takes up the oxygen and replaces the hydrogen - then we shall obtain the latter gas from the water. Thus with sodium, water gives hydrogen, and with chlorine, which takes up the hydrogen, oxygen is obtained.

Hydrogen is evolved from water by many metals, which are capable 
of forming oxides (rusts or earths, as Stahl called them) in air-that is, which are capable of burning or combining with oxygen. The capacity of metals for combining with oxygen, and therefore for decomposing water, or for the evolution of hydrogen, is very dissimilar. ${ }^{7}$ Among metals, potassium and sodium have the greatest energy in this respect. The first occurs in potash, the second in soda. They are both lighter than water, soft, and easily change in air. By bringing one or the other of them in contact with water at the ordinary temperature, ${ }^{8}$ a quantity of

7 In order to demonstrate the difference of the affinity of oxygen for different elements, it is enough to compare the amounts of heat which are evolved in their combination with 16 parts by weight of oxygen; in the case of sodium (when $\mathrm{Na}_{2} \mathrm{O}$ is formed, or 46 parts of $\mathrm{Na}$ combine with 16 parts of oxygen, according to Beketoff) 100,000 calories (or units of heat) are evolved, for hydrogen (when water, $\mathrm{H}_{2} \mathrm{O}$, is formed) 69,000 calories, for iron (when the oxide, $\mathrm{FeO}$, is formed) 69,000, and if the oxide $\mathrm{Fe}_{2} \mathrm{O}_{3}$ is formed, 64,000 calories, for zinc ( $\mathrm{ZnO}$ is formed) 86,000 calories, for lead (when $\mathrm{PbO}$ is formed) 51,000 calories, for copper (when $\mathrm{CuO}$ is formed) 38,000 calories, and for mercury ( $\mathrm{HgO}$ is formed) 31,000 calories.

These figures cannot correspond directly with the magnitude of the affinities, for the physical and mechanical side of the matter is very different in the different cases. Hydrogen is a gas, and, in combining with oxygen, gives a liquid; consequently it changes its physical state, and, in doing so, evolves beat. But zinc and copper are solids, and, in combining with oxygen, give solid oxides. The oxygen, previously a gas, now passes into a solid or liquid state, and, therefore, also must have given up its store of heat in forming oxides. As we shall afterwards see, the degree of contraction (and consequently of mechanical work) was different in the different cases, and therefore the figures expressing the heat of combination cannot directly depend on the affinities, on the loss of internal energy previously in the elements. Nevertheless, the figures above cited correspond, in a certain degree, with the order in which the elements stand in respect to their affinity for oxygen, as may be seen from the fact that the mercury oxide, which evolves the least heat (among the above examples), is the least stable, is easily decomposed, giving up its oxygen; whilst sodium, the formation of whose oxide is accompanied by the greatest evolution of heat, is able to decompose all the other oxides, taking up their oxygen. In order to generalise the connection between affinity and the evolution and the absorption of heat, which is evident in its general features, and was firmly established by the researches of Favre and Silberman (about 1840), and then of Thomsen (in Denmark) and Berthelot (in France), many investigators, especially the one last mentioned, established the law of maximum work. This states that only those chemical reactions take place of their own accord in which the greatest amount of chemical (latent, potential) energy is transformed into heat. But, in the first place, we are not able, judging from what has been said above, to distinguish that heat which corresponds with purely chemical action from the sum total of the heat observed in a reaction (in the calorimeter); in the second place, there are evidently endothermal reactions which proceed under the same circumstances as exothermal (carbon burns in the vapour of sulphur with absorption of heat, whilst in oxygen it evolves heat); and, in the third place, there are reversible reactions, which when taking place in one direction evolve heat, and when taking place in the opposite direction absorb it; and, therefore, the principle of maximum work in its elementary form is not supported by science. But the subject continues to be developed, and will probably lead to a general law, such as thermal chemistry does not at present possess.

8 If a piece of metallic sodium be thrown into water, it floats on it (owing to its lightness), keeps in a state of continual movement (owing to the evolution of hydrogen on all sides), and immediately decomposes the water, evolving hydrogen, which can be 


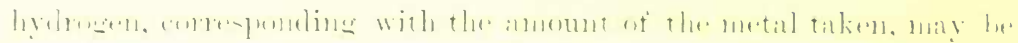

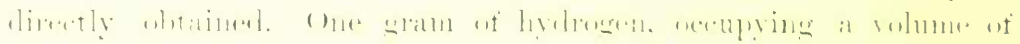

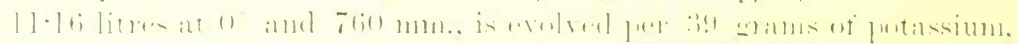

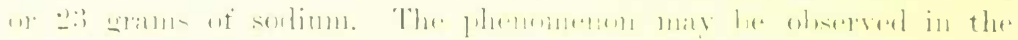

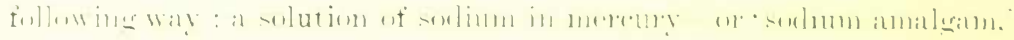

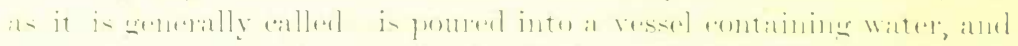

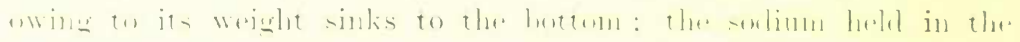

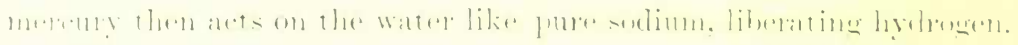

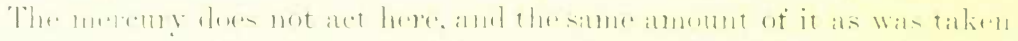

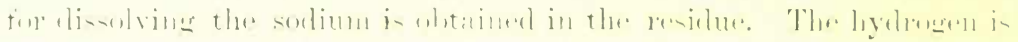

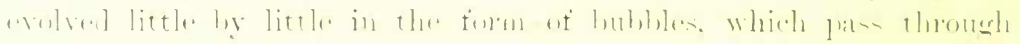
the linguiel.

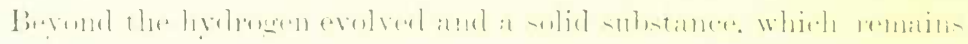

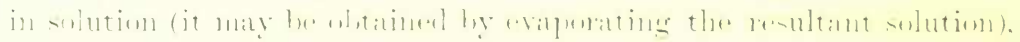

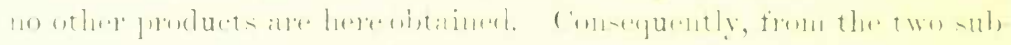

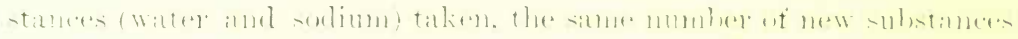

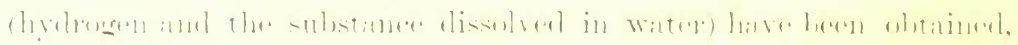

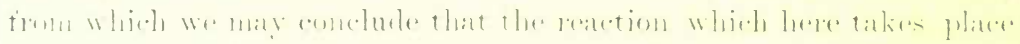

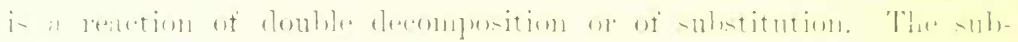

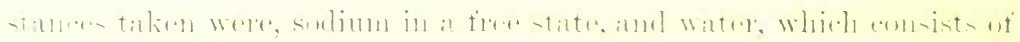

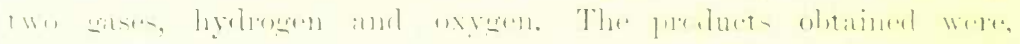

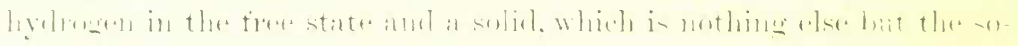

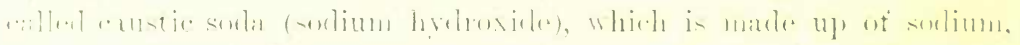

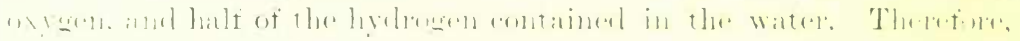

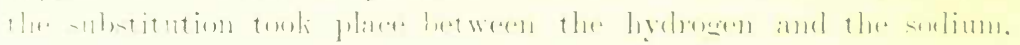

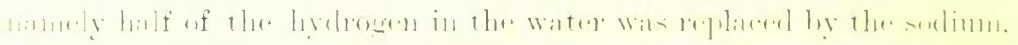

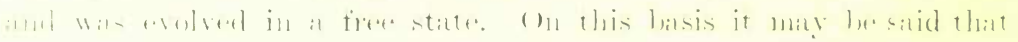

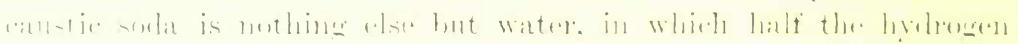

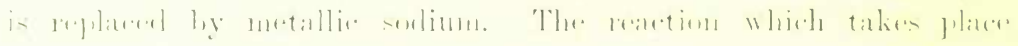

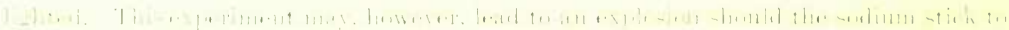

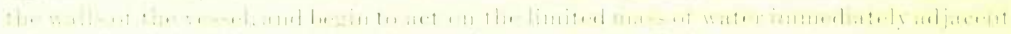

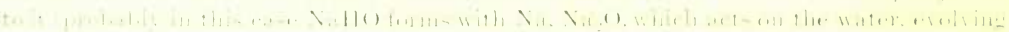

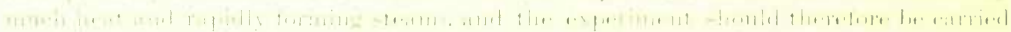

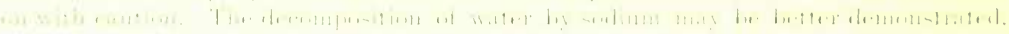

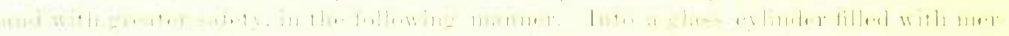

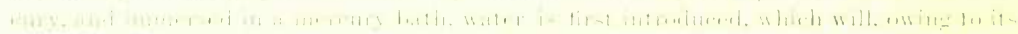

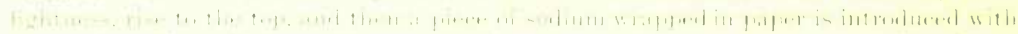

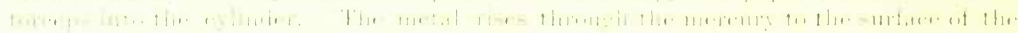

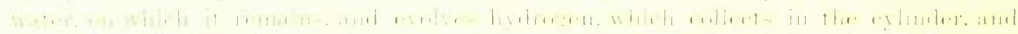

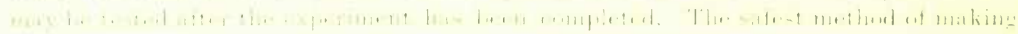

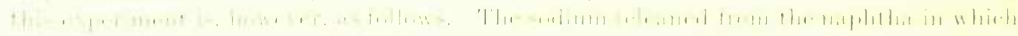

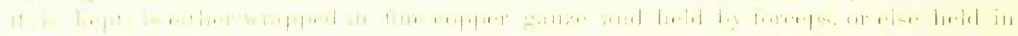

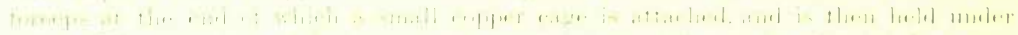

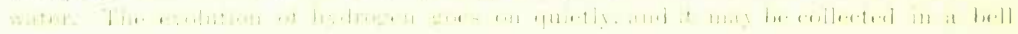

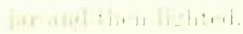


may be expressed by the equation : $\mathrm{H}_{2} \mathrm{O}+\mathrm{Na}=\mathrm{NaHO}+\mathrm{H}$; the meaning of this is clear from what has been already said. ${ }^{9}$

Sodium and potassium act on water at the ordinary temperature. Other heavier metals only act on it with a rise of temperature, and then not so rapidly or vigorously. Thus magnesium and calcium only liberate hydrogen from water at its boiling point, and zine and iron only at a red heat, whilst a whole series of heavy metals, such as copper, lead, mercury, silver, gold, and platinum, do not in the least decompose water at any temperature, and do not replace its hydrogen.

From this it is clear that hydrogen may be obtained by the decomposition of steam by the action of iron (or zinc) with a rise of temperature. The experiment is conducted in the following manner: pieces of iron (filings, nails, drc.), are laid in a porcelain tube, which is then

9 This reaction is vigorously exothermal. If a sufficient quantity of water be taken the whole of the sodium hydroxide, $\mathrm{NaHO}$, formed is dissolved, and about 42,500 units of heat are evolved per 23 grams of sodium taken. As 40 grams of sodium hydroxide are produced, and they in dissolving, judging from direct experiment, evolve about 10,000 calories; therefore, without an excess of water, and without the formation of a solution, the reaction $\mathrm{Na}+\mathrm{H}_{2} \mathrm{O}=\mathrm{H}+\mathrm{NaHO}$ would evolve abont 32,500 calories. We shall afterwards learn that hydrogen contains in its smallest isolable particles $\mathrm{H}_{2}$ and not $\mathrm{H}$, and therefore it follows that the reaction should be written thus $-2 \mathrm{Na}+2 \mathrm{H}_{2} \mathrm{O}=\mathrm{H}_{2}+$ $2 \mathrm{NaHO}$, and it then corresponds with an evolution of heat of $+65,000$ calories. And as $\mathrm{N}$. N. Beketoff showed that $\mathrm{Na}_{2} \mathrm{O}$, or anhydrous oxide of sodium, forms the hydrate, or sodium hydroxide (caustic soda), $2 \mathrm{NaHO}$, with water, evolving about 35,500 calories, therefore the reaction $2 \mathrm{Na}+\mathrm{H}_{2} \mathrm{O}=\mathrm{H}_{2}+\mathrm{Na}_{2} \mathrm{O}$ corresponds to 29,500 calories. This quantity of heat is less than that which is evolved in combining with water, in the formation of caustic soda, and therefore it is not to be wondered at that the hydrate, $\mathrm{NaHO}$, is always formed and not the anhydrous substance $\mathrm{Na}_{2} \mathrm{O}$. That such a conclusion, which agrees with facts, is inevitable is also seen from the fact that, according to Beketoff, the anhydrous sodium oxide, $\mathrm{Na}_{2} \mathrm{O}$, acts directly on hydrogen, with separation of sodium $\mathrm{Na}_{2} \mathrm{O}+\mathrm{H}=$ $\mathrm{NaHO}+\mathrm{Na}$. This reaction is accompanied by an evolution of heat equal to about 3,000 calories, because $\mathrm{Na}_{2} \mathrm{O}+\mathrm{H}_{2} \mathrm{O}$ gives, as we saw, 35,500 calories and $\mathrm{Na}+\mathrm{H}_{2} \mathrm{O}$ evolves 32,500 calories. However, an opposite reaction also takes place- $\mathrm{NaHO}+\mathrm{Na}=\mathrm{Na}_{2} \mathrm{O}+\mathrm{H}$ (both with the aid of heat)-consequently, in this case heat is absorbed. In this we see an example of calorimetric calculations and the small use of the law of maximum work for the general phenomena of reversible reactions, to which the case just considered belongs. But it must be remarked that all reversible reactions evolve or absorb but little heat, and judging from what has been said in Note 6 (and in Note 25 of Chap. I.), the reason of the discrepancy between the law of maximum work and reality must before all be looked for in the fact that we have no means of separating the heat which corresponds with the purely chemical process from the sum total of the lieat observed, and as the structure of a number of substances is altered by heat alone and also by contact, we can scarcely hope that the time approaches when such a distinction will be possible. A heated substance, in point of fact, has no longer the original energy of its atoms - that is, the act of heating not only alters the store of movement of the molecules but also of the atoms forming the molecules, in other words, it makes the beginning of or preparation for chemical change. From this it must be concluded that thermo-chemistry, or the study of the heat accompanying chemical transformations, cannot be identified with chemical mechanics. Thermo-chemical data form a part of it, but they alone cannot give it. 
subjected to a strong heat and steam passed through it. The steam, coming into contact with the iron, gives up its oxygen to it, and thus the hydrogen is set free and passes out at the other end of the tube together with undecomposed steam. This method, which is historically very significant, ${ }^{10}$ is practically inconvenient, as it requires a rather high temperature. Further, this reaction, as a reversible one (a redhot mass of iron decomposes a current of steam, forming oxide and hydrogen ; and a mass of oxide of iron, heated to redness in a stream of hydrogen, forms iron and steam), does not proceed in virtue of the comparatively small difference between the affinity of oxygen for iron (or zinc), and for hydrogen, but only because the hydrogen escapes, as it is formed, in virtue of its elasticity. ${ }^{11}$ If the oxygen compounds-that is, the oxides - which are obtained from the iron or zinc, be able to pass into solution, then the affinity acting in solution is added, and the reaction may become non-reversible, and proceed with comparatively much greater facility. ${ }^{12}$ As the oxides of iron and zinc, by themselves

10 The composition of water, as we saw above, was determined by passing steam over red-hot iron; the same method has been used for making hydrogen for filling balloons. An oxide having the composition $\mathrm{Fe}_{3} \mathrm{O}_{4}$ is formed in the reaction, so that it is expressed by the equation $3 \mathrm{Fe}+4 \mathrm{H}_{2} \mathrm{O}=\mathrm{Fe}_{3} \mathrm{O}_{4}+8 \mathrm{H}$. It is very important to remark that this reaction is reversible. By heating the scoria in a current of hydrogen, water and iron are obtained. From this it follows, from the principle of chemical equilibria, that if there be taken iron and hydrogen, and also oxygen, but in such a quantity that it is insufficient for combination with both substances, then it will divide itself between the two; part of it will combine with the iron and the other part with the lhydrogen, but a portion of both will remain in an uncombined state. Here again (see note 9) the reversibility is connected with the small heat effect, and here again both reactions (direct and reverse) proceed at a red heat. But if, in the above-described reaction, the hydrogen escapes as it is evolved, then its partial pressure does not increase with its formation, and therefore all the iron can be oxidised by the water, which could not take place were the iron and water heated to the temperature of reaction in a closed vessel. In this we see the elements of that influence of mass to which we shall have occasion to return later.

11 Therefore, if iron and water be placed in a closed space, decomposition of the water will proceed on leating to the temperature at which the reaction $3 \mathrm{Fe}+4 \mathrm{H}_{2} \mathrm{O}=\mathrm{Fe}_{3} \mathrm{O}_{4}+8 \mathrm{H}$ commences; but it ceases, does not go on to the end, because the conditions for a reverse reaction are attained, and a state of equilibrium will ensue after the decomposition of a certain quantity of water. Judging from what has been said in Note 9, something of the same kind takes place if the iron be replaced by sodium, only then the mass of the water decomposed will be greater, and equilibrium will ensue, with the formation of the hydrate, $\mathrm{NaHO}$, and not of anhydrous oxide, $\mathrm{Na}_{2} \mathrm{O}$-that is, the water will remain in the form of hydrate only. With copper and lead there will be no decomposition, either at the ordinary or at a high temperature, because the affinity of these metals for oxygen is much less than that of hydrogen.

12 In general, if reversible as well as non-reversible reactions can take place between substances acting on each other, then, judging by our present knowledge, the nonreversible reactions take place in the majority of cases, which obliges one to acknowledge the action, in this case, of compuratively strong affinities. The reaction, $\mathrm{Zn}+\mathrm{H}_{2} \mathrm{SO}_{4}=$ $\mathrm{H}_{2}+\mathrm{ZnSO}_{4}$, which takes place in solutions at the ordinary temperature, is scarcely reversible under these conditions, but at a certain ligh temperature it becomes reversible, 
insoluble in water, are capable of combining with (have an affinity for) acid oxides (as we shall afterwards fully consider), and form saline and soluble substances, with acids, or hydrates having acid properties, hence by the action of such hydrates, or of their aqueous solutions, ${ }^{13}$ iron and zinc are able to liberate hydrogen with great ease at the ordinary temperature - that is, they act on solutions of acids just as sodium acts on water. ${ }^{14}$ Sulphuric acid, or oil of vitriol, $\mathrm{H}_{2} \mathrm{SO}_{4}$, is usually chosen

because at this temperature zinc sulphate and sulphuric acid split up, and the action must take place between the water and zinc. From the preceding proposition results proceed which are in some cases verified by experiment. If the action of zinc or iron on a solution of sulphuric acid presents a non-reversible reaction, then we may by this means obtain hydrogen in a very compressed state, and compressed lyydrogen will not act on solutions of sulphates of the above-named metals. This is verified in reality as far as was possible in the experiments to keep up the compression or pressure of the hydrogen. Those metals which do not evolve hydrogen with acids, on the contrary, should, at least at an increase of pressure, be displaced by hydrogen. And in fact Brunner showed that gaseous hydrogen displaces platinum and palladium from the aqueous solutions of their chlorine compounds, but not gold, and Beketoff succeeded in showing that silver and mercury, under a considerable pressure, are separated from the solutions of certain of their compounds by means of hydrogen. Reaction already commences under a pressure of six atmospheres, if a weak solution of silver sulphate be taken; with a stronger solution a much greater pressure is required, however, for the separation of the silver.

13 For the same reason, many metals in acting on solutions of the alkalis displace hydrogen. Aluminium acts particularly clearly in this respect, because its oxide gives a soluble compound with alkalis. For the same reason tin, in acting on hydrochloric acid, evolves hydrogen, and silicon does the same with hydrofluoric acid. It is evident that in such cases the sum of all the affinities plays a part; for instance, taking the action of zinc on sulphuric acid, we have the affinity of zinc for oxygen (forming zinc oxide, $\mathrm{ZnO}$ ), the affinity of its oxide for sulphuric anhydride, $\mathrm{SO}_{3}$ (forming zinc sulphate, $\mathrm{ZnSO}_{4}$ ), and the affinity of the resultant salt, $\mathrm{ZnSO}_{4}$, for water. It is only the first-named affinity that acts in the reaction between water and the metal, if no account is taken of those forces (of a physico-mechanical character) which act between the molecules (for instance, the cohesion between the molecules of the oxide) and those forces (of a chemical character) which act between the atoms forming the molecule, for instance, between the atoms of hydrogen giving the molecule $\mathrm{H}_{2}$ containing two atoms. I consider it necessary to remark, that the hypothesis of the affinity or endeavour of heterogeneous atoms to enter into a common system and in harmonious movement (i.e., to form a compound molecule) must inevitably be in accordance with the hypothesis of forces inducing homogeneous atoms to form complex molecules (for instance, $\mathrm{H}_{2}$ ), and to build up the latter into solid or liquid substances, in which the existence of an attraction between the homogeneous particles must certainly be admitted. Therefore, those forces which bring about solution must also be taken into consideration. These are all forces of one and the same series, and in this may be seen the great difficulties surrounding the study of molecular mechanics and its province-chemical mechanics.

14 The representation given above of the cause of the easy action of iron or zinc on sulphuric acid, naturally forms a hypothesis which explains only what is observed. It is only at first sight that this hypothesis exhibits any similarity to the hypothesis of predisposing affinity which reigned in past times. According to that, it was supposed that reaction takes place (and hydrogen is evolved) by reason of the affinity for the sulphuric acid of the oxide of zinc which might be produced, and that decomposition could not take place without this. The influence of a force in respect to a substance which has not been produced, but which is capable of being formed, is not clear. In the representation introduced by me, it is acknowledged that zinc already acts on water by 
for this purpose ; from it the hydrogen is displaced by many metals with incomparably greater facility than directly from water, and such a displacement is accompanied by the evolution of a large amount of heat. ${ }^{15}$ By the action of zine or iron on sulphuric acid, hydrogen is evolved, because the metal replaces it. When the hydrogen in sulphuric acid is replaced by a metal, a substance is obtained which is called a salt of sulphuric acid or a sulphate. Thus, by the action of zinc on sulphuric acid, hydrogen and zine sulphate, $\mathrm{ZnSO}_{4}$, are obtained. The latter is a solid substance, soluble in water. In order that the action of the metal on the acid should go on regularly, and to the end, it is necessary that the acid should be diluted with water, which dissolves the salt as it is formed; otherwise the salt covers the inetal, and hinders the acid from attacking it. Usually the acid is diluted with from three to five times its volume of water, and the metal is covered with this solution. In order that the metal should act rapidly on the acid, it should present a large surface, so that a maximum amount of the reacting substances may come into contact in a given time. For this purpose the zinc is used as strips of sheet zinc, or in the granulated form (that is, zinc which has been poured from a certain height, in a molten state, into water). The iron should be in the form of wire, nails, filings, or cuttings.

The usual method of obtaining hydrogen is as follows : A certain quantity of granulated zinc is put into a double-necked, or Woulfe's, bottle. Into one neck a funnel is placed, reaching to the bottom of the bottle, so that the liquid poured in may prevent the hydrogen from

itself, even at the ordinary temperature, but that the action is limited by small masses and only proceeds at the surface. In reality, zinc, in the form of a very fine powder, or so called 'zinc dust,' is capable of decomposing water with the formation of oxide (hydrated) and hydrogen. The oxide formed acts on sulphuric acid, water then dissolves the salt produced, and the action continues because one of the products of the action of water on zinc, zinc oxide, is removed from the surface. One might naturally imagine that the reaction does not proceed directly between the metal and water, but between the metal and the acid, but such a simple representation, which we shall cite afterwards, hides the mechanism of the reaction, and does not permit of its actual complexity being seen.

15 According to Thomsen the reaction between zinc and a very weak solution of sulphuric acid evolves about 38,000 calories (zinc sulphate being formed) per 65 parts by weight of zinc; and 56 parts by weight of iron-which combine, like 65 parts by weight of zinc, with 16 parts by weight of oxygen-evolve about 25,000 calories (forming ferrous sulphate, $\mathrm{FeSO}_{4}$ ). Paracelsus observed the action of metals on acids in the seventeenth century; but it was not until the eighteenth century that Lémery determined that the gas which is evolved in this action is a particular one which differs from air and is capable of burning. Even Boyle confused it with air. Cavendish determined the chief properties of the gas discovered by Paracelsus. At first it was called 'inflammable air'; later, when it was recognised that in burning it gives water, it was called hydrogen, from the Greek words for water and generator. 
The first part of the gas evolved should not be collected, as ${ }^{-}$it is mixed with the air originally in the apparatus. This precaution

phuric acid in the other. The neck of the former is closed by a cork, which is fitted with a gas-conducting tube with a stop-cock. If the two bottles are put in communication with each other and the cock be opened, the acid will flow to the zinc and evolve hydrogen. If the cock be closed, the hydrogen will force out the acid from the bottle containing the zinc, and the action will cease. Or the vessel containing the acid may be placed at a lower level than that containing the zinc, when all the liquid will flow into it, and in order to start the action the acid vessel may be placed on a higher level than the other, and the acid will flow to the zinc. Such an arrangement presents the simplest form of a continuously-acting apparatus, which is of great use in chemical work. It can also be employed for collecting gases (as an aspirator or gasometer).

In laboratory practice, however, other forms of apparatus are generally employed for

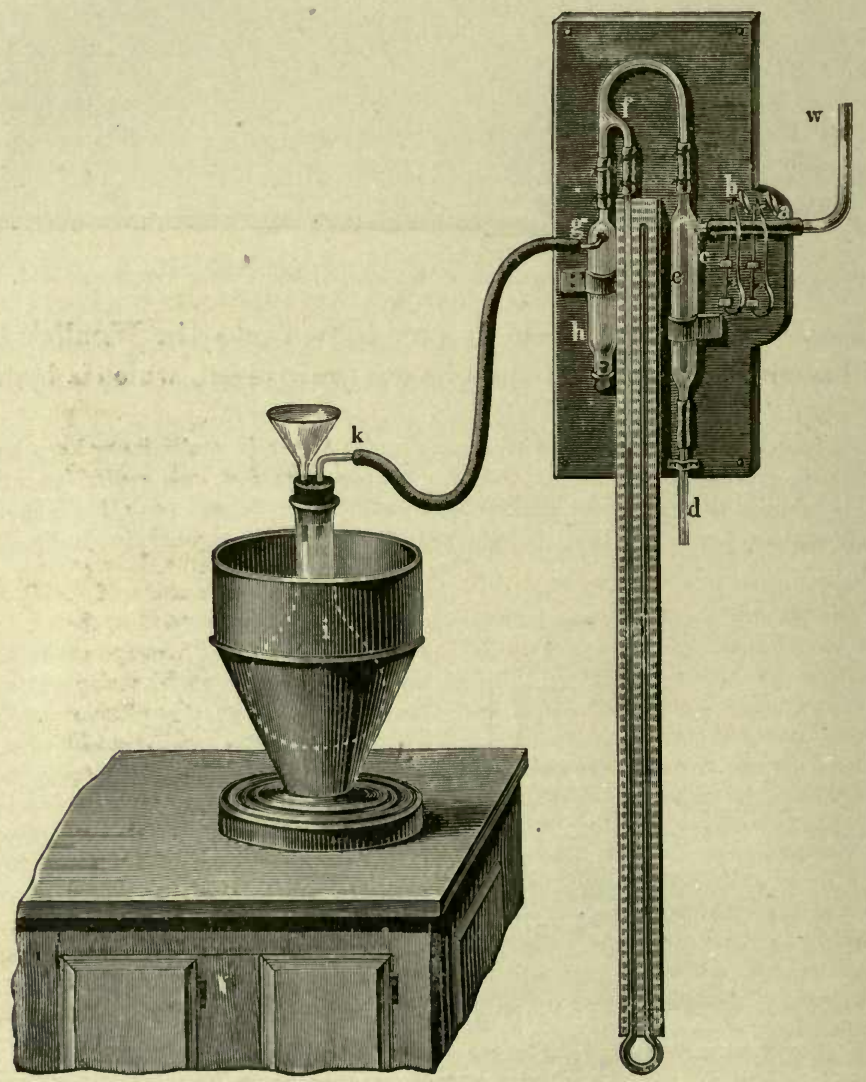

FIG. 22.-Constant-acting aspirator. The tube $d$ should be long (over 32 feet).

exhausting, collecting, and holding gases. We will here cite the most usual forms. An aspirator usually consists of a vessel furnished with a stop-cock at the bottom. A stout 
should be taken in the preparation of all gases. Time must be allowed for the gas evolved to displace all the air from the apparatus, other-

cork, through which a glass tube passes, is fixed into the neck of this vessel. If the vessel be filled up with water to the cork and the bottoin stop-cock be opened, then the water will run out and draw gas in. For this purpose the glass tube is connected with the apparatus from which it is desired to pump out or exhaust the gas.

The aspirator represented in fig. 22 may be recommended for its continuous action. It consists of a tube $d$ which widens out at the top, the lower part being long and narrow. In the expanded upper portion $c$, two tubes are sealed; one, $e$, for drawing in the gas, whilst the other, $b$, is connected to the water supply $w$. The amount of water supplied through the tube $b$ must be less than the amount which can be carried off by the tube $d$. Owing to this the water in the tube $d$ will flow through it in cylinders alternating with cylinders of gas, which will be thus carried away. The gas which is drawn throngh may be collected from the end of the tube $d$, but this form of pump is usually employed where the air or gas aspirated is not to be collected. If the tube $d$ is of considerable length, say $40 \mathrm{ft}$. or more, a very fair vacuum will be produced, the amount of which is shown by the gauge $g$; it is often used for filtering under reduced pressure, as shown in the figure. If water be replaced by mercury, and the length of the tube $d$ be greater than $760 \mathrm{~mm}$., the aspirator may be enployed as an air-pump, and all the air may be exhausted from a limited space; for instance, by connecting $g$ with a hollow sphere.

Gasholders are often used for collecting and holding gases. They are made of glass, copper, or tin plate. The usual form is shown in fig. 23. The lower vessel $B$ is made hermetically tight - i.e., impervious to gases-and is filled with water. A funnel is attached to this vessel (on several supports). The vessel $B$ communicates with the bottom of the funnel by a stop-cock $b$ and a tube $a$, reaching to the bottom of the vessel $B$. If water be poured into the funnel and the stop-cocks $a$ and $b$ opened, the water will run through $a$, and the air escape from the vessel $B$ by $b$. A glass tube $f$ runs up the side of the vessel $B$, with which it communicates at the top and bottom, and shows the amount of water and gas the gasholder contains. In order to fill the gasholder with a gas, it is first filled with water, the cocks $a, b$ and $e$ are closed, the nut $d$ unscrewed, and the end of the tube conducting the gas from the apparatus in which it is generated is passed into $d$. As the gas fills the gasholder, the water runs out at $d$. If the pressure of a gas be not greater than the atmospheric pressure and it be required to collect it in the gasholder, then the cock $e$ is put into communication with the space containing the gas. Then, having opened the orifice $d$, the gasholder acts like an aspirator; the gas will pass through $e$, and the water run out at $d$. If

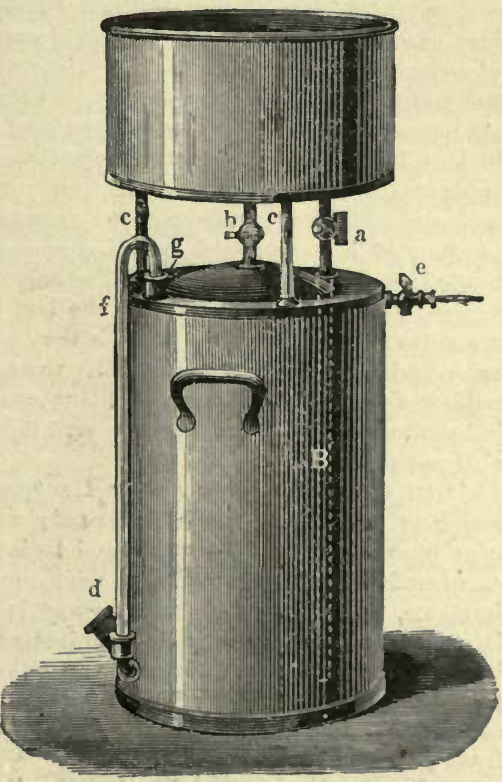

Fig. 23.-Gasholder. the cocks be closed, the gas collected in the gasholder may be easily preserved and transported. If it be desired to transfer this gas into another vessel, then a gas-conducting tube is attached to $e$, the cock $a$ opened, $b$ and $d$ closed, and then the gas will pass out at $e$, owing to its pressure in the apparatus being greater than the atmospheric pressure 


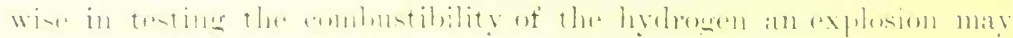

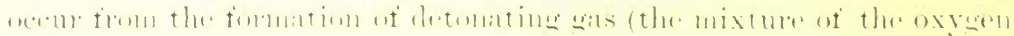

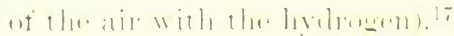

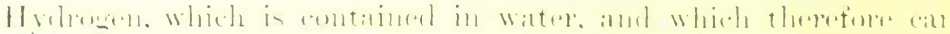

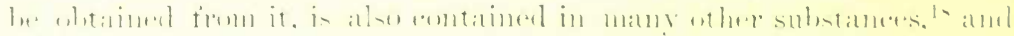

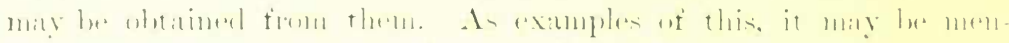

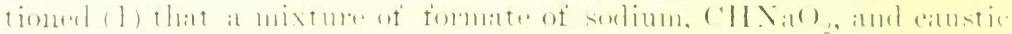

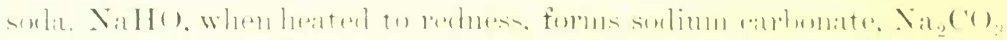

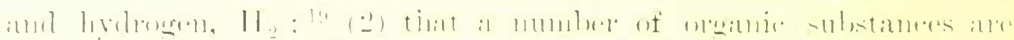
deand]

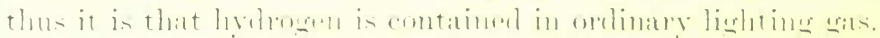

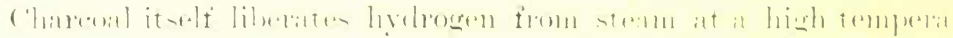

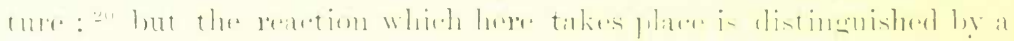

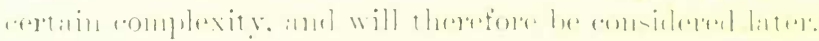

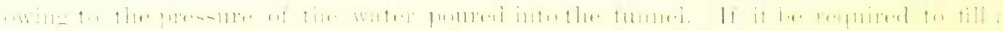

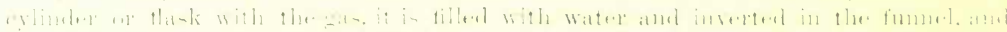

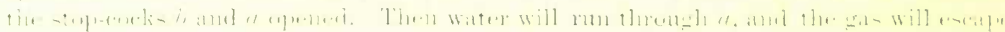

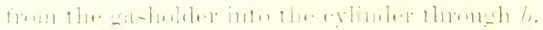

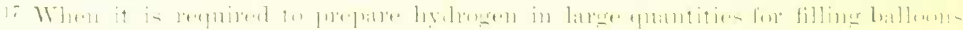

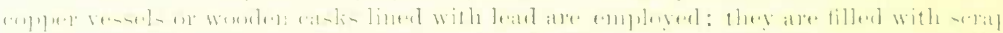

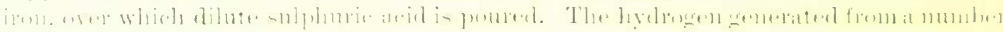

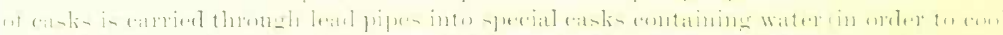

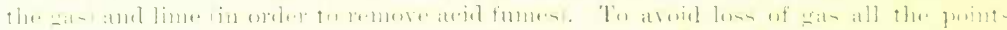

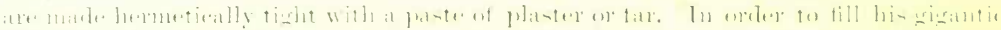

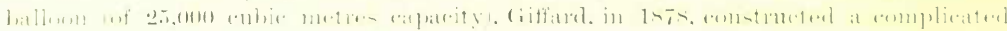

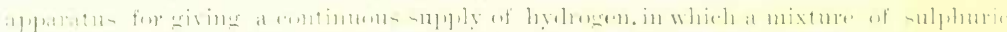

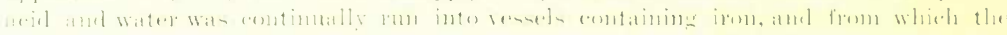

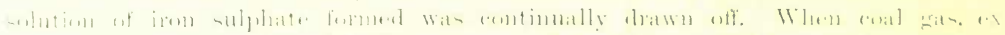

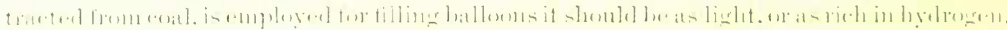

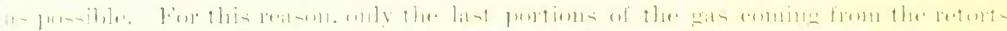

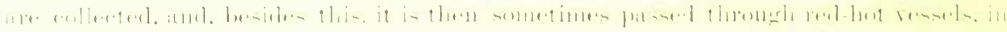

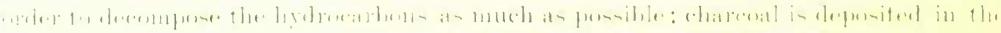

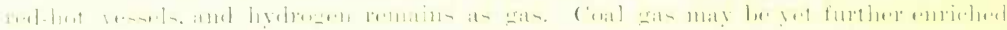

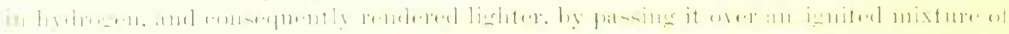

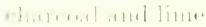

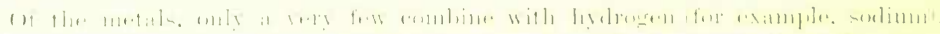

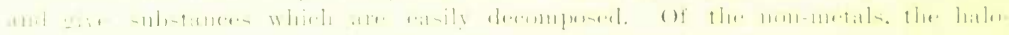

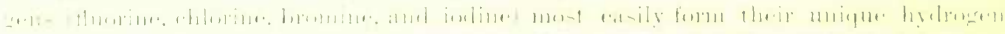

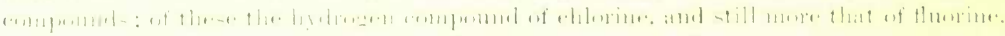

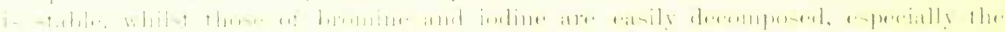

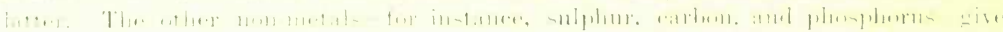

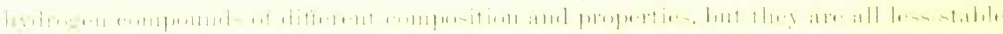

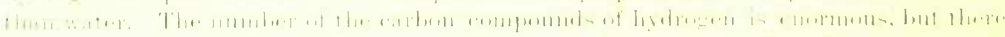

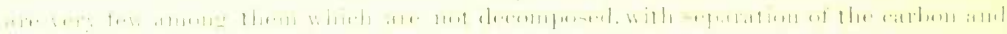

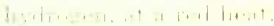

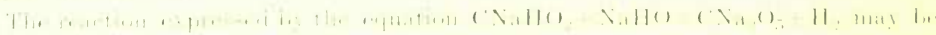

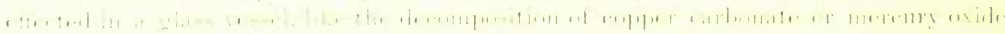

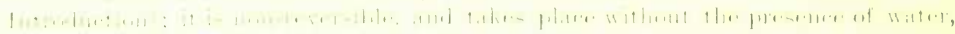

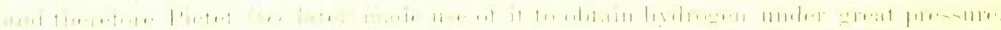

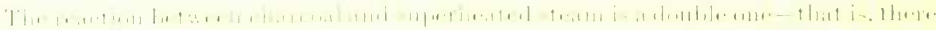

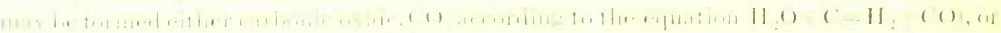


The properties of hydrogen.- Hydrogen presents us with an example of a gas which at first sight does not differ from air. It is not surprising, therefore, that Paracelsus, having discovered that an aëriform substance is obtained by the action of metals on sulphuric acid, did not quite determine its difference from air. In fact, hydrogen, like air, is colourless, and has no smell ${ }^{21}$ but a more intimate acquaintance with its properties proves it to be entirely different from air. The first sign which distinguishes hydrogen from air is its combustibility. This property is so easily observed that it is the one to which recourse is usually had in order to recognise hydrogen, if it is evolved in a reaction, although there are many other combustible gases. But before speaking of the combustibility and other chemical properties of hydrogen, we will first describe the physical properties of this gas, as we did in the case of water. It is easy to show that hydrogen is one of the lightest gases. ${ }^{22}$ If passed into the bottom of a flask full of air,

carbonic anhydride $\mathrm{CO}_{2}$ (according to the equation $2 \mathrm{H}_{2} \mathrm{O}+\mathrm{C}=2 \mathrm{H}_{2}+\mathrm{CO}_{2}$ ), and the resulting mixture is called water-gas; we shall speak of it in describing the oxides of carbon.

"1 Hydrogen obtained by the action of zinc or iron on sulphuric acid generally smells of hydrogen sulphide (like rotten eggs), which it contains in admixture. As a rule such hydrogen is not so pure as that obtained by the action of an electric current or of sodium on water. The impurity of the hydrogen depends on the impurities contained in the zinc, or iron, and sulphuric acid, and on secondary reactions which take place simultaneously with the main reaction. Thus iron sulphide gives hydrogen sulphide $\left(\mathrm{FeS}+\mathrm{H}_{2} \mathrm{SO}_{4}=\mathrm{H}_{2} \mathrm{~S}+\mathrm{FeSO}_{4}\right)$. However, the hydrogen obtained in this manner may be easily freed from the impurities it contains: some of them-namely those haring acid properties-are absorbed by caustic soda, and therefore may be removed by passing the hydrogen through a solution of this substance; another series of impurities is absorbed by a solution of mercuric chloride; and, lastly, a third series is absorbed by a solution of potassium permanganate. The hydrogen may be dried by passing it over sulphuric acid or calcium chloride. The substances serving for purifying the hydrogen are either placed in Woulfe's bottles, or in tubes containing pumice stone moistened with the purifying agent. The surface of contact is then greater, and the purification proceeds more rapidly. If it be desired to procure completely pure hydrogen, it is sometimes obtained by the decomposition of water (previously boiled to expel all air, and mixed with pure sulphuric acid), by the galvanic current. Only the gas evolved at the negative electrode is collected. Or else, an apparatus like that which gives detonating gas is used, only the positive electrode being immersed under mercury containing zine in solution. The oxygen which is evolved at this electrode then immediately, at the moment of its evolution, combines with the zinc, and this compound dissolves in the sulphuric acid and forms zinc sulphate, which remains in solution, and therefore the hydrogen generated will be quite free from oxygen.

22 An inverted beaker is attached to one arm of the beam of a rather sensitive balance, and its weight counterpoised by weights in the pan attached to the other arm. If the beaker be then filled with hydrogen it rises, owing to the air being replaced by hydrogen. Thus, at the ordinary temperature of a room, a litre of air weighs about 1.2 grams, and on replacing the air by hydrogen a decrease in weight of about 1 gram per litre is obtained. Moist hydrogen is heavier than dry-for aqueous vapour is nine times heavier than hydrogen. In filling balloons it is usually calculated that (it being impossible to have perfectly dry hydrogen or to obtain it quite free from air) the lifting force is equal to 1 kilogram $(=1,000$ grams) per cubic metre (=1,000 litres). 
hydrogen will not remain in it, but, owing to its lightness, rapidly escapes and mixes with the atmosphere. If, however, a cylinder whose orifice is turned downwards be filled with hydrogen, it will not escape, or, more correctly, it will only siowly mix with the atmosphere. This may be demonstrated by the fact that a lighted taper sets fire to the hydrogen at the orifice of the cylinder, and is itself extinguished inside the cylinder. Hence hydrogen, being itself combustible, does not support combustion. The great lightness of hydrogen is taken advantage of for balloons. Ordinary coal gas, which is often also used for the same purpose, is only about twice as light as air, whilst hydrogen is $14 \frac{1}{2}$ times lighter than air. A very simple experiment with soap bubbles very well illustrates the application of hydrogen for filling balloons. Charles, of Paris, showed the lightness of hydrogen in this way, and constructer a balloon filled with hydrogen almost simultaneously with Montgolfier. One litre of hydrogen ${ }^{23}$ at $0^{\circ}$ and $760 \mathrm{~mm}$. pressure weighs

23 The density of hydrogen in relation to the air has been determined by accurate experiments. The first determination, made by Lavoisier, was not entirely exact ; taking the density of air as unity, he obtained 0.0769 for that of hydrogen - that is, hydrogen as thirteen times lighter than air. Later determinations have corrected this figure, the most accurate determinations being due to Thomsen, who obtained the figure 0.0693 ; Berzelius and Dulong, who obtained 0.0688; and Dumas and Bunsen, who obtained 0.06945 . But the most exact determination of all is, without doubt, due to Regnault. $\mathrm{He}$ took two spheres of considerable capacity, which contained equal volumes of air (thus avoiding the necessity of any correction for weighing them in air). Both spheres were attached to the scale pans of a balance. One was sealed up, and the other first weighed empty and then full of hydrogen. Thus, knowing the weight of the hydrogen filling the sphere, and the capacity of the sphere, it was easy to find the weight of a litre of hydrogen; and, knowing the weight of a litre of air at the same temperature and pressure, it was easy to calculate the density of hydrogen. Regnault, by these experiments, found the average density of hydrogen to be 0.06926 in relation to air, or including the necessary corrections 0.06949 .

In this book I shall always refer the densities of all gases to hydrogen, and not to air ; therefore, for the sake of clearness, I will cite the weight of a litre of dry pure hydrogen in grams at a temperature $t^{\circ}$ and under a pressure $H$ (measured in millimetres of mercury at $0^{\circ}$, in long. $45^{\circ}$ ). The weight of a litre of hydrogen

$$
=0.08958 \times \frac{H}{760} \times \frac{1}{1+0.00367 t} \text { gram. }
$$

For aeronauts it is very useful to know, besides this, the weight of the air at different heights, and I therefore insert the adjoining table, constructed on the basis of Glaisher's

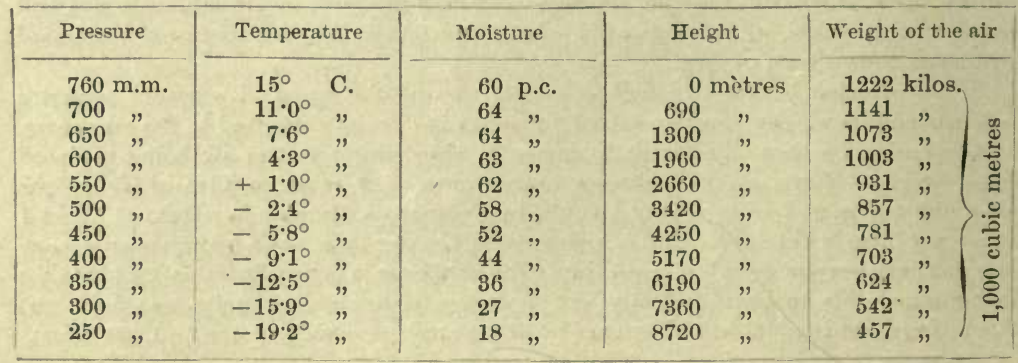


0.089578 gram ; that is, hydrogen is almost $14 \frac{1}{2}$ (more exactly, 14.43 ) times lighter than air. It is the lightest of all gases. The small density of hydrogen determines many remarkable properties which it shows; thus, hydrogen flows exceedingly rapidly from fine orifices, its molecules (Chap. I.) being endued with the greatest velocity of movement. ${ }^{24}$ At pressures somewhat higher than the atmospheric pressure, all other gases exhibit a greater compressibility and co-efficient of expansion than they should according to the laws of Mariotte and Gay-Lussac ; whilst hydrogen, on the contrary, is less compressed than should follow from the law of Mariotte, ${ }^{25}$ and with a rise of pressure it expands slightly

data, for the temperature and moisture of the atmospheric strata in clear weather. All the figures are given in the metrical system -1000 millimetres $=39 \cdot 37$ inches, 1000 kilograms $=2204.3375 \mathrm{lbs}$., 1000 cubic metres $=35316.5$ cubic feet. The starting temperature at the earth's surface is taken as $=15^{\circ} \mathrm{C}$., its moisture 60 p.c., pressure 760 millimetres. The pressures are taken as indicated by an aneroid barometer, assumed to be corrected at the sea level and at long. $45^{\circ}$.

Although the figures of this table are calculated with every possible care from average data, yet they can only be taken for an elementary judgment of the matter, for in every separate case the conditions, both at the earth's surface and in the atmosphere, will differ from those here taken. In calculating the height to which a balloon can ascend, it is evident that the density of gas in relation to air must be known. This density for ordinary coal gas is from $0 \cdot 6$ to $0 \cdot 35$, and for hydrogen with its ordinary contents of moisture and air from $0 \cdot 1$ to $0 \cdot 15$.

Hence, for instance, it may be calculated that a balloon of 1000 cubic metres capacity filled with pure hydrogen, and weighing (the envelope, tackle, people, and ballast) 727 kilograms, will ascend to a height of not much more than 4250 metres.

24 If a cracked flask be filled with hydrogen and its neck immersed under water or mercury, then the liquid will rise up into the flask, owing to the hydrogen passing through the cracks about 3.8 times quicker than the air is able to pass through these cracks into the flask. The same thing may be better seen if, instead of a flask, a tube whose end is closed by a porous substance, such as graphite, unglazed earthenware, or a gypsum plate, be employed.

25 According to Boyle and Mariotte's law, for a given gas at a constant temperature the volume decreases by as many times as the pressure increases; that is, this law requires that the product of the volume $v$ and the pressure $p$ for a given gas should be a constant quantity: $p v=C$, a constant quantity which does not vary with a change of pressure. In reality this equation does very nearly and exactly express the observed relation between the volume and pressure, but only within comparatively small variations of pressure, density, and volume. If these variations be in any degree considerable, the quantity $p v$ proves to be dependent on the pressure, and it either increases or diminishes with an increase of pressure. In the former case the compressibility is less than it should be according to Mariotte's law, in the latter case it is greater. We will call the first case a positive discrepancy (because then $d(p v) / d(p)$ is greater than zero), and the second case a negative discrepancy (because then $d(p v) / d(p)$ is less than zero). Determinations made by myself, M. L. Kirpicheff, and Hemilian showed that all known gases at low pressures, when considerably rarefied, present positive discrepancies. On the other hand it appears from the researches of Cailletet, Natterer, and Amagat that all gases under great pressures (when the volume obtained is 500-1000 times less than under the atmospheric pressure) also present positive discrepancies. Thus under a pressure of 2700 atmospheres air is compressed, not 2700 times, but only 800 , and hydrogen 1000 times. Hence the positive kind of discrepancy is, so to say, normal to gases. And this is easily understood. Did a gas follow Mariotte's law, or were it compressed to a 


\section{less than at the atmospheric pressure. ${ }^{26}$ However, hydrogen, like air and many other gases which are permanent at the ordinary tem-}

greater extent than is shown by this law, then under great pressures it would attain a density greater than that of solid and liquid substances, which is in itself improbable and even impossible by reason of the fact that solid and liquid substances are themselves but little compressible. For instance, a cubic centimetre of oxygen at $0^{\circ}$ and under the atmospheric pressure weighs about 0.0014 gram, and at a pressure of 3000 atmospheres (this pressure is attained in guns) it would, if it followed Mariotte's law, weigh $4 \cdot 2$ gramsthat is, would be about four times heavier than water-and at a pressure of 10000 atmospheres it would be heavier than mercury. Besides this, positive discrepancies are probable in the sense that the molecules of a gas themselves must occupy a certain volume. Admitting that Mariotte's law only applies to the intermolecular space still we find the necessity of positive discrepancies. If we designate the volume of the molecules of a gas by $b$ (like Van der Waals, see Chap. I. note 34), then it must be expected that $p(v-b)=C$. Hence $p v=C+b p$, which expresses a positive discrepancy. Supposing that for hydrogen $p v=1000$, at a pressure of one metre of mercury, according to the results of Regnault's, Amagat's, and Natterer's experiments, we obtain $b$ as approximately 0.7 to 0.9 .

Thus the increase of $p v$ with the increase of pressure must be considered as the normal law of the compressibility of gases. Hydrogen presents such a positive compressibility at all pressures, for it presents positive discrepancies from Mariotte's law, according to Regnault, at all pressures above the atmospheric pressure. Hence hydrogen is, so to say, a sample gas. No other gas behaves so simply with a change of pressure. All other gases at pressures from 1 to 30 atmospheres present negative discrepancies-that is, they are then compressed to a greater degree than should follow from Mariotte's law, as was shown by the determinations of Regnault, which were verified when repeated by myself and Boguzsky. Thus, for example, on changing the pressure from 4 to 20 metres. of mercury - that is, on increasing the pressure five times-the volume only decreased 4.93 times when hydrogen was taken, and 5.06 when air was taken.

The discrepancies from the law of Boyle and Mariotte for considerable pressures (from 1 to 3000 atmospheres) are well expressed (for constant temperatures) by the above-mentioned formula of Van der Waals (Chap. I. Note 34); Clausius' formula is more closely approximate, but as it and Van der Waals' formula also do not in any way express the existence of positive discrepancies from the law at low pressures, and as, according to the above-mentioned determinations made by myself, Kirpicheff, and Hemilian and verified (by two methods) by K. D. Kraevitch, they are proper to all gases (even to those which are easily compressed into a liquid state, such as carbonic and sulphurous anhydrides); therefore these formulæ, whilst accurately interpreting the phenomena of condensation and even of liquefaction, do not answer in the case of a high rarefaction of gases-that is, to that instance where a gas approaches to a condition of maximum dispersion of its molecules, and perhaps presents a passage towards the substance termed 'luminiferous ether' which fills up interplanetary and interstellar space. If we suppose that gases are rarefiable to a definite limit only, having attained which they (like solids) do not alter in volume with a decrease of pressure, then on the one hand the passage of the atmosphere at its upper limits into a homogeneous ethereal medium becomes com. prehensible, and on the other hand it would be expected that gases would, in a state of high rarefaction (i.e., when small masses of gases occupy large volumes, or when furthest removed from a liquid state) present positive discrepancies from Boyle and Mariotte's law. Our present acquaintance with this province of highly rarefied gases is most limited, and its further development promises to elucidate much in respect to natural phenomena. To the three states of matter (solid, liquid, and gaseous) it is evident a fourth must be yet added, the ethereal or ultra-gaseous (as Crookes proposed), understanding by this matter in its highest possible state of rarefaction.

26 The law of Gay-Lussac states that all gases in all conditions present one coefficient of expansion 0.00367 ; that is, when heated from $0^{\circ}$ to $100^{\circ}$ they expand like air; namely, a thousand volumes of a gas measured at $0^{\circ}$ will occupy 1367 volumes at $100^{\circ}$. 
perature, does not pass into a liquid state under a very considerable pressure, ${ }^{27}$ but is compressed into a lesser volume than would

Regnault, about 1850, showed that Gay-Lussac's law is not entirely correct, and that different gases, and also one and the same gas at different pressures, have not quite the same coefficients of expansion. Thus the expansion of air between $0^{\circ}$ and $100^{\circ}$ is 0.367 under the ordinary pressure of one atmosphere, and at three atmospheres it is 0.371 , the expansion of hydrogen is 0.366 , and of carbonic anhydride 0.37 . Regnault, however, did not directly determine the change of volume between the $0^{\circ}$ and $100^{\circ}$, but measured the variation of tension with the change of temperature; but as gases do not entirely follow Mariotte's law, therefore the change of volume cannot be directly judged by the variation of tension. The investigations carried on by myself and Kayander, about 1870, showed the direct variation of volume on heating from $0^{\circ}$ to $100^{\circ}$. These investigations confirmed Regnault's conclusion that Gay-Lussac's law is not entirely correct, and further showed (1) that the expansion per volume from $0^{\circ}$ to $100^{\circ}$ under a pressure of one atmosphere, for air $=0.368$, for hydrogen $=0.367$, for carbonic anhydride $=0.373$, for hydrogen bromide $=0.386$, \&c.; (2) that for gases which are more compressible than should follow from Mariotte's law the expansion by heat increases with the pressure-for example, for air at a pressure of three and a half atmospheres, it equals 0.371 , for carbonic anhydride at one atmosphere it equals 0.373 , at three atmospheres 0.389 , and at eight atmospheres 0.413 ; (3) that for gases wlich are less compressible than should follow from Mariotte's law, the expansion by heat decreases with an increase of pressurefor example, for hydrogen at one atmosphere 0.367 , at eight atmospheres 0.369 , for air at a quarter atmosphere 0.370 , at one atmosphere 0.368 ; and hydrogen like air (and all gases) is less compressed at low pressures than should follow from Mariotte's law (air at higher pressures than the atmospheric pressure gives a contrary result), as investigations made by myself, aided by Kirpicheff and Hemilian, showed. Hence, hydrogen, starting from zero to the highest pressures, exhibits a gradually, although only slightly, varying coefficient of expansion, whilst for air and other gases at the atmospheric and higher pressures, the coefficient of expansion increases with the increase of pressure, so long as their compressibility is greater than should follow from Mariotte's law. But when at considerable pressures, this kind of discrepancy passes into the normal (see Note $25)$, then the coefficient of expansion of all gases decreases with an increase of pressure, as is seen from the researches of Amagat. The difference between the two coefficients of expansion, for a constant pressure and for a constant volume, is explained by these relations. Thus, for example, for air at a pressure of one atmosphere the true coefficient of expansion (the volume varying at constant pressure) $=0.00368$ (according to Mendeléeff and Kayander) and the variation of tension (at a constant volume, according to Regnault) $=0 \cdot 00367$.

27 Permanent gases are such as cannot be liquefied by an increase of pressure alone. With a rise of temperature, all gases and vapours become permanent gases. As we shall afterwards learn, carbonic anhydride becomes a permanent gas at temperatures above $31^{\circ}$, and at lower temperatures it has a maximum tension, and may be liquefied by pressure alone.

The liquefaction of gases, accomplished by Faraday (see Ammonia) and others, in the first half of this century, showed that a number of substances are capable, like water, of taking all three physical states, and that there is no essential difference between vapours and gases, the only distinction being that the boiling points (or the temperature at which the tension $=760 \mathrm{~mm}$.) of liquids lie above the ordinary temperature, and those of liquefied gases below, and consequently a gas is a superheated vapour, or vapour heated above the boiling point, or removed from saturation, rarefied, having a lower tension than that maximum which is proper to a given temperature and substance. We will here cite, as we did for water (p. 54), the maximum tensions of certain liquids and gases at various temperatures, because they may be taken advantage of for obtaining constant temperatures by changing the pressure at which boiling or the formation of 


\section{follow from Mariotte's law. ${ }^{28}$ From this it may be concluded that the absolute boiling point of hydrogen, and of gases resembling}

saturated vapours takes place. The temperatures (according to the air thermometer) are placed on the left, and the tension in millimetres of mercury (at $0^{\circ}$ ) on the right, hand side of the equations. Carbon bisulphide, $\mathrm{CS}_{2}, 0^{\circ}=127.9 ; 10^{\circ}=198.5 ; 20^{\circ}=298.1$; $30^{\circ}=431 \cdot 6 ; \quad 40^{\circ}=617 \cdot 5 ; \quad 50^{\circ}=857 \cdot 1$. Chlorobenzene, $\mathrm{C}_{6} \mathrm{H}_{5} \mathrm{Cl}, 70^{\circ}=97 \cdot 9 ; 80^{\circ}=141.8 ;$ $90^{\circ}=208.4 ; 100^{\circ}=292.8 ; 110^{\circ}=402.6 ; 120^{\circ}=542.8 ; 130^{\circ}=719.0 . \quad$ Aniline, $\mathrm{C}_{6} \mathrm{H}_{7} \mathrm{~N}$, $150^{\circ}=283.7 ; 160^{\circ}=387.0 ; 170^{\circ}=515 \cdot 6 ; 180^{\circ}=677 \cdot 2 ; 185^{\circ}=771.5$. Methyl salicylate, $\mathrm{C}_{8} \mathrm{H}_{8} \mathrm{O}_{3}, 180^{\circ}=249.4 ; 190^{\circ}=330.9 ; 200^{\circ}=432.4 ; 210^{\circ}=557.5 ; 220^{\circ}=710^{\circ} 2 ; 224^{\circ}=779.9$. Mercury, $\mathrm{Hg}, 300^{\circ}=246.8 ; 310^{\circ}=304.9 ; \quad 320^{\circ}=373.7 ; 330^{\circ}=454.4 ; 340^{\circ}=548.6$; $350^{\circ}=658^{\circ} 0 ; 359^{\circ}=770.9$. Sulphur, S, $395^{\circ}=300 ; 423^{\circ}=500 ; 443^{\circ}=700 ; 452^{\circ}=800 ;$ $459^{\circ}=900$. These figures (Ramsay and Young) show the possibility of fixing constant temperatures in the vapours of boiling liquids. The tension of liquefied gases is expressed in atmospheres. Sulphurous anhydride, $\mathrm{SO}_{4},-50^{\circ}=0.4 ;-20^{\circ}=0.6$; $-10^{\circ}=1 ; \quad 0^{\circ}=1.5 ; \quad+10^{\circ}=2.3 ; 20^{\circ}=3.2 ; 30^{\circ}=5.3 . \quad$ Ammonia, $\mathrm{NH}_{3},-40^{\circ}=0.7 ;$ $-30^{\circ}=1 \cdot 1 ;-20^{\circ}=1 \cdot 8 ;-10^{\circ}=2.8 ; 0^{\circ}=4 \cdot 2 ;+10^{\circ}=6.0 ; 20^{\circ}=8.4$. Carbonic anhydride, $\mathrm{CO}_{2},-115^{\circ}=0.033 ;-80^{\circ}=1 ;-70^{\circ}=2 \cdot 1 ;-60^{\circ}=3.9 ;-50^{\circ}=6.8 ;-40^{\circ}=10 ;-20^{\circ}=23 ;$ $0^{\circ}=35 ;+10^{\circ}=46 ; 20^{\circ}=58$. Nitrous oxide, $\mathrm{N}_{2} \mathrm{O},-125^{\circ}=0.033 ;-92^{\circ}=1 ;-80^{\circ}=1 \cdot 9 ;$ $-50^{\circ}=7 \cdot 6 ; \quad-20^{\circ}=23.1 ; 0^{\circ}=36.1 ; \quad+20^{\circ}=55.3$. Ethylene, $\mathrm{C}_{2} \mathrm{H}_{4}, \quad-140^{\circ}=0.033 ;$ $-130^{\circ}=0.1 ;-103^{\circ}=1 ;-40^{\circ}=13 ;-1^{\circ}=42 . \quad$ Air, $-191^{\circ}=1 ;-158^{\circ}=14 ;-140^{\circ}=39$. Nitrogen, $\mathrm{N}_{2},-203^{\circ}=0.085 ;-193^{\circ}=1 ;-160^{\circ}=14 ;-146^{\circ}=32$. The methods of liquefying gases (by pressure and cold) will be described under ammonia, nitrous oxide, sulphurous anhydride, and in later footnotes. We will now turn our attention to the fact that the evaporation of volatile liquids, under various, and especially under low, pressures, gives an easy means for obtaining low temperatures. Thus liquefied carbonic anhydride, under the ordinary pressure, reduces the temperature to $-80^{\circ}$, and when it evaporates in an atmosphere rarefied (in an air-pump) to $25 \mathrm{~mm} .(=0.033$ atmospheres) the temperature, judging by the above-cited figures, falls to $-115^{\circ}$ (Dewar). Even the evaporation of liquids of common occurrence, under low pressures easily attainable in an air-pump, may produce low temperatures, which may be again taken advantage of for obtaining still lower temperatures. Water boiling in a vacuum becomes cold, and under a pressure of less than $4.5 \mathrm{~mm}$. it freezes, because its tension at $0^{\circ}$ is $4.5 \mathrm{~mm}$. A sufficiently low temperature may be obtained by forcing fine streams of air through common ether, or liquid carbon bisulphide, $\mathrm{CS}_{2}$, or methyl chloride, $\mathrm{CH}_{3} \mathrm{Cl}$, and other similar volatile liquids. In the adjoining table are given, for certain gases, (1) the number of atmospheres necessary for their liquefaction at $15^{\circ}$, and (2) the boiling points of the resultant liquids under a pressure of $760 \mathrm{~mm}$.

$\begin{array}{ccccccccccc} & \mathrm{C}_{2} \mathrm{H}_{4} & \mathrm{~N}_{2} \mathrm{O} & \mathrm{CO}_{2} & \mathrm{H}_{2} \mathrm{~S} & \mathrm{AsH}_{3} & \mathrm{NH}_{3}^{\circ} & \mathrm{HCl} & \mathrm{CH}_{3} \mathrm{Cl} & \mathrm{C}_{2} \mathrm{~N}_{2} & \mathrm{SO}_{3} \\ (1) & 42 & 31 & 52 & 10 & 8 & 7 & 25 & 4 & 4 & 3 \\ (2) & -103^{\circ} & -92^{\circ} & -80^{\circ} & -74^{\circ} & -58^{\circ} & -38^{\circ} & -35^{\circ} & -24^{\circ} & -21^{\circ} & -10^{\circ}\end{array}$

28 Natterer's determinations (1851-1854), together with Amagat's results (1880-1888), show that the compressibility of hydrogen, under high pressures, may be expressed by the following figures :-

$\begin{array}{lrrrrr}p & = & 1 & 100 & 1000 & 2500 \\ v & = & 1 & 0.0107 & 0.0019 & 0.0018 \\ p v & = & 1 & 1.07 & 1.9 & 3.25 \\ 8 & = & 0.11 & 10.3 & 58 & 85\end{array}$

where $p=$ the pressure in metres of mercury, $v=$ the volume, if the volume taken under a pressure of 1 metre $=1$, and $s$ the weight of a litre of hydrogen at $20^{\circ}$ in grams. If hydrogen followed Mariotte's law, then under a pressure of 2500 metres, one litre would contain not 85 , but 265 , grams. It is evident from the above figures that the weight of a litre of the gas approaches a limit as the pressure increases, which is doubtless the density of the gas when liquefied, and therefore the weight of a litre of liquid hydrogen will probably be near 100 grams (density about $0 \cdot 1$, being less than that of all other liquids). 
it, ${ }^{29}$ lies very much below the ordinary temperature ; that is, that the liquefaction of this gas is only possible at low temperatures, and under

2) Cagniard de Latour, on heating ether in a closed tube to about $190^{\circ}$, observed that at this temperature the liquid is transformed into vapour occupying the original volume -that is, having the same density as the liquid. The further investigations made by Drion and myself, showed that every liquid has such an absolute boiling point, above which it cannot exist as a liquid and is transformed into a dense gas. In order to grasp the true signification of this absolute boiling temperature, it must be remembered that the liquid state is characterised by a colesion of its particles which does not exist in vapours and gases. The cohesion of liquids is expressed in their capillary phenomena (the breaks in a column of liquid, drop formation, and rise in capillary tubes, \&c.), and the product of the density of a liquid into the height to which it rises in a capillary tube (of a definite diameter) may serve as the measure of the magnitude of cohesion. Thus, in a tube of $2 \mathrm{~mm}$. diameter, water at $15^{\circ}$ rises (the height being corrected for the meniscus) $14.8 \mathrm{~mm}$., and ether at $t^{\circ}$ to a height $5 \cdot 35-0 \cdot 028 t^{\circ} \mathrm{mm}$. 'The cohesion of a liquid is lessened by heating, and therefore the capillary heights are also diminished. It has been shown by experiment that this decrement is proportional to the temperature, and hence by the aid of capillary observations we are able to form an idea that at a certain rise of temperature the cohesion may become $=0$. For ether, according to the above formula, this would happen at $191^{\circ}$. If the cohesion disappear from a liquid it becomes a gas, for cohesion is the only point of difference between these two states. A liquid in evaporating and overcoming the force of cohesion absorbs heat. Therefore, the absolute boiling point was defined by me (1861) as that temperature at which $(a)$ a liquid cannot exist as a liquid, but forms a gas which cannot pass into a liquid state under any pressure whatever; $(b)$ cohesion $=0$; and $(c)$ the latent heat of evaporation $=0$.

These ideas were but little spread until Andrews (1869) explained the matter from another aspect. Starting from gases, he discovered that carbonic anhydride cannot be liquefied by any degree of compression at temperatures above $31^{\circ}$, whilst at lower temperatures it can be liquefied. He called this temperature the critical temperature. It is evident that it is the same as the absolute boiling point. We shall afterwards designate it by $t c$. At low temperatures a gas which is subjected to a pressure greater than its maximum tension (Note 27) is completely transformed into a liquid, which, in evaporating, gives a saturated vapour which possesses this maximum tension; whilst at temperatures above $t c$ the pressure to which the gas is subjected may increase indefinitely. However, under these conditions the volume of the gas does not change indefinitely but approaches a definite limit (see Note 28)-that is, it resembles in this respect a liquid or a solid which is altered but little in volume by pressure. The volume which a liquid or gas occupies at $t c$ is termed the critical volume, which corresponds with the critical pressure, which we will designate by $p c$ and express in atmospheres. It is evident from what has been said that the discrepancies from Mariotte and Boyle's law, the absolute boiling point, the density in liquid and compressed gaseous states, and the properties of liquids, must all be intimately connected together. We will consider these relations in one of the following notes. At present we will supplement the above observations by the values of $t c$ and $p c$ for certain liquids and gases which have been investigated in this respect-

\begin{tabular}{|c|c|c|c|c|c|}
\hline & t.c. & p.c. & & t.c. & p.c. \\
\hline $\begin{array}{l}\mathrm{N}_{2} \\
\mathrm{CO} \\
\mathrm{O}_{2} \\
\mathrm{CH}_{4} \\
\mathrm{NO} \\
\mathrm{C}_{2} \mathrm{H}_{4} \\
\mathrm{CO}_{2} \\
\mathrm{~N}_{2} \mathrm{O} \\
\mathrm{C}_{2} \mathrm{H}_{2} \\
\mathrm{HCl}\end{array}$ & $\begin{array}{l}-146^{\circ} \\
-140^{\circ} \\
-119^{\circ} \\
-100^{\circ} \\
-\quad 93^{\circ} \\
+\quad 10^{\circ} \\
+\quad 32^{\circ} \\
+\quad 53^{\circ} \\
+\quad 37^{\circ} \\
+\quad 52^{\circ}\end{array}$ & $\begin{array}{l}33 \\
39 \\
50 \\
50 \\
71 \\
51 \\
77 \\
75 \\
68 \\
86\end{array}$ & $\begin{array}{l}\mathrm{H}_{2} \mathrm{~S} \\
\mathrm{C}_{2} \mathrm{~N}_{2} \\
\mathrm{NH}_{3} \\
\mathrm{CH}_{3} \mathrm{Cl} \\
\mathrm{SO}_{2} \\
\mathrm{C}_{5} \mathrm{H}_{10} \\
\mathrm{C}_{4} \mathrm{H}_{10} \mathrm{O} \\
\mathrm{CHCl}_{3} \\
\mathrm{CS}_{2} \\
\mathrm{C}_{6} \mathrm{H}_{6}\end{array}$ & $\begin{array}{l}+108^{\circ} \\
+124^{\circ} \\
+131^{\circ} \\
+141^{\circ} \\
+155^{\circ} \\
+192^{\circ} \\
+193^{\circ} \\
+268^{\circ} \\
+278^{\circ} \\
+292^{\circ}\end{array}$ & $\begin{array}{r}92 \\
62 \\
114 \\
73 \\
79 \\
\mathbf{3 4} \\
40 \\
55 \\
78 \\
60\end{array}$ \\
\hline
\end{tabular}


great pressures. ${ }^{30}$ This conclusion was verified (1879) by the experiments of Pictet and Cailletet. ${ }^{31}$ They compressed gases at a

30 This conclusion was arrived at by me in 1870 (Ann. Pliys. Chem. 141, 623).

31 Pictet, in his researches, effected the direct liquefaction of many gases which up to that time had not been liquefied. He employed the apparatus used for the manufacture of ice on a large scale, employing the vaporisation of liquid sulphurous anhydride which may be liquefied by pressure alone. This anhydride is a gas which is transformed into a liquid at the ordinary temperature under a pressure of several atmospheres (see Note 27), and boils at $-10^{\circ}$ at the ordinary atmospheric pressure. This liquid, like all others, boils at a lower temperature under a diminished pressure, and by continually pumping out the gas which comes off by means of a powerful air-pump its boiling point falls as low as $-75^{\circ}$. Consequently, if we on the one hand force liquid sulphurous anhydride into a vessel, and on the other hand pump out the gas from the same ressel by powerful air-pumps, then the liquefied gas will boil in the vessel, and cause the temperature in it to fall to $-75^{2}$. If a second vessel is placed inside this vessel, then another gas may be easily liquefied in it at the low temperature produced by the boiling liquid sulphurous anhydride. Pictet in this manner easily liquefied carbonic anhydride, $\mathrm{CO}_{2}$ (at $-60^{\circ}$ under a pressure of from four to six atmospheres). This gas is more refractory to liquefaction than sulphurous anhydride, but for this reason it gives on evaporating a still lower temperature than can be attained by the evaporation of sulphurous anhydride. A temperature of $-80^{\circ}$ may be obtained by the evaporation of liquid carbonic anhydride at a pressure of $760 \mathrm{~mm}$., and in an atmosphere rarefied by a powerful pump the temperature falls to $-140^{\circ}$. By employing such low temperatures, it was possible, with the aid of pressure, to liquefy the majority of the other gases. It is evident that special pumps which are capable of rarefying gases are necessary to reduce the pressure in the chambers in which the sulphurous and carbonic anhydride boil; and that, in order to re-condense the resultant gases into liquids, special force pumps are required for pumping the liquid anhydrides into the refrigerating chamber. Thus, in Pictet's apparatus (fig. 24), the carbonic anhydride was liquefied by the aid of the pumps E F, which com-

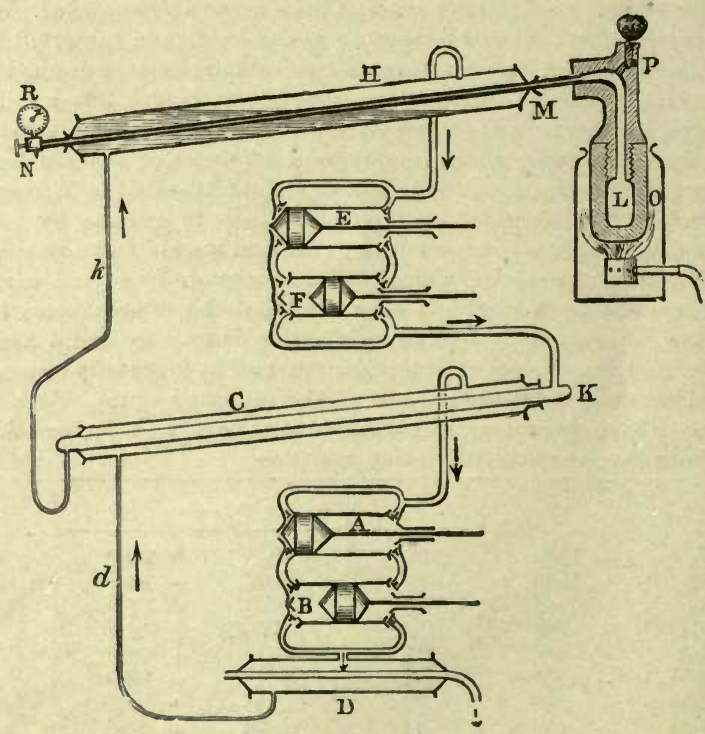

FIG. 24.-General arrangement of the apparatus employed by Pictet for liquefying gases. 
very low temperature, and then allowed them to expand, either by directly decreasing the pressure or by allowing them to escape into the air, by which means the temperature fell still lower, and then, just as steam when rapilly rarefied $^{32}$ deposits liquid water in the form of a

pressed the gas (at a pressure of 4-6 atmospheres) and forced it into the tube $K$, vigorously cooled by being surrounded by boiling liquid sulphurous anhydride, which was condensed in the tube $\mathrm{C}$ by the pump $\mathrm{B}$, and rarefied by the pump $\mathrm{A}$. The liquefied carbonic anhydride flowed down the tube $\mathrm{K}$ into the tube $\mathrm{H}$, in which it was subjected to a low pressure by the pump $\mathrm{E}$, and thus gave a very low temperature of about $140^{\circ}$. The pump $\mathrm{E}$ carried off the vapour of the carbonic anhydride, and conducted it to the pump F, by which it was again liquefied. The carbonic anhydride thus made an entire circuit-that is, it passed from a rarefied vapour of small tension and low temperature into a compressed and cooled gas, which was transformed into a liquid, which again vaporised and produced a low temperature.

Inside the wide inclined tube $\mathrm{H}$, where the carbonic acid evaporated, was placed a second and narrow tube M containing hydrogen, which was evolved in the vessels $\mathrm{L}$ from a mixture of sodium formate and caustic soda $\left(\mathrm{CHO}_{2} \mathrm{Na}+\mathrm{NaHO}=\mathrm{Na}_{2} \mathrm{CO}_{3}+\mathrm{H}_{2}\right)$. This mixture gives hydrogen on heating the vessel $L$. This vessel and the tube $\mathbf{M}$ were made of thick copper, and could withstand great pressures. They were, besides, hermetically connected together and closed up. Thus the hydrogen which was evolved had no outlet, accumulated in a limited space, and its pressure increased in proportion to the amount of it evolved. The magnitude of this pressure was recorded on a metallic manometer $\mathrm{R}$ attached to the end of the tube M. As the hydrogen in this tube was submitted to a very low temperature and a powerful pressure, there were all the necessary conditions for its liquefaction. When the pressure in the tube $\mathrm{H}$ became steady-i.e., when the temperature had fallen to $-140^{\prime}$, and the manometer $R$ indicated a pressure of 650 atmospheres in the tube $\mathrm{M}$ - then this pressure did not rise with a further evolution of hydrogen in the vessel $\mathrm{L}$. This served as an indication that the tension of the vapour of the hydrogen had attained a maximum corresponding with $-140^{\circ}$, and that consequently all the excess of the gas was condensed to a liquid. Pictet convinced himself of this by opening the cock $\mathrm{N}$, when the liquid hydrogen rushed out from the orifice. But, on leaving a space where the pressure was equal to 650 atmospheres, and coming into contact with air under the ordinary pressure, the liquid or powerfully-compressed hydrogen expanded, began to boil, absorbed still more heat, and became still colder. In doing so a portion of the liquid hydrogen, according to Pictet, passed into a solid state, and did not fall in drops into a vessel placed under the outlet $\mathrm{N}$, but as pieces of solid matter, which struck against the sides of the vessel like shot and immediately vaporised. Thus, although it was impossible to see and keep the liquefied hydrogen, still it was admitted that it passed not only into a liquid, but also into a solid, state, because Pictet in his experiments obtained other gases which had not previously been liquefied, especially oxygen and nitrogen, in a liquid and solid state. Pictet supposed that liquid and solid hydrogen have the properties of a metal, like iron.

32. At the same time (1879) as Pictet was working on the liquefaction of gases in Switzerland, Cailletet, in Paris, was occupied on the same subject, and his results, although not so convincing as Pictet's, still showed that the majority of gases, previously unliquefied, were capable of passing into a liquid state. Cailletet subjected gases to a pressure of several hundred atmospheres in thin glass tubes (fig. 25); he then cooled the compressed gas as far as possible by surrounding it with a freezing mixture; a cock was then rapidly opened for the outlet of mercury from the tube containing the gas, which consequently rapidly and vigorously expanded. This rapid expansion of the gas would producc great cold, just as the rapid compression of a gas evolves heat and causes a rise in temperature. This cold was produced at the expense of the gas itself, for in rapidly expanding its particles were not able to absorb heat from the walls of the tube, and in cooling a portion of the expanding gas was transformed into liquid. This 
fog, hydrogen in expanding forms a fog, thus indicating its passage into a liquid state. But as yet it has been impossible to preserve this liquid, even for a short time, to determine its properties, notwithstanding the employment of a temperature of $-200^{\circ}$ and a pressure of 200 atmospheres, ${ }^{33}$ although by these means the gases of the atmosphere may be kept in a liquid state for a long time. This is naturally dependent on the fact that the absolute boiling point of hydrogen lies lower than that of all other known gases, which is related to the extreme lightness of hydrogen. ${ }^{34}$

was seen from the formation of cloud-like drops, like a fog, which rendered the gas opaque. Thus Cailletet proved the possibility of the liquefaction of gases, but he did not isolate

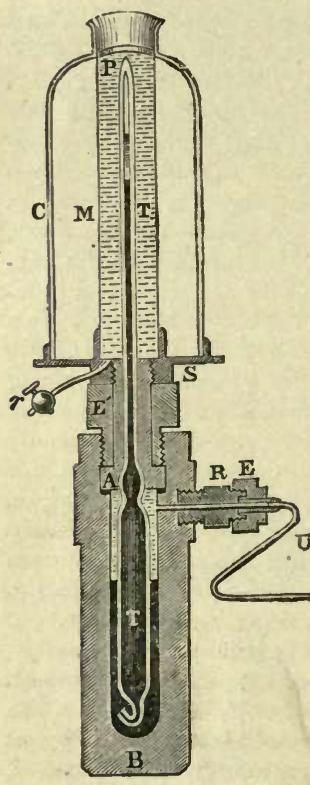

FIG. 25.-Cailletet's apparatus for liquefying gases.

the liquids. The method of Cailletet allows the passage of gases into liquids being observed with greater facility and simplicity than Pictet's method, which requires a very complicated and expensive apparatus.

The methods of Pictet and Cailletet were afterwards improved by Olszewski, Wroblewski, Dewar, and others. In order to obtain a still lower temperature they employed liquid ethylene or nitrogen instead of carbonic acid gas, whose evaporation at low pressures produces a much lower temperature (to $-200^{\circ}$ ). They also improved on the methods of determining such low temperatures, but the methods were not essentially altered; they obtained nitrogen and oxygen in a liquid, and nitrogen even in a solid, state, but no one has yet succeeded in seeing hydrogen in a liquid form.

35 The investigations of C. Wrollewski in Cracow clearly proved that Pictet could not have obtained liquid hydrogen in the interior of his apparatus, and that if he did obtain it, it could only have been at the moment of its outrush due to the fall in temperature following its sudden expansion. Pictet calculated that he obtained a temperature of $-140^{\circ}$, but in reality it hardly fell below $-120^{\circ}$, judging from the latest data for the vaporisation of carbonic anhydride under low pressure. The difference lies in the method of determining low temperatures. Judging from other properties of hydrogen (see Note 34 ), one would think that its absolute bolling point lies far below $-120^{\circ}$, and even $-140^{\circ}$ (according to the calculation of Sarrau, on the basis of its compressibility, at $-174^{\circ}$ ). But even at $-200^{\circ}$

(if the methods of determining such low temperatures be correct) hydrogen does not give a liquid even under a pressure of several hundred atmospheres. However, on expansion a fog is formed and a liquid state attained, but the liquid does not separate.

34 After the conception of the absolute temperature of ebullition ( $t c$, note 29) had been worked out (about 1870), and its connection with the deviations from Mariotte's law had become evident, and especially after the liquefaction of permanent gases, general attention was turned to the development of the fundamental conceptions of the gaseous and liquid states of matter. Some investigators directed their energies to the further study of vapours (for instance, Ramsay and Young), gases (for instance, Amagat), and liquids (for instance, Zaencheffsky, Nadeschdin, and others), especially to liquids near tc and $p c$; others (for instance, Konovaloff and De Haen) endeavoured to discover the relation between liquids under ordinary conditions (removed from $t c$ and $p c$ ) and gases, 


\section{Although a substance which passes with great difficulty into a} liquid state by the action of physico-mechanical forces, hydrogen loses

while a third class of investigators (Van der Waals, Clausius, and others), starting from the already generally-accepted principles of the mechanical theory of heat and the kinetic theory of gases, and having made the self-evident proposition of the existence in gases of those forces which clearly act in liquids, deduced the connection between the properties of one and the other. It would be out of place in an elementary landbook like the present to enunciate the whole mass of conclusions arrived at by this method, but it is necessary to give an idea of the results of Van der Waals' considerations, for they explain the gradual uninterrupted passage from a liquid into a gaseous state in the simplest form, and, although the deduction cannot be considered as complete and decisive (see note 25), nevertheless it penetrates so deeply into the essence of the matter that its signification is not only reflected in a great number of physical investigations, but also in the province of chemistry, where instances of the passage of substances from a gaseous to a liquid state are so common, and where the very processes of dissociation, decomposition, and combination must be identified with a change of physical state of the participating substances.

For a given quantity (weight, mass) of a definite substance, its state is expressed by three variables-volume $v$, pressure (elasticity, tension) $p$, and temperature $t$. Although the compressibility-[i.e., $d(v) d(p)]$-of liquids is small, still it is clearly expressed, and varies not only with the nature of liquids but also with their pressure and temperature (at $t c$ the compressibility of liquids is very considerable). Although gases, according to Mariotte's law, with small variations of pressure, are uniformly compressed, nevertheless the dependence of their volume $v$ on $t$ and $p$ is very complex. The same applies to the coefficient of expansion $[=d(v) d(t)$, or $d(p) d(t)]$, which also varies with $t$ and $p$, both for gases (see Note 26), and for liquids (at $t c$ it is very considerable, and often exceeds that of gases, 0.00367 ). Hence the equation of state must include three variables- $v, p$, and $t$. For a so-called perfect (ideal) gas, or for ineonsiderable variation of density, the elementary expression $p v=R \alpha(t+a t)$, or $p v-R(273+t)$ should be accepted, where $R$ is a constant varying with the mass and nature of a gas, as expressing this dependence, because it includes in itself the laws of Gay-Lussac and Mariotte, for at a constant pressure the volume varies proportionally to $1+a t$, and when $t$ is constant the product of $t v$ is constant. In its simplest form the equation may be expressed thus:

$$
p v=R T \text {; }
$$

where $T$ denotes what is termed the absolute temperature, or the ordinary temperature +273 - that is, $\mathrm{T}=t+273$.

Starting from the supposition of the existence of an attraction or internal pressure (expressed by $a$ ) proportional to the square of the density (or inversely proportional to the square of the volume), and of the existence of a volume or length of path (expressed by $b$ ) of gaseous molecules, Van der Waals gives for gases the following more complex equation of state:-

$$
\left(p+\begin{array}{c}
a \\
v^{2}
\end{array}\right)(v-b)=1+0.00367 t
$$

if at $0^{\circ}$ under a pressure $p=1$ (for instance, under the atmospheric pressure), the volume (for instance, a litre) of a gas or vapour be taken as 1 , and therefore $v$ and $b$ be expressed by the same units as $p$ and $a$. The deviations from both the laws of Mariotte and Gay. Lussac are expressed by the above equation. Thus, for hydrogen $a$ must be taken as infinitely small, and $b=0.0009$, judging by the data for 1000 and 2500 metres pressure (Note 28). For other permanent gases, for which (Note 28) I showed (about 1870) from Regnault's and Natterer's data, a decrement of $p v$, followed by an increment, which was confirmed (about 1880) by fresh determinations made by Amagat, this phenomena may be expressed in definite magnitudes of $a$ and $b$ (although Van der Waals' formula is not applicable for minimum pressures) with sufficient accuracy for contemporary requirements. It is evident that Van der Waals' formula can also express the difference of the 
its gaseous state (that is, its elasticity, or the physical energy of its molecules, or their rapid progressive movement) with comparative ease

coefticients of expansion of gases with a change of pressure, and according to the methods of determination (Note 26). Besides this, Van der Waals' formula shows that at temperatures above $273\left(\begin{array}{c}8 a \\ 27 b\end{array}-1\right)$ only one actual volume (gaseous) is possible, whilst at lower temperatures, by varying the pressure, three different volumes-liquid, gaseous, and partly liquid partly saturated-vaporous-are possible. It is evident that the above temperature is the absolute boiling point-that is, $(t c)=273\left(\frac{8 a}{27 b}-1\right)$. It is found under the condition that all three possible volumes (the three roots of Van der Waals' cubic equation) are then similar and equal $(v c=3 b)$. The pressure in this case $(p c)=\frac{a}{27 b^{2}}$. These ratios between the constants $a$ and $b$ and the conditions of critical state-i.e. $(t c)$ and $(p c)$-give the possibility of determining the one magnitude from the other. Thus for ether (Note 29), $(t c)=193^{\circ},(t p)=40$, from whence $a=0.0307, b=0.00533$. From whence $(v c)=0 \cdot 016$. That mass of ether which at a pressure of one atmosphere at $0^{\circ}$ occupies one volume-for instance, a litre-occupies, according to the above-mentioned condition, this critical volume. And as the density of the vapour of ether compared with hydrogen $=37$, and a litre of hydrogen at $0^{\circ}$ and under the atmospheric pressure weighs $0.0896 \mathrm{grams}$, then a litre of ether vapour weighs 3.32 grams; therefore, in a critical state (at $193^{\circ}$ and 40 atmospheres), 3.32 grams occupy 0.016 litres, or 16 c.c.; therefore 1 gram occupies a volume of about 5 c.c., and the weight of 1 c.c. of ether will then be $0 \cdot 21$. According to the investigations of Ramsay and Young (1887), the critical volume of ether was approximately such at about the absolute boiling point, but the compressibility of the liquid is so great that the slightest change of pressure or temperature acts considerably on the volume. But the investigations of the above savants gave another indirect demonstration of the true composition of Vun der Waals' equation. They also found for ether that the isochords, or the lines of equal volumes, are generally straight lines if the temperatures and pressures vary. For instance, the volume of 10 c.c. for 1 gram of ether corresponds with pressures (expressed in metres of mercury) equal to $0.135 t-3.3$ (for instance, at $180^{\circ}$ and 21 metres pressure, at $280^{\circ}$ and 34.5 metres pressure). The rectilinear form of the isochord (then $v=$ a constant quantity) is a direct result of Van der Waals' formuk.

When, in 1883, I demonstrated that the specific gravity of liquids decreases in proportion to the rise of temperature $\left[\mathrm{S}_{t}=\mathrm{S}_{o}-\mathrm{K} t\right.$ or $\left.\mathrm{S}_{t}=\mathrm{S}_{o}(1-\mathrm{K} t)\right]$, or that the volumes increase in inverse proportion to the binomial $1-\mathrm{K} t$, that is, $\mathrm{V}_{t}=\mathrm{V}_{o}(1-\mathrm{K} t)^{-1}$, where $\mathrm{K}$ is the modulus of expansion, which varies with the nature of the liquid (an exactitude of the same kind as that by which for gases the volumes increase proportionately to the binomial $1+a t$ ), then, in general, not only does a connection arise betwecn gases and liquids with respect to a change of volume, but also it would appear possible, by availing oneself of Van der Waals' formula, to judge, from the phenomena of the expansion of liquids, as to their transition into vapour, and to connect together all the principal properties of liquids, which up to this time had not been considered to be in direct dependence. Thus Thorpe and Riicker found that $2(t c)+273=1 / K$, where $K$ is the modulus of expansion in the above-mentioned formula. For example, the expansion of ether is expressed with sufficient accuracy from $0^{\circ}$ to $100^{\circ}$ by the equation $\mathrm{S}_{t}=0.736(1-0.00154 t)$, or $\mathrm{V}_{t}$ $=1(1-0.00154 t)$, where 0.00154 is the modulus of expansion, and therefore $(t c)=188^{\circ}$, or by direct observation $193^{\circ}$. For silicon tetrachloride, $\mathrm{SiCl}_{4}$, the modulus equals $0^{\circ} 00136$, from whence $(t c)=231^{\circ}$, and by experiment $230^{\circ}$. On the other hand, D. P. Konovoloff, admitting that the external pressure $p$ in liquids is insignificant when compared with the internal ( $a$ in Van der Waals' formula), and that the work in the expansion of liquids is proportional to their temperature (as in gases), directly deduced, from Van der Waals' formula, the above-mentioned formula for the expansion of liquids, $V_{t}=1$ / $(1-K t)$, and 
under the influence of chemical attraction, ${ }^{35}$ which is not only shown from the fact that hydrogen and oxygen (two permanent gases) form liquid water, but also from many phenomena of the absorption of hydrogen.

Hydrogen is vigorously condensed by certain solids; for example, by charcoal and by spongy platinum. If a piece of freshly-ignited charcoal be introduced into a cylinder full of hydrogen standing in a mercury bath, then the charcoal absorbs as much as twice its volume of hydrogen Spongy platinum condenses still more hydrogen. But palladium, a grey metal which occurs with platinum, absorbs more hydrogen than any other metal. Graham showed that when heated to a red heat and cooled in an atmosphere of hydrogen, palladium retains as much as 600 volunies of hydrogen. When once absorbed it retains the hydrogen at the ordinary temperature, and only parts with it when heated to a red heat. ${ }^{36}$ This capacity of certain dense metals for the absorption of hydrogen explains the property of hydrogen of passing through metallic tubes. ${ }^{37}$ It is termed occlusion, and presents a

also the magnitude of the latent heat of evaporation, cohesion, and compressibility under pressure. In this way Van der Waals' formula embraces the gaseous, critical, and liquid states of substances, and shows the connection between them. On this account, although Van der Waals' formula cannot be considered as perfectly general and accurate, yet it is not only very much more exact than $p v=R T$ but is also more comprehensive, because it applies to both gases and liquids. Further research will naturally give further proximity to truth, and will show the connection between composition and the constants $(a$ and $b$ ); but a great scientific progress is seen in this form of the equation of state.

Clausins (in 1880), taking into consideration the variability of $a$, in Van der Waals formula, with the temperature, gave the following equation of state:-

$$
\left(p+\frac{a}{T(v+c)^{2}}\right)(v-b)=R T .
$$

Sarrau applied this formula to Amagat's data for hydrogen, and found $a=0.0551$, $c=-0.00043, b=0.00089$, and therefore calculated its absolute boiling point as $-174^{\circ}$, and $(p c)=99$ atmospheres. But as similar calculations for oxygen $\left(-105^{\circ}\right)$, nitrogen $\left(-124^{\circ}\right)$, and marsh gas $\left(-76^{\circ}\right)$ gave $t c$ higher than it really is, therefore the absolute boiling point of hydrogen must lie below $-174^{\circ}$.

35 This and a number of similar cases clearly show how great are the internal chemical forces compared with physical and mechanical forces.

36 The capacity of palladium to absorb hydrogen, and in so doing to increase in volume, may be easily demonstrated by taking a sheet of palladium varnished on one side, and using it as a cathode. The hydrogen which is evolved by the action of the current is retained by the unvarnished surface, as a consequence of which the sheet curls up. By attaching a pointer (for instance, a quill) to the end of the sheet this bending effect is rendered strikingly evident, and on reversing the current (when oxygen will be evolved and combine with the absorbed hydrogen, forming water) it may be shown that on losing the hydrogen the palladium regains its original form.

37 Deville discovered that iron and platinum become pervious to lydrogen at a red heat. He speaks of this in the following terms:- 'The permeability of such homogeneous substances as platinum and iron is quite different from the passage of gases through such non-compact substances as clay and graphite. The permeability of metals depends 
similar phenomenon to solution ; it is based on the capacity of metals of forming unstable easily dissociating compounds ${ }^{38}$ with hydrogen similar to those which salts form with water.

At the ordinary temperature hydrogen very feebly and rarely enters into chemical reaction. The capacity of gaseous hydrogen for reaction becomes evident only under a change of circumstances - by compression, heating, or the action of light, or at the moment of its evolution. However, under these circumstances it combines directly with only a very few of the elements. Hydrogen combines directly with oxygen, sulphur, carbon, potassium, and certain other elements, but it does not combine directly with either the majority of the metals or with nitrogen, phosphorus, \&c. Compounds of hydrogen with certain elements on which it does not act directly are, however, known; they are not obtained by a direct method, but by reactions of decomposition, or of double decomposition, of other hydrogen compounds. The property of hydrogen of combining with oxygen at a red heat determines its combustibility. We have already seen that hydrogen easily takes fire, and that it then

on their expansion, brought about by heat, and proves that metals and alloys have a certain porosity.' However, Graham proved that it is only hydrogen which is capable of passing through the above-named metals in this manner. Oxygen, nitrogen, ammonia, and many other gases, only permeate through in extremely minute quantities. Graham showed that at a red heat about 500 c.c. of hydrogen pass per minute through a surface of one square metre of platinum $1.1 \mathrm{~mm}$. thick, but that with other gases the amount transmitted is hardly perceptible. Indiarubber has the same capacity for allowing the transference of hydrogen through its substance (see Chap. III.), but at the ordinary temperature one square metre, $0.014 \mathrm{~mm}$. thick, transmits only 127 c.c. of lydrogen per minute. In the experiment on the decomposition of water by heat in porous tubes, the clay tube may be exchanged for a platinum one with advantage. Graham slowed that by placing a platinum tube containing hydrogen under these conditions, and surrounding it by a tube containing air, the transference of the hydrogen may be observed by the decrease of pressure in the platinum tube. In one hour almost all the hydrogen ( 97 p.c.) had passed from the tube, without being replaced by air. It is evident that the occlusion and passage of hydrogen through metals capable of occluding it are not only intimately connected together, but are dependent on the capacity of metals to form compounds of various degrees of stability with hydrogen-like salts with water.

58 Palladium, as it appeared on further investigation, gives a definite compound, $\mathrm{Pd}_{2} \mathrm{H}$ (see further) with hydrogen; but what was most instructive was the investigation of sodium hydride, $\mathrm{Na}_{2} \mathrm{H}$, which clearly showed that the origin and properties of such compounds are in entire accordance with the conceptions of dissociation. In the chapter devoted to sodium we shall therefore speak more fnlly of this substance.

Being a gas which is difficult to condense, hydrogen is little soluble in water and other liquids. At $0^{\circ}$ a hundred volumes of water dissolve 1.9 volumes of hydrogen, and alcohol 6.9 volumes measurer at $0^{\circ}$ and $760 \mathrm{~mm}$. Molten iron absorbs hydrogen, but in solidifying, it expels it. The solution of hydrogen by metals is to a certain degree based on its affinity for metals, and must be likened to the solution of metals in mercury and to the formation of alloys. In its chemical properties hydrogen, as we shall see later, has much of a metallic character. Pictet (see Note 31 ) even affirms that liquid hydrogen has metallic properties. The metallic properties of hydrogen are also evinced in the fact that it is a good conductor of heat, which is not the case with other gases (Magnus). 
burns with a pale- - that is, non-luminous - flame. ${ }^{39}$ Hydrogen does not combine with the oxygen of the atmosphere at the ordinary temperature ; but this combination takes place at a red heat, ${ }^{40}$ and is accompanied by the evolution of much heat. The product of this combination is water - that is, a compound of oxygen and hydrogen. This is the synthesis of water, and we have already noticed its analysis or decomposition into its component parts. The synthesis of water may be very easily observed if a cold glass bell jar be placed over a burning hydrogen flame, and, better still, if the hydrogen flame be lighted in the tube of a condenser. The water will condense in drops as it is formed on the walls of the condenser and trickle down. ${ }^{41}$

Light does not aid the combination of hydrogen and oxygen, so that a mixture of these two gases does not change when exposed to the action of light; but an electric spark acts just like a flame, and this is taken advantage of for inflaming a mixture of oxygen and hydrogen, or detonating gas, mside a vessel, as will be explained in the following chapters. As hydrogen (and oxygen also) is condensed by spongy platinum, by which a rise of temperature ensues, and as platinum acts by contact (p. 38), therefore hydrogen also combines with oxygen, under the influence of platinum, as Döbereiner showed. If spongy platinum be thrown into a mixture of hydrogen and oxygen, an explosion takes place. If a mixture of the gases be passed over spongy platinum, combination also ensues, and the platinum becomes red-hot. ${ }^{42}$

59 If it be desired to obtain a perfectly colourless hydrogen flame, it must issue from a platinum nozzle, as the glass end of a gas-conducting tube imparts a yellow tint to the flame, owing to the presence of sodium in the glass.

40 Let us imagine that a stream of hydrogen passes along a tube, and let us mentally divide this stream into several parts, consecutively passing ont from the orifice of the tube. The first part is lighted - that is, brought to a state of incandescence, in which state it combines with the oxygen of the atmosphere. A considerable amount of heat is evolved in the combination. The heat evolved then, so to say, ignites the second part of hydrogen coming from the tube, and, therefore, when once ignited, the hydrogen continues to burn, if there be a continual supply of it, and if the atmosphere in which it burns be unlimited and contains oxygen.

41 The combustibility of hydrogen may be shown by the direct decomposition of water by sodium. If a pellet of sodium be thrown into a cup containing water, then it floats on the water and evolves hydrogen, which may be lighted. The presence of sodium imparts a yellow tint to the flame. If potassium be taken, the hydrogen bursts into flame of itself, because sufficient heat is evolved in the reaction for the ignition and inflammation of the hydrogen. The flame is rendered violet by the potrssium. If sodium be thrown not on water, but on an acid, it will evolve more heat, and the hydrogen will then also burst into flame. These experiments must be carried on with caution, as sometimes towards the end a mass of sodium oxide (Note 8 ) is produced, and flies about; therefore it is best to corer the vessel in which the experiment is carried on.

42 This property of spongy platinum is made use of in the so-called hydrogen cigarlight. It consists of a glass cylinder or beaker, inside which there is a small lead stand (which is not acted on by sulphuric acid), on which a piece of zinc is laid. This zinc is covered by a bell, which is open at the bottom and furnished with a cock at the top. 
Although gaseous hydrogen does not act directly ${ }^{43}$ on many substances, yet in a nascent state reaction often takes place. Thus, for instance, water on which sodium amalgam is acting contains hydrogen in a nascent state. The hydrogen is here evolved from a liquid, and at the first moment of its formation it must be in a condensed form. ${ }^{44}$

Sulphuric acid is poured into the space between the bell and the sides of the outer glass cylinder, and will thus compress the gas in the bell. If the cock of the cylinder be opened the gas will escape by it, and will be replaced by the acid, which, coming into contact with the zinc, evolves hydrogen, and it will escape through the cock. If the cock be closed, then the hydrogen evolved will increase the pressure of the gas in the bell, and thus again force the acid into the space between the bell and the walls of the outer cylinder. Thus the action of the acid on the zinc may be stopped or started at will by opening or shutting the cock, and consequently a stream of hydrogen may be always turned on. Now, if a piece of spongy platinum be placed in this stream, the hydrogen will take light, because the spongy platinum becomes hot in condensing the hydrogen and inflames it. The considerable rise in temperature of the platinum depends, among other things, on the fact that the hydrogen condensed in its pores comes into contact with previously absorbed and condensed atmospheric oxygen, with which hydrogen combines with great facility in this form. In this manner the hydrogen cigar-light gives a stream of burning hydrogen when the cock is open. In order that it should work regularly it is necessary that the spongy platinum should be quite clean, and it is best enveloped in a thin sheet of platinum foil, which protects it from dust. In any case, after some time it will be necessary to clean the platinum, which may be easily done by boiling it in nitric acid, which does not dissolve the platinum, but clears it of all dirt. This imperfection has given rise to several other forms, in which an electric spark is made to pass before the orifice from which the hydrogen escapes. This is arranged in such a manner that the zinc of a galvanic element is immersed when the cock is turned, or a small coil giving a spark is put into circuit on turning the hydrogen on.

43 Under conditions the same as those in which hydrogen combines with oxygen it is also capable of combining with chlorine. A mixture of hydrogen and chlorine explodes on the passage of an electric spark through it, or on contact with an incandescent substance, and also in the presence of spongy platinum; but, besides this, the action of light alone is enough to bring about the combination of hydrogen and chlorine. If a mixture of equal volumes of hydrogen and chlorine be exposed to the action of sunlight, complete combination rapidly ensues, accompanied by a report. Hydrogen does not combine directly with carbon, neither at the ordinary temperature nor by the action of heat and pressure. But if an electric current be passed through carbon electrodes at a short distance from each other (as in the elecric light or voltaic arc), so as to form an electric arc in which the particles of carbon are carried from one pole to the other, then, in the intense heat to which the carbon is subjected in this case, it is capable of combining with hydrogen. A peculiar-smelling gas, called acetylene, $\mathrm{C}_{2} \mathrm{H}_{2}$, is thus formed from carbon and hydrogen.

44 There is another explanation for the facility of the reactions which proceed at the moment of separation. We shall afterwards learn that the molecule of hydrogen contains two atoms, $\mathrm{H}_{2}$, but there are elements the molecules of which only contain one atomfor instance, mercury. Therefore, every reaction of gaseous hydrogen must be accompanied by the dissolution of that bond which exists between the atoms forming a molecule. At the moment of evolution, however, it is supposed that free atoms exist, and for this reason, according to the hypothesis, act energetically. This hypothesis is not borne out by facts, and the conception of hydrogen being condensed at the moment of its evolution is more natural, and is in accordance with the fact (Note 12) that compressed hydrogen displaces palladium and silver (Brunner, Beketoff)-that is, acts as at the moment of its evolution. 
In this condensed form it is capable of reacting on substances on which it does not act in a gaseous state. There is a very intimate and evident relation between the phenomena which take place in the action of spongy platinum and the phenomena of the action in a nascent state. The combination of hydrogen with aldehyde may be taken as an example. Aldehyde is a volatile liquid with an aromatic smell, boiling at $21^{\circ}$, soluble in water, and absorbing oxygen from the atmosphere, and in this absorption forming acetic acid-the substance which is found in ordinary vinegar. If sodium amalgam be thrown into an aqueous solution of aldehyde, the greater part of the hydrogen evolved combines with the aldehyde, forming alcohol - a substance which is also soluble in water, which forms the principle of all spirituous liquors, boils at $78^{\circ}$, and which contains the same amount of oxygen and carbon as aldehyde, but more hydrogen. The composition of aldehyde is $\mathrm{C}_{2} \mathrm{H}, \mathrm{O}$, and of alcohol $\mathrm{C}_{2} \mathrm{H}_{6} \mathrm{O}$. Reactions of substitution or displacement of metals by hydrogen at the moment of its evolution are particularly numerous. ${ }^{4.5}$

Metals, as we shall afterwards see, are in many cases able to replace each other; they also, and in some cases still more easily, replace and are replaced by hydrogen. We have already seen examples of this in the formation of hydrogen from water, sulphuric acid, sc. In all these cases the metals sodium, iron, or zinc displace the hydrogen which occurs in these compounds. Hydrogen may be displaced from many of its compounds by metals by exactly the same method as it is displaced

45 When, for instance, an acid and zinc are added to a salt of silver, the silver is reduced; but this may be explained as a reaction of the zinc, and not of the hydrogen at the moment of its evolution. There are, however, examples to which this explanation is entirely inapplicable; thus, for instance, hydrogen, at the moment of its evolution, easily takes up oxygen from its compounds with nitrogen if they be in solution, and converts the nitrogen into its combination with hydrogen. Here the nitrogen and hydrogen, so to speak, meet at the moment of their evolution, and in this state combine together.

It is evident from this that the elastic gaseous state of hydrogen fixes the limit of its energy : hinders it from entering into those combinations of which it is capable. In the nascent state we have hydrogen which is not in a gaseous state, and its action is then much more energetic. This is rendered very clear from the conception of chemical energy, because the process of passing into a gas requires a certain amount of heat, and consequently absorbs a certain amount of work. If gaseous hydrogen is produced, it shows that there are already conditions sufficient for the transmission of heat to the hydrogen evolved in order to convert it into a gas. It is evident at the moment of erolution that heat, which would be latent in the gaseous hydrogen, is transmitted to its molecules, and consequently they are in a state of potential, and can hence act on many substances.

Let us here remark the circumstance, which will be clearly understood from what has been said above, that hydrogen condensed in the pores of certain metals, like palladium and platinum, acts as a reducing agent on many substances. It will afterwards be understood that substances containing much hydrogen, and easily parting with it, can also act vigorously in effecting a reduction.

VOL. I. 
from water; so, for example, hydrochloric acid, which is formed directly by the combination of hydrogen with chlorine, gives hydrogen by the action of a great many metals, just as sulphuric acid does. Potassium and sodium also displace hydrogen from its compounds with nitrogen ; it is only from its compounds with carbon that hydrogen is not displaced by metals. Hydrogen, in its turn, is able to replace metals ; this is accomplished most easily on heating, and with those metals which do not themselves displace hydrogen. If hydrogen be passed over the compounds of many metals with oxygen at a red heat, it takes up the oxygen from the metals and displaces them just as it is itself displaced by metals. If hydrogen be passed over the compound of oxygen with copper at a red heat, then metallic copper and water are obtained $-\mathrm{CuO}+\mathrm{H}_{2}=\mathrm{H}_{2} \mathrm{O}+\mathrm{Cu}$. This kind of double decomposition is called reduction with respect to the metal, which is thus reduced to a metallic state from its combination with oxygen. But it must be recollected that all metals do not displace hydrogen from its compound with oxygen, and, conversely, hydrogen is not able to displace all metals from their compounds with oxygen; thus it does not displace potassium, calcium, or aluminium from their compounds with oxygen. If the metals be arranged in the following series: $\mathrm{K}, \mathrm{Na}, \mathrm{Ca}, \mathrm{Al} \ldots \mathrm{Fe}, \mathrm{Zn}, \mathrm{Hg} \ldots \mathrm{Cu}, \mathrm{Pb}, \mathrm{Ag}, \mathrm{Au}$, then the first are able to take up oxygen from water - that is: displace hydrogen - whilst the last do not act thus, but are, on the contrary, reduced by hydrogen-that is, have, as is said, a less affinity for oxygen than hydrogen, whilst potassium, sodium, calcium have more. This is also expressed by the amount of heat evolved in the act of combination with oxygen, and is shown by the fact that potassium and sodium and other similar metals evolve heat in decomposing water; but copper, silver, and the like do not do this, because in combining with oxygen they evolve less heat than hydrogen does, and therefore it happens that when hydrogen reduces these metals heat is evolved. Thus, for example, if 16 grams of oxygen combine with copper, 38000 units of heat are evolved; and when 16 grams of oxygen combine with hydrogen, forming water, 69000 units of heat are evolved; whilst 23 grams of sodium, in combining with 16 grams of oxygen, evolve 100000 units of heat. This example clearly shows that chemical reactions which proceed directly and unaided evolve heat. Sodium decomposes water and hydrogen reduces copper, because they are exothermal reactions, or those which evolve heat; copper does not decompose water, because such a reaction would be accompanied by an absorption (or secretion) of heat, or belongs to the class.of endothermal reactions, in which heat is absorbed; and such reactions do not generally proceed directly, 
although they may take place with the aid of energy (electrical, thermal, dc.) borrowed from some foreign source. ${ }^{46}$

The reduction of metals by hydrogen is taken advantage of for determining the exact composition of water by weight. Copper oxide is usually chosen for this purpose. It is heated to redness in hydrogen, and the quantity of water thus formed is determined, then the quantity of oxygen which occurs in it is found from the loss in weight of the copper oxide. This loss will depend on the fact that the oxygen has entered into the water. The copper oxide must be weighed immediately before and after the experiment. The difference shows the weight of the oxygen which entered into the composition of the water formed. In this manner only solids have to be weighed, which is a very great gain in the accuracy of the results obtained. ${ }^{47}$ Dulong and Berzelius (1819) were the first to determine the composition of water by this method, and they found that water contains 88.91 of oxygen and 11.09 of hydrogen in 100 parts, or 8.008 parts of oxygen per one part of hydrogen. Dumas (1842) improved on this method, ${ }^{48}$ and found that

46 Several numerical data and reflections bearing on this matter are enumerated in Notes 7, 9, and 11. It must be observed that the action of iron or zinc on water, or, conversely, of hydrogen on the oxides of iron or zinc, forms a reversible reaction, which proceeds in one or the other direction, according to which is removed from the sphere of action; the hydrogen or the water act according to which is present in a predominating mass. The influence of mass is clearly evinced in this case. . But the reaction $\mathrm{CuO}+\mathrm{H}_{2}=\mathrm{Cu}+\mathrm{H}_{2} \mathrm{O}$ is not reversible; the difference between the degrees of affinity is very great in this case, and, therefore, as far as is at present known, no hydrogen is evolved even in the presence of a large excess of water. It is to be further remarked, that under the conditions of the dissociation of water, copper is not oxidised by water, most probably because the oxide of copper itself is decomposable by heat.

47 This determination may be carried on in an apparatus like that mentioned in Note 13 of Chapter I.

48 We will proceed to describe Dumas' method and results. For this determination pure and dry copper oxide is necessary. Dumas took a sufficient quantity of copper oxide for the formation of 50 grams of water in each determination. As the oxide of copper was weighed before and after the experiment, and as the amount of oxygen contained in water was determined by the difference between these weights, it was essential that no other substance besides the oxygen forming the water should be evolved from the oxide of copper during its ignition in hydrogen. It was necessary, also, that the hydrogen should be perfectly pure, and free not only from traces of moisture, but from any other impurities which might dissolve in the water or combine with the copper and form some other compound with it. The bulb containing the oxide of copper (fig. 26), and which was heated to redness, should be quite free from air, as otherwise the oxygen in the air might, in combining with the hydrogen passing through the vessel, form water in addition to the oxygen of the oxide of copper. The water formed should be entirely absorbed in order to accurately determine the quantity of the resultant water. The hydrogen was evolved in the three-necked bottle. The sulphuric acid, for acting on the zinc, is poured through funnels into the middle neck. The hydrogen evolved in the Woulfe's bottle passes through $\mathbf{U}$ tubes, in which it is purified, to the bulb, where it comes into contact with the copper oxide, forms water, and reduces the oxide to metallic copper; the water formed is condensed in the second bulb, and any passing off is absorbed in the second set of $U$ tubes. This is the general arrangement of the apparatus. The bulb 
water contains 12.575 parts of hydrogen per 100 parts oxygen, that is-7.990 parts of oxygen per 1 part of hydrogen, and therefore it is usually

with the copper oxide is weighed before and after the experiment. The loss in weight, shows the quantity of oxygen which went into the composition of the water formed,

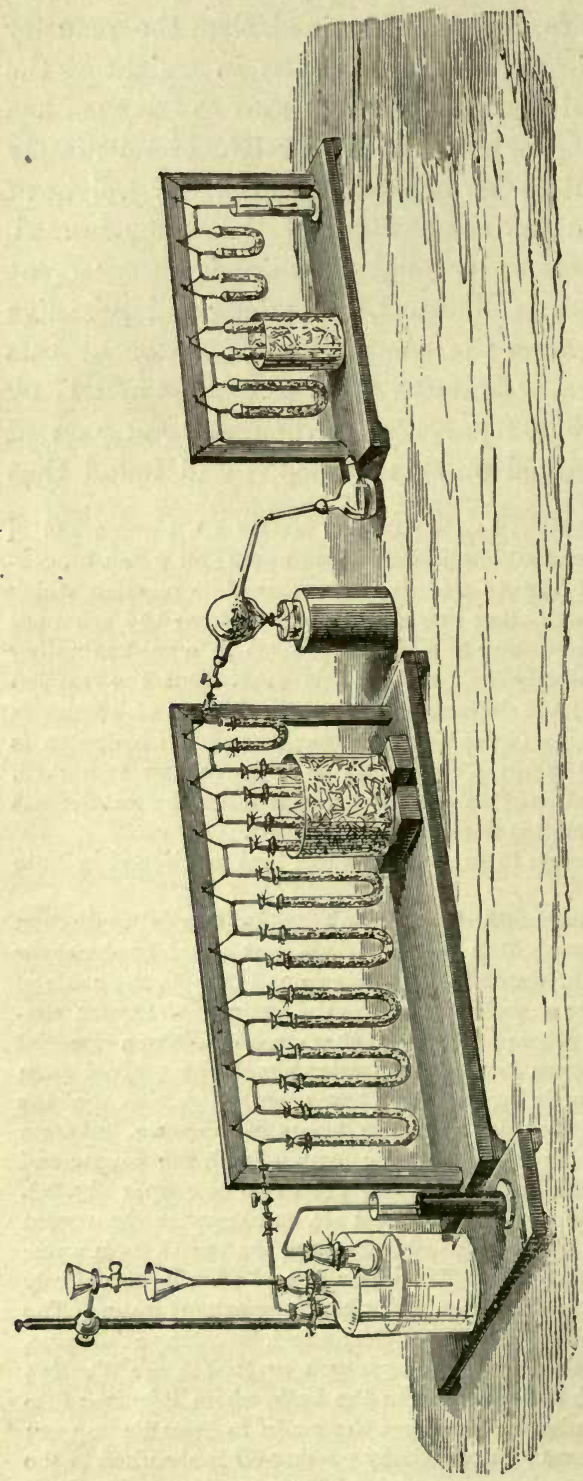

the weight of the latter being shown by the gain in weight of the absorbing apparatus. Knowing the amount of oxygen in the water formed, we also know the quantity of hydrogen contained in it, and consequently we determine the composition of water by weight. This is the essence of the determination. We will now turn to particulars. In one neck of the three-necked bottle there is placed a tube immersed in mercury. This serves as a safety-valve to prevent the pressure inside the apparatus becoming too great from the rapid evolution of hydrogen. Did the pressure rise to any considerable extent, the current of gases and vapours would be very rapid, and, as a consequence, the hydrogen would not be perfectly purified, or the water be entirely absorbed in the tubes placed for this purpose. In the third neck of the Woulfe's bottle there is a tube leading the hydrogen to the purifying apparatus, consisting of eight $U$ tubes, destined for the purification and testing of the hydrogen. The hydrogen, evolved $\vec{b}$ by zinc and sulphuric acid, is purified by passing it first through c a tube full of pieces of glass moistened with a solution of lead nitrate, next through silver sulphate; the lead nitrate retains sulphuretted hydrogen, and arseniuretted hydrogen is retained I. by the tube with silver sulphate. \& Caustic potash in the next $U$ tube retains any acid which might come over. The two following tubes are filled with lumps of dry caustic potash in order to absorb any carbonic anhydride and moisture which the hydrogen might contain. The next two tubes are, to completely dry the gas, filled with a powder of phosphoric 
received that water contains eight parts by weight of oxygen per one part by weight of hydrogen. By whatever method water be obtained, it will

anhydride, intermingled with lumps of pumice-stone. They are immersed in a freezing mixture. The small $U$ tube contains hygroscopic substances, and is weighed before the experiment: this is in order to know whether the hydrogen passing through still retains any moisture. If it does not, then the weight of this tube will not vary during the whole experiment, but if the hydrogen evolved still retains moisture, the tube will increase in weight. The copper oxide is dropped into the bulb, which is, previous to the experiment, dried with the copper oxide during a long period of time. The air is then exhausted from it, in order to weigh the oxide of copper in a vacuum and to avoid making any correction for weighing in air. The bulb is made of infusible glass, that it may be able to withstand a lengthy ( 20 hours) exposure to a red heat without changing in form. The weighed bulb is only connected with the purifying apparatus after the hydrogen has already passed through for a long time, and after experiment has shown that the hydrogen passing from the purifying apparatus is pure and does not contain any air. When the bulb is connected with the purifying apparatus, its cock is opened and the hydrogen fills the bulb. The drawn-out end of the bulb is joined by an indiarubber tube with the second bulb, in which the water formed is condensed. When this connection is made, the thread binding up the india-rubber tube is untied, and then the hydrogen can pass freely through the apparatus. On passing from the condensing bulb the gas and vapour enter into an apparatus for absorbing the last traces of moisture. The first $U$ tube contains pieces of ignited potash, the second and third tubes phosphoric anhydride or pumice-stone moistened with sulphuric acid. The last of the two is employed for determining whether all the moisture is absorbed, and is therefore weighed separately. The final tube only serves as a safety-tube for the whole apparatus, in order that the external moisture should not penetrate into it. The glass cylinder contains sulphuric acid, through which the excess of hydrogen passes; it enables the rate at which the hydrogen is evolved to be judged, and whether its amount should be decreased or increased.

When the apparatus is set up it must be seen that all its parts are hermetically tight before commencing the experiment. When the previously weighed parts are joined up together and the whole apparatus put into communication, then the bulb containing the copper oxide is heated with a spirit lamp (reduction does not take place without the aid of heat), and the reduction of the copper oxide then takes place, and water is formed, which condenses in the absorbing apparatus. When nearly all the copper oxide is reduced the lamp is removed and the apparatus allowed to cool, the current of hydrogen being kept up all the time. When cool, the drawn-out end of the bulb is fused up, and the hydrogen remaining in it is exhausted, in order that the copper may be again weighed in a vacuum. The absorbing apparatus remains full of hydrogen, and would therefore present a less weight than if it were full of air, as it was before the experiment, and, therefore, having disconnected the copper oxide bulb, a current of dry air is passed through it until the gas passing from the glass cylinder is quite free from hydrogen. The condensing bulb and the two tubes next to it are then weighed, in order to determine the quantity of water formed. Dumas repeated this experiment many times. The average result was that water contains $1253^{*} 3$ parts of hydrogen per 10000 parts of oxygen. Making a correction for the amount of air contained in the sulphuric acid employed for producing the hydrogen, Dumas obtained the average figure 1251.5 , between the extremes $1247^{\cdot 2}$ and 1256.2 . This proves that per 1 part of hydrogen water contains 7.9904 parts of oxygen, with a possible error of not more than $\frac{1}{2} \frac{1}{50}$, or 0.03 , in the amount of oxygen per 1 part of hydrogen.

Erdmann and Marchand, in eight determinations, found that per 10000 parts of oxygen water contains an average of 1252 parts of hydrogen, with a difference of from 1258.5 to 1248.7 ; hence per 1 part of hydrogen there would be 7.9952 of oxygen, with an error of at least 0.05 , because, taking the figure 1258.5 , the amount of oxygen per 1 part of hydrogen would be $7 \cdot 944$. 
always present the same composition. Whether it be taken from nature and purified, or whether it be obtained from hydrogen by oxidation, or whether it be separated from any of its compounds, or obtained by some double decomposition - it will in every case contain one part of hydrogen and eight parts of oxygen. This is because water is a definite chemical compound. Detonating-gas, from which it may be formed, is a simple mixture of oxygen and hydrogen, although a mixture of the same composition as water. All the properties of both constituent gases are preserved in detonating-gas. Either one or the other gas may be added to it without destroying its homogeneity. The fundamental properties of oxygen and hydrogen are not found in water, and neither of the gases can be added to it. But they may be evolved from it. In the formation of water there is an evolution of heat ; for the decomposition of water heat is required. All this is expressed by the words, Water is a definite chemical compound of hydrogen with oxygen. Taking the symbol of hydrogen, $\mathrm{H}$, as expressing a unit quantity by weight of this substance, and by expressing 16 parts by weight of oxygen by $O$, we can express all the above statements by the chemical symbol of water, $\mathrm{H}_{2} \mathrm{O}$. As only definite chemical compounds are denoted by formulæ, having denoted the formula of a compound substance, we express by it the entire series of conceptions which are connected with the representation of a definite compound, and, at the same time, the quantitative composition of the substance by weight. Further, as we shall afterwards see, formulæ express the volume of the gases contained in a substance. Thus the formula of water shows that it contains two volumes of hydrogen and one volume of oxygen. Besides which, we shall learn that the formula expresses the density of the vapour of a compound, and on this, as we have seen, many properties of substances depend. This vapour density, as we shall learn, also determines the quantity of a substance entering into a reaction. Thus the letters $\mathrm{H}_{2} \mathrm{O}$ tell the chemist the entire history of the substance. This is an international language, which endows chemistry with a simplicity, clearness, stability, and trustworthiness founded on the investigation of the laws of nature.

Keiser (1888), in America, by employing palladium hydride, and by introducing various new precautions for obtaining accurate results, found the composition of water to be 15.95 parts of oxygen per 2 of hydrogen.

Certain of the latest determinations of the composition of water are hardly less exact. than the analysis made by Dumas, and always give less than 8 , and on the average $7 \cdot 98$, of oxygen per 1 part of hydrogen. At present, therefore, the atomic weight of oxygen is taken as $\mathbf{1 5} \cdot 96$. However, this figure is not to be entirely depended on, and for ordinary accuracy it may be considered that $\mathrm{O}=16$. 


\section{CHAPTER III}

OXYGEN AND THE CHIEF ASPECTS OF ITS SALINE COMBINATIONS.

ON the earth's surface there is no other element which is so widely distributed as oxygen in its various compounds. ${ }^{1}$ It makes up eight-ninths of the weight of water, which occupies the greater part of the earth's surface. Nearly all earthy substances and rocks consist of compounds of oxygen with metals and other elements. Thus, the greater part of sand is formed of silica, $\mathrm{SiO}_{2}$, which is a compound of oxygen with silicon, and contains 53 p.c of oxygen; clay contains water, alumina (formed of aluminium and oxygen), and silica. It may be considered that earthy substances and rocks contain up to one-third of their weight of oxygen ; animal and vegetable substances are also very rich in oxygen. Without counting the water present in them, plants contain up to 40 , and animals up to 20 p.c. by weight of oxygen. Thus, oxygen cornpounds predominate on the earth's surface, and form about one-half of the whole of the solid and liquid matters of the earth's crust. Besides this, a portion yet remains free, and is contained in admixture with nitrogen in the atmosphere, forming about one-fourth of its mass, or one-fifth of its volume.

Being so widely distributed in nature, oxygen plays a very important part in it, for a number of the phenomena which take place before us are mainly dependent on it. Animals breathe air in order to obtain only oxygen from it, the oxygen entering into their respiratory organs (the lungs of human beings and animals, the gills of fishes, and the trochæ of insects) ; they, so to say, drink in air in order to absorb the oxygen. The oxygen of the air (or dissolved in water) passes through the membranes of the respiratory organs into the blood, is retained in it by the blood corpuscles, is transmitted by their means to all parts of the body, aids their transformations, bringing

1 As regards the interior of the earth, it probably contains far less oxygen compounds than the surface, judging by the accumulated evidences of the earth's origin, of meteorites, of the earth's density, \&c., as set forth in the fourth chapter of my work on the 'Naphtha Industry,' 1877, in speaking of the origin of naphtha. 
about chemical processes in them, and chiefly extracting carbon from them in the form of carbonic anhydride, the greater part of which passes into the blood, is dissolved by it, and is thrown off by the lungs during the absorption of the oxygen. Thus, in the process of respiration carbonic anhydride (and water) is given off, and the oxygen of the air absorbed, by which means the blood is changed from a dark-red venous to a bright-red arterial blood. The cessation of this process causes leath, because then all those chemical processes, and the consequent heat and work which the oxygen introduced into the system brought about, ceases. For this reason suffocation and death ensue in a vacuum, or in a gas which does not contain free oxygen (which does not support combustion). If an animal be placed in an atmosphere of free oxygen, then at first its movements are very active and a general invigoration is remarked, but a reaction soon sets in, and perhaps death may ensue. The oxygen of the air, when it enters the lungs, is diluted with four volumes of nitrogen, which is not absorbed into the system, and therefore the blood absorbs but a small quantity of oxygen from the air, whilst in an atmosphere of pure oxygen a large quantity of oxygen would be absorbed, which would produce a very rapid change of all parts of the organism, and destroy it. From what has been said, it will be understood that oxygen may be employed in respiration, at, least for a limited time, when the respiratory organs suffer under certain forms of suffocation and impediment to breathing. ${ }^{2}$

The combustion of organic substances-that is, substances which make up the composition of plants and animals-proceeds in the same manner as the combustion of many inorganic substances, such as sulphur, phosphorus, iron, \&c., from the combination of these substances with oxygen, as was described in the Introduction. The decomposition, rotting, and similar transformations of substances, which

2 It is evident that the partial pressure (see Chap.II.) acts in respiration. The researches of Paul Bert showed this with particular clearness. Under a pressure of one-fifth of an atmosphere consisting of oxygen only, animals and human beings remain under the ordinary conditions of the partial pressure of oxygen, but organisms cannot support air rarefied to onefifth, for then the partial pressure of the oxygen falls to one-twenty-fifth of an atmosphere. Even under a pressure of one-third of an atmosphere the regular life of human beings is impossible, by reason of the impossibility of respiration (of the decrease of solubility of oxygen in the blood), owing to the small partial pressure of the oxygen, and not from the mechanical effect of the decrease of pressure. Paul Bert illustrated all this by many experiments, some of which he conducted on himself. This explains, among other things, the discomfort felt in the ascent of high mountrins or in balloons when the height reached exceeds eight kilometres, and at pressures below $250 \mathrm{~mm}$. (Chap. II. note 23). It is evident that an artificial atmosphere has to be employed in the ascent to great heights, just as in submarine work. The cure by compressed and rarefied air which is practised in certain illnesses is based partly on the mechanical action of the change of pressure, and partly on the alteration in the partial pressure of the respired oxygen. 
proceed around us, are also very often dependent on the action of the oxygen of the air, and also reduce it from a free to a combined state. The majority of the compounds of oxygen are, like water, very stable, and do not give up their oxygen under the ordinary conditions of nature. As these processes are taking place everywhere, therefore the amount of free oxygen in the atmosphere should decrease, and this decrease should proceed somewhat rapidly. This is, in fact, observed where combustion or respiration proceeds in a closed space. Animals suffocate in a closed space because in consuming the oxygen the air remains unfit for respiration. In the same manner combustion, in time, ceases in a closed space, which may be proved by a very simple experiment. An ignited substance - for instance a piece of burning sulphur-has only to be placed in a glass flask, which is then closed with a stout cork to prevent the access of the external air ; combustion will proceed for a certain time, so long as the flask contains any free oxygen, but it will cease, although there still remain unburnt sulphur, when all the oxygen of the enclosed air has combined with the sulphur. From what has been said, it is evident that regularity of combustion or respiration requires a constant renewal of air - that is, that the burning substance or respiring animal should have access to a fresh supply of oxygen. This is attained in human habitations by having many windows, outlets, and ventilators, and by the current of air produced by fires and stoves. As regards the air over the entire earth's surface, its amount of oxygen hardly decreases, because in nature there is a process going on which renews the supply of free oxygen. Plants, or rather their leaves, during daytime ${ }^{3}$ - that is, under the intluence of light-evolve free oxygen. Thus the loss of oxygen which occurs in consequence of the respiration of animals and of combustion is made good by plants. If a leaf be placed in a bell jar containing water, and carbonic anhydride (because this gas is absorbed and oxygen evolved from it by plants) be passed into the bell, and the whole apparatus be placed in sunlight, then oxygen will accumulate in the bell jar. This experiment was first made by Priestley at the end of the last century. Thus the life of plants on the earth not only serves for the formation of food for animals, but also for keeping up a constant percentage of oxygen in the atmosphere. In the long period of the life of the earth that equilibrium has been attained between the processes ab-

3 At night, without the action of light, without the absorption of that energy which is required for the decomposition of carbonic anhydride into free oxygen and carbon, which is retained by the plants, they breathe like animals, absorbing oxygen and evolving carbonic anhydride. This process also goes on side by side with the reverse process in daytime, but then it is far feebler than that which gives oxygen. This obserration is a necessary consequence of an aggregate of data referring to the physiological processes of plants. 
sorbing and envolving oxygen, by which a definite quantity of free oxygen is preserved in the entire mass of the atmosphere. ${ }^{4}$

Free oxygen may be obtained by one or another method from all the substances in which it occurs. Thus, for instance, the oxygen of many substances may be transferred into water, from which, as we have already seen, oxygen may be obtained.5 We will tirst consider the methods of extracting oxygen from air as being a substance everywhere distributed. The separation of oxygen from it is, however, hampered by many difficulties.

From air, which contains a mixture of oxygen and nitrogen, the nitrogen alone cannot be removed, because it has no inclination to combine directly or readily with any substance; and although it does combine with certain substances (boron, titanium), these substances combine simultaneously with the oxygen of the atmosphere. ${ }^{6}$ However,

4 The earth's surface is equal to about 510 million square kilometres, and the mass of the air (at a pressure of $760 \mathrm{~mm}$.) on each kilometre of surface is about $10 \frac{1}{3}$ thousand millions of kilograms, or about $10 \frac{1}{3}$ million tons; therefore the whole weight of the atmosphere is about 5100 million million $\left(=51 \times 10^{11}\right)$ tons. Consequently there are about $2 \times 10^{13}$ tons of free oxygen in the earth's atmosphere. The innumerable series of processes which absorb a portion of this oxygen are compensated for by the plant processes. Count. ing that 100 million tons of vegetable matter, containing 40 p.c. of carbon, formed from carbonic acid, are produced (and the same process proceeds in water) per year on the 100 million square kilometres of dry land (ten tons of roots, leaves, stems, \&c. per hectare, or $\frac{1}{100}$ of a square kilometre), we find that the plant life of the dry land gives about 100,000 tons of oxygen, which is an insignificant fraction of the entire mass of the oxygen of the air.

5 The extraction of oxygen from water may evidently be accomplished by two processes : either by the decomposition of water into its constituent parts by the action of a galvanic current (Chap. II.), or by means of the removal of the hydrogen from water. But, as we have seen and already know, hydrogen enters into direct combination with very few substances, and then only under special circumstances; whilst oxygen, as we shall soon learn, combines with nearly all substances. Only gaseous chlorine (and especially, fluorine) is capable of decomposing water, taking up the hydrogen from it, without combining with the oxygen. Chlorine is soluble in water, and if an aqueous solution of chlorine, so-called chlorine water, be poured into a flask, and this flask be inverted in a basin containing the same chlorine water, then we shall have an apparatus by means of which oxygen may be extracted from water. At the ordinary temperature and in the dark, chlorine does not act on water, or only acts very feebly; but under the action of direct sunlight chlorine decomposes water, with the evolution of oxygen. The chlorine then combines with the hydrogen, and gives hydrochloric acid, which dissolves in the water, and therefore free oxygen only will be separated from the liquid and it will only contain a small quantity of chlorine in admixture, which ean be easily removed by passing the gas through a solution of caustic potash, which retains the chlorine.

6 A difference in the physical properties of both gases cannot be here taken advantage of, because they are very similar in this respect. Thus the density of oxygen is 16, and of nitrogen 14 times greater than the density of hydrogen, and therefore porous vessels cannot be here employed-the difference between the times of their passage through a porous surface would be too insignificant.

Graham, however, succeeded in enriching air in oxygen by passing it through india- 
oxygen may be separated from air by causing it to combine with substances which may be easily decomposed by the action of heat, and, in

rubber. This may be done in the following way:-A common india-rubber cushion, $\mathrm{E}$ (Fig. 27), is taken, and its orifice hermetically connected with an air-pump, or, better still, a mercury aspirator (the Sprengel pump is designated by the letters A, c, B). When the aspirator (Chap. II. note 16) pumps out the air, which will be seen by the mercury running out in an almost uninterrupted stream, and from its standing at near the barometric height, then it may be clearly remarked that gas passes through the india-rubber. This is also seen from the fact that bubbles of gas continually pass along with the mercury. A small pressure of air may be constantly kept up in the cushion by pouring mercury into the funnel $\mathrm{A}$, and screwing up the cock $\mathrm{c}$, so that the stream flowing from it be small, and then a portion of the air passing through the indiarubber will be carried along with the mercury. This air may be collected in the cylinder $\mathbf{r}$. Its composition proves to be about 42 volumes of oxygen with 57 volumes of nitrogen, and one volume of carbonic anhydride, whilst ordinary air contains only 21 volumes of oxygen in 100 volumes. A square metre of india-rubber surface (of the usual thickness) passes about 45 c.c. of such air per hour. This experiment clearly shows that indiarubber is permeable to gases. This may, by the way, be observed in common toy balloons filled with coal-gas. They fall after a day or two, not because there are holes in them, but because air penetrates into, and the gas from, their interior,

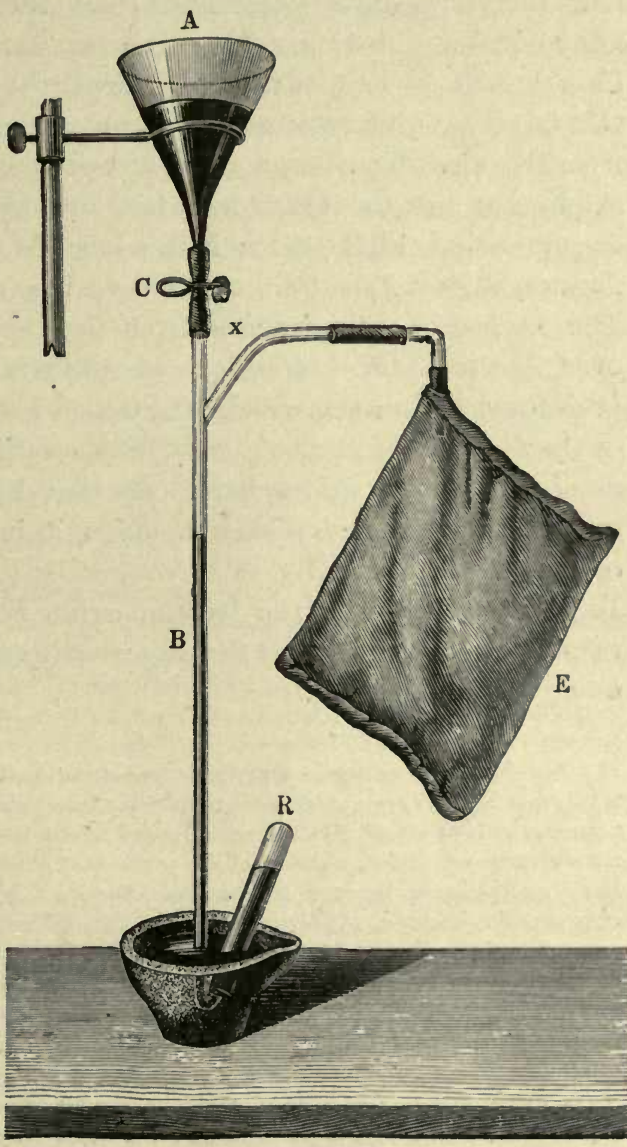

FrG. 27. -Graham's apparatus for the decomposition of air by pumping it through india-rubber. through the surface of the indiarubber of which they are made. The rate of the passage of gases through indiarubber does not, as Mitchell and Graham showed, depend on their densities, and consequently its permeability is not determined by orifices. It more resembles dialysis - that is, the penetration of liquids through colloid surfaces. Equal volumes of gases penetrate through india-rubber in periods of time which are related to each other as follows :-carbonic anhydride, 100 ; hydrogen, 247 ; oxygen, 532 ; marsh gas, 633 ; carbonic oxide, 1220 ; nitrogen, 1358 . Hence nitrogen penetrates more slowly than oxygen, and carbonic anhydride more quickly than other gases. 2.556 volumes of oxygen and 


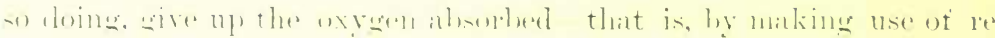

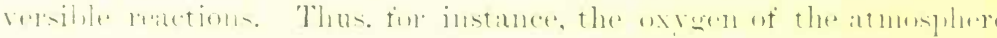

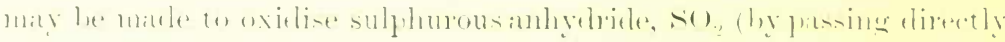

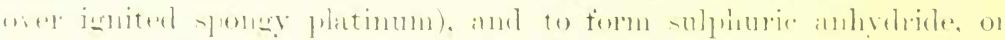

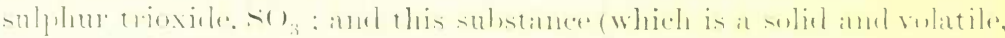

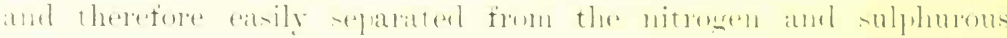

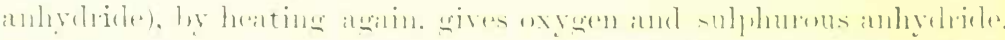

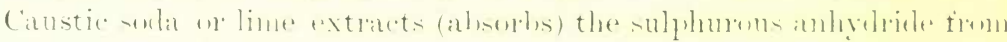
this mixture. whilst the oxyen is not aburbed, and thus it is isolater

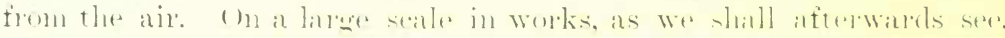

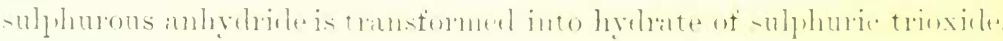

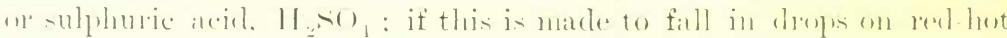

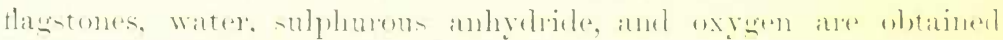

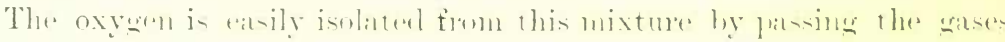

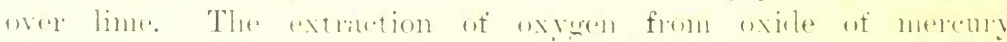

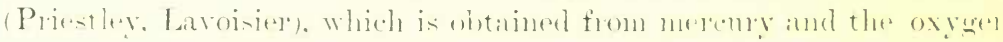

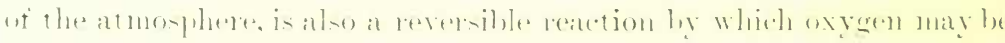

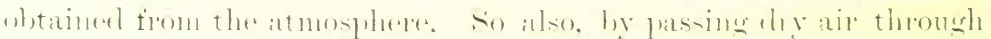

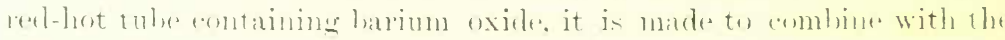

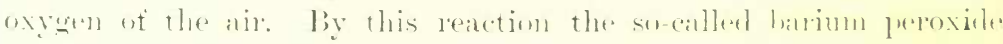

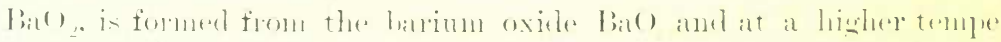

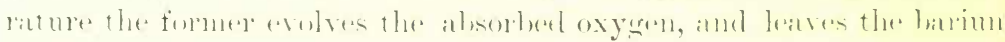
oxithe miginally takent

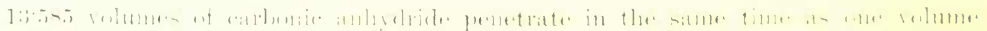

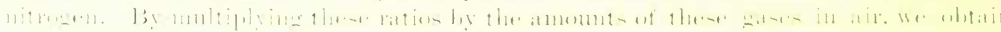

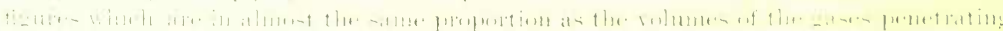

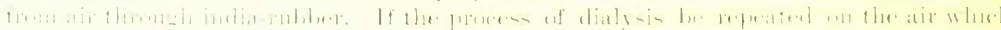

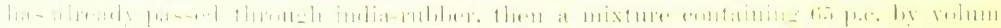

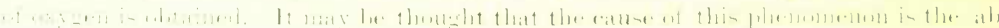
aty

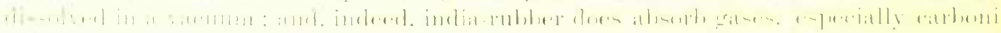

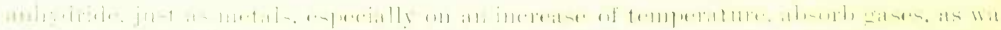

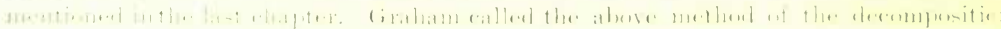

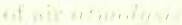

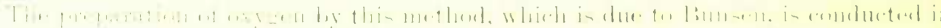

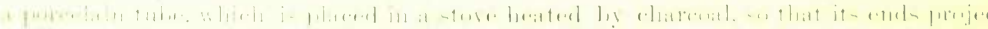

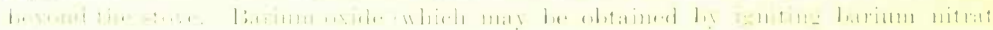

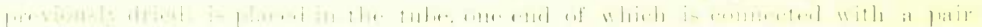

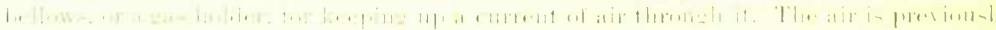

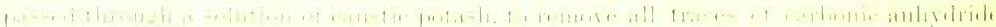

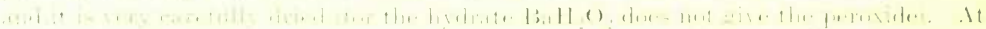

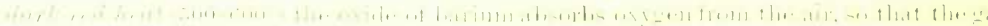

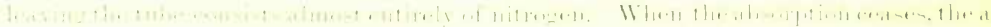

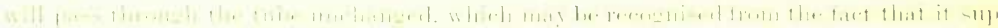

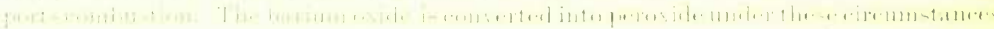

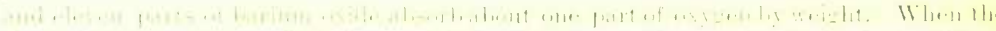
1 A 
Oxygen is evolved with particular ease by a whole series of unstable oxygen compounds, of which we will proceed to take a general survey, remarking that many of these reactions, although not all, belong to the number of reversible reactions $;^{8}$ so that in order to obtain many of these substances (for instance, potassium chlorate) rich in oxygen, recourse must be had to indirect methods (see Introduction), with which we shall become acquainted in the course of this book.

1. The compounds of oxygen with certain metals, and especially with the so-called noble metals - that is, mercury, silver, gold, and platinum - having been once obtained, retain their oxygen at the ordinary temperature, but part with it at a red heat. The compounds are solids, generally amorphous and infusible, and are easily decomposed by heat into the metal and oxygen. We have seen an example of this in speaking of the decomposition of mercury oxide. Priestley, in 1774, obtained pure oxygen for the first time by heating mercury oxide by means of a burning-glass, and clearly showed its difference from air. He showed its characteristic property of supporting combustion ' with remarkable vigour,' and named it dephlogisticated air.

into the other end, and the heat of the stove is increased to a bright-red heat $\left(800^{\circ}\right)$. At this temperature the barium peroxide gives up all that oxygen which it acquired at a darkred heat-i.e., about one part by weight of oxygen is evolved from twelve parts of barium peroxide. After the evolution of the oxygen there remains the barium oxide which was originally taken, so that air may be again passed over it, and thus the preparation of oxygen from one and the same quantity of barium oxide may be repeated many times. Oxygen has been procured one hundred times from one mass of oxide by this method; all the necessary precautions being taken, as regards the temperature of the mass and the removal of moisture and carbonic acid from the air. Unless these precautions be taken, the mass of oxide soon spoils.

As oxygen may become of considerable technical use, from its capacity for giving high temperatures and intense light in the combustion of substances, its preparation directly from air by practical methods forms a problem whose solution many inrestigators continue to work at up to the present day. The most practical method is that of Tessie du Motoy. It is based on the fact that a mass of equal weights of manganese peroxide and caustic soda at an incipient red heat (about $350^{\circ}$ ) absorbs oxygen from air, with the separation of water, according to the equation $\mathrm{MnO}_{2}+2 \mathrm{NaHO}+\mathrm{O}=\mathrm{Na}_{2} \mathrm{MnO}_{4}$ $+\mathrm{H}_{2} \mathrm{O}$. If superheated steam, at a temperature of about $450^{\circ}$, be then passed through the mixture, the manganese peroxide and caustic soda orginally taken are regenerated, and the oxygen held by them is evolved, sccording to the reverse equation $\mathrm{Na}_{2} \mathrm{MnO}_{4}$ $+\mathrm{H}_{2} \mathrm{O}=\mathrm{MnO}_{2}+2 \mathrm{NaHO}+\mathrm{O}$. This mode of preparing oxygen may be repeated for an infinite number of times. The oxygen in combining separates out water, and steam, acting on the resultant substance, evolves oxygen. Hence all that is required for the preparation of oxygen by this method is fuel and the alternate cutting off the supply of air and steam.

8 Even the decomposition of manganese peroxide is reversible, and it may be reobtained from that suboxide (or its salts), which is formed in the evolution of oxygen (Chap. XI. note 6). The compounds of chromic acid containing the trioxide $\mathrm{CrO}_{3}$ in evolving oxygen give chromium oxide, $\mathrm{Cr}_{2} \mathrm{O}_{5}$, but they re-form the salt of chromic acid when heated at a red heat in air with an alkali. 


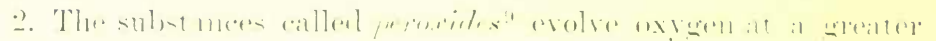

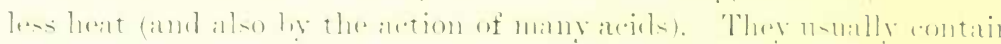

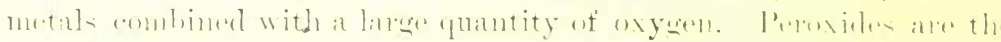

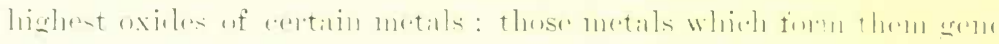

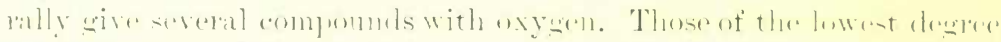

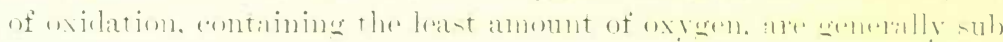

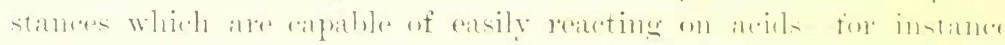

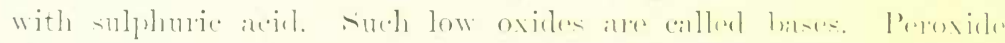

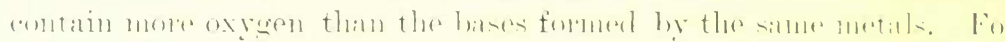

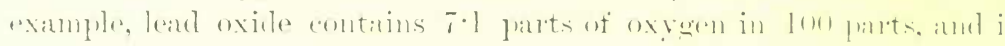

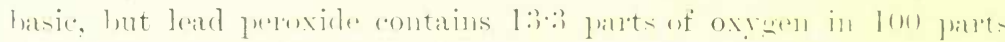

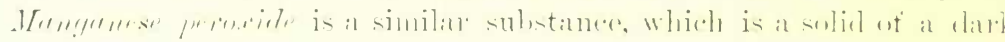

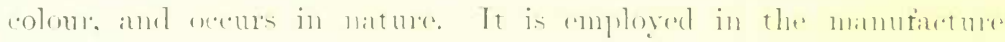

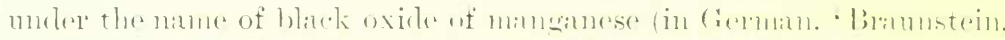

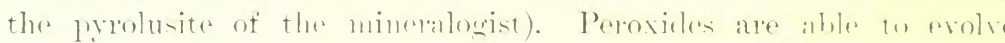

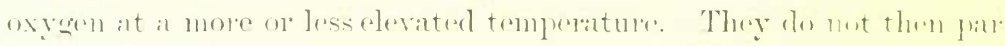
with all their oxygen, hut witle only a portion of it, amel are consereter into a lower oxideor hase. Thus, for eximple, leat peroxide. on heat

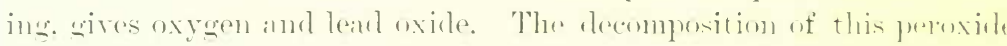

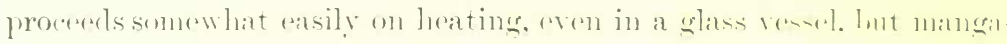

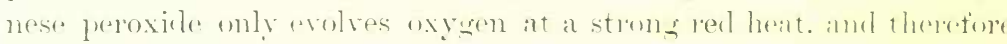

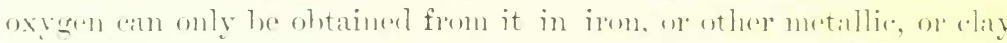

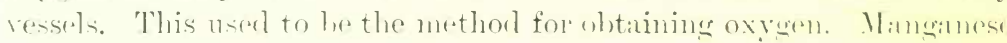

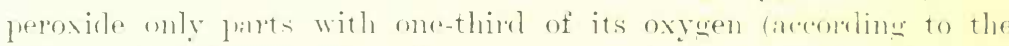

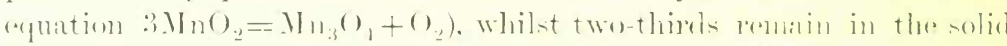

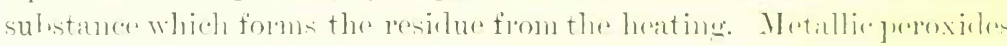

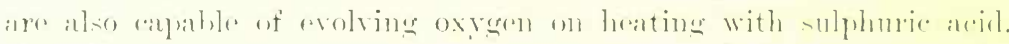

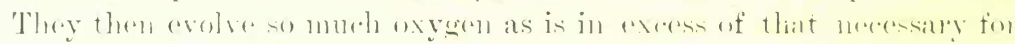
the formation of the halse, the latter leateting on the silphure acill

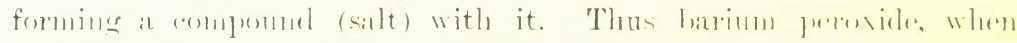

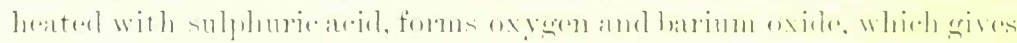

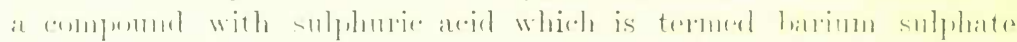

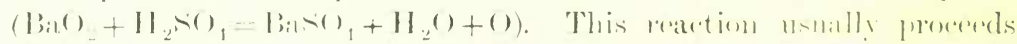

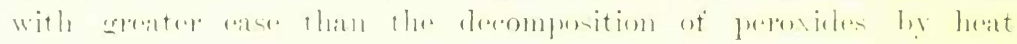

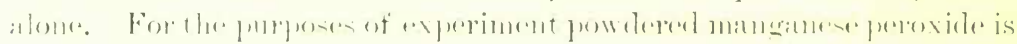

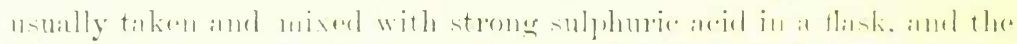

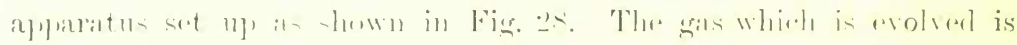

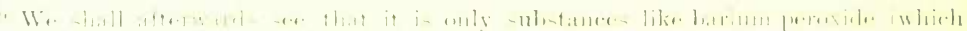

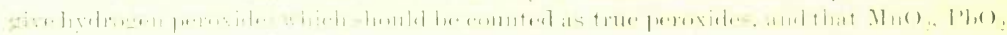

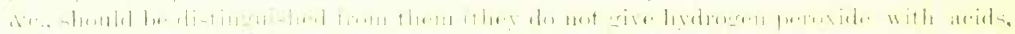

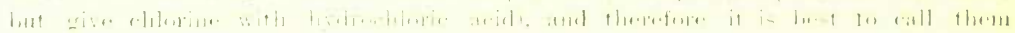
lintiste. 
passed through a Woulfe's bottle containing a solution of caustic potash, to purify it from carbonic anhydride and chlorine, which accompany the evolution of oxygen from commercial manganese peroxide, and the gas is not collected until a thin smouldering taper placed in front of the escape orifice bursts into Hame, which shows that the gas coming off is oxygen. By this method of decomposition of the manganese peroxide by sul-

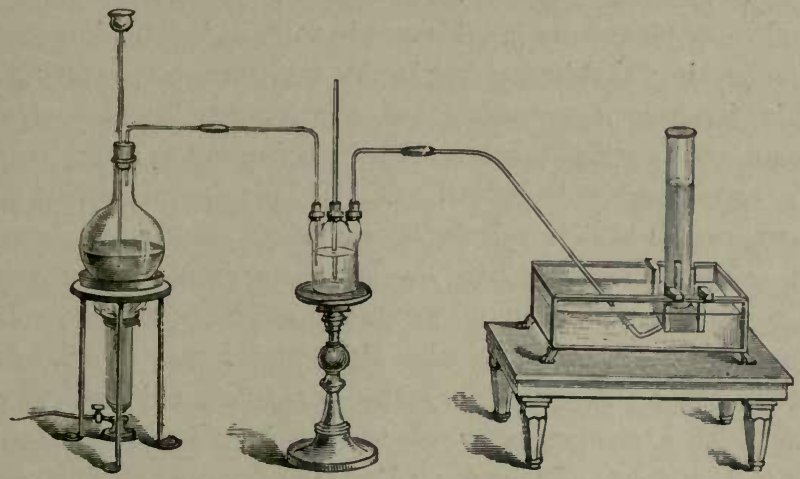

FIG. 28.-Preparation of oxygen from manganese peroxide and sulphuric acid. The gas evolved is passed through a Woulfe's bottle containing caustic potash.

phuric acid there is evolved, not, as in heating, one-third, but one-half of the oxygen contained in the peroxide $\left(\mathrm{MnO}_{2}+\mathrm{H}_{2} \mathrm{SO}_{4}=\mathrm{MnSO}_{4}+\right.$ $\mathrm{H}_{2} \mathrm{O}+\mathrm{O}$ ) - that is, from 50 grams of peroxide about $7 \frac{1}{5}$ grams, or about $5 \frac{1}{2}$ litres, of oxygen, ${ }^{10}$ whilst by heating only about $3 \frac{1}{2}$ litres are obtained. The chemists of Lavoisier's time generally obtained oxygen by heating manganese peroxide. Now there are more convenient methods known.

3. A third source to which recourse may be had for obtaining oxygen is represented in acids and salts containing much oxygen, and which are capable, by parting with a portion or all of their oxygen, of being converted into other compounds (lower products of oxidation) which are more difficultly decomposed. These acids and salts (like peroxides) evolve oxygen either on heating alone, or when heated with some other substance. Sulphuric acid may be taken as an example of an acid which is decomposed by the action of heat alone, ${ }^{11}$ for it breaks up at a red heat into water, sulphurous anhydride,

10 Scheele, in 1785 , discovered the method of obtaining oxygen by treating manganese peroxide with sulphuric acid.

11 All acids rich in oxygen, and especially those whose elements form lower oxides, evolve oxygen either directly at the ordinary temperature (for instance, ferric acid), or on heating (for instance, nitric, manganic, chromic, chloric, and others), or if basic lower oxides are formed from them, by heating with sulphuric acid. Thus the salts 
and oxygen, as was mentioned before. Priestley, in 1772, and Scheele, somewhat later, obtained oxygen by heating nitre to a red heat. The best examples of the formation of oxygen by the heating of salts is given in potassium chlorate, or Berthollet's salt, so called after the French chemist who discovered it. Potassium chlorate is a salt composed of the elements potassium, chlorine, and oxygen, $\mathrm{KClO}_{3}$. It occurs as transparent colourless plates, is soluble in water, especially in hot water, and resembles common table salt in some of its physical properties; it melts on heating, and in melting begins to decompose, evolving oxygen gas. This decomposition ends in all the oxygen being evolved from the potassium chlorate, potassium chloride being left as a residue, according to the equation $\mathrm{KClO}_{3}=\mathrm{KCl}+\mathrm{O}_{3} \cdot{ }^{12}$ This decomposition proceeds at a temperature which allows of its being conducted in a vessel made of glass. However, in decomposing, the molten potassium chlorate swells up and boils, and gradually solidifies, so the evolution of the oxygen is not regular, and the glass vessel may crack. In order to overcome this inconvenience, the potassium chlorate is crushed and mixed with a powder of a substance which is incapable of combining with the oxygen evolved, and which is a good conductor of heat. Usually it is mixed with manganese peroxide. ${ }^{13}$ The decomposition of the potassium chlorate is then considerably facilitated, and proceeds at a lower temperature (because the entire mass is then better heated, both externally and internally), without swelling up, and is therefore more convenient than the decomposition of the salt alone. This method for the preparation of oxygen is very convenient; it is generally employed when a small quantity of oxygen is required. Further, potassium chlorate is easily obtained pure, and it evolves much oxygen. 100 grams of the salt give as much as 39 grains, or 30 litres, of oxygen. This method is so simple and easy, ${ }^{14}$ that a course of practical chemistry

of chromic acid (for instance, potassium dichromate, $\mathrm{K}_{2} \mathrm{Cr}_{2} \mathrm{O}_{7}$ ) give oxygen with sulphuric acid ; first potassium sulphate, $\mathrm{K}_{2} \mathrm{SO}_{4}$, is formed, and then the chromic acid set free gives a sulphuric acid salt of the lower oxide, $\mathrm{Cr}_{2} \mathrm{O}_{3}$.

12 This reaction is not reversible, and is exothermal-that is, it does not absorb heat, but, on the contrary, evolves 9713 calories per molecular weight $\mathrm{KClO}_{3}$, equal to 122 parts of salt (according to the determination of Thomsen, who burnt hydrogen in a calorimeter either alone or with a definite quantity of potassium chlorate mixed with oxide of iron). It does not proceed at once, but first forms perchlorate, $\mathrm{KClO}_{4}$ (see Chlorine and Potassium). It is to be remarked that potassium chlcride melts at $738^{\circ}$, potassium chlorate at $372^{\circ}$, and potassium perchlorate at $610^{\circ}$.

${ }_{15}$ The peroxide does not evolve oxygen in this case. It may be replaced by many oxides -for instance, by oxide of iron. It is necessary to take the precaution that no combustible substances (such as bits of paper, splinters, sulphur, \&c.) fall into the mixture, as they might cause an explosion.

14 The decomposition of a mixture of melted and well-crushed potassium chlorate 
is often commenced by the preparation of oxygen by this method, and of hydrogen by the aid of zinc and sulphuric acid, all the more as these gases enable many interesting and striking experiments to be made. ${ }^{15}$

A solution of bleaching pouder, which contains calcium hypochlorite, $\mathrm{CaCl}_{2} \mathrm{O}_{2}$, evolves oxygen when gently heated with the addition of a small quantity of certain oxides - for instance, cobalt oxide, which in this case acts by contact (see Introduction). Of itself, a solution of bleaching powder does not evolve oxygen when heated, but it oxidises the cobalt oxide to a higher degree of oxidation ; this higher oxide of cobalt in contact with the bleaching powder, decomposes into oxygen and lower oxidation products, and the resultant lower oxide of cobalt with bleaching powder again gives the higher oxide, which again gives up its oxygen, and so on. ${ }^{16}$ The calcium hypochlorite is here decomposed according to the equation $\mathrm{CaCl}_{2} \mathrm{O}_{2}=$ $\mathrm{CaCl}_{2}+\mathrm{O}_{2}$. In this manner a small quantity of cobalt oxide ${ }^{17}$ is sufficient for the decomposition of an indefinitely large quantity of bleaching powder.

with powdered manganese peroxide proceeds at so low a temperature (the salt does not melt) that it may be effected in an ordinary glass flask. As the reaction is exothermal, the decomposition of potassium chlorate with the formation of oxygen may probably be accomplished, under certain conditions (for example under contact action), at very low temperatures. Substances mixed with the potassium chlorate probably act partially in this manner.

15 Many other salts evolve oxygen by heat, like potassium chlorate, but they only part with it either at a very strong heat (for instance, common nitre) or else are unsuited for use on account of their cost (for instance, potassium manganate), or evolve impure oxygen at a high temperature (for instance, zinc sulphate at a red heat gives a mixture of sulphurous anhydride and oxygen), and are not therefore used in practice.

16 Such is, at present, the only possible method of explaining the phenomenon of contact action. In many cases, as here, it is supported by observations based on facts. Thus, for instance, it is known, as regards oxygen, that often two substances rich in oxygen retain it so long as they are separate, but directly they come into contact free oxygen is evolved from both of them. Thus, an aqueous solution of hydrogen peroxide (containing twice as much oxygen as water) acts in this manner on silver oxide (containing silver and oxygen). This reaction takes place at the ordinary temperature, and the oxygen is evolved from both compounds. To this class of phenomena may be also referred the fact that a mixture of barium peroxide and potassium manganate with water and sulphuric acid evolves oxygen at the ordinary temperature. It would seem that the essence of phenomena of this kind is entirely and purely a property of contact; the distribution of the atoms is changed by contact, and if the equilibrium be unstable it is destroyed. This is especially clear for substances which change exothermally - that is, for those reactions which are accompanied by an evolution of heat. The decomposition $\mathrm{CaCl}_{2} \mathrm{O}_{2}=\mathrm{CaCl}_{2}+\mathrm{O}_{2}$ belongs to this class (like the decomposition of potassium chlorate).

17 Generally a solution of bleaching powder is alkaline (contains free lime), and, there-

VOL. I. 


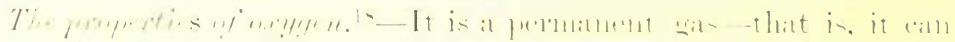

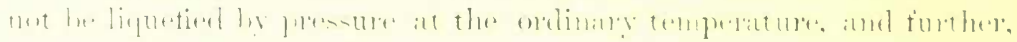

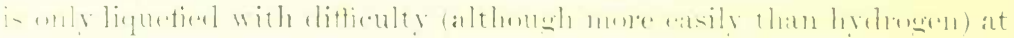

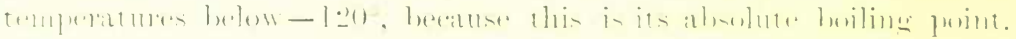

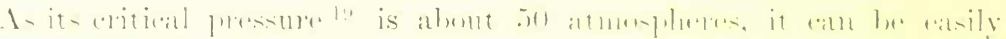

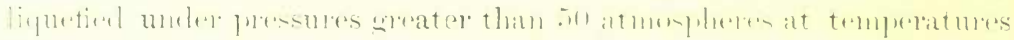

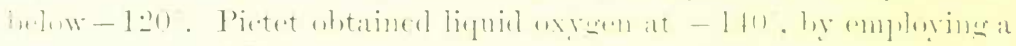

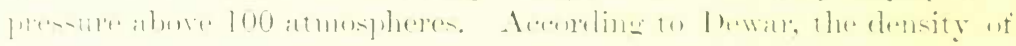

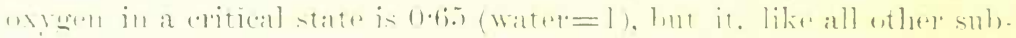

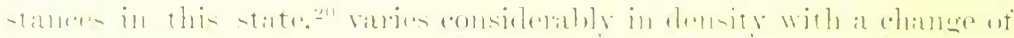

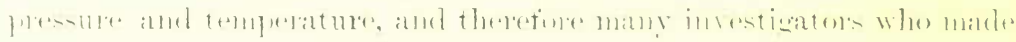

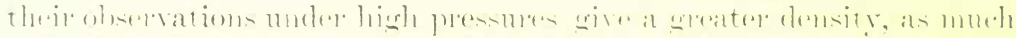

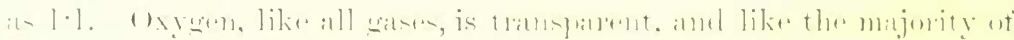

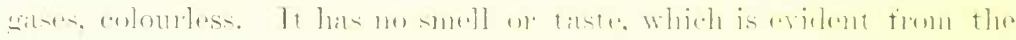

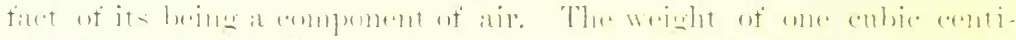

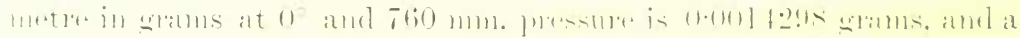

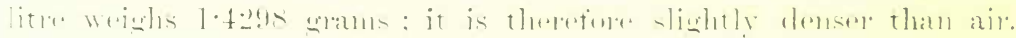

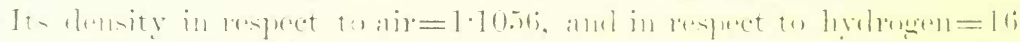

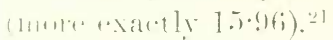

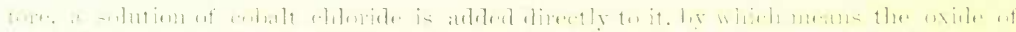

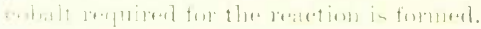

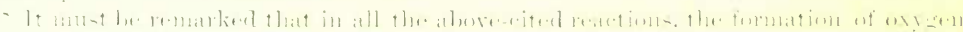

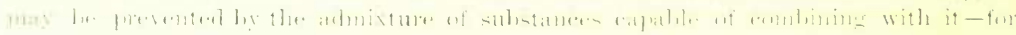

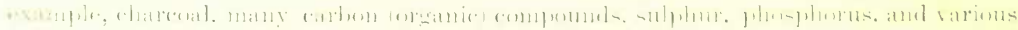

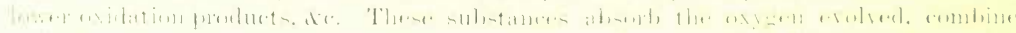

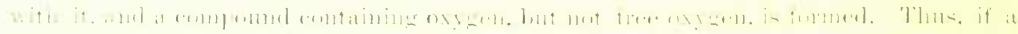

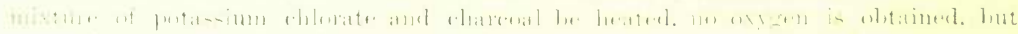

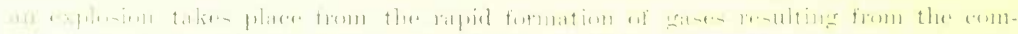

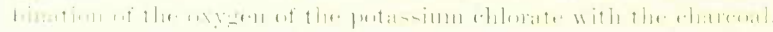

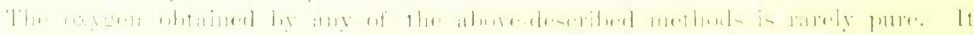

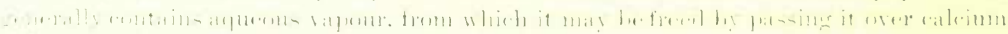

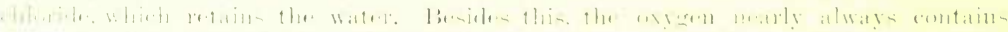

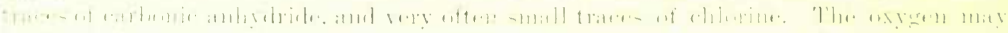

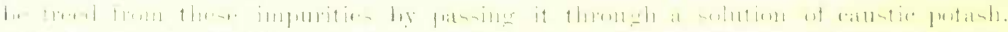

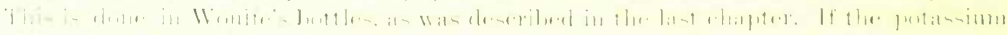

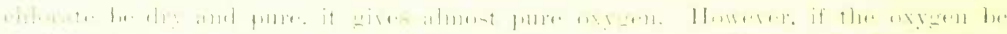

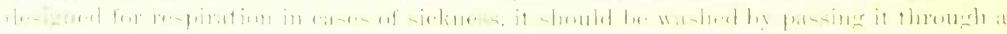

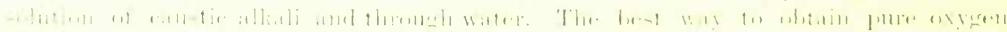

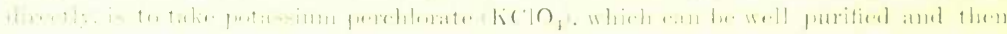

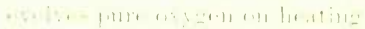

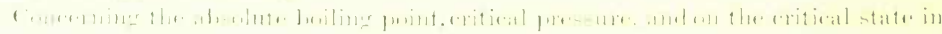

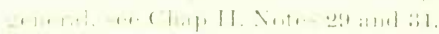

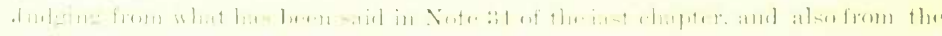

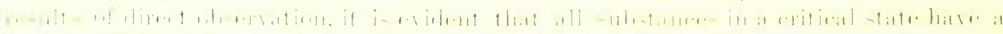

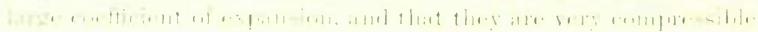

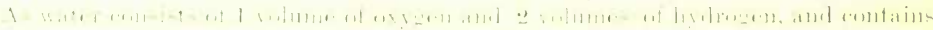

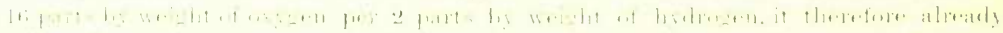

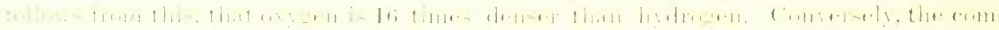


In its chemical properties oxygen is remarkable from the fact that it very easily - and, in a chemical sense, vigorously - reacts on a number of substances, forming oxygen compounds. However, only a few substances and mixtures of substances (for example, phosphorus, copper with ammonia, decomposing organic matter, aldehyde, pyrogallol with an alkali, \&c.) combine directly with oxygen at the ordinary temperature, whilst many substances easily combine with oxygen at a red heat, and often this combination presents a rapid chemical reaction accompanied by the evolution of a large quantity of heat. Every reaction which takes place rapidly, if it be accompanied by so great an evolution of heat as to produce incandescence, is termed combustion. Thus combustion ensues when many metals are plunged into chlorine, or oxide of sodium or barium into carbonic anhydride, or when a spark falls on gunpowder. A great many substances are combustible in oxygen, and, owing to its presence, in air also. In order to start combustion it is generally necessary ${ }^{22}$ that the combustible substance should be brought to a state of incandescence. When once startedi.e. when once the incandescent portion of the substance begins to combine with oxygen - then combustion will proceed uninterruptedly until either all the combustible substance or all the oxygen is consumed. The continuation of the process does not require the aid of fresh external heat, because sufficient heat ${ }^{23}$ is evolved to raise the temperature of the remaining parts of the combustible substance to the required

position of water by weight may be deduced from the densities of hydrogen and oxygen, and the volumetric composition of water. This kind of mutual and opposite correction is a method which strengthens the practical data of the exact sciences, whose conclusions require, above all things, the greatest possible exactitude and variety of corrections.

It must be observed that the specific heat of oxygen at constant pressure is 0.2175 , consequently it is to the specific heat of hydrogen $\left(3^{\cdot 409)}\right.$ as 1 is to $\mathbf{1 5}^{\cdot 6}$. Hence, the specific heats are inversely proportional to the weights of equal volumes. This signifies that equal volumes of both gases have (nearly) equal specific heats-that is, they require an equal quantity of heat for raising their temperature by $1^{\circ}$. We shall afterwards consider the specific heat of different substances more fully, and we will not, therefore, linger over it at present.

Oxygen, like the majority of difficulty-liquefiable gases, is but slightly soluble in water and other liquids. At the ordinary temperature, 100 volumes of water dissolve about 3 volumes of oxygen, or more exactly, at $0^{\circ} 4 \cdot 1$ vols., at $10^{\circ} 3.3$, and at $20^{\circ} 3.0$ (measuring the volumes at the same temperature as the water). From this it is evident that water standing in air must absorb-i.e., dissolve-oxygen. This oxygen serves for the respiration of fishes. Fishes cannot exist in boiled water, because it does not contain the oxygen necessary for their respiration (see Chap. I.).

22 Certain substances (with which we shall afterwards become acquainted), however, inflame of themselves in air; for example, impure phosphuretted hydrogen, silicon, hydride, zinc ethyl, and pyrophorus (very finely divided iron, \&c.).

25 If so little heat is evolved that the adjacent parts are not heated to the temperature of combustion, then combustion will cease. 
degree. Examples of this are familiar to all from every-day experience. Combustion proceeds in oxygen with greater rapidity, and is accompanied by a more powerful incandescence, than in ordinary air. This may be demonstrated by a number of very convincing experiments. If a piece of charcoal, attached to a wire and previously brought to redheat, be plunged into a flask full of oxygen, it rapidly burns at a white heat-i.e., it combines with the oxygen, forming a gaseous product of combustion called carbonic anhydride, or carbonic acid gas. This is the same gas that is evolved in the act of respiration, for charcoal is one of the substances which is obtained by the decomposition of all organic substances which contain it, and in the process of respiration part of the constituents of the body, so to speak, slowly

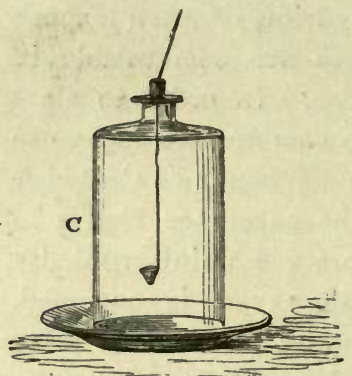

FIG. 29.-Mode of burning sulphur, phosphorus, sodium, \&c., in oxygen. burn. If a piece of burning sulphur be laid on a small cup attached to a wire and be placed in a flask full of oxygen, then the sulphur, which burns in air with a very feeble flame, burns in the oxygen with a violet flame, which, although pale, is much larger than in air. If the sulphur be exchanged for a piece of phosphorus, ${ }^{24}$ then, unless the phosphorus be heated, it combines very slowly with the oxygen; but, if heated, although on only one spot, it burns with a very brilliant white flame, which is unbearable to the sight. In order to heat the phosphorus inside the flask, the most simple way is to bring a red-hot wire into contact with it. Before the charcoal can burn, it must be brought to a state of incandescence. Sulphur also will not burn under $100^{\circ}$, whilst phosphorus inflames at $40^{\circ}$. Phosphorus which has been already lighted in air cannot so well be introduced into the flask, because it burns very rapidly and with a large flame in air. If a small lump of metallic sodium be put in a small cup made of lime, ${ }^{25}$ melted, and inflamed, ${ }^{26}$ then it burns very feebly in air. But if burning sodium be immersed in oxygen, the

24 The phosphorus must be dry; it is usually kept in water, as it oxidises in air. It should be cut under water, as otherwise the freshly-cut surface oxidises. It must be dried carefully and quickly by wrapping it in blotting-paper. If damp, it splutters in burning. A small piece should be taken, as otherwise the iron spoon-will melt. In this and the other experiments on combustion, water should be poured over the bottom of the vessel containing the oxygen, to prevent it from cracking. The cork closing the vessel should not fit tightly, otherwise it may fly off with the spoon and burning substance, owing to the expansion due to the heat of the combustion.

25 An iron cup will melt with sodium in oxygen.

26 In order to rapidly heat the lime crucible with the sodium, they are heated in the flame of a blow-pipe described in Chap. VIII. 
combustion is invigorated and is accompanied by a brighter yellow flame. Metallic magnesium, which burns brightly in air, continues to burn with still greater vigour in oxygen, forming a white powder, which is a compound of magnesium with oxygen (magnesium oxide; magnesia). A strip of iron or steel does not burn in air, but an iron wire or steel spring may be easily burnt in oxygen. A much larger piece of iron might naturally be burnt if it only were convenient to heat it to the required degree. ${ }^{27}$ The combustion of steel or iron in oxygen is not accompanied by a flame, but sparks of oxide fly in all directions from the burning portions of the iron. ${ }^{28}$

In order to demonstrate by experiment the combustion of hydrogen in oxygen, a gasconducting tube, bent so as to form a convenient jet, is led from the vessel evolving hydrogen. The hydrogen is first set light

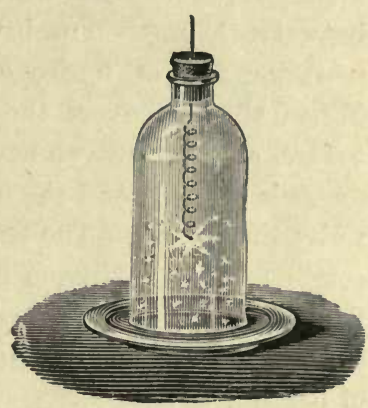

Fig. 30.-Mode of burning a steel spring in oxygen. to in air, and then the gas-conducting tube is let down into a flask containing oxygen. The combustion in oxygen will be similar to that in air ; the flame remains pale, notwithstanding the fact that its temperature rises considerably. It is instructive to remark that oxygen may burn in hydrogen, just as hydrogen in oxygen. In order to show the combustion of oxygen in hydrogen, a tube bent vertically upwards and ending in a fine orifice is attached to the stop-cock of a gas holder full of oxygen. Two wires, placed at such a distance from

27 In order to burn a watch spring, a piece of tinder (or paper soaked in a solution of nitre, and dried) is attached to one end. The tinder is lighted, and the spring is then plunged into the oxygen. The burning tinder heats the end of the spring, the heated part burns, and in so doing heats the further portions of the spring, which thus entirely burns if enough oxygen is present.

28 The sparks of rust are produced by reason of the volume of the oxide of iron being nearly twice that of the volume of the iron, and as the heat evolved is not sufficient to entirely melt the oxide or the iron, the particles must be torn off and fly about. Similar sparks are formed in the combustion of iron, in other cases also. We saw the combustion of iron filings in the Introduction. In the welding of iron small iron splinters fly off in all directions and burn in the air, as is seen from the fact that whilst flying through the air they remain red hot, and also because, on cooling, they are seen to be no longer iron, but a compound of it with oxygen. The same thing takes place when the hammer of a gun strikes against the flint. Small scales of steel are heated by the friction, and glow and burn in the air. The combustion of iron is still better seen by taking it as a very fine powder, such as is obtained by the decomposition of certain of its compounds - for instance, by heating Prussian blue, or by the reduction of its compounds with oxygen by hydrogen; when this fine powder is strewn in air, it burns by itself, even without being previously heated (it forms a pyrophorus). This obviously depends on the fact that the powder of iron presents a larger surface of contact with air than an equal weight in a compact form. 


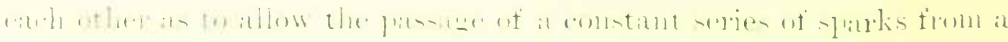

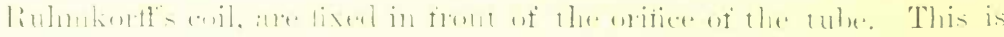

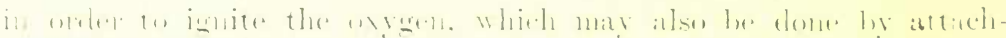

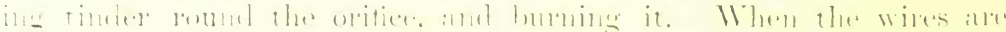

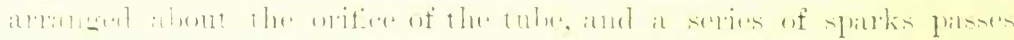

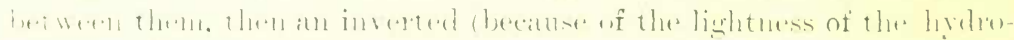

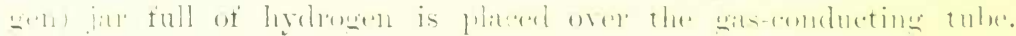

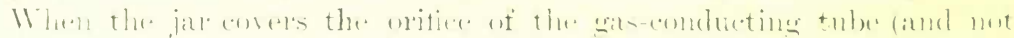

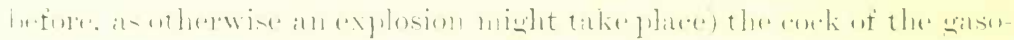

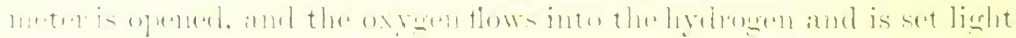

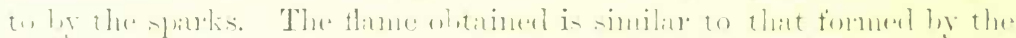

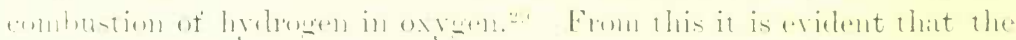

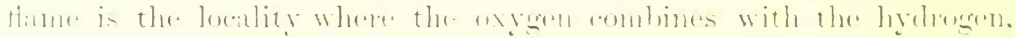

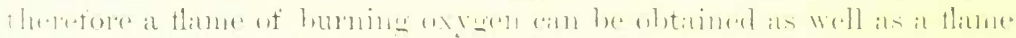

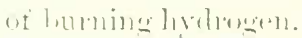

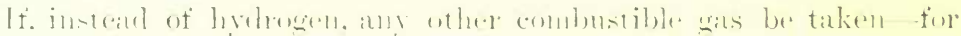

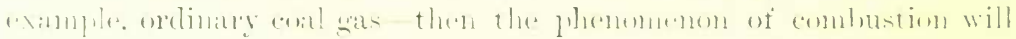

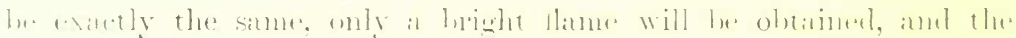

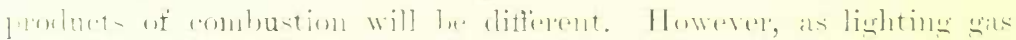
ontatim a

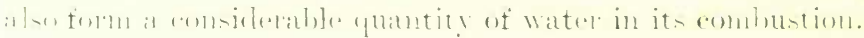

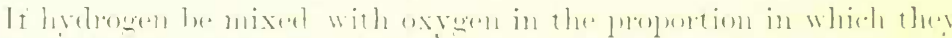

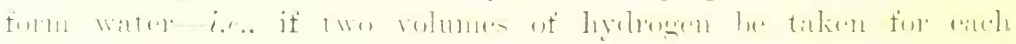

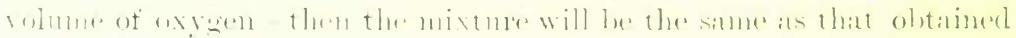

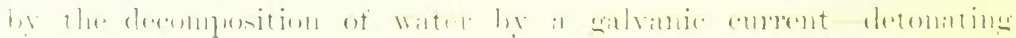
Li:

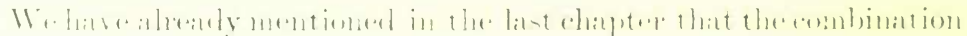

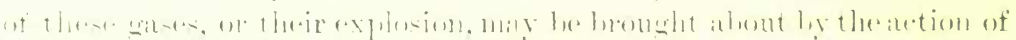

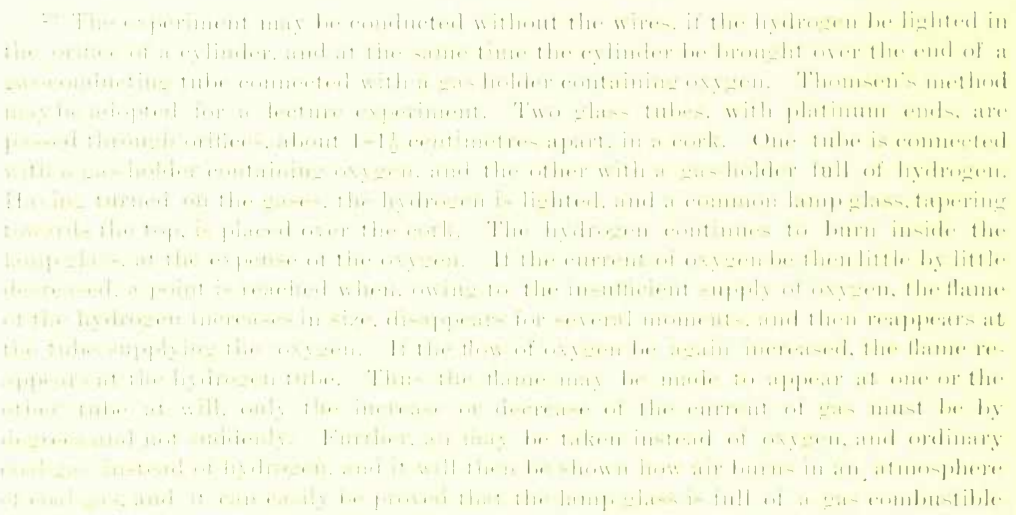


an electric spark, because the spark heats the space through which it passes, and acts consequently in a manner similar to ignition by means of contact with an incandescent or burning substance. In fact, instead of a spark a fine wire simply may be taken, and ån electric current passed through it to bring it to a state of incandescence; in this case there will be no sparks, but the gases will inflame if the wire be fine enough to become red hot by the passage of the current. Cavendish made this experiment on the ignition of detonating gas, at the end of the last century, in the apparatus shown in fig. 31. Ignition by the aid of the electric spark is convenient, for the reason that it may then be brought about in a closed ressel, and hence chemists still employ this method when it is required to ignite a mixture of oxygen with a combustible gas in a closed vessel. For this purpose they now, especially since Bunsen's time, ${ }^{30}$ employ an eudiometer.

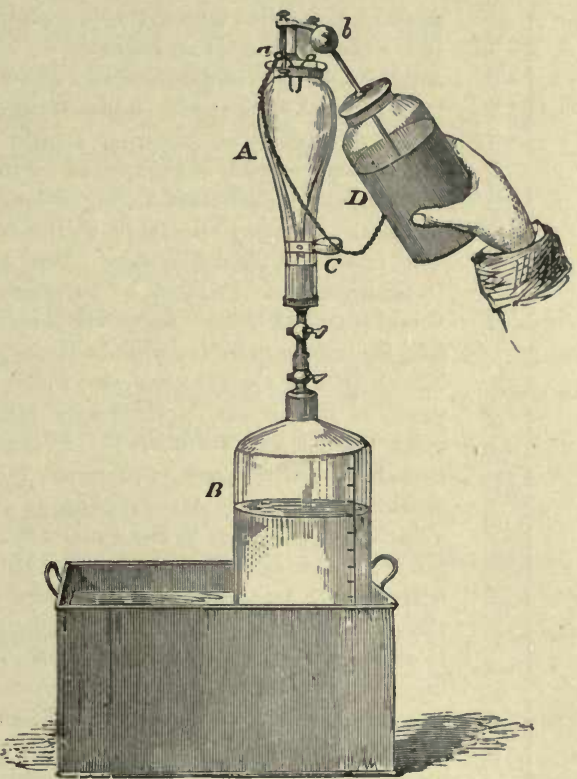

FIG. 31.-Cavendish's apparatus for exploding detonating gas. The bell jar standing in the bath is filled with a mixture of two volumes of hydrogen and one volume of oxygen, and the thick glass vessel $\mathrm{A}$ is then screwed into it. The air is first pumped out of this vessel, so that when the stop-cock $\mathrm{c}$ is opnned, it becomes filled with detonating gas. The stop cock is then re-closed, and the explosion producerl by means of a spark from a Leyden jar. After the explosion has taken place the stop-cock is again opened, and the water rises into the vessel $A$. It consists of a thick glass tube graduated along its length in millimetres (for indicating the height of the mercury column), and calibrated for a definite volume (weight of mercury). Two platinum wires are fused into the upper closed end of the tube, as shown in fig. 32. They must be hermetically sealed into the tube, so that there be no aperture left between them and the glass. ${ }^{31}$

30 Now, a great many other different forms of apparatus, sometimes designed for special purposes, are employed in the laboratory for the investigation of gases. Detailed descriptions of the methods of gas unalysis, and of the apparatus employed, must be looked for in works on analytical and applied chemistry.

31 In order to test this, the eudiometer is filled with mercury, and its open end inverted into mercury. If there be the smallest orifice at the wires, the external air will enter into the cylinder and the mercury will fall, although not rapidly if the orifice be very fine. 


\section{By the aid of the eudiometer we may not only determine the volu. metric composition of water, ${ }^{32}$ and the quantitative contents of oxygen}

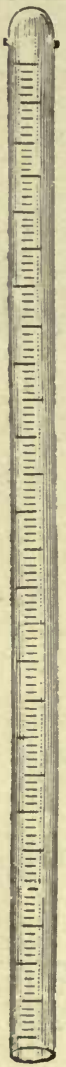

32 The eudiometer is used for determining the composition of combustible gases. A detailed account of gas analysis would be out of place in this work (see Note 30 ), but, as an example, we will give a short description of the determination of the composition of water by the eudiometer.

Pure and dry oxygen is first introduced into the eudiometer. When the eudiometer and the gas in it acquire the temperature of the surrounding atmosphere-which is recognised by the fact of the meniscus of the mercury not altering its position during a long period of time-then the heights at which the mercury stands in the eudiometer and in the bath are observed. The difference (in millimetres) gives the height of the column of mercury in the eudiometer. It must be reduced to the height at which the mercury would stand at $0^{\circ}$ and deducted from the atmospheric pressure, in order to find the pressure under which the oxygen is measured (see Chap. I. Note 29). The height of the mercury also shows the volume of the oxygen. The temperature of the surrounding atmosphere and the height of the barometric column must also be observed, in order to know the temperature of the oxygen and the atmospheric pressure. When the volume of the oxygen has been measured, pure and dry hydrogen is introduced into the endiometer, and the volume of the gases in the eudiometer again measured. They are then exploded. This is done by a Leyden jar, whose outer coating is connected by a chain with one wire, so that a spark passes when the other wire, fused into the eudiometer, is touched by the terminal of the jar. Or else an electrophorus is used, or, better still, a Ruhmkorff's coil, which has the advantage of working equally well in damp or dry air, whilst a Leyden jar or electrical machine does not act in damp weather. Further, it is necessary to close the lower orifice of the eudiometer before the explosion (for this purpose the eudiometer, which is fixed in a stand, is firmly pressed down from above on to a piece of india-rubber placed at the bottom of the bath), as otherwise the mercury and gas would be thrown from the apparatus by the explosion. It must also be remarked that to ensure complete combustion the proportion between the volumes of oxygen and hydrogen must not exceed twelve volumes of hydrogen to one volume of oxygen, or fifteen volumes of oxygen to one volume of hydrogen, because no explosion will take place if one of the gases be in great excess. It is best to take a mixture of one volume of hydrogen with several volumes of oxygen. The combustion will then be complete. It is

FIG. 32. - evident that water is formed, and that the volume (or tension) is diminished, Eurliometer. so that on opening the end of the eudiometer the mercury will rise in it.

But the tension of the aqueous vapour is now added to the tension of the g as remaining after the explosion. This must be taken into account (Chap. I. Note 1 ). If there remain but little gas, the water which is formed will be sufficient for its saturation with aqueous vapour. This may be learnt from the fact that drops of water are visible on the sides of the eudiometer after the mercury has risen in it. If there be none, a certain quantity of water must be introduced into the eudiometer. Then the number of millimetres expressing the pressure of the vapour corresponding with the temperature of the experiment must be subtracted from the atmospheric pressure at which the remaining gas is measured, otherwise the result will be inaccurate.

This is essentially the method of the determination of the composition of water which was made for the first time by Gay-Lussac' and Humboldt with sufficient accuracy. Their determinations led them to the conclusion that water consists of two volumes of hydrogen and one volume of oxygen. Every time they took a greater quantity of oxygen, the gas remaining after the explosion was oxygen. When they took an excess of hydrogen, the remaining gas was hydrogen; and when the oxygen and hydrogen were taken in 
in air, ${ }^{33}$ but also make a number of experiments explaining the phenomenon of combustion.

Thus, for example, it may be demonstrated, by the aid of the eudiometer, that for the ignition of detonating gas a definite temperature is required. If the temperature be below that required, combination will not take place, but if at any spot within the tube it rises to the temperature of inflammation, then combination will ensue at that spot, and evolve enough heat for the ignition of the adjacent portions of the detonating mixture. If to 1 volume of detonating gas there be added 10 volumes of oxygen, or 4 volumes of hydrogen, or 3 volumes of carbonic anhydride, then we shall not obtain an explosion by passing a spark through the diluted mixture. This depends on the fact that the temperature falls with the dilution of the detonating gas by another gas, because the heat evolved by the combination of the small quantity of hydrogen and oxygen brought to incandescence by the spark is not only transmitted to the water proceeding from the combination, but also to the foreign substance mixed with the detonating gas. ${ }^{34}$ The necessity of a definite temperature for the ignition of detonating gas is also seen from the fact that pure detonating gas explodes in the presence of a red-hot iron wire, or of charcoal so feebly incandescent as to be hardly distinguishable by day light, bui with a lower degree of incandescence there is not any explosion. It may also be brought about by rapid compression, when, as is known, heat is evolved.3.5 Experiments made in the eudiometer showed that the ignition of detonating gas takes place at a temperature between $450^{\circ}$ and $500^{\circ} .^{36}$

exactly the above proportion neither one nor the other remained. The composition of water was thus definitely confirmed.

33 Concerning this application of the eudiometer, see the chapter on nitrogen.

34 Thus $\frac{1}{4}$ volume of carbonic oxide, an equal volume of marsh gas, two volumes of hydrogen chloride or of ammonia, and six volumes of nitrogen or twelve volumes of air added to one volume of detonating gas, prevent its explosion.

35 If the compression be brought about slowly, so that the heat evolved succeeds in passing to the surrounding space, then the combination of the oxygen and hydrogen does not take place, even when the mixture is compressed by 150 times; for the gases are not heated. If paper soaked with a solution of platinum (in aqua regia) and sal ammoniac be burnt, then the ash obtained contains very finely-divided platinum, and in this form it is best fitted for setting light to hydrogen and detonating gas. Platinum wire requires to be heated, but platinum in so finely divided, a state as it occurs in this ash inflames hydrogen, even at $-20^{\circ}$. Many other metals, such as palladium, iridium, and gold, act with a slight rise of temperature, like platinum; charcoal, like the majority of finely divided substances, inflames detonating gas at $350^{\circ}$, but mercury, at its boiling point, does not inflame detonating gas. All data of this kind show that the explosion of detonating gas presents one of the many cases of contact phenomena.

36 From the very beginning of the diffusion of the idea of dissociation, it might have been imagined that reversible reactions of combination (the formation of $\mathrm{H}_{2}$ and $\mathrm{O}$ belongs to this number) start at the same temperature as that at which dissociation begins. And so it is in many cases, but not always, as may be seen from the facts (1) that 
The combination of hydrogen with oxygen is accompanied by the evolution of a very considerable amount of heat; according to the determinations of Favre and Silbermann, ${ }^{37} 1$ part by weight of hydrogen in forming water evolves 34462 units of heat. Many of the most recent determinations are very near this figure, so that it may be taken that in the formation of 18 parts of water $\left(\mathrm{H}_{2} \mathrm{O}\right)$ there are evolved 69 major calories, or 69000 units of heat. ${ }^{38}$ If the specific heat

at $450-560^{\circ}$, when detonating gas explodes, the density of aqueous vapour not only does not vary (and it hardly varies at higher temperatures, probably because the amount of the products of dissociation is small), but there are not, as far as is yet known, any traces of dissociation; (2) that under the influence of contact the temperature at which combination takes place falls even to the ordinary temperature, when water and similar compounds naturally are not dissociated and, judging from the data communicated by D. P. Konovaloff (Introduction, Note 39) and others, it is impossible to escape the phenomena of contact; all vessels, whether of metal or glass, show the same influence as spongy platinum although to a much less degree. The phenomena of contact, judging from the mass of the data referring to it, must be especially sensitive in reactions which are powerfully exothermal, and the explosion of detonating gas is of this kind.

37 The amount of heat evolved in the combustion of a known weight (for instance, 1 gram) of a given substance is determined by the rise in temperature of water, to which the whole of the heat evolved in the combustion is transmitted. A calorimeter, for example, that shown in fig. 33, is employed for this purpose. It consists of a thin (in order that it may absorb less heat), polished (that it should transmit a minimum of heat) metallic vessel, surrounded by down (c), or some other bad conductor of heat, and an outer metallic vessel. This is necessary in order that the least possible amount of heat should be lost from the vessels; nevertheless, there is always a certain loss, whose magnitude

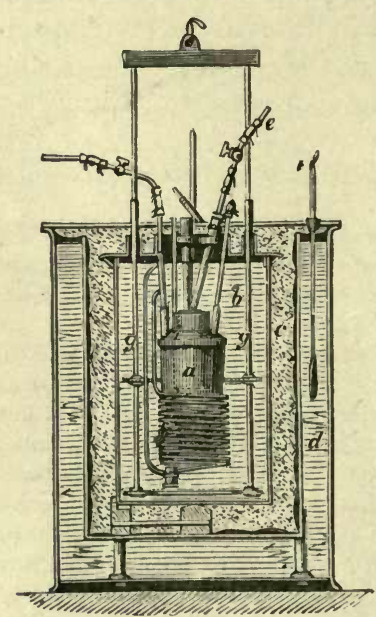
is determined by preliminary experiment (by taking warm water, and determining its fall in temperature after a definite period of time) as a correction for the results of observations. The water to which the heat of the burning substance is transmitted is poured into the vessel. The stirrer $g$ allows of all the layers of water being brought to an equal temperature, and the thermometer serves for the determination of the temperature of the water. The heat evolved passes, naturally, not to the water only, but to all the parts of the apparatus. The quantity of water corresponding with the whole amount of those objects (the vessels, tubes, \&c.) to which the heat is transmitted is previously determined, and in this manner another most important correction is made in the calorimetric determinations. The combustion itself is carried on in the vessel $a$. The ignited substance is introduced through the tube at the top, which closes tightly. In fig. 33 the apparatus is arranged for the combustion of a gas, introduced by a tube. The oxygen required for the combustion is led into $a$ by the tube $e$, and the pro-

FIG. 33.-Favre and Silbermann's calo- ducts of combustion either remain in the vessel $a$ (if rimeter for determining the heat liquid or solid), or escape by the tube $f$ into an appara-
evolved in combustion.

tus in which their quantity and properties can easily

be determined. Thus the heat evolved in combustion passes to the walls of the vessel $a$, and to the gases which are furmed in it, and these transmit it to the water of the calorimeter.

38 This quantity of heat corresponds with the formation of liquid water at the ordinary 


\section{of aqueous vapour $(0.48)$ remained constant from the ordinary tempera- ture to that at which the combustion of detonating gas takes place (but}

temperature from detonating gas at the same temperature. If the water be as vapour the heat evolved $=58$ major calories; if as ice $=70.4$ major calories. A portion of this heat is due to the fact that 1 vol. of hydrogen and $\frac{1}{2}$ vol. of oxygen give 1 vol. of aqueous vapour - that is to say, contraction ensues - and this evolves heat. This quantity of heat may be calculated, but it cannot be said how much is expended in the tearing apart of the atoms of oxygen from each other, and therefore, strictly speaking, we do not know the quantity of heat which is evolved in the combination of hydrogen with oxygen; although the number of units of heat evolved in the combustion of detonating gas is accurately known.

The construction of the calorimeter and even the method of determination vary considerably in different cases. The greatest number of calorimetric determinations were made by Berthelot and Thomsen. They are given in their works Essai de mécanique chimique fondée sur la thermochimie, by M. Berthelot, 1879. (2 vols.), and thermochemische Untersuchungen, by J. Thomsen, 1886 (4 vols.). The student must refer to works on theoretical and physical chemistry for a description of the elements and methods of thermochemistry, into the details of which it is impossible to enter in this work, all the more so because, as las been shown of late, both the theoretical side of this subject and its practical methods are still in an elementary state of development, and must be subjected to improvement in many aspects before thermochemical study can be of that enormous utility to chemical mechanics which was expected from it at the time of the appearance of the first researches in its province. One of the originators of thermochemistry was a member of the St. Petersburg Academy of Sciences, Hess. Since 1870 a mass of researches have appeared in this province of chemistry, especially in France and Germany, after the leading works of the French Academician, Berthelot, and the Copenhagen professor, Thomsen. Among Russians, Beketoff, Luginin, Cheltzoff, Chroustchoff, and others are known by their thermo-chemical researches. The present epoch of thermochemistry, in the absence of a steadfast foundation (and the principle of maximum work cannot be counted as such), must be considered rather as a collective one, wherein the material of facts is amassed, and the first consequences arising from them are noticed. In my opinion three essential circumstances prevent the possibility of extracting any exact consequences, of importance to chemical mechanics, from the amassed and already immense store of thermochemical data: (1) The majority of the determinations are conducted in weak aqueous solutions, and, the heat of solution being known, are referred to the substances in solution; yet there is much (Chap. I.) which forces one to consider that in solution water does not play the simple part of a diluting medium, but of itself acts independently in a chemical sense on the substance dissolved. (2) The other chief portion of thermochemical determinations is conducted by the ignition of substances at high temperatures, and as yet we do not know the specific heat of many substances at these temperatures. (3) Physical and mechanical changes (decrease of volume, diffusion, and others) inevitably proceed side by side with chemical changes, and for the present it is impossible, in a number of cases, to distinguish the thermal effect of the one and the other kind of change. It is evident that the one kind of change (chemical) is essentially inseparable and incomprehensible without the other (mechanical and physical); and therefore it seems to me that thermochemical data will only acquire their true meaning when the connection between the phenomena of both kinds (on the one hand chemical and atomic, and on the other hand mechanical and molecular or between entire masses) is explained more clearly and fully than is the case at present. As there is no doubt that the simple mechanical contact, or the action of heat alone, on substances sometimes causes an evident and always a latent (incipient) chemical change-that is, a different distribution or movement of the atoms in the molecules-it follows that purely chemical phenomena are inseparable from physical and mechanical phenomena. This is because the atomic relations forming the essence of the chemical relations of a substance are not observable, and at present are incomprehensible, without the molecular relations 
most probably it increases), were the combustion concentrated at one point $^{39}$ (but it occurs as a flame), were there no loss from radiation and heat conduction, and, chiefly, did dissociation not take place-that is, did not a state of equilibrium between the hydrogen, oxygen, and water come about-then it would be possible to calculate the temperature of the flame of detonating gas. It would then be $10000^{\circ} .{ }^{40}$ In reality it is very much lower, but it is nevertheless higher than the temperature attained in furnaces and flames, and reaches up to $2000^{\circ}$. The explosion of detonating gas is explained by this high temperature, because the aqueous vapour formed must occupy a volume at least 5 times greater than that occupied by the detonating gas at the ordinary temperature. Detonating gas emits a sound, not only as a consequence of the commotion which occurs from the rapid expansion of the heated vapour, but also because it is immediately followed by a cooling effect, the conversion of the vapour into water, and a rapid contraction. ${ }^{41}$

forming the essence of the physical relations, and even without the relations of the entire masses of molecules evincing themselves in purely mechanical relations, inasmuch as an individual atom is something umreal and fantastic. A mechanical change may be imagined without a physical change, and a physical without a chemical change (although such a representation would be artificial), but it is impossible to imagine a chemical change without a physical and mechanical one, for without them we should not perceive it, and through them we attain it. There was a time when the province of physics embraced the whole of chemistry and mechanics. In the present day they have been developed independently and been isolated from each other, but in the future a fresh conjunction is imminent, and is heralded by the laws of the conservation of matter and of energy.

59 The flame, or locality where the combustion of gases and vapours is accomplished, is a complex plenomenon, 'an entire factory,' as Faraday says, and therefore we will consider flame in some detail in one of the following notes.

40 If 34500 units of heat are evolved in the combustion of 1 part of hydrogen, and this heat is transmitted to the resulting 9 parts by weight of aqueous vapour, then we find that, taking the specific heat of the latter as 0.475 , each unit of heat raises the temperature of 1 part by weight of aqueous vapour $2 \cdot 1^{\circ}$ and 9 parts by weight $(2 \cdot 1 \div 9)$ $0.23^{\circ}$; hence the 34500 units of heat raise its temperature $7935^{\circ}$. If detonating gas is converted into water in a closed space, then the aqueous vapour formed cannot expand, and therefore, in calculating the temperature of combustion, the specific heat at a constant volume must be taken into consideration; it is 0.36 for aqueous vapour. This figure gives a still higher temperature for the flame. In reality it is much lower, but the results given by different observers are very contradictory (from $1700^{\circ}$ to $2400^{\circ}$ ), the discrepancies depending on the fact that flames of different sizes are cooled by radiation to a different degree, but mainly on the fact that the methods and apparatus (pyrometrrs) for the determination of high temperatures, although they enable relative changes of temperature to be judged, are of little use for determining their absolute magnitude. By taking the temperature of the flame of detonating gas as $2000^{\circ}$, I give, I think, the average of the most trustworthy determinations.

41 It is evident that not only hydrogen, but every other combustible gas, will give an explosive mixture with oxygen. For this reason conl-gas mixed with air explodes when the mixture is ignited. The pressure obtained in the explosions serves as the motive pouer of gas engines. In this case advantage is taken, not only of the pressure produced by the explosion, but also of that contraction which takes place after the explosion. On this is based the construction of several motors, of which Lenoir's was 
Mixtures of hydrogen and of various other gases with oxygen are taken advantage of for obtaining high temperatures. By the aid of such high temperatures metals like platinum may be melted on a large scale, which cannot be done in furnaces heated with charcoal and fed by a current of air. The burner, shown in fig. 34 , is constructed for the application of detonating gas to the purpose. It consists of two brass tubes, one fixed inside the other, as shown in the drawing. The internal central tube $\mathrm{C} \mathrm{C}$ conducts oxygen, and the outside, enveloping, tube $\mathrm{E}^{\prime} \mathrm{E}^{\prime}$ conducts hydrogen. Previous to their egress the gases do not mix together, so that there can be no explosion inside. the apparatus. When this burner is in use $\mathrm{C}$ is connected with a gasholder containing oxygen, and $\mathrm{E}$ with a gas holder containing hydrogen (or sometimes coal-gas). The flow of the gases can be easily regulated by

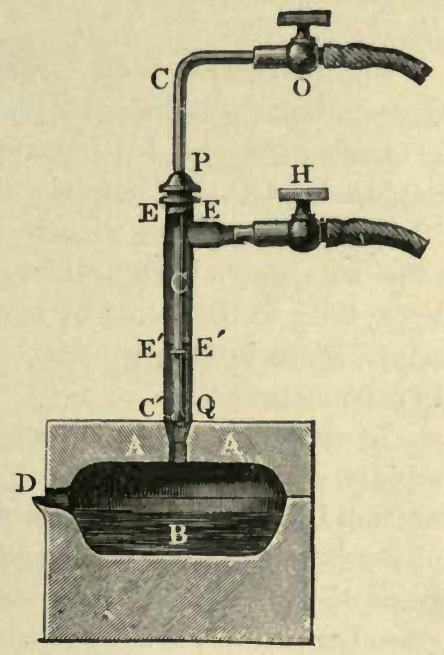

FIG. 34.- Safety burner for detonating gas, described in text.

the stop-cocks $\mathrm{OH}$. The flame is shortest and evolves the greatest heat when the gases burning are in the proportion of 1 volume of oxygen to 2 volumes of hydrogen. The degree of heat may he easily judged from the fact that a thin platinum wire placed in the flame easily melts. By placing the burner in the orifice of a hollow piece of lime, a crucible A B is obtained in which platinum may be easily melted, even in large quantities if the current of oxygen and hydrogen be sufficiently great (Deville). The flame of detonating gas. may also be used for illuminating purposes. It is by itself very pale, but owing to its high temperature it may serve for rendering infusible objects incandescent, and at the very high temperature produced by the detonating gas the incandescent substance gives a most intense light. For this purpose lime, magnesia, or oxide of zirconium are used, as they are not fusible at the very high temperature evolved by the detonating gas. A small cylinder of lime placed in the flame of detonating gas, if regulated to the required point, gives a very brilliant white

formerly, and Otto's is now, the best known. The explosion is usually produced by coalgas and air, but of late the vapours of combustible liquids (kerosene, benzene) are also being employed in place of gas (Chap. IX.). In Lenoir's engine a mixture of coalgas and air is ignited by means of sparks from a Ruhmkorff's coil, but in the most recent machines the gases are ignited by the direct action of a gas jet. 


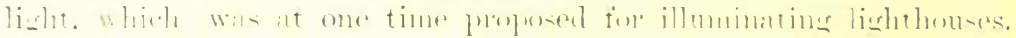

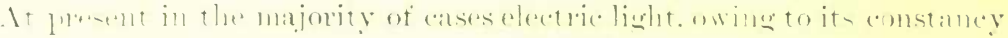

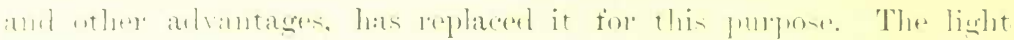

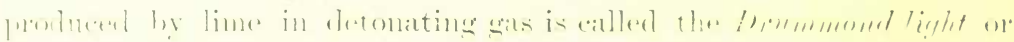
li,...li,flit.

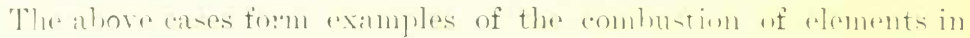

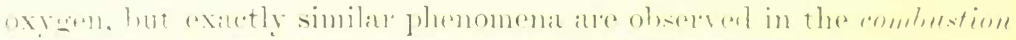

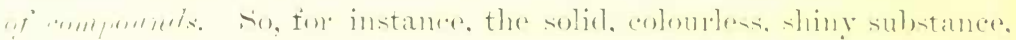

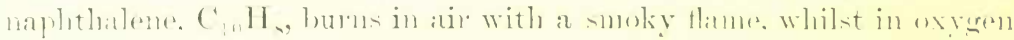
it contimmes to bum with a very brilliant thane. Ileohol, oil. and

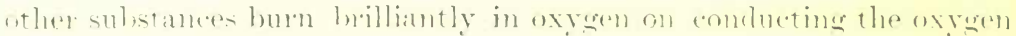
ly a cube to the thame of lampe bumbing these-uhatanees. A high

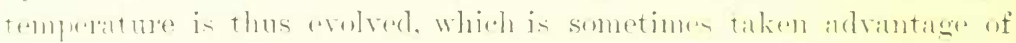
in eleminal pratetice.

In oreler to molerstanel why ambustim in oxyen proceeds more

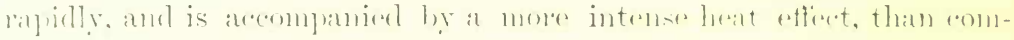
hustion in air, it must he recolleceted that air is os yegen tiluted with

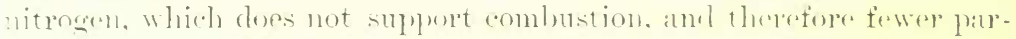

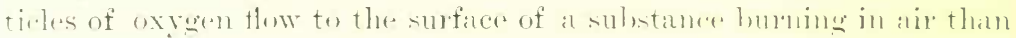

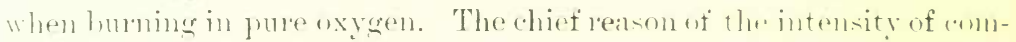
hustion in oxyen is the high temperature ate niled hy the substance

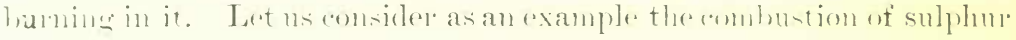

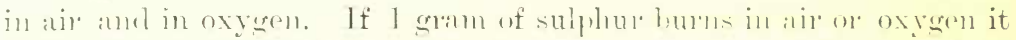

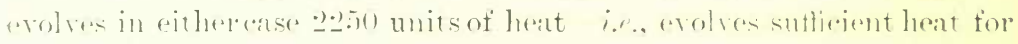

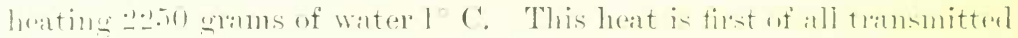

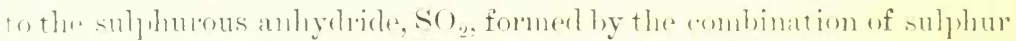

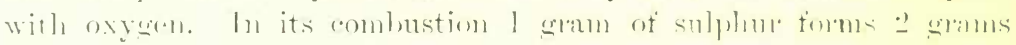

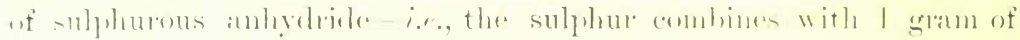

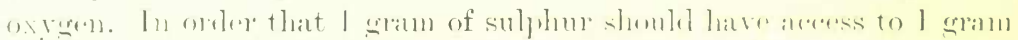

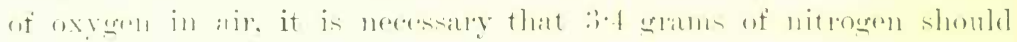

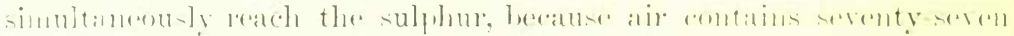

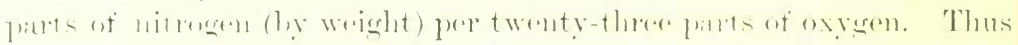

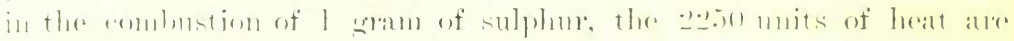

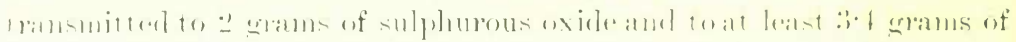

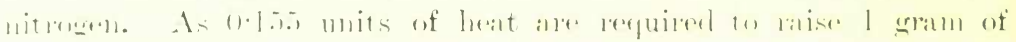

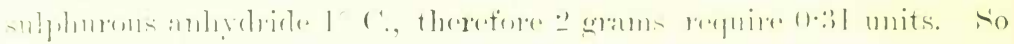

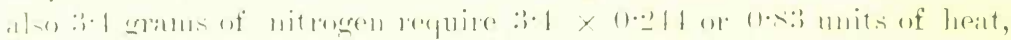

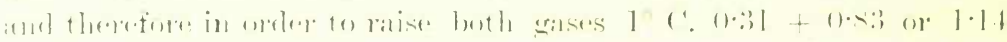

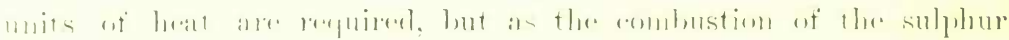

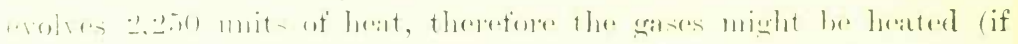

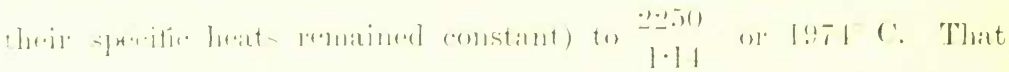


is, the maximum possible temperature of the flame of the sulphur burning in air will be $1974^{\circ} \mathrm{C}$. In the combustion of the sulphur in oxygen the heat evolved ( 2250 units) can only pass to the 2 grams of sulphurous anhydride, and therefore the highest possible temperature of the flame of the sulphur in oxygen will be $=\frac{2250}{0.31}$ or $7258^{\circ}$. In the same manner it may be calculated that the temperature of charcoal burning in air cannot exceed $2700^{\circ}$, while in oxygen it may attain $10100^{\circ} \mathrm{C}$. For this reason the temperature in oxygen will always be higher than in air, although (judging from what has been said respecting detonating gas) neither one nor the other temperature will nearly approach the theoretical quantities.

Among the phenomena accompanying the combustion of certain substances, the phenomenon of flame attracts attention. Sulphur, phosphorus, sodium, magnesium, naphthalene, drc., burn like hydrogen with a flame, whilst in the combustion of other substances no flame is observed, as, for instance, in the combustion of iron and of charcoal. The appearance of flame depends on the capacity of the combustible substance to yield gases or vapours at the temperature of combustion. At the temperature of combustion, sulphur, phosphorus, sodium, and naphthalene pass into vapour, whilst wood, alcohol, oil, dc., are decomposed into gaseous and vaporous substances. The combustion of gases and vapours forms flames, and therefore a flame is composed of the hot and incandescent gases and vapours produced by combustion. It may be easily proved that the flames of such nun-volatile substances as wood contain volatile and combustible substances formed from them, by placing a tube in the flame and drawing air from it with an aspirator. Besides the products of combustion, combustible gases and liquids, previously in the flame as vapours, collect in the aspirator. For this experiment to succeed-i.e., in order to really extract combustible gases and vapours from the flame-it is necessary that the suction tube should be placed inside the flame. The combustible gases and vapours can only remain unburnt inside the flame, for at the surface of the flame they come into contact with the oxygen of the air and burn. ${ }^{42}$ Flames are of different degrees of

42. Faraday proved this by a very convincing experiment on a candle flame. If one arm of a bent glass tube be placed in a candle flame above the wick in the dark portion of the flame, then the products of the partial combustion of the stearin will pass up the tube, condense in the other arm, and collect in a flask placed under it (fig. 35) as heavy white fumes which burn when lighted. If the tube be raised into the upper luminous portion of the flame, then a dense black smoke which will not inflame accumulates in the flask. Lastly, if the tube be let down until it tonches the wick, then little but stearic acid condenses in the flask. 
brilliancy, according to whether solid incandescent particles occur in the combustible gas or vapour, or not. Incandescent gases and vapours emit but little light by themselves, and therefore give a paler

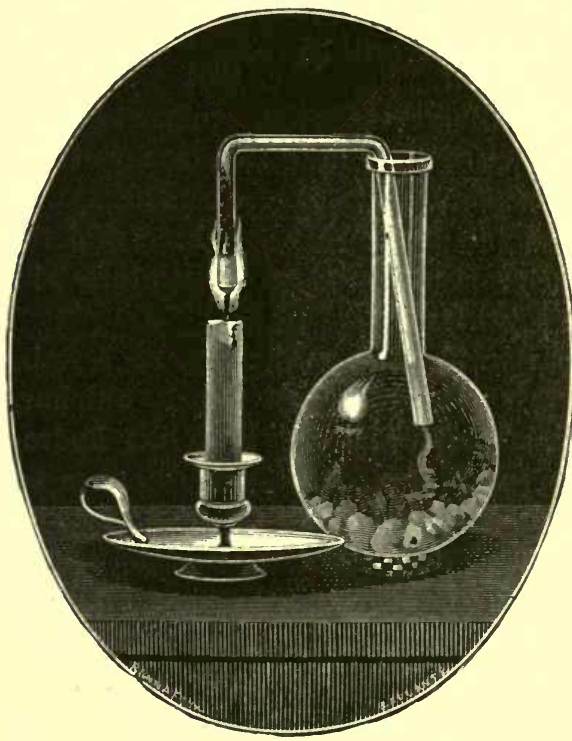

FIf $x$, 35.-Faraday's experiment for investigating the different parts of a candle flame. flame. ${ }^{43}$ If a flame does not. contain solid particles it is transparent, pale, and emits but little light.44 The flames of burning alcohol, sulphur, and hydrogen are of this kind. A pale flame may be rendered luminous by placing fine particles of solid matter in it. Thus, if a very fine platinum wire he placed in the pale flame of burning alcohol-or, better still, of hydrogen - then the flame emits a bright light. This is still better seen by sifting the powder of an incombustible substance, such as fine sand, into the flame, or by placing a bunch of asbestos threads in it. Every brilliant flame always contains some kind of solid particles, or at least some very dense vapour. The flame of sodium burning in oxygen has a brilliant yellow colour, from the presence of particles of solid sodium oxide. The flame of magnesium is brilliant from the fact that in burning it forms solid magnesia, which becomes white hot, and similarly the brilliancy of the Drummond light is due to the heat of the flame raising the solid non-volatile lime to a state of incandescence. The flames of a candle, wood, and similar substances are brilliant, because they contain particles of charcoal or soot. It is not the flame itself which is luminous, but the incandescent soot it contains. These particles of charcoal which occur in flames may be easily observed by introducing a cold object, like a knife, into the

43 All transparent substances which transmit light with great ease (that is, which absorb but little light) are but little luminous when heated; so also substances which absorb but few heat rays, when heated transmit few rays of heat.

44 There is, however, no doubt but that very heavy dense vapours or gases under pressure (according to the experiments of Frankland) are luminous when heated, because, as they become denser they approach a liquid or solid state. Thus detonating gas when exploded under pressure is brightly luminous. 
flame. ${ }^{45}$ The particles of charcoal burn at the outer surface of the flame if the supply of air be sufficient, but if the supply of air - that is, of oxygen-be insufficient for their combustion the flame smokes, because these unconsumed particles of charcoal are carried off by the current of air. ${ }^{46}$

45 If hydrogen gas be passed through a volatile liquid hydrocarbon-for instance, through benzene (the benzene may be poured directly into the vessel in which hydrogen is generated) - then its vapour burns with the hydrogen and gives a very bright flame, because the resultant particles of carbon (soot) are powerfully ignited. Benzene, or platinum gauze, introduced into a hydrogen flame may be employed for illuminating purposes.

46 In flames the separate parts may be distinguished with more or less distinctness. That portion of the flame whither the combustible vapours or gases flow, is not luminous because its temperature is still too low for the process of combustion to take place in it. This is the space which in a candle surrounds the wick, or in a gas jet. is immediately above the orifice from which the gas escapes. In a candle the combustible vapours and gases which are formed by the action of heat on the melted tallow or stearin, rise in the wick, and are lieated by the high temperature of the flame. By the action of the heat, the solid or liquid substance is here, as in other cases, decomposed, forming products of dry distillation. These products occur in the central portion of the flame of a candle. The air travels to the flame from the outside, and is not able to intermix with the vapours and gases in all parts of the flame; consequently, in the outer portion of the flame the amount of oxygen flowing to it will be greater than in the interior portions of the flames. But, owing to diffusion, the oxygen, naturally together with nitrogen, flowing to the combustible substance penetrates inside the flame, when the combustion takes place in ordinary air. The combustible vapours and gases combine with this oxygen, evolve a considerable amount of heat, and bring about that state of red heat which is so necessary both for keeping up the combustion and also for the uses to which the flame is applied. Passing from the colder envelope of air to the interior of the flame, to the source of the combustible vapours (for instance, the wick), we evidently first traverse layers of high temperature, and then layers of lower and lower temperature, in which the combustion is less complete, owing to the limited supply of oxygen.

Thus, yet unburnt products of the decomposition of organic substances occur in the interior of the flame. But there is always free hydrogen in the interior of the flame, even when oxygen is introduced there, or when a mixture of hydrogen and oxygen burns, because the temperature evolved in the combustion of hydrogen or the carbon of organic matter is so high that the products of combustion are themselves partially decomposed-that is, dissociated-

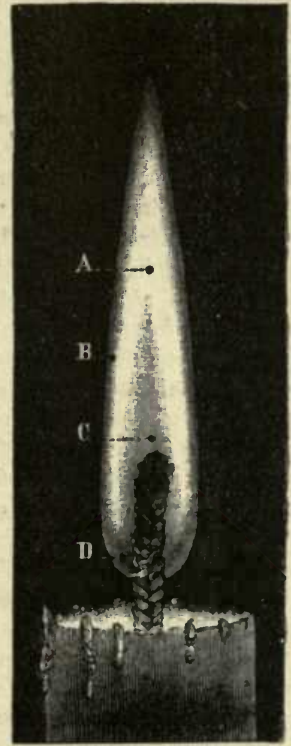

Fig. 36. - In the candle flame the portion $\mathrm{C}$ contains the vapours and products of decomposition: in the bright zone $\mathrm{A}$ the combustion has com. menced, and particles of carbon are emitted; and in the pale zone $B$ the combustion is com. pleted.

at this temperature. Hence, in a flame a portion of the hydrogen and of the oxygen which might combine with the combustible substances must always occur in a free state. If a hydrocarbon burns, and we imagine that a portion of the hydrogen occurs in a free state, then a portion of the carbon must aiso occur in the same form in

VOL. I. 


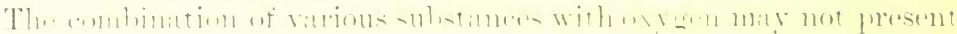

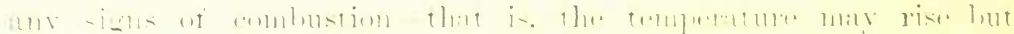

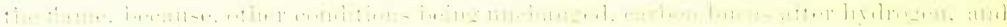

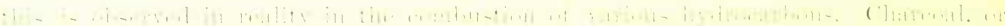

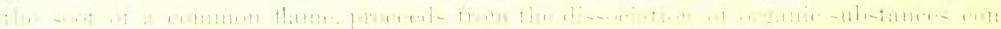

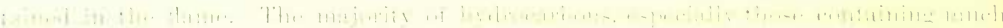

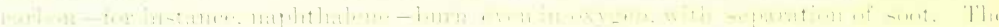

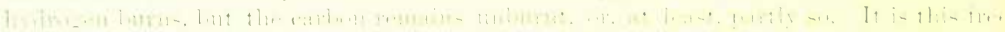

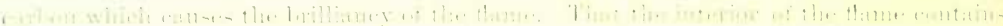

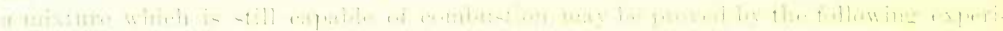

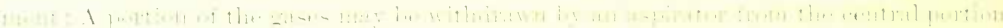

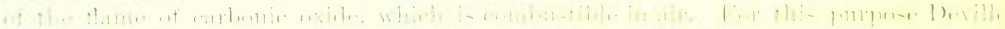

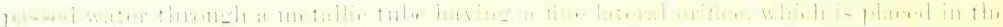

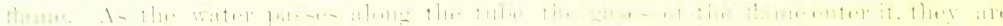
LA⿱

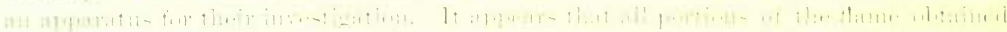

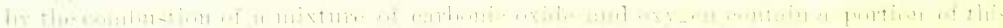

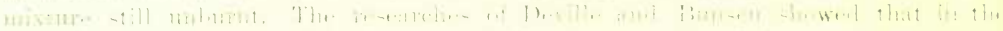

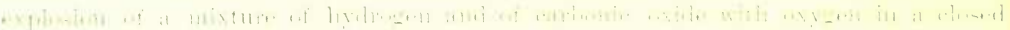

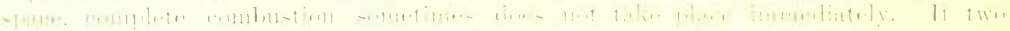

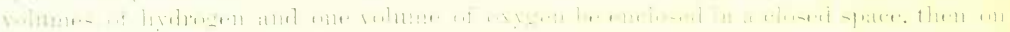

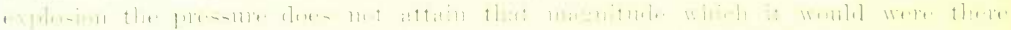

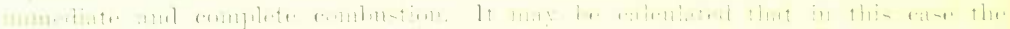

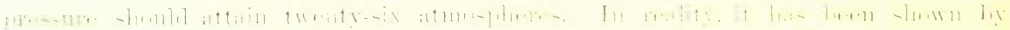

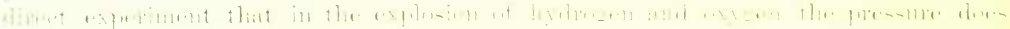

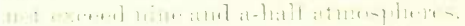

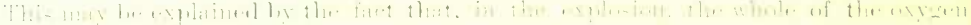

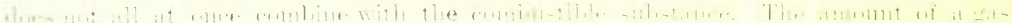

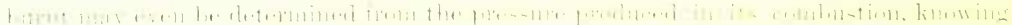

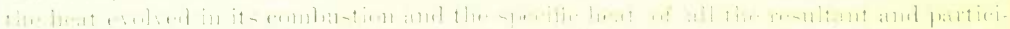

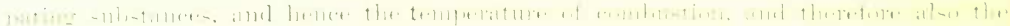

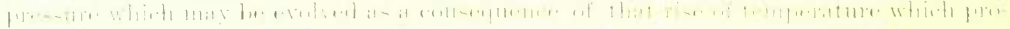

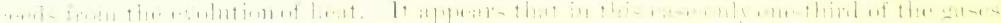

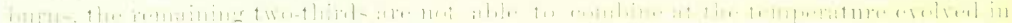

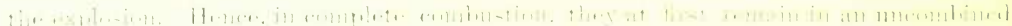

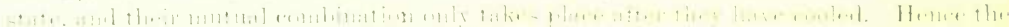

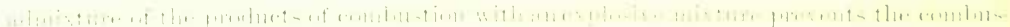

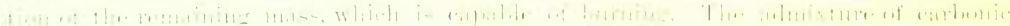

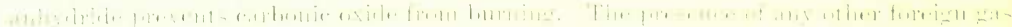

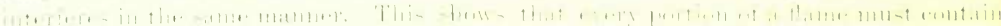

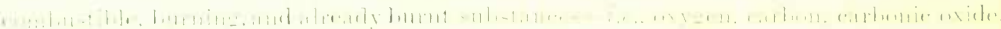

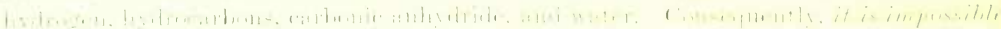

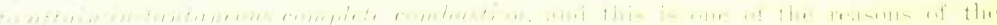

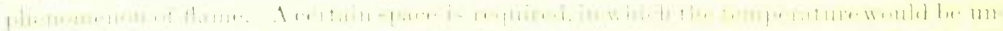

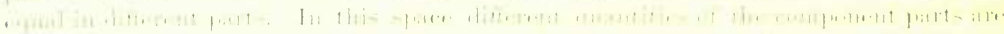

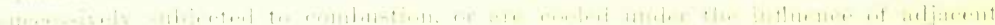

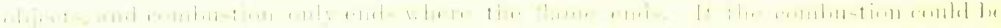

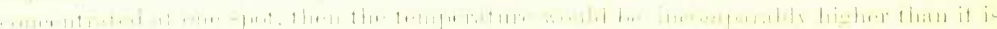

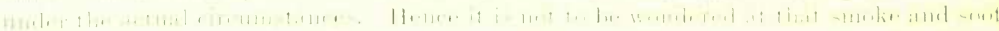

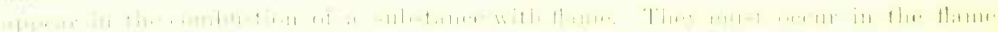

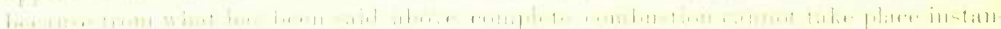

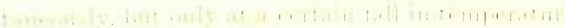

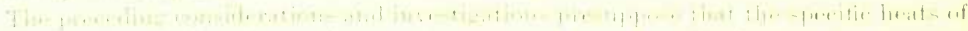

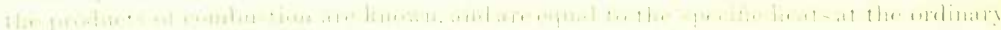


inconsiderably. This may either proceed from the fact that the reaction of the substance (for example, tin, mercury, lead at a high temperature, or a mixture of pyrogallol with caustic potash at the ordinary temperature) evolves but little heat, or that the heat evolved is transmitted to good conductors of heat, like metals, or that the combination with oxygen takes place so slowly that the heat evolved succeeds in passing to the surrounding objects. Combustion is only a particular, intense, and evident case of combination with oxygen. Respiration is also an act of combination with oxygen ; it also serves, like combustion, for the development of heat by those chemical processes which are its consequences (the transformation of oxygen into carbonic anhydride). Lavoisier enunciated this in the clear expression, 'respiration is slow combustion.'

Reactions of slow combination of substances with oxygen are termed oxidations. Combination of this kind (and also combustion) often results in the formation of acid substances, and hence the name oxygen (Sauerstoff). Combustion is only rapid oxidation. Phosphorus, iron, and wine may be taken as examples of substances which slowly oxidise in air at the ordinary temperature. If such a substance be left in contact with a definite volume of air or oxygen, it little by little absorbs the oxygen, as may be seen by the decrease in volume of the gas. This slow oxidation is, as a rule, rarely accompanied by a sensible evolution of heat ; but an evolution of heat really occurs, only it is not apparent to our senses, owing to the inconsiderable rise of temperature which takes place; this is owing to the slow rate of the reaction and to the transmission of the heat formed as radiant heat, \&c. Thus, in the oxidation of wine and its transformation into vinegar by the usual method of its preparation, the heat evolved cannot be observed because it extends over whole weeks, but in the so-called rapid process of the manufacture of vinegar, when a large quantity of wine is comparatively rapidly oxidised, the evolution of heat is quite apparent.

Such slow processes of oxidation are always taking place in nature by the action of the atmosphere. Dead organisms and the substances obtained from them-such as bodies of animals, wood, wool, grass, dc.-

temperature. If they vary (as Berthelot and Vieille affirm), the portion of a substance which remains unburnt on explosion cannot be calculated from the pressure, and therefore the quantitative side of the subject should be considered as doubtful. But the qualitative side of the subject cannot be subject to doubt, because the dissociation of the products of combustion at high temperatures is proved clearly by the most varied experiments. 
are especially subject to this action. They rot and putrefy that is, their solid matter is transformed into gases, under the influence of moisture, and atmospheric oxygen, and often under the influence of other organisms, such as moulds, worms, micro-organisms (bacteria), and such like. These are processes of slow combustion, of slow combination with oxygen. Everyone knows that manure rots and evolves heat, that stacks of damp hay, damp flour, straw, \&c., become heated and are changed in the process. ${ }^{47}$ In all these transformations there are formed the same chief products of combustion as are contained in smoke; the carbon gives carbonic anhydride, and the hydrogen water. Hence these processes require oxygen just like combustion. This is the reason why the entire prevention of access of air hinders these transformations, ${ }^{48}$ and an increased supply of air accelerates them. The mechanical treatment of arable lands by the plough, harrow, and other similar means has not only the object of facilitating the spread of roots in the ground, and of making the soil more permeable to water, but it also serves to facilitate the access of the air to the component parts of the soil ; as a consequence of which the organic remains of soil rot-so to speak, breathe air and evolve carbonic anhydride. One acre of good garden land in summer evolves more than six tons of carbonic anhydride.

It is not only vegetable and animal substances which are subject to slow oxidation in the presence of water. The very metals are rusted under these conditions. Copper very easily absorbs oxygen in the presence of acids. Many metallic sulphides (for example, pyrites) are very easily oxidised with access of air and moisture. Thus processes of slow oxidation proceed throughout nature.

There are many elements which do not, under any circumstances, combine directly with gaseous oxygen; nevertheless their compounds with oxygen may be obtained. Platinum, gold, iridium, chlorine, and iodine are examples of such elements. In this case recourse is had to a so-called indirect method-i.e., the given substance is

47 Cotton waste (it is used in factories for cleaning machines from lubricating oil) soaked in oil and lying in heaps is self-combustible, being oxidised by the air.

48 Wlien it is desired to preserve a supply of vegetable and animal food, the access of the oxygen of the atmosphere (and also of the germs of organisms borne in the air) is often prevented. For this reason articles of food are often kept in hermetically closed vessels, from which the air is withdrawn; vegetables are dried and soldered up while hot in tin boxes; sardines are immersed in oil, \&c. The removal of water from substances is also sometimes resorted to with the same object (the drying of hay, corn, fruits), as also is saturation with substances which absorb oxygen (suclı as sulphurous anhydride), or which hinder the growth of organisms forming the first cause of putrefaction, as in processes of smoking, embalming, and in the keeping of fishes and other animal specimens in spirit, \&c. 
combined with another element, and by a method of double decomposition this element is replaced by oxygen, or a substance is taken which easily evolves oxygen, and is brought into contact with the given substance. The oxygen then acts at the moment of its evolution. If the conditions are such that the substance to be oxidised is liberated at the same moment, then oxidation proceeds with greater ease. (The explanation of this phenomenon was given in the last chapter.) It must be remarked that substances which do not directly combine with oxygen, but form compounds with it by an indirect method, often readily lose the oxygen which was absorbed by them by double decomposition or at the moment of its evolution. Such, for example, are the compounds of oxygen with chlorine, nitrogen, and platinum, which evolve oxygen on heating. They, like other substances which easily evolve oxygen on heating, may serve as a means for obtaining oxygen, or for oxidation. They, in the presence of substances which are capable of combining with oxygen, are decomposed, give up their oxygen to them, and may thus be themselves employed for indirect oxidation. In this respect oxidising agents, or those compounds of oxygen which are employed in chemical and technical practice for transferring oxygen to other substances, are especially remarkable. The most important anong these is nitric acid or aqua fortis - a substance rich in oxygen, and capable of evolving it when heated, and which easily oxidises a great number of substances. Thus nearly all metals and organic substances containing carbon and hydrogen are more or less oxidised when heated with nitric acid. If strong nitric acid be taken, and a piece of burning charcoal be inmersed in the acid, it continues to burn, the combustion proceeding in this case at the expense of the oxygen contained in the liquid nitric acid. Chromic acid acts like nitric acid; alcohol burns when mixed with it. Although the action is not so marked, even water may oxidise with its oxygen. Sodium is not oxidised in perfectly dry oxygen at the ordinary temperature, but it burns very easily in water and aqueous vapour. Charcoal can burn in carbonic anhydride-a product of combustion - forming carbonic oxide. Magnesium burns in the same gas, separating carbon from it. Generally, combined oxygen can pass from one compound to another.

The products of combustion or oxidation - and in general the definite compounds of oxygen-are termed oxides. Some oxides are not capable of combining with other oxides - or combine with only a few, and then form unstable compounds with the evolution of very little heat; others, on the contrary, enter into combination with very many other oxides, and in general have remarkable chemical energy. The oxides incapable of combining with others, or only showing this quality in a 


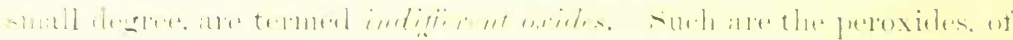

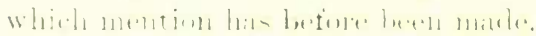

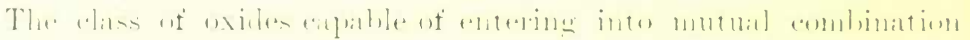

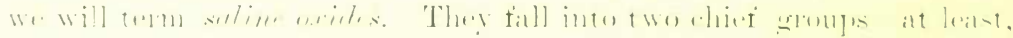

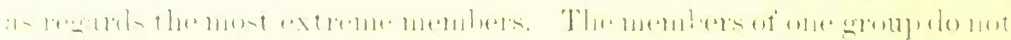

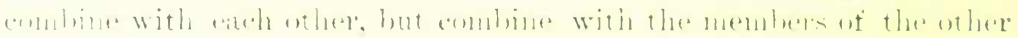

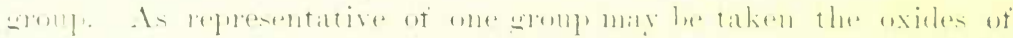

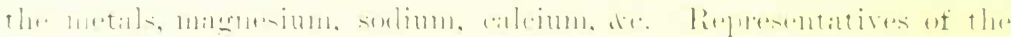

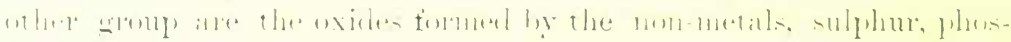

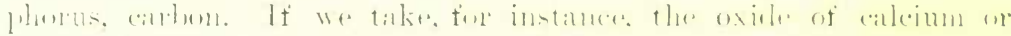

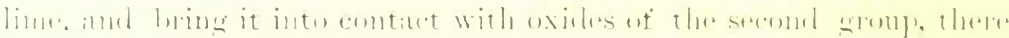

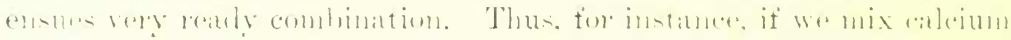

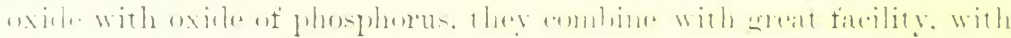

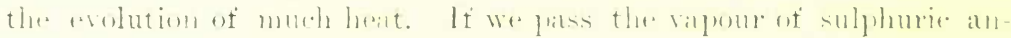

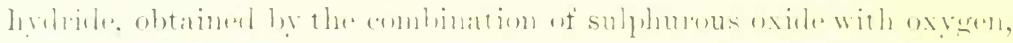

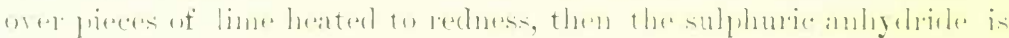

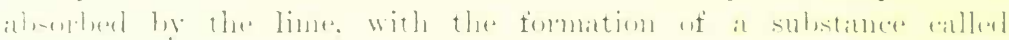
"ablimm sulfhate. The oxiles of the tirst kind. Which andain

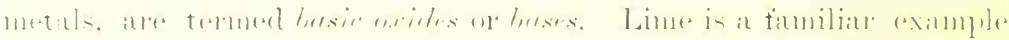

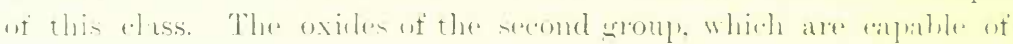

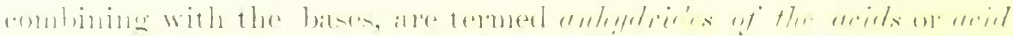

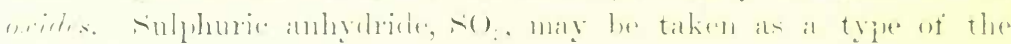

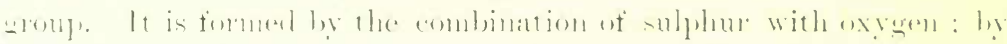

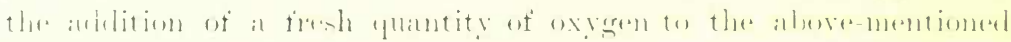

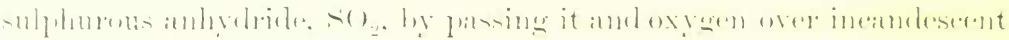

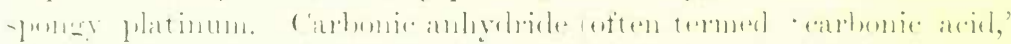

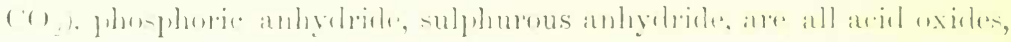

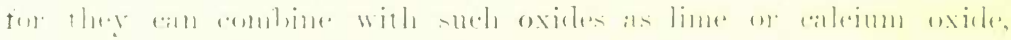

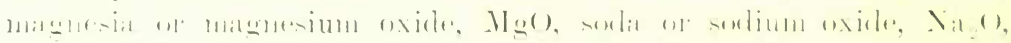
心.

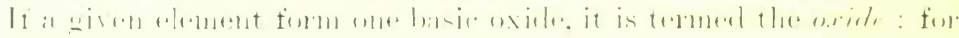

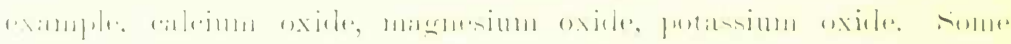

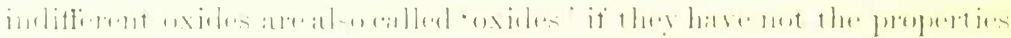

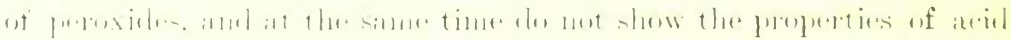

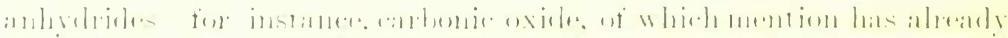

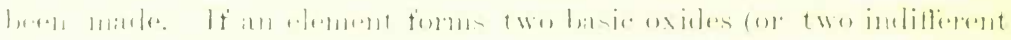

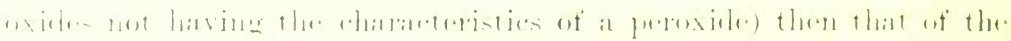

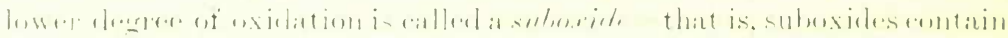

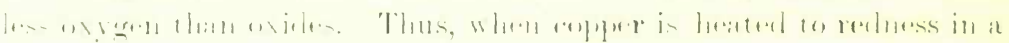

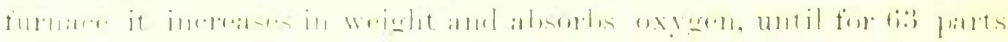

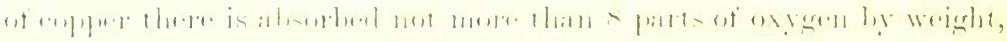

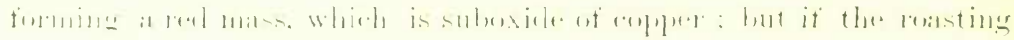


be prolonged, and the draught of air be increased, 63 parts of copper absorb 16 parts of oxygen, and form black oxide of copper. Some. times to distinguish between the degrees of oxidation a change of suffix is made in the oxidised element - ic oxide naming the higher degree of oxidation, and -ous oxide the lower degree. Thus ferrous oxide and ferric oxide are the same as suboxide of iron and oxide of iron. This nomenclature is convenient in some cases, but cannot always be employed. If an element forms one anhydride only, then it is named by an adjective formed from the name of the element made to end in -ic and the word anhydride. When an element forms two anhydrides, then the suffixes - ous and -ic are used to distinguish them : - ous signifying less oxygen than - ic ; for example, sulphurous and sulphuric anhydrides. ${ }^{49}$ When several oxides are formed from the same element, the prefixes mon, di, tri, tetra are used, thus : chlorine monoxide, chlorine dioxide, chlorine trioxide, and chlorine tetroxide or chloric anhydride.

Chemical transformations of the oxides themselves are rarely accomplished, and in the few cases where they are subject to such changes a particularly important part is played by their combinations with water. The majority of, if not ull, basic and acid oxides combine with water, either by a direct or an indirect method forming hydrates - that is, such compounds as split up into water and an oxide of the same kind only. We already know that many substances are capable of combining with water. Oxides possess this property in the highest degree. We have already seen examples of this (Chap. I.) in the combination of lime, and of sulphuric and phosphoric anhydrides, with water. Hence the results of such combination are basic and acid hydrates. Acid hydrates are called acids, because they have an acid

49 It must be remarked that certain elements form oxides of all three kinds-i.e., indifferent, basic, and acid; for example, manganese forms manganous oxide, manganic oxide, peroxide of manganese, red oxide of manganese, and manganic anhydride, although some of them are not known in a free state but only in combination. It is, then, always to be remarked that the basic oxide contains less oxygen than the peroxides, and the peroxides less than the acid anhydride. Thus they must be placed in the following general normal order with respect to the amount of oxygen entering into their composition-(1) basic oxides, suboxides, and oxides; (2) peroxides; (3) acid anhydrides. The majority of elements, however, do not give all three kinds of oxides, some giving only one degree of oxidation. It must further be remarked that there are oxides formed by the combination of acid anhydrides with basic oxides, or, in general, of oxides with oxides. For every oxide having a higher and a lower degree of oxidation, it might be said that the intermediate oxide was formed by the combination of the higher with the lower oxide. But this is not true in all cases-for instance, when the oxide under consideration forms a whole series of independent compounds-for oxides which are really formed by the combination, of two other oxides do not give such independent compounds, but in many cases decompose into the higher and lower oxides. 
taste when dissolved in water (or saliva, for then only can they act on the palate). Vinegar, for example, has an acid taste because it contains acetic acid dissolved in water. Sulphuric acid, of which we have made mention many times, because it is the acid of the greatest importance both in practical chemistry and for its technical applications, is really a hydrate formed by the combination of sulphuric anhydride with water. Besides their acid taste, dissolved acids or acid hydrates have the property of changing to red the blue colour of certain vegetable dyes. Of these dyes litmus is particularly remarkable and much used. It is the blue substance extracted from certain lichens, and is used for dyeing tissues blue; it gives a blue infusion with water. This infusion, on the addition of an acid, changes from blue to red. ${ }^{50}$

Basic oxides, in combining with water, form hydrates, of which, however, very few are soluble in water. Those which are soluble in water have an alkaline taste like that of soap or of water in which ashés have been boiled, and are called alkalis. Further, alkalis have the

50 Blotting or unsized paper, soaked in a solution of litmus, is usually employed for detecting the presence of acids. This paper is cut into strips, and is called test paper; when dipped into acid it immediately turns red. This is a most sensitive reaction, and may be employed for testing for the least traces of acids. If 10000 parts by weight of water be mixed with 1 part of sulphuric acid, the coloration is distinctly perceptible, and it is quite distinguishable on the addition of ten times more water. Certain precautions must, however, be taken in the preparation of such very sensitive litmus paper. Litmus is sold in lumps. Take, say, 100 grams of it; pound it, and add it to cold pure water in a flask. Shake and decant the water. Repeat this three times. This is done to wash away easily-soluble impurities, especially alkalis. Transfer the washed litmus to a flask, and pour in 600 grams of water, heat, and allow the hot infusion to remain for some hours in a warm place. Then filter, and divide the filtrate into two parts. Add a few drops of nitric acid to one portion, so that a faint red tinge is obtained, and then mix the two portions. Add spirit to the mixture, and keep it thus in a stoppered bottle (it soon spoils if left open to the air). This infusion may be employed directly; it reddens in the presence of acids, and turns blue in the presence of alkalis. If evaporated, a solid muss is obtained which is soluble in water, and may be kept unchanged for any length of time. The test paper may be prepared as follows:-Take a strong infusion of litmus, and soak blotting-paper with it; dry it, and cut it into strips, and use it as testpaper for acids. For the detection of alkalis, the paper must be soaked in a solution of litmus just reddened by a few drops of acid; if too much acid be taken, the paper will not be sensitive. Such acids as sulphuric acid colour litmus, and especially its infusion, a brick-red colour, whilst more feeble acids, such as carbonic, give a faint red-wine tinge. Test-paper of a yellow colour is also employed; it is dyed by an infusion of turmeric roots in spirit. In alkalis it turus brown, but regains its original hue in acids. Many blue and other vegetable colouring matters may be used for the detection of acids and alkalis; for example, infusions of cochineal, violets, log-wood, \&c. Certain artificially-prepared substances and dyes may also be employed. Thus rosolic acid, $\mathrm{C}_{20} \mathrm{H}_{10} \mathrm{O}_{3}$, and phenolplithalein, $\mathrm{C}_{26} \mathrm{H}_{14} \mathrm{O}_{4}$, are colourless in an acid, and red in an alkaline, solution. Cyanine is also colourless in the presence of acids, and gives a blue coloration with alkalis. These are very sensitive tests. Their behaviour in respect to various acids, alkalis and salts sometimes gives the means of distinguishing substances from each other. 
property of restoring the blue colour to litmus which has been reddened by the action of acids. The hydrates of the oxides of sodium and potassium, $\mathrm{NaHO}$ and $\mathrm{KHO}$, are examples of basic hydrates easily soluble in water. They are true alkalis, and are termed caustic, because they act very powerfully on the skin of animals and plants. Thus $\mathrm{NaHO}$ is called 'caustic' soda.

Thus, the saline oxides are capable of combining together and with water. Water itself is an oxide, and not an indifferent one, for it can, as we have seen, combine with basic and acid oxides ; it is a representative of a whole series of saline oxides, intermediate oxides, capable of combining with both basic and acid oxides. There are many such oxides, which, like water, combine with basic and acid anhydrides-for instance, the oxides of aluminium and tin, drc. From this it may be concluded that all oxides might be placed, in respect to their capacity for combining with one another, in one uninterrupted series, at one extremity of which would stand those oxides which do not combine with the bases - that is, the alkalis - while at the other end would be the acid oxides, and in the interval those oxides which combine with one another and with both the acid and basic oxides. The further apart are the members of this series the more stable are the compounds they form together, the more energetically do they act on each other, the greater the quantity of heat evolved in their reaction, and the clearer is their saline chemical character.

We said above that basic and acid oxides combine together, but rarely react on each other; this depends on the fact that the majority of them are solids or gases - that is, they occur in the state least prone to chemical reaction. The gaseo-elastic state is with difficulty destroyed, because it necessitates overcoming the elasticity proper to the gaseous particles. The solid state is characterised by the immobility of its particles; whilst chemical action requires contact, and hence a displacement and mobility. If solid oxides be heated, and especially if they be melted, then reaction proceeds with great ease. But such a change of state rarely occurs in nature or in practice. In a few furnace processes only is this the case. For example, in the manufacture of glass, the oxides contained in it combine together in a molten state. But when oxides combine with water, and especially when they form hydrates soluble in water, then the mobility of their particles increases to a considerable extent, and their reaction is greatly facilitated. Reaction then takes place at the ordinary temperature-easily and rapidly ; so that this kind of reaction belongs to the class of those which take place with unusual facility, and are, therefore, very often taken advantage of in practice, and also have been and are going on in nature at 
every step. We will now consider the reactions of oxides in the state of hydrates, not losing sight of the fact that water is itself an oxicle with definite properties, and has, therefore, no little intluence on the course of those changes in which it takes part.

If we take a definite quantity of an acid, and add an infusion of litmus to it, it turns red; the addition of an alkaline solution does not at once alter the red colour of the litmus, but on adding more and more of the alkaline solution a point is reached when the red colour changes to violet, and then the further addition of a fresh quantity of the alkaline solution changes the colour to blue. This change of the colour of the litmus is a consequence of the formation of a new compound. This reaction is termed the saturation or neutralisation of the acid by the base, or vice versa. The solution in which the acid properties of the acid are saturated by the alkaline properties of the base is termed a neutral solution. Such a solution, although derived from the mixture of a base with an acid, does not, however, exhibit either the acid or basic reaction on litmus, yet it preserves many other signs of the acid and alkali. It is observed that in such a definite admixture of an acid with an alkali, besides the change in the colour of litmus, there is a heating effect-i.e., an evolution of heat-which is alone sufficient to prove that there was chemical action. And, indeed, if the resultant violet solution be evaporated, there separates out, not the acid nor the alkali originally taken, but a substance which has neither acid nor alkaline properties, but is usually solid and crystalline, having a saline appearance ; this is a salt in the chemical sense of the word. Hence it is derived from the reaction of an acid on an alkali, and through a definite relation between the acid and alkali. The water here taken for solution plays no other part than merely facilitating the progress of the reaction. This is seen from the fact that the anhydrides of the acids are able to combine with basic oxides, and give the same salts as do the acids with the alkalis or hydrates. Hence, a salt is a compound of definite quantities of an acid with an alkali. In the latter reaction, water is separated out if the substance formed be the same as is produced by the combination of anhydrous oxides together. ${ }^{51}$ Examples of the formation of salts from acids and bases are easily observed, and are very often applied in

31 That water really is separated in the reaction of acid on alkaline hydrates, may be shown by taking some other intermediate hydrate-for instance, alumina-instead of water. Thus, if a solution of alumina in sulphuric acid be taken, it will have, like the acid, an acid reaction, and will therefore colour litmus red. If, on the other hand, a solution of alumina in an alkali-for instance, potash-be taken, it will liave an alkaline reaction, and will turn red litmus blue. On adding the alkaline to the acid solution until neither an alkaline nor an acid reaction is produced, a salt is formed, consisting of 
practice. If we take, for instance, insoluble magnesium oxide, it is easily dissolved in sulphuric acid, and on evaporation gives a saline substance, bitter, like all the salts of magnesium, and familiar to all under the name of Epsom salts, used as a purgative. If a solution of caustic soda - which is obtained, as we saw, by the action of water on sodium oxide-be poured into a flask in which charcoal has been burnt; or if carbonic anhydride, which is produced under so many circumstances, be passed through a solution of caustic soda, then sodium carbonate or soda, $\mathrm{Na}_{2} \mathrm{CO}_{3}$, is obtained, of which we have spoken several times, and which is prepared on a large scale and often used in manufactures. This reaction is expressed by the equation, $2 \mathrm{NaHO}+\mathrm{CO}_{2}=$ $\mathrm{Na}_{2} \mathrm{CO}_{3}+\mathrm{H}_{2} \mathrm{O}$. Thus, the various bases and acids form an innumerable number of different salts. ${ }^{52}$ Salts constitute an example of definite chemical compounds which, both in the history and practice of science,

sulphuric anhydride and potassium oxide. In this, as in the reaction of hydrates, an intermediate oxide is separated out-namely, alumina. Its separation will be very evident in this case, as alumina is insoluble in water, whilst its compounds with the acid and alkali, like the compound of an alkali with an acid-i.e., a salt-are soluble in water, and therefore on mixing the solutions of alumina in an acid and an alkali, it is precipitated as a gelatinous hydrate.

52 The mutual interaction of hydrates, and their capacity of forming salts, may be taken advantage of for determining the character of such hydrates as are insoluble in water. Let us imagine that a given hydrate, whose chemical character is unknown, is insoluble in water. It is therefore impossible to test its reaction on litmus. It is then mixed with water, and an acid-for instance, sulphuric acid-is added to the mixture. If the hydrate taken be basic, reaction will take place, either directly or by the aid of heat, with the formation of a salt. In certain cases, the resultant salt is soluble in water, and this will at once show that combination has taken place between the insoluble basic hydrate and the acid, with the formation of a soluble saline substance. In those cases where the resultant salt is insoluble, still the water loses its acid reaction, and therefore it may be ascertained, by the addition of an acid, whether a given liydrate has a basic character, like the hydrates of oxide of copper, lead, \&c. If the acid does not act on the given insoluble hydrate (at any temperature), then it has not a basic character, and it should be tested as to whether it has an acid character. This is done by taking an alkali, instead of the acid, and by observing whether the unknown hydrate then dissolves, or whether the alkaline reaction disappears. Thus it may be proved that hydrate of silica is acid, because it dissolves in alkalis and not in acids. If it be a case of an insoluble intermediate hydrate, then it will be observed to react on both the acid and alkali. Hydrate of alumina is an instance in question, which is soluble both in caustic potash and in sulphuric acid. But it must be remarked that intermediate oxides, in an anhydrous state, often evince great resistance to the formation of saline compounds. Thus alumina or aluminium oxide, in the anhydrous form in which it is met with in nature, and which forms a crystalline substance, is insoluble in this form both in solntions of alkalis and of acids. In order to convert it into a soluble form, it must be ground into a fine powder and fused together with certain acid compounds, which are unchanged by heat, such as acid potassium sulphate.

The degree of affinity or chemical energy proper to oxides and their hydrates is very dissimilar; some extreme members of the series have it to a great extent. When acting on each other they evolve a large quantity of heat, and when acting on intermediate hydrates they also evolve heat to a considerable degree, as we saw in the combi- 


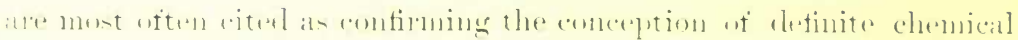

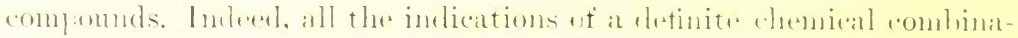

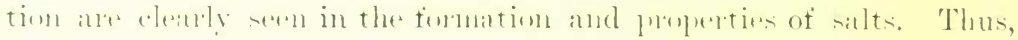

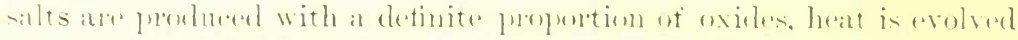

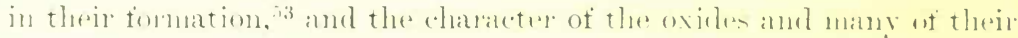

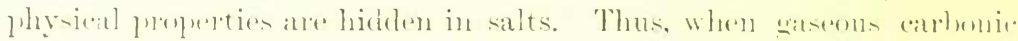

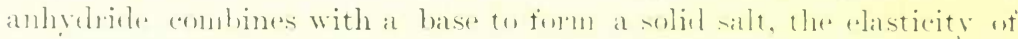

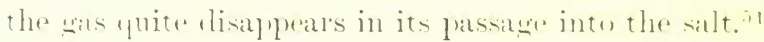

Intering from the abure, a salt in a eompoumel of hasic and

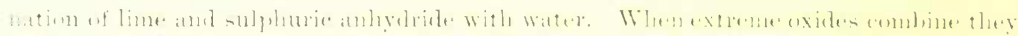

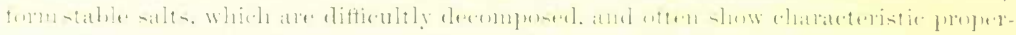

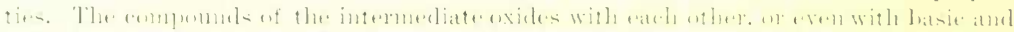

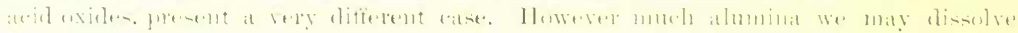

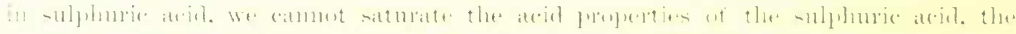

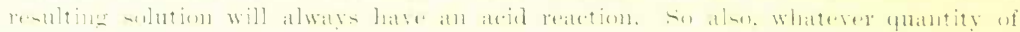

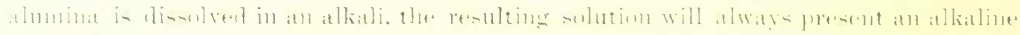
Ireillotion.

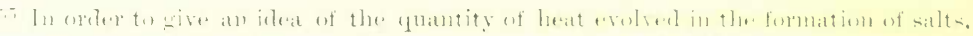

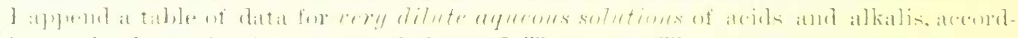

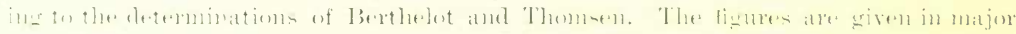

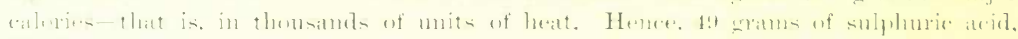

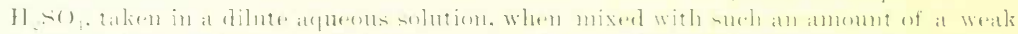

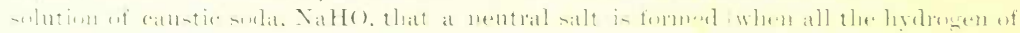

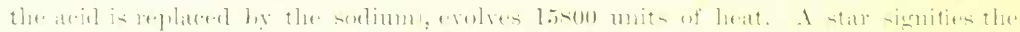
formation of an insolub]e sult.

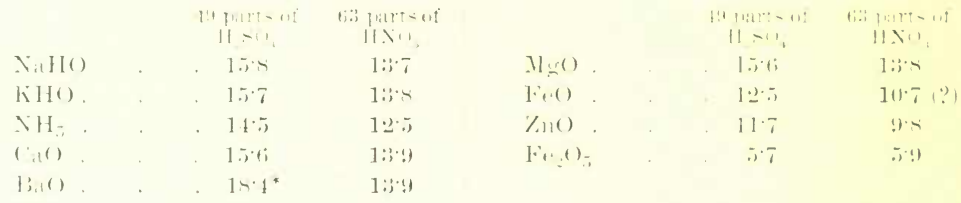

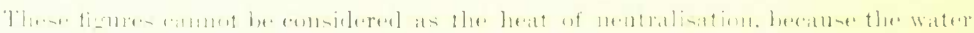

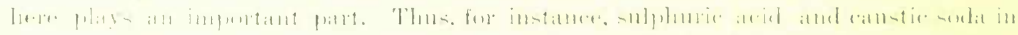

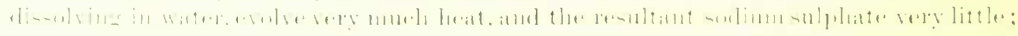

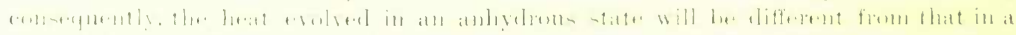

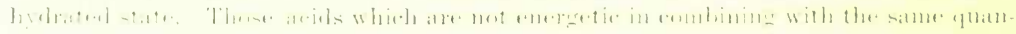

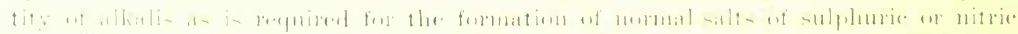

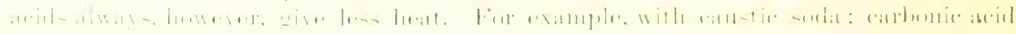

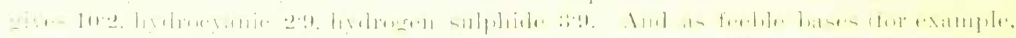

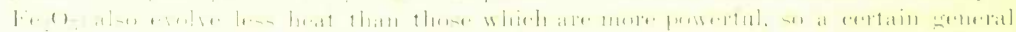

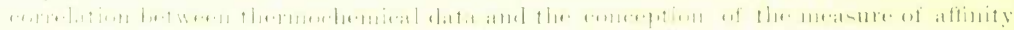

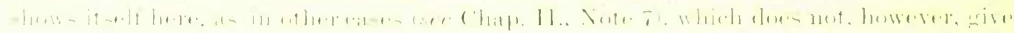

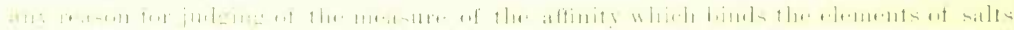

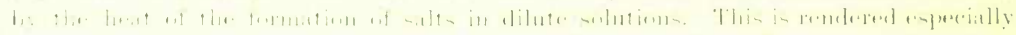

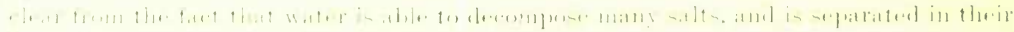

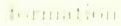

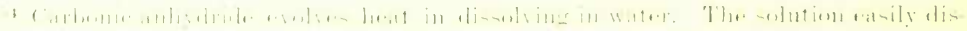

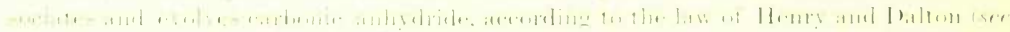

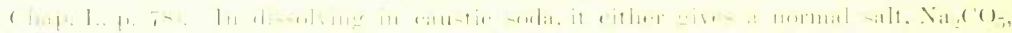

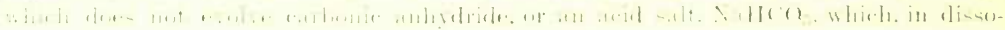


acid oxides, or the result of the action of hydrates of these classes on each other. with separation of water. But salts may be obtained by other methods. Let us not forget that basic oxides are formed by metals, and acid oxides often by non-metals. But metals and non-metals are capable of combining together, and a salt is frequently formed by the oxidation of such a compound. For example, iron very easily combines with sulphur, forming iron sulphide (as we saw in the Introduction); this in air, and especially moist air, absorbs oxygen, with the formation of the same salt as may be obtained by the combination of the oxides of iron and sulphur, or of the hydrates of these oxides. Hence, it cannot be said or supposed that a salt contains the principles of the oxides, or that a salt must necessarily contain two kinds of oxides in itself. The same conclusion may be arrived at by investigating the different other methods of the formation of saltsthus, for instance, many salts enter into double decomposition with the metals, in which case the acting metal replaces that which originally occurred in the salt. As we saw in the Introduction, iron, when placed in a solution of copper sulphate, separates out the copper, and forms an iron salt. Thus, the derivation of salts from oxides, is only one of the methods of their preparation, there being many others, and, therefore, it cannot be affirmed that a salt is simply the compound of two oxides. We saw, for instance, that, in sulphuric acid it was possible to replace the hydrogen by zinc, and that by this means zinc sulphate was formed; so likewise the hydrogen in many other acids may be replaced by zinc, iron, potassium, sodium, and a whole series of similar metals, corresponding salts being obtained. The hydrogen in the water of the acid, in this case, is exchanged for a metal, and a salt is obtained from the hydrate. In this sense of a salt it may be said, that $a$ salt is ar acid in which hydrogen is replaced by a metal. Such a definition will be much niore exact than that previously given, for it refers directly to elements and not to their compounds with oxygen. It shows that a salt and an acid are essentially compounds of the same series, with the difference that the latter contains hydrogen and the former a metal. Such a definition is still more exact than the first definition of salts in respect to its referring likewise to those acids which do not contain oxygen, and, as we shall afterwards learn, there is a series of such acids. Such elements as chlorine and bromine form

ciating, evolves carbonic anhydride. The same gas, when dissolved in solutions of salts, acts in one or the other manner (see Chap. II., Note 38). Here it is seen what a successire series of relations exists between compounds of a different order, between substances of different degrees of stability. Were solutions distinctly separated from chemical compounds, we should not be able to see those natural transitions which exist. in reality. 
compounds with hydrogen, in which the hydrogen may be replaced by a metal forming substances which, in their reactions and external characters, resemble the salts formed from oxides. Table salt, $\mathrm{NaCl}$, is an example of this. It may be obtained by the replacement of hydrogen in hydrochloric acid, $\mathrm{HCl}$, by the metal sodium, just as sulphate of sodium, $\mathrm{Na}_{2} \mathrm{SO}_{4}$, may be obtained by the replacement of hydrogen in sulphuric acid, $\mathrm{H}_{2} \mathrm{SO}_{4}$, by sodium. The exterior appearance of the resulting products, their neutral reaction, and even their saline taste, show their mutual resemblance; as the acid reaction, the property of saturating bases, the capacity of exchanging their hydrogen for some metal, and the acid taste, show the common properties belonging to hydrochloric and sulphuric acids.

To the fundamental properties of salts yet another must be addednamely, that they are more or less decomposed by the action of a galvanic current. The results of this decomposition are very different, according to whether the salt be taken in a fused or dissolved state. But the decomposition may be so represented, that the metal appears at the electro-negative pole (like hydrogen in the decomposition of water, or its mixture with sulphuric acid), and the remaining parts of the salt appear at the electro-positive pole (where the oxygen of water appears). If, for instance, an electric current acts on an aqueous solution of sodium sulphate, then the sodium appears at the negative pole, and oxygen and the anhydride of sulphuric acid at the positive pole. But in the solution itself the result is different, for sodium, as we know, decomposes water with evolution of hydrogen, forming caustic soda ; consequently hydrogen will be erolved, and caustic soda appear at the negative pole: while at the positive pole the sulphuric anhydride immediately combines with water and forms sulphuric acid, and therefore oxygen will be evolved and sulphuric acid formed round this pole. ${ }^{55}$ In other cases, when the metal separated is not able to decompose water, it will be deposited in a free state. Thus, for example, in the decomposition of copper sulphate, copper separates out at the cathode, and oxygen and sulphuric acid appear at the anode, and if a copper plate be attached to the positive pole, then the oxygen evolved will oxidise the copper, and the oxide of copper will dissolve in the sulphuric acid which is formed around this pole; hence the copper will be dissolved at the positive, and deposited at the negative, pole-

35 This kind of decomposition may be easily observed by pouring a solution of sodium sulphate in a $U$-shaped tube and inserting electrodes in both branches. If the solution be coloured with an infusion of litmus, it will easily be seen that it turns blue round the electro-negative pole, owing to the formation of sodium hydroxide, and red at the electro-positive pole, from the formation of sulphuric acid. 
that is, a transfer of copper from the positive to the negative pole ensues. The galvanoplastic art (electrotyping) is based on this principle. ${ }^{56}$ Therefore the most radical and general properties of salts (including also such salts as table salt, which contains no oxygen) may be expressed by representing the salt as composed of a metal $\mathrm{M}$ and a haloid $\mathrm{X}$ - that is, by expressing the salt by MX. In common table salt the metal is sodium, and the haloid an elementary body, chlorine. In sodium sulphate, $\mathrm{Na}_{2} \mathrm{SO}_{4}$, sodium is again the metal, but the complex group, $\mathrm{SO}_{4}$, is the haloid. In sulphate of copper, $\mathrm{CuSO}_{4}$, the metal is copper, and the haloid the same as in the preceding salt. Such a representation of salts expresses with great simplicity the capacity of every salt to enter into saline double decompositions with other salts; consisting in the mutual replacement of the metals in the salts. This exchange of their metals forms the fundamental property of salts. If there be two salts with different metals and haloids, and they be in solution or fusion, or any other manner, brought into contact, then the metals of these salts will always partially or wholly exchange places. If we designate one salt by MX, and the other by NY, then we either partially or wholly obtain from them new salts, MY and NX. Thus we saw in the Introduction, that on mixing solutions of table salt, $\mathrm{NaCl}$, and silver nitrate, $\mathrm{AgNO}_{3}$, a white insoluble precipitate of silver chloride, $\mathrm{AgCl}$, is formed, and a new salt, sodium nitrate, $\mathrm{NaNO}_{3}$, is obtained in solution. If the metals of salts exchange places in reactions of double decomposition, it is clear that metals themselves, taken in a separate state, are able to act on salts, as zinc evolves hydrogen from acids, and as iron separates copper from copper sulphate. When, to what extent, and which metals displace each other, and how the metals are distributed between the haloids, all this we will discuss later on, guided by those reflections and deductions which Berthollet introduced into the science at the beginning of this century.

According to the above observations, an acid is nothing more than a salt of hydrogen. Water itself may be looked on as a salt in which

56 In other cases the decomposition of salts by the electric current may be accompanied by much more complex results. Thus, when the metal of the salt is capable of a higher degree of oxidation, such a higher oxide may be formed at the positive pole by the oxygen which is evolved there. This takes place, for instance, in the decomposition of salts of silver and manganese by the galvanic current, peroxides of these metals being formed. If the metal separated at the negative pole acts on a salt occurring in the solution, then it may do so at this pole, and in this manner the phenomena of the action of a current on a salt are in many cases rendered remarkably complicated. But all the phenomena as yet known may be expressed by the above law-that the current decomposes salts into metals, which appear at the negative pole, and into the remaining component parts, which appear at the positive pole. 
the hydrogen is combined with either oxyen or the agueous radiele.

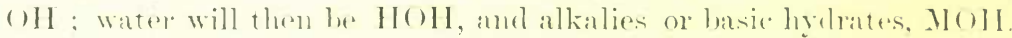

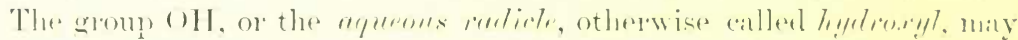

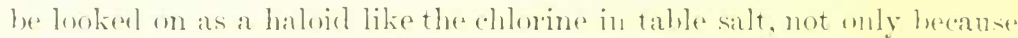
the element ('l and the group ()H very often chamere places, and combine with one and the silue element, but also becaluse frees ehlorine is

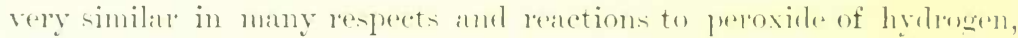
which is the same in composition an the arueous lateliele, as we shall afterwarels see. Alkalis and basic hrellates ale also salts comsisting of a metal and hylloxyl-for instance, (austieserla, Na()H ; this is therefore

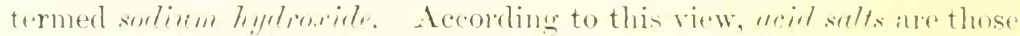
in which a portion only of the hyehereen is replaced hy a metal, anel a portion of the lyetrogen of the aciel remains. Thus sulphuric $\left(\mathrm{H}_{2} \mathrm{~s}()_{1}, 1\right.$

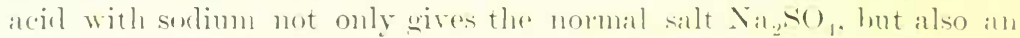
acid salt, Nalls(). Alusire selt is one in which the metal is combined unt only with the haloids of acifls. but also with the alpueous latieles of hasie hydrates - for example, bismuth gires not only a mormal salt

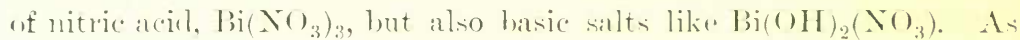
hasic ant acid salts contesponting with the oxygen acids contain hydrogen and oxyeren, they are therefore able to part with these as water anf to give anhyder-salts, which it is evilent will be erpual to (emprounts of nemand salts with anhydrites of the acids or with bases. Thus the above-mentioned acid sodium sulphate conesponts with

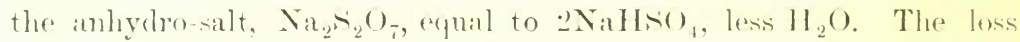
of water is here, and frequently in other cases, bremglit about lye

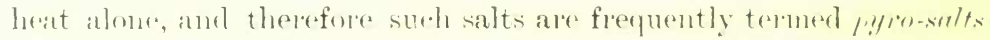
for instanee, the preceding is sodimu pyrosulphate ( $\left.\mathrm{Na}_{2} \mathrm{~s}_{2} \mathrm{C}_{7}\right)$, or it mily

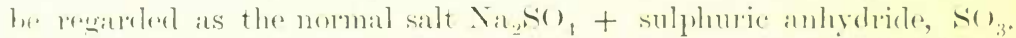

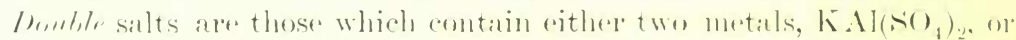
two hathinls.it

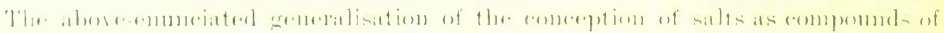

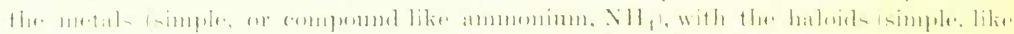

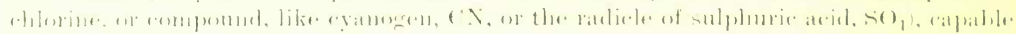

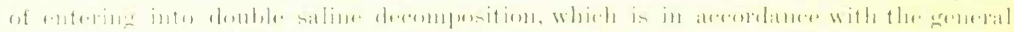

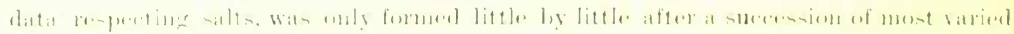

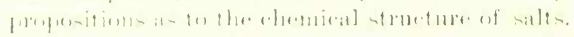

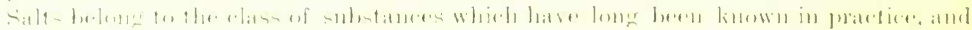

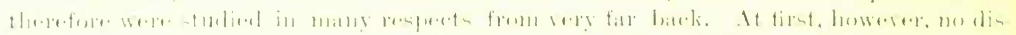

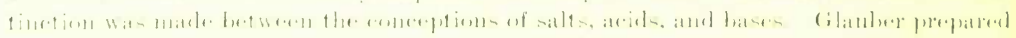

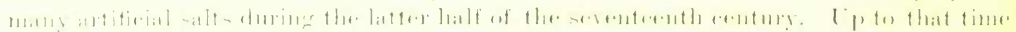

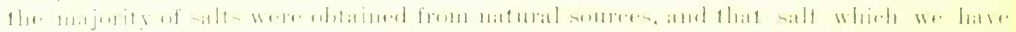

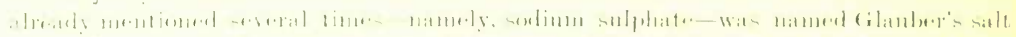

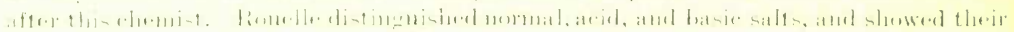

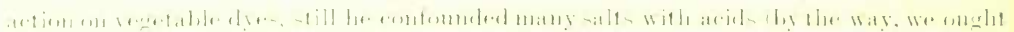

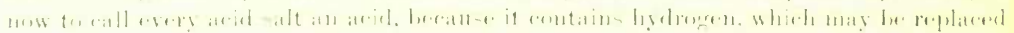


Inasmuch as oxygen compounds predominate in nature, it should be expected, from what has been said above, that the occurrence of salts, rather than of acids or bases, would be most frequent in nature, for the latter on meeting, especially under the medium of the all-per-

by metals-that is, it is the hydrogen of an acid). Baumé disputed Rouelle's opinion concerning the subdivision of salts, contending that normal salts only are true salts, and that basic salts are simple mixtures of normal salts with bases and acid salts with acids, considering that washing alone could remove the base or acid from them. Rouelle, in the middle of the last century, however, rendered a great service to the study of salts and the diffusion of knowledge respecting this class of compounds in his attractive lectures. $\mathrm{He}$, like the majority of the chemists of that period, did not employ the balance in his researches, but satisfied himself with purely qualitative data. The first quantitative researches on salts were carried on by Wenzel about this time. He was the director of the Freiburg mines, in Saxony. Wenzel studied the double decomposition of salts, and he observed that in the double decomposition of neutral salts a neutral salt was always obtained. He proved, by a method of weighing, that this is due to the fact that the saturation of a given quantity of a base requires such relative quantities of different acids as are capable of saturating every other base. Having taken two neutral salts-for example, sodium sulphate and calcium nitrate-let us mix their solutions together. Double decomposition takes place, because the almost insoluble calcium sulphate is formed. However much we might add of each of the salts, the neutral reaction will still be preserved, consequently the neutral character of the salts is not destroyed by the interchange of metals; that is to say, that quantity of sulphuric acid which saturated the sodium is sufficient for the saturation of the calcium, and that amount of nitric acid which saturated the calcium is enough to saturate the sodium contained in combination with sulphuric acid in sodium sulphate. Wenzel was even convinced that matter does not disappear in nature, and on this principle he corrects, in his Doctrine of Affinity, the results of his experiments when he remarked that he obtained less than he had originally taken. Although Wenzel deduced the law of the double decomposition of salts quite correctly, he did not determine those quantities in which acids and bases act on each other. This was done quite at the end of the last century by Richter. He determined the quantities by weight of the basea which saturate acids and of the acids which saturate bases, and he obtained comparatively correct results, although his conclusions were not correct, for he states that the quantity of a base saturating a given acid varies in arithmetical progression, and the quantity of an acid saturating a given base in geometrical progression. Richter studied the deposition of metals from their salts by other metals, and observed that the neutral reaction of the solution is not destroyed by this exchange. He also determined the quantities by weight of the metals replacing one another in salts. He showed that copper displaces silver from its salts, and that zinc displaces copper and a whole series of other metals. Those quantities of metals which were capable of replacing one another were termed equivalents.

Richter's teaching found no followers, because, although he fully believed in the discoveries of Lavoisier, yet he still held to the phlogistic reasonings which rendered his expositions very obscure. The works of the Swedish savant Berzelius freed the facts discovered by Wenzel and Richter from the obscurity of former conceptions, and led to their being explained in accordance with Lavoisier's views, and in the sense of the law of multiple proportions which had already been discovered by Dalton. On applying to salts those conclusions which Berzelius arrived at by a whole series of researches of remarkable accuracy, we are obliged to acknowledge the following law of equivalentsone part by weight of hydrogen in an acid is replaced by the corresponding equivalent weight of any metal ; and, therefore, when metals replace each other their weights are in the same ratio as their equivalents. Thus, for instance, one part by weight of hydrogen is replaced by 23 parts of sodium, 39 parts of potassium, 12 parts of nagnesium, 20 parts 
vading water, form salts. And, indeed, salts are found everywhere in nature. In animals and plants they occur, although in but small

of calcium, 28 parts of iron, 108 parts of silver, 33 parts of zinc, \&c.; and therefore, if zine replaces silver, then 33 parts of zinc will take the place of 108 parts of silver, or 33 parts of zinc will be substituted by 23 parts of sodium, \&c.

The doctrine of equivalents would be precise and simple did every metal only give one oxide or one salt. It is rendered complicated from the fact that many metals form several oxides, and consequently offer different equivalents in their different degrees of oxidation. For example, there are oxides containing iron in which its equivalent is 28 -this is in the salts formed by the suboxide; and there is another series of salts in which the equivalent of iron equals $18 \frac{2}{3}$-which contain less iron, and consequently more oxygen, and correspond with a higher degree of oxidation-ferric oxide. It is true that the former salts are easily formed by the direct action of metallic iron on acids, and the latter only by a further oxidation of the compound formed already; but this is not always so. In the case of copper, mercury, and tin, under different circumstances, there are formed salts which correspond with different degrees of oxidation of these metals, and many metals have two equivalents in their different salts - that is, in salts corresponding with the different degrees of oxidation. Thus it is impossible to endow every metal with one definite equivalent weight. Therefore the conception of equivalents, while playing an important part from an historical point of view, appears, with a fuller study of chemistry, to be but an incidental conception, subordinate to a higher one, with which we shall afterwards become acquainted.

The fate of the theoretical views of chemistry was for a long time bound up with the history of salts. The clearest representation of this subject dates back to Lavoisier, and was very severely developed by Berzelius. This representation is called the binary theory. All compounds, and especially salts, are represented as consisting of two parts. Salts are represented as a compound of a basic oxide (a base) and an acid (that is, an anhydride of an acid, then termed an acid), whilst hydrates are represented as compounds of anhydrous oxides with water. They employed such an expression not only to denote the most usual method of formation of these substances (which would be quite true), but also to express that internal distribution of the elements by which they proposed to explain all the properties of these substances. They supposed copper sulphate to contain two most intimate component parts-copper oxide and sulphuric anhydride. This is an hypothesis. It arose from the so-called electro-chemical hypothesis, which supposed the two component parts to be held in mutual union, because one component (the anhydride of the acid) has electro-negative properties, and the other (the base in salts) electro-positive. Both parts are attracted together, like substances having opposite electrical charges. But as the decomposition of salts in a state of fusion by an electric current always gives a metal, therefore the representation of the constitution and decomposition of salts, called the hydrogen theory of acids, is more probable than that considering salts as made up of a base and an anhydride of an acid. But the hydrogen theory of acids is also a binary hypothesis, and does not even contradict the electro-chemical hypothesis, but is rather a modification of it. The binary theory dates from Rouelle and Lavoisier, the electro-chemical representation was developed with great power by Berzelius, and the hydrogen theory of acids is due to Davy and Liebig.

These hypothetical representations simplified and generalised the study of a complicated subject, and gave support to arguments, but when salts were in question it was equally convenient to follow one or the other of these hypotheses. But these theories were brought to bear on all other substances, on all compound substances. Those holding the binary and electro-chemical lyppotheses searched for two anti-polar component parts, and endeavoured to express the process of chemical reactions by electrochemical and similar differences. If zinc replaces hydrogen, they concluded that it is 
amount, because, as forming the last stage of chemical reaction, they are capable of only a few chemical transformations, the energy of the elements being evolved (passing into heat) both in the formation of oxides and in their mutual combinations; hence in salts there remains but little energy. Organisms are bodies in which a series of uninterrupted, varied, and active chemical transformations proceed, whilst salts, which only enter into double decompositions between each other, are incapable of such changes. But organisms always contain salts. Thus, for instance, bones contain calcium phosphate, the juice of grapes, potassium tartrate (cream of tartar), certain lichens, calcium oxalate, and the shells of mollusca, calcium carbonate, \&c. As regards water and soil, portions of the earth in which the chemical processes are less active, they are full of salts. Thus the waters of the oceans, and all others (Chap. I.), abound in salts, and in the soil, in the rocks of the earth's crust, in the upheaved lavas, and in the falling meteorites the salts of silicic acid, and

more electro-positive than hydrogen, whilst they forgot that hydrogen may, under different circumstances, displace zinc-for instance, at a red heat. Chlorine and oxygen were considered as being of opposite polarity to hydrogen because they easily combine with it, whilst one and the other are capable of replacing hydrogen, and, what is very characteristic, in the replacement of hydrogen by chlorine in carbon compounds, not only does the chemical character often remain unaltered, but even the external form remains unchanged, as Laurent and Dumas demonstrated. These considerations undermine the binary theory, and especially the electro-chemical system. An explanation of known reactions then began to be sought for not in the difference of the polarity of the different substances, but in the joint influences of all the elements on the properties of the compound formed. This is the reverse of the preceding hypotheses.

This reversal was not, however, limited to the destruction of the tottering founda. tions of the preceding theory; it projected a new doctrine, and laid the foundation for the whole contemporary direction of our science. This doctrine may be termed the unitary theory - that is, it is such as strictly acknowledges the joint influences of the elements in a compound substance, denies the existence of separate and contrary components in them, regards copper sulphate, for instance, as a strictly definite compound of copper, sulphur, and oxygen; then seeks for compounds which are analogous in their properties, and, placing them side by side, endeavours to express the influence of each element on the united properties of its compound. In the majority of cases it arrives at systems of consideration similar to those which are obtained by the above-mentioned hypotheses but in certain special cases the conclusions of the unitary theory are in entire opposition to the binary theory and its consequences. Cases of this kind are most often met with in the consideration of compounds of a more complex nature than salts, especially organic compounds containing hydrogen. But it is not in this revolution from an artificial to a natural system, important as it is, that the chief service and strength of the unitary doctrine lies. By a simple review of the vast store of data regarding the reactions of typical substances, it succeeded from its first appearance in establishing a new and important law, it introduced a new conception into science-namely, the conception of molecules, with which we shall soon become acquainted. The deduction of the law and of the conception of molecules bas been verified by facts in a number of cases, and was the cause of the majority of chemists of our times deserting the binary theory and accepting the unitary theory, which forms the basis of the present work. Laurent and Gerhardt must be looked on as the propagators of this doctrine. 


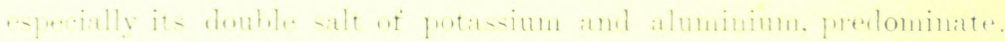

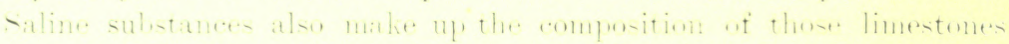

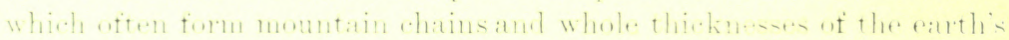

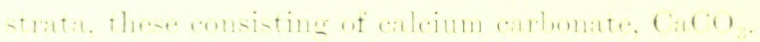

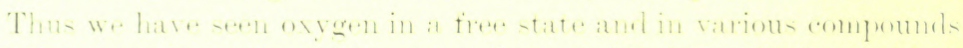

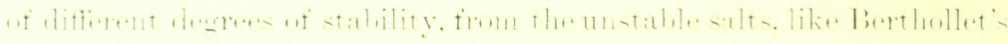

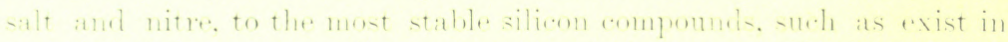

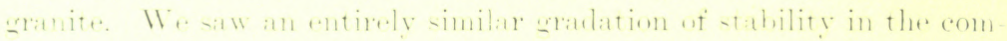

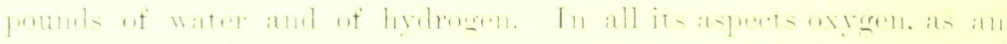

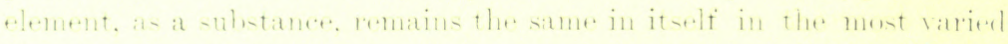

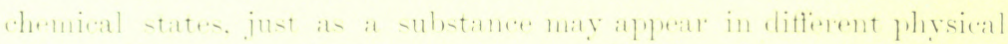

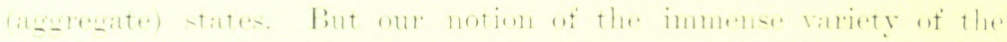

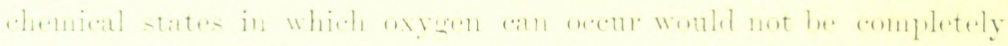

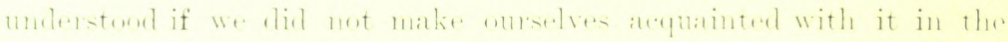

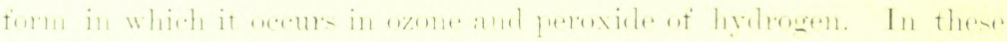

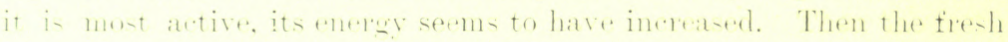

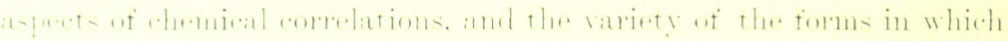

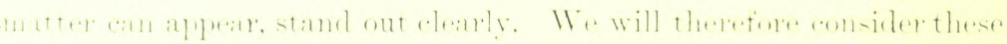
wo-uhistances somewhat in detail. 


\section{CHAPTER IV}

OZONE AND HYDROGEN PEROXIDE. DALTON'S LAW

VAN-MARUM, during the last century, observed that oxygen in a glass tube, when subjected to the action of a series of electric sparks, acquired a peculiar smell and the property of combining with mercury at the ordinary temperature. This was afterwards confirmed by a number of fresh experiments. Even in the simple revolution of an electrical machine, when electricity diffuses into the air or passes through it, the peculiar and characteristic smell proper to ozone, proceeding from the action of the electricity on the oxygen of the atmosphere, is recognised. In 1840 Prof. Schönbein, of Basle, turned his attention to this odoriferous substance, and showed that it is also formed, with the oxygen evolved at the positive pole, in the decomposition of water by the action of a galvanic current; in the oxidation of phosphorus in damp air, and also in the oxidation of a number of substances, in consequence of which it is found in the atmosphere, although it is distinguished for its instability and capacity for oxidising other substances. The characteristic smell of this substance (which is always mixed with unaltered oxygen) gave it its name, from the Greek ő $\zeta$, ' to emit an odour.' Schönbein pointed out the characterjstic properties of ozone, and especially its power of oxidising many substances, even silver, acting like oxygen, but with this difference-that there are a number of substances on which oxygen does not act at the ordinary temperature, whilst ozone does so very energetically. It will be enough to point out, for instance, that it oxidises silver, mercury, charcoal, and iron with great energy at the ordinary temperature. It might be thought that ozone was some new substance, simple or compound, as it was at first supposed to be; but careful observations made in this direction have long led to the conclusion that ozone is noth ing but oxygen altered in its properties. This is most strikingly proved by the complete transformation of oxygen containing ozone into ordinary oxygen when it is passed through a tube heated to $250^{\circ}$. Further, at a low temperature pure oxygen gives ozone when electric 


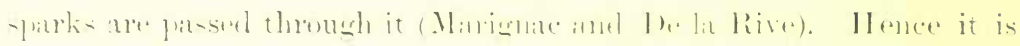

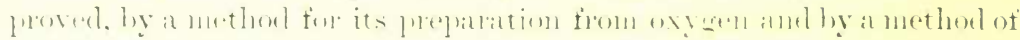

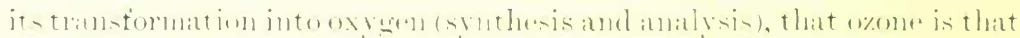

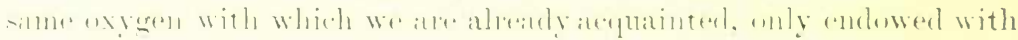

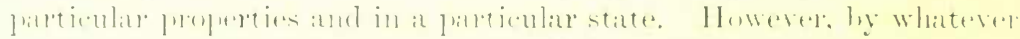

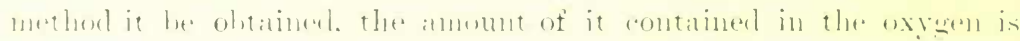

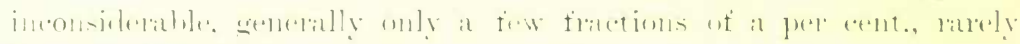

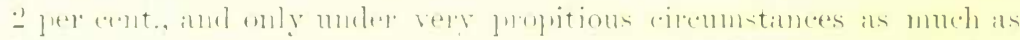

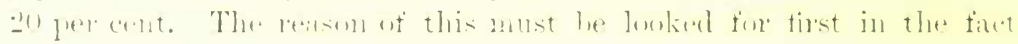

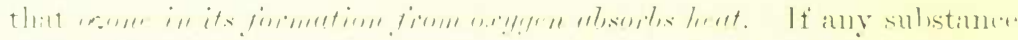

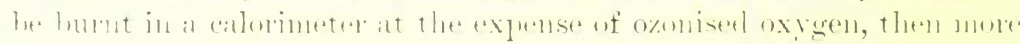

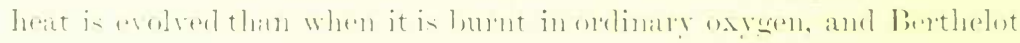

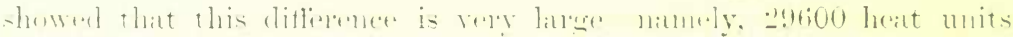

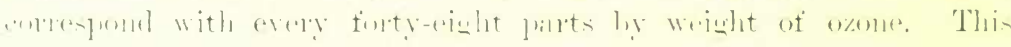

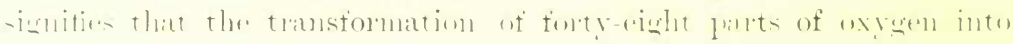

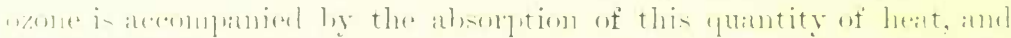

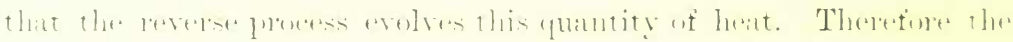

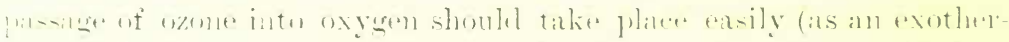
mat latetion). like (a)

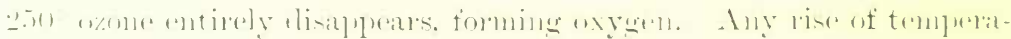

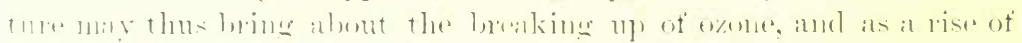

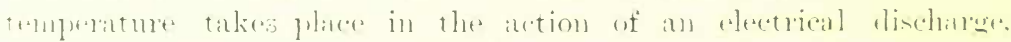

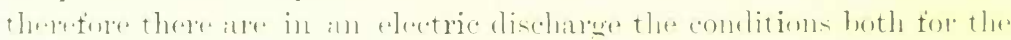

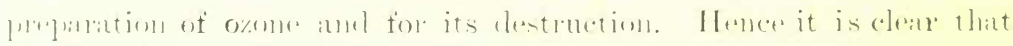

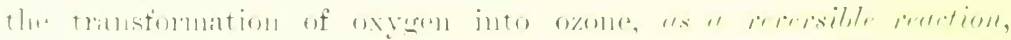

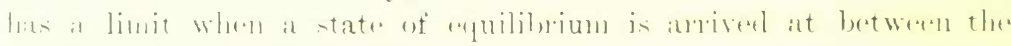

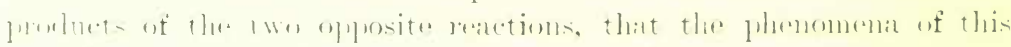

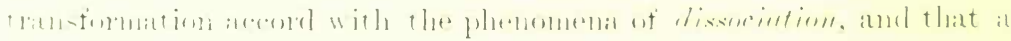

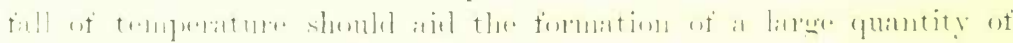

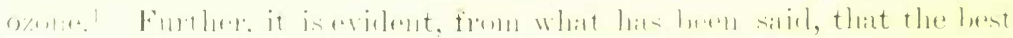

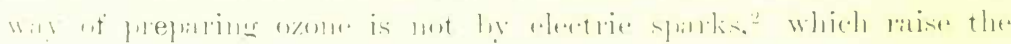

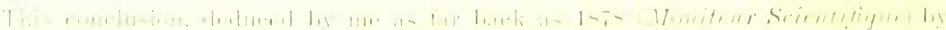

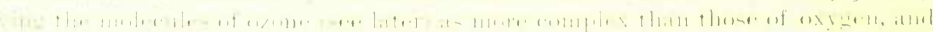

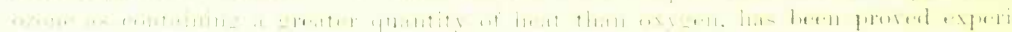

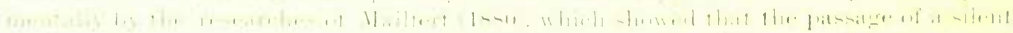

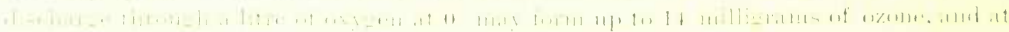

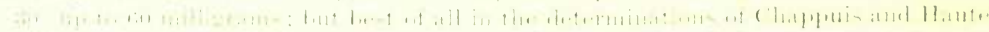

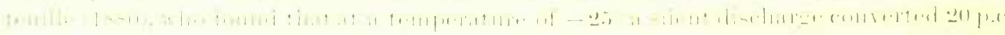

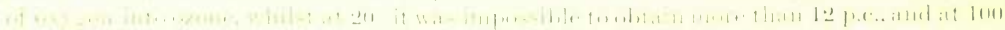

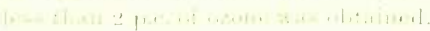

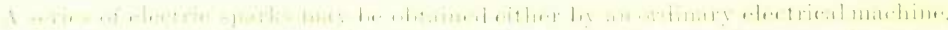

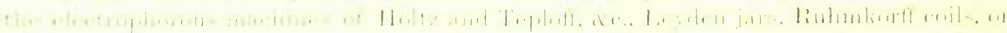

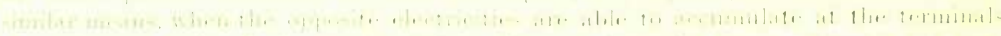

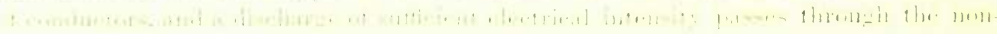


temperature, but by the employment of a continual discharge or flow of electricity - that is, to transform the oxygen by the action of a silent discharge. ${ }^{3}$ For this reason all ozonisers (which are of most varied construction), or forms of apparatus for the preparation of ozone from oxygen (or air) by the action of electricity, now usually consist of conductors (sheets of metal - for instance, tinfoil - or a solution of sulphuric acid with chromic acid, \&c.) separated by thin glass surfaces placed at short distances from each other, and between which

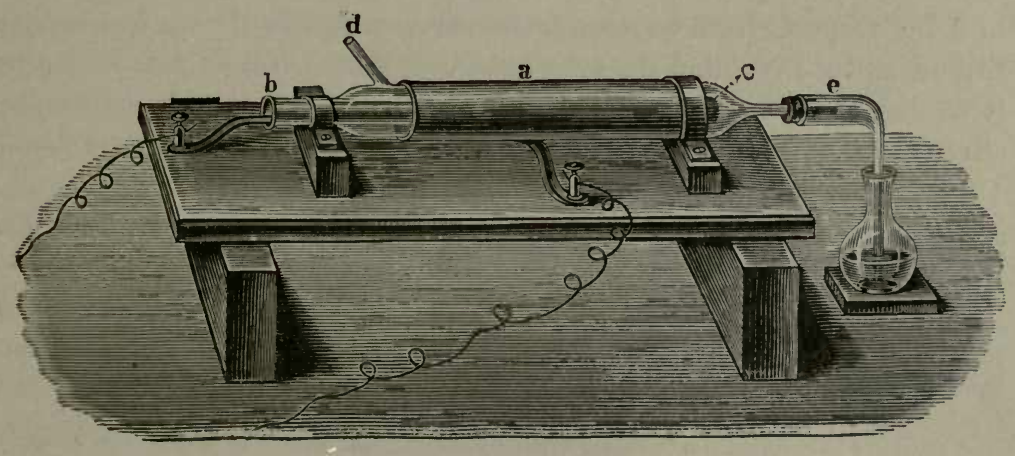

FIG. 37.-Siemens' apparatus for preparing ozone by means of a silent discharge.

the oxygen or air to be ozonised is introduced and subjected to the action of a silent discharge. ${ }^{4}$ Thus in Siemens' apparatus (fig. 37 ) the

3 A silent discharge is such a combination of opposite statical (potential) electricities as takes place (generally between large surfaces) regularly, without sparks, slowly, and quietly (as in the dispersion of electricity). The discharge is only luminous in the dark; there is no observable rise of temperature, and therefore a larger amount of ozone is formed. But, nevertheless, on continuing the passage of a silent discharge through ozone it is destroyed. For the action to be observable a large surface is necessary, and consequently a powerful source of electrical potential. For this reason the slent discharge is best produced by a Ruhmkorff coil, as the most handy means of obtaining a considerable potential of statical electricity with the employment of the comparatively feeble current of a galvanic battery.

$4 V$. Babo's apparatus was one of the first constructed for ozonising oxygen by means of a silent discharge (and it is still one of the best). It is composed of a number (twenty and more) of long, thin capillary glass tubes closed at one end. A platinum wire, extending along their whole length, is introduced into the other end of each tube, and this end is then fused up round the wire, the end of which protrudes outside the tube. The protruding ends of the wires are arranged alternately in two sides in such a manner that on one side there are ten closed ends and ten wires. A bunch of such tubes (forty should make a bunch of not more than $1 \mathrm{c.m}$. diameter) is placed in a glass tube, and the ends of the wires are connected into two conductors, and are fused to the ends of the surrounding tube. The discharge of a Rulunkorff coil is passed through these ends of the wires, and the dry air or oxygen to be ozonised is passed through the tube. If oxygen be passed through, ozone is obtained in large quantities, and free from oxides of 
exterior of the tube $a$ and the interior of the tube $b c$ are coated with tinfoil and connected with the poles of a source of electricity (with the terminals of a Ruhmkorff's coil). A silent discharge passes through the thin walls of the glass cylinders $a$ and $b c$ over all their surfaces, and eonsequently, if oxygen be passed through the apparatus by the tube $d$, fused into the side of $a$, it will be ozonised in the annular space between $a$ and $b c$. The ozonised oxygen escapes by the tube $e$, and may be introduced into any other apparatus. ${ }^{5}$

1. The properties of ozone obtained by such a method ${ }^{6}$ distinguish it in many respects from oxygen. Ozone very rapidly decolorises indigo, litmus, and many other dyes by oxidising them. Silver is oxidised by it at the ordinary temperature, whilst oxygen is not able to oxidise silver even at high temperatures; a bright silver plate rapidly turns

nitrogen, which are partially formed when air is acted on. It is remarked that at low temperatures ozone is formed in large quantities. As ozone is acted on by corks and india-rubber, the apparatus should be made entirely of glass. With a powerful Rulumkorff coil and forty tubes the ozonation is so powerful that the gas, when passed through a solution of iodide of potassium, not only sets the iodine free, but even oxidises it into potassium iodate, so that in five minutes the gas-conducting tube is choked up with crystals of the insoluble iodate.

5 In order to connect the ozoniser with any other apparatus it is impossible to make use of india-rubber, mercury, or cements, \&c., because they are themselves acted on by, and act on, ozone. All connections must, as was first proposed by Brodie, be hermetically closed by sulphuric acid, which is not acted on by ozone. Thus, a cork is passed over the vertical end of a tube, over which a wide tube passes so that the end of the first tube protrudes above the cork; mercury is first poured over the cork (to prevent its being acted on by the sulphuric acid), and then sulphuric acid is poured over the mercury. The protruding end of the first tube is covered by the lower end of a third tube immersed in the sulphuric acid.

6 The above-described method is the only one which has been well investigated. The admixture of nitrogen, or even of hydrogen, and especially of silicon fluoride, appears to aid the formation and preservation of ozone. Amongst other methods for preparing ozone we may mention the following:-1. In the action of oxygen on phosphorus at the ordinary temperaturc a portion of the oxygen is converted into ozone. At the ordinary temperature a stick of phosphorus, partially immersed in water and partially in air in a large glass vessel, causes the air to acquire the odour of ozone. It must further be remarked that if the air be left for long in contact with the phosphorus, or without the presence of water, the ozone formed is destroyed by the phosphorus. 2. By the action of sulphuric acid on peroxide of barium. If the latter be covered with strong sulphuric acid (the acid, if diluted with only one-tenth of water, does not give ozone), then at a low temperature the oxygen evolved contains ozone, and in much greater quantities than that in which ozone is obtained by the action of electric sparks or phosphorus. 3. Ozone may also be obtained by decomposing strong sulphuric acid by potassium manganate, especially with the addition of barium peroxide. Gorup-Besanez stated (but it requires confirmation) that ozone is formed in the slow evaporation of large quantities of water. In the near proximity of salt-gardens (salterns) the atmosphere is considerably richer in ozone than in the surrounding neighbourhood. In connection with this is the fact that the air of the sea-shore is rich in ozone. Ozone is also stated to be formed in the ordinary process of the respiration of plants. This is, however, denied by many to be the case. 
black (from oxidation) in ozonised oxygen. It is rapidly absorbed by mercury, forming oxide ; it transforms the lower oxides into higher-for instance, sulphurous anhydride into sulphuric, nitrous oxide into nitric, arsenious anhydride $\left(\mathrm{As}_{2} \mathrm{O}_{3}\right)$ into arsenic anhydride $\left(\mathrm{As}_{2} \mathrm{O}_{5}\right)$ \&c. ${ }^{7}$ But what is especially characteristic in ozone is the decomposing action it exerts on potassium iodide. Oxygen does not act on it, but ozone passed into a solution of potassium iodide liberates iodine, whilst the potassium is obtained as caustic potash, which remains in solution, $2 \mathrm{KI}+\mathrm{H}_{2} \mathrm{O}+\mathrm{O}=2 \mathrm{KHO}+\mathrm{I}_{2}$. As the presence of minute traces of free iodine may be discovered by means of starch paste, with which it forms a very dark blue coloured substance, a mixture of potassium iodide with starch paste will detect the presence of very small traces of ozone. $^{8}$ Ozone is destroyed or converted into ordinary oxygen not only by heat, but also by long keeping, especially in the presence of alkalis, peroxide of manganese, chlorine, drc.

Hence ozone, although it has the same composition as oxygen, differs

7 Ozone takes up the hydrogen from hydrochloric acid; the chlorine is set free, and can dissolve gold. Chromium and iodine are directly oxidised by ozone, but not by oxygen, and so also with a number of other substances. Ammonia, $\mathrm{NH}_{3}$, is oxidised by ozone into anmonium nitrite (and nitrate), $2 \mathrm{NH}_{3}+\mathrm{O}_{3}=\mathrm{NH}_{4} \mathrm{NO}_{2}+\mathrm{H}_{2} \mathrm{O}$, and therefore a drop of ammonia, on falling into the gas, gives a thick cloud of the salts formed. Ozone converts lead oxide into peroxide, and suboxide of thallium (which is colourless) into oxide (which is brown), so that this reaction is made use of for discovering the presence of ozone. Lead sulphide, $\mathrm{PbS}$, is converted into sulphate, $\mathrm{PbSO}_{4}$, by ozone. A neutral solution of manganese sulphate gives a precipitate of manganese peroxide, and an acid solution may be oxidised into permanganic acid, $\mathrm{HMnO}_{4}$. With respect to the oxidising action of ozone on organic substances, it may be mentioned that with ether, $\mathrm{C}_{4} \mathrm{H}_{10} \mathrm{O}$, ozone gives ethyl peroxide, which is capable of decomposing with explosion (according to Berthelot), and is decomposed by water into alcohol, $2 \mathrm{C}_{2} \mathrm{H}_{e} \mathrm{O}$, and hydrogen peroxide, $\mathrm{H}_{2} \mathrm{O}_{2}$.

8 This reaction is the one usually made use of for detecting the presence of ozone. In the majority of cases paper is soaked in solutions of potassium iodide and starch. Such ozonometrical or iodised starch-paper when damp turns blue in the presence of ozone, and the tint obtained varies considerably, according to the length of time it is exposed and to the amount of ozone present. The amount of ozone in a given gas may eren to a certain degree be judged by the shade of colour acquired by the paper, if prelininary tests be made.

Test-paper for ozone is prepared in the following manner:-One gram of neutral potassium iodide is dissolved in 100 grams of distilled water; 10 grams of starch are then shaken up in the solution, and the mixture is boiled until the starch is converted into a jelly. This jelly is then smeared over blotting-paper and left to dry. The colour of iodised starch-paper is changed not only by the action of ozone, but of many other oxidisers; for example, by the oxides of nitrogen and hydrogen peroxide. Houzeau proposed soaking common litmus-paper with a solution of potassium iodide, which in the presence of iodine would turn blne, owing to the formation of KHO. In order to find if the blue colour is not produced by an alkali (ammonia) in the gas, a portion of the paper is not soaked in the potassium iodide, but moistened with water; this portion will then also turn blue if ammonia be present. A reagent for distinguishing ozone from hydrogen peroxide with certainty is not known, and therefore these substances in very small quantities (for instance, in the atmosphere) may easily be confounded. 
from it in stability, and by the fact that it oxidises a number of substances very energetically at the ordinary temperature. In this respect ozone resembles the oxygen of certain unstable compounds, or oxygen at the moment of its liberation.

In ordinary oxygen and ozone we see an example of one and the same substance, in this case an element, appearing in two states. This indicates that the properties of a substance, and even of an element, may vary without its composition varying. Very many such cases are known. Such cases of a chemical transformation which determines a difference in the properties of one and the same element are termed isomerism. The cause of isomerism evidently lies deep within the essence of the nature of a substance, and its investigation has already led to a number of results of unexpected importance and of immense scientific significance. It is easy to understand the difference between substances containing different elements or the same elements in different proportions. That a difference should exist in the latter case necessarily follows, if, as our knowledge compels us, we admit that there is a radical difference in the simple bodies or elements. But when the quality and quantity of the elements (the composition) in a substance are the same and yet its properties are different, then it becomes clear that the conceptions of the elements and of the composition of compounds, alone, are insufficient for the expression of all the diversity of the properties of the matter of nature. Something else, still more profound and internal than the composition of substances, must, judging from isomerism, determine the properties and transformation of substances.

On what is the isomerism of ozone with oxygen, and the peculiarities of ozone, dependent? In what, besides the store of energy, which in its way expresses the peculiarities of ozone, resides the causes of its difference from oxygen? These questions for long occupied the minds of investigators, and were the motive for the most varied, exact, and accurate researches, which were chiefly directed to the study of the volumetric relations exhibited by ozone. In order to acquaint the reader with the previous researches of this kind, I cite the following from a memoir by Soret, in the 'Transactions of the French Academy of Sciences' for 1866:

' Our present knowledge of the volumetric relations of ozone may be expressed at the present time in the following manner :

'1. "Ordinary oxygen in changing into ozone under the action of electricity shows a diminution in volume." This was discovered by Andrews and Tait.

'2. "In acting on ozonised oxygen with potassium iodide and other substances capable of being oxidised, we destroy the ozone, but the 
volume of the gas remains unchanged." Indeed, the researches of Andrews, Soret, v. Babo, and others showed that the quantity of oxygen absorbed by the potassium iodide is equal to the original contraction of the volume of the oxygen-that is, in the absorption of the ozone the volume of the gas remains unchanged. From this it might be imagined that ozone, so to say, does not occupy any room-is indefinitely dense.

'3. "By the action of heat ozonised oxygen increases in volume, and is transformed into ordinary oxygen. This increase in volume corresponds with the quantity of oxygen which is given up to the potassium iodide in its decomposition "(the same observers).

'4. These indubitable experimental results lead to the conclusion that ozone is denser than oxygen, and that ozone in its oxidising action gives off that portion of its substance which distinguishes it by its density from ordinary oxygen.'

If we imagine (says Weltzien) that $n$ volumes of ozone consist of $n$ volumes of oxygen combined with $m$ volumes of the same substance, and that ozone in oxidising gives up $m$ volumes of oxygen and leaves $n$. volumes of oxygen gas, then all the above facts can be explained; otherwise it must be supposed that ozone is indefinitely dense. "In order to determine the density of ozone (we again cite Soret) recourse cannot be had to the direct determination of the weight of a given volume of the gas, because ozone cannot be obtained in a pure state. It is always mixed with a very large quantity of oxygen. It was necessary, therefore, to have recourse to such substances as would absorb ozone without absorbing oxygen and without destroying the ozone. Then the density might be deduced from the decrease of volume produced in the gas by the action of this solvent in comparison with the quantity of oxygen given up to potassium iodide. Advantage must also be taken of the determination of the increase of volume produced by the action of heat on ozone, if the volume previously occupied by the ozone before heating be known.' Soret found two such substances, turpentine and oil of cinnamon. 'Ozone disappears in the presence of turpentine. This is accompanied by the appearance of a dense vapour, which fills a vessel of small capacity ( 0.14 litre) to such an extent that it is impenetrable to direct sun-rays. On then leaving the vessel at rest, it is observed that the cloud of vapour settles; the clearing is first remarked at the upper portion of the vessel, and the brilliant colours of the rainbow are seen on the edge of cloud of vapour.' Oil of cinnamon - that is, the volatile or odoriferous substance of the well-known spice, cinnamon-gives under similar circumstances the same kind of vapours, but they are much less voluminous. On 


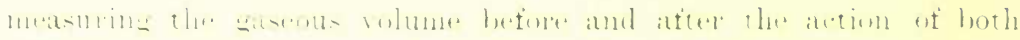

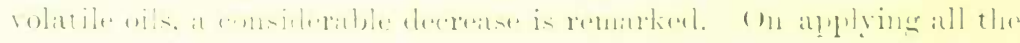

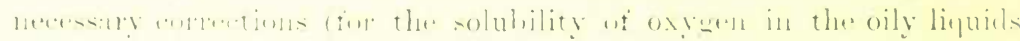

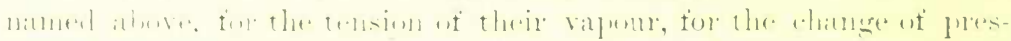

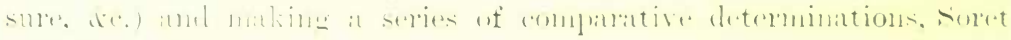

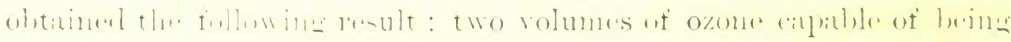

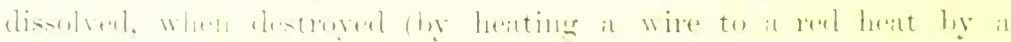

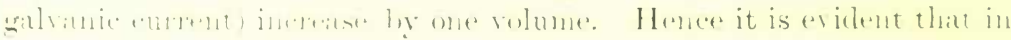

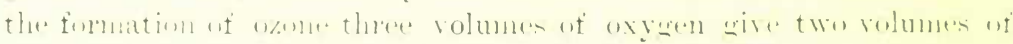

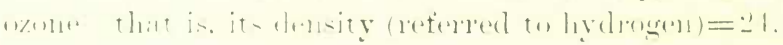

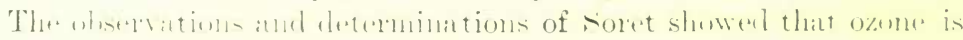

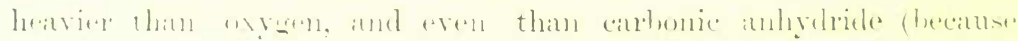

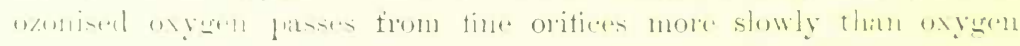

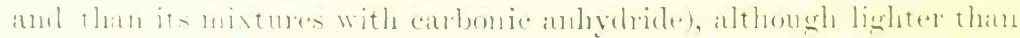

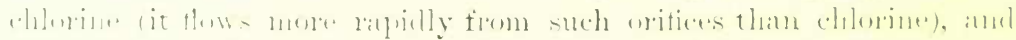

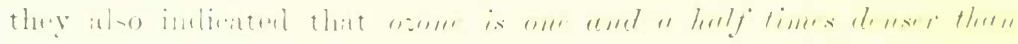

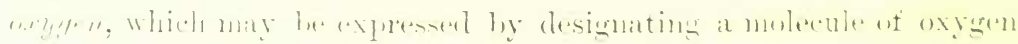

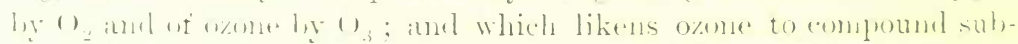

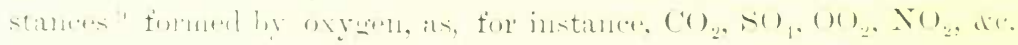

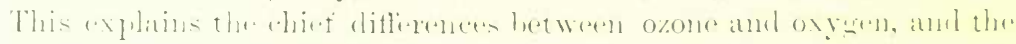

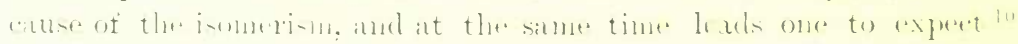

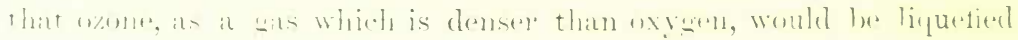

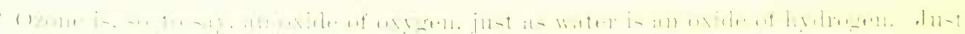

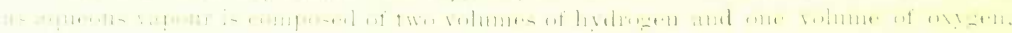

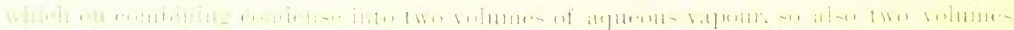

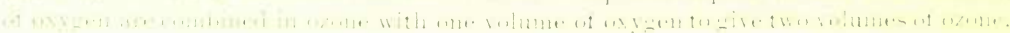

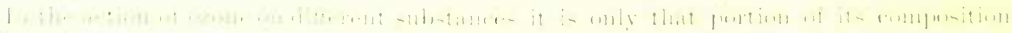

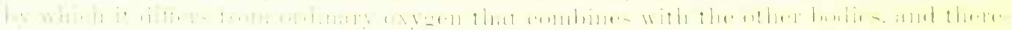

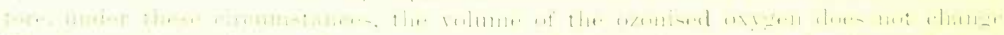

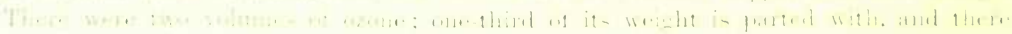

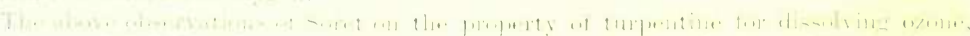

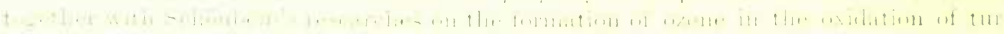

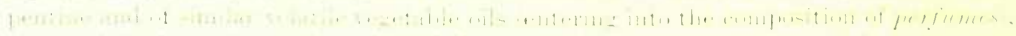

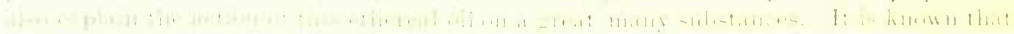

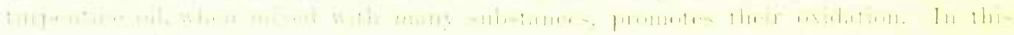

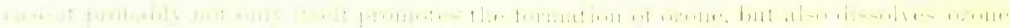

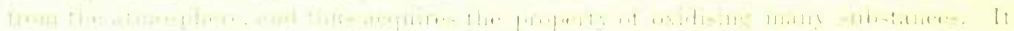

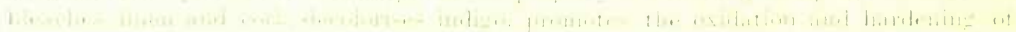

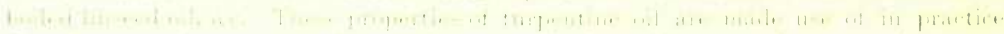

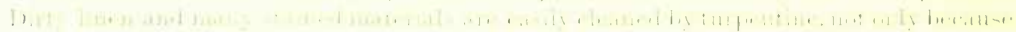

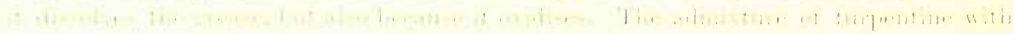

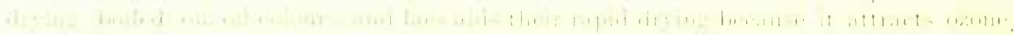

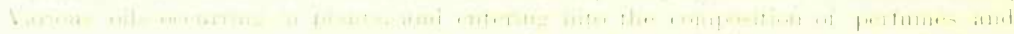

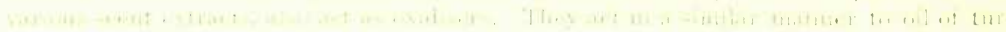

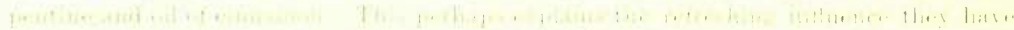

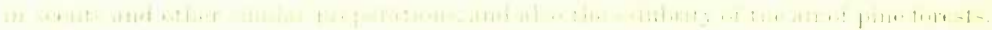

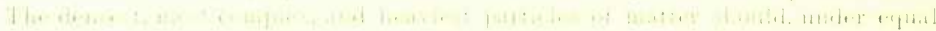


much more easily. This was actually shown to be the case, in 1880, by Chappuis and Hautefeuille in their researches on the physical properties of oxygen. Its absolute boiling point is about $-106^{\circ}$, and consequently compressed and refrigerated ozone when rapidly expanded gives drops, is liquefied. Liquid and compressed "ozone is blue. In dissolving in water ozone partly passes into oxygen. Ozone violently explodes when suddenly compressed and heated, changing into ordinary oxygen, and evolving, like all explosive substances, ${ }^{12}$ that heat which distinguishes it from oxygen.

Thus, judging by what has been said above, ozone should be formed in nature not only in the many processes of oxidation which go on, but also by the condensation of atmospheric oxygen. The significance of ozone in nature has often arrested the attention of observers. There is a series of ozonometrical observations which show the different amounts of ozone in the air at different localities, at different times of the year, and under different circumstances-for instance, on the appearance of epidemics. But the observations made in this direction cannot be considered as sufficiently exact, because the methods in use for determining ozone were not quite accurate. It is however indisputable ${ }^{13}$ that the amount of ozone in the atmosphere is subject to variation; that the air of dwellings contains no ozone (it disappears in oxidising organic matter) ; that the air of fields and forests always contains ozone, or substances (peroxide of hydrogen) which act like it; that the amount of ozone increases after storms; and that miasms, \&c., are destroyed by ozonising the atmosphere. It may be imagined that the influence exerted by ozone on animal life is due to the fact that it easily oxidises organic substances, and miasms are formed of organic substances and the germs of organisms, which are easily changed and oxidised. Indeed, many miasms-for instance,

conditions, evidently be less capable of passing into a state of gaseous movement, should sooner attain a liquid state, and have a greater cohesive force.

11 The blue colour proper to ozone may be seen through a tube one metre long containing oxygen 10 p.c. ozonised. The density of liquid ozone has not, as far as I am aware, been determined.

12 All explosive bodies and mixtures (gunpowder, detonating gas, \&c.) evolve heat in exploding (in giving a greater number of molecules from one molecule, and sometimes several substances from one substarce, as in the explosion of nitro-compounds; see later)that is, the reactions which accompany explosions are exothermal. In this manner ozone in decomposing evolves latent heat, although generally heat is absorbed in decomposition. This shows the meaning and cause of explosion.

13 In Paris it has been found that the further from the centre of the town the greater the amount of ozone in the air. The reason of this is evident: in a city there are many conditions for the destruction of ozone. This is why we distinguish country air as being fresh. In spring the air contains more ozone than in autumn; the air of fields more than the air of towns. 
the volatile substance of decomposing organisms - are clearly destroyed or changed not only by ozone, but also by many powerfully oxidising. substances, such as chlorine with water, potassium permanganate, and the like. ${ }^{14}$

Thus in ozone we see (1) the capacity of elements (and it must be all the more marked in compounds) of changing in properties without altering in composition; this is termed isomerism $;^{15}$ (2) the capacity of elements for arranging themselves in molecules of.different densities ; this forms a special case of isomerism called polymerism; (3) the capacity of oxygen for appearing in a still more intense and energetic chemical state than that in which it occurs in ordinary gaseous oxygen; and (4) the formation of unstable equilibria, or chemical states, which are expressed both by the ease with which ozone acts as an oxidiser and in its capacity for decomposing with explosion. ${ }^{16}$

Hydrogen peroxide.-Many of those properties which we have seen in ozone belong also to a peculiar substance containing oxygen and hydrogen, and called hydrogen peroxide, or oxygenated water. This substance was discovered in 1818 by Thénard. When heated it is decomposed into water and oxygen, evolving as much oxygen as is contained in the water remaining after the decomposition. That portion of oxygen by which hydrogen peroxide differs from water behaves in a number of cases just like the active oxygen in ozone, which distinguishes it from ordinary oxygen. In $\mathrm{H}_{2} \mathrm{O}_{2}$, and in $\mathrm{O}_{3}$, one atom of oxygen acts in a powerfully oxidising manner, and on separating out

14 The oxidising action of ozone may be taken advantage of for technical ends; for instance, for destroying colouring matters. It has even been employed for bleaching tissues and for the rapid preparation of vinegar, although these methods have not yet received wide application.

15 Isomerism in elements is termed allotropism.

16 A number of substances resemble ozone in one or another of these respects. Thus cyanogen, $\mathrm{C}_{2} \mathrm{~N}_{2}$, nitrogen chloride, \&c., decompose with an explosion and evolution of heat. Nitrous anhydride, $\mathrm{N}_{2} \mathrm{O}_{3}$, forms a blue liquid like ozone, and in a number of cases oxidises like ozone. Red phosphorus is to white phosphorus, in a certain sense, what oxygen is to ozone, and in other respects the reverse; this is also a case of allotropism. Thus a chemical analogy is diffused in different and most varied directions, and it is only after an acquaintance with the diverse relations of substances that an idea can be formed of the complexity of chemical changes, whilst their general system is still wanting; that is to say, there is nothing analogous to and explaining the correlation of liquid to gaseous substances. But there is reason to think that in this case also an explanation will arise with the accumulation of data, as we see from the fact that the conception of dissociation explained in the simplest manner a number of chemical relations which without it were not at all clear. It should be here observed that the transition between oxygen and ozone under the conditions of a silent discharge forms a reversible reaction which is subject to the conception of dissociation, whilst, exempt from the conditions of a silent discharge, the passage of ozone into oxygen is not reversible, and forms an instance of decomposition in the strictest sense. 
it leaves $\mathrm{H}_{2} \mathrm{O}$ or $\mathrm{O}_{2}$, which do not act so sharply, although they still contain oxygen. ${ }^{17}$ Both contain the oxygen in a compressed state, so to speak, and when freed from pressure by the forces (internal) of the elements in another substance, this oxygen is easily evolved, and therefore acts like oxygen at the moment of its liberation. Both substances in decomposing, with the separation of a portion of their oxygen, evolve heat, while an absorption of heat is usually required for decomposition.

Hydrogen peroxide is formed under many circumstances by combustion and oxidation, but in very limited quantities ; thus, for instance, it is sufficient to shake up zinc with sulphuric acid, or even with water, tn remark the formation of a certain quantity of hydrogen peroxide in the water. ${ }^{18}$ From this cause, probably, a series of diverse oxidation processes are accomplished in nature, and, according to Prof. Schöne, of Moscow, hydrogen peroxide occurs in the atmosphere, although in variable and small quantities, and probably its formation is connected with ozone, with which it has much in common. The usual case of the formation of hydrogen peroxide, and the means by which it may be in-

17 It is evident that there is a want of words here for distinguishing oxygen, $O$, as an ultimate element, from oxygen, $\mathrm{O}_{2}$, as a free element. It should be called oxygen gas, did not habit and the length of the expression render it inconvenient.

18 Schönbein states that the formation of hydrogen peroxide is to be remarked in every oxidation in water or in the presence of aqueous vapour. According to Struve, hydrogen peroxide is contained in snow and in rain-water, and its formation, together with ozone and ammonium nitrate, is even probable in the processes of respiration and combustion. A solution of tin in mercury, or liquid tin amalgam, when shaken up in water containing sulphuric acid gives rise to the formation of hydrogen peroxide, whilst iron under the same circumstances does not give rise to its formation. The presence of small quantities of hydrogen peroxide in these and similar cases is recognised by many reactions. Amongst them, its action on chromic acid in the presence of ether is very characteristic. $\mathrm{Hydrogen}$ peroxide converts the chromic acid into a higher oxide, $\mathrm{Cr}_{2} \mathrm{O}_{7}$, which is of a dark-blue colour, and dissolves in ether. This ethereal solution is to a certain degree stable, and therefore the presence of hydrogen peroxide may be recognised by mixing the liquid to be tested with ether and adding several drops of a solution of chromic acid. On shaking the mixture the ether dissolves the higher oxide of chromium which is formed, and acquires a blue colour. The formation of bydrogen peroxide in the combustion and oxidation of substances containing or evolving hydrogen must be understood in the sense of the conception, to be considered later, of molecules occupying equal volumes in a gaseous state. At the moment of its evolution a molecule $\mathrm{H}_{2}$ combines with a molecule $\mathrm{O}_{2}$ and gives $\mathrm{H}_{2} \mathrm{O}_{2}$. As this substance is unstable, a large proportion of it is decomposed, a small amount only remaining unchanged. If it is obtained, water is easily formed from it; this reaction evolves heat, and the reverse action is not very pro. bable. Direct determinations show that the reaction $\mathrm{H}_{2} \mathrm{O}_{2}=\mathrm{H}_{2} \mathrm{O}+\mathrm{O}$ evolves 22000 heat units. From this it will be understood how easy is the decomposition of hydrogen peroxide, as well as the fact that a number of substances which are not directly oxidised by oxygen are oxidised by hydrogen peroxide and by ozone, which also evolres heat on decomposition. Such a representation of the origin of hydrogen peroxide has been developed by me since 1870 . In recent times Traube has pronounced a similar opinion. 
directly obtained, ${ }^{19}$ is by the double decomposition of an acid and the peroxides of certain metals, especially those of potassium. calcium, and barium. ${ }^{20}$ Among these peroxides, that of barium is the most conveniently obtained, it being enough, as we saw when speaking of oxygen (Chap. III.), to heat the anhydrous oxide of barium to a red heat in a current of air or oxygen ; or, better still, to heat it with potassium ehlorate, and then to wash away the potassium chloride also formed. ${ }^{21}$ Barium peroxide gives hydrogen peroxide by the action of acids in the cold. ${ }^{22}$ The process of decomposition is very clear in this case; the hydrogen of the acid replaces the barium of the peroxide, a barium salt of the acid being formed, while the hydrogen peroxide formed by the

19 The formation of hydrozen peroxide from barium peroxide by a method of double decomposition is an instance of a number of indirect methods of preparation. A substance $\mathrm{A}$ does not combine with $\mathrm{B}$, but $\mathrm{AB}$ is obtained from $\mathrm{AC}$ in its action on $\mathrm{BD}$ (see Introduction) when $\mathrm{CD}$ is formed. Water does not combine with oxygen, but as a hydrate of acids it acts on the compound of oxygen with barium oxide, because this oxide gives a salt with an acid anhydride; or, what is the same, hydrogen with oxygen does not directly form hydrogen peroxide, but when combined with a haloid (for example, chlorine), under the action of barium peroxide, $\mathrm{BaO}_{2}$, it leads to the formation of a salt of barium and $\mathrm{H}_{2} \mathrm{O}_{2}$. It is to be remarked that the passage of barium oxide, $\mathrm{BaO}$, into the peroxide, $\mathrm{BaO}_{2}$, is accompanied by the evolution of 121000 heat units per 16 parts of oxygen by weight combined, and the passage of $\mathrm{H}_{2} \mathrm{O}$ into the peroxide $\mathrm{H}_{2} \mathrm{O}_{2}$ does not proceed directly, because it would be accompanied by the absorption of 22000 units of heat by 16 parts by weight of oxygen combined. Barium peroxide, in acting on an acid, evidently evolves less heat than the oxide, and it is this difference of heat that is absorbed in the hydrogen peroxide. Its energy is obtained from the energy evolved in the formation of the salt of barium.

20 Peroxides of lead and manganese, and other analogous peroxides (see Chapter III., Note 9), do not give hydrogen peroxide under these conditions, but yield chlorine with hydrochloric acid.

21 The impure barium peroxide obtained in this manner may be easily purified. For this purpose it is dissolved in a dilute solution of nitric acid. There will always remain a certain quantity of an insoluble residue, from which the solution is separated by filtration. The solution will contain not only the compound of the barium peroxide, but also a compound of the barium oxide itself, a certain quantity of which always remains uncombined with oxygen. The acid compounds of the peroxide and oxide of barium are easily distinguishable by their stability. The peroxide gives an unstable compound, and the oxide a stable salt. By adding an aqueous solution of barium oxide to the resultant solution, the whole of the peroxide contained in the solution may be precipitated as a pure aqueous compound. The first portions of the precipitate will consist of impuritiesfor instance, oxide of iron. The barium peroxide separates out, and is collected on a filter and washed; it then forms a substance having an entirely definite composition, $\mathrm{BaO}_{2}, 8 \mathrm{H}_{2} \mathrm{O}$, and is very pure. Pure hydrogen peroxide should always be prepared from such purified barium peroxide.

22 In the cold, strong sulphuric acid with barium peroxide gives ozone; when diluted with a certain amount of water it gives oxygen (see Note 6), and hydrogen peroxide is only obtained by the action of very weak sulphuric acid. The acids hydrochloric, hydrofluoric, carbonic, and hydrosilicofluoric, and others, when diluted with water also give hydrogen peroxide with barium peroxide. Professor Schöne, who investigated hydrogen peroxide with great detail, showed that it is formed by the action of many of the above-mentioned acids on barium peroxide. 
barium peroxide remains in solution. ${ }^{23}$ The reaction is expressed by the equation $\mathrm{BaO}_{2}+\mathrm{H}_{2} \mathrm{SO}_{4}=\mathrm{H}_{2} \mathrm{O}_{2}+\mathrm{BaSO}_{4}$. It is best to take a weak cold solution of sulphuric acid and to almost saturate it with barium peroxide, so that a small excess of acid remains; insoluble barium sulphate is formed. A more or less dilute aqueous solution of hydrogen peroxide is obtained. This solution may be concentrated in a vacuum over sulphuric acid. In this way the water may even be entirely evaporated from the solution of the hydrogen peroxide; only in this case it is necessary to work at a low temperature, and not to keep the peroxide for long in the rarefied atmosphere, as otherwise it decomposes. ${ }^{24}$

When pure, hydrogen peroxide is a colourless liquid, without smell, and having a very unpleasant taste - such as belongs to the salts of many metals - the so-called 'metallic' taste. Water held in zinc vessels has this taste, which is probably due to its containing hydrogen peroxide. The tension of the vapour of hydrogen peroxide is less than that of aqueous vapour; this enables its solutions to be concentrated in a vacuum. The specific gravity of anhydrous hydrogen peroxide is $1 \cdot 455$. Pure hydrogen peroxide decomposes, with the evolution of oxygen, when heated even to $20^{\circ}$ (by the action of light?). But the more dilute its aqueous solution the more stable it is. Very weak solutions may be distilled without the hydrogen peroxide decomposing. It decolorises solutions of litmus and turmeric, and acts in a similar manner on many colouring matters of organic origin (for which reason it is employed for bleaching tissues).

Many substances decompose hydrogen peroxide, forming water and oxygen, without apparently suffering any change. In this case substances in a state of fine division evince an incomparably quicker action

23 With the majority of acids, that salt of barium which is formed remains in solution; thus, for instance, by employing hydrochloric acid, hydrogen peroxide and barium chloride remain in solution. Complicated processes would be required to obtain pure bydrogen peroxide from such a solution. It is much more convenient to take advantage of the action of carbonic anhydride on the pure hydrate of barium peroxide. For this purpose the hydrate is stirred up in water, and a rapid stream of carbonic anhydride is passed through the water. Barium carbonate, insoluble in water, is formed, and the hydrogen peroxide remains in solution, so that it may be separated from the carbonate by filtering only. On a large scale hydrofluosilicic acid is employed, because its barium salt is also insoluble in water.

24 Hydrogen peroxide may be extracted from very dilute solutions by means of ether, which dissolves it, and when mixed with it the hydrogen peroxide may even be distilled. A solution of hydrogen peroxide in water may be enriched by cooling it to a low temperature, when the water crystallises out-that is, is converted into ice-whilst the hydrogen peroxide remains in solution, as it only freezes at very low temperatures. It must be observed that hydrogen peroxide, in a strong solution in a pure state, is exceedingly unstable even at the ordinary temperature, and therefore it must be preserved in vessels always kept cold, as otherwise it evolves oxygen and forms water.

VOL. I. 


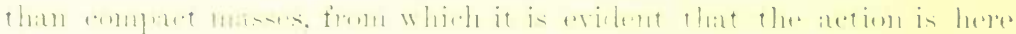

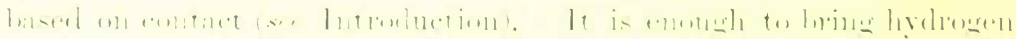

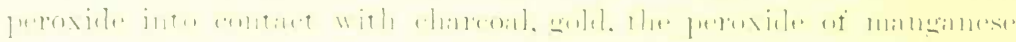

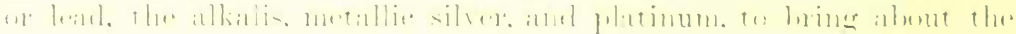

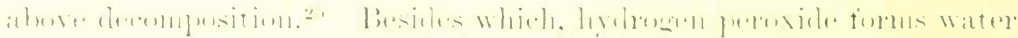

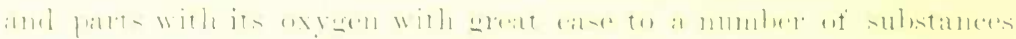

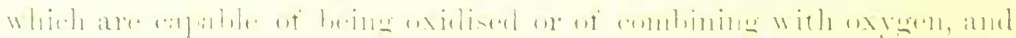

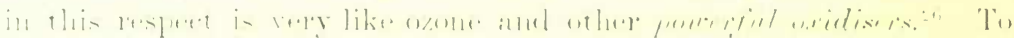

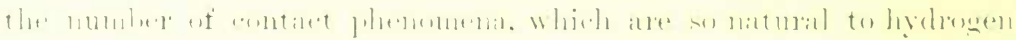

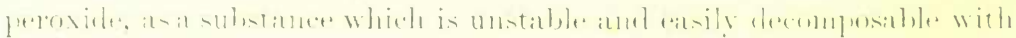

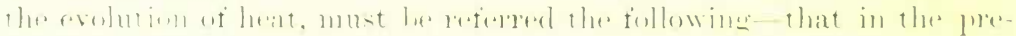

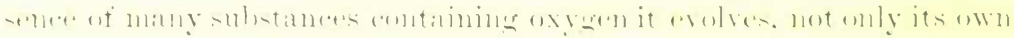

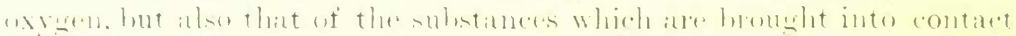

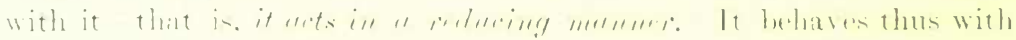

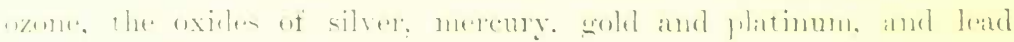

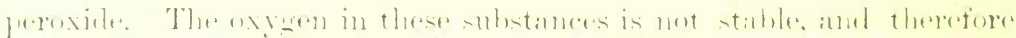

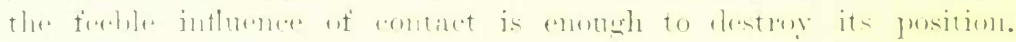

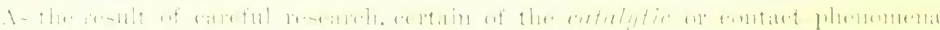

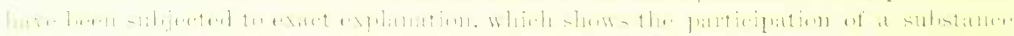

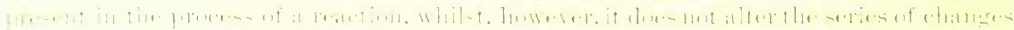

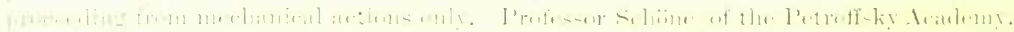

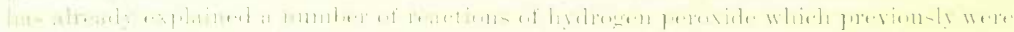

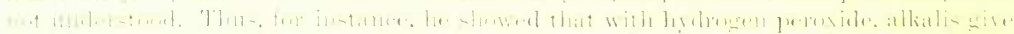

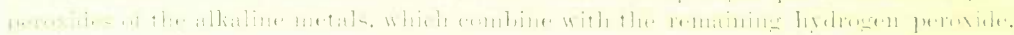

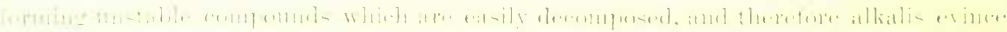

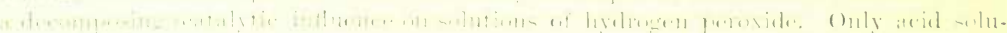

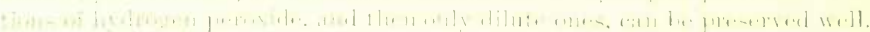

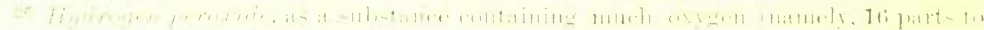

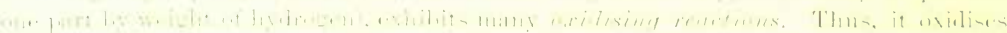

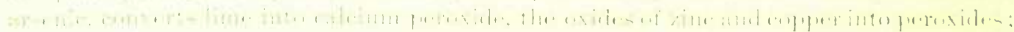

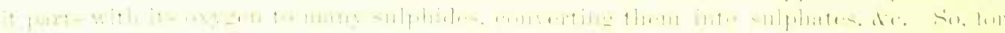

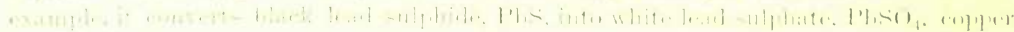

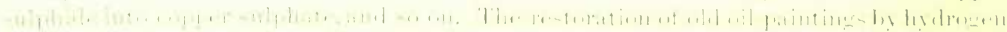

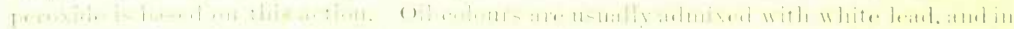

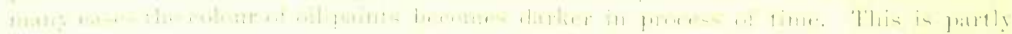

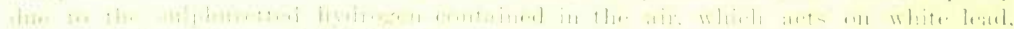

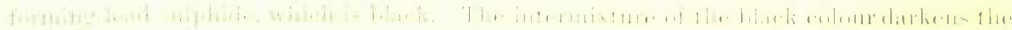

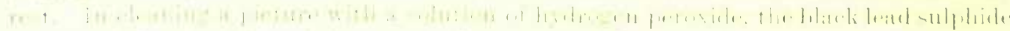

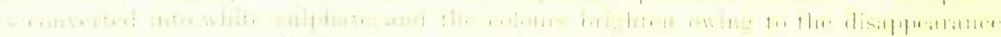

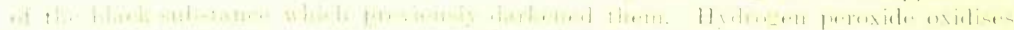

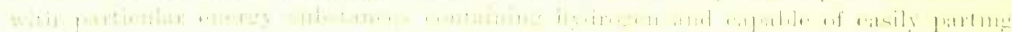

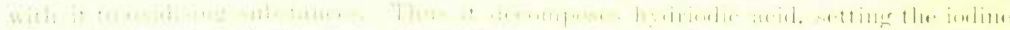

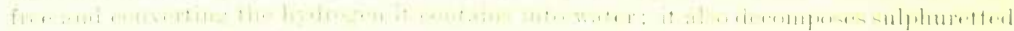

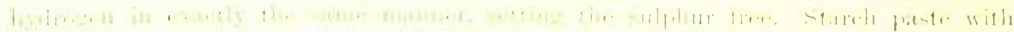

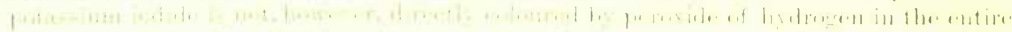

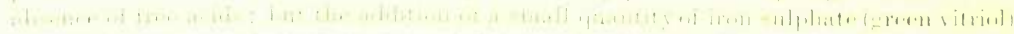

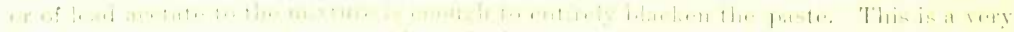

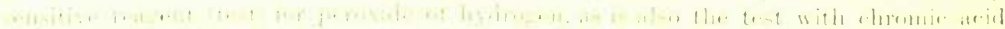

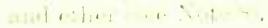


Hydrogen peroxide, especially in a concentrated form, in contact with these substances, evolves an immense quantity of oxygen, so that an explosion takes place and an exceedingly powerful evolution of heat is observed if hydrogen peroxide in a concentrated form be made to fall in drops upon these substances in dry powder. An exactly similar decomposition takes place in dilute solutions. ${ }^{27}$

Just as a whole series of metallic compounds, and especially the oxides and their hydrates, correspond with water, so also there are many substances analogous to hydrogen peroxide. Thus, for instance, calcium peroxide is related to hydrogen peroxide in exactly the same way as calcium oxide or lime is related to water. In both cases the hydrogen is replaced by a metal-namely, by calcium. But it is most important to remark that the nearest approach to the properties of hydrogen peroxide is afforded by a non-metallic element, chlorine; its action on colouring matters, its capacity for oxidising, and for evolving oxygen from many oxides, is analogous to that exhibited by hydrogen peroxicle. Even the very formation of chlorine is closely analogous to the formation of peroxide of hydrogen ; chlorine is obtained from manganese peroxide, $\mathrm{MnO}_{2}$, and hydrochloric acid, $\mathrm{HCl}$, and hydrogen peroxide from barium peroxide, $\mathrm{BaO}_{2}$, and the same acid. The result in one case is essentially water, chlorine, and manganese chloride; and in the other case there is produced barium chloride and hydrogen peroxide. Hence water + chlorine corresponds with hydrogen peroxide, and the action of chlorine in the presence of water is analogous to the action of hydrogen peroxide. This analogy between chlorine and hydrogen peroxide is expressed in the conception of an aqueous radicle, which (Chap. III.) has been already mentioned. This aqueous radicle (or hydroxyl) is that which is left from water if it be imagined as deprived of half of its hydrogen. According to this method of expression, caustic soda will be a compound of sodium with the aqueous radicle, because it is formed from water with the evolution of half the hydrogen. This is expressed by the following formula: water, $\mathrm{H}_{2} \mathrm{O}$, caustic soda, $\mathrm{NaHO}$,

27 To explain the phenomenon an hypothesis has been put forward by Brodie, Clausius, and Schönbein which supposes ordinary oxygen to be an electrically neutral substance, composed of, so to speak, two electrically opposite aspects of oxygen-positive and negative. It is supposed that hydrogen peroxide contains one kind of such polar oxygen, whilst in the oxides of the above-named metals the oxygen is of opposite polarity. It is supposed that in the oxides of the metals the oxygen is electro-negative, and in hydrogen peroxide electro-positive, and that on the mutual contact of these substances ordinary neutral oxygen is evolved as a consequence of the mutual attraction of the oxygens of opposite polarity. Brodie admits the polarity of oxygen in combination, but not in an uncombined state, whilst Schönbein supposes uncombined oxygen to be polar also, considering ozone as electro-negative oxygen. The supposition of the oxygen of ozone being other than that of hydrogen peroxide is contradicted by the fact that in acting on barium peroxide strong sulphuric acid forms ozone, and dilute acid forms hydrogen peroxide. 
just as hydrochloric acid is $\mathrm{HCl}$ and sodium chloride $\mathrm{NaCl}$. Hence the aqueous radicle $\mathrm{HO}$ is a compound radicle, just as chlorine, $\mathrm{Cl}$, is a simple radicle. They give hydrogen compounds, $\mathrm{HHO}$, water, and $\mathrm{HCl}$, hydrochloric acid ; sodium compounds, $\mathrm{NaHO}$ and $\mathrm{NaCl}$, and a whole series of analogous compounds. Free chlorine in this sense will be $\mathrm{ClCl}$, and hydrogen peroxide $\mathrm{HOHO}$, which indeed expresses its composition, because it contains twice as much oxygen as water.

Thus in ozone and hydrogen peroxide we see examples of very unstable, easily decomposable (by time, spontaneously, and on contact) substances, full of the energy necessary for change, ${ }^{28}$ capable of being easily reconstructed (in this case decomposing with the evolution of heat); therefore they are examples of unstable chemical equilibria. If a substance exists, it signifies that it already presents a certain form of equilibrium between those elements of which it is built up. But chemical, like mechanical, equilibria exhibit different degrees of stability or solidity. ${ }^{29}$

28 The lower oxides of nitrogen and chlorine and the higher oxides of manganese are also formed with the absorption of heat, and therefore, like hydrogen peroxide, act in a powerfully oxidising manner, and are not formed by the same methods as the majority of other oxides. It is evident that, being endowed with a richer store of energy (acquired in combination or absorption of heat), such substances, compared with others poorer in energy, will exhibit the greatest diversity of cases of chemical action with other substances.

29 If the point of support of a body lies in a vertical line below the centre of gravity, the equilibrium is entirely unstable. If the centre of gravity lies below the point of support, the state of equilibrium is very stable, and a vibration may take place about this position of stable equilibrium, as in a pendulum or balance, which ends in the body passing to its position of stable equilibrium. But if, keeping to the same mechanical example, the body be supported not on a point, in the geometrical sense of the word, but on a small plane, then the state of unstable equilibrium may be preserved, unless destroyed by external influences. Thus a man stands upright supported on the plane, or several points of the surfaces of his feet, having the centre of gravity above the points of support. Vibration is then possible, but it is limited, otherwise on passing outside the limit of possible equilibrium another more stable position is attained about which vibration becomes more possible. A prism immersed in water may have several more or less stable positions of equilibrium. It is the same with the atoms in molecules. Some molecules present a state of more stable equilibrium than others. Hence from this simple comparison it will be already clear that the stability of molecules may vary considerably, that one and the same elements, taken in the same number, may give isomerides of different stability, and, lastly, that there may exist states of equilibria which are so unstable, so ephemeral, that they will only arise under particularly special conditions-such, for example, as certain hydrates mentioned in the first chapter (see Notes 57, 67, and others). And if in one case the instability of a given state of equilibrium is expressed by its instability with a change of temperature or physical state, then in other cases it is expressed by the case of decomposition under the influence of contact or of the purely chemical influence of other substances. However clearly the greater or less stability of the elementary structure of substances be depicted to us in these general considerations, still at present there is no possibility of presenting them in a sufficiently concrete form to enable purely mechanical conceptions to be applied to them; that is, to subject them to mathematical analysis, and to master the subject to such an extent 
Besides this, hydrogen peroxide indicates another side of the subject which is not less important, and is much clearer and more general.

Hydrogen unites with oxygen in two degrees of oxidation : water or hydrogen oxide, and oxygenated water or hydrogen peroxide ; for a given quantity of hydrogen the peroxide contains twice as much oxygen as does water. This is a fresh example confirming the correctness of the law of multiple proportions, of which we have already made mention in speaking of the water of crystallisation of salts. Now we can formulate this law with entire clearness-the law of multiple proportions. If two radicles $\mathrm{A}$, and $\mathrm{B}$ (either simple or compound substances), unite together to form several compounds, $\mathrm{A}_{n} \mathrm{~B}_{m}, \mathrm{~A}_{q} \mathrm{~B}_{r}$. . . ., then having expressed the compositions of all these compounds in such a way that the quantity (by weight or volume) of one of the component parts will be a constant quantity $\mathrm{A}$, it will be observed that in all the compounds $\mathrm{AB}_{a}, \mathrm{AB}_{b} \ldots$. the quantities of the other component part, $\mathrm{B}$, wili always be in commensurable relation: generally in simple multiple proportion - that is, that $a: b \ldots$, or $m / n$ is to $r / q$ as whole numbers, for instance as $2: 3$ or $3: 4$. . .

The analysis of water shows that in 100 parts by weight it contains 11.112 parts by weight of hydrogen and 88.888 of oxygen, and the analysis of peroxide of hydrogen shows that it contains 94.112 parts of oxygen to 5.888 parts of hydrogen. In this the analysis is expressed, as analyses generally are, in percentages ; that is, it gives the amounts of the elements in a hundred parts by weight of the substance. The direct comparison of the percentage compositions of water and hydrogen peroxide does not give any simple relation. But such a relation is immediately observed if we calculate the composition of water and of hydrogen peroxide, having taken either the quantity of oxygen or the quantity of hydrogen as a constant quantity - for instance, as unity. The most simple proportions show that in water there are contained eight parts of oxygen to one part of hydrogen, and in hydrogen peroxide sixteen parts of oxygen to one part of hydrogen ; or one-eighth part of hydrogen in water and one-sixteenth part of hydrogen in hydrogen peroxide to one part of oxygen. Naturally, the analysis does not give these figures with absolute exactness - it gives them within a certain degree of error-but they approximate, as the error diminishes, to that limit which is here given. The comparison of the quantities of hydrogen and oxygen in the two substances above named, taking one of the components as a constant quantity, gives an example of the application of

as to foretell the degree of stability of different chemical states of equilibrium. The commencement of elementary generalisations has been apprehended in only a few cases. 
the law of multiple proportions, because water contains eight parts and hydrogen peroxide sixteen parts of oxygen to one part of hydrogen, and these figures are commensurable and are in simple proportion as $1: 2$.

An exactly similar multiple proportion is observed in the composition of all other well-investigated definite chemical compounds, ${ }^{30}$ and therefore the law of multiple proportions is accepted in chemistry as the starting point from which other considerations are judged.

The law of multiple proportions was discovered at the very beginning of this century by John Dalton, of Manchester, in investigating the compounds of carbon with hydrogen. It appeared that two gaseous compounds of these substances-marsh gas, $\mathrm{CH}_{4}$, and olefiant gas, $\mathrm{C}_{2} \mathrm{H}_{4}$, contain for one and the same quantity of hydrogen quantities of carbon which stand in multiple proportion ; namely, marsh gas contains relatively half as much carbon as olefiant gas. Although the analysis of that time was not exact, and did not give Dalton results in complete accordance with truth, still the accuracy of this law, recognised by Dalton, was confirmed by further more accurate investigations. On establishing the law of multiple proportions, Dalton gave a hypothetical explanation for it. This explanation is based on the atomic theory of matter. In fact, the law of multiple proportions is understood with unusual ease by admitting the atomic structure of matter.

3n When, for example, any element forms several oxides, they are subject to the law of multiple proportions. For a given quantity of the non-metal or metal the quantities of oxygen in the different degrees of oxidation will stand as $1: 2$, or as $1: 3$, or as $2: 3$, or as $2: 7$, and so on. Thus, for instance, copper combines with oxygen in at least two proportions, forming the oxides found in nature, and called the suboxide and the oxide of copper, $\mathrm{Cu}_{2} \mathrm{O}$ and $\mathrm{CuO}$; the oxide contains twice as much oxygen as the suboxide. Lead also presents two degrees of oxidation, the oxide and peroxide, and in the latter there is twice as much oxygen as in the former, $\mathrm{PbO}$ and $\mathrm{PbO}_{2}$. The substance known under the name of minium, and which is somewhat widely used as a red paint, is only a mixture of the mutual compounds of these oxides, which is proved not only by the inconstancy of its composition, but also by the fact that reagents capable of extracting the oxide of lead, especially acids, do actually extract it and leave lead peroxide. When a base and an acid are capable of forming several kinds of salts, normal, acid, basic, and anhydro-, it is found that they also clearly exemplify the law of multiple proportions. This was demonstrated by Wollaston soon after the discovery of the law in question. We saw in the first chapter that salts show different degrees of combination with water of crystallisation, and that they obey the law of multiple proportions. And, more than this, the indefinite chemical compounds existing as solutions may, as we saw in the same chapter, be brought under the law of multiple proportions by the hypothesis that solutions are unstable hydrates formed according to the law of multiple proportions, but occurring in a state of dissociation. By means of this hypothesis the law of multiple proportions becomes still more general, and all the aspects of chemical compounds are subject to it. The direction of the whole contemporary state of chemistry was determined by the discoveries of Lavoisier and Dalton. By bringing indefinite compounds also under the law of multiple proportions we arrive at that unity of chemical conceptions 
The essence of the atomic theory is that matter is supposed to consist of an agglomeration of small and indivisible parts - atoms - which do not fill up the whole space occupied by a substance, but stand apart from each other, as the sun, planets, and stars do not fill up the whole space of the universe, but are at a distance from each other. The form and properties of substances are determined by the position of their atoms in space and by their state of movement, while the phenomena accomplished by substances are understood as redistributions of the relative positions of atoms and changes in their movement. The atomic representation of matter arose in very ancient times, ${ }^{31}$ and up to recent times was at strife with the dynamical hypothesis, which considers matter as only a manifestation of forces. At the present time, however, the majority of scientific men uphold the atomic hypothesis, although the present conception of an atom is quite different from that of the ancient

which was impossible so long as definite compounds were separated from indefinite by a sharp line of demarcation.

51 Leucippus, Democritus, and especially Lusretius, in the classical ages, represented matter as made up of atoms-that is, of parts incapable of further division. The geometrical impossibility of such an admission, as well as the conclusions which were deduced by the ancient atomists from their fundamental propositions, prevented other philosophers from following them, and the atomic doctrine, like very many others, lived, without being ratified by fact, in the imaginations of its followers. Between the present atomic theory and the doctrine of the above-named ancient philosophers there is naturally a remote historical connection, as between the doctrine of Pythagoras and Copernicus, but they are essentially profoundly different. For us the atom is indivisible, not in the geometrical abstract sense, but only in a physical and chemical sense. It would be better to call the atoms indivisible individuals. The Greek atom=the Latin individual, according to both the sum and sense of the words, but historically these two words are endowed with a different meaning. The individual is mechanically and geometrically divisible, but only indivisible in a definite sense. The earth, the sun, a man or fly are individuals, although geometrically divisible. Thus the atoms of contemporary science, indivisible in a physico-chemical sense, form those units which are concerned in the investigation of the natural phenomena of matter, just as a man is an indivisible unit in the investigation of social relations, or as the stars, planets, and luminaries serve as units in astronomy. The formation of the vortex hypothesis, in which, as we shall afterwards see, atoms are entire whirls mechanically complex, although physico-chemically indivisible, already shows that the scientific men of our time in holding to the atomic theory have only borrowed the word and form from the ancient philosophers, and not the essence of their atomic doctrine. It is erroneous to imagine that the contemporary conceptions of the atomists are nothing but the repetition of the metaphysical reasonings of the ancients. As a geometrician in reasoning about curves represents them as formed of a sum total of straight lines, because such a method enables him to analyse the subject under investigation, so the scientific man applies the atomic theory as a method of analysing the phenomena of nature. Naturally there are people now, as in ancient times, and as there always will be, who apply reality to imagination, and therefore there are to be found atomists of extreme views; but it is not in their spirit that we should acknowledge the great services rendered by the atomic doctrine to all science, which, while it has been essentially independently dereloped, is, if it be desired to reduce all ideas to the doctrines of the ancients, a union of the ancient dynamical and atomic doctrines. 


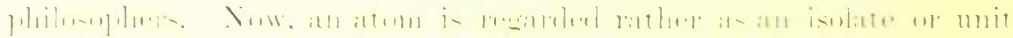

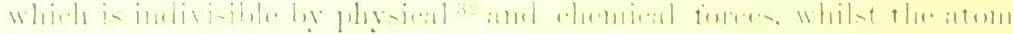

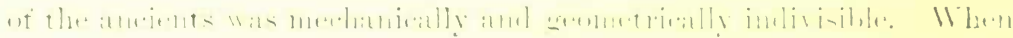

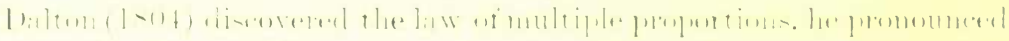

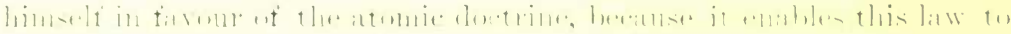

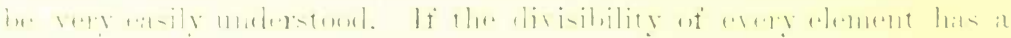

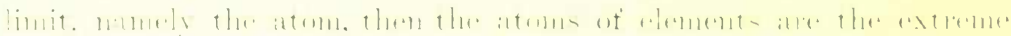

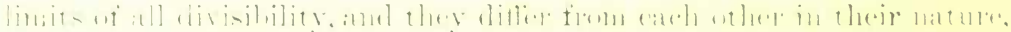

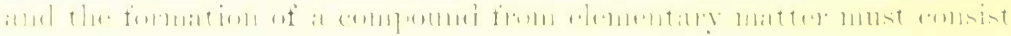

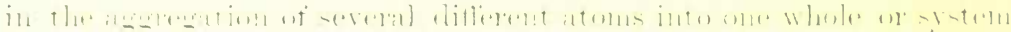

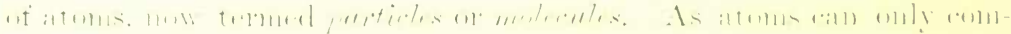

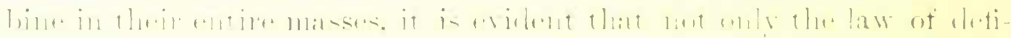

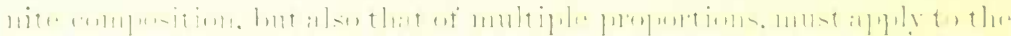

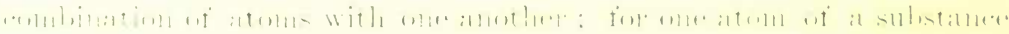

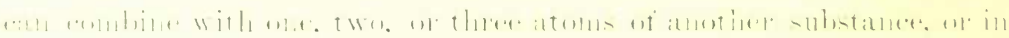

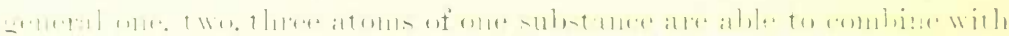

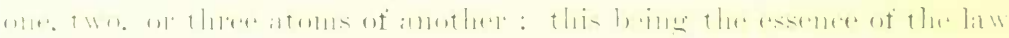

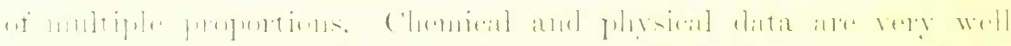

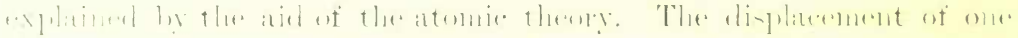

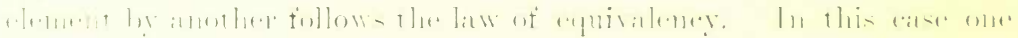

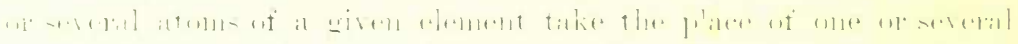

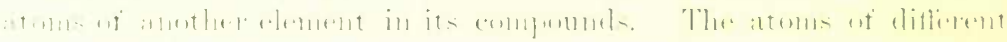

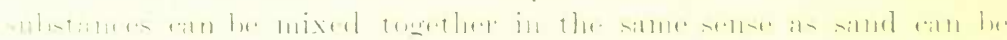

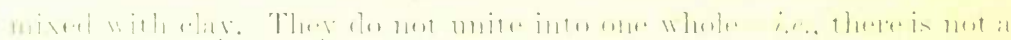

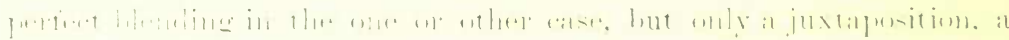

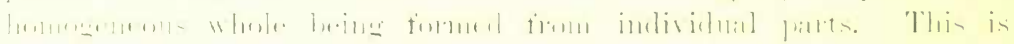

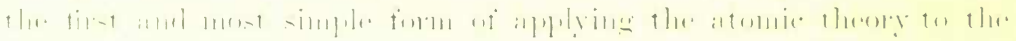

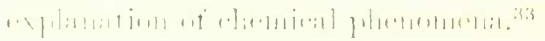

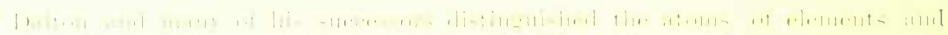

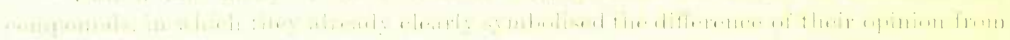

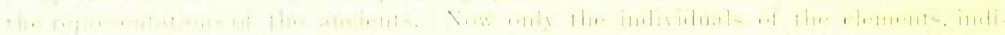

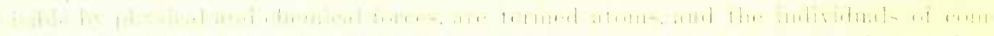

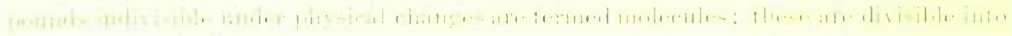

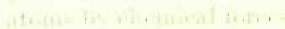

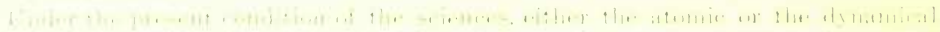

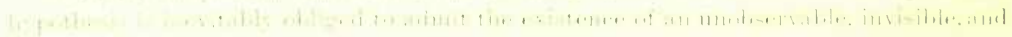
H.

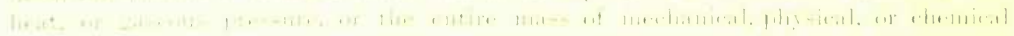

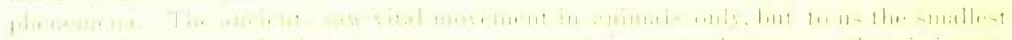

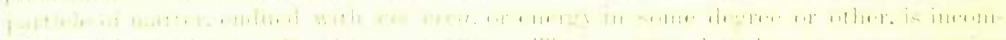

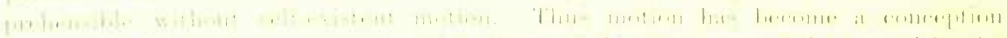

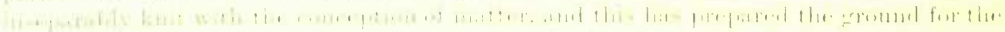

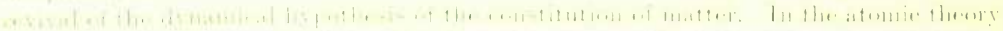

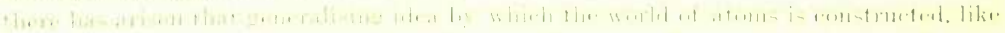

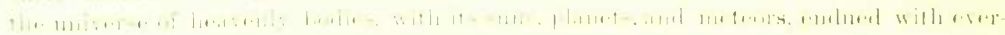




\section{A certain number of atoms $n$ of an element $\mathbf{A}$ in combining with} several atoms $m$ of another element $\mathbf{B}$ give a compound $\mathbf{A}_{n} \mathbf{B}_{m}$, each molecule of which will contain the atoms of the elements $A$ and $B$ in this ratio, and therefore the compound will present a definite composition, expressed by the formula $A_{n} B_{m}$, where $A$ and $B$ are the weights of the

lasting force of motion, forming molecules as the heavenly bodies form systems, like the solar system, which molecules are only relatively indivisible in the same way as the planets of the solar system are inseparable, and stable and lasting as the solar system is lasting. Such a representation, without necessitating the absolute indivisibility of atoms, expresses all that science can require for an hypothetical representation of the constitution of matter. In closer proximity to the dynamical hypothesis of the constitution of matter is the oft-times revived vortex hypothesis. Descartes first endeavoured to raise it; Helmholtz and Thomson gave it a fuller and more modern form; many scientific men applied it to physics and chemistry. The idea of vortex rings serves as the starting point of this hypothesis; these are familiar to all as the rings of tobacco smoke, and may be artificially obtained by giving a sharp blow to the sides of a cardboard box having a circular orifice and filled with smoke. Phosphine, as we shall see later on, when bubbling from water always gives very perfect vortex rings in a still atmosphere. In such rings it is easy to observe a constant circular motion about their axes, and to remark the stability the rings possess in their motion of translation. This unchangeable mass, endued with a rapid internal motion, is likened to the atom. In a medium deprived of friction, such a ring, as is shown by theoretical considerations of the subject from a mechanical point of view, would be perpetual and unchangeable. The rings are capable of grouping together, and combining, being indivisible, remain indivisible. The vortex hypothesis has been established in our times, but it has not been fully developed; its application to chemical phenomena is not clear, although not impossible; it does not satisfy a doubt in respect to the nature of the space existing between the rings (just as it is not clear what exists between atoms, and between the planets), neither does it tell us what is the nature of the moving substance of the ring, and therefore for the present it only presents the germ of an hypothetical conception of the constitution of matter, consequently, I consider that it would be superfluous to speak of it in greater detail. However, the thoughts of investigators are now (and naturally will be in the future), as they were in the time of Dalton, often turned to the question of the limitation of the mechanical division of matter, and the atomists have searched for an answer in the most diverse spheres of nature. I select one of the methods tried, which does not in any way refer to chemistry, in order to show how closely all the provinces of natural science are bound together. Wollaston proposed the investigation of the atmosphere of the heavenly bodies as a means for confirming the existence of atoms. If the divisibility of matter be infinite, then air must extend throughout the entire space of the heavens as it extends all over the earth by its elasticity and diffusion. If the infinite divisibility of matter be admitted, it is impossible that any portion of the whole space of the universe can be entirely void of the component parts of our atmosphere. But if matter be divisible up to a certain limit only-namely, up to the atom-then there can exist a heavenly body void of an atmosphere; and if such abody be discovered, it would serve as an important factor for the acceptation of the validity of the atomic doctrine. The moon has long been considered as such a luminary, and this circumstance, especially from its proximity to the earth, has been cited as the best proof of the validity of the atomic doctrine. This proof is apparently (Poisson) deprived of some of its force from the possibility of the transformation of the component parts of our atmosphere into a solid or liquid state at immense heights above the earth's surface, where the temperature is exceedingly low; but a series of researches (Poulé) has shown that the temperature of the heavenly space is, comparatively, not so rery low, and is attainable by experimental means, so that at the low existing pressure the liquefaction 
atoms and $m$ and $n$ their relative number. If the same elements $\mathbf{A}$ and $\mathrm{B}$, in addition to $\mathrm{A}_{n} \mathrm{~B}_{m}$, also yield another compound $\mathrm{A}_{r} \mathrm{~B}_{q}$, then by expressing the composition of the first compound by $\mathbf{A}_{n}, \mathbf{B}_{m r}$ (and this is the same composition as $\mathbf{A}_{n} \mathbf{B}_{m}$ ), and of the second compound by $\mathbf{A}_{r n} \mathbf{B}_{q n}$, we have the law of multiple proportions, because for a given

of gases camnot be expected. Therefore the absence of an atmosphere about the moon, if it were not subject to doubt, would be counted as a forcible proof of the atomic theory. As a proof of the absence of a lunar atmosphere, it is cited that the moon, in its independent movement between the stars, when eclipsing a star-that is, when passing between the eye and the star-does not show any signs of refraction at its edge; the image of the star does not alter its position in the heavens on approaching the moon's surface, consequently there is no atmosphere on the moon's surface. capable of refracting the rays of light. Such is the conclusion by which the absence of a lunar atmosphere is acknowledged. But this conclusion is most feeble, and there are even facts in exact contradiction to it, by which the existence of a lunar atmosphere may be proved. The entire surface of the moon is covered with a number of mountains, having in the majority of cases the conical form natural to volcanoes. The volcanic character of the lunar mountains was confirmed in October 1866, when a change was observed in the form of one of them (the crater Linnea). These mountains must be on the edge of the lunar disc. Seen in profile, they screen one another and interfere with making observations on the surface of the moon, so that when looking at the edge of the lunar disc we are obliged to make our observations not on the moon's surface, but at the summits of the lunar mountains. These mountains are higher than those on our earth, and consequently at their summits the lunar atmosphere must be exceedingly rarefied even if it possess an observable density at the surface. Knowing the mass of the moon to be eighty-two times less than the mass of the earth, we are able to approximately determine that our atmosphere at the moon's surface would be about twentyeight times lighter than it is on the earth, and consequently at the very surface of the moon the refraction of light by the lunar atmosphere must be very slight, and at the heights of the lunar mountains it must be imperceptible, and would be lost within the limits of experimental error. Therefore the absence of refraction of light at the edge of the moon's disc cannot yet plead in favour of the absence of a lunar atmosphere. There is even a series of observations obliging us to admit the existence of this atmosphere. These researches are due to Sir John Herschel. This is what he writes :- 'It has often been remarked that during the eclipse of a star by the moon there occurs a peculiar optical illusion; it seems as if the star before disappearing passed over the edge of the moon and is seen through the lunar disc, sometimes for a rather long period of time. I myself have observed this phenomenon, and it has been witnessed by perfectly trustworthy observers. I ascribe it to optical illusion, but it must be admitted that the star might have been seen on the lunar disc through some deep ravine on the moon.' Geniller, in Belgium (1856), following the opinion of Kassiné, Eiler, and others, gave an explanation to this phenomenon; he considers it due to the refraction of light in the valleys of the lunar mountains which occur on the edge of the lunar disc. In fact, although these valleys do not probably present the form of straight ravines, yet it may sometimes happen that the light of a star is so refracted that its image might be seen, notwithstanding the absence of a direct path for the light-rays. He then goes on to remark that the density of the lunar atmosphere must be variable in different parts, owing to the very long nights on the moon. On the dark, or non-illuminated, portion, owing to these long nights, which last thirteen of our days and nights, there must be excessive cold, and hence a denser atmosphere, while, on the contrary, at the illuminated portion the atmosphere must be much more rarefied. This variation in the temperature of the different parts of the moon's surface explains also the absence of clouds, notwithstanding the possible presence of air and aqueous vapour, on the visible portion of the moon. The 
quantity of the first element, $\mathbf{A}_{r n}$, there occur quantities of the second element bearing the same ratio to each other as $m r$ is to $q n$; and as $m$, $r, q$, and $n$ are whole numbers, therefore their products are also whole numbers, and this is also expressed by the law of multiple proportions. Consequently the atomic theory is in accordance with and evokes the first laws of definite chemical compounds : the law of definite composition and the law of multiple proportions.

So, also, is the relation of the atomic theory to the third law of definite chemical compounds, the law of reciprocal combining weights, which is as follows :- If a certain weight of a substance $C$ combine with a weight $a$ of a substance $\mathbf{A}$, and with a weight $b$ of a substance $\mathrm{B}$, then, also, the substances $\mathrm{A}$ and $\mathrm{B}$ will combine together in quantities $a$ and $b$ (or in multiples of them). This should be the case from the conception of atoms. Let $\mathrm{A}, \mathrm{B}$, and $\mathrm{C}$ be the weights of the atoms of the three substances, and for simplicity of reasoning let combination proceed in the quantity of one atom. It is evident that if the substance gives $\mathrm{AC}$ and $\mathrm{BC}$, then the substances $A$ and $B$ will give a compound $A B$, or their multiple, $A_{n} B_{m}$.

Sulphur combines with hydrogen and with oxygen. Sulphuretted hydrogen contains thirty-two parts by weight of sulphur to two parts by weight of hydrogen, which is expressed by the formula $\mathrm{H}_{2} \mathrm{~S}$. Sulphur dioxide, $\mathrm{SO}_{2}$, contains thirty-two parts of sulphur and thirty-two parts of oxygen, and therefore we conclude, from the law of combining weights, that oxygen and hydrogen will combine in the proportion of two parts of hydrogen and thirty-two parts of oxygen, or multiple numbers of them. And we have seen this to be the case. Hydrogen peroxide contains thirty-two parts of oxygen, and water sixteen parts, to two parts of hydrogen ; and so it is in all other cases. This consequence of the atomic theory is in accordance with nature, with the results of analysis, and is one of the most important laws of chemistry. It is a law, because it indicates the relation between the weights of substances entering into chemical combination. Further it is an eminently exact law, and not an approximate one. The law of combining weights is a law of nature, and by no means an hypothesis, for let the entire theory of atoms be cast down, still the laws of multiple proportions and of combining weights will remain, inasmuch as they deal with facts. They may be guessed at from the sense of the atomic theory, and historically

presence of an atmosphere round the sun and planets, judging from astronomical observations, may be considered as fully proved. On Jupiter and Mars there may be even distinguished bands of clouds. Thus the atomic doctrine, admitting a finite mechanical divisibility only, must be, as yet at least, only accepted as a means, similar to that means which a mathematician employs when he breaks up a continuous curvilinear line into a number of straight lines. There is a simplicity of representation in atoms, but there is no absolute necessity to have recourse to them. The conception of the individuality of the parts of matter exhibited in chemical elements only is necessary and trustworthy. 
the law of combining weights is intimately connected with this theory; but they are not identical, but only connected, with it. The law of combining weights is formulated with great ease, and is an immediate consequence of the atomic theory, without it, it is even difficult to understand. Data for its evolution existed previously, but it was not seen until those data were interpreted by the atomic theory. Such is the property of hypotheses. They are indispensable to science; they bestow an order and simplicity which are difficultly attainable without their aid. The whole history of science is a proof of this. And therefore one may boldly say that it is better to hold to an hypothesis which may afterwards prove untrue than to have none at all. Hypotheses facilitate scientific work and render it uniform. The search for truth, like the plough of the husbandman, helps forward the work of the labourer, regulates it, and forces him to think of the further improvement both of the work itself and of its implements. 


\section{CHAPTER V}

\section{NITROGEN AND AIR}

GASEOUS nitrogen forms about four-fifths (by volume) of the atmosphere ; consequently the air contains an exceedingly large mass of it. Whilst entering in so considerable a quantity into the composition of air, nitrogen does not seem to play any active part in the atmosphere, the chemical action of which is mainly dependent on the oxygen it contains. But this is not an entirely correct idea, because animal life cannot exist in pure oxygen, in which animals pass into an abnormal state and die; and the nitrogen of the air, although slowly, forms diverse compounds, many of which play a most important part in nature, especially in the life of organisms. However, neither plants nor animals directly absorb the nitrogen of the air, but take it up from already prepared nitrogenous compounds; further, plants are nourished by the nitrogenous substances contained in the soil and water, and animals by the nitrogenous substances contained in plants and in other animals. Atmospheric electricity is capable of aiding the passage of gaseous nitrogen into nitrogenous compounds, as we shall afterwards see, and the resultant substances are carried to the soil by rain, where they serve for the nourishment of plants. Plentiful harvests, fine crops of hay, vigorous growth of trees-other conditions being equalare only obtained when the soil contains ready prepared nitrogenous compounds, consisting either of those which occur in air and water, or of the residues of the decomposition of other plants or animals (as in manure). The nitrogenous substances contained in animals have their origin in those substances which are formed in plants. Thus the nitrogen of the atmosphere is the origin of all the nitrogenous substances occurring in animals and plants, although not directly so, but after first combining with the other elements of air.

The nitrogenous compounds which enter into the composition of plants and animals are of primary importance; no vegetable or animal cell - that is, the elementary form of organism-exists without containing a nitrogenous substance ; organic life, before all, evinces itself in 


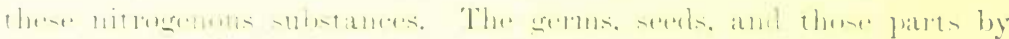

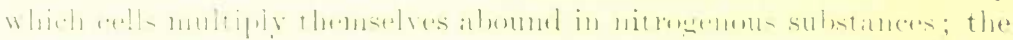

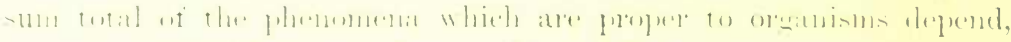

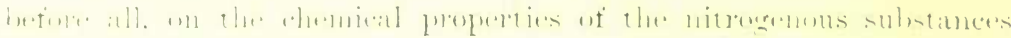

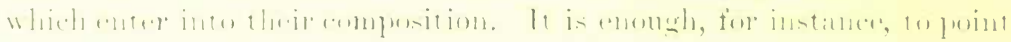

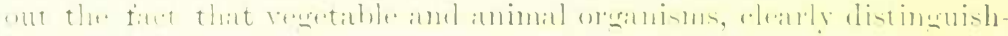

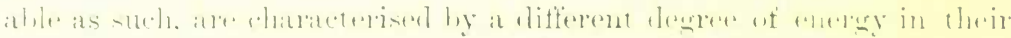

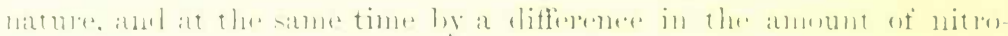

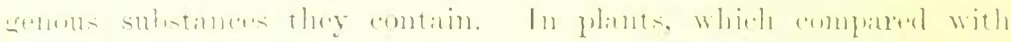

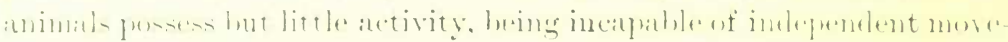

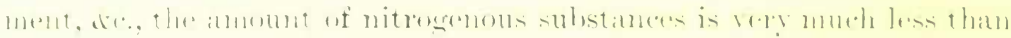

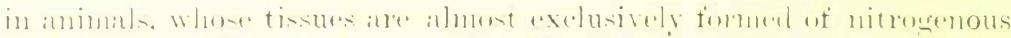
subtaneres. It is remarkahle that the nitrosemens parts of plants,

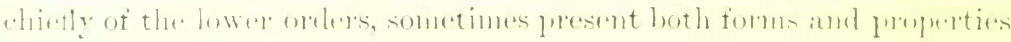

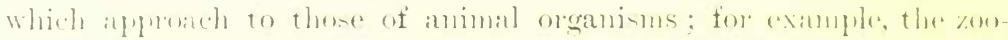

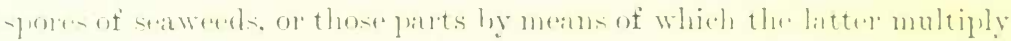

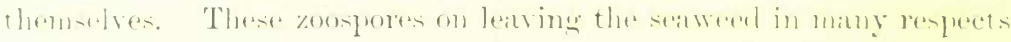

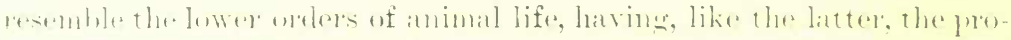

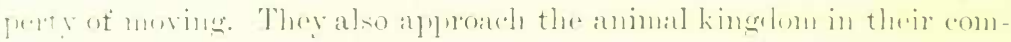

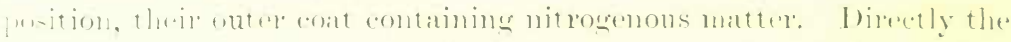

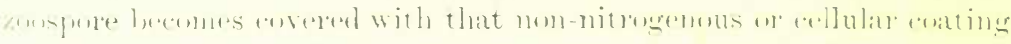

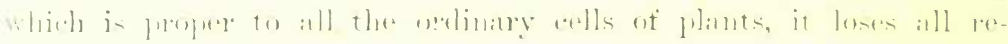

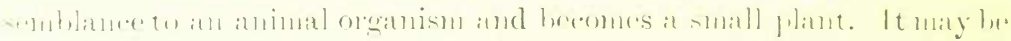

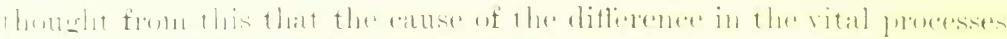

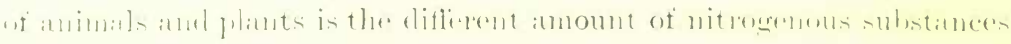

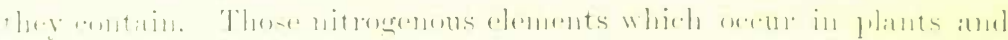

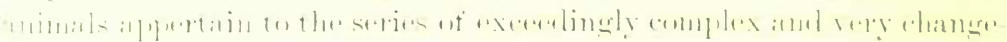

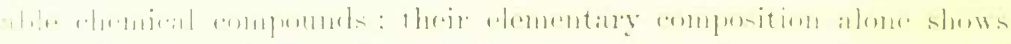

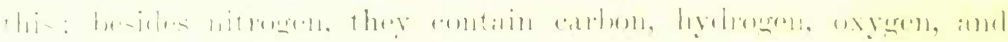

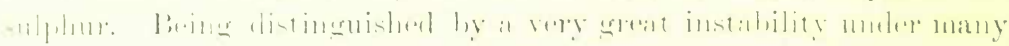

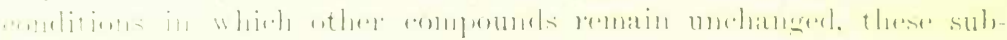

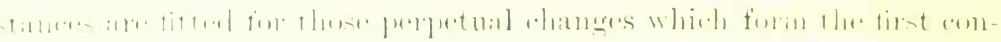

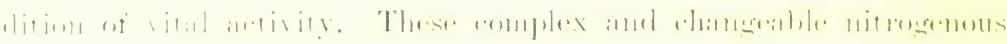

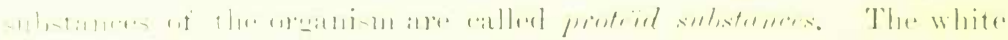

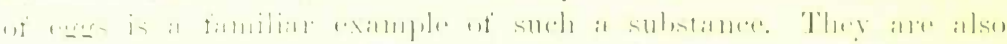

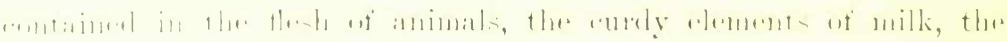

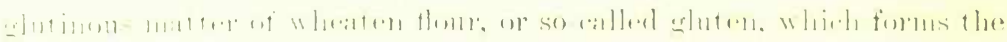

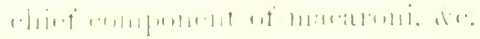

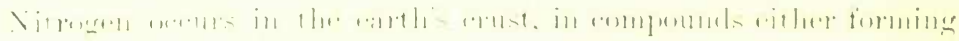

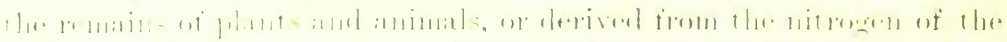

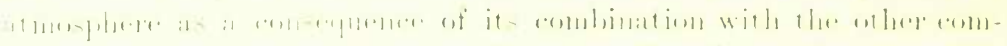

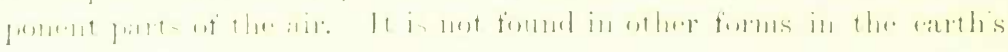


crust; so that nitrogen must be considered, in contradistinction to oxygen, as an element which is purely superficial, and does not extend to the depths of the earth. ${ }^{1}$

Nitrogen is liberated in a free state in the decomposition of the nitrogenous oryanic substances entering into the composition of organisms - for instance, on their combustion. All organic substances burn when heated to redness with oxygen (or substances readily yielding it, such as oxide of copper); the oxygen combines with the carbon, sulphur, and hydrogen, and the nitrogen is evolved in a free state, because at a high temperature it does not form any stable compound, but remains free. Carbonic anhydride and water are formed from the carbon and hydrogen respectively, and therefore to obtain pure nitrogen it is necessary to remove the carbonic anhydride from the gaseous products obtained. This may be done very easily by the action of alkalis - instance, caustic soda. The amount of nitrogen in organic substartees is determined by a method founded on this.

It is also very easy to obtain nitrogen from air, because oxygen combines with many substances. Either phosphorus or metallic copper are usually employed for removing the oxygen from air, but, naturally, a number of other substances may also be used. If a small saucer on which a piece of phosphorus is laid be placed on a cork floating on water, and the phosphorus be lighted, and the whole be covered with a glass bell jar, then the air under the jar will be deprived of its oxygen, and nitrogen only will remain, owing to which, on cooling the water will rise to a certain extent in the bell jar. The same object (procuring nitrogen from air) is attained much more conveniently and perfectly when air is passed through a red-hot tube containing copper filings. At a red heat, metallic copper combines with oxygen and gives a black powder of copper oxide. If the layer of copper be sufficiently long and the current of air slow, all the oxygen of the air will be absorbed, and nitrogen alone will pass from the tube. ${ }^{2}$

1 The reason why there are no other nitrogenous substances within the earth's mass beyond those which have come there with the remains of organisms, and from the air with rain-water, must be looked for in two circumstances. In the first place, in the instability of many nitrogenous compounds, which are liable to break up with the formation of gaseous nitrogen; and in the second place in the fact that the salts of nitric acid, forming the product of the action of air on many nitrogenous and especially organic compounds, are very soluble in water, and on penetrating into the depths of the earth (with water) give up their oxygen. The result of the changes of the nitrogenous organic substances which fall into the earth is without doubt frequently, if not always, the formation of gaseous nitrogen. Thus the gas evolved from coal always contains much nitrogen (together with marsh gas, carbonic anhydride, and other gases).

2 Copper (best as shavings, which present a large surface) absorbs oxygen, forming $\mathrm{CuO}$, at the ordinary temperature in the presence of solutions of acids, or, better still, in 
Nitrogen may also be procured from many of its compounds with oxygen ${ }^{3}$ and hydrogen, ${ }^{4}$ but the best fitted for this purpose is a saline mixture containing, on the one hand, a compound of nitrogen with oxygen, termed nitrous anhydride, $\mathrm{N}_{2} \mathrm{O}_{3}$, and on the other hand, ammonia, $\mathrm{NH}_{3}$-that is, a compound of nitrogen with hydrogen. By heating such a mixture the oxygen of the nitrous anhydride combines with the hydrogen of the ammonia, forming water, and gaseous nitrogen is evolved, $2 \mathrm{NH}_{3}+\mathrm{N}_{2} \mathrm{O}_{3}=3 \mathrm{H}_{2} \mathrm{O}+\mathrm{N}_{4}$. Nitrogen is procured by this method in the following manner:-A solution of caustic potash is saturated with nitrous anhydride, by which means potassium nitrite is formed. On the other hand, a solution of hydrochloric acid saturated with ammonia is prepared; a saline substance called sal-ammoniac, $\mathrm{NH}_{4} \mathrm{Cl}$, is thus formed in the solution. The two solutions thus prepared are mixed together and heated. Reaction takes place according to the equation $\mathrm{KNO}_{2}+\mathrm{NH}_{4} \mathrm{Cl}=\mathrm{KCl}+2 \mathrm{H}_{2} \mathrm{O}+\mathrm{N}_{2}$. This reaction proceeds in virtue of the fact that potassium nitrite and ammonium chloride are salts which, on interchanging their metals, give potassium chloride and ammonium nitrite, $\mathrm{NH}_{4} \mathrm{NO}_{2}$, which breaks up into water and nitrogen. This reaction does not take place without the aid of heat, but it proceeds very easily at a moderate temperature. Of the resultant substances, the nitrogen only is gaseous, the potassium chloride is non-volatile, and is left behind in the vessel in which the solutions are heated. Pure nitrogen may be obtained by drying the resulting gas and passing it through a solution of sulphuric acid (to absorb. a certain quantity of ammonia which is evolved in the reaction).

Nitrogen is a gaseous substance which does not much differ in physical properties from air; its density, referred to hydrogen, is approximately equal to 14 --that is, it is slightly lighter than air ; one litre of nitrogen weighs 1.256 grams. Nitrogen mixed with oxygen,

the presence of a solution of ammonia, when it forms a bluish-violet solution of oxide of copper in ammonia. Nitrogen is very easily procured by this method. A flask is filled with copper shavings and closed with a cork furnished with a funnel and stopcock. A solution of ammonia is poured into the funnel, and caused to slowly drop upon the copper. If at the same time a current, of air be slowly passed through the flask (from a gasholder), then all the oxygen will be absorbed from it and the nitrogen will pass from the flask. It should be washed with water to retain any ammonia that. may be carried off with it.

3 The oxygen compounds of nitrogen (for example, $\mathrm{N}_{2} \mathrm{O}, \mathrm{NO}, \mathrm{NO}_{2}$ ) are decomposed at a red heat by themselves, and under the action of red-hot copper, sodium, \&c., they give up their oxygen to the metals, leaving the nitrogen free. According to Meyer and Langer (1885), nitrous oxide, $\mathrm{N}_{2} \mathrm{O}$, decomposes below $900^{\circ}$, although not completely, whilst. the decomposition of nitric oxide, NO, does not start at $1200^{\circ}$, but is complete at $1700^{\circ}$.

4 Chlorine and bromine (in excess), as well as bleaching-powder (hypochlorites), take up the hydrogen from ammonia, $\mathrm{NH}_{3}$, leaving nitrogen. Nitrogen is best procured from ammonia by the action of a solution of sodium hypobromite on solid sal-ammoniac. 
which is slightly heavier than air, forms air. It is a gas which, like oxygen and hydrogen, is difficultly liquefied, and but little soluble in water and other liquids. Its absolute boiling point ${ }^{5}$ is about $-140^{\circ}$; above this temperature it is not liquefiable by pressure, and at lower temperatures it remains a gas at a pressure of 50 atmospheres. Liquid nitrogen boils at $-193^{\circ}$, so that it may be employed as a source of great cold. At about $-203^{\circ}$, in vaporising under a decrease of pressure, nitrogen solidifies into a colourless snow-like mass. Nitrogen does not burn, does not support combustion, is not absorbed by any of the reagents used in gas analysis, at least at the ordinary temperature - in a word, it presents a whole series of negative chemical properties ; this is expressed by saying that this element has no energy for combination. Although it is capable of forming compounds both with oxygen and hydrogen as well as with carbon, yet these compounds are only formed under particular circumstances, to which we will directly turn our attention. At a red heat nitrogen combines with boron, titanium, and silicon, forming very stable nitrogenous compounds, ${ }^{6}$ whose properties are entirely different from those of nitrogen with hydrogen, oxygen, and carbon. However, the combination of nitrogen with carbon, although it does not take place directly between the elements at a red heat, yet proceeds with comparative ease by heating a mixture of charcoal with an alkaline carbonate, especially potassium carbonate or barium carbonate, to redness, carbo-nitrides or cyanides of the metals being formed ; for instance, $\mathrm{K}_{2} \mathrm{CO}_{3}+4 \mathrm{C}+\mathrm{N}_{2}=2 \mathrm{KCN}+3 \mathrm{CO} .^{7}$

Nitrogen is found with oxygen in the air, but they do not readily combine. Cavendish, however, in the last century, showed that nitrogen combines with oxygen under the influence of a series of electric sparks. Electric sparks in passing through a moist ${ }^{8}$ mixture of nitrogen and oxygen-for instance, through air-cause these elements to combine,

5 See Chapter II. note 29.

6 The combination of boron with nitrogen is accompanied by the evolution of sufficient heat to raise the mass to redness; titanium combines so easily with nitrogen that it is difficult to obtain it free from that element. It is a remarkable and instructive fact that the compounds of nitrogen with these non-volatile elements are very stable, and are themselves non-volatile. Probably in this case the physical state of the substance with which the nitrogen combines, and the state in which the nitrogenous substance is obtained, evinces its influence. Thus carbon $(C=12)$ with nitrogen gives cyanogen, $\mathrm{C}_{2} \mathrm{~N}_{2}$, which is gaseous and very unstable, and whose molecule is not large, whilst boron $(B=11)$ forms a nitrogenous compound which is solid, non-volatile, and very stable. Its composition, BN, is essentially like that of cyanogen, but its molecular weight is probably greater.

7 This reaction, as far as is known, does not proceed beyond a certain limit, probably because cyanogen, $\mathrm{CN}$, itself breaks up into carbon and nitrogen.

8 Frémy and Becquerel took dry air, and observed the formation of brown vapours of oxides of nitrogen on the passage of sparks.

vor. I. 


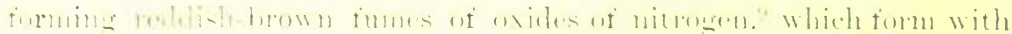

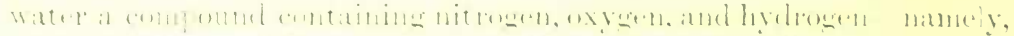

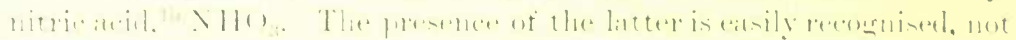

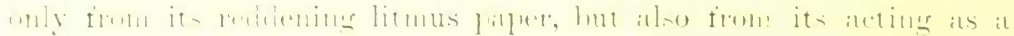

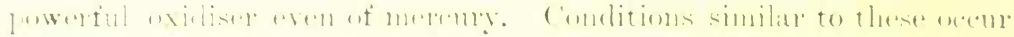

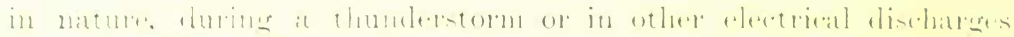

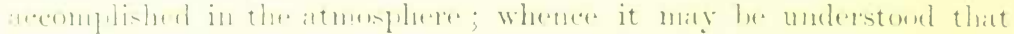

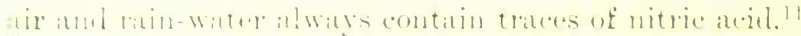

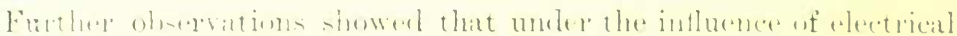

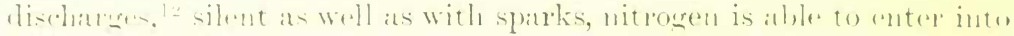

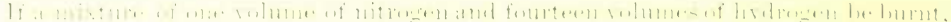

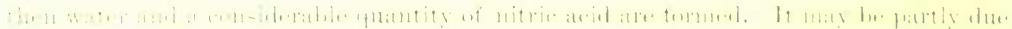

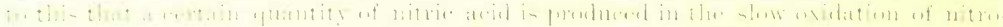

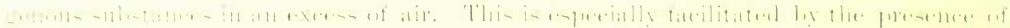

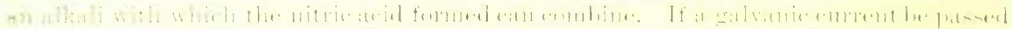

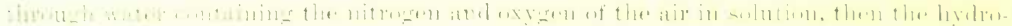

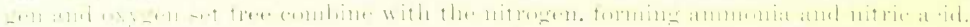

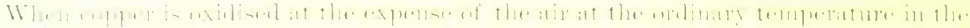

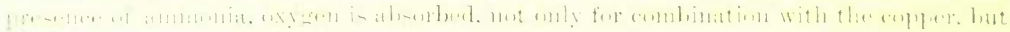

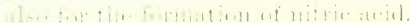

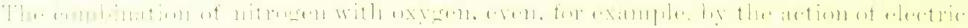

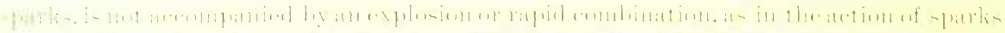

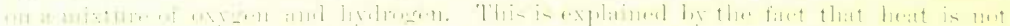

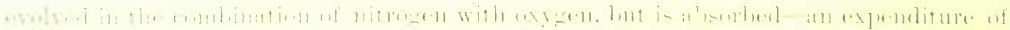

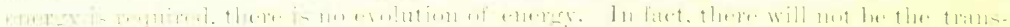

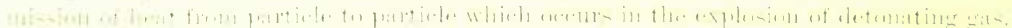

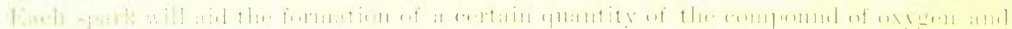

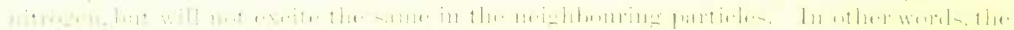

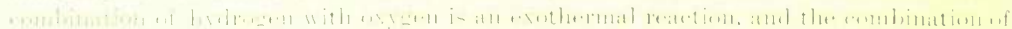
D. I.

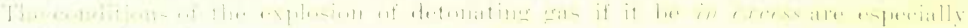

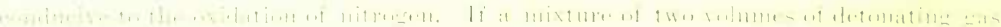

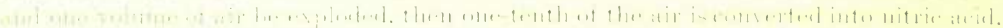

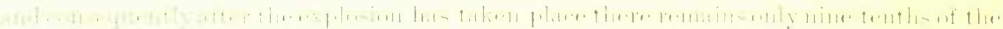

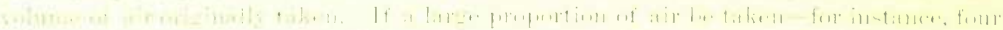

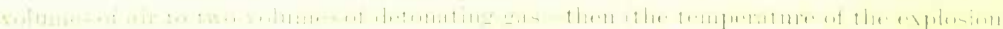

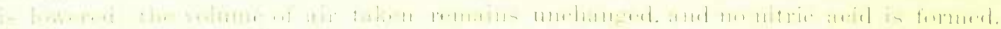

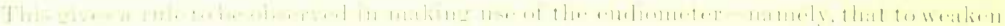

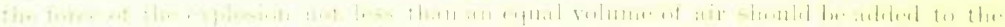

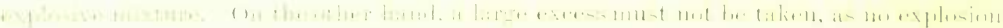

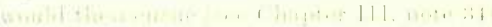

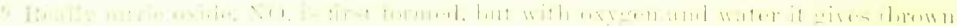

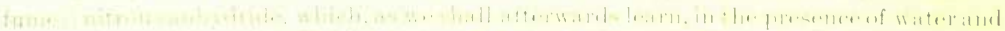

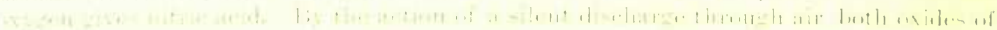

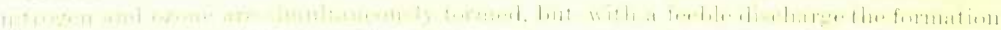

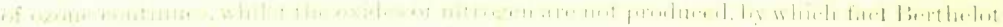

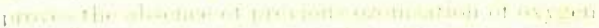

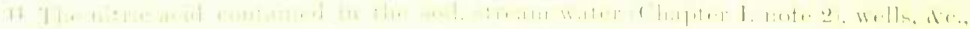

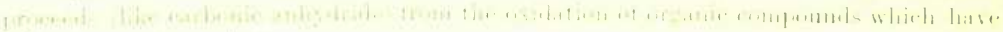

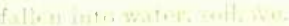

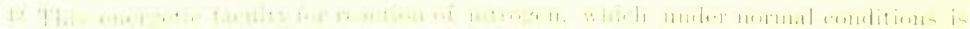

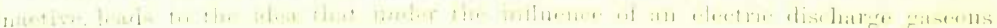


many reactions with hydrogen itself and with many hydrocarbons; although these reactions cannot be effected by exposure to a red heat. Thus, for instance, a series of electric sparks passed through a mixture of nitrogen and hydrogen causes them to combine and form ammonia ${ }^{13}$ or nitrogen hydride, $\mathrm{NH}_{3}$, composed of one volume of nitrogen and three volumes of hydrogen. This combination is limited to the formation of 6 per cent. of ammonia, because ammonia is decomposed, although not entirely $\left(\frac{94}{100}\right)$ by electric sparks. This signifies that under the action of electric sparks the reaction $\mathrm{NH}_{3}=\mathrm{N}+3 \mathrm{H}$ is reversible, consequently it is a dissociation, and in it a state of equilibrium is arrived at. The equilibrium may be destroyed by the addition of gaseous hydrochloric acid, $\mathrm{HCl}$, because with ammonia it forms a solid saline compound, sal-ammoniac, $\mathrm{NH}_{4} \mathrm{Cl}$, which (being formed from a gaseous mixture of $3 \mathrm{H}, \mathrm{N}$, and $\mathrm{HCl}$ ) fixes the ammonia. The remaining mass of nitrogen and hydrogen, under the action of the sparks, again forms ammonia, and in this manner solid sal-ammoniac is obtained to the end by the action of a series of electric sparks on a mixture of gaseous $\mathrm{N}, \mathrm{H}_{3}$, and $\mathrm{HCl} .{ }^{14}$ Berthelot (1876) showed that under the action of a silent discharge many non-nitrogenous organic substances (benzene, $\mathrm{C}_{6} \mathrm{H}_{6}$, cellulose in the form of paper, resin, glucose, $\mathrm{C}_{6} \mathrm{H}_{10} \mathrm{O}_{5}$, and others) absorb nitrogen and form complex nitrogenous compounds, which are capable, like albuminous substances, of evolving their nitrogen as ammonia when heated with alkalis. ${ }^{15}$

nitrogen changes in its properties; if not permanently like oxygen (electrolysed oxygen or ozone does not react on nitrogen, according to Berthelot), it may be temporarily at the moment of the action of the discharge, just as some substances under the action of heat are durably affected (that is, when once changed remain so-for instance, mercuric oxide is decomposed, white phosphorus passes into red, \&c.), whilst others are only temporarily altered (the dissociation of $\mathrm{S}_{6}$ into $\mathrm{S}_{2}$ or of sal-ammoniac into ammonia and hydrochloric acid). Such a proposition is favoured by the fact of nitrogen giving two kinds of spectra, with which we shall afterwards become acquainted. It may be that the molecules $\mathrm{N}_{2}$ then give less complex molecules, $\mathbf{N}$ containing one atom. Probably under a silent discharge the molecules of oxygen, $\mathrm{O}_{2}$, are partly decomposed and the individual atoms $\mathrm{O}$ combine with $\mathrm{O}_{2}$, forming ozone, $\mathrm{O}_{3}$.

13 This reaction, discovered by Chabrié and investigated by Thenard, was only rightly understood when Deville applied the principles of dissociation to it.

14 The action of nitrogen on acetylene (Berthelot) resembles this reaction. A mixture of these gases under the influence of a silent discharge gives hydrocyanic acid, $\mathrm{C}_{2} \mathrm{H}_{2}+\mathrm{N}_{2}$ $=2 \mathrm{CNH}$. This reaction cannot proceed beyond a certain limit because it is reversible.

- 15 Berthelot successfully employed electricity of even feeble potential in these experiments, which fact led him to think that in nature, where the action of electricity takes place very frequently, a part of the complex nitrogenous substances may proceed from the gaseous nitrogen of the air by this method.

As the nitrogenous substances of organisms play a very important part in them (organic life cannot exist without them), and as the nitrogenous substances introduced into the soil are capable of invigorating its crops (naturally in the presence of the other nourishing principles required by plants), therefore the question of the means of converting the atmospherid nitrogen into the nitrogenous compounds of the soil, or 
By such and, it may be, other similar indirect methods does gaseous nitrogen yield its primary compounds, in which form it enters into plants, and is elaborated in them into complex albuminous substances. But, starting from a given compound of nitrogen with hydrogen or oxygen, we may, without the aid of organisms, obtain, as will afterwards be partially indicated, most diverse and complex nitrogenous substances, which cannot by any means be formed directly from gaseous nitrogen. In this we see an example not only of the difference between an element in the free state and an intrinsic element, but also of those circuitous or indirect methods by which substances are formed in nature. The discovery, prognostication, and, in general, study of such indirect methods of the preparation and formation of substances forms one of the existing problems of chemistry. From the fact that $\mathbf{A}$ does not act at all on $B$, it must not be concluded that a compound $A B$ is not to be formed. The substances $\mathrm{A}$ and $\mathrm{B}$ contain atoms which occur in $\mathrm{AB}$, but their state, the nature of their movement and union, may not be at all that which is required for the formation of $A B$, and in this substance, although it contains the same elements in mass and quality of them as in A and B, yet their chemical state may be as different as the state of the atoms of oxygen in ozone and in water. Thus free nitrogen is inactive; but in its compounds it very easily enters into changes and is distinguished by great activity. An acquaintance with the compounds of nitrogen confirms this. But, before entering on this subject, let us consider air as a mass containing free nitrogen.

Judging from what has been already stated with respect to water, oxygen, ozone, and nitrogen, it will be evident that atmospheric air ${ }^{16}$

into assimilable nitrogen capable of being absorbed by plants and of forming complex (albuminous) substances in them, forms a question of great theoretical and practical interest. The artificial (technical) conversion of the atmospheric nitrogen into nitrogenous compounds, notwithstanding repeated trials, cannot yet be considered as fulfilled in a practical, remunerative manner, although its possibility is already evident. Electricity will probably aid in solving this problem of great practical importance. When the theoretical side of the question is further advanced, then without doubt an advantageous means will be found for the manufacture of nitrogenous substances from the nitrogen of the air ; and this is needed, before all, for the agriculturist, to whom nitrogenous fertilisers form an expensive item, and are more important than all other manures.

One thousand tons of farmyard manure do not generally contain more than four tons of nitrogen in the form of complex nitrogenous substances, and this amount of nitrogen is contained in twenty tons of ammonium sulphate, therefore the action evinced by the mass of manure in respect to the introduction of nitrogen may be produced by small quantities of artificial nitrogenous fertilisers. Over 160000 tons of guano are imported into Europe from South America, because guano (the excrement of sea and other birds) contains many nitrogenous compounds which are required by the agriculturist.

16 Under the name of atmospheric air the chemist and physicist understand ordinary air containing nitrogen and oxygen only, notwithstanding that the other component parts of air have a very important significance for the vitality of the earth's surface. That air 
contains a mixture of several gases and vapours. Some of them are met with in it in nearly constant proportions, whilst others, on the contrary, are very variable in their amount. The chief component parts of air, placed in the order of their relative amounts, are the following : nitrogen, oxygen, aqueous vapour, carbonic anhydride, nitric acicl, salts of ammonia, uzone, hydrogen peroxide, and complex nitrogenous substances. Besides these, air generally contains water, as spray, drops, and snow, and particles of solids, perhaps of cosmic origin in certain instances but in the majority of cases proceeding from the mechanical translation of solid particles from one locality to another by the wincl. These small solid and liquid particles (having a large surface and little weight) hang in air as solid matter hangs in turbid water; they often settle on the surface of the earth, but the air is never entirely free from them, because they are never in a state of complete rest. Then, air not unfrequently contains incidental traces of various substances, as everyone knows by experience. These incidental substances sometimes belong to the order of those which act injuriously (miasmas), the germs of lower organisms - for instance, of moulds-and to the class of carriers of infectious diseases.

In the air of the diverse countries of the earth, at different longitudes and at different altitudes above its surface, on the ocean or on the dry land-in a word, in the air of most diverse localities of the earth-the oxygen and nitrogen are everywhere in a constant ratio. This is, moreover, self-evident from the fact that the air constantly diffuses (intermixes in virtue of the internal movement of the gaseous particles) and is put in a state of movement and intermixed by the wind, and therefore it is equalised in its composition over the entire surface of the

is so represented in science is based on the fact that only the two above-named components are met with in air in a constant quantity, whilst the others are variable. The solid impurities may be separated from air required for chemical or physical research by simple filtration through a long layer of cotton-wool placed in a tube. Organic impurities are removed by passing the air through a solution of potassinm permanganate. The carbonic anhydride contained in air is absorbed by alkalis-best of all, soda lime, which in a dry state in porous lumps absorbs it with exceeding rapidity and completeness. Aqueous vapour is removed by passing the air over calcium chloride, strong sulphuric acid, or phosphoric anhydride. Air thus purified is accepted as containing only nitrogen and oxygen, although in reality it still contains a certain quantity of hydrogen and hydrocarbons, from which it may be purified by passing over copper oxide heated to redness. The copper oxide then oxidises the hydrogen and hydrocarbons-it burns them, forming water and carbonic anhydride, which may be removed as above described. Such purified air differs in many respects from ordinary air. Thus, for instance, it does not support plant life. When it is said that in the determination of the density of gases the weight of air is taken as unity, then it is understood to be such air, containing only nitrogen and oxygen. It is a litre of such air that weighs 1.293 grams at $0^{\circ}$ and $760 \mathrm{~mm}$. pressure at long. $45^{\supset}$, and 1.294 grams at St. Petersburg. 
earth. In those localities where the air is subject to change, being in a more or less enclosed space, or, at least, an unventilated space, it may alter very considerably in its composition. For this reason the air of dwellings, cellars, and wells, in which there are substances absorbing oxygen, contains less of this gas, whilst the air on the surface of standing water, abounding in the lower orders of plant life evolving oxygen, holds an excess of this gas. ${ }^{17}$ The constant composition of air over the whole surface of the earth has been proved by a number of most careful researches. ${ }^{18}$

17 As a proof of the fact that certain circumstances may actually change the composition of air, it will be enough to point out that the air contained in the cavities of glaciers (of permanent mountain ice) contains only up to 10 p.c. of oxygen. This depends on the fact that at low temperatures oxygen is much more soluble in snow-water and snow than nitrogen. When shaken up with water the composition of air should change, because the water dissolves an unequal quantity of oxygen and nitrogen. We have already seen (Chapter I.) that the air boiled off from water saturated at about $0^{\circ}$ contains about thirtyfive volumes of oxygen and sixty-five volumes of nitrogen, and we have considered the reason of this. It is remarkable that the solubility of oxygen and nitrogen in water decreases so uniformly with the temperature that the proportion of oxygen and nitrogen held in an aqueous solution remains almost constant at the most varied temperatures. This explains the circumstance that the air over the sea (especially arctic) is, as certain observers have found, poorer in oxygen than on dry land-the water dissolves more oxygen than nitrogen. The difference does not, however, exceed 0.3 p.c., and sometimes does not exist.

18 The analysis of air by weight conducted by Dumas and Boussingault in Paris, and which they repeated many times between April 27 and September 22, 1841, under various. conditions of weather, showed that the amount by weight of oxygen only varies between 22.89 p.c. and 23.08 p.c., the average amount being 23.07 p.c. Brunner, at Bern in Switzerland, and Bravais, at Faulhorn in the Bernese Alps, at a height of two kilometres above the level of the sea, made analyses of the air at the same season of the year as Dumas, and found that the composition of the air at these places did not exceed the limits determined for Paris. Marignac at Geneva, Lewy at Copenhagen, and Stas at Brussels, confirmed this. The analyses of air taken from different parts of the world, at the surface of the ocean and at different heights above the level of the sea, lead to the conclusion that air everywhere contains an equal amount of oxygen, or that if it does vary it does so within very inconsiderable limits.

As there is some basis (which will be mentioned shortly) for considering that the composition of the air at great altitudes is different from that at attainable heights-namely, that it is richer in nitrogen-several fragmentary observations made at Munich and in America gave reason for thinking that in the upward currents (that is in the region of minimum barometric pressure or at the centres of meteorological cyclones) the air is richer in oxygen than in the descending currents of air (in the regions of anticyclones or of barometric maxima); but more carefully conducted observations showed this proposition to be incorrect. Improved methods for the analysis of air have shown that certain slight variations in the composition of air do actually occur, but in the first place they depend on incidental local influences (on the passage of the air over mountains and large surfaces of water, regions of forest and vegetation, and the like), and in the second place are limited by quantities which are scarcely distinguishable from possible errors in the analyses.

The considerations which make one think that the atmosphere at great altitudes contains less oxygen than at the surface of the earth are based more particularly on the law of partial pressures (page 81). It obliges one to consider that the equilibrium of the oxygen in the strata of the atmosphere is not dependent on the equilibrium of the nitro- 
The analysis of air is effected by converting the oxygen into a nongaseous compound, so as to separate it from the air. The original volume of the air is first measured, and then the volume of the remaining nitrogen. The quantity of oxygen is calculated either from the difference between these volumes or by the weight of the oxygen compound formed. All the volumetric measurements have to be corrected for pressure, temperature, and moisture (Chapters I. and II.). The medium employed for converting the oxygen into a non-gaseous substance should enable its being taken up from the nitrogen to the very end without evolving any gaseous substance. So, for instance, ${ }^{19}$ a mixture of pyrogallol, $\mathrm{C}_{6} \mathrm{H}_{6} \mathrm{O}_{3}$, and a solution of a caustic alkali absorbs oxygen with great ease at the ordinary temperature (the solution turns black), but it is unsuited for accurate analysis because it requires an aqueous solution of an alkali, and it alters the composition of the air by acting on it as a solvent. ${ }^{20}$ However, for approximate determinations this simple method gives entirely satisfactory results.

The determinations in a eudiometer (Chapter III.) give much more exact results, if all the necessary corrections for changes of pressure, temperature, and moisture be taken into account. This determination is essentially carried on as follows :- A certain amount of air is introduced into the eudiometer, and its volume is determined. Then about

gen, and that the variation in the densities of both gases with the height is determined by the pressure of each gas separately. Details of the calculations and considerations here involved are contained in my work On Barometric Levellings, 1876, p. 48.

On the basis of the law of partial pressure and of hypsometrical formulæ, expressing the laws of the variation of pressures at different altitudes, the conclusion may be deduced that at the upper strata of the atmosphere the proportion of the nitrogen with respect to the oxygen increases, but the increase will not exceed a fraction per cent., even at altitudes of four and a half to six miles, the greatest height within the reach of men either by climbing mountains or by means of balloons. This conclusion is confirmed by the analyses of air collected by Welsh in England during his aëronautic ascents. The question of the distribution of gases in the upper strata of the atmosphere is of particular importance for understanding in what state the gaseous or vaporous masses occur which are borne in space, and are one of the elementary forms of the heavenly bodies (according to Laplace's and Kant's theory). I touch on this subject in speaking of the origin of naphtha in my work On the Naphtha Industry, 1879.

19 The complete absorption of the oxygen may be attained by introducing moist phosphorus into a definite volume of air; this is recognised by the fact of the phosphorus becoming non-luminous in the dark. The amount of oxygen may be determined by measuring the volume of nitrogen remaining. This method, however, cannot gire accurate results, owing to a portion of the air being dissolved in the water; to the combination of some nitrogen with oxygen; to the necessity of introducing and withdrawing the phosphorus, which cannot be accomplished without introducing bubbles of air; and to the numerous corrections of the volume (for moisture, temperature, and pressure), \&c.

${ }^{20}$ For rapid and approximate analyses (especially technical and hygienic), such a mixture is very suitable for determining the amount of oxygen in mixtures of gases, from which the substances absorbed by alkalis have first been removed. According to certain observers, this mixture evolves a small quantity of carbonic oxide after absorbing oxygen. 
an equal volume of dry hydrogen is passed into the eudiometer, and the volume again determined. The mixture is then exploded, as was described in the determination of the composition of water. The remaining volume of the gaseous mixture is again measured; it will be less than the second of the previously measured volumes. Out of three volumes which have disappeared, one appertains to the oxygen and two to the hydrogen, consequently one-third of the loss of volume indicates the amount of oxygen held in the air. ${ }^{21}$

The most accurate method for the analysis of air, and one which is accompanied by the least amount of error, consists in the direct weighing, as far as is possible, of the oxygen, nitrogen, water, and carbonic

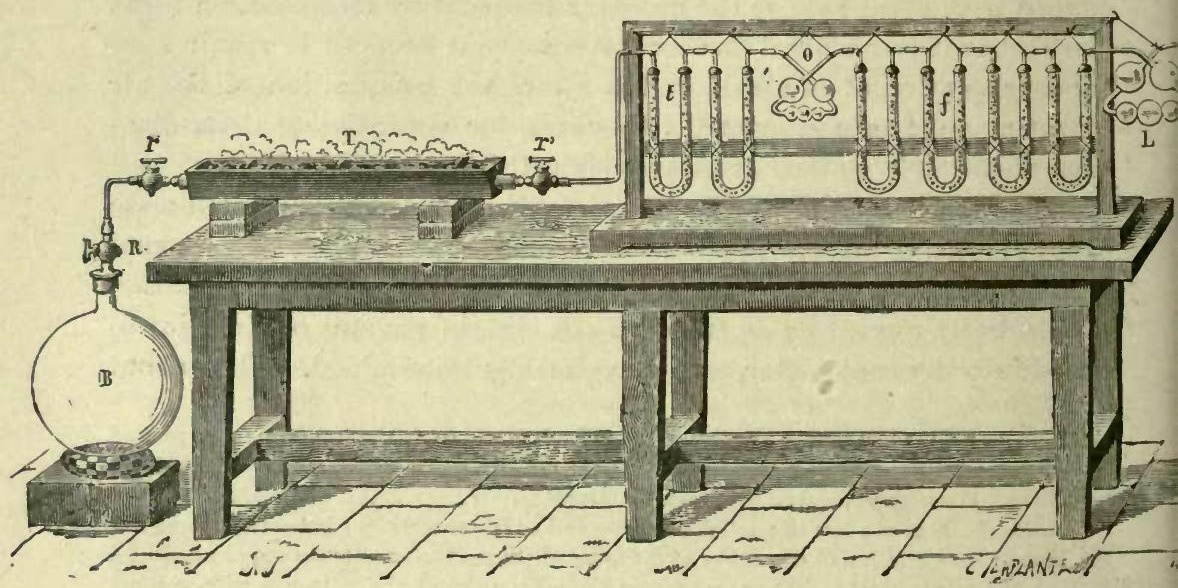

Frg. 38.-Dumas and Bousingault's apparatus for the analysis of air by weight. The globe $\mathbf{B}$ contains 10-15 litres. The air is first pumpel out of it, and it is weighed empty. The tube $T$ connected with it is flled with copper, and is weighed empty. It is heated in a charcoal furnacc. When the copper has become red-hot, the stop-cock $r$ (near $\mathrm{R}$ ) is slightly opened, and the air passes through the vessels $\mathrm{L}$, containing a solution of potash; $f$, containing solutions and pieces of caustic potash, which remove the carbonic anhydride from the air, and thell through 0 and $t$, containing sulphuric acid (which has been previously boiled to expel dissolved air) and pumice-stone, which removes the moisture from the air. The pure air then gives up its oxygen to the copjer in T. When the air passes into $T$ the stop-cock $\mathrm{R}$ of the globe $\mathrm{B}$ is opened, and it becomes filled with nitrogen. When the air ceases to flow in, the stop-cocks are closed, and the globe $B$ and tube $\mathrm{T}$ weighed. The nitrogen is then pumped out of the tube, and it is weighed agsin. The increase in weight of the tube slows the amount of oxygen, and the difference of the second and third weighings of the tube, with the increase in weight of the globe, gives the weight of the nitrogen.

anhydride contained in air. For this purpose, the air is first passed through an apparatus for retaining the moisture and carbonic anhydride. (which will be considered presently), and is then led through

21 Details of eudiometrical analysis must, as was pointed out in Chap. III. note $\mathbf{3 2}$, be looked for in works on analytical chemistry. The same must be remarked in reference to the other analytical methods mentioned in this work. They are only described for the sake of showing the diversity of the methods of chemical research. 
a tube, which is previously weighed, and contains shavings of metallic copper. A long layer of such copper heated to redness absorbs all the oxygen from the air, and leaves pure nitrogen, whose weight must be determined. This is done by collecting it in a weighed and exhausted globe, and the amount by weight of oxygen is shown by the increase in weight of the tube with the copper after the experiment.

Air free from moisture and carbonic anhydride ${ }^{22}$ contains $23 \cdot 15$ parts of oxygen and 76.85 of nitrogen by weight, ${ }^{23}$ which, taking the density of oxygen $=16$ and of nitrogen $=14$, gives the volumetric composition of air as $20 \cdot 84$ volumes of oxygen and $79 \cdot 16$ of nitrogen. ${ }^{24}$

The possibility of the composition of air being altered by the mere action of a solvent very clearly shows that the component parts of air are in a state of mixture, in which any gases may occur; they do not in this case form a definite compound, although the composition of the atmosphere does appear constant under ordinary conditions. The fact that its composition varies under different conditions confirms the truth of this conclusion, and therefore the constancy of the composition of air must not be considered as in any way dependent on the nature of the gases entering into its composition, but only as proceeding from cosmic phenomena co-operating towards this constancy. It must be admitted, therefore, that the processes evolving oxygen, and chiefly the processes of the respiration of plants, are of equal force with those processes which absorb oxygen over the entire surface of the earth. ${ }^{25}$

22 Air free from carbonic anhydride indicates after explosion the presence of a small quantity of carbonic anhydride, as De Saussure remarked, and air free from moisture, after being passed over red-hot copper oxide, seems invariably to contain a small quantity of water, as Boussingault has observed. These observations cause one to think that air always contains a certain quantity of gaseous hydrocarbons, like marsh gas, which, as we shall afterwards learn, is evolved from the earth, marshes, \&c. Its amount, however, does not exceed some hundredths of a per cent.

23 The analyses of air are accompanied by errors, and there are variations of composition attaining hundredths per cent.; therefore one must limit oneself to the first places in decimals in expressing the average normal composition of air.

24 The weight of a litre of hydrogen at $0^{\circ}$ and $760 \mathrm{~mm}$. pressure is $0.08958 \mathrm{gram}$, therefore 20.8 litres of oxygen weigh $29 \cdot 87$ grams, and $79^{\cdot 2}$ litres of nitrogen $99 \cdot 28$ grams, which gives the weight of a litre of air as $1 \cdot 2914$, instead of $1 \cdot 293$. This difference corresponds with the possible errors of both the analysis of air and of the other data entering into the calculation.

${ }^{25}$ In Chapter III. note 4, an approximate calculation made for the determination of the amount of oxygen in the entire atmosphere is evidently without solid foundationthat is, it may be supposed that the composition of air varies from time to time when the relation between vegetation and the processes absorbing oxygen changes; but such a supposition may be met by an argument of the following kind: the atmosphere of the earth has not, and should not have, a definite limit, and we have already seen (Chapter IV. note 33 ) that there are observations confirming this, consequently ouratmosphere should vary in its component parts with the entire heavenly space. If the equilibrium now existing were destroyed, it would be rectified by means of the immense mass of rarefied air 
Air always contains more or less moisture ${ }^{26}$ and carbonic anhydride, proceeding from the respiration of animals and the combustion of carbon and carboniferous compounds. The latter shows the properties.

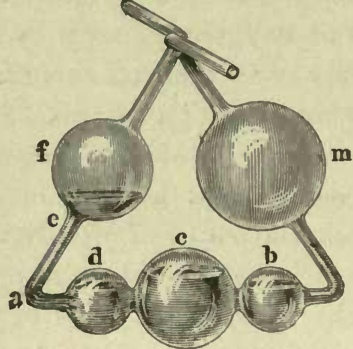

Fra. 39.-Apparatus for the absorption and washing of gases, known as Liebig's bulbs. The gas enters $m$, presses on the absorptive liquid, and passes from $m$ into $b, c, d$, and $e$ consecutively, and escapes through $f$.

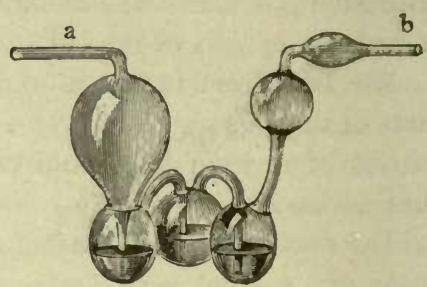

Fig. 4\%.-Geisler's potash bulbs. The gas enters. at $a$, and passes through a solution of potash in the lower bulbs, where the carbonic anhydride is absorbed, and the gas escapes from $b$. The lower bulbs are arranged in a triangle, so that the apparatus can stand without. support.

of an acid anhydride. In order to determine the amount of carbonic anhydride in air, substances are employed which absorb it-namely, alkalies either in solution or solid. A solution of caustic potash, $\mathrm{KHO}$,

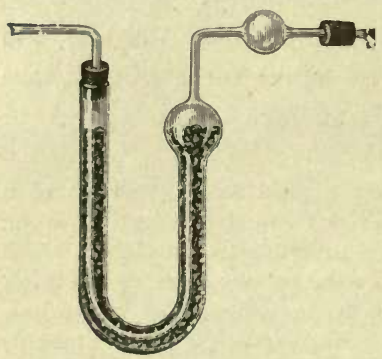

FrG. 41.-Tube for the absorption of carbonic acid. A wad of cotton wool is placed in the bulb to prevent the powder of soda-lime being carried off by the gas. The tube contains soda-lime and chloride of calcium. is poured into light glass vessels, through which the air is passed, and the amount of carbonic anhydride is determined by the increase in weight of the vessel. But it is. best to take a solid porous alkaline mass, such as soda-lime. ${ }^{27}$ With a feeble current of air a layer of soda-lime $20 \mathrm{c.m}$. in length is sufficient to completely deprive the air of the carbonic anhydride it contains. A series of tubes containing calcium which occurs in the heavenly space. If, for instance, the amount of oxygen were diminished, then it would be replenished at the expense of the oxygen pervad. ing the space of the heavens.

26 The amount of moisture contained in the air is considered in greater detail by physics and meteorology, and the subject has been mentioned above, in Chapter I. note 1, where the methods of absorbing moisture from gases were pointed out.

27 Soda-lime is prepared in the following manner :-Unslaked lime must be reduced to a fine powder and mixed with a slightly warmed and very strong solution of caustic soda. The mixing should be done in an iron dish, and they should be well stirred together until the lime begins to slack. When the mass becomes hot, it boils, swells up, and solidifies, forming a porousmass very rich in alkali and capable of absorbing carbonic anhydride. A lump of caustic soda or potash presents a much smaller surface for absorption, and therefore acts much less rapidly. It is necessary to place an apparatus for absorbing water after the apparatus for absorbing the carbonic anhydride, because the alkali in absorbing the latter evolves water. 
chloride for absorbing the moisture ${ }^{28}$ is placed before the apparatus for the absorption of the carbonic anhydride, and a measured mass of air is caused to pass through the whole apparatus by means of an aspirator. In this manner the determination of the moisture is combined with the absorption of the carbonic acid. The arrangement shown in fig. 38 is such a combination.

The amount of carbonic anhydride ${ }^{29}$ in free air is incomparably more constant than the amount of moisture. The average amount of carbonic anhydride in 100 volumes of dry air is approximately $\frac{3}{100}$ p.c. that is, 10000 volumes of air contain about 3 volumes of carbonic anhydride, most frequently about 2.95 volumes. As the specific gravity of carbonic anhydride, referred to air $=1.52$, therefore 100 parts by weight of air contain 0.045 part by weight of carbonic anhydride. This quantity varies according to the time of year (more in winter), the altitude above the level of the sea (less at high altitudes), on the proximity to. forests and fields (less) or cities (greater), \&c. But the variation is small and rarely exceeds limits of $2 \frac{1}{2}$ to 4 ten-thousandths by volume. ${ }^{30}$ As there are many natural local influences which either increase the

${ }^{28}$ It is evident that the calcium chloride employed for absorbing the water slould be free from lime or other alkalis in order that it should not retain earbonic anhydride. Such calcium chloride may be prepared in the following manner. An entirely neutral solution of calcium chloride is prepared from lime and hydrochloric acid; it is then evaporated first. over a water-bath and then over a sand-bath with great care. When the solution attains a certain strength a scum is formed, which solidifies on reaching the top. This scum is collected, and will be found to be free from caustic alkalis. It is necessary in any case to test it before use, as otherwise a large error may be introduced into the results, owing ta the presence of free alkali (lime). It is better still to pass carbonic anhydride through the tube containing the calcium chloride for some time before the experiment, in order to saturate any free alkali that may remain from the decomposition of a portion of the calcium chloride by water, $\mathrm{CaCl}_{2}+2 \mathrm{H}_{2} \mathrm{O}=\mathrm{CaOH}_{2} \mathrm{O}+2 \mathrm{HCl}$.

${ }^{29}$ Recourse is had to special methods when the determination regards only the carbonic. anhydride of the air. For instance, it is absorbed by an alkali which does not contain carbonates (by a solution of baryta or caustic soda mixed with baryta), and then the carbonic anhydride is expelled by an excess of an acid, and its amount determined by the volume given off. During the last ten years the question as to the amount of carbonic anhydride present in the air has been submitted to many voluminous and exact researches, especially those of Reiset, Schloesing, Miintz, and Aubin, who showed that the amount of carbonic anhydride is not subject to such variations as was at first supposed on the basis of incomplete and insufficiently accurate determinations.

30 It is a different case in enclosed spaces in dwellings, cellars, wells, caves, and mines, where the renewal of air is hampered. Under these circumstances large quantities of carbonic anhydride may accumulate. Even in cities, where there are many conditions for the evolution of carbonic anhydride (respiration, decomposition, combustion), its amount is greater than in free air, yet even in still weather the difference does not often exceed one ten-thousandth (that is, rarely attains 4 instead of $2 \cdot 4$ vols. in 10000 vols. of air). Hundreds of very careful comparative determinations made simultaneously around Paris and in the city itself, and constant daily determinations conducted at certain meteorological stations (for instance, at Montsouris, near Paris), confirm this conclusion. On high mountains and in deep valleys, as has been proved in the Pyrenees, the difference of 
amount of carbonic anhydride in the air (respiration, combustion, rotting, volcanic eruptions, \&c.), or diminish it (absorption by plants and water), therefore the reason of the great constancy in the anount of this gas in air must be looked for, in the first place, in the fact that the wind mixes the air of various localities together, and, in the second place, in the fact that the waters of the ocean, holding carbonic acid in solution, ${ }^{31}$ form an immense reservoir for regulating the supply of this gas in the atmosphere. As immediately the partial pressure of the carbonic anhydride in the air decreases, the water evolves it, and when the partial pressure increases, it absorbs it, nature consequently supplies the conditions for a natural mobile state of equilibrium in this instance, as in a number of others. ${ }^{32}$

Beyond nitrogen, oxygen, moisture, and carbonic acid, all other substances occurring in air are found in infinitesimally small quantities by weight, and therefore the weight of a cubic measure of air depends, to a sensible degree, on the above-named components alone. We have already mentioned that at $0^{\circ}$ and $760 \mathrm{~mm}$. pressure the weight of a cubic litre of air is 1.293 grams $^{33}$ the air being understood to be

the amount of carbonic anhydride is also very slight (at a height it is, however, less, as would be expected).

31 In the sea as well as fresh water the carbonic acid occurs in two forms, directly dissolved in the water, and combined with lime, as calcium bicarbonate (hard waters sometimes contain very much carbonic acid in this form). If the tension of the carbonic anhydride in the first form varies with the temperature, and its amount with the partial pressure, that in the form of acid salts is under the same conditions, because direct experiments have shown a similar dependence in this case, although the quantitative relations are different in the two cases.

32 In studying the phenomena of nature one inevitably arrives at the idea that the universally reigning state of mobile equilibrium forms the chief reason of that harmonious order which impresses all observers. It not unfrequently happens that we do not see the causes regulating the order and harmony ; in the particular instance of carbonic anhydride it is a striking circumstance that in the first instance a search was made for an harmonious and strict uniformity, and in incidental (insufficiently accurate and fragmentary) observations conditions were even found for concluding it to be absent. When, later, the rule of this uniformity was confirmed, then the causes regulating such order were also discovered. The researches of Schloesing were of this character. Deville's idea of the dissociation of the acid carbonates of sea-water is suggested in them. In much else, also, a right understanding can only be looked for in detailed investigation.

33 The difference of the weight of a litre of dry air (free from carbonic anhydride) at $0^{\circ}$ and $760 \mathrm{~mm}$., at different longitudes and altitudes, depends on the fact that the force of gravity varies under these conditions, and with it the pressure of the barometrical column also varies. This is treated in detail in my works On the Elasticity of Gases and On Barometric Levellings.

In reality the weight is not measured in absolute units of weight (in pressure-refer to works on mechanics and physics), but in relative units (grams, scale weights) whose mass is one and the same, and therefore the variation of the weight of the weiglits itself with the change of gravity must not be here taken into account, for the matter deals with weights proportional to the masses, and with a change of locality the weight of the weights varies as the weight of a given volume of air does. 
dry and free from carbonic anhydride. Taking the amount of the latter as 0.03 per 100 volumes, we obtain a greater weight-namely, 1.000156 times greater (consequently at $0^{\circ}$ and $760 \mathrm{~mm}$., instead of $1 \cdot 29300$ grams, the weight of a litre will then be $1 \cdot 29319$ grams). The weight of moist air in which the tension ${ }^{34}$ of the aqueous vapour (partial pressure) $=f \mathrm{~mm}$., and consequently the volume of vapour (its density referred to air $=0 \cdot 62)=\frac{f}{760}$ f the whole pressure of the moist air $=760 \mathrm{~mm}$.) is to the weight of an equal volume of dry air as $\frac{760-f}{760}+0.62 \underset{760}{f}$, or as $\frac{760-0.38 f}{760}$, is to 1 ; that is, the weight of air containing carbonic anhydride and moisture at $0^{\circ}$ and $760 \mathrm{~mm}$. pressure is not 1.2930 grams, but this weight multiplied by 1.000156 and by $\frac{1-0.38 f}{760}$. At a pressure (total) of air of $\mathrm{H}$ millimetres, a temperature $t$, and elasticity of vapour $f$, the weight of a litre of air will be (i.e., if at $0^{\circ}$ and $760 \mathrm{~mm}$. the weight of dry air $=1.293$ ) equal to. $\frac{1.29319}{1+0.00367 t} \times \frac{\mathrm{H}-0.38 \mathrm{f}}{760}$. For instance, if $\mathrm{H}=730 \mathrm{~mm} ., t=20^{\circ}$, and $f=10 \mathrm{~mm}$. (the moisture is then slightly below 60 p.c.), the weight of a litre of air $=1 \cdot 1512$ gram..$^{35}$

The presence of ammonia, a compound of nitrogen and hydrogen, in the air, is indicated by the fact that all acids exposed to the air absorb ammonia from it after a time. De Saussure observed that aluminium sulphate is converted by air into a double sulphate of ammonium and aluminium, or the so-called ammonia alum. Quantitative determinations have shown that the amount of ammonia ${ }^{36}$ contained in air varies at different periods. However, it may be accepted that 100 cubic

54 The tension of the aqueous vapour in the air is determined by hygrometers and other similar methods. It may also be determined by analysis (see Chapter I. note 1).

55 In determining the weight of small and heavy objects (crucibles, \&c. in analysis, and in determining the specific gravities of liquids, \&c.) a correction may be introduced for the loss of weight in the air of the room, by taking the weight of a litre of air displaced as 1.2 gram, and consequently 0.0012 gram for every cubic centimetre. But if gases or, in general, large vessels are weighed, and the weighings have to be accurate, it is necessary to take into account all the data for the determination of the density of the air $(t, \mathrm{H}$, and $f)$, because sensitive balances can determine the possible variations of the weight of air, as in the case of a litre the weight of air varies in centigrams, even at a constant temperature, with variations of $\mathrm{H}$ and $f$. The following method was long ago (1859) proposed and applied by me for this purpose. A large light and closed vessel is taken, and its volume and weight in a vacuum are accurately determined, and verified from time to time. On weighing it we obtain the weight in air of a given density, and by subtracting this weight from its absolute weight and dividing by its volume we obtain the density of the air.

36 Schloesing studied the equilibrium of the ammonia of the atmosphere and of the rivers, seas, \&c., and he showed that its amount is interchangeable between them. The 


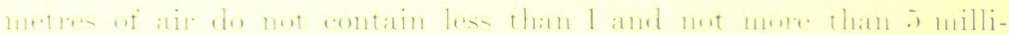

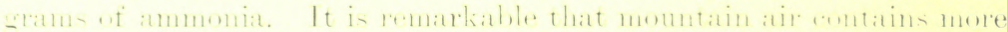

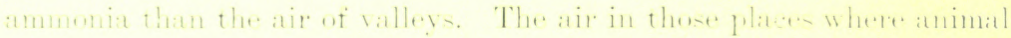

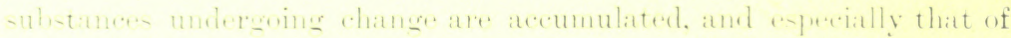

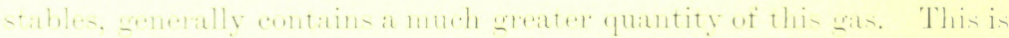

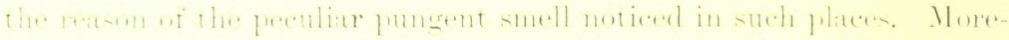

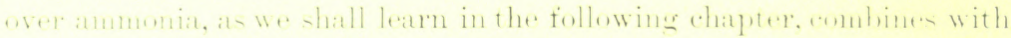

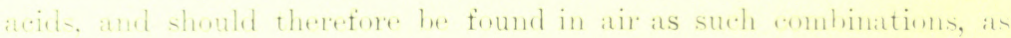

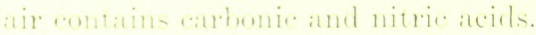

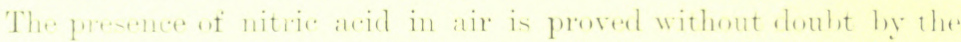

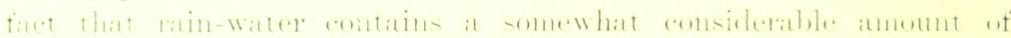
nitrie arid. as we shall learn when speaking of this acid.

Furthere (as has heen mentioned in Chateter IV.), air (antains asome and hydrentell perexide.:

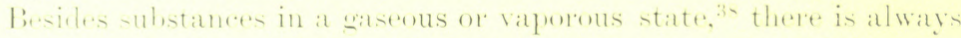

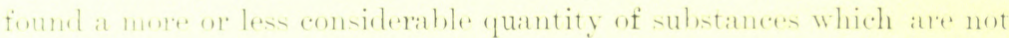
known in a state of vapour. These substances ale carrient in the air as most. If a linen surface moistened with an aceirl, be platerel in perfectly bure ate then the washines are found to contain sodium, aaleium, iron,

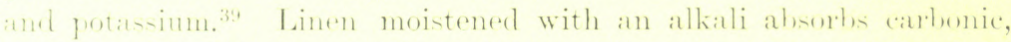
-olphurie, phosphorie, and hyolochloric acists. Further. the presence

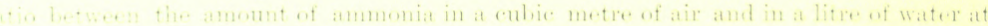

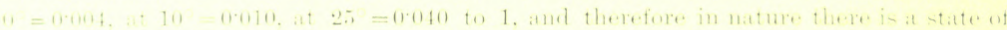

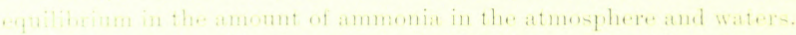

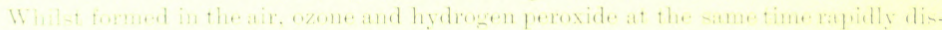

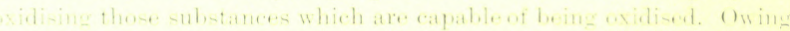

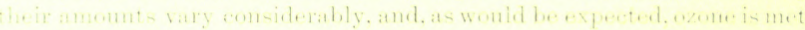

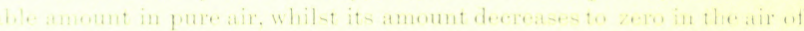

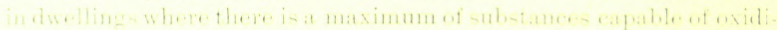

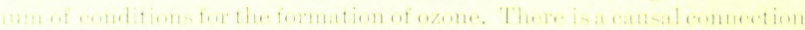

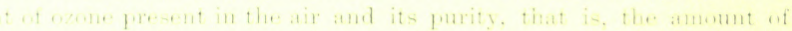

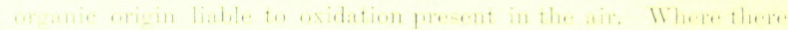

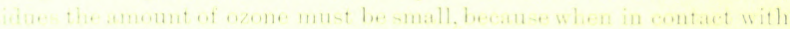

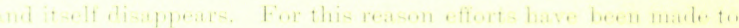

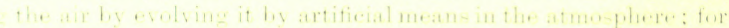

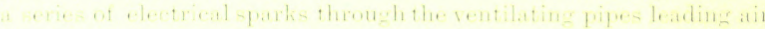

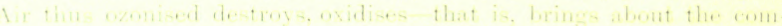

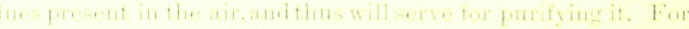

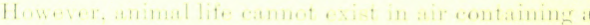

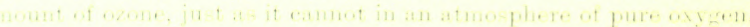

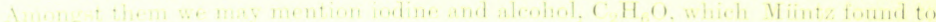

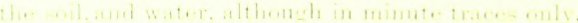

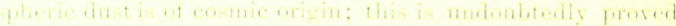

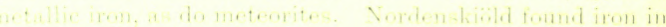

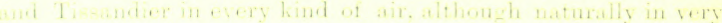


of organic substances in air has been proved by a similar experiment. If a glass globe be filled with ice and placed in a room where are a number of people, then the presence of organic substances, like albuminous substances, may be proved in the water which condenses on the surface of the globe. It may be that the miasmas causing infection in marshy localities, hospitals, and in epidemic illnesses proceed from the presence of such substances in the air, as well as from the presence of germs of lower organisms borne in the air as a minute dust. Pasteur proved the presence of such germs in the air by the following experiment:- He placed gun-cotton (pyroxylin), which has the appearance of ordinary cotton, in a glass tube. Gun-cotton is soluble in a mixture of ether and alcohol, forming the so-called collodion. A current of air was passed through the tube for a long period of time, and the gun-cotton was then dissolved in a mixture of ether and alcohol. An insoluble residue was thus obtained which actually contained the germs of organisms, as was shown by microscopical observations, and by their capacity to develop into organisms (mould, \&c.) under favourable conditions. The presence of these germs determines the property of air of bringing about the processes of rotting and fermentation-that is, the fundamental alteration of organic substances, which is accompanied by an entire change in their properties. The appearance of lower organisms, both vegetable and animal, is frequently to be remarked in these processes. Thus, for instance, in the process of fermentation, when, for example, wine is procured from the sweet juice of grapes, a sediment separates out which is known under the name of lees, and contains peculiar yeast organisms. Germs are required before these organisms can appear. ${ }^{40}$ They are borne in the air, and fall into such substances from it. Finding themselves under favourable conditions, the germs develop into organisms ; they are nourished at the expense of the organic substance, and during growth change and destroy it, and bring about corruption and rotting. This is why, for instance, the juice of the grape when contained in the skin of the fruit, which allows access of the air but is impenetrable to the germs, does not ferment, does not alter so long as the skin remains intact. This is also the reason why animal substances when kept from the access of air may be preserved for a great length of time. Conserves for long sea

40 The idea of the spontaneous growth of organisms in a suitable medium, although still upheld by many, has since the work of Pasteur and his followers (and to a certain extent of his predecessors) been discarded, because it has been proved how, when, and whence (from the air, water, \&c.) the germs appear; that fermentation as well as infectious diseases cannot take place without them; and mainly because it has been shown that any change accompanied by the development of the organisms introduced may be brought about at will by the introduction of the germs into a suitable medium. 
voyages are preserved in this way. ${ }^{41}$ Hence it is evident that however infinitesimal the quantity the germs carried in the atmosphere may be, still they have an immense significance in nature. ${ }^{42}$

Thus we see that air contains a great variety of substances. The nitrogen, which is found in it in the largest quantity, has the least. influence on those processes which are accomplished by the action of air. The oxygen, which is met with in a lesser quantity than the nitrogen, on the contrary, takes a very important part in a number of reactions ; it supports combustion and respiration, it brings about corruption and every process of slow oxidation. The part played by the moisture of air is known to everyone. The carbonic anhydride, which is met with in still smaller quantities, has an immense significance in nature, inasmuch as it serves for the nourishment of plants. The importance of the ammonia and nitric acid is immense, because they are the sources of the nitrogenous substances comprising an indispensable element in all living organisms. And, lastly, the infinitesimal quantity of germs evinces their significance in a number of processes. Thus it is not the quantitative but the qualitative relations of the component parts of the atmosphere which determine its importance in nature. ${ }^{43}$

Air, being a mixture of various substances, may suffer considerable changes in consequence of incidental circumstances. It is particularly important to remark that change in the composition of air which takes place in dwellings and in various localities where human beings have to remain during a lengthy period of time. The respiration of human beings and animals alters the air. ${ }^{44}$ A similar deterioration of air is produced by the influence of decomposing organic substances, and

41 In further confirmation of the fact that putrefaction and fermentation depend on germs carried in the air, we may cite the circumstance that poisonous substances destroying the life of organisms stop or hinder the appearance of the above processes. Air which has been heated to redness or passed through sulphuric acid no longer contrins the germs of organisms, and loses the faculty of producing fermentation and putrefaction.

42 Their presence in the air is naturally due to the diffusion of germs into the atmosphere, and owing to their microscopical dimensions they, as it were, hang in the air in virtue of their large surfaces compared to their weight. In Paris the amount of dust hanging in the air equals from 6 (after rain) to 23 grams per 1000 c.m. of air.

43 We see similar cases everywhere. For example, the predominating mass of sand and clay in the soil takes hardly any chemical part in the economy of the soil in respect to the nourishment of plants. The plants by their roots search for substances which are diffused in comparatively small quantities in the soil. If a large quantity of these nourishing substances be taken, then the plants will not develop in the soil, just as animals die in oxygen.

44 A man in breathing burns about 10 grams of carbon per hour-that is, he produces about 880 grams, or (as 1 c.m. of carbonic anhydride weighs about 2000 grams) about $\frac{5}{12}$ c.m. of carbonic anhydride. The air coming from the lungs contains 4 p.c. of carbonic anhydride by volume. The exhaled air acts as a direct poison, owing to this gas and to other impurities. 
especially of substances burning in it. ${ }^{45}$. Hence it is necessary to have regard to the purification of the air of dwellings. The renewal of air, the replacing of respired by fresh air, is termed 'ventilation,' 46 and the

45 For this reason candles, lamps, and gas change the composition of air almost in the same way as respiration. In the burning of 1 kilogram of stearin candles, 50 cubic metres of air are changed as by respiration-that is, 4 p.c. of carbonic acid will be formed in this volume of air. The respiration of animals and exhalations from their skins, and especially from the intestine and the excrements and the transformations taking place in them, spoil the air to a still greater extent, because they introduce other volatile substances besides carbonic anhydride into the air. At the same time that carbonic anhydride is formed the amount of oxygen in the air decreases, and consequently the relative amount of nitrogen increases, together with which there is noticed the appearance of mias. mata which occur in but small quantity, but which are noticeable in passing from fresh air into space full of such adulterated air. The researches of Schmidt and Leblanc and others show that with $20^{\circ} 6$ p.c. of oxygen (instead of $20^{\circ} 9$ p.c.), when the diminution is due to respiration, air already becomes noticeably heavy and unfit for respiration, and that the heavy feeling experienced in such air increases with a lesser percentage of oxygen. It is difficult to remain for a few minutes in air containing $17 \cdot 2$ p.c. of oxygen. These observations were chiefly obtained by observations on the air of different mines, at different depths below the surface. The air of theatres and buildings full of people also proves to contain less oxygen; it was once found that at the end of a theatrical representation the air at the stalls contained $20 \cdot 75$ p.c. of oxygen, whilst the air at the upper part of the theatre contained only 20.36 p.c. The amount of carbonic anhydride in the air may be taken as a measure of its purity (Pettenkofer). When it reaches 1 p.c. it is very difficult for human beings to remain long in such air, and it is necessary to set up a vigorous ventilation for the removal of the adulterated air. In order to keep the air in dwellings in a uniformly good state, it is necessary to introduce at least 10 cubic metres of fresh air per hour per person. We saw that a man exhales about five-twelfths cubic metres of carbonic anhy. dride per day. Accurate observations have shown that air containing one-tenth p.c. of exhaled carbonic anhydride (and consequently also a corresponding amount of the other substances evolved together with it) is not yet felt as spoilt; and therefore the five-twelfth cubic metres of carbonic anhydride should be diluted with 420 cubic metres of fresh air if it be desired to keep not more than one-tenth p.c. (by volume) of carbonic anhydride in the air. Hence a man requires 420 cubic metres of air per day, or 18 cubic metres per hour. With the introduction of only 10 cubic metres of fresh air per person, the amount of carbonic anhydride may reach one-fifth p.c., and the air will not then be of the required freshness.

46 The ventilation of inhabited buildings is most necessary, and is even indispensable in hospitals, schools, and similar buildings. In winter it is carried on by the so-called calorifiers or stores heating the air before it enters. The best kind of calorifiers in this respect are those in which the fresh cold air is led through a series of channels heated by the hot gases coming from a stove In ventilation, particularly during winter, care is taken that the incoming air sliall be moist, because in winter the amount of moisture in the air is very sinall. Ventilation, besides introducing fresh air into a dwelling-place, must also withdraw the air already spoilt by respiration and other causes-that is, it is necessary to construct channels for the escape of the bad air, besides those for the introduction of fresh air. In ordinary dwelling-places, where not many people are congregated, the ventilation is conducted by natural means, in the heating by fires, through erevices, windows, and various orifices in walls, doors, and windows. In mines, factories, and works ventilation is of the greatest importance.

Animal vitality may still continue for a period of several minutes in air containing up to 30 p.c. of carbonic anhydride, if the remaining 70 p.c. consist of ordinary air, but respiration ceases after a certain time, and death may even ensue. The flame of a candle is extinguished in an atmosphere containing from 5 to 6 p.c. of carbonic anhydride,

VOL. I. 


\section{removal of impurities from the air is called 'disinfection.' ${ }^{47}$ The accu-} mulation of all kinds of impurities in the air of dwellings and cities is the reason why the air of mountains, forests, seas, and non-marshy localities, covered with vegetation or snow, is distinguished for its freshness, and, in all respects, beneficial action.

but animal vitality can be sustained in it for a somewhat long time, although the effect of such air is exceedingly painful to even the lower animals. There are mines in which a lighted candle easily goes out from the excess of carbonic anhydride, but in which the miners have to remain for a long time. The properties of air may be considerably vitiated by the combustion of charcoal, wood, and similar substances in it, even when it contains a comparatively small amount of carbonic anhydride. This doubtless depends on the fact that certain gaseous substances are formed in the act of combustion (carbonic oxide, acetylene, hydrocyanic acid, and others) which are positively injurious to breathe. The action of charcoal fumes and smoke is based on this fact. The presence of 1 p.c. of carbonic oxide is deadly even to cold-blooded animals. The air of explosive mines, where explosions are made, is known to produce a state of insensibility resembling that produced by charcoal fumes. Deep wells and vaults not unfrequently contain similar substances, and their atmosphere often causes suffocation. The atmospheres of such places cannot be tested by lowering a lighted candle into it, as these poisonous gases would not extinguish the flame. This method only suffices to indicate the amount of carbonic anhydride. If a candle keeps alight, it signifies that there is less than 5 p.c. of this gas. In doubtful eases it is best to lower a dog or other animal into the air to be tested.

47 Different so-called disinfectants are capable of purifying the air, and of preventing the injurious action of certain of its components by changing or destroying them. Disinfection is especially necessary in those places where a considerable amount of volatile substances are evolved into the air, and where organic substances are decomposed; for instance, in hospitals, closets, \&c. The numerous disinfectants are of the most varied nature. They may be divided into the following chief categories: oxidising substances, antiseptic substances, and absorbent substances. To the oxidising substances used for disinfection belong chlorine, and various substances evolving it, because chlorine in the presence of water oxidises the majority of organic substances. Further, to this class belong the permanganates of the alkalis, as substances easily oxidising matters dissolved in water; these salts are not volatile like chlorine, and therefore act much more slowly, and in a much more limited sphere. Antiseptic substances are those which convert organic substances into such as are little prone to change, and prevent putrefaction and fermentation. They most probably kill the germs of organisms occurring in miasmata. The most important of these substances are creosote and phenol (carbolic acid), which occur in tar, and which act in preserving smoked meat. Phenol is a substance little soluble in water, volatile, oily, and having the characteristic smell of smoked objects. Its action on animals in considerable quantities is injurious, but in small quantities, used in the form of a weak solution, it prevents the change of animal matter. The smell of privies, which depends on the change of excremental matter, may be easily removed by means of chlorine or phenol. Salicylic acid, thymol, common tar, \&c., are also substances having the same property. They are used in special cases, but naturally not so generally. Absorbent substances are of no less importance than the preceding two classes of disinfectants, inasmuch as they act regularly and are innocuous. They are such substances as absorb the odoriferous gases and vapours emitted during putrefaction, which are chiefly ammonia, sulphuretted hydrogen, and other volatile compounds. To this class belong charcoal, certain salts of iron, gypsum, salts of magnesia, and such like substances, as well as peat, mould, and clay. Their employment is profitable, not only for removing the odour, but also in the radical destruction of miasmata. The questions both of disinfection and ventilation appertain to the most serious problems of common life and hygiene. These questions are so vast that we are here able only to give a short outline of their nature. 


\section{CHAPTER VI}

\section{THE COMPOUNDS OF NITROGEN WITH HYDROGEN AND OXYGEN}

Is the last chapter we saw that nitrogen does not immediately combine with hydrogen, but that a mixture of these gases in the presence of hydrochloric acid gas, $\mathrm{HCl}$, forms ammonium chloride, $\mathrm{NH}_{4} \mathrm{Cl}$, on the passage of a series of electric sparks. ${ }^{1}$ In ammonium chloride, $\mathrm{HCl}$ is combined with $\mathrm{NH}_{3}$, consequently $\mathrm{N}$ with $\mathrm{H}_{3}$ forms ammonia. ${ }^{2}$ Almost all the nitrogenous substances of plants and animals evolve ammonia when heated with an alkali. But even without the presence of an alkali the majority of nitrogenoussubstances, when decomposed or heated with a limited supply of air, evolve their nitrogen, if not entirely, at all events partially, in the form of ammonia. Thus, when animal substances such

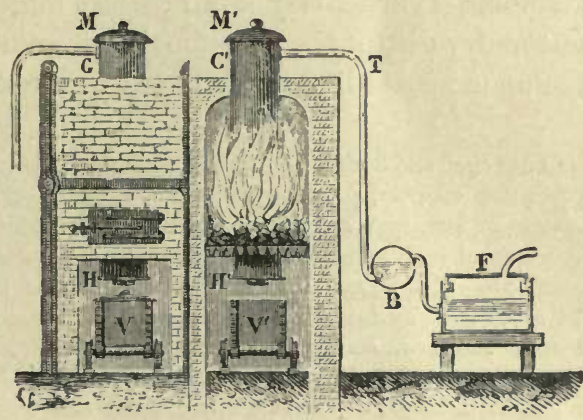

F1G. 42. - The dry distillation of bones on a large scale. The boues are heated in the vertical cylinders $C$ (about $1 \frac{1}{2}$ metres high and 30 centimetres in diameter). The products of distillation pass through the tubes $\mathrm{T}$, into the condenser $\mathrm{B}$, and receiver $\mathrm{F}$. When the distillation is completed the trap $H$ is opened, and the burnt bones are loaded into trucks $V$. The roof $M$ is then opened, and a fresh quantity of bones charged into the cylinders. The ammonia water is preserved, and goes to the preparation of ammoniacal salts, as described in the following drawing.

as skins, bones, flesh, hair, horns, \&c., are heated without access of air in iron retorts-or, as it is termed, are subjected to dry distil-

1 The ammonia in the air, water, and soil proceeds from the decomposition of the nitrogenous substances of plants and animals, and also probably from the reduction of nitrates. Ammonia is always formed in the rusting of iron. Its formation in this case depends in all probability on the decomposition of water, and on the action of the hydrogen at the moment of its evolution on the nitric acid contained in the air (Cloez), or on the formation of ammonium nitrite, which takes place under many circumstances. The evolution of vapours of ammonia compounds is sometimes observed in the vicinity of volcanoes.

2 If a silent discharge (as in the ozonisation of oxygen), or a series of electric sparks (for instance, in a eudiometer), be passed through ammonia gas, it is decomposed 
lation, they decompose. A portion of the substances proceeding from the decomposition remains in the retort and forms a carbonaceous residue, whilst the other portion, in virtue of its volatility, escapes through the tube leading from the retort. The vapours given off, on cooling, form a liquid which separates into two layers; the one, which is oily, is composed of the so-called animal oils (oleum animale), the other, an aqueous layer, contains a solution of ammonia salts. If this solution be mixed with lime and heated, the lime takes up the elements of carbonic acid from the ammonia salts, and ammonia is evolved as a gas. $^{3}$ In ancient times ammonia compounds were imported into Europe from Egypt, where they were prepared from the soot obtained in the employment of camels' dung as fuel in the locality of the temple of Jupiter Ammon (in Lybia), and therefore the salt obtained was called 'sal-ammoniacale,' from which the name of ammonia is derived. Now ammonia is exclusively obtained, on a large scale, either from the products of the dry distillation of animal or vegetable refuse, from urine, or from the ammoniacal liquors collected in the destructive distillation of coal

into nitrogen and hydrogen. This is a phenomenon of dissociation, as was explained in the preceding chapter, p. 227. Therefore, a series of sparks do not totally decompose the ammonia, but leave a certain portion undecomposed. One volume of nitrogen and three volumes of hydrogen are obtained from two volumes of ammonia. The presence of free ammonia - that is, ammonia not combined with acids-in a gas or aqueous solution may be recognised by its characteristic smell. But many ammonia salts do not possess this smell. However, on the addition of an alkali (for instance, caustic lime, potash, or soda), they evolve ammonia gas, especially when heated. The presence of ammonia may be made visible by introducing a substance moistened with strong hydrochloric acid into its neighbourhood. A white cloud, or visible white vapour, then makes its appearance. This depends on the fact that both ammonia and hydrochloric acid are volatile, and on coming into contact with each other form solid sal-ammoniac, $\mathrm{NH}_{4} \mathrm{Cl}$, which forms a cloud. This test is usually made by dipping a glass rod into hydrochloric acid, and holding it over the ressel from which the ammonia is evolved. With small amounts of ammonia this test is, however, untrustworthy, as the white vapour is scarcely observable. In this case it is best to take paper moistened with mercurous nitrate, $\mathrm{HgNO}_{3}$. This paper turns black in the presence of ammonia, owing to the formation of a black compound of ammonia with mercurous oxide. The smallest traces of ammonia, for instance, in river water, may be discovered by means of the so-called Nessler's reagent, containing a solution of mercuric chloride and potassium iodide, which forms a brown coloration or precipitate with the smallest quantities of ammonia. Here it will be useful to give the thermo-chemical data (in thousands of units of heat, according to Thomsen), or the quantities of heat evolved in the formation of ammonia and its compounds in quantities expressed by their formulæ. Thus, for instance $\left(\mathrm{N}+\mathrm{H}_{3}\right) 26.7$ indicates that 14 grams of nitrogen in combining with 3 grams of hydrogen develop sufficient heat to raise the temperature of 26.7 kilograms of water $1^{\circ} \cdot\left(\mathrm{NH}_{3}+\mathrm{nH}_{2} \mathrm{O}\right) 8.4$ (heat of solution); $\left(\mathrm{NH}_{3}, \mathrm{nH}_{2} \mathrm{O}+\mathrm{HCl}, \mathrm{nH}_{2} \mathrm{O}\right) 12 \cdot 3 ;\left(\mathrm{N}+\mathrm{H}_{4}+\mathrm{Cl}\right) 90 \cdot 6 ;\left(\mathrm{NH}_{3}+\mathrm{HCl}\right) 41.9$.

3 The same ammonia water is obtained, although in smaller quantities, in the dry distillation of plants and of coal, which consists of the remains of fossil plants. In all these cases the ammonia proceeds from the destruction of the complex nitrogenous substances occurring in plants and animals. The ammonia salts employed in practice are prepared by this method. 
for the preparation of coal gas. This ammoniacal liquor is placed in a retort with lime and heated; the ammonia is then evolved together with steam. ${ }^{4}$ In practice, only a small amount of ammonia is used in a free state - that is, in an aqueous solution; the greater portion of it is converted into different salts having technical uses, especially salammoniac, $\mathrm{NH}_{4} \mathrm{Cl}$, and ammonia sulphate, $\left(\mathrm{NH}_{4}\right)_{2} \mathrm{SO}_{4}$. They are saline substances which are formed because amnonia, $\mathrm{NH}_{3}$, combines with all acids, $\mathrm{HX}$, forming ammonia salts, $\mathrm{NH}_{4} \mathrm{X}$. Sal-ammoniac, $\mathrm{NH}_{4} \mathrm{Cl}$, is

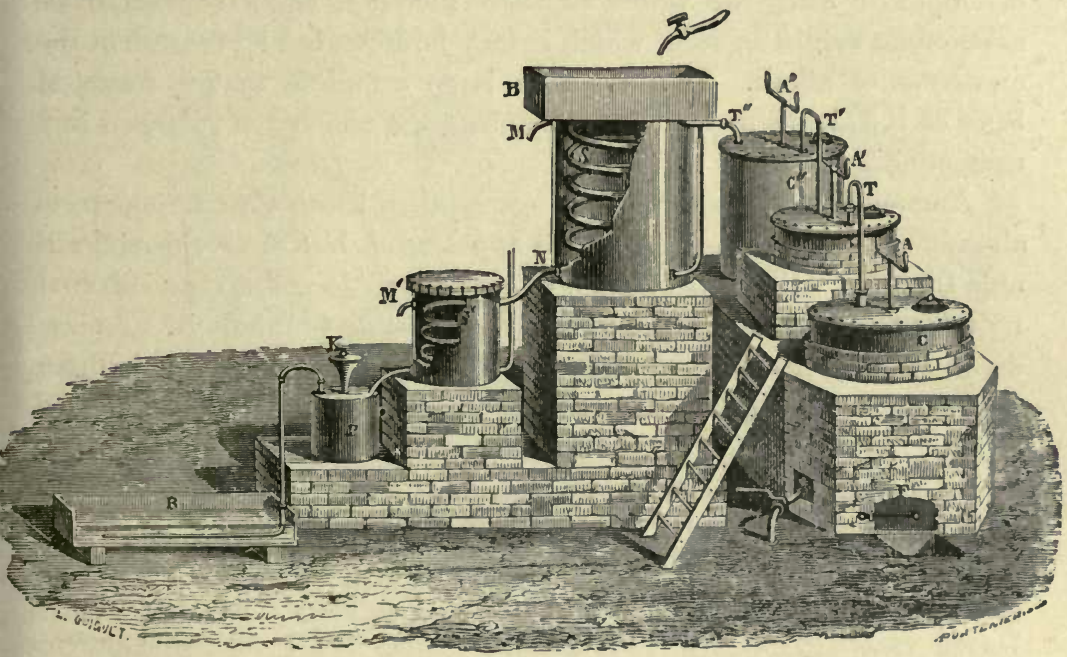

FIG. 43.-Method of abstracting ammonia, on a large scale, from ammonia water obtainel at gas works by the dry distillation of coal, or by the fermentation of urine, scc. This water is mixed with lime and poured into the boiler $\mathbf{C}^{\prime \prime}$, and from thence into $\mathbf{C}^{\prime}$ and $\mathbf{C}$ consecutively. The last boiler is heated directly over a furnace, and therefore no ammonia remains in solution after the liquid has been boiled in it. The liquid is therefore then thrown away. The ammonla vapour and steam pass from the boiler $C$, through the tube $T$, into the boiler $C^{\prime}$, and then into $C^{\prime \prime}$, so that the solution in $\mathrm{C}^{\prime}$ becomes stronger than that in $\mathrm{C}$, and still stronger in $\mathrm{C}^{\prime \prime}$. The boilers are furnished with stirrers $\mathbf{A}, \mathbf{A}^{\prime}$, and $\mathbf{A}^{\prime \prime}$ to prevent the lime settling. From $\mathbf{C}^{\prime \prime}$ the ammonia and steam pass through the tube $T^{\prime \prime}$ into worm condensers surrounded with cold water, from thence into the Woulfe's bottle P, where the solution of ammonia is collected, and from whence the still uncondensed ammonia vapour is led into the flat vessel $R$, containing acid which retains traces of ammonia.

a compound of ammonia with hydrochloric acid. It is prepared by passing the vapours of ammonia and water, evolved, as above described, from ammoniacal liquor, into an aqueous solution of hydrochloric acid, and on evaporating the solution sal-ammoniac is obtained in the form of soluble crystals ${ }^{5}$ resembling common salt in appearance and pro-

4 The technical methods for the preparation of ammonia water, and for the extraction of ammonia from it, are to a certain extent explained in the figures accompanying the text.

3 Usually these crystals are sublimed by heating them in crucibles or pots, when the vapours of sal-ammoniac condense on the cold covers as a crust, in which form it comes into the market. 
perties. Ammonia may be very easily prepared from this sal-ammoniac, $\mathrm{NH}_{4} \mathrm{Cl}$, as from any other ammoniacal salt, by heating it with lime. Calcium hydroxide, $\mathrm{CaH}_{2} \mathrm{O}_{2}$, as an alkali takes up the acid and sets free the ammonia, forming calcium chloride, according to the equation $2 \mathrm{NH}_{4} \mathrm{Cl}+\mathrm{CaH}_{2} \mathrm{O}_{2}=2 \mathrm{H}_{2} \mathrm{O}+\mathrm{CaCl}_{2}+2 \mathrm{NH}_{3}$. In this reaction the ammonia, as a gas, is evolved. ${ }^{6}$

It must be observed that all the complex nitrogenous substances of plants, animals, and soils are decomposed when heated with an excess of sulphuric acid, the whole of their nitrogen being converted into ammonium sulphate, from which it may be liberated by treatment with an excess of alkali. This reaction is so complete that it forms the basis of Kjeldahl's method for estimating the amount of nitrogen in its compounds.

Ammonia is a colourless gas, resembling those with which we are already acquainted in its outward appearance but clearly distinguishable from any other gas by its very characteristic and strong smell. It irritates the eyes, and it is positively impossible to inhale it. Animals die in it. Its density, referred to hydrogen, is 8.5 ; hence it is lighter than air. It belongs to the class of gases which are easily liquefied. ${ }^{7}$

6 On a small scale ammonia may be prepared in a glass flask by mixing equal parts by weight of slacked lime and finely-powdered sal-ammoniac, the neck of the flask being connected with an arrangement for drying the gas obtained. In this instance neither calcium chloride nor sulphuric acid can be used for drying the gas, because they absorb ammonia, and therefore solid caustic potash, which is capable of retaining the water, is employed. The gas conducting tube leading from the desiccating apparatus is introduced into a mercury bath, if dry gaseous ammonia be required, because water cannot be employed in collecting ammonia gas. Ammonia was first obtained in this dry state by Priestley, and its composition was investigated by Berthollet at the end of the last century. Oxide of lead mixed with sal-ammoniac (Isambert) evolves ammonia with still greater ease than lime. The cause and process of the decomposition is almost the same, $2 \mathrm{PbO}+2 \mathrm{NH}_{4} \mathrm{Cl}=\mathrm{Pb}_{2} \mathrm{OCl}_{2}+\mathrm{H}_{2} \mathrm{O}+2 \mathrm{NH}_{3}$. Lead oxychloride is (probably) formed.

7 This is evident from the fact that its absolute boiling point lies about $+130^{\circ}$ (Chap. II. Note 29). Consequently, it may be liquefied by pressure alone at the ordinary, and even much higher, temperatures. The latent heat of evaporation of 17 parts by weight of ammonia equals 4400 units of heat, and therefore liquid ammonia may be employed for the production of cold. Strong aqueous solutions of ammonia, which in parting with their ammonia act in a similar manner, are not unfrequently employed for this purpose. Suppose a saturated solution of ammonia to be held in a closed vessel furnished with a receiver. If the ammoniacal solution be heated, the ammonia, with a small quantity of water, will pass off from the solution, and in accumulating in the apparatus will produce a considerable pressure, and will therefore liquefy in the cooler portions of the receiver. Hence liquid ammonia will be obtained in the receiver. The heating of the vessel containing the aqueous solution of ammonia is then stopped. After having been heated it contains only water, or a solution poor in ammonia. When once it begins to cool, the ammonia vapours commence dissolving in it, the space becomes rarefied, and a rapid vaporisation of the liquefied ammonia left in the receiver takes place. In evaporating in the receiver it will cause the temperature in it to fall considerably, and will itself pass into 
Faraday employed the following method for liquefying ammonia. Ammonia, when passed over dry silver chloride, $\mathrm{AgCl}$, is absorbed by it to a considerable extent, especially at low temperatures. ${ }^{8}$ The solid

the aqueous solution. In the end, the same ammoniacal solution as was originally taken is re-obtained. Thus, in this case, on heating the vessel the pressure increases of itself, and on cooling it diminishes, so that here heat directly replaces mechanical work. This is the principle of the simplest forms of Carré's ice-making machines, shown in fig. 44. $\mathrm{C}$ is a vessel made of boiler plates into which the saturated solution of ammonia is poured; $m$ is a tube conducting the ammonia vapour to the receiver A. All parts of the apparatus should be hermetically joined together, and should be able to withstand a pressure reaching ten atmospheres. The apparatus should be freed from air, which
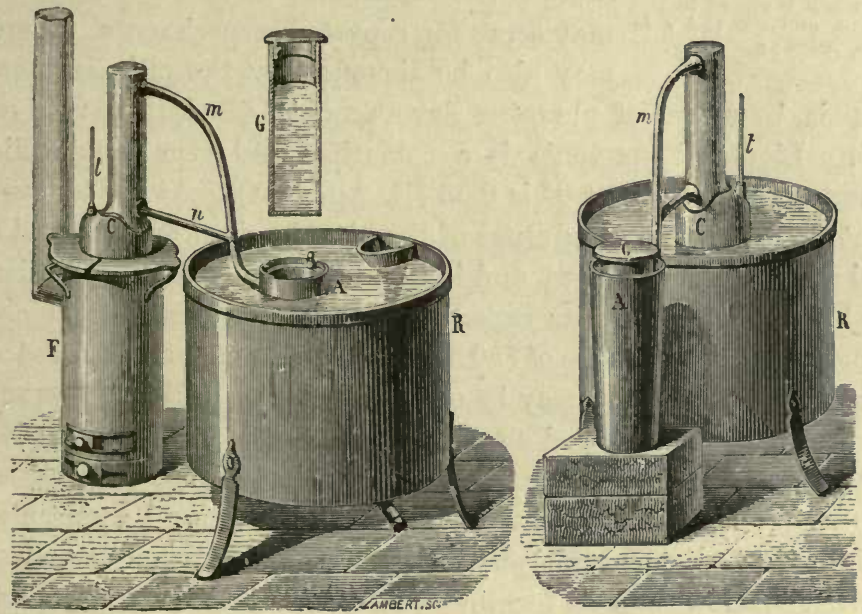

FIG. 44.-Carré's apparatus. Described in text.

would otherwise hinder the liquefaction of the ammonia. The process is carried on as follows:-The apparatus is first so inclined that any liquid remaining in A may flow into $\mathrm{C}$. The vessel $\mathrm{C}$ is then placed upon a stove $\mathrm{F}$, and heated until the thermometer $t$ indicates a temperature of $130^{\circ} \mathrm{C}$. During this time the ammonia has been expelled from $\mathbf{C}$, and has liquefied in $\mathbf{A}$. In order to facilitate the liquefaction, the receiver $\mathbf{A}$ should be immersed in a tank of water $R$ (see the left-hand drawing in fig. 44). After about half an hour, when it may be supposed that the ammonia has been expelled, the fire is removed from under $C$, and this is now immersed in the tank of water $R$. The apparatus is represented in this position in the right-hand drawing of fig. 44. Then the liquefied ammonia evaporates, and passes over into the water in C. This causes the temperature of $\mathbf{A}$ to fall considerably. The substance to be refrigerated is placed in a vessel $\mathrm{G}$, in the cylindrical space inside the receiver $\mathrm{A}$. The refrigeration is also kept on for about half an hour, and with an apparatus of ordinary dimensions (containing about two litres of ammonia solution), five kilograms of ice are produced by the consumption of one kilogram of coal. In industrial works more complicatedtypes of Carré's machines are employed.

${ }^{8}$ Below $15^{\circ}$ (according to Isambert), the compound $\mathrm{AgCl}_{3}, 3 \mathrm{NH}_{3}$ is formed, and above $20^{\circ}$ the compound $2 \mathrm{AgCl}, 3 \mathrm{NH}_{3}$. The tension of the ammonia evolved from the latter 


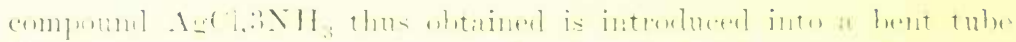

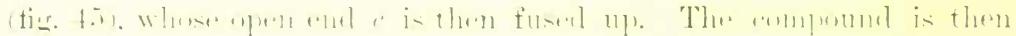

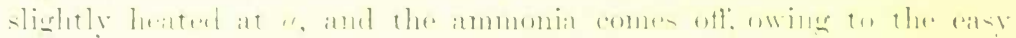

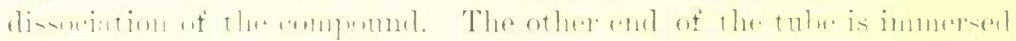

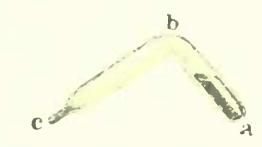

Yho, :

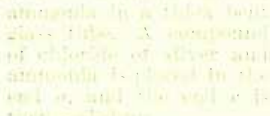
in a thereing mixtme. The purente of the was

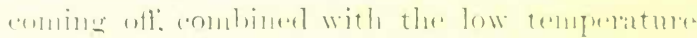

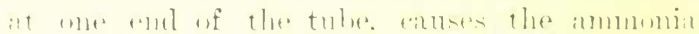
soulverl to condene into al liquiel, in which fom

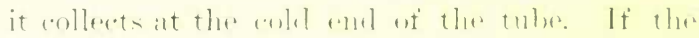

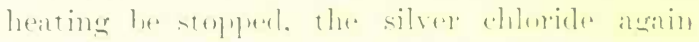

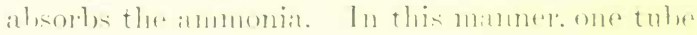

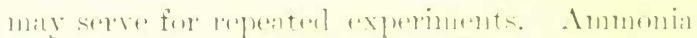
mat also be liquefienl hy the omelinamy methents

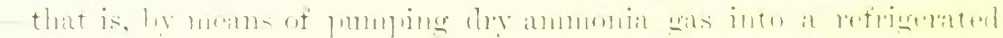

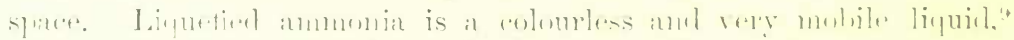

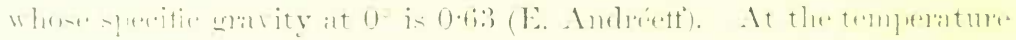

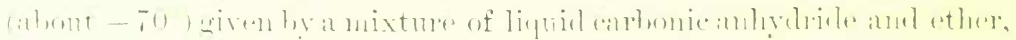

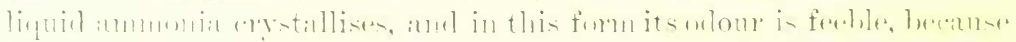

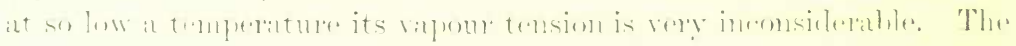

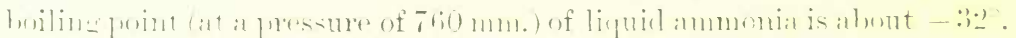

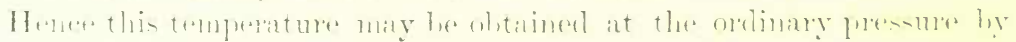

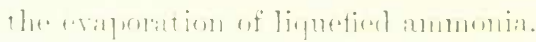

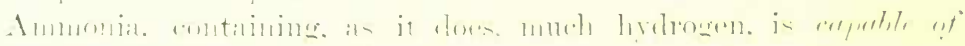

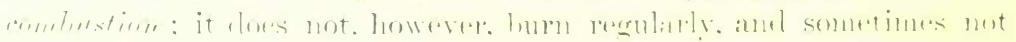

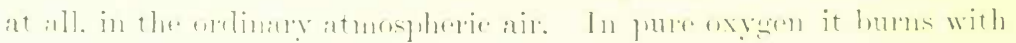

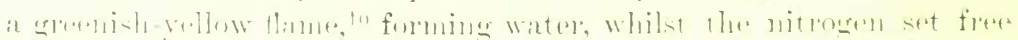

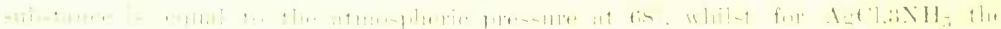

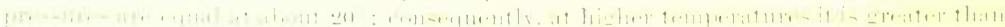

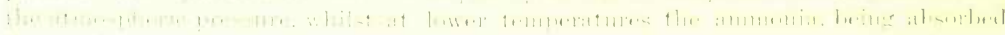

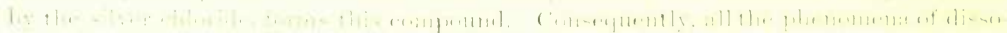

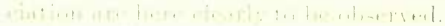

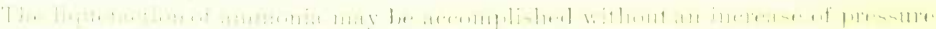

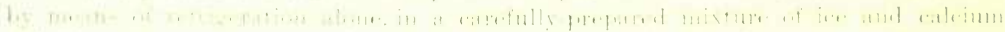

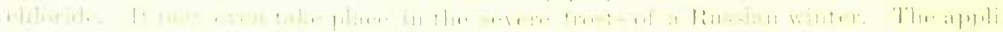

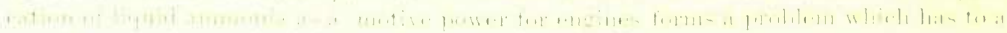

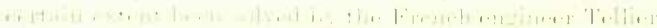

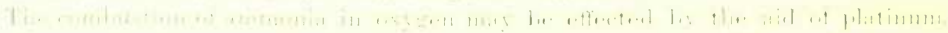

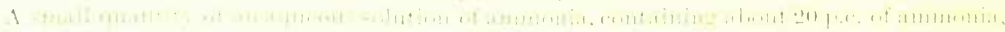

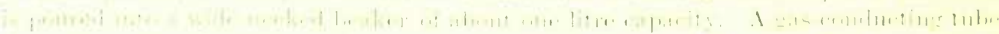

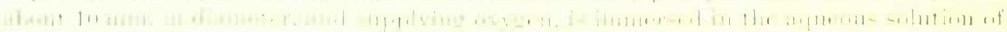

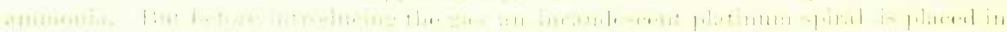

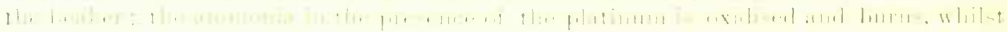

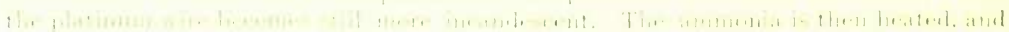

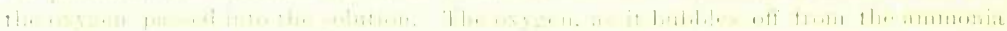

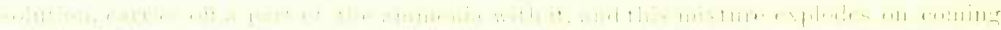

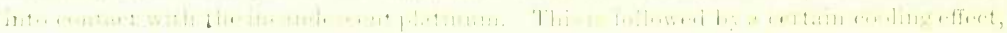


gives its oxygen compound - that is, oxide of nitrogen. The decomposition of ammonia into hydrogen and nitrogen not only takes place at a red heat and under the action of electric sparks, but also by means of many oxidising substances ; for instance, by passing ammonia through a tube containing red-hot copper oxide. The water thus formed may be collected by substances absorbing it, and the quantity of nitrogen may be measured in a gaseous form, and thus the composition of ammonia determined. In this manner it is very easy to prove that ammonia contains 3 parts by weight of hydrogen to 14 parts by weight of nitrogen; and, by volume, 3 vols. of hydrogen and 1 vol. of nitrogen form 2 vols. of ammonia."

Ammonia is capable of combining with a number of substances, forming, like water, substances of various degrees of stability. It is more soluble than any other known gas, both in water and in many aqueous solutions. We have already seen, in the first chapter, that one volume of water, at the ordinary temperature, dissolves about 700 vols. of ammonia gas. The great solubility of ammonia enables it to be always kept ready for use in the form of an aqueous solution ${ }^{12}$ which is commercially known as spirits of hartshorn. Ammonia water

owing to the combustion ceasing, but after a short interval this is, however, renewed, so that one feeble explosion follows after another. During the period of oxidation without explosion, white vapours of ammonium nitrite and red-brown vapours of oxides of nitrogen make their appearance, while during the explosion there is complete combustion, and consequently water and nitrogen are formed.

11 This may be verified by their densities. Nitrogen is 14 times denser than hydrogen, and ammonia is $8 \frac{1}{2}$ times. If 3 volumes of hydrogen with 1 volume of nitrogen gave 4 volumes of ammonia, then these 4 volumes would weigh 17 times more than 1 volume of hydrogen ; consequently, 1 volume of ammonia would weigh $4 \frac{1}{4}$ times heavier than the same volume of hydrogen. But as these 4 volumes only give 2 volumes of ammonia, therefore they weigh $8 \frac{1}{2}$ times heavier than hydrogen, which we find to be actually the case.

12 Aqueous solutions of ammonia are lighter than water, and at $15^{\circ}$, taking water at $4^{\circ}=10000$, their specific gravity, as dependent on $p$, or the percentage amount (by weight) of ammonia, is given by the expression $s=9992-42.5 p+0.21 p^{2}$; for instance, with 10 p.c. $s=9587$. If the temperature be $=t^{\circ}$, but not less than $10^{\circ}$ or above $20^{\circ}$, then the expression $(15-t)(1 \cdot 5+0 \cdot 14 p)$ must be added to the formula for the specific gravity. Solutions containing above 24 p.c. have not been sufficiently investigated in respect to the variation of their specific gravity. It is, however, easy to obtain more concentrated solutions, and at $0^{\circ}$ solutions approaching $\mathrm{NH}_{5}, \mathrm{H}_{2} \mathrm{O}\left(48 \cdot 6\right.$ p.c. $\left.\mathrm{NH}_{3}\right)$ in their composition, and of sp. gr. 0.85 , may be prepared. But such solutions give up the bulk of their ammonia at the ordinary temperature, so that more than 24 p.c. $\mathrm{NH}_{3}$ is rarely contained in solution. Ammoniacal solutions containing a considerable amount of ammonia give ice-like crystals at temperatures far below $0^{\circ}$ (for instance, an 8 p.c. solution at $-14^{\circ}$, the strongest solutions at $-48^{\circ}$ ) which seem to contain ammonia. The whole of the ammonia may be expelled from a solution by heating, even at a comparatively low temperature; therefore, in beating aqueous solutions containing ammonia a very strong solution of ammonia is obtained in the distillate. Alcohol, ether, and many other liquids are also capable of dissolving ammonia. Solutions of ammonia, when exposed to the atmosplicre, 
is continually evolving ammoniacal vapour, and so has the characteristic smell of ammonia itself. It is a very characteristic and important fact that ammonia has an alkaline reaction, and colours litmus paper blue, just like caustic potash or lime ; it is therefore sometimes called caustic ammonia (volatile alkali). Acids may be saturated by ammonia water or gas in exactly the same way as by any other alkali. In so doing, ammonia combines directly with acids, and this forms the most essential chemical reaction of this substance. If sulphuric, nitric, acetic, or any

give off a part of their ammonia, in accordance with the laws of the solution of gases in liquids already considered by us. But the ammoniacal solutions at the same time absorb carbonic anhydride from the air, and ammonium carbonate remains in the solution.

Solutions of ammonia are required both in the laboratory and in practice, and have therefore to be frequently prepared. For this purpose the arrangement shown in fig. 46 is employed in the laboratory. In works the same arrangement is used, only on a larger scale (with earthenware or metallic vessels). The gas is prepared in the retort, from

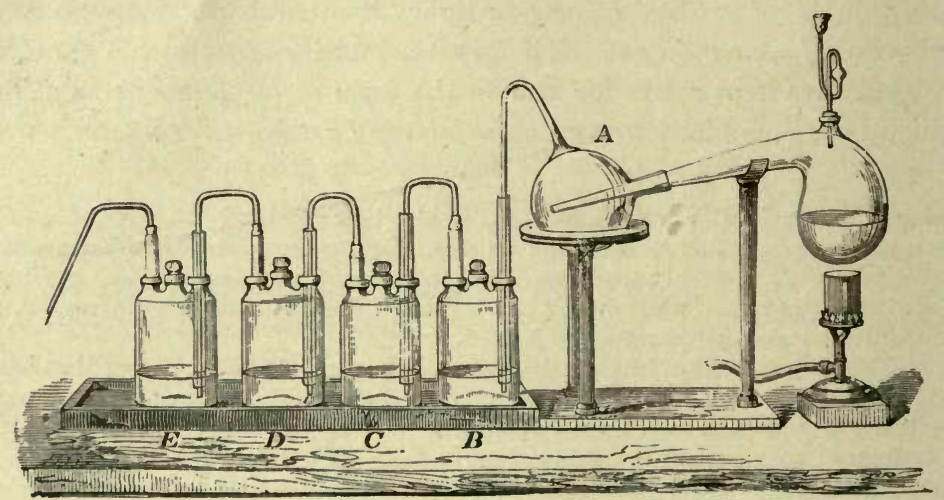

FIG. 46.-Apparatus for preparing solutions of ummonia.

whence it is led into the two-necked globe A, and then through a series of Woulfe's bottles, B, C, D, E. The impurities spurting over collect in A, and the gas is dissolved in B, but the solution soon becomes saturated, and a purer (washed) ammonia passes over into the following vessels, in which only a pure solution is obtained. The bent funnel tube in the retort preserves the apparatus from the possibility both of the pressure of the gas evolved in it becoming too great (when the gas escapes through it into the air), and also from the pressure incidentally falling too low (for instance, owing to a cooling effect, or from the reaction stopping). If this takes place, the air passes into the retort, otherwise the liquid from $B$ would be drawn into A. The safety tubes in each Woulfe's bottle, open at both ends, and immersed in the liquid, serve for the same purpose. Without them, in case of an accidental stoppage in the evolution of so soluble a gas as ammonia, the solution would be sucked from one vessel to another-for instance from $\mathrm{E}$ into D, \&c. In order to clearly see the necessity of the safety tubes in a gas apparatus, it must be considered that the gaseous pressure in the interior of the arrangement must exceed the atmospheric pressure by the height of the sum of the columns of liquid through which the gas has to pass. 
other acid be brought into contact with ammonia it absorbs it, and in so doing evolves a large amount of heat and forms a compound having all the properties of a salt. Thus, for example, sulphuric acid, $\mathrm{H}_{2} \mathrm{SO}_{4}$, in absorbing ammonia, forms (on evaporating the solution) two salts, according to the relative quantities of ammonia and acid. One salt is formed from $\mathrm{NH}_{3}+\mathrm{H}_{2} \mathrm{SO}_{4}$, and consequently has the composition $\mathrm{NH}_{5} \mathrm{SO}_{4}$, and the other is formed from $2 \mathrm{NH}_{3}+\mathrm{H}_{2} \mathrm{SO}_{4}$, and its composition is therefore $\mathrm{N}_{2} \mathrm{H}_{8} \mathrm{SO}_{4}$. The former has an acid reaction and the latter a neutral reaction, and they are called respectively acid ammonium sulphate (ammonium hydrogen sulphate), and normal ammonium sulphate, or simply ammonium sulphate. The same takes place in the action of all other acids ; but certain of them are able to form normal ammonium salts only, whilst others give both acid and normal ammonium salts. This depends on the nature of the acid and not on the ammonia, as we shall afterwards see. Ammonium salts are very similar in appearance and in many of their properties to metallic salts; for instance, sodium chloride, or table salt, resembles sal-ammoniac, or ammonium chloride, not only in its outward appearance but even in crystalline form, in its property of giving precipitates with silver salts, in its solubility in water, and in its evolving hydrochloric acid when heated with sulphuric acid-in a word, a most perfect analogy is to be remarked in an entire series of reactions. An analogy in composition is seen if sal-ammoniac, $\mathrm{NH}_{4} \mathrm{Cl}$, be contrasted with table salt, $\mathrm{NaCl}$; and the ammonium hydrogen sulphate, $\mathrm{NH}_{4} \mathrm{HSO}_{4}$, with the sodium hydrogen sulphate, $\mathrm{NaHSO}_{4}$; or ammonium nitrate, $\mathrm{NH}_{4} \mathrm{NO}_{3}$, with sodium nitrate, $\mathrm{NaNO}_{3} .{ }^{13} \mathrm{It}$ is seen, on comparing the above compounds, that the part which sodium takes in the sodium salts is played in ammonium salts by a group $\mathrm{NH}_{4}$, which is called ammonium. If table salt, as the product obtained by the action of caustic soda or sodium hydroxide on hydro-

13 The analogy between the ammonium and sodium salts might seem to be destroyed by the fact that the latter are formed from the alkali or oxide and an acid, with the separation of water, whilst the ammonium salts are directly formed from ammonia and an acid, without the separation of water; but the analogy is restored if we compare soda to ammonia water, and liken caustic soda to a compound of ammonia with water. Then the very preparation of ammonia salts from such a hydrate of ammonia will completely resemble the preparation of sodium salts from soda. We may cite as an example the action of hydrochloric acid on both substances.

$$
\begin{gathered}
\underset{\text { Sodium hydroxide }}{\mathrm{NaHO}}+\underset{\text { Hydrochloric acid }}{\mathrm{HCl}}=\underset{\text { Water }}{\mathrm{H}_{2} \mathrm{O}}+\underset{\text { Table salt. }}{\mathrm{NaCl}} \\
\underset{\text { Ammonium hydroxide }}{\mathrm{NH}_{4} \mathrm{HO}}+\underset{\text { Hydrochloric acid }}{\mathrm{HCl}}=\begin{array}{c}
\mathrm{H}_{2} \mathrm{O} \\
\text { Water }
\end{array}+\underset{\text { Sal-ammoniac. }}{\mathrm{NH}_{4} \mathrm{Cl}}
\end{gathered}
$$

Just as in soda the hydroxyl or aqueous radicle $\mathrm{OH}$ is replaced by chlorine, so it is in ammonia hydrate. 
chloric acid, be called 'sodium chloride,' then sal-ammoniac, as the product obtained from caustic ammonia or ammonium hydroxide, is called ' ammonium chloride.'

The hypothesis that ammoniacal salts correspond with a complex metal ammonium bears the name of the ammonium theory. It was enunciated by the famous Swedish chemist Berzelius after the proposition made by Ampère. The analogy admitted between ammonium and metals is probable, owing to the fact that mercury is able to form an amalgam with ammonium similar to that which it forms with sodium or many other metals. The only difference between ammonium amalgam and sodium amalcam consists in the instability of the ammonium, which easily decomposes into ammonia and hydrogen. ${ }^{14}$ Ammonium amalgam may be prepared from sodium amalgam. If the latter be shaken up with a strong solution of sal-ammoniac, the mercury swells up violently and loses its mobility while preserving its metallic appearance. In so doing, the mercury dissolves ammonium - that is, the sodium in the mercury is replaced by the ammonium, and replaces it in the salammoniac, forming sodium chloride, $\mathrm{NH}_{4} \mathrm{Cl}+\mathrm{HgNa}=\mathrm{NaCl}+\mathrm{HgNH}_{4}$. Naturally, ammonium amalgam does not entirely prove the existence of ammonium itself in a separate state; but it shows the possibility of this substance existing, and, what is more important, its analogy with the metals, because only metals dissolve in mercury, forming compounds. termed 'amalgams,' without altering its metallic form. ${ }^{15}$ Ammonium amalgam crystallises in cubes, three times heavier than water; it is only stable in the cold, and particularly at very low temperatures. It. begins to decompose at the ordinary temperature, evolving ammonia and hydrogen in the proportion of two volumes of ammonia and one

14 Weyl, by working at considerable pressures, obtained the compound $\mathrm{NH}_{3} \mathrm{~K}$, and then ammonium itself-by the action of sal-ammoniac on this substance-in the form of a blue liquid, but his researches require confirmation. Ammonium amalgam was originally obtained in exactly the same way as sodium amalgam (Davy); namely, a piece of sal-ammoniac was taken, and moistened with water (in order to render it a conductor of electricity). A cavity was made in it, into which mercury was poured, and it was laid on a sheet of platinum connected with the positive pole of a galvanic battery, while the negative pole was put into connection with the mercury. On passing a current the mercury increased considerably in volume, and became plastic, while preserving its metallic appearance, just as would be the case were the sal-ammoniac replaced by a lump of a sodium salt or of many other metals. In the analogous decomposition of common metallic salts, the metal contained in a given salt separates out at the negative pole, immersed in mercury, by which the metal is dissolved. A similar phenomenon is observed in the case of sal-ammoniac; the elements of ammonium, $\mathrm{NH}_{4}$, in this case are also collected in the mercury, and are retained by it for a certain time.

15 It would seem that hydrogen is also capable of forming an amalgam resembling the amalgam of ammonium. If an amalgam of zinc be shaken up with an aqueous solution of platinum chloride, without access of air, then a spongy mass is formed which easily decomposes, with the evolution of hydrogen. 
volume of hydrogen, $\mathrm{NH}_{4}=\mathrm{NH}_{3}+\mathrm{H}$. By the action of water, ammonium amalgam gives hydrogen and ammonia water, just as sodium amalgam gives hydrogen and sodium hydroxide; and therefore, in accordance with the ammonium theory, ammonia water must be looked on as containing ammonium hydroxide, $\mathrm{NH}_{4} \mathrm{OH},{ }^{16}$ just as an aqueous solution of sodium hydroxide contains $\mathrm{NaOH}$. The ammonium. hydroxide, like ammonium itself, is an unstable substance, which easily dissociates, and which can only exist in a free state at low temperatures. $^{17}$ Ordinary solutions of ammonia must be looked on as the products of the dissociation of this hydroxide, inasmuch as $\mathrm{NH}_{4} \mathrm{OH}$ $=\mathrm{NH}_{3}+\mathrm{H}_{2} \mathrm{O}$. The liability to a greater or less decomposition is proper, in the same degree, to substances containing $\mathrm{NH}_{3}$ as to substances containing water.

All ammoniacal salts decompose at a red heat into ammonia and an acid, which, on cooling in contact with each other, re-combine together. If the acid be non-volatile, the ammoniacal salt, when heated, evolves the ammonia, leaving the non-volatile acid behind; if the acid be volatile, then, on heating, both the acid and ammonia volatilise together, and on cooling re-combine into the salt which originally served for the formation of their vapours. ${ }^{18}$

Ammonia is not only capable of combining with acids, but also with many salts, as was seen from its forming definite compounds, $\mathrm{AgCl}, 3 \mathrm{NH}_{3}$ and $2 \mathrm{AgCl}, 3 \mathrm{NH}_{3}$, with silver chloride. So, also, ammonia is absorbed by the chlorine, iodine, and bromine compounds of many metals, and in so doing evolves heat. Certain of these com-

16 We saw above that the solubility of ammonia in water at low temperatures attains to the molecular ratio $\mathrm{NH}_{3}+\mathrm{H}_{2} \mathrm{O}$, in which these substances are contained in caustic ammonia, and perhaps it may be possible at exceedingly low temperatures to obtain ammonium hydroxide, $\mathrm{NH}_{4} \mathrm{HO}$, in a solid form. By regarding solutions as dissociated definite compounds, we should see a confirmation of this view in the property shown by ammonia of being extremely soluble in water, and in so doing of approaching to the limit $\mathrm{NH}_{4} \mathrm{HO}$.

17 In confirmation of the truth of this conclusion we may cite the remarkable fact that there exist, in a free state and as comparatively stable compounds, a series of alkaline hydroxides, $\mathrm{NR}_{4} \mathrm{HO}$, which are perfectly analogous to ammonium hydroxide, and present a striking resemblance to it and to sodium hydroxide, with the only difference that the hydrogen in $\mathrm{NH}_{4} \mathrm{HO}$ is replaced by complex groups, $\mathrm{R}=\mathrm{CH}_{3}, \mathrm{C}_{2} \mathrm{H}_{5}$, \&c., for instance $\mathrm{N}\left(\mathrm{CH}_{3}\right)_{4} \mathrm{HO}$.

18 The fact that ammoniacal salts are decomposed when ignited, and not simply sublimed, may be proved by a direct experiment with sal-ammoniac, $\mathrm{NH}_{4} \mathrm{Cl}$, which in a state of vapour is decomposed into ammonia, $\mathrm{NH}_{3}$, and hydrochloric acid, $\mathrm{HCl}$, as will be explained in the following chapter. The readiness with which ammonium salts decompose is seen from the fact that a solution of ammonium oxalate is decomposed with the evolution of ammonia even at $-1^{\circ}$. Dilute solutions of ammonium salts, when boiled, give aqueous vapour, having an alkaline reaction, owing to the presence of free ammonia given off from the salt. 
pounds part with their ammonia even when left exposed to the air, but others only do so at a red heat; many give up their ammonia when dissolved, whilst others dissolve without decomposition, and when evaporated separate from their solutions unchanged. All these facts only indicate that ammoniacal, like aqueous, compounds dissociate with greater or lesser facility. ${ }^{19}$ Certain metallic oxides also absorb ammonia and are dissolved in ammonia water. Such are, for instance, the oxides of zinc, nickel, copper, and many others ; the majority of such compounds are unstable. The property of ammonia of combining with the oxides of certain metals explains its action on certain metals. ${ }^{20}$ For this reason, copper vessels are not suitable for holding liquids containing ammonia. Iron is not acted on by such liquids.

The relation of ammonia and water to salts and other substances becomes especially clear in the case when the salt is capable of combining with both ammonia and water. Take, for example, copper sulphate, $\mathrm{CuSO}_{4}$. As we saw in Chapter I., it gives with water blue crystals, $\mathrm{CuSO}_{4}, 5 \mathrm{H}_{2} \mathrm{O}$; but it also absorbs ammonia in the same molecular proportion, forming a blue substance, $\mathrm{CuSO}_{4}, 5 \mathrm{NH}_{3}$, and therefore the ammonia combining with salts may be termed ammonia of crystallisation.

Such are the reactions of combination proper to ammonia. Let us now turn our attention to the reactions of substitution proper to this substance. If ammonia be passed through a heated tube containing metallic potassium, then hydrogen is evolved, and a compound is obtained containing ammonia in which one atom of hydrogen is replaced by an atom of potassium, $\mathrm{NH}_{2} \mathrm{~K}$ (according to the equation $\mathrm{NH}_{3}+\mathrm{K}=\mathrm{NH}_{2} \mathrm{~K}+\mathrm{H}$ ). This body is termed potassamide. We shall afterwards see that iodine and chlorine are also capable of directly displacing hydrogen from ammonia, and of replacing it; we shall also see that in hydrocyanic acid, $\mathrm{NCH}$, carbon has replaced hydrogen. Hence the hydrogen of ammonia may be replaced in many ways by different elements. If in so doing $\mathrm{NH}_{2}$ remains, the resultant substances are called amides, if only $\mathrm{NH}$ imides, and those in which the whole of the hydrogen is displaced are termed nitrides. It may be imagined that the albuminous substances - that is, the complex organic

19 Isambert studied the dissociation of ammoniacal compounds, as we have seen in Note 8 , and he showed that at low temperatures many salts are able to combine with a still greater amount of ammonia, which proves an entire analogy with aqueous compounds; and as in this case it is easy to isolate the definite compounds, and as the least possible tension of ammonia is greater than that of water, therefore the ammoniacal compounds present a great and peculiar interest, both as a means for explaining the nature of aqueous solutions, and as a confirmation of the conception of the formation of definite compounds in them; for these reasons we shall frequently turn to these compounds in the further exposition of this work.

20 Chapter V. Note 2. 
substances which we have already had occasion to mention-are the amide compounds corresponding with saccharine substances. The most important point to be remarked is that in the action of different substances on ammonia it is the hydrogen that is substituted; the reactions proceed at the expense of the hydrogen and not of the nitrogen, which remains in the resultant compound, so to say, untouched. The same is to observed in the action of various substances on water. In the majority of cases the reactions of water consist in the hydrogen being evolved, and in its being replaced by different elements. The same takes place, as we have seen, in acids, in which the hydrogen is easily displaced by metals. This chemical mobility of hydrogen is distinctly connected with the great lightness of the atoms of this element.

In chemical practice ${ }^{21}$ ammonia is often employed, not only for saturating acids, but also for accomplishing reactions of double decomposition with salts, and especially in separating insoluble basic hydroxides from soluble salts. Let $\mathrm{MHO}$ stand for an insoluble basic hydroxide, and $\mathrm{HX}$ for an acid. The salt formed by them will have a composition $\mathrm{MHO}+\mathrm{XH}-\mathrm{H}_{2} \mathrm{O}=\mathrm{MX}$. If aqueous ammonia, $\mathrm{NH}_{4} \mathrm{OH}$, be added to a solution of this salt, then the ammonia will change

21 In practice, the applications of ammonia are very varied. The use of ammonia as a stimulant, in the forms of the so-called 'smelling salts' or of spirits of hartshorn, in cases of faintness, \&c., is known to everyone. The volatile carbonate of ammonium, or a mixture of an ammonium salt with an alkali, is also employed for this purpose. Ammonia also produces a well-known stimulating effect when rubbed on the skin, for which reason it is sometimes employed for outward applications. Thus, for instance, the well-known volatile salve is prepared from any liquid oil shaken up with a solution of ammonia. A portion of the oil is thus transformed into a soapy substance. The solubility of greasy substances in ammonia, which proceeds from the formation both of emulsions and soaps, explains its use in extracting grease spots. It is also employed as an external application for stings from insects, and for bites from poisonous snakes, and in general in medicine. It is also remarkable that in cases of drunkenness a few drops of ammonia in water taken internally rapidly renders a person sober. A large quantity of ammonia is used in dyeing, either for the solution of certain dyes-for example, carmine-or for changing the tints of others, or else for neutralising the action of acids. It is also employed in the manufacture of artificial pearls. For this purpose the small scales of a peculiar small fish are mixed with ammonia, and the liquid so obtained is blown into small hollow glass beads, shaped like pearls.

In nature and the arts, however, the ammonium salts, not free ammonia, are most frequently employed. In this form a portion of that nitrogen which is necessary for the formation of albuminous substances 28 supplied to plants. Owing to this, a large quantity of ammonium sulphate is now employed as a fertilising substance. But the same part may be fulfilled by nitre, or by animal refuse, which in putrefying gives ammonia. The nitre of the soil is formed from these ammonia-giving substances, because nitrogen in combination with hydrogen is easily converted into oxygen compounds of nitrogen, as we shall afterwards see. For this reason, if an ammoniacal (lydrogen) compound be introduced into the soil in the spring, it will be converted into a nitrate (oxygen salt) in the summer. 


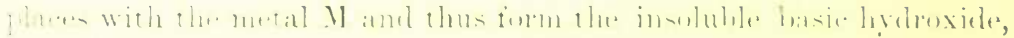

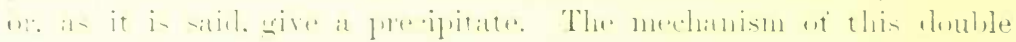

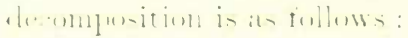

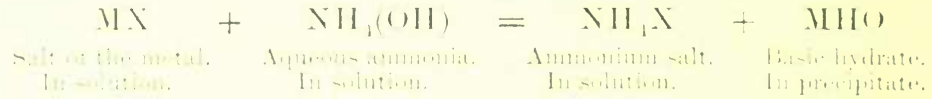

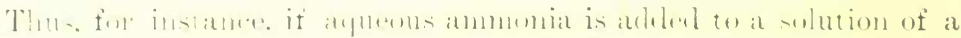

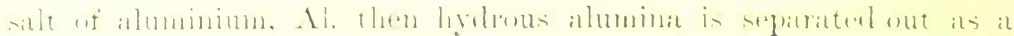

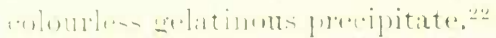

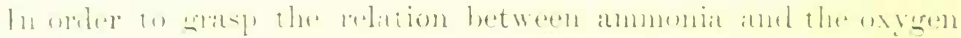

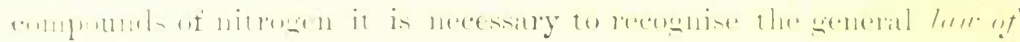

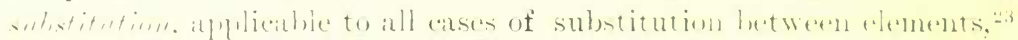

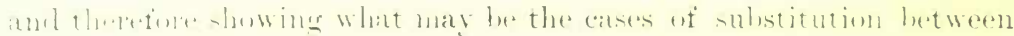

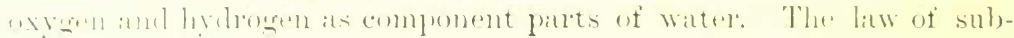

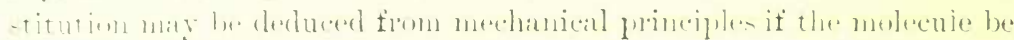

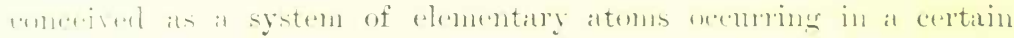

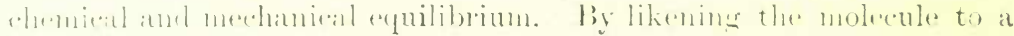

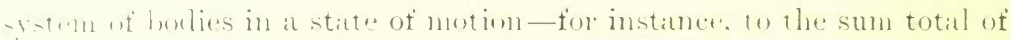
the sume planet-and satellites, ocemring in eonditions ot mohile equi-

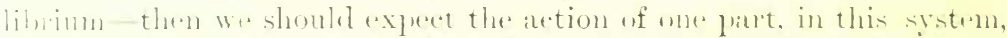

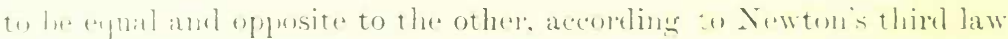

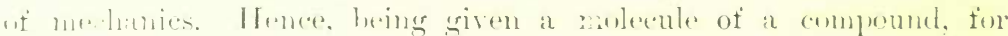

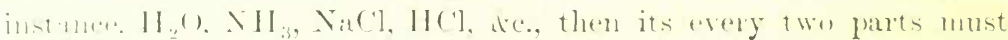

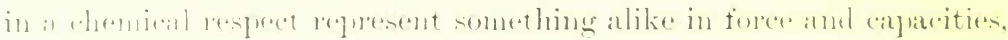

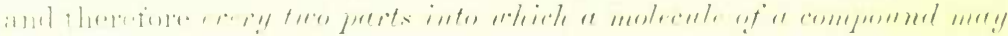

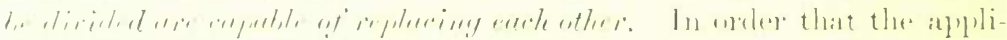

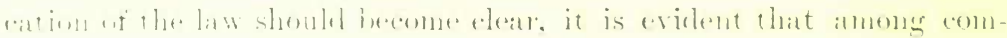

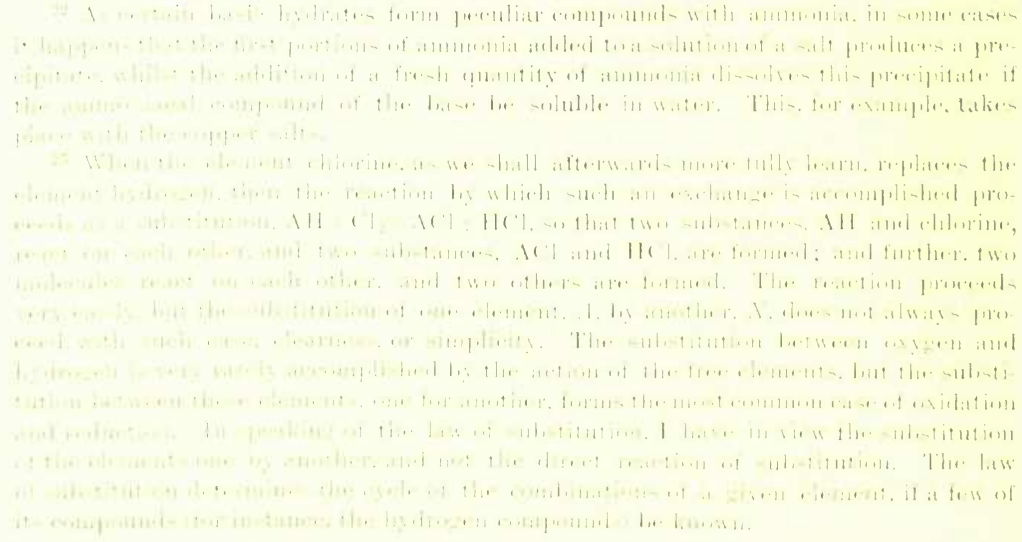


pounds the most stable should be chosen. We will, therefore, take water as the most stable compound of hydrogen and oxygen, ${ }^{24}$ in order to see the cases of substitution between hydrogen and oxygen; but for the sake of clearness we will start by taking the very stable molecule of hydrochloric acid, $\mathrm{HCl}$, as one which can be divided into $\mathrm{H}$ and $\mathrm{Cl}$ only. According to the law of substitution, if these elements are able to form a molecule, and a stable one, then they are able to replace each other. And, indeed, we shall afterwards see that in a number of instances a substitution between hydrogen and chlorine conversely takes place. Given $\mathrm{RH}$, then $\mathrm{RCl}$ is possible, because $\mathrm{HCl}$ exists and is stable. The molecule of water, $\mathrm{H}_{2} \mathrm{O}$, may be divided in two ways, because it contains 3 atoms : into $\mathrm{H}$ and (HO) on the one hand, and into $\mathrm{H}_{2}$ and $\mathrm{O}$ on the other. Consequently, being given $\mathrm{RH}$, its substitution products will be $\mathrm{R}(\mathrm{HO})$ according to the first form, and $\mathrm{R}_{2} \mathrm{O}$ according to the second; being given $\mathrm{RH}_{2}$, its corresponding substitution products will be $\mathrm{RH}(\mathrm{OH}), \mathrm{R}(\mathrm{OH})_{2}, \mathrm{RO}$, $(\mathrm{RH})_{2} \mathrm{O}$, dc. The group $(\mathrm{OH})$ is the same hydroxyl or aqueous radicle which we have already mentioned in the third chapter as a component part of hydroxides and alkalis-for instance, $\mathrm{Na}(\mathrm{OH})$, $\mathrm{Ca}(\mathrm{OH})_{2}$, dc. It is evident, judging from $\mathrm{HCl}$, that $(\mathrm{OH})$ can be substituted by $\mathrm{Cl}$, because both are replaceable by $\mathrm{H}$; and this is of common occurrence in chemistry, because metallic chlorides-for instance, $\mathrm{NaCl}$ and $\mathrm{NH}_{4} \mathrm{Cl}$-correspond with hydroxides of the alkalis $\mathrm{Na}(\mathrm{OH})$ or $\mathrm{NH}_{4}(\mathrm{OH})$. In hydrocarbons - for instance, $\mathrm{C}_{2} \mathrm{H}_{6}$ - the hydrogen is replaceable by chlorine and by hydroxyl. Thus common alcohol is $\mathrm{C}_{2} \mathrm{H}_{6}$, in which one atom of $\mathrm{H}$ is replaced by $(\mathrm{OH})$; that is, $\mathrm{C}_{2} \mathrm{H}_{5}(\mathrm{OH})$. It is evident that the replacement of hydrogen by hydroxyl essentially forms the phenomenon of oxidation, because $\mathrm{RH}$ gives $\mathrm{R}(\mathrm{OH})$, or $\mathrm{RHO}$. Hydrogen peroxide may in this sense be regarded as water in which the hydrogen is replaced by hydroxyl; $\mathrm{H}(\mathrm{OH})$ gives $(\mathrm{OH})_{2}$ or $\mathrm{H}_{2} \mathrm{O}_{2}$. For this reason chlorine, as we shall afterwards see, exhibits in its reactions much analogy to hydrogen peroxide, which may be termed free hydroxyl. The other form of substitution - namely, that of $\mathrm{O}$ in the place of $\mathrm{H}_{2}$-is also a common chemical phenomenon. Thus common alcohol, $\mathrm{C}_{2} \mathrm{H}_{6} \mathrm{O}$, or $\mathrm{C}_{2} \mathrm{H}_{5}(\mathrm{OH})$, when oxidising in the air, gives, as every one knows, acetic acid, $\mathrm{C}_{2} \mathrm{H}_{4} \mathrm{O}_{2}$, or $\mathrm{C}_{2} \mathrm{H}_{3} \mathrm{O}(\mathrm{OH})$, in which $\mathrm{H}_{2}$ is replaced by $\mathrm{O}$.

24 If hydrogen peroxide be taken as a starting point, then still higher forms of oxidation than those corresponding with water should be looked for. They should possess the properties of hydrogen peroxide, especially that of parting with their oxygen with extreme ease (even by contact). Such compounds are known. Pernitric, persulphuric, and similar acids present these properties, as we shall see in describing them.

vOL. I. 


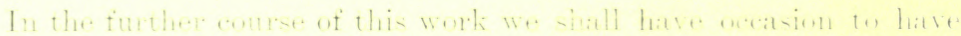

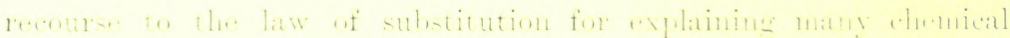

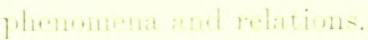

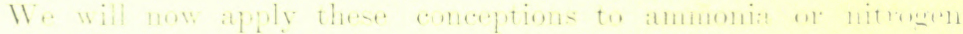

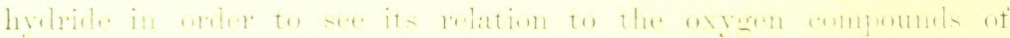

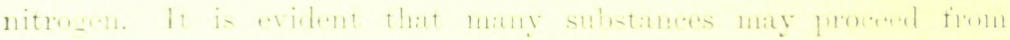

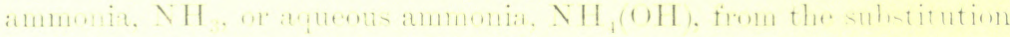

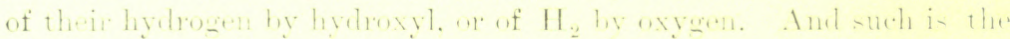

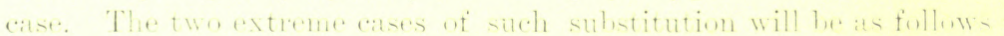

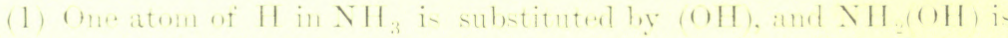

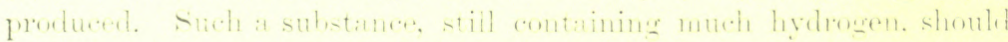
have many of the properties of ammonia. It is knows molere the

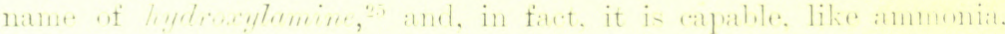

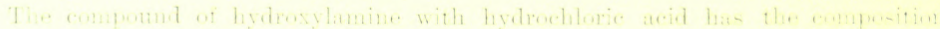

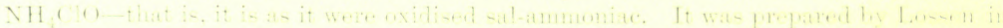

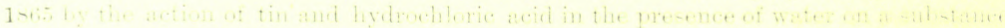

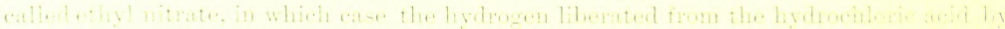
the :

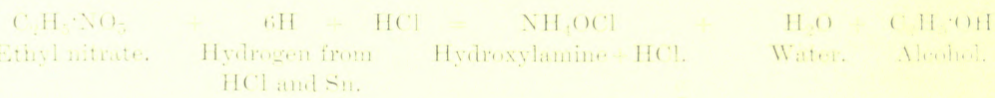

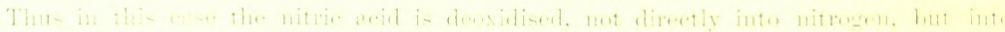

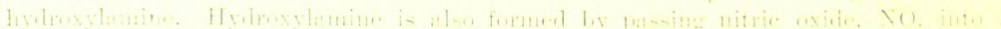

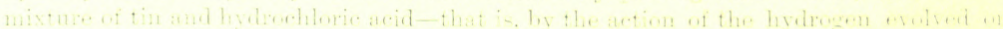

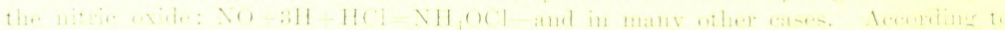

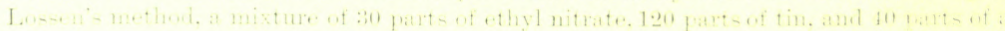

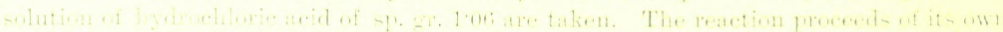

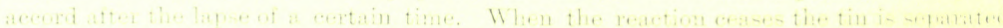

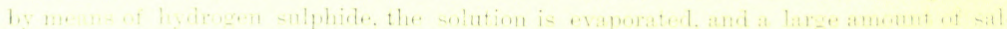

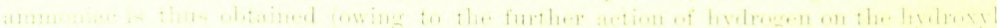

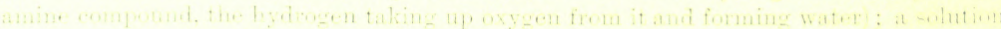

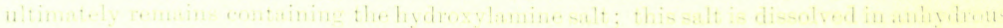

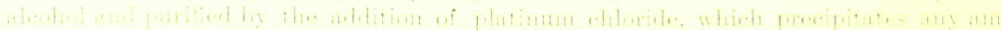

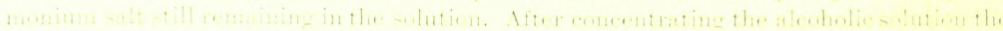

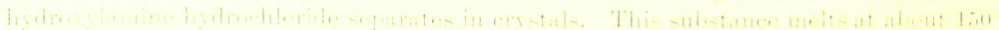

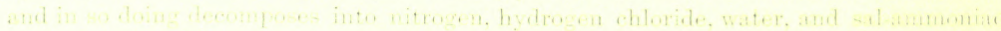

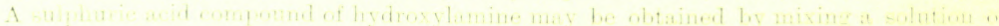

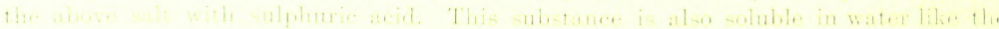

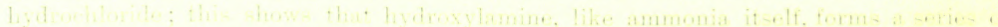
aft:

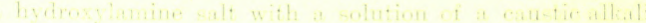

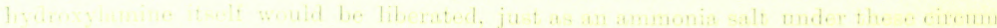

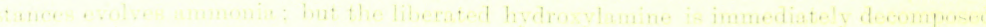

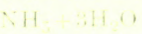


of giving salts with acids; for example, with hydrochloric acid, $\mathrm{NH}_{3}(\mathrm{OH}) \mathrm{Cl}$-which is a substance corresponding with sal-ammoniac, in which one atom of hydrogen is replaced by hydroxyl. (2) The other extreme case of substitution given by ammonium hydroxide, $\mathrm{NH}_{4}(\mathrm{OH})$, is when the whole of the hydrogen of the ammonium is replaced by oxygen ; and as ammonium contains 4 atoms of hydrogen, the highest oxygen compound should be $\mathrm{NO}_{2}(\mathrm{OH})$, or $\mathrm{NHO}_{3}$, as we find to be really the case, because $\mathrm{NHO}_{3}$ is nitric acid, or the highest degree of the oxidation of nitrogen. ${ }^{26}$ If instead of the two extreme aspects of substitution we take an intermediate one, then we obtain one of the intermediate oxygen compounds of nitrogen. For instance, $\mathrm{N}(\mathrm{OH})_{3}$ is orthonitrous acid, ${ }^{27}$ with which corresponds nitrous acid, $\mathrm{NO}(\mathrm{OH})$, or $\mathrm{NHO}_{2}$, equal to $\mathrm{N}(\mathrm{OH})_{3}-\mathrm{H}_{2} \mathrm{O}$, and nitrous anhydride $\mathrm{N}_{2} \mathrm{O}_{3}=2 \mathrm{~N}(\mathrm{OH})_{3}-3 \mathrm{H}_{2} \mathrm{O}$. Thus nitrogen gives a series of oxygen

tion, like ammonia, precipitates basic hydrates, and it deoxidises the oxides of copper, silver, and other metals. Hydroxylamine is obtained, in a great number of cases, for instance, by the action of tin on dilute nitric acid, and also by the action of zinc on ethyl nitrate and dilute hydrochloric acid, \&c. The relation between hydroxylamine, $\mathrm{NH}_{2}(\mathrm{OH})$, and nitrous acid, $\mathrm{NO}(\mathrm{OH})$, which is so clear in the sense of the law of substitutions, becomes a reality in those cases when reducing agents act on salts of nitrous acid. Thus Raschig (1888) proposed the following method for the preparation of the hydroxylamine sulphate. A mixture of strong solutions of potassium nitrite, $\mathrm{KNO}_{2}$, and lyydroxide, KHO, in molecular proportions, is prepared and cooled. An excess of sulphurous anliydride is then passed into the mixture, and the solution boiled for a long time. A mixture of the sulphates of potassium and hydroxylamine is thus obtained: $\mathrm{KNO}_{2}+\mathrm{KHO}+2 \mathrm{SO}_{2}+2 \mathrm{H}_{2} \mathrm{O}=\mathrm{NH}_{2}(\mathrm{OH}), \mathrm{H}_{2} \mathrm{SO}_{4}+\mathrm{K}_{2} \mathrm{SO}_{4}$. The salts may be separated from each other by crystallisation.

With respect to substances intermediate between $\mathrm{NH}_{3}$ and the oxides of nitrogen, we must turn our attention to hyponitrous acid, $\mathrm{NHO}$, and amidogen, which are mentioned in Note 67.

${ }_{26}$ Nitric acid corresponds with the anhydride $\mathrm{N}_{2} \mathrm{O}_{5}$, which will afterwards be described, but which must be regarded as the highest saline oxide of nitrogen, just as $\mathrm{Na}_{2} \mathrm{O}$ (and the hydroxide $\mathrm{NaHO}$ ) in the case of sodium, although sodium forms a peroxide possessing the property of parting with its oxygen with the same ease as hydrogen peroxide, if not on heating, at all events in reactions - for instance, with acids. So also nitric acid has its corresponding peroxide, which may be called pernitric acid. Its composition is not well known-probably $\mathrm{NHO}_{4}$-so that its corresponding anhydride would be $\mathrm{N}_{2} \mathrm{O}_{7}$. It is formed by the action of a silent discharge on a mixture of nitrogen and oxygen, so that a portion of its oxygen is in a state similar to that in ozone. The instability of this substance (obtained by Hautefeuille, Chappuis, and Berthelot), which easily splits up with the formation of nitric peroxide, and its resemblance to persulphuric acid, which we shall afterwards describe, will permit our passing over the consideration of the little that is further known concerning it.

27 Phosphorus, as we shall afterwards find, gives the hydride $\mathrm{PH}_{3}$, corresponding with ammonia, $\mathrm{NH}_{3}$, and forms phosphorous acid, $\mathrm{PH}_{3} \mathrm{O}_{3}$, which is analogous to nitrous acid, just as phosphoric acid is to nitric acid; but phosphoric (or, better, orthophosphoric) acid, $\mathrm{PH}_{3} \mathrm{O}_{4}$, is able to lose water and give pyro- and meta-phosphoric acids. The latter is equal to the ortho-acid minus water $=\mathrm{PHO}_{3}$, and therefore nitric acid, $\mathrm{NHO}_{3}$, is really meta-nitric acid. So also nitrous acid, $\mathrm{HNO}_{2}$, is meta-nitrous (anhydrous) acid, and then the ortho-acid is $\mathrm{NH}_{3} \mathrm{O}_{3}=\mathrm{N}(\mathrm{OH})_{3}$. 
compounds, which we will proceed to describe. Only let us first show that the passage of ammonia into the oxygen compounds of nitrogen up to nitric acid, as well as the converse preparation of ammonia from nitric acid, are reactions which proceed directly and easily under many circumstances. In nature the matter is complicated by a number of influences and circumstances, but in the law the relations are presented in their simplest aspect. The bond between this simplicity of laws and this complexity of phenomena forms the essence of a scientific understanding of things.

It is easy to prove the possibility of the oxidation of ammonia into nitric acid by passing a mixture of ammonia and air over heated spongy platinum. This causes the oxidation of the ammonia, nitric acid being formed, which partially combines with the excess of ammonia.

The converse passage of nitric acid into ammonia is accomplished by the action of hydrogen at the moment of its evolution. ${ }^{28}$ Thus metallic aluminium, evolving hydrogen from caustic soda, is able to completely convert nitric acid added to the mixture (really as a salt, because the alkali gives a salt with the nitric acid) into ammonia, $\mathrm{NHO}_{3}+8 \mathrm{H}=\mathrm{NH}_{3}+3 \mathrm{H}_{2} \mathrm{O}$.

The compounds of nitrogen with oxygen present an excellent example of the law of multiple proportions, because they contain for 14 parts by weight of nitrogen $8,16,24,32$, and 40 parts, respectively, by weight of oxygen. The composition of these compounds is as follows :-

$$
\begin{aligned}
& \mathrm{N}_{2} \mathrm{O} \text {, nitrous oxide; hydrate } \mathrm{NHO} \text {. } \\
& \mathrm{N}_{2} \mathrm{O}_{2} \text {, nitric oxide, NO. } \\
& \mathrm{N}_{2} \mathrm{O}_{3} \text {, nitrous anhydride ; hydrate } \mathrm{NHO}_{2} \text {. } \\
& \mathrm{N}_{2} \mathrm{O}_{4} \text {, peroxide of nitrogen, } \mathrm{NO}_{2} . \\
& \mathrm{N}_{2} \mathrm{O}_{5} \text {, nitric anhydride ; hydrate } \mathrm{N}_{\mathrm{HO}_{3}} .
\end{aligned}
$$

Of these compounds, ${ }^{29}$ nitrous and nitric oxides, peroxide of nitrogen, and nitric acid, $\mathrm{NHO}_{3}$, are characterised as being the most stable. The lower oxides, when coming into contact with the higher, may give the intermediate forms ; for instance, $\mathrm{NO}$ and $\mathrm{NO}_{2}$ form $\mathrm{N}_{2} \mathrm{O}_{3}$, and the intermediate oxides may, in splitting up, give a higher and lower oxide.

28 The formation of ammonia is remarked in many cases of oxidation by means of nitric acid. This sulstance is even formed in the action of nitric acid on tin, especially if dilute acid be employed in the cold. A still more considerable annount of ammonia is obtained if, in the action of nitric acid, there are conditions directly tending to the evolu. tion of hydrogen, which then reduces the acid to ammonia; for instance, in the action of zinc on a mixture of nitric and sulphuric acids.

29 According to the determinations of Favre, Thomsen, and more especially of Berthelot, on thermochemical data, it follows that in the formation of such quantities of the oxides of nitrogen as express their formulie, if gaseou nitrogen and oxygen be taken as the 
So $\mathrm{N}_{2} \mathrm{O}_{4}$ gives $\mathrm{N}_{2} \mathrm{O}_{3}$ and $\mathrm{N}_{2} \mathrm{O}_{5}$, or, in the presence of water, their hydrates.

We have already seen that, under certain conditions, nitrogen combines with oxygen, and we know that ammonia may be oxidised. In these cases various oxidation products of nitrogen are formed, but in the presence of water and an excess of oxygen they always give nitric acid. Nitric acid, as corresponding with the highest oxide, is able, in deoxidising, to give the lower oxides, and for this reason we will begin with it.

Nitric acid, $\mathrm{NHO}_{3}$, is likewise known as aqua fortis. In a free state it is only met with in nature in small quantities, in the air and rain-water after storms; but even in the atmosphere nitric acid does not long remain free, but combines with anmonia, traces of which are always found in air. On falling on the soil and into running water, \&c., the nitric acid everywhere comes into contact with bases (or their carbonates), which easily act on it, and therefore it is converted into the nitrates of these bases. Ammonia and other compounds of nitrogen, if oxidised in the soil, are always in the presence of bases, and therefore also give salts of nitric acid, and not the free acid itself. Hence nitric acid is always met with in the form of salts in nature. These salts are called nitres. This name is derived from the Latin sal nitri. The potassium salt $\mathrm{KNO}_{3}$ is common nitre, and the sodium salt $\mathrm{NaNO}_{3}$ Chili saltpetre, or cubic nitre. Nitres are formed when a nitrogenous substance is slowly oxidised in the presence of an alkali by means of the oxygen of the atmosphere. In nature there are very frequent instances of such oxidation. For this reason, certain soils and

starting points, and if the compounds formed be also gaseous, the following amounts of heat, expressed in thousands of heat units, are absorbed (hence a minus sign):-

$$
\begin{array}{lllll}
\mathrm{N}_{2} \mathrm{O} & \mathrm{N}_{2} \mathrm{O}_{2} & \mathrm{~N}_{2} \mathrm{O}_{3} & \mathrm{~N}_{2} \mathrm{O}_{4} & \mathrm{~N}_{2} \mathrm{O}_{5} \\
-21 & -43 & -22 & -5 & -1
\end{array}
$$

The difference is given in the lower line. For example, if $\mathrm{N}_{2}$, or 28 grams of nitrogen, combine with $\mathrm{O}$-that is, with 16 grams of oxygen - then 21000 units of heat are absorbed, or sufficient heat is assimilated to heat 21000 grams of water through $1^{\circ}$. Naturally, direct observations are impossible in this case; but if charcoal, phosphorus, or similar substances, are burnt both in nitrous oxide and in oxygen, and the heat evolved is observed in both cases, then the difference (more heat will be evolved in burning in nitrous oxide) gives the figures required. If, then, $\mathrm{N}_{2} \mathrm{O}_{2}$, by combining with $\mathrm{O}_{3}$, gives $\mathrm{N}_{2} \mathrm{O}_{4}$, then, as is seen from the table, heat should be developed, namely 38000 units of heat, or $\mathrm{NO}+\mathrm{O}=$ 19000 units of heat. The differences given in the table show that the maximum absorption of heat corresponds with nitric oxide, and that the higher oxides are formed from it with evolution of heat. If liquid nitric acid, $\mathrm{NHO}_{3}$, were decomposed into $\mathrm{N}+\mathrm{O}_{3}+\mathrm{H}$, then 41000 heat units would be required ; that is, an evolution of heat takes place in its formation from the gases. It should be observed that the formation of ammonia, $\mathrm{NH}_{3}$, from the gases $\mathrm{N}+\mathrm{H}_{3}$ evolves $12 \cdot 2$ thousand heat units. 


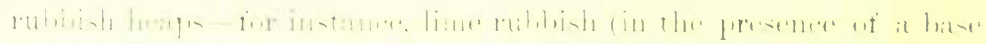

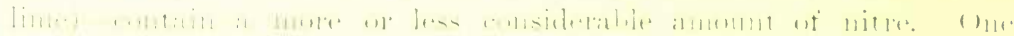

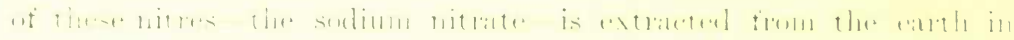

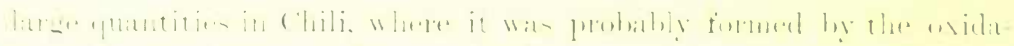

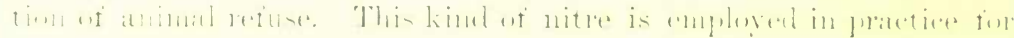

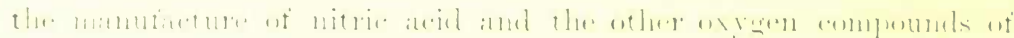

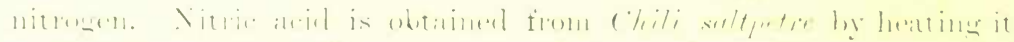

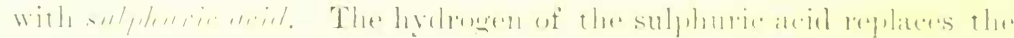

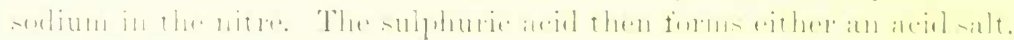

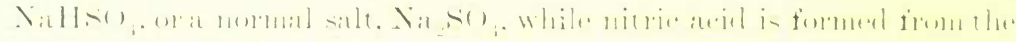

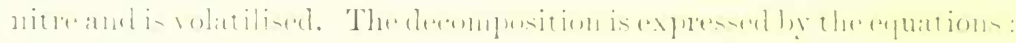

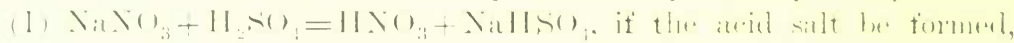

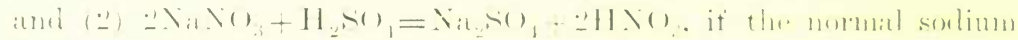

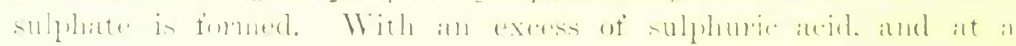

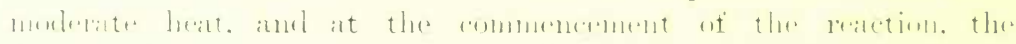

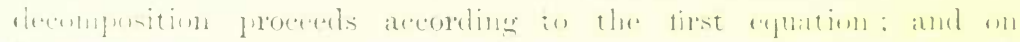
further heating with a sufficient amount of nitre, aceording to the

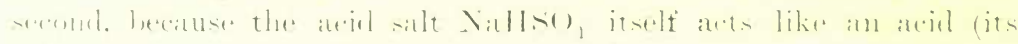

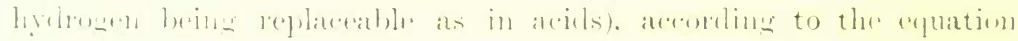
$\lambda_{i 1} \lambda_{1}-\lambda_{i 1} l 1-()_{1}=\lambda_{i 1}-()_{1}+11 \lambda_{1}()_{3}$.

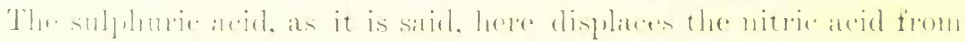

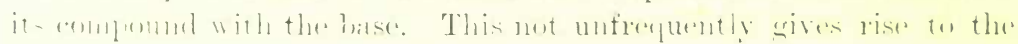

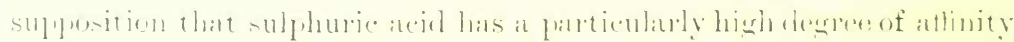

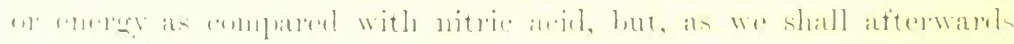

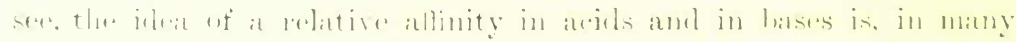

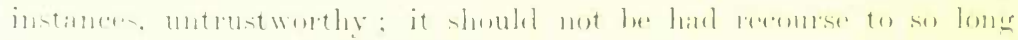

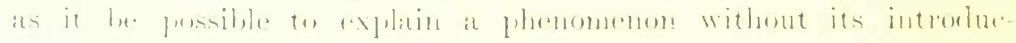

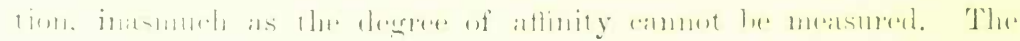

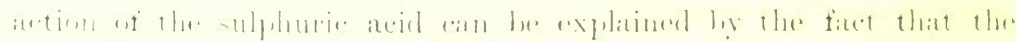

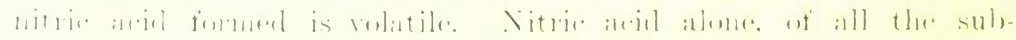

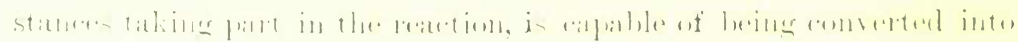

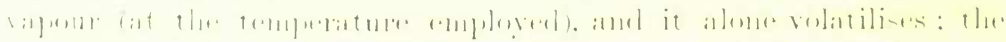

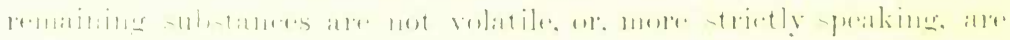

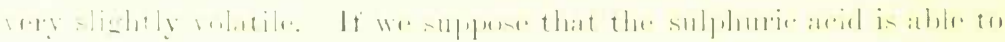

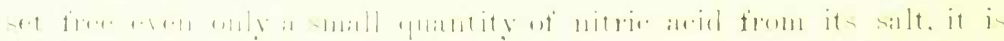

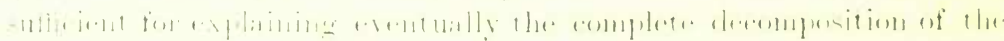

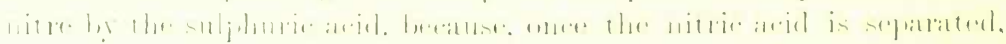

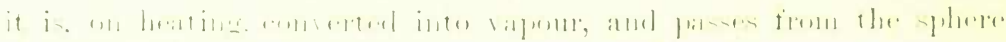

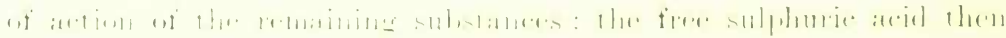

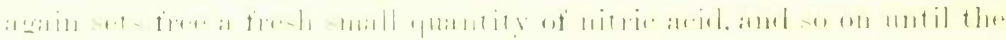

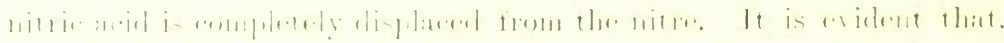

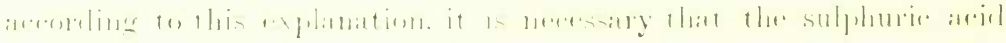


should be in excess (although but small) to the end of the reaction. According to the equation expressing the reaction, it is required that there should be 98 parts of sulphuric acid to 85 parts of sodium nitrate ; but if these quantities be taken, the nitric acid is not entirely displaced by the sulphuric acid. It is necessary that an excess of the latter should be taken; generally, 80 parts of nitre are taken to 98 parts of sulphuric acid, and therefore a portion of the sulphuric acid remains in a free state to the end of the reaction. Thus, in the reaction of sulphuric acid on nitre there is formed a non-volatile salt of sulphuric acid, which remains, together with an excess of this acid, in the distilling apparatus, and nitric acid, which is converted

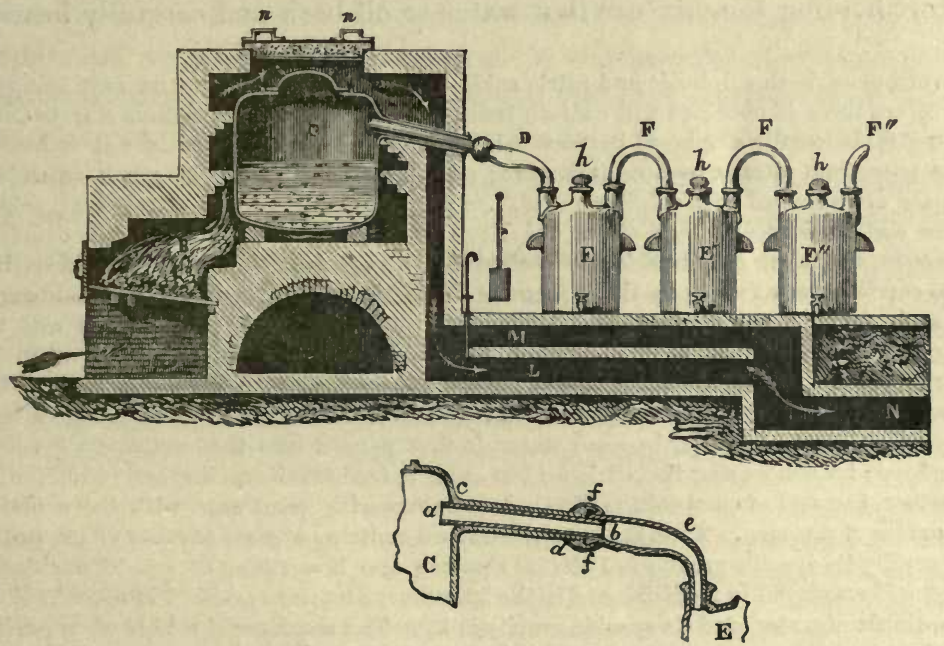

FIG. 47.-Method of preparing nitric acid on a large scale. A cast-iron retort, $\mathrm{C}$, is fixed into the furnace, and heatel by the fire, B. The flame and products of combustion are at first led along the flue, M (in order to heat the receivers), and afterwards into L. The retort is charged with Chili saltpetre and sulphuric acil, and the cover is luted on with clay and gypsum. A clay tube, $a$, is fixed into the neck of the retort (in order to prevent the nitric acid from corroding the cast iron), and a bent glass tube, D, is luted on to it. This tube carries the vapours into a series of earthenware receivers, E. Nitric acid mixed with sulpluric acid collects in the first. The purest nitric acid is procured from the second, whilst that which condenses in the third receiver contains hydrochloric acid, and that in the fourth nitrous oxide. Water is pcured into the last receiver in orler to condense the residual vapours.

into vapour, and may be condensed, because it is a liquid and volatile substance. On a small scale, this reaction may be carried on in a glass retort with a glass condenser. On a large scale, in chemical works, the process is exactly similar, only iron retorts are employed for holding the mixture of nitre and sulphuric acid, and earthenware three-necked bottles are used instead of a condenser, ${ }^{30}$ as shown in fig. 47.

30 It must be observed that sulphuric acid, at least when undiluted ( $60^{\circ}$ Baumé), corrodes cast iron with difficulty, so that the acid may be heated in cast-iron boilers. 
Nitric acid so obtained always contains water. It is extremely difficult to deprive it of all the admixed water without destroying a portion of the acid itself and partially converting it into lower oxides, because without the presence of an excess of water it is very unstable. When rapidly distilled a portion is decomposed, and there are obtained free oxygen and lower oxides of nitrogen, which, together with the water, remain in solution with the nitric acid. Therefore it is necessary to work with great care in order to obtain a pure hydrate of nitric acid, $\mathrm{HNO}_{3}$, and especially to mix the nitric acid obtained from nitre, as above described, with sulphuric acid, which takes up the water, and to distil it at the lowest possible temperature - that is, by placing the retort holding the mixture in a water or oil bath and carefully heating

Nevertheless, both sulphuric and nitric acids evince a certain action on cast iron, and therefore the acid obtained will contain traces of iron. In practice sodium nitrate (Chili saltpetre) is usually employed because it is cheaper, but in the laboratory it is best to take potassimm nitrate, because it is purer and does not froth up so much as sodium nitrate when heated with sulphuric acid. In the action of an excess of sulphuric acid on nitre and nitric acid a portion of the latter is decomposed, forming lower oxides of nitrogen, which are dissolved in the nitric acid. A portion of the sulphuric acid itself is also carried over as spray by the vapours of the nitric acid. Hence sulphuric acid occurs as an impurity in commercial nitric acid. A certain amount of hydrochloric acid will also be found to be present in it, because sodium chloride is generally found as an impurity in nitre, and under the action of sulphuric acid it forms hydrochloric acid. Commercial acid further contains a considerable excess of water above that necessary for the formation of the hydrate, because water is first poured into the earthenware vessels employed for condensing the nitric acid in order to facilitate its cooling and condensation. Further, the acid of composition $\mathrm{HNO}_{3}$ decomposes with great ease, with the evolution of oxides of nitrogen. Thus the commercial acid contains a great number of impurities. Generally its specific gravity is $1.33\left(36^{\circ}\right.$ Baumé), and it contains 53 p.c. of nitric acid. The acid employed in medicine and in the laboratory contains one-third of nitric acid and two-thirds of water, and its specific gravity is $1 \cdot 2$. The commercial acid is often purified in the following manner:-Lead nitrate is first added to the acid because it forms nonvolatile and almost insoluble (precipitated) substances with the free sulphuric and hydrochloric acids, and liberates nitric acid in so doing, according to the equations $\mathrm{Pb}\left(\mathrm{NO}_{3}\right)_{2}+2 \mathrm{HCl}=\mathrm{PbCl}_{2}+2 \mathrm{NHO}_{3}$ and $\mathrm{Pb}\left(\mathrm{NO}_{3}\right)_{2}+\mathrm{H}_{2} \mathrm{SO}_{4}=\mathrm{PbSO}_{4}+2 \mathrm{NHO}_{5}$. . Potas sium chromate is then added to the impure nitric acid, by which means oxygen is liberated from the chromic acid, and this oxygen, at the moment of its evolution, oxidises the lower oxides of nitrogen and converts them into nitric acid. A pure nitric acid, containing no impurities other than water, may be then obtained by distilling the acid, manipulated as above described, with care, and particularly if only the middle portions of the distillate are collected. Such acid should give no precipitate, either with a solution of barium chloride (a precipitate shows the presence of sulphuric acid) or with a solution of silver nitrate (a precipitate shows the presence of hydrochlorio acid), nor should it, after being diluted with water, give a coloration with starch containing potassium iodide (a coloration shows the admixture of other oxides of nitrogen). The oxides of nitrogen may be most easily removed from impure nitric acid by heating for a certain time with a small quantity of pure charcoal. By the action of nitric acid on the charcoal carbonic anhydride is evolved, which carries off the $\mathrm{NO}, \mathrm{NO}_{2}$, and other volatile substances. On redistilling, pure acid is obtained. The oxides of nitrogen occurring in solution may also be removed by passing air through the nitric acid. 
it. The first portion of the nitric acid thus distilled boils at $86^{\circ}$, has a specific gravity at $15^{\circ}$ of 1.526 , and solidifies at $-50^{\circ}$; it is very unstable at higher temperatures. This is the normal hydrate, $\mathrm{HNO}_{3}$, which corresponds with the salts, $\mathrm{NMO}_{3}$, of nitric acid. When diluted with water nitric acid presents a higher boiling point, not only as compared with that of the nitric acid itself, but also with that of water ; so that, if very dilute nitric acid be distilled, the first portions passing over will consist of almost pure water, until the boiling point in the vapours reaches $121^{\circ}$. At this temperature a compound of nitric acid with water, containing about 70 p.c. of nitric acid, ${ }^{31}$ distils over; its specific gravity at $15^{\circ}=1.521$. If the solution contain less than 25 p.c. of water, then, the specific gravity of the solution being above $1 \cdot 44, \mathrm{HNO}_{3}$ evaporates off and fumes in the air, forming the above hydrate, whose vapour tension is less than that of water. Such solutions form fuming nitric acid. On distilling it gives monohydrated acid, ${ }^{32} \mathrm{HNO}_{3}$; it is a hydrate boiling at $121^{\circ}$, so that it is obtained from both weak and strong solutions. Fuming nitric acid, under the action not only of organic substances, but even of heat, loses a portion

31 Dalton, Smith, Bineau, and others considered that the hydrate of constant boiling point (see Chapter I. Note 60) for nitric acid was the compound $2 \mathrm{HNO}_{3}, 3 \mathrm{H}_{2} \mathrm{O}$, but Roscoe showed that its composition changes with a variation of the pressure and temperature under which the distillation proceeds. Thus, at a pressure of 1 atmosphere the solution of constant boiling point contains $68^{\circ} 6$ p.c., and at one-tenth atmosphere $66^{\circ} 8$ p.c. Judging from what has been said concerning solutions of hydrochloric acid, and from the variation of specific gravity, I think that the comparatively large decrease of the tensions of the vapours depends on the formation of a hydrate, $\mathrm{NHO}_{3}, 2 \mathrm{H}_{2} \mathrm{O}$ (=63.6 p.c.). Such a hydrate may be expressed by $\mathrm{N}(\mathrm{HO})_{5}$, that is as $\mathrm{NH}_{4}(\mathrm{HO})$ in which all the equivalents of hydrogen are replaced by hydroxyl. The constant boiling point will then be the temperature of the decomposition of this hydrate.

Besides which, judging by the variation of the specific gravity (see my work cited in Chapter I. Note 29), at least one more hydrate, $\mathrm{NHO}_{3}, 5 \mathrm{H}_{2} \mathrm{O}\left(41 \cdot 2\right.$ p.c. $\left.\mathrm{HNO}_{3}\right)$, must be acknowledged. Starting from water $(p=0)$ to this hydrate, the specific gravities of the solutions at $15^{\circ}$ is well expressed by $s=9992+57 \cdot 4 p+0.16 p^{2}$, if water $=10000$ at $4^{\circ}$. For example, when $p=30$ p.c., $s=11860$. For more concentrated solutions, at least, the above-mentioned hydrate, $\mathrm{HNO}_{3}, 2 \mathrm{H}_{2} \mathrm{O}$, must be taken, up to which the specific gravity $s=9570+84 \cdot 18 p-0 \cdot 240 p^{2} ;$ but perhaps (the results of observations of the specific gravity of the solutions are not in sufficient agreement to make a decision) the hydrate $\mathrm{HNO}_{3}, 3 \mathrm{H}_{2} \mathrm{O}$ should be recognised, as is indicated by many nitrates ( $\mathrm{Al}, \mathrm{Mg}, \mathrm{Co}$. , \&c.), which crystallise with this amount of water of crystallisation. From $\mathrm{HNO}_{3}, 2 \mathrm{H}_{2} \mathrm{O}$ to $\mathrm{HNO}_{3}$ the specific gravity of the solutions (at $15^{\circ}$ ) $s=10652+62 \cdot 08 p-0 \cdot 160 p^{2}$. The pentahydrated hydrate is recognised by Berthelot on the basis of the thermo-chemical data for solutions of nitric acid, because on approaching to this composition there is a rapid change in the amount of heat evolved by mixing nitric acid with water. This hydrate solidifies at about $-19^{\circ}$. One would think that a more detailed study of the reactions of hydrated nitric acid would show the existence of change in the process and rapidity of reaction in approaching these hydrates.

32 The normal hydrate $\mathrm{HNO}_{3}$, corresponding with the ordinary salts, may be termed the monohydrated acid, because the anhydride $\mathrm{N}_{2} \mathrm{O}_{5}$ with water forms this normal nitric acid. In this sense the hydrate $\mathrm{HNO}_{3}, 2 \mathrm{H}_{2} \mathrm{O}$ is the pentahydrated acid. 


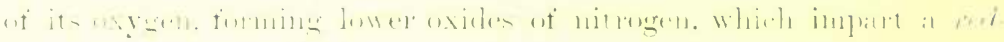

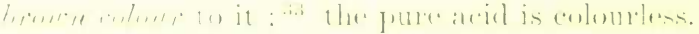

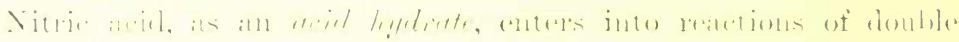

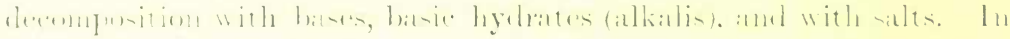

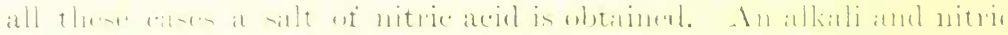

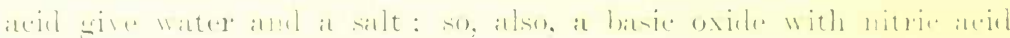

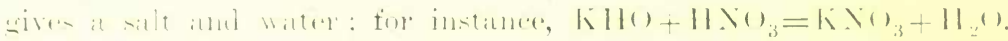

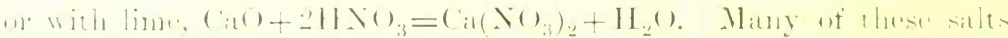

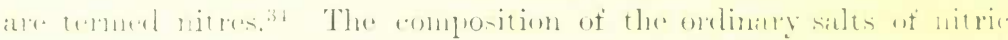

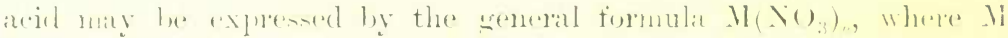

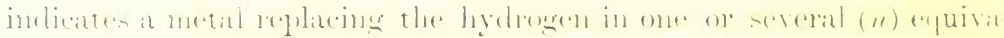

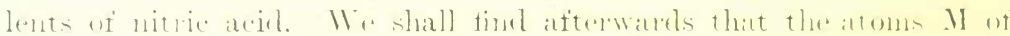

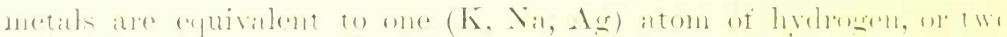

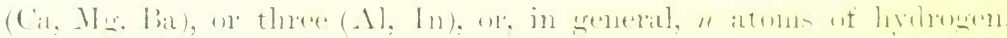

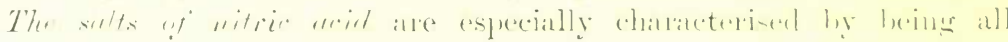

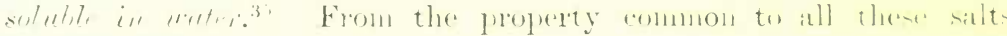

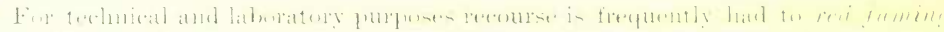

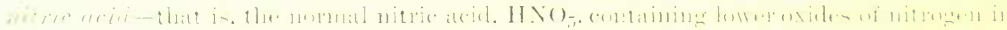

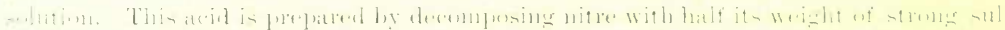

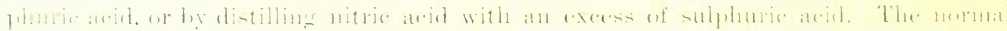

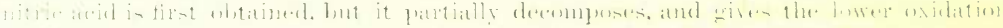

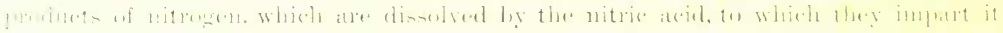

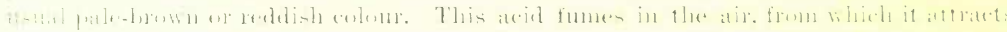

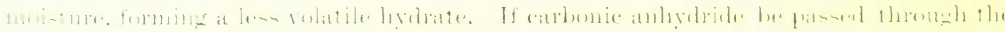

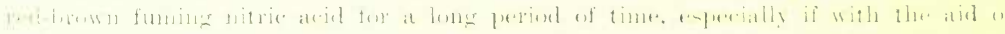

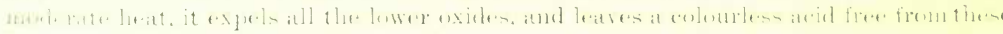

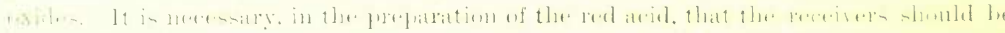

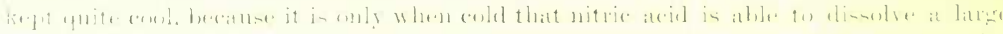

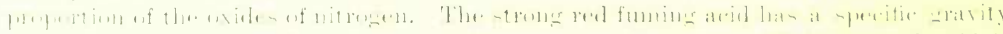

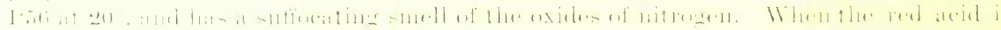

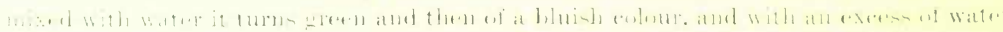

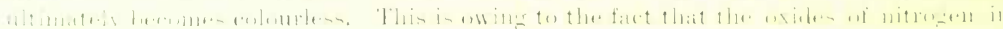

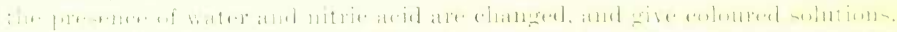

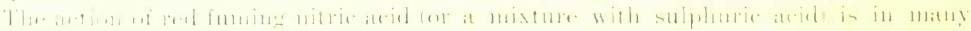

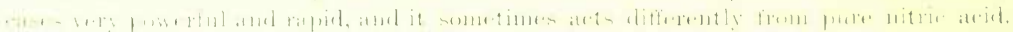

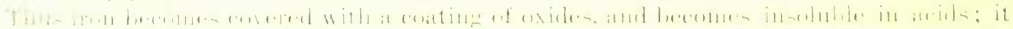

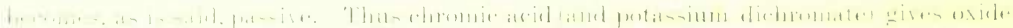

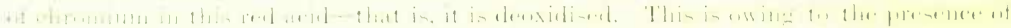

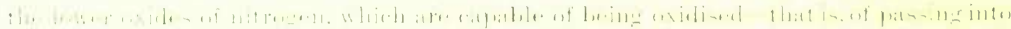

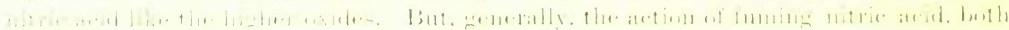
1.

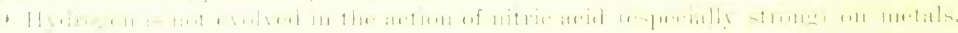

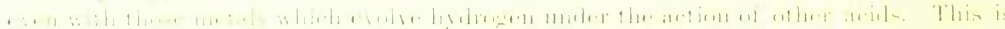

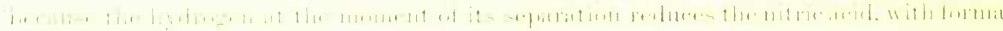

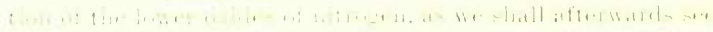

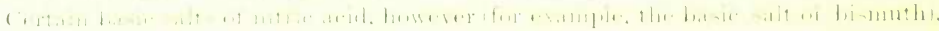

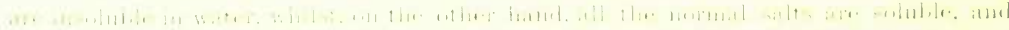

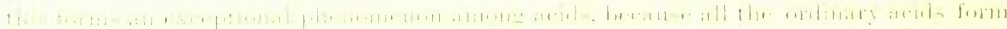

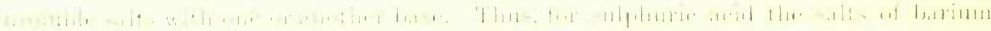


of entering into double decompositions, and owing to the volatility of nitric acid, they, like cubic nitre, evolve nitric acid when heated with sulphuric acid. They all, like nitric acid itself, are capable of evolving oxygen when heated, and consequently of acting like oxidising substances, and therefore, for instance, deflagrate with ignited carbon, the carbon burning at the expense of the oxygen of the salt and forming gaseous products of combustion. ${ }^{36}$

Nitric acid also enters into double decompositions with a number of hydrocarbons not in any way possessing alkaline characters and not reacting with other acids. Under these circumstances, the nitric acid gives water and a new substance termed a nitro-compound. The chemical character of the nitro-compound is the same as that of the original substance ; for example, if an indifferent substance be taken, then the nitro-compound obtained from it will also be indifferent; if an acid be taken, then an acid is obtained also. Benzene, $\mathrm{C}_{6} \mathrm{H}_{6}$, for instance, acts according to the equation $\mathrm{C}_{6} \mathrm{H}_{6}+\mathrm{HNO}_{3}$ $=\mathrm{H}_{2} \mathrm{O}+\mathrm{C}_{6} \mathrm{H}_{5} \mathrm{NO}_{2}$. Nitrobenzene is produced. The substance taken, $\mathrm{C}_{6} \mathrm{H}_{6}$, is a liquid hydrocarbon having a faint tarry smell, boiling at $80^{\circ}$, and lighter than water; by the action of nitric acid nitrobenzene is obtained, which is a substance boiling at about $210^{\circ}$, heavier than water, and having an alinond-like odour; it is employed in large quantities for the preparation of aniline and aniline dyes. ${ }^{37}$ As they contain both combustible elements (hydrogen and carbon), as

lead, \&c., for hydrochloric acid the salts of silver, \&c., are insoluble in water. However, the normal salts of acetic and certain other acids are all soluble.

56 Ammonium nitrate, $\mathrm{NH}_{4} \mathrm{NO}_{3}$, is easily obtained by adding a solution of ammonia or of ammonium carbonate to nitric acid until it becomes neutral. On evaporating this solution crystals of the salt are formed which contain no water of crystallisation. It crystallises in prisms like those formed by common nitre, and has a refreshing taste; 100 parts of water at $t^{\circ}$ dissolve $54+0 \cdot 61 t$ parts by weight of the salt. It is soluble in alcohol, melts at $160^{\circ}$, and is decomposed at about $180^{\circ}$, forming water and nitrous oxide, $\mathrm{NH}_{4} \mathrm{NO}_{3}=2 \mathrm{H}_{2} \mathrm{O}+\mathrm{N}_{2} \mathrm{O}$. If ammonium nitrate be mixed with sulphuric acid, and the mixture be heated at about the boiling point of water, then nitric acid is evolved, and ammonium hydrogen sulphate remains in solution; but if the mixture be heated rapidly to $160^{\circ}$, then nitrous oxide is evolved. In the first case the sulphuric acid takes up ammonia, and in the second place water. Ammonium nitrate is employed in practice for the artificial production of cold, because in dissolving in water it lowers the temperature very considerably. For this purpose it is best to take equal parts by weight of the salt and water. The salt must first be reduced to a powder and then rapidly stirred up in the water, when the temperature will fall from $+15^{\circ}$ to $-10^{\circ}$, so that the water freezes.

Ammonium nitrate absorbs ammonia, with which it forms unstable compounds resembling compounds containing water of crystallisation. At $-10^{\circ} \mathrm{NH}_{4} \mathrm{NO}_{3}, 2 \mathrm{NH}_{3}$ is formed : it is a liquid of sp.gr. 1.50, which loses all its ammonia under the influence of heat. At $+28^{\circ} \mathrm{NH}_{4} \mathrm{NO}_{3}, \mathrm{NH}_{3}$ is formed : it is a solid which easily parts with its anmonia when heated, especially in solution.

37 The action of nitric acid on cellulose, $\mathrm{C}_{6} \mathrm{H}_{10} \mathrm{O}_{5}$, is similar. This substance, which forms the outer coating of all plant cells, occurs in an almost pure state in cotton, in 


\section{well as oxygen in unstable combination with nitrogen, in the form of the radicle $\mathrm{NO}_{2}$ of nitric acid, the nitro-compounds, when ignited or}

common writing-paper, and in flax, \&c.; under the action of nitric acid it forms water and nitrocellulose, which, although it has the same appearance as the cotton originally taken, differs from it entirely in properties. It explodes when struck, bursts into flame very easily under the action of sparks, and acts like gunpowder, whence its name of pyroxylin, or gun-cotton. The composition of gun-cotton is $\mathrm{C}_{6} \mathrm{H}_{7} \mathrm{~N}_{3} \mathrm{O}_{11}=\mathrm{C}_{6} \mathrm{H}_{10} \mathrm{O}_{5}+3 \mathrm{NHO}_{3}$ $-3 \mathrm{H}_{2} \mathrm{O}$. The proportion of the group $\mathrm{NO}_{2}$ in nitrocellulose may be decreased by limiting the action of the nitric acid, and a compound is obtained which burns without explosion, although it is capable of bursting into flame. This substance when dissolved in a mixture of alcohol and ether is called collodion. The solution when poured on to any surface loses all the ether and alcohol by evaporation, and leaves an amorphous mass in the form of a transparent membrane insoluble in water. A solution of collodion is employed in medicine for covering wounds, and in wet-plate photography for giving on glass an even coat of a substance into which the various reagents employed in the process are introduced.

The property possessed by nitroglycerin (occurring in dynamite), nitrocellulose, and the other nitro-compounds, of burning with an explosion depends on the reasons in virtue of which a mixture of nitre and charcoal deflagrates and explodes; in both cases the elements of the nitric acid occurring in the compound are decomposed, the oxygen in burning unites with the carbon, and the nitrogen is set free; thus a very large volume of gaseous substances (nitrogen and oxides of carbon) is rapidly formed from the solid substances originally taken. These gases occupy an incomparably larger volume than the original substance, and therefore produce a powerful pressure and explosion. It is evident that in exploding with the development of heat (that is, in decomposing, not with the absorption of energy, as is generally the case, but with the evolution of energy) the nitro-compounds form stores of energy which are easily set free, and that consequently their elements occur in a state of particularly energetic movement, which is especially strong in the group $\mathrm{NO}_{22}$; this group is common to all nitrocompounds, and all the oxygen compounds of nitrogen are unstable, easily decomposable, and (Note 29) absorb heat in their formation. On the other hand, the nitrocompounds are instructive as an example and proof of the fact that the elements and groups forming compounds are united in definite order in the molecules of a compound. A blow, concussion, or rise of temperature is necessary to bring the combustible elements $\mathrm{C}$ and $\mathrm{H}$ into the most intimate contact with $\mathrm{NO}_{2}$, and to distribute the elements in a new order in new compounds.

As regards the composition of the nitro-compounds, it will be seen that the hydrogen of a given substance is replaced by the complex group $\mathrm{NO}_{2}$ of the nitric acid. The same is observed in the passage of alkalis into nitrates, so tliat the reactions of substitution of nitric acid-that is, the formation of salts and nitro-compounds-may be expressed in the following manner. In these cases the hydrogen is replaced by the so-called radicle of nitric acid $\mathrm{NO}_{2}$, as is evident from the following table :-

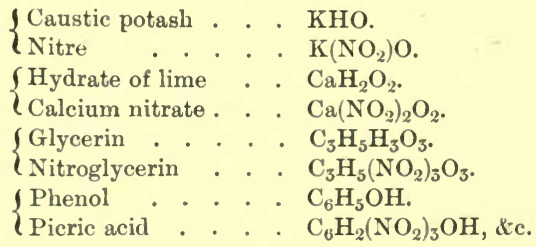

The difference between the salts formed by nitric acid and the nitro-compounds consists in the fact that nitric acid is very easily separated from the salts of nitric acid by 
even struck, decompose with an explosion, owing to the pressure of the vapours and gases formed-free nitrogen, carbonic anhydride, and aqueous vapour. In the explosion of nitro-compounds much heat is evolved, as in the combustion of gunpowder or detonating gas, and in this case the force of explosion in a closed space is great, because from a solid or liquid nitro-compound occupying a small space there proceed vapours and gases whose elasticity is great not only from the small space in which they are formed, but owing to the high temperature corresponding to the combustion of the nitro-compound. ${ }^{38}$

The combustion of nitro-compounds, as well as that which nitrates bring about (in gunpowder), originates in the weakness of the bond which holds together the oxygen and nitrogen in nitric acid itself, as well as in all the oxygen compounds of nitrogen. If the vapour of nitric acid is passed through an even moderately heated glass tube, the formation of dark-brown fumes of the lower oxides of nitrogen and the separation of free oxygen may be observed-2 $\mathrm{NHO}_{3}=\mathrm{H}_{2} \mathrm{O}+2 \mathrm{NO}_{2}+\mathrm{O}$. The decomposition is complete at a white heat that is, nitrogen is formed, $2 \mathrm{NHO}_{3}=\mathrm{H}_{2} \mathrm{O}+\mathrm{N}_{2}+\mathrm{O}_{5}$. Hence it is easily understood that nitric acid may part with its oxygen to a number of substances capable of being oxidised. ${ }^{39}$ It is consequently an oxidising agent. Charcoal, as we have already seen, burns in nitric acid; phosphorus, sulphur, iodine, and the majority of metals also decompose nitric acid,

means of sulphuric acid (that is, by a method of double saline decomposition), whilst nitric acid is not displaced by sulphuric acid from true nitro-compounds; for instance, nitrobenzene, $\mathrm{C}_{6} \mathrm{H}_{5} \cdot \mathrm{NO}_{2}$. As nitro-compounds are formed exclusively from hydrocarbons, they are described with them in organic chemistry.

The group $\mathrm{NO}_{2}$ of nitro-compounds in many cases (like all the oxidised compounds of nitrogen) passes into the ammonia group or into the ammonia radicle $\mathrm{NH}_{2}$. It is evident that this requires the action of reducing substances evolving hydrogen: $\mathrm{RNO}_{2}+6 \mathrm{H}$ $=\mathrm{RNH}_{3}+2 \mathrm{H}_{2} \mathrm{O}$. Thus Zinin converted nitrobenzene, $\mathrm{C}_{6} \mathrm{H}_{5} \cdot \mathrm{NO}_{2}$, into aniline, $\mathrm{C}_{6} \mathrm{H}_{5} \cdot \mathrm{NH}_{2}$, by the action of hydrogen sulphide.

Adnitting the existence of the group $\mathrm{NO}_{2}$, replacing hydrogen in various compounds, then nitric acid may be considered as water in which half the hydrogen is replaced by the radicle of nitric acid. In this sense nitric acid is nitro-water, $\mathrm{NO}_{2} \cdot \mathrm{OH}$, its anhydride dinitro-water, $\left(\mathrm{NO}_{2}\right)_{2} \mathrm{O}$, and nitrous acid nitro-hydrogen, $\mathrm{NO}_{2} \mathrm{H}$. In nitric acid the radicle of nitric acid is combined with hydroxyl, just as in nitrobenzene it is combined with the radicle of benzene.

It should here be remarked that the group $\mathrm{NO}_{3}$ may be recognised in the salts of nitric acid, because the salts have the composition $\mathrm{M}\left(\mathrm{NO}_{3}\right)_{n}$, just as the metallic chlorides have the composition $\mathrm{MCl}_{n}$. But the group $\mathrm{NO}_{3}$ does not form any other compounds beyond the salts, and therefore it should be considered as hydroxyl, $\mathrm{HO}$, in which $\mathrm{H}$ is replaced by $\mathrm{NO}_{2}$.

38 The nitro-compounds play a very important part in mining and artillery. Detailed accounts of them must be looked for in special works. The most important and historical work in this connection is due to Berthelot, who elucidated much in connection with explosive compounds by a series of both experimental and theoretical researches.

39 Nitric acid may be entirely decomposed by passing its vapour over highly incandescent copper, because the oxides of nitrogen first formed give up their oxygen to the 
some on heating and others even at the ordinaty temperature: the

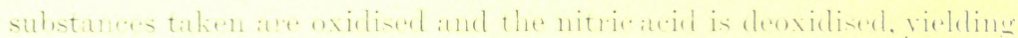

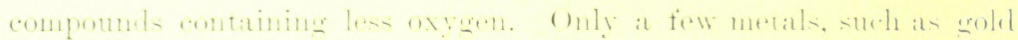

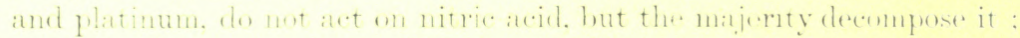
in so doing. an ovile of the motal is formed. Which. if it has the

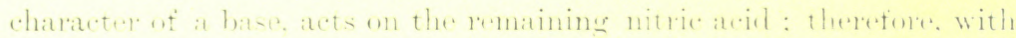

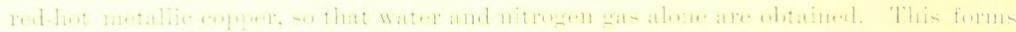

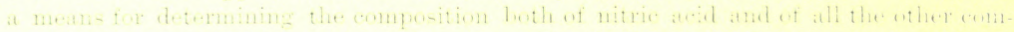

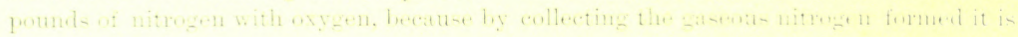

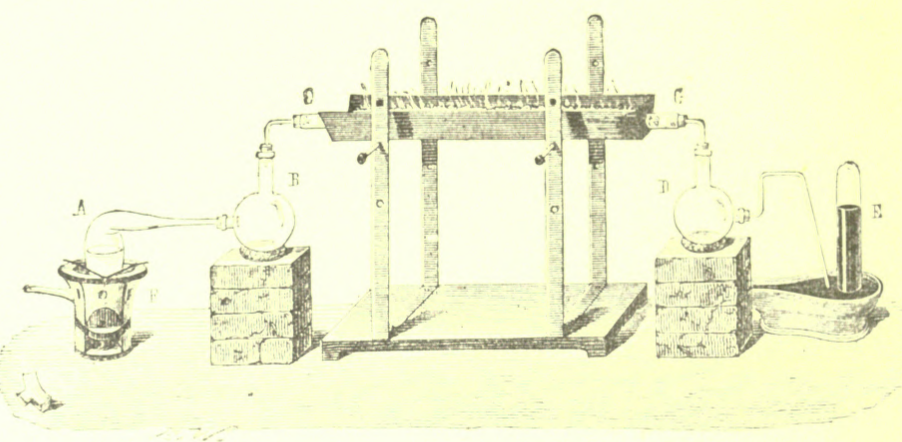

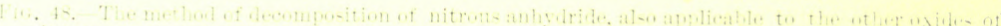

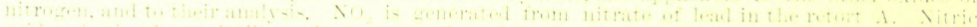

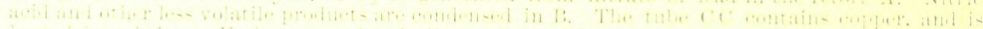

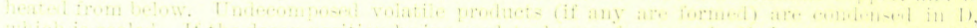

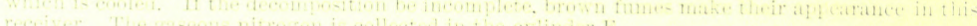

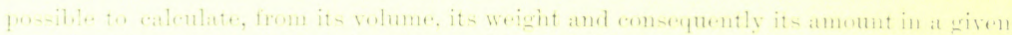

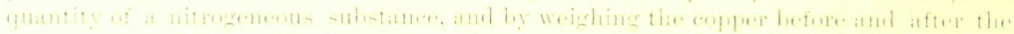

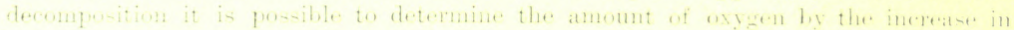

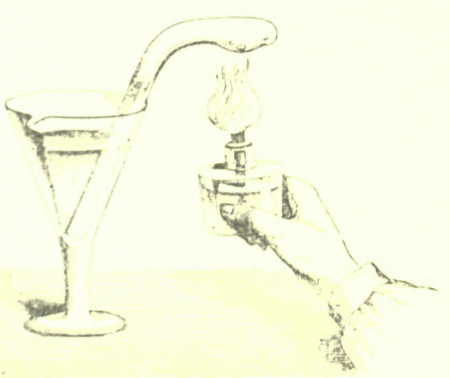
weighe Fin nitrie ateid this de.

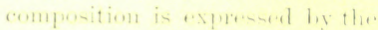

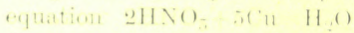

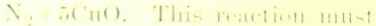

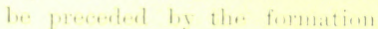

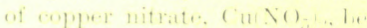

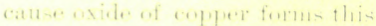
sitlt with nitrig ate id. This millt is

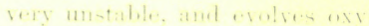

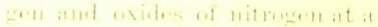

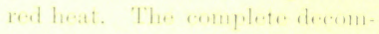

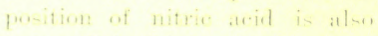

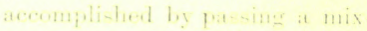
$+3$

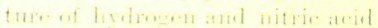

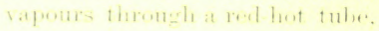

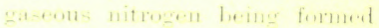

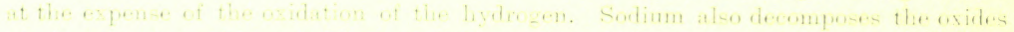

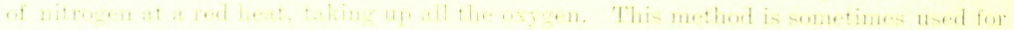

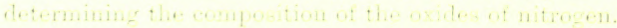


metals the result of the reaction is usually not an oxide of the metal, but the corresponding salt of nitric acid, and, at the same time, one of the lower oxides of nitrogen. The resulting salts of the metals are soluble, and hence it is said that nitric acid dissolves nearly all metals. ${ }^{40}$ This case is termed the solution of mevals by acids, although it is not a case of simple solution, but a complex chemical change of the substances taken. When treated with this acid, those metals whose oxides do not combine with nitric acid yield the oxide itself, and not a salt; for example, tin acts in this manner on nitric acid, forming a hydrous oxide, $\mathrm{SnH}_{2} \mathrm{O}_{3}$, which is obtained in the form of a white powder, $\mathrm{Sn}+4 \mathrm{NHO}_{3}=\mathrm{H}_{2} \mathrm{SnO}_{3}+4 \mathrm{NO}_{2}+\mathrm{H}_{2} \mathrm{O}$. Silver is able to take up still more oxygen, and to convert a large portion of nitric acid into nitrous anhydride, $4 \mathrm{Ag}+6 \mathrm{HNO}_{3}=4 \mathrm{AgNO}_{3}+\mathrm{N}_{2} \mathrm{O}_{3}+3 \mathrm{H}_{2} \mathrm{O}$. Copper takes up still more oxygen from nitric acid, converting it into nitric oxide, a perfectly colourless gas; and, by the action of zinc, nitric acid is able to give up a still further quantity of nitrogen, forming nitrous oxide, $4 \mathrm{Zn}+10 \mathrm{NHO}_{3}=4 \mathrm{Zn}\left(\mathrm{NO}_{3}\right)_{2}+\mathrm{N}_{2} \mathrm{O}+5 \mathrm{H}_{2} \mathrm{O}$. ${ }^{41}$ Sometimes, and especially with dilute solutions of nitric acid, the deoxidation proceeds as far as the formation of hydroxylamine and ammonia, and

40 The application of this acid for etching copper or steel in engraving is based on this fact. The copper is covered with a coating of wax, resin, \&c. (etching ground), on which nitric acid does not act, and then the ground is removed in certain parts with a needle, and the whole is washed in nitric acid. The parts covered with the ground remain untouched, whilst the uncovered portions are eaten into by the acid. Copper plates for etchings, aquatints, \&c., are prepared in this manner.

41 The formation of such complex equations as the above often presents some difficulty to the beginner. It should be observed that if the reacting and resultant substances be known, it is easy to form an equation for the reaction. Thus, if we wish to form an equation expressing the reaction that nitric acid acting on zinc gives nitrous oxide, $\mathrm{N}_{2} \mathrm{O}$, and zinc nitrate, $\mathrm{Zn}\left(\mathrm{NO}_{3}\right)_{2}$, we must reason as follows :-Nitric acid contains hydrogen, whilst the salt and nitrous oxide do not; hence water is formed, and therefore it is as though anhydrous nitric acid, $\mathrm{N}_{2} \mathrm{O}_{5}$, were acting. For its conversion into nitrous oxide it parts with four equivalents of oxygen, and hence it is able to oxidise four equivalents of zinc and to convert it into zinc oxide, $\mathrm{ZnO}$. These four equivalents of zinc oxide require for their conversion into the salt four more equivalents of nitric anhydride, consequently five equivalents in all of the latter are required, or ten equivalents of nitric acid. Consequently ten equivalents of nitric acid are necessary for four equivalents of zinc in order to express the reaction in whole equivalents. It must not be forgotten, however, that there are very few such reactions which can be entirely expressed by simple equations. The majority of equations of reactions only express the chief and ultimate products of reaction, and thus none of the three preceding equations express all that in reality occurs in the action of metals on nitric acid. In no one of them is only one oxide of nitrogen formed, but always several together or consecutively-one after the other, according to the temperature and strength of the acid. And this is easily understood. The resulting oxide is itself capable of influencing metals and of being deoxidised, and in the presence of the nitric acid it may change the acid and be itself changed. The equations given must be looked on as a systematic expression of the main aspects of reactions. Further, these reactions vary considerably with different temperatures and varying strengths of acid. 
sometimes it leads to the formation of ammonia itself. The formation of one or other nitrogenous substance from nitric acid is determined not only by the nature of the reacting substances, but also by the relative mass of water and nitric acid, and also by the temperature and pressure, or the sum total of the conditions of reaction; and as in a given mixture these conditions even vary (the temperature and the relative mass vary), therefore a mixture of different products of the deoxidation of nitric acid is not unfrequently formed.

Thus the action of nitric acid on metals cousists in their being oxidised, whilst it is itself converted, according to the temperature, concentration in which it is taken, and the nature of the metal, \&c., into either lower oxides, or even into ammonia. ${ }^{42}$ Many compounds are oxidised by nitric acid like metals and other elements ; for instance, lower oxides are converted into higher oxides. Thus, arsenious acid is converted into arsenic acid, suboxide of iron into oxide, sulphurous acid into sulphuric acid, the sulphides of the metals, $\mathrm{M}_{2} \mathrm{~S}$, into sulphates, $\mathrm{M}_{2} \mathrm{SO}_{4}$, dc. ; in a word, nitric acid brings about oxidation, its oxygen is taken up and transferred to many other substances. Certain substances are oxidised by strong nitric acid so rapidly and with so great an evolution of heat that they defiagrate and burst into flame. Thus turpentine, $\mathrm{C}_{10} \mathrm{H}_{16}$, bursts into flame when poured into fuming nitric acid. In virtue of its oxidising property, nitric acid removes the hydrogen from many substances. Thus it decomposes hydriodic acid, separating the iodine and forming water; and if fuming nitric acid be poured into a flask containing gaseous hydriodic acid, then a rapid reaction takes place, accompanied by flame and the separation of violet vapours of iodine and brown fumes of oxides of nitrogen. ${ }^{43}$

42 It is observed that normal nitric acid oxidises many metals with much greater difficulty than when dilnted with water; iron, copper, and tin are very easily oxidised by dilute nitric acid, but remain unaltered under the influence of monohydrated nitric acid or of the pure hydrate $\mathrm{NHO}_{5}$. Nitric acid diluted with a large quantity of water does not oxidise copper, but it oxidises tin; dilute nitric acid also does not oxidise either silver or mercury; but, on the addition of nitrous acid, even dilute acid acts on the above metals. This naturally depends on the smaller stability of nitrous acid, and on the fact that after the commencement of the action the nitric acid is itself converted into nitrous acid, which continues to act on the silver and mercury.

43 When nitric acid acts on many organic substances it often happens that not only is hydrogen removed, but also oxygen is combined; thus, for example, nitric acid converts toluene, $\mathrm{C}_{7} \mathrm{H}_{8}$, into benzoic acid, $\mathrm{C}_{7} \mathrm{H}_{6} \mathrm{O}_{2}$. In certain cases, also, a portion of the carbon contained in an organic snbstance burns at the expense of the oxygen of the nitric acid. So, for instance, phthalic acid, $\mathrm{C}_{8} \mathrm{H}_{6} \mathrm{O}_{4}$, is obtained from naphthalene, $\mathrm{C}_{10} \mathrm{H}_{8}$. Thus the action of nitric acid on the hydrocarbons is often most complex; there takes place (besides nitrification) the separation of carbon, the displacement of hydrogen, and the combination of oxygen. There are few organic sulsstances which can withstand the action of nitric acid. Hence nitric acid acts in a powerfully transforming manner on a number of crganic substances. It leaves a yellow stain on the skin, and in 
As nitric acid is very easily decomposed with the separation of oxygen, it was for a long time supposed that it was not capable of forming the corresponding nitric anhydride, $\mathrm{N}_{2} \mathrm{O}_{5}$; but first Deville, and then Weber and others, discovered the methods of its formation. Deville obtained nitric anhydride by decomposing silver nitrate by chlorine under the influence of a moderate heat. Chlorine acts on the above salt at a temperature of $95^{\circ}\left(2 \mathrm{AgNO} \mathrm{N}_{3}+\mathrm{Cl}_{2}=2 \mathrm{AgCl}+\mathrm{N}_{2} \mathrm{O}_{5}+\mathrm{O}\right)$, and when once the reaction is started it continues by itself without further heating. Brown fumes are given off, which are condensed in a tube surrounded by a freezing-mixture. A portion condenses in this tube and a portion remains in a gaseous state. The latter contains free oxygen. A crystalline mass and a liquid substance are obtained in the tube ; the liquid is poured off, and a current of dry carbonic acid gas is passed through the apparatus in order to remove all traces of volatile substances (liquid oxides of nitrogen) adhering to the crystals of nitric anhydride. These form a voluminous mass of rhombic crystals (density 1.64), which sometimes are of rather large size; they melt at about $30^{\circ}$ and distil at about $47^{\circ}$. In distilling, a portion of the substance is decomposed. With water these crystals give nitric acid. Nitric anhydride is also obtained by the action of phosphoric anhydride, $\mathrm{P}_{2} \mathrm{O}_{5}$, on cold pure nitric acid (below $0^{\circ}$ ). During the very careful distillation of equal parts by weight of these two substances a portion of the acid decomposes, giving a liquid compound, $\mathrm{H}_{2} \mathrm{O}, 2 \mathrm{~N}_{2} \mathrm{O}_{5}$ $=\mathrm{N}_{2} \mathrm{O}_{5}, 2 \mathrm{HNO}_{3}$, whilst the greater part of the nitric acid gives the anhydride according to the equation $2 \mathrm{NHO}_{3}+\mathrm{P}_{2} \mathrm{O}_{5}=2 \mathrm{PHO}_{3}+\mathrm{N}_{2} \mathrm{O}_{5}$. On heating, and sometimes even spontaneously with explosion, nitric anhydride decomposes into nitric peroxide and oxygen, $\mathrm{N}_{2} \mathrm{O}_{5}$ $=\mathrm{N}_{2} \mathrm{O}_{4}+\mathrm{O}$.

Nitrogen peroxide, $\mathrm{N}_{2} \mathrm{O}_{4}$, and nitrogen dioxide, $\mathrm{NO}_{2}$, express one and the same composition, but they should be distinguished like ordinary oxygen and ozone, although in this case their mutual conversion is more easily accomplished, even by vaporisation; also, $\mathrm{O}_{3}$ loses heat in passing into $\mathrm{O}_{2}$, whilst $\mathrm{N}_{2} \mathrm{O}_{4}$ absorbs heat in forming $\mathrm{NO}_{2}$.

Nitric acid in acting on tin and on many organic substances (for example, starch) gives brown vapours, consisting of a mixture of $\mathrm{N}_{2} \mathrm{O}_{3}$ and $\mathrm{NO}_{2}$. A purer product is obtained by the decomposition of lead nitrate by heat, $\mathrm{Pb}\left(\mathrm{NO}_{3}\right)_{2}=2 \mathrm{NO}_{2}+\mathrm{O}+\mathrm{PbO}$, when non-volatile lead

a large quantity causes a wound and entirely eats away the membranes of the body. The membranes of plants are eaten into with the greatest ease by strong nitric acid in just the same manner. One of the most durable blue vegetable dyes which is employed in dyeing tissues is indigo, yet it is easily converted into a yellow substance by the action of nitric acid, and small traces of free nitric acid may be recognised by this means. 
oxide, oxygen gas, and nitrogen peroxide are formed. The latter, in a strongly cooled vessel, condenses into a brown liquid, which boils at about $22^{\circ}$. The purest peroxide of nitrogen, solidifying at $-9^{\circ}$, is obtained when dry oxygen is mixed in a freezing-mixture with twice its volume of dry nitric oxide, NO, when transparent prisms of nitrogen peroxide are formed in the receiver; they melt into a colourless liquid at about $-10^{\circ}$. When the temperature of the receiver is above $-9^{\circ}$, the crystals melt, ${ }^{44}$ and at $0^{\circ}$ give a reddish-yellow liquid, like that obtained in the decomposition of lead nitrate. The vapours of nitrogen peroxide have a characteristic odour, and at the ordinary temperature are of a dark-brown colour, but at lower temperatures the colour of the vapour is much fainter. When heated, especially above $50^{\circ}$, the colour becomes a very dark brown, so that the vapours almost lose their transparency.

The causes of these peculiarities of nitrogen peroxide were not clearly understood until Deville and Trooste determined the density and dissociation of the vapour of this substance at different temperatures, and showed that the density varies. If the density be referred to that of hydrogen at the same temperature and pressure, then it is found to vary from 38 at the boiling point, or about $27^{\circ}$, to 23 at $135^{\circ}$, after which the density remains constant up to those high temperatures at which the oxides of nitrogen are decomposed. As, on the basis of the laws enunciated in the following chapter, the density 23 corresponds with the compound $\mathrm{NO}_{2}$ (because the weight corresponding with this molecular formula $=46$, and the density referred to hydrogen as unity is equal to half the molecular weight), therefore at temperatures above $135^{\circ}$ the existence of nitrogen dioxide only must be recognised. It is this gas which is of a brown colour. At a lower temperature it forms nitrogen peroxide, $\mathrm{N}_{2} \mathrm{O}_{4}$, whose molecular weight, and therefore density, is twice that of the dioxide. This substance, which is isomeric with nitrogen dioxide, as ozone is isomeric with oxygen, and has twice as great a vapour density (46 referred to hydrogen), is formed in greater quantity the lower the temperature, and crystallises at $-10^{\circ}$. The reasons both of the variation of the colour of the gas $\left(\mathrm{N}_{2} \mathrm{O}_{4}\right.$ gives colourless and transparent vapours, whilst those of $\mathrm{NO}_{2}$ are brown and opaque) and the variation of the vapour density with the variation of

44 According to certain investigations, if a brown liquid is formed from the melted crystals by heating above $-9^{\circ}$, then they no longer solidify at $-10^{\circ}$, probably because a certain amount of $\mathrm{N}_{2} \mathrm{O}_{3}$ (and oxygen) is formed, and this substance remains liquid at $-30^{\circ}$, or it may be that the passage from $2 \mathrm{NO}_{2}$ into $\mathrm{N}_{2} \mathrm{O}_{4}$ is not so easily accomplished as the passage from $\mathrm{N}_{2} \mathrm{O}_{4}$ into $2 \mathrm{NO}_{2}$.

Liquid nitrogen peroxide (that is, a mixture of $\mathrm{NO}_{2}$ and $\mathrm{N}_{2} \mathrm{O}_{4}$ ) is employed in admixture with hydrocarbons as an explosive. 
temperature are thus made quite clear, and as at the boiling point a density 38 was obtained, therefore at that temperature the vapours consist of a mixture of 79 parts by weight of $\mathrm{N}_{2} \mathrm{O}_{4}$ with 21 parts by weight of $\mathrm{NO}_{2} \cdot{ }^{45} \quad$ It is evident that a decomposition here takes place whose peculiarity consists in the fact that the product of decomposition, $\mathrm{NO}_{2}$, is polymerised (i.e. becomes denser, combines with itself) at a lower temperature ; that is, the reaction,

$$
\mathrm{N}_{2} \mathrm{O}_{4}=\mathrm{NO}_{2}+\mathrm{NO}_{2}
$$

is a reversible reaction, and consequently the whole phenomenon represents a dissociation in a homogeneous gaseous medium, where the original substance, $\mathrm{N}_{2} \mathrm{O}_{4}$, and the resultant, $\mathrm{NO}_{2}$, are both gases. The measure of dissociation will be expressed if we find the proportion of the quantity of the substance decomposed to the whole amount of the substance. At the boiling point, therefore, the measure of the decomposition of nitrogen peroxide will be $21 /(79+21)=0 \cdot 21$, or 21 p.c. ; at $135^{\circ}$ it $=1$, and at $10^{\circ}$ it $=0$-that is, the $\mathrm{N}_{2} \mathrm{O}_{4}$ is not then decomposable. Consequently here the limits of dissociation are $-10^{\circ}$ and $135^{\circ}$ at the atmospheric pressure. ${ }^{46}$ Within the limits of these temperatures the vapours of nitrogen peroxide have not a constant density, and above and below these limits definite substances exist. Thus above $135^{\circ} \mathrm{N}_{2} \mathrm{O}_{4}$ has ceased to exist and $\mathrm{NO}_{2}$ alone remains. It is

45 Because if $x$ equal the amount by weight of $\mathrm{N}_{2} \mathrm{O}_{4}$, its volume will $=x / 46$, and the amount of $\mathrm{NO}_{2}$ will $=100-x$, and consequently its volume will $=(100-x) / 23$. But the nixture, having a density 38 , will weigh 100 , consequently its volume will $=100 / 38$. Hence $x / 46+(100-x) / 23=100 / 36$, or $x=79^{\circ} 0$.

46 The phenomena and laws of dissociation, considered by us in only separate and particular instances, are discussed in detail in works on theoretical chemistry. Besides, certain points in the doctrine of chemical equilibria are still subject to some doubt owing to the recent date at which the exact study of this subject commenced. Nevertheless, in respect to nitrogen peroxide, as an historically important example of dissociation in a homogeneous gaseous medium, we will cite the results of the careful investigations (1885-1886) of E. and L. Natanson, who determined the densities under variations of temperature and pressure. The measure of dissociation, expressed as above (it may also be expressed otherwise-for example, by the ratio of the substance decomposed to that unaltered), proves to increase at all temperatures as the pressure diminishes, which would be expected for a homogeneous gaseous medium, as a decreasing pressure aids the formation of the lightest product of dissociation (that having the least density or largest volume). Thus, in Natansons' experiments the measure of dissociation at $0^{\circ}$ increases from 10 p.c. to 30 p.c., with a decrease of pressure of from 251 to $38 \mathrm{~mm}$. ; at $49 \cdot 7^{\circ}$ it increases from 49 p.c. to 93 p.c., with a fall of pressure of from 498 to $27 \mathrm{~mm}$., and at $100^{\circ}$ it increases from $89 \cdot 2$ p.c. to $99 \cdot 7$ p.c., with a fall of pressure of from 732.5 to $11 \cdot 7 \mathrm{~mm}$. At $130^{\circ}$ and $150^{\circ}$ the decomposition is complete-that is, $\mathrm{NO}_{2}$ only remains at the low pressures (less than the atmospheric) at which the Natansons made their determinations; but it is probable that at considerable pressure (of several atmospheres) molecules of $\mathrm{N}_{2} \mathrm{O}_{4}$ would still be formed, and it would be exceedingly interesting to trace the phenomena under the conditions of both very considerable pressures and of relatively large volumes. 
evident that at the ordinary temperature there is a partially dissociated system or mixture of nitrogen peroxide, $\mathrm{N}_{2} \mathrm{O}_{4}$, and nitrogen dioxide, $\mathrm{NO}_{2}$. In the brown liquid boiling at $22^{\circ}$ probably a portion of the $\mathrm{N}_{2} \mathrm{O}$, has already passed into $\mathrm{NO}_{2}$, and it is only the colourless liquid and crystalline substance at $-10^{\circ}$ that can be considered as pure nitrogen peroxide. ${ }^{47}$

The above explains the action of nitrogen peroxide on water at low temperatures. $\mathrm{N}_{2} \mathrm{O}_{4}$ then acts on water like a mixture of the anhydrides of nitrous and nitric acids. The first, $\mathrm{N}_{2} \mathrm{O}_{3}$, may be looked on as water in which the two atoms of hydrogen are replaced by the radicle $\mathrm{NO}$, while in the second the hydrogen is replaced by the radicle $\mathrm{NO}_{2}$, proper to nitric acid; and in nitrogen peroxide one atom of the hydrogen of water is replaced by $\mathrm{NO}$ and the other by $\mathrm{NO}_{2}$, as is seen from the formula-

or

$$
\begin{aligned}
& \left.\left.\left.\left.\begin{array}{l}
\mathrm{H} \\
\mathrm{H}
\end{array}\right\} \mathrm{O} ; \begin{array}{l}
\mathrm{NO} \\
\mathrm{NO}
\end{array}\right\} \mathrm{O} ; \begin{array}{l}
\mathrm{NO}_{2} \\
\mathrm{NO}_{2}
\end{array}\right\} \mathrm{O} ; \begin{array}{l}
\mathrm{NO} \\
\mathrm{NO}_{2}
\end{array}\right\} \mathrm{O} \text {; } \\
& \mathrm{H}_{2} \mathrm{O} ; \quad \mathrm{N}_{2} \mathrm{O}_{3} ; \quad \mathrm{N}_{2} \mathrm{O}_{5} ; \quad \mathrm{N}_{2} \mathrm{O}_{4} \text {. }
\end{aligned}
$$

In fact, nitrogen peroxide at low temperatures gives with water (ice) both nitric, $\mathrm{HNO}_{3}$, and nitrous, $\mathrm{HNO}_{2}$, acids. The latter, as we shall afterwards see, splits up into water and the anhydride, $\mathrm{N}_{2} \mathrm{O}_{3}$. If, however, warm water act on nitrogen peroxide, only nitric acid and oxide of nitrogen are formed: $3 \mathrm{NO}_{2}+\mathrm{H}_{2} \mathrm{O}=\mathrm{NO}+2 \mathrm{NHO}_{3}$.

Although $\mathrm{NO}_{2}$ is not decomposed into $\mathrm{N}$ and $\mathrm{O}$ even at $500^{\circ}$, still in many cases it acts as an oxidising agent. Thus, for instance, it oxidises mercury, converting it into mercurous nitrate, $2 \mathrm{NO}_{2}+$ $\mathrm{Hg}=\mathrm{HgNO}_{3}+\mathrm{NO}$, it being itself deoxidised into nitric oxide, into

47 The fact that the presence of a portion of dioxide, $\mathrm{NO}_{2}$, must be acknowledged in liquid nitrogen peroxide, $\mathrm{N}_{2} \mathrm{O}_{4}$, at temperatures of from $0^{\circ}$ to $22^{\circ}$, is not only of great significance for the theory which regards solutions as liquid systems of equilibrium, consisting of combined and decomposed substances, but it also shows the nature of solutions of gaseous substances, because the $\mathrm{NO}_{2}$ must be regarded as a gas dissolved in the volatile liquid $\mathrm{N}_{2} \mathrm{O}_{4}$.

Liquid nitrogen peroxide is said by Geuther to boil at $22^{\circ}-26^{\circ}$, and to have a sp. gr. at $0^{\circ}=1.494$ and at $15^{\circ}=1.474$. It is evident that, in the liquid as in the gaseous state, the variation of density with the temperature depends not only on physical, but also on chemical changes, as the amount of $\mathrm{N}_{2} \mathrm{O}_{4}$ decreases and the amount of $\mathrm{NO}_{2}$ increases with the temperature, and they (as polymeric substances) should have different densities, as we find, for instance, in the hydrocarbons $\mathrm{C}_{5} \mathrm{H}_{10}$ and $\mathrm{C}_{10} \mathrm{H}_{20}$.

It may not be superfluous to here mention that the measurement of the specific heat of a mixture of the vapours of $\mathrm{N}_{2} \mathrm{O}_{4}$ and $\mathrm{NO}_{2}$ enabled Berthelot to determine that the transformation of $2 \mathrm{NO}_{2}$ into $\mathrm{N}_{2} \mathrm{O}_{4}$ is accompanied by the evolution of about 13000 units of heat, and as the reaction proceeds with equal facility in either direction, it will be exothermal in the one direction and endothermal in the other; and this clearly demonstrates the possibility of reactions of both aspects proceeding in either direction, although, as a rule, reactions evolving heat proceed with greater ease. 
which nitrogen dioxide in many other instances passes, and from which it is easily formed. ${ }^{48}$

Nitrous anhydride, $\mathrm{N}_{2} \mathrm{O}_{3}$, corresponds ${ }^{49}$ with nitrous acid, $\mathrm{NHO}_{2}$, and with the latter corresponds a series of salts, the nitrites-for example, the sodium salt $\mathrm{NaNO}_{2}$, the potassium salt $\mathrm{KNO}_{2}$, the ammonium salt $\left(\mathrm{NH}_{4}\right) \mathrm{NO}_{2},{ }^{50}$ the silver salt $\mathrm{AgNO}_{2},{ }^{51}$ \&c. Neither the anhydride nor the hydrate of the acid is known in a perfectly pure state. The anhydride has only been obtained as a very unstable substance, and has not yet been investigated with proper fulness; and when efforts are made to obtain the acid $\mathrm{NHO}_{2}$ from its salts, it always gives water and the anhydride, whilst the latter, as an intermediate oxide, easily splits up into $\mathrm{NO}+\mathrm{NO}_{2}$. But the salts of nitrous acid are distinguished for their great stability. Potassium nitrate, $\mathrm{KNO}_{3}$, may be converted into potassium nitrite by depriving it of a portion of its oxygen; for instance, by fusing it (at a not too great heat) with metals, such as lead, $\mathrm{KNO}_{3}+\mathrm{Pb}=\mathrm{KNO}_{2}+\mathrm{PbO}$. The resultant salt is soluble in water, whilst the oxide of lead is insoluble. With sulphuric and other acids the solution of potassium nitrite ${ }^{52}$ immediately evolves a brown gas, nitrous anhydride : $2 \mathrm{KNO}_{2}+\mathrm{H}_{2} \mathrm{SO}_{4}=\mathrm{K}_{2} \mathrm{SO}_{4}+\mathrm{N}_{2} \mathrm{O}_{3}+\mathrm{H}_{2} \mathrm{O}$. The same gas $\left(\mathrm{N}_{2} \mathrm{O}_{3}\right)$ is obtained by passing nitric oxide at $0^{\circ}$ through liquid peroxide of nitrogen, ${ }^{53}$ or by heating starch with nitric acid of sp.

48 Nitric acid of sp. gr. 1.51 in dissolving nitrogen peroxide becomes brown, whilst nitric acid of sp.gr. 1.32 is coloured greenish blue, and acid of sp. gr. below $1 \cdot 15$ remains colourless on absorbing nitrogen peroxide.

49 Nitrogen peroxide as a mixed substance has no corresponding independent salts.

50 Ammonium nitrite may be easily obtained in solution by a similar method of double decomposition (for instance, of the barium salt with ammonium sulphate) to the other salts of nitrous acid, but it decomposes with great ease when evaporated, with the evolution of gaseous nitrogen, as has been already mentioned (Chap. V.). If the solution, however, be evaporated at the ordinary temperature under the receiver of an air-pump, a solid saline mass is obtained, which is easily decomposed when heated. The dry salt even decomposes with an explosion when struck, or when heated to about $70^{\circ}-\mathrm{NH}_{4} \mathrm{NO}_{2}=$ $2 \mathrm{H}_{2} \mathrm{O}+\mathrm{N}_{2}$. It is also formed by the action of aqueous ammonia on a mixture of nitric oxide and oxygen, or by the action of ozone on ammonia, and in many other instances.

51 Silver nitrite, $\mathrm{AgNO}_{2}$, is obtained as a very slightly soluble substance, as a precipitate, on mixing solutions of silver nitrate, $\mathrm{AgNO}_{3}$, and potassium nitrite, $\mathrm{KNO}_{2}$. It is soluble in a large volume of water, and this is taken advantage of to free it from silver oxide, which is also present in the precipitate, owing to the fact that potassium nitrite always contains a certain amount of oxide, which with water gives the hydroxide, forming oxide of silver with silver nitrate. The solution of silver nitrite gives, by double decomposition with metallic chlorides (for instance, barium chloride), insoluble silver chloride and the nitrite of the metal taken (for instance, barium nitrite, $\left.\mathrm{Ba}\left(\mathrm{NO}_{2}\right)_{2}\right)$.

52 Probably potassium nitrite, $\mathrm{KNO}_{2}$, when strongly heated, especially with metallic oxides, evolves $\mathrm{N}$ and $\mathrm{O}$, and gives potassium oxide, $\mathrm{K}_{2} \mathrm{O}$, because nitre is liable to such a decomposition, but it has, as yet, been but little investigated.

$33 \mathrm{It}$ is evident that the reaction $\mathrm{N}_{2} \mathrm{O}_{3}=\mathrm{NO}_{2}+\mathrm{NO}$ is reversible, and that it resembles the conversion of $\mathrm{N}_{2} \mathrm{O}_{4}$ into $\mathrm{NO}_{2}$, but as yet this reaction has not been thoroughly 
gr. 1·3. At a very low temperature it condenses into a blue liquid boiling below $0^{\circ},{ }^{54}$ but then partially decomposing into $\mathrm{NO}+\mathrm{NO}_{2}$. Nitrous anhyclride evinces a remarkable capacity for oxidising. Ignited bodies burn in it, nitric acid absorbs it, and then acquires the property of acting on silver and other metals, even when diluted. Potassium iodide is oxidised by this gas just as it is by ozone (and by peroxide of hydrogen, chromic and other acids, but not by dilute nitric acid nor by sulphuric acid), with the separation of iodine. This iodine may be recognised (see Ozone, Chap. IV.) by its turning starch blue. The smallest traces of nitrites may be easily discovered by this method. If, for example, starch and potassium iodide are added to a solution of potassium nitrite (there will be no change, there being no free nitrous acid), and then sulphuric acid be added, then the nitrous acid (or its anhydride) immediately set free evolves iodine, which communicates a blue colour to the starch. Nitric acid does not act in this manner, but in the presence of zinc the coloration takes place, which proves the formation of nitrous acid in the deoxidation of nitric acid. ${ }^{55}$ Nitrous acid (or even a mixture of $\mathrm{HNO}_{2}+\mathrm{NO}$ ) acts directly on ammonia, forming nitrogen and water, $\mathrm{HNO}_{2}+\mathrm{NH}_{3}=\mathrm{N}_{2}+2 \mathrm{H}_{2} \mathrm{O} .56$

As nitrous anhydride easily splits up into $\mathrm{NO}_{2}+\mathrm{NO}$, so with warm water it, like $\mathrm{NO}_{2}$, gives nitric acid and nitric oxide, according to the equation $3 \mathrm{~N}_{2} \mathrm{O}_{3}+\mathrm{H}_{2} \mathrm{O}=4 \mathrm{NO}+2 \mathrm{NHO}_{3}$.

Being in a lower degree of oxidation than nitric acid, nitrous acid

studied. The brown colour of the rapours of nitrous anhydride probably depends on the presence of $\mathrm{NO}_{2}$.

If nitrogen peroxide be cooled to $-20^{\circ}$, and half its weight of water be added to it drop by drop, then the peroxide is decomposed, as we have already said, into nitrous and nitric acids; the former does not then remain as a hydrate, but straightway passes into the anhydride, and, therefore, if the resultant liquid be slightly warmed vapours of nitrous anhydride, $\mathrm{N}_{2} \mathrm{O}_{5}$, are evolved, and condense into a blue liquid, as Fritzsche showed. This method of preparing nitrous anlydride evidently gives the purest product.

54 According to Thorpe, $\mathrm{N}_{2} \mathrm{O}_{3}$, boils at $+18^{\circ}$. According to Geuther, at $+3 \cdot 5^{\circ}$, and its sp. gr. at $0^{\circ}=1 \cdot 449$.

${ }^{5} \mathrm{In}$ its oxidising action nitrous anhydride gives nitric oxide, $\mathrm{N}_{2} \mathrm{O}_{5}=2 \mathrm{NO}+\mathrm{O}$. Thus its analogy to ozone becomes still closer, because in ozone it is only one-third of the oxygen that acts in oxidising; from $\mathrm{O}_{3}$, there is obtained $\mathrm{O}$, which acts as an oxidiser, and common oxygen $\mathrm{O}_{22}$. In a physical aspect the affinity between $\mathrm{N}_{2} \mathrm{O}_{5}$ and $\mathrm{O}_{3}$ is expressed by both substances being of a blue colour when in the liquid state.

56 This reaction is taken advantage of for converting the amides, $\mathrm{NH}_{2} \mathrm{R}$ (where $\mathrm{R}$ is an element or a complex group) into hydroxides, $\mathrm{RHO}$. In this case $\mathrm{NH}_{2} \mathrm{R}+\mathrm{NHO}_{2}$ forms $2 \mathrm{~N}+\mathrm{H}_{2} \mathrm{O}+\mathrm{RHO} ; \mathrm{NH}_{2}$ is replaced by $\mathrm{HO}$, the radicle of ammonia by the radicle of water. This reaction is employed for transforming many nitrogenous organic substances having the properties of amides into their corresponding hydroxides. Thus aniline, $\mathrm{C}_{6} \mathrm{H}_{5} \cdot \mathrm{NH}_{2}$, which is ol,tained from nitrobenzene, $\mathrm{C}_{6} \mathrm{H}_{5} \cdot \mathrm{NO}$, (Note 37), is converted by nitrous anhydride into phenol, $\mathrm{C}_{6} \mathrm{H}_{5} \cdot \mathrm{OH}$, which occurs in the creosote extracted from coal tar. Thus the $\mathrm{H}$ of the benzene is successively replaced by $\mathrm{NO}_{3}, \mathrm{NH}_{2}$, and $\mathrm{HO}-\mathrm{a}$ method which is suitable for other cases also. 
and its anhydride are oxidised in solutions by many oxidising substances - for example, by potassium permanganate - into nitric acid..$^{57}$

Nitric oxide, NO.-This permanent gas ${ }^{58}$ (that is, unliquefiable by pressure without the aid of cold) may be obtained from all the abovedescribed compounds of nitrogen with oxygen. The deoxidation of nitric acid by metals is the usual method employed for its preparation. Dilute nitric acid (sp. gr. $1 \cdot 18$, but not stronger, as then $\mathrm{N}_{2} \mathrm{O}_{3}$ and $\mathrm{NO}_{2}$ are produced) is poured into a flask containing metallic copper. ${ }^{59}$ The reaction commences at the ordinary temperature. Mercury and silver also give nitric oxide with nitric acid. In these reactions with metals one portion of the nitric acid is employed in the oxidation of the metal, whilst the other, and by far the greater, portion combines with the metallic oxide so obtained, with formation of the nitrate corresponding with the metal taken. The first action of the copper on the nitric acid is thus expressed by the equation

$$
2 \mathrm{NHO}_{3}+3 \mathrm{Cu}=\mathrm{H}_{2} \mathrm{O}+3 \mathrm{Cu} \mathrm{O}+2 \mathrm{NO} .
$$

The second reaction consists in the formation of copper nitrate-

$$
6 \mathrm{NHO}_{3}+3 \mathrm{CuO}=3 \mathrm{H}_{2} \mathrm{O}+3 \mathrm{Cu}\left(\mathrm{NO}_{3}\right)_{2} \text {. }
$$

Nitric oxide is a colourless gas which is only slightly soluble in water ( $\frac{1}{20}$ of a volume at the ordinary temperature). Reactions of double decomposition in which nitric oxide readily takes part are not known - that is to say, it is an indifferent, not a saline, oxide. Like the other oxides of nitrogen, it is decomposed into its elements at a red heat. The most characteristic property of nitric oxide consists in its capacity for directly and easily combining with oxygen (owing to the evolution of heat in the combination). With oxygen it forms nitrous anhydride

37 The action of a solution of potassium permanganate, $\mathrm{KMnO}_{4}$, on nitrous acid in the presence of sulphuric acid is determined by the fact that the higher oxide of manganese, $\mathrm{Mn}_{2} \mathrm{O}_{7}$, contained in the permanganate is converted into the lower oxide $\mathrm{MnO}$, which as a base forms manganese sulphate, $\mathrm{MnSO}_{4}$, and the oxygen serves for the oxidation of the $\mathrm{N}_{2} \mathrm{O}_{3}$ into $\mathrm{N}_{2} \mathrm{O}_{5}$, or its hydrate. As the solution of the permanganate is of a red colour, whilst that of manganese sulphate is almost colourless, this reaction is clearly seen, and may be employed for the recognition and determination of nitrous acid and its salts.

58 The absolute boiling point $=93^{\circ}$ (see Chap. II. Note 29).

59 Kammerer proposed preparing nitric oxide, NO, by pouring a solution of sodium nitrate over copper shavings, and adding sulphuric acid drop by drop. The oxidation of ferrous salts by nitric acid also gives NO. One part of strong hydrochloric acid is taken and iron is dissolved in it $\left(\mathrm{FeCl}_{2}\right)$, and then an equal quantity of liydrochloric acid and nitre is added to the solution. On heating, nitric oxide is erolved. When nitric oxide is prepared by either of the above methods, the apparatus first becomes full of brown fumes of nitrogen peroxide, formed by the oxygen of the air and the nitric oxide, and therefore the pure gas can only be collected after it has displaced all the air in the apparatus, and when the latter becomes full of colourless gas. 
and nitrogen peroxide, $2 \mathrm{NO}+\mathrm{O}=\mathrm{N}_{2} \mathrm{O}_{3}, 2 \mathrm{NO}+\mathrm{O}_{2}=2 \mathrm{NO}_{2}$. If nitric oxide is mixed with oxygen and immediately shaken up with caustic potash, it is almost entirely converted into potassium nitrite, whilst after a certain time, when the formation of nitric peroxide has already commenced, a mixture of potassium nitrite and nitrate is obtained. If oxygen is passed into a bell jar filled with nitric oxide, then brown fumes of nitrous anhydride and nitric peroxide are formed, which in the presence of water give, as we already know, nitric acid and nitric oxide, so that in the presence of an excess of water and oxygen the whole of the nitric oxide is easily and directly converted into nitric acid. This reaction of the re-formation of nitric acid from nitric oxide, air, and water, $2 \mathrm{NO}+\mathrm{H}_{2} \mathrm{O}+\mathrm{O}_{3}=2 \mathrm{HNO}_{3}$, is frequently made use of in practice. The experiment showing the conversion of nitric oxide into nitric acid is very striking and instructive. As the intermixture of the oxygen with the oxide of nitrogen proceeds, the nitric acid formed dissolves in water, and if an excess of oxygen has not been added the whole of the gas (nitric oxide), being converted into $\mathrm{HNO}_{3}$, is absorbed, and the water entirely fills the bell jar previously containing the gas. ${ }^{60}$ It is evident that nitric oxide ${ }^{61}$ in combining with oxygen

50 This transformation of the permanent gases nitric oxide and oxygen into liquid nitric acid in the presence of water, and with the evolution of heat, presents a most striking instance of liquefaction produced by the action of chemical forces. They perform with ease the work which physical (cooling) and mechanical (pressure) forces do with difficulty. In this the motion, which is so clearly the property of the gaseous molecules, is extinguished. In other cases of chemical action its appearance arises from latent energy - that is, in all probability, from the movement of the atoms in the molecules.

61 Nitric oxide is capable of entering into many characteristic combinations; it is absorbed by the solutions of many acids (for instance, tartaric, acetic, phosphoric, sulphuric), and also by the solutions of many salts, especially those formed by suboxide of iron (for instance, ferrous sulphate). In this case a brown compound is formed which is exceedingly unstable, like all the analogous compounds of nitric oxide. The amount of nitric oxide combined in this manner is in atomic proportion with the amount of the substance taken; thus ferrous sulphate, $\mathrm{FeSO}_{4}$, absorbs it in the proportion of $\mathrm{NO}$ to $2 \mathrm{FeSO}_{4}$. Anmonia is obtained by the action of a caustic alkali on the resultant compound, because the oxygen of the nitric oxide and water are transferred to the ferrous oxide, forming ferric oxide, whilst the nitrogen combines with the hydrogen of the water. According to the investigations of Gay (188.5), the compound is formed with the evolution of a large quantity of heat, and is easily dissociated, like a solution of ammonia in water. This subject must be regarded as not sufficiently studied. On passing nitric oxide through nitric acid, nitrogen peroxide and nitrous anhydricle are formed, whose solutions in the nitric acid are, as we have already mentioned, of various colours. It is evident that oxidising substances (for example, potassium permangunate, $\mathrm{KMnO}_{4}$, Note 57) are able to convert it into nitric acid. If the presence of a radicle $\mathrm{NO}_{2,2}$, composed like nitrogen peroxide, must be recognised in the compounds of nitric acid, then a radicle NO, having the composition of nitric oxide, may be admitted in the compounds of nitrous acid. The compounds in which the radicle $\mathrm{NO}$ is recognised are culled nitroso-compounds. The compounds are described in Prof. Bunge's work (Kief, 1868). 
has a strong tendency to give only the higher types of nitrogen compounds, which we see in nitric acid, $\mathrm{HNO}_{3}$ or $\mathrm{NO}_{2}(\mathrm{OH})$, in nitric anhydride, $\mathrm{N}_{2} \mathrm{O}_{5}$ or $\left(\mathrm{NO}_{2}\right)_{2} \mathrm{O}$, and in ammonium chloride, $\mathrm{NH}_{4} \mathrm{Cl}$. If $\mathrm{X}$ stand for an atom of hydrogen, or its equivalents, chlorine, hydroxyl, \&c., and if $\mathrm{O}$, which is, according to the law of substitution, equivalent to $\mathrm{H}_{2}$, be indicated by $\mathrm{X}_{2}$, then the three above-named compounds of nitrogen should be considered as compounds of the type or form $\mathbf{N X}_{5}$. For example, in nitric acid $\mathrm{X}_{5}=\mathrm{O}_{2}+(\mathrm{OH})$, where $\mathrm{O}_{2}=\mathrm{X}_{4}$, and $\mathrm{OH}=\mathrm{X}$; whilst nitric oxide is a compound of the form $\mathrm{NX}_{2}$. Hence this lower form, as is true of lower forms in general, strives by combination to attain to the higher forms proper to the compounds of a given element. $\mathrm{NX}_{2}$ passes consecutively into $\mathrm{NX}_{3}$-namely, into $\mathrm{N}_{2} \mathrm{O}_{3}$ and $\mathrm{NHO}_{2}, \mathrm{NX}_{4}$ (for instance $\mathrm{NO}_{2}$ ) and $\mathrm{NX}_{5}$.

As the decomposition of nitric oxide begins at temperatures above $600^{\circ}$, many substances burn in it ; for instance, ignited phosphorus continues to burn in nitric oxide, but sulphur and charcoal are extinguished in it. This is due to the fact that the heat evolved in the combustion of these two substances is insufficient for the entire decomposition of the nitric oxide, whilst the heat developed by burning phosphorus suffices to produce this decomposition. That this is the true explanation of the behaviour of nitric oxide in these cases is proved by the fact that charcoal when very strongly ignited will burn in the gas. ${ }^{62}$

The compounds of nitrogen with oxygen which we have so far considered may all be prepared from nitric oxide, and may themselves be converted into it. Thus nitric oxide stands in intimate connection with them. ${ }^{63}$ The passage of nitric oxide into the higher degrees of

${ }^{62}$ A mixture of nitric oxide and hydrogen is inflammable. If a mixture of both gases be passed over spongy platinum, the nitrogen and hydrogen even combine, forming ammonia. A mixture of nitric oxide with many combustible vapours and gases is very inflammable. A very characteristic flame is obtained in burning a mixture of nitric oxide and the vapour of the combustible carbon bisulphide, $\mathrm{CS}_{2}$. The latter substance is very volatile, so that it is sufficient to pass the nitric oxide through a layer of the carbon bisulphide (for instance, in a Woulfe's bottle) in order that the gas escaping should contain a considerable amount of the vapours of this substance. This mixture continues to burn when set light to, and the flame emits a large quantity of the so-called ultra-violet rays, which are capable of bringing about chemical combinations and decompositions, and therefore the flame may be employed in photography in the absence of sufficient daylight (magnesium and electric light have the same property). A mixture of nitric oxide with many gases (for instance, ammonia) explodes in a eudiometer.

63 The oxides of nitrogen do not proceed directly from oxygen and nitrogen by contact alone, naturally because their formation is accompanied by the absorption of a large quantity of heat, namely (see Note 29), about 21500 heat units are absorbed when 16 parts of oxygen and 14 parts of nitrogen combine, consequently the decomposition of nitric oxide into oxygen and nitrogen is accompanied by the evolution of this amount of heat; and therefore with nitric oxide, as with all explosive substances and mixtures, the reaction once started is able to proceed by itself. In fact, Berthelot remarked the decomposition of nitric oxide in the explosion of fulminate of mercury. This decomposition does not take 
oxidation and the converse reaction is employed in practice as a means for transferring the oxygen of the air to substances capable of being oxidised. Having nitric oxide, it may easily be converted, with the aid of the oxygen of the atmosphere and water, into nitric acid, nitrous anhydride, and nitric peroxide, and by their means employed to oxidise other substances. In this oxidising action nitric oxide is again formed, and it may again be converted into nitric acid, and so on without end, if only there be oxygen and water. Hence the fact, which at first appears to be a paradox, that by means of a small quantity of nitric oxide in the presence of oxygen and water it is possible to oxidise an indefinitely large quantity of substances which cannot be directly oxidised either by the action of the atmospheric oxygen or by the action of nitric oxide itself. The sulphurous anhydride, $\mathrm{SO}_{2}$, which is obtained in the combustion of sulphur and in roasting many metallic sulphides in the air, is an example of this kind. In practice this gas is obtained by burning sulphur or iron pyrites, the latter being thereby converted into oxide of iron and sulphurous anhydride. In contact with the oxygen of the atmosphere this gas does not pass into the higher degree of oxidation sulphuric anhydride, $\mathrm{SO}_{3}$, and if it does form sulphuric acid with water and the oxygen of the atmosphere, $\mathrm{SO}_{2}+\mathrm{H}_{2} \mathrm{O}+\mathrm{O}=\mathrm{H}_{2} \mathrm{SO}_{4}$, it does so very slowly. With nitric acid (and especially with nitrous acid, but not with nitrogen peroxide) and water, sulphurous anhydride, on the contrary, very easily forms sulphuric acid, and especially so when slightly heated (about $40^{\circ}$ ), the nitric acid (or, better still, nitrous acid) being converted into nitric oxide-

$$
3 \mathrm{SO}_{2}+2 \mathrm{NHO}_{3}+2 \mathrm{H}_{2} \mathrm{O}=2 \mathrm{H}_{2} \mathrm{SO}_{4}+2 \mathrm{NO} \text {. }
$$

The presence of water is absolutely indispensable here, otherwise sulphuric anhydride is formed, which combines with the oxides of nitrogen (nitrous anhydride), forming a crystalline substance containing oxides of nitrogen (chamber crystals, which will be described in the chapter on sulphur). Water destroys this compound, forming sulphuric acid and separating the oxides of nitrogen. The water must be taken in a greater quantity than that required for the formation of the hydrate $\mathrm{H}_{2} \mathrm{SO}_{4}$, because the latter absorbs oxides of nitrogen. With an excess of water, however, solution does not take place. If, in the above reaction, only water, sulphurous anhydride, and nitric or nitrous acid be taken in

place spontaneously ; substances even burn with difficulty in nitric oxide, probably because a certain portion of the nitric oxide in decomposing gives oxygen, which combines with another portion of nitric oxide and forms nitric peroxide, a somewhat more stable compound of nitrogen and oxygen. The further combinations of nitric oxide with oxygen all proceed with the evolution of heat, and take place spontaneously by contact with air alone. From these examples it is seen how the use of thermochemical data is limited by facts. 
a definite quantity, then a definite quantity of sulphuric acid and nitric oxide will be formed, according to the preceding equation; but there the reaction ends, the excess of sulphurous anhydride, if there be any, will remain unchanged. But if we add air and water, then the nitric oxide will unite with the oxygen to form nitrogen peroxide, and the latter with water to form nitric and nitrous acids, which again give sulphuric acid from a fresh quantity of sulphurous anhydride. Nitric oxide is again formed, which is able to start the oxidation afresh if there be sufficient air. Thus it is possible with a definite quantity of nitric oxide to convert an indefinitely large quantity of sulphurous anhydride into sulphuric acid, water and oxygen only being required. ${ }^{64}$ This may be easily demonstrated by an experiment on a small scale, if a certain quantity of nitric oxide be first introduced into a flask, and sulphurous anhydride, steam, and oxygen be then continually passed in. Thus the above-described reaction may be expressed in the following manner :

$$
n \mathrm{SO}_{2}+n \mathrm{O}+(n+m) \mathrm{H}_{2} \mathrm{O}+\mathrm{NO}=n \mathrm{H}_{2} \mathrm{SO}_{4}, m \mathrm{H}_{2} \mathrm{O}+\mathrm{NO},
$$

if we consider only the original substances and those finally formed. Thus a definite quantity of nitric oxide may serve for the conversion of an indefinite quantity of 'sulphurous anhydride, oxygen, and water into sulphuric acid. In reality, however, there is a limit to this, because a portion of the resulting oxides of nitrogen are dissolved by the sulphuric acid, so that in employing even pure oxygen the amount of free (undissolved) or active nitric oxide decreases little by little. If air, and not pure oxygen, be employed for the oxidation, as it is necessary to do in practice, then it is necessary to remove the nitrogen of the air and to introduce a fresh quantity of air. A certain quantity of nitric oxide will pass away with this nitrogen, and will in this way be lost. ${ }^{65}$

64 The instance of the action of a small quantity of $\mathrm{NO}$ in inciting a definite chemical reaction between large masses $\left(\mathrm{SO}_{2}+\mathrm{O}+\mathrm{H}_{2} \mathrm{O}=\mathrm{H}_{2} \mathrm{SO}_{4}\right)$ is very instructive, because the particulars relating to it have been studied, and show that intermediate forms of reaction may be discovered in the so-called contact or catalytic phenomena. The essence of the matter here is that $\mathrm{A}\left(=\mathrm{SO}_{2}\right)$ reacts upon $\mathrm{B}\left(=\mathrm{O}\right.$ and $\left.\mathrm{H}_{2} \mathrm{O}\right)$ in the presence of $\mathrm{C}$, because it gives $\mathrm{BC}$, a substance which forms $\mathrm{AB}$ with $\mathrm{A}$, and again liberates C. Consequently $\mathrm{C}$ is a medium, a transferring substance, without which the matter does not proceed of its own accord. Many similar phenomena may be found in other departments of life. Thus the merchant is an indispensable medium between the producer and the consumer; thus experiment is a medium between the phenomena of nature and the cognisant faculties; thus language, forms, and laws are media which are as necessary for the consolidation of social intercourse as nitric oxide for the relations between sulphurous anhydride and oxygen and water.

${ }^{65}$ If the sulphurous anhydride be prepared by roasting iron pyrites, $\mathrm{FeS}_{2}$, then each equivalent of pyrites (equivalent of iron 56, of sulphur 32, of pyrites 120) requires six equivalents of oxygen (that is 96 parts) for the conversion of its sulphur into sul- 
The preceding series of changes serve as the basis of the manufacture of sulphuric acid or so called chamber acid. This acid is prepared on a very large scale in chemical works because it is the cheapest acid whose action can be applied in a great number of cases. It is thus used in immense quantities.

The process is carried on in a series of chambers (or in one divided by partitions as in fig. 50, which shows the beginning and end of a chamber) constructed of sheet lead. These chambers are placed one

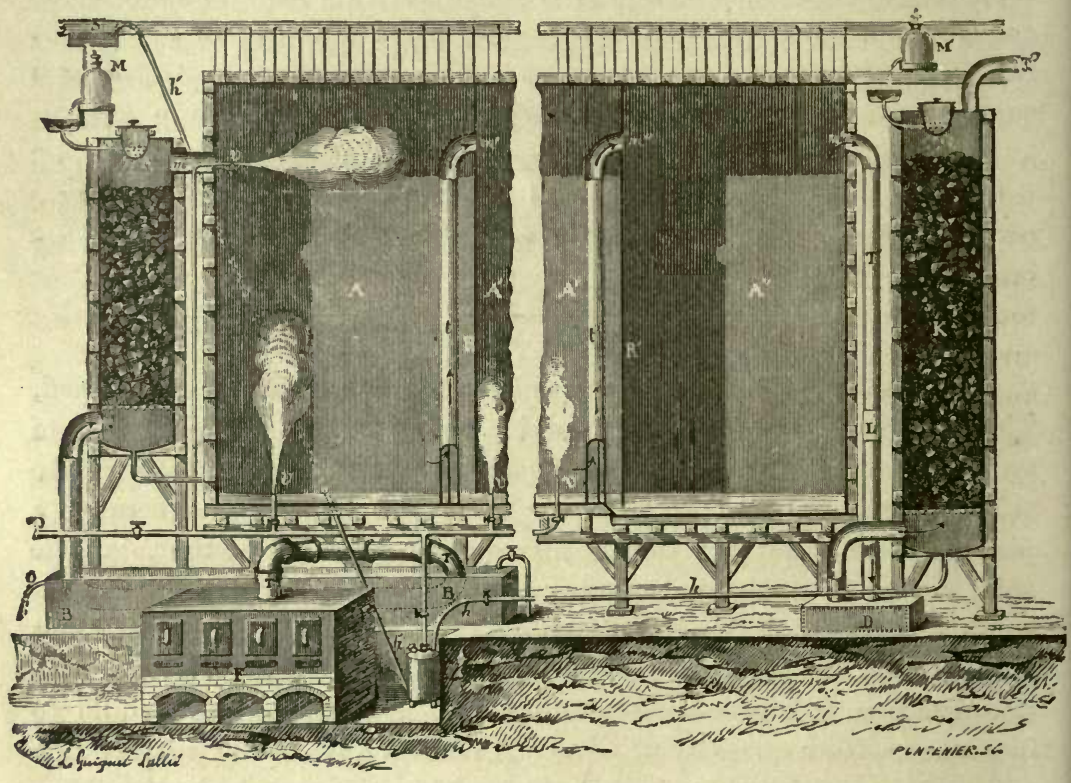

FIG. 50.- Section of sulphuric acid chambers, the first and last chambers only being represented. The tower to the left is called the Glover's tower, and that on the right the Gay-Lussac's tower. Less than $\frac{1}{100}$ of the natural size.

after the other, and communicate with one another by tubes or special orifices so placed that the inlet tubes are in the upper portion of the chamber, and the outlet in the lower and opposite end. The current of

phuric acid (for forming $2 \mathrm{H}_{2} \mathrm{SO}_{4}$ with water), besides $1 \frac{1}{2}$ equivalents (24 parts' for converting the iron into oxide, $\mathrm{Fe}_{2} \mathrm{O}_{3}$; hence the combustion of the pyrites for the formation of sulphuric acid and ferric oxide requires the introduction of an equal weight of oxygen (120 parts of oxygen to 120 parts of pyrites), or five times its weight of air, whilst four parts by weight of nitrogen will remain inactive, and in the removal of the exhausted air will carry off the remaining nitric oxide. If not all, at least a large portion of the nitric oxide may be collected by passing the escaping air, still containing some oxygen, through substances which absorb oxides of nitrogen. Sulphuric acid itself may be employed for this purpose if it be taken as the hydrate $\mathrm{H}_{2} \mathrm{SO}_{4}$, or containing only a small amount of water, because such sulphuric acid dissolves the oxides of nitrogen. 
steam and gases necessary for the preparation of the sulphuric acid passes through these chambers and tubes. The acid as it is formed falls to the bottom of the chambers, or runs down their walls, and flows from one chamber to another (from the last to the first, to permit of which the partitions do not reach to the bottom of the chambers), and therefore the floor and walls of the chambers should be made of a material on which the sulphuric acid will not act. Among the ordinary metals lead is the only one suitable. The other metals, such as iron, zinc, or copper, are corroded by the acid; glass and earthenware are not acted on, but would not withstand the changes of temperature which occur in the chambers, and would be difficult to join closely ; whilst wood and similar materials are destroyed by the acid.

For the formation of the sulphuric acid it is necessary to introduce sulphurous anhydride, steam, air, and nitric acid, or some oxide of nitrogen, into the chambers. The sulphurous anhydride is produced by burning sulphur or iron pyrites. This is carried on in the furnace with four hearths to the left of the drawing. Air is led into the chambers and furnace through orifices in the furnace doors. The current of air and oxygen is regulated by opening or closing these orifices to a greater or less extent. The ingoing draught in the chambers is brought about by the fact that heated gases and vapours pass into the chambers whose temperature is further raised by the reaction itself, and also by the remaining nitrogen being continually withdrawn from the outlet (above the tower $\mathrm{K}$ ) by a tall chimney situated near the chambers. Nitric acid is prepared from a mixture of sulphuric acid and Chili saltpetre, in the same furnaces in which the sulphurous anhydride is evolved (or in special furnaces). Not more than 8 parts of nitre are taken to 100 parts of sulphur burnt. On leaving the furnace the vapours of nitric acid and oxides of nitrogen mixed with air and sulphurous anhydride first pass along the horizontal tubes $\mathrm{T}$ into the receiver $\mathrm{B} \mathrm{B}$, which is partially cooled by water flowing in on the right-hand side and running out on the left by $o$, in order to reduce the temperature of the gases entering the chamber. The gases then pass up a tower filled with coke, and shown to the left of the drawing. In this tower are placed lumps of

They may be easily expelled from this solution by heating or by dilution with water, as they are only slightly soluble in aqueous sulphuric acid. Besides which, sulphurous anhydride acts on such sulphuric acid, being oxidised at the expense of the nitrous anhydride, and forming nitric oxide from it, which again enters into the cycle of action. Therefore the sulphuric acid which has absorbed the oxides of nitrogen escaping from the chambers in the tower $\mathrm{K}$ (see fig. 50 ) is led back into the first chamber, where it comes into contact with sulphurous anhydride, by which means the oxides of nitrogen are reintroduced into the reaction which proceeds in the chambers. This is the use of the towers (Gay-Lussac's and Glover's) which are erected at either end of the chambers. 


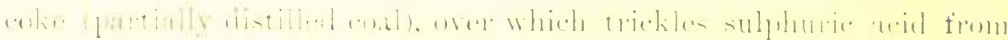

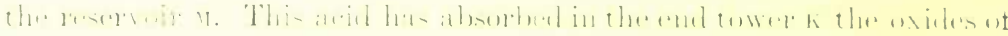

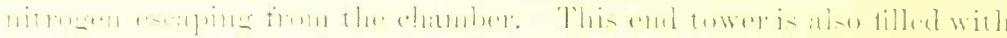

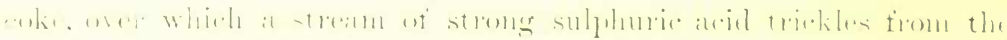

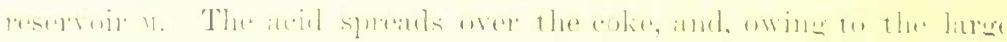

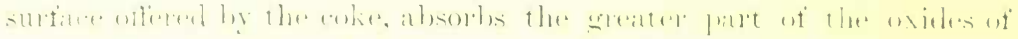

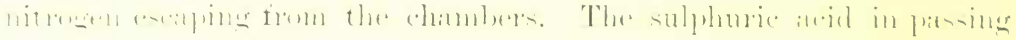

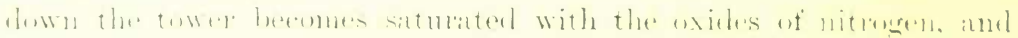

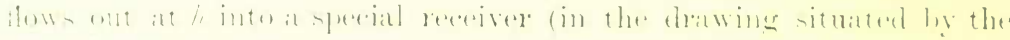

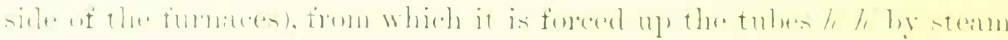

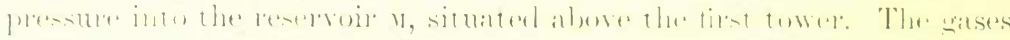

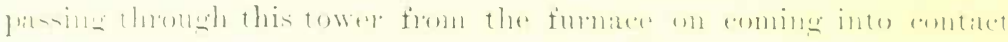

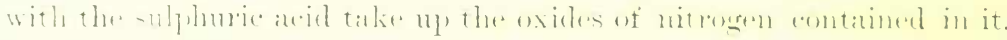

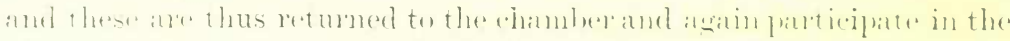

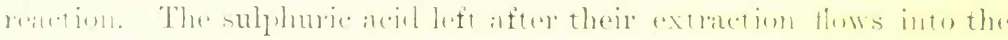

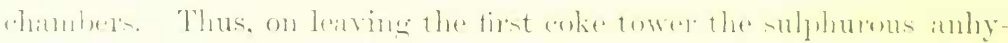

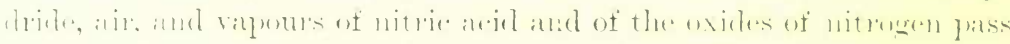

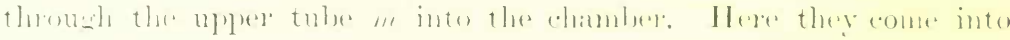

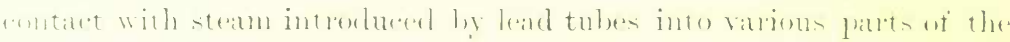

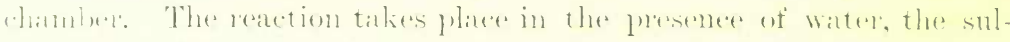

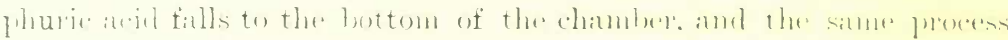

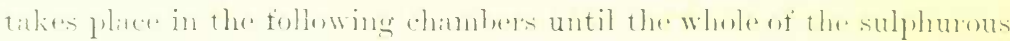

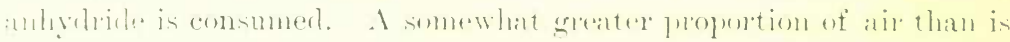

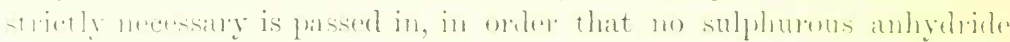

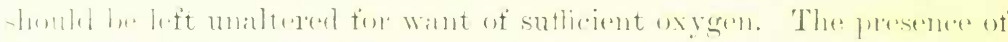

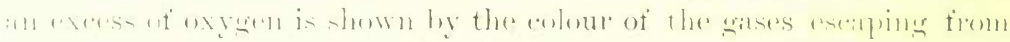

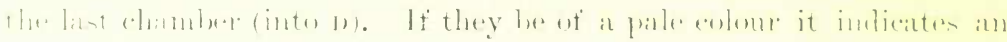

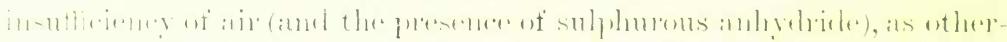

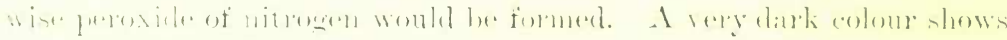

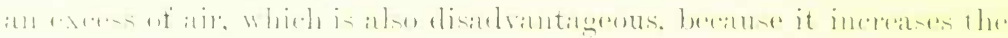

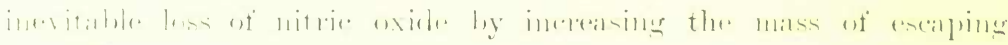
trit-1..'

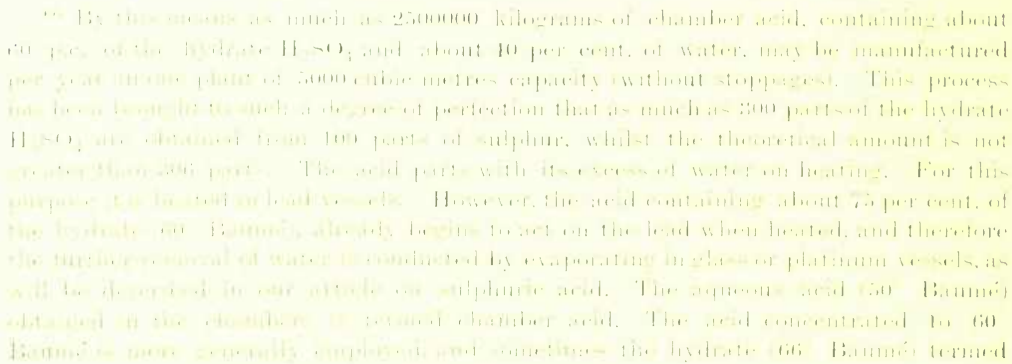


Nitrous oxide, $\mathrm{N}_{2} \mathrm{O}, 67$ is similar to water in its volumetric composition. Two volumes of nitrous oxide are formed from two volumes of nitrogen and one volume of oxygen, which may be shown by the common

vitriol acid is also used. In England alone more than 1000 million kilograms of chamber acid are prodnced by this method. The formation of sulphuric acid by the action of nitric acid was discovered by Drebbel, and the first lead chamber was erected by Roebuck, in Scotland, in the middle of the last century. The essence of the process was ably brought to light at the beginning of this century, when many improvements were introduced into practice.

67 Hyponitrous acid, corresponding with nitrous oxide (as its anhydride), is not known in a pure state, but its salts are known. They are prepared by the reduction of nitrous (and consequently of nitric) salts by sodium amalgam. If this amalgam be added to a cold solution of an alkaline nitrite until the evolution of gas ceases, and the excess of alkali saturated with acetic acid, an insoluble yellow precipitate of silver hyponitrite, NAgO, will be obtained on adding a solution of silver nitrate. The hyponitrite is insoluble in cold acetic acid, and decomposes when heated, with the evolution of nitrous oxide. If rapidly heated it decomposes with an explosion. It is dissolved unchanged by feeble mineral acids, whilst the stronger acids (for example, sulphuric and hydrochloric acids) decompose it, with the evolution of nitrogen, nitric and nitrous acids remaining in solution. Among the other salts of hyponitrous acid, HNO, the salts of lead, copper, and mercury are insoluble in water. This is almost all that is at present known (according to the researches of Divers) concerning this compound, which in its composition and reactions presents a certain analogy to hypochlorous acid. There is even reason to think that the composition of silver hyponitrite, $\mathrm{AgNO}$, is more complex than was at first supposed. As a substance which has not been sufficiently fully investigated, hyponitrous acid must be ranked with those compounds which yet present much that is doubtful. It is evident from the very method of its formation that it belongs to the class of compounds which are intermediate between the oxygen and hydrogen compounds of nitrogen. If its composition be $\mathrm{NHO}$, then perhaps it $=\mathrm{NH}_{3}$, in which two equivalents of lydrogen are replaced by oxygen (see p. 258). A substance of this composition, containing the hydrogen combined with the nitrogen, should most probably be isomeric, and not identical, with the true hydrate of nitrous oxide, because in the latter the hydrogen would be in the form of hydroxyl. Among such insufficiently investigated compounds - which, however, are of great interest-we must rank amidogen, or hydrazine, $\mathrm{N}_{2} \mathrm{H}_{4}$, which was prepared by Curtius (1887) by means of ethyl diazoacetate, or triazoacetic acid. Curtius and Jay (1889) showed that triazoacetic acid, $\mathrm{CHN}_{2} \cdot \mathrm{COOH}$ (the formula should be tripled), when heated with water or a mineral acid gives (quantitatively) oxalic acid and amidogen (lyddrazine), $\mathrm{CHN}_{2} \cdot \mathrm{COOH}+$ $2 \mathrm{H}_{2} \mathrm{O}=\mathrm{C}_{2} \mathrm{O}_{2}(\mathrm{OH})_{2}+\mathrm{N}_{2} \mathrm{H}_{4}-i . e$. (empirically), the oxygen of the water replaces the nitrogen of the azoacetic acid. The amidogen is thus obtained in the form of a salt. With acids amidogen forms very stable salts of the two types, $\mathrm{N}_{2} \mathrm{H}_{4} \mathrm{HX}$, and $\mathrm{N}_{2} \mathrm{H}_{4} \mathrm{H}_{2} \mathrm{X}_{2}$, as, for example, with $\mathrm{HCl}, \mathrm{H}_{2} \mathrm{SO}_{4}$, \&c. These salts are easily crystallised; in acid solutions they act as powerful reducing agents, evolving nitrogen; when ignited they are decomposed into ammoniacal salts, nitrogen, and hydrogen; with nitrites they evolve nitrogen. The sulphate, $\mathrm{N}_{2} \mathrm{H}_{4}, \mathrm{H}_{2} \mathrm{SO}_{4}$, is sparingly soluble in cold water ( 3 parts in 100 of water), but is very soluble in hot water; its specific gravity is 1.378 , it fuses at $254^{\circ}$, with decomposition. The hydrochloride, $\mathrm{N}_{2} \mathrm{H}_{4}, 2 \mathrm{HCl}$, crystallises in octahedra, is very soluble in water, but not in alcohol; it fuses at $198^{\circ}$, evolving hydrogen chloride, and forming the salt $\mathrm{N}_{2} \mathrm{H}_{4} \mathrm{HCl}$; when rapidly heated it decomposes with an explosion; with platinic chloride it immediately evolves nitrogen, forming platinous chloride. By the action of the alkalis the salts $\mathrm{N}_{2} \mathrm{H}_{4}, 2 \mathrm{HX}$ give hydrate of amidogen, $\mathrm{N}_{2} \mathrm{H}_{4}, \mathrm{H}_{2} \mathrm{O}$, which is a fuming liquid, boiling at $119^{\circ}$, almost without odour, and whose aqueous solution corrodes glass and india-rubber, has an alkaline taste and poisonous properties. The reducing caprcities of the hydrate are clearly seen from the fact that it reduces the 
method of the analysis of the oxides of nitrogen (by passing them over red-hot copper or sodium). In contradistinction to the other oxides of nitrogen, it is not directly oxidised by oxygen, but it may be obtained from the higher oxides of nitrogen by the action of certain deoxidising substances; thus, for example, a mixture of two volumes of nitric oxide and one volume of sulphurous anhydride if left in contact with water and spongy platinum is converted into sulphuric acid and nitrous oxide, $2 \mathrm{NO}+\mathrm{SO}_{2}+\mathrm{H}_{2} \mathrm{O}=\mathrm{H}_{2} \mathrm{SO}_{4}+\mathrm{N}_{2} \mathrm{O}$. Nitric acid, also, under the action of certain metals - for instance, of zinc ${ }^{68}$ - gives nitrous oxide, although in this case mixed with nitric oxide. The usual method of preparing nitrous oxide consists in the decomposition of ammonium nitrate by the aid of heat, because in this case only water and nitrous oxide are formed, $\mathrm{NH}_{4} \mathrm{NO}_{3}=2 \mathrm{H}_{2} \mathrm{O}+\mathrm{N}_{2} \mathrm{O}$ (a mixture of $\mathrm{NH}_{4} \mathrm{Cl}$ and $\mathrm{KNO}_{3}$ is sometimes taken). The decomposition ${ }^{69}$ proceeds very easily in an apparatus like that used for the preparation of ammonia or oxygen that is, in a retort or flask with a gas-conducting tube. The decomposition must, however, be carried on carefully, as otherwise nitrogen is formed from the decomposition of the nitrous oxide. ${ }^{70}$

Nitrous oxide is not a permanent gas (absolute boiling point $+36^{\circ}$ ), it is easily liquefied by the action of cold under a high pressure; at $15^{\circ}$ it may be liquefied by a pressure of about 40 atmospheres. This gas is usually liquefied by means of the force pump ${ }^{71}$ shown in fig. 51 .

metals platinum and silver from their solutions. With mercuric oxide it explodes. It reacts directly with the aldehydes $\mathrm{RO}$, forming $\mathrm{N}_{2} \mathrm{R}_{2}$ and water ; for example, with benzaldehyde it gives the very stable insoluble benzalazine $\left(\mathrm{C}_{6} \mathrm{H}_{5} \mathrm{CHN}\right)_{2}$, of a yellow colour. Further research should explain the relation of these very interesting salts to amidogen $\left(\mathrm{N}_{2} \mathrm{H}_{4}\right)$ itself, which has not yet been isolated. Amidogen must be regarded as a substance which stands to ammonia in the same relation as hydrogen peroxide stands to water. Water, $\mathrm{H}(\mathrm{OH})$, gives, according to the law of substitution, as was clearly to be expected, $(\mathrm{OH})(\mathrm{OH})$ - that is, peroxide of hydrogen is the free radicle of water (hydroxyl). So also ammonia, $\mathrm{H}\left(\mathrm{NH}_{2}\right)$, forms hydrazine, $\left(\mathrm{NH}_{2}\right)\left(\mathrm{NH}_{2}\right)$-that is, the free radicle of ammonia, $\mathrm{NH}_{2}$, or amidogen. In the case of phosphorus a similar substance, as we shall afterwards see, has long been known under the name of liquid phosphuretted hydrogen, $\mathrm{P}_{2} \mathrm{H}_{4}$.

68 It is remarkable that electro-deposited copper shavings give nitrous oxide with a 10 p.c. solution of nitric acid, whilst ordinary copper gives nitric oxide. It is here evident that the physical and mechanical structure of the substance effects the course of the reaction-that is to say, it is a case of contact.

69 This decomposition is accompanied by the evolution of about 25000 calories per molecular quantity $\mathrm{NH}_{4} \mathrm{NO}_{3}$, and therefore takes place with ease, and sometimes with an explosion.

70 In order to remove any nitric oxide that might be present, the gas obtained is passed through a solution of ferrous sulphate. As nitrous oxido is very soluble in cold water (at $0^{\circ} 100$ volumes of water dissolve 130 volumes of $\mathrm{N}_{2} \mathrm{O}$, at $20^{\circ}, 67$ volumes), it. must be collected over warm water. The nitrous oxide is much more soluble than nitric oxide, which is in agreement with the fact that the nitrous oxide is much more easily liquefied than the nitric oxide.

71 Faraday obtained liquid nitrous oxide by the same method as liquid ammonia, 
As it is liquefied with comparative ease, and as the cold produced by its vaporisation is very considerable, ${ }^{72}$ it (as also liquid carbonic anhydride) is often employed in investigations requiring a low temperature.

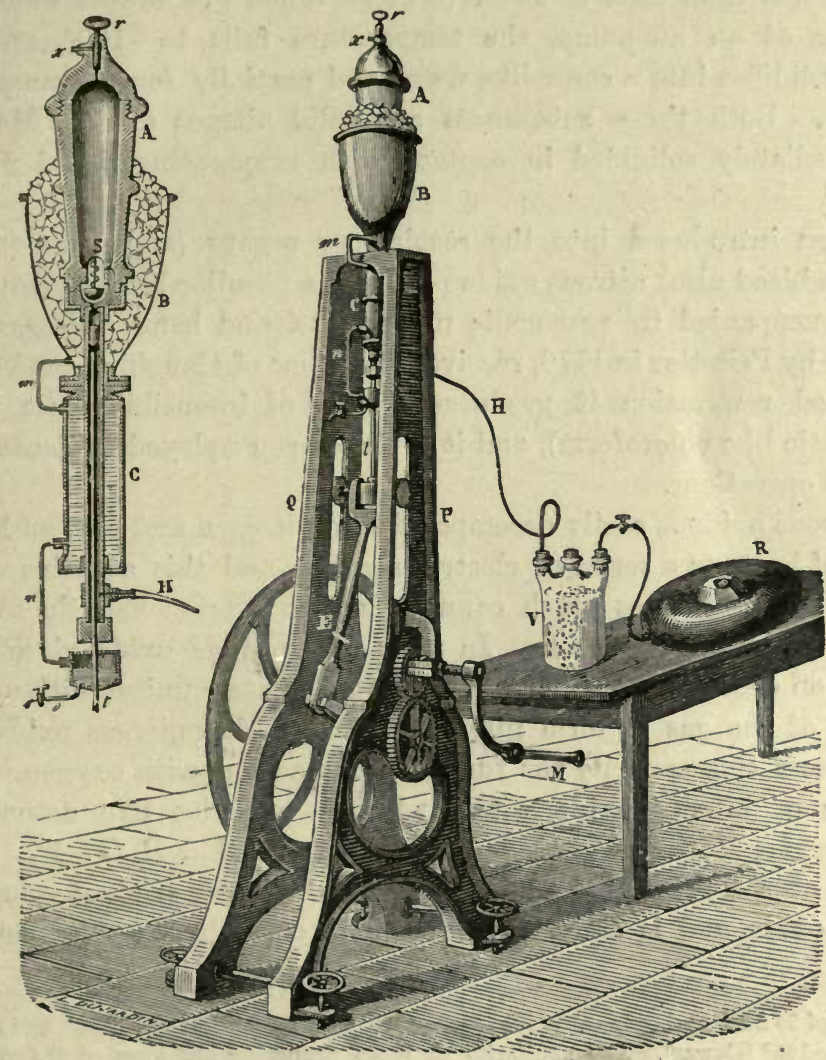

FiG. 51.-Natterer's apparatus for the preparation of liquid nitrous oxide and carbonic anhydride. The gas first passes through the vessel V, for drying, and then into the pump (a section of the upper part of the apparatus is given on the left). The piston $t$ of the force pump is moved by the crank $\mathrm{E}$ and fly-wheel turned by hand. The gas is pumped into the iron chamber $\mathrm{A}$, where it is liquefied. The valve $\mathrm{S}$ allows the gas to enter $\mathrm{A}$, but not to escape from it. The chamber and pump are cooled by the jacket $B$, filled with ice. When the gas is liquefied the vessel $A$ is uuscrewed from the pump, and the liquid may be poured from it by inverting it and unscrewing the valve $v$, and the liquid then runs out of the tube $x$.

by heating dry ammonium nitrate in a closed bent tube, one arm of which was immersed in a freezing mixture. In this case two layers of liquid are obtained at the cooled end, a lower layer of water and an upper layer of nitrous oxide. This experiment should be conducted with great care, as the pressure of the nitrous oxide in a liquid state is considerable, namely (according to Regnault), at $+10^{\circ}=45$ atmospheres, at $0^{\circ}=36$ atmospheres, at $-10^{\circ}=29$ atmospheres, and at $-20^{\circ}=23$ atmospheres. It boils at $-92^{\circ}$, and the pressure is then therefore $=1$ atmosphere.

72 Liquid nitrous oxide, in vaporising at the same pressure as liquid carbonic anhydride, gives rise to almost equal or even slightly lower temperatures. Thus at a

VOL. I. 
Nitrous oxide forms a very mobile, colourless liquid, which acts on the skin, and which is incapable in a cold state of oxidising either metallic potassium, phosphorus, or carbon; its specific gravity is slightly less than that of water $(0 \cdot 94)$. When evaporated under the receiver of an air-pump, the temperature falls to $-100^{\circ}$, and the liquid solidifies into a snow-like mass, and partially forms transparent crystals. Both these substances are solid nitrous oxide. Mercury is immediately solidified in contact with evaporating liquid nitrous oxide. ${ }^{73}$

When introduced into the respiratory organs (and consequently into the blood also) nitrous oxide produces a peculiar kind of drunkenness accompanied by spasmodic movements, and hence this gas, discovered by Priestley in 1776, received the name of 'laughing gas.' On a prolonged respiration it produces a state of insensibility (it is an anæsthetic like chloroform), and it is therefore employed in dental and surgical operations.

Nitrous oxide is easily decomposed into nitrogen and oxygen by the action of heat, or a series of electric sparks; and this explains why a number of substances which cannot burn in nitric oxide do so with great ease in nitrous oxide. In fact, when nitric oxide gives some oxygen on decomposition, this oxygen immediately unites with a fresh portion of the gas to form nitric peroxide, whilst nitrous oxide does not possess this capacity for further combination with oxygen. ${ }^{74} \mathrm{~A}$ mixture of nitrous oxide with hydrogen explodes like detonating gas, gaseous nitrogen being formed, $\mathrm{N}_{2} \mathrm{O}+\mathrm{H}_{2}=\mathrm{H}_{2} \mathrm{O}+\mathrm{N}_{2}$. The volume of the remaining nitrogen is equal to the original volume of nitrous oxide, and is equal to the volume of hydrogen entering into

pressure of $25 \mathrm{~mm}$. carbonic anhydride gives a temperature as low as $-115^{\circ}$, and nitrous oxide of $-125^{\circ}$ (Dewar). The similarity of these properties and even of the absolute boiling point $\left(\mathrm{CO}_{2}+32^{\circ}, \mathrm{N}_{2} \mathrm{O}+36^{\circ}\right)$ is all the more remarkable because these gases have the same molecular weight $=44$ (Chap. IV. Note 10, and Chap. VII.).

73 A very characteristic experiment of simultaneous combustion and of intense cold may be conducted by means of liquid nitrous oxide; if liquid nitrous oxide be poured into a test tube containing some mercnry, then the mercury will solidify, and if a piece of red-hot charcoal be thrown upon the surface of the nitrous oxide it will continue to burn very brilliantly, giving rise to a high temperature.

74 In the following chapter we shall consider the volumetric composition of the oxides of 'nitrogen. It explains the difference between nitric and nitrous oxide. Nitrous oxide is formed with a diminution of volumes (contraction), nitric oxide without contraction, its volume being equal to the sum of the volumes of nitrogen and oxygen of which it is composed. By oxidation, if it could be directly accomplished, two volumes of nitrous oxide and one volume of oxygen would not give three but four volumes of nitric oxide. These facts must be taken into consideration in comparing the calorific equivalents of formation, the capacity for supporting combustion, and other properties of nitrous and nitric oxides, $\mathrm{N}_{2} \mathrm{O}$ and $\mathrm{NO}$. 
combination with the oxygen; hence in this reaction equal volumes of nitrogen and hydrogen replace each other. Nitrous oxide is also very easily decomposed by red-hot metals ; and sulphur, phosphorus, and charcoal burn in it, although not so brilliantly as in oxygen. An equal quantity of a substance in burning in nitrous oxide evolves more heat than in burning in oxygen; which most clearly shows that in the formation of nitrous oxide by the combination of nitrogen with oxygen there was not an evolution but an absorption of heat, there being no other source for the excess of heat in the combustion of substances in nitrous oxide. If a given volume of nitrous oxide be decomposed by a metal-for instance, sodium--then there remains, after cooling and total decomposition, an exactly equal volume of nitrogen to that of the nitrous oxide taken ; consequently, the oxygen is, so to say, distributed between the atoms of nitrogen without producing an increase in the volume of the nitrogen. 


\section{CHAPTER VII}

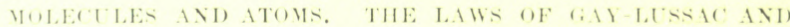
IVOR:ALRO-GERHARIT

Hreforias ambines with oxyente in the proportion of two volumes to one. Tlere componition hy volume of nitrous oxide is exactly similar it is entupesed of two volumes of nitrogen and one volume of oxyenes. by decomposing ammonia by the action of an electric spark it is atsy (1) prove that it eontains one volume of nitrogen to thee volumes of hydrogen. So, similarly, it is found, whenever at compound is decom. pered and the volumes of the gases proceeding firom it are measured. that the volumes of the gases or vapours entering into combination are in a very simple proportion one to another. With water, nitrous oxide, de.e this may be pored hy direct observation, but in the majority of cases, and epereially with substances which, although volatile that is, capable of passing into a gaseous (or vaporous) state are liquid al the ordinaty temperature, such a diect method of observation pre sents many diffieulties. But then, if the densities of the vapours and Eastes he known, the same simplieity in their ration is shown by cateulation. Ther wolume of a substance is propertional to its weight and inver-ely prepentional to its density and therefore by divioing the amoment hy wright of each substance entering inte the composition of at eomponmel hy its demsity in the gaseous or vaporous state we shatl ohtain factors which will be in the same proportion as the volumes of the subtanewe enterine inte the composition of the compound. so.

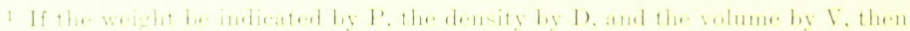

$$
\text { 1. Ki. }
$$

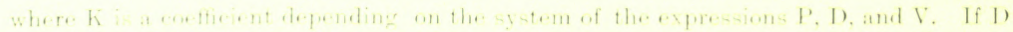

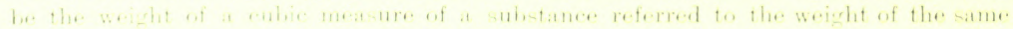

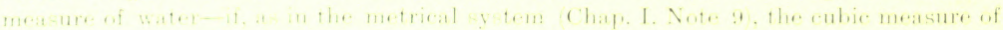

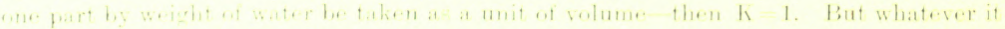

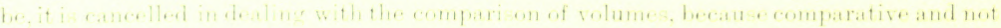

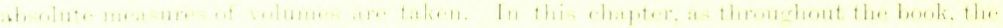

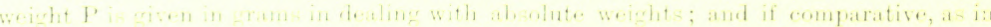


for example, water contains eight parts by weight of oxygen to one part by weight of hydrogen, and their densities are 16 and 1, consequently their volumes (or the above-mentioned factors) 1 and $\frac{1}{2}$, and therefore it is seen without direct experiment that water contains two volumes of hydrogen for every one volume of oxygen. So also, knowing that nitric oxide contains fourteen parts of nitrogen and sixteen parts of oxygen, and knowing that the specific gravities of both these gases are fourteen and sixteen, we find that the volumes in which nitrogen and oxygen combine for the formation of nitric oxide are in the proportion of $1: 1$. Consequently, nitric oxide is a combination of equal volumes of nitrogen and oxygen. We will cite another example. In the last chapter we saw that the density of $\mathrm{NO}_{2}$ only becomes constant and equal to twenty-three (referred to hydrogen) above $135^{\circ}$, and therefore a method of direct observation of the volumetric composition of this substance would be very difficult at so high a temperature. But it may be easily calculated. $\mathrm{NO}_{2}$, as is seen from its formula and analysis, contains thirty-two parts by weight of oxygen to fourteen parts by weight of nitrogen, forming forty-six parts by weight of $\mathrm{NO}_{2}$, and knowing the densities of these gases we find that one volume of nitrogen with two volumes of oxygen gives two volumes of nitrogen peroxide. Therefore, knowing the amounts by weight of the substances participating in a reaction or forming a given substance, and knowing the density of the gas or vapour, ${ }^{2}$ the volumetric relations of the sub-

the expression of chemical composition, then the weight of an atom is taken as unity. The density of gases, D, is also taken in reference to the density of hydrogen, and the volume $\mathrm{V}$ in metrical units (cubic centimetres or cubic metres, \&c.), if it be a matter of absolute magnitudes of volumes, and if it be a matter of chemical transformations-that is, of relative volumes - then the volume of an atom of hydrogen, or of one part by weight of hydrogen, is taken as unity, and all volumes are expressed according to these units.

${ }^{2}$ As the volumetric relations of vapours and gases, next to the relations of substances by weight, form the most important province of chemical learning and the most important means for the attainment of chemical conclusions, and inasmuch as these volumetric relations are determined by the densities of gases and vapours, therefore the methods of determining the densities of vapours (and also of gases) form important means in chemical research. These methods are described in detail in works on physics and physical and analytical chemistry, and therefore we here only touch on the general principles of the subject.

If we know the weight $p$ and volume $v$, occupied by the vapour of a given substance at a temperature $t$ and pressure $h$, then its density may be directly obtained by dividing $p$ by the weight of a volume $v$ of hydrogen (if the density be expressed according to hydrogen, see Chap. II. Note 23) at $t$ and $h$. Hence, the methods of determining the density of vapours and gases are based on the determination of $p, v, t$, and $h$. The two last data (the temperature $t$ and pressure $h$ ) are given by the thermometer and barometer and the heights of mercury or other liquid confining the gas, and therefore do not require further explanation. It need only be remarked that: (1) In the case of easilyvolatile liquids there is no difficulty in procuring a bath with ${ }^{\circ}$ constant temperature, but that it is, nevertheless, best (especially considering the inaccuracy of thermo- 


\section{stances acting in a reaction or entering into the composition of a com- pound, nay be also determined.}

meters) to have a medium of absolutely constant temperature, and therefore to take either a bath in which some substance is melting - such as melting ice at $0^{\circ}$ or crystals "of sodium acetate, melting at $+56^{\circ}$-or, as is more generally practised, to place the containing the substance to be experimented with in the vapour of a liquid boiling at a definite temperature, and, knowing the pressure under which it is boiling, to determine the temperature of the vapour. For this purpose the boiling points of water at different pressures are given in Chap. I. Note 11, and the boiling points of certain easily procurable liquids at various pressures are given in Chap. II. Note 27. (2) With respect to temperatures above $300^{\circ}$ (below which mercurial thermometers may be conveniently employed), they are most simply obtained constant (to give time for the weight and volume of a substance being observed in a given space, and to allow that space to attain the calculated temperature $t$ ) by means of substances boiling at a high temperature. Thus, for instance, at the ordinary atmospheric pressure the temperature $t$ of the vapour of sulphur is about $448^{\circ}$, of phosphorus pentasulphide $518^{\circ}$, of tin chloride $606^{\circ}$, of cadmium $770^{\circ}$, of zinc $930^{\circ}$ (according to Violle and others), or $1040^{\circ}$ (according to Deville), \&c. (3) The indications of the hydrogen thermometer must be considered as the most exact (but hydrogen diffuses through ignited platinum, and therefore nitrogen is then employed). (4) The temperature of the vapours used as the bath should in every case be several degrees higher than the boiling point of the liquid whose density is to be determined, in order that no portion should remain in a liquid state. But even in this case, as is seen from the example of nitric peroxide (Chap. VI.), the vapour density does not always remain constant with a change of $t$, as it should were the law of the expansion of gases and vapours absolutely exact (Chap. II. Note 26). If variations of a chemical and physical nature similar to that which we saw in nitric peroxide take place in the vapours, the main interest is centred in constant densities, which do not vary with $t$, and therefore the possible effect of $t$ on the density must always be kept in mind in having recourse to this means of investigation. (5) Usually, for the sake of convenience of observation, the vapour density is determined at the atmospheric pressure which is read on the barometer; but in the case of substances which are volatilised with difficulty, and also of substances which decompose, or, in general, vary at temperatures near their boiling points, it is best or indispensable to conduct the determination at low pressures, whilst for substances which decompose at low pressures the observations have to be conducted under a more or less considerably increased pressure. (6) In many cases it is convenient to determine the vapour density of a substance in admixture with other gases, and consequently under the partial pressure, which may be found, knowing the volume of the mixture and that of the intermixed gas (see Chap. I. Note 1). This method is especially important for substances which are easily decomposable, because, judging by the phenomena of dissociation, a substance is able to remain unchanged in the atmosphere of one of its products of decomposition. Thus, Wurtz determined the density of phosphoric chloride, $\mathrm{PCl}_{5}$, in admixture with the vapour of phosphorous chloride, $\mathrm{PCl}_{3}$. (7) It is evident, from the example of nitric peroxide, that a change of pressure may alter the density and aid decomposition, and therefore identical results are sometimes attained (if the density be variable) by raising $t$ and lowering $h$; .but if the density does not vary under these variable conditions (at least, not to an observable degree exceeding the limits of experimental error), then this constant density indicates the gaseous and invariable state of a substance. The laws hereafter laid down refer only to such vapour densities. But the majority of volatile substances show such a constant density at a certain distance from their boiling points up to the starting point of decomposition. Thus, the density of aqueous vapour does not vary for $t$ between the ordinary temperature and $1000^{\circ}$ (there are no trustworthy determinations beyond this) and for pressures varying from fractions of an atmosphere up to several atmospheres. If, however, the density does vary considerably with a variation of $h$ and $t$, 


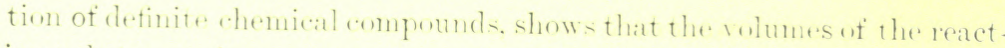

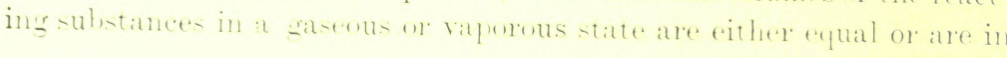

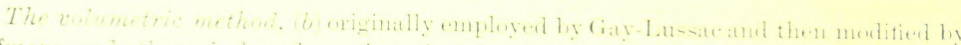

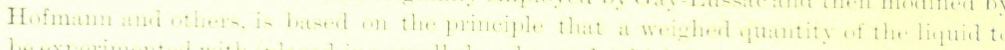

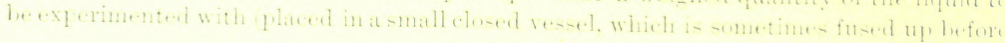

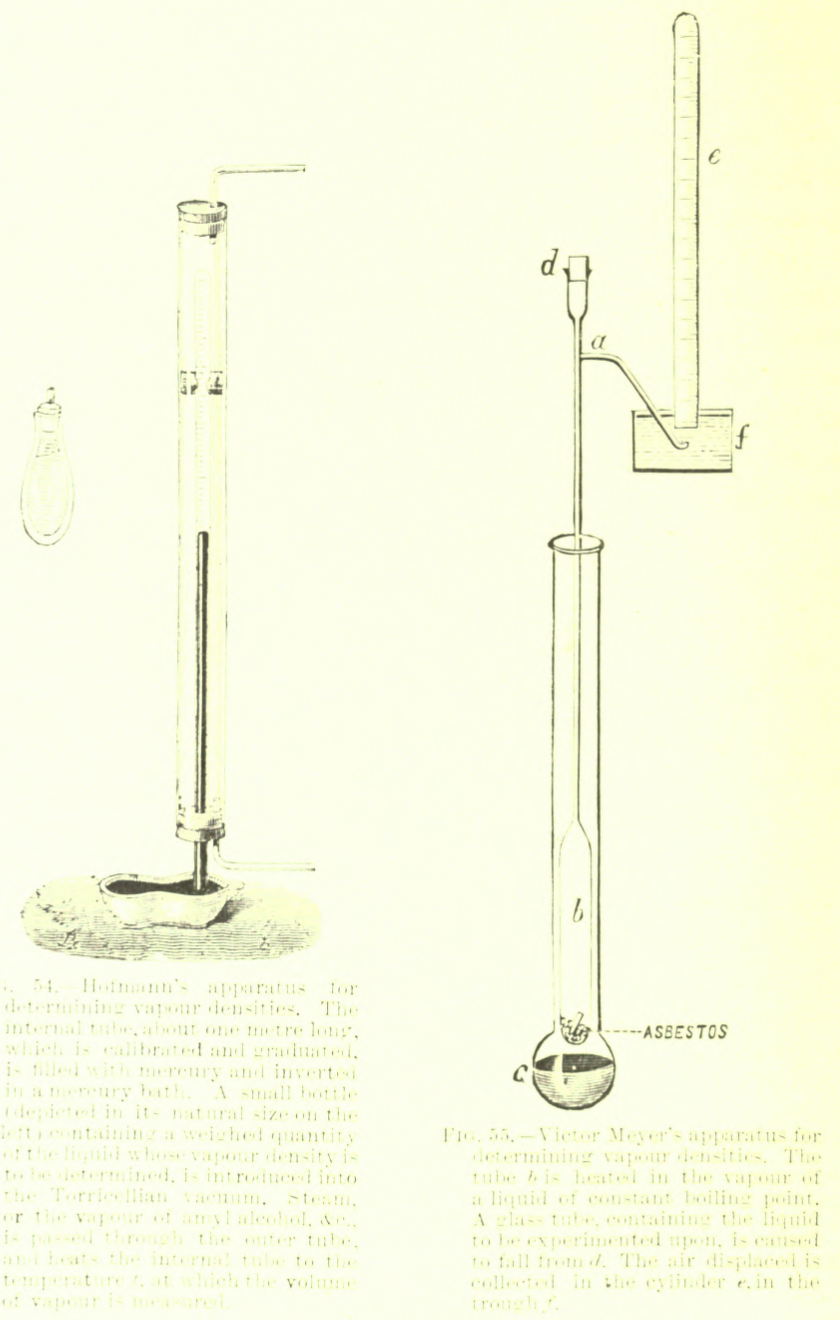

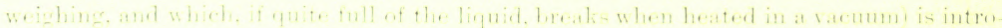

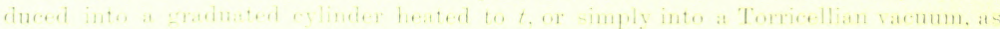

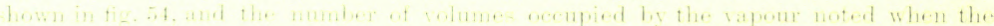

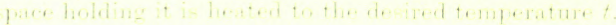

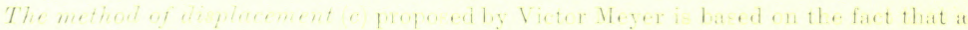


simple multiple proportion. ${ }^{3}$ This forms the first law of those discovered by Gay-Lussac. This law may be formulated as follows: The amounts of substances entering into chemical reaction, occupy under similar physical conditions, in a gaseous or vaporous state, equal or multiple volumes. This law not only refers to elements, but also to compounds entering into mutual chemical combination ; thus, for example, one volume of ammonia gas combines with one volume of hydrogen chloride. For in the formation of sal-ammoniac, $\mathrm{NH}_{4} \mathrm{Cl}$, there enters into reaction 17 parts by weight of ammonia, $\mathrm{NH}_{3}$, which is $8 \frac{1}{2}$ times denser than hydrogen, and 36.5 parts by weight of hydrogen chloride, whose vapour density is $18 \frac{1}{4}$ times that of hydrogen, as has been proved by direct experiment. By dividing the weights by the respective densities we find that the volume of ammonia, $\mathrm{NH}_{3}$, is equal to two, and so also the volume of hydrogen chloride. Hence the volumes of the compounds which here combine together are equal to each other. By taking into consideration that the law of Gay-Lussac holds good, not only for elements, but also for compounds, it should be expressed as follows: Substances interact with one another in commensurable volumes of their vapours. ${ }^{4}$

space $b$ is heated to a constant temperature $t$ (by the surrounding vapours of a liquid of constant boiling point), and the air (or other gas enclosed in this space) is allowed to attain this temperature, and when it has done so a glass bulb containing a weighed quantity of the liquid to be experimented with is dropped into the space. The liquid is immediately converted into vapour, and displaces the air into the graduated cylinder $e$. The amount of this air is calculated from its volume, and consequently the volume at $t$, and, therefore, also the volume occupied by the vapour, is found. The general arrangement of the apparatus is given in fig. 55.

3 Vapours and gases, as was explained in the second chapter, are subject to the same laws, which are, however, only approximate. It is evident that for the deduction of the laws which will presently be enunciated it is only possible to take into consideration a perfect gaseous state (removed from the liquid state) and chemical invariability in which the vapour density is constant-that is, the volume of a given gas or vapour varies like a volume of hydrogen, air, or other gas, with the pressure and temperature.

It is necessary to make this statement in order that it may be clearly seen that the laws of gaseous volumes, presently to be described, are in the most intimate connection with the laws of the variations of volumes with pressure and temperature. And as these latter laws (Chap. II.) are not infallible but only approximately exact, the same, therefore, applies to the laws presently to be described. And as it is possible to find more exact laws (a second approximation) for the variation of $v$ with $p$ and $t$ (for example, Van der Waals' formula, Chap. II. Note 33), so also a more exact expression of the relation between the composition and the density of vapours and gases is also possible. But to prevent any doubt arising at the very beginning as to the breadth and general application of the laws of volumes, it will be sufficient to mention that the density of such gases as oxygen, nitrogen, and carbonic anhydride is already known to remain constant (within the limits of experimental error) hetween the ordinary temperature and a white heat; whilst, judging from what is said in my work on the 'Tension of Gases' (vol. i. p. 9), it may be said that, as regards pressure, the density remains very constant even when the deviations from Mariotte's law are very considerable. However, in this respect the number of data is yet too small for forming an exact conclusion.

4 We must recollect that this law is only approximate, like Boyle and Mariotte's law, 


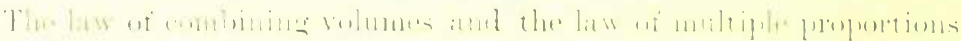

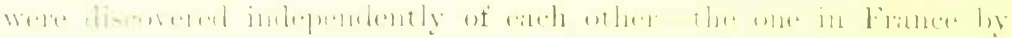

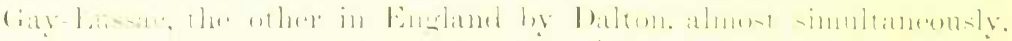

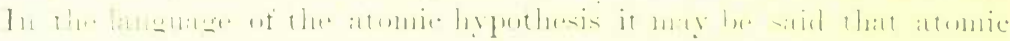

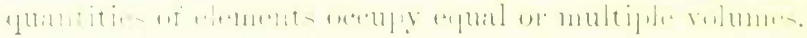

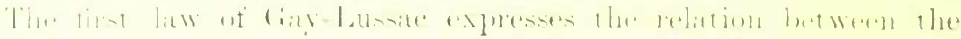

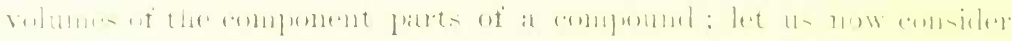

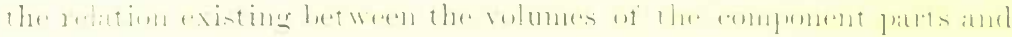

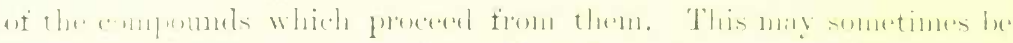

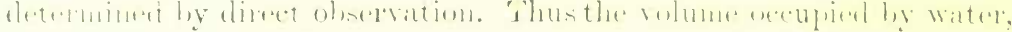

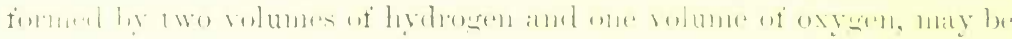

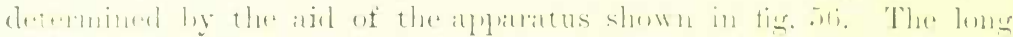

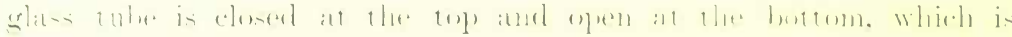

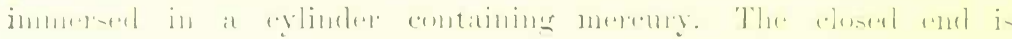

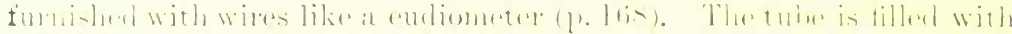

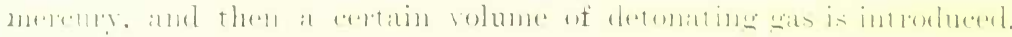

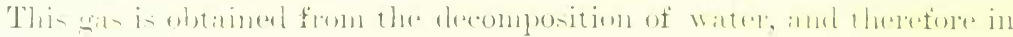

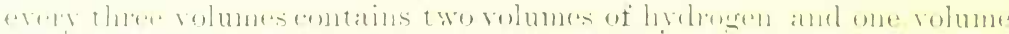

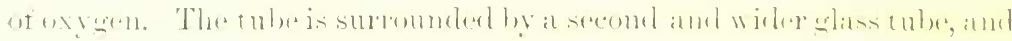

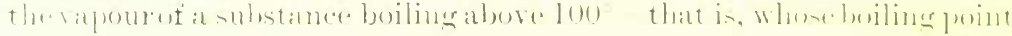

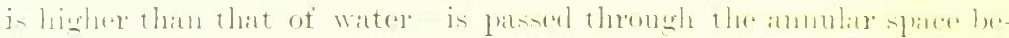

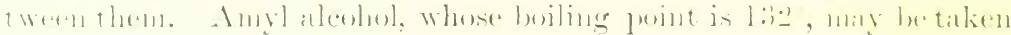

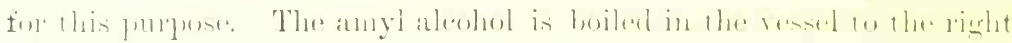

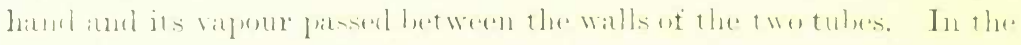

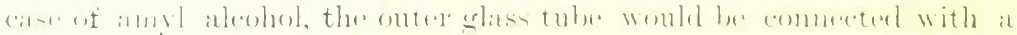

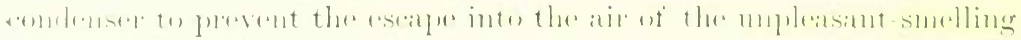

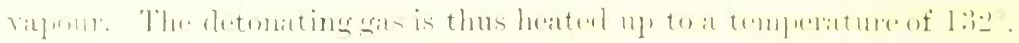

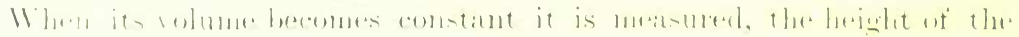

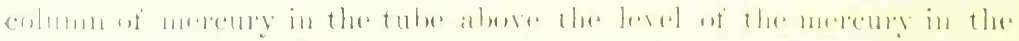

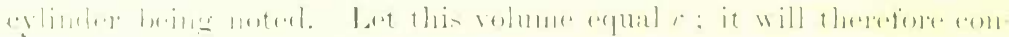

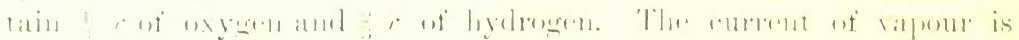

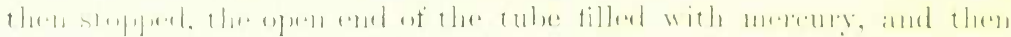

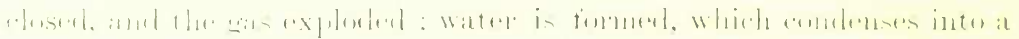

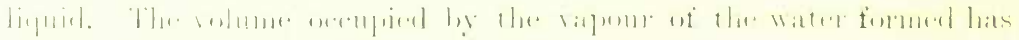

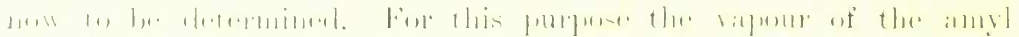

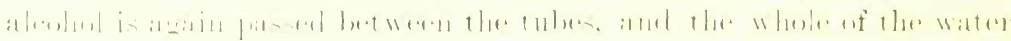

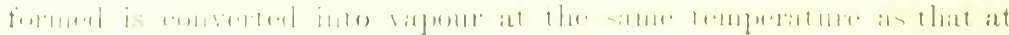

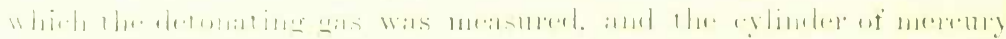

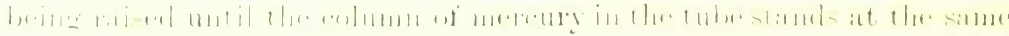

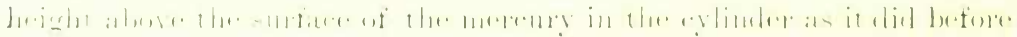




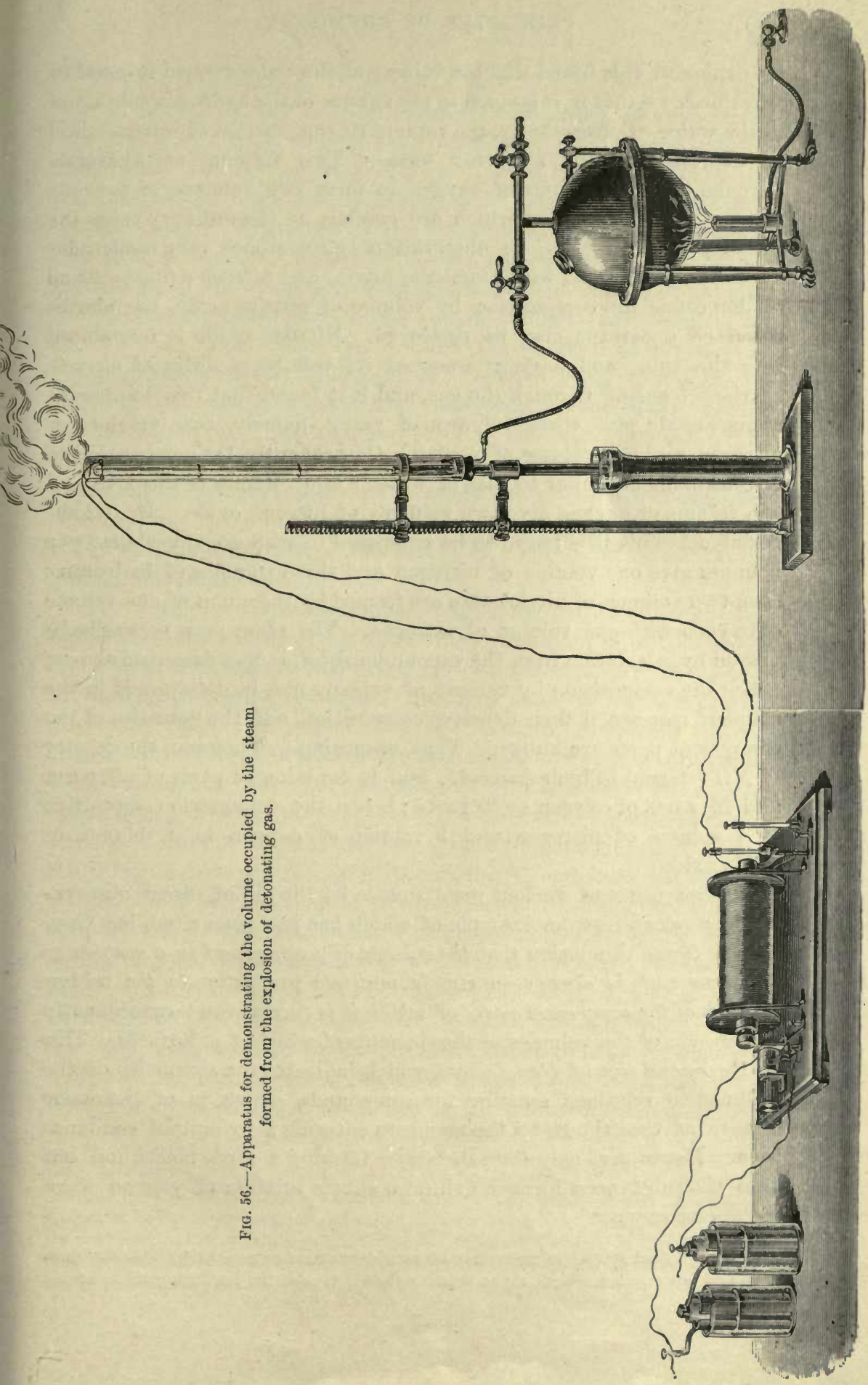




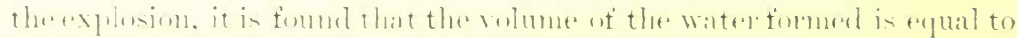

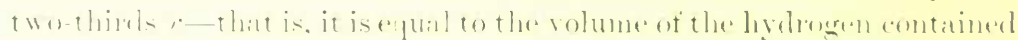

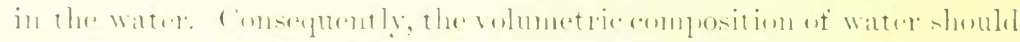

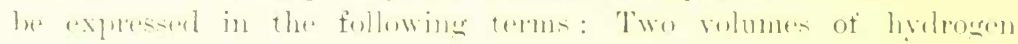

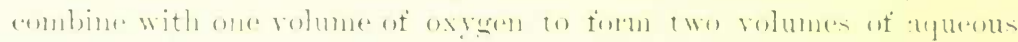
valume. For substanees which are gareous at the orelinaly tempera-

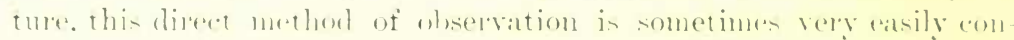
ductert : for instanere, with anmonia, nitrie. and nitrous oxirles. Thus to deternime the composition by volume of nitrous oxide. the above-

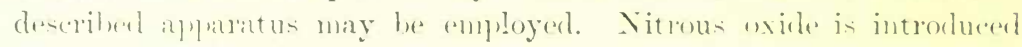
into the tulee and having measured its volume a series of electrie

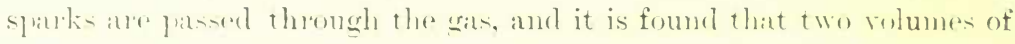
nitrous oxirle erive thres volumes of erases namely. two volumes of

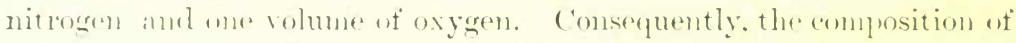
nitrous axile is similar to that of water : two volumes of nitrogem and mes volume of oxyeren grive two volumes of nitrous oxide. liv decomfosing anmonotia it is fomml lo he compusent in such a mamner that two volumes erive one volume of nitrogen and these volumes of hyeleogen : il he, two volumes of nitric oxide are formed by the mion of ane volume of exyene with one volume of nitrogen. The sime may naturally he

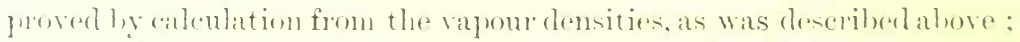

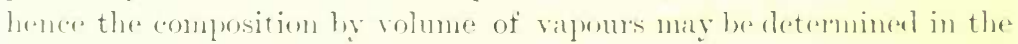
simplent manmer, if theil density, composition, and the densities of the (omploment pates are knwwn. Thus, aceording to Thomsen, the density

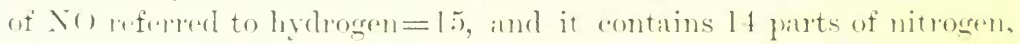

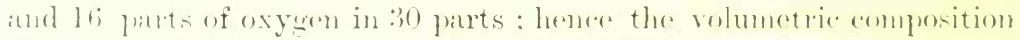
i. 1 volums of nitrogen and 1 volume of oxygen in : volunes of nitive oxille.

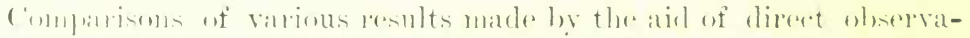

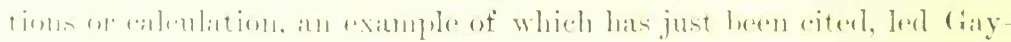

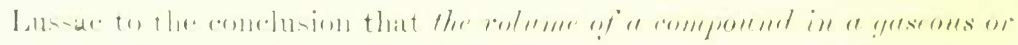

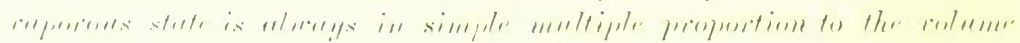

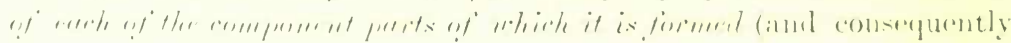

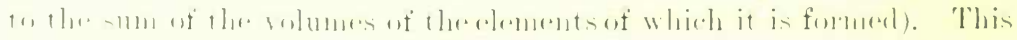

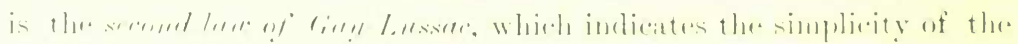

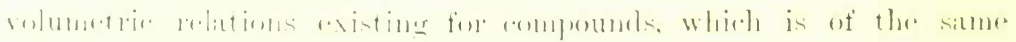

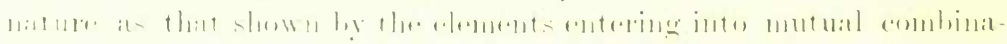

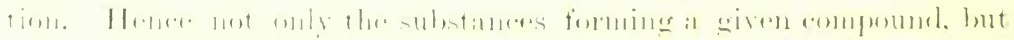

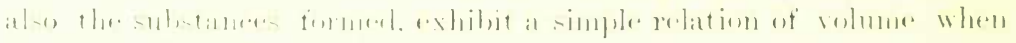

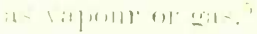

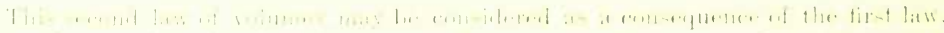

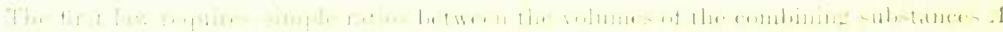


When a compound is formed from two or more components, there is or is not a contraction; the volume of the acting substances is in this case either equal to or greater than the volume of the resultant compound. The reverse is naturally observed in the case of decompositions, when from one substance there are produced several of simpler nature. Therefore in the future we shall term combination a reaction in which a contraction is observed - that is, a diminution in the volume of the component bodies in a state of vapour or gas ; and we shall term decomposition a reaction in which an expansion is produced; while those reactions in which the volumes in a gaseous or vaporous state remain constant (the volumes being naturally compared at the same temperature and pressure) we shall term reactions of substitution or of replacement or of double decomposition. Thus the transition of oxygen into ozone is a reaction of combination, the formation of nitrous oxide from oxygen and nitrogen will also be a combination, the formation of nitric oxide from the same will be a reaction of substitution, the action of oxygen on nitric oxide a combination, and so on.

The degree of contraction proceeding in the formation of chemical compounds not unfrequently leads to the possibility of distinguishing the degree of change which takes place in the chemical character of the components when combined. In those cases in which a contraction takes place, the properties of the resultant compound are very different from the properties of the substances of which it is composed. Hence ammonia bears no resemblance in its physical or chemical properties to the elements from which it is derived; a contraction takes place in a state of vapour, and consequently approachment of partsthe distance between the atoms is diminished, and from gaseous substances there is formed a liquid substance, or at least one which is easily liquefied. For this reason nitrous oxide formed by the condensation of two permanent gases is a substance which is somewhat easily converted into a liquid; again nitric acid, which is formed from elements which are permanent gases, is a liquid, whilst, on the contrary, nitric oxide, which is formed without contraction and is decomposed without expansion, remains a gas which is as difficult to liquefy as nitrogen and oxygen. In order to obtain a still more com-

and $B$. A substance $A B$ is produced by their combination. It may, according to the law of multiple proportion, combine not only with substances $C, D, \&$ c., but also with $A$ and with $B$. In this new combination the volume of $A B$, combining with the volume of $A$, should be in simple multiple proportion with the volume of $A$; hence the volume of the compound $A B$ is in simple proportion to the volume of its component parts. Therefore, only one law of volumes need be accepted. We shall afterwards see that the third law of volumes may also embrace in itself the two first laws. 
plete idea of the dependence of the properties of a compound on the properties of the component substances, it is further necessary to know the quantity of heat which is developed in the formation of the compound. If this quantity be large-as, for example in the formation of water-then the amount of energy in the resultant compound will be considerably less than the energy of the elements entering into its composition ; whilst, on the contrary, if the amount of heat evolved in the formation of a compound be small, or if there even be an absorption of heat, as in the formation of nitrous oxide, then the energy of the elements is not destroyed or is only altered to a slight extent: hence, notwithstanding the contraction (compression) involved in its formation, nitrous oxide supports combustion.

The preceding laws were deduced from purely experimental and empirical data, and as such evoke further consequences, as the law of multiple proportions evoked the atomic theory and the law of equivalents (Chapter IV.). In point of view of the atomic conception of the constitution of substances, the question naturally arises as to what then are the relative volumes proper to those physically-indivisible molecules which chemically react on each other and consist of the atoms of elements. The simplest possible hypothesis in this respect would be that the volumes of the molecules of substances are equal ; or, what is the same thing, to suppose that equal volumes of vapours and gases contain an equal number of molecules. This proposition was first enunciated by the Italian savant, Avogadro, in 1810, It was also admitted by the French physico-mathematician, Ampere (1815), for the sake of simplifying all kinds of physico-mathematical conceptions respecting gases. But Avogadro and Ampère's propositions were not generally received in science until Gerhardt in the forties had applied them to the generalisation of chemical reactions, and had demonstrated by aid of a series of phenomena that the reactions of substances are actually accomplished with the greatest simplicity, and before all, that such reactions take place between those quantities of substances which occupy equal volumes, and until he had stated the hypothesis in an exact manner and deduced the consequences that necessarily flow from it. Following Gerhardt, Clausius, in the fifties, placed the hypothesis of the equality of the number of molecules in equal volumes of gases and vapours on the basis of the kinetic theory of gases. Since then the hypothesis of Avogadro and Gerhardt has become the basis of contemporary physical, mechanical, and chemical conceptions ; the consequences arising from it have often been subject to doubt, but in the end have been verified by the most diverse methods, and now, when all efforts to refute those consequences have proved 
fruitless, the hypothesis must be considered as verified, ${ }^{6}$ and the law of Avogadro-Gerhardt must be spoken of as fundamental, and as of great importance for the comprehension of the phenomena of nature. The law may now be formulated in two aspects. In the first place, from a physical aspect: equal volumes of gases (or vapours) at equal temperatures and pressures contain ar equal number of molecules-or of such quantities of matter which are neither mechanically nor physically divisible-previous to chemical change. In the second place, from a chemical aspect, the same law may be expressed thus : the quantities of substances entering into chemical reactions occupy, in a state of vapour, equal volumes. For our purpose, the chemical aspect is the most important, and therefore, before developing the law and its consequences, we will stay to consider the chemical phenomena from which the law is deduced or by which it may be explained.

When two isolated substances interact with each other directly and easily - as, for instance, an alkali on an acid - then it is found that the reaction is accomplished between quantities which in a gaseous state (at equal temperatures and pressures) occupy equal volumes. Thus, ammonia, $\mathrm{NH}_{3}$, reacts directly with hydrochloric acid, $\mathrm{HCl}$, forming sal-ammoniac, $\mathrm{NH}_{4} \mathrm{Cl}$, and in this case the $\mathrm{NH}_{3}$, or 17 parts by weight of ammonia, occupy the same volume as the 36.5 parts by weight of hydrochloric acid. ${ }^{7}$ Ethylene, $\mathrm{C}_{2} \mathrm{H}_{4}$, combines with chlorine, $\mathrm{Cl}_{2}$, in only one proportion, forming ethylene dichloride, $\mathrm{C}_{2} \mathrm{H}_{4} \mathrm{Cl}_{2}$, and this combination proceeds directly and with great facility; the reacting quantities occupying equal volumes. Chlorine reacts with hydrogen in only one proportion, forming hydrochloric acid, $\mathrm{HCl}$, and in this case

- It must not be forgotten that Newton's law of gravity, or of the unity of the forces causing a body to fall to the earth, and the planets to be attracted to and revolve round the sun, was first a hypothesis, but it became a trustworthy, perfect theory, and acquired the qualities of a fundamental law owing to the concord between its consequences and reality. All laws, all theories of natural phenomena, are first hypotheses. Some are rapidly established by their exact consequences, which agree with facts; others only take root by slow degrees.

7 This is not only seen from the above calculations, but may be proved by experiment. A glass tube, divided in the middle by a stop-cock, is taken, and one portion filled with $d r y$ hydrogen chloride (the dryness of the gases is strictly necessary, because ammonia and hydrogen chloride are both very soluble in water, and hence a small trace of water may contain a large amount of these gases in solution) and the other with dry ammonia, under the atmospheric pressure. One orifice (for instance, of that portion which contains the ammonia) is firmly closed, and the other is immersed under mercury, and the cock is then opened. Solid sal-ammoniac is formed, but if the volume of one gas be greater than that of the other, some of the first gas will remain. By immersing the tube in the mercury in order that the internal pressure shall equal the atmospheric pressure, it may easily be shown that the volume of the remaining gas is equal to the difference between the volumes of the two portions of the tube, and that the remaining gas is that whose volume was the greater. 
equal volumes interact with each other. If an equality of volumes is observed in cases of combination, it should be all the more frequently encountered in cases of decomposition taking place in substances which split up into two others. Indeed, acetic acid breaks up into marsh gas, $\mathrm{CH}_{4}$, and carbonic anhydride, $\mathrm{CO}_{2}$, and in the proportions in which they are formed from acetic acid they occupy equal volumes. Also from phthalic acid, $\mathrm{C}_{8} \mathrm{H}_{6} \mathrm{O}_{4}$, there may be obtained benzoic acid, $\mathrm{C}_{7} \mathrm{H}_{6} \mathrm{O}_{2}$, and carbonic anhydride, $\mathrm{CO}_{2}$, and as all the elements of phthalic acid enter into the composition of these substances, therefore, although they cannot re-form it by their direct action on each other (the reaction is not reversible), still they form the direct products of its decomposition, and they occupy equal volumes. But benzoic acid, $\mathrm{C}_{7} \mathrm{H}_{6} \mathrm{O}_{2}$, is also composed of benzene, $\mathrm{C}_{6} \mathrm{H}_{6}$, and carbonic anhydride, $\mathrm{CO}_{2}$, which also occupy equal volumes. ${ }^{8}$ There is an immense number of similar examples among those organic substances to whose study Gerhardt consecrated his whole life and work, and he did not allow such facts as these to escape his attention. Still more frequently in the phenomena of substitution, when two substances act on one another, and two are produced without a change of volumes - that is, when the first definition given (p. 4) .corresponds with that given on p. $301-i t$ is found that the two substances acting on each other occupy equal volumes as well as each of the two resultant substances. Thus, in general, reactions of substitution take place between volatile acids, $\mathrm{HX}$, and volatile alcohols, $\mathrm{R}(\mathrm{OH})$, with the formation of ethereal salts, $\mathrm{RX}$, and water, $\mathrm{H}(\mathrm{OH})$, and the volume of the vapour of the reacting quantities, $\mathrm{HX}, \mathrm{R}(\mathrm{OH})$, and $\mathrm{RX}$, is the same as that for water $\mathrm{H}(\mathrm{OH})$, whose weight, corresponding with the formula, 18, occupies 2 volumes, if 1 part by weight of hydrogen occupy 1 volume and the density of aqueous vapour referred to hydrogen is 9 . Such general examples, of which there are many, ${ }^{9}$ show that the reaction of equal volumes forms a chemical phenomenon. of frequent occurrence, indicating the necessity of acknowledging the law of AvogadroGerhardt.

$\checkmark$ Let us demonstrate this by figures. From 122 grams of benzoic acid there is obtained (a) 78 grams of benzene, whose density referred to hydrogen $=39$, hence the relative volume $=2$, and $(b) 44$ grams of carbonic anhydride, whose density $=22$, and hence the volume $=2$. It is the same in other cases.

9 A large number of such generalised reactions, showing reaction by equal volumes, occur in the case of the hydrocarbon derivatives, because many of these compounds are volatile. The reactions of alkalis on acids, or anhydrides on water, \&c., which are so frequent between mineral substances, present but few such examples, because many of these substances are not volatile and their vapour densities are unknown. But essentially, the same is seen in these cases also; for instance, sulphuric acid, $\mathrm{H}_{2} \mathrm{SO}_{4}$, breaks up into the anhydride, $\mathrm{SO}_{3}$, and water, $\mathrm{H}_{2} \mathrm{O}$, which exhibit an equality 
But the question arises: What is the relation of volumes if the reaction of two substances takes place in different proportions, according to the law of multiple proportions? A clefinite answer can only be given in cases which have been very thoroughly studied. Thus chlorine, in acting on marsh gas, $\mathrm{CH}_{4}$, forms four compounds, $\mathrm{CH}_{3} \mathrm{Cl}, \mathrm{CH}_{2} \mathrm{Cl}_{2}$, $\mathrm{CHCl}_{3}$, and $\mathrm{CCl}_{4}$, and it may be established by direct experiment that the substance $\mathrm{CH}_{3} \mathrm{Cl}$ (methylic chloride) precedes the remainder, and that the latter proceed from it by the further direct action of chlorine. And this substance, $\mathrm{CH}_{3} \mathrm{Cl}$, is formed by the reaction of equal volumes of marsh gas, $\mathrm{CH}_{4}$, and chlorine, $\mathrm{Cl}_{2}$, according to the equation $\mathrm{CH}_{4}+$ $\mathrm{Cl}_{2}=\mathrm{CH}_{3} \mathrm{Cl}+\mathrm{HCl}$. A great number of similar cases are met with amongst organic - that is, carbon - compounds. Gerhardt was led to the discovery of his law by having investigated many such reactions, and from observing that in them the reaction of equal volumes precedes all others.

But if nitrogen or hydrogen give several compounds with oxygen, the above proposed question cannot be answered with complete clearness, because the consecutive formation of one degree of combination after the other cannot be so strictly defined. It may be supposed, but neither affirmed with assurance nor confirmed by experiment, that nitrogen and oxygen first give nitric oxide, $\mathrm{NO}$, and only afterwards the brown vapours $\mathrm{N}_{2} \mathrm{O}_{3}$ and $\mathrm{NO}_{2}$. Such a sequence in the combination of nitrogen with oxygen can only be supposed on the basis of the fact that $\mathrm{NO}$ forms $\mathrm{N}_{2} \mathrm{O}_{3}$ and $\mathrm{NO}_{2}$ directly with oxygen. If it be admitted that $\mathrm{NO}$ (and not $\mathrm{N}_{2} \mathrm{O}$ or $\mathrm{NO}_{2}$ ) be first formed, then this instance would also confirm the law of Avogadro-Gerhardt, because nitric oxide contains equal volumes of nitrogen and oxygen. So, also, it may be admitted that, in the combination of hydrogen with oxygen, hydrogen peroxide is first formed (equal volumes of hydrogen and oxygen), which is decomposed by the heat evolved into water and oxygen, all the more as this explains the presence of traces of hydrogen peroxide (Chap. IV.) in almost all cases of the combustion or oxidation of hydrogenous sub. stances ; for it cannot be supposed that water is first formed and then the peroxide of hydrogen, because up to now such a reaction has not been accomplished, whilst the formation of $\mathrm{H}_{2} \mathrm{O}$ from $\mathrm{H}_{2} \mathrm{O}_{2}$ is very easily reproduced. ${ }^{10}$

of volumes. Let us take another example where three substances combine in equal volumes : carbonic anhydride, $\mathrm{CO}_{2}$, ammonia, $\mathrm{NH}_{3}$, and water, $\mathrm{H}_{2} \mathrm{O}$ (the volumes of all are equal to 2), form acid ammonium carbonate, $\left(\mathrm{NH}_{4}\right) \mathrm{HCO}_{3}$.

10 This opinion as to the primary origin of hydrogen peroxide and of the formation of water by means of its decomposition, which was always held by me (in the first editions of this work), has in latter days become more generally accepted, thanks more especially to the work of Traube. Probably, it explains most simply the necessity for 
Thus a whole series of phenomena show that the chemical reaction of substances actually takes place, as a rule, between equal volumes, but this does not remove the possibility of the frequent reaction of unequal volumes, although, in this case, it is often possible to discover a preceding reaction between equal volumes. ${ }^{11}$

the presence of traces of water in many reactions, as, for instance, in the explosion of carbonic oxide with oxygen, and perhaps the very theory of the explosion of detonating gas and of the combustion of hydrogen gain in clearness and truth if we take into consideration the preliminary formation of hydrogen peroxide and its decomposition. We may here point out the fact that Ettingen (at Dorpat, 1888) observed the existence of currents and waves in the explosion of detonating gas by taking photographs, which showed the periods of combustion and the waves of explosion, which should be taken into consideration in the theory of this subject. As the formation of $\mathrm{H}_{2} \mathrm{O}_{2}$ from $\mathrm{O}_{2}$ and $\mathrm{H}_{2}$ corresponds with a less amount of heat than the formation of water from $\mathrm{H}_{2}$ and $\mathrm{O}$, it may be that the temperature of the flame of detonating gas depends on the pre-formation of hydrogen peroxide.

11 The possibility of reactions between unequal volumes, notwithstanding the general application of the law of Avogadro-Gerhardt, may, in addition to what has been said above, depend on the fact that the participating substances, at the moment of reaction, undergo a preliminary modification, decomposition, isomeric (polymeric) transformation, \&c. Thus, if $\mathrm{NO}_{2}$ seems to proceed from $\mathrm{N}_{2} \mathrm{O}_{3}$, if $\mathrm{O}_{2}$ is formed from $\mathrm{O}_{3}$, and the converse, then it cannot be denied that the production of molecules containing only one atom is also possible-for instance, of oxygen-as also of higher polymeric forms-as the molecule $\mathrm{N}$ from $\mathrm{N}_{2}$, or $\mathrm{H}_{3}$ from $\mathrm{H}_{2}$. In this manner it is naturally possible, by means of a series of hypotheses, to explain the cases of the formation of ammonia, $\mathrm{NH}_{3}$ from 3 vols. of hydrogen and 1 vol. of nitrogen. But it must be observed that perhaps our information in similar instances is, as yet, far from being complete. If the existence of hydrazine or diamide $\mathrm{N}_{2} \mathrm{H}_{4}$ (Chap. VI. Note 6) be verified, then, perhaps, an omide $\mathrm{N}_{2} \mathrm{H}_{2}$ will be discovered [this substance is now stated to.have been obtained] in which 2 vols. of hydrogen are combined with 2 vols. of nitrogen-that is, the reaction is accomplished between equal volumes. If it be shown that diamide gives nitrogen and ammonia $\left(3 \mathrm{~N}_{2} \mathrm{H}_{4}=\mathrm{N}_{2}+4 \mathrm{NH}_{3}\right)$ under the action of sparks, heat, or the silent discharge, \&c., then it will be possible to admit that it is formed before ammonia. And, perhaps, the still less stable amide $\mathrm{N}_{2} \mathrm{H}_{2}$, which may also decompose with the formation of ammonia, is produced before the amide $\mathrm{N}_{2} \mathrm{H}_{4}$.

I mention this to show that the fact of there being apparent exceptions to the law of reactions between equal volumes does not prove the impossibility of their being included under the law on further study of the subject. Having admitted a certain law or hypothesis, then consequences must be deduced from it, and if by their means a clearness and harmony is arrived at-and all the more, if by their means that which could not otherwise be known can be recognised-then the consequences verify the hypothesis. This was the case with the law now under discussion. The simplicity alone of the deduction of the weight. proper to the atoms of the elements, or the fact alone that having admitted the law it. follows, as will afterwards be shown, that the vis viva of the molecules of all gases is a constant quantity, is alone a sufficient reason for retaining the hypothesis if not for believing in it as a fact beyond doubt. And as by the acceptance of the law it became possible to foretell even the properties and atomic weights of elements which had not yet been discovered, and as these predictions afterwards proved to be in agreement with the actual facts, it is evident that the law of Avogadro-Gerhardt. penetrates deeply into the nature of the chemical relation of substances. This being granted, it is possible at the present time to expound and deduce the truth under consideration in many ways, and in every case it, like all that is highest in science (for example, the law of the indestructibility of matter, the law of the conservation of energy, 
The law of Avogadro-Gerhardt may also be easily expressed in an algebraical form. If the weight of a molecule, or of that quantity of a substance which enters into chemical reaction, and occupies in a state of vapour, according to the law, a volume equal to the molecules of other bodies, be indicated by the letters $\mathbf{M}_{1}, \mathbf{M}_{2} \ldots \ldots \ldots \ldots$ or, in general, $\mathrm{M}$, and if the letters $\mathrm{D}_{1}, \mathrm{D}_{2} \ldots \ldots \ldots \ldots \ldots$, or, in general, $\mathrm{D}$, stand for the density or weight of a given volume of the gases or vapours of the corresponding substances under certain definite conditions of temperature and pressure, then the law requires that

$$
\frac{M_{1}}{D_{1}}=\frac{M_{2}}{D_{2}} \ldots \ldots \ldots \ldots=\frac{M}{D}=C
$$

where $\mathrm{C}$ is a certain constant. This expression directly shows that the volumes corresponding with the weights $M_{1}, M_{2} \ldots \ldots \ldots . . M$, are equal to a certain constant, because the volume is proportional to the weight and inversely proportional to the density. The magnitude of $\mathrm{C}$ is naturally conditioned by and dependent on the units taken for the expression of the weights of the molecules and the densities. The weights of the molecules (equal to the sum of the atomic weights of the elements forming a given substance) are usually expressed by taking the weight of an atom of hydrogen as unity, and hydrogen is now also chosen as the unit for the expression of the densities of gases and vapours ; it is therefore only needful to find the magnitude of the constant for any one compound, as it will be the same for all others. Let us take water. Its reacting mass is expressed (conditionally) by the formula or molecule $\mathrm{H}_{2} \mathrm{O}$, for which $\mathrm{M}=18$, if $\mathrm{H}=1$, as we already know from the composition of water. Its vapour density, or $\mathrm{D}$, compared to hydrogen $=9$, and consequently for water $\mathrm{C}=2$, and therefore and in general for the molecules of all substance $\frac{M}{D}=2$.

Consequently, the weight of a molecule is equal to twice its vapour density expressed in relation to hydrogen, and conversely the density of a gas is equal to half the molecular weight referred to hydrogen.

The truth of this may be seen from an infinitely large number of

and the law of gravity, \&c.), proves to be not an empirical deduction from direct observation and experiment, not a direct result of analysis, but a creation, a penetration of an inquiring mind, which is guided and directed only by experiment and observation-a synthesis to which the exact sciences are capable to an equal extent with the highest forms of art. Without such a synthetical process of reasoning, science would only be a collection of the results of arduous labour, and would not be distinguished by that force with which it is really endowed when once it succeeds in attaining a synthesis, or concordance of outward form with the inner nature of things, without departing from the analysis of individual parts; in short, when it discovers by means of outward forms, which are apparent to the sense of touch, to observation, and to the mind, the internal signification of things-discovering simplicity in complexity and uniformity in diversity. 
observed vapour densities by comparing them with the results obtained by calculation. As an illustration, we may point out that for ammonia, $\mathrm{NH}_{3}$, the weight of the molecule or quantity of the reacting substance, as well as the composition and weight corresponding with the formula, is expressed by the figures $14+3=17$. Consequently, $M=17$. Hence, according to the law, $\mathrm{D}=8 \cdot 5$. And this result is also obtained by experiment. The density, according to the formula and experiment, of nitrous oxide, $\mathrm{N}_{2} \mathrm{O}$, is 22 , of nitric acid 15 , and of nitric peroxide 23 . In the case of nitrous anhydride, $\mathrm{N}_{2} \mathrm{O}_{3}$, as a substance which dissociates into $\mathrm{NO}+\mathrm{NO}_{2}$, the density should vary between 38 (so long as the $\mathrm{N}_{2} \mathrm{O}_{3}$ remains unchanged), and 19 (when $\mathrm{NO}+\mathrm{NO}_{2}$ is obtained). There are no figures of constant density for $\mathrm{H}_{2} \mathrm{O}_{2}, \mathrm{NHO}_{3}, \mathrm{~N}_{2} \mathrm{O}_{4}$, and many similar compounds which are either wholly or partially decomposed in passing into vapour. Salts and similar substances either have no vapour density because they do not pass into vapour (for instance, potassium nitrate, $\mathrm{KNO}_{3}$ ) without decomposition, or if they pass into vapour without decomposing, their vapour density is observed with difficulty only at very high temperatures. The practical determination of the vapour density at these high temperatures (for example, for sodium chloride, ferrous chloride, stannous chloride, drc.), requires special methods which have been worked out by Sainte-Claire, Deville, Crafts, Nilson and Pettersson, Meyer, Scott, and others. Having overcome the difficulties of experiment, it is found that the law of Avogadro-Gerhardt holds good for such salts as potassium iodide, beryllium chloride, aluminium chloride, ferrous chloride, dc.that is, the density obtained by experiment proves to be equal to half the molecular weight-naturally within the limits of experimental error or of possible deviation from the law.

Gerhardt deduced his law from a great number of examples of volatile carbon compounds. We shall become acquainted with certain of them in the following chapters ; their entire study, from the complexity of the subject, and from long-established custom, forms the subject of a special branch of chemistry, termed 'organic' chemistry. With all these suhstances the observed and calculated densities are very similar.

When the consequences of a law are verified by a great number of observations, it should be considered as confirmed by experiment. But this does not exclude the possibility of apparent deviations. They may evidently be of two kinds : the fraction $\frac{M}{D}$ may be found to be either greater or less than 2-that is, the calculated density may be either greater or less than the observed density. When the difference between the results of experiment and calculation fall within the possible errors 
of experiment (for example, equal to hundredths of the density), or within a possible error owing to the laws of gases having an only approximate significance (as is seen from the deviations, for instance, from the law of Boyle and Mariotte), then the fraction $\frac{M}{D}$ proves but slightly different from 2 (between $1 \cdot 9$ and $2 \cdot 2$ ), and such cases as these may be classed among those which ought to be expected from the nature of the subject. It is a different matter if the quotient of $\frac{M}{D}$ be several times, and in general a multiple, greater or less than

2. The application of the law must then be explained or it must be laid aside, because the laws of nature admit of no exceptions. We will therefore take two such cases, and first one in which the quotient $\frac{\mathrm{M}}{\mathrm{D}}$ is greater than 2, or the density obtained by experiment is less than is in accordance with the law.

It must be admitted as a consequence of the law of AvogadroGerhardt, that there is a decomposition in those cases where the volume of the vapour corresponding with the weight of the amount of a substance entering into reaction is greater than the volume of two parts by weight of hydrogen. Suppose the density of the vapour of water to be determined at a temperature above that at which it is decomposed, then, if not all, at all events a large portion of the water will be decomposed into hydrogen and oxygen. The density of such a mixture of gases, or of detonating gas, will be less than that of aqueous vapour; it will be equal to 6 (compared with hydrogen), because 1 volume of oxygen weighs 16 , and 2 volumes of hydrogen 2 ; and, consequently, 3 volumes of detonating gas weigh 18 , and 1 volume 6 , whilst the density of aqueous vapour $=9$. Hence, if the density of aqueous vapour be determined after its decomposition, the quotient $\frac{M}{D}$ would be found to be 3 and not 2 . This phenomenon might be considered as a deviation from Gerhardt's law, but this would not be correct, because it may be shown by means of diffusion through porous substances, as described in Chap. II., that water is decomposed at such high temperatures. In the case of water itself there can naturally be no doubt, because its vapour density agrees with the law at all temperatures at which it has been determined. ${ }^{12}$ But there are

12 As the density of aqueous vapour remains constant within the limits of experimental accuracy, even at $1000^{\circ}$, when dissociation has certainly commenced, it must be admitted that only a very small amount of water is decomposed at these temperatures. If even 10 p.c. of water were decomposed, then the density would be 8.57 and the quotient $\mathrm{M} / \mathrm{D}=2 \cdot 1$, but at the high temperatures here concerned the error of experiment is not greater than the difference of this quantity. And probably at $1000^{\circ}$ the dissocia- 
many substances which decompose with great ease directly they are volatilised, and which only exist as solids or liquids, but not in a state of vapour. There are, for example, many salts of this kind, besides all definite solutions having a constant boiling point, all the compounds of ammonia-for example, all ammonium salts-\&rc. Their vapour densities, determined by Bineau, Deville, and others, show that they do not agree with Gerhardt's law. Thus, the vapour density of sal-ammoniac, $\mathrm{NH}_{4} \mathrm{Cl}$, is nearly 14 (compared with hydrogen), whilst its molecular weight is not less than 53.5 , whence the vapour density should be nearly 27 according to the law. The molecule of sal-ammoniac cannot be less than $\mathrm{NH}_{4} \mathrm{Cl}$, because it is formed from the molecules $\mathrm{NH}_{3}$ and $\mathrm{HCl}$, and contains single atoms of nitrogen and chlorine, and therefore it cannot be divided; it, further, never enters into reactions with the molecules of other substances (for instance, potassium hydroxide or nitric acid) in quantities of less than 53.5 parts by weight, \&c. The calculated density $(27)$ is here double the observed density (about 14), hence $\frac{M}{D}=4$ and not 2. For this reason the vapour density of sal-ammoniac for a long time served as an argument for doubting the truth of the law. But it proved otherwise, after the matter had been fully studied. The low density depends on the decomposition of sal-ammoniac, on volatilising, into ammonia and hydrogen chloride. The observed density is not that of sal-ammoniac, but of a mixture of $\mathrm{NH}_{3}$. and $\mathrm{HCl}$, which should be nearly 14 , because the density of $\mathrm{NH}_{3}=8.5$ and $\mathrm{HCl}=18.5$, and therefore the density of their mixture (in equal volumes) should be $13 \cdot 3 .{ }^{13}$ The actual decomposition of the vapours of sal-ammoniac was demonstrated by Pebal and Than by the same method as the decomposition of water, by passing the vapour of sal-ammoniac through a porous substance. The experiment demonstrating the decomposition during volatilisation of sal-ammoniac may be made very easily, and is a very instructive point in the history of the law of Avogadro-Gerhardt, because without its aid it would never have been imagined that salammoniac decomposed in volatilising, as this decomposition bears all the signs of simple sublimation; consequently, the knowledge of the decomposition itself was forestalled by the law. The whole force

tion is far from being equal to 10 p.c. Therefore, the variation in the vapour density of water cannot be the means of ascertaining the amount of its dissociation.

13 This explanation of the vapour density of sal-ammoniac, sulphuric acid, and similar substances which decompose in being distilled was the most natural to resort to as soon as the application of the law of Avogadro-Gerhardt to chemical relations was begun; it was, for instance, given in my work on Specific volumes, 1856, p. 99. The formula, $M / D=2$, which later was applied by many other investigators, had already been applied in that work. 
and visible use of the discovery of the laws of nature lies in, and is expressed by, the fact that they enable the unknown to be foretold, and the unobserved to be foreseen. The arrangement of the experiment is based on the following reasoning. ${ }^{14}$ According to the law and to experiment the density of ammonia, $\mathbf{N H}_{3}$, is $8 \frac{1}{2}$, and of hydrochloric acid, $\mathrm{HCl}, 18 \frac{1}{4}$, if the density of hydrogen=1. Consequently, in a mixture of $\mathrm{NH}_{3}$ and $\mathrm{HCl}$, the ammonia will penetrate much more rapidly through a porous mass, or a fine orifice, than the heavier hydrochloric acid, just as in a former experiment the hydrogen penetrated more rapidly than oxygen. Therefore, if the vapour of sal-ammoniac comes into contact with a porous mass, the ammonia will pass through it in greater quantities than the hydrochloric acid, and this excess of ammonia may be discovered by means of moist red litmus paper, which should be turned blue. If the vapour of sal-ammoniac were not decomposed, it would pass through the porous mass as a whole, and the colour of the litmus paper would not be altered, because sal-ammoniac is a neutral salt. Thus, by testing with litmus the substance passing through the porous mass, it may be decided whether the sal-ammoniac is decomposed or not when passing into vapour. Sal-ammoniac rolatilises at so moderate a temperature that the experiment may be conducted in a glass tube heated by means of a lamp, an asbestos plug being placed in the central portion of a glass tube. ${ }^{15}$ The asbestos forms a porous mass, which is not changed at a high temperature. A piece of dry sal-ammoniac is placed at one side of the asbestos plug, and is heated by a Bunsen's burner. The vapours formed are driven by a current of air forced from a gasometer or bag through two tubes containing pieces of moist litmus paper, one blue and one red paper in each. If the sal-ammoniac be heated, then the ammonia appears on the opposite side of the asbestos plug, and the corresponding litmus turns blue. And as an excess of hydrochloric acid remains on the side where the sal-ammoniac is heated, therefore it turns the litmus at that end red. This proves that the sal-ammoniac, when converted into vapour, splits up into ammonia and hydrochloric acid, and at the same time gives an instance of the possibility of correctly conjecturing a fact on the basis of the law of Avogadro-Gerhardt.

So also the fact of a decomposition may be proved in the other instances where $\frac{M}{D}$ proved greater than 2, and hence the apparent

14 The beginner must remember that an experiment and the mode in which it is carried out must be determined by the principle or fact which it is intended to illustrate, and not vice versâ as some suppose. The idea which determines the necessity of an experiment is the chief consideration.

15 It is important that the tubes, asbestos, and sal-ammoniac should be dry, as otherwise the moisture retains the ammonia and hydrogen chloride. 
deviations appear in reality as an excellent proof of the general application and significance of the law of Arogadro-Gerhardt.

In those cases where the quotient $\frac{\mathrm{M}}{\mathrm{D}}$ proves to be less than 2, or the observed density greater than that calculated by a multiple number of times, the matter is evidently more simple, and the fact observed only indicates that the weight of the molecule is as many times greater, as that taken as the quotient obtained is less than 2. So, for instance, in the case of ethylene, whose composition is expressed by $\mathrm{CH}_{2}$, the density was found by experiment to be 14, and in the case of amylene, whose composition is also $\mathrm{CH}_{2}$, the density proved to be 35 , and consequently the quotient for ethylene $=1$, and for amylene $=2$. If the molecular weight of ethylene be taken, not as 14 , as might be imagined from its composition, but as twice as great-namely, as 28and for amylene as five times greater-that is as 70 -then the molecular composition of the first will be $\mathrm{C}_{2} \mathrm{H}_{4}$, and of the second $\mathrm{C}_{3} \mathrm{H}_{10}$, and for both of them $\frac{M}{D}$ will be equal to 2. This application of the law, - which at first sight may appear perfectly arbitrary, is nevertheless strictly correct, because the amount of ethylene which reacts-for example, with sulphuric and other acids-is not equal to 14 , but to 28 parts by weight. Thus with $\mathrm{H}_{2} \mathrm{SO}_{4}, \mathrm{Br}_{2}$, or $\mathrm{HI}$, \&c., ethylene combines in a quantity $\mathrm{C}_{2} \mathrm{H}_{4}$, and amylene in a quantity $\mathrm{C}_{5} \mathrm{H}_{10}$, and not $\mathrm{CH}_{2}$. On the other hand, ethylene is a gas which liquefies with difficulty (absolute boiling point $=+10^{\circ}$ ), whilst amylene is a liquid boiling at $35^{\circ}$ (absolute boiling point $=+192^{\circ}$ ), and by admitting the greater density of the molecules of amylene $(M=70)$ its difference from the lighter molecules of ethylene $(M=28)$ becomes clear. Thus, the smaller quotient $\frac{\mathrm{M}}{\mathrm{D}}$ is an indication of polymerisation, as the larger quotient is of decomposition. The difference between the densities of oxygen and ozone (Chap. IV.) is a case in point.

On turning to the elements, it is found in certain cases, especially with metals - for instance, mercury, zinc, and cadmium-that that weight of the atoms which must be acknowledged in their compounds (of which mention will be afterwards made) appears to be also the molecular weight. Thus, the atomic weight of mercury must be taken as $=200$, but the vapour density $=100$, and the quotient $=2$. Consequently, the molecule of mercury contains one atom, $\mathrm{Hg}$. It is the same with sodium, cadmium, and zinc. This is the simplest possible molecule, which necessarily is only possible in the case of elements, as the molecule of a compound must contain at least two atoms. However, the 
molecules of many of the elements prove to be complex-for instance, the weight of an atom of oxygen $=16$, and its density $=16$, so that its molecule must contain two atoms $\mathrm{O}_{2}$, which might already be concluded by comparing its density with that of ozone, whose molecule contains $\mathrm{O}_{3}$ (Chap. IV.). So also, the molecule of hydrogen equals $\mathrm{H}_{2}$, of chlorine $\mathrm{Cl}_{2}$, of nitrogen $\mathrm{N}_{2}$, dc. If chlorine react with hydrogen, the volume remains unaltered after the formation of hydrochloric acid, $\mathrm{H}_{2}+\mathrm{Cl}_{2}=\mathrm{HCl}+\mathrm{HCl}$. It is a case of substitution between the one and the other, and, therefore, the volumes remain constant. There are elements whose molecules are much more complex-for instance, sulphur, $\mathrm{S}_{6}$-although by heating the density is reduced to a third, and $\mathrm{S}_{2}$ is formed. Judging from the vapour density of phosphorus $(D=62)$ the molecule contains four atoms $P_{4}$. Hence, many elements, when polymerised, appear in molecules which are more complex than the simplest possible. In carbon, as we shall afterwards find, a very complex molecule must be admitted, as otherwise its nonvolatility and other properties cannot be understood. And if compounds are decomposed by a more or less powerful heat, and if polymeric substances are depolymerised (that is, the weight of the molecule diminishes) by a rise of temperature as $\mathrm{N}_{2} \mathrm{O}_{4}$ passes into $\mathrm{NO}_{2}$, or ozone, $\mathrm{O}_{3}$, into ordinary oxygen, $\mathrm{O}_{2}$, then we might expect to find the splitting-up of the complex molecules of elements into the simplest molecule containing a single atom only-that is to say, if $\mathrm{O}_{2}$ be obtained from $\mathrm{O}_{3}$, then the formation of $\mathrm{O}$ might also be looked for. The likelihood of such a proposition is indicated by the vapour of iodine. Its normal density $=127$ (Dumas, Deville, and others), which corresponds with the molecule $I_{2}$. At temperatures above $800^{\circ}$ (up to which the density remains almost constant), this density distinctly decreases, as is seen from the verified results obtained by Victor Meyer, Crafts, and Troost. At the ordinary pressure and $1000^{\circ}$ it is about 100 , at $1250^{\circ}$ about 80 , at $1400^{\circ}$ about 75 , and it apparently strives to reduce itself to one-half - that is, to 63 . Under a reduced pressure this splitting-up, or depolymerisation, of iodine vapour actually reaches a density ${ }^{16}$ of 66 , as Crafts demonstrated by reducing the pressure to $100 \mathrm{~mm}$. and raising the temperature to $1500^{\circ}$. From this it may be concluded that at high temperatures and low pressures the molecule $I_{2}$ gradually passes into molecule I containing one atom like mercury, and that something similar occurs with other elements at a considerable rise of temperature, which tends to bring about

16 Just as we saw (Chap. VI. Note 46) an increase of the dissociation of $\mathrm{N}_{2} \mathrm{O}_{4}$ and the formation of a large proportion of $\mathrm{NO}_{2}$, witl a decrease of pressure. The splitting-up of $I_{2}$ into $I+I$ is a similar dissociation. 


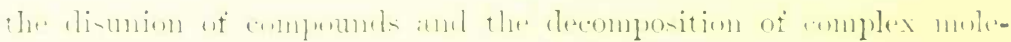
, ules. 17

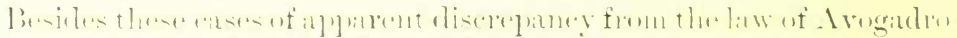

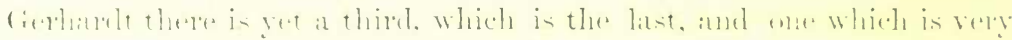

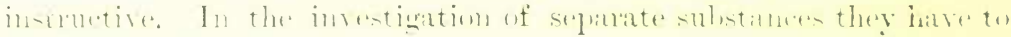

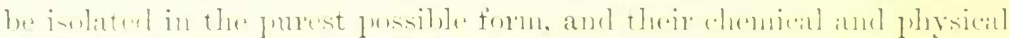

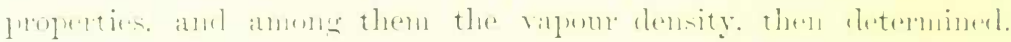

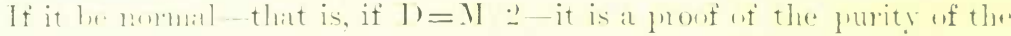

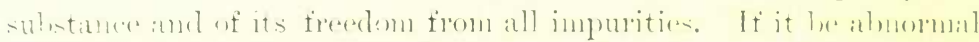

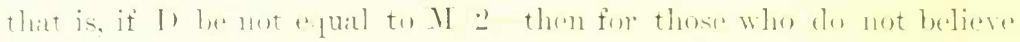

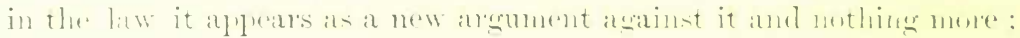

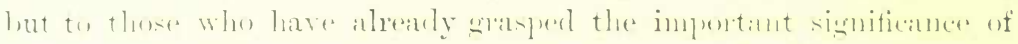

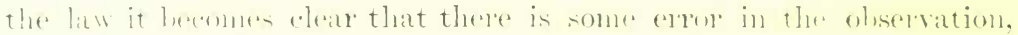
or that the density wats determinerl muler eomlitions in which the

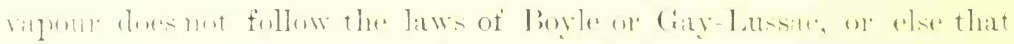

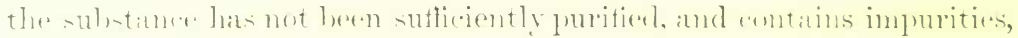

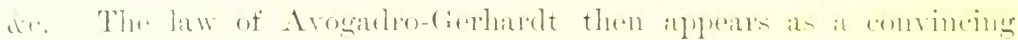
wirlence of the necessity of a flesh and mone exate rexeareh. Anelas

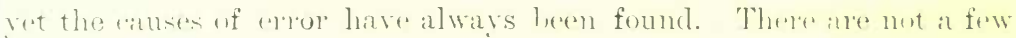

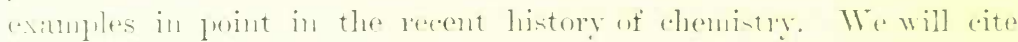

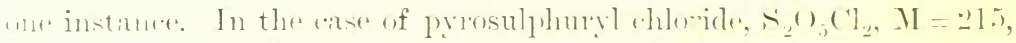
and (e)

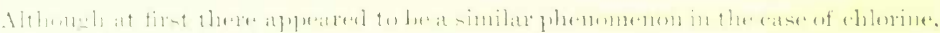

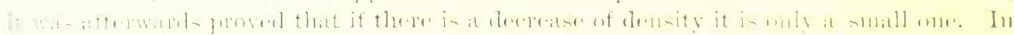

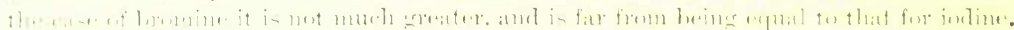

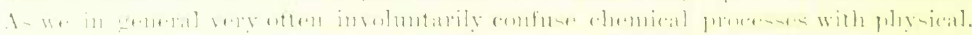

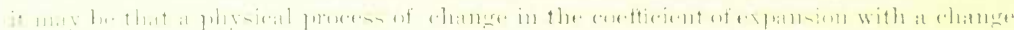

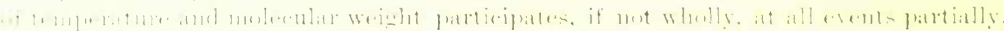

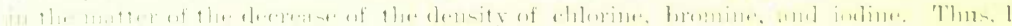

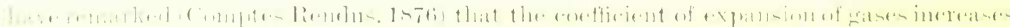

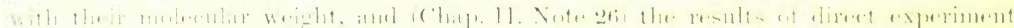

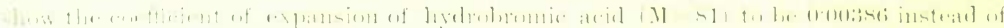

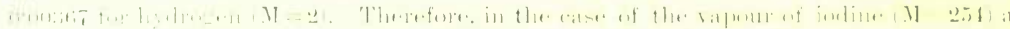

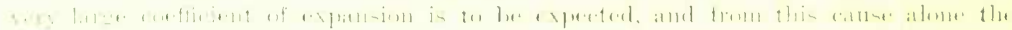

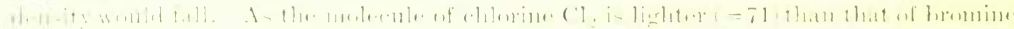

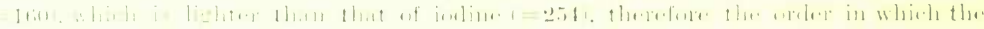

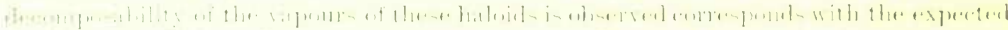

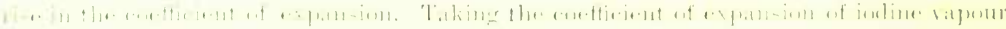

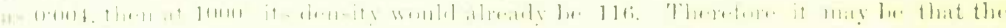

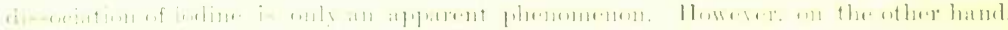

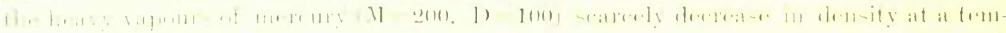

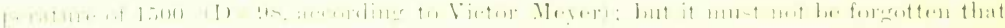

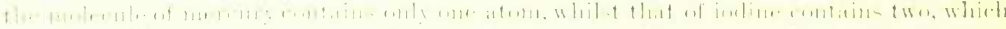

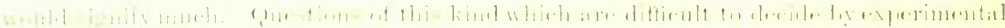

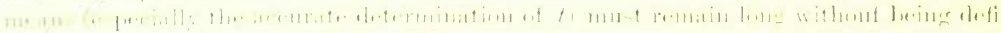

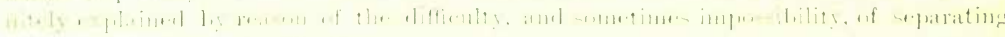

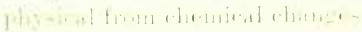


obtained 53.8 - that is, a density half as great; and further, Ogier (1882) demonstrated clearly that the substance is not dissociated by distillation into $\mathrm{SO}_{3}$ and $\mathrm{SO}_{2} \mathrm{Cl}_{2}$, or any other two products, and thus the abnormal density of $\mathrm{S}_{2} \mathrm{O}_{5} \mathrm{Cl}_{2}$ remained unexplained until D. P. Konovaloff (1885) showed that the previous investigators were working with a mixture (containing $\mathrm{SO}_{3} \mathrm{HCl}$ ), and that pyrosulphuryl chloride has a normal density of approximately 107. Had not the law of AvogadroGerhardt served as a guide, the impure liquid would have still passed as pure; all the more as the determination of the amount of chlorine could not aid in the discovery of the impurity. Thus, by following a true law of nature we are aided in the attainment of true deductions.

All cases which have been studied confirm the law of AvogadroGerhardt, and as by it a deduction is obtained, from the determination of the vapour density (a purely physical property), as to the size of the inolecule or quantity of a substance entering into chemical reaction, therefore, this law links together the two provinces of learning - physics and chemistry - in the most powerful manner. Besides which, the law of Avogadro-Gerhardt places the conceptions of molecules and atoms on a firm foundation, which was previously wanting. Although since the days of Dalton it had become evident that it was necessary to admit the existence of the atom (the chemical individual indivisible by chemical or other forces) of elements, and the groups of atoms or molecules of compounds indivisible by mechanical and physical forces; still the relative magnitude of the molecule and atom was not defined with sufficient clearness. So, for instance, the atomic weight of oxygen might be taken as 8 or 16 , or any multiple of these numbers, and nothing indicated a means for the acceptation of one or another of these magnitudes $;^{18}$ whilst as regards the weight of the molecules of elements and compounds there was no trustworthy conception whatever.

18 And so it was in the fifties. Some took $O=8$, others $O=16$. Water in the first case would be $\mathrm{HO}$ and hydrogen peroxide $\mathrm{HO}_{2}$, and in the second case, as is now generally accepted, water $\mathrm{H}_{2} \mathrm{O}$ and hydrogen peroxide $\mathrm{H}_{2} \mathrm{O}_{2}$ or $\mathrm{HO}$. Discussion and confusion were reigning. In 1860 the chemists of the whole world met at Carlsrïhe for the purpose of arriving at some agreement and uniformity of opinion. I was present at this Congress, and well remember how great was the difference of opinion, and how a conditional agreement was defended with the greatest acumen by the ranks of science, and with what warmth the followers of Gerhardt, at whose head stood the Italian professor, Canizzaro, followed up the consequences of the law of Avogadro. In the reign of scientific freedom (without which science would make no progress, and would remain petrified as in the middle ages) and with the simultaneous necessity of scientific conservatism (without which the roots of past study could give no fruit) a conditional agreement was not arrived at, and ought not to have been, but instead of it truth, in the form of the law of Arogadro-Gerhardt, received by means of the Congress a wider development, and soon afterwards conquered all minds. Then the new so-called Gerhardt atomic weights established themselves, and in the seventies they had already become generally used. 
With the establishment of the law the conception of the molecule was fully defined, and from it the conception of the magnitude of the atom of elements.

The particle or chemical particle or molecule must be considered as the quantity of a substance entering into chemical reaction with other. molecules, and which in a state of vapour occupies the same volume as two parts by weight of hydrogen.

The molecular weight (which has been indicated by M) of a substance is determined by its composition, transformations, and vapour density.

The molecule is not divisible by the mechanical and physical changes of substances, but in chemical reaction it is either altered in its properties, or quantity, or structure, or in the nature of the movement of its parts.

An agglomeration of molecules, which are alike in all chemical respects, makes up the masses of definite homogeneous substances in all states. ${ }^{19}$

Molecules consist of atoms in a certain state of distribution and movement, just as the solar system ${ }^{20}$ is made up of inseparable parts. (the sun, planets, satellites, comets, \&c.). The greater the number of

19 A volume of gas, a drop of a liquid, or the smallest crystal, presents an agglomeration of a number of molecules, in a state of movement, continuously repeated (like the stars of the milky way), distributing themselves in order or forming their new systems. If the aggregation of all kinds of heterogeneous molecules be possible in a gaseous state, where the molecules are considerably removed from each other, then in a liquid state, where the molecules are already close together, such an aggregation becomes possible only in that aspect of mutual reaction between the molecules which appears in their chemical attraction, and especially in the faculty of heterogeneous molecules for combining together. Solutions and other so-called indefinite chemical compounds should be regarded in this light. According to the representation evolved in this work we should regard them as containing both the compounds of the heterogeneous molecules themselves and the products of their decomposition, as in peroxide of nitrogen $\mathrm{N}_{2} \mathrm{O}_{4}$ and $\mathrm{NO}_{2}$. And we must consider that those molecules $A$, which at a given moment are combined with $B$ in $A B$, will in the following moment become free in order to again enter into a combined form. The instances of chemical equilibria proper to dissociated systems cannot be regarded in any other sense.

20 This strengthens the fundamental idea of the unity and harmony of the type of creation which forms one of those ideas which impress themselves on man in all ages, and give rise to a hope of arriving in time, by means of a lengthy labour of discoveries, observations, experiments, laws, hypotheses, and theories, at a comprehension of the internal and invisible structure of concrete substances with the same degree of clearness and exactitude as has been attained in the visible structure of the heavenly bodies. It is not many years ago since the law of Avogadro-Gerhardt took root in science. It is within the memory of many living scientific men. It is not surprising, therefore, that as yet little progress has been made in the province of molecular mechanics; but the theory of gases alone, which is intimately connected with the conception of molecules, shows by its success that the time is approaching when our knowledge of the internal structure of matter will grow rapidly. 
atoms in a molecule, the more complex is the resultant substance. The equilibrium between the dissimilar atoms may then be more or less stable, and may for this reason give more or less stable substances. Physical and mechanical transformations alter the velocity of the movement and the distances between the individual molecules, or of the atoms in the molecules, or of their sum total, but they do not alter the original equilibrium of the system ; whilst chemical changes, on the other hand, alter the molecules themselves, that is, the velocity of movement, the relative distribution, and the quality and quantity of the atoms in the molecules.

Atoms are the smallest quantities or indivisible chemical masses of the elements forming the molecules of elements and compounds.

Atoms have weight, the sum of their weights forms the weight of the molecule, and the sum of the weights of the molecules forms the weight of masses, and is the cause of gravity, and of all the phenomena which depend on the mass of a substance.

The elements are characterised, not only by their independent exist. ence, their incapacity of being converted into each other, \&c., but also by the weight of their atoms.

Chemical and physical properties depend on the weight, composition, and properties of the molecules forming a substance, and on the weight and properties of the atoms forming the molecules.

This is the substance of those conceptions of molecular mechanics which lie at the basis of all contemporary physical and chemical constructions since the establishment of the law of Avogadro-Gerhardt. The fecundity of the principles enunciated is encountered at every step in the entire sum of the particular cases forming the present store of chemical data. We will here cite a few examples of the application of the law.

As the weight of an atom must be understood as the minimum quantity of an element entering into the composition of all the molecules formed by it, therefore, in order to find the weight of an atom of oxygen, let us take the molecules of those of its compounds which have been already described, together with the molecules of certain of those carbon compounds which will be described in the following chapter :-

\begin{tabular}{lcclcc} 
& $\begin{array}{c}\text { Molecular } \\
\text { Weight. }\end{array}$ & $\begin{array}{c}\text { Amount of } \\
\text { Oxygen. }\end{array}$ & \multicolumn{2}{c}{$\begin{array}{c}\text { Molecular } \\
\text { Weight. }\end{array}$} & $\begin{array}{c}\text { Amount of } \\
\text { Oxygen. }\end{array}$ \\
$\mathrm{H}_{2} \mathrm{O}$ & 18 & 16 & $\mathrm{HNO}_{3}$ & 63 & 48 \\
$\mathrm{~N}_{2} \mathrm{O}$ & 44 & 16 & $\mathrm{CO}$ & 28 & 16 \\
$\mathrm{NO}$ & 30 & 16 & $\mathrm{CO}_{2}$ & 44 & 32 \\
$\mathrm{NO}_{2}$ & 46 & 32 & & &
\end{tabular}


The number of substances taken might be considerably increased, but the result would be the same-that is, the molecules of the compounds of oxygen would never be found to contain less than 16 parts by weight of this element, but always $n 16$, where $n$ is a whole number. The molecular weights of the above compounds are found either directly from the density of their vapour or gas, or from their reactions. Thus, the vapour density of nitric acid (as a substance which easily decomposes above its boiling point) cannot be accurately determined, but the fact of its containing one part by weight of hydrogen, and all its properties and reactions, indicate the above molecular composition and no other. In this manner it is very easy to find the atomic weight of all the elements, knowing the molecular weight and composition of their compounds. It may, for instance, be easily proved that less than $n 12$ parts of carbon never enters into the molecules of carbon compounds, and therefore $\mathrm{C}$ must be taken as 12, and not as 6 which was the case before Gerhardt. In similar manner the atomic weights now accepted for the elements oxygen, nitrogen, carbon, chlorine, sulphur, \&c., were found and indubitably established, and they are even now termed the Gerhardt atomic weights. As regards the metals, many of which do not give a single volatile compound, we shall afterwards see that there are also methods by which their atomic weights may be established, but nevertheless the law of Avogadro-Gerhardt is here also ultimately rosorted to, in order to remove any doubt which may be encountered. Thus, for instance, although much that was known concerning the compounds of beryllium necessitated its atomic weight being taken as $\mathrm{Be}=9$ - that is, the oxide as $\mathrm{BeO}$ and the chloride $\mathrm{BeCl}_{2}$ - still certain analogies gave reason for considering its atomic weight to be $\mathrm{Be}=13.5$, in which case its oxide would be expressed by the composition $\mathrm{Be}_{2} \mathrm{O}_{3}$, and the chloride $\mathrm{BeCl}_{3}{ }^{21}$ It was then found that the vapour density of beryllium chloride was approximately 40 , when it became quite clear that its molecular weight was 80 , and as this satisfies the formula $\mathrm{BeCl}_{2}$ but does not suit the formula $\mathrm{BeCl}_{3}$, it therefore became necessary to regard the atomic weight of $\mathrm{Be}$ as 9 and not as $13 \frac{1}{2}$.

21 If $\mathrm{Be}=9$, and beryllium chloride be $\mathrm{BeCl}_{2}$, then for every 9 parts of beryllium there are 71 parts of chlorine, and the molecular weight of $\mathrm{BeCl}_{2}=80$; hence the vapour density should be 40 or $n 40$. If $\mathrm{Be}=13.5$, and beryllium chloride be $\mathrm{BeCl}_{3}$, then to 13.5 of beryllium there are 106.5 of chlorine; hence the molecular weight would be 120 , and the vapour density 60 or $n 60$. The composition is evidently the same in both cases, because $9: 71 ;: 13.5: 106.5$. Thus, if the symbol of an element designate different atomic weights, what seem to be very different formulæ may equally well express both the percentage composition of compounds, and those properties which are required by the laws of multiple proportions and equivalents. The chemists of former days accurately expressed the composition of substances, and accurately applied Dalton's laws, by taking $\mathrm{H}=1, \mathrm{O}=8, \mathrm{C}=6, \mathrm{Si}=14$, \&c. The Gerhardt equivalents are also satisfied by 
With the establishment of a true conception of molecules and atoms, chemical formula became direct expressions, not only of composition, ${ }^{22}$ but also of molecular weight or vapour density, and consequently

then, because $\mathrm{O}=16, \mathrm{C}=12, \mathrm{Si}=28$, \&c., are multiples of them. The choice of one or the other multiple quantity for the atomic weight is impossible without a firm and concrete conception of the molecule and atom, and this is only obtained as a consequence of the law of Arogadro-Gerhardt, and therefore the contemporary atomic weights are the results of this law.

${ }_{22}$ In order to calculate the percentage amounts of the elements contained in a given compound from its formula, it is necessary to use a very simple proportion sum. Thus, for example, to find the percentage amount of hydrogen in hydrochloric acid we reason as follows:- $\mathrm{HCl}$ shows that hydrochloric acid contains 35.5 of chlorine and 1 part of hydrogen. Hence, in 36.5 parts of hydrochloric acid there is 1 part by weight of hydrogen, consequently 100 parts by weight of hydrochloric acid will contain as many more units of hydrogen as 100 is greater than $36^{\circ} 5$; therefore, the proportion is as follows $-x: 1:: 100: 36 \cdot 5$ or $x=\frac{100}{36.5}=2 \cdot 739$. Therefore 100 parts of hydrochloric acid contain 2.739 parts of hydrogen. In general, when it is required to transfer a formula into its percentage composition, we must replace the symbols by their corresponding atomic weights and find their sum, and knowing the amount by weight of a given element in it, it is easy by proportion to find the amount of this element in 100 or any other quantity of parts by weight. If, on the contrary, it be required to find the formula from a given percentage composition, we must proceed as follows: Divide the percentage amount of each element entering into the composition of a substance by its atomic weight, and compare the figures thus obtained together-they should be in simple multiple proportion to each other. Thus, for instance, from the percentage composition of hydrogen peroxide, 5.88 of hydrogen and $94 \cdot 12$ of oxygen, it is easy to find its formula; it is only necessary to divide the amount of hydrogen by unity and the amount of oxygen by 16 . The numbers 5.88 and 5.88 are thus obtained, which are in the ratio of $1: 1$, which means that in hydrogen peroxide there is one atom of hydrogen to one atom of oxygen.

The following is a proof of the above practical rule:-That to find the ratio of the number of atoms from the percentage composition, it is necessary to divide the percentage amounts by the atomic weights of the corresponding substances, and to find the ratio which these numbers bear to each other. Let us suppose that two radicles (simple or compound), whose symbols and combining weights are $\mathrm{A}$ and $\mathrm{B}$, combine together, forming a compound composed of $x$ atoms of A and $y$ atoms of B. The formula of the substance will be $\mathrm{A} x \mathrm{~B} y$. From this formula we know that our compound contains $x \mathrm{~A}$ parts by weight of the first element, and $y \mathrm{~B}$ of the second. In 100 parts of our compound there will be (by proportion) $\frac{100 . x \mathrm{~A}}{x \mathrm{~A}+y \mathrm{~B}}$ of the first element, and $\frac{100 . y \mathrm{~B}}{x \mathrm{~A}+y \mathrm{~B}}$ of the second. Let us divide these quantities, expressing the percentage amounts by the corresponding combining weights; we then obtain $\frac{100 x}{x \mathrm{~A}+y \mathrm{~B}}$ for the first element and $\frac{100 y}{x \mathrm{~A}+y \mathrm{~B}}$ for the second element. And these numbers are in the ratio $x: y$-that is, in the ratio of the number of atoms of both substances.

It may be further observed that even the very language or nomenclature of chemistry acquires a particular clearness and conciseness by means of the conception of molecules, because then the names of substances may directly indicate their composition. Thus the term 'carbon dioxide' tells more about and expresses $\mathrm{CO}_{2}$ better than carbonic acid gas, or even carbonic anhydride. Such nomenclature is already employed by many. But expressing the composition without an indication or even hint as to the properties, would be neglecting the advantageous sides of the present nomenclature. Sulphur dioxide, $\mathrm{SO}_{2}$, expresses the same as barium dioxide, $\mathrm{BaO}_{2}$, but sulphurous anhydride indicates the acid properties of $\mathrm{SO}_{2}$. Probably in time one harmonious chemical language will succeed in embracing both advantages, 
of a series of fundamental chemical and physical data, inasmuch as a number of the properties of substances are dependent on their vapour density, or molecular weight and composition. Therefore, the vapour density $\mathrm{D}=\frac{\mathrm{M}}{2}$. For instance, the formula of ethyl ether is $\mathrm{C}_{4} \mathrm{H}_{10} \mathrm{O}$, hence it corresponds with the molecular weight 74 , and the vapour density of 37 , which is the fact. Therefore, the density of vapours and gases ceased to be an empirical magnitude obtained by experiment only, and it acquired a rational meaning. It is only then needful to remember that 2 grams of hydrogen, or the molecular weight of this primary gas in grams, occupies, at $0^{\circ}$ and $760 \mathrm{~mm}$. pressure, a volume of $22 \cdot 3$ litres (or 22300 cubic centimetres), in order to directly reduce the weights of cubical measures of gases and rapours from their formulae, because the molecular veights of all other vapours at $0^{\circ}$ and $760 \mathrm{~mm}$. occupy the same volume, $22 \cdot 3$ litres. Thus, for example, in the case of carbonic anhydride, $\mathrm{CO}_{2}$, the molecular weight $\mathrm{M}=44$, hence 44 grams of carbonic anhydride at $0^{\circ}$ and $760 \mathrm{~mm}$. occupy a volume of $22 \cdot 3$ litres-consequently, a litre weighs 1.97 grams. By combining the laws of gases - Gay-Lussac's, Mariotte's, and Avogadro-Gerhardt's -we obtain ${ }^{23}$ a general formula for gases

$$
6255 s(273+t)=\mathbf{M} p
$$

where $s$ is the weight in grams of a cubic centimetre of a vapour or gas at a temperature $t$ and pressure $p$ (expressed in centimetres of mercury) if the molecular weight of the gas $=M$. Thus, for instance, at $100^{\circ}$ and 760 millimetres pressure (i.e., at the atmospheric pressure) the weight of a cubic centimetre of the vapour of ether $(\mathrm{M}=74)$ is equal to $s=0.0024 .{ }^{24}$

As the molecules of many elements (hydrogen, oxygen, nitrogen,

25 This formula (which is given in my work on 'The Tension of Gases,' and in a somewhat modified form in the 'Comptes Rendus,' Feb. 1876) is deduced in the following manner. According to the law of Avogadro-Gerhardt, $M=2 D$ for all gases, where $M$ is the molecular weight and $\mathrm{D}$ the density referred to hydrogen. But they equal the weight $\mathrm{S}_{0}$ of a cubic centimetre of a gas in grams at $0^{\circ}$ and $76 \mathrm{c.m}$. pressure, divided by 0.0000896 , for this is the weight in grams of a cubic centimetre of hydrogen. But the weight $s$ of a cubic centimetre of a gas at a temperature $t$ and under a pressure $p$ (in centimetres) is equal to $s p / 76(1+\alpha t)$. Therefore, $s_{0}=s .76(1+\alpha t) p$; hence $\mathrm{D}=76 . S(1+\alpha t) / 0.0000896 p$, whence $\mathrm{M}=152 s(1+\alpha t) / 0 \cdot 0000896 p$, which gives the above expression, because $1 / \alpha=273 . m / v$, where $m$ is the weight and $v$ the volume of $a$ vapour, may be taken instead of $s$.

24 The above formula may be applied in order to ascertain directly the molecular weight for a given vapour density, as $8=$ the weight of vapour $m$, divided by the volume $v$, and consequently by experiment, $\mathrm{M}=6,255 \mathrm{~m}(273+t) / p v$. Therefore, instead of the formula (see Chap. II. Note 33), $p v=\mathrm{R}(273+t)$, where $\mathrm{R}$ varies with the mass and nature of a gas, we may apply the formula $p v=6,255(\mathrm{~m} / \mathrm{M})(273+t)$, and taking a weight of a gas $m$ equal to its molecular weight, $p v=6,255(273+t)$ for all gases. 
chlorine, bromine, sulphur - at least at high temperatures) are of uniform composition, therefore, the formulie of the compounds formed by them, directly indicates the composition by volume. So, for example, the formula $\mathrm{HNO}_{3}$ directly shows that in the decomposition of nitric acid there is obtained 1 vol. of hydrogen, 1 vol. of nitrogen, and 3 vols. of oxygen.

And as a great number of mechanical, physical, and chemical properties are directly dependent on the elementary and volumetric composition, and on the vapour density; so the accepted system of atoms and molecules gives the possibility of simplifying a number of most complex relations. For instance, it may be easily demonstrated that the vis viva of the molecules of all vapours and gases is alike. For it is proved by mechanics that the vis viva of a moving mass $=\frac{1}{2} m v^{2}$, where $m$ is the mass and $v$ the velocity. For a molecule $m=\mathbf{M}$, or the molecular weight, and the velocity of the movement of gaseous molecules = a constant which we will designate by $\mathrm{C}$, divided by the square root of the density of the gas ${ }^{25}=\mathrm{C} / \mathrm{D} \frac{1}{2}$, and as $\mathrm{D}=\mathrm{M} / 2$, therefore, the vis viva of molecules $=\mathrm{C}^{2}$ - that is, a constant for all molecules. Q.E.D. ${ }^{26}$ The specific heat of gases (as we shall afterwards see), and many other of their properties, are determined by their density, and consequently by their molecular weight. Gases and vapours in passing into a liquid state evolve the so-called latent heat, which also proves to be in connection with the molecular weight. The observed latent heats of] carbon bisulphide, $\mathbf{C S}_{2}=90$, of ether.

25 Chap. I. Note 34.

26 The velocity of the transmission of sound through gases and vapours closely bears on this. It $=\sqrt{ } \mathrm{Kpg} / \mathrm{D}(1+a t)$ where $\mathrm{K}$ is the ratio between the two specific heats (it is approximately 1.4 for gases containing 2 atoms in a molecule), $p$ the pressure of the gas expressed by weight (that is, the pressure expressed by the height of a column of mercury multiplied by the density of mercury), $g$ the acceleration of gravity, D the weight of a cubic measure of the gas, $\alpha=0.00367$, and $t$ the temperature. Hence, if $\mathrm{K}$ be known, and as $\mathrm{D}$ can be found from the composition of a gas, we can calculate the velocity of the transmission of sound in that gas. Or if this velocity be known, we can find $\mathrm{K}$. The relative velocities of sound in two gases can be determined with peculiar ease (Kundt).

If a horizontal glass tube (about 1 metre long and closed at both ends) be full of a gas, and be firmly fixed in the middle, then it is easy to bring the tube and gas into a state of vibration, by rubbing it from centre to end with a damp cloth. The vibration of the gas is easily rendered visible, if the interior of the tube be dusted with lycopodium (the yellow powder-dust or spores of the lycopodium plant is often employed in medicine), before the gas is introduced and the tube fused up. The fine lycopodium powder forms itself into figures, whose number depends on the velocity of sound in the gas. If there be 10 figures, then the velocity of sound in the gas is ten times slower than in glass. It is evident that this is an easy method of comparing the velocity of sound in gases. It has been demonstrated by experiment that the velocity of sound in oxygen is four times less than in hydrogen, and the square roots of the densities and molecular weights of lydrogen and oxygen stand in this ratio.

VOL. I. 


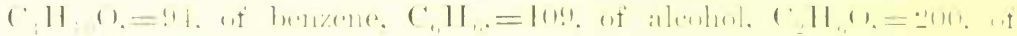

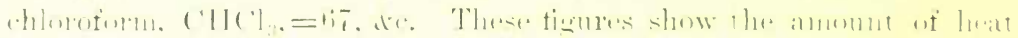

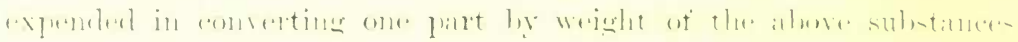

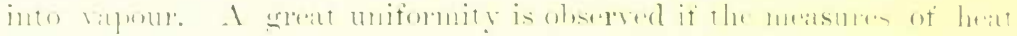

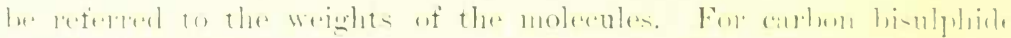

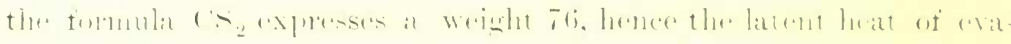

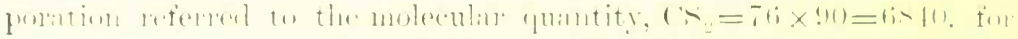

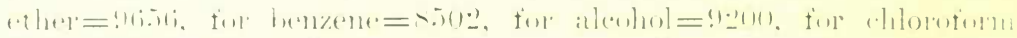

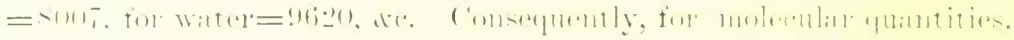

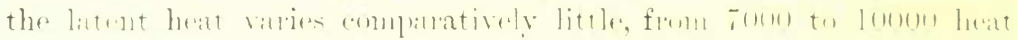

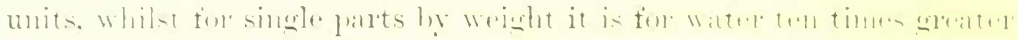
thath ton elelerofonm and many others. ${ }^{27}$

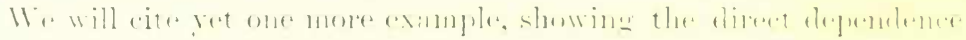

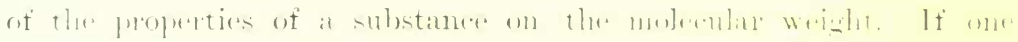

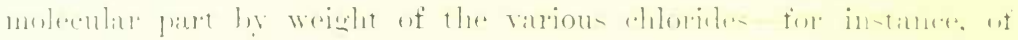

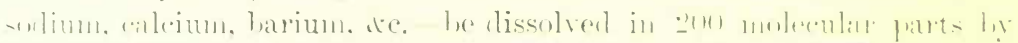

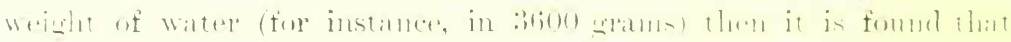

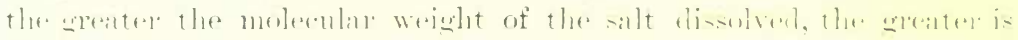

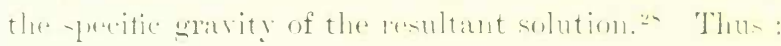

\begin{tabular}{|c|c|c|c|c|c|}
\hline & 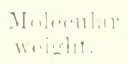 & - & & 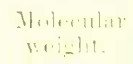 & $-1+\cdots+1$ \\
\hline $\left.110^{\circ}\right)$ & $: i t i \cdot i$ & $1 \cdot(1) 111$ & $\left({ }^{a}{ }_{1}()_{2}\right.$ & 111 & 1.0.2:ari \\
\hline$N_{i 1}(y$ & 动石 & $|\cdot 1| 111 ;$, & Iir'l, & $1: 311$ & ] $11: 3: 2$ \\
\hline lir & $i+i$ & $1 \cdot(1) 1: 1$ & $\left.1 / 11 C^{\prime}\right]_{2}$ & $1: i 1 ;$ & 1.11:3:; \\
\hline$[\because, 1 \cdot]$ & -11 & 1.111:3: & li:l'l & $\because 11$ & $1 \cdot 111<!$ \\
\hline $11 \div$ & $4 . \overline{7}$ & 1.(1):-2):3 & & & \\
\hline
\end{tabular}

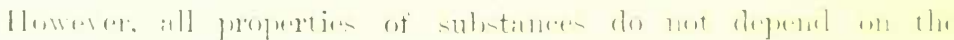

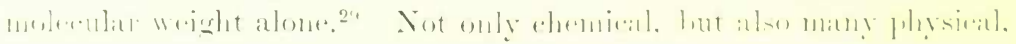

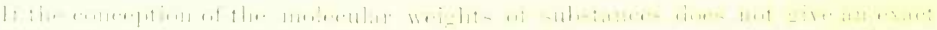

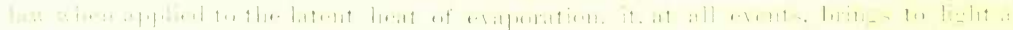

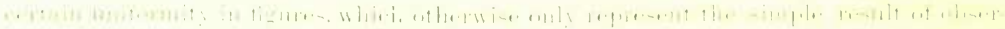

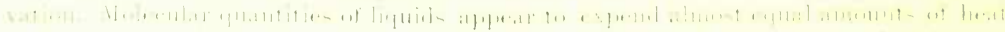

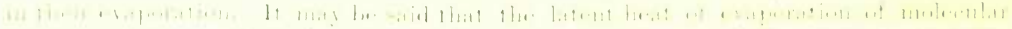

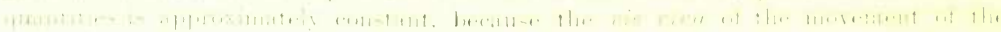

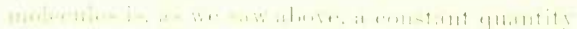

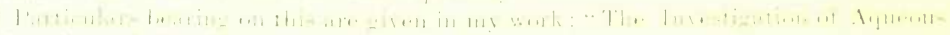

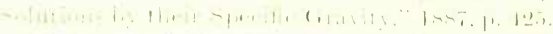

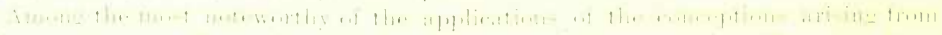

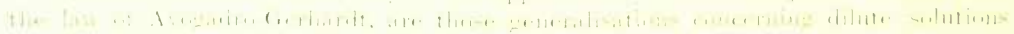

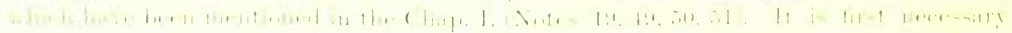

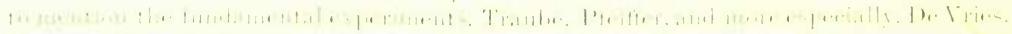

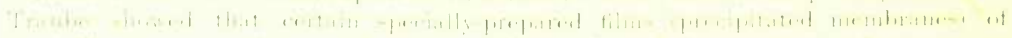

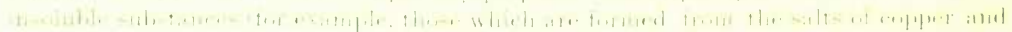

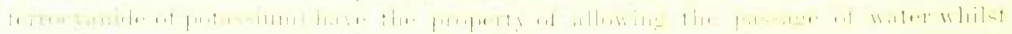

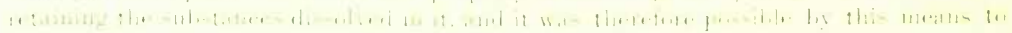


properties are determined by the composition of the molecules, and by the properties of the elements forming them. Thus the density of solids

determine the osmotic pressure, as in the experiment described in Chap. I. Note 19. De Vries found in vegetable cells a convenient means of determining such (isotonic) solutions, which produce indentical osmotic pressures. A thin slice of a coloured plant tissue-for instance, of Tradescantia discolor-is moistened under the microscope with the solution to be tested. If its osmotic pressure be equal, or less than that of the liquid contrined in the cells, no visible change occurs; but if the liquid taken be endued with a greater osmotic pressure than the cellular sap, then the water will pass from the cell, and the coloured matter of the cell will shrink away from the envelope, and this process is easily observed under the microscope. Knowing, then, the osmotic pressure for any one substance-for instance, for sugar-with different strengths of solutions, it is possible to find the osmotic pressure of all other substances investigated, because it is shown by direct experiment that the osmotic pressure increases in proportion to the strength of the solution. Thus having fixed on any one substance-for instance, sugar-and on one of its solutions, we may see the substance of the results which have been attained.

If (as on p. 64) a one per cent. solution of sugar be taken, then according to the experiments made by Pfeiffer (1877) its osmotic pressure $=53.5$ centimetres at $14^{\circ}$. According to the formula of sugar, $\mathrm{C}_{12} \mathrm{H}_{22} \mathrm{O}_{11}$, its molecular weight $\mathrm{M}=342$, and as the weight of a cubic centimetre of a ne per cent. solution of sugar $=1.003 \mathrm{gram}$, therefore the weight of sugar in a cubic centimetre of the solution, or $s$ in the preceding formula (p. $320: 6255 s(273+t)=\mathbf{M} p$ ), is equal to 0.01003 gram, and therefore, according to this formula (as $M=342$ and $t=14$ ), $p=52 \cdot 6$ centimetres. This shows that if the sugar were, instead of being in solution, in a state of vapour, then in following the law of AvogadroGerhardt it would produce a pressure equal to the osmotic pressure. This deduction (whose sense is at present not clear) forms the substance of Van't Hoff's doctrine (Chap. I. Note 19), when $i=1$ (Chap. I. Note 49).

Consequently, the molecular weight determines the osmotic pressure (and together with it the vapour tension and temperature of freezing, according to Note 49, Chap. I.), and therefore the molecular weight itself may be determined by the osmotic pressure as well as by the vapour density.

But so simple a relation only exists for dilute solutions of substances like sugar, which do not conduct an electric current, for which $i=1$. For salts and acids which conduct a current, this factor varies up to $i=4$ (Chap. I. Note 49). Arrhenius explains this phenomenon by supposing (partially after Hittorf and Clausius) that a portion of such substances in solutions, and especially in dilute solutions, occurs in a state of dissociation, and owing to this the number of molecules is multiplied (Chap. I. Note 45). As the conceptions of this order have been as yet but very little developed, and as in regarding solutions from this point of view, which is warmly supported by Ostwald, the water or solvent in general, which certainly plays an important part in solutions, and especially dilute ones, is entirely lost sight of, I consider it premature at present to explain the theory of Arrhenius, but think that it contains the seeds for further development and for its being merged into a fuller theory of solutions.

For the matter now under our consideration we need not see in the fact of the variability of $i$ any hindrance to employing $(a)$ the determination of the osmotic pressure, (b) the freezing point of a solvent (the so-called Raoult's method), and (c) the variation of the vapour density as means for finding the molecular weight of a substance in solution, not only in the ordinary cases when $i=1$, but even in those cases where $i>1$. These methods have already proved useful for solving the question of the molecular weight in many particular instances among the hydrocarbons, and some portion of their application to inorganic compounds will be mentioned in the further course of this work.

It may not be superfluous to remark that the osmotic pressure in the cells of organisms attains several atmospheres, and probably forms one of the causes determining the 
and liquids (as will afterwards be shown) is chiefly determined by the weights of the atoms of the elements entering into their composition, inasmuch as heavy (free) elements and compounds are only met with among substances containing elements with large atomic weights, such as gold, platinum, and uranium. And these elerrents themselves, in a free state, are the heaviest of all elements. Substances containing such light elements as hydrogen, carbon, oxygen, and nitrogen (like many organic substances) never have a high specific gravity; in the majority of cases it scarcely exceeds that of water. The density generally decreases with the augmentation of the amount of hydrogen, as the lightest element, and a substance is often obtained lighter than water. The refractive power of substances also entirely depends on the composition and the properties of the component elements. ${ }^{30}$ The history

individual functions of the cells. The further development of the questions touching on this subject should in this manner not only aid the perfecting of the theory of solutions but also the further progress of physiological science.

30 With respect to the optical refractive power of substances, it must first be observed that the coefficient of refraction is determined by two methods: $(a)$ either all the data are referred to one definite ray-for instance, to the Frauenhofer (sodium) line D of the solar spectrum - that is, to a ray of definite wave length, and often to that red ray (of the hydrogen spectrum) whose wave length is 656 million parts of a millimeire; $(b)$ or Cauchy's formula is used, showing the relation between the coefficient of refraction and dispersion to the wave length $n=\mathrm{A}+\frac{\mathrm{B}}{\alpha^{2}}$, where $\mathrm{A}$ and $\mathrm{B}$ are two constants varying for every substance but constant for all rays of the spectrum, and $\alpha$ is the wave length of that ray whose coefficient of refraction is $n$. In the latter method the investigation usually concerns the magnitudes of $\mathrm{A}$, which are independent of dispersion. We shall afterwards cite the data, investigated by the first method, by which Gladstone, Landolt, and others established the conception of the refraction equivalent.

The coefficient of refraction $n$ for a given substance decreases, as has long been known, with the density of a substance $\mathrm{D}$, so that the magnitude $(n-1) \div \mathrm{D}=c$ is almost constant for a given ray (having a definite wave length) and for a given substance. This constant is called the refractive energy, and its product with the atomic or molecular weight of a substance the refraction equivalent. The coefficient of refraction of oxygen is $1 \cdot 00021$, of hydrogen, $1 \cdot 00014$, their densities (referred to water) are 0.00143 and 0.00009 , and their atomic weights, $\mathrm{O}=16, \mathrm{H}=1$; hence their refraction equivalents are 3 and 1.5 . Water contains $\mathrm{H}_{2} \mathrm{O}$, consequently the sum of the equivalents of refraction is $(2 \times 1.5)+$ $3=6$. But as the coefficient of refraction of water $=1 \cdot 331$, therefore its refraction equivalent $=5 \cdot 958$, or nearly 6 . The comparison shows that, approximately, the sum of the refraction equivalents of the atoms forming compounds (or mixtures) is equal to the refraction equivalent of the compound. According to the researches of Gladstone, Landolt, Hagen, Briihl and others, the refraction equivalent of the elements are $-\mathrm{H}=1 \cdot 3, \mathrm{Li}=3 \cdot 8$, $\mathrm{B}=4 \cdot 0, \mathrm{C}=5 \cdot 0, \mathrm{~N}=4 \cdot 1$ (in its highest state of oxidation, $5 \cdot 3$ ), $\mathrm{O}=3 \cdot 0, \mathrm{~F}=1 \cdot 4, \mathrm{Na}=4 \cdot 8$, $\mathrm{Mg}=7 \cdot 0, \mathrm{Al}=8 \cdot 4, \mathrm{Si}=6 \cdot 8, \mathrm{P}=18 \cdot 3, \mathrm{~S}=16^{\circ} 0, \mathrm{Cl}=9 \cdot 9, \mathrm{~K}=8 \cdot 1, \mathrm{C} \mathrm{a}=10^{\circ} 4, \mathrm{Mn}=12 \cdot 2, \mathrm{Fe}=12^{\cdot} 0$, (in the salts of its higher oxides $20^{\circ} 1$ ), $\mathrm{Co}=10 \cdot 8, \mathrm{Cu}=11 \cdot 6, \mathrm{Zn}=10^{\cdot} 2, \mathrm{As}=15 \cdot 4, \mathrm{Bi}=15^{\bullet}$, $\mathrm{Ag}=15 \cdot 7, \mathrm{Cd}=13 \cdot 6, \mathrm{I}=24 \cdot 5, \mathrm{Pt}=26^{\circ} 0, \mathrm{Hg}=20^{\circ} 2, \mathrm{~Pb}=24 \cdot 8$, \&c. The refraction equivalents of many elements could only be calculated from the solutions of their compounds. The composition of a solution being known it is possible to calculate the refraction equivalent of one of its component parts, those for all its other components being known. The results are founded on the acceptance of a law which cannot be strictly applied. Nevertheless the conception of the refraction equivalents gives an easy means for directly, 
of chemistry presents a striking example in point-Newton foresaw from the high refractive index of the diamond that it would contain a combustible substance since so many combustible oils have a high refractive power.

As regards purely chemical relations, especially the understanding of reactions and the structure of substances, the fecundity of the law of Avogadro-Gerhardt is evinced at every step of the contemporary path of chemistry. Starting from the laws and conceptions of Lavoisier the chemistry of our time is entirely founded on the laws of Dalton and Avogadro-Gerhardt, on the doctrine of Berthollet respecting the equilibria brought about in chemical actions, and on the conceptions of dissociation introduced into the science by Saint-Claire Deville.

although only approximately, obtaining the coefficient of refraction from the chemical composition of a substance. For instance, the composition of carbon bisulphide, $\mathrm{CS}_{2}=\mathbf{7 6}$, and from its density, $1 \cdot 27$, we find its coefficient of refraction to be 1.618 (because the refraction equivalent $=5+2 \times 16=37$ ), which is very near the actual figure. It is evident that in the above representation compounds are looked on as simple mixtures of atoms, and the physical properties of a compound as referable to the properties present in the elementary atoms forming it. If this representation of the presence of simple atoms in compounds did not exist, then the effort to combine by a few figures a whole mass of data relating to the coefficient of refraction of different substances would hardly arise. 


\title{
('H.APTER V'II
}

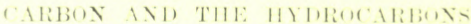

It is neceneary to aleatly distinguish between the two closely-allied

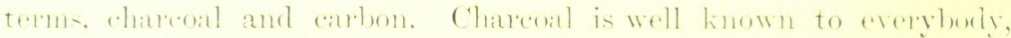
although it is no easy matter to obtain it in at ehemically pure state. Pure charenal is a simple, insoluble infu-ible. combustible substance moduced by heating organic matter, and has the familiar a-pect of a black mass, devoid of any arstalline structure, and completely in- luble. C'hareoal is a substance posecsing a certain combination of phrysical and ehemical properties. This substance, whilst in a state of ignition, combines directly with oxygen: in oreanie substances it is found in combination with hydrogen, oxyen, sitrogen, and sulphur.

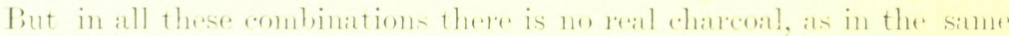
seree there is no ice in steans. What is found in such comblinations is termed 'artion' - that is, an element eommon to ehatrenal, to thome subsances which ean he formed hy it, and also to those substances from

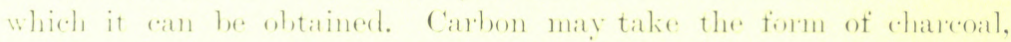
but alpears also as diamond and as graphite. It is true that no

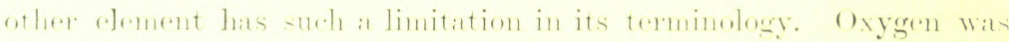

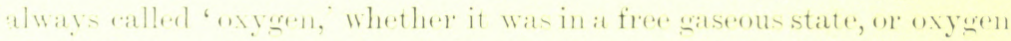

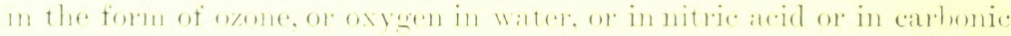

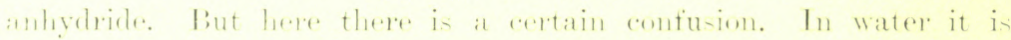
evident that there is an oxyegen in a caterous forme, such as and be

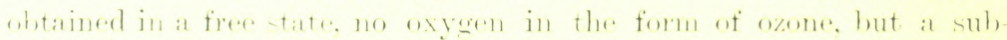

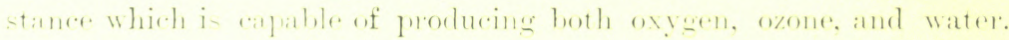

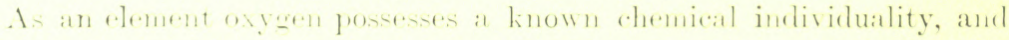

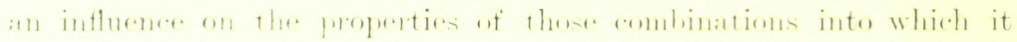

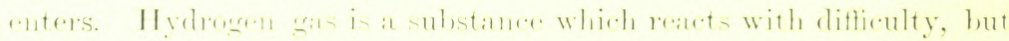

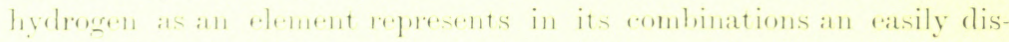

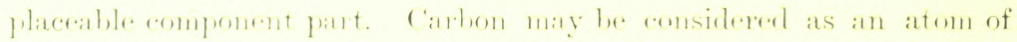

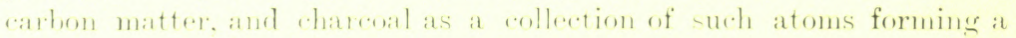
whole substance, as molecules in the mase of the substance. The

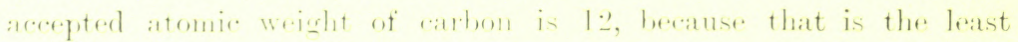


quantity of carbon which enters into combination in molecules of its compounds; but the weight of the molecules of charcoal is probably very great. This weight remains unknown, because charcoal is capable of but few direct reactions, and those only at a high temperature (when the weight of its molecules probably changes, as when ozone changes into oxygen), when it does not turn into vapour. Carbon exists in nature, both in a free and combined state, in most varied forms and aspects. Carbon in a free state is found in at least three different forms, as charcoal, graphite, and the diamond. In a combined state it enters into the composition of what are called organic substances-a multitude of substances which are found in all plants and animals. It exists as carbonic anhydride both in air and in water, and in the soil and crust of the earth as salts of carbonic acid and as organic remains.

The variety of the substances of which the structure of plants and animals is built up is familiar to all. Wax, oil, turpentine, and tar, cotton and albumin, the tissue of plants and the muscular fibre of animals, vinegar and starch, are all vegetable and animal matters, and all carbon compounds. ${ }^{1}$ The sphere of carbon compounds is so vast

1 Wood is the non-vital part of ligneous plants; the vital part of ordinary trees is situated between the bark and the lignin. Every year a layer of lignin is deposited on this part by the juices which are absorbed by the roots and worked up by the leaves; for this reason the age of trees may be determined by the number of lignin layers deposited. The following year the juices travel over a new layer, and in this way the layer already deposited serves only as a support for the vital parts of the tree. A living tree may be regarded as an agglomeration of many plants living on one support. The woody matter consists principally of fibrous tissue on to which the lignin or so-called incrusting matter has been deposited. The tissue has the composition $\mathrm{C}_{6} \mathrm{H}_{10} \mathrm{O}_{5}$, the substance deposited on it contains more carbon and hydrogen and less oxygen. This matter is saturated with moisture when the wood is in a fresh state. Fresh birch wood contains about 31 p.c. of water, lime wood 47 p.c., oak 35 p.c., pine and fir about 37 p.c. When dried in the air the wood loses a considerable quantity of water and not more than 19 p.c. remains. By artificial means this loss of water may be increased. If water be driven into the pores of wood it becomes heavier than water, as the lignin of which it is composed lias a density of about $1 \cdot 6$. One cubic centimetre of birch wood does not weigh inore than 0.901 grams, fir 0.894 , lime tree 0.817 , poplar 0.765 when in a fresh state ; when in a dry state birch weighs 0.622 , pine 0.550 , fir 0.355 , lime 0.430 , guaiacum 1.342 , ebony 1.226 . It is not out of place to remark here that on one deciatin $(2 \cdot 7$ acres $)$ of woodland the yearly growtl averages an amount of 3000 kilograms, or 180 poods of wood, but rarely reaches as much as 5000 . The average chemical composition of wood dried in air may be expressed as follows:- Hygroscopic water 15 p.c., carbon 42 p.c., hydrogen 5 p.c., oxygen and nitrogen 37 p.c., ash 1 p.c. Wood parts with its hygroscopic water at $150^{\circ}$, and decomposes at about $300^{\circ}$, giving a brown, brittle, so-called red charcoal; above $350^{\circ}$ black charcoal is produced. From the above-mentioned average composition of wood it is evident that the hydrogen may be accepted as being in about the quantity to combine with the oxygen, as the lyddrogen contained in the wood requires for its combustion about forty parts by weight of oxygen. Therefore all that burns of the wood is the carbon which it contains, 100 parts of wood only giving out as much heat as forty parts of charcoal. Charcoal gives out much more useful heat than wood because the water contained in tlie wood, or formed by the combination of its oxygen and hydrogen, has to be 


\section{that it forms a separate branch of chemistry, which treats of the com- pounds of carbon, part of which are met with in plants and animals.}

evaporated during its combustion. The above-mentioned composition of wood shows that it would be far more profitable to use charcoal for heating purposes than wood, if it were possible to obtain it in such quantities as correspond with its percentage ratio-that is, forty parts per 100 parts of wood. Generally, however, the quantity produced is far less, not more than 30 p.c., because part of the carbon is given off as gas, tar, \&c. If wood has to be transported great distances, or if it is necessary to obtain a very high temperature by burning it, then even as little as 25 p.c. of charcoal from 100 parts of wood may be advantageous. Charcoal (wood) develops on burning 8000 heat units, whilst wood dried in air does not develop more than 2800 units of heat; therefore seven parts of charcoal give as much heat as twenty parts of wood; but twenty parts of wood are only capable of yielding five parts of charcoal. This datum may be used for comparing the heating power of wood and charcoal. As regards the temperature of combustion, it is far higher with charcoal than with wood, because twenty parts of buming wood give, besides the carbonic anhydride which is also formed with charcoal, eleven parts of water, the eraporation of which requires a considerable amount of heat.

The composition of the growing parts of plants, the leaves, young branches, shoots, \&c., differs from the composition of the wood in that these vital parts contrin a considerable quantity of sap which contains much nitrogenous matter (in the wood itself there is very little), mineral salts, and a large amount of water. Taking, for example, the composition of clover and pasture hay in the green and dry state. In 100 parts of green clover there is about 80 p.c. of water and 20 p.c. of dry matter, in which there are about 3.5 parts of nitrogenous matter, about $9 \cdot 5$ parts of soluble and about 5 parts of insoluble non-nitrogenous matter, and about 2 p.c. of ash. In dry clover or clover-hay there is about 15 p.c. of water, 13 p.c. of nitrogenous matter, and 7 p.c. of ash. This composition of grassy substances shows that they are capable of forming the same sort of charcoal as wood itself. It also shows the difference of nutritive properties existing between wood and the substances mentioned. These latter serve as food for animals, because they contain those substances which are capable of being dissolved (entering into the blood) and forming the body of animals; such substances are proteids, starch, \&c. Let us remark here that with a good harvest an acre of land gives in the form of grass as much organic substance as it yields in the form of wood.

One hundred parts of dry wood are capable of giving, by means of dry distillation, besides 25 p.c. of charcoal and 10 p.c. or more of tar, 40 p.c. of watery liquid, containing acetic acid and wood spirit, and about 25 p.c. of gases, which may be used for heating or lighting purposes, because they do not differ from ordinary illuminating gas, which can indeed be obtained from wood. As wood-charcoal and tar are costly products, in some cases the dry distillation of wood is carried on principally for producing them. For this purpose those kinds of woods are particularly advantageous which contain resinous substances, especially coniferous trees, such as fir, pine, \&c.; birch, oak, and ash give much less tar, but on the other hand they yield more watery liquid. The latter is used for the manufacture of wood spirit, $\mathrm{CH}_{4} \mathrm{O}$, and acetic acid, $\mathrm{C}_{2} \mathrm{H}_{4} \mathrm{O}_{2}$. In such cases the dry distillation is carried on in stills. Stills are nothing more than horizontal or vertical cylindrical retorts, made of boiler plate, heated with fuel and having apertures at the top and sometimes also at the bottom for the exit of the light and heavy products of distillation. The dry distillation of wood in stoves is carried on in two ways, either by burning a portion of the wood inside the stove in order to submit the remainder to dry distillation by means of the heat obtained in this manner, or by placing the wood in a stove the thin sides of which are surrounded with a flue leading from the fuel, placed in a space below.

The first means does not give such a large amount of liquid products of the dry distillation as the latter. In the latter process there is generully an outlet below for 
This branch of chemistry is known under the name of organic chemistry - that is, the chemistry of carbon compounds, or, more strictly, of the hydrocarbons and their derivatives.

If any one of these organic compounds be strongly heated without access of air-or, better still, in a vacuum-it decomposes with more or less facility. When organic substances are heated in air, it is well known that they burn; but if the supply of air be insufficient, or the temperature be too low for combustion, and if the first volatile products of transformation of the organic matter are subjected to condensation (for example, if the door of a stove be opened), an imperfect combustion takes place, and smoke, with charcoal or soot, is formed. ${ }^{2}$

emptying out the charcoal at the close of the operation. For the dry distillation of 100 parts of wood from forty to twenty parts of fuel are used.

There are many steps between the method of burning wood in stacks (Note 4), and that of burning it in an enclosed space-namely, those in which the burning of the char-

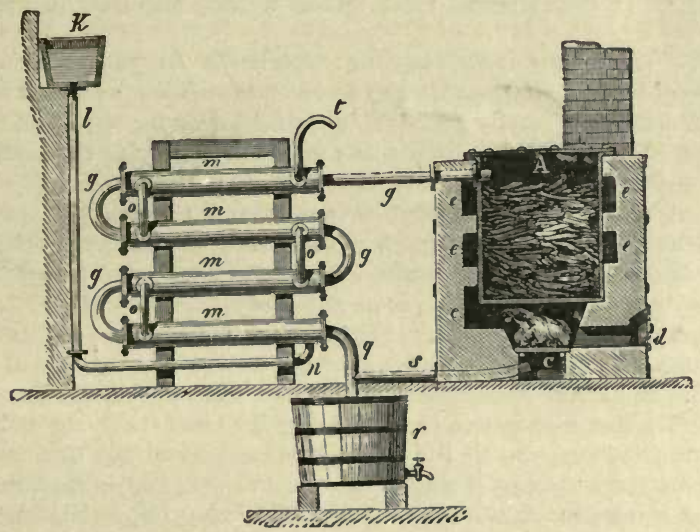

FrG. 57.-Apparatus for the dry distillation of wood. The retort $a$ containing the wood is heated by the flues $c e$. The steam and volatile products of distillation pass along the tube $g$ through the condenser $m$, where tliey are condensed. The form, distribution, and dimensions of the apparatus vary.

coal is accompanied by a certain production of tar. This is effected by means of trenches dug in the earth and having sloping bottoms, by which contrivance the tar separated by the charring of the wood flows into special receivers. This process is much used in the north of Russia.

In the north of Russia wood is so plentiful and cheap that this locality is admirably fitted to become the centre of a general trade in the products of the dry distillation of wood. Coal (Note 6), sea-weed, turf, animal substances (Chap. VI.), \&c., are also submitted to the process of dry distillation.

2 The result of imperfect combustion is not only the loss of a part of the fuel and the production of smoke, which in some respects is inconvenient and injurious to health, but also a low flame temperature, which means that a less amount of heat is transmitted to the object heated. Imperfect combustion is not only always accompanied by the formation of soot or unburnt particles of charcoal, but also by that of carbonic oxide, $\mathrm{CO}$, in the 
The nature of the phenomenem, and the products arising from it, are the sime as those produced hy heating alone, as that part which is in at state of combustion serves to heat the remaindere of the fuel. 'The rlecomposition which takes plate on heating a compromel composed of cambor:, hydrogen, and oxyenen is as follows:- A part of the hydrogen is sepalrated in a gaseous state, another part in conbination witl oxygen, and a thirel part separates in combination with carbon, and sometimes in combination with carlon and oxygen in the form of gaseous or volatile products, or, as they are also called, the products of dry distillation. If the vapours of these products are passerl through a highly-heated tube, then they are changed again in a similar manner, and finally resolve themselves into hydrogen and chareal. All these various products of decomposition contain a smaller amount of carbon than the minnary olganic matter; prat of the carbon seluarates in combination with hydrogen and oxygen, hut part of it remains in a free state, forming chatrenal. ${ }^{3}$ It remains in that space where the elecomposition takes

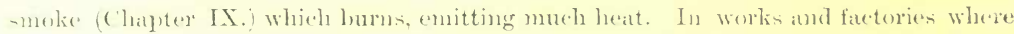

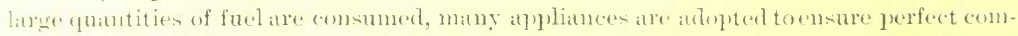
bustion, and to combut arainst such a rumons pratetice as the inuperfect eombustion of fuel. The ment effective and raclical means consists in employing the ('ombustible gatres

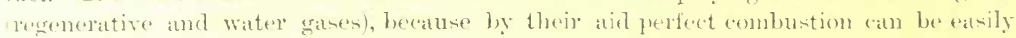

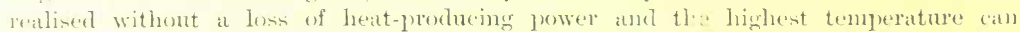
be reached. Whem solid fuel is used (such as coal, work, and turf), imperfect combustion

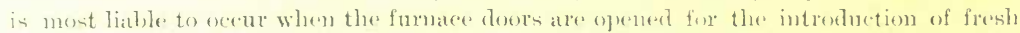

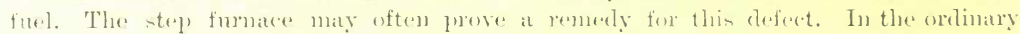

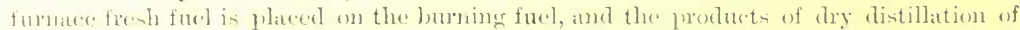

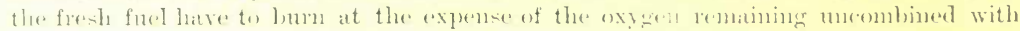

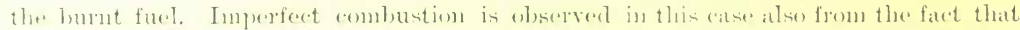

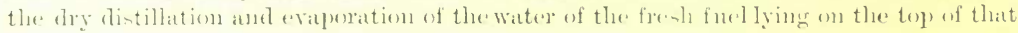
burnt, lewers the tenuperature of the flame, beranse part of the heat beennes latent.

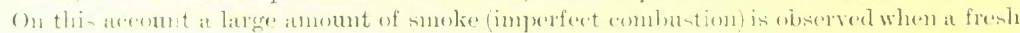

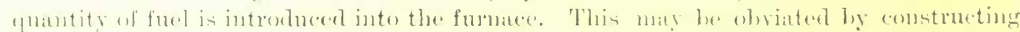

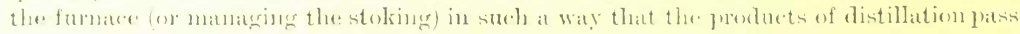

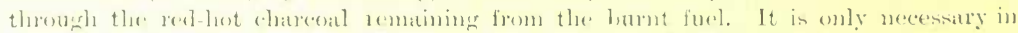

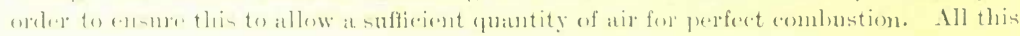

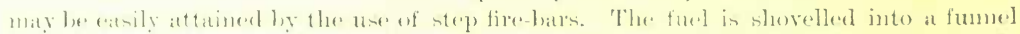

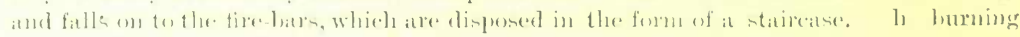

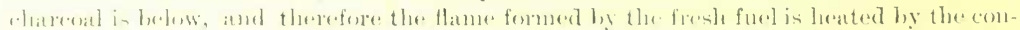

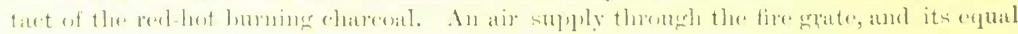

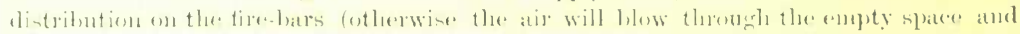

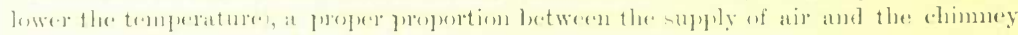

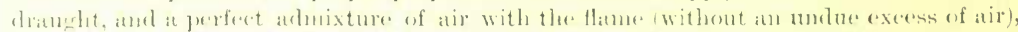

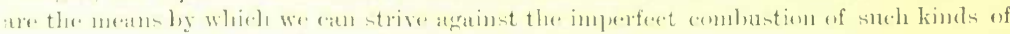

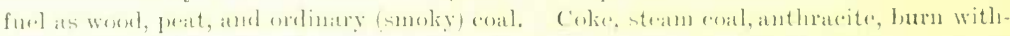

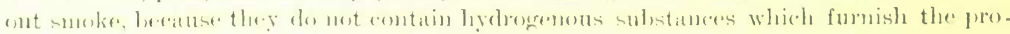

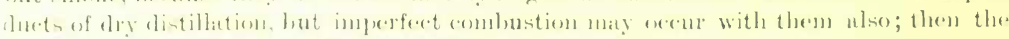

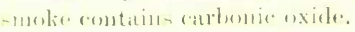

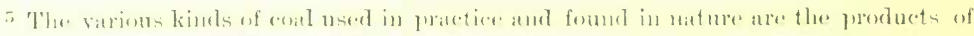

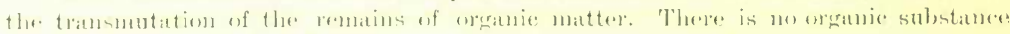

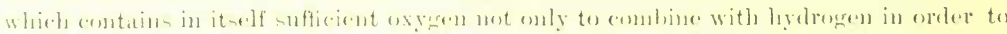


place in the shape of a black, infusible, non-volatile charcoal familiar to all. The earthy matter and all non-volatile substances (ash) forming a part of the organic matter, remain behind with the charcoal. The tar-like substances, which require a high temperature in order to decompose them, also remain mixed with charcoal. If a volatile organic substance, such as a gaseous compound containing oxygen and hydrogen, be taken, the carbon separates on passing the vapour through a

form water, but also to unite with the carbon to form carbonic anhydride. The greater part of regetable tissue consists of the cellulose, $\mathrm{C}_{6} \mathrm{H}_{10} \mathrm{O}_{5}$; from its composition it is evident that the oxygen is insufficient to transform the hydrogen into water and the carbon into carbonic anhydride, because for this purpose it would require 17 equivalents and it only contains 5 . This reasoning also refers to all the remaining organic substances. Under the action of air, organic substances are capable of oxidising to such an extent that all the carbon and all the hydrogen they contain will be transformed into carbonic anhydride and water. The refuse of plants and animals are subjected to such a change whether they slowly decompose and putrefy, or rapidly burn, with direct access to air. But if the supply of air be limited, then, in virtue of the abore-stated reasoning, there can be no complete trunsformation into water and carbonic anhydride, and therefore, if organic matter decomposes under these conditions, charcoal must remain, as it is a non-volatile substance. All organic substances are unstable, they do not resist heat, and in time easily change at ordinary temperatures, particularly if water be present. Therefore it is easy to understand that charcoal may be obtained in many eases through the transformation of substances entering into the composition of organisms, but that it is never found in a pure state.

The transformation of organic matter is not, however, so simple as would appear from the preceding statements; that is to say, water and carbonic anhydride are not the only products separated from organic substances. Carbon, hydrogen, and oxygen are capable of giving a multitude of compounds; some of these are volatile compounds, gaseous, soluble in water-they are carried off from organic matter, undergoing change without access of air. Others, on the contrary, are non-volatile, rich in carbon, constant under the influence of heat and other agents. The latter remain in admixture with charcoal where the decomposition takes place; such, for example, are tarry substances. The quantity of those bodies which are found mixed with the charcoal is very varied, and depends on the energy and duration of the decomposing influence. For instance, when wood is first acted on by heat, the moisture separates; it then turns brown, but still contains a large amount of oxygen and hydrogen. If the action be further continued the quantity of these latter elements diminishes, and the proportion of carbon in the residue increases, although a part of it is carried off in the shape of the volatile products of decomposition. The greater the heat, the less the quantity of charcoal obtained, and the less the amount of hydrogen and oxygen contained in the remaining charcoal. The annexed table shows, according to the data of Violette, those changes to which wood is subjected at various temperatures when submitted to dry distillation by means of superheated steam :-

\begin{tabular}{|c|c|c|c|c|c|}
\hline Temperature & $\begin{array}{c}\text { Residue } \\
\text { from } 100 \text { parts } \\
\text { of alder wood }\end{array}$ & \multicolumn{4}{|c|}{ In 100 parts of the residual charcoal } \\
\hline $\begin{array}{r}150^{\circ} \\
350^{\circ} \\
1032^{\circ} \\
1500^{\circ}\end{array}$ & $\begin{array}{r}100 \cdot 0 \\
29 \cdot 7 \\
18 \cdot 7 \\
17 \cdot 3\end{array}$ & $\begin{array}{c}\mathrm{C} \\
47 \cdot 5 \\
76 \cdot 6 \\
81 \cdot 9 \\
95 \cdot 0\end{array}$ & $\begin{array}{l}H \\
6 \cdot 1 \\
4 \cdot 1 \\
2 \cdot 3 \\
0 \cdot 7\end{array}$ & $\begin{array}{c}O \text { and } N \\
46 \cdot 3 \\
18 \cdot 4 \\
14 \cdot 1 \\
3 \cdot 8\end{array}$ & $\begin{array}{l}\text { Ash } \\
0.1 \\
0.6 \\
1 \cdot 6 \\
1 \cdot 7\end{array}$ \\
\hline
\end{tabular}


tube heated to a high temperature. Organic substances, when burning with an insufficient supply of air, give off soot - that is, charcoal-proceeding from carbon compounds in a state of vapour, the hydrogen of which has, by combustion, been converted into water ; so, for instance, turpentine, naphthalene, and other hydrocarbons which are with difficulty decomposed by heat, easily yield carbon in the form of soot during combustion. Chlorine and other substances, which, like oxygen, are capable of taking up hydrogen, and also substances which are capable of taking up water, can also separate carbon from (or char) most organic substances.

When organic substances are burning in an insufficient supply of air, they both separate and leave charcoal. Part of it remains behind as a residue, as, for instance, after burning wood in a stove some remains in the form of charcoal. Wood charcoal is prepared in large quantities. in a similar manner - that is, by the partial combustion of wood. ${ }^{4}$ In nature a like process of carbonisation of vegetable refuse takes place in its transformation under water, as shown by the marshy vegetation which forms peat.5 In this manner, doubtless, the enormous masses

4 The object of producing charcoal from wood has been explained in Footnote 1. Wood charcoal is obtained in so-called stacks by partially burning the wood, or by means of dry distillation-that is, by heating wood in all enclosed space (in retorts, Note 1)-without the access of air. It is principally manufactured for metallurgical processes, especially for smelting and forging iron-that is, for use in smithies. The preparation of charcoal in stacks has one advantage, and that is that it may be done on any spot in the forest. But in this way all the products of dry distillation are lost. For charcoal burning, a pile or stack is generally built, in which the logs are closely placed, either horizontally, vertically, or inclined, forming a stack of from six to fifty feet in diameter and even larger. Under the stack there are several horizontal air passages, and an opening in the middle to let out the smoke. The surface of the stack is covered with sods to a considerable thickness, especially the upper part, in order to hinder the free passage of air and to concentrate the lieat inside. When the stack is kindled, the pile begins to settle down by degrees, and it is then necessary to look after the turf casing and keep it in repair. As the combustion spreads throughout the whole pile, the temperature rises and real dry distillation commences. It is then necessary to stop the air holes, in order as much as possible to prevent unnecessary combustion. The nature of the process is, that part of the fuel burns and develops the heat required for subjecting the remainder to dry distillation. The charring of the stack lasts about a fortnight, and is brought to a termination by scattering earth over the incandescent charcoal to stop the combustion. The charring is stopped when the products of dry distillation, which are emitted, no longer burn with a brilliant flame, but the pale blue flame of carbonic oxide appears. Dry wood in stacks yields about one-fourth of its weight of charcoal.

5 When dead vegetable matter undergoes transformation in air, in the presence of moisture, there remains a substance much richer in carbon-namely, humus, black earth or mould. 100 parts of humus in a dry state contain about 70 p.c. of carbon. The roots, leaves, and stems of plants which wither and fall off form a soil rich in humus. The nori-vital vegetable substances (ligneous tissue) first form brown matter (ulmic compounds), and then black matter (humic substances), which are both insoluble in water; after this a brown acid is produced, which is soluble in water (apocrenic acid), 


\section{of coal were formed, ${ }^{6}$ which, following the example set by England, are now utilised everywhere as the principal material for heating steam}

and lastly a colourless acid also soluble in water (crenic acid). Alkali dissolves a part of the original brown and black substances, forming solutions of a brown tint (ulmic and humic acids), which sometimes communicate their colour to springs and rivers. The composition of the humus in the soil is generally in connection with its fertility; firstly, because putrefying plants develop carbonic anhydride and ammonia, and yield the saline substances which are necessary to vegetation; secondly, because humus is capable of attracting the moisture of the air and of absorbing water (twice its weight) and in this way keeps the soil in a damp condition, which is indispensable for nourishment; thirdly, humus renders the soil porous, and, fourthly, renders it more capable of absorbing the heat of the sun's rays. On this account black earth is often most remarkable for its fertility. One object of manuring is to increase the quantity of humus in the soil, and any easily changeable vegetable or any animal matter (composts) may be used. The boundless tracts of black earth soil in Russia are capable of bestowing countless wealth on the country.

The origin and extent of black earth soil is treated in detail in Professor Dokouchaeff's works.

If those substances which produce humus undergo decomposition under water, less carbonic anhydride is formed, a quantity of marsh gas, $\mathrm{CH}_{4}$, is evolved, and the solid residue forms an acid humus, found in great quantities in marshy places, and called peat. Peat especially abounds in the lowlands of Holland, North Germany, Ireland, and Bararia. In Russia it is likewise found in large quantities, especially in the North-west districts. The old hard forms of peat resemble in composition and properties brown coal; the newest formations, as yet unhardened by pressure, form very porous masses which retain traces of the vegetable matter from which they have been formed. Dried (and sometimes pressed) peat is used as fuel. The composition of peat varies considerably with the locality in which it is found. When dried in air it does not contain less than 15 p.c. of water and 8 p.c. of ash; the remainder consists of 45 p.c. of carbon, 4 p.c. of hydrogen, 1 p.c. of nitrogen, and 28 p.c. of oxygen. Its heating power is about equivalent to that of wood. The brown earthy varieties of coal were probably formed from peat. In other cases they have a marked woody structure, and are then known as lignites. The composition of the brown sorts of coal resembles in a marked degree that of peat-namely, in a dried state brown coal contains on an average 60 p.c. of carbon, 5 p.c. of hydrogen, 26 p.c. of oxygen and nitrogen, and 9 p.c. of ash. In Russia brown coal is met with in many districts near Moscow, in the governments of Toula and Tver and the neighbourhood; it is very generally used as fuel, particularly when found in thick seams. The brown coals generally burn with a flame like wood and peat, and are akin to them in heating power, which is two or three times less than that of the best coal.

6 Grass and wood, the vegetation of primæval seas and similar refuse of all geological periods, must have been, in many cases, subjected to the same changes they now undergo-that is, under water they formed peat and lignites. Such substances, preserved for a long time underground, subjected to the action of water, compressed by the new strata formed above them, transformed by the separation of their more volatile component parts (peat and lignites, even in a finished state, still continue to evolve nitrogen, carbonic anhydride, and marsh gases), form coal. Coal is a dense homogeneous mass, dark brown or black, with an oily or glassy lustre, or, more rarely, dull, without any evident vegetable structure; this distinguishes it in appearance from the majority of lignites. The density of coal (not counting the admixture of pyrites, \&c.) varies from 1.25 (dry bituminous coal) to 1.6 (anthracite, flameless), and even reaches 1.9 in the very dense variety of coal found in the Olonetzky government (termed thungite), which, according to the investigations of Professor Inostrantzeff, may be regarded as the extreme member of the various forms of coal.

In order to explain the formation of coal from vegetable matter, Caigniard de la Tour 


\section{boilers, and in general for all purposes of heating and burning. ${ }^{7}$ Russia}

enclosed pieces of dried wood in a tube and heated them to the boiling point of mercury, when the wood was changed into a semi-liquid black mass from which a substance exceedingly like coal separated. In this manner some kinds of wood formed coal, which, on being heated, left caking coke, others non-caking; precisely as we find with the natural varieties of coal. Violette repeated these experiments with wood dried at $150^{\circ}$, and showed that when wood is decomposed in this way a gas, a watery liquid, and a residue are formed. The latter, at a temperature of $200^{\circ}$, has the properties of wood charcoal incompletely burnt; at $300^{\circ}$ and higher a homogeneous mass like coal is formed, which at $340^{\circ}$ is dense and without cavities. At $400^{\circ}$ the residue resembles anthracite. In nature probably the decomposition was effected by heat alone in rare cases; more generally it was effected by means of water and heat, but in either case the result ought to be almost the same.

The average composition of coal compiled from many analyses, disregarding the ash, is as follows : 84 parts of carbon, 5 parts of hydrogen, 1 part of nitrogen, 8 parts of oxygen, 2 of sulphur. The medium quantity of ash is 5 p.c., but there are coals which contain a large quantity, and naturally they are not so advantageous for use as fuel. As regards the amount of water, coal in this respect is much more profitable than lignite or peat, as it does not usually contain more than 10 p.c. of water. The anthracites form a remarkable variety of coals, and are indeed sometimes placed in a separate category; they do not give any volatile products, or but a very small amount, as they contain but little hydrogen compared to oxygen. In the average composition of coal we saw that for 5 parts of hydrogen there were 8 parts of oxygen; therefore 4 parts by weight of the hydrogen are capable of forming hydrocarbons, because 1 part of hydrogen is necessary in order to form water with the 8 parts of oxygen. These 4 parts by weight of hydrogen can convert 48 parts of carbon into the form of benzene or similar volatile products, because 1 part of hydrogen by weight in these substances combines with 12 parts of carbon. The anthracites differ essentially from this; neglecting the ash, their average composition is as follows: 94 parts of carbon, 3 of hydrogen, and 3 of oxygen and nitrogen. According to the analyses of A. A. Voskresensky, the Grousheffsky anthracite (Don district) contains: $\mathrm{C}=93 \cdot 8, \mathrm{H}=1 \cdot 7$, ash $=1 \cdot 5$. Therefore the anthracites contain but little hydrogen capable of combining with the carbon to form hydrocarbons, which burn with a flame. Anthracites are the most ancient forms of coal. The newest and least tranformed, which resemble some of the brown varieties, are the dry coals (Griiner's first group). They burn with a flame like wood, and leave a coke having the appearance of lumps of coal, half their component parts being absorbed by the flame (they contain much hydrogen and oxygen). The remaining varieties of coal (2nd group, gas coal; 3rd, smithy coal ; 4th, coking; and 5th, anthracite, according to Griiner) in all respects form connecting links between the dry coals and the anthracites. These coals burn with a very smoky flame, and on being heated leave coke, which bears the same relation to coal as charcoal does to wood. The quantity and quality of coke varies considerably with the different sorts of coal from which it is formed. In practice coals are most often distinguished by the properties and quantity of the coke which they give. In this particular the so-called bituminous coals are especially valuable, as even small coal (baked) of this kind gives by dry distillation large spongy masses of coke. If large pieces of these kinds of coal are subjected to dry distillation, they, as it were, melt, flow together, and form caking masses of coke. The best coking coals give 65 p.c. of dense caking coke. Such coal is very valuable for metallurgical purposes (see Note 8 ). Besides coke, the dry distillation of coal produces gas (see further, illuminating gas), coal-tar (which gives benzene, carbolic acid, naphthalene, tar for artificial asphalt, \&c.), and also a watery alkaline liquid (with wood and lignites the liquid is acid from acetic acid) which contains ammonium carbonate (see Note 6).

7 In England in 1850 the output of coal was already 48 million tons, and in latter years (1884-1888) it rose to about 160 millions. Besides this the other countries con- 
possesses many very rich coalfields, amongst which the Don district is most worthy of remark. ${ }^{8}$

During the imperfect combustion of volatile substances containing carbon and hydrogen, the hydrogen and part of the carbon first burn, and the remainder of the carbon forms soot. If oil of turpentine,

tribute 230 millions-Russia about $3 \frac{1}{2}$ millions, the United States of America come next to England with an output of 75 million tons, then Germany 60 millions; France produces but little ( 20 millions), and takes about 5 million tons from England. Besides household purposes, coal is chiefly used as fuel for steam-engines. As every horse-power (=75 kilogrammetres per second) of a steam-engine expends on the average more than 25 kilograms in 24 hours, or in a year (counting stoppages) not less than 5 tons per horse-power, and there are not less than 40 million horse-power at work in the world, the consumption of coal for motive-power is at least equal to half the whole production. For this reason coal serves as a standard for industrial development. About 15 p.c. of coal is used for the manufacture of cast iron, wrought iron, steel, and articles made of them.

8 The principal workable coal beds of Russia are : The Don basin (115 million poods per annum, 62 poods=1 ton), the Polish basin (Dombrovo and others 110 million poods per annum), the Toula and Riazan beds of the Moscow basin (up to 25 million poods), the Ural basin (10 million poods), the Caucasian (Kliboul, near Kutais), the Khirjhis steppes, the smithy coal basin (Gov. of Tomsk), the Sahaline, \&c. The Polish and Moscow basins do not give any coking coals. The presence of every variety of coal (from the dry coal near Lisichanena on the Donetz to the anthracites of the entire south-east basin), the great abundance of excellent metallurgical coal (coking, see Note 6) in the western part of the basin, its vast extent (as much as 25,000 sq. versts), the proximity of the seams to the surface (the shafts are now from 20 to 100 fathoms deep, and in England and Belgium as deep as 500 fathoms), the fertility of the soil (black earth), the proximity of the sea (about 100 versts from the Sea of Azoff) and of the rivers Donetz, Don, and Dnieper, the most abundant seams of excellent iron ore (Korsan Mogila, Krivoy rog, Soulin, \&c., \&c.), copper ore, mercury ore (near Nikitooka, in the Bakhmouth district of the Ekaterinoslav Gov.), and other ores, the richest probably in the whole world, the beds of rock-salt (near the stations of the Stoupka and Brianzovka), the excellent clay of all kinds (china, fire-clay), gypsum, slate, sandstone, and other wealth of the Don coal basin, give complete assurance of the fact that with the growth of industrial activity in Russia this bountiful land of the Cossacks and New Russia will become the centre of the most extensive productive enterprise, not only for the requirements of Russia alone, but of the whole world, because in no other place can be found such a concentration of favourable conditions. The growth of enterprise and knowledge, together with the extinction of the forests, which compels Russia to foster the production of coal, will help to bring about this desired result. The forest wealth of North Russia and the naphtha treasures of Caucasus can only contribute to its advancement, and have not the power to check that influence which the Don coal basin should have on the industrial state of Russia. England with a whole fleet of merchant vessels exports annually about 25 million tons of coal, the price of which is higher than on the Donetz (where a pood of worked coal costs less than 5 copecks on the average), where anthracites and semi-anthracites (like Cardiff or steam coal, which burns without smoke) and coking and metallurgical coals are able both in quantity and quality to satisfy the most. fastidious requirements of the industry already existing and rapidly increasing every. where. In 1850 the world's consumption and production of coal was only 5000 million poods, and now the quantity has risen to 25000 million poods. The coal mines of England and Belgium are approaching to a state of exhaustion, whilst in those of the Don basin, only at a depth of 100 fathoms 1200000 million poods of coal lies waiting. to be worked. 
or naphthalene or other hydrocarbons, be burned in the open air, soot is formed in large quantities, because these substances contain a large amount of carbon. Tar, pitch, and similar substances for this reason burn with a smoky flame. Thus, soot is finely-divided charcoal, separated during the imperfect combustion of the vapours and gases of carbonaceous substances rich in carbon. Specially-prepared soot (lampblack) is very largely used as a black paint, and a large quantity goes for the manufacture of printers' ink. The quantity of organic matter remaining undecomposed in the charcoal depends on the temperature to which it has been submitted. Charcoal, prepared at the lowest temperature, still contains a considerable quantity of hydrogen and oxygen-even as much as 4 p.c. of hydrogen and 20 p.c. of oxygen. Such charcoal still preserves the structure of the substance from which it was obtained. Ordinary charcoal, for instance, in which the layers of the tree are yet visible, is of this kind. On submitting it to further heating, a fresh quantity of hydrogen with carbon and oxygen (in the form of gases or volatile matter) may be separated, and the purest charcoal will be obtained on submitting it to the greatest heat. ${ }^{9}$ If it be required to prepare pure charcoal from soot, it is necessary first to wash it with alcohol and ether, in order to get rid of the soluble tarry products, and then submit it to a powerful heat to drive off the impurities containing hydrogen and oxygen. Charcoal, however, when completely purified does not change in appearance. Everybody knows that charcoal is a black amorphous substance, without any signs whatever of crystallisation (probably a colloid). Its porosity, ${ }^{10}$ bad conducting power for heat, capability of absorbing the

9 As it is difficult to separate from the charcoal the admixture of ash-that is, the earthy matter contained in the vegetable substance used for producing charcoal-in order to obtain it in its purest condition, it is necessary to use such organic substances as do not contain any ash, for example, completely refined or purified crystallised sugar, crystallised tartaric acid, \&c.

10 The cavities in charcoal are the passages through which those volatile products formed at the same time as the charcoal have passed. The degree of porosity of charcoal varies considerably, and has a technical significance, in different kinds of charcoal. The most porous charcoal is very light; a cubic metre of wood charcoal weighs about 200 kilograms. Many of the properties of charcoal which depend exclusively on its porosity are shared by many other porous substances, and vary with the density of the charcoal and depend on the way it was prepared. The power which charcoal has of absorbing gases, liquids, and many substances in solution, has reference to this. The densest kind of charcoal is formed by the action of great heat on sugar. The lustrous gray dense charcoal formed in gas retorts is also of this character. This dense charcoal collects on the internal walls of the retorts subjected to great heat, and is produced by the vapours and gases separated from the heated coal in the retorts. In virtue of its density, such charcoal becomes a good conductor of the galvanic current and approaches graphite. It is principally used in galvanic batteries. Coke, or the charcoal remaining from the imperfect combustion of coal and tarry substances, is also but 
luminous rays (due to its blackness and opacity), and many other qualities are familiar from everyday experience. ${ }^{11}$ The specific gravity of charcoal varies from 1.4 to 1.9 , and that it floats on water is due to the air contained in its pores. If charcoal be reduced to a powder and moistened with spirit, it immediately sinks in water. It is infusible in the furnace and even at the temperature of the oxyhydrogen flame. In the heat generated by means of a strong galvanic current, charcoal only softens, but does not completely melt, and on cooling it is found to have undergone a complete change both in properties and appearance, and is more or less transformed into graphite. The physical stability

slightly porous, brilliant, does not soil or mark paper, is dense, almost devoid of the faculty of retaining liquids and solids, and does not absorb gases. The light sorts of charcogl produced from charred wood, on the other hand, show this absorptive power in a most marked degree. This property is particularly developed in that very fine and friable charcoal prepared by heating animal substances, such as hides and bones. The absorptive power of charcoal with reference to gases, is similar to the condensation of gases in spongy platinum. Here evidently there is a phenomenon of the adherence of gases to a solid, precisely as liquids have the property of adhering to various solids. One volume of charcoal will absorb the following volumes of gases (charcoal is capable of absorbing an immense amount of chlorine, almost equal its own weight) :-

\begin{tabular}{|c|c|c|}
\hline $\begin{array}{c}\text { Saussure } \\
\text { Boxwood Charcoal. }\end{array}$ & $\begin{array}{c}\text { Favre } \\
\text { Cocoanut Cbarcoal. }\end{array}$ & $\begin{array}{l}\text { Heat emitted } \\
\text { per gram of gas. }\end{array}$ \\
\hline $\begin{array}{ll}\mathrm{NH}_{3} & 90 \\
\mathrm{CO}_{2} & 35 \\
\mathrm{~N}_{2} \mathrm{O} & 40 \\
\mathrm{HCl} & 85\end{array}$ & $\begin{array}{rc}172 & \text { vols. } \\
97 & " \\
99 & " \\
165 & "\end{array}$ & $\begin{array}{l}494 \text { units. } \\
158 \text { " } \\
169 \text { " } \\
274\end{array}$ \\
\hline
\end{tabular}

The quantity of gas absorbed by the charcoal increases with the pressure, and is approximately proportional to it. The quantity of heat given out by the absorption nearly approaches that set free on dissolving, or passing into a liquid condition.

Charcoal absorbs not only gases, but a number of other substances. For instance, alcohol, which contains disagreeably smelling fusel oil, on being mixed with charcoal or filtered though it, loses the bulk of the fusel oil. The practice of filtering substances through charcoal in order to get rid of foreign matters is often applied in chemical and manufacturing processes. Oils, spirits, various extracts, and vegetable and other solutions containing an admixture of colouring or odorous matters, and also water, are filtered through charcoal in order to purify them. The bleaching power of charcoal may be tested by using various coloured solutions-such as aniline dyes, litmus, \&c. Charcoal, which has absorbed one substance to saturation, is still capable of absorbing certain other substances. The more porous charcoal is, the greater its surface, and this is the reason why animal charcoal, produced in a very finely-divided state, especially by heating bones, makes the best sort for the purposes of absorption. Bone charcoal is used in large quantities in sugar works for filtering syrups, and all saccharine solutions, in order to purify them, not only from colouring and odorous matter, but also from lime. which is mixed with the syrups in order to render them less unstable during boiling, The absorption of lime by animal charcoal depends, in all probability, in a great degree on the mineral component parts of bone charcoal.

11 Charcoal is a very bad conductor of heat, and therefore forms an excellent insulator or packing to prevent the transmission of heat. A charcoal lining is often used in crucibles for heating many substances, as it does not melt and resists a far greater heat than many other substances.

VOL. I. 
of charcoal is, without doubt, allied to its chemical stability. It is evidently a substance devoid of energy, as charcoal is insoluble in all known liquids, and at an ordinary temperature does not combine with anything; it is an inactive substance, like nitrogen. ${ }^{12}$ But these properties of charcoal change with a rise of temperature ; thus, in contradistinction from nitrogen, charcoal, at a high temperature, combines directly with oxygen. This is well known, as charcoal burns in air. Indeed, not only does oxygen combine with charcoal at a red heat, but sulphur, hydrogen, and also iron and some other metals do so at a very high temperature - that is, when the molecules of the charcoal have reached a state of great instability - whilst at ordinary temperatures neither oxygen, sulphur, nor metals act on charcoal in any way. When burning in oxygen, charcoal forms carbonic anhydride, $\mathrm{CO}_{2}$, whilst in the vapours of sulphur carbon bisulphide, $\mathrm{CS}_{2}$, is formed, and wrought iron, when acted on by carbon, becomes cast iron. At the great heat obtained by passing the galvanic current through carbon electrodes, the charcoal combines with hydrogen, forming acetylene, $\mathrm{C}_{2} \mathrm{H}_{2}$. Charcoal does not combine directly with nitrogen, but in the presence of metals and alkaline oxides, nitrogen is absorbed, forming a metallic cyanide, as, for instance, potassium cyanide, KCN. From these few direct combinations which charcoal is capable of entering into, may be derived those numerous carbonaceous compounds which enter into the composition of plants and animals, and which are obtained artificially. Certain substances containing oxygen give up a part of it to carbon at a relatively low temperature. For instance, nitric acid when boiled with charcoal gives carbonic anhydride and nitric peroxide. Sulphuric acid is reduced to sulphurous anhydride when heated with carbon. When heated to redness charcoal absorbs oxygen from a large number of the oxides. Even such oxides as those of sodium and potassium, when heated to redness, yield their oxygen to charcoal, although they do not part with it to hydrogen. Only a few of the oxides, like silica (oxide of silicon), and lime (calcium oxide), resist the reducing action of charcoal. Charcoal is capable of changing its physical condition without undergoing any alteration in its essential chemical properties - that is, it passes into isomeric or

12 The unalterability of charcoal under the action of atmospheric influences, which produce changes in the majority of stony and metallic substances, is often made use of in practice. For example, charcoal is frequently strewn in boundary ditches. The surface of wood is often charred to render it durable in those places where the soil is damp, and wood itself would soon rot. The chambers (or in some works towers) through which acids pass (for example, sulphuric and hydrochloric) in order to bring them into contact with gases or liquids, are filled with charcoal or coke, because, at ordinary temperatures, it resists the action of even the most energetic acids. 
allotropic forms. The two particular forms in which carbon appears are the diamond and graphite. The identity of the composition of these with charcoal is proved by burning an equal quantity of all three separately in oxygen (at a great heat), when each gives the same quantity of carbonic anhydride - namely, 12 parts of charcoal, diamond, or graphite in a pure state, yield on burning 44 parts by weight of carbonic anhydride. The physical properties present a marked contrast; the densest sorts of charcoal have a density of only 1.9 , whilst the density of graphite is about $2 \cdot 3$, and that of the diamond $3 \cdot 5$. A great many other properties depend on the density, for instance, com bustibility. The lighter charcoal is, the more easily it burns ; graphite burns with considerable difficulty even in oxygen, and the diamond burns only in oxygen and at a very high temperature. On burning, charcoal, the diamond, and graphite develop different quantities of heat. One part by weight of wood charcoal converted by burning into carbonic anhydride develops 8080 heat units;. dense charcoal separated in gas retorts develops 8050 heat units; natural graphite, 7800 heat units; and the diamond, 7770 . The greater the density the less the heat evolved by the combustion of the carbon. ${ }^{13}$

By means of intense heat charcoal may be transformed into graphite. If a charcoal rod $4 \mathrm{~mm}$. in diameter and $5 \mathrm{~mm}$. long be enclosed in an exhausted receiver and the current from 600 Bunsen's elements, placed in parallel series of 100 , be passed through it, the charcoal becomes strongly incandescent, partially volatilises, and is deposited in the form of graphite. If sugar be placed in a charcoal crucible and a powerful galvanic current passed through it, it is baked into a nuass similar to graphite. If charcoal be mixed with wrought iron and heated, cast iron is formed, which absorbs as much as fire per cent. of charcoal. If molten cast iron be suddenly chilled, the carbon remains in combination with the iron, forming so called white cast iron ; but if the cooling proceeds slowly the greater part of the carbon separates in the form of graphite, and if such cast iron (so called grey cast iron) be dissolved in acid, the carbon remains in the form of graphite. Graphite is met with in nature, sometimes in the form of large compact masses, sometimes it permeates rocky formations like the schists or slates, and in fact is met with in those places which, in all

15 When subjected to pressure, charcoal loses heat, hence the densest form stands to the less dense as a solid to a liquid, or as a compound to an element. From this the conclusion might be drawn, that the molecules of graphite are more complex than those of charcoal, and those of the dimmond still more so. The specific heat shows the same thing, because, as we shall see on further exposition, the complexity of a molecule leads to a diminution of the specific heat. At ordinary temperatures, the specific heat of charcoal is $0 \cdot 24$, graphite $0 \cdot 20$, the diamond $0 \cdot 147$. 
probability, were submitted to the action of subterranean heat. ${ }^{14}$ The graphite in cast iron, and sometimes also natural graphite, occasionally appears in a crystalline form in the shape of six-sided plates, but more often it occurs as a compact amoruhous mass having the characteristic properties of the familiar black-lead pencil. ${ }^{15}$

The diamond is a crystalline and transparent form of carbon. It crystallises in octahedra, dodecahedra, cubes, and other forms of the regular system. ${ }^{16}$ The efforts which have been made to produce diamonds artificially, although they have not been absolutely fruitless, have not as yet led to the production of large-sized crystals, because those means by which crystals are generally formed are inapplicable to carbon. Indeed, carbon in all its forms being insoluble and infusible does not pass into a liquid condition by means of which crystalli-

14 There are places where anthracite gradually changes into graphite, as the strata sink. I myself had the opportunity of observing this gradual transformation in the valley of Aosta, near Mont Blanc, not far from Courmayeur, near the warm mineral waters.

is Pencils are made of graphite worked up into a homogeneous mass by disintegrating, powdering, and cleansing it from earthy impurities; the best kinds are made of completely homogeneous graphite sawn up into the requisite sticks. Graphite is found in many places. In Russia the so-called Aliberoffsky graphite is particularly renowned; it is found in the Altai mountains near the Chinese frontier; in many places in Finland and likewise on the banks of the Little Tungouska, Sidoroff also found a considerable quantity of graphite.

Graphite, like most forms of charcoal, still contains a certain quantity of hydrogen, oxygen, and ash, so that in its natural state it does not contain more than 98 p.c. of carbon.

In practice graphite is purified simply by washing it when in a finely-ground state, by which means the bulk of the earthy matter may be separated. The following pre. cess, proposed by Brodie, consists in mixing the powdered graphite with $\frac{1}{14}$ part of its weight of potassium chlorate. The mixture is then heated with twice its weight of strong sulphuric acid until no more odoriferous gases are emitted; on cooling, the mixture is thrown into water and washed; the graphite is then dried and heated to a red heat; after this it considerably shrinks in volume, and turns into a very fine powder, which is then washed. When mixed with clay, graphite is used for making crucibles and pots for melting metals. By acting on graphite several times with a mixture of potassium chlorate and nitric acid heated up to $60^{\circ}$, Brodie transformed it into a yellow insoluble acid substance which he called graphitic acid, $\mathrm{C}_{11} \mathrm{H}_{4} \mathrm{O}_{5}$. The diamond remains unchanged when subjected to this treatment, whilst amorphous charcoal completely oxidises. Availing himself of this possibility of distinguishing graphite from the diamond or amorphous charcoal, Berthelot showed that when compounds of carbon and hydrogen are decomposed by heat, amorphous charcoal is mainly formed, whilst when compounds of carbon with chlorine, sulphur, and boron are decomposed, graphite is principally deposited.

16 Diamonds are sometimes found in the shape of small balls, and in that case it is impossible to cut them, because directly the surface is ground or broken they fall into minute pieces. Sometimes minute diamond crystals form a dense mass like sugar, and this is generally reduced to diamond powder and used for grinding. Some known varieties of the diamond are almost opaque and of a black colour. Such diamonds are as hard as the ordinary ones, and are used for polishing diamonds and other precious stones, and also for rock boring and tunnelling. 
sation could take place. Diamonds have several times been successfully produced in the shape of minute microscopic crystals having the appearance of a black powder, but when viewed under the microscope they appeared transparent, and possessed that hardness which is the peculiar characteristic of the diamond. This diamond powder is deposited on the negative electrode, when a weak galvanic current is passed through liquid chloride of carbon. ${ }^{17}$

Judging from the fact that carbon forms a number of gaseous (carbonic oxide, carbonic anhydride, methane, ethylene, acetylene, \&c.) and volatile (such are, for instance, many hydrocarbons and their most simple derivatives) substances, and also because the atomic weight of carbon, $\mathrm{C}=12$, approaches that of nitrogen, $\mathrm{N}=14$, and that of oxygen, $\mathrm{O}=16$, and the compounds $\mathrm{CO}$ (carbonic oxide) and $\mathrm{N}_{2} \mathrm{C}_{2}$ (cyanogen) are gases, it must be argued that if carbon formed the molecule $\mathrm{C}_{2}$ like $\mathrm{N}_{2}$ and $\mathrm{O}_{2}$, it would be a gas. And as through polymerism, or the mutual combination of molecules (as $\mathrm{O}_{2}$ passes into $\mathrm{O}_{3}$ or $\mathrm{NO}_{2}$ into $\mathrm{N}_{2} \mathrm{O}_{4}$ ) the temperatures of ebullition and fusion rise (which is particularly clearly proved with the hydrocarbons of the $\mathrm{C}_{n} \mathrm{H}_{2 n}$ series), it ought to be considered that the molecules of charcoal, graphite, and the diamond are very complex, seeing that they are not soluble, not volatile, and not fusible. The aptitude which the atoms of carbon show for combining together and forming complex molecules appears in all carbon compounds. Among the volatile compounds of carbon many are well known, the molecules of which contain $\mathrm{C}_{5} \ldots \mathrm{C}_{10} \ldots \mathrm{C}_{20} \ldots \mathrm{C}_{30}$, \&c., in general $\mathrm{C}_{n}$, where $n$ may be very large, and in none of the other elements is this faculty of complexity so developed as in carbon. ${ }^{18} \mathrm{Up}$

17 Hannay, in 1880, obtained diamonds by heating a mixture of heavy liquid hydrocarbons (paraffin oils) with magnesium in a thick iron tube. This investigation was, however, not repeated. Diamonds are found in a particular dense rock, known by the name of itacolumnite, and are dug out of the débris produced by the destruction of the itacolumnite by water. When the débris is washed the diamonds remain behind; they are principally found in Brazil, in the provinces of Rio and Bahia, and at the Cape. of Good Hope. The débris gives the black or amorphous diamond, carbonado, and the ordinary colourless or yellow translucent diamond. As the diamond possesses a very marked cleavage, the first operation consists in splitting it, and then roughly and finely polishing it with diamond powder. It is very remarkable that Professors P. A. Jatchinoff and Erofeeff found (1887) diamond powder in a meteoric stone which fell in the govern. ment of Penza, in the district of Krasnoslobodsk, near the settlement of Novo Urei (Sept. 10, 1886). Up to that time charcoal and graphite (a special variety, cliftonite) had been found in meteorites and the diamond only conjectured to be therein. The Novo Urei meteorite was composed of siliceous matter and metallic iron (with nickel), like many other meteorites.

18 The existence of a molecule $S_{6}$ is known, and it must be held that this accounts for the formation of hydrogen persulphide $\mathrm{H}_{2} \mathrm{~S}_{5}$. Phosphorus appears in the molecule $\mathrm{P}_{4}$ and gives $\mathrm{P}_{4} \mathrm{H}_{2}$. When expounding the data on specific heat we shall have occasion to return to the question of the complexity of the carbon molecule. 
to the present time there are no grounds for determining the degree of polymerism of the charcoal, graphite, or diamond molecules, and it can only be supposed that they contain $\mathrm{C}_{n}$ where $n$ is a large quantity. Therefore charcoal and those complex non-volatile organic substances which represent the gradual transitions to charcoal ${ }^{19}$ and which form the principal masses of the organisms, contain a store or accumulation of internal power in the form of the energy binding the atoms into complex molecules. When charcoal or complex compounds of carbon burn, the energy of the carbon and oxygen is turned into heat, and this fact is taken advantage of at every turn for the generation of heat from fuel. ${ }^{20}$

No other two elements are capable of combining together in such variety as carbon and hydrogen. The hydrocarbons of the $\mathrm{C}_{n} \mathrm{H}_{2 m}$ series in many eases widely differ from each other, although they have some properties in common. All hydrocarbons, whether gaseous, liquid, or solid, are combustible substances sparingly soluble or insoluble in water. The liquefied gaseous hydrocarbons, also those which are liquid at ordinary temperatures, and those solid hydrocarbons which have been liquefied by fusion, have the appearance and property of oily liquias, more or less viscid or fluid.21 The solid hydrocarbons

19 The hydrocarbons, poor in hydrogen (far from the limit) and containing many atoms of carbon, like chrysene and carbopetrocene, \&c., $\mathrm{C}_{n} \mathrm{H}_{2(n-m)}$ are solids, and less fusible as $n$ and $m$ increase. They present a marked approach to the properties of the diamond. And in proportion to the diminution of the water in the carbohydrates $\mathrm{C}_{n} \mathrm{H}_{2 m} \mathrm{O}_{m}$-for example, in the humic compounds (Note 5) - the transition of complex organic substances to charcoal is very evident. That residue resembling charcoal and graphite which is obtained by the separation (by means of copper sulphate and sodium chloride) of iron from white cast-iron containing carbon, chemically combined with the iron, also seems, especially after the researches of G. A. Zaboudsky, to be a complex substance containing $\mathrm{C}_{12} \mathrm{H}_{6} \mathrm{O}_{3}$. The endeavours which have been directed towards determining the measure of complexity of the molecules of charcoal, graphite, and the diamond will probably at some period lead to the solution of this problem, and will most likely prove that the various forms of charcoal, graphite, and the dianond contain molecules of different and very considerable complexity. The constancy of the grouping of benzene, $\mathrm{C}_{6} \mathrm{H}_{6}$, and the wide diffusion and facility of formation of the carbohydrates, containing $\mathrm{C}_{6}$ (for example, cellulose, $\mathrm{C}_{6} \mathrm{H}_{10} \mathrm{O}_{5}$, glucose, $\mathrm{C}_{6} \mathrm{H}_{12} \mathrm{O}_{6}$ ), give reason for thinking that the group $\mathrm{C}_{6}$ is the first and simplest of those possible to free carbon, and it may be hoped that some time or other it may be possible to get charcoal in this group. Perhaps in the diamond there may be found such a relation between the atoms as in the benzene group, and in charcoal such as in the carbohydrates.

20 When charcoal burns, the complex molecule $\mathrm{C}_{n}$ is resolved into the simple molecules $n \mathrm{CO}_{2}$, and therefore part of the heat-probably no small amount-is expended in the destruction of the complex molecule $\mathrm{C}_{n}$. Perhaps by burning the most complex substances, which are the poorest as regards hydrogen, it may be possible to form an idea of the work required to split up $\mathrm{C}_{n}$ into separate atoms.

21 The viscosity, or the degree of mobility, of liquids is determined by their internal friction. It is estimated by passing the liquids through narrow (capillary) tubes, the mobile liquids passing through with greater facility and speed than the viscid ones. The 
more or less resemble wax in their properties, although ordinary oils and wax generally contain oxygen in addition to carbon and hydrogen, but in relatively small proportion. There are also a few hydrocarbons which have the appearance of tar-as, for instance, metacinnamene and gutta-percha. Those liquid hydrocarbons which boil at a high temperature are like oils, and those which have a low boiling point resemble ether, whilst the gaseous hydrocarbons in many of their properties are akin to hydrogen. All this tends to show that, in hydrocarbons physically considered, the properties of solid non-volatile charcoal are strongly modified and hidden, whilst those of the hydrogen predominate. All hydrocarbons are neutral substances (neither basic nor acid), but under certain conditions they enter into peculiar reactions. It has been seen in those hydrogen compounds which have been already considered (water, nitric acid, ammonia) that the hydrogen in almost all cases enters into reaction, being displaced by metals. The hydrogen of the hydrocarbons, it may be said, has no metallic character-that is to say, it is not directly ${ }^{22}$ displaced by metals, even by such as sodiun and

viscosity varies with the temperature and nature of the liquids, and, in the case of solutions, changes with the amount of the substance dissolved, but is not proportional to it. So that, for example, with alcohol at $20^{\circ}$ the viscosity will be 69 , and for a 50 p.c. solution 160 , the viscosity of water being taken as 100 . The volume of the liquid which passes through by experiment (Poiseuille) and theory (Stokes) is proportional to the time, the pressure, and the fourth power of the diameter of the (capillary) tube, and inversely proportional to the length of the tube; this renders it possible to form comparative estimates of the coefficients of internal friction and viscosity.

As the complexity of the molecules of hydrocarbons and their derivatives increases by the addition of carbon ( or $\mathrm{CH}_{2}$ ), so does the degree of viscosity also rise. The extensive series of investigations referring to this subject still await the necessary generalisation. That connection which (already partly observed) ought to exist between the viscosity and the other physical and chemical properties, forces us to conclude that the magnitude of internal friction plays an important part in molecular mechanics. In investigating organic compounds and solutions, similar researches ought to stand foremost. Many observations have already been made, but not much has yet been done with them; the bare facts and some mechanical data exist, but their relation to molecular mechanics has not been cleared up in the requisite degree. It has already been seen from existing data that the viscosity at the temperature of the absolute boiling point becomes as small as in gases.

22 In a number of hydrocarbons and their derivatives such a substitution of metals for the hydrogen may be arrived at by indirect means. The property shown by acetylene, $\mathrm{C}_{2} \mathrm{H}_{2}$, and its analogues of forming metallic derivatives is in this respect particularly characteristic. Judging from the fact that carbon is an acid element (that is, gives an acid anhydride with oxygen), but comparatively slightly acid (because carbonic acid is not at all an energetic acid; compounds of chlorine and carbon, even $\mathrm{CCl}_{4}$, are not decomposed by water as is the case with phosphorus chloride, and even silicic chloride or boric chloride, although they correspond with acids of but little energy), one might expect to find in the lydrogen of hydrocarbons this faculty for being substituted by metals. The metallic compounds which correspond with hydrocarbons are known under the name of organo-metallic compounds. Such, for instance, is zinc ethyl, $\mathrm{Zn}\left(\mathrm{C}_{2} \mathrm{H}_{5}\right)_{2}$, which corresponds with ethyl hydride or ethane, $\mathrm{C}_{2} \mathrm{H}_{6}$, where two atoms of hydrogen have been exchanged for one of zinc. 


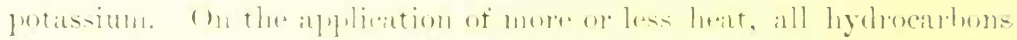

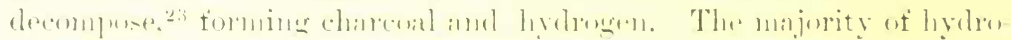

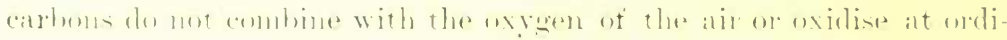
naly temperatures. hut under the antiom of nitrie ale del and many other oxili-ing-ubstande most of them are sublect to widation, in whicle

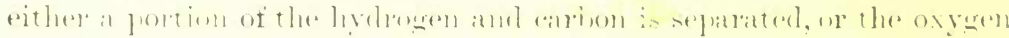

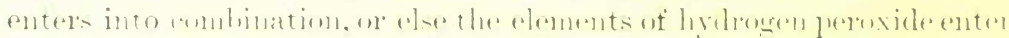

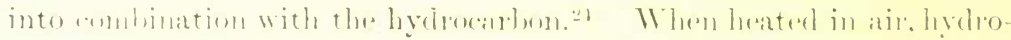

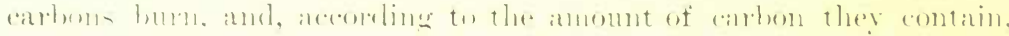

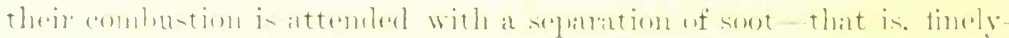

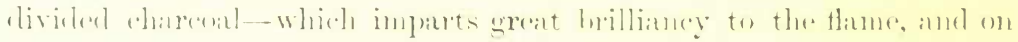

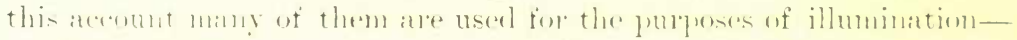

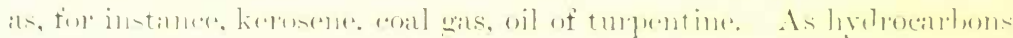

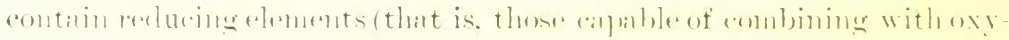

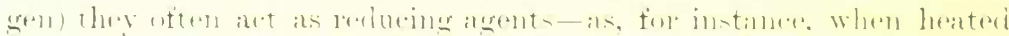

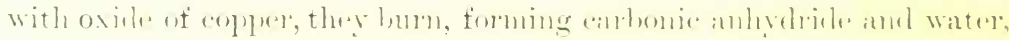

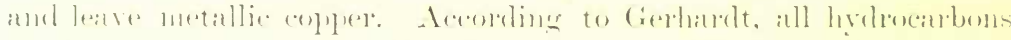

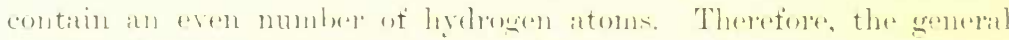

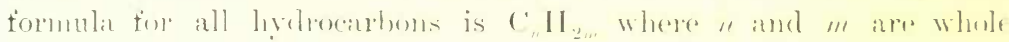

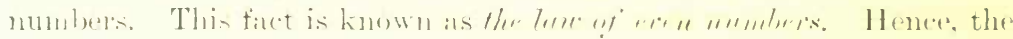

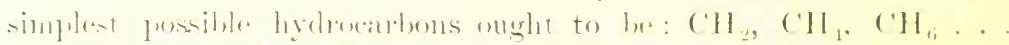

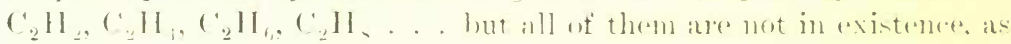

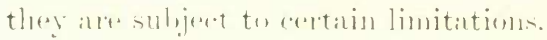

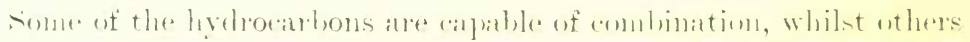

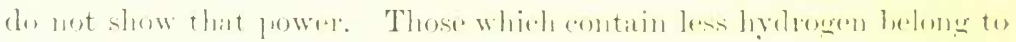

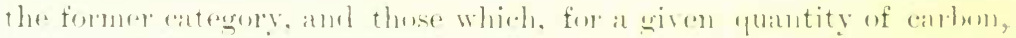

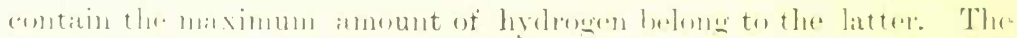

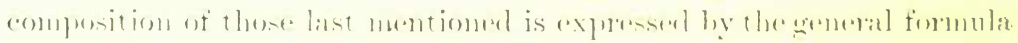

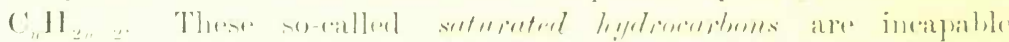

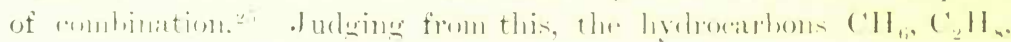

:- (

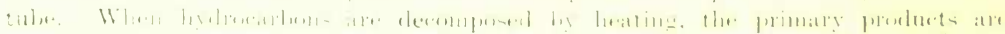

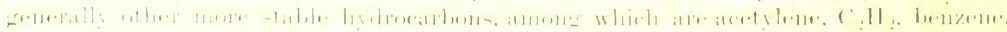

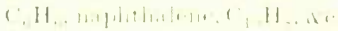

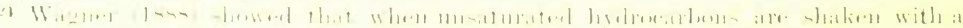

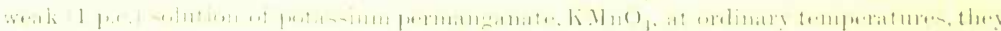

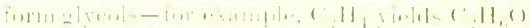

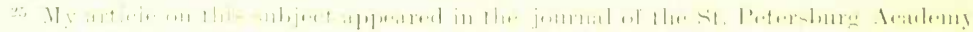

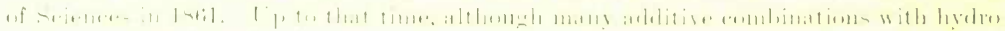

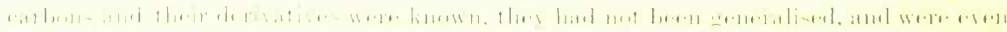

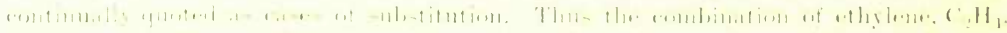

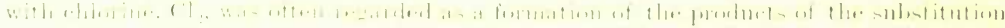

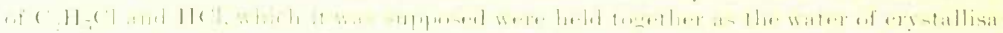

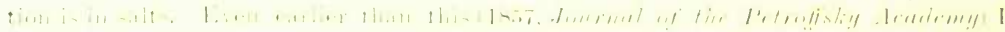

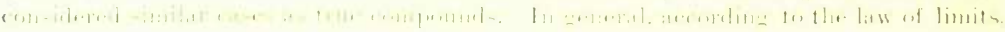


$\mathrm{C}_{3} \mathrm{H}_{10}$, dr. . . do not exist. Those containing the maximum amount of hydrogen will be represented by $\mathrm{CH}_{4}(n=1,2 n+2=4), \mathrm{C}_{2} \mathrm{H}_{6}(n=2)$, $\mathrm{C}_{3} \mathrm{H}_{8}(n=3), \mathrm{C}_{4} \mathrm{H}_{10}$, dic. This may be termed the law of limits. Placing this in juxtaposition with the law of even numbers, it is easy to perceive that the possible hydrocarbons can be ranged in series, the terms of which may be expressed by the general formula $\mathrm{C}_{n} \mathrm{H}_{2 n+2}$, $\mathrm{C}_{n} \mathrm{H}_{2 n}, \mathrm{C}_{n} \mathrm{H}_{2 n-2}$, dc. . . Those hydrocarbons which belong to any oneof the series expressible by a general formula are said to be homologous with one another. Thus, the hydrocarbons $\mathrm{CH}_{4}, \mathrm{C}_{2} \mathrm{H}_{6}, \mathrm{C}_{3} \mathrm{H}_{8}, \mathrm{C}_{4} \mathrm{H}_{10}$, dc. . . are members of the limiting (saturated) homologous series $\mathrm{C}_{n} \mathrm{H}_{2 n+2}$. That is, the difference between the members of the series is $\mathrm{CH}_{2} \cdot{ }^{26}$ Not only the composition, but also the properties, of the members of a series tend to classification in one group. For instance, the members of the series $\mathrm{C}_{n} \mathrm{H}_{2 n+2}$ are not capable of forming additive compounds, whilst those of the series $\mathrm{C}_{n} \mathrm{H}_{2 n}$ are capable of combining with chlorine, sulphuric anhydride, dc.; and the members of the $\mathrm{C}_{n} \mathrm{H}_{2 n-6}$ group, belonging to the coal tar series, are acted on by nitric acid, and have other properties in common. The physical properties of the members of a given homologous series vary in such a manner, the boiling point generally rises and the internal friction increases as $n$ increases ${ }^{27}$-that is, with an increase in the atomic weight; the specific gravity also successively changes as $n$ becomes greater. ${ }^{28}$

an unsaturated hydrocarbon, or its derivative, on combining with $r \mathrm{X}_{2}$, gives a substance which is saturated or else approaching the limit. The investigations of Frankland with many organo-metallic compounds clearly showed the limit in the case of metallic compounds, which we shall constantly refer to later on.

26 The conception of homology has been applied by Gerhardt to all organic compounds in his classical work, 'Traité de Chimie Organique,' finished in 1855 (4 vols.), where he divided all organic compounds into fatty and aromatic, which is in principle still adhered to at the present time, although the latter are more often called benzene derivatives, on account of the fact that Kekule, in his beautiful investigations on the structure of aromatic compounds, showed the link which unites them all with the 'nucleus' benzene, $\mathrm{C}_{6} \mathrm{H}_{6}$.

27 This is always true for hydrocarbons, but for derivatives of the lower homologues it is sometimes different; for instance, in the series of saturated alcohols $\mathrm{C}_{n} \mathrm{H}_{2 n+1}(\mathrm{OH})$, when $n=0$, we obtain water, $\mathrm{H}(\mathrm{OH})$, which boils at $100^{\circ}$, and whose specific gravity at $15^{\circ}=0.9992$; when $n=1$, wood spirit $\mathrm{CH}_{3}(\mathrm{OH})$, which borls at $66^{\circ}$, and at $15^{\circ}$ has a specific gravity $=0.7964$; when $n=2$, ordinary alcohol $\mathrm{C}_{2} \mathrm{H}_{5}(\mathrm{OH})$, boiling at $78^{\circ}$, specific gravity at $15^{\circ}=0.7936$, and with further increase of $\mathrm{CH}_{2}$ the specific gravity increases. For the glycols $\mathrm{C}_{n} \mathrm{H}_{2^{n}}(\mathrm{OH})_{2}$ the phenomenon of a similar kind is still more striking; at first the temperature of the boiling pointand the density increase, and then for higher (more complex) members of the series diminish. The reason for this phenomenon, it is evident, must be sought for in the influence and properties of water, and that strong affinity which, acting between hydrogen and oxygen, determines many of the exceptional properties of water (Chap. I.).

$28 \mathrm{As}$, for example, in the saturated series of hydrocarbons $\mathrm{C}_{n} \mathrm{H}_{2 n+2}$, the lowest member $(n=0)$ must be taken as hydrogen $\mathrm{H}_{2}$, a gas which $\left(t . c\right.$. below $\left.-190^{\circ}\right)$ is liquefied with great difficulty, and when in a liquid state has doubtless a very small 
Many of the hydrocarbons which are met with in nature are the products of organisms, and do not belong to the mineral kingdom. A still greater number are produced artificially. These are formed by what is termed the combination of residues. For instance, if a mixture of the vapours of hydrogen sulphide and carbon bisulphide be passed through a tube in which copper is heated, this latter absorbs the sulphur from both the compounds, and the liberated carbon and hydrogen combine to form a hydrocarbon, methane. If a mixture of bromobenzene, $\mathrm{C}_{6} \mathrm{H}_{5} \mathrm{Br}$, and ethyl bromide, $\mathrm{C}_{2} \mathrm{H}_{5} \mathrm{Br}$, be heated with metallic sodium, the sodium combines with the bromine of both compounds, forming sodium bromide. From the first combination the group $\mathrm{C}_{6} \mathrm{H}_{5}$ remains, and from the second $\mathrm{C}_{2} \mathrm{H}_{5}$. Having an odd number of hydrogen atoms, they in virtue of the law of even numbers cannot exist alone, therefore they combine together forming the compound $\mathrm{C}_{6} \mathrm{H}_{5} \cdot \mathrm{C}_{2} \mathrm{H}_{5}$ or $\mathrm{C}_{8} \mathrm{H}_{10}$ (ethylbenzene). Hydrocarbons are also produced by the breaking up of more complex organic or hydrocarbon compounds, especially by heating - that is, by dry distillation. For instance, gum-benzoin contains an acid called benzoic acid, $\mathrm{C}_{7} \mathrm{H}_{6} \mathrm{O}_{2}$, the vapours of which, when passed through a heated tube, split up into carbonic anhydride, $\mathrm{CO}_{2}$, and benzene, $\mathrm{C}_{6} \mathrm{H}_{6}$. Carbon and hydrogen only unite directly in one degree of combination - namely, to form acetylene, having the composition $\mathrm{C}_{2} \mathrm{H}_{2}$, which, as compared with other hydrocarbons, exhibits a very great constancy at a high temperature. ${ }^{29}$.

density. Where $n=1,2,3$, the hydrocarbons $\mathrm{CH}_{4}, \mathrm{C}_{2} \mathrm{H}_{6}, \mathrm{C}_{5} \mathrm{H}_{8}$ are gases, more and more readily liquefiable. The temperature of the absolute boiling point for $\mathrm{CH}_{4}=-100^{\circ}$, and for ethane $\mathrm{C}_{2} \mathrm{H}_{6}$, and in the higher members it rises. The hydrocarbon $\mathrm{C}_{4} \mathrm{H}_{10}$, already liquefies about $0^{\circ} . \quad \mathrm{C}_{5} \mathrm{H}_{12}$ boils at from $+9^{\circ}$ (Livoff) to $37^{\circ}, \mathrm{C}_{6} \mathrm{H}_{14}$ from $58^{\circ}$ to $78^{\circ}$, ic. The specific gravities in a liquid state at $15^{\circ}$ are :-

$\begin{array}{ccccc}\mathrm{C}_{5} \mathrm{H}_{12} & \mathrm{C}_{6} \mathrm{H}_{14} & \mathrm{C}_{7} \mathrm{H}_{16} & \mathrm{C}_{10} \mathrm{H}_{22} & \mathrm{C}_{16} \mathrm{H}_{34} \\ 0.63 & 0.66 & 0.70 & 0.75 & 0.85\end{array}$

29 If, at the ordinary temperature (assuming therefore that the water formed will be in a liquid state, , a gram molecule ( 26 grams) of acetylene, $\mathrm{C}_{2} \mathrm{H}_{2}$, be burnt, 310 thousand calories will be emitted (Thomsen), and as 12 grams of charcoal produce 97 thousand calories, and 2 grams of hydrogen 69 thousand calories, therefore, if the liydrogen and carbon of the acetylene were burnt there would be only $2 \times 97+69$, or 263 thousand calories produced. It is evident, then, that acetylene in its formation absorbs $310-263$, or 47 thousand calories - that is, the reaction of charcoal and hydrogen is endothermal and the product is in this respect a substance resembling nitrous oxide, lydrogen peroxide, \&c. Such reasoning is, however, open to certain defects of one kind or another, especially when a reaction, which can really only take place at a high temperature, is supposed to be effected at ordinary temperatures or vice versâ. Calculations cannot be made for high temperatures, on account of insufficient evidence as to specific heat.

For considerations referring to the combustion of carbon compounds, we will first enumerate the quantity of heat separated by the combustion of definite chemical carbon compounds, and then a few figures touching the kinds of fuel applied in a practical way. 


\section{There is one known substance among the saturated hydrocarbons} composed of 1 atom of carbon and 4 atoms of hydrogen ; this is a com-

I. For molecular quantities in perfect combustion, the following amounts of heat are given out (when gaseous carbonic anhydride and liquid water are formed), according to the data of Thomsen: (1) for gaseous $\mathrm{C}_{n} \mathrm{H}_{2 n+2}: 52 \cdot 8+158 \cdot 8 n$ thousand calories; (2) for $\mathrm{C}_{n} \mathrm{H}_{2 n}: 17 \cdot 7+158 \cdot 1 n$ thousand calories; (3) according to Stohmann (1888) for liquid satuated alcohols, $\mathrm{C}_{n} \mathrm{H}_{2 n+2} \mathrm{O}: 11 \cdot 8+156 \cdot 3 n$, and as the latent heat of evaporation $=$ about $8 \cdot 2+0 \cdot 6 n$, in a gaseous state, $20^{*} 0+156 \cdot 9 n ;(4)$ for monobasic saturated liquid acids, $\mathrm{C}_{n} \mathrm{H}_{2 n} \mathrm{O}_{2}: 95 \cdot 3+154 \cdot 3 n$, and as their latent heat of evaporation is about $5 \cdot 0+1 \cdot 2 n$, in a gaseous form, about $90+155 n$; (5) for solid saturated bibasic acids, $\mathrm{C}_{n} \mathrm{H}_{2 n-2} \mathrm{O}_{4}$ : $253 \cdot 8+152 \cdot 6 n$, if they are expressed as $\mathrm{C}_{n} \mathrm{H}_{2}{ }_{n} \mathrm{C}_{2} \mathrm{H}_{2} \mathrm{O}_{4}$, then $51 \cdot 4+152 \cdot 6 n$; (6) for benzene and its liquid homologues (still according to Stohmann) $\mathrm{C}_{n} \mathrm{H}_{2 n}-{ }_{6}: 158 \cdot 6+156 \cdot 3 n$, and in a gaseous form about $155+157 n ;(7)$ for the gaseous homologues of acetylene, $\mathrm{C}_{n} \mathrm{H}_{2 n}-2$, (according to Thomsen), $5+157 n$. It is evident from the preceding figures that the group $\mathrm{CH}_{2}$, or the substitution of $\mathrm{CH}_{3}$ for $\mathrm{H}$, on burning gives out from 152 to 159 thousand calories. This is less than that given out by $\mathrm{C}+\mathrm{H}_{2}$, which is $97+69$ or 166 thousand; the reason of this difference (it would be still greater if charcoal were gaseous) is the amount of heat separated during the formation of $\mathrm{CH}_{2}$. The heat of combustion of the following solids (determined by Stohmann) is expressed, not in terms of the molecular weight of the substance, but per unit of weight: $\mathrm{C}_{6} \mathrm{H}_{10} \mathrm{O}_{5}$, cellulose, 4146 ; starch, 4123 ; dextrose, $\mathrm{C}_{6} \mathrm{H}_{12} \mathrm{O}_{6}, 3692$; cane sugar, $\mathrm{C}_{12} \mathrm{H}_{22} \mathrm{O}_{11}$, 3866 ; naphthalene, $\mathrm{C}_{10} \mathrm{H}_{8}$, 9621 ; urea, $\mathrm{CN}_{2} \mathrm{H}_{1} \mathrm{O}, 2465$; white of eggs, 5579; dry rye bread, 4421 ; wheaten bread, 4302 ; tallow, 9365 ; butter, 9192 ; linseed oil, 9323.

II. The number of units of heat given out during the complete combustion and cooling of the following ordinary kinds of fuel in their usual state of dryness and purity are :-(1) for wood charcoal, anthracite, semi-anthracite, tarry coal and coke, from 7200 to 8200 ; (2) for dry, long flaming coals, and the best brown coals, from 6200 to 6800 ; (3) for perfectly dry wood, 3500 ; hardly dry, 2500 ; (4) perfectly dry peat, best kind, 4500 ; compressed and dried, 3000 ; (5) petroleum refuse and similar liquid hydroearbons, about 11000 ; (6) illuminating gas of the ordinary composition (about 45 vols. $\mathrm{H}, 40$ vols. $\mathrm{CH}_{4}, 5$ vols. $\mathrm{CO}$, and 5 vols. N), about 12000 ; (7) generator gas (see next Chap.), containing 2 vols. carbonic anhydride, 30 vols. carbonic oxide, and 68 vols. nitrogen for one part by weight of the whole carbon burnt, 5300, and for one part by weight of the gas, 910, units of heat; and (8) water gas (see next Chap.) containing 4 vols. carbonic anhydride, 8 vols. $\mathrm{N}_{2}, 24$ vols. carbonic oxide, and 46 vols. $\mathrm{H}_{2}$, for one part by weight of the carbon consumed in the generator 10900, and for one part by weight of the gas, 3600 , units of heat. In these figures, as in all calorimetric observations, the water produced by the combustion of the fuel is supposed to be liquid. As regards the temperature reached by the fuel, it is important to remark that for solid fnel it is indispensable to admit (to ensure complete combustion) twice the amount of air required, but liquid, pulverised fuel, and especially gaseous fuel, does not require an excess of air ; therefore, a kilogram of charcoal, giving 8000 units of heat, requires about 24 kilograms of air (3 kilograms of air per 1000 calories) and a kilogram of generator gas requires only 0.77 kilogram of air $(0.85$ kilo. of air per 1000 calories), 1 kilogram of water gas about 4.5 of air (1.25 kilos. of air per 1000 calories).

III. As one of the most important and extensive applications of combustible matter is the formation of steam and conversion of it into work, we will take as an example the calculation for a boiler burning per hour (=3600 seconds) 100 kilograms of anthracite, giving out 8000 units of heat, and containing 90 p.c. C, 3 p.c. $\mathrm{H}, 3$ p.c. $\mathrm{O}$, and 4 p.c. of ash and nitrogen. For the combustion of 100 kilos. of such coal, giving 800 thousand units of heat, about 2400 kilos. (counting double the umount) of air will be required, therefore in the smoke there will be 2500 kilos., and as the specific heat of the products of combustion is about $0^{*} 25$, so, taking the temperature of the smoke passing from underneath the boiler, $t=200^{\circ}$ (higher than the temperature of the air), the smoke will carry off 125 thousand units of heat, and $187 \cdot 5$ units of heat per second will be communi- 
pound containing the highest percentage of hydrogen $\left(\mathrm{CH}_{4}\right.$ contains 25 per cent. of hydrogen), and at the same time it is the only hydrocarbon whose molecule contains a single atom of carbon This saturated hydrocarbon, $\mathrm{CH}_{4}$, is called marsh-gas or methane. If vegetable or animal refuse suffers decomposition in a space where the air has not free access, or no access at all, then the decomposition is accompanied with the formation of marsh gas, and this either at an ordinary temperature, or at a comparatively much higher one. On this account plants, when decomposing under water in marshes, give out this gas. It is well known that if the mud in bogs be stirred up, the act is accompanied with the evolution of a large quantity of gas bubbles; these may, although slowly, also separate of their own accord. The gas which is evolved consists principally of marsh-gas. ${ }^{30}$. If wood, coal, or many other vegetable or animal substances are decomposed by the action of heat without access of air - that is, are subjected to dry distillation - they, in addition to many other gaseous products of decomposition (carbonic anhydride, hydrogen, and various other substances), evolve a great deal of methane. Generally, the gas which is used for lighting purposes is obtained by this means, and, therefore, always contains marsh gas, mixed with hydrogen and other vapours and gases, although it is subsequently purified from many of them. ${ }^{31}$ As the-

cated to the boiler, and to the surrounding air. Subtracting 27.5 units of heat for radiation, we have 160 units of heat per second communicated to the water. If the pressure in the boiler be 5 atmospheres, $t=152^{\circ}$ (page 54), and the temperature of the feed water $12^{\circ}$, then (the heat of warming and evaporation of 640 units of heat, see page 53) 0.25 kilo. of steam at $150^{\circ}$ per second (900 kilos. per hour) will be produced. If this steam does its work without waste of any kind, and be condensed in the condenser at $50^{\circ}$, then, according to the second law of the mechanical theory of heat, not more in any case than $160(150-50) \div(150+273)$ or 37.8 units of heat, will be converted into work, which (according to the first law of the mechanical theory of heat) will equal $424 \times 87.8$ or 15927 kilogrammetres of work per second, or 212 horse-power (one horse power $=75$ kilogrammetres per second).

But as at least 35 p.c. (in the best machines) is lost in inevitable loss of heat and through friction, only about 140 horse-power can be obtained with the above-mentioned quantity of fuel in a steam-engine of excellent construction. Generally, 1 kilo. of coal per horse-power per hour is consumed in the best steam-engines (with expansion and condensation). Only in gas engines at present does the work of the fuel approach the theoretical limit.

30 It is easy to collect the gas which is evolved in marshy places if a glass bottle be inverted in the water and a funnel put into it (in water); if the mud of the bottom be now agitated, the bubbles which rise may be easily caught in the funnel.

31 Illuminating gas is generally prepared by heating gas coal (see Note 6) in oval cylindrical horizontal cast-iron or clay retorts. Several such retorts $B B$ (fig. 58) are disposed in the furnace $A$, and heated together. When the retorts are heated to a. red heat, lumps of coal are thrown into them, and they are then closed with a closelyfitting cover. The illustration shows the furnace, with five retorts. Coke (see Note 1 , dry distillation) remains in the retorts, and the volatile products in the form of vapours and gases travel along the pipes $d$, rising from each retort. These pipes branch above the stove, and are hermetically sealed into the receiver $f$ (hydraulic main) placed above 


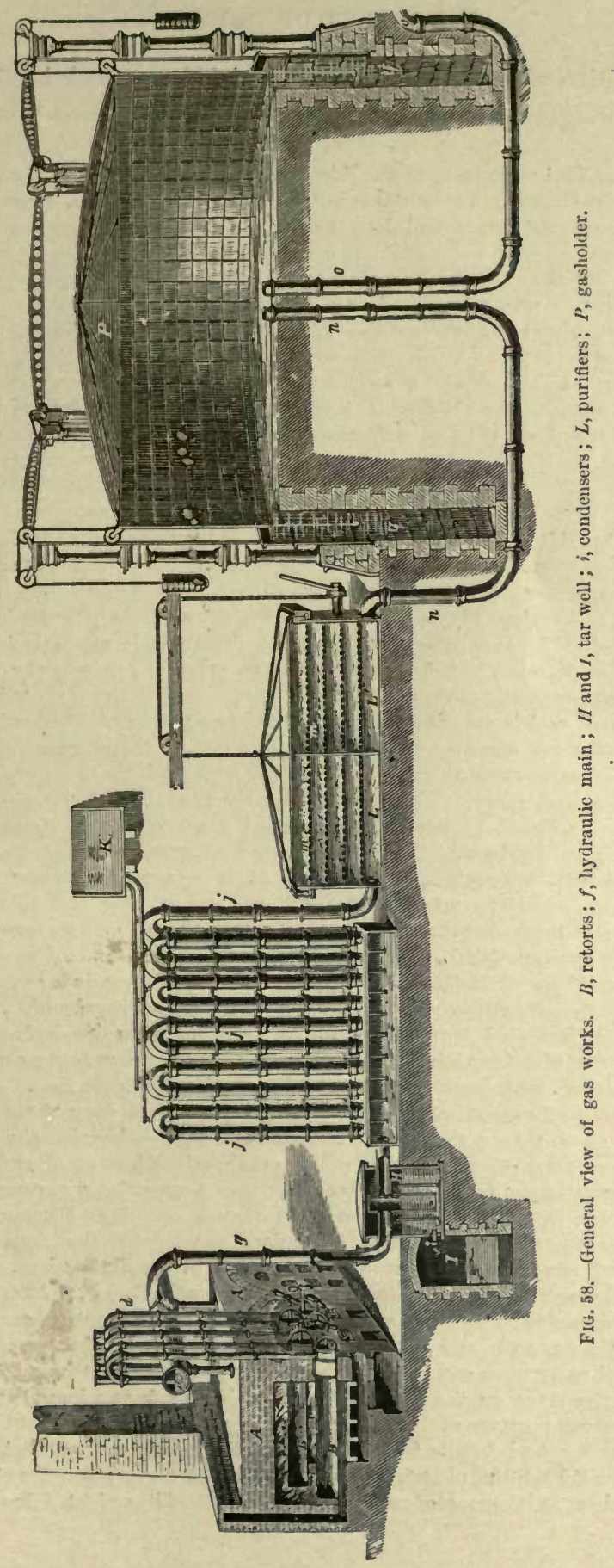




\section{decomposition of the organic matter, which forms coal, is still going on underground, the evolution of large quantities of marsh gas frequently}

the furnace. Those products of the dry distillation which most easily pass from the gaseous into the liquid and solid states collect in the hydraulic main. From the hydraulic main the vapours and gases travel along the pipe $g$ and the series of vertical pipes $j$ (which are sometimes cooled by water trickling over the surface), where the vapours and gases cool from the contact of the colder surface, and a fresh quantity of vapour condenses. The condensed liquids pass from the pipes $g$ and $j$ and into the troughs $H$. These troughs always contain liquid at a constant level (the excess flowing away), so that the gas cannot escape, and thus forms, as it is termed, a hydraulic joint. In the state in which it leaves the condensers, the gas consists principally of the following vapours and gases: (1) vapour of water, (2) ammonium carbonate, (3) liquid hydrocarbons, (4) hydrogen sulphide, $\mathrm{H}_{22} \mathrm{~S}$, (5) carbonic anhydride, $\mathrm{CO}_{2},(6)$ carbonic oxide, $\mathrm{CO},(7)$ sulphurous anhydride, $\mathrm{SO}_{2}$, but a great part of the illuminating gas consists of (8) hydrogen, (9) marsh gas, (10) olefiant. gas, $\mathrm{C}_{2} \mathrm{H}_{4}$, and other gaseous hydrocarbons. The hydrocarbons $(3,9$, and 10$)$, the hydrogen, and carbonic oxide are capable of combustion, and are useful component parts, but the carbonic anhydride, the hydrogen sulphide, and sulphurous anhydride, as well as the vapours of ammonium carbonate, form an injurious admixture, because they do not burn $\left(\mathrm{CO}_{2}, \mathrm{SO}_{2}\right)$ and lower the temperature and brilliancy of the flume, or else, althongh they are capable of burning, they give out during combustion sulphurous anhydride (for example, $\mathrm{H}_{2} \mathrm{~S}$, CS., and others), which has a disagreenble smell, is injurious when inhaled, and spoils many smrounding objects. In order to separute the injurious products, the gas is washed with water, a cylinder (not shown in the illustration) filled with coke continually moistened with water serving for this purpose. The water coming into contact with the gas dissolves the ammonium carbonate; hydrogen sulphide, carbonic anhydride, and sulphurons anlydride, being only partly soluble in water, have to be got rid of by a special means. For this purpose the gas is passed through moist lime or another alkaline liquid, as the above-mentioned gases have acid properties and are therefore retained by the alkali. In the case of lime, calcium carbonate, sulphite and sulphide, all solid substances, are formed. It is necessary to renew the purifying material, as its absorbing power ceases. A mixture of lime and sulphate of iron, $\mathrm{FeSO}_{4}$, acts still better, hecause the latter, with lime, $\mathrm{Ca}(\mathrm{HO})_{2}$, forms ferrous hydroxide, $\mathrm{Fe}(\mathrm{HO})_{2,}$ and gypsum, $\mathrm{CaSO}_{4}$. The suboxide (partly turning into oxide) of iron absorbs $\mathrm{H}_{2} \mathrm{~S}$, forming $\mathrm{FeS}$ and $\mathrm{H}_{2} \mathrm{O}$, and the gypsum retains the remainder of the ammonia, the excess of lime absorbing carbonic anbydride and sulphuric anhydride. [In English works a native hydrated ferric hydroxide is used for removing lydrogen sulphide.] This purification of the gas takes place in the apparatus $L$, where the gas passes through netting $m$, covered with sawdust mixed with lime and sulphate of iron. It is necessary to remark that in the mannfacture of gas it is indispensable to draw off the vapours from the retorts, so that they should not remain there long (otherwise the hydrocarbons. would in a considerable degree be resolved into charcoal and hydrogen), and also to avoid a great pressure of gas in the apparatus, otherwise a quantity of gas would escape at all cracks such as must inevitably exist in such a complicated arrangement. For this purpose there are special pumps (exhausters) so regulated that they only pump off the quantity of gas formed (the pump is not shown in the illustration). The purified gas passes through the pipe $n$ into the gasometer (gasliolder) $P$, a dome made of iron plate. The edges of the dome dip into water poured into a ring-shaped channel $g$, in which the sides of the dome rise and fall. The gas is collected in this holder, and distributed to its destimation by pipes commuricating with the pipe $o$, issuing from the dome. The pressure of the dome on the gas enables it, on issuing from a long pipe, to penetrate through the small aperture of the burner. 100 kilograms of coal give about 20 to 30 cubic metres of gas, having a density from four to nine times greater than that of hydrogen. $A$ culic metre (1000 litres) of hydrogen weighs about 87 grams; therefore 100 kilograms of coal give about 18 kilograms of gas, or about one-sixtl of its weight. Illuminating gas is 


\section{occurs in coal-mines. ${ }^{32}$ When mixed with air it forms an explosive} mixture, which forms one of the great dangers of coal mining, as subterranean work has always to be carried on by lamp-light. This danger is, however, overcome by the use of Humphry Davy's safety

generally lighter than marsh gas, as it contains a considerable amount of hydrogen, and is only heavier than marsh gas when it contains much of the heavier hydrocarbons. Thus olefiant gas, $\mathrm{C}_{2} \mathrm{H}_{4}$, is fourteen times, and the vapours of benzene thirty-nine times, heavier than hydrogen, and illuminating gas sometimes contains 15 p.c. of its volume of them. The brilliancy of the flame of the gas increases with the quantity of olefiant gas and similar heavy hydrocarbons, as it then contains more carbon for a given volume and a greater number of carbon particles are separated. Gas usually contains from 35 to 60 p.c. of its volume of marsh gas, from 30 to 50 p.c. of hydrogen, from 3 to 5 p.c. of carbonic oxide, from 2 to 10 p.c. heavy hydrocarbons, and from 3 to 10 p.c. of nitrogen. Wood gives almost the same sort of gas as coal, and almost the same quantity, but the wood gas contains a great deal of carbonic anhydride, and the vapours of water and tarry liquids, but on the other hand there is almost complete absence of sulphur compounds. Tar, oils, naphtha, and such like materials furnish a large quantity of good illuminating gas. An ordinary burner of 8 to 12 candle power burns 5 to 6 cubic feet of coal gas per hour, but only 1 cubic foot of naphtha gas. One pood (36 lbs. Eng.) of naphtha gives 500 cubic feet of gas-that is, one kilogram of naphtha produces about one cubic metre of gas. The formation of combustible gas by heating coal was discovered in the beginning of the last century, but only put into practice towards the end by Le-Bon in France and Murdoch in England. In England, Murdoch, together with the renowned Watt, built the first gas works in 1805 .

It is worthy of remark that 1 candle-power per hour entails a consumption of not less than 7 grams of coal gas, or 1 \} grams of naplitha gas, whilst lamps consume but 4 grams of kerosene per candle-power, and ordinary-sized candles burn 6-9 grams of stearin, wax, or paraffin, or 13 grams of tallow per hour. In practice illuminating gas is not only used for lighting (electricity and kerosene are cheaper in Russia), but also as the motive-power for gas engines (see p. 172), which consume about half a cubic metre per horsepower per hour ; gas is also used in laboratories for heating purposes. When it is necessary to concentrate the heat, either the ordinary blowpipe (fig. 59) is applied, placing the end in the flame and blowing through the mouthpiece; or, in other forms, gas is passed through the blowpipe; when a large, hot, smokeless flame is required for heating crucibles or glass-blowing, a foot-blower is used. High temperatures, which are often required for laboratory and manufacturing purposes, are most easily attained by the use of gaseous fuel (illuminating gas, generator gas, and water gas, which will be treated of in the following chapter) because complete combustion may be effected without an excess of air. It is evident that for obtaining high temperatures means must be taken to diminish the loss of heat by radiation.

32 The gas which is set free in coal mines contains a good deal of nitrogen, some carbonic anhydride, and a large quantity of marsh gas. The best means of avoiding an explosion consists in efficient ventilation. It is best to

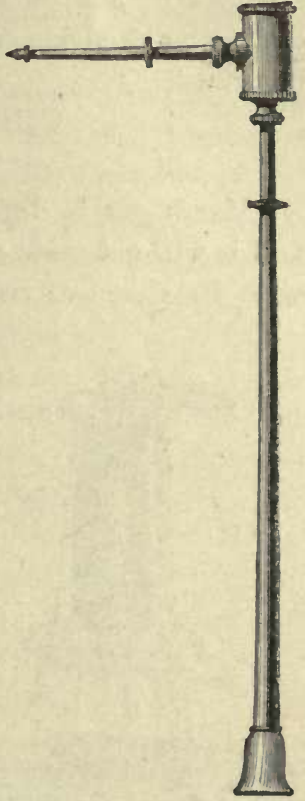

Fr: 59.- Blowpipe. Air is: blown in at the trumpetshapel mouthpiece, and escapes in fine stream from the platinum jet placed at the extrenity of the side tube. light coal mines with electric lamps. 


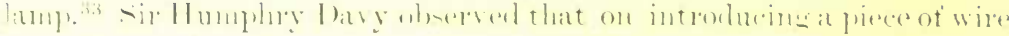

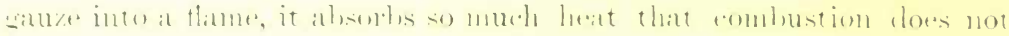

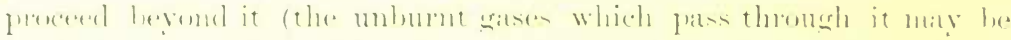
kindled on the other side). In aceorelanes with this, the flame of the

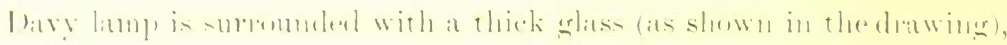

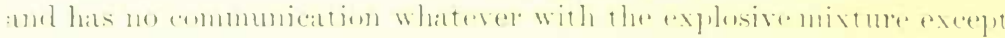

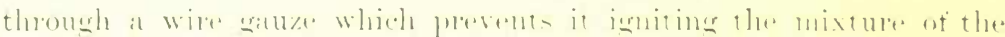

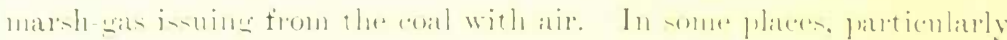

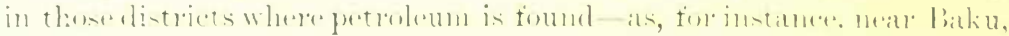

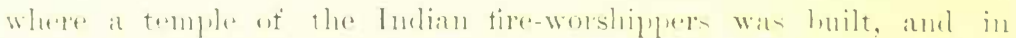

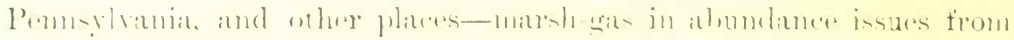

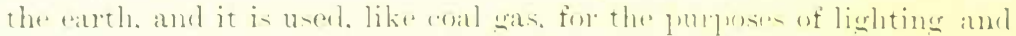

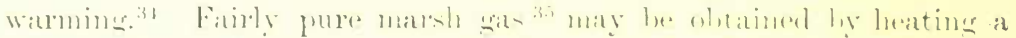

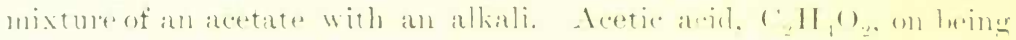

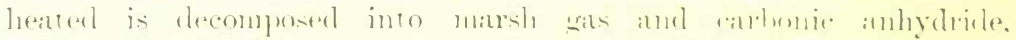

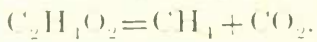

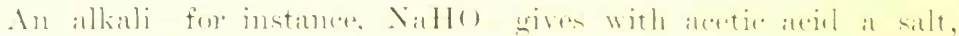

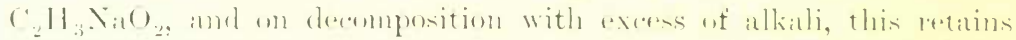

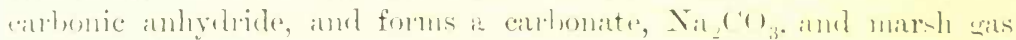
is firmerel.

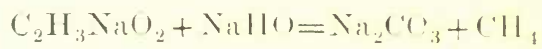

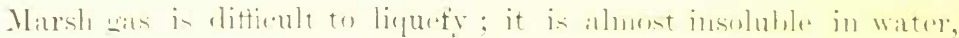
and is without taste or sumble The most impertant puint in eommetion

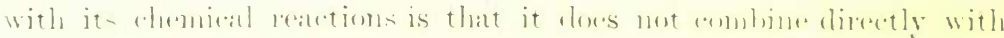
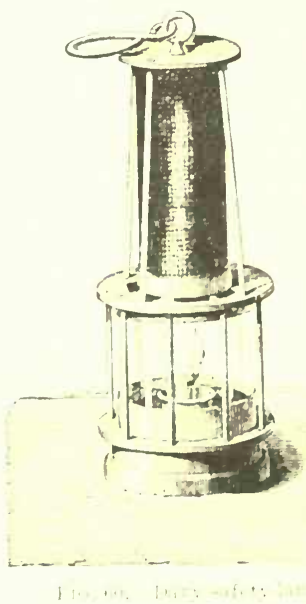

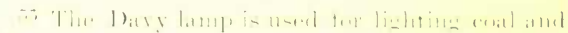

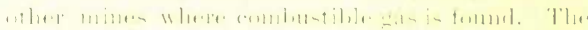

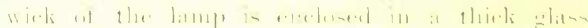

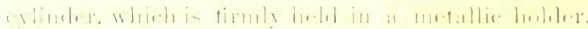

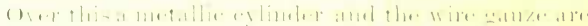

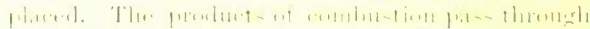

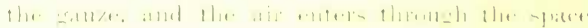

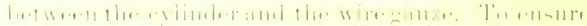

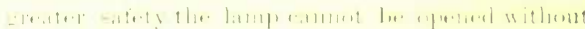

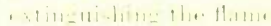

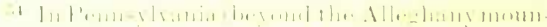

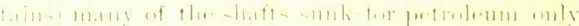

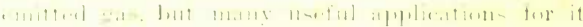

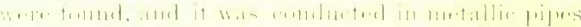

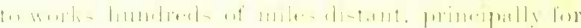

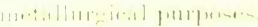

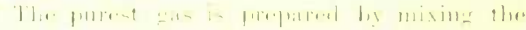

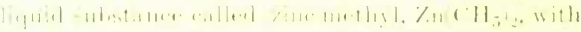

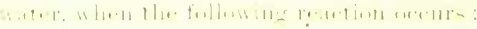

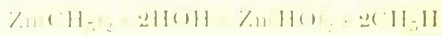


anything, whilst the other hydrocarbons which contain less hydrogen than expressed by the formula $\mathrm{C}_{n} \mathrm{H}_{2 n+2}$ are capable of combining with hydrogen, chlorine, certain acids, dre.

Marsh gas is the only hydrocarbon which contains one atom of carbon in its molecule, ${ }^{36}$ and stands, therefore, to that element in the same relation as water does to oxygen or ammonia to nitrogen. The multitude of hydrocarbons which carbon has the power of forming must be derived from this unique and simplest hydrocarbon. By arranging the molecules (and consequently equal volumes) in the following order -

$\mathrm{HH} ; \mathrm{OH}_{2} ; \mathrm{NH}_{3} ; \mathrm{CH}_{4}$;

$\mathrm{HCl} ; \mathrm{SH}_{2} ; \mathrm{PH}_{3}: \mathrm{SiH}_{4}$;

it is evident that hydrogen and chlorine are univalent, oxygen and sulphur bivalent, nitrogen and phosphorus trivalent, carbon and silicon quadrivalent. And if the law of substitution gives a very simple explanation of the formation of hydrogen peroxide as a compound containing two aqueous residues $(\mathrm{OH})(\mathrm{OH})$, then on the basis of this law all hydrocarbons ought to be derived from methane, $\mathrm{CH}_{4}$, as being the simplest hydrocarbon. The increase in complexity of a molecule of methane is brought about by the faculty of mutual combination which exists in the atoms of carbon, and, as a consequence of the most detailed study of the subject, much of that which might be foreseen and conjectured from the law of substitution has been actually brought about in such manner as might have been predicted. Although this subject, on account of its magnitude, appertains, as has been already stated, to the sphere of organic chemistry, yet it has been touched on here in order to show, although only in part, the best investigated example of the application of the law of substitution. According to this law, a molecule of methane, $\mathrm{CH}_{4}$, is capable of undergoing substitution in the four following ways:-(1) Methyl substitution, when the radicle equivalent to hydrogen $\mathrm{CH}_{3}$, called methyl, replaces the hydrogen. In $\mathrm{CH}_{4}$ this radicle is combined with $\mathrm{H}$, and therefore can replace it, as $(\mathrm{OH})$ replaces $\mathrm{H}$ because with it it gives water ; (2) methylene substitution, or the exchange between $\mathrm{H}_{2}$ and $\mathrm{CH}_{2}$ (this radicle is called methylene) is founded on a similar division of the molecule $\mathrm{CH}_{4}$ into two equivalent parts, $\mathrm{H}_{2}$ and $\mathrm{CH}_{2} ;(3)$ acetylene substitution, or the exchange between $\mathrm{CH}$ on the one hand and $\mathrm{H}_{3}$ on the other; and (4) carbon substitution - that is, the substitution of $\mathrm{H}_{4}$ by an atom of carbon $\mathrm{C}$, which is founded on the law of substitution similarly to the methyl substitution. These four cases of substitution render it possible to understand the principal relations of the hydrocarbons. For instance,

36 Methylene, $\mathrm{CH}_{2}$, does not exist. When attempts are made to obtain it (for example, by removing $\mathrm{X}_{2}$ from $\mathrm{CH}_{2} \mathrm{X}_{2}$ ), $\mathrm{C}_{2} \mathrm{H}_{4}$ or $\mathrm{C}_{3} \mathrm{H}_{6}$ are produced-that is to say, it undergoes polymerisation. 
the law of even numbers is seen from the fact that in all the cases of substitution mentioned the hydrogen atoms increase or decrease by an even number; but, as in $\mathrm{CH}_{2}$ they are likewise even, it follows that no matter how many substitutions they produce there will always be an even number of hydrogen atoms obtained. Indeed, when $\mathrm{H}$ is replaced by $\mathrm{CH}_{3}$ there is an increase of $\mathrm{CH}_{2}$; when $\mathrm{H}_{2}$ is replaced by $\mathrm{CH}_{2}$ there is no increase of hydrogen; in the acetylene substitution $\mathrm{CH}$ replaces $\mathrm{H}_{3}$, therefore there is an increase of $\mathrm{C}$ and a decrease of $\mathrm{H}_{2}$; in the carbon substitution there is a decrease of $\mathrm{H}_{4}$. In a similar way the law of limit may be deduced as a corollary of the law of substitution. In fact, the largest possible quantity of hydrogen introduced corresponds with the methyl substitution, but it leads to the introduction of $\mathrm{CH}_{2}$; therefore, no matter how many times this exchange may be effected with $\mathrm{CH}_{4}$, and, denoting the number of times by $(n-1)$, the result will always be $\mathrm{CH}_{4}(n-1)\left(\mathrm{CH}_{2}\right)$, or $\mathrm{C}_{n} \mathrm{H}_{2 n+2}$ as the highest hydrogen compound. Unsaturated hydrocarbons, containing less hydrogen, are evidently only formed when the increase of the new molecule derived from methane proceeds from one of the other forms of substitution. When the methyl substitution alone takes place with methane, $\mathrm{CH}_{4}$, it is evident that the saturated hydrocarbon formed is $\mathrm{C}_{2} \mathrm{H}_{6}$ or $\left(\mathrm{CH}_{3}\right)\left(\mathrm{CH}_{3}\right) \cdot{ }^{37}$ This is called ethane. Directly, by means of the methylene substitution alone, ethylene, $\mathrm{C}_{2} \mathrm{H}_{4}$, or $\left(\mathrm{CH}_{2}\right)\left(\mathrm{CH}_{2}\right)$ may be obtained from $\mathrm{CH}_{4}$, and by the acetylene substitution, $\mathrm{C}_{2} \mathrm{H}_{2}$ or $(\mathrm{CH})(\mathrm{CH})$, or acetylene, both the latter being unsaturated hydrocarbons. Thus we have all the possible hydrocarbons with two atoms of carbon in the molecule, $\mathrm{C}_{2} \mathrm{H}_{6}$, ethane, $\mathrm{C}_{2} \mathrm{H}_{4}$, ethylene, and $\mathrm{C}_{2} \mathrm{H}_{2}$, acetylene. But with them, according to the law of substitution, the same forms of substitution may be repeated - that is, the methyl,

37 Although the methods of formation and the reactions connected with hydrocarbons are not described in this work, because they are dealt with in organic chemistry, yet, in order to clearly show the mechanism of those transformations by which the carbon atoms are built up in the molecules of the carbon compounds, we here give a general example of reactions of this kind. From marsh gas, $\mathrm{CH}_{4}$, on the one hand, the substitution of chlorine or iodine, $\mathrm{CH}_{3} \mathrm{Cl}, \mathrm{CH}_{3} \mathrm{I}$, for the hydrogen may be effected, and, on the other hand, such metals as sodium may be substituted for the hydrogen, $\mathrm{CH}_{3} \mathrm{Na}$. Such and other similar products of substitution are exceedingly characteristic of hydrocarbons, and serve as a means of obtaining other more complex substances from given carbon compounds. If we place the two above-named products of substitution of marsh gas (metallic and haloid) in mutual contact, the metal combines with the halogen, forming a very stable compound-namely, common salt or sodium chloride and the carbon-groups which were in combination with them separate in mutual combination, as shown by the equation:

$$
\mathrm{CH}_{3} \mathrm{Cl}+\mathrm{CH}_{5} \mathrm{Na}=\mathrm{NaCl}+\mathrm{C}_{2} \mathrm{H}_{6} .
$$

This is the most simple example of the formation of a complex hydrocarbon from these

- radicles. The cause of the reaction must be sought for in the property which chlorine and sodium have of entering into mutual combination. 
methylene, acetylene, and even carbon substitutions (because $\mathrm{C}_{2} \mathrm{H}_{6}$ will still contain hydrogen when $\mathrm{C}$ replaces $\mathrm{H}_{4}$ ) and therefore further substitutions will serve as a source for the production of a set of saturated and unsaturated hydrocarbons, all containing more and more carbon in the molecule and, in the case of the acetylene substitution and carbon substitution, containing less and less hydrogen. Thus by means of the law of substitution we can foresee not only the limit $\mathrm{C}_{n} \mathrm{H}_{2 n+2}$, but an unlimited number of unsaturated hydrocarbons, $\mathrm{C}_{n} \mathrm{H}_{2 n}$, $\mathrm{C}_{n} \mathrm{H}_{2 n-2} \ldots \mathrm{C}_{n} \mathrm{H}_{2}\left({ }_{n-m}\right)$, where $m$ varies from 0 to $n-1,{ }^{38}$ and where $n$ increases indefinitely. From these facts not only does the existence of a multitude of polymeric hydrocarbons, differing in molecular weight, become evident, but it is also seen that there is a possibility of cases of isomerism with the same molecular weight. Already in the first unsaturated series $\mathrm{C}_{n} \mathrm{H}_{2 n}$ that polymerism so common to hydrocarbon compounds is apparent, because all the terms of this series $\mathrm{C}_{2} \mathrm{H}_{4}, \mathrm{C}_{3} \mathrm{H}_{6}, \mathrm{C}_{4} \mathrm{H}_{8} \ldots \mathrm{C}_{30} \mathrm{H}_{60} \ldots$ have one and the same composition $\mathrm{CH}_{2}$, but different molecular weights, as has been already explained in Chapter VII. (page 312). The differences in the vapour density, boiling points, and melting points, of the quantities entering into reactions, ${ }^{39}$ and the means of preparation ${ }^{40}$ also so clearly tally with the doctrine of polymerism, that this example will always be the clearest and most conclusive for the conception of polymerism and molecular weight. Such a case is also met with among other hydrocarbons. Thus benzene, $\mathrm{C}_{6} \mathrm{H}_{6}$, and cinnamene, $\mathrm{C}_{8} \mathrm{H}_{8}$, correspond with the composition of acetylene or to a compound of the composition $\mathrm{CH}^{41}$ The first boils at $81^{\circ}$, the second at $144^{\circ}$;

38 When $m=n-1$, we have the series $\mathrm{C}_{n} \mathrm{H}_{2}$. The lowest member is acetylene, $\mathrm{C}_{2} \mathrm{H}_{2}$. Amongst the hydrocarbons with $\mathrm{C}_{3}$, there ought to be two of the formula $\mathrm{C}_{3} \mathrm{H}_{2}$, one with the structure $\mathrm{CH}, \mathrm{CCH}$, and the other $\mathrm{CCH}_{2} \mathrm{C}$. But one of these is unknown. For $\mathrm{C}_{4} \mathrm{H}_{2}, \mathrm{CHCCCH}$ is known the diacetylene of $\mathrm{v}$. Baeyer, an explosive, easily destructible, gaseous substance. Nothing more is known of the series $\mathrm{C}_{n} \mathrm{H}_{2}$.

39 For instance, ethylene, $\mathrm{C}_{2} \mathrm{H}_{4}$, combines with $\mathrm{Br}_{2}, \mathrm{HI}, \mathrm{H}_{2} \mathrm{SO}_{4}$, as a whole molecule, as also does amylene, $\mathrm{C}_{5} \mathrm{H}_{10}$, and, in general, $\mathrm{C}_{n} \mathrm{H}_{2 n}$-that is, the reacting masses are proportional to the molecular weight or $n$, or to the density of the vapours (page 307).

40 For instance, ethylene is obtained by removing the water from ethyl alcohol, $\mathrm{C}_{2} \mathrm{H}_{5}(\mathrm{OH})$, and amylene, $\mathrm{C}_{5} \mathrm{H}_{10}$, from amyl alcohol, $\mathrm{C}_{5} \mathrm{H}_{11}(\mathrm{OH})$, or in general $\mathrm{C}_{2} \mathrm{H}_{2 n}$ from $\mathrm{C}_{n} \mathrm{H}_{2 n+1}(\mathrm{OH})$.

41 Acetylene and its polymerides have an empirical composition $\mathrm{CH}$, ethylene and its homologues (and polymerides) $\mathrm{CH}_{2}$, ethane $\mathrm{CH}_{3}$, methane $\mathrm{CH}_{4}$. This series presents a good example of the law of multiple proportions, but such diverse proportions are met with between the number of atoms of the carbon and hydrogen in the hydrocarbons already known that the accuracy of Dalton's law might be doubted. Thus the difference in weight between the substances $\mathrm{C}_{30} \mathrm{H}_{62}$ and $\mathrm{C}_{30} \mathrm{H}_{60}$ is so slight that it is within the inevitable errors of analysis, but their reactions and properties are so distinct that they can be distinguished beyond a doubt. Without Dalton's law, chemistry could not have been brought to its present condition, but it cannot alone express all those shades which are quite clearly understood and predicted by the law of Avogadro-Gerhardt. 
the specific gravity of the first is 0.899 ; that of the second, 0.925 , at $0^{\circ}$ - that is, here also the boiling point rises with the increase of molecular weight, and so also, as might be expected, does the density.

Cases of isomerism in the exact sense of the word - that is, when, with an identity of composition and of molecular weight, the properties of the substances are different-are very numerous among the hydrocarbons and their derivatives. Such cases are particularly important for the comprehension of molecular structure, and they also, like the polymerides, may be predicted from the above-mentioned conceptions, expressing the principles of the structure, of the carbon compounds ${ }^{42}$ based on the law of substitution. According to it, for example, it is evident that there can be no isomerism in the cases of the saturated hydrocarbons $\mathrm{C}_{2} \mathrm{H}_{6}$ and $\mathrm{C}_{3} \mathrm{H}_{8}$, because the former is $\mathrm{CH}_{4}$, where methyl has taken the place of $\mathrm{H}$, and as all parts of the hydrogen atoms of methane ought to be supposed to have the same relation to carbon, it is all the same which of them be subjected to the methyl substitution - the resulting product will only be ethane, $\mathrm{CH}_{3} \mathrm{CH}_{3} ;{ }^{43}$ and therefore the same argument applies in the case of propane, $\mathrm{CH}_{3} \mathrm{CH}_{2} \mathrm{CH}_{3}$, where one compound only can be imagined. It

42 The conception of the structure of hydrocarbon compounds-that is, the expression of those unions and correlations which their atoms have in the molecules-was for a long time limited to the representation that organic substances contained complex radicles (for instance, ethyl $\mathrm{C}_{2} \mathrm{H}_{5}$, methyl $\mathrm{CH}_{3}$, phenyl $\mathrm{C}_{6} \mathrm{H}_{5}$, \&c.) ; then about the year 1840 the phenomena of substitution and the correspondence of the products of substitution with the primary bodies (nuclei and types) were observed, but it was not until about the year 1860 and later when, on the one hand, the teaching of Gerhardt about molecules was spreading, and, on the other hand, the materials had accumnlated for discussing the transformations of the simplest hydrocarbon compounds, that conjectures began to appear as to the mutual connection of the atoms of carbon in the molecules of the complex hydrocarbon compounds. Then Kekule and A. M. Butleroff began to express the connection loetween the separate atoms of carbon, regarding it as a quadrivalent element. Although in their methods of expression and in some of their views they differ from each other, and also from the way in which the subject is treated in this work, yet the essence of the matter-namely, the comprehension of the causes of isomerism and of the union between the separate atoms of carbon-remains the same. In addition to this, starting from the year 1870, there appears a tendency, which from year to year increases, to discover the actual spacial distribution of the atoms in the molecules. Thanks to the endeavours of Le Bel (1874), Van't Hoff (1874), and Wislicenus (1887) in observing cases of isomerism-such as the effect of different isomerides on the direction of the rotation of the plane of polarisation of light-this tendency promises much for chemical mechanics, but the details of the still imperfect knowledge in relation to this matter must be sought for in special works devoted to organic chemistry.

43 Direct experiment shows that however $\mathrm{CH}_{3} \mathrm{X}$ is prepared (where $\mathrm{X}=$ for instance $\mathrm{Cl}, \& \mathrm{c}$.) it is always one and the same. If, for instance, in $\mathrm{CX}_{4}, \mathrm{X}$ is gradually replaced by hydrogen until $\mathrm{CH}_{3} \mathrm{X}$ is produced, or in $\mathrm{CH}_{4}$ the hydrogen by various means is replaced by $\mathrm{X}$, or else, for instance, if $\mathrm{CH}_{3} \mathrm{X}$ be obtained by the decomposition of more complex compounds, the same product is always obtained.

This was shown in the year 1860 , or there about, by many methods, and is the fundamental conception of the structure of hydrocarbon compounds. If the atoms of hydro- 
is to be expected, however, that there should be two butanes, $\mathrm{C}_{4} \mathrm{H}_{10}$, and this is actually the case. In one methyl may be considered as replacing the hydrogen of one of the methyls, $\mathrm{CH}_{3} \mathrm{CH}_{2} \mathrm{CH}_{2} \mathrm{CH}_{3}$; and in the other $\mathrm{CH}_{3}$ may be considered as substituted for $\mathrm{H}$ in $\mathrm{CH}_{2}$, and there it will consist of $\mathrm{CH}_{3} \mathrm{CH}_{\mathrm{CH}_{3}}^{\mathrm{CH}_{3}}$. It may be also regarded as methane, in which three of hydrogen are exchanged for three of methyl. On going further in the series it is evident that the number of possible isomerides will be still greater. But we have limited ourselves to the simplest examples, showing the possibility and actual existence of isomerides. $\mathrm{C}_{2} \mathrm{H}_{4}$ and $\mathrm{CH}_{2} \mathrm{CH}_{2}$ are, it is evident, identical, but there ought to be, and are, two hydrocarbons of the composition $\mathrm{C}_{3} \mathrm{H}_{6}$, propylene and trimethylene ; the first is ethylene $\mathrm{CH}_{2} \mathrm{CH}_{2}$, in which one part of hydrogen is exchanged for methyl $\mathrm{CH}_{2} \mathrm{CHCH}_{3}$, and trimethylene is ethane, $\mathrm{CH}_{3} \mathrm{CH}_{3}$, only with the exchange of methylene for two hydrogen atoms from two methyl groups - that is, $\mathrm{CH}_{2} \mathrm{CH}_{2}$, ${ }^{44}$ where the methyl introduced is united to both the atoms of carbon existing in $\mathrm{CH}_{3} \mathrm{CH}_{3}$. It is evident that the cause of isomerism here is, on the one hand, the difference of the amount of hydrogen in union with the particular atoms of carbons, and, on the other, the different connection between the separate atoms of carbon. In the first case they may be said to be chained together (more generally to form 'an open chain'), and in the second case, to be locked up (to form a ' closed chain' or 'ring '). Here, also, it is easily understood that on increasing the quantity of carbon atoms the number of conjectured and existing isomerides will greatly increase. If, also, in addition to the substitution of one of the radicles of methane for hydrogen a further exchange of part of the hydrogen for some of the other groups of elements X, Y .... occurs, the quantity of possible isomerides still further increases in a considerable degree. For instance, there are even two possible isomerides for the derivatives of ethane, $\mathrm{C}_{2} \mathrm{H}_{6}$ : if two parts of the hydrogen be exchanged for $\mathrm{X}_{2}$, one

gen in methyl were not absolutely identical in value and position (as, for instance, in $\mathrm{CH}_{3} \mathrm{CH}_{2} \mathrm{CH}_{3}$, or $\mathrm{CH}_{3} \mathrm{CH}_{2} \mathrm{X}$ ), then there would be as many different forms of $\mathrm{CH}_{3} \mathrm{X}$ as there were diversities in the atoms of hydrogen in $\mathrm{CH}_{4}$. The scope of this work does not permit of a more detailed account of this matter. It is given in works on organic chemistry.

44 The union of carbon atoms in closed chains or rings was first suggested by Kekulé as an explanation of the structure and isomerism of the derivatives of benzene, $\mathrm{C}_{6} \mathrm{H}_{6}$, forming aromatic compounds (Note 26). It is now incontestable, although the question of isomerism between the benzene derivatives cannot be considered as finally solved, that this closed connection exists, and gives a peculiar character to these compounds. 


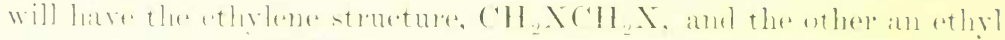

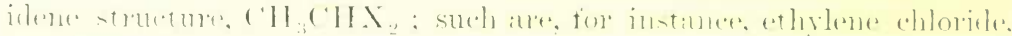

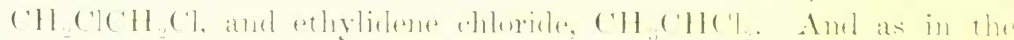

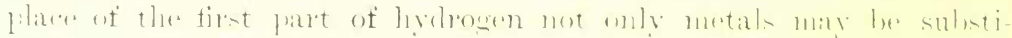

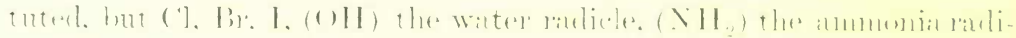

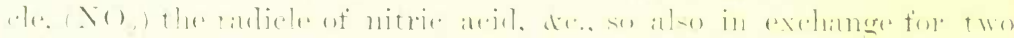

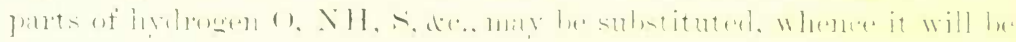

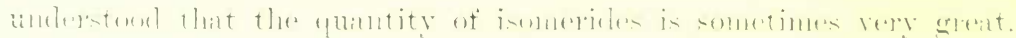

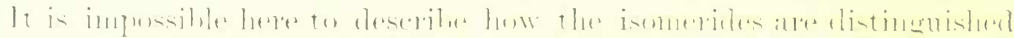

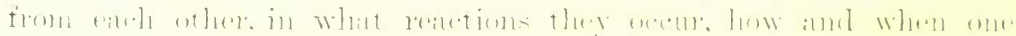

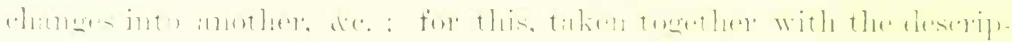

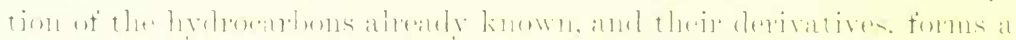

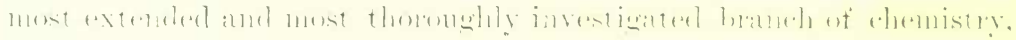

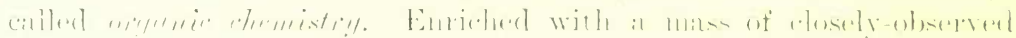

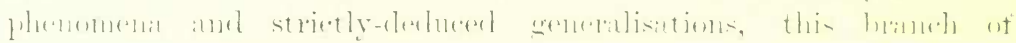

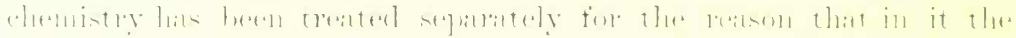

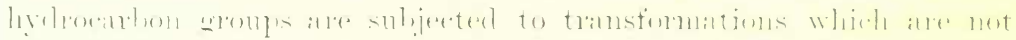

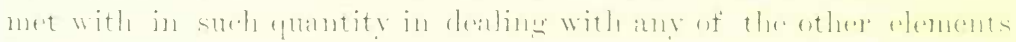

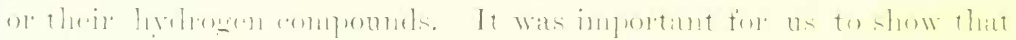

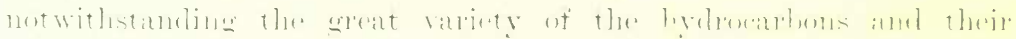

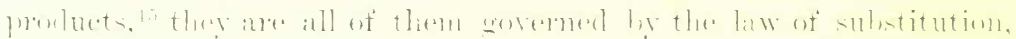

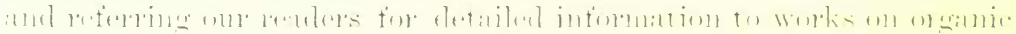

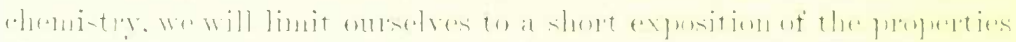

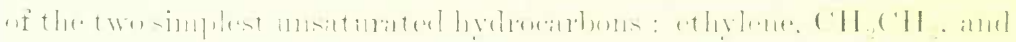

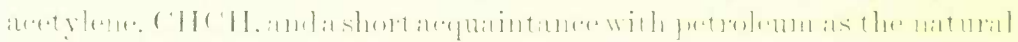

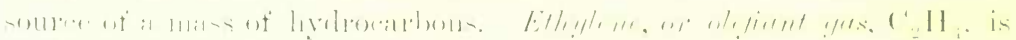

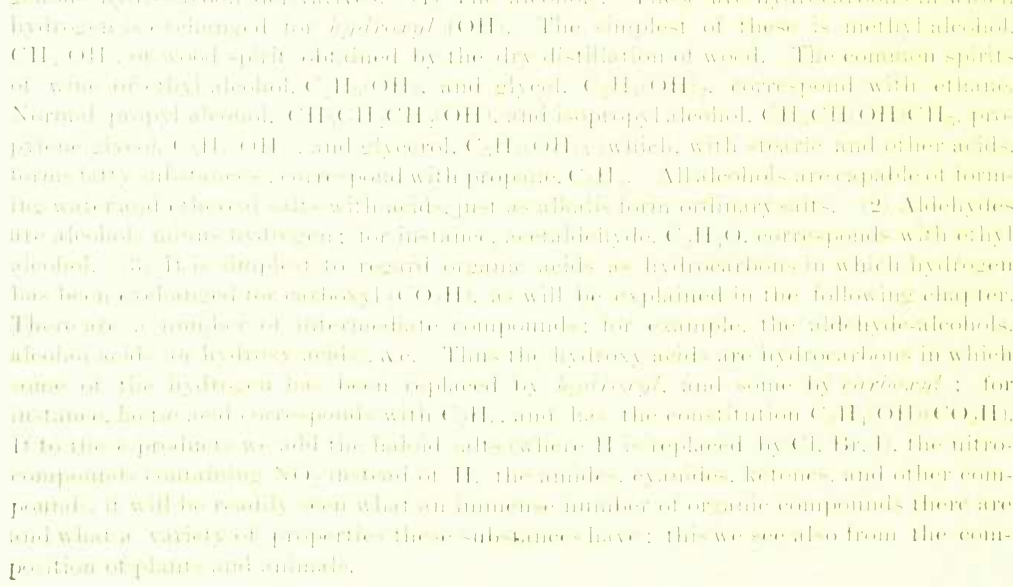


the lowest known member of the unsaturated hydrocarhon series of the composition $\mathrm{C}_{n} \mathrm{H}_{2 n}$. As in composition it is equal to two molecules of marsh gas deprived of two molecules of hydrogen, it is evident that it might be, and actually can, together with hydrogen, be produced, although but in small quantities, by heating marsh gas. On being heated, however, olefiant gas is decomposed into charcoal and marsh gas : $\mathrm{C}_{2} \mathrm{H}_{4}=\mathrm{CH}_{4}+\mathrm{C}$; and therefore in those cases where marsh gas is produced by heating, olefiant gas, hydrogen, and charcoal will also be formed, although only in small quantities. The lower the temperature at which complex organic substances are heated, the greater the quantity of olefiant gas found in the gases given off; at a white heat it is entirely decomposed into charcoal and marsh gas. If coal, wood, and more particularly petroleum, tars and fatty substances are subjected to dry distillation, they give off illuminating gas, which contains more or less olefiant gas.

Olefiant gas, almost free from other gases, ${ }^{46}$ may be obtained from ordinary alcohol (if possible, free from water), if it be mixed with five parts of strong sulphuric acid and the mixture heated to slightly above $100^{\circ}$. Under these conditions, the sulphuric acid removes the elements of water from the alcohol, $\mathrm{C}_{2} \mathrm{H}_{5}(\mathrm{OH})$, and gives olefiant gas, $\mathrm{C}_{2} \mathrm{H}_{6} \mathrm{O}=\mathrm{H}_{2} \mathrm{O}+\mathrm{C}_{2} \mathrm{H}_{4}$. The greater molecular weight of olefiant gas compared with marsh gas points out that it may be comparatively easily converted into a liquid by means of pressure or great cold; this may be effected, for example, by the evaporation of liquid nitrous oxide. Its absolute boiling-point is $+10^{\circ}$, it boils at $-103^{\circ}$ ( 1 atmosphere), liquefies at $0^{\circ}$, at a pressure of 43 atmospheres, and solidifies at $-160^{\circ}$. Ethylene is colourless, has a slight ethereal smell, is slightly soluble in water, and somewhat more soluble in alcohol and in ether (in five volumes of spirit and six volumes of ether). ${ }^{47}$

Like other unsaturated hydrocarbons, olefiant gas readily enters

46 Ethylene bromide, $\mathrm{C}_{2} \mathrm{H}_{4} \mathrm{Br}_{2}$, when gently heated in alcoholic solution with finely divided zinc, yields pure ethylene, the zinc merely taking up the bromine (Sabaneyeff).

17 Ethylene decomposes somewhat easily under the influence of the electric spark, or a high temperature. In this case the volume of the gas formed may remain the same when olefiant gas is decomposed into carbon and marsh gas, or may increase to double its volume when hydrogen and carbon are formed, $\mathrm{C}_{2} \mathrm{H}_{4}=\mathrm{CH}_{4}+\mathrm{C}=2 \mathrm{C}+2 \mathrm{H}_{2}$. A mixture of olefiant gas and oxygen is highly explosive; two volumes of this gas require six volumes of oxygen for its perfect combustion. The eight volumes thus taken then resolve themselves into a mixture of eight volumes of the products of combustion, water and carbonic anhydride, $\mathrm{C}_{2} \mathrm{H}_{4}+3 \mathrm{O}_{2}=2 \mathrm{CO}_{2}+2 \mathrm{H}_{2} \mathrm{O}$. On cooling, after the explosion, diminution of volume occurs because the water becomes liquid. For two volumes of the olefiant gas taken, the diminution will be equal to four volumes, and the same for marsh gas. The quantity of carbonic anhydride formed by both gases is not the same. Two volumes of marsh gas give only two volumes of carbonic anhydride, and two volumes of ethylene give four volumes of carbonic anhydride $\left(2 \mathrm{CO}_{2}\right)$. 
into combination with certain substances, such as chlorine, bromine, iodine, fuming sulphuric acid, or sulphuric anhydride. If olefiant gas be sealed up with a small quantity of sulphuric acid in a glass vessel, and be subjected to continued shaking (as, for instance, by suspending it from a moving part of a machine), the prolonged contact and repeated mixing causes the olefiant gas, little by little, to combine with the sulphuric acid, forming $\mathrm{C}_{2} \mathrm{H}_{4} \mathrm{H}_{2} \mathrm{SO}_{4}$. If, after this absorption, the sulphuric acid be diluted with water and distilled, alcohol separates, which is produced in this case by the olefiant gas combining with the elements of water, $\mathrm{C}_{2} \mathrm{H}_{4}+\mathrm{H}_{2} \mathrm{O}=\mathrm{C}_{2} \mathrm{H}_{6} \mathrm{O}$. In this reaction (Berthelot) we see an excellent example of the fact that if a given substance, like olefiant gas, is produced by the decomposition of another, then in the reverse way this substance, entering into combination, is capable of forming the original substance-in our example, alcohol. Therefore the reaction, $\mathrm{CH}_{3} \mathrm{CH}_{2}(\mathrm{OH})=\mathrm{H}(\mathrm{OH})+\mathrm{CH}_{2} \mathrm{CH}_{2}$, ought to be classed as a reversible reaction. In combination with various molecules, $\mathrm{X}_{2}$, ethylene gives saturated compounds, $\mathrm{C}_{2} \mathrm{H}_{4} \mathrm{X}_{2}$-that is, either $\mathrm{CH}_{2} \mathrm{XCH}_{2} \mathrm{X}$, or $\mathrm{CH}_{3} \mathrm{CHX}_{2}$, which correspond with ethane, $\mathrm{CH}_{3} \mathrm{CH}_{3}$, or $\mathrm{C}_{2} \mathrm{H}_{6}$. ${ }^{48}$

Acetylene, $\mathrm{C}_{2} \mathrm{H}_{2}=\mathrm{CHCH}$, is a gas; it was first prepared by Berthelot (1857). It has a very pungent smell, is characterised by its great constancy under the action of heat, and is the only product of the direct combination of carbon with hydrogen. This combination takes place under the action of great heat, such as is produced by a very strong electric current between carbon points. A brilliant arc (voltaic) is then formed between the carbon electrodes, which contains particles of carbon passing from one pole to the other. If the carbons be surrounded with an atmosphere of hydrogen, the carbon in part combines with the hydrogen, forming $\mathrm{C}_{2} \mathrm{H}_{2}$. Acetylene may be formed from olefiant gas if two atoms of hydrogen be taken from it. This may be effected in the following way : the olefiant gas is first made to combine with bromine, giving $\mathrm{C}_{2} \mathrm{H}_{4} \mathrm{Br}_{2}$; from this the hydrobromic acid is taken by means of an alcoholic solution of caustic potash, leaving the volatile product $\mathrm{C}_{2} \mathrm{H}_{3} \mathrm{Br}$; and from this yet another part of hydrobromic acid is withdrawn by passing it through anhydrous alcohol in which metallic sodium has been dissolved, or by heating it with a strong alcoholic solution of caustic potash. Under these cir-

48 The homologues of ethylene, $\mathrm{C} \mathrm{H}_{2 n}$, are also capable of direct combination with halogens, \&c., but with various degrees of facility. The composition of these homologues can be expressed thus: $\left(\mathrm{CH}_{3}\right)_{x}\left(\mathrm{CH}_{2}\right)_{y}(\mathrm{CH})_{2} \mathrm{C}_{r}$ where the sum of $x+z$ is always an even number, and the sum of $x+z+r$ is equal to half the sum of $3 x+z$, whence $z+2 r=x$; by this means the possible isomerides are determined. For example, for butylenes, $\mathrm{C}_{4} \mathrm{H}_{8}$, $\left(\mathrm{CH}_{3}\right)_{2}(\mathrm{CH})_{2},\left(\mathrm{CH}_{3}\right)_{2}\left(\mathrm{CH}_{2}\right) \mathrm{C},\left(\mathrm{CH}_{2}\right)\left(\mathrm{CH}_{2}\right)_{2} \mathrm{CH}$, and $\left(\mathrm{CH}_{2}\right)_{4}$ are possible. 
cumstances (Berthelot, Sawitsch, Miasnikoff) the alkali takes up the hydrobromic acid from $\mathrm{C}_{n} \mathrm{H}_{2 n-1} \mathrm{Br}$, forming $\mathrm{C}_{n} \mathrm{H}_{2 n-2}$.

Acetylene is also produced in all those cases where organic substances are decomposed by the action of a high temperature - for example, by dry distillation. On this account a certain quantity is always found in coal gas, and gives to it, at all events in part, its peculiar smell, but the quantity of acetylene in coal gas is very small. If the vapour of alcohol be passed through a heated tube a certain quantity of acetylene is formed. It is also produced by the imperfect combustion of olefiant and marsh gas-for example, if the flame of coal gas has not free access to air. ${ }^{49}$ The inner part of every flame contains gases in imperfect combustion, and in them some amount of acetylene.

Acetylene, being further removed than ethylene from the limit $\mathrm{C}_{n} \mathrm{H}_{2 n+2}$ of hydrocarbon compounds, has a still greater faculty of combination than is shown by olefiant gas, and therefore can be more readily separated from any mixture containing it. Actually, acetylene not only combines with one and two molecules of $\mathrm{I}_{2}, \mathrm{HI}, \mathrm{H}_{2} \mathrm{SO}_{4}, \mathrm{Cl}_{2}, \mathrm{Br}_{2}$, \&c. . . . (many other unsaturated hydrocarbons combine with them) but also with cuprous chloride, $\mathrm{CuCl}$, forming a red precipitate. If a gaseous mixture containing acetylene be passed through a solution of cuprous chloride (or $\mathrm{AgNO}_{3}$ ) and ammonia water, the other gases do not combine, but the acetylene gives a red precipitate (or gray with silver), which on being struck with a hammer decomposes with an explosion. This red precipitate gives off acetylene under the action of acids. In this manner pure acetylene may be produced. Acetylene and its homologues also readily react with corrosive sublimate, $\mathrm{HgCl}_{2}$ (Koucheroff, Favorsky). Acetylene burns with a very brilliant flame, which is accounted for by the comparatively large amount of carbon it contains. ${ }^{50}$

The formation and existence in nature of large masses of petroleum or a mixture of liquid hydrocarbons, principally of the series $\mathrm{C}_{n} \mathrm{H}_{2+2}$ and $\mathrm{C}_{n} \mathrm{H}_{2 n}$ is in most respects remarkable. ${ }^{51}$ In some mountainous dis-

49 This is easily accomplished with those gas lamps which are used in laboratories and mentioned in the Introduction, page 11. In these lamps the gas is first mixed with air in a long tube, above which it is kindled. But if it be lighted inside the pipe it does not fully burn, but forms acetylene, on account of the cooling effect of the walls of the metallic tube; this is observed by the smell, and may be shown by passing the issuing gas (by aid of an aspirator) into an ammoniacal solution of cuprous chloride.

50 Amongst the homologues of acetylene, $\mathrm{C}_{n} \mathrm{H}_{2 n-2}$, the lowest is $\mathrm{C}_{5} \mathrm{H}_{4}$; allylene, $\mathrm{CH}_{3} \mathrm{CCH}$, and allene, $\mathrm{CH}_{2} \mathrm{CCH}_{2}$, are known, but the closed structure, $\mathrm{CH}_{2}(\mathrm{CH})_{2}$, is unknown.

51 The saturated hydrocarbons predominate in American petroleum, especially in its more volatile parts; in Baku naphtha the hydrocarbons of the composition $\mathrm{C}_{n} \mathrm{H}_{2 n}$ form 
tricts--as, for instance, by the slopes of the Caucasian chain, on inclines. in a parallel direction to the chain - an oily liquid issues from the earth together with salt water and hot gases (methane and others) ; it has a tarry smell, dark brown colour, and is lighter than water. This liquid is called naphtha or rock oil (petroleum), and is obtained in large quantities by making wells and deep bore-holes in those places where traces of naphtha are observed, the naphtha being sometimes thrown up from the wells in fountains of considerable height. ${ }^{52}$ The evolution of naphtha is always accompanied by salt water and marsh gas. Naphtha has from ancient times been worked in Russia in the Apsheron peninsula near Baku, and is also now worked in Burmah (India), in Galicia near the Carpathians, and in America, especially in Pennsylvania and Canada. Naphtha does not consist of one definite hydrocarbon, but of a mixture of many, and its density, external appearance, and other qualities vary with the amount of the different hydrocarbons of which it is composed. The light kinds of naphtha have a specific gravity about, 0.8 and the heavy kinds up to $0 \cdot 98$. The former are very mobile liquids, and are more volatile ; the latter contain less of the volatile hydrocarbons and are less mobile. When the light kinds of naphtha are distilled the boiling point in the vapours constantly changes, beginning at $0^{\circ}$ and going up to above $350^{\circ}$. That which passes over first is a very mobile, colourless ethereal liquid, from which the hydrocarbons whose boiling points start from $0^{\circ}$ may be extracted - namely, the hydrocarbons $\mathrm{C}_{4} \mathrm{H}_{10}, \mathrm{C}_{5} \mathrm{H}_{12}$ (which boils at $30^{\circ}$ ), $\mathrm{C}_{6} \mathrm{H}_{14}$ (boils at $62^{\circ}$ ), $\mathrm{C}_{7} \mathrm{H}_{16}$ (boils about $\left.90^{\circ}\right)$, drc. Those fractions of the distillate of naphtha which boil

the main part (Lisenko, Markovinkoff, Beilstein), but doubtless (Mendeléeff) it also contains saturated ones, $\mathrm{C}_{n} \mathrm{H}_{2 n+2}$. The strueture of the naphtha hydrocarbons is only known for the lower homologues, but doubtless the distinction between the hydrocarbons of the Pennsylvanian and Baku naphthas, boiling at the same temperature (after the requisite refining by repeated, methodical distillation, which can be very conveniently done by means of steam, passing the steam through the dense mass-that is, by means of rectification), depends not only on the predomination of saturated hydrocarbons in the former, and naphthenes, $\mathrm{C}_{n} \mathrm{H}_{n}$, in the latter, but also on the diversity of composition and structure of the corresponding portions of the distillation. The products of the Baku naphtha are richer in earbon (therefore, in a suitably constructed lamp they ought to give a brighter light), they are of greater specific gravity, and have greater internal friction (and are therefore more suitable for lubricating macininery) than the American produsts. collected at the same temperature.

52 The formation of naphtha fountains (which burst forth after the higher clay strata covering the layers of sand impregnated with naphtha lave been bored through) is without doubt eaused by the pressure or tension of the combustible hydrocarbon gases which accompany the naphtha, and are soluble in it under pressure. Sometimes these naphtha fountains reach a height of 100 metres-for instance, the fountain of 1887 , near Baku. Naphtha fountains generally act periodically, and their force diminishes with the lapse of time, which might be expected, because the gases which cause the fountains find an outlet, and the naphtha issuing from the bore-hole carres away the sand which was partially choking it up. 
above $130^{\circ}$, and contain hydrocarbons with $\mathrm{C}_{9}, \mathrm{C}_{10}, \mathrm{C}_{11}$, \&c., enter into the composition of the oily substance, universally used for lighting, called kerosene or photogen or photonaphthalene and other names. The specific gravity of kerosene is from 0.78 to 0.84 , and it smells like naphtha. Those products of the distillation of naphtha which pass off below $130^{\circ}$ and have a specific gravity below 0.75 , enter into the composition of light petroleum (benzoline, ligroin, petroleum spirit, \&c.); which is used as a solvent for india-rubber, for taking out grease spots, dc. Those portions of naphtha (which can only be distilled without change by nieans of superheated steain, otherwise they are largely decomposed) which boil above $275^{\circ}$ to $300^{\circ}$ and have a specific gravity higher than $0 \cdot 85$, form an excellent oil, ${ }^{53}$ safe as regards inflammability (which is very important as diminishing the risks of fire), and may be used in lamps as an effective substitute for kerosene. ${ }^{54}$ Those portions of naphtha which pass over at a still higher temperature and have a higher specific gravity than 0.9 , which are found in abundance (about 30 p.c.) in the Baku naphtha, make excellent lubricating or machine oils. Naphtha

55 This is a so-called intermediate (between kerosene and lubricating oils) oil or pyronapht. Lamps are already being manufactured for burning it, but still require. improvement. Above all, however, it requires a more extended market, and at present is likely to want it owing to the following two reasons: (1) The products of the American petroleum, which are the most widely spread and almost universally consumed, contain but little of this intermediate oil, and what there is is partly introduced into the kerosene and partly into the lubricating oils; (2) the Baku naphtha, which is capable of yielding a great deal (up to 30 p.c.) of intermediate oil, is produced in enormous quantities, about 165 million poods (1887), but has no regular markets abroad, and for the consumption in Russia (about 20 million poods of kerosene per annum) and for the limited export. (20 millions poods per annum) into Western Europe (by the Trans-Caucasian Railway) those volatile and more dangerous parts of the naphtha which enter into the composition of the American petroleumare sufficient, because Baku naphtha yields about 20 p.c of such kerosene. For this reason pyronapht is not manufactured in sufficient quantities, and the whole world is consuming the unsafe kerosene. When a pipe line has been laid from Baku to the Black Sea (in America there are many which carry the raw naphtha to the sea-shore, where it is made into kerosene and other products) then the whole mass of the Baku naphtha will furnish safe illuminating oils, which, without doubt, will find an immense application. A mixture of the intermediate oil with kerosene or Baku oil (specific gravity 0.84 to 0.85 ) may be considered (on removing the benzoline) to be the best illuminating oil, because it is safe (flashing point from $40^{\circ}$ to $60^{\circ}$ ), cheaper (Baku naphtha gives as much as 60 p.c. of Baku oil), and burns perfectly well in lamps, differing but little from those made for burning American kerosene (unsafe, flashing point $20^{\circ}$ to $25^{\circ}$ to $30^{\circ}$ ).

54 The substitution of Baku pyronapht, or intermediate oil, or Baku oil (see Note 53), would not only be a great advantage as regards safety from fire, but would also be highly economical. A ton ( 62 poods) of American crude petroleun costs there at the coast considerably more than 24s. (12 roubles), and yields two-thirds of a ton of kerosene suitable for ordinary lamps. A ton of raw naphtha in Baku costs less than 4s. (1 rouble 80 copecks), and with a pipe line to the slore of the Black Sea would not cost more than 8 roubles, or $16 s$. And a ton of Baku naphtha will also yield as much as two-thirds of a ton of kerosene, Baku oil, and pyronapht suitable for illuminating purposes. 
has many important applications, and the naphtha industry is now of great commercial importance, especially as naphtha and its refuse may be used as fuel. ${ }^{5.5}$ Whether naphtha was formed from organic matter is very doubtful, as it is found in the most ancient Silurian strata which correspond with epochs of the earth's existence when there was little organic matter; it could not penetrate from the higher to the lower strata (more ancient) as it floats on water (and water penetrates through all strata). It therefore tends to rise to the surface of the earth, and it is always found in highlands parallel to the direction of the mountains. ${ }^{56}$ It is much more probable that its formation should be attributed to the action of water, penetrating through the crevices formed on the mountain slopes to the heart of the earth, on that kernel of heated metallic matter which must be accepted as existing in the interior of the earth. And as meteoric iron often contains carbon (like cast iron), so, accepting the existence of such carburetted iron at unattainable depths in the interior of the earth, it may be sup-

$\therefore$ Naphtha has been applied to heating purposes on a large scale in Russia only, not only on account of the low cost of naphtha and of the refuse from the preparation of kerosene, but also because the products of all the Baku naphtha do not find an outlet for universal consumption. Naphtha itself and its various residues form excellent fuel, burning without smoke and giving a high temperature (steel and iron may be easily inelted in the flame). A hundred poods of good coal (for instance, Don coal) used as fuel for heating boilers are equivalent to 36 cubic feet (about 250 poods) of dry wood, while only 70 poods of naphtha will be required; and, moreover, there is no need for stoking, as the liquid can be readily and evenly supplied in the required quantity. The economical and other questions dealing with American and Baku petroleums have been discussed more in detail in some separate works of mine (D. Mendeléeff): (1) 'The Naphtha Industry of Pennsylvania and the Caucasus,' 1870 ; $(2)$ 'Where to Build Naphtha Works,' 1880; (3) 'On the Naphtha Question,' 1883; (4) 'The Baku Naphtha Question,' 1886.

56 As during the process of the dry distillation of wood, seaweed, and similar vegetable débris, and also when fats are decomposed by the action of heat (in closed vessels), hydrocarbons similar to those of naphtha are formed, it was natural that this should have been turned to account to explain the formation of the latter. But the hypothesis of the formation of naphtha from vegetable debris inevitably assumes coal to be the chief element of decomposition, and nuphtha is met with in Pennsylvania and Canada, in the Silurian and Devonian strata, which do not contain coal, and correspond to an epoch not abounding in orgunic matter. Coal was formed from the vegetable débris of the Carboniferous, Jurassic, and other recent strata, but, judging from the composition and structure, it was subjected to the sume decomposition as peat; neither could liquid hydrocarbons have been formed to such an extent as we see in naphtha. If we ascribe the derivation of naphtha to the decomposition of fat (adipose, animal fat), we encounter three almost insuperable difficulties: (1) Animal remains would furnish a great deal of nitrogenous matter, whilst there is but very little in naphtha; (2) the enormous quantity of naphtha already discovered as compared with the insignificant amount of fat in the animal carcase; (3) the sources of naphtha always running parallel to mountain chains is completely inexplicable. Being struck with this last-mentioned circumstance in Pennsylvania, and finding that the sources in the Caucasus surround the whole Caucasian range (Baku, Tiflis, (rouria, Kouban, Taman, Groznoe, Dagestan), I developed in 1876 the hypothesis of the mineral origin of naphtha expounded further on. 
posed that naphtha was produced by the action of water penetrating through the crevices of the strata during the upheaval of mountain chains, ${ }^{57}$ because water with iron carbide ought to give iron oxide and lyydrocarbons. ${ }^{58} \quad$ Direct experiment proves that the so-called spiegeleisen (manganiferous iron, rich in chemically-combined carbon) when treated with acids gives liquid hydrocarbons, ${ }^{59}$ which in

37 During the upheaval of mountain ranges crevasses would be formed at the peaks with openings upwards, and at the foot of the mountains with openings downwards. These cracks in course of time fill up, but the younger the mountains (the Alleghany mountains are, without doubt, more recent; they were formed during the tertiary epoch) the fresher the cracks; through them water must gain access deep into the recesses of the earth to an extent that could not occur on the level (on plains). The situation of naphtha at the foot of mountain chains is the principal argument in my hypothesis.

Another fundamental reason is the consideration of the mean density of the earth. Cavendish, Airy, Cornu, and many others who have investigated the subject by various methods, found that, taking water $=1$, the mean density of the earth is nearly $5 \cdot 5$. As: at the surface water and all rocks (sand, clay, limestone, granite, \&c.) have a density less than 3, it is evident (as solid substances are but slightly compressible even under the greatest pressure) that inside the earth there are substances of a greater densitynamely, not less than 7 or 8 . What conclusion can then be arrived at? Something heary contained in the bosom of the earth must be scattered not only on its surface, but in the whole solar system, because everything tends to show that the sun and planets proceed from the same material, and, according to the hypothesis of Laplace and Kant, it is most probable, and even ought to be held, that the earth and planets are but fragments of the solar atmosphere which have had time to cool considerably and become masses semi-liquid inside and solid outside, forming planets and satellites. The sun, amongst other heavy elements, contains a great deal of iron, as shown by spectrum analysis. There is also much of it in an oxidised condition on the surface of the earth. Meteoric stones, carried as fragments of the planets in the solar system and sometimes falling upon the earth, consisting of siliceous rocks similar to terrestrial ones, often contain either dense masses of iron (for example, the Pallosovo iron preserved in the St. Petersburg Academy of Sciences) or granular masses (for instance, the Okhansk meteorite of 1886). For this reason it is possible that the interior of the earth contains much iron in a metallic state. This might be expected from the hypothesis of Laplace, for the iron must have been compressed into a liquid at that period when the other component parts of the earth were still strongly heated, and oxides of iron could not yet be formed. The iron was covered with slags (mixtures of silicates, like glass fused together with rocky matter), which did not allow it to burn at the expense of the oxygen of the atmosphere and water, just at that time when the temperature of the earth was. very high. Carbon was in the same state; its oxides were also capable of dissociation (Deville); it is also but slightly volatile, and has an affinity for iron, and iron carbide is found in meteoric stones. On this account the supposition of the existence of iron carbides in the interior of the earth was drawn by me from many indications, some of which are confirmed by the fact that granular pieces of iron have been found in some basalts (arcient lava) as well as in meteoric stones.

38 The following is the typical equation for this formation :

$$
3 \mathrm{Fe}_{m} \mathrm{C}_{n}+4_{m} \mathrm{H}_{2} \mathrm{O}=m \mathrm{Fe}_{3} \mathrm{O}_{4} \text { (magnetic oxide) }+\mathrm{C}_{5 n} \mathrm{H}_{8 m} \text {. }
$$

59 Cloez investigated the hydrocarbons formed when cast-iron is dissolved in hydrochloric acid, and found $\mathrm{C}_{n} \mathrm{H}_{2 n}$ and others. I treated crystalline nanganiferous cast-iron with the same acid, and obtained a liquid mixture of hydrocarbons exactly similar to natural naphtha in taste, smell, and reaction. The occurrence of iron with carbon during the formation' of the earth is all the more probable because those elements predominate in nature which have small atomic weights, and among them the most widely-diffused, the most difficultly-fusible, and therefore the most easily-condensed (Chap. XV.) are 


\section{composition, appearance, and properties are completely identical with naphtha. ${ }^{60}$}

carbon and iron. They passed into the liquid state when all compounds were at a temperature of dissociation.

69 Probably naphtha was produced during the upheaval of all mountain cliains, but only in some cases were the conditions favourable to its being preserved underground. The water penetrating below formed there a mixture of naphtha and watery vapours, and this mixture issued through fissures to the cold parts of the earth's crust. The naphtha vapours, on condensing, formed naphtha, which, if there were no obstacles, appeared on the surface of land and water. Here part of it soaked through formations (perhaps the bituminous slates, schists, domites, \&c., were thus formed), another part was carried away on the water, became oxidised, evaporated, and was driven to the shores (the Caucasian naphtha probably in this way, during the existence of the AraloCaspian sea, was carried as far as the Sisran banks of the Volga, where many strata are impregnated with naphtha and products of its oxidation resembling asphalt and pitch); a great part of it was burnt in one way or another-that is, gives carbonic anhydride and water. If the mixture of vapours, water, and naphtha formed inside the earth had no free outlet to the surface, it nevertheless would find its way through fissures to the superior and colder strata, and there become condensed. Some of the formations (clays) which do not absorb naphtha were only washed away by the warm water, and formed mud, which we also now observe issuing from the earth in the form of mud volcanoes. All the suburbs of Baku and the whole of the Caucasus in the neighbourhood of the naphtha districts are full of such volcanoes, still from time to time in a state of eruption. In old naphtha beds (such as the Pennsylvanian) even these blow-holes are closed, and the mud volcanoes have had time to be washed away. The naphtha and the gaseous hydrocarbons formed with it under the pressure of the overlying earth and water impregnated the layers of sand, which are capable of absorbing a great quantity of such liquid, and if above this there were strata impermeable to naphtha (dense, clayey, damp strata) the naphtha would accumulate in them. It is thus preserved from remote geological periods up to the present day, compressed and dissolved under the pressure of the gases which burst out in places forming naphtha fountains. If this be granted, it may be thought that in the comparatively new (geologically speaking) mountain chains, such as the Caucasian, naphtha is even now being formed. Such a supposition may explain the remarkable fact that, in Pennsylvania, localities where naphtha had been rapidly worked for five years have become exhausted, and it becomes necessary to constantly have recourse to sinking new wells in fresh places. Thus, from the year 1859 , the workings were gradually transferred along a line running parallel to the Alleghany mountains for a distance of more than 200 miles, whilst in Baku the industry dates from time immemorial (the Persians worked near the village of Ballaghana) and up to the present time keeps to one and the same place. The amounts of the Pennsylvanian and Baku annual outputs are at present equal-namely, about 150 million poods ( $2 \frac{1}{2}$ million tons). It may be that the Baku beds, as being of more recent geological formation, are not so exhausted by nature as those of Pennsylvania, and perhaps in the neighbourhood 'of Baku naphtha is still being formed, which is partially indicated by the continued activity of the mud volcanoes. As many varieties of naphtha contain in solution solid, slightly volatile hydrocarbons like paraffin and mineral wax, the production of ozocerite, or mountain wax, is accounted for in conjunction with the formation of naphtha. Ozocerite is found in Galicia, also in the neighbourhood of Novorossisk, in the Caucrsus, and on the islands of the Caspian Sea (particularly in the Chileken and Holy Islands); it is met with in large masses, and is used for the production of paraffin and cercsene, for the manufacture of candles, and similar purposes.

As the naphtha treasures of the Caucasus are hardly broached (near Baku and near Kouban and Novorossisk), and as naphtha finds manifold uses, the subject presents most interesting features to chemists and geologists, and is worthy of the close attention of practical men. 


\section{CHAPTER IX}

\section{COMPOUNDS OF CARBON WITH OXYGEN AND NITROGEN}

Carboxic anhydride (or carbonic acid or carbon dioxide, $\mathrm{CO}_{2}$ ) was the first of all gases distinguished from atmospheric air. Paracelsus and Van Helmont, in the sixteenth century, knew that on heating limestone a particular gas separated, which is also formed during the alcoholic fermentation of saccharine solutions (for instance, in the manufacture of wine) ; they knew that it was identical with the gas which is produced by the combustion of charcoal, and that in some cases it is found in nature. In course of time it was found that this gas is absorbed by alkali and saturates it, forming a salt which, under

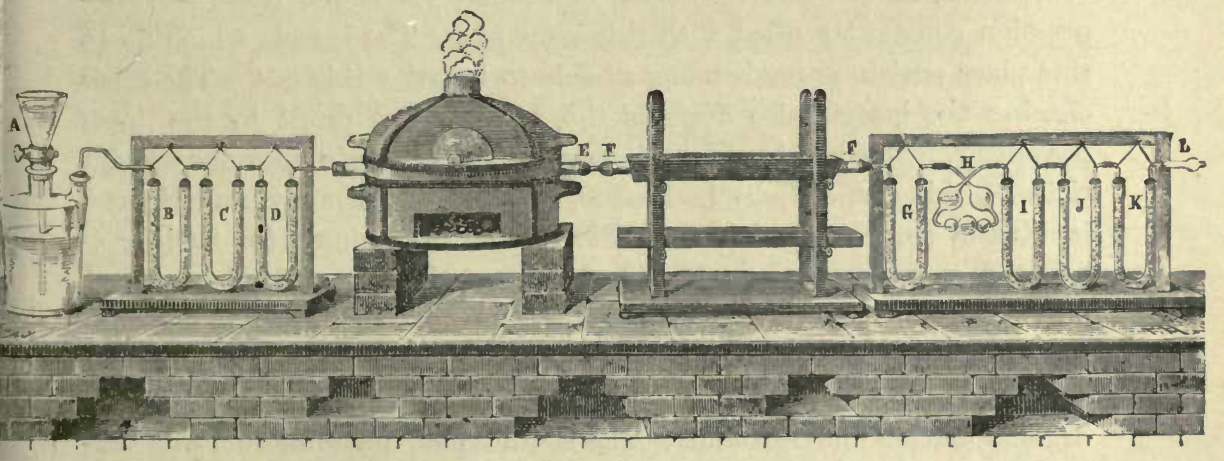

Fig. 61.-Dumas and Stas' apparatus for determining the compnsition of carbonic anhydride. Carbon, graphite, or a cliamond is placed in the tube $\mathrm{E}$ in the furuace, and heated in a stream of oxygen displaced from the bottle by water flowing from $\mathrm{A}$. The oxygen is purified from carbonic anhydride and water in the tubes $\mathrm{B} C \mathrm{C}$. Carbonic anhydride, togetler with a certain amount of carbon monoxide, is formed in $\mathrm{E}$. The latter is converted into carbonic acid by passing the products of combustion through a tube $\mathbf{F}$, containing cupric oxide heated in a furuace. The cupric oxide oxidises this $\mathrm{CO}$ into $\mathrm{CO}_{2}$, forming metallic copper. The potash bulbs $\mathrm{H}$ and tubes I, J, $\mathrm{K}$ retain the carbonic anhydride. Thus, knowing the weight of carbon taken and the weight of the resultant carbonic anliydrile (by weighing $\mathrm{H}, \mathrm{I}, \mathrm{J}, \mathrm{K}$ before and after the experiment), the composition of carbonic anhydride and the equivalent of carbon may be determined.

the action of acid, again yields this same gas. Priestley found that this gas exists in air, and Lavoisier determined its formation during respiration, combustion, putrefaction, and during the reduction of metals by charcoal; he determined its composition, and showed that it only contains oxygen and carbon. Berzelius, Dumas with Stas, and Roscoe, determined its composition, showing that it contains twelve 
parts of carbon to thirty-two of oxygen. The composition by volume of this gas is determined from the fact that during the combustion of charcoal in oxygen, the volume remains unchanged; that is to say, carbonic antydride occupies the same volume as the oxygen which it contains - that is, the atoms of the carbon are, so to speak, squeezed in between the atoms of the oxygen. $\mathrm{O}_{2}$ occupies two volumes and is a molecule of ordinary oxygen; $\mathrm{CO}_{2}$ likewise occupies two volumes, and expresses the composition and molecular weight of the gas. Carbonic anhydride exists in nature, both in a free state and in the most varied compounds. In a free state it is always contained (Chap V.) in the air, and in solution is in all kinds of water. It is evolved from volcanoes, from mountain fissures, and in some caves. The well known Dog grotto, near Agnano on the bay of Bair, near Naples, is the best known example of such an evolution. Similar sources of carbonic anhydride are also found in other places. In France, for instance, there is a well-known poisonous fountain in Auvergne. It is a round hole, surrounded with luxurious vegetation and constantly evolving carbonic anhydride. In the woods surrounding the Lacher See, near the Rhine, in the neighbourhood of extinct volcanoes, there is a depression constantly filled with this same gas. The insects which fly to this place perish, animals being unable to breathe this gas. The birds chasing the insects also die, and this is turned to profit by the local peasantry. Many mineral springs carry into the air enormous quantities of this gas. Vichy in France, Sprüdel in Germany, and Narzan in Russia (in Kislovodsk near Piatigorsk) are known for their carbonated gaseous waters. Much of this gas is also evolved in mines, cellars, diggings, and wells. For this reason sometimes people descending into such places are suffocated and die. The evolution of carbonicanhydricle in the earth is accounted for by the slow oxidation of organic matter underground. The combustion, putrefaction, and fermentation of organic substances give rise to the formation of carbonicanhydride. It is also introduced into the atmosphere during the respiration of animals at all times and during the respiration of plants in darkness and also during their growth. Very simple experiments prove the formation of carbonic anhydride under these circumstances ; thus, for example, if the air expelled from the lungs be passed through a glass tube into a transparent solution of lime (or baryta) in water a white precipitate will soon be formed which contains an insoluble compound of lime and carbonic anhydride. If a funnel be held over a substance in a state of combustion-for instance, over a lighted candle or brazier--and the air be collected from it by means of an aspirator, the presence of a large amount of carbonic 
anhydricle in this air may be detected by its action on lime water. By allowing the seeds of plants to grow under a bell jar, or in a closed vessel, the formation of carbonic anhydride may be similarly confirmed. By forcing an animal, for instance a mouse, to remain under a bell jar, the quantity of carbonic acid which it evolves may be exactly determined, and it will be found to be many grams per day for a mouse. Such experiments on the respiration of animals have been also made with great exactitude with large animals, such as men, bulls, sheep, \&c. By means of enormous hermetically-closed bell receivers and the analysis of the gases evolved during respiration it was found that a man expels about 900 grams (more than two pounds) of carbonic anhydride per diem, and absorbs during this time 700 grams of oxygen. ${ }^{1}$ It must be remarked that the carbonic anhy-

1 The quantity of carbonic acid gas exhaled by a man during the twenty-four hours is not evenly distributed; during the night more oxygen is taken in than during the day (by night, in twelve hours, about 450 grams), and more carbonic anhydride is separated by day than during night-time and repose; thus, of the 900 grams separated during the twenty-four hours about 375 are given out during the night and 525 by day. This depends on the separation of carbonic anhydride during any kind of work performed by the man. During the day-time his activity in many respects is comparatively greater than by night. Every movement is the result of some change of matter, because force cannot be produced by itself (in accordance with the law of the conservation of energy). Proportionally to the amount of carbon consumed an amount of force is stored $\mathrm{up}$ in the organism and is consumed in the various movements performed by animals. This is proved by the fact that during work-time, in twelve hours, a man exhales 900 grams of carbonic anhydride instead of 525, absorbing the same amount of oxygen as before. In a working day a man exhales by night almost the same amount of carbonic anhydride as in a day of rest, so that during a total twenty-four hours' work a man exhales about 1300 grams of carbonic anhydride and absorbs about 950 grams of cxygen. Therefore in work the change of matter increases. The carbon expended on the work is obtained from the food; on this account the food of animals ought certainly to contain carbonaceous substances capable of dissolving under the action of the digestive fluids, and of passing into the blood, or, in other words, capable of being digested. Such food for man and all other animals is formed of vegetable matter, or of parts of other animals. The latter in every case obtain their carbonaceous matter from plants; in which it is formed by the separation of the carbon from the carbonic anhydride taken up during the day by the respiration of the plants. The green parts of plants during the day absorb the carbonic anhydride of the air, and find it ample notwithstanding the small proportion of it in the air, and give out oxygen. The volume of the oxygen exhaled is almost equal to the volume of the carbonic anhydride absorbed; that is to say, nearly all the oxygen entering in to the plant in the form of carbonic anhydride is separated in a free state, whilst the carbon from the carbonic anhydride remains in the plant. At the same time, the plant absorbs moisture by its leaves and roots. By a process which is unknown to us, this absorbed moisture and carbon remaining from the carbonic acid enter into the composition of the plants in the form of so-called carbohydrates, composing the greater part of the vegetable tissues, starch and cellulose of the composition $\mathrm{C}_{6} \mathrm{H}_{10} \mathrm{O}_{5}$ being representatives of them. We may represent their composition as a compound of carbon remaining from carbonic acid and water, $6 \mathrm{C}+5 \mathrm{H}_{2} \mathrm{O}$. In this way a circulation of the carbon goes on in nature by means of vegetable and animal organisms, in which changes the principal item is the carbonic anhydride of the air. 
dride of the air constitutes the fundamental food of plants (Chaps. III., V., and VIII.). Carbonic anhydride, in a state of combination with most varied substances, is perhaps even more widely diffused in nature than in a free state. Some of these substances are very stable, and form a large portion of the earth's crust. In this state carbonic anhydride enters into the composition of limestones. Limestones, calcium carbonate $\mathrm{CaCO}_{3}$, were formed as precipitates in the seas existing previously on the earth; this is proved by their stratified structure and the number of remains of sea animals which they frequently contain. It may be concluded, judging from the enormous quantity of these limestones, that the amount of carbon in the form of carbonic anhydride in the atmosphere was in former periods much greater than at present. Chalk, lithographic stone, limestone, marls (a mixture of limestone and clay), and many other rocks are examples of such sedimentary formations. Carbonates with various other basessuch as, for instance, magnesia, ferrous oxide, zinc oxide, ftc.-are often found in nature. The shells of molluses have also the composition $\mathrm{CaCO}_{3}$, and many limestones were exclusively formed from the shells of minute organisms.

For the preparation of carbonic anhydride in laboratories and often in manufactories, various kinds of calcium carbonate are used, being treated with some acid ; it is, however, most usual to employ the so-called muriatic acid-that is, an aqueous solution of hydrochloric acid, $\mathrm{HCl}$-because, in the first place, the substance formed, calcium chloride, $\mathrm{CaCl}_{2}$, is soluble in water and does not hinder the further action of the acid on the calcium carbonate, and secondly because, as we shall see further on, muriatic acid is a common product of chemical works and one of the cheapest. For calcium carbonate, either limestone, chalk, or marble is used. ${ }^{2}$

$$
\mathrm{CaCO}_{3}+2 \mathrm{HCl}=\mathrm{CaCl}_{2}+\mathrm{H}_{2} \mathrm{O}+\mathrm{CO}_{2} \text {. }
$$

2 Other acids may be used instead of hydrochloric; for instance, acetic, or even sulphuric, although this latter is not suitable, because it forms as a product insoluble calcium sulphate (gypsum) surrounding the untouched calcium carbonate, and thus preventing a further evolution of gas. But if porous limestone-for instance, chalk-be treated with sulphuric acid diluted with an equal volume of water, the latter is absorbed, and, acting on the mass of the salt, the evolution of carbonic anhydride continues evenly for a long time. Instead of calcium carbonate, other carbonates may of course be used; for instance, washing-soda $\mathrm{Na}_{2} \mathrm{CO}_{3}$, which is often chosen when it is required to produce a rapid stream of carbonic anhydride (for example, for liquefying it). But natural crystalline magnesium carbonate and similar salts are with difficulty decomposed by hydrochloric and sulphuric acids. When for manufacturing purposes-for instance, in precipitating lime in sugar-works-a large quantity of carbonic acid gas is required; generally charcoal is burnt, and the products of combustion, rich in carbonic anhydride, are pumped into the liquid containing the lime, and the carbonic anhydride is thus 
The nature of the reaction in this case is the same as in the decomposition of nitre by sulphuric acid ; only in the latter ease a hydrate is formed, but in the former an anhydride of the acid, because the hydrate, carbonic acid, $\mathrm{H}_{2} \mathrm{CO}_{3}$, is unstable and, as soon as it separates, decomposes into water and its own anhydride. It is evident from the explanation of the cause of the action of sulphuric acid on nitre that not every acid can be employed for obtaining carbonic anhydride; namely, those will not set it free which chemically are but slightly energetic, or those which are insoluble in water, or are themselves as volatile as carbonic anhydride. ${ }^{3}$ But as almost all the known acids are characterised by their great solubility in water and are less volatile than carbonic anhydride, the latter is evolved by the action of most acids on its salts, and this reaction takes place at ordinary temperatures, provided the acid be soluble in water and that the calcium salt formed is also soluble in water. ${ }^{4}$

For the preparation of carbonic anhydride in laboratories marble is generally used. It is placed in a Woulfe's bottle and treated with hydrochloric acid in an apparatus similar to the one used for the production of hydrogen. The gas evolved carries away through the tube part of the volatile hydrochloric acid, and it is therefore necessary to wash the gas by passing it through another Woulfe's bottle containing water. If

absorbed. Another method is also practised, which consists in using the carbonic anhydride separated during fermentation, or that evolved from lime-kilns. During the fermentation of sweet-wort, grape-juice, and other similar saccharine solutions, the glucose $\mathrm{C}_{6} \mathrm{H}_{12} \mathrm{O}_{6}$ changes under the influence of the yeast organism, forming alcohol $\left(2 \mathrm{C}_{2} \mathrm{H}_{6} \mathrm{O}\right)$ and carbonic anhydride $\left(2 \mathrm{CO}_{2}\right)$, which separates in the form of gas; if the fermentation proceeds in closed bottles sparkling wine is obtained. When carbonic acid gas is prepared for saturating water and other beverages it is necessary to use it in a pure state. Whilst in the state in which it is evolved from ordinary limestones by the aid of acids it contains, besides a certain quantity of acid, the organic matters of the limestone; in order to diminish the quantity of these substances the densest kinds of dolomites are used, which contain less organic matter, and the gas formed is passed through various washing apparatus, and then through a solution of potassium permanganate, which absorbs organic matter and does not take up carbonic anhydride.

3 Hypochlorous acid, $\mathrm{HClO}$, and its anhydride, $\mathrm{Cl}_{2} \mathrm{O}$, do not displace carbonic acid, and hydrogen sulphide has the same relation to carbonic acid as nitric acid to hydrochlorican excess of either one displaces the other.

4 Thus, in preparing the ordinary effervescing powders, sodium bicarbonate (or acid carbonate of soda) is used, and mixed with powdered citric or tartaric acid. In a dry state these powders do not evolve carbonic anhydride, but when mixed with water the evolution takes place briskly, which is due to the substance passing into solution. The salts of carbonic acid may be recognised from the fact that they evolve carbonic acid with a hissing noise when treated with acids. If vinegar, which contains acetic acid, be poured upon limestone, marble, malachite (contains copper carbonate), \&c., carbonic anhydride is evolved with a hissing noise. It is necessary to remark that without the presence of water neither hydrochloric acid, nor even sulphuric acid nor acetic acid, acts on limestone. We shall refer to this hereafter. 
it be necessary to obtain dry carbonic anhydride, it must be passed through chloride of calcium. ${ }^{5}$

Carbonic anhydride may also be prepared by heating many of the salts of carbonic acid; for instance, by heating magnesium carbonate, $\mathrm{MgCO}_{3}$ (in dolomite), the separation is easily effected, particularly in the presence of the vapours of water. The acid salts of carbonic acid (see further on) readily give much carbonic anhydride when heated. Carbonic anhydride, together with water, is produced during the combustion of all organic compounds in a stream of oxygen or by heating them with substances which readily part with oxygen to them-for instance, with copper oxide, potassium chlorate (readily causing an explosion), drc.

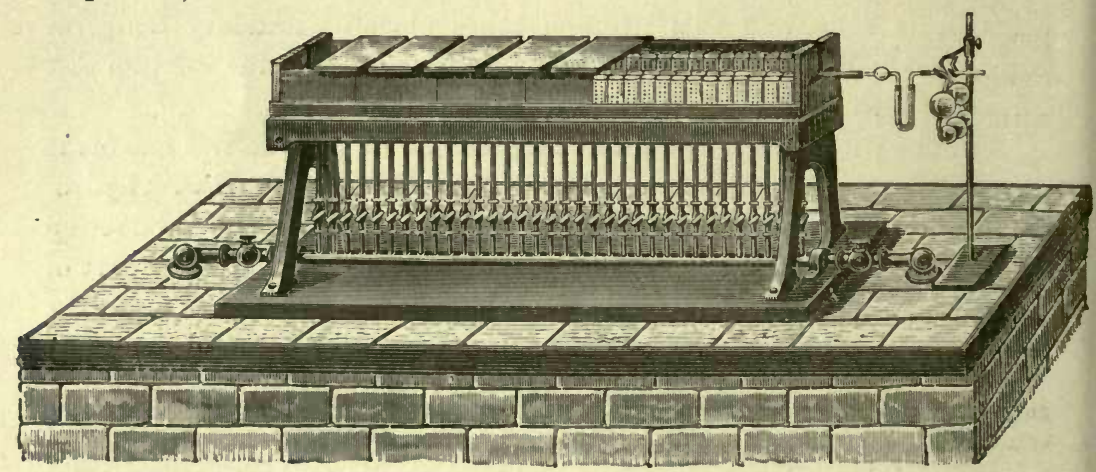

FIG. 62. - Apparatus for the combustion of organic substances by igniting them with oxide of copper.

The method of estimating the amount of carbon in organic compounds is founded on this property. For this purpose a glass tube, closed at one end, is filled with a mixture of the organic substance (about 0.2 grams) and copper oxide. The open end of the tube is fitter? with a

5 The direct observations made (1876) by Messrs. Bogouski and Kayander lead to the conclusion that the quantity of carbonic anhydride evolved by the action of acids on marble (as homogeneous as possible) is directly proportional to the time of action, the extent of surface, and the degree of concentration of the acid, and inversely proportional to the molecular weight of the acid. If the surface of a piece of Carrara marble be equal to one decimetre, the time of action one minute, and one cubic decimetre or litre contains one gram of hydrochloric acid, then about 0.02 gram of carbonic anhydride will be evolved. If the litre contains $n$ grams of hydrochloric acid, then by experiment the amount will be $n \times 0.02$ of carbonic anhydride. Therefore, if the litre contains 36.5 $(=\mathrm{HCl})$ grams, about 0.73 grams of carbonic anhydride (about half a litre) would be evolved per minute. If nitric acid or hydrobromic acid be used instead of hydrochloric, then, with a combining proportion of the acid, the same quantity of carbonic anhydride will be evolved; thus, if the litre contains $63\left(=\mathrm{HNO}_{\overline{3}}\right)$ grams of nitric acid, or $81(=\mathrm{HBr})$ grams of hydrobromic acid, the quantity of carbonic anhydride evolved will still be 0.78 gram. 
cork and tube containing calcium chloride, for absorbing the water formed by the oxidation of the substance. This tube is hermetically connected (by a caoutchouc tube) with potash bulbs or other weighing apparatus (Chap. V.) containing alkali destined to absorb the carbonic anhydride. The increase in weight of this apparatus shows the amount of carbonic anhydride formed during the combustion of the given substance, and the quantity of carbon may be determined from this, because three parts of carbon give eleven parts of carbonic anhydride.

Carbonic anhydride is colourless, has a slight smell and a feeble acid taste ; its density in a gaseous state is twenty-two times greater than that of hydrogen, because its molecular weight is forty-four. ${ }^{6}$ It is an example of those gaseous substances which have been long ago transformed into all the three states. In order to obtain liquid carbonic anhydride, the gas must be submitted to a pressure of thirty-six atmospheres at $0^{\circ} .7$ Its absolute boiling point $=+32^{\circ} .{ }^{8}$ Liquid carbonic anhydride is colourless, does not mix with water, but is soluble in alcohol, ether, and oils ; at $0^{\circ}$ its specific gravity is 0.83 . When poured into a tube, which is then sealed up, liquid carbonic anhydride is easily preserved, because a thick tube easily resists the pressure which the liquid entails at an ordinary temperature-namely, about fifty atmospheres. The boiling point of this liquid lies at $-80^{\circ}$ - that is to say, the pressure of carbonic acid gas at that temperature does not exceed that of the atmosphere. At the ordinary temperature the liquid remains as such for some time under ordinary pressure, on account of its requiring a considerable amount of heat for its evaporation. If the evaporation takes place rapidly, especially if the liquid issues in a stream, such a decrease of temperature occurs that

6 As carbonic anhydride is one and a, half times heavier than air, it diffuses with difficulty, and therefore does not easily mix with air, but sinks in it. This may be shown in various ways; for instance, it may be carefully poured from one vessel into another containing air. If a lighted taper be plunged into the vessel containing carbonic anhydride it is extinguished, and then, after pouring the gas into the other cylinder, it will burn in the former and be extinguished in the latter. If a certain quantity of carbonic anhydride be poured into a vessel containing air, and soap-bubbles be introduced, they will only sink as far as the line where the atmosphere of carbonic anhydride commences, as this latter is heavier than the soap-bubbles filled with air. Naturally, after a certain lapse of time, the carbonic anhydride will be diffused throughout the vessel, and form a uniform mixture with the air, just as salt in water.

7 This liquefaction was first observed by Faraday, who sealed up in a tube a mixture of a carbonate and sulphuric acid. Afterwards this method was very considerably improved by Thilorier and Natterer, whose apparatus is described on page 289 . It is, however, necessary to remark here that in working with liquid carbonic anhydride it is indispensable to have good liquefying apparatus, constant cooling, and in particular a rapid preparation of large masses of pure carbonic anhydride.

s Carbonic anhydride, having the same molecular weight as nitrous oxide, very much resembles it when in a liquid state. 
a part of the carbonic anhydride is transformed into a solid snowy mass. Water, mercury, and many other liquids freeze on coming into contact with snowlike carbonic anhydride. ${ }^{9}$ In this form carbonic anhydride may be preserved for a long time in the open air, because it requires still more heat to turn it into a gas than when in a liquid state. Solid carbonic anhydride, notwithstanding its very low temperature, can be safely placed on the hand, because it constantly evolves gas which prevents its coming into actual contact with the skin; but if a piece be squeezed between the fingers, it produces a severe frost bite similar to a burn. If the snowlike solid be mixed with ether a semiliquid mass is obtained, which may be used for artificial refrigeration. This mixture may be used for liquefying many other gases-such as chlorine, nitrous oxide, hydrogen sulphide, and others. The evaporation of such a mixture proceeds with far greater rapidity under the receiver of an air-pump, and consequently the refrigeration is more intense. By this means many gases may be liquefied which resist other methods-namely, olefiant gas, hydrochloric acid gas, and others. Liquid carbonic anhydride in this case congeals in the tube into a glassy transparent mass. Pictet availed himself of this method for liquefying many permanent gases (see Chap. II.).

The capacity which carbonic anhydride has of being liquefied stands in connection with its solubility in considerable quantity in water, alcohol, and other liquids. Its solubility in water has been already spoken of in the first chapter. Carbonic anhydride is still more soluble in alcohol than in water, namely at $0^{\circ}$ one volume of alcohol dissolves 4.3 volumes of this gas, and at $20^{\circ} 2.9$ volumes.

Aqueous solutions of carbonic anhydride, under a pressure of several atmospheres, are now prepared artificially, because water saturated with this gas is a good means of promoting digestion and quenching thirst. For this purpose the carbonic anhydride is pumped by means of a force pump into a closed vessel containing the liquid, and then bottled off, taking special means to ensure rapid and air-tight corking. Various effervescing drinks and artificially effervescing wines are thus prepared. The presence of carbonic anhydride has an important

9 When a fine stream of liquid carbonic anhydride is discharged into a closed metallic vessel, about one-third of its mass solidifies and the remainder evaporates. In employing solid carbonic anhydride for making experiments at low temperatures, it is best to use it mixed with ether, otherwise there will be few points of contact. If a stream of air be blown through a mixture of liquid carbonic anhydride and ether, the evaporation proceeds rapidly, and great cold is obtained. At present in some special manufactories (and for making artificial mineral waters) carbonic anhydride is liquefied on the large scale, poured into wrought-iron cylinders provided with screwed taps, and in this manner it can be transported and safely preserved for a long time. 
significance in nature, because by this means water acquires the property of destroying and dissolving many substances which are not acted on by pure water; for instance, calcium phosphates and carbonates are soluble in water containing carbonic acid. If the water in the interior of the earth is saturated with carbonic acid under pressure, the quantity of calcium carbonate in solution may reach three grams per litre, and on issuing at the surface, as the carbonic anhydride escapes, the calcium carbonate will be deposited. ${ }^{10}$ Water charged with carbonic anhydride brings about the destruction of many rocky formations by removing the lime, alkali, \&c., from them. This process has been going on and continues on an enormous scale. Rocky formations contain silica and the oxides of various metals, amongst others oxides of aluminium, calcium, and sodium. Water charged with carbonic acid dissolves both the latter, transforming them into carbonates. The waters of the ocean ought, as the evolution of the carbonic anhydride proceeds, to precipitate salts of lime; these are actually found everywhere on the surface of the ground in those places which previously formed the bed of the ocean.

The presence of carbonic anhydride in solution in water is essential to the nourishment and growth of water plants. Although carbonic anhydride is soluble in water, yet no definite hydrate is formed ; ${ }^{11}$ nevertheless an idea of the composition of this hydrate may be

10 If such water trickles through crevices and enters a cavern, the evaporation will be slow, and therefore in those places from whence the water drips growths of calcium carbonate will be formed, just like the icicles formed on the roof-gutters in winter-time. Similar conical and cylindrical stony growths form the so-called stalactites or pendants hanging from above and stalagmites formed on the bottom of caves. Sometimes these two kinds meet together, forming entire columns filling the cave. Many of these caves are remarkable for their picturesqueness; for instance, the cave of Antiparos, in the Grecian Archipelago. This same cause also forms spongy masses of calcium carbonate in those places where the springs come to the surface of the earth. It is therefore very evident that a calcareous solution is sometimes capable of penetrating plants and filling the whole of their mass with calcium carbonate. This is one of the forms of petrified plants. Calcium phosphate in solution in water containing carbonic acid plays an important part in nourishing plants, because all plants contain both lime and phosphoric acid.

11 The crystallohydrate $\mathrm{CO}_{2}, 8 \mathrm{H}_{2} \mathrm{O}$ of Wroblewski (Chapter I. Note 67), in the first place, is only formed under special conditions; in the second place, it still requires confirmation; and in the third place, it does not correspond with that hydrate $\mathrm{H}_{2} \mathrm{CO}_{3}$ which should occur, judging from the composition of the salts.

It is easy to demonstrate the acid properties of carbonic anhydride by taking a long tube, closed at one end, and filling it with this gas; a test-tube is then filled with a solution of an alkali (for instance, sodium hydroxide), which is then poured into the long tube and the open end is corked. The solution is then well shaken in the tube, and the corked end plunged into water. If the cork be now withdrawn under water the water will fill the tube. The vacuum obtained by the absorption of the carbonic anhydride by an alkali is so complete that even an electric discharge will not pass through it. This method is often applied to produce a vacuum. 
formed from the composition of the salts of carbonic acid, because a hydrate is nothing but a salt in which the metal is replaced by hydrogen. As carbonic anhydride forms salts of the composition $\mathrm{K}_{2} \mathrm{CO}_{3}, \mathrm{Na}_{2} \mathrm{CO}_{3}, \mathrm{HNaCO}_{3}$, \&c., therefore carbonic acid ought to have the composition $\mathrm{H}_{2} \mathrm{CO}_{3}$-that is, it ought to be formed from $\mathrm{CO}_{2}+\mathrm{H}_{2} \mathrm{O}$. Whenever this substance is formed, it decomposes into its component parts - that is, into water and carbonic anhydride. The acid properties of carbonic anhydride are demonstrated by its being directly absorber by alkaline solutions and forming salts with them. In distinction from nitric, $\mathrm{HNO}_{3}$, and similar monobasic acids which with univalent metals (exchanging one atom for one atom of hydrogen) give salts such as those of potassium, sodium, and silver containing only one atom of the metal $\left(\mathrm{NaNO}_{3}, \mathrm{AgNO}_{3}\right)$, and with bivalent ${ }^{12}$ metals (such as calcium, barium, lead) salts containing two acid groups - for example $\mathrm{Ca}\left(\mathrm{NO}_{3}\right)_{2}$, $\mathrm{Pb}\left(\mathrm{NO}_{3}\right)_{2}$-carbonic acid, $\mathrm{H}_{2} \mathrm{CO}_{3}$, is bibasic, that is, contains two atoms of hydrogen in the hydrate or two atoms of univalent metals in their salts : for example $\mathrm{Na}_{2} \mathrm{CO}_{3}$ is washing soda, a normal salt; $\mathrm{NaHCO}_{3}$ is the bicarbonate or an acid salt. Therefore, if $\mathbf{M}^{\prime}$ be a univalent metal, its carbonates in general are the normal carbonate $\mathrm{MI}_{2} \mathrm{CO}_{3}$ and the acid carbonate $\mathrm{M}^{\prime} \mathrm{HCO}_{3}$; or if $\mathrm{M}^{\prime \prime}$ be a bivalent metal (replacing $\mathrm{H}_{2}$ ) its normal carbonate will be $\mathrm{MI}^{\prime \prime} \mathrm{CO}_{3}$; these metals do not usually form acid salts, as we shall see further on. The bibasic character of carbonic acid is akin to that of sulphuric acid, $\mathrm{H}_{2} \mathrm{SO}_{4}{ }^{13}$ but the latter, in distinction

12 The reasons for distinguishing the uni-, bi-, tri-, and quadri-valent metals will be examined hereafter on passing from the univalent metals $(\mathrm{Na}, \mathrm{K}, \mathrm{Li})$ to the divalent $(\mathrm{Mg}$, $\mathrm{Ca}, \mathrm{Ba})$.

13 Up to the year 1840 , or thereabout, acids were not distinguished by their basicity. Graham, while studying phosphoric acid, $\mathrm{H}_{3} \mathrm{PO}_{4}$, and Liebig, while studying many organic acids, distinguished mono-, bi-, and tri-basic acids. Gerhardt and Laurent generalised these relations, showing that this distinction extends over many reactions (for instance, to the faculty of bibasic acids of forming acid salts with alkalis, $\mathrm{KHO}$ or $\mathrm{NaHO}$, or with alcohols RHO, \&c.); but now, from the determination of a hard-and-fast conception as to atoms and molecules, the basicity of an acid is determined by the number of hydrogen atoms contained in a molecule of the acid which can be exchanged for metals. If carbonic acid forms acid salts, $\mathrm{NaHCO}_{3}$, and normal salts, $\mathrm{Na}_{2} \mathrm{CO}_{5}$, it is evident that the hydrate is $\mathrm{H}_{2} \mathrm{CO}_{3}$, a bibasic acid. Otherwise it is at present impossible to account for the composition of these salts. But when $\mathrm{C}=6$ and $\mathrm{O}=8$ were taken, then the formula $\mathrm{CO}_{2}$ expressed the composition, but not the molecular weight, of carbonic anhydride; and the composition of the normal salt would be $\mathrm{Na}_{2} \mathrm{C}_{2} \mathrm{O}_{6}$ or $\mathrm{NaCO}_{3}$, therefore carbonic acid might have been considered as a monobasic acid. Then the acid salt would have been represented by $\mathrm{NaCO}_{3}, \mathrm{HCO}_{3}$. Such questions were the cause of much argument and difference of opinion among chemists about forty years ago. At present there cannot be two opinions on the subject if the law of Avogadro-Gerhardt and its sequences be strictly adhered to. Let us, however, remark here that the monobasic acids $\mathrm{R}(\mathrm{OH})$ were for a long time considered to be incapable of being decomposed into water and anhydride, and this property was ascribed to the bibasic acids $\mathrm{R}(\mathrm{OH})_{2}$ as containing the elements necessary for the separation of the molecule of water $\mathrm{H}_{2} \mathrm{O}$. This $\mathrm{H}_{2} \mathrm{SO}_{4}$ or $\mathrm{SO}_{2}(\mathrm{OH})_{2}, \mathrm{H}_{2} \mathrm{CO}_{3}$, 
from the former, is an example of the energetic or strong acids (such as nitric or hydrochloric), whilst in carbonic acid we observe but feeble development of the acid properties ; hence carbonic acid must be considered a weak acid. This conception must, however, be taken as only comparative, as up to this time there is no definitely established rule for measuring the energy ${ }^{14}$ of acids. The feeble acid properties of carbonic

or $\mathrm{CO}(\mathrm{OH})_{2}$, and other bibasic acids decompose into the anliydride, RO, and water, $\mathrm{H}_{2} \mathrm{O}$. But as nitrous, $\mathrm{HNO}_{2}$, iodic, $\mathrm{HIO}_{3}$, hypochlorous, $\mathrm{HClO}$, and other monobasic acids easily give their anhydrides $\mathrm{N}_{2} \mathrm{O}_{3}, \mathrm{I}_{2} \mathrm{O}_{5}, \mathrm{Cl}_{2} \mathrm{O}$, \&c., that method of distinguishing the basicity of acids, although it fairly well satisfies the requirements of organic chemistry, must not be considered correct. It may also be remarked that up to the present time not one of the bibasic acids has been found to have the faculty of being distilled without being decomposed into anhydride and water (even $\mathrm{H}_{2} \mathrm{SO}_{4}$, on being evaporated and distilled, gives $\mathrm{SO}_{5}+\mathrm{H}_{2} \mathrm{O}$ ), and the decomposition of acids into water and anhydride proceeds particularly easily in dealing with feebly energetic acids, such as carbonic, nitrous, boric, and hypochlorous. Let us add that carbonic acid, as a hydrate corresponding to marsh gas, $\mathrm{C}(\mathrm{HO})_{4}=\mathrm{CO}_{2}+2 \mathrm{H}_{2} \mathrm{O}$, ought to be tetrabasic. But in general it does not form such salts. Basic salts, however, such as $\mathrm{CuCO}_{3} \mathrm{CuO}$, may be regarded in this sense, because $\mathrm{CCu}_{2} \mathrm{O}_{4}$ corresponds with $\mathrm{CH}_{4} \mathrm{O}_{4}$, as $\mathrm{Cu}$ corresponds with $\mathrm{H}_{2}$. Amongst the ethereal salts (alcoholic derivatives) of carbonic acid corresponding cases are, however, observed; for instance, ethylic orthocarbonate, $\mathrm{C}\left(\mathrm{C}_{2} \mathrm{H}_{5} \mathrm{O}\right)_{4}$ (obtained by the action of chloropicrin, $\mathrm{C}\left(\mathrm{NO}_{2}\right) \mathrm{Cl}_{3}$, on sodium ethoxide, $\mathrm{C}_{2} \mathrm{H}_{5} \mathrm{ONa}$; boiling point, $158^{\circ}$; specific gravity, 0.92$)$. The name orthocarbonic acid for $\mathrm{CH}_{4} \mathrm{O}_{4}$ is taken from orthophosphoric acid, $\mathrm{PH}_{3} \mathrm{O}_{4}$, which corresponds with $\mathrm{PH}_{3}$ (see Chapter on Phosphorus).

14 Long ago endeavours were made to find a measure of affinity of acids and bases, because some of the acids, such as sulphuric or nitric, form comparatively stable salts, decomposed with difficulty by heat and water, whilst others, like carbonic and hypochlorous acids, do not combine with feeble bases, and with many form salts which are easily decomposed. The same may be said with regard to bases, among which those of potassium, $\mathrm{K}_{2} \mathrm{O}$, sodium, $\mathrm{Na}_{2} \mathrm{O}$, and barium, $\mathrm{BaO}$, may serve as examples of the most powerful, because they combine with the most feeble acids and form a mass of salts of great stability, whilst as examples of the feeblest bases alumina, $\mathrm{Al}_{2} \mathrm{O}_{3}$, or bismuth oxide, $\mathrm{Bi}_{2} \mathrm{O}_{3}$, may be taken, because they form salts easily decomposed by heat and water if the acid be volatile. Such a division of acids and bases into the feeblest and most powerful is justified by all evidence concerning them, and is quoted in this work. But in recent years the teaching of this subject has acquired quite a new tone, which, in my opinion, cannot be accepted without certain reservations and remarks, although it comprises many interesting features. The fact is that Thomsen, Ostwald, and others proposed to express the measure of affinity of acids to bases by figures drawn from data of the measure of displacement of acids in aqueous solutions, judging (1) from the amount of heat developed by mixing a solution of the salt with a solution of another acid (the avidity of acids, according to Thomsen); (2) from the change of the volumes accompanying such a mutual action of solutions (Ostwald); (3) from the change of the index of refraction of solutions (Ostwald), \&c. Besides this there are many other methods which allow us to form an opinion about the distribution of bases among various acids in aqueous solutions. Some of these methods will be described hereafter. It ought, however, to be remarked that in making investigations in aqueous solutions the affinity to water is generally left out of sight. If a base $\mathrm{N}$, combining with acids $\mathrm{X}$ and $\mathrm{Y}$ in presence of them both, divides in such a way that onethird of it combines with $\mathrm{X}$ and two-thirds with $\mathrm{Y}$, a conclusion is formed that the affinity, or power of forming salts, of the acid $\mathrm{Y}$ is twice as great as that of $\mathrm{X}$. But the presence of the water is not taken into account. If the acid $\mathrm{X}$ has an affinity for water and for $\mathrm{N}$ it will be distributed between them; and if $\mathrm{X}$ has a greater affinity for water than $\mathrm{Y}$, then less of $\mathrm{X}$ will combine with $\mathrm{N}$ than of $\mathrm{Y}$. If, in addition to this, the acid $\mathrm{X}$ is 
acid may, however, be judged from the accumulation of many indications. With such energetic alkalis as soda and potash, carbonic acid forms.

capable of forming an acid salt $\mathrm{NX}_{2}$, and $\mathrm{Y}$ is not, the conclusion of the relative strength of $\mathrm{X}$ and $\mathrm{Y}$ will be still more erroneous, because the $\mathrm{X}$ set free will form such a salt on the addition of $\mathrm{Y}$ to $\mathrm{NX}$. We shall see in Chapter $\mathrm{X}$. that when sulphuric and nitric acids in weak aqueous solution act on sodium they are distributed exactly in this way: namely, one-third of it combines with the sulphuric and two-thirds with the nitric acid; but, in my opinion, this does not show that sulphuric acid, compared with nitric acid, possesses but half the degree of affinity for bases similar to soda, and only demonstrates the greater affinity of sulphuric acid for water compared with that of nitric acid. In this way the methods of studying the distribution in aqueous solutions probably only sliows the difference of the relation of the acid to a base and to water. In general, it is impossible to hope to be able to determine the direct relative degree of affinity of acids for bases by studying aqueous solutions without taking into account the relation of the acids, bases, and salts to water, and by regarding the water as a passive medium, because the water itself forms saline and all other compounds with substances. This refers more especially to those weak solutions by means of which investigations of this kind are most often conducted, because the weak solutions contain a large mass of water, and its influence is then great even when there is but little affinity, in accordance with the law of the action of masses, and from the fact that water itself is a saline oxide.

In deference to these considerations, although the teaching of the distribution of saltforming elements in aqueous solutions is an object of great and independent interest, it can hardly serve to determine the measure of affinity between bases and acids. Similar considerations ought to be kept in view whon determining the energy of acids by means of the electrical conductivity of their weak solutions. This method, proposed by Arrhenius (1884), and applied on an extensive scale by Ostwald (who developed it in great detail in his Lehrbuch d. allgemeinen Chemie, v. ii., 1887), is founded on the fact that the relation of the so-called molecular electrical-conductivity of weak solutions of various acids. (I) coincides with the relation in which the same acids stand according to the distribution, (II) found by one of the above-mentioned methods, and with the relation deduced for them from observations upon the velocity of reaction, (III) for instance, according to the rate of the splitting up of an ethereal salt (into alcohol and acid), or from the rate of the socalled inversion of sugar - that is, its transformation into glucose-as is seen by comparing the annexed figures, in which the energy of hydrochloric acid is taken as equal to 100 :-

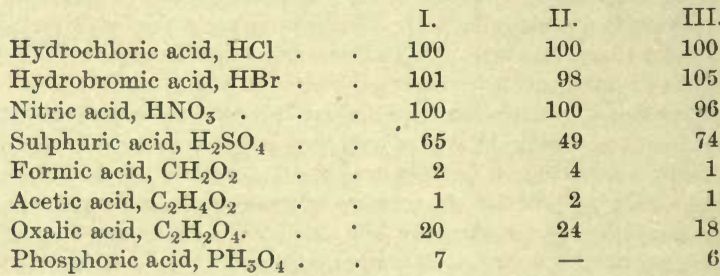

The coincidence of these figures, obtained by so many various methods, presents a most important and most instructive relation between phenomena of a different kind, but. in my opinion it does not permit us to assert that the degree of affinity existing between bases and various acids is determined by all these various methods, becuuse the influence of the water must be taken into consideration. On this account, until the theory of solution is more thoroughly worked out, this subject (which now ought to be treated of in special treatises on chemical mechanics) must be treated with great caution. But now we may hope to decide this question by exact methods, which we shall partially touch on when speaking about the velocity of reaction.

There is no means of determining the degree of energy of carbonic acid by any one of the above-mentioned methods 
normal salts, soluble in water, but having an alkaline reaction and in many cases acting themselves as alkalis. ${ }^{15}$ The acid salts of these alkalis, $\mathrm{NaHCO}_{3}$ and $\mathrm{KHCO}_{3}$, have a neutral reaction on litmus, although they, like acids, contain hydrogen, which may be exchanged for metals. The acid salts of such acids - as, for instance, of sulphuric acid, $\mathrm{NaHSO}_{4}$ have a clearly-defined acid reaction, and therefore carbonic acid is unable to saturate the powerful basic properties of such alkalis as potash or soda. Carbonic acid does not even combine at all with feeble bases, such as alumina, $\mathrm{Al}_{2} \mathrm{O}_{3}$, and therefore if a strong solution of sodium carbonate, $\mathrm{Na}_{2} \mathrm{CO}_{3}$, be added to a strong solution of aluminium sulphate $\mathrm{Al}_{2}\left(\mathrm{SO}_{4}\right)_{3}$, then (according to double saline decompositions) aluminium carbonate, $\mathrm{Al}_{2}\left(\mathrm{CO}_{3}\right)_{3}$, ought to be formed, but the carbonic acid separates. as this salt splits up in the presence of water into aluminium hydroxide and carbonic anhydride: $\mathrm{Al}_{2}\left(\mathrm{CO}_{3}\right)_{3}+3 \mathrm{H}_{2} \mathrm{O}=\mathrm{Al}_{2}(\mathrm{OH})_{6}+3 \mathrm{CO}_{2}$. Therefore feeble bases are unable to retain carbonic acid even at ordinary temperatures. For this reason in the case of bases of medium energy, although they form carbonates, these are comparatively easily decomposed by heating, as is shown by the decomposition of copper carbonate, $\mathrm{CuCO}_{3}$ (see Introduction), and even calcium carbonate, $\mathrm{CaCO}_{3}$. Only the normal (but not the acid) salts of such powerful bases as potassium or sodium are capable of standing a red heat without decomposition. But the acid salts, for instance $\mathrm{NaHCO}_{3}$, decompose even on heating their solutions $\left(2 \mathrm{NaHCO}_{3}=\mathrm{Na}_{2} \mathrm{CO}_{3}+\mathrm{H}_{2} \mathrm{O}+\mathrm{CO}_{2}\right)$, evolving carbonic anhydride. The amount of heat given out by the combination of carbonic acid with bases also shows its feeble acid properties, because it is considerably less than with energetic acids. Thus if a weak solution of forty grams of sodium hydroxide be saturated (until the formation of a normal salt) with sulphuric or nitric acid or another powerful acid, from thirteen to fifteen thousand calories are given out, but with carbonic acid only about ten thousand calories. $^{16}$ The majority of carbonates are insoluble in water, and

15 Thus, for instance, in the washing of fabrics the caustic alkalis, such as sodium hydroxide, in weak solutions, act in removing the fatty matter just in the same way as carbonate solutions; for instance, a solution of soda crystals, $\mathrm{Na}_{2} \mathrm{CO}_{3}$. Soap, acts in the same way, being composed of feeble acids, either fatty or resinous, combined with alkali. On this account all such substances are applied to manufacturing purposes, and used equally well in practice for bleaching and washing fabrics. Soda crystals or soap are preferred to caustic alkali, because an excess of the latter may have a destructive effect on the fabrics. It may be supposed that in aqueous solutions with soap or with soda crystals part of the base will form caustic alkali ; that is to say, the water will compete with the weak acids, and the alkali will be distributed between them and the water.

16 Although carbonic acid is reckoned among the feeble acids, yet there are evidently many others still feebler-for instance, hydrogen sulphide, prussic acid, hypochlorous acid, many organic acids, \&c. Bases like alumina, or such feeble acids as silica, when in combination with alkalis are decomposed in aqueous solutions by carbonic acid, 


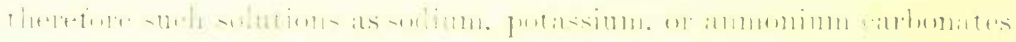

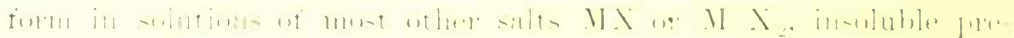

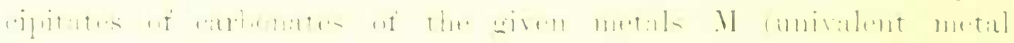

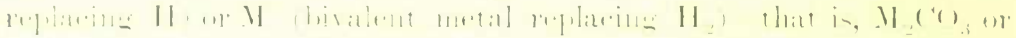

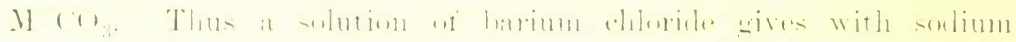

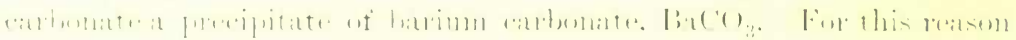

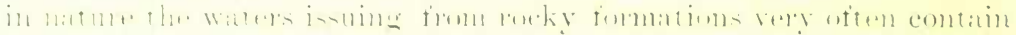
(a)

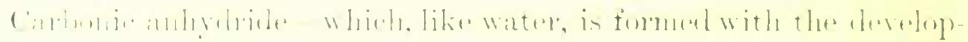

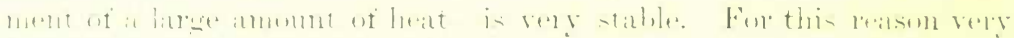

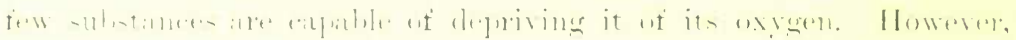

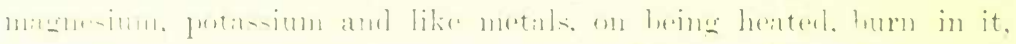

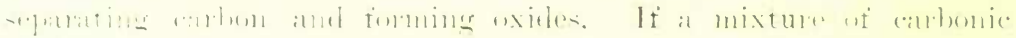

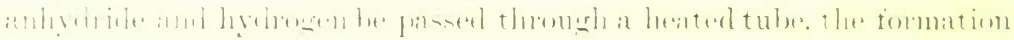

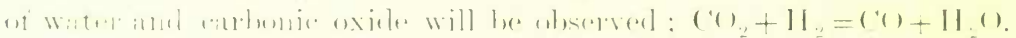

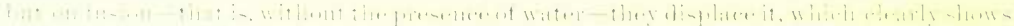

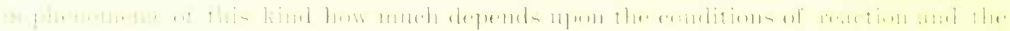

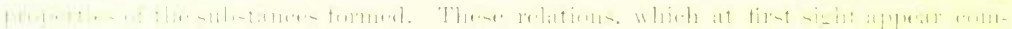

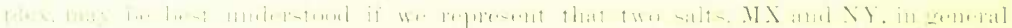

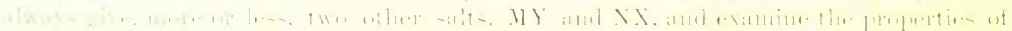

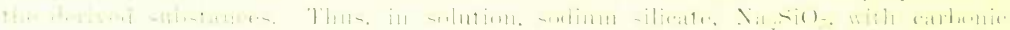

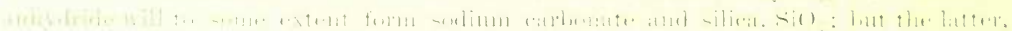

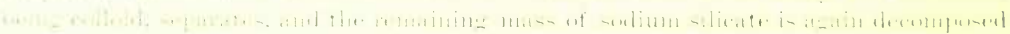

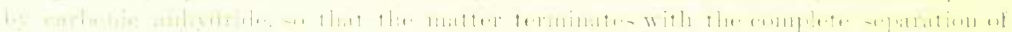

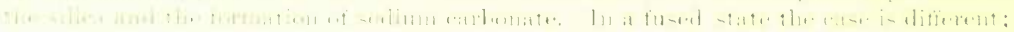

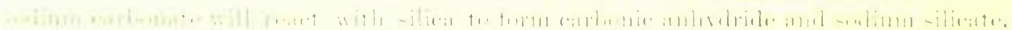

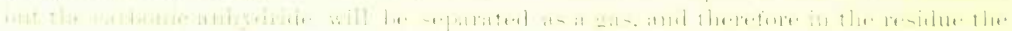

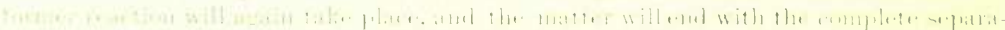

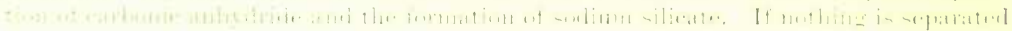

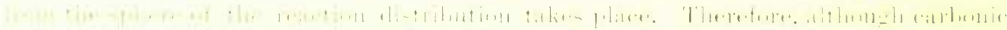

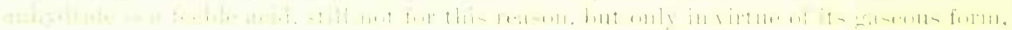

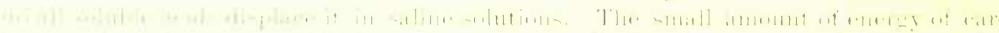

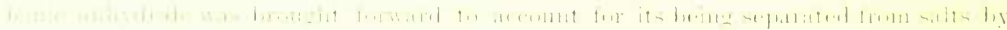

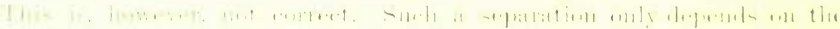

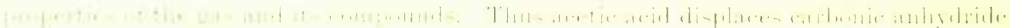

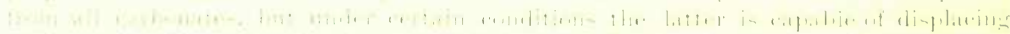

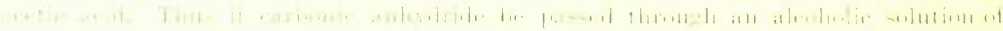

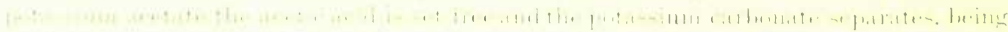

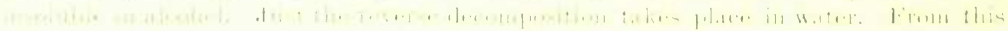

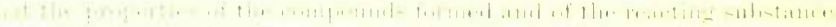

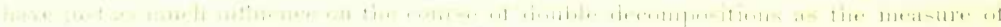

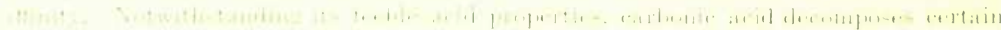

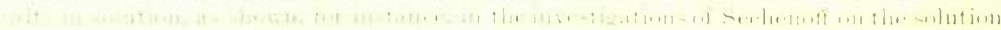

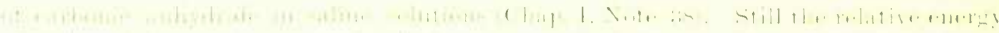

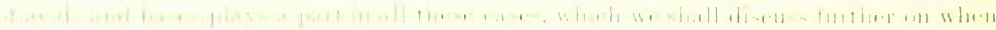

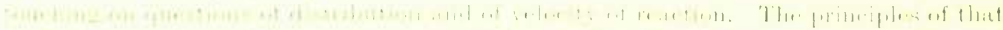

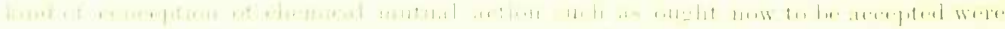

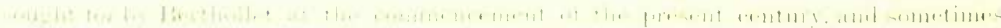

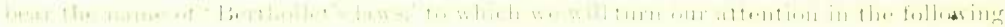
Whition. 
But only a portion of the carbonic acid gas undergoes this change, and therefore the result will be a mixture of carbonic anhydride, carbonic oxide, hydrogen, and water, which does not change under the action of heat. ${ }^{17}$ Although, like water, carbonic anhydride is exceedingly stable, still on being heated it partially decomposes into carbonic oxide and oxygen. Deville showed that such is the case if carbonic anhydride be passed through a long heated tube containing pieces of porcelain and heated to $1300^{\circ}$. If the products of decomposition-namely, the carbonic oxide and oxygen-be suddenly cooled, they can be collected separately, although they partly reunite together. A similar decomposition of carbonic anhydride into carbonic oxide and oxygen takes place on passing a series of electric sparks through it (for instance, in the eudiometer). With this an increase of volume occurs, because two volumes of $\mathrm{CO}_{2}$ give two volumes of $\mathrm{CO}$ and one volume of $\mathrm{O}$. The decomposition reaches a certain limit (less than one-third) and does not proceed further, so that the result is a mixture of carbonic anhydride, carbonic oxide, and oxygen, which is not altered in composition by the continued action of the sparks. This is readily understood, as it is a reversible reaction. If the carbonic anhydride be removed, then the mixture explodes when a spark is passed and forms carbonic anhydride. If from an identical mixture the oxygen (and not the carbonic anhydride) be removed, and a series of sparks again be passed, the decomposition is renewed,

17 Hydrogen and carbon are near akin to oxygen as regards affinity, but it ought to be considered that the affinity of hydrogen is slightly greater than that of carbon, because during the combustion of hydrocarbons the hydrogen burns first. Some idea of this similarity of affinity may be formed by the quantity of heat evolved. Gaseous hydrogen, $\mathrm{H}_{2}$, on combining with an atom of oxygen, $\mathrm{O}=16$, develops 69000 heat-units if the water formed be condensed to a liquid state. If the water remains in the form of a gas (steam) the latent heat of evaporation must be subtracted, and then 58000 calories will be developed. Charcoal taken in the solid form on combining with $\mathrm{O}_{2}=32$ develops about 97000 calories, forming gaseous $\mathrm{CO}_{2}$. If the charcoal were gaseous like the hydrogen, and only contained $\mathrm{C}_{2}$ in its molecule, much more heat would be developed, and judging by other substances, whose molecules on passing from the solid to the gaseous state absorb about 10000 to 15000 calories, it must be held that gaseous carbon on forming gaseous carbonic anhydride would develop not less than 110,000 calories-that is, approximately twice as much as is developed in the formation of water. And as in a molecule of carbonic anhydride there is twice as much oxygen as in a molecule of water, the oxygen develops approximately the same quantity of heat on combining with hydrogen and carbon. That is to say, that here we find the same close. affinity (see Chap. II. Note 7) determined by the quantity of heat as between hydrogen, zinc, and iron. For this reason here also, as in relation to hydrogen and iron, we ought to expect an evident distribution of oxygen between hydrogen and carbon, if they are both in excess compared with the amount of oxygen; but if there be an excess of carbon it will decompose water, whilst an excess of hydrogen will decompose carbonic anliydride. If similar relations of mutual action are made clear in isolated cases, still the full theory of the subject is wanting in the present condition of chemical knowledge. 
and terminates with the compiete decomposition of the carbonic anhydride. Phosphorus is used in order to attain the complete absorption of the oxygen. In these examples we see that a known mixture of changeable substances is capable of arriving at a state of stable equilibrium, destroyed, however, by the removal of one of the substances composing the mixture. This represents one of the instances of the influence of mass. And as on rapid cooling the combustible carbonic oxide does not succeed in combining with the oxygen, with which it totally combines if slowly cooled, forming carbonic anhydride, here, as in all chemical phenomena, the action of time is apparent-that is, the existence of a definite velocity of reaction.

Although carbonic anhydride is decomposed on heating, yielding oxygen, it is, nevertheless, like water, an unchangeable substance at ordinary temperatures. The decomposition of carbonic anhydride, as effected by plants, is on this account all the more remarkable ; in this case the whole of the oxygen of the carbonic anhydride is separated in the free state. The mechanism of this decomposition is that the heat and light absorbed by the plants are expencled in the decomposition of the carbonic anhydride. This accounts for the enormous influence of temperature and light on the growth of plants. But it is at present not clearly understood how this takes place, or by what separate intermediate reactions the whole process of decomposition of carbonic anhydride in plants into oxygen and the carbohydrates (Chapter VIII.) remaining in them, takes place. It is known that sulphurous anhydride (in many ways resembling carbonic anhydride) under the action of light (and also of heat) forms sulphur and sulphuric anhydride, $\mathrm{SO}_{3}$, and in the presence of water sulphuric acid. But no similar decomposition has been directly obtained with carbonic anhydride, indeed it forms the highest degree of oxidation of carbon; perhaps for that reason the oxygen separates. On the other hand, it is known that plants always form and contain organic acids, and these must be regarded as derivatives of carbonic acid, as is seen by all their reactions, which we will shortly treat of. For this reason it might be thought that the carbonic acid absorbed by the plants first forms organic acids in them, and that these latter in their final transformation form all the other complex organic substances of the plants. Many organic acids are found in plants in considerable quantity ; for instance, tartaric acid, $\mathrm{C}_{4} \mathrm{H}_{6} \mathrm{O}_{6}$, found in grape-juice and in the acid juice of many plants; malic acid, $\mathrm{C}_{4} \mathrm{H}_{6} \mathrm{O}_{5}$, not only found in unripe apples but in still greater quantities in mountain ash berries; citric acid, $\mathrm{C}_{6} \mathrm{H}_{8} \mathrm{O}_{7}$, found in the acid juice of lemons, in gooseberries, cranberries, drc.; oxalic acid, $\mathrm{C}_{2} \mathrm{H}_{2} \mathrm{O}_{4}$, found in wood-sorrel and 
many other plants. Sometimes these acids exist in a free state in the plants, and sometimes in the form of salts ; for instance, tartaric acid is met with in grapes as the salt known as cream of tartar, but in the impure state called argol, or tartar, $\mathrm{C}_{4} \mathrm{H}_{5} \mathrm{KO}_{6}$. In sorrel we find the so-called salts of sorrel, or acid potassium oxalate, $\mathrm{C}_{2} \mathrm{HKO}_{4}$. There is a very clear connection between carbonic anhydride and the abovementioned organic acids-namely, they all, under one condition or another, yield carbonic anhydride, and can all be formed by means of it from substances destitute of acid properties. The following examples afford the best demonstration of this fact : if acetic acid, $\mathrm{C}_{2} \mathrm{H}_{4} \mathrm{O}_{2}$, the acid of vinegar, be passed in the form of vapour through a heated tube, it splits up into carbonic anhydride and marsh gas $=\mathrm{CO}_{2}+\mathrm{CH}_{4}$. But it can also be obtained conversely from those components into which it decomposes. If one equivalent of hydrogen in marsh gas be replaced (by indirect means) by sodium, and the compound $\mathrm{CH}_{3} \mathrm{Na}$ is obtained, this directly absorbs carbonic anhydride, forming a salt of acetic acid, $\mathrm{CH}_{3} \mathrm{Na}+\mathrm{CO}_{2}=\mathrm{C}_{2} \mathrm{H}_{3} \mathrm{NaO}_{2}$; from this acetic acid itself may be easily obtained in a similar way to that by which nitric acid may be obtained from nitre. Therefore acetic acid decomposes into marsh gas and carbonic anhydride, and conversely is obtainable from them. The hydrogen of marsh gas does not, like that in acids, show the property of being directly replaced by metals; the gas itself does not show any acid character whatever, but on combining with the elements of carbonic anhydride it acquires the properties of an acid. The investigation of all other organic acids shows similarly that their acid character depends on their containing the elements of carbonic anhydride. For this reason there is no organic acid containing less oxygen in its molecule than there is in carbonic anhydride; every organic acid contains in its molecule at least two atoms of oxygen. In order to express the relation between carbonic acid, $\mathrm{H}_{2} \mathrm{CO}_{3}$, and organic acids, and in order to understand the reason. of the acidity of these latter, it is simplest to turn to that law of substitution which shows (Chapter VI.) the relation between the hydrogen and oxygen compounds of nitrogen, and permits us (Chapter VIII.) to regard all hydrocarbons as derived from methane. The facts of the matter are as follows : if we have a given organic compound, $\mathrm{A}$, which has not the properties of an acid, but as the derivative of a hydrocarbon contains hydrogen combined with carbon, then $\mathrm{ACO}_{2}$ will be a monobasic organic acid, $\mathrm{A}_{2} \mathrm{CO}_{2}$ a bibasic, $\mathrm{A} 3 \mathrm{CO}_{2}$ a tribasic, and so on - that is, each molecule, $\mathrm{CO}_{2}$, transforms one atom of hydrogen into that state in which it may be replaced by metals, as in acids. This already furnishes proof that in organic acids it is necessary to recognise the group $\mathrm{HCO}_{2}$, or carboxyl. 
If the addition of $\mathrm{CO}_{2}$ raises the basicity the removal of $\mathrm{CO}_{2}$ lowers it. Thus from the bibasic oxalic acid, $\mathrm{C}_{2} \mathrm{H}_{2} \mathrm{O}_{4}$, or phthalic acid, $\mathrm{C}_{8} \mathrm{H}_{6} \mathrm{O}_{4}$, by eliminating $\mathrm{CO}_{2}$ (easily effected experimentally) we obtain the monobasic formic acid, $\mathrm{CH}_{2} \mathrm{O}_{2}$, or benzoic acid, $\mathrm{C}_{7} \mathrm{H}_{6} \mathrm{O}_{2}$., respectively. The nature of carboxyl is directly explained by the law of substitution. Judging from what has been stated in Chapters V. and VIII. concerning this law, it should already be evident that $\mathrm{CO}_{2}$ is $\mathrm{CH}_{4}$, with the exchange of $\mathrm{H}_{4}$ for $\mathrm{O}_{2}$, and the hydrate of carbonic acid, $\mathrm{H}_{2} \mathrm{CO}_{3}$, is $\mathrm{CO}(\mathrm{OH})_{2}$, that is, methane, where two parts of hydrogen are replaced by two parts of the water radicle $(\mathrm{OH}$, hydroxyl), and the other two by oxygen. Therefore the group $\mathrm{CO}(\mathrm{OH})$, or carboxyl, $\mathrm{HCO}_{2}$, is a part of carbonic acid, and is equivalent to $(\mathrm{OH})$, and therefore also to $\mathrm{H}$. That is, $i t$ is a univalent residue of carbonic acid capable of replacing one atom of hydrogen. Carbonic acid itself is a bibasic acid, both hydrogen atoms in it being replaceable by metals, therefore carboxyl, which contains one of the hydrogen atoms of carbonic acid, represents a group in which the hydrogen is exchangeable with metals. And therefore if $1,2 \ldots n$ atoms of non-metallic hydrogen are exchanged $1,2 \ldots n$ times for carboxyl we ought to obtain $1,2 \ldots n$-basic acids. Organic acids are the products of the carboxyl substitution of hydrocarbons. ${ }^{18}$ If in the saturated hydrocarbons, $\mathrm{C}_{n} \mathrm{H}_{2 n+2}$, one part of hydrogen is replaced by carboxyl the monobasic saturated (or fatty) acids, $\mathrm{C}_{n} \mathrm{H}_{2 n+1}\left(\mathrm{CO}_{2} \mathrm{H}\right)$, will be obtained, as, for instance, formic,

18 If $\mathrm{CO}_{2}$ is the anhydride of a bibasic acid, and carboxyl corresponds with it, replacing the hydrogen of hydrocarbons, and giving them the character of comparatively feeble acids, then $\mathrm{SO}_{3}$ is the anhydride of an energetic bibasic acid, and sulphoxyl, $\mathrm{SO}_{2}(\mathrm{OH})$, corresponds with it, being capable of replacing the hydrogen of hydrocarbons, and forming comparatively energetic sulphur oxyacids (sulphonic acids); for instance, $\mathrm{C}_{6} \mathrm{H}_{5}(\mathrm{COOH})$, benzoic acid, and $\mathrm{C}_{6} \mathrm{H}_{5}\left(\mathrm{SO}_{2} \mathrm{OH}\right)$, benzenesulphonic acid, are derived from $\mathrm{C}_{6} \mathrm{H}_{6}$. As the exchange of $\mathrm{H}$ for methyl, $\mathrm{CH}_{3}$, is equivalent to the addition of $\mathrm{CH}_{2}$, the exchange of carboxyl, $\mathrm{COOH}$, is equivalent to the addition of $\mathrm{CO}_{2}$; so the exchange of $\mathrm{H}$ for sulphoxyl is equivalent to the addition of $\mathrm{SO}_{3}$. The latter proceeds directly, for instance: $\mathrm{C}_{6} \mathrm{H}_{6}+\mathrm{SO}_{3}=\mathrm{C}_{6} \mathrm{H}_{5}\left(\mathrm{SO}_{2} \mathrm{OH}\right)$.

In Chapter VIII. we saw that the structure of hydrocarbons might and ought to be represented by the presence of $\mathrm{CH}_{3}, \mathrm{CH}_{2}, \mathrm{CH}$, and $\mathrm{C}$-radicles of methane, starting from methane itself; therefore the atoms of hydrogen are retained by separate atoms of carbon, which refers also to $\mathrm{OH}, \mathrm{COOH}$, and other radicles. Although with one atom of carbon, $\mathrm{H}_{4}, \mathrm{H}_{3}$, and $\mathrm{H}_{2}$, or several, $\mathrm{CH}_{3}, \mathrm{Cl}$, \&c., may be retained, yet not more than one of hydroxyl may be retained, and therefore there is no alcohol $\mathrm{CH}_{2}(\mathrm{OH})_{2}$ or $\mathrm{C}_{3} \mathrm{H}_{4}(\mathrm{OH})_{4}$. The carboxyl, as well as the sulphoxyl, substitution of hydrogen in hydrocarbons, leads to the formation of acids, because the derivative acids $\mathrm{H}_{2} \mathrm{CO}_{3}$ and $\mathrm{H}_{2} \mathrm{SO}_{4}$ are bibasic. Nitric acid, being monobasic, is unable to form a similar radicle. Its radicle, $\mathrm{NO}_{3}$, does not contain hydrogen, and does not produce acidity, but has the same relation to the acid $\mathrm{NO}_{2}(\mathrm{OH})$ as carboxyl to carbonic acid.

As, according to the determinations of Thomsen, the heat of combustion of the vapours of acids $\mathrm{RCO}_{2}$ is known where $\mathrm{R}$ is a hydrocarbon, and the heat of combustion of the hydrocarbons $\mathrm{R}$ themselves, it may be seen that the formation of acids, $\mathrm{RCO}_{2}$, from $\mathrm{R}+\mathrm{CO}_{2}$ is always accompanied by a small absorption or development of heat. We give 
$\mathrm{HCO}_{2} \mathrm{H}$, acetic acid, $\mathrm{CH}_{3} \mathrm{CO}_{2} \mathrm{H}, \ldots$ stearic acid, $\mathrm{C}_{17} \mathrm{H}_{35}, \mathrm{CO}_{2} \mathrm{H}$, \&c. The double substitution will give bibasic acids, $\mathrm{C}_{n} \mathrm{H}_{2 n}\left(\mathrm{CO}_{2} \mathrm{H}\right)\left(\mathrm{CO}_{2} \mathrm{H}\right)$; for instance, oxalic $n=0$, malonic $n=1$, succinic acid $n=2$, \&c. To benzene, $\mathrm{C}_{6} \mathrm{H}_{6}$, correspond benzoic acid, $\mathrm{C}_{6} \mathrm{H}_{5}\left(\mathrm{CO}_{2} \mathrm{H}\right)$, phthalic acid (and its isomerides), $\mathrm{C}_{6} \mathrm{H}_{4}\left(\mathrm{CO}_{2} \mathrm{H}\right)_{2}$, up to melitic acid, $\mathrm{C}_{6}\left(\mathrm{CO}_{2} \mathrm{H}\right)_{6}$, in all of which the basicity is equal to the number of carboxyl groups. As many isomerides exist in hydrocarbons, it is readily understood not only that such can exist also in organic acids, but that their number and structure may be foreseen. As in hydrocarbons the hydrogen may be replaced by chlorine, hydroxyl, drc., evidently the same is possible with organic acids. And, therefore, the number and transformations of such compounds are exceedingly great. This complex and most interesting branch of chemistry is treated separately in organic chemistry.

Carbonic Oxide.-This gas is formed whenever the combustion of organic substances takes place in the presence of a large excess of incandescent charcoal ; the air first burns the carbon into carbonic anhydride, but this in penetrating through the red-hot charcoal is transformed into carbonic oxide, $\mathrm{CO}_{2}+\mathrm{C}=2 \mathrm{CO}$. By this reaction carbonic oxide is prepared by passing carbonic anhydride through charcoal at a red heat. It may be separated from the excess of carbonic anhydride by passing it through a solution of alkali, which does not absorb carbonic oxide. This reduction of carbonic anbydride explains why carbonic oxide is formed in ordinary clear fires, where the incoming air passes over a large surface of heated coal. A blue flame is then observed burning above the coal; this is the burning carbonic oxide. When charcoal is burnt in stacks, or when a thick layer of coal is burning in a brazier, and under many similar circumstances, carbonic oxide is also formed. In metallurgical processes, for instance, when iron is smelted from the ore, very often the same process of conversion of carbonic anhydride into carbonic oxide occurs, especially if the combustion of the coal be effected in high, so-called blast, furnaces and ovens, where the air enters from the lower part and is compelled to pass through a thick layer of coal. In this way, also, combustion with flame may be

the heats of combustion in thousands of calories, referring to the molecular weights of the burning substances:-

$\begin{array}{llccc}\mathrm{R}= & \mathrm{H}_{2} & \mathrm{CH}_{4} & \mathrm{C}_{2} \mathrm{H}_{6} & \mathrm{C}_{6} \mathrm{H}_{6} \\ & 68^{\circ} 4 & 212 & 370 & 777 \\ \mathrm{RCO}_{2}= & 69 \cdot 4 & 225 & 387 & 766\end{array}$

Thus $\mathrm{H}_{2}$ corresponds with formic acid, $\mathrm{CH}_{2} \mathrm{O}_{2}$; benzene, $\mathrm{C}_{6} \mathrm{H}_{6}$, with benzoic acid, $\mathrm{C}_{7} \mathrm{H}_{6} \mathrm{O}_{2}$. The data for the latter are taken from Stohmann, and refer to the solid condition. For formic acid Stohmann gives the heat of combustion as 59000 calories in a liquid state, but in a state of vapour, 64.6 thousand units, which is much less than according to Thomsen. 
obtained from those kinds of fuel which under ordinary conditions burn without flame : for instance, anthracite, coke, charcoal. Heating by means of a gas-producer - that is, an apparatus producing carbonic oxide from fuel-is carried on in the same manner. ${ }^{19}$ In transforming one part of charcoal into carbonic oxide 2420 heat units are given out, and on burning to carbonic anhydride 8080 heat units. It is evident that on transforming the charcoal first into carbonic oxide we obtain a gas which in burning is capable of giving out 5660 heat units for one part of charcoal. This preparatory transformation of fuel into carbonic oxide, or producer gas containing a mixture of carbonic oxide (about $\frac{1}{3}$ by volume) and nitrogen ( $\frac{2}{3}$ volume), in many cases presents most important advantages, as it is easy to completely burn gaseous fuel without an excess of air, which would lower the temperature. ${ }^{20}$ In stoves where solid fuel is burnt it is impossible to effect the complete combustion of the various kinds of fuel without admitting an excess of air. Gaseous fuel, such as carbonic oxide,

19 In gas-producers all carbonaceous fuels are transformed into inflammable gas. In those which (on account of their slight density and large amount of water, or incombustible admixtures which absorb heat) are not as capable of giving a high temperature

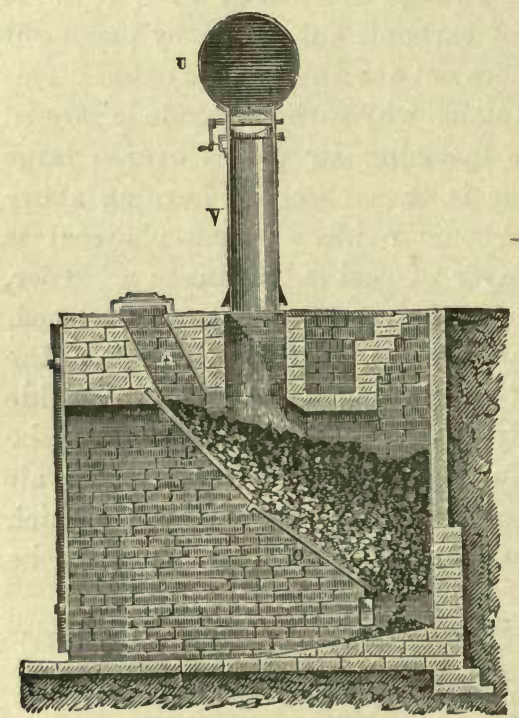

FIG. 63.-Gas-producer for the formation of carbon monoxide for heating purposes. in ordinary furnaces-for instance, fir cones, peat, the lower kinds of coal, \&c. - the same gas is obtained as with the best kinds of coal, because the water condenses on cooling, and the ashes and earthy matter remain in the gas-producer. The construction of a gas-producer is seen from the accompanying drawing. The fuel lies on the fire-bars $\mathrm{O}$, the air enters through them and the ashhole (drawn by the draught of the chimney of the stove where the gas burns, or else forced by a blowing apparatus), the quantity of air being exactly regulated by means of valves. The gases formed are then led by the tube $\mathrm{V}$, provided with a valve, into the gas main, $U$. The addition of fuel ought to proceed in such a way as to avoid the separation of carbonic anhydride; hence the space $\mathbf{A}$ is kept filled with the combustible and covered with a lid.

20 An excess of air lowers the temperature of combustion, because it becomes heated itself, as explained in Chapter III. In ordinary furnaces the excess of air is three or four times greater than the quantity required for perfect combustion. In the best furnaces (with fire-bars, regulated air supply, and corresponding chimney draught) it is necessary to introduce twice as much air as is necessary, otherwise the smoke contains much carbonic oxide. 
is easily completely mixed with air and burnt without excess of it. If, in addition to this, the air and gas required for the combustion be previously heated by means of the heat which would otherwise be uselessly carried off in the products of combustion (smoke) ${ }^{21}$ it is easy to reach a high temperature, so high (about $1800^{\circ}$ ) that platinum may be melted. Such an arrangement is known as a regenerative furnace. ${ }^{22}$ By means of this process not only may there be obtained the high temperatures indispensable in many industries (for instance, glassworking, steel-melting, \&c.), but also great advantage ${ }^{23}$ as regards the quantity of fuel, because the transmission of heat to the object to be heated, conditions being equal, is determined by the difference of temperatures.

\section{The transformation of carbonic anhydride, by means of charcoal,}

${ }^{21}$ If in manufactories it is necessary, for instance, to maintain the temperature in a furnace at $1000^{\circ}$, the flame passes out at this or a higher temperature, and therefore much fuel is lost in the smoke. For the draught of the chimney a temperature of $100^{\circ}$ to $150^{\circ}$ is sufficient, and therefore the remaining heat ought to be utilised. For this purpose the flues are carried under boilers or other heating apparatus. The preparatory heating of the air is the best means of utilisation, as by this means the highest temperature is attained and the quickest heating and greatest economy are effected.

${ }_{22}$ Regenerative furnaces were introduced by the Brothers Siemens about the year 1860 in many industries, and mark a most important progress in the use of fuel, especially in obtaining high temperatures. The principle is as follows: The products of combustion from the furnace are led into a chamber, I, and heat up the bricks in it, and then pass into the outlet flue; when the bricks are at a red heat the products of combustion are passed (by altering the valves) into another adjoining chamber, II, and air requisite for the combustion of the generator gases is passed through I. In passing round about the incandescent bricks the air is heated, and the bricks cool-that is, the heat of the smoke is returned into the furnace. The air is then passed through II, and the smoke through I. The regenerative burners for illuminating gas are founded on this same principle, the products of combustion heat the incoming air and gas, the temperature is higher, the light brighter, and an economy of gas is effected in lighting. Absolute perfection in these appliances has of course not yet been attained; amelioration is still further possible, but dissociation imposes a limit because at a certain high temperature combinations do not ensue, possible temperatures being limited by reverse reactions. Here, as in a number of other cases, the ultimate conception of the subject ought to be limited to the sphere of general external and visible advantages.

23 At first sight it appears absurd, useless, and paradoxical to lose nearly one-third of the heat which fuel develops by turning it into gas. Actually the advantage is enormous, especially for producing high temperatures, as is already seen from the fact that fuel rich in oxygen (for instance, wood) when damp is unable, with any kind of hearth whatever, to give the temperature required for glass-melting or steel-casting, whilst in the gas-producer they furnish exactly the same gas as the driest and most carbonaceous fuel. In order to understand the principle which is here involved, it is sufficient to turn one's attention to the fact that the only heat employed with advantage is that which is concentrated in the shape of products of combustion having a high temperature. A large amount of heat, but having a low temperature, in many cases is of no use whatever. We are unable here to enter into all the details of the complicated matter of the application of fuel, and further particulars must be sought for in special technical treatises. The following footnotes, however, contain certain fundamental figures for calculations concerning combustion. 
into carbonic oxide $\left(\mathrm{C}+\mathrm{CO}_{2}=\mathrm{CO}+\mathrm{CO}\right)$, is considered a reversible reaction, because at a high temperature the carbonic oxide splits up into carbon and carbonic anhydride, as Sainte-Claire Deville showed by using the method of the 'cold and hot tube.' Inside a tube heated in a furnace another thin metallic (silvered copper) tube is fitted, through which a constant stream of cold water flows. The carbonic oxide coming into contact with the heated walls of the exterior tube forms charcoal, and its minute particles settle in the form of lampblack on (the lower part) of the cold tube, and, since they are cooled, they do not act further on the oxygen or carbonic anhydride formed. ${ }^{24}$ A series of electric sparks also decomposes carbonic oxide into carbonic anhydride and carbon, and if the carbonic anhydride be removed by alkali complete decomposition may be obtained (Deville). Aqueous vapour, which is so similar to carbonic anhydride in many respects, acts, at a high temperature, on charcoal in an exactly similar way, $\mathrm{C}+\mathrm{H}_{2} \mathrm{O}=\mathrm{H}_{2}+\mathrm{CO}$. From 2 volumes of carbonic anhydride with charcoal 4 volumes of carbonic oxide ( 2 molecules) are obtained, and precisely the same from 2 volumes of water vapour with charcoal

24 The first product of combustion of charcoal is always carbonic anhydride, and not carbonic oxide. This is seen from the fact that with a shallow layer of charcoal (less than a decimetre if the charcoal be closely packed) carbonic oxide is not formed at all. It is not even produced with a deep layer of charcoal if the temperature is not above $500^{\circ}$, and the current of air or oxygen is very slow. With a rapid current of air the charcoal becomes red hot, and the temperature rises, and then carbonic oxide appears (Lang 1888). Naumann and Pistor determined that the reaction of carbonic anhydride with carbon commences at about $550^{\circ}$, and that between water and carbon at about $500^{\circ}$. At the latter temperature carbonic anhydride is formed, and only with a rise of temperature is carbonic oxide formed (Lang) from the action of the carbonic anhydride on the carbon, and from the reaction $\mathrm{CO}_{2}+\mathrm{H}_{2}=\mathrm{CO}+\mathrm{H}_{2} \mathrm{O}$. Rathke (1881) showed that at no temperature whatever is the reaction as expressed by the equation $\mathrm{CO}_{2}+\mathrm{C}=2 \mathrm{CO}$ complete; a part of the carbonic anhydride remains, and Lang determined that at about $1000^{\circ}$ not less than 3 p.c. of the carbonic anhydride remains untransformed into carbonic oxide, even after the action has been continued for several hours. The endothermal reactions, $\mathrm{C}+2 \mathrm{H}_{2} \mathrm{O}=$ $\mathrm{CO}_{2}+2 \mathrm{H}_{2}$, and $\mathrm{CO}+\mathrm{H}_{2} \mathrm{O}=\mathrm{CO}_{2}+\mathrm{H}_{2}$, are just as incomplete. This is made clear if we note that on the one hand the above-mentioned reactions are all reversible, and therefore bounded by a limit; and, on the other hand, that at about $500^{\circ}$ oxygen begins to combine with hydrogen and carbon, and also that the lower limits of dissociation of water, carbonic anhydride, and carbonic oxide lie near one another between $500^{\circ}$ and $1200^{\circ}$. For water and carbonic oxide the lower limit of the commencement of dissociation is unknown, but judging from the accumulated data (according to Le Chatelier, 1888) that of carbonic anhydride may be taken as about $1050^{\circ}$. Even at about $200^{\circ}$ half the carbonic anhydride dissociates if the pressure be small, about 0.001 atmosphere. At the atmospheric pressure, not more than 0.05 of the carbonic anhydride decomposes. The influence of pressure is here evident, for the reason that the splitting up of carbonic anhydride into carbonic oxide and oxygen is accompanied by an increase in volume (as in the case of the dissociation of nitric peroxide. See Chapter VI. Note 46). As in stoves and lamps, and also with explosive substances, the temperature is not higher than $2000^{\circ}$ to $2500^{\circ}$, it is evident that although the partial pressure of carbonic anhydride is small, still its dissociation cannot be considerable, and probably does not exceed 5 p.c. 
4 volumes of a gas consisting of hydrogen and carbonic oxide $\left(\mathrm{H}_{2}+\mathrm{CO}\right)$ is formed. This mixture of combustible gases is called water gas. ${ }^{25}$ But aqueous vapour (and only when strongly superheated, otherwise it cools the charcoal) only acts on charcoal to form a large amount of carbonic oxide at a very high temperature (when carbonic anhydride dissociates); it begins to react at about $500^{\circ}$, forming carbonic anhydride, according to the equation $\mathrm{C}+2 \mathrm{H}_{2} \mathrm{O}=\mathrm{CO}_{2}+2 \mathrm{H}_{2}$. Besides this, carbonic oxide on splitting up forms carbonic anhydride, and therefore water gas always contains a mixture ${ }^{26}$ in which hydrogen pre-

25 A molecular weight of this gas, or 2 volumes CO (28 grams), on combustion (forming $\mathrm{CO}_{2}$ ) gives out 68,000 heat units (Thomsen 67960 calories). A molecular weight of hydrogen, $\mathrm{H}_{2}$ (or 2 volumes), develops on burning into liquid water 69000 heat units (according to Thomsen 68300 ), but if it forms aqueous vapour 58000 heat units. Charcoal, resolving itself by combustion into the molecular quantity of $\mathrm{CO}_{2}$ (2 volumes), develops 97000 heat units. From the data furnished by these exothermal reactions it follows: (1) that the oxidation of solid charcoal into carbonic oxide develops 29000 heat units; (2) that the reaction $\mathrm{C}+\mathrm{CO}_{2}=2 \mathrm{CO}$ absorbs 39000 heat units; (3) $\mathrm{C}+\mathrm{H}_{2} \mathrm{O}=\mathrm{H}_{2}+\mathrm{CO}$ absorbs (if the water be in a state of vapour) 29000 calories, but if the water be liquid 40000 calories (almost as much as $\mathrm{C}+\mathrm{CO}_{2}$ ); (4) $\mathrm{C}+\mathrm{H}_{2} \mathrm{O}=\mathrm{CO}_{2}+2 \mathrm{H}_{2}$ absorbs (if the water be in a state of vapour) 19000 heat units; (5) the reaction $\mathrm{CO}+\mathrm{H}_{2} \mathrm{O}=\mathrm{CO}_{2}+\mathrm{H}_{2}$ develops 10000 heat units if the water be in the state of vapour.

Therefore it follows that 2 volumes of $\mathrm{CO}$ or $\mathrm{H}_{2}$ burning into $\mathrm{CO}_{2}$ or $\mathrm{H}_{2} \mathrm{O}$ develop almost the same amount of heat, just as also the heat effects corresponding with the equations

$$
\begin{aligned}
& \mathrm{C}+\mathrm{H}_{2} \mathrm{O}=\mathrm{CO}+\mathrm{H}_{2} \\
& \mathrm{C}+\mathrm{CO}_{2}=\mathrm{CO}+\mathrm{CO}
\end{aligned}
$$

are nearly equal.

26 Water gas, obtained from steam at a white heat, contains about 50 p.c. of hydrogen, about 40 p.c. of carbonic oxide, about 5 p.c. of carbonic anhydride, the remainder being nitrogen from the charcoal and air. Compared with producer gas, which contains much nitrogen, this is a gas much richer in combustible matter, and therefore capable of giving high temperatures, and is for this reason of the greatest utility. If carbonic anhydride could be as readily obtained in as pure a state as water, there would be no difference in the heating powers of producer gas and water gas. As regards the utilisation of the heat of the charcoal there is no difference, because on burning carbonic oxide gives out almost as much heat as hydrogen-even more if the smoke has a temperature above $100^{\circ}$, and the water remains as vapour (Note 25). But water gas stands higher than producer gas as regards the concentration of the fuel in its transformation into gas, and therefore in places where a particularly high temperature is required (for instance, for lighting by means of incandescent lime or magnesia, or for steel melting, \&c.), and for distributing through pipes at great distances, water gas is at present held in high estimation, but when (in ordinary furnaces, re-heating, glass-melting, and other furnaces) a very high temperature is not required, and there is no need to convey the gas in pipes, producer gas is generally preferred on account of the simplicity of its preparation, especially as for water gas such a high temperature is required that the plant soon becomes damaged.

Water gas is prepared (there are very many systems, but the American patent of $T$. Lowe, of Norristown, Pennsylvania, is much used) in a cylindrical geuerator, into which heated air is introduced, in order, by partial combustion of the coke, to heat the remainder, \&c., to a white heat. The products of combustion containing carbonic oxide are utilised in a contrivance for superheating steam, which is then passed over the white hot coke. Water gas, or a mixture of hydrogen and carbonic oxide, is thus obtained. The practical 
dominates, the volume of carbonic oxide being comparatively less, whilst the amount of carbonic anhydride increases as the temperature of the reaction decreases (generally it is more than 3 per cent.).

Metals, like iron and zinc, which at a red heat are capable of decomposing water with the formation of hydrogen, also decompose carbonic anhydride with the formation of carbonic oxide; so both the ordinary products of complete combustion, water and carbonic anhydride, are very similar in their reactions-and hydrogen may be compared with carbonic oxide. The metallic oxides of the abovementioned metals, when reduced by charcoal, also give carbonic oxide. Priestley obtained it by heating charcoal with zinc oxide. It must be held that here carbonic anhydride is first produced, but that, combining with carbon, it forms carbonic oxide. As free carbonic anhydride may be transformed into carbonic oxide, so, in the same way precisely, may that carbonic acid which is in a state of combination; therefore, if magnesium or barium carbonates $\left(\mathrm{MgCO}_{3}\right.$ or $\left.\mathrm{BaCO}_{3}\right)$ be heated to a red heat with charcoal, oriron or zinc, carbonic oxide will be producedfor instance, it is obtained by heating an intimate mixture of 9 parts of chalk and 1 part of charcoal in a clay retort.

Many organic substances ${ }^{27}$ on being heated, or under the action of different agents, yield carbonic oxide ; amongst these are many organic or carboxylic acids. The simplest are formic and oxalic acids. Formic acid, $\mathrm{CH}_{2} \mathrm{O}_{2}$, on being heated to $200^{\circ}$, easily decomposes into carbonic oxide and water, $\mathrm{CH}_{2} \mathrm{O}_{2}=\mathrm{CO}+\mathrm{H}_{2} \mathrm{O}$. This reaction may be effected

side of the question resolves itself into utilising all the heat given out by the charcoal, and contained in the incandescent gas, for heating up the air, for the formation and superheating of the steam.

Water gas is sometimes called 'the fuel of the future,' because it is applicable to all purposes, develops a high temperature, and is therefore available, not only for domestic and industrial uses, but also for gas-motors (page 172) and for lighting. For the latter purpose platinum, lime, magnesia, zirconia, and similar substances (as in the Drummond light, Chapter III.), are rendered incandescent in the flame, or else the gas is carburetted - that is, mixed with the vapours of volatile hydrocarbons (generally benzene or naphtha, naphthalene, or simply naphtha gas), which communicate to the pale flame of carbonic oxide and hydrogen a great brilliancy, owing to the high temperature developed by the combustion of the non-luminous gases. As water gas, possessing these properties, may be prepared at central works and conveyed in pipes to the consumers, may be produced from any kind of fuel, and ought to be much cheaper than ordinary gas, it may actually be considered that in course of time (when practice will have determined the cheapest and best way to prepare it) it will not only supplant ordinary gas but will with advantage everywhere replace the ordinary forms of fuel, which in many respects are inconvenient. At present its consumption spreads principally for lighting purposes, and for use in gas-engines instead of ordinary illuminating gas.

27 The so-called yellow prussiate, $\mathrm{K}_{4} \mathrm{FeC}_{6} \mathrm{~N}_{6}$, on being heated with ten parts of strong sulphuric acid forms a considerable quantity of very pure carbonic oxide completely free from carbonic anhydride. 
by mixing formic acid with glycerin, because in a separate state it volatilises much earlier, and therefore cannot be heated up to the required temperature. The salts of formic acid, on being heated with sulphuric acid, yield carbonic oxide. Usually, however, carbonic oxide is prepared in laboratories, not from formic but from oxalic acic, $\mathrm{C}_{2} \mathrm{H}_{2} \mathrm{O}_{4}$, the more so as formic acid is itself prepared from oxalic acid. The latter acid is easily obtained by the action of nitric acid on starch, sugar, \&c. ; it is also found in nature. Oxalic acid is easily decomposed by heat; its crystals first lose water, then partly volatilise, but the greater part is decomposed. The decomposition is of the following nature : it splits up into water, carbonic oxide, and carbonic anhydride, ${ }^{28} \quad \mathrm{C}_{2} \mathrm{H}_{2} \mathrm{O}_{4}=\mathrm{H}_{2} \mathrm{O}+\mathrm{CO}_{2}+\mathrm{CO}$. This decomposition is generally practically effected by mixing oxalic acid with strong sulphuric acid, because the latter favours the decomposition by taking up the water. On heating a mixture of oxalic and sulphuric acids a mixture of carbonic oxide and carbonic anhydride is evolved. This mixture is passed through a solution of an alkali in order to absorb the carbonic anhydride, whilst the carbonic oxide passes on. Oxalic acid in admixture with glycerin, on being heated first to $100^{\circ}$ and then to $140^{\circ}$, decomposes in a similar manner.

In its physical properties carbonic oxide resembles nitrogen; this is explained by the equality of their molecular weights. The absence of colour and smell, the low temperature of the absolute boiling point, $-140^{\circ}$ (nitrogen $-146^{\circ}$ ), the faculty of solidifying at $-200^{\circ}$ (nitrogen, $-202^{\circ}$ ), the boiling point of $-190^{\circ}$ (nitrogen, $-203^{\circ}$ ), and the slight solubility (page 78 ), of carbonic oxide are almost the same as in those of nitrogen. The chemical properties of both gases are, however, very different, and in these carbonic oxide resembles hydrogen. Carbonic oxide burns with a blue flame, giving 2 volumes of carbonic anhydride from 2 volumes of carbonic oxide, just as 2 volumes of hydrogen give 2 volumes of aqueous vapour. It explodes with oxygen, in the eudiometer, like hydrogen. ${ }^{29}$ When

28 The decomposition of formic and oxalic acids, with the formation of carbonic oxide, considering these acids as carboxyl derivatives, may be explained as follows:-The first is $\mathrm{H}(\mathrm{COOH})$, and the second $(\mathrm{COOH})_{2}$, or $\mathrm{H}_{2}$, in which one or both parts of hydrogen are exchanged for carboxyl; therefore they are equal to $\mathrm{H}_{2}+\mathrm{CO}_{2}$ and $\mathrm{H}_{2}+2 \mathrm{CO}_{2}$; but $\mathrm{H}_{2}$ reacts with $\mathrm{CO}_{2}$, as has been stated above, forming $\mathrm{CO}$ and $\mathrm{H}_{2} \mathrm{O}$. From this it is also evident that oxalic acid on losing $\mathrm{CO}_{2}$ forms formic acid, and alsu that the latter may proceed from $\mathrm{CO}+\mathrm{H}_{2} \mathrm{O}$, as we shall see further on.

${ }^{29} \mathrm{It}$ is remarkable that, according to the investigations of Dixon, perfectly dry carbonic oxide does not explode with oxygen when a spark of low intensity is used, but an explosion takes place if there is the slightest admixture of moisture. L. Meyer, however, showed that sparks of an electric discharge of considerable intensity produce an explosion. I think that this may be explained by the fact that water with 
breathed it acts as a strong poison, because it is absorbed by the blood $;^{30}$ this explains the action of charcoal fumes, the products of the incomplete combustion of charcoal and other carbonaceous fuels. Owing to its faculty of combining with oxygen, carbonic oxide acts as a powerful reducing agent, taking up the oxygen from many compounds at a red heat, and being itself transformed into carbonic anhydride. The reducing action of carbonic oxide, however, is (like that of hydrogen, Chapter II.) naturally confined to those oxides which easily part with their oxygen-as, for instance, copper oxide-whilst the oxides of magnesium or potassium are not reduced. Metallic iron itself is capable of reducing carbonic anhydride into carbonic oxide, just as it liberates the hydrogen from water. Copper, which does not decompose water, does not decompose carbonic oxide. If a platinum wire heated to $300^{\circ}$, or spongy platinum at the ordinary temperature, be plunged into a mixture of carbonic oxide and oxygen, or of hydrogen and oxygen, the mixture explodes. These reactions remind one exceedingly of those which are peculiar to hydrogen. The following important distinction, however, exists between them, namely : the molecule of hydrogen is composed of $\mathrm{H}_{2}$, a group of elements divisible into two like parts, whilst, as the molecule of carbonic oxide, $\mathrm{CO}$, contains unlike atoms of carbon and oxygen, in none of its reactions of combination can it give two molecules of matter containing its elements. This is particularly noticeable in the action of chlorine on hydrogen and on carbonic oxide respectively; with the former chlorine forms hydrogen chloride, and with the latter it produces the so-called carbonyl chloride, $\mathrm{COCl}_{2}$; that is to say, the molecule of hydrogen, $\mathrm{H}_{2}$, under the action of chlorine divides, forming two molecules of hydrochloric acid, whilst the molecule of carbonic oxide entirely enters into the molecule of carbonyl chloride. This characterises the socalled diatomic or bivalent reactions of radicles or residues. $\mathrm{H}$ is a monatomic residue or radicle, like $\mathrm{K}, \mathrm{Cl}$, and others, whilst carbonic oxide, $\mathrm{CO}$, is an indivisible (without decomposition) bivalent radicle, equivalent to $\mathrm{H}_{2}$ and not to $\mathrm{H}$, and therefore combining with $\mathrm{X}_{2}$ and

carbonic oxide gives carbonic anhydride and hydrogen, but hydrogen with oxygen gives hydrogen peroxide (Chapter VII. p. 305), which with carbonic oxide forms carbonic anhydride and water. The water, therefore, is renewed, and again serves the same purpose. But it may be that here it is necessary to acknowledge a simple contact influence.

30 Carbonic oxide is a very quick poison, because it is absorbed by the blood in the same way as oxygen. In addition to this, the spectrum of the absorption of the blood changes so that by the help of blood it is easy to detect the slightest traces of carbonic oxide in the air. M. A. Kapoustin found that linseed oil (and therefore oil paints) are capable of giving off carbonic oxide while drying (absorbing oxygen). 
interchangeable with $\mathrm{H}_{2}$. This distinction is evident from the annexed comparison :

$\begin{array}{ll}\mathrm{HH}, \text { hydrogen. } & \mathrm{CO}, \text { carbonic oxide. } \\ \mathrm{HCl} \text {, hydrochloric acid. } & \mathrm{COCl} \text {, carbonyl chloride. } \\ \mathrm{HKO}, \text { potash. } & \mathrm{CO}(\mathrm{KO})_{2}, \text { potassium carbonate. } \\ \mathrm{HNH}_{2}, \text { ammonia. } & \mathrm{CO}\left(\mathrm{NH}_{2}\right)_{2} \text {, urea. } \\ \mathrm{HCH}_{3}, \text { methane. } & \mathrm{CO}\left(\mathrm{CH}_{3}\right)_{2} \text {, acetone. } \\ \mathrm{HHO}, \text { water. } & \mathrm{CO}(\mathrm{HO})_{2} \text {, carbonic acid. }\end{array}$

Such monatomic (univalent) residues, $\mathrm{X}$, like $\mathrm{H}, \mathrm{Cl}, \mathrm{Na}, \mathrm{NO}_{2}, \mathrm{NH}_{4}$, $\mathrm{CH}_{3}, \mathrm{CO}_{2} \mathrm{H}$ (carboxyl), $\mathrm{OH}$, and others, in accordance with the law of substitution, combine together, forming compounds, $\mathrm{XX}^{\prime}$; with oxygen, or in general with diatomic (bivalent) residues, $\mathrm{Y}$ - for instance, with $\mathrm{O}, \mathrm{CO}, \mathrm{CH}_{2}, \mathrm{~S}, \mathrm{Ca}$, \&c.-compounds $\mathrm{XX}^{\prime} \mathrm{Y}$; but diatomic residues, $\mathrm{Y}$, sometimes capable of existing separately, combine together, $\mathrm{YY}^{\prime}$ and with $\mathrm{X}_{2}$ or $\mathrm{XX}^{\prime}$, as we see from the transition of $\mathrm{CO}$ into $\mathrm{CO}_{2}$ and $\mathrm{COCl}_{2}$. This combining faculty of carbonic oxide appears in many of its reactions. Thus it is very easily absorbed by cuprous chloride, $\mathrm{CuCl}$, dissolved in fuming hydrochloric acid, forning a crystalline compound, $\mathrm{COCu}_{2} \mathrm{Cl}_{2}, 2 \mathrm{H}_{2} \mathrm{O}$, decomposable by water; it combines directly with potassium (at $90^{\circ}$ ), forming $(\mathrm{KCO})_{n}{ }^{31}$ with platinum dichloride, $\mathrm{PtCl}_{2}$, with chlorine, $\mathrm{Cl}_{2}$, \&c.

But the compounds of carbonic oxide with the alkalis are still more remarkable - for instance, with potassium or barium hydroxides, \&c.although it is not directly absorbed by them, as it has no acid properties. Berthelot (1861) showed that potash in the presence of water is capable of absorbing carbonic oxide, but the absorption takes place slowly, little by little, and it is only after being heated for many hours that the whole of the carbonic oxide is absorbed by the potash. The salt, $\mathrm{CHKO}_{2}$, is obtained by this absorption ; it corresponds with an acid found in nature, namely, the simplest organiccarboxylic - acid, formic acid, $\mathrm{CH}_{2} \mathrm{O}_{2}$. It can be extracted from the potassium salt by means of distillation with dilute sulphuric acid, just as nitric acid is prepared from sodium nitrate. The same acid is found in ants, in nettles (when the points of the nettles enter

31 The molecule of metallic potassium (Scott, 1887), like that of mercury, contains one. atom, and it is probably in virtue of this that the molecules $\mathrm{CO}$ and $\mathrm{K}$ combine together. But as in the majority of cases potassium acts as a univalent radicle, the polymeride $\mathrm{K}_{2} \mathrm{C}_{2} \mathrm{O}_{2}$ is formed, and probably $\mathrm{K}_{10} \mathrm{C}_{10} \mathrm{O}_{10}$, because products containing $\mathrm{C}_{10}$ are formed by the action of hydrochloric acid. The black mass formed by the combination of carbonic oxide with potassium explodes with great ease, and oxidises in the air. Although Brodie and Lerch have greatly extended our knowledge of this compound, much still remains unexplained. 
the skin they break, and the corrosive formic acid enters into the body); it is obtained during the action of oxidising agents on many organic substances; it is formed from oxalic acid, and under many conditions splits up into carbonic oxide and water. In the formation of formic acid from carbonic oxide we observe an example of the synthesis of organic compounds, such as are now very numerous, and are treated of in detail in organic chemistry.

Formic acid, $\mathrm{H}\left(\mathrm{CHO}_{2}\right)$, carbonic acid, $\mathrm{HO}\left(\mathrm{CHO}_{2}\right)$, and oxalic acid $\left(\mathrm{CHO}_{2}\right)_{2}$, are the simplè organic or carboxylic acids, $\mathrm{R}\left(\mathrm{CHO}_{2}\right)$ corresponding with $\mathrm{HH}$ and $\mathrm{HOH}$. Proceeding from carbonic oxide, $\mathrm{CO}$, the formation of carboxylic acids is clearly seen from the fact that $\mathrm{CO}$ is capable of combining with $\mathrm{X}_{2}$, that is, forming $\mathrm{COX}_{2}$. If, for instance, one $\mathrm{X}$ is an aqueous residue, $\mathrm{OH}$ (hydroxyl), and the other $\mathrm{X}$ hydrogen, then the simplest organic acid-formic acid, $\mathrm{H}(\mathrm{COOH})$-is obtained. As all hydrocarbons (Chapter VIII.) correspond with the simplest, $\mathrm{CH}_{4}$, so all organic acids may be considered to proceed from formic acid.

In a similar way it is easy to explain the relation to other compounds of carbon of those compounds which contain nitrogen. By way of an example we will examine one class of such nitrogenous compounds. There are ammonium salts, $\mathrm{R}\left(\mathrm{CNH}_{4} \mathrm{O}_{2}\right)$ corresponding with every carboxylic acid, $\mathrm{R}\left(\mathrm{CHO}_{2}\right)$, which salts contain the elements of water; this, with the assistance of substances taking up the water, may be separated with formation of a special class of nitrogenous organic, or, as they are termed, cyanogen compounds, $\mathrm{RCN},{ }^{32}$ because the group or monovalent residue, or radicle, $\mathrm{CN}$, is called cyanogen. From this the connection of the most varied organic compounds is apparent, and it is not only expressed in relation to their composition but by a multitude of reactions. It is most important to turn our attention to the two following circumstances: (1) We have already noticed the various mutual transformations of the simplest organic acids, clearly explained by the existence of carboxyl in their composition. In a similar way, in many reactions, the various cyanogen compounds undergo mutual transformation, especially hydrocyanic acid, $\mathrm{H}(\mathrm{CN})$, corresponding with formic acid, $\mathrm{H}\left(\mathrm{CHO}_{2}\right)$; cyanic acid, $\mathrm{OH}(\mathrm{CN})$, corresponding to carbonic acid, $\mathrm{OH}\left(\mathrm{CHO}_{2}\right)$; and cyanogen, $(\mathrm{CN})_{2}$, corresponding with oxalic acid, $\left(\mathrm{CHO}_{2}\right)_{2}$. The mutual transitions of cyanogen compounds into one another were known much earlier than the doctrine of carboxylic acids, and therefore Gay-Lussac acknowledged cyanogen,

32 The connection of the immense series of cyanogen compounds with the rest of the bydrocarbons by means of carboxyl was enunciated by me, about the year 1860 , at the first Annual Meeting of the Russian Naturalists. 
$\mathrm{CN}$, as the radicle of cyanic acid or hydrocyanic acid, HCN, forming salts, for instance of mercury, which latter on being heated, as Gay-Lussac showed, gives cyanogen itself, $(\mathrm{CN})_{2}$. (2) As the ammonium salt of a carboxylic acid, $\mathrm{R}\left(\mathrm{CNH}_{4} \mathrm{O}_{2}\right)$, contains the elements of two molecules of water, and molecular quantities are those which act in reactions; then, previously to forming a cyanogen compound, or nitrile, by parting with two molecules water, an ammonium salt ought to form an amide by losing one molecule of water : $\mathrm{R}\left(\mathrm{CO}_{2} \mathrm{NH}_{4}\right)-\mathrm{H}_{2} \mathrm{O}=$ $\mathrm{R}\left(\mathrm{CNH}_{2} \mathrm{O}\right)$. Amides are therefore compounds containing the univalent amide residue, $\mathrm{NH}_{2}$, of ammonia, or a compound, $\mathrm{COX}_{2}$, in which one $\mathrm{X}=\mathrm{R}$ and the other $=\mathrm{NH}_{2}$. Such are, for instance, formamide, or the amide of formic acid, $(\mathrm{CO}) \mathrm{H}\left(\mathrm{NH}_{2}\right)$, or $\mathrm{H}\left(\mathrm{CONH}_{2}\right)$. The amides compose a most numerous series of nitrogen compounds, and are obtained in many ways ${ }^{33}$ they are met with in plants and animals, and, as has already been shown, by parting with water they form nitriles or cyanogen compounds, so that they serve as transitional terms between $\mathrm{R}\left(\mathrm{CO}_{2} \mathrm{NH}_{4}\right)$ and $\mathrm{RCN}$. The varieties, reactions, and properties of the amides and nitriles of organic acids, and therefore also of cyanogen compounds, are, as carbon compounds, examined in greater detail in organic chemistry, and here we will only dwell on the simplest of them; and, in order to clearly explain the derived ammonia compounds, we will first dwell on the ammonium salts and amides of carbonic acids.

As carbonic acid is bibasic, its ammonium salts ought to have the following composition : acid carbonate of ammonium, $\mathrm{H}\left(\mathrm{NH}_{4}\right) \mathrm{CO}_{3}$, and the normal carbonate, $\left(\mathrm{NH}_{4}\right)_{2} \mathrm{CO}_{3}$; they represent compounds of one or two molecules of ammonia with carbonic acid. The acid salt appears in the form of a non-odoriferous and (when tested with litmus) neutral substance, soluble at the ordinary temperature in six parts of water, insoluble in alcohol, and obtainable in a crystalline form either without water of crystallisation or with various proportions of it. If an aqueous solution of ammonia be saturated with carbonic anhydride, and then evaporated over sulphuric acid in the bell jar of an air-pump, crystals of this salt are separated. Solutions of all other ammonium carbonates, when evaporated under the air-pump, yield crystals of this salt. A solution of this salt, even at the ordinary temperature, gives off carbonic anhydride, as do all the acid salts of earbonic acid (for

33 Thus, for instance, oxamide, or the amide of oxalic acid, $\left(\mathrm{CNH}_{2} \mathrm{O}\right)_{2}$, is obtained in the form of an insoluble precipitate on adding a solution of ammonia to an alcoholic solution of ethyl oxalate $\left(\mathrm{CO}_{2} \mathrm{C}_{2} \mathrm{H}_{5}\right)_{2}$, which is formed by the action of oxalic acid on alcohol: $\left(\mathrm{CHO}_{2}\right)_{2}+2\left(\mathrm{C}_{2} \mathrm{H}_{5}\right) \mathrm{OH}=2 \mathrm{HOH}+\left(\mathrm{CO}_{2} \mathrm{C}_{2} \mathrm{H}_{5}\right)_{2}$. As the nearest derivatives of ammonia, the amides with alkalis yield ammonia and form the salt of the acid. The nitriles do not, however, give similar reactions so readily. 
instance, $\mathrm{NaHCO}_{3}$ ), and at $38^{\circ}$ the separation of carbonic anhydride takes place with great rapidity. For this reason the formation of the acid salt in the solution, and the evaporation, must be conducted at a low temperature, and with an excess of carbonic acid, as the product of dissociation of this salt. On losing carbonic anhydride and water, the acid salt is converted into the normal salt, $2\left(\mathrm{NH}_{4}\right) \mathrm{HCO}_{3}=$ $\mathrm{H}_{2} \mathrm{O}+\mathrm{CO}_{2}+\left(\mathrm{NH}_{4}\right)_{2} \mathrm{CO}_{3}$; the latter, however, decomposes in solution, and therefore can only be obtained in crystals, $\left(\mathrm{NH}_{4}\right)_{2} \mathrm{CO}_{3}, \mathrm{H}_{2} \mathrm{O}$, at low temperatures, and from solutions containing an excess of ammonia as the product of dissociation of this salt: $\left(\mathrm{NH}_{4}\right)_{2} \mathrm{CO}_{3}=$ $\mathrm{NH}_{3}+\left(\mathrm{NH}_{4}\right) \mathrm{HCO}_{3}$. But the normal salt, ${ }^{34}$ according to the general type, is capable of decomposing with separation of water, and forming ammonium carbamate, $\mathrm{NH}_{4} \mathrm{O}\left(\mathrm{CONH}_{2}\right)=\left(\mathrm{NH}_{4}\right)_{2} \mathrm{CO}_{3}-\mathrm{H}_{2} \mathrm{O}$; this still further complicates the history of the carbonates of ammonium. It is evident that, in reality, on changing the quantity of water, ammonia, and carbonic acid, various intermediate salts will be formed containing mixtures or combinations of those mentioned above, or, in other words, various states of equilibrium of reactions reverse to one another will be produced. Thus the ordinary commercial carbonate of ammonia, obtained by distillation of a mixture of chalk and sulphate of ammonia (Chapter VI.), or sal-ammoniac, $2 \mathrm{NH}_{4} \mathrm{Cl}+\mathrm{CaCO}_{3}=$ $\mathrm{CaCl}_{2}+\left(\mathrm{NH}_{4}\right)_{2} \mathrm{CO}_{3}$, does not remain as a normal salt, but, through loss of part of the ammonia, partly contains the acid salt, and, through loss of water, cortains some carbamate, and most frequently presents the composition $\mathrm{NH}_{4} \mathrm{O}\left(\mathrm{CONH}_{2}\right)+2 \mathrm{OH}\left(\mathrm{CO}_{2} \mathrm{NH}_{4}\right)=4 \mathrm{NH}_{3}+3 \mathrm{CO}_{2}+2 \mathrm{H}_{2} \mathrm{O}$. Indeed, this salt, from having under various conditions parted with ammonia, carbonic anhydride, and water, does not present a constant composition, and ought rather to be regarded as a mixture of acid salt and amide salt. The latter must be recognised as entering into the composition of the ordinary carbonate of ammonia, because it contains less water than is required for the normal or acid salt $;^{35}$ but on being dissolved in water this salt gives a mixture of acid and normal salts. Ammonium carbamate itself, whose composition (and formation) is equal to $2 \mathrm{NH}_{3}+\mathrm{CO}_{2}$, when dissolved in water, does not entirely precipitate a calcium salt, for instance $\mathrm{CaCl}_{2}$, which produces a normal salt, $\left(\mathrm{NH}_{4}\right)_{2} \mathrm{CO}_{3}+\mathrm{CaCl}_{2}=2 \mathrm{NH}_{4} \mathrm{Cl}+\mathrm{CaCO}_{3}$, probably because a calcium carbamate is produced which is soluble in water.

5t The acid salt $\left(\mathrm{NH}_{4}\right) \mathrm{HCO}_{3}$ on losing water ought to form the carboxylic acid, $\left(\mathrm{NH}_{2}\right) \mathrm{HCO}_{2}$, or, better, $\mathrm{OH}\left(\mathrm{CNH}_{2} \mathrm{O}\right)$, as we wrote it before ; but it is not formed, which is accounted for by the instability of the acid salt itself. Carbonic anlyydride is given off and ammonia is produced, which gives a carboxylic ammonium salt.

33 In the normal salt, $2 \mathrm{NH}_{3}+\mathrm{CO}_{2}+\mathrm{H}_{2} \mathrm{O}$, in the acid salt, $\mathrm{NH}_{3}+\mathrm{CO}_{2}+\mathrm{H}_{2} \mathrm{O}$, but in the commercial salt to $3 \mathrm{CO}_{2}$ only $2 \mathrm{H}_{2} \mathrm{O}$ 
This ammonium carbamate is one of the most remarkable of the ammonium carbonates, because it is the simplest in its composition, and is easily and immediately formed by mixing two volumes of $d r y$ ammonia with one volume of dry carbonic anhydride, $2 \mathrm{NH}_{3}+\mathrm{CO}_{2}=$ $\mathrm{NH}_{4} \mathrm{O}\left(\mathrm{CONH}_{2}\right)$; it is a solid substance, smells strongly of ammonia, attracts moisture from the air, and entirely decomposes at $60^{\circ}$. The fact of this decomposition may be judged ${ }^{36}$ by the density of its vapour, which $=13(\mathrm{H}=1)$; this exactly corresponds with the density of a mixture of 2 volumes of ammonia and 1 volume of carbonic anhydride. It is easily understood that such a combination will take place with any ammonium carbonate under the action of salts which take up the water - for instance, sodium or potassium carbonate. ${ }^{37}$ As ammonia and carbonic anhydride in an anhydrous state only form one compound, $\mathrm{CO}_{2} 2 \mathrm{NH}_{3},{ }^{38}$ ammonium carbamate, as has been already stated, may be regarded as $\mathrm{COX}_{2}$, where $\mathrm{X}$ has been replaced by the residues $\mathrm{NH}_{2}$ and $\mathrm{NH}_{4} \mathrm{O}$ (that is, $\mathrm{HO}$ in which $\mathrm{H}$ is replaced by $\mathrm{NH}_{1}$ ), and therefore is still capable of losing water and forming the symmetrical amide $\mathrm{CO}\left(\mathrm{NH}_{2}\right)_{2}$. This must be termed carbamide. It is identical with urea, $\mathrm{CN}_{2} \mathrm{H}_{4} \mathrm{O}$, which, contained in the urine (about 2 per cent. in human urine), is for the higher animals (especially the carnivorous) the ordinary product of excretion ${ }^{39}$ of the nitrogenous substances found in the organism. If ammonium carbamate be heated to $140^{\circ}$ (in a sealed tube, Bazarnff), or if carbonyl chloride, $\mathrm{COCl}_{2}$, be treated with ammonia (Natanson), urea will be obtained, which

36 Naumann determined the following densities of the vapour of ammonium carbamate (in millimetres of mercury) :-

$\begin{array}{rrrrrrrr}-10^{\circ} & 0^{\circ} & +10^{\circ} & 20^{\circ} & 30^{\circ} & 40^{\circ} & 50^{\circ} & 60^{\circ} \\ 5 & 12 & 30 & 62 & 124 & 248 & 470 & 770\end{array}$

Horstmann and Isambert studied the densities corresponding with excess of $\mathrm{NH}_{3}$ or $\mathrm{CO}_{2}$, and found, as might have been expected, that with such excess the mass of the salt formed (in a solid state) increases and the decomposition (transition into vapour) decreases.

37 Calcium chloride enters into double decomposition with ammonium carbamate. Acids (for instance sulphuric) take up ammonia, and set free carbonic anhydride; alkalis (for instance, potash) take up carbonic anhydride and set free ammonia, and therefore, for removing water here only sodium or potassium carbonate may be taken.

38 It must be imagined that the reaction takes place at first between equal volumes (Chapter VII.); but then carbamic acid, $\mathrm{HO}\left(\mathrm{CNH}_{2} \mathrm{O}\right)$, is produced, which, as an acid, immediately combines with the ammonia, forming $\mathrm{NH}_{4} \mathrm{O}\left(\mathrm{CNH}_{2} \mathrm{O}\right)$.

39 Urea is undoubtedly a product of the oxidation of complex nitrogenous matters (albumin) of the animal body. It is found in the blood. It is absorbed from the blood by the kidneys. A man excretes about 30 grams of urea per day. As a derivative of carbonic anhydride, into which it is readily converted, urea is in a sense a product of oxidation. 
shows its direct connection with carbonic acid - that is, the presence of carbonic acid and ammonia in it. From this it will be understood how urea during the putrefaction of urine is converted into ammonium carbonate, $\mathrm{CN}_{2} \mathrm{H}_{4} \mathrm{O}+\mathrm{H}_{2} \mathrm{O}=\mathrm{CO}_{2}+2 \mathrm{NH}_{3}$.

Thus urea, both by its origin and reactions, is an amide of carbonic acid. Representing as it does ammonia (two molecules) in which the hydrogen (two atoms) is replaced by the bivalent radicle of earbonic acid, urea retains the property of ammonia of entering into combination, with acids (for instance, with nitric acid, $\mathrm{CN}_{2} \mathrm{H}_{4} \mathrm{O}, \mathrm{HNO}_{3}$ ), with bases (for instance, with mercury oxide), and with salts (for instance, sodium chloride, ammonium chloride), but it has no alkaline properties. It is soluble in water without change, but at a red heat loses ammonia and forms cyanic acid, CNHO, and its polymeride, cyanuric acid, $\mathrm{C}_{3} \mathrm{~N}_{3} \mathrm{H}_{3} \mathrm{O}_{3}$. The first is a nitrile of carbonic acid-that is to say, is a cyanogen compound. This conformity is evident from the fact that the acid ammonium carbonate, $\mathrm{OH}\left(\mathrm{CNH}_{4} \mathrm{O}_{2}\right)$, on parting with $2 \mathrm{H}_{2} \mathrm{O}$, ought to form cyanic acid, $\mathrm{CNOH}$. There is a relation of direct polymerisation between cyanuric acid (a solid, crystalline, and very stable substance) and cyanic acid (a liquid, very unstable substance, easily changeable in several ways). Both have the same composition, and they pass one into another at different temperatures. If crystals of cyanuric acid be heated to a temperature, $t^{\circ}$, then the vapour tension, $p$, in millimetres of mercury (Troost and Hautefeuille) will be :

$$
\begin{aligned}
& \text { t. } 160^{\circ}, 170^{\circ}, 200^{\circ}, 250^{\circ}, 300^{\circ}, 350^{\circ} \text {. } \\
& \text { p. 56, 68, 130, 220, 430, } 1200 .
\end{aligned}
$$

The vapour contains cyanic acid, and, if it be rapidly cooled, it condenses into a mobile volatile liquid (specific gravity at $0^{\circ}=1 \cdot 14$ ). If the liquid cyanic acid be gradually heated, it passes into a new amorphous polymeride (cyamelide), which, on being heated, like cyanuric acid, forms vapours of cyanic acid. If these fumes are heated above $150^{\circ}$, they directly pass into cyanuric acid. Thus, at a temperature of $350^{\circ}$, the pressure does not rise above $1200 \mathrm{~mm}$. on the addition of vapours of cyanic acid, because the whole excess is transformed into cyanuric acid. Therefore, the above-mentioned figures give the tension of dissociation of cyanuric acid, or the greatest pressure which the vapours of $\mathrm{HOCN}$ are able to attain at a given temperature, whilst at a greater pressure, or by the introduction of a large mass of matter into the given volume, the whole of the excess is converted into cyanuric acid. The properties of cyanic acid which we have described were principally observed by Wöhler, and clearly show the faculty of polymerism of cyanogen compounds. This is observed in many other 
cyanogen derivatives, and is regarded as the consequence of the abovementioned explanation of their nature. All cyanogen compounds are ammonium salts, $\mathrm{R}\left(\mathrm{CNH}_{4} \mathrm{O}_{2}\right)$, deprived of water, $2 \mathrm{H}_{2} \mathrm{O}$; therefore their molecules ought to show the faculty of combining with two molecules of water or with other molecules in exchange for it (for instance, with $\mathrm{H}_{2} \mathrm{~S}$, or $\mathrm{HCl}$, or $2 \mathrm{H}_{2}$, drc.), and are therefore capable of combining together. The combination of molecules of the same kind to form more complex ones is nothing else but polymerisation. ${ }^{40}$

Besides being a substance very apt to form polymerides, cyanic acid presents many other features of interest, expounded in greater detail in organic chemistry. First of all, the transition of ammonium cyanate into urea ought to be considered. The reason is that the salts of cyanic acid, which are easily split up, by the action of acids and even of water, into ammonia and carbonic anhydride, are produced by the oxidation of saline metals, as we shall see further on. Potassium cyanate, $\mathrm{KCNO}$, for instance, is most often obtained in this way. Solutions of cyanates, by the addition of sulphuric acid, yield cyanic acid, which, however, immediately decomposes : $\mathrm{CNHO}+\mathrm{H}_{2} \mathrm{O}$ $=\mathrm{CO}_{2}+\mathrm{NH}_{3}$. A solution of ammonium cyanate, $\mathrm{CN}\left(\mathrm{NH}_{4}\right) \mathrm{O}$, behaves in the same manner, but only in the cold. On being heated it completely changes, because it is transformed into urea. The composition of both substances is identical, $\mathrm{CN}_{2} \mathrm{H}_{4} \mathrm{O}$, but the structure or disposition and the connection between the elements is different: in the ammonium cyanate one atom of nitrogen exists in the form of cyanogen, $\mathrm{CN}$ - that is, united with carbon-and the other as ammonium, $\mathrm{NH}_{4}$, but, as cyanic acid contains the hydroxyl radicle of carbonic acid, $\mathrm{OH}(\mathrm{CN})$, the ammonium in this salt is united with oxygen; whilst in urea both the nitrogen atoms are symmetrically and uniformly disposed as regards the radicle $\mathrm{CO}$ of carbonic acid: $\mathrm{CO}\left(\mathrm{NH}_{2}\right)_{2}$. For this reason, urea is much more stable than ammonium cyanate, and therefore the latter, on being slightly heated in solution, is converted into

40 Just as the aldehydes (for instance, $\mathrm{C}_{2} \mathrm{H}_{4} \mathrm{O}$, page 145) are alcohols (for instance, $\mathrm{C}_{2} \mathrm{H}_{6} \mathrm{O}$ ) which have lost hydrogen.

The aldehydes are also capable of entering into combination with many substances, and of polymerising, forming slightly-volatile polymerides, which depolymerise on heating. Although there are also many similar phenomena (for instance, the transformation of yellow into red phosphorus, the transition of cinnamene into metacinnamene, \&c.) of polymerisation, in no other case are they so clearly and simply expressed as in cyanic acid. The details relating to this must be sought for in treatises on organic and theoretical chemistry. If we touch on certain sides of this question it is principally with the view of showing the phenomenon of polymerisation by typical examples (this phenomenon being met with more frequently than was formerly imagined, see Silica), and also to show the faculty which cyanogen derivatives have of forming the most varied compounds. 
urea. This remarkable isomeric transformation was discovered by Wöhler in 1828. It had an important historical signification, because at that time such an easy formation outside the organism of those substances which are met with in it was quite unexpected, it being at that time supposed that the vital force of the organism produced substances which could not be formed outside it. But in addition to the abolition of this presupposition, the easy transition of $\mathrm{NH}_{4} \mathrm{OCN}$ into $\mathrm{CO}\left(\mathrm{NH}_{2}\right)_{2}$ is the best example of the transition of one state of atomic equilibrium into another more stable one. Every carboxyl acid, $\mathrm{RCOOH}$, has its corresponding amide, $\mathrm{RCONH}_{2},{ }^{41}$ and nitrile, as is the case with carbonic acid. In this way, formamide, $\mathrm{HCONH}_{2}$, and hydrocyanic acid, $\mathrm{HCN}$, as a nitrile, correspond with formic acid, $\mathrm{HCOOH}$, and therefore ammonium formate, $\mathrm{HCOONH}_{4}$, and formamide, under the action of heat and substances which take up water (phosphoric anhydride), form hydrocyanic acid, HCN, whilst, under many conditions (for instance, on combining with hydrochloric acid in presence of water), this hydrocyanic acid forms formic acid and ammonia. Although containing hydrogen in the presence of two acid-forming elements-namely, carbon and nitrogen ${ }^{42}$ -hydrocyanic acid does not give an acid reaction with litmus (cyanic acid has very marked acid properties), but forms salts, $M C N$, and therefore presents the properties of a feeble acid, and for this reason is called an acid. The small amount of energy which it has is shown by the fact that the cyanides of the alkali metals - for instance, potassium cyanide $\left(\mathrm{KHO}+\mathrm{HCN}=\mathrm{H}_{2} \mathrm{O}+\mathrm{KCN}\right)$ in solution - have a strongly

41 The majority of the amides corresponding with acids, being represented by the composition $\mathrm{RNH}_{2}$, very easily undergo the inverse change; they combine with water, even when simply boiled with it, and still more so under the action of alkalis and acids. Ammonia is evolved by the action of alkalis on amides, naturally in virtue of the water combining with the amide, in addition to which there is formed a salt of the acid, which had served to form the amide according to the equation: $\mathrm{RNH}_{2}+\mathrm{KHO}=\mathrm{RKO}+\mathrm{NH}_{3}$. The same thing takes place under the action of acids, only, naturally, an ammonium salt of the given acid is formed, and the acid, previously in the condition of an amide, separates in the free state: $\mathrm{RNH}_{2}+\mathrm{HCl}+\mathrm{H}_{2} \mathrm{O}=\mathrm{RHO}+\mathrm{NH}_{4} \mathrm{Cl}$. Thus the amides, in the majority of cases, easily pass back into ammonium salts in the presence of water, alkalis, and acids; but they differ in a marked way from them. None of the ammonium salts are distillable, nor volatilise without change; in most cases ammonia salts, on being heated, lose water and form amides ; many amides volatilise without change, and sometimes are crystalline volatile substances easily distilled. Such are, for instance, the amides of acetic, benzoic, formic, and a large number of other organic acids. Judging from the preceding facts, amides may be regarded as acids RHO, in which the hydroxyl HO is replaced by the ammonia radicle (amidogen) $\mathrm{NH}_{2}$.

42 If ammonia and methane (marsh gas) do not show any acid properties, that is in all probability due to the presence of a large amount of hydrogen in both; but in hydrocyanic acid one atom of hydrogen is under the influence of two acid-forming elements. Acetylene, $\mathrm{C}_{2} \mathrm{H}_{2}$, which contains but little hydrogen, presents acid properties in certain respects, because its hydrogen is easily replaced by metals. 
alkaline reaction. ${ }^{43}$ If ammonia be passed over charcoal at a red heat, especially in the presence of an alkali, or if gaseous nitrogen be passed through a mixture of charcoal and an alkali (especially potash, KHO), and also if a mixture of nitrogenous organic substances and alkali be heated to a red heat, in all these cases the alkali metal combines with the carbon and nitrogen, forming a metallic cyanide, MCN - for example, KCN. Potassium cyanide is much used in the arts, and is obtained, as above stated, under many circumstances-as, for instance, in iron smelting, especially with the assistance of wood charcoal, the ash of which contains much potash. The nitrogen of the air, the alkali of the ash, and the charcoal are brought into contact at a high temperature during iron smelting, and therefore, under these conditions, a considerable quantity of potassium cyanide is formed. In practice it is not usual to prepare potassium cyanide directly, but a peculiar compound of it containing potassium, iron, and cyanogen. This compound is potassium ferrocyanide, and is also known as yellow prussiate of potash, because with ferric salts it forms a blue colour-Prussian blue. This saline substance, having the composition $\mathrm{K}_{4} \mathrm{FeC}_{6} \mathrm{~N}_{6}+3 \mathrm{H}_{2} \mathrm{O}$, is prepared on the large scale in cast-iron pots (the iron of which enters into the salt), by heating with alkali leather cuttings, horn filings and shavings, and similar animal matter, composed of complex organic substances containing carbon and nitrogen; the alkali which is added is composed of potashes containing potassium ; thus all the necessary elements for producing the yellow prussiate are brought into contact at a high temperature, and the above-mentioned substance is formed. The name of cyanogen (кúavos) is derived from the property which the yellow prussiate possesses of forming, with a solution of a ferric salt, $\mathrm{FeX}_{3}$, the familiar pigment Prussian blue. The yellow prussiate is generally used as the source of the other cyanogen compounds (because it is manufactured on a large scale), and we will therefore describe the method of forming the cyanogen compounds derived from it.

If yellow prussiate be mixed with two parts of water and three-

4 Solutions of cyanides-for instance, of those of potassium or barium-are decomposed by carbonic acid. Even the carbonic anhydride of the air acts in a sinilar way, and for this reason these solutions do not keep, because, in the first place, free hydrocyanic acid itself decomposes and polymerises, and, in the second place, with alkaline liquids it forms ammonia and formic acid. Hydrocyanic acid does not liberate carbonic anhydride from solutions of sodium or potassium carbonates. But a mixture of solutions of potassium carbonate and hydrocyanic acid yields carbonic anliydride on the addition of oxides similar to zinc oxide, mercuric oxide, \&c. This is due to the great inclination which the cyanides evince to form double salts. For instance, $\mathrm{ZnK}_{2}(\mathrm{CN})_{4}$ is formed, which is a kind of soluble double salt. 
quarter part of sulphuric acid, and the mixture be heated, it decomposes, volatile hydrocyanic acid separating. This was obtained for the first time by Scheele in 1782 , but it was only known to him in solution. In 1809 Ittner prepared anhydrous prussic acid, and in 1815 Gay-Lussac finally settled its properties and showed that it contains only hydrogen, carbon, and nitrogen, $\mathrm{CNH}$. If the distillate be redistilled (a weak solution of $\mathrm{HCN}$ ), and the first part be collected, the anhydrous acid may be prepared from this stronger solution. In order to do this, pieces of calcium chloride are added to the concentrated solution, and the anhydrous acid floats as a separate layer, because it is not soluble in an aqueous solution of calcium chloride. If this layer be then distilled over a new portion of calcium chloride at the lowest temperature possible, the prussic acid may be obtained completely free from water. It is, however, necessary to use the greatest caution in work of this kind, because prussic acid, besides being extremely poisonous, is exceedingly volatile. ${ }^{44}$

Anhydrous prussic acid is a very mobile and volatile liquid ; its. specific gravity is 0.697 at $18^{\circ}$; at lower temperatures, especially when mixed with a small quantity of water, it easily congeals; it boils at $26^{\circ}$, and therefore very easily evaporates, and at ordinary temperatures may be regarded as a gas. On this account the greatest caution is necessary in dealing with it, as an insignificant amount, when inhaled or brought into contact with the skin, causes death. It is soluble in all proportions in water, alcohol, and ether; weak aqueous solutions. are used in medicine. ${ }^{45}$

44 The mixture of the vapours of water and hydrocyanic acid, evolved on heating yellow prussiate with sulphuric acid, may be directly passed through vessels or tubes filled with calcium chloride. These tubes must be cooled, because, in the first place, hydrocyanic acid easily changes on being heated, and, in the second place, the calcium chloride, when warm, would absorb less water. The mixture of hydrocyanic acid and aqueous vapour on passing over a long layer of calcium chloride gives up water, and hydrocyanic acid alone remains in the vapour. It ought to be cooled as carefully as possible in order to bring it into a liquid condition. The method which Gay-Lussac employed for obtaining pure hydrocyanic acid consisted in the action of hydrochloric acid gas on mercuric cyanide. The latter may be obtained in a pure state if a solution of yellow prussiate be boiled with a solution of mercuric nitrate, then filtered and crystallised by cooling; the mercuric cyanide is then obtained in the form of colourless crystals $\mathrm{Hg}(\mathrm{CN})_{2}$.

If a strong solution of hydrochloric acid be poured upon these crystals, and the mixture of vapours evolved, consisting of aqueous vapour, hydrochloric acid, and hydrocyanic acid, be passed through a tube containing, first marble (for absorbing the hydrochloric acid), and then lumps of calcium chloride, on cooling, the hydrocyanic acid will be condensed. In order to obtain the hydrocyanic acid in an anhydrous form, the decomposition of heated mercury cyanide by hydrogen sulphide may be made use of. Here the sulphur and cyanogen change places, and hydrocyanic acid and mercury sulphide are formed: $\mathrm{Hg}(\mathrm{CN})_{2}+\mathrm{H}_{2} \mathrm{~S}=2 \mathrm{HCN}+\mathrm{HgS}$.

45 A weak (up to 2 p.c.) aqueous solution of hydrocyanic acid is obtained by the dis- 
The salts MCN - for instance, potassium, sodium, ammonium-as well as the salts $\mathrm{M}(\mathrm{CN})_{2}$ - for instance, barium, calcium, mercury - are soluble in water, but the salts of manganese, zinc, lead, and many others are insoluble in water. They form clouble salts with potassium cyanide and similar metallic cyanides, an example of which we will consider in a further description of the yellow prussiate. Not only are some of the double salts remarkable for their constancy and comparative stability, but so also are the soluble salt $\mathrm{HgC}_{2} \mathrm{~N}_{2}$, the insoluble silver cyanide $\mathrm{AgCN}$, and even potassium cyanide in the absence of water. This salt, ${ }^{46}$ when fused, acts as a reducing agent with its elements, $\mathrm{K}$ and $\mathrm{C}$, and oxidises when fused with lead oxide, forming potassium cyanate, KOCN, which establishes the connection between $\mathrm{HCN}$ and $\mathrm{OHCN}$ - that is, between the nitriles of formic and carbonic acidswhich connection is the same as between the acids themselves, because formic acid, on oxidation, yields carbonic acid. The relation which exists between hydrocyanic acid, metallic cyanides, and the salts of cyanic acid, as well as between all the above-mentioned compounds, may be expressed by granting the existence of a radicle in them containing carbon and nitrogen-cyanogen, CN. In this sense, prussic acid is hydrocyanic acid, and cyanic acid a cyanogen hydroxide, in exactly the same way as for chlorine we have hydrochloric acid, $\mathrm{HCl}$, and hypochlorous acid, $\mathrm{ClOH}$. Free cyanogen ought to be represented as prussic acid in which the hydrogen has been replaced by cyanogen, $(\mathrm{CN})_{2}$ or CNCN. This composition, judging from what has been

tillation of certain vegetable substances. The so-called laurel water in particular enjoys considerable notoriety from its containing hydrocyanic acid. It is obtained by the steeping and distillation of laurel leaves. A similar kind of water is formed by the infusion and distillation of bitter almonds. It is well known that bitter almonds are poisonous, and have a peculiar characteristic taste. This bitter taste is due to the presence of a certain substance called amygdalin, which can be extracted by alcohol. This amygdalin decomposes in an infusion of bruised almonds, forming the so-called bitter almond oil, glucose, and hydrocyanic acid :

\begin{tabular}{|c|c|c|c|c|c|c|}
\hline $\begin{array}{c}\mathrm{C}_{10} \mathrm{H}_{27} \mathrm{NO}_{11} \\
\text { Amygdalin in } \\
\text { bitter almonds. }\end{array}$ & + & $\begin{array}{c}\mathrm{H}_{2} \mathrm{O} \\
\text { Water. }\end{array}$ & $=$ & $\begin{array}{l}\mathrm{C}_{7} \mathrm{H}_{6} \mathrm{O} \\
\text { Bitter } \\
\text { almond oil. }\end{array}$ & + & $\begin{array}{c}\text { CNH } \\
\text { Hydrocyanic } \\
\text { acid. }\end{array}$ \\
\hline
\end{tabular}

If after this the infusion of bitter almonds be distilled with water, the hydrocyanic acid and the volatile bitter almond oil are carried over with the aqueous vapour. The latter is insoluble in water, or only sparingly soluble, while the hydrocyanic acid remains as an aqueous solution. Bitter almond water is similar to laurel water, and is used, like the former, in medicine, naturally only in small quantities, because a considerable amount has poisonous effects. Perfectly pure anhydrous hydrocyanic acid keeps without change, just like the weak solutions, but the strong solutions only keep in the presence of other acids; but in the presence of many admixtures these solutions easily give a brown polymeric substance, which is also formed from a solution of potassium cyanide.

46 This salt will be described under potassium, as it is one of those which are often used. 
already stated, exactly expresses that of the nitrile of oxalic acid, and, as a matter of fact, oxalate of ammonia and the amide corresponding with it (oxamide), on being heated with phosphoric anhydride, which takes up the water, yield cyanogen. This substance is also produced by simply heating some of the metallic cyanides. Mercuric cyanide is particularly adapted for this purpose, because it is easily obtained in a pure state and is very stable in a separate form. If mercuric cyanide be heated, it decomposes, in like manner to mercury oxide, into metallic mercury and cyanogen: $\mathrm{HgC}_{2} \mathrm{~N}_{2}=\mathrm{Hg}+\mathrm{C}_{2} \mathrm{~N}_{2} \cdot{ }^{47}$ When cyanogen is formed, part of it always polymerises into a dark brown insoluble substance called paracyanogen, capable of forming cyanogen when heated to redness. ${ }^{48}$ Cyanogen is an odorous, colourless, poisonous gas, easily condensed by cooling into a colourless liquid, insoluble in water and having a specific gravity of 0.86 . It boils at about $-21^{\circ}$, and therefore cyanogen may be easily condensed into a liquid by a strong freezing mixture. At $-35^{\circ}$ liquid cyanogen solidifies. This gas is soluble in water and in alcohol to a considerable extent-namely, 1 volume of water absorbs as much as $4 \frac{1}{2}$ volumes, and alcohol 23 volumes. Cyanogen resists the action of heat without decomposing, but, under the action of the electric spark, the carbon is separated, leaving a

47 For the preparation it is necessary to take completely dry mercuric cyanide, because when heated in the presence of moisture it gives ammonia, carbonic anhydride, and hydrocyanic acid. Instead of mercuric cyanide, a mixture of perfectly dry yellow prussiate and mercuric chloride may be used, then double decomposition and the formation of mercuric cyanide take place in the retort. Silver cyanide also disengages cyanogen on being heated.

48 Paracyanogen is a brown substance having the composition of cyanogen, formed during the preparation of cyanogen by all metlods, and remaining as a residue. Silver cyanide, on being slightly heated, fuses, and on being further heated evolves a gas; in the residne a considerable quantity of paracyanogen is formed. Here it is remarkable that exactly half the cyanogen becomes gaseous, and the other half is transformed into paracyanogen. Metallic silver will be found in the residue with the paracyanogen; it may be extracted with mercury or nitric acid, which does not act on paracyanogen. If paracyanogen be lieated in a vacuum, it decomposes, forming cyanogen; but here the pressure $p$ for a given temperature $t$ exceeds the determined magnitude, so that the phenomenon presents all the external appearance of a plysical transformation into vapour; but, nevertheless, it is a complete change in the nature of the substance, but limited by the pressure of dissociation, as we saw before in the transformation of cyanuric acid into hydrocyanic, and as ought to be expected from the fundamental principles of dissociation. Troost and Hautefeuille (1868) found that for paracyanogen

$$
\begin{array}{lllc}
t=530^{\circ} & 581^{\circ} & 600^{\circ} & 633^{\circ} \\
p=90 & 143 & 296 & 1089 \mathrm{~mm} .
\end{array}
$$

However, already at $550^{\circ}$ part of the cyanogen decomposes into carbon and nitrogen. The reverse transition of cyanogen into paracyanogen commences at $350^{\circ}$, and at $600^{\circ}$ proceeds rapidly. And if the transition of the first kind is likened to evaporation, then the reverse transition, or polymerisation, presents a likeness to the transition of vapours into the solid state. 
volume of nitrogen equal to the volume of the gas taken. As it contains carbon it burns, and the colour of the flame is reddish-violet, which is due to the presence of nitrogen, all compounds of which impart more or less of this reddish-violet hue to the flame. During the combustion of cyanogen, carbonic anhydride and nitrogen are formed. This also takes place in the eudiometer with oxygen and during the action of cyanogen on many oxides at a red heat.

The relation of cyanogen to the metallic cyanides is seen not only from the fact that it is formed from mercuric cyanide, but also by its forming cyanide of sodium or potassium on being heated with sodium or potassium, the sodium or potassium taking fire in the cyanogen. On heating a mixture of hydrogen and cyanogen to $500^{\circ}$ (Berthelot), ${ }^{49}$ or under the action of the silent discharge (Boilleau), hydrocyanic acid is formed, so that the reciprocity of the transitions does not leave any doubt in the matter that all the nitriles of the organic acids contain cyanogey-m as all the organic acids contain carboxyl, and in it the elements arbonic anhydride. Besides the amides, ${ }^{50}$ the nitriles (or cyanogen compounds, RCN), and nitro-compounds (containing the radicle of nitric acid, $\mathrm{RNO}_{2}$ ), there are a great number of other substances containing, at the same time, carbon and nitrogen, particulars of which must be sought for in special works on organic chemistry.

49 Cyanogen (like chlorine) is absorbed by a solution of sodium hydroxide, sodium cyanide and cyanate being produced: $\mathrm{C}_{2} \mathrm{~N}_{2}+2 \mathrm{NaHO}=\mathrm{NaCN}+\mathrm{CNNaO}+\mathrm{H}_{2} \mathrm{O}$. However, the latter salt easily decomposes, just as also part of the cyanogen liberated by heat from its compounds is subjected to a more complex transformation.

50 If, in general, compounds containing the radicle $\mathrm{NH}_{2}$ are called amides, some of the amines ought to be ranked with them; that is, the hydrocarbons, $\mathrm{C}_{n} \mathrm{H}_{2 m}$, in which the hydrogen is replaced by $\mathrm{NH}_{2}$; for instance, methylamine, $\mathrm{CH}_{3} \cdot \mathrm{NH}_{2}$, aniline, $\mathrm{C}_{6} \mathrm{H}_{5} \cdot \mathrm{NH}_{2}$, \&c. In general the amines represent ammonia in which part or all of the hydrogen is replaced by hydrocarbon radicles-such as, for instance, trimethylamine $\mathrm{N}\left(\mathrm{CH}_{3}\right)_{3}$. They, like ammonia, combine with acids and form crystalline salts. Related substances are sometimes met with in nature, and bear the general name of alkaloids; such are, for instance, quinine in cinchona bark nicotine in tobacco, \&c. 


\section{CHAPTER X}

SODIUM CHLORIDE-BERTHOLLET'S LAWS-HYDROCHLORIC ACID

Is the preceding chapters we have become acquainted with the most important properties of the four elements, hydrogen, oxygen, nitrogen, and carbon. They are sometimes termed the organogens, because they enter into the composition of organic substances. Their mutual combinations may serve as types for all other chemical compounds - that is, they present the same atomic relations (types, forms, or grades of combinations) as those in which the other elements also combine together.

$\begin{array}{llll}\text { Hydrogen, } \mathrm{HH} \text {, or, in general, } \mathrm{HR} \text {. } \\ \text { Water, } \mathrm{H}_{2} \mathrm{O}, \quad " & , & \mathrm{H}_{2} \mathrm{R} \text {. } \\ \text { Ammonia, } \mathrm{H}_{3} \mathrm{~N}, \quad " & , & \mathrm{H}_{3} \mathrm{R} \text {. } \\ \text { Marsh gas, } \mathrm{H}_{4} \mathrm{C}, \quad " & , & \mathrm{H}_{4} \mathrm{R} \text {. }\end{array}$

One, two, three, and four atoms of hydrogen enter into these molecules for one atom of another element. No compounds of one atom of oxygen with three or four atoms of hydrogen are known; hence the atom of oxygen is without certain properties which are found in the atoms of carbon and nitrogen.

The faculty of an element to form a compound of definite composition with hydrogen (or an element analogous to it) gives the possibility of foretelling the composition of many other of its compounds. Thus, if we know that an element, M, combines with hydrogen, forming HM, and not forming $\mathrm{H}_{2} \mathrm{M}, \mathrm{H}_{3} \mathrm{M}, \mathrm{H}_{\mathrm{n}} \mathrm{M}_{\mathrm{m}}$, then we must conclude, on the basis of the law of substitution, that this element will give compounds $\mathrm{M}_{2} \mathrm{O}, \mathrm{M}_{3} \mathrm{~N}, \mathrm{MHO}, \mathrm{MH}_{3} \mathrm{C}$, drc. Chlorine is an example of this kind. If we know that another element, $\mathrm{R}$, like oxygen, gives with hydrogen a molecule, $\mathrm{H}_{2} \mathrm{R}$, then we may expect that it will form compounds like hydrogen peroxide, or the metallic oxides, or carbonic anhydride, or carbonic oxide, and others. Sulphur is an instance of this kind. Hence the elements may be classified according to their resemblance to hydrogen, oxygen, nitrogen, and carbon, and in conformity with - this analogy it is possible to foretell, if not the properties (for example, 


\section{the acidity or basicity), at all events, the composition ${ }^{1}$ of their com-} pounds. This forms the substance of the conception of the valency or

1 But it is impossible to foretell all the compounds formed by an element from its atomicity or valency, because the atomicity of the elements is variable, and furthermore this variability is not identical for different elements. In $\mathrm{CO}_{2}, \mathrm{COX}_{2}, \mathrm{CH}_{4}$, and the multitude of carbon compounds corresponding with them, the $\mathbf{C}$ is quadrivalent, but in $\mathrm{CO}$ either the carbon must be taken as bivalent or the atomicity of oxygen be accounted as variable. And carbon, nevertheless, is an example of an element which preserves its atomicity to a greater degree than most of the other elements. Nitrogen in $\mathrm{NH}_{3}, \mathrm{NH}_{2}(\mathrm{OH})$, $\mathrm{N}_{2} \mathrm{O}_{3}$, and even in $\mathrm{CNH}$, must be considered as trivalent, but in $\mathrm{NH}_{4} \mathrm{Cl}, \mathrm{NO}_{2}(\mathrm{OH})$, and in all their corresponding compounds it is necessarily pentavalent. In $\mathrm{N}_{2} \mathrm{O}$, if the atomicity of oxgen $=2$, nitrogen has an uneven atomicity $(1,3,5)$, whilst in NO it is bivalent. If sulphur be bivalent, like oxygen, in many of its compounds (for example, $\mathrm{H}_{2} \mathrm{~S}, \mathrm{SCl}_{2}, \mathrm{KNS}, \& \mathrm{c}$.), then it could not be foreseen from this that it would form $\mathrm{SO}_{2}$, $\mathrm{SO}_{3}, \mathrm{SCl}_{4}, \mathrm{SOCl}_{2}$, and a series of similar compounds in which its atomicity must be acknowledged as greater than 2 . Thus in $\mathrm{SO}_{2}$, sulphurous anhydride, there are a multitude of points in common with $\mathrm{CO}_{2}$, and if carbon be quadrivalent then the $\mathrm{S}$ in $\mathrm{SO}_{2}$ is quadrivalent. Therefore the principle of the atomicity (valency) of the elements cannot be established as the basis for the study of the elements, although it gives an easy possibility for grasping many analogies. Although a definite atomicity cannot be considered as a radical property of atoms and elements, it may, however, be made use of with great advantage in investigating the compounds of such elements as carbon, because carbon in all its usual compounds, and especially in the saturated compounds and those approaching them, acts as a quadrivalent element. I consider the four following as the chief obstacles to acknowledging the atomicity of the elements as a primary conception for the consideration of the properties of the elements: 1 . Such univalent elements as $\mathrm{H}, \mathrm{Cl}$, \&c., appear in a free state as molecules $\mathrm{H}_{2}, \mathrm{Cl}_{2}$, \&c., and are consequently like the univalent radicles $\mathrm{CH}_{3}, \mathrm{OH}, \mathrm{CO}_{2} \mathrm{H}, \&$ c., which, as might be expected, appear as $\mathrm{C}_{2} \mathrm{H}_{6}$, $\mathrm{O}_{2} \mathrm{H}_{2}, \mathrm{C}_{2} \mathrm{O}_{4} \mathrm{H}_{2}$ (ethane, hydrogen peroxide, oxalic acid), whilst on the other hand, potassium and sodium (perhaps also iodine at a high temperature) contain only one atom, $\mathrm{K}, \mathrm{Na}$, in the molecule in a free state. Hence it follows that free affinities may exist. And then nothing obliges us not to admit free affinities in all unsaturated compounds; for example, it may be said that in $\mathrm{C}_{2} \mathrm{H}_{4}$ each atom of carbon is bound to the other by one affinity, two other affinities holding the hydrogen, and that the fourth affinity of each carbon atom is free. If such instances of free affinities be admitted, then all the possible advantages to be gained by the application of the doctrine of atomicity (valency) are lost. 2. There are instances-for example, $\mathrm{Na}_{2} \mathrm{H}$-where univalent elements are gathered together in molecules which are more complex than $\mathrm{R}_{2}$, and form molecules, $R_{3}, R_{4}$, \&c.; which may again be either taken as evidence of the existence of free affinities, or else necessitates such primary univalent elements as sodium and hydrogen being considered as variable in their atomicity. 3. The periodic system of the elements, with which we shall afterwards become acquainted, shows that there is a law or rule for the variation of the forms of oxygen and hydrogen compounds; chlorine is univalent with respect to hydrogen, and septavalent with respect to oxygen; sulphur is bivalent to lydrogen, and sexavalent to oxygen; phosphorus is trivalent to hydrogen and pentavalent in respect to oxygen - the sum is in every case equal to 8 . Only carbon and its analogues (for example, silicon) are quadrivalent to both hydrogen and oxygen. Hence the property of the elements to change their atomicity lies in the essence of their nature, and consequently atomicity cannot be considered as a fundamental property. 4. Crystallohydrates (for instance, $\mathrm{NaCl}, 2 \mathrm{H}_{2} \mathrm{O}$, or $\mathrm{NaBr}, 2 \mathrm{H}_{2} \mathrm{O}$ ), double salts (for instance, $\mathrm{PtCl}_{4}, 2 \mathrm{KCl}$, $\mathrm{H}_{2} \mathrm{SiF}_{6}, \& \mathrm{c}$.), and similar complex compounds (and, according to Chap. I., solutions also) demonstrate the capacity not only of the elements themselves, but also of their saturated and limiting compounds, of entering into further combination. Therefore the admission of a definite limited atomicity of the elements includes in itself an admision of limitation which is not in accordance with the nature of chemical reactions. 
atomicity of the elements. Hydrogen is taken as the representative of the univalent elements, giving compounds, $\mathrm{RH}, \mathrm{R}(\mathrm{OH}), \mathrm{R}_{2} \mathrm{O}, \mathrm{RCl}$, $\mathrm{R}_{3} \mathrm{~N}, \mathrm{R}_{4} \mathrm{C}$, dc. Oxygen, in that form in which it gives water, is the representative of the bivalent elements, forming $\mathrm{RH}_{2}, \mathrm{RO}, \mathrm{RCl}_{2}$, $\mathrm{RHCl}, \mathrm{R}(\mathrm{OH}) \mathrm{Cl}, \mathrm{R}(\mathrm{OH})_{2}, \mathrm{R}_{2} \mathrm{C}, \mathrm{RCN}$, dc. Nitrogen in ammonia is the representative of the trivalent elements, giving compounds $\mathrm{RH}_{3}$, $\mathrm{R}_{2} \mathrm{O}_{3}, \mathrm{R}(\mathrm{OH})_{3}, \mathrm{RCl}_{3}, \mathrm{RN}, \mathrm{RHC}$, \&c. In carbon is expressed the properties of the quadrivalent elements, $\mathrm{RH}_{4}, \mathrm{RO}_{2}, \mathrm{RO}(\mathrm{OH})_{2}, \mathrm{R}(\mathrm{OH})_{4}$, $\mathrm{RHN}, \mathrm{RCl}_{4}, \mathrm{RHCl}_{3}$, drc. We meet with these forms of combination or aspects of union of atoms in all other elements, some being analogous to hydrogen, others to oxygen, and others to nitrogen or to carbon. But besides these quantitative analogies or resemblances, which are foretold by the law of substitution (Chapter VI.), there exist among the elements qualitative analogies and relations which are not exhausted in the compounds of the elements which have been considered, but are most distinctly expressed in the formation of bases, acids, and salts of different types and properties. Therefore, for a complete study of the nature of the elements and their compounds it is especially important to become acquainted with the salts as substances of a peculiar character, corresponding with acids and bases. Common table salt, or sodium chloride, $\mathrm{NaCl}$, may in every respect be taken as a type of salts in general, and therefore we will pass to the consideration of this substance, of hydrochloric acid, and of the base sodium hydroxide, of the non-metal chlorine, and the metal sodium, which correspond with it.

Sodium chloride, $\mathrm{NaCl}$, or the familiar table salt, occurs in the primary formations of the earth's crust, ${ }^{2}$ from which it is washed away by the atmospheric waters, and is held in small quantities in all waters flowing through these formations, and is in this manner collected in the oceans and seas. The immense mass of salt in the oceans has been accumulated by this process from the remote ages of the earth's crea-

2 The primary formations are those which do not bear any distinct traces of having been deposited from water (have not a stratified formation, contain no remains of animal or vegetable life), occur under the sedimentary formations of the earth, and are everywhere uniform in composition and structure, which is generally distinctly crystalline. If the origin of the earth were of a molten nature, the first primary formations are those which formed the first solid crust of the earth. But even with this hypothesis of the earth's origin, it is necessary to admit that the first aqueous deposits must have re-formed the crust of the earth, and therefore under the head of the primary formations must be understood the most ancient of the products of decomposition (mostly by atmospheric, aqueous, and organic agency, \&c.), from which all the rocks and substances of the earth's surface have arisen. In speaking of the origin of one or another substance, we can only, on the basis of facts, descend to the primary formation, of which granite, gneiss, and trachyte may be taken as examples. 
tion, because the water has evaporated from them while the salt has remained in solution. The salt of sea water serves as the source not only for its direct extraction, but also for the formation of other masses of workable salt, such as rock salt and of saline springs and lakes.

The extraction of salt from sea water is carried on in several ways. In southern climes, especially on the shores of the Atlantic Ocean, the Mediterranean and Black Seas, the summer heats are taken advantage of. A convenient, low-lying sea shore is chosen, and a whole series of basins, communicating with each other, are constructed along it. The upper of these basins are filled with sea water by pumping, or else advantage is taken of high tides. These basins are sometimes separated from the sea by natural sand-banks (limans) or by artificial means, and in April the water already begins to evaporate considerably. As the solution becomes more concentrated, it is run into the succeeding basins, and the upper ones are supplied with a fresh quantity of sea water, or else an arrangement is made enabling the salt water to flow by degrees through the series of basins. It is evident that the bed of the basins should be, as far as possible, impervious to water, and for this purpose they are made of beaten clay. The crystals of table salt begin to separate out when the concentration attains 28 p.c. of salt (which corresponds to $28^{\circ}$ of Baumé's hydrometer). It is raked off, and employed for all those purposes to which table salt is applicable. In the majority of cases only the first half of the sodium chloride which can be separated from the sea water is extracted, because the second half has a bitter taste, from the presence of magnesium salts which separate out together with the table salt. But in certain localities - as, for instance, in the estuary of the Rhone, on the island of Camarga ${ }^{3}$ - the evaporation is carried on to the very end, in order to obtain those magnesium and potassium salts which separate out at the end of the evaporation of sea water. Various salts are separated from sea water in its evaporation. In the water of oceans (Chapter I.) there is held so large an amount of sodium chloride that on evaporation this salt, notwithstanding its great solubility in water, soon reaches saturation and separates out. From 100 parts of sea water there separates out, by natural and artificial evaporation, about one part of tolerably pure table salt at the very commencement of the operation; the total amount held in solution

3 The extraction of the potassium salts (or so-called summer salts) was carried on at the Isle of Camarga about 1870, when I had occasion to visit that spot. If I mistake not, this industry is now no longer carried on, because the deposits of Stassfurt provide a much cheaper salt, owing to the evaporation and separation of the salt being there carried on by natural means and only requiring a treatment and refining, which is also necessary in addition for the 'summer salt' obtained from sea water. 
being about $2 \frac{1}{2}$ p.c. The remaining portion of the table salt separates out, intermixed with the bitter salts of magnesium which form the chief admixture with table salt in sea water. These salts of magnesium, owing to their degree of solubility and the small amount in which they are present (less than 1 p.c.), only separate out, in the first crystallisations, in traces with the table salt; whilst, in the succeeding crystallisations, they are deposited together with the table salt. Gypsum, or calcium sulphate, $\mathrm{CaSO}_{4} 2 \mathrm{H}_{2} \mathrm{O}$, owing to its sparing solubility, separates together with or even before the table salt. When about half of the table salt has separated, then, on further evaporation, the sea water gives a mixture of table salt and magnesium sulphate, and, on still further evaporation, the chlorides of potassium and magnesium begin to separate in a state of combination, forming the double salt $\mathrm{KMgCl}_{3}, 6 \mathrm{H}_{2} \mathrm{O}$, which occurs in nature as carnallite. This is a crystallo-hydrated compound of $\mathrm{KCl}$ and $\mathrm{MgCl}_{2}{ }^{4}$ After the separation of this salt from sea water, there remains a mother liquor, containing a large amount of magnesium chloride in admixture with various other salts. ${ }^{5}$. The extraction of sea salt is usually carried on for the purpose of procuring table salt, and therefore directly it begins to separate mixed with a considerable proportion ${ }^{6}$ of magnesium salts (when it acquires a bitter taste) the remaining liquor is run back into the sea.

The same process which is employed for artificially obtaining salt in a crystalline form from sea water has been repeatedly accomplished during the geological evolution of the earth on a gigantic scale; upheavals of the earth have cut off portions of the sea from the remainder (as the Dead Sea was formerly a part of the Mediterranean, and the Sea

4 The double salt, $\mathrm{KCl}, \mathrm{MgCl}_{2}$, is only formed from solutions containing an excess of magnesium chloride, because water decomposes this double salt, extracting the more soluble magnesium chloride from it.

5 Owing to the fundamental property of salts of interchanging their metals, it cannot be said that sea water contains this or that salt, but only that it contains certain amounts of certain metals $\mathrm{M}$ (univalent like $\mathrm{Na}$ and $\mathrm{K}$, and bivalent like $\mathrm{Mg}$ and $\mathrm{Ca}$ ), and haloids $\mathrm{X}$ (univalent like $\mathrm{Cl}, \mathrm{Br}$, and bivalent like $\mathrm{SO}_{4}, \mathrm{CO}_{3}$ ), which are disposed in every possible kind of grouping; for instance, $\mathrm{K}$ as $\mathrm{KCl}, \mathrm{KBr}, \mathrm{K}_{2} \mathrm{SO}_{4}, \mathrm{Mg}$ as $\mathrm{MgCl}_{2}$, $\mathrm{MgBr}_{2}, \mathrm{MgSO}_{4}$, and so on for all the other metals. In evaporation different salts separate out consecutively only because they reach saturation. A proof of this may be seen in the fact that a solution of a mixture of sodium chloride and magnesium sulphate (both of which salts are obtained from sea water, as was mentioned above), when evaporated, separates out crystals of these salts, but when refrigerated (if the solution be sufficiently saturated) the salt $\mathrm{Na}_{2} \mathrm{SO}_{4}, 10 \mathrm{H}_{2} \mathrm{O}$ is first deposited because it is the first to arrive at saturation at low temperatures. Consequently, this solution contains $\mathrm{MgCl}_{2}$ and $\mathrm{Na}_{2} \mathrm{SO}_{4}$, besides $\mathrm{MgSO}_{4}$ and $\mathrm{NaCl}$. So it is with sea water.

6 The salt extracted from water is piled up in heaps and left under the action of rain water, which purifies the salt, owing to its becoming saturated with table-salt and then no longer dissolving it, but washing out the impurities. 
of Aral of the Caspian), and their water has evaporated and formed (if the mass of the inflowing fresh water were less than that of the mass evaporated) deposits of rock salt. It is always accompanied by gypsum, because the latter is separated from sea water before the sodium chloride. For this reason rock salt may always be looked for in those localities where there are deposits of gypsum. But inasmuch as the gypsum may remain on the spot where it has been deposited (as it is a sparingly soluble salt), whilst the rock salt (as one which is very soluble) may be washed away by rain or fresh running water, it may sometimes happen that although gypsum is found still there may be no salt; but, on the other hand, where there is rock salt there will always be gypsum. As the geological changes of the earth's surface are still proceeding to the present day, so in the midst of the dry land sait lakes are met with, which are sometimes scattered over vast districts formerly covered by seas now dried up. Such is the origin of many of the salt lakes about the lower portions of the Volga and in the Kirghiz steppes, where at a geological epoch preceding the present the Aralo-Caspian Sea extended. Such are the Baskunchaksky (in the Government of Astrakhan, 112 square kilometres superficial area), the Eltousky (140 versts from the left bank of the Volga, and 200 square kilometres in superficial area), and upwards of 700 other salt lakes lying about the lower portions of the Volga. In those in which the inflow of fresh water is less than that yearly evaporated, and in which the concentration of the solution has reached saturation, the selfdeposited salt is found already deposited on their beds, or is being yearly deposited during the summer months. Certain limans, or sea-side lakes, of the Azoff Sea are essentially of the same character-as, for instance, those in the neighbourhood of Henichesk and Berdiansk. The saline soils of certain Central Asian steppes, which suffer from a want of atmospheric fresh water, are of the same origin. Their salt originally proceeded from the salt of seas which previously covered these localities, and has not yet been washed away by fresh water. The main result of the above described process of nature is the formation of masses of rock salt, which are, however, being gradually washed away by the subsoil waters flowing in their neighbourhood, and afterwards rising to the surface in certain places as saline springs, which indicate the presence of masses of deposited rock salt in the depths of the earth. If the subsoil water flows along a strata of salt for a sufficient length of time it becomes saturated; but in flowing in its further course along an impervious strata (clay) it becomes diluted by the fresh water leaking through the upper soil, and therefore the greater the distance of source of a saline spring from the deposit of rock salt, the poorer will it be in 
salt. A perfectly-saturated brine, however, may be procured from the depths of the earth by means of bore holes. The deposits of rock salt themselves, which are sometimes hidden at great depths below the earth's strata, may be arrived at by the guidance of bore-holes and the arrangement of the strata of the district. Deposits of rock salt, about 35 metres thick and 20 metres below the surface, were discovered in this manner in the neighbourhood of Briaustcheffky and Dekonoffky, in the Bakhmut district of the Government of Ekaterinoslav. Large quantities of most excellent rock salt are now (since 1880) won from these deposits, whose presence was indicated by the neighbouring salt springs (near Slaviansk and Bakhmut), and bore-holes which had been sunk at these localities for procuring strong (saturated) brines. But the Stassfurt deposits of rock salt, near Magdeburg in Germany, are distinguished as being the first discovered in this manner, and for their many remarkable peculiarities. ${ }^{7}$ The considerable distribution of saline springs in this and the neighbouring districts suggested the presence of deposits of rock salt in the near vicinity. Deep bore-holes sunk in this locality in fact did give a richer brine - even quite saturated with salt. On sinking to a still greater depth, the deposits of salt were themselves in the end arrived at. But the first salt which was met with was a bitter salt unfit for consumption, and which was, therefore, called refuse salt (abraumsalz). On sinking still deeper through fresh bands of earth, vast beds of real rock salt were struck. In this instance, the presence of these upper strata containing salts of potassium, magnesium, and sodium, is an excellent proof of the formation of rock salt from sea water. It is a self-evident fact that not only a case of evaporation to the end-for instance, to the separation of carnallitebut also the conservation of such soluble salts as those which separate out from sea water after the sodium chloride, forms a very excep. tional phenomenon, which is not repeated in all deposits of rock salt. The Stassfurt deposits, therefore, are of particular interest, not only from a scientific point of view, but also because they form a rich source of potassium salis which have many practical uses. In Western

7 When the German savants pointed out the exact locality of the Stassfurt saltbeds and their depth below the surface on the basis of information collected from various quarters respecting bore-holes and the direction of the strata, and when the borings, conducted by the Government, struck out a salt-bed which was bitter and unfit for use, then there was a great outcry against science, and the doubtful result even caused the cessation of the further work of deepening the shafts. It required a great effort to persuade the Government to continue the work. Now, when the pure salt. encountered below forms one of the important riches of Germany, and when those 'refuse salts' have proved to be most precious (as a source of potassium and magnesium), we should see in the utilisation of the Stassfurt deposits one of the conquests of science for the common welfare. 
Europe, deposits of rock salt have long been known at Wieliczka, near Cracow, and at Cardona in Spain. In Russia the following deposits are known : ( $x$ ) the vast masses of rock salt (3 square kilometres area and up to 140 metres thick) lying directly on the surface of the earth at Iletzky Zastchit, on the left bank of the river. Ural, in the Government of Orenburg; $(b)$ the Chingaksky deposit, 90 versts from the river Volga, in the Enotaeffisky district of the Government of Astrakhan; (c) the Kulepinsky (and other) deposits (whose thickness attains 150 metres), on the Araks, in the Government of Erivan in the Caucasus; $(d)$ the Katchiezmansky deposit in the province of Kars; and (e) the Krasnovodsky deposit in the Trans-Caspian province.

A saturated brine, formed by the continued contact of subsoil water with rock salt, is extracted by means of bore-holes, as, for instance, in the Governments of Perm, Kharkoff, and Ekaterinoslav. Sometimes, as at Wergtesgaden in Austria (at Salzkammerhutte), spring water is run on underground beds of rock salt containing much clay.

If a saline spring or the salt water pumped from bore-holes contains but little salt, then the first concentration of the natural solution is not carried on by the costly consumption of fuel, but by the cheaper method of evaporation by means of the wind. For this purpose the so-called graduators are constructed : they consist of long and lofty sheds, which are sometimes several versts long, and generally extend in a direction at right angles to that of the usual course of the wind in the district. These sheds are open at the sides, and are filled with brushwood as shown in fig. 64 . Troughs, A B, C D, into which the salt water is pumped, run along the top. On flowing from these troughs, through the openings, $a$, the water spreads over the brushwood and distributes.itself in a thin layer over it, so that it presents a very large surface for evaporation, in consequence of which it rapidly becomes concentrated in warm or windy weather. After trickling over the brushwood, the solution collects in a reservoir under the graduator, from whence it is usually pumped up by the pumps $\mathbf{P} \mathbf{P}^{\prime}$, and again run a second and third time through the graduator, until the solution reaches a degree of concentration at which it becomes profitable to extract the salt by direct heating. Generally the evaporation in the graduator is not carried beyond a concentration of 15 to 20 parts of salt in 100 parts of solution. Strong natural solutions of salt, and also the graduated solutions, are evaporated in large shallow metallic vessels, which are either heated by the direct action of the flame from below or from above. These vessels are made of boiler plate, and are called salt pans. Various means are employed for accelerating the evapora- 
tion and for economising fuel, which are mainly based on an artificial draught to carry off the steam as it is formed, and on subjecting the saline solution to a preliminary heating by the waste heat of the steam and furnace gases. Furthermore, the first portions of the salt which

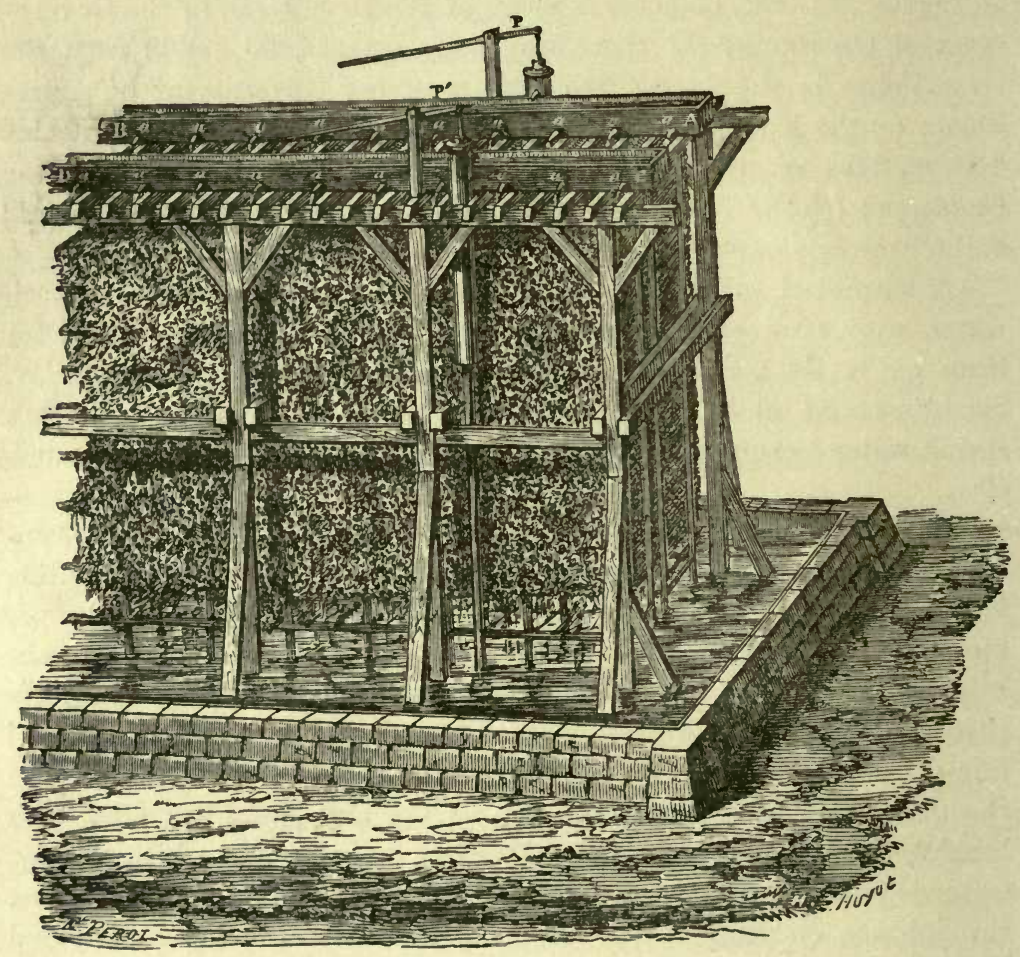

FIG. 64.-Graduator for the evaporation of the water of saline springs.

crystallise out in the salt pans always contain gypsum, owing to the water of saline springs always containing this substance. It is only the portions of the salt which separate later that are distinguished by their great purity. The salt is ladled out as it is deposited, and left to drain on inclined tables and then dried, and in this manner the socalled bay salt is obtained. Since it has become possible to discover the saline deposits themselves, the extraction of table salt from the water of saline springs by evaporation, which was before in general use, has begun to be rejected, and is only able to hold its ground in cases where fuel is cheap.

In order to understand the full importance of the extraction of salt, it will be enough to mention that on the average $20 \mathrm{lbs}$. of table 
salt are consumed per head of population, directly in food or for cattle. In those countries where common salt is employed in technical processes, and especially in England, almost an equal quantity is consumed in the production of substances containing chlorine and sodium, and especially in the manufacture of washing soda, \&c., and of chlorine compounds (bleaching powder and hydrochloric acid).

Although certain lumps of rock salt and crystals of bay salt sometimes consist of almost pure sodium chloride, still the ordinary commercial salt contains various impurities, the most common of which are magnesium salts. If the salt be pure, its solution gives no precipitate with sodium carbonate, $\mathrm{Na}_{2} \mathrm{CO}_{3}$, showing the absence of magnesium salts, because magnesium carbonate, $\mathrm{MgCO}_{3}$, is insoluble in water. Rock salt, which is ground for use, further generally contains a large admixture of clay and other insoluble impurities. ${ }^{8}$. For common use the bulk of the salt obtained is quite suitable without further purification; but some salts are purified by solution and crystallisation of the solution after standing, in which case the evaporation is not carried on to the end, and the impurities remain in the mother liquor or in the sediment. When perfectly pure salt is required for chemical purposes it is best to proceed as follows : a saturated solution of table salt is prepared, and hydrochloric acid gas is passed through it; this precipitates the sodium chloride (which is not soluble in a strong solution of hydrochloric acid), while the impurities remain in solution. By repeating the operation and fusing the salt (when adhering hydrochloric acid is volatilised) a pure salt is obtained, which is again crystallised from its solution by evaporation. ${ }^{9}$

Pure sodium chloride, in the form of well-formed crystals (slowly formed at the bottom of the liquid) or in compact masses (in which form rock salt is sometimes met with), is a colourless and transparent substance resembling, but more brittle and less hard than, glass. ${ }^{10}$ Common

8 The fracture of rock salt generally shows the presence of interlayers of impurities, which are sometimes very small in weight, but visible owing to their refraction. In the excellently laid out salt mines of Briansk, belonging to Mr. Letunoffsky, I counted (1888), if my memory does not deceive me, on an average ten interlayers per metre of thickness, between which the salt was in general very pure, and in places quite transparent. If this be the case, then there would be 350 interlayers for the whole thickness (about 35 metres) of the bed. They probably correspond with the yearly deposition of the salt. In this case the deposition would have extended over more than 300 years. This should be observable at the present day in lakes where the salt is saturated and in course of deposition.

9 I have personally convinced myself that by this method not only the sulphates, but also the potassium salts are entirely removed.

10 According to the determinations of Klodt, the Briansk rock salt withstands a pressure of 340 kilograms per square centimetre, whilst glass withstands 1700 kilos. 
salt always crystallises in the cubic system, most frequently in cubes, and more rarely in octahedra. Large transparent cubes of common salt, having edges up to 10 centimetres long, are sometimes found in masses of rock salt. ${ }^{11}$ By rapid evaporation common salt is deposited from its solutions in the form of very small crystals, but with slow evaporation the crystals attain a considerable size. When evaporated in open spaces the salt often separates out on the surface ${ }^{12}$ as cubes, which grow on to each other in the form of pyramidal square funnels. In still weather, these clusters are able to support themselves on the surface of the water for a long time, and sometimes go on increasing to a considerable degree, but they sink directly the water penetrates inside them. Salt fuses into a colourless liquid (sp. gr. 1.602, according to Quincke) at about $774^{\circ}$ (Carnelley), and if pure it solidifies into a noncrystalline mass, and if impure into an opaque mass whose surface is not smooth. In fusing, sodium chloride commences to volatilise (its

In this respect salt is twice as secure as bricks, and therefore immense masses may be extracted from underground workings with perfect safety, without having recourse to brickwork supports, but only taking advantage of the properties of the salt itself.

11 To obtain well-formed crystals, a saturated solution is mixed with ferric chloride, and several small crystals of sodium chloride are placed at the bottom, and the solution is allowed to slowly evaporate in a closed vessel. Octahedral crystals are obtained in the presence of borax, urea, \&c., in the solution. Very fine crystals are formed in a mass of gelatinous silice.

12 If a solution of sodium chloride be slowly heated from above, where the evaporation is accomplished, then the upper layer will become saturated before the lower and cooler layers, and therefore crystallisation will then begin on the surface, and the crystals first formed will be held up, having also dried from above, on the surface until they become quite soaked. Being heavier than the solution the crystals are partially immersed under it, and the following crystallisation, also proceeding on the surface, will only form crystals along the side of the original crystals. The funnels are formed in this manner. It will be borne on the surface like a boat (if there be no waves), because it will grow more from the upper edges. We can thus understand this, at first sight, strange funnel form of crystallisation of salt. In explanation why the crystallisation under the above conditions begins at the surface and not at the lower layers, it must be mentioned that the specific gravity of a crystal of sodium chloride $=2 \cdot 16$, and that of a solution saturated at $25^{\circ}$ contains 26.7 p.c. of salt and has a specific gravity at $25.4^{\circ}$ of 1.2004 ; at $15^{\circ}$ a solution contains 26.5 p.c. of salt and has a sp. gr. 1.203 at $15.4^{\circ}$. Hence a solution saturated at a higher temperature is specifically lighter, notwithstanding the greater amount of salt it contains. Surface crystallisation cannot take place with many substances, because their solubility increases more rapidly with the temperature than their specific gravity decreases. In this case the saturated solution will always be in the lower layers, where also the crystallisation will take place. Besides which, it may be added that, as a consequence of the properties of water and solutions, when they are heated from above (for instance, by the sun's rays) the warmer layers being the lightest remain above, whilst when heated from below they rise to the top. For this reason the water at great depths below the surface is always cold, as has long been known. These circumstances, as well as those observed by Soret (Chapter I. Note 19), explain the great differences of density, temperature, and in the amount of salts held in the oceans at different latitudes (in polar and tropical climes) and at various depths. 
weight decreases), and at a white heat it volatilises with great ease and entirely; but at the ordinary temperature it may, like all ordinary salts, be considered as non-volatile, although as yet no exact experiments have been made in this respect.

A saturated ${ }^{13}$ solution of table salt (containing $26 \cdot 4$ per cent.) has at the ordinary temperature a specific gravity of about 1.2. The specific gravity of the crystals is $2 \cdot 16$. The salt which separates out at the ordinary and higher temperatures contains no water of crystallisation $;^{14}$ but if the crystals are formed at a low temperature, especially from a saturated solution cooled to $-12^{\circ}$, then they present a prismatic form, and contain two equivalents of water, $\mathrm{NaCl}, 2 \mathrm{H}_{2} \mathrm{O}$. At the ordinary temperature, these crystals split up into sodium chloride and its solution. ${ }^{15}$ Unsaturated solutions of table salt when cooled below $0^{\circ}$ give $^{16}$ crystals of ice, but when the solution has a

13 By combining the results of Poggiale, Miuller, and Karsten (they are evidently more accurate than those of Gay-Lussac and others), I found that a saturated solution at $t^{\circ}$, from $0^{\circ}$ to $108^{\circ}$, contains $35 \cdot 7+0.024 t+0^{\circ} 0002 t^{2}$ grams of salt per 100 grams of water. This formula gives a solubility at $0^{\circ}=35 \cdot 7$ grams $(=26 \cdot 3$ p.c. $)$, whilst according to Karsten it is $36^{\circ} 09$, Poggiale $35^{\circ} 5$, and Müller $35^{\circ} 6$ grams. The somewhat large diversity in the data respecting so common a substance as sodium chloride shows the necessity of fresh and most exact determinations.

14 Perfectly pure fused salt is not hygroscopic, according to Karsten, whilst the crystallised salt, even when quite pure, attracts as much as 0.6 p.c. of water from moist air, according to Stas. In the Briansk mines, where the temperature throughout the whole year is about $+10^{\circ}$, it may be observed, as Baron Klodt informed me, that in the summer during damp weather the walls become moist, while in winter they are dry. This is in accordance with the fact that the vapour tension of solutions has a definite magnitude, which is less than that of water.

If the salt contain impurities-such as magnesium sulphate, \&c.-it is more hygroscopic. If it contain any magnesium chloride it partially effloresces in a damp atmosphere. The crystallised and not perfectly pure salt decrepitates when heated, owing to its containing water. The pure salt, and also the transparent rock salt, or that which has been once fused, does not decrepitate. Fused sodium chloride gives a feeble alkaline reaction with litmus, as has been shown by many observers, which is due to the formation of sodium oxide (probably by the action of the oxygen of the atmosphere). According to A. Stcherbakoff very sensitive litmus (washed in alcohol and neutralised with oxalic acid) shows an alkaline reaction with even the crystallised salt.

It may be observed that rock salt sometimes contains cavities filled with a colourless liquid. Certain kinds of rock salt emit an odour like that of hydrocarbons. These phenomena have as yet received very little attention.

$15 \mathrm{By}$ cooling a solution of table salt saturated at the ordinary temperature to $-15^{\circ}$ I first obtained well-formed tabular (six-sided) crystals, which on arriving at the ordinary temperature disintegrated (with the separation of anhydrous sodium chloride), and then prismatic needles up to $20 \mathrm{~mm}$. long were formed from the same solution. I have not yet investigated what is the reason of the difference in crystalline form. It is known (Mitscherlich) that $\mathrm{NaI}, 2 \mathrm{H}_{2} \mathrm{O}$ also crystallises in plates or prisms. Sodium bromide also crystallises with $2 \mathrm{H}_{2} \mathrm{O}$ at the ordinary temperature.

16 Notwithstanding the great simplicity (p. 91) of the observations on the formation of ice from solution, still even for sodium chloride they cannot yet be considered as sufficiently harmonious. According to Blagden and Raoult the temperature of the formation 
composition $\mathrm{NaCl}, 10 \mathrm{H}_{2} \mathrm{O}$ it solidifies completely at a temperature of $-23^{\circ}$. A solution of table salt saturated at its boiling point boils at about $109^{\circ}$, and contains about 42 parts of salt per 100 parts of water.

Of all its physical properties the specific gravity of solutions of sodium chloricle is the one which has been the most fully investigated. A comparison of the results of Kremers, Gerlach, Schmidt, Marignac, Thomsen, Nicol, and Bender proves ${ }^{17}$ that the specific gravity (in vacuum, taking water at $4^{\circ}$ as 10000 ) at $15^{\circ}$ in relation to $p$, or the percentage amount of the salt in solution, is expressed by the equation $\mathrm{S}_{15}=9991 \cdot 6+\tau 1 \cdot 1 \tau p+0 \cdot 2140 p^{2}$. For instance, for a solution $200 \mathrm{H}_{2} \mathrm{O}+\mathrm{NaCl}$, in which case $p=1 \cdot 6, \mathrm{~S}_{15}=10106$. It is seen from the curve that the addition of water to the solution produces a contraction, ${ }^{18}$ and that the addition of salt (or thedifferential $\frac{d s}{d p}$ ) causes, at $15^{\circ}$, a change of specific gravity which is expressed by the straight line $71 \cdot 17+0.428 \mathrm{p}$. At $0^{\circ}$ and $100^{\circ}$ (when the specific

of ice from a solution containing $c$ grams of salt per 100 grams of water $=-0 \cdot 6 c$ to $c=10$, according to Rosetti $=-0.649 c$ to $c=8 \cdot 7$, according to De Coppet (to $c=10)=-0.762 c$ $+0 \cdot 0084 c^{2}$, and according to Guthrie a much lower figure. By taking Rosetti's figure and applying the rule given on p. 91 (Chapter I. Note 49) we obtain-

$$
i=0.649 \times \frac{58.5}{18.5}=2.05 \text {. }
$$

The data for strong solutions are not less contradictory. Thus with 20 p.c. of salt, ice is formed at $-14.4^{\circ}$ according to Karsten, $-17^{\circ}$ according to Guthrie, $-17 \cdot 6^{\circ}$ according to De Coppet. Riidorff says that for strong solutions the temperature of the formation of ice descends in proportion to the contents of the compound, $\mathrm{NaCl}, 2 \mathrm{H}_{2} \mathrm{O}$ (per 100 grams: of water) by $0.342^{\circ}$ per 1 gram of salt, and De Coppet shows that there is no proportionality, in a strict sense, for either a percentage of $\mathrm{NaCl}$ or of $\mathrm{NaCl}, 2 \mathrm{H}_{2} \mathrm{O}$. The data respecting the vapour tension and boiling point of solutions of sodium chloride are as untrustworthy as the preceding.

17 A collection of observations on the specific gravity of solutions of sodium chloride and all other aqueous solutions which have been more or less investigated up to the present time is given in my work cited in Chapter I. Note 50.

Solutions of common salt have also been frequently investigated as regards rate of diffusion (p. 62), but as yet there are no complete data in this respect. It may be mentioned that Graham and De Vries demonstrated that diffusion in gelatinous masses (for instance, gelatin jelly or gelatinous silica) proceeds in the same manner as in water, which may probably lead to a convenient and accurate method for the investigation of the phenomena of diffusion. N. Umoff (Odessa, 1888) investigated the diffusion of common salt by means of glass globules of definite density. Having poured water into a cylinder over a layer of a solution of sodium chloride, he observed during a period of several months the position (height) of the globules, which floated up higher and higher as the salt permeated upwards. Umoff found that at a constant temperature the distances of the globules (that is, the length of a column limited by layers of definite concentration) remain constant; that at a given moment of time the concentration, $q$, of different layers situated at a depth $z$ is expressed by the equation $\mathrm{B}-\mathrm{K} z=\log$. $(\mathrm{A}-q)$, where $\mathrm{A}, \mathrm{B}$, and $K$ are constants; that at a given moment the velocity of the different layers is proportional to their depth, \&c. The information respecting diffusion is considerably extended by these researches, but still this subject, from its importance for the theory of solutions and of liquids in general, yet awaits a perfectly-detailed investigation.

18 If $S_{0}$ be the specific gravity of water, and $S$ the specific gravity of a solution containing 
gravity of water is $9998 \cdot 7$ and 9585$)$ this augmentation is expressed if $75 \cdot 4$ and $65 \cdot 7$ be substituted for $71 \cdot 17$, and 0.31 and 0.72 for 0.428 . In this manner the specific gravity ${ }^{19}$ at

$\begin{array}{rcccr} & 0^{\circ} & 15^{\circ} & 30^{\circ} & 100^{\circ} \\ p=5 & 10372 & 10353 & 10307 & 9922 \\ 10 & 10768 & 10728 & 10669 & 10278 \\ 15 & 11164 & 11107 & 11043 & 10652 \\ 20 & 11568 & 11501 & 11429 & 11043\end{array}$

when water at $0^{\circ}=10000 .{ }^{20}$

$p$ p.c. of salt, then by mixing, for instance, equal weights of water and the solution, we shall obtain a solution containing $\frac{1}{2} p$ of the salt, and if it beformed without contraction, then its specific gravity $x$ will be determined by the equation $\frac{2}{x}=\frac{1}{S_{o}}+\frac{1}{S}$, because the volume is equal to the weight divided by the density. In reality, the specific gravity always comes out greater than that calculated on the supposition of an absence of contraction, as may be shown by substituting $S$ by its parabolic expression, $S=S_{o}+A p+B p^{2}$, and $x$ by $S_{o}+A_{2} p+$ $B \frac{1}{4} p^{2}$. By this means it may be easily shown that the contraction, $c$, in the formation of 100 grams of solution, is not in so simple a relation to the composition of the solution as G.T. Gerlach (1888) takes it, on the supposition that $c=A p(100-p)$, where $A$ is a constant for all solutions of a given substance. The magnitude of $c$ is $\epsilon$ vidently determined by the equation $p / B+(100-p) / S_{o}=100 / S+c$, where $B$ is the specific gravity of the substance dissolved, on the supposition of its being in a liquid state. If for sodium chloride at $15^{\circ}$ with $p=10$ and $p=20$, the mean observed specific gravities be taken as 10726 and 11501, then (as $S_{o}=9991 \cdot 6$ ) $A=2851 \times 10^{-10}$, and $B=17476$, and therefore for $p=5$, the calculated specific gravity will be 10377 , and the observed is 10353 , with a probable error of not more than \pm 2 : hence the difference greatly exceeds the possible error. A similar un. adaptability of the above-mentioned supposition is evinced in the investigation of all other solutions. The hypothesis under consideration resembles in this respect the hypothesis of Michel and Crafts or Grosjean, which are examined in my work on the specific gravities of solutions. In a first rough approximation solutions may be regarded as mechanical aggregates, and then a general law of their formation may be looked for, but a detailed study of the subject necessitates the search for chemical reactions in them, and such a representation of the nature of solutions leads to the conclusions enunciated in the first chapter and more fully developed in my above-mentioned work. This naturally does not exclude the desire to find laws to which solutions may be subjected, but under the inevitable condition of the consideration of their chemical composition. Such are, for example, the deductions of Van't Hoff, who does not, however, touch on the specific gravity of solutions. With respect to the sp. gr. of weak solutions of metallic chlorides, it may, for instance, be supposed that, having a composition $\mathrm{RCl}_{n}+200 \mathrm{OH}_{2}$, they would all have a specific gravity at $15^{\circ} / 4^{\circ}$, approaching to $9951+2.585 M$, where $M$ is the molecular weight of the metallic chloride dissolved. For instance, for $\mathrm{SrCl}_{2}, M=158$, and the formula gives $S=10361$, and experiment 10364 ; for $\mathrm{LiCl}, M=42 \cdot 5$, and $S=10061$, and experiment 10060 . But similar rules, without the existence of a complete theory of solutions, can only serve as material for the construction of a theory, and too great an importance should not be attaclied to them.

19 Generally the specific gravity is observed by weighing in air, dividing the weight in grams by the volume in cubic centimetres, found from the weight of water displaced, divided by its density at the temperature under which the experiment is carried on. If we call this specific gravity $S_{1}$, then as a cubic centimetre of air under the usual conditions weighs about $0.0012 \mathrm{gram}$, the sp. gr. in a vacuum $S=S_{1}-0.0012\left(S_{1}-1\right)$, if the density of water $=1$.

${ }^{20}$ If the sp. gr. $S_{2}$ be found directly by dividing the weight of a solution by the 
It should be remarked that Baumés hydrometer is graduated by taking a 10 per cent. solution of sodium chloride as $10^{\circ}$ on the scale, and therefore it gives approximately the percentage amount of the salt in a solution. Table salt is somewhat soluble in alcohol, ${ }^{21}$ but it is insoluble in ether and in oils.

Table salt gives very few compounds ${ }^{22}$ (double salts), and these are very readily decomposed; it is also decomposed with great difficulty and its dissociation is unknown. ${ }^{23}$ But it is easily decomposed, both when fused and in solution, by the action of a galvanic current. If the dry salt be fused in a crucible and an electric current be passed through it by immersing carbon or platinum electrodes in it (the positive electrode is made of carbon and the negative of platinum or mercury), it is decomposed into two substances: a malodorous gas called chlorine appears at the positive pole and metallic sodium at the negative pole, which shows that table salt consists of these two elements. Both of them act on water at the moment of their evolution; the sodium, as we already know, evolves hydrogen from water and forms caustic soda, and the chlorine evolves oxygen from water and forms hydrochloric acid, and therefore on passing a current through a solution of table salt metallic sodium will not be formed-but oxygen, chlorine, and hydrochloric acid will appear at the positive pole, and hydrogen and caustic soda at the negative pole. The presence of hydrochloric acid is easily recognised by its acid properties, and the presence of caustic soda by its alkaline

weight of water at the same temperature and in the same volume, then the true sp. gr. $S$ referred to water at $4^{\circ}$ is found by multiplying $S_{2}$ by the sp. gr. of water at the temperature of observation. All the necessary corrections for the specific gravity of liquids are considered in my two works, On the Compounds of Alcohol with Water, 1865, and The Investigation of Aqueous Solutions by their Specific Gravity, 1887.

It may not be superfluous to remark that the data respecting the sp. gr. for solutions of sodium chloride near saturation do not sufficiently agree, and there is reason for thinking that in strong solutions containing more sodium chloride than in $\mathrm{NaCl}, 10 \mathrm{H}_{2} \mathrm{O}$ $(p=24 \cdot 53)$ a different curve should be adopted.

21 According to Schiff 100 grams of alcohol, containing $p$ p.c. by weight of $\mathrm{C}_{2} \mathrm{H}_{6} \mathrm{O}$, dissolves at $15^{\circ}-$

$$
\begin{array}{rllll}
p=10 & 20 & 40 & 60 & 80 \\
25 & 22 \cdot 6 & 13 \cdot 2 & 5 \cdot 9 & 1 \cdot 2 \text { grams } \mathrm{NaCl} .
\end{array}
$$

22 Amongst the double salts formed by sodium chloride that obtained by Ditte (1870) by the evaporation of the solution remaining after heating sodium iodate with hydrochloric acid until chlorine ceases to be liberated, is a remarkable one. Its composition is $\mathrm{NaIO}_{3}, \mathrm{NaCl}, 4 \mathrm{H}_{2} \mathrm{O}$. Rammelsberg obtained a similar (perhaps the same) salt in wellformed crystals by the direct reaction of both salts.

25 But it already gives sodium in the flame of a Bunsen's burner (see Spectrum Analysis), doubtless under the reducing action of the elements carbon and hydrogen. In the presence of an excess of hydrochloric acid in the flame (when the sodium would give sodium chloride), there is no sodium formsd in the flame and the salt does not communicate its usual coloration. 
reaction. Thus table salt, like other salts, is decomposed by the action of an electric current into a metal and a haloid, and presents a composition of great simplicity in comparison with the composition of many salts containing oxygen, which fact supports the hydrogen theory of acids discussed in Chapter III. Naturally, like all other salts, it may be formed from the corresponding base and acid with the separation of water. In fact if we mix caustic soda (alkali) with hydrochloric acid (acid) then table salt is formed, $\mathrm{NaHO}+\mathrm{HCl}=\mathrm{NaCl}+\mathrm{H}_{2} \mathrm{O}$.

With respect to the double decompositions of sodium chloride it should be observed that they are most varied, and serve as the means for obtaining nearly all the other compounds of sodium and chlorine.

The double decompositions of sodium chloride, as an example of the double decompositions of salts, are almost exclusively based on the possibility of the metal sodium being exchanged for hydrogen and other metals. But neither hydrogen nor any other metal is able to directly displace the sodium from table salt. This would result in the separation of metallic sodium, which itself displaces hydrogen and the majority of other metals from their compounds, and is not, as far as is known, ever separated by them. The replacement, then, of the sodium in sodium chloride by hydrogen and different other metals is accomplished by the transference of the sodium into some other sodium compound. If hydrogen or another metal, M, were combined with an element $\mathrm{X}$, then the double decomposition $\mathrm{NaCl}+\mathrm{MX}=\mathrm{NaX}+\mathrm{MCl}$ takes place. Such double decompositions proceed under particular conditions, sometimes completely and sometimes only partially, as we shall endeavour to explain. In order to acquaint ourselves with the double decompositions of sodium chloride, we will follow the methods actually employed in practice to procure compounds of sodium and chlorine from table salt. For this purpose we will first describe the treatment of sodium chloride by sulphuric acid for the preparation of hydrochloric acid and sodium sulphate. We will then describe the substances obtained from hydrochloric acid and sulphate of sodium. Chlorine itself, and nearly all the other compounds of this element, may be procured from hydrochloric acid, whilst sodium carbonate, caustic soda, metallic sodium itself and all its compounds may be obtained from sodium sulphate.

Even in the laboratory of animal organisms table salt is subjected to similar changes, furnishing the sodium, alkali, and hydrochloric acid, which take part in the processes of animal life.

Its necessity as a constituent in the food of both human beings and animais becomes evident when we consider that both hydrochloric acid and salts of sodium are found in the substances which are separated out from the blood into the stomach and intestines. So, for example, 
sodium salts are found in the blood and in the bile elaborated in the liver, and acting on the food in the alimentary canal, whilst hydrochloric acid is found in the acid juices of the stomach. Chlorides of the metals are always found in considerable quantities in the urine, and if they are excreted they must be replenished in the organism; and for the replenishment of the loss substances containing chlorine compounds must be taken in food. Not only do animals consume those amounts of sodium chlorine which are found in drinking water or in plants or other animals, but experience has shown that many wild animals travel long distances in search of salt springs, and that domestic animals, which in their natural condition do not require table salt, willingly take it, and that the functions of their organisms become much more regular from doing so.

The action of sulphuric acid on sodium chloride.-If sulphuric acid be poured over table salt, then even at the ordinary temperature, as Glauber observed, an odorous gas, hydrochloric acid, is evolved. The reaction which takes place consists in the sodium of the table salt and the hydrogen of the sulphuric acid changing places.

$\underset{\text { Sodium chloride. }}{\mathrm{NaCl}}+\underset{\text { Sulphuric acid. }}{\mathrm{H}_{2} \mathrm{SO}_{4}}=\underset{\text { Hydrochloric acid. Acid sodium sulphate. }}{\mathrm{HCl}}+\underset{\mathrm{NaHSO}_{4}}{\mathrm{NaH}^{2}}$

At the ordinary temperature this reaction is not complete, but soon ceases. If the mixture be heated, the decomposition proceeds until, if there be sufficient table salt present, all the sulphuric acid taken is converted into acid sodium sulphate. If there be an excess of acid it will remain unaltered. If 2 molecules of sodium chloride (117 parts) be taken per molecule of sulphuric acid (98 parts), then on heating the mixture to a moderate temperature only one-half $(58 \cdot 5)$ of the table salt will suffer change. Complete decomposition, after which neither hydrogen nor chlorine is left in the residual salt, proceeds (when 117 parts of table salt are taken per 98 parts of sulphuric acid) at a red heat only. Then-

$$
\underset{\text { Table salt. }}{2 \mathrm{NaCl}}+\underset{\text { Sulphuric acid. }}{\underset{\mathrm{H}}{\mathrm{H}_{2} \mathrm{SO}_{4}}}=\underset{\text { Hydrochloric acid. }}{2 \mathrm{HCl}}+\underset{\text { Sodium sulphate. }}{\mathrm{Na}_{2} \mathrm{SO}_{4}}
$$

This double decomposition is the result of the action of the acid salt, $\mathrm{NaHSO}_{4}$, first formed on sodium chloride, because the acid salt, as it contains hydrogen, itself acts like an acid, $\mathrm{NaCl}+\mathrm{NaHSO}_{4}=$ $\mathrm{HCl}+\mathrm{Na}_{2} \mathrm{SO}_{4}$. By adding this equation to the first we obtain the second, which expresses the ultimate reaction. Then all the hydrogen of the sulphuric acid and chlorine of the salt are evolved as gaseous 
hydrochloric acid, and the residue will be entirely free from them. Hence in the above reaction, non-volatile or sparingly-volatile table salt and sparingly-volatile sulphuric acid are taken, and as the result of their reaction, after the hydrogen and sodium have exchanged places, there is obtained non-volatile sodium sulphate and gaseous hydrochloric acid. The fact of the latter being a gaseous substance forms the main reason for the reaction proceeding to the very end. The mechanism of this kind of double decomposition, and the cause of the course of the reaction, are exactly the same as those we saw in the decomposition of nitre (Chapter VI.) by the action of sulphuric acid. The sulphuric acid in each displaces the other, volatile, acid.

Not only in these two, but in every instance, if a volatile acid can be formed through the substitution for a metal of the hydrogen of sulphuric acid, then this volatile acid will be formed. From this it may be concluded that the volatility of the acid should be considered as the cause of the progress of the reaction ; and, indeed, if the acid be soluble but not volatile, or if the reaction takes place in an enclosed space where the resulting acid cannot volatilise, or at the ordinary temperature when it does not pass into an elastic state of vapour-then the decomposition does not proceed to the end, but only up to a certain limit. In this respect the explanations given at the beginning of this century by the French chemist Berthollet in his work ' Essai de Statique Chimique,' are very important. The doctrine of Berthollet starts from the supposition that the chemical reaction of substances is accomplished in consequence not only of the measure of affinity between the different parts, but also under the influence of the relative masses of the reacting substances and of those physical conditions under which the reaction takes place. Two substances containing the elements MX and NY, being brought into mutual contact, form by double decomposition the compounds MY and NX, but the formation of these two new compounds will not proceed to the end unless one of the resulting substances is removed from the sphere of action. But it can only be removed if it possesses different physical properties from those of the other substances which are present together with it. Either it will be a gas while the others are liquid or solid, or it will be an insoluble solid while the others are liquid or soluble. The relative amounts of the resultant substance lepend, if nothing be separated out from their mutual contact, only on the relative quantities of the substances MX and NY, and upon the measure of attraction existing between the elements $\mathrm{M}, \mathrm{N}, \mathrm{X}$, and $\mathrm{Y}$; but however great their mass may be, and however considerable the attraction, still in any case, if nothing be separated out from the sphere of action, the decomposition will cease, a state of equilibrium will be 
established, and instead of two there will remain four substances in the mass : namely, a portion of the original bodies MX and NY, and a certain quantity of the newly-formed substances MY and NX, if it be admitted that neither MN nor XY, nor any other substances, are produced, which may for the present ${ }^{24}$ be admitted in the case of the double decomposition of salts, for which $\mathrm{M}$ and $\mathrm{N}$ are metals and $\mathrm{X}$ and $\mathrm{Y}$ haloids. As the ordinary double decomposition consists exclusively in the exchange of metals, the above simplification is applicable in this case. The sum total of the existing data concerning the double decomposition of salts leads to the conclusion that from salts MX $+\mathrm{NY}$ there always proceeds a certain quantity of $\mathrm{NX}$ and MY, as it should be according to Berthollet's doctrine. A portion of the historical data concerning this subject will be afterwards mentioned, and we will now proceed to point out the observations made by Spring (1888), which show that even in a solid state salts are subject to a similar interchange of metals if under a condition of sufficiently close contact (which requires time, a finely-divided state, and intimate mixture). Spring took two non-hygroscopic salts, potassium nitrate, $\mathrm{KNO}_{3}$, and well-dried sodium acetate, $\mathrm{C}_{2} \mathrm{H}_{3} \mathrm{NaO}_{2}$, and left a mixture of their powders for several months in a desiccator. An interchange of metals took place, as was seen from the fact that the resultant mass vigorously attracted the moisture of the air owing to the formation of sodium nitrate, $\mathrm{NaNO}_{3}$, and potassium acetate, $\mathrm{C}_{2} \mathrm{H}_{3} \mathrm{KO}_{2}$, both of which are highly hygroscopic. ${ }^{24^{b}}$

When Berthollet enunciated his doctrine the present views of atoms and molecules had yet to be developed, but it is now necessary to submit the examination of the matter to these conceptions, and we will therefore consider the reaction of salts, taking $\mathrm{M}$ and $\mathrm{N}, \mathrm{X}$ and $\mathrm{Y}$ as

24 If $\mathrm{M} \mathrm{X}$ and $\mathrm{N} \mathrm{Y}$ represent the molecules of two salts, and if there be no third substance present (such as water in a solution), the formation of XY would also be possible ; for instance, cyanogen, iodine, \&c., are capable of combining with simple haloids, and with the complex groups which play the part of haloids in salts. Besides which the salts MX and NY or MY with NX may form double salts. If the number of molecules be unequal, or if the valency of the elements contained be different, as in $\mathrm{NaCl}+\mathrm{H}_{2} \mathrm{SO}_{4}$, where $\mathrm{Cl}$ is a univalent haloid and $\mathrm{SO}_{2}$ is bivalent, then the matter may be complicated by the formation of other compounds besides MY and NX, and when a solvent participates in the action, and especially if in a large proportion, then the phenomena must evidently become still more complex; and this is actually the case in reality. Therefore in placing before the reader a certain portion of the existing store of matter concerning the phenomena of double saline decompositions I cannot consider the theory of the subject as complete, and have therefore limited myself to a few data, the completion of which must be looked for, without losing sight of what has been said above, in more detailed works on the subject of theoretical chemistry.

$24 b$ When the mixture of potassium nitrate and sodium acetate was heated by Spring to $100^{\circ}$ it was completely fused into one mass, although potassium nitrate fuses at about $340^{\circ}$, and sodium nitrate at about $320^{\circ}$. 
equivalent to each other-that is, as capable of replacing each other ' in toto,' as $\mathrm{Na}$ or $\mathrm{K}, \frac{1}{2} \mathrm{Ca}$ or $\frac{1}{2} \mathrm{Mg}$ (bivalent elements), replace hydrogen.

And as, according to Berthollet's doctrine, when $m \mathrm{MX}$ of one salt comes into contact with $n \mathrm{NY}$ of another salt a certain quantity $x \mathrm{MY}$ and $x \mathrm{NX}$ is formed, therefore there remains $m-x$ of the salt $\mathrm{MX}$, and $n-x$ of the salt $\mathrm{NY}$. If $m$ be greater than $n$, and the mass of $\mathrm{M}$ and $\mathrm{N}$ and $\mathrm{X}$ and $\mathrm{Y}$ be equivalent, then the maximum interchange could lead to $x=n$, whilst from the salts taken there would ensue $n M Y+$ $n \mathrm{NX}+(m-n) \mathrm{MX}$ - that is, a portion of one of the salts taken would remain unchanged only because the reaction could only proceed between $n \mathrm{MX}$ and $n \mathrm{NY}$. If $x$ were actually equal to $n$ or 0 , the mass of the salt MX would not have any influence on the modus operandi of the reaction, which is essentially according to the teaching of Bergman, who supposed double reactions to be independent of the mass but determined by affinity only. If $\mathrm{M}$ had more affinity to $\mathrm{X}$ than to $\mathrm{Y}$, and $\mathrm{N}$ more affinity to $\mathrm{Y}$ than to $\mathrm{X}$, then, according to Bergman, there would be no decomposition whatever, and $x$ would equal 0 . If the affinity of $\mathrm{M}$ to $\mathrm{Y}$ and of $\mathrm{N}$ to $\mathrm{X}$ were greater than in the original grouping, then the affinities of $\mathrm{M}$ for $\mathrm{X}$ and of $\mathrm{N}$ for $\mathrm{Y}$ would act, and, according to Bergman's doctrine, complete interchange would take place-i.e., $x$ would equal $n$. According to Berthollet's teaching, a distribution of $\mathrm{M}$ and $\mathrm{N}$ between $\mathrm{X}$ and $\mathrm{Y}$ will take place in every case, not only in proportion to the measure of affinity, but also in proportion to the mass, so that with a small affinity and a large mass the same action can be produced as with a large affinity and a small mass. Therefore, (1) $x$ will always be less than $n$ and their ratio $\frac{x}{n}$ less than unity-that is, the decomposition will be expressed by the equation, $m \mathrm{MX}+n \mathrm{NY}=$ $(m-x) \mathrm{MX}+(n-x) \mathrm{NY}+x \mathrm{MY}+x \mathrm{NX} ;(2)$ by increasing the mass $m$. we increase the decomposition-that is, the measure of $x$ and the ratio $\frac{x}{(n-x)}$ - until with an infinitely large quantity $m$ the fraction $\frac{x}{n}$ will equal 1 and the fraction $\frac{x}{(n-x)}$ be infinite, and the decomposition will be complete, however small the affinities MY and NX may be; and (3) (if $m=n$ ) by taking $\mathrm{MX}+\mathrm{NY}$ or $\mathrm{MY}+\mathrm{NX}$ we arrive at one and the same system in both cases : $(n-x) \mathrm{MX}+(n-x) \mathrm{NY}+x^{\prime} \mathrm{MY}+x \mathrm{NX}$. These direct consequences of Berthollet's teaching are verified in reality. Thus, for example, a mixture of the solutions of sodium nitrate and potassium chloride in all cases has the same sum of properties as a mixture composed of the solutions of potassium nitrate and sodium chloride, naturally under the condition of the mixed solutions being of 
identical composition. But this similarity of properties may either proceed from the fact that one system of salts passes into the other (as Bergman professed), in conformity with the predominating affinities (for instance, from $\mathrm{KCl}+\mathrm{NaNO}_{3}$ there proceeds $\mathrm{KNO}_{3}+\mathrm{NaCl}$, if it be admitted that the affinity of the elements of the latter system be greater than in the former); or, on the other hand, because both systems by the interchange of a portion of their elements give one and the same state of equilibrium, as according to Berthollet's teaching. Experiment proves the latter. But before citing the most historically important experiments verifying Berthollet's doctrine, we must stop to consider the conception of the mass of the reacting substances. Berthollet by mass understood the direct relative quantity of substances; but now it is impossible to understand this term otherwise than as the number of molecules, for they act as chemical units, and in the special case of double saline decompositions it is better to take the number of equivalents. Thus in the reaction $\mathrm{NaCl}+\mathrm{H}_{2} \mathrm{SO}_{4}$, the salt is taken in one equivalent and the $\mathrm{H}_{2} \mathrm{SO}_{4}$ in two. If $2 \mathrm{NaCl}+\mathrm{H}_{2} \mathrm{SO}_{4}$ act, then the number of equivalents are equal, and so on. The influence of mass on the measure of decomposition $\frac{x}{n}$ forms the root of Berthollet's doctrine, and therefore we will first of all turn our attention to the establishment of this conception with regard to the double decomposition of salts.

About $1840 \mathrm{H}$. Rose showed that water decomposes metallic sulphides like calcium sulphide, CaS, forming hydrogen sulphide, $\mathrm{H}_{2} \mathrm{~S}$, notwithstanding the fact that the affinity of hydrogen sulphide, as an acid, for lime, $\mathrm{CaH}_{2} \mathrm{O}_{2}$, as a base, causes them to react on each other, forming calcium sulphide and water, $\mathrm{CaS}+2 \mathrm{H}_{2} \mathrm{O}$. Furthermore, Rose showed that the greater the amount of water acting on the calcium sulphide, the more complete is the decomposition. The results of this reaction are evident from the fact that the hydrogen sulphide formed may be expelled from the solution by heating, and that the resulting lime is sparingly soluble in water. Rose clearly saw from this that such feeble agents, in a chemical sense, as carbonic anhydride and water, by acting in a mass and for long periods of time in nature on the durable rocks, which resist the action of the most powerful acids, are able to bring about chemical change - to extract, for example, from rocks the bases, lime, soda, potash. The influence of the mass of water on antimonious chloride, bismuth nitrate, \&c., is essentially of the same character. These substances give up to the water a mass of acid which is greater according as the mass of the water acting on them is greater. ${ }^{25}$

25 Historically the influence of the mass of water was the first well-observed phenomenon in support of Berthollet's teaching, and it should not now be forgotten. In double 
Barium sulphate, $\mathrm{BaSO}_{4}$, which is insoluble in water, when fused with sodium carbonate, $\mathrm{Na}_{2} \mathrm{CO}_{3}$, gives, but not completely, barium carbonate, $\mathrm{BaCO}_{3}$ (also insoluble), and sodium sulphate, $\mathrm{Na}_{2} \mathrm{SO}_{4}$. If a solution of sodium carbonate acts on precipitated barium sulphate, then the same decomposition is also accomplished (Dulong, Rose), but it is restricted by a limit and requires time. A mixture of sodium carbonate and sulphate is obtained in the solution and a mixture of barium carbonate and sulphate in the precipitate. If the solution be decanted off and a fresh solution of sodium carbonate be poured over the precipitate, then a fresh portion of the barium sulphate passes into barium carbonate, and so by increasing the mass of sodium carbonate it is possible to entirely convert the barium sulphate into barium carbonate. If a definite quantity of sodium sulphate be added to the solution of sodium carbonate, then the sodium carbonate will have no action whatever on the barium sulphate, because then an equilibrated system, determined by the reverse action of the sodium sulphate on the barium carbonate and by the presence of the sodium carbonate and sulphate in the solution, is at once arrived at. On the other hand, if the mass of the sodium sulphate in the solution be great, then the barium carbonate is reconverted into sulphate until a definite state of equilibrium is attained between the reverse reactions, producing the barium carbonate by the aid of the sodium carbonate or the barium sulphate by the aid of the sodium sulphate.

Another most important conception of Berthollet's teaching consists in the existence of a limit of exchange decomposition, or in the attainment of a state of equilibrium. In this respect the determinations of Malaguti (1857) are historically the most important. He took a mixture of the solutions of equivalent quantities of two salts $M X$ and $\mathrm{NY}$, and judged the amount of the resulting exchange from the composition of the precipitate produced by the addition of alcohol. When, for example, zinc sulphate and sodium chloride $\left(\mathrm{ZnSO}_{4}\right.$ and $2 \mathrm{NaCl}$ ) were taken, then there were produced by exchange sodium sulphate and zinc chloride. A mixture of zinc sulphate and sodium sulphate was precipitated by an excess of alcohol, and it appeared from the composition of the precipitate that 72 per. cent. of the salts taken had been decomposed. When, however, a mixture of solutions of

decompositions taking place in dilute solutions where the mass of water is large, its inHuence, notwithstanding the weakness of affinities, must be great, according to the very spirit of Berthollet's doctrine.

As explaining the action of the mass of water, the experiments of Pattison Muir (1879) are very instructive. These experiments demonstrate that bismuth chloride is decomposed the more the greater the relative quantity of water, and the less the mass of hydrochioric acid forming one of the products of the reaction. 


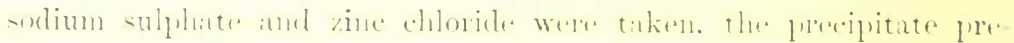

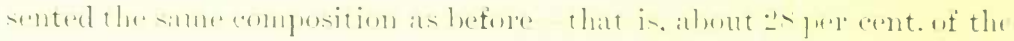

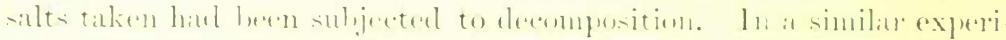

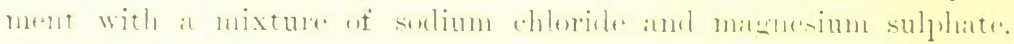

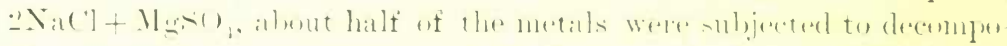

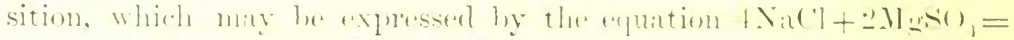

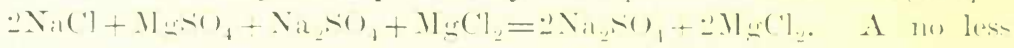

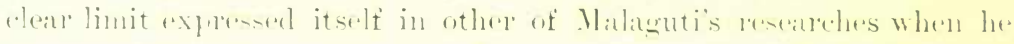

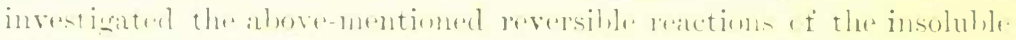

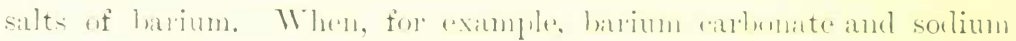

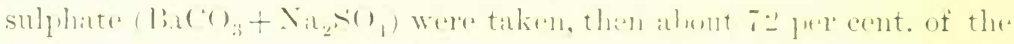

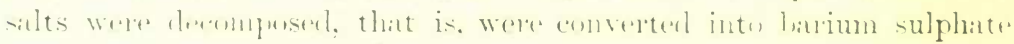

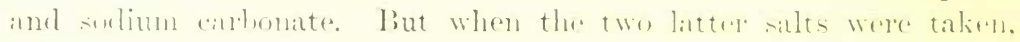

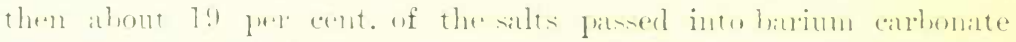

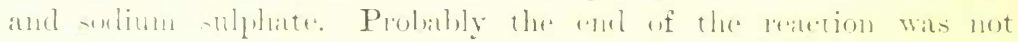

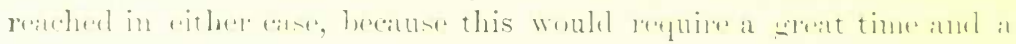

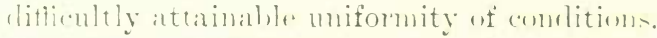

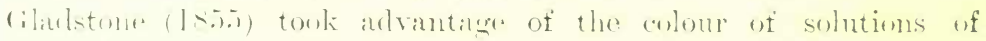

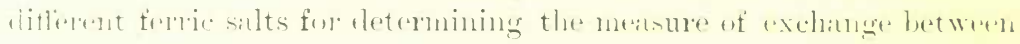
metals. Thus a solution of ferrice thioeganate has an exeecelingly

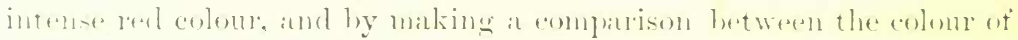

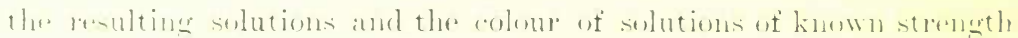

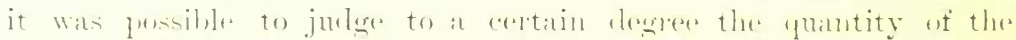

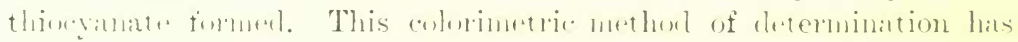
an ingultant signiticanere as lering the tirst in which a mether was alp-

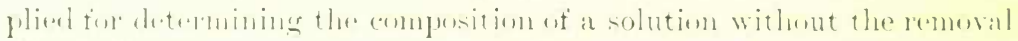

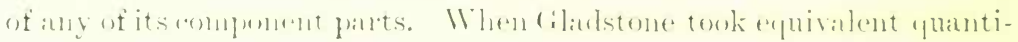

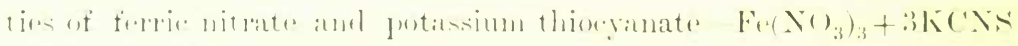

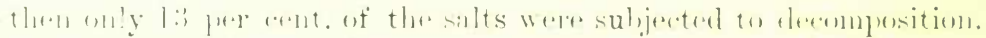
(1) inereateine the matse of the latter salt the quantity of forre thio-

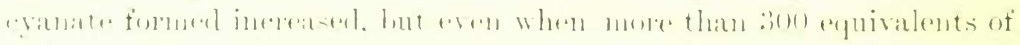

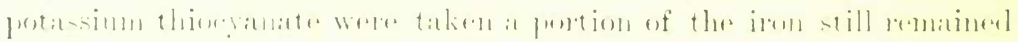

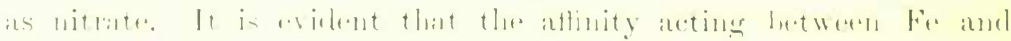

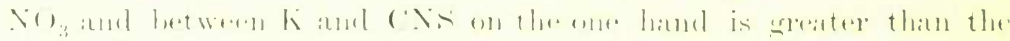

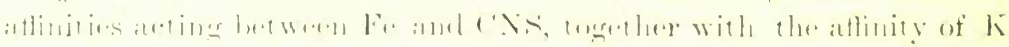

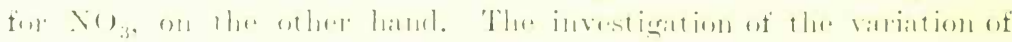

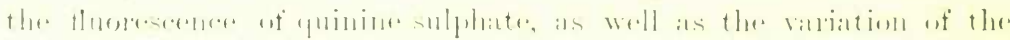

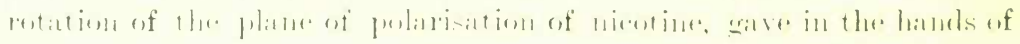

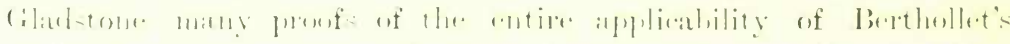

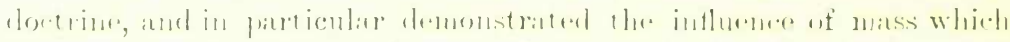

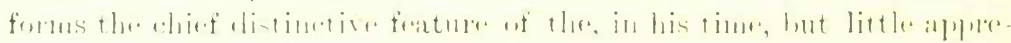
eiallel touthion of berthellet. 
At the beginning of the year 1860, the doctrine of the limit of reaction and of the influence of mass on the process of chemical transformations received a very important support in the researches of Berthelot and P. de Saint-Gilles on the formation of the ethereal salts RX from the alcohols $\mathrm{ROH}$ and acids $\mathrm{HX}$, when water is also formed. This conversion is essentially very similar to the formation of salts, but riffers in that it proceeds slowly at the ordinary temperature, extending over whole years and is not complete - that is, it has a distinct limit determined by a reverse reaction; thus an ethereal salt RX with water gives an alcohol $\mathrm{ROH}$ and an acid $\mathrm{HX}$ - up to that limit generally corresponding with two thirds of the alcohol taken, if the action proceed between molecular quantities of alcohol and acid. Thus common alcohol, $\mathrm{C}_{2} \mathrm{H}_{5} \mathrm{OH}$, with acetic acid, $\mathrm{HC}_{2} \mathrm{H}_{3} \mathrm{O}_{2}$, gives the following system rapidly when heated, or slowly at the ordinary temperature, $\mathrm{ROH}+\mathrm{HX}+2 \mathrm{RX}+2 \mathrm{H}_{2} \mathrm{O}$, whether we start from $3 \mathrm{RHO}+3 \mathrm{HX}$ or from $3 \mathrm{RX}+3 \mathrm{H}_{2} \mathrm{O}$. The process and completion of the reaction in the above-described instance are very easily observed, because the quantity of free acid is easily determined from the amount of alkali requisite for its saturation, as neither alcohol nor ethereal salt acts on litmus and other reagents for acids. Under the influence of an increased mass of alcohol the reaction proceeds further. If two molecules of alcohol, $\mathrm{RHO}$, be taken for every one molecule of acetic acid, $\mathrm{HX}$, then instead of 66 p.c., 85 p.c. of the acid passes into ethereal salt, and with fifty molecules of $\mathrm{RHO}$ nearly all the acid is etherised. The researches of Menschutkin in their details touched on many essential aspects of the same subject, such as the influences of the composition of the alcohol and acid on the limit and rate of exchange-but these, as well as other details, must be looked for in special treatises on organic and theoretical chemistry. In any case the study of etherification supplied chemical mechanics with clear and valuable data, which directly confirm the two fundamental propositions of Berthollet : the influence of mass, and the limit of reaction-that is, the equilibrium between opposite reactions. The study of numerous instances of dissociation which we have already touched on, and which we shall yet meet with on several occasions, gave the same results. With respect to double saline decompositions, it is necessary to further mention the researches of Wiedemann on the decomposing action of the mass of water on the ferric salts, which could be judged of by measuring the magnetism of the solutions, because the ferric oxide (soluble colloid) set free by the water is less magnetic than the ferric salts.

A very important epoch in the history of Berthollet's doctrine was attained when, in $186 \pi$, the Norwegian chemists, Guldberg and Waage, 


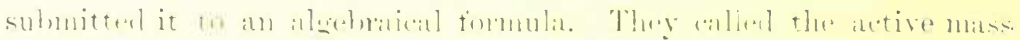

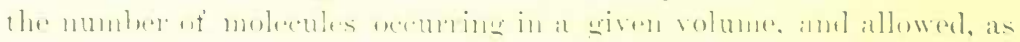

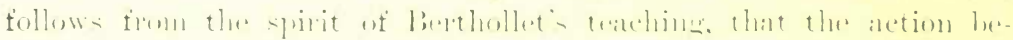

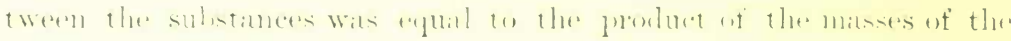

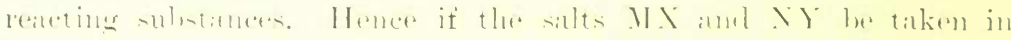

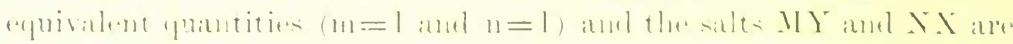

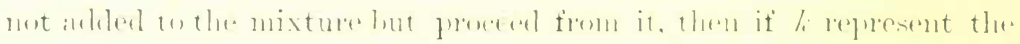

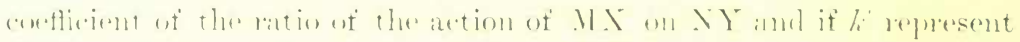

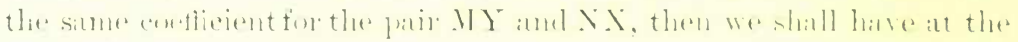

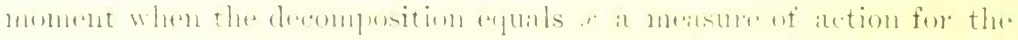

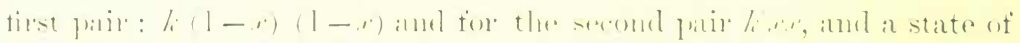

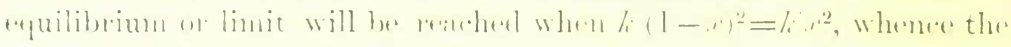

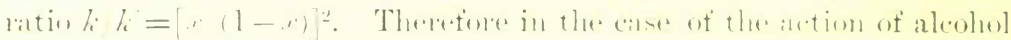

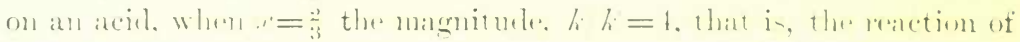

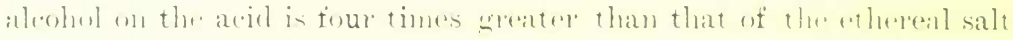

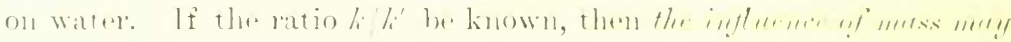

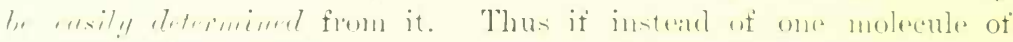

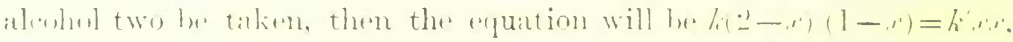

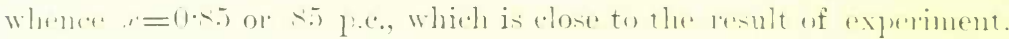

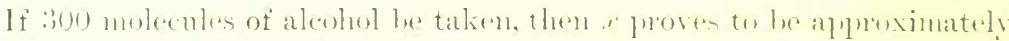

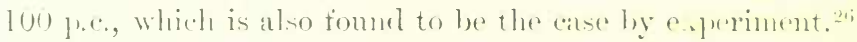

but it is impussilule to subject the formation of salles to any process

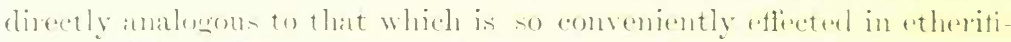

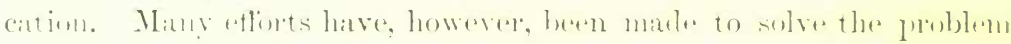
of the measure of reatetion in this ciste also. Thus, for eximulue.

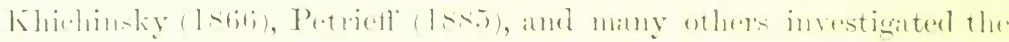

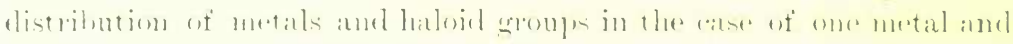

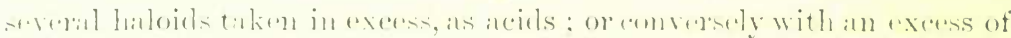

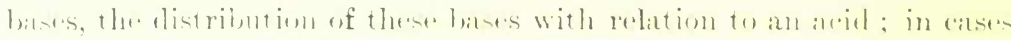

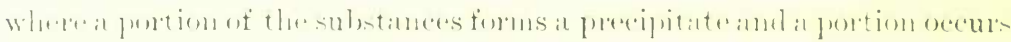

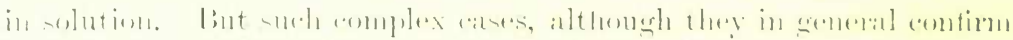

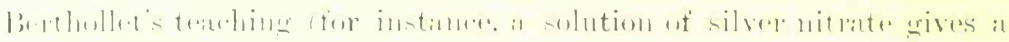

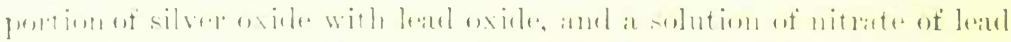

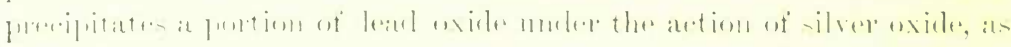

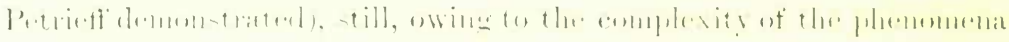

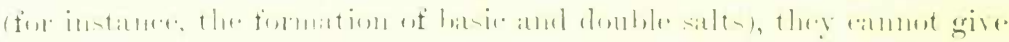

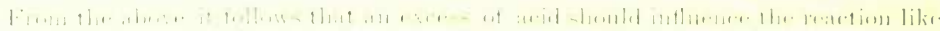

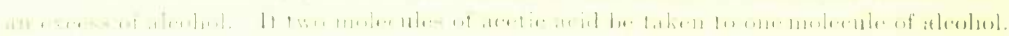

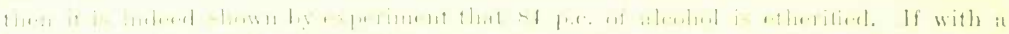

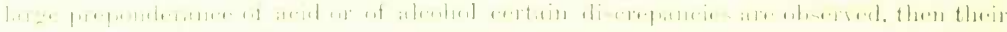

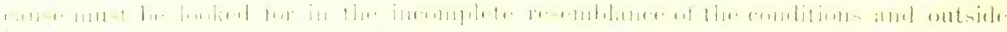
intitititi. 
simple results. But much more instructive and complete are researches similar to those made by Pattison Muir (1876), who took the simple case of the precipitation of calcium carbonate, $\mathrm{CaCO}_{3}$, by the mixture of solutions of calcium chloride and sodium or potassium carbonate, and found in this case that not only was the rate of action (for example, in the case of $\mathrm{CaCl}_{2}+\mathrm{Na}_{2} \mathrm{CO}_{3}, 75$ per cent. of $\mathrm{CaCO}_{3}$ was precipitated in five minutes, 85 per cent. in thirty minutes, and 94 in two days) determined by the temperature, relative mass, and amount of water (the mass of water decreases the rate and limit), but that the limit of decomposition was also dependent on these influences. However, even in researches of this kind the conditions of reaction are complicated by the non-uniformity of the media, inasmuch as a portion of the substance is obtained or remains in the precipitate, so that the system is heterogeneous. The investigation of double saline decompositions offers many difficulties, which cannot be considered as yet entirely overcome. Many efforts have, however, long since been made. In virtue of their historical interest, I will cite two of these efforts, which are due to Thomsen (1869) and Ostwald (1876).

Thomsen applied a thermo-chemical method to exceedingly dilute solutions without taking the water into further consideration. $\mathrm{He}$ took solutions containing $100 \mathrm{H}_{2} \mathrm{O}$ per $\mathrm{NaHO}$, and sulphuric acid containing $\frac{1}{2} \mathrm{H}_{2} \mathrm{SO}_{4}+100 \mathrm{H}_{2} \mathrm{O}$. If these solutions be mixed in such proportions that atomic proportions of acid and alkali would act, then for forty grams of caustic soda (which answers to its equivalent) there should be employed 49 grams of sulphuric acid, and then +15689 heat units would be evolved. If the normal sodium sulphate so formed be mixed with $n$ equivalents of sulphuric acid, then a certain amount of heat is absorbed, namely a quantity equal to $\frac{n .1650}{(n+0 \cdot 8)}$ heat units. An equivalent of caustic soda, in combining with an equivalent of nitric acid, evolves +13617 units of heat, and the augmentation of the amount of nitric acid entails an absorption of heat for each equivalent equal to -27 units ; so also in combining with hydrochloric acids +13740 heat units are absorbed, and for each equivalent of hydrochloric acid beyond this amount there are absorbed 32 heat units. Thus sulphuric acid evolves a somewhat greater quantity of heat than nitric and hydrochloric acids ; the difference being approximately equal to 2000 heat units per equivalent of caustic soda. From this it might be concluded that nitric acid or hydrochloric acid would not act on sodium sulphate. In reality, Thomsen found this not to be the case. Nitric and hydrochloric acids decompose sodium sulphate to a much more considerable extent than sulphuric acid decomposes sodium 
chloride or sodium nitrate. The following are the data from which Thomsen deduced this conclusion. He mixed any one of these three neutral salts with an acid which is not contained in it ; for instance, he mixed a solution of sodium sulphate with a solution of nitric acid and determined the number of heat units then absorbed. An absorption of heat ensued because a normal salt was taken in the first instance, and the mixture of all the above normal salts with acid produced an absorption of heat. The amount of heat absorbed enabled him to obtain an insight into the process taking place in this intermixture, for sulphuric acid added to sodium sulphate absorbs a considerable quantity of heat, whilst hydrochloric and nitric acids absorb a very small amount of heat in this case. By mixing an equivalent of sodium sulphate with various numbers of equivalents of nitric acid Thomsen observed that the amount of heat absorbed increases more and more as the amount of nitric acid was increased; thus when $\mathrm{HNO}_{3}$ was taken per $\frac{1}{2} \mathrm{Na}_{2} \mathrm{SO}_{4}$, 1752 heat units were absorbed per equivalent of soda contained in the sodium sulphate. When twice as much nitric acid was taken 2026 heat units, and when three times as much 2050 heat units were absorbed. Had the double decomposition been complete in this case when one equivalent of nitric acid was taken, then the heat evolved would be determined by the sum of $13617-15689-1650 / 1 \cdot 8$, or would equal -2989 heat units, if it be admitted that sulphuric acid when mixed with $\mathrm{NaNO}_{3}$ absorbs as much heat as when mixed with $\frac{1}{2} \mathrm{Na}_{2} \mathrm{SO}_{4}$. But as in reality only 1752 heat units were absorbed instead of 2989 units, therefore a displacement of only about two-thirds of the sulphuric acid had taken place-that is, the ratio $k: k^{\prime}$ for the reaction $\frac{1}{2} \mathrm{Na}_{2} \mathrm{SO}_{4}+\mathrm{HNO}_{3}$ and $\mathrm{NaNO}_{3}+\frac{1}{2} \mathrm{H}_{2} \mathrm{SO}_{4}$ is equal, as for ethereal salts, to 4 . By taking this figure and admitting the above supposition, Thomsen found that for all mixtures of sodium with nitric acid, and of sodium nitrate with sulphuric acid, the amounts of heat followed Guldberg and Waage's law ; that is, the limit of decomposition reached was greater the greater the mass of acid added. The relation of hydrochloric to sulphuric acid gave the same results. Thus on mixing $\frac{1}{2} \mathrm{Na}_{2} \mathrm{SO}_{4}$ with $\mathrm{HCl}$, the thermal result of experiment was -1682 , and hy calculation -1690 ; when mixed with $2 \mathrm{HCl}$ the experimental result was -1878 and by calculation -1870 , with $4 \mathrm{HCl}$ the result of experiment was -1896 and by calculation -1917 . When $\mathrm{NaCl}+{ }_{2}^{1} \mathrm{H}_{2} \mathrm{SO}_{4}$ was taken, then experiment showed an evolution of +244 heat units, whilst from calculation it should have been +257 heat units; on doubling the quantity of acid experiment gave +336 and calculation +292 . The slight differences between the results obtained by experiment and .calculation are due to the unavoidable errors of calorimetric determi- 


\section{nations. Therefore the researches of Thomsen fully confirm the bypotheses of Guldberg and Waage and the doctrine of Berthollet. ${ }^{27}$}

27 Thoonsen concludes his investigation with the words: $(a)$ 'When equivalent quantities of $\mathrm{NaHO}, \mathrm{HNO}_{3}$ (or $\mathrm{HCl}$ ) and $\frac{1}{2} \mathrm{H}_{2} \mathrm{SO}_{4}$ react on one another in an aqueous solution, then two-thirds of the soda combines with the nitric and one-third with the sulphuric acid; (b) this subdivision repeats itself, whether the soda be taken combined with nitric or with sulphuric acid; $(c)$ and therefore nitric acid has double the tendency to combine with the base that sulphuric acid has, and therefore in the wet way it is a stronger acid than the latter.'

'It is therefore necessary,' Thomsen afterwards remarks, 'to have an expression indicating the tendency of an acid for the saturation of bases. This idea cannot be expressed by the word affinity, because by this term is most often understood that force which it is necessary to overcome in order to decompose a substance into its component parts. This force should therefore be measured by the amount of work or heat employed for the decomposition of the substance. The above-mentioned phenomenon is of an entirely different nature,' and Thomsen introduces the term avidity, by which he designates the tendency of acids for neutralisation. 'Therefore the avidity of nitric acid with respect to soda is twice as great as the avidity of sulphuric acid. An exactly similar result is obtained with hydrochloric acid, so that its avidity with respect to soda is also double the avidity of sulphuric acid. Experiments conducted with other acids showed that not one of the acids investigated had so great an avidity as nitric acid; some had a greater avidity than sulphuric acid, others less, and in some instances the avidity $=0$.' The reader will naturally clearly see that the path chosen by Thomsen deserves being worked out, because his results concern important questions of chemistry, but great faith camnot be placed in the deductions as yet arrived at by Thomsen, because great complexity of relations is to be seen in the very method of his investigation. It is especially important to turn attention to the fact that all the reactions investigated are reactions of double decomposition. In them $\mathrm{A}$ and $\mathrm{B}$ do not combine with $\mathrm{C}$ and distribute themselves according to their affinity or avidity for combination, but reversible reactions are indnced. MX and NY give MY and NX, and conversely; therefore, the affinity or avidity for combination is not here directly determined, but only the difference or relation of the affinities or avidities. The affinity of nitric acid not only for the water of constitution, but also for that serving for solution, is much less than that of sulphuric acid. This is seen from thermal data. The reaction $\mathrm{N}_{2} \mathrm{O}_{5}+\mathrm{H}_{2} \mathrm{O}$ gives +3600 heat units, and the solution of the resultant hydrate, $2 \mathrm{NHO}_{3}$, in a large excess of water evolves +14986 heat units. The formation of $\mathrm{SO}_{3}+\mathrm{H}_{2} \mathrm{O}$ evolves +21308 heat units, and the solution of $\mathrm{H}_{2} \mathrm{SO}_{4}$ in an excess of water 17860 - that is, sulphuric acid gives more heat in both cases. The interchange between $\mathrm{Na}_{2} \mathrm{SO}_{4}$ and $2 \mathrm{HNO}_{3}$ is not only accomplished at the expense of the production of $\mathrm{NaNO}_{3}$, but also at the expense of the formation of $\mathrm{H}_{2} \mathrm{SO}_{4}$, hence the affinity of sulphuric acid for water plays its part in the phenomena of displacement. Therefore in determinations like those made by Thomsen the water does not form a medium which is present without participating in the process, but surely plays its own part. No less essential is the circumstance that sodium sulphate is able to combine with an excess of sulphuric acid to form the acid salt, whilst the salts of nitric and hydrochloric acids do not have this property. But with that method of interpretation of Guldberg and Waage's deduction which is given in the text, the adaptability of their formula to the phenomena observed by Thomsen may be expected, notwithstanding these incidental actions, although the idea of the relative tendencies of acids for combining with alkalis cannot be deduced from the coincidence of the results of experiment with those of calculation. If sodium oxide $\left(\mathrm{Na}_{2} \mathrm{O}\right)$ were brought into contact with sulphuric anhydride and nitric anhydride $\left(\mathrm{SO}_{3}\right.$ and $\left.\mathrm{N}_{2} \mathrm{O}_{5}\right)$ the distribution would probably be different, and even if stronger solutions than those employed by Thomsen were taken perhaps the result would be different, more especially judging from what is said in the following note. (Compare also Chapter IX. Note 14.)

VOL. I. 
Whilst retaining essentially the methods of Thomsen, Ostwald determined the variation of the sp. gr. (and afterwards of volume) proceeding in the same dilute solutions, on the saturation of acids by bases, and in the decomposition of the salts of one acid by the other, and arrived at conclusions of just the same nature as Thomsen did. Ostwald's method will be clearly understood from an example. A solution of caustic soda, containing an almost molecular (40 grams) weight per litre, had a specific gravity of $1 \cdot 04051$. The specific gravities of solutions of equal volume and equivalent composition of sulphuric and nitric acids were 1.02970 and 1.03084 respectively. On mixing the solutions of $\mathrm{NaHO}$ and $\mathrm{H}_{2} \mathrm{SO}_{4}$ there was formed a solution of $\mathrm{Na}_{2} \mathrm{SO}_{4}$ of sp. gr. 1.02959; hence there ensued a decrease of specific gravity which we will term $Q$, equal to $1 \cdot 04051+1 \cdot 02970-2(1 \cdot 02959)=0.01103$. So also the specific gravity after mixture of the solutions of $\mathrm{NaHO}$ and $\mathrm{HNO}_{3}$ was $1 \cdot 02633$, and therefore $\mathrm{Q}=0.01869$. When one volume of the solution of nitric acid was added to two volumes of the solution of sodium sulphate, a solution of sp. gr. 1.02781 was obtained, and therefore the resultant decrease of sp. gr.

$$
\mathrm{Q}_{1}=2(1 \cdot 02959)+1 \cdot 03084-3(1 \cdot 02781)=0.00659 \text {. }
$$

Had there been no chemical reaction between the salts, then according to Ostwald's reasoning the specific gravity of the solutions would not have changed, and if the nitric acid had displaced the sulphuric acid $Q_{2}$ would be $=0.01869-0.01103=0.00766$. It is evident that a portion of the sulphuric acid was displaced by the nitric acid. But the measure of displacement is not equal to the ratio between $Q_{1}$ and $Q_{2}$, because a decrease of sp. gr. also occurs on mixing the solution of sodium sulphate with sulphuric acid, whilst the mixing of the solutions of sodium nitrate and nitric acid only produces a slight variation of sp. gr. which falls within the limits of error of experiment. Ostwald deduces. from similar data the same conclusions as Thomsen, and thus reconfirms the formula deduced by Guldherg and Waage, and the teaching of Berthollet. ${ }^{28}$

28 The participation of water is seen still more clearly in the methods adopted by Ostwald than in those of Thomsen, because in the saturation of solutions of acids by alkalis (which Kremers, Reinhold, and others had previously studied) there is observed, not a contraction, as might have been expected from the quantity of heat which is then evolved, but an expansion, of volume (a decrease of specific gravity, if we calculate as Ostwald did in his first investigations). Thus by mixing 1180 grams of a solution of sulphuric acid of the composition $\mathrm{SO}_{3}+100 \mathrm{H}_{2} \mathrm{O}$, occupying a volume of 1815 c.c., with a corresponding quantity of a solution $2\left(\mathrm{NaHO}+5 \mathrm{H}_{2} \mathrm{O}\right)$, whose volume $=1793$ c.c., we obtain not 3608 but 3633 c.c., an expansion of 25 c.c. per gram molecule of the resulting salt, $\mathrm{Na}_{2} \mathrm{SO}_{4}$. It is the same in other cases. Nitric and hydrochloric acids give a still greater expansion than sulphuric acid, and potassium hydroxide than sodium hydroxide, 
The majority of the above-mentioned researches were carried on in aqueous solutions, and as water is itself a saline compound, and is able to combine with salts and enter into double decomposition with them, such reactions taking place in solutions in reality present very complex cases. In this sense the reaction between alcohols and acids is much more simple, and therefore its significance in confirmation of Berthollet's doctrine is of particular importance. The only cases which can be compared with these reactions from their simplicity are those exchange decompositions investigated by G. G. Gustavson, and which take place between $\mathrm{CCl}_{4}$ and $\mathrm{RBr}_{n}$ on the one hand and $\mathrm{CBr}_{4}$ and $\mathrm{RCl}_{n}$ on the the other. This case is convenient for investigation in the sense that the $\mathrm{RCl}_{n}$ and $\mathrm{RBr}_{n}$ taken belong (like $\mathrm{BCl}_{3}, \mathrm{SiCl}_{4}$, $\mathrm{TiCl}_{4}, \mathrm{POCl}_{3}$, and $\mathrm{SnCl}_{4}$ ) to the number of substances which are decomposed by water, whilst $\mathrm{CCl}_{4}$ and $\mathrm{CBr}_{4}$ are not decomposed by water ; and therefore, by heating, for instance, a mixture $\mathrm{CCl}_{4}+\mathrm{SiBr}_{4}$ it is possible to arrive at a conclusion as to the amount of interchange by treating the product with water, which decomposes the $\mathrm{SiBr}_{4}$ left unchanged and the $\mathrm{SiCl}_{4}$ formed by the exchange, and therefore by determining the composition of the product acted on by the water it is possible to form a conclusion as to the amount of decomposition.

whilst a solution of ammonia gives a contraction. The relation to water must be considered as the cause of these phenomena. When sodium hydroxide and sulphuric acid dissolve in water they develop heat and give a vigorous contraction; the water is separated from such solutions with great difficulty. After mutual saturation they form the salt $\mathrm{Na}_{2} \mathrm{SO}_{4}$, which retains the water but feebly and evolves but little heat with it, which, in a word, has little affinity for water. The water in the saturation of sulphuric acid by soda is, so to say, displaced from a stable combination and passes into an unstable combination; hence an expansion (decrease of sp. gr.) takes place. It is not the reaction of the acid on the alkali, but the reaction of water, that produces the phenomenon by which Ostwald desires to measure the degree of salt formation. The water, which escaped attention, itself has affinity, and influences those phenomena which are being investigated. Furthermore, in the given instance its influence is very great because its mass is large. When it is not present, or only present in small quantities, the affinity of the base to the acid leads to contraction, and not expansion. $\mathrm{Na}, \mathrm{O}$ has a sp. gr. $2 \cdot 8$, hence its volume $=22$; the sp. gr. of $\mathrm{SO}_{3}$ is 1.9 and volume 41 , hence the sum of their volumes is 63 , and for $\mathrm{Na}_{2} \mathrm{SO}_{4}$ the sp. gr. is $2 \cdot 65$ and volume $53 \cdot 6$, consequently a contraction of 10 c.c. per gram molecule of salt. The volume of $\mathrm{H}_{2} \mathrm{SO}_{4}=53 \cdot 3$, that of $2 \mathrm{NaHO}=37 \cdot 4$; there is produced $2 \mathrm{H}_{2} \mathrm{O}$, volume $=36+\mathrm{Na}_{2} \mathrm{SO}_{4}$, volume $=53 \cdot 6$. There react $90 \cdot 7$ c.c. , and on saturation there results $89^{\circ} 6$ c.c. ; consequently contraction again ensues, although less, and although this reaction is one of substitution and not of combination. Consequently the phenomena studied by Ostwald hardly depend on the measure of the reaction of the salts, but more on the relation of the substances dissolved to water. In substitutions, for instance, $2 \mathrm{NaNO}_{3}+\mathrm{H}_{2} \mathrm{SO}_{4}=2 \mathrm{HNO}_{3}+\mathrm{Na}_{2} \mathrm{SO}_{4}$, the volumes rary but slightly ; in the above example they are $2(38 \cdot 8)+53 \cdot 3$ and $2(41 \cdot 2)+53 \cdot 6$; lence 131 volumes act, and 136 volumes are produced. It may be thought, therefore, on the basis of what has been said, that on taking water into consideration the plenomena studied by Thomsen and Ostwald prove to be much more complex than they at first appear, and that this method can scarcely lead to a right judgment as to the distribution of acids between bases. 
The mixture was always formed with equivalent quantities - for instance, $4 \mathrm{BCl}_{3}+3 \mathrm{CBr}_{4}$. It appeared that there was no exchange whatever on simple intermixture, but that it proceeds, and then slowly, when the mixture is heated (for example with the above-cited mixture at $123^{\circ} 4.06$ per cent. of $\mathrm{Cl}$ was replaced by $\mathrm{Br}$ after 14 days' heating, and 6.83 per cent. after 28 days, and 10.12 per cent. when heated at $150^{\circ}$ for 60 days) A limit was always reached which corresponded with the complemental system ; for example, in the given instance to the system $4 \mathrm{BBr}_{3}+3 \mathrm{CCl}_{4}$. So in this last $89 \cdot 97$ per cent. of bromine in the $\mathrm{BBr}_{3}$ was replaced by chlorine ; that is, there was obtained 89.97 molecules of $\mathrm{BCl}_{3}$ and there remained 10.02 molecules of $\mathrm{BBr}_{3}$, and therefore the same state of equilibrium was reached as that given by the system $4 \mathrm{BCl}_{3}+3 \mathrm{CBr}_{4}$. Both systems gave one and the same state of equilibrium at the limit, as follows from Berthollet's doctrine. ${ }^{28 \mathrm{~b}}$

$28 \mathrm{~b}$ G. G. Gustarson's researches, which were conducted in the laboratory of the St. Petersburg University in 1871-72, are among the first in which the measure of the affinity of the elements for haloids appears with perfect clearness in the limit of substitution and in the rate of reaction. The researches conducted by $\mathrm{A}$. L. Potilitzin (of which mention will be made in Chapter XI. Note 66) in the same laboratory touch on another aspect of the same problem which has not yet made much progress, notwithstanding its importance and the fact that the theoretical side of the subject (thanks especially to Guldberg and Van't Hoff) has since been rapidly pushed forward. It would be very important if the researches of Gustavson touched on the influence of mass, and were more fully supplied with data concerning velocities and temperatures, because of the great significance which the instance considered has for the understanding of double saline decompositions in the ' absence of water.'

Furthermore, Gustavson showed that the greater the atomic weight of the element $(\mathrm{B}, \mathrm{Si}, \mathrm{Ti}, \mathrm{As}, \mathrm{Sn})$ combined with chlorine the greater the amount of chlorine replaced by bromine by the action of $\mathrm{CBr}_{4}$, and consequently the less the amount of bromine replaced by chlorine by the action of $\mathrm{CCl}_{4}$ on bromine compounds. For instance, for chlorine compounds the percentage of substitution (at the limit) is-

$\begin{array}{ccccc}\mathrm{BCl}_{5} & \mathrm{SiCl}_{4} & \mathrm{TiCl}_{4} & \mathrm{AsCl}_{3} & \mathrm{SnCl}_{4} \\ 10 \cdot 1 & 12 \cdot 5 & 43 \cdot 6 & 71 \cdot 8 & 77 \cdot 5\end{array}$

It should be observed, however, that Thorpe, on the basis of his experiments, denies the universality of this. I may mention one conclusion which it appears to me may be drawn from the ubove cited figures of Gustavson, if they further verify themselves even within narrow limits. If $\mathrm{CBr}_{4}$ be heated with $\mathrm{RCl}_{4}$, then an exchange of the bromine by chlorine takes place. But what would be the result if it were mixed with $\mathrm{CCl}_{4}$ ? Judging by the magnitude of the atomic weights, $\mathrm{B}=11, \mathrm{C}=12, \mathrm{Si}=28$, about 11 p.c. of the chlorine would be replaced by bromine. But what does this signify? I think that this shows the existence of a movement of the atoms in the molecule. The mixture of $\mathrm{CCl}_{4}$ and $\mathrm{CBr}_{4}$ does not remain in a state of static equilibrium; not only are the molecules contained in it in a state of movement but also the atoms in the molecules, and the above figures show the measure of their translation under these conditions. The bromine in the $\mathrm{CBr}_{4}$ is, within the limit, substituted by the chlorine of the $\mathrm{CCl}_{4}$ in a quantity of about 11 out of 100 ; that is, a portion of the atoms of bromine previously to this moment in combination with one atom of carbon pass over to the other atom of carbon, and the chlorine passes over from this second atom of carbon to replace it. Therefore, also, in the homogeneous mass $\mathrm{CCl}_{4}$ all the atoms of $\mathrm{Cl}$ do not remain constantly combined with the same atoms of 
Thus we now find ample confirmation from various quarters for the following rules of Berthollet, on referring them to double saline decompositions : 1. From two salts MX and NY containing different haloids and metals there result from their reaction two others, MY and NX, but such a substitution will not proceed to the end unless one product passes from the sphere of action. 2. This reaction is limited by the existence of an equilibrium between MX, NY, MY, and NX, because a reverse reaction is quite as possible as the direct reaction. 3. This limit is determined by both the measure of the active affinities and by the relative masses of the substances measured by the number of the reacting molecules. 4. Other conditions being constant, the chemical action is proportional to the product of the chemical masses in action..$^{29}$

Thus if the salts MX and NY after reaction partly formed salts MY and $\mathrm{NX}$, then a state of equilibrium is reached and the reaction ceases ; but if one of the resultant compounds, in virtue of its physical properties, passes from the sphere of action of the remaining substances, then the reaction will continue. This exit from the sphere of action depends on the physical properties of the substance and on the conditions under which the reaction takes place. Thus, for instance, the salt NX may, in the case of reaction between solutions, separate as a precipitate, is an insoluble substance, while the other three substances remain in solution, or it may pass into vapour, and in this manner also pass away from the sphere of action of the remaining substances. Let us now suppose that it passes away in some form or other from the sphere of action of the remaining substances - for instance, that it is transformed into a precipitate or vapour - then a fresh reaction will set in and a

carbon, and there is an exchange of atoms between different molecules in a homogeneous medium also. This hypothesis may, in my opinion, explain certain phenomena of dissociation, but in mentioning it I do not consider it possible to linger on it. I will only observe that a similar hypothesis suggested itself to me in my researches on solutions, and that Pfaundler essentially enunciated a similar hypothesis, and in recent times a like view is beginning to diffuse itself with respect to the electrolysis of saline solutions.

29 Berthollet's doctrine can hardly be in any way undermined when it is shown that there are cases in which there is no decomposition between salts, because in principle the affinity may be so small that a large mass would still give no observable displacements. The fundamental condition of the adaptability of Berthollet's doctrine, as well as Deville's doctrine, of dissociation lies in the reversibility of reactions. As there are practically unreversible reactions (for instance, $\mathrm{CCl}_{4}+2 \mathrm{H}_{2} \mathrm{O}=\mathrm{CO}_{2}+4 \mathrm{HCl}$ ), and as non-volatile substances do exist, so whilst accepting the doctrine of reversible reactions and retaining the doctrines of the evaporation of liquids, it is possible to admit the existence of nonvolatile substances, and in just the same way of reaction without any visible conformity to Berthollet's doctrine. This doctrine evidently approaches nearer than the opposite doctrine of Bergman to the solution of the complex problems of chemical mechanics, for the successful resolution of which at the present time the richest fruits are to be expected from the working out of data concerning dissociation, the influence of mass, and the equilibrium and velocity of reactions. 
reformation of the salt $\mathrm{NX}$. If it be removed, then, although the quantity of the elements $\mathrm{N}$ and $\mathrm{X}$ in the mass will be diminished, still, according to Berthollet's law, a certain amount of NX should be again formed. When this substance is again formed, then, owing to its physical properties, it will again pass away; hence the reaction, in consequence of the physical properties of the resultant substance, is able to proceed to completion notwithstanding the possible weakness of the attraction existing between the elements entering into the composition of the resultant substance NX. Naturally, if the resultant substance is also formed of elements having a considerable degree of affinity, then the complete decomposition is considerably facilitated.

Such a representation of the modus operandi of chemical transformations is applicable with great clearness to a number of reactions studied in chemistry, and, what is especially important, the application of this aspect of Berthollet's teaching does not in any way require the determination of the measure of affinity acting between the substances present. For instance, the action of ammonia on solutions of salts; the displacement, by its means, of basic hydrates soluble in water; the separation of volatile nitric acid by the aid of non-volatile sulphuric acid, as well as the decomposition of table salt by means of sulphuric acid, when gaseous hydrochloric acid is formed-may be taken as examples of reactions which proceed to the end, inasmuch as one of the resultant substances is entirely removed from the spliere of action, but they in no way indicate the measure of affinity. ${ }^{30}$

30 Common salt not only enters into double decomposition with acids but also with every salt. However, as clearly follows from Berthollet's doctrine, this form of decomposition will only in a few cases render it possible for new metallic chloricies to be obtained, because the decomposition will not be carried on to the end unless the metallic chloride formed separates from the mass of the active substances. Thus, for example, if a solution of common salt be mixed with a solution of magnesium sulphate, double decomposition ensues, but not completely, because all the substances remain in the solution. In this case the decomposition may result in the formation of sodium sulphate and magnesium chloride, subsitances which are soluble in water; nothing is disengaged, and therefore the decomposition $2 \mathrm{NaCl}+\mathrm{MgSO}_{4}=\mathrm{MgCl}_{2}+\mathrm{Na}_{2} \mathrm{SO}_{4}$ cannot proceed to the end. However, the sodium sulphate formed in this manner may be separated by freezing the mixture. Sulphate of sodium is sparingly soluble in the cold, and therefore if a sufficiently strong solution of common salt and magnesium sulphate be taken, the resulting sodium sulphate will separate out in the cold as crystals containing water of crystallisation. The complete separation of the sodium sulphate will naturally not take place, owing to a portion of the salt remaining in the solution in the cold. Nevertheless, this kind of decomposition is made use of for the preparation of sodium sulphate from the residues left after the evaporation of sea-water, which contain a mixture of magnesium sulphate and common salt. Such a mixture is encountered at Stassfurt in a natural form. The separation is sometimes carried on by means of artificial cooling by refrigerating machines. It might be suid that this form of double deconıposition is only accomplished with a change of temperature; but this would not be true, as may be concluded from other analogous cases. Thus, for instance, a solution of copper sulphate is of a blue 
As a proof that double decompositions like the above are actually accomplished in the sense of Berthollet's doctrine, the fact may be cited that table salt may be entirely decomposed by nitric acid, and nitre may be completely decomposed by hydrochloric acid, just as they are decomposed by sulphuric acid; but this only takes place when, in the first instance, an excess of nitric acid is taken, and in the second instance with in excess of hydrochloric acid for a given quantity of the sodium salt, and if the resultant acid passes off. If sodium chloride be put into a porcelain evaporating basin, nitric acid be added to it, and the mixture be heated, then both hydrochloric and nitric acids are expelled by the heat. Thus the nitric acid partially acts on the sodium chloride, but on heating, as both acids are volatile, they are both converted into vapour ; and therefore the residue will contain a mixture of a certain quantity 'of the sodium chloride taken and of the sodium nitrate formed. If a fresh quantity of nitric acid be then added, reaction will again set in, a certain portion of hydrochloric acid is again evolved, and on heating is expelled together with nitric acid. If this be repeated several times, it is possible to expel all the hydrochloric acid, and to obtain sodium nitrate only in the residue. If, on the contrary, we take sodium nitrate and add hydrochloric acid to it in an aqueous solution, then reaction again sets in, a certain quantity of the hydrochloric acid displaces a portion of the nitric acid, and on heating the excess of hydrochloric acid passes away with the nitric acid formed. On repeating this process, it is possible to displace the nitric acid with an excess of hydrochloric acid, just as it was possible to displace the hydrochloric acid by an excess of nitric acid. The influence of the mass of the substance in action and the influence of volatility is here very distinctly

colour, while a solution of copper chloride is green. If we mix both salts together the green tint is distinctly visible, so that by this means the presence of the copper chloride in the solution of copper sulphate is clearly seen. If now we add a solution of common salt to a solution of copper sulphate a green coloration is obtained, which indicates the formation of copper chloride. In this instance it is not separated, but it is immediately formed on the addition of common salt, as it should be according to Berthollet's doctrine.

The complete formation of a metallic chloride from common salt can only occur, judging from the above, when it separates from the sphere of action. The salts of silver are instances in question, because the silver chloride is insoluble in water; and therefore if we add a solution of sodium chloride to a solution of a silver salt, then silver chloride and the sodium salt of that acid which was in the silver salt are formed. The amount of silver chloride formed immediately separates from the solution, because it is insoluble in water, and the reaction in consequence is further directed to the end-that is, either the whole quantity of the silver is separated or all the chlorine passes into silver chloride. This method is made use of for separating silver from its solutions, and also for determining the quantity of chlorine. It must not, however, be forgotten that silver chloride is slightly soluble in water and still more so in a solution of sodium chloride, and that, therefore, a certain trace of silver escapes precipitation. 
seen. Hence it may be affirmed that sulphuric acid does not displace hydrochloric acid because of an especially high degree of affinity, but that this reaction is only carried on to the end because the sulphuric acid is not volatile whilst the hydrochloric acid which is formed is volatile.

The preparation of hydrochloric acid in the laboratory and on a large scale is based upon these data. In the first instance, an excess of sulphuric acid is employed in order that the reaction may proceed easily and at a low temperature, whilst on a large scale, when it is necessary to economise every material, equivalent quantities are taken in order to obtain the normal salt $\mathrm{Na}_{2} \mathrm{SO}_{4}$ and not the acid salt (p. 422), which would require twice as much acid. It may be remarked that dry hydrochloric acid is a gas which is very soluble in water. It is most frequently used in practice in this state of solution under the name of muriatic acid. ${ }^{31}$

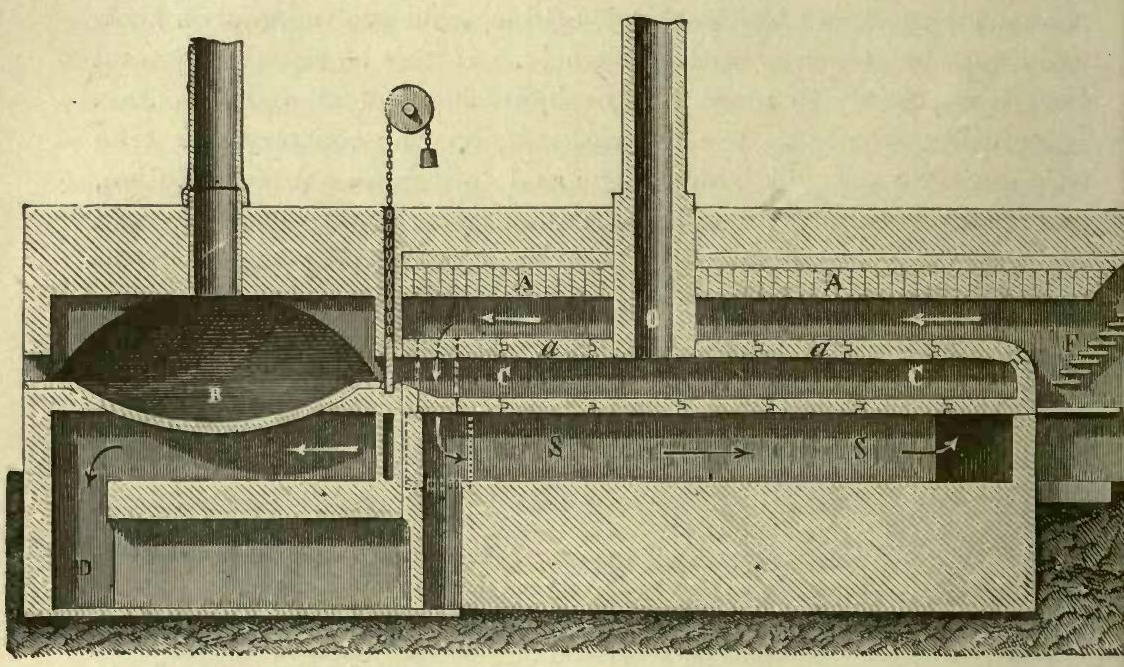

F1G. 65.- Secticn of a salt cake furnace. B, pan in which the solium chloride and sulpluric acid are first mixed and heated. C, muffle for the ultimate decomposition.

In chemical works the decomposition of sodium chloride by means of sulphuric acid is carried on on a very large scale, chiefly with a view to the preparation of normal sodium sulphate, the hydrochloric acid being a bye-product. The furnace employed is termed a salt cake furnace. It is represented in fig. 65 , and consists of the following two parts : the

31 The apparatus shown in fig. 46 (p. 250) is generally employed for the preparation of small quantities of hydrochloric acid. Common salt is placed in the retort; the salt is generally previously fused, as it otherwise froths and boils over the apparatus. When the 
pan B and the roaster C, or enclosed space built up of large bricks $a$. and enveloped on all sides by the smoke and flarnes from the fire grate, F. The ultimate decomposition of the salt by the sulphuric acid is accomplished in the roaster. The hydrochloric acid gas evolved in this reaction escapes through the tube $\mathrm{O}$ as will presently be described. But the first decomposition of sodium chloride by sulphuric acid does not require so high a temperature as the ultimate decomposition, and is therefore carried on in the front and cooler portion, B, whose bottom is heated by gas flues. When the reaction in this portion ceases and the evolution of hydrochloric acid stops, then the mass, which contains about half of the sodium chloride yet undecomposed and the sulphuric acid in the form of acid sodium sulphate, is removed from $\mathrm{B}$ and thrown into the roaster $\mathrm{C}$, where the action is completed. Normal sodium sulphate, which we shall afterwards describe, remains in the roaster. It is employed both directly in the manufacture of glass, and in the preparation of other sodium compounds-for instance, in the preparation of soda ash, as will afterwards be described. For the present we will only turn our attention to the hydrochloric acid evolved in B and C. In chemical works, where sulphuric acid of $60^{\circ}$ Baumé (22 p. c. of water) is employed, 117 parts of sodium chloride are taken to about 125 parts of sulphuric acid.

The hydrochloric acid gas evolved is subjected to condensation by dissolving it in water. ${ }^{32}$ If the apparatus in which the decomposition apparatus is placed in order sulphuric acid mixed with water is poured down the thistle funnel into the retort. About one and a half times the weight of the salt of strong sulphuric acid is usually taken, and it is diluted with a small quantity of water (half) if it be desired to retard the action, as in using strong sulphuric acid the action immediately begins with great vigour. The mixture, at first without the aid of heat and then at a moderate temperature (in a water-bath), evolves hydrochloric acid. Commercial hydrochloric acid contains many impurities; it is usually purified by distillation, the middle portions being collected. It is purified from arsenic by adding $\mathrm{FeCl}_{2}$, distilling, and casting aside the first third of the distillate. If free hydrochloric acid gas be required, it is passed through a ressel containing strong sulphuric acid to dry it, and it is collected over a mercury bath.

Phosphoric anhydride absorbs hydrogen chloride (Bailey and Fowler, 1888; $\left.2 \mathrm{P}_{2} \mathrm{O}_{5}+3 \mathrm{HCl}=\mathrm{POCl}_{3}+3 \mathrm{HPO}_{5}\right)$ at the ordinary temperature, and therefore the gas cannot be dried by this substance.

32 As at works which treat common salt in order to obtain sodium sulphate, the hydrochloric acid is sometimes held in no value, they would readily allow it to escape into smoke and into the atmosphere, which would greatly injure the air of the neighbourhood and destroy all vegetation, and therefore in all countries there are laws forbidding the works to proceed in this manner, and requiring the absorption of the hydrochloric acid by water at the works themselves, and not permitting the solution to be run into rivers and streams, whose waters it would spoil. It may be remarked that the absorption of hydrochloric acid presents no particular difficulties (the absorption of sulphurous acid is much more difficult), because hydrochloric acid has a great affinity for water and gives a hydrate which boils above $100^{\circ}$. Hence, even steam and hot water, as well as weaker solutions, can be used for absorbing the acid. However, Warder (1888) showed that weak 
is accomplished were hermetically closed, and only presented one outlet, then the escape of the hydrochloric acid would only proceed through the escape pipe intended for this purpose. But as it is impossible to construct a perfectly hermetically closed furnace, it is necessary to increase the draught by artificial means, or to oblige the hydrochloric acid gas to pass through those arrangements in which it is condensed. This is done by connecting the ends of the tubes through which the hydrochloric acid gas escapes from the furnace with high chimneys, where the strong draught proceeding from the combustion of the fuel carries off the hydrochloric acid and air which is mixed with it. This causes a current of hydrochloric acid gas to pass through the absorbing apparatus in a definite direction. Here it encounters a current of water flowing in the opposite direction, by which it is absorbed. It is not customary to cause the acid to pass through the water, but only to bring it into contact with the surface of the water. The absorption apparatus consists of large earthenware vessels having four orifices, two above and two lateral ones in the wide central portion of each vessel. The upper orifices serve for connecting the vessels together, and the hydrochloric acid gas escaping from the furnace passes through these tubes. The water for absorbing the acid enters at the upper, and flows out from the lower, vessel, and passes through the lateral orifices in the vessels. The water flows from the chimney towards the furnace, and it is therefore evident that the outflowing water will be the most saturated with acicl, of which it actually contains about 20 per cent. The absorption in these vessels is not complete. The ultimate absorption of the hydrochloric acid is carried on in the so-called coke towers. These are high chimneys, like that shown in fig. 66. They are frequently divided into two portions, or consist of two adjacent chimneys. A lattice work of bricks, C, is laid on the bottom of these towers, on which coke is piled up to the top of the tower. Water, distributing itself over the coke, trickles down to the bottom of the tower, and in so doing absorbs the hydrochloric acid gas rising upwards.

It will be readily understood that hydrochloric acid may be

solutions of composition $\mathrm{H}_{2} \mathrm{O}+n \mathrm{HCl}$ when boiled (the residue will be almost $\mathrm{HCl}, 8 \mathrm{H}_{2} \mathrm{O}$ ) evolve (not water but) a solution of the composition $\mathrm{H}_{2} \mathrm{O}+445 n^{4} \mathrm{HCl}$; for example, on distilling $\mathrm{HCl}, 10 \mathrm{H}_{2} \mathrm{O}, \mathrm{HCl}, 23 \mathrm{H}_{2} \mathrm{O}$ is first obtrined in the distillate. As the strength of the residue becomes greater, so also does that of the distillate, and therefore in order to completely absorb hydrochloric acid it is necessary in the end to have recourse to water.

As in Russia the manufacture of sodium sulphate from sodium chloride has not yet been sufficiently developed, and as hydrochloric acid is required for many technical purposes (for instance, for the preparation of zinc chloride, which is employed for soaking railway sleepers), therefore the salt is often treated mainly for the manufacture of hydrochloric acid. 
obtained from all other metallic chlorides. ${ }^{33}$. It is

35 Thus the metallic chloricles, which are decomposed to a greater or less degree by water,correspond with feeble bases. Such are, for example, $\mathrm{MgCl}_{2}, \mathrm{AlCl}_{3}, \mathrm{SbCl}_{3}$, $\mathrm{BiCl}_{3}$. The decomposition of magnesium chloride (and also carnallite) by sulphuric acid proceeds at the ordinary temperature and may be employed as a convenient method for the production of hydrochloric acid. The vapour of common salt in the presence of steam and silica (which is capable of combining with sodium oxide) gives hydrochloric acid and sodium silicate. Hydrochloric acid is also produced in many reactions of decomposition of carbon compounds containing chlorine and hydrogen; for instance, when the vapour of ethyl chloride is passed through a redhot tube, it gives olefiant gas and hydrochloric acid. It is also produced by the ignition of certain metallic chlorides in a stream of hydrogen, especially of those metals which are easily reduced and difficultly oxidised-for instance, silver chloride. Lead chloride, when heated to redness in a stream of steam, gives hydrochloric acid and lead oxide. In general $2 \mathrm{MCl}_{n}+n \mathrm{H}_{2} \mathrm{O}$ frequently gives $\mathrm{M}_{2} \mathrm{O}_{n}+2{ }_{n} \mathrm{HCl}$, and $\mathrm{MCl}_{n}+\mathrm{H}_{n}$ sometimes $\mathrm{MI}+n \mathrm{HCl}$, although both these reactions also proceed in the opposite direction, that is, $\mathrm{M}_{2} \mathrm{O}_{n}+n 2 \mathrm{HCl}$ frequently forms $2 \mathrm{MCl}_{n}+n \mathrm{H}_{2} \mathrm{O}$ and sometimes $\mathrm{M}+n \mathrm{HCl}=\mathrm{MCl}_{n}+$ $\mathrm{H}_{n}$, where $\mathrm{M}$ is a metal. The multitude of the cases of formation of hydrochloric acid are understood from the fact that it is a substance which is comparatively very stable, resembling water in this respect, and even most probably more stable than water, because, at a high temperature and even under the action of light, chlorine decom-
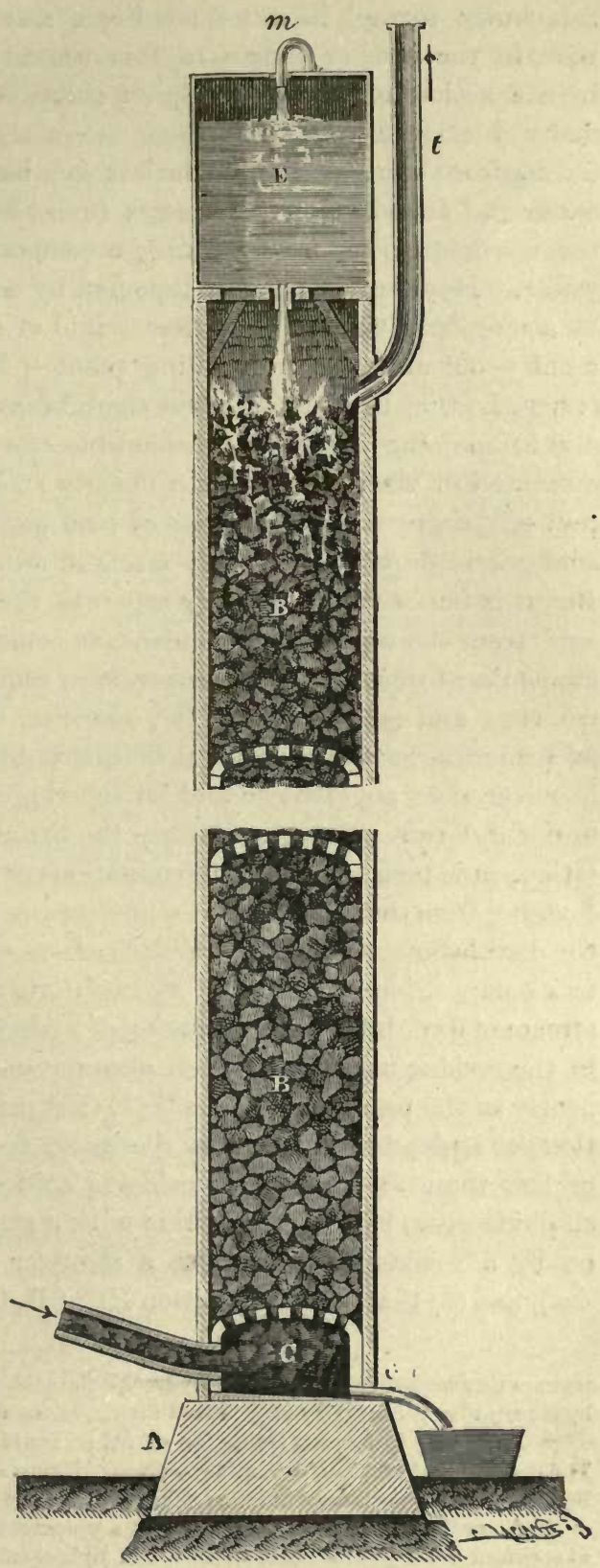

FIa. 66.-Coke tower. 


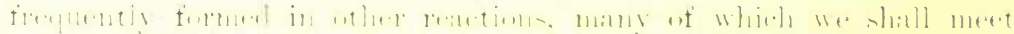

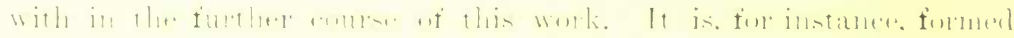

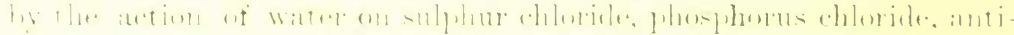

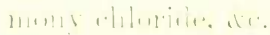

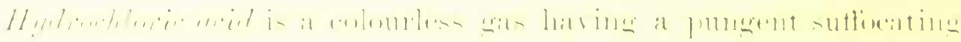

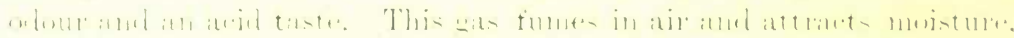

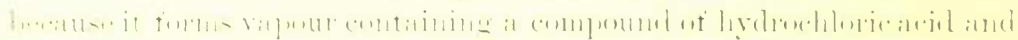

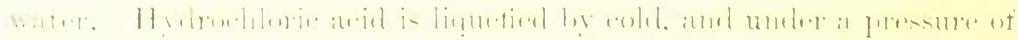

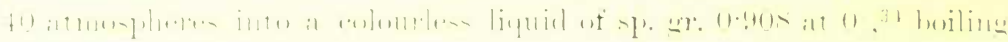

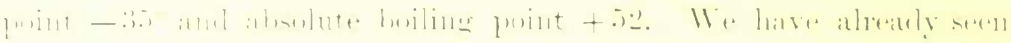

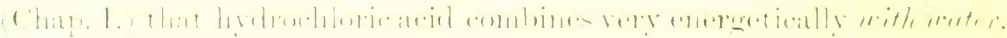

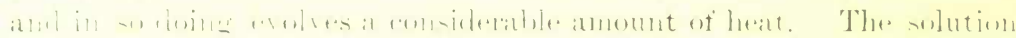

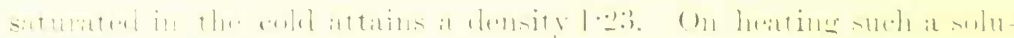

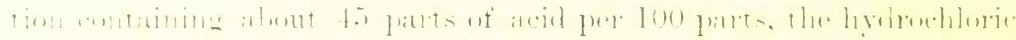

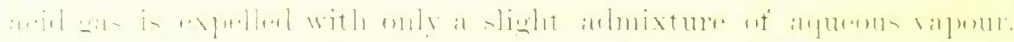

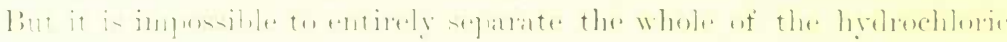

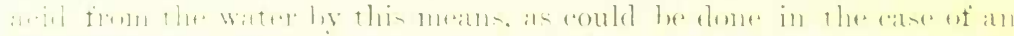

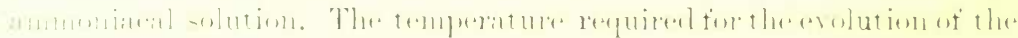

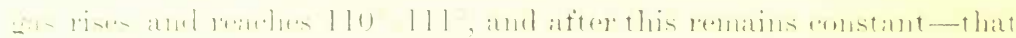

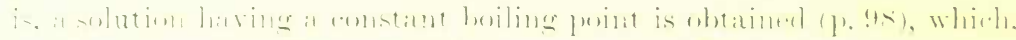

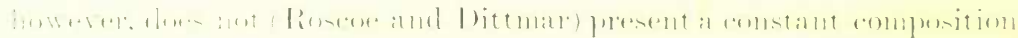

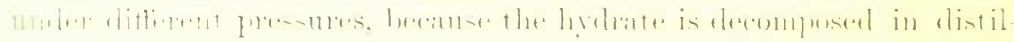

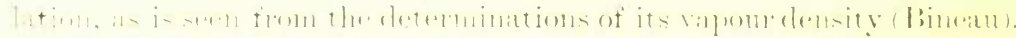

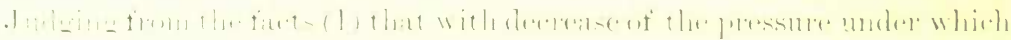

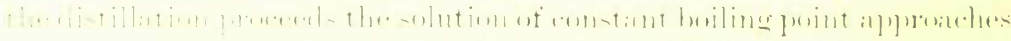

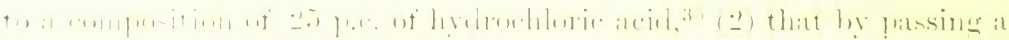

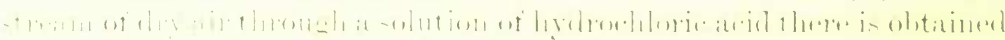

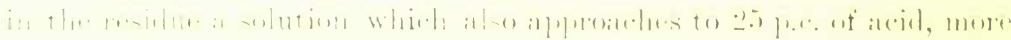

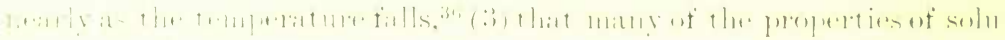

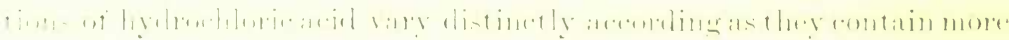

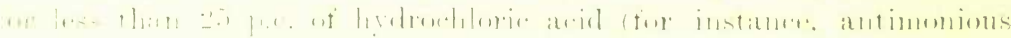

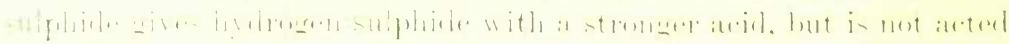

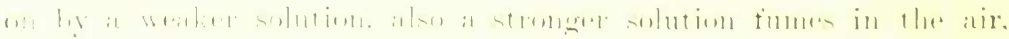

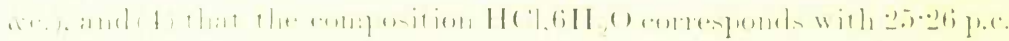

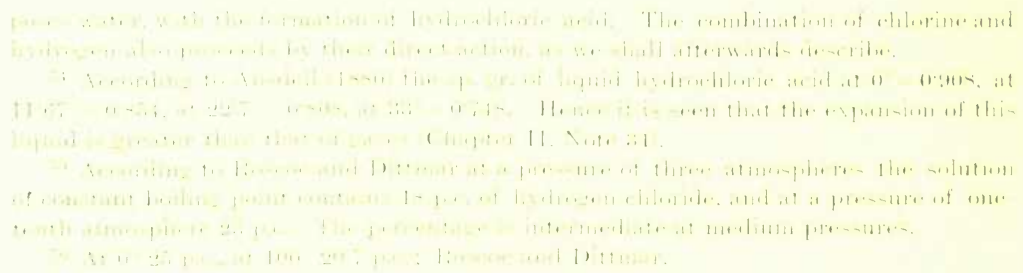


$\mathrm{HCl}$-judging from all these data, and also from the loss of tension which ocours in the combination of hydrochloric acid with water, it may be said that they form a definite hydrate of the composition $\mathrm{HCl}, 6 \mathrm{H}_{2} \mathrm{O}$. The conclusions to be drawn from the densities of solutions of hydrochloric acid also point to the same fact, as will presently be explained. Besides this hydrate there exists also a crystallo-hydrate $\mathrm{HCl}, 2 \mathrm{H}_{2} \mathrm{O},{ }^{37}$ which is formed by the absorption of hydrochloric acid by a saturated solution at a temperature of $-23^{\circ}$. It crystallises and melts at $-18^{\circ} .^{38}$

si This crystallo-hydrate (obtained by Pierre and Puchot, and investigated by Roozeboom) is analogous to $\mathrm{NaCl}, 2 \mathrm{H}_{2} \mathrm{O}$. The crystals $\mathrm{HCl}, 2 \mathrm{H}_{2} \mathrm{O}$ at $-22^{\circ}$ have a specific gravity $1 \cdot 46$, the vapour tension (dissociation) of the solution having a composition $\mathrm{HCl}, 2 \mathrm{H}_{2} \mathrm{O}$ at $-24^{\circ}=760$, at $-19^{\circ}=1010$, at $-18^{\circ}=1057$, at $-17^{\circ}=1112 \mathrm{~mm}$. of mercury. In a solid state the crystallo-hydrate at $-17.7^{\circ}$ has the same tension, whilst at lower temperatures it is much less: at $-24^{\circ}$ about 150 , at $-19^{\circ}$ about $580 \mathrm{~mm}$., at $-17 \cdot 2^{\circ}$ about 10 atmo spheres ; at $-13^{\circ}$ about 150 atmospheres. A mixture of fuming hydrochloric acid with snow reduces the temperature to $-38^{\circ}$.

58 According to Roscoe at $0^{\circ}$ a hundred grams of water at a pressure $p$ (in millimetres of mercury) dissolves-

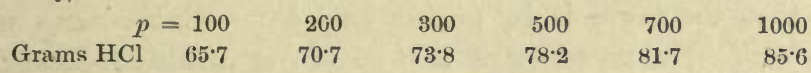

At a pressure of 760 millimetres and temperature $t$, a hundred grams of water dissolves

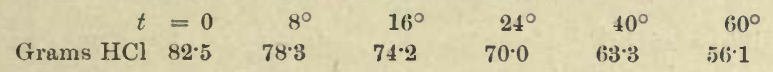

Roozeboom (1886) showed that at $t^{\circ}$ solutions containing $c$ grams of hydrogen chloride per 100 grams of water may (with the variation of the pressure $p$ ) be formed together with the crystallo-hydrate $\mathrm{HCl}, 2 \mathrm{H}_{2} \mathrm{O}$ :

$\begin{array}{lrrrc}t=-28 \cdot 8^{\circ} & -21^{\circ} & -19^{\circ} & -18^{\circ} & +17 \cdot 7^{\circ} \\ c=84.2 & 86.8 & 92.6 & 98.4 & 101 \cdot 4 \\ p=-1 & 334 & 580 & 900 & 1073 \mathrm{~mm} .\end{array}$

The last composition answers to the melted crystallo-hydrate $\mathrm{HCl}, 2 \mathrm{H}_{2} \mathrm{O}$, which splits up at temperatures above $-177^{\circ}$, and at a constant atmospheric pressure when there are no crystals-

$$
\begin{array}{lrrrc}
t=-24^{\circ} & -21^{\circ} & -18^{\circ} & -10^{\circ} & 0 \\
c=.101^{\circ} 2 & 98^{\circ} 3 & 95 \cdot 7 & 89 \cdot 8 & 84 \cdot 2
\end{array}
$$

From these data it is seen that the hydrate $\mathrm{HCl}, 2 \mathrm{H}_{2} \mathrm{O}$ can exist in a liquid state, which is not the case for the hydrates of carbonic and sulphurous anhydrides, chlorine, \&c.

According to Marignac, the specific heat $c$ of a solution $\mathrm{HCl}+m \mathrm{H}_{2} \mathrm{O}$ (at about $30^{\circ}$, taking the specific heat of water $=1$ ) is given by the expression-

$$
\mathrm{C}(36 \cdot 5+m 18)=18 m-28 \cdot 39+140 / m-268 / m^{2}
$$

if $m$ be not less than 6.25 . For example, for $\mathrm{HCl}+25 \mathrm{H}_{2} \mathrm{O} \mathrm{C}=0.877$.

According to Thomsen's data, the amount of heat $Q$, expressed in thousands of calories, evolved in the solution of 36.5 grams of gaseous hydrochloric acid in $m \mathrm{H}_{2} \mathrm{O}$ or $18 \mathrm{~m}$ grams of water-

$$
\begin{array}{lcccc}
m=2 & 4 & 10 & 50 & 400 \\
Q=11 \cdot 4 & 14 \cdot 3 & 16 \cdot 2 & 17 \cdot 1 & 17 \cdot 3
\end{array}
$$

In these quantities the latent heat of liquefuction is included, which, judging by analogy (page 321), must be taken as 5-9 thousand calories per molecular quintity of hydrogen chloride.

The researches of Scheffer (1888) on the rate of diffusion (in water) of solutions of 


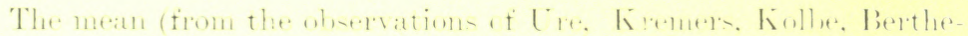

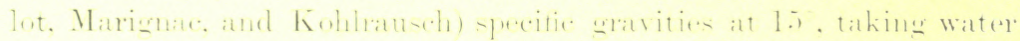

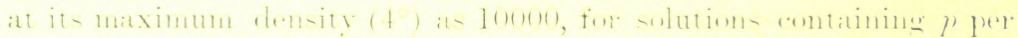

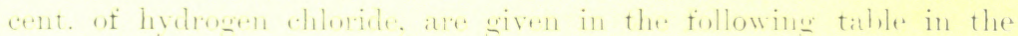

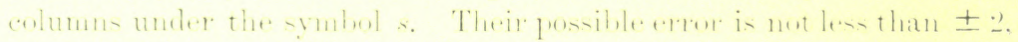

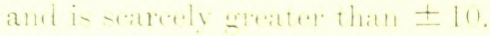

\begin{tabular}{|c|c|c|c|c|c|}
\hline I. & $s$ & $\therefore$ & 11 & $s$ & $s$ \\
\hline$\therefore$ & $1101=$ & $11 \div+10$ & 25 & $11266 ;$ & $11 \div 6: 3$ \\
\hline 10 & 1114901 & 1114512 & $: 11$ & 11.7 .0 & 11.7 .12 \\
\hline $1 . i$ & $1117+1$ & $117+6$ & 3.5 & $1177: 3$ & 11770 \\
\hline$\therefore 1$ & $1 \mid 10111$ & $110110: 3$ & $f(1$ & 115197 & 1200.5 \\
\hline
\end{tabular}

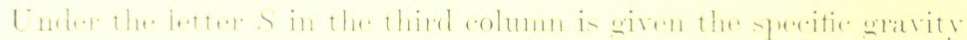

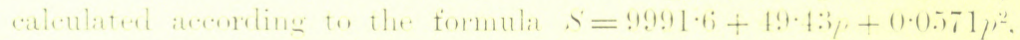

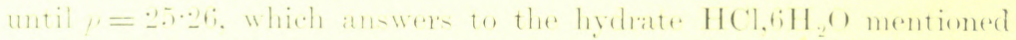

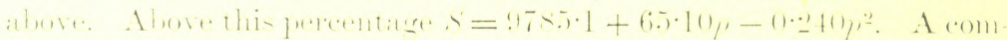

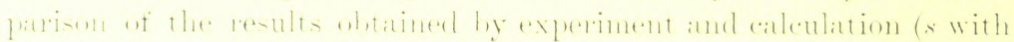

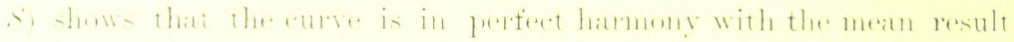

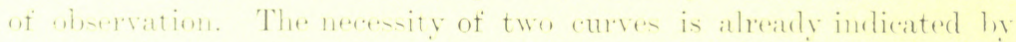

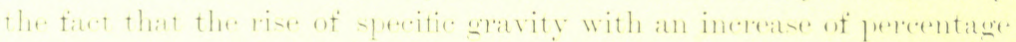

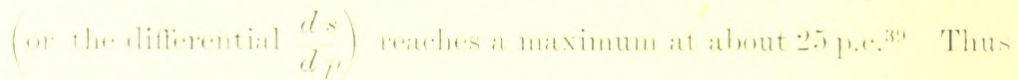

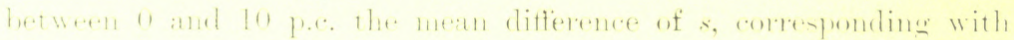

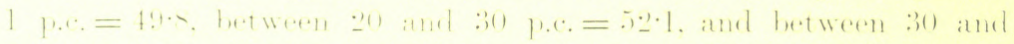

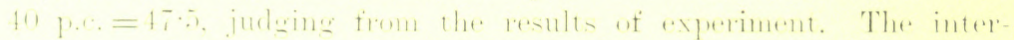

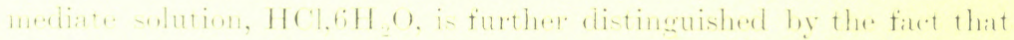

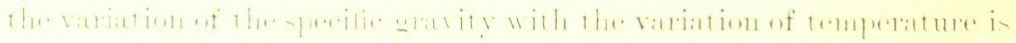

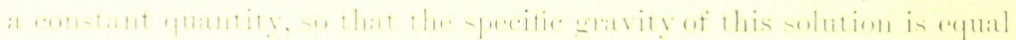

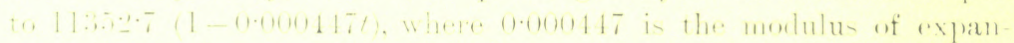

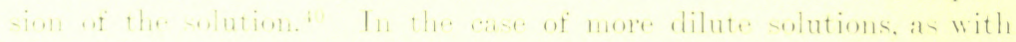

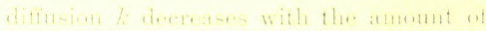
$11,1,11,+11:$

\begin{tabular}{|c|c|c|c|c|}
\hline & $A ;$ & $\because \cdots$ & 11 & 2,1 \\
\hline $2 \ldots, i 1$ & & 1 thit & $17 i 7$ & $1 \ldots 2$ \\
\hline
\end{tabular}


water, the specific gravity at $1^{\circ}\left(\right.$ or the differential $\left.\frac{d s}{d t}\right)$ rises with a rise of temperature, for the difference between $S_{0}-S_{15}$ and $S_{15}-S_{30}$ (i.e. the difference between the specific gravities at $0^{\circ}, 15^{\circ}$, and $30^{\circ}$ ) varies as follows : ${ }^{41}$

$\begin{array}{rccccc}p & =0 & 5 & 10 & 15 & 20 \\ S_{0}-S_{15}= & 7 \cdot 1 & 23 & 38 & 52 & 64 \\ S_{15}-S_{30} & =33 \cdot 9 & 42 & 50 & 59 & 67\end{array}$

Whilst for solutions which contain a greater proportion of hydrogen chloride than $\mathrm{HCl}, 6 \mathrm{H}_{2} \mathrm{O}$, these coefficients decrease with a rise of temperature ; for instance, for 30 p.c. of hydrogen chloride $S_{0}-S_{15}=88$ and $S_{15}-S_{30}=87$ (according to Marignac's data). In the case of $\mathrm{HCl}, 6 \mathrm{H}_{2} \mathrm{O}$ these differences are constant, and equal 76 .

Thus the formation of two definite hydrates, $\mathrm{HCl}, 2 \mathrm{H}_{2} \mathrm{O}$ and $\mathrm{HCl}, 6 \mathrm{H}_{2} \mathrm{O}$, between hydrochloric acid and water may be accepted upon the basis of many facts. But both of them, inasmuch as they occur in a liquid state, dissociate with great facility into hydrogen. chloride and water, and are completely decomposed when distilled.

All solutions of hydrochloric acid present the properties of an energetic acid. They not only transform blue vegetable colouringmatter into red, and disengage carbonic acid gas from carbonates, \&c., but they also entirely saturate bases, even such energetic ones as pot-

uniform, then the modulus of expansion must be taken as - ds / dt $S_{0}$ ) attains a magnitude 0.000447 at about $48^{\circ}$, it might be thought that at $48^{\circ}$ all solutions of hydrochloric acid would have the same modulus of expansion, but in reality this is not the case. At low and the ordinary temperatures the modulus of expansion of aqueous solutions is greater than that of water, and the greater it is the greater the amount of the substance dissolved (for this reason the temperature of maximum density, when the modulus $=0$, is lowered by solution). But for water the modulus of expansion $\left(-d s / d t S_{n}\right)$ rises rapidly with the temperature, whilst for solutions the increase is much slower (or even falls, as for sulphuric acid or fuming hydrochloric acid), and therefore at a certain temperature $t$ the modulus of solutions becomes equal to that of water. This temperature may be termed the 'characteristic temperature.' For solutions of sodium chloride it is about $58^{\circ}$, for lithium chloride $30^{\circ}$, for potassimm nitrate about $80^{\circ}$, for weak solutions of sulphuric acid about $68^{\circ}$. In order to illustrate this by an example I will insert the magnitudes of denomination of expansion (multiplied by 10000) of solutions of sodium chloride :-

$\begin{array}{lcclllll} & & 0^{\circ} & 20^{\circ} & 50^{\circ} & 60^{\circ} & 80^{\circ} & 100^{\circ} \\ \text { Water } . & -065 & 2 \cdot 07 & 3 \cdot 64 & 5 \cdot 11 & 6 \cdot 25 & 7 \cdot 09 \\ 10 \text { p.c. } \mathrm{NaCl} & . & 2 \cdot 3 & 3 \cdot 4 & 4 \cdot 3 & 5 \cdot 0 & 5 \cdot 7 & 6 \cdot 3 \\ 20 \text { p.c. } \quad . \quad & . & 8 \cdot 6 & 4 \cdot 0 & 4 \cdot 5 & 5 \cdot 0 & 5 \cdot 4 & 5 \cdot 8\end{array}$

41 The figures cited above may serve for the direct determination of the rariation of the specific gravity of solutions of hydrochloric acid with the temperature, because we may take $S_{t}=S_{t}-t(A-B t)$. Thus, knowing that at $15^{\circ}$ the specific gravity of a 10 p.c. solution of hydrocliloric acid $=10492$, we find that at $t^{\circ}$ it $=10530-t(2 \cdot 13+0027 t)$. Whence also may be found the modulus of expansion (Note $4 n$ ). 
ash, lime, drc. In a dry state, however, hydrochloric acir does not alter vegetable dyes, and does not accomplish many double decompositions which easily take place in the presence of water. This is explained by the fact that the gaso-elastic state of the hydrochloric acid prevents its entering into reaction. However, incandescent iron, zinc, sodium, dc., act on gaseous hydrochloric acid, displacing the hydrogen and leaving half a volume of hydrogen against one volume of hydrochloric acid gas; this reaction may serve for determining the composition of hydrochloric acid. Combined with water the hydrochloric acid gas is deprived of its elasticity, and loses a considerable amount of heat in its passage into a liquid and combined state. It then acts as an acid which much resembles nitric acid ${ }^{42}$ in its energy and in many of its reactions ; lowever, the latter contains oxygen, which is disengaged with great ease, and so very frequently acts as an oxidiser, which hydrochloric is not capable of doing. The majority of metals (even those which do not displace the $\mathrm{H}$ from $\mathrm{H}_{2} \mathrm{SO}_{4}$ but which, like copper, decompose it to the limit of $\mathrm{SO}_{2}$ ) displace the hydrogen from hydrochloric acid. Thus hydrogen is disengaged by the action of zinc, and even of copper and tin. Only a few metals withstand its action; for example, gold and platinum. Lead is only acted on feebly in compact masses, because the lead chloride formed is insoluble and prevents the further action of the acid on the metal. The same is to be remarked with respect to the feeble action of hydrochloric acid on mercury and silver, because the compounds of these metals, $\mathrm{AgCl}$ and $\mathrm{HgCl}$, are insoluble in water. Metallic chlorides are not only formed by the action of hydrochloric acid on the metals, but also by many other methods ; for instance, by the action of hydrochloric acid on the carbonates, oxides, and hydroxides, and also by the action of chlorine on metals and certain of their compounds. Metallic chlorides have a composition $\mathrm{MCl}$; for example, $\mathrm{NaCl}, \mathrm{KCl}, \mathrm{AgCl}, \mathrm{HgCl}$, if the metal replaces hydrogen equivalent for equivalent, or, as it is said, if it be monatomic or univalent. In the case of bivalent metals, they have a composition $\mathrm{MCl}_{2}$; for example, $\mathrm{CaCl}_{2}$, $\mathrm{CuCl}_{2}, \mathrm{PbCl}_{2}, \mathrm{HgCl}_{2}, \mathrm{FeCl}_{2}, \mathrm{MnCl}_{2}$. The composition of the haloid salts of other metals presents a further variation ; for example, $\mathrm{AlCl}_{3}$, $\mathrm{Fe}_{2} \mathrm{Cl}_{6}, \mathrm{PtCl}_{4}$, \&c. Many metals, as will have been remarked in the preceding examples, give several degrees of combination with chlorine, as with hydrogen. In their composition the metallic chlorides differ

42 Thus, for instance, with feeble bases they evolve in dilute solutions (Chapter III. Note 53) almost equal amounts of heat; their relation to sulphuric acid is quite identical. They both form fuming solutions as well as hydrates; they both form solutions of constant boiling point; in the solutions of both (with $\mathrm{HCl}, 6 \mathrm{H}_{2} \mathrm{O}$ and with $\mathrm{NHO}_{3}, 5 \mathrm{H}_{2} \mathrm{O}$ ) the direction of the differential $c_{s} / d p$ varies, \&c. 
from the corresponding oxides in that the $\mathrm{O}$ is replaced by $\mathrm{Cl}_{2}$, as should follow from the law of substitution, because oxygen gives $\mathrm{OH}_{2}$ and is consequently bivalent, whilst chlorine forms $\mathrm{HCl}$, and is therefore univalent. So, for instance, ferrous oxide, $\mathrm{FeO}$, corresponds with ferrous chloride, $\mathrm{FeCl}_{2}$, and the oxide $\mathrm{Fe}_{2} \mathrm{O}_{3}$ with ferric chloride, which is also seen from the origin of these compounds, because $\mathrm{FeCl}_{2}$ is obtained by the action of hydrochloric acid on ferrous oxide or carbonate and $\mathrm{FeCl}_{3}$ by its action on ferric oxide. The action of hydrochloric acid, like that of every acid, on basic oxides, MO, and in general on $\mathrm{M}_{n} \mathrm{O}_{m}$, consists in a double decomposition, water, $m \mathrm{H}_{2} \mathrm{O}$, and a metallic chloride, $\mathrm{M}_{n} \mathrm{Cl}_{2 m}$, being formed. Hydrochloric acid, $\mathrm{HCl}$, acts in the same manner on the hydroxides of the bases $\mathbf{M}_{n}(\mathrm{OH})_{2 m}+2 m \mathrm{HCl}$ $=2 m \mathrm{H}_{2} \mathrm{O}+\mathrm{M}_{n} \mathrm{Cl}_{2 m}$, and on carbonates, for instance, $\mathrm{Na}_{2} \mathrm{CO}_{3}+2 \mathrm{HCl}$ $=2 \mathrm{NaCl}+\mathrm{H}_{2} \mathrm{O}+\mathrm{CO}_{2}$. In a word, all the typical properties of acids are shown by hydrochloric acid, and all the typical properties of salts in the metallic chlorides derived from it. Acids and salts composed like $\mathrm{HCl}$ and $\mathrm{M}_{n} \mathrm{Cl}_{2 m}$ without any oxygen bear the name of haloid salts; for instance, $\mathrm{HCl}$ is a haloid acid, $\mathrm{NaCl}$ a haloid salt, chlorine a halogen. Certain higher degrees of combination of chlorine with the metals disengage chlorine when heated to redness; for instance, the salt $\mathrm{CuCl}_{2}$ is converted into $\mathrm{CuCl}$. The capacity of hydrochloric acid to give, by its action on bases, MO, a metallic chloride, $\mathrm{MCl}_{2}$, and water, is limited at high temperatures by the reverse reaction $\mathrm{MCl}_{2}+\mathrm{H}_{2} \mathrm{O}=\mathrm{MO}+2 \mathrm{HCl}$, and the more pronounced are the basic properties of MO the feebler is the reverse reaction, while for feebler bases such as $\mathrm{Al}_{2} \mathrm{O}_{3}, \mathrm{MgO}$, dc., this reverse reaction proceeds with ease. In this manner Deville obtained crystals of the amorphous oxides $\mathrm{Fe}_{2} \mathrm{O}_{3}, \mathrm{SnO}_{2}$, \&c., by passing a slow current of hydrogen chloride over them at a red heat; $\mathrm{Fe}_{2} \mathrm{Cl}_{6}$ and $\mathrm{SnCl}_{4}$ were momentarily formed, but were again decomposed by the water liberated and the liberated oxides were separated as crystals. Metallic chlorides corresponding with the peroxides either do not exist, or are easily decomposed with the disengagement of chlorine. Thus there is no compound $\mathrm{BaCl}_{4}$ corresponding with the oxide $\mathrm{BaO}_{2}$. Metallic chlorides having the general aspect of salts, like their representative sodium chloride, are, as a rule, easily fusible, more so than the oxides (for instance, $\mathrm{CaO}$ is infusible whilst $\mathrm{CaCl}_{2}$ is easily fused), and many other salts. Under the action of heat many are more stable than the oxides, many are even converted into vapour ; thus corrosive sublimate, $\mathrm{HgCl}_{2}$, is particularly volatile, whilst the oxide $\mathrm{HgO}$ decomposes at a red heat. Silver chloride, $\mathrm{AgCl}$, is fusible and is decomposed with difficulty, whilst $\mathrm{Ag}_{2} \mathrm{O}$ is easily decomposed. The majority of the metallic chlorides are soluble in 
water, but silver chloride, cuprous chloride, mercurous chloride, and lead chloricle are sparingly soluble in water, and are therefore easily obtained as precipitates when a solution of the salts of these metals is mixed with a solution of any chloride or even with hydrochloric acid. The metal contained in a haloid salt may often be replaced by another metal, or even by hydrogen, just as is the case with a metal in an oxide. Thus copper displaces mercury from a solution of mercuric chloride, $\mathrm{HgCl}_{2}+\mathrm{Cu}=\mathrm{CuCl}_{2}+\mathrm{Hg}$; thus hydrogen at a red heat displaces silver from silver chloride, $2 \mathrm{AgCl}+\mathrm{H}_{2}=\mathrm{Ag}_{2}+2 \mathrm{HCl}$. These, and a whole series of similar reactions, form the typical methods of double saline decompositions. The measure of decomposition and the conditions under which one or the other side of reactions of double saline decompositions proceeds (for instance, whether a metal and an acid give hydrogen and a salt or the reverse) are determined by the properties of the compounds which are in action, and which are able to be formed at the temperature, drc., as was shown when speaking of sodium chloride, and as will be frequently found hereafter.

If hydrochloric acid enters into double decomposition with basic oxides and their hydrates, this is only due to its acid properties; and for the same reason it rarely enters into double decomposition with acids and acid anhydrides. Sometimes, however, it combines with the latter, as, for instance, with the anhydride of sulphuric acid, forming the compound $\mathrm{SO}_{3} \mathrm{HCl}$; and in other cases it acts on acids, giving up its hydrogen to their oxygen and forming chlorine, as will be seen in the following chapter.

Hydrochloric acid, as may already be concluded from the compo. sition of its molecule, belongs to the monobasic acids, and does not, therefore, give true acid salts (like $\mathrm{HNaSO}_{4}$ or $\mathrm{HNaCO}_{3}$ ); nevertheless many metallic chlorides, formed from powerful bases, are capable of combining with hydrochloric acid, just as they combine with water, or with ammonia, and as they give double salts. Compounds have long been known of hydrochloric acid with auric, platinic, and antimonious chlorides, and other similar metallic chlorides corresponding with very feeble bases. But Berthelot, Engel, and others have shown that the capacity of $\mathrm{HCl}$ for combining with $\mathrm{M}_{n} \mathrm{Cl}_{m}$ is much more frequently encountered than was before supposed. Thus, for instance, dry hydrochloric acid when passed into a solution of zine chloride (containing an excess of the salt) gives in the cold $\left(0^{\circ}\right)$ a compound $\mathrm{HCl}, \mathrm{ZnCl}_{2}, 2 \mathrm{H}_{2} \mathrm{O}$, and at the ordinary temperature $\mathrm{HCl}, 2 \mathrm{ZnCl}, 2 \mathrm{H}_{2} \mathrm{O}$, just as it is able at low temperatures to form the crystallo-hydrate $\mathrm{ZnCl}_{2}, 3 \mathrm{H}_{2} \mathrm{O}$ (Engel, 1886). Similar compounds are obtained with $\mathrm{CdCl}_{2}, \mathrm{CuCl}_{2}, \mathrm{HgCl}_{2}, \mathrm{Fe}_{2} \mathrm{Cl}_{6}$, drc. (Berthelot, Ditte, Cheltzoff, Lachinoff, 
and others). These compounds with hydrochloric acid are generally more soluble in water than the metallic chlorides themselves, so that whilst hydrochloric acid decreases the solubility of $\mathbf{M C l}_{m}$, corresponding with energetic bases (for instance, sodium or barium chlorides), it increases the solubility of the metallic chlorides corresponding with feeble bases (cadmium chloricle, ferric chloride, dcc.). Silver chloride, which is insoluble in water, is soluble in hydrochloric acid. Hydrochloric acid also combines with certain unsaturated hydrocarbons (for instance with turpentine, $\mathrm{C}_{10} \mathrm{H}_{16} 2 \mathrm{HCl}$ ) and their derivatives. Salammoniac, or ammonia hydrochloride, $\mathrm{NH}_{4} \mathrm{Cl}=\mathrm{NH}_{3}, \mathrm{HCl}$, also belongs to this class of compounds. ${ }^{43}$ If dry hydrogen chloride be mixed with dry ammonia, a solid compound formed from equal volumes of each is immediately formed. The same compound is formed on mixing solutions of the two gases. It is also produced by the action of hydrochloric acid on ammonium carbonate. Sal-ammoniac is usually prepared by the last method in practice. ${ }^{44}$ The specific gravity of salammoniac is 1.55 . We have already seen (p. 253) that sal-ammoniac,

45 When an unsaturated hydrocarbon, or, in general, an unsaturated compound, assimilates to itself the molecules $\mathrm{Cl}_{2}, \mathrm{HCl}, \mathrm{SO}_{3}, \mathrm{H}_{2} \mathrm{SO}_{4}, \& \mathrm{c}$., the cause of the reaction is most simple. As nitrogen, besides the type $\mathrm{NX}_{3}$ to which $\mathrm{NH}_{3}$ belongs, forms compounds of the form $\mathrm{NX}_{5}$-for example, $\mathrm{NO}_{2}(\mathrm{OH})$-therefore the formation of the salts of ammonium should be understood in this sense. $\mathrm{NH}_{3}$ gives $\mathrm{NH}_{4} \mathrm{Cl}$ because $\mathrm{NX}_{3}$ is capable of giving $\mathrm{NX}_{5}$. But as saturated compounds-for instance, $\mathrm{HCl}, \mathrm{H}_{2} \mathrm{O}, \mathrm{NaCl}$, Sc.-are also capable of combination even between themselves, therefore it is impossible to deny the capacity of $\mathrm{HCl}$ also for combination. $\mathrm{SO}_{3}$ combines with $\mathrm{H}_{2} \mathrm{O}$, and also with $\mathrm{HCl}$ and the unsaturated hydrocarbons. It is impossible to see the limit here, which it was lately wished to establish, by distinguishing atomic from molecular compounds, and regarding, for instance, $\mathrm{PCl}_{5}$ as an atomic compound and $\mathrm{PCl}_{5}$ as a molecular one, only because it easily splits up into molecules $\mathrm{PCl}_{3}$ and $\mathrm{Cl}_{2}$.

44 Sal-ammoniac is prepared from ammonium carbonate, obtained in the dry distillation of nitrogenous substances (Chapter VI.), by saturating the resultant solution with hydrochloric acid. A solution of sal-ammoniac is thus produced, it is evaporated, and in the residue a mass is obtained containing a mixture of various other, especially tarry, products of dry distillation. The sal-ammoniac is generally purified by sublimation. For this purpose iron vessels covered with semispherical metallic covers are employed, or else simply clay crucibles covered by other crucibles. The upper portion, or helmet, of the apparatus of this kind will have a lower temperature than the lower portion, which is under the direct action of the flame. The sal-ammoniac volatilises when heated, and settles on the cooler portion of the apparatus. It is thus purified from many impurities, and is obtained as a crystalline crust, generally several centimetres thick, in which form it is generally sold.

In order to demonstrate the very instructive formation of solid sal-ammoniac from the gases $\mathrm{NH}_{3}+\mathrm{HCl}$ we may proceed as follows: A thin glass tube is filled with ammonia and closed with a cork, and placed in a thick glass cylinder into which a rapid stream of hydrogen chloride is introduced and is then closed with a cork; the inside tube is then broken by violently shaking the apparatus. The ammonium chloride is obtained in white flakes. A glass rod, or paper moistened with hydrochloric acid, also gives a distinct cloud of sal-ammoniac when held over the mouth of a bottle containing a strong solution of ammonia. 
like all other ammonium salts, easily decomposes; for instance, by volatilisation with alkalis, and even partially when its solution is boiled. But at higher temperatures ammonia decomposes with the liberation of hydrogen, and therefore ammonia is then able to act as a reducing agent-as, for example, on metallic oxides. The other properties and reactions of sal-ammoniac, especially in solution, fully bring to mind what has been already mentioned in speaking of sodium chloride. Thus, for instance, with silver nitrate it gives a precipitate of silver chloride; with sulphuric acid it gives hydrochloric acid and ammonium sulphate, and it forms double salts with certain metallic chlorides and other salts. ${ }^{45}$

45 The solubility of sal-ammoniac in 100 parts of water (according to Alluard) is-

$\begin{array}{ccccccccc}0^{\circ} & 10^{\circ} & 20^{\circ} & 30^{\circ} & 40^{\circ} & 60^{\circ} & 80^{\circ} & 100^{\circ} & 110^{\circ} \\ 28 \cdot 40 & 32.48 & 37 \cdot 28 & 41 \cdot 72 & 46 & 55 & 64 & 73 & 77\end{array}$

A saturated solution boils at $115 \cdot 8^{\circ}$. The specific gravity at $15^{\circ} / 4^{\circ}$ of solutions of salammoniac (water $4^{\circ}=10000$ ) $=9991 \cdot 6+31 \cdot 26 p-0 \cdot 085 p^{2}$, where $p$ is the amount by weight of ammonium chloride in 100 parts of solution. With the majority of salts the differential $d s d p$ increases, but here it decreases with the increase of $p$. For (unlike the sodium and potassium salts) a solution of the alkali plus a solution of acid occupy a greater volume than that of the resultant ammonium salt. In the solution of solid ammonium chloride a contraction, and not expansion, generally takes place. It may further be remarked that solutions of sal-ammoniac have an acid reaction even when prepared from the salt remaining 'after prolonged washing of the sublimed salt with water (A. Stcherbakoff). 


\section{CHAPTER XI}

THE HALOGENS : CHLORINE, BROMINE, IODINE, AND FLUORINE

Although hydrochloric acid, like water, is one of the most stable substances, it is nevertheless decomposed not only by the action of a galvanic current ${ }^{1}$ but also by a rise of temperature. Sainte-Claire Deville showed that decomposition already occurs at $1300^{\circ}$, because a cold tube (p. 388) covered with an amalgam of silver absorbs chlorine in a redhot tube, and the escaping gas contains hydrogen. Meyer and Langer (1885) observed the decomposition of hydrochloric acid at $1690^{\circ}$ in a platinum vessel; the decomposition in this instance was proved not only from the fact that hydrogen permeated through the platinum (p. 141), owing to which the volume was diminished, but also from chlorine being obtained in the residue (the hydrogen chloride was mixed with nitrogen), which liberated iodine from potassium iodide. ${ }^{2}$ The usual method for the preparation of chlorine consists in the abstraction of the hydrogen by oxidising agencies.

The acid properties of hydrochloric acid were known when Lavoisier pointed out the formation of acids by the combination of water with the oxides of the non-metals, and therefore there was reason for thinking that hydrochloric acid was formed by the combination of water with the oxide of some element. Therefore when Scheele obtained chlorine by the action of hydrochloric acid on manganese peroxide he considered it as the acid contained in common salt. When it became known that chlorine gives hydrochloric acid with hydrogen, Lavoisier and Berthollet.supposed it to be a compound with oxygen of an anhydride contained in hydrochloric acid. They supposed that hydrochloric acid contained water and the oxide of a particular radicle

1 The decomposition of fused sodium chloride by an electric current has been proposed in America and Russia (N. N. Beketoff) as a means for the preparation of chlorine and sodium. A strong solution of hydrochloric acid is decomposed into equal volumes of chlorine and hydrogen by the action of an electric current.

2 To obtain so high a temperature (at which the best kinds of porcelain soften) Langer and Meyer employed the dense graphitoidal carbon from gas retorts, and a strong draught. They determined the temperature by, the alteration of the volume of nitrogen in the platinum vessel, for it does not permeate through platinum, and is not altered by heat. 
and that chlorine was a higher degree of oxidation of this radicle murias (from the Latin name of hydrochloric acid, acidum muriaticum). It was only in 1811 that Gay-Lussac and Thenard in France and Davy in England arrived at the conclusion that the substance obtained by Scheele does not contain oxygen, nor under any conditions give water with hydrogen, and that there is no water in hydrochloric acid gas, and therefore concluded that chlorine is an elementary substance. They named it 'chlorine' from the Greek word $x^{\lambda}$ woós, signifying a green colour, because of the peculiar colour by which this gas is characterised.

An aqueous solution of hyclrochloric acid is generally employed for the evolution of chlorine. The hydrogen has to be abstracted from the hydrochloric acid. This is accomplished by nearly all oxidising substances, and especially by those which are able to evolve oxygen at a red heat (besides bases, such as mercury and silver oxides, which are able to give salts with hydrogen chloride); for example, manganese peroxide, potassium chlorate, chromic acid, \&c. The decomposition essentially consists in the oxygen of the oxidising substance displacing the chlorine from $2 \mathrm{HCl}$, forming water, $\mathrm{H}_{2} \mathrm{O}$, or taking up the hydrogen and setting the chlorinefree, as is sometimes said, $2 \mathrm{HCl}+\mathrm{O}$ (disengaged by the oxidising substances) $=\mathrm{H}_{2} \mathrm{O}+\mathrm{Cl}_{2}$. Even nitric acid partially produces a like reaction, but as we shall afterwards see its action is more complicated, and it is therefore not suitable for the preparation of pure chlorine. But other oxidising substances which do not give any other volatile products with hydrochloric acid may be employed for the preparation of chlorine. Among these may be mentioned: potassium chlorate, acid potassium chromate, sodium manganate, manganese peroxide, \&c. Manganese peroxide is commonly employed in the laboratory, and on a large scale, for the preparation of chlorine. The chemical process which proceeds in this case may be represented as follows: an exchange takes place between $4 \mathrm{HCl}$ and $\mathrm{MnO}_{2}$, in which the manganese takes the place of the four atoms of hydrogen, or the chlorine and oxygen exchange places - that is, $\mathrm{MnCl}_{4}$ and $2 \mathrm{H}_{2} \mathrm{O}$ are produced. The chlorine compound, $\mathrm{MnCl}_{4}$, obtained is very unstable ; it splits up into chlorine, which as a gas passes from the sphere of action, and a lower compound containing less chlorine than the substance first formed, and which remains in the apparatus in which the mixture is heated, $\mathrm{MnCl}_{4}=\mathrm{MnCl}_{2}+\mathrm{Cl}_{2} \cdot{ }^{3}$ The action of hydro-

3 This representation of the process of the reaction is the most natural. However, this decomposition is generally represented as if chlorine gave only one degree of combination with manganese, $\mathrm{MnCl}_{2}$, and therefore directly reacts in the following manner$\mathrm{MnO}_{2}+4 \mathrm{HCl}=\mathrm{MnCl}_{2}+2 \mathrm{H}_{2} \mathrm{O}+\mathrm{Cl}_{2}$, in which ease it is, as it were, supposed that 


\section{chloric acid requires a temperature of about $100^{\circ}$. In the laboratory} the preparation of chlorine is carried on in flasks, heated over a water bath, by acting on manganese peroxide with hydrochloric acid or a

manganese peroxide, $\mathrm{MnO}_{2}$, breaks up into manganous oxide, $\mathrm{MnO}$, and oxygen, both of which react with hydrochloric acid, the manganous oxide acting as a base, $\mathrm{MnO}+2 \mathrm{HCl}=$ $\mathrm{InCl}_{2}+\mathrm{H}_{2} \mathrm{O}$, and at the same time $2 \mathrm{HCl}+\mathrm{O}=\mathrm{H}_{2} \mathrm{O}+\mathrm{Cl}_{2}$. In reality a mixture of oxygen and hydrochloric acid does give chlorine at a red heat, and this may also take place at the moment of its evolution in this case.

All the oxides of manganese $\left(\mathrm{Mn}_{2} \mathrm{O}_{3}, \mathrm{MnO}_{2}, \mathrm{MnO}_{5}, \mathrm{Mn}_{2} \mathrm{O}_{7}\right)$, with the exception of manganous oxide, $\mathrm{MnO}$, disengage chlorine from hydrochloric acid, because manganous chloride, $\mathrm{MnCl}_{2}$, is the only compound of chlorine and manganese which exists as a stable compound, all the higher chlorides of manganese being unstable and evolving chlorine. The reaction between hydrochloric acid and salts containing much oxygen is explained according to the law of substitution by the double decomposition and instability of the higher chlorides; thus, for example, by taking potassium dichromate, $\mathrm{K}_{2} \mathrm{Cr}_{2} \mathrm{O}_{7}$, a compound, $\mathrm{K}_{2} \mathrm{Cr}_{2} \mathrm{Cl}_{1,1}$, might be formed, but it is unknown, and if it is formed it in all probability immediately decomposes into chlorine, $6 \mathrm{Cl}$, potassium chloride, $2 \mathrm{KCl}$, and chromium chloride, $\mathrm{Cr}_{2} \mathrm{Cl}_{6}$. Hence we here encounter two circumstances: (1) a substitution between oxygen and chlorine, and (2) the instability of those higher chlorine compounds. Both these circumstances have a very important signification for the comprehension of the relation of such elements as chlorine and oxygen. As (according to the law of substitution) in the substitution of oxygen by chlorine, $\mathrm{Cl}_{2}$ takes the place of $\mathrm{O}$, therefore the chlorine compounds will contain in themselves more atoms than the corresponding oxygen compounds. It is not surprising, therefore, that certain of the chlorine compounds corresponding with oxygen compounds do not exist, or if they are formed are very unstable. And furthermore, an atom of chlorine is heavier than an atom of oxygen, and therefore a given element would have to retain a large mass of chlorine if in the higher oxides the oxygen were replaced by chlorine. For this reason equivalent compounds of chlorine do not exist for all oxygen compounds. Many of the former are immediately decomposed, when formed, with the evolution of chlorine. Therefore chlorine is evolved by the action of hydrochloric acid on compounds which contain much oxygen. From this it is evident that there should exist such chlorine compounds as would evolve chlorine as peroxides evolve oxygen, and indeed a large number of such compounds are known. Amongst them may be mentioned antimony pentachloride, $\mathrm{SbCl}_{5}$, which splits up into chlorine and antimony trichloride when heated. Cupric chloride, corresponding with copper oxide, and having a composition $\mathrm{CuCl}_{2}$, similar to $\mathrm{CuO}$, when heated parts with half its chlorine, just as barium peroxide evolves half its oxygen. This method may even be taken advantage of for the preparation of chlorine and cuprous chloride, $\mathrm{CuCl}$. The latter attracts oxygen from the atmosphere, and in so doing is converted from a colourless substance into a green compound whose composition is $\mathrm{Cu}_{2} \mathrm{Cl}_{2} \mathrm{O}$. With hydrochloric acid this substance gives cupric chloride $\left(\mathrm{Cu}_{2} \mathrm{Cl}_{2} \mathrm{O}+2 \mathrm{HCl}=\mathrm{H}_{2} \mathrm{O}+2 \mathrm{CuCl}_{2}\right)$, which has only to be dried and heated, and it again evolves chlorine. Thus, in solution, and at the ordinary temperature, the compound $\mathrm{CuCl}_{2}$ is constant, but when heated it splits up. On this property is founded Deacon's process for the preparation of chlorine from hydrochloric acid by the aid of air and copper salts, by passing a mixture of air and hydrochloric acid at about $440^{\circ}$ over bricks soaked with a solution of a copper salt (a mixture of solutions of $\mathrm{CuSO}_{4}$ and $\mathrm{Na}_{2} \mathrm{SO}_{4}$ ). $\mathrm{CuCl}_{3}$ is then formed by the double decomposition of the salt of copper and the hydrochloric acid; the $\mathrm{CuCl}_{2}$ liberates chlorine, and the $\mathrm{CuCl}$ forms $\mathrm{Cu}_{2} \mathrm{Cl}_{2} \mathrm{O}$ with the oxygen of the air, which again gives $\mathrm{CuCl}_{2}$ with $2 \mathrm{HCl}$, and so on.

Magnesium chloride, which is obtained from sea-water, carnallite, \&c., may serve not only as a means for the preparation of hydrochloric acid, but also of chlorine, because (Weldon-Pechiny's process) its basic salt (magnesium oxychloride) when heated in the air gives magnesium oxide and chlorine. Chlorine is now prepared on a large scale by this method. 
mixture of common salt and sulphuric acid ${ }^{4}$ and washing the gas with water to remove hydrochloric acid. The action of hydrochloric acid on bleaching powder (p. 161) gives chlorine without the aid of heat: $\mathrm{CaCl}_{2} \mathrm{O}_{2}+4 \mathrm{HCl}=\mathrm{CaCl}_{2}+2 \mathrm{H}_{2} \mathrm{O}+2 \mathrm{Cl}_{2}$, and is therefore also taken advantage of in the laboratory for the preparation of chlorine. ${ }^{5}$ Chlorine cannot be collected over mercury, because it combines with it as with many other metals, and it is soluble in water ; however, it is but slightly soluble in hot water or brine. Owing to its great weight, chlorine may be directly collected in a dry vessel by introducing the gas-conducting tube into the bottom of the vessel. The chlorine will lie in a heavy layer at the bottom of the vessel, displace the air, and the extent to which it fills the vessel may be followed by its colour. ${ }^{6}$

4 The following proportions are then taken by weight: 3 parts of powdered manganese peroxide, 4 parts of salt (best fused, to prevent its frothing), and 9 parts of sulphuric acid previously mixed with 5 parts of water. It is heated in a salt bath, so as to obtain a temperature above $100^{\circ}$. The corks in the apparatus must be soaked in paraffin (otherwise they are corroded by the chlorine), and black india-rubber tubing must be taken, and not vulcanised (which contrins sulphur, and becomes brittle under the action of the chlorine).

The reaction which proceeds may be expressed thus: $\mathrm{InO}_{2}+2 \mathrm{NaCl}+2 \mathrm{H}_{2} \mathrm{SO}_{4}=$ $\mathrm{MnSO}_{4}+\mathrm{Na}_{2} \mathrm{SO}_{4}+2 \mathrm{H}_{2} \mathrm{O}+\mathrm{Cl}_{2}$. The preparation of $\mathrm{Cl}_{2}$ from manganese peroxide and hydrochloric acid was discovered by Scheele, and from sodium chloride by Berthollet.

5 The reaction of hydrochloric acid on bleaching powder is very violent if all the acid be added at once; it should be poured in drop by drop (Mermé, Kammerer). C. Winkler proposed mixing bleaching powder with one quarter of burnt and powdered gypsum, and having damped the mixture with water, to press and cut it up into cubes and dry at the ordinary temperature. These cubes can be used for the preparation of chlorine in the same apparatus as that used for the evolution of hydrogen and carbonic anhydride - the disengagement of the chlorine proceeds uniformly.

A mixture of potassium dichromate and hydrochloric acid evolves chlorine perfectly free from oxygen (V. Meyer and Langer).

6 Chlorine is manufactured on a large scale from manganese peroxide and lydrochloric acid. It is most conveniently prepared in the arrangement shown in fig. 67 , which con-

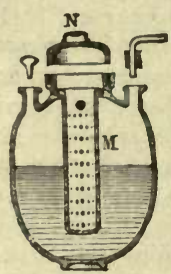

FIG. 67.-Clay retort for the preparation of clilorine on a large scale. sists of a three-necked earthenware vessel whose central orifice is the largest. A clay or lead funnel, furnished with a number of orifices, is placed in the central wide neck of the ressel. Roughly-ground lumps of natural manganese peroxide are placed in the funnel, which is then closed by the cover $\mathrm{N}$, and luted with clay. One orifice is closed by a clay stopper, and is used for the introduction of the hydrochloric acid and withdrawal of the residues. The chlorine disengaged passes along a leaden gasconducting tube placed in the other orifice. A row of these vessels is surrounded by a water-bath, to ensure their being uniformly heated. Manganese chloride is found in the residue. In Weldon's process lime is added to the acid solution of manganese chloride. A double decomposition takes place, resulting in the formation of manganous hydroxide and calcium chloride. When the insoluble manganous hydroxide has settled, a further excess of milk of lime is added (to make a mixture $2 \mathrm{Mn}(\mathrm{OH})_{2}+\mathrm{CaO}+x \mathrm{CaCl}_{2}$, which is found to be the best proportion, judging from experiment), and then air is forced through the mixture. The hydroxide is then converted from a colourless to a brown substance, containing peroxide, $\mathrm{MnO}_{2}$, and oxide of manganese, $\mathrm{Mn}_{2} \mathrm{O}_{3}$. This is due to the manganous oxide absorbing oxy 
Chlorine is a gas of a yellowish green colour, and has a very suffocating and characteristic odour. On lowering the temperature to $-50^{\circ}$ or increasing the pressure to six atmospheres (at $0^{\circ}$ ) chlorine condenses ${ }^{7}$ into a liquid which has a yellowish green colour and a density of $1 \cdot 3$, and boils at $-34^{\circ}$. The density and atomic weight of chlorine is 35.5 times greater than that of hydrogen, hence the molecule contains $\mathrm{Cl}_{2} \cdot{ }^{8}$ At $0^{\circ}$ one volume of water dissolves about $1 \frac{1}{2}$ volumes of chlorine, at $10^{\circ}$ about three volumes, at $50^{\circ}$ again $1 \frac{1}{2}$ volumes. ${ }^{9}$ Such a solution of chlorine is termed ' chlorine water' ; and is employed in a diluted form in medicine and laboratory practice. It is prepared

air. Under the action of hydrochloric acid this mixture evolves chlorine, because of all the compounds of chlorine and manganese the chloride $\mathrm{MnCl}_{2}$ is the only one which is stable (see Note 3 ). Thus one and the same mass of manganese may be repeatedly used for the preparation of chlorine. The same result is attained in other ways. If manganous oxide be subjected to the action of oxides of nitrogen and air (Coleman's process), then manganese nitrate is formed, which at a red heat gives oxides of nitrogen (which are again used in the process) and manganese peroxide, which is thus renewed for the fresh evolution of chlorine.

7 Davy and Furaday liquefied chlorine in 1823 by heating the crystallo-hydrate $\mathrm{Cl}_{2} 8 \mathrm{H}_{2} \mathrm{O}$ in a bent tube (like that shown in fig. 45, p. 248), in warm water, while the other end of the tube was immersed in a freezing mixture. Meselan condensed chlorine in freshly-burnt charcoal (placed in a glass tube), which when cold absorbs an equal weight of chlorine. The tube was then fused up, the bent end cooled, and the charcoal heated, by which means the chlorine was expelled from the charcoal, and the pressure increased.

8 Judging from Ludwig's observations (1868), and from the fact that the coefficient of expansion of gases increases with their molecular weight (Chapter II. Note 26, for hydrogen $=0.367$, carbonic anhydride $=0.373$, hydrogen bromide $=0.386)$, it might be expected that the expansion of chlorine would be greater than that of air or of the gases composing it. V. Meyer and Langer(1885) having remarked that at $1400^{\circ}$ the density of chlorine (taking its expansion as equal to that of nitrogen) $=29$, consider that the molecules of chlorine split up and partially give molecules $\mathrm{Cl}$, but it might be thought that the decrease in density observed only depends on the increase of the coefficient of expansion.

9 Investigations on the solubility of chlorine in water (the solutions evolve all their chlorine on boiling and passing air through them) show many different peculiarities. First Gay-Lussac, and then Pelouze, determined that the solubility increases between $0^{\circ}$ and $8^{\circ}$ to $10^{\supset}$ (from $1 \frac{1}{2}$ to 2 vols. of chlorine per 100 vols. of water at $0^{\supset}$ to 3 to $2 \frac{3}{4}$ at $10^{\circ}$ ). In the following note we shall see that this is not due to the breaking-up of the hydrate at about $8^{\circ}$ to $10^{\circ}$, but to its formation below $9^{\circ}$. Roscoe observed an increase in the solubility of chlorine in the presence of hydrogen-even in the dark. Berthelot determined an increase of solubility with the progress of time. Schönebein and others suppose that chlorine acts on water, forming hypochlorous and hydrochloric acids $(\mathrm{HClO}+\mathrm{HCl})$.

The equilibrium between chlorine, steam as a gas, between water, liquid chlorine, ice, and the solid crystallo-hydrate of chlorine is evidently very complex. Guldberg (1870) gave a theory for similar states of equilibrium, which was afterwards developed by Roozeboom (1887), but it would be inopportune here to enter into its details. It will be enough in the first place to mention, that there is now no duubt (according to the theory of heat, and the direct observations of Ramsay and Young) that the vapour tension at one and the same temperature are different for the liquid and solid states of substances; secondly, to call attention to the following note; and, thirdly, to state that, in the presence of the crystallo-hydrate, water between $0.24^{\circ}$ and $+28 \cdot 7^{\circ}$ (when the hydrate and a solution may occur simultaneously) dissolves a different amount of chlorine than it does in the absence of the crystallo-hydrate. 
by passing chlorine through a series of Woulfe's bottles, or into an inverted retort filled with water. Under the action of light, chlorine water gives oxygen and hydrochloric acid. At $0^{\circ}$ a saturated solution of chlorine yields a crystallo-hydrate, $\mathrm{Cl}_{2}, 8 \mathrm{H}_{2} \mathrm{O}$, which easily splits up into chlorine and water when heater, so that if it be fused up in a tube and heated to $35^{\circ}$ two layers of liquids are formed-a lower stratum of chlorine containing a small quantity of water, and an upper stratum of water containing a small quantity of chlorine. ${ }^{10}$

Chlorine explodes with hydrogen, if a mixture of equal volumes be exposed to the direct action of the sun's rays ${ }^{11}$ or brought into contact with spongy platinum, or a strongly-heated substance, or subjected to the action of an electric spark. The explosion in this case takes place

10 According to Faraday's data the hydrate of chlorine contains $\mathrm{Cl}_{2}, 10 \mathrm{H}_{2} \mathrm{O}$, but Roozeboom (1885) showed that it is poorer in water $=\mathrm{Cl}_{2}, 8 \mathrm{H}_{2} \mathrm{O}$. At first small, almost. colourless, crystals are obtained, but they gradually form (if the temperature be below their critical point $28^{\circ} 7^{\circ}$, above which they do not exist) large yellow crystals, like those of potassium chromate. The specific gravity is $1 \cdot 23$. The hydrate is formed if there be more chlorine in a solution than it is able to dissolve under the dissociation pressure corresponding with a given temperature. In the presence of the hydrate the percentage amount of chlorine at $0^{\circ}=0.5,9^{\circ}=0.9$, and at $20^{\circ}=1.82$. At temperatures below $9^{\circ}$ the solubility (determined by Gray-Lussac and Pelouze, see Note 9) is dependent on the formation of the hydrate; whilst at higher temperatures under the ordinary pressure the hydrate cannot be formed, and the solubility of chlorine falls, as it does for all gases (Chapter I.). If the crystallo-hydrate is not formed, then below $9^{\circ}$ the solubility follows the same rule $\left(6^{\circ} 1.07\right.$ p.c. $\mathrm{Cl}, 9^{\circ} 0.95$ p.c.). According to Roozeboom the chlorine evolved by the hydrate presents the following tensicns of dissociation at $0^{\circ}=249 \mathrm{~mm}$., at $4^{\circ}=398$, at $8^{\circ}=620$, at $10^{\circ}=797$, at $14^{\circ}=1400 \mathrm{~mm}$. In this case a portion of the crystallo-hydrate remains solid. At $9 \cdot 6$ the tension of dissociation is equal to the atmospheric pressure. At a higher pressure the crystallo-hydrate may form at temperatures above $9^{\circ}$ up to $28.7^{\circ}$, when the vapour tension of the hydrate equals the tension of the chlorine. It is evident that the equilibrium which is established is on the one hand a case of a complex heterogeneous system, and on the other hand a case of the solution of solid and gaseous substances in water.

The crystallo-hydrate or chlorine water must be kept in the dark, or the access of light be prevented by coloured glass, otherwise oxygen is evolved and hydrochloric acid formed.

11 The chemical action of light on a mixture of chlorine and hydrogen was discovered by Gay-Lussac and Draper (1809). It has been investigated by many, and especially by Draper, Bunsen, and Roscoe. Electric or magnesium light, or the light emitted by the combustion of carbon bisulphide in nitric oxide, and in general that which forms photographic images, acts in the same manner as sunlight, according to the intensity. At temperatures below $-12^{\circ}$ light no longer brings about reaction, or at all events does not give an explosion. It was long supposed that chlorine that had been subjected to the action of light was afterwards able to act on hydrogen in the dark, but it was shown that this only takes place with moist chlorine, and depends on the formation of oxides of chlorine. The presence of foreign gases, and even of excess of chlorine or of hydrogen, very much enfeebles the explosion, and therefore the experiment is conducted with a detonating mixture prepared by the action of an electric current on a strong solution (sp. gr. 1.15) of hydrochloric acid, in which case the water is not decomposed-that is, no oxygen is mixed with the chlorine. 
from exactly the same reasons-i.e. the evolution of heat and expansion of the resultant product - as in detonating gas (Chap. III.). Diffused light acts in the same way, but slowly, whilst the direct sunlight excites an explosion. ${ }^{12}$ The hydrochloric acid gas produced by the reaction of chlorine on hydrogen occupies (at the same temperature and pressure) a volume equal to the sum of the original volumes; that is, a reaction of substitution here takes place: $\mathrm{H}_{2}+\mathrm{Cl}_{2}=\mathrm{HCl}+\mathrm{HCl}$, the atoms of chlorine and hydrogen change places - there were two molecules and two molecules are also obtained, although different molecules were first taken and like molecules are formed. In this reaction twenty-two thousand heat units are evolved for one part by weight of hydrogen. ${ }^{13}$

These relations show that the affinity of chlorine for hydrogen is very great and analogous to the affinity between hydrogen and oxygen.

12 The quantity of chlorine and hydrogen which combine is proportional to the intensity of the light-not of all the rays, but only those so-termed chemical (actinic) rays which produce chemical action. Hence a mixture of chlorine and hydrogen, when exposed to the action of light in vessels of known capacity and surface, may be employed as a means for estimating the intensity of the chemical rays (as an actinometer), the influence of the heat rays being previously destroyed, which may be done by passing the rays through water. Investigations of this kind (photo-chemical) showed that chemical action is chiefly limited to the violet end of the spectrum, and that even the invisible ultra-violet rays produce this action. A colourless gas flame contains no chemically-active rays, the flame coloured green by a salt of copper evinces more chemical action than the colourless flame, but the flame brightly coloured yellow by salts of sodium has no more chemical action than that of the colourless flame.

As the chemical action of light becomes evident in plants, photography, the bleaching of tissues, and the fading of colours in the sunlight, and as a means for studying the phenomenon is given in the reaction of chlorine on hydrogen, this subject has been the most fully investigated in photo-chemistry. The researches of Bunsen and Roscoe in the fifties and sixties are the most complete in this respect. Their actinometer contains hydrogen and chlorine, and is enclosed by a solution of chlorine in water. The hydrochloric acid is absorbed as it forms, and therefore the variation in volume indicates the progress of the combination. As was to be expected, the action of light proved to be proportional to the time of exposure and intensity of the light, so that it was possible to conduct detailed photometrical investigations respecting the time of day and season of the year, various sources of light, its absorption, \&c. This subject is considered in detail in special works, and we only stop to mention one circumstance, that a small quantity of a foreign gas decreases the action of light; for example, $\frac{1}{3} \frac{1}{3} \pi$ of hydrogen by 38 p.c., $\frac{1}{200}$ of oxygen by 10 p.c., $\frac{1}{100}$ of chlorine by 60 p.c., \&c. According to the researches of Klimenko and Pekatoros (1889), the photo-chemical alteration of chlorine water is retarded by the presence of traces of metallic chlorides, and this influence varies with different metals.

As much heat is evolved in the reaction of chlorine on hydrogen, and as this reaction, being exothermal, may proceed by itself, therefore the action of light is essentially the same as that of heat-that is, it brings the chlorine and hydrogen into the condition necessary for the reaction-it, as we may say, shakes the original equilibrium; this is the work done by the luminous energy. It seems to me that the action of light on the mixed gases should be understood in this sense, as Pringsheim (1877) pointed out.

13 In the formation of steam (from one part by weight of hydrogen) 29000 heat units are evolved. The following are the quantities of heat (thousands of units) evolved in 
Thus ${ }^{14}$ on the one hand by passing a mixture of steam and chlorine through a red-hot tube, or by exposing water and chlorine to the sunlight, oxygen is disengaged, whilst on the other hand, as we saw above, oxygen in many cases displaces chlorine from its compound with hydrogen, and therefore the reaction $\mathrm{H}_{2} \mathrm{O}+\mathrm{Cl}_{2}=2 \mathrm{HCl}+\mathrm{O}$ belongs to the number of reversible reactions, and hydrogen will distribute itself between oxygen and chlorine if it comes into contact with both these elements. These relations determine many of the properties of chlorine - that is, its relation to substances containing hydrogen and its reactions in the presence of water, to which we shall turn our attention after having pointed out the relation of chlorine to other elements.

Many metals when brought into contact with chlorine immediately combine with it, and form those metallic chlorides which correspond with hydrogen chloride and with the oxide of the metal taken. With a large surface of metal, and if it be slightly heated, this combination may proceed rapidly with the evolution of heat and light; that is, metals are able to burn in chlorine. Thus, for example, sodium ${ }^{15}$ burns in chlorine, thus synthesising common salt. Metals in the form of powders burn without the aid of heat, and become highly incandescent in the process ; for instance, antimony, which is a metal easily converted into a powder. ${ }^{16}$ Even such metals as gold and platinum, ${ }^{17}$

the formation of various other corresponding compounds of oxygen and of chlorine (from Thomsen's, and, for $\mathrm{Na}_{2} \mathrm{O}$, Beketoff's results) :

$$
\left\{\begin{array}{rrrc}
2 \mathrm{NaCl}, 195 ; & \mathrm{CaCl}_{2}, 170 ; & \mathrm{HgCl}_{2}, 63 ; & 2 \mathrm{AgCl}, 59 . \\
\mathrm{Na}_{2} \mathrm{O}, 100 ; & \mathrm{CaO}, 131 ; & \mathrm{HgO}_{42} ; & \mathrm{Ag}_{2} \mathrm{O}, 6 . \\
2 \mathrm{AsCl}_{5}, 143 ; & 2 \mathrm{PCl}_{5}, 210 ; & \mathrm{CCl}_{4}, 21 ; & 2 \mathrm{HCl}, 44 \text { (gas). } \\
\mathrm{As}_{2} \mathrm{O}_{3}, 155 ; & \mathrm{P}_{2} \mathrm{O}_{5}, 370 ; & \mathrm{CO}_{2}, 97 ; & \mathrm{H}_{2} \mathrm{O}, 58 \text { (gas). }
\end{array}\right.
$$

With the first four elements the formation of the chlorine compound gives the most heat, and with the four following the formation of the oxygen compound evolves the greater amount of heat. The first four chlorides are true salts formed from $\mathrm{HCl}$ and the oxide, whilst the remainder have other properties, as is seen from the fact that they are not formed from hydrochloric acid and the oxide, but give hydrochloric acid with water. If affinity be measured by heat, then in the former the affinity for chlorine is greater than that for oxygen, whilst in the latter it is the reverse. But as the physical states of the substances are different, and as chlorine displaces oxygen as well as oxygen displaces chlorine, the affinity cannot be determined from thermo-chemical data without a number of corrections which are still subject to doubt.

14 This has been already pointed out in Chap. III. Note 5 .

15 Sodium remains unaltered in perfectly dry chlorine at the ordinary temperature, and even when slightly warmed; but the combination is exceedingly violent at a red heat.

16 An instructive experiment on combustion in chlorine may be conducted as follows : leaves of Dutch metal (used for gilding instead of gold) are placed in a glass globe, and a gas-conducting tube furnished with a glass cock is placed in the cork closing it, and the air is pumped out of the globe. The gas-conducting tube is then connected with a vessel containing chlorine, and the cock opened; the chlorine rushes in, and the metallic leaves are consumed.

17 The behaviour of platinum to chlorine at a high temperature $\left(1400^{\circ}\right)$ is very 
which do not combine directly with oxygen and give very unstable compounds with it, unite directly with chlorine to form metallic chlorides. Either chlorine water or aqua regia may be einployed for this purpose instead of gaseous chlorine. These dissolve gold and platinum, converting them into metallic chlorides. Aqua regia is a mixture of 1 part of nitric acid with 2 to 3 parts of hydrochloric acid. This mixture converts into soluble chlorides not only those metals which are acted on by hydrochloric and nitric acids, but also gold and platinum, which are insoluble in either acid separately. This action of aqua regia depends on the fact that nitric acid in acting on hydrochloric acid deprives it of its hydrogen, and evolves chlorine little by little as the action proceeds. If the chlorine evolved be transferred to a metal, then a fresh quantity is formed from the remaining acids and combines with the metal. ${ }^{18}$ Thus the aqua regia acts in virtue of the chlorine which it contains and disengages.

The majority of non-metals also react directly on chlorine; sulphur and phosphorus burn in it, and combine with it directly when heated or even at the ordinary temperature. Only nitrogen, carbon, and oxygen do not form any direct compounds with it. The chlorine compounds formed by the non-metals - for instance, phosphorus trichloride, $\mathrm{PCl}_{3}$, and sulphurous chloride, \&c., do not have the properties of salts, and if the metallic chlorides $\mathrm{M}_{n} \mathrm{Cl}_{2 m}$ correspond with bases $\mathrm{M}_{n} \mathrm{O}_{m}$ and their hydrates $\mathrm{M}_{n}(\mathrm{OH})_{2 m}$, then, as we shall afterwards see more fully, the chlorides of the non-metals bear the same relation to acid anhydrides. and acids :

$$
\begin{array}{lllll}
\mathrm{NaCl} & \mathrm{FeCl}_{2} & \mathrm{SnCl}_{4} & \mathrm{PCl}_{3} & \mathrm{HCl} \\
\mathrm{Na}(\mathrm{HO}) & \mathrm{Fe}(\mathrm{HO})_{2} & \mathrm{Sn}(\mathrm{HO})_{4} & \mathrm{P}(\mathrm{HO})_{3} & \mathrm{H}(\mathrm{HO})
\end{array}
$$

remarkable, because platinous chloride, $\mathrm{PtCl}_{2}$, is then formed, whilst this substance decomposes at a much lower temperature into chlorine and platinum. Therefore, when chlorine comes into contact with platinum at such high temperatures it forms fumes of platinous chloride, and they on conling decompose with the liberation of platinum, so that the phenomenon appears to be dependent on the volatility of platinum. Deville proved the formation of platinous chloride by inserting a cold tube inside a red-hot one (as in the experiment on carbonic oxide, p. 388). However, Meyer was able to observe the density of chlorine in a platinum vessel at $1690^{\circ}$, at which temperature chlorine does not exert this action on platinum, or at least only to an insignificant degree.

18 When left exposed to the air aqua regia disengages chlorine, and afterwards it no longer acts on gold. Gay-Lussac, in explaining the action of aqua regia, showed that when heated it evolves, besides chlorine, the vapours of two chloranhydrides-that of nitric acid, $\mathrm{NO}_{2} \mathrm{Cl}$ (nitric acid, $\mathrm{NO}_{2} \mathrm{OH}_{2}$ in which $\mathrm{HO}$ is replaced by chlorine, see chapter on Phosphorus), and that of nitrous acid, $\mathrm{NOCl}$ (ibid.)-but these do not act on gold. The formation of aqua regia may therefore be expressed by $4 \mathrm{NHO}_{3}+8 \mathrm{HCl}=2 \mathrm{NO}_{2} \mathrm{Cl}+2 \mathrm{NOCl}+$ $6 \mathrm{H}_{2} \mathrm{O}+2 \mathrm{Cl}_{2}$. The formation of the cllorides $\mathrm{NO}_{2} \mathrm{Cl}$ and $\mathrm{NOCl}$ is explained by the fact that the nitric acid is deoxidised, gives the oxides $\mathrm{NO}$ and $\mathrm{NO}_{2}$, and they directly combine with chlorine to form the above anhydrides. 
As the above-mentioned relation in composition exists between many chlorine compounds and their corresponding hydrates and as furthermore some (acid) hydrates are obtained from chlorine compounds by the action of water, for instance

$\underset{\begin{array}{c}\text { Phosphorus } \\ \text { trichloride }\end{array}}{\mathrm{PCl}_{3}}+\underset{\text { Water }}{3 \mathrm{H}_{2} \mathrm{O}}=\underset{\begin{array}{c}\text { Phosphorous } \\ \text { acid }\end{array}}{\mathrm{P}(\mathrm{HO})_{3}}+\underset{\begin{array}{c}\text { Hydrochloric } \\ \text { acid; }\end{array}}{3 \mathrm{HCl}}$

whilst other chlorine compounds (basic) are formed from hydroxides and hydrochloric acid, with the liberation of water, for instance

$$
\mathrm{NaHO}+\mathrm{HCl}=\mathrm{NaCl}+\mathrm{H}_{2} \mathrm{O} \text {; }
$$

therefore this intimate connection between the hydrates and chlorine compounds is endeavoured to be expressed by calling the latter chloranhydrides. In general terms, if the hydrate be basic, then,

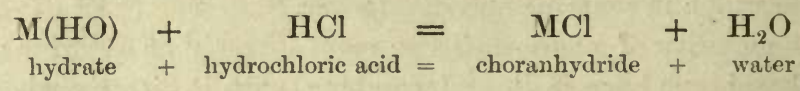

and if the hydrate $\mathrm{ROH}$ be acid, then

$$
\begin{gathered}
\mathrm{RCl}+\mathrm{H}_{2} \mathrm{O}=\mathrm{R}(\mathrm{HO})+\underset{\text { hydrate }}{\mathrm{HCl}} \\
\text { Choranhydride }+ \text { water }=\text { hydrochloric acid. }
\end{gathered}
$$

In this manner a distinct equivalency is remarked between the compounds of chlorine and the so-called hydroxyl radicle $(\mathrm{HO})$, which is also expressed in the analogy existing between chlorine, $\mathrm{Cl}_{2}$, and hydrogen peroxide, $(\mathrm{HO})_{2}$.

As regards the chloranhydrides corresponding with acids and nonmetals, they bear but little resemblance to metallic salts. They are nearly all volatile, and have a powerful suffocating smell which irritates the eyes and respiratory organs. They react on water like many anhydrides of the acids, with the evolution of heat and liberation of hydrochloric acid, forming acid hydrates. For this reason they cannot usually be obtained from hydrates-that is, acids-by the action of hydrochloric acid, as then water would be formed together with then, and water decomposes them, converting them into hydrates. There are many intermediate chlorine compounds between true saline metallic chlorides like sodium chloride and true acid chloranhydrides, just as there are all kinds of transitions between bases and acids ; feeble bases not unfrequently present feeble acid properties. These relations will become gradually clearer as we become acquainted with elements of different character, and for the sake of greater clearness we will not enter into an intimate acquaintance with the saline character of acid .chloranhydrides, and will only remark that compounds of this type are 
not only obtained from chlorine and non-metals, but also from many lower oxides, by the aid of chlorine. Thus, for example, CO, NO, $\mathrm{NO}_{2}, \mathrm{SO}_{2}$, and other lower oxides which are capable of combining with oxygen may also assimilate to themselves a corresponding quantity of chlorine. Thus $\mathrm{COCl}_{2}, \mathrm{NOCl}, \mathrm{NO}_{2} \mathrm{Cl}, \mathrm{SO}_{2} \mathrm{Cl}_{2}$, \&c., are obtained. They correspond with the hydrates $\mathrm{CO}(\mathrm{OH})_{2}, \mathrm{NO}(\mathrm{OH}), \mathrm{NO}_{2}(\mathrm{OH}), \mathrm{SO}_{2}(\mathrm{OH})_{2}$, Sc., and to the anhydrides $\mathrm{CO}_{2}, \mathrm{~N}_{2} \mathrm{O}_{3}, \mathrm{~N}_{2} \mathrm{O}_{5}, \mathrm{SO}_{3}$, \&c. In this we should evidently see two sides of the matter : (1) chlorine combines with that with which oxygen is able to combine, because it is in many respects equally if not more energetic than oxygen and replaces it in the proportion $\mathrm{Cl}^{2}: \mathrm{O}$ or $(\mathrm{Cl}: \mathrm{OH})$; (2) that lighest limit of possible combination which is proper to a given element or grouping of elements is very easily and often attained by combination with chlorine. If phosphorus gives $\mathrm{PCl}_{3}$ and $\mathrm{PCl}_{5}$, it is evident that $\mathrm{PCl}_{5}$ is the higher form of combination compared with $\mathrm{PCl}_{3}$. To the form $\mathrm{PCl}_{5}$ or in general $\mathrm{PX}_{5}$, correspond $\mathrm{PH}_{4} \mathrm{I}, \mathrm{PO}(\mathrm{OH})_{3}, \mathrm{POCl}_{3}$, dc. If chlorine does not always directly give compounds of the highest possible forms for a given element, then generally the lower forms combine with it in order to reach or approach the limit. This is particularly clear in hydrocarbons, where we see the limit $\mathrm{C}_{n} \mathrm{H}_{2 n+2}$ very distinctly. The unsaturated hydrocarbons are sometimes able to combine with chlorine with the greatest ease and thus reach the limit. Thus ethylene, $\mathrm{C}_{2} \mathrm{H}_{4}$, combines with $\mathrm{Cl}_{2}$ forming the so-called Dutch liquid or ethylene chloride, $\mathrm{C}_{2} \mathrm{H}_{4} \mathrm{Cl}_{2}$, because it then reaches the limit $\mathrm{C}_{u} \mathrm{X}_{2 n+2}$. In all like cases the combined chlorine is able by reactions of substitution to give a hydroxide and a whole series of other derivatives. Thus a hydroxide called glycol, $\mathrm{C}_{2} \mathrm{H}_{4}(\mathrm{OH})_{2}$, is obtained from $\mathrm{C}_{4} \mathrm{H}_{4} \mathrm{Cl}_{2}$.

In this way chlorine whilst entering with great ease into combination with simple gases, in a number of cases converts lower forms of combination into higher. Very often chlorine in the presence of water acts directly as an oxidising agent. A substance A combines with chlorine and gives, for example, $\mathrm{ACl}_{2}$, and this in turn a hydroxide $\mathrm{A}(\mathrm{OH})_{2}$, which on losing water forms $\mathrm{AO}$. Hence the chlorine oxidised the substance $\mathrm{A}$. This frequently happens in the simultaneous action of water and chlorine: $\mathrm{A}+\mathrm{H}_{2} \mathrm{O}+\mathrm{Cl}_{2}=2 \mathrm{HCl}+\mathrm{AO}$. Examples of this oxidising action of chlorine may frequently be observed both in chemical practice and technical processes. Thus chlorine, for instance, in the presence of water oxidises sulphur and metallic sulphides. In this case the sulphur is converted into sulphuric acid, and the chlorine into hydrochloric acid or a metallic chloride if a metallic sulphide be taken. A mixture of carbonic oxide and chloriñe passed into water gives carbonic anhydride 
and hydrochloric acid. Sulphurous anhydride is oxidised by chlorine in the presence of water into sulphuric acid, just as it is by the action of nitric acid: $\mathrm{SO}_{2}+2 \mathrm{H}_{2} \mathrm{O}+\mathrm{Cl}_{2}=\mathrm{H}_{2} \mathrm{SO}_{4}+2 \mathrm{HCl}$. The oxidising action of chlorine in the presence of water is taken advantage of in practice for the rapid bleaching of tissues and fibres. The colouring matter of the fibres is altered by oxidation and converted into a colourless substance, but the chlorine afterwards acts on the tissue itself. Bleaching by means of chlorine therefore requires a certain amount of technical skill in order that the chlorine should not act on the fibres themselves, but that its action should be limited to the colouring matter only. The fibre for making writing paper, for instance, is bleached in this manner. The property of chlorine of bleaching was discovered by Berthollet, and forms an important acquisition to the arts, because it has in the majority of cases replaced that which before was the universal method of bleaching, namely, exposure to the sun of the fabrics damped with water, which is still employed for linens, \&c. Time and great trouble, and therefore money also, have been considerably saved by this change. ${ }^{19}$

The power of chlorine for combination is intimately connected with its capacity for substitution, because, according to the law of substitution, if chlorine combines with hydrogen, then it also replaces hydrogen, and furthermore the combination and substitution are accomplished in the same quantities. Therefore the atom of chlorine which combines with the atom of hydrogen is also able to replace the atom of hydrogen. We mention this property of chlorine not only because it illustrates the adaptation of the law of substitution in clear and historically important examples, but more especially because reactions of this kind explain those indirect methods of the formation of many substances which we have often mentioned and to which recourse is had in many cases in chemistry. Thus chlorine does not act on carbon, ${ }^{20}$ oxygen, or nitrogen, but nevertheless its compounds with these elements may be obtained by the indirect method of the substitution of hydrogen by chlorine.

As chlorine easily combines with hydrogen and does not act on

19 The oxidising property of chlorine shows itself when it destroys the majority of organic tissues, and proves fatal to organisms. This property of chlorine is taken advantage of in quarantine stations. But the simple fumigation by chlorine must be carried on with great care in dwelling places, because chlorine disengaged into the atmosphere renders it harmful to the health by attacking the respiratory organs and the tissues of the lungs.

20 A certain propensity of carbon to attract chlorine must be seen in the immense absorption of chlorine by charcoal (Note 7), but, as far as is at present known (no one has tried, if I do not mistake, to have recourse to the aid of light), no combination then takes place between the chlorine and carbon. 
carbon, it decomposes hydrocarbons (and many of their derivatives) at a high temperature, depriving them of their hydrogen and liberating the carbon, as, for example, is clearly seen when a lighted candle is placed in a vessel containing chlorine. The flame becomes smaller but - continues for a certain time, a large amount of soot is obtained, and hydrochloric acid is formed. In this case the gaseous and incandescent substances of the flame are decomposed by the chlorine, the hydrogen combines with it, and the carbon is disengaged as soot. ${ }^{21}$ This action of chlorine on hydrocarbons, dc., proceeds otherwise at lower temperatures, as we will proceed to consider.

A very important epoch in the history of chemistry was formed by the discovery of Dumas and Laurent that chlorine is able to displace and replace hydrogen. This discovery is important from the fact that chlorine proved to Le an element which combines with great ease simultaneously with both the hydrogen and the element with which the hydrogen was combined. This clearly proved that there is no opposite polarity between elements forming stable compounds. Chlorine does not combine with hydrogen because it has opposite properties, as Dumas and Laurent stated previously, accounting hydrogen to be electro-positive and chlorine electro-negative ; this is not the reason of their combining together, because the same chlorine which combines with hydrogen is also able to replace it without altering many of the properties of the resultant substance. This substitution of hydrogen by chlorine is termed metalepsis. The mechanism of this substitution is very constant. If we take a hydrogen compound, preferably a hydrocarbon, and if chlorine act directly on it, then there is produced on the one hand hydrochloric acid and on the other hand a compound containing chlorine in the place of the hydrogen - so that the chlorine divides itself into two equal portions, one portion is evolved as hydrochloric acid, and the other portion takes the place of the hydrogen thus liberated. Hence this metalepsis is always accompanied by the formation of hydrochloric acid. ${ }^{22}$ The scheme of the process is as follows :
$\mathrm{C}_{n} \mathrm{H}_{m} \mathrm{X}$
$+$
$\mathrm{Cl}_{2}=$
$\mathrm{C}_{n} \mathrm{H}_{m-1} \mathrm{ClX}$
$\mathrm{HCl}$
Hydrocarbon.
Free chlorine.
Product of metalepsis. Hydrochloric acid.

21 The same takes place under the action of oxygen, with the difference that it burns the carbon, which chlorine is not able to do. If chlorine and oxygen compete together at a high temperature, the oxygen will unite with the carbon, and the chlorine with the hydrogen; if pure hydrogen be taken with a sufficient quantity of chlorine, it will all combine with the chlorine without forming any water with the oxygen.

23 This division of chlorine into two portions may at the same time be taken as a clear confirmation of the conception of molecules. According to Avogadro-Gerhardt's law, the molecule of chlorine (p. 303) contains two atoms of this substance; one atom replaces the hydrogen, and the other combines with it.

VOL. I. 
Or, in general terms-

\section{$\mathrm{RH}+\mathrm{Cl}_{2}=\mathrm{RCl}+\mathrm{HCl}$.}

The conditions under which metalepsis takes place are also very constant. In the dark chlorine does not usually act on hydrogen compounds, but the action commences under the influence of light. The direct action of the sun's rays is particularly propitious to metalepsis. It is also remarkable that the presence of traces of certain substances ${ }^{23}$ promotes the action (especially of iodine, aluminium chloride, antimony chloride, \&c.). A trace of iodine added to the substance subjected to metalepsis often produces the same effect as sunlight. ${ }^{24}$

If marsh gas be mixed with chlorine and the mixture ignited, then the hydrogen is entirely taken up from the marsh gas and hydrochloric acid and carbon formed, but there is no metalepsis. ${ }^{25}$ But if a mixture of equal volumes of chlorine and marsh gas be exposed to the action of diffused light, then the greenish yellow mixture gradually becomes colourless, and hydrochloric acid and the first product of metalepsis, namely, methyl chloride, are formed :

$\underset{\text { Marsh gas. }}{\mathrm{CH}_{4}}+\underset{\text { Chlorine. }}{\mathrm{Cl}_{2}}=\underset{\text { Methyl chloride. }}{\mathrm{CH}_{3} \mathrm{Cl}}+\underset{\text { Hydrochloric acid. }}{\mathrm{HCl}}$

23 Such carriers or media for the transference of chlorine and the halogens in general' were long known to exist in iodine and antimonious chloride, and have been most fully studied by Gustavson and Friedel, of the Petroffsky Academy-the former with respect. to aluminium bromide, and the latter with respect to aluminium chloride. Gustavson showed that if a trace of metallic aluminium be dissolved in bromine (it floats on bromine, and when combination takes place much heat and light are evolved), the latter becomes endowed with the property of entering into metalepsis, which it is not able to do of its own accord. When pure, for instance, it acts very slowly on benzene, $\mathrm{C}_{6} \mathrm{H}_{6}$, but in the presence of a trace of aluminium bromide the reaction proceeds violently and easily, so that each drop of the hydrocarbon gives a mass of hydrobromic acid, and of the product of metalepsis. Gustavson showed that the modus operandi of this instructive reaction is based on the property of aluminium bromide to enter into combination with hydrocarbons and their derivatives. The details of this and all researches concerning the metalepsis of the hydrocarbons must be looked for in works on organic chemistry.

24 As small admixtures of iodine, aluminium bromide, \&c., aid the metalepsis of large quantities of a substance, just as nitric oxide aids the reaction of sulphurous anhydride on oxygen and water, so the matter is essentially the same in both cases. Effects of this kind (which should also be explained by a chemical reaction proceeding at the surfaces) only differ from true contact phenomena in that the latter are produced by solid bodies and are accomplished at their surfaces, whilst in the former all is in solution. Probably the action of iodine is founded on the formation of indine chloride, which reacts more easily than chlorine.

25 Metalepsis belongs to the number of delicate reactions-if it may be so expressedas compared with the energetic reaction of combustion. Many cases of substitution are of this kind. Reactions of metalepsis are accompanied by the evolution of heat, but in a less quantity than that evolved in the formation of the resulting quantity of the halogen acids. Thus the reaction $\mathrm{C}_{2} \mathrm{H}_{6}+\mathrm{Cl}_{2}=\mathrm{C}_{2} \mathrm{H}_{3} \mathrm{Cl}+\mathrm{HCl}$, judging from the data given by 
The volume of the mixture remains unaltered. The methyl chloride which is formed is a gas. If it be separated from the hydrochloric acid (it is soluble in acetic acid, in which hydrochloric is but sparingly soluble) and be again mixed with chlorine then it may be subjected to a further metalepsical substitution - the second atom of hydrogen may be substituted by chlorine, and a liquid substance $\mathrm{CH}_{2} \mathrm{Cl}_{2}$, called methylene chloride, will be obtained. In the same manner the substitution may be carried on still further, and $\mathrm{CHCl}_{3}$, or chloroform, and, lastly, carbon tetrachloride, $\mathrm{CCl}_{4}$, will be produced. Of these substances the best known is chloroform, owing to its being formed in many cases from organic substances (by the action of bleaching powder) and to its being used in medicine as an anæsthetic; chloroform boils at $62^{\circ}$ and carbon tetrachloride at $78^{\circ}$. They are both colourless odoriferous liquids, heavier than water. The progressive substitution of hydrogen by chlorine is thus evident, and it can be clearly seen that the double decompositions are accomplished between molecular quantities of the substance - that is, between equal volumes in a gaseous state. Chloroform may be obtained directly from marsh gas, but this, judging from the above, will be the third product of the metalepsis of marsh gas. Between it and marsh gas there are two more intermediate products, and the first of them is produced in the action of one molecule of marsh gas on one molecule of chlorine.

Carbon tetrachloride, which is obtained by the metalepsis of marsh gas, is not obtained directly from chlorine and carbon, but it may be obtained from certain compounds of carbon - for instance, from carbon bisulphide-if its vapour mixed with chlorine be passed through a red-hot tube. Both the sulphur and carbon then combine with the chlorine. It is evident that by ultimate metalepsis a corresponding earbon chloride may be obtained from any hydrocarbon-indeed, the number of chlorides of carbon already known is very large.

As a rule, the fundamental chemical characters of hydrocarbons are not changed by metalepsis; that is, if a neutral substance be taken, then the product of metalepsis is also a neutral substance, or if an acid be taken the product of metalepsis also has acid properties. Even the crystalline form not unfrequently remains unaltered after metalepsis. The metalepsis of acetic acid, $\mathrm{CH}_{3} \cdot \mathrm{COOH}$, is historically the most important. It contains three of the atoms of the hydrogen of marsh gas, the fourth being replaced by carboxyl, and therefore by the action of chlorine it gives three products of metalepsis (according to the mass of the chlorine and conditions under which the reaction takes place),

Thomsen, evolves about 20000 heat units, whilst the formation of hydrochloric acid evolves 22000 units. 
mono-, di-, and tri-chloracetic acids $-\mathrm{CH}_{2} \mathrm{Cl} \cdot \mathrm{COOH}, \mathrm{CHCl}_{2} \cdot \mathrm{COOH}$, and $\mathrm{CCl}_{3} \cdot \mathrm{COOH}$; they are all, like acetic acid, monobasic. Without entering into the further description of cases of like reaction (they must be looked for in organic chemistry), it is necessary to turn special attention first to the fact that in this manner by an indirect method, carbon compounds (for instance, $\mathrm{CCl}_{4}, \mathrm{C}_{2} \mathrm{Cl}_{4}, \mathrm{C}_{6} \mathrm{Cl}_{6}$, \&c.) are formed, which cannot be obtained directly from the elements, and secondly, that the resulting products of metalepsis, in containing an element which so easily acts on metals as chlorine, give the possibility of attaining a further complexity of molecules for which the original hydrocarbon is often in no way capable. Thus on treating with an alkali (or first with a salt and then with an alkali, or with a basic oxide and water, \&c.) the chlorine forms a salt with its metal, and the hydroxyl radicle takes the place of the chlorine - for example, $\mathrm{CH}_{3} \cdot \mathrm{OH}$ is obtained from $\mathrm{CH}_{3} \mathrm{Cl}$. By the action of metallic derivatives of hydrocarbons-for instance, $\mathrm{CH}_{3} \mathrm{Na}$ - the chlorine also gives a salt, and the hydrocarbon radicle-for instance, $\mathrm{CH}_{3}$-takes the place of the chlorine. Thus, or in a similar manner, $\mathrm{CH}_{3} \cdot \mathrm{CH}_{3}$ or $\mathrm{C}_{2} \mathrm{H}_{6}$ is obtained from $\mathrm{CH}_{3} \mathrm{Cl}$ or $\mathrm{C}_{6} \mathrm{H}_{5} \cdot \mathrm{CH}_{3}$ from $\mathrm{C}_{6} \mathrm{H}_{6}$. The products of metalepsis also often react on ammonia, forming hydrochloric acid (and thence $\mathrm{NH}_{4} \mathrm{Cl}$ ) and an amide; that is, the product of metalepsis with the ammonia radicle $\mathrm{NH}_{2}$ in the place of chlorine. Thus by means of metalepsical substitution a method is found in chemistry for an artificial and general means of the formation of complex carbon compounds from more simple compounds which are often totally incapable of direct reaction. Besides which, this key opened the doors of that secret edifice of the structure of complex organic compounds into which man had up to then feared to enter, supposing it to be the dwelling of the spirits of organisms under the influence of whose magical force that was united which by other means could not be brought together. ${ }^{26}$

26 With the predominance of the representation of compound radicles (this doctrine dates from Lavoisier and Gay-Lussac) in organic chemistry, it was a very important moment in its history when it became possible to gain an insight into the structure of the radicles themselves. It was clear, for instance, that ethyl, $\mathrm{C}_{2} \mathrm{H}_{5}$, or the radicle of common alcohol, $\mathrm{C}_{2} \mathrm{H}_{5} \cdot \mathrm{OH}$, passes, without changing, into a number of ethyl derivatives, but its relation to the still simpler hydrocarbons was not clear, and occupied the attention of science in the 'forties' and 'fifties.' Having obtained ethyl hydride, $\mathrm{C}_{2} \mathrm{H}_{5} \mathrm{H}=\mathrm{C}_{2} \mathrm{H}_{6}$, it was looked on as containing the same ethyl, just as methyl hydride, $\mathrm{CH}_{4}=\mathrm{CH}_{3} \mathrm{H}$, was considered as existing in methane. Having obtained free methyl, $\mathrm{CH}_{3} \mathrm{CH}_{3}=\mathrm{C}_{2} \mathrm{H}_{6}$, from it, it was considered as a derivative of methyl alcohol, $\mathrm{CH}_{5} \mathrm{OH}$, and as only isomeric with ethyl hydride. By means of the products of metalepsis it was proved that this is not a case of isomerism but of strict identity, and it therefore became clear that ethyl is methylated methyl, $\mathrm{C}_{2} \mathrm{H}_{5}=\mathrm{CH}_{2} \mathrm{CH}_{3}$. In its time a still greater impetus was giren by the study of the reactions of monochloracetic acid, $\mathrm{CH}_{2} \mathrm{Cl} \cdot \mathrm{COOH}$, or $\mathrm{CO}\left(\mathrm{CH}_{2} \mathrm{Cl}\right)(\mathrm{OH})$. It appeared that metalepsical chlorine, like the chlorine of chloranhydrides-for instance 
It is not only hydrocarbons which are subjected to metalepsis. Certain other hydrogen compounds, under the action of chlorine, also give corresponding chlorine derivatives in exactly the same manner; for instance, ammonia, caustic potash, caustic lime, and a whole series of alkaline substances. ${ }^{27}$ In fact, just as the hydrogen in marsh gas can be replaced by chlorine and form methyl chloride, so the hydrogen in caustic potash, $\mathrm{KHO}$, ammonia, $\mathrm{NH}_{3}$, and calcium hydroxide, $\mathrm{CaH}_{2} \mathrm{O}_{2}$ or $\mathrm{Ca}(\mathrm{OH})_{2}$, may be replaced by chlorine and give potassium hypochlorite, $\mathrm{KClO}$, calcium hypochlorite, $\mathrm{CaCl}_{2} \mathrm{O}_{2}$, and the so-called chloride of nitrogen, $\mathrm{NCl}_{3}$. Not only is the correlation in composition the same as in the substitution in marsh gas, but the whole mechanism of the reaction is the same. Here, also, two atoms of chlorine act : one takes the place of the hydrogen whilst the other is evolved as hydrochloric acid, only in the former case the hydrochloric acid evolved remained free, and in the latter, in presence of alkaline substances, the hydrochloric acid formed reacts on them. Thus, in the action of chlorine on caustic potash, the hydrochloric acid formed acts on another quantity of caustic potash and gives potassium chloride and water, and, therefore, not only $\mathrm{KHO}+\mathrm{Cl}_{2}=\mathrm{HCl}+\mathrm{KClO}$, but also $\mathrm{KHO}+\mathrm{HCl}=\mathrm{H}_{2} \mathrm{O}+\mathrm{KCl}$, and therefore the result of both simultaneous phases will be $2 \mathrm{KHO}+\mathrm{Cl}_{2}=\mathrm{H}_{2} \mathrm{O}+\mathrm{KCl}+\mathrm{KClO}$. We will here enter into certain special cases.

The action of chlorine on ammonia may either result in the entire breaking up of the ammonia with the evolution of gaseous nitrogen, or in a product of metalepsis (as with $\mathrm{CH}_{4}$ and $\mathrm{H}_{2} \mathrm{O}$ ). With an excess of chlorine and the aid of heat the ammonia is decomposed, with the disengagement of free nitrogen. ${ }^{28}$ This reaction is evidently accom-

of methyl chloride, $\mathrm{CH}_{3} \mathrm{Cl}$, or ethyl chloride, $\mathrm{C}_{2} \mathrm{H}_{5} \mathrm{Cl}$-is capable of substitution ; for instance, glycollic acid, $\mathrm{CH}_{2}(\mathrm{OH})\left(\mathrm{CO}_{2} \mathrm{H}\right)$, or $\mathrm{CO}\left(\mathrm{CH}_{2} \cdot \mathrm{OH}\right)(\mathrm{OH})$, was obtained from it, and it appeared that the $\mathrm{OH}$ in the group $\mathrm{CH}_{2}(\mathrm{OH})$ reacted like that in alcohols, and it became clear, therefore, that it was necessary to examine the radicles themselves by analysing them from the point of view of the bonds connecting the constituent atoms. Whence arose the present doctrine of the structure of the carbon compounds. (See Chapter. VIII. Note 42.)

${ }^{27} \mathrm{By}$ embracing many instances of the action of chlorine under metalepsis we not only explain the indirect formation of $\mathrm{CCl}_{4}, \mathrm{NCl}_{3}$, and $\mathrm{Cl}_{2} \mathrm{O}$ by one method, but we also arrive at the fact that the reactions of the metalepsis of the hydrocarbons lose that exclusiveness which was often ascribed to them. Also by subjecting the chemical representations to the law of substitution we may foretell metalepsis as a particular case of a general law.

${ }_{28}$ This may be taken advantage of in the preparation of nitrogen. If a large excess of chlorine water be poured into a beaker, and a small quantity of a solution of ammonia be added, then, after shaking, nitrogen is evolved. If chlorine act on a dilute solution of ammonia, then the volume of nitrogen doos not correspond with the volume of the chlorine taken, because ammonium hypochlorite is formed. If ammonia gas be passed through a.fine orifice into a vessel containing chlorine, then the reaction of the formation 
panied by the formation of sal-ammoniac, $8 \mathrm{NH}_{3}+3 \mathrm{Cl}_{2}=6 \mathrm{NH}_{4} \mathrm{Cl}+\mathrm{N}_{2}$. But if the ammonium salt be in excess, then the reaction proceeds with the replacement of the hydrogen in the ammonia by chlorine. The essence of the matter is that $\mathrm{NH}_{3}+3 \mathrm{Cl}_{2}$ forms $\mathrm{NCl}_{3}+3 \mathrm{HCl} .{ }^{29}$ The resulting product of metalepsis, or chloride of nitrogen, $\mathrm{NCl}_{3}$, discovered by Dulong, is a liquid having the property of decomposing with excessive ease not only when heated, but even under the action of mechanical influences, as by a blow or the contact of certain solid substances. The explosion which accompanies the decomposition is due to the fact that the liquid chloride of nitrogen gives gaseous products, nitrogen and chlorine; a large volume of gas is evolved instantaneously and causes the explosion. It is even dangerous to prepare this substance in any considerable quantity. Whenever an ammoniacal substance comes into contact with chlorine great care must be taken, because it might be a case of the formation of such products and a very dangerous explosion. The liquid product of the metalepsis of ammonia may be most safely prepared in the form of small drops by the action of a galvanic current on a slightly warm solution of sal-ammoniac; chlorine is then evolved at the positive pole, and this chlorine, acting on the ammonia, gradually forms the product of metalepsis, which floats on the surface of the liquid (because it is borne up by the gas), and if a layer of turpentine be poured on to it these small drops, on

of nitrogen is accompanied by the emission of light and the appearance of a cloud of salammoniac. In all these instances there must be an excess of chlorine.

29 The hydrochloric acid formed combines with ammonia, and therefore the result is $4 \mathrm{NH}_{3}+3 \mathrm{Cl}_{2}=\mathrm{NCl}_{5}+3 \mathrm{NH}_{4} \mathrm{Cl}$. Consequently, more ammonia enters into the reaction, but the metalepsical aspect of the reaction in reality only takes place with an excess of ammonia in the form of the salt. If bubbles of chlorine be passed through a fine tube into a vessel containing ammonia gas, then each bubble gives rise to an explosion. If, however, chlorine be passed into a solution of ammonia, then the reaction first directs itself towards the formation of nitrogen, because chloride of nitrogen acts on ammonia like chlorine. But when sal-anmoniac begins to form, then the reaction directs itself towards the formation of chloride of nitrogen. The first action of chlorine on a solution of sal-ammoniac always consists in the formation of chloride of nitrogen, which reacts on ammonia thus: $\mathrm{NCl}_{3}+4 \mathrm{NH}_{3}=\mathrm{N}_{2}+3 \mathrm{NH}_{4} \mathrm{Cl}$. Therefore, so long as the liquid is alkaline from the presence of ammonia the chief product will be nitrogen. The reaction $\mathrm{NH}_{4} \mathrm{Cl}+3 \mathrm{Cl}_{2}=\mathrm{NCl}_{3}+4 \mathrm{HCl}$ is reversible; with a dilute solution it proceeds in the abovedescribed direction (perhaps owing to the affinity of the hydrochloric acid for the excess of water), but with a strong solution of hydrochloric acid it takes the opposite direction (probably in virtue of the affinity of hydrochloric acid for ammonia). Therefore there must exist a very interesting case of equilibrium between ammonia, hydrochloric acid, chlorine, water, and chloride of nitrogen which has not yet been investigated. The reaction $\mathrm{NCl}_{3}+4 \mathrm{HCl}=\mathrm{NH}_{4} \mathrm{Cl}+3 \mathrm{Cl}_{2}$ enabled Deville and Hautefeuille to determine the composition of chloride of nitrogen. When slowly decomposed by water, chloride of nitrogen gives, like a chloranhydride, nitrous acid or its anhydride $2 \mathrm{NCl}_{3}+3 \mathrm{H}_{2} \mathrm{O}$ $=\mathrm{N}_{2} \mathrm{O}_{3}+6 \mathrm{HCl}$. From these observations it is evident that chloride of nitrogen presents great chemical interest, which is strengthened by its analogy with trichloride of phosphorus. 
coming into contact with the turpentine, give feeble explosions, which are in no way dangerous, owing to the small mass of the substance formed. The drops of chloride of nitrogen may, with great precaution, be collected for investigation in the following manner. The neck of a funnel is immersed in a basin containing mercury, and, first, a saturated solution of common salt is poured into the funnel, and above it a solution of sal-ammoniac in 9 parts of water. Chlorine is then slowly passed through the solutions, when drops of chloride of nitrogen fall into the salt water. It is a yellow oily liquid of sp. gr. $1 \cdot 65$, which boils at $71^{\circ}$, and breaks up into $\mathrm{N}+\mathrm{Cl}_{3}$ at $97^{\circ}$. The contact of phosphorus, turpentine, india-rubber, \&c., causes an explosion, which is sometimes so violent that a small drop will pierce through a thick board. The exceeding facility of the decomposability of chloride of nitrogen is connected with the fact that it is formed with an absorption of heat, which it evolves when decomposed, to the amount of about 38000 heat units for $\mathrm{NCl}_{3}$, as Deville and Hautefeuille determined.

Chlorine, when absorbed by a solution of caustic soda (and also of other alkalis) at the ordinary temperature causes the replacement of the hydrogen in the caustic soda by the chlorine, with the formation of sodium chloride by the hydrochloric acid formed, so that the reaction may be represented in two phases, as was described above. In this manner, sodium hypochlorite, $\mathrm{NaClO}$, and sodium chloride are simultaneously formed: $2 \mathrm{NaHO}+\mathrm{Cl}_{2}=\mathrm{NaCl}+\mathrm{NaClO}+\mathrm{H}_{2} \mathrm{O}$. The resultant solution is termed 'eau de Javelle.' An exactly similar reaction takes place when chlorine is passed over dry hydrate of lime at the ordinary temperature : $2 \mathrm{Ca}(\mathrm{HO})_{2}+2 \mathrm{Cl}_{2}=\mathrm{CaCl}_{2} \mathrm{O}_{2}+\mathrm{CaCl}_{2}+2 \mathrm{H}_{2} \mathrm{O}$. A mixture of the product of metalepsis and calcium chloride is obtained. This mixture is employed in practice on a large scale, and is termed 'bleaching powder,' owing to its acting, especially when mixed with acids, as a bleaching agent on tissues, so that it resembles chlorine in this respect, but is preferable to chlorine, because the destructive action of the chlorine may be moderated in this case, and because it is much more convenient to deal with a solid substance than with gaseous chlorine. Bleaching powder is also called chloride of lime, because this substance is obtained from chlorine and hydrate of lime, and contains ${ }^{30}$ both

50 Quicklime, $\mathrm{CaO}$ (or calcium carbonate, $\mathrm{CaCO}_{3}$ ), does not absorb chlorine when cold, but at a red heat, in a current of chlorine, it forms calcium chloride, with the evolution of oxygen. This reaction corresponds with the decomposing action of chlorine on methane, ammonia, and water. Slaked lime (calcium hydroxide, $\mathrm{CaH}_{2} \mathrm{O}_{2}$ ) also, when dry, does not absorb chlorine at $100^{\circ}$. The absorption proceeds at the ordinary temperature (below $40^{\circ}$ ). The dry mass thus obtained contains not less than three equivalents of calcium hydroxide to four equivalents of chlorine, so that its composition is $\left[\mathrm{Ca}(\mathrm{HO})_{2}\right]_{3} \mathrm{Cl}_{4}$. In all probability a simple absorption of chlorine by the lime at first takes place in this case, as may be seen from the fact that even carbonic anhydride, when 
these substances. It may be prepared in the laboratory by passing a stream of chlorine through a cold mixture of water and lime (milk of lime). The mixture must be kept cold, as otherwise $3 \mathrm{Ca}(\mathrm{ClO})_{2}$ passes. into $2 \mathrm{CaCl}_{2}+\mathrm{Ca}\left(\mathrm{ClO}_{3}\right)_{2}$. In the manufacture of bleaching powder in large quantities at chemical works, the purest possible slaked lime is.

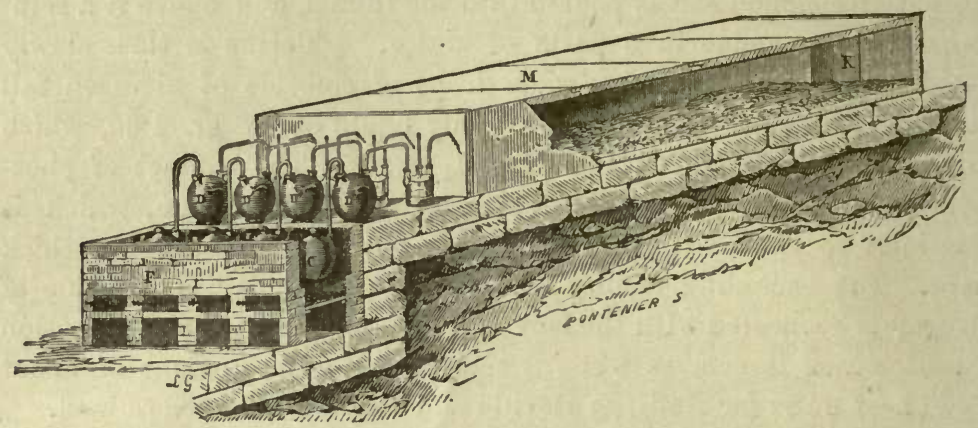

Fic. 68.- Apparatus for the manufacture of bleaching powder (at small works) by the action of chlorine, which is generater in the vessels $C$, on lime, which is charged into $M$.

taken and laid in a thin layer in large flat chambers, M (whose walls. are made of Yorkshire flags or tarred wood, on which chlorine has no. action), and into which chlorine gas is introduced by lead tubes. The distribution of the plant is shown in the annexed drawing (fig. 68).

acting on the dry mass obtained as above, disengages all the chlorine from it, forming only calcium carbonate. But if the bleaching-powder be obtained by a wet method, or if it be dissolved in water (it is very soluble), and carbonic anhydride be passed into it, then chlorine is no longer disengaged, but chlorine oxide, $\mathrm{Cl}_{2} \mathrm{O}$, and only lialf of the chlorine is converted into this oxide while the other half remains in the liquid as calcium chloride. From this it may be supposed that calcium chloride is formed by the action of water on bleaching powder, and this is proved to be the case by the fact that small quantities of water extract much calcium chloride from bleaching-powder. If a large quantity of water act on bleaching-powder there remains an excess of calcium hydroxide, a portion of which is not subjected to change. The action of the water may be expressed by the following formulæ: From the dry mass $\mathrm{Ca}_{5}(\mathrm{HO})_{6} \mathrm{Cl}_{4}$ there is formed lime, $\mathrm{Ca}(\mathrm{HO})_{2}$, calcium chloride, $\mathrm{CaCl}_{2}$, and a saline substance, $\mathrm{Ca}(\mathrm{ClO})_{2}$. $\mathrm{Ca}_{5} \mathrm{H}_{6} \mathrm{O}_{6} \mathrm{Cl}_{4}$ $=\mathrm{CaH}_{2} \mathrm{O}_{2}+\mathrm{CaCl}_{2} \mathrm{O}_{2}+\mathrm{CaCl}_{2}+2 \mathrm{H}_{2} \mathrm{O}$. The resulting substances are not equally soluble;: water first extracts the calcium chloride, which is the most soluble, then the compound $\mathrm{Ca}(\mathrm{ClO})_{2}$, and calcium hydroxide is ultimately left. A mixture of calcium chloride and hypochlorite passes into solution. On evaporation there remains $\mathrm{Ca}_{2} \mathrm{O}_{2} \mathrm{Cl}_{4}, 3 \mathrm{H}_{2} \mathrm{O}$. The dry bleaching-powder does not absorb more chlorine, but the solution is able to absorb it in considerable quantity. If the liquid be boiled, a considerable amount of chlorine. monoxide is evolved. After this calcium chloride alone remains in solution, and the decomposition may be expressed as follows: $\mathrm{CaCl}_{2}+\mathrm{CaCl}_{2} \mathrm{O}_{2}+2 \mathrm{Cl}_{2}=2 \mathrm{CaCl}_{2}+2 \mathrm{Cl}_{2} \mathrm{O}$. Chlorine monoxide may be prepared in this manner.

It is sometimes said that bleaching-powder contains a substance, $\mathrm{Ca}(\mathrm{OH})_{2} \mathrm{Cl}_{2}$, like calcium peroxide, $\mathrm{CaO}_{2}$, in which one atom of oxygen is replaced by $(\mathrm{OH})_{2}$, and the other by $\mathrm{Cl}_{2}$; but, judging from what has been said above, this can only be admitted in thedry mass, and not in solutions.

After being kept for some time, bleaching-powder sometimes decomposes, with the: evolution of oxygen (page 161); the same takes place when it is heated. 
The products of the metalepsis of alkaline hydrates, $\mathrm{NaClO}$ and $\mathrm{Ca}(\mathrm{ClO})_{2}$, which are held in solutions of 'Javelle salt,' and bleaching powder (they are not obtained free from metallic chlorides), must be counted as salts, because their metals are liable to substitution. . But the hydrate $\mathrm{HClO}$ corresponding with these salts, or hypochlorous acid, is not obtained in a free or pure state, for two reasons: in the first place, because this hydrate, as a very feeble acid, splits up (like $\mathrm{H}_{2} \mathrm{CO}_{3}$ or $\mathrm{HNO}_{3}$ ) into water and the anhydride, or chlorine monoxide, $\mathrm{Cl}_{2} \mathrm{O}=2 \mathrm{HClO}-\mathrm{H}_{2} \mathrm{O}$; and, in the second place, because, in a number of instances, it evolves oxygen with great facility, forming hydrochloric acid: $\mathrm{HClO}=\mathrm{HCl}+\mathrm{O}$. Both hypochlorous acid and chlorine monoxide may be regarded as the product of the metalepsis of water, because $\mathrm{HOH}$ corresponds with $\mathrm{ClOH}$ and $\mathrm{ClOCl}$. Hence, in many instances, bleaching salts (a mixture of hypochlorites and chlorides) break up, with the evolution of (1) chlorine, under the action of an excess of a powerful acid, capable of evolving hydrochloric acid from sodium or calcium chlorides, and which is most simple under the action of hydrochloric acid itself, because (p. 456) $\mathrm{NaCl}+\mathrm{NaClO}+3 \mathrm{HCl}$ $=2 \mathrm{NaCl}+\mathrm{HCl}+\mathrm{Cl}_{2}+\mathrm{H}_{2} \mathrm{O}$; (2) oxygen, as we saw in Chapter III. (p. 161) - the bleaching properties and, in general, oxidising action of bleaching salts is based on this evolution of oxygen (or chlorine); oxygen is also disengaged on heating the dry salts--for instance, $\mathrm{NaCl}+\mathrm{NaClO}=2 \mathrm{NaCl}+\mathrm{O} ;(3)$ and, lastly, chlorine monoxide, which contains both chlorine and oxygen. Thus, if a little sulphuric, nitric, or similar acid (in order that hydrochloric acid should not yet be produced) be added to a solution of a bleaching salt (which has an alkaline reaction, owing either to an excess of alkali or to the feeble acid properties of $\mathrm{HClO}$ ), then the hypochlorous acid set free gives water and chlorine monoxide. If carbonic anhydride (or boracic or a similar very feeble acid) act on the solution of a bleaching salt, then hydrochloric acid is not evolved from the sodium or calcium chlorides, but the hypochlorous acid is displaced and gives chlorine monoxide, ${ }^{31}$ because hypochlorous acid is one of the most feeble acids (p. 371). An excellent method for the preparation of chlorine monoxide is based on these feeble acid properties of hypochlorous acid. Zinc oxide and mercury oxide, under

51 For this reason it is necessary that in the formation of bleaching powder the chlorine should be free from hydrochloric acid, and even the lime from calcium cliloride. An excess of chlorine, in acting on a solution of bleaching-powder, may also give chlorine. monoxide, because calcium carbonate also gives chlorine monoxide under the action of chlorine. This reaction may be brought about by treating freshly-precipitated calcium. carbonate with a stream of chlorine in water: $2 \mathrm{Cl}_{2}+\mathrm{CaCO}_{3}=\mathrm{CO}_{2}+\mathrm{CaCl}_{2}+\mathrm{Cl}_{2} \mathrm{O}$. From this we may conclude that, although carbonic anhydride displaces hypochlorous anhydride, it may be itself displaced by an excess of the latter. 
the action of chlorine in the presence of water, do not give a salt of hypochlorous acid, but form a chloride and hypochlorous acid, which fact shows the incapacity of this acid to combine with the given bases. Therefore, if such oxides as those of zine or mercury be shaken up in water, and chlorine be passed through the turbid liquid, ${ }^{32}$ a reaction occurs which may be expressed in the following manner: $2 \mathrm{HgO}+2 \mathrm{Cl}_{2}$ $=\mathrm{Hg}_{2} \mathrm{OCl}_{2}+\mathrm{Cl}_{2} \mathrm{O}$. In this case, a compound of mercury oxide with mercury chloride, or the so-called mercury oxychloride, is obtained : $\mathrm{Hg}_{2} \mathrm{OCl}_{2}=\mathrm{HgO}+\mathrm{HgCl}_{2}$. This is insoluble in water, and is not affected by hypochlorous anhydride, so that the solution will contain hypochlorous acid only, but the greater part of it splits up into the anhydride and water.

A solution of hypochlorous anhydride is also obtained by the action of chlorine on many salts ; for example, in the action of chlorine on a solution of sodium sulphate the following reaction takes place: $\mathrm{Na}_{2} \mathrm{SO}_{4}+\mathrm{H}_{2} \mathrm{O}+\mathrm{Cl}_{2}=\mathrm{NaCl}+\mathrm{HClO}+\mathrm{NaHSO}_{4}$. Hence here the hypochlorous acid is formed, together with $\mathrm{HCl}$, at the expense of the reaction of chlorine on water, for $\mathrm{Cl}_{2}+\mathrm{H}_{2} \mathrm{O}=\mathrm{HCl}+\mathrm{HClO}$. If the crystallo-hydrate of chlorine be mixed with mercury oxide, then the hydrochloric acid formed in the reaction gives mercury chloride, and hypochlorous acid remains in solution. A dilute solution of hypochlorous acid or chlorine monoxide may be concentrated by distillation, and if a substance which takes up water (without destroying the acid) -for instance, calcium nitrate-be added to the stronger solution then the anhydride of hypochlorous acid-i.e. chlorine monoxide-is disengaged.

In the bleaching salts and hypochlorous salts which correspond with chlorine monoxide and contain the two elements oxygen and chlorine, which both act in an oxidising manner, we see a characteristic example of a compound of elements which, in the majority of cases, act chemically in an analogous manner. Chlorine monoxide, as prepared from an aqueous solution by the abstraction of water or by the action of dry chlorine on cold mercury oxide, is, at the ordinary temperature, a gas or vapour which condenses into a red liquid boiling at $+20^{\circ}$ and giving a vapour whose density (43 referred to hydrogen) shows that

52 Dry red mercury oxide acts on chlorine, forming dry hypochlorous anhydride (chlorine monoxide) (Balard); when mixed with water, red mercury oxide acts feebly on chlorine, and when freshly precipitated it evolves oxygen and chlorine. An oxide of mercury which easily and abundantly evolves chlorine monoxide under the action of chlorine in the presence of water may be prepared as follows: the oxide of mercury, precipitated from a mercuric salt by an alkali, is heated to $300^{\circ}$ and cooled (Pelouze). If a salt, $\mathrm{MClO}$, be added to a solution of mercuric salt, $\mathrm{HgX}_{2}$, then mercuric oxide is liberated, because the hypochlorite is decomposed. 
2 vols. of chlorine and 1 vol. of oxygen give 2 vols. of chlorine monoxide. In an anhydrous form the gas or liquid easily explodes, splitting up into chlorine and oxygen. This explosiveness is determined by the fact that heat is evolved in the decomposition to the amount of about 15000 heat units for $\mathrm{Cl}_{2} \mathrm{O}{ }^{33}$ The explosion may even take place accidentally, and also in the presence of many oxidisable substances (for instance, sulphur, organic compounds, drc.), but the solution, although unstable and showing strong oxidising properties, does not give any explosion. ${ }^{34}$

Hypochlorous acid, its salts, and chlorine monoxide serve as a transition between hydrochloric acid, chlorides, and chlorine, and a whole series of compounds containing the same elements combined with a still greater quantity of oxygen. The higher oxides of chlorine, even in their origin, are closely connected with hypochlorous acid and its salts :

$\mathrm{Cl}_{2}, \mathrm{NaCl}, \mathrm{HCl}$, hydrochloric acid.
$\mathrm{Cl}_{2} \mathrm{O}, \mathrm{NaClO}, \mathrm{HClO}$, hypochlorous acid.
$\mathrm{Cl}_{2} \mathrm{O}_{3}, \mathrm{NaClO}_{2}, \mathrm{HClO}_{2}$, chlorous acid. ${ }^{35}$
$\mathrm{Cl}_{2} \mathrm{O}_{5}, \mathrm{NaClO}_{3}, \mathrm{HClO}_{3}$, chloric acid.
$\mathrm{Cl}_{2} \mathrm{O}_{7}, \mathrm{NaClO}_{4}, \mathrm{HClO}_{4}$, perchloric acid.

When heated, solutions of hypochlorites undergo a remarkable change. Themselves so unstable, they, without the addition of any-

53 All explosive substances are of this kind-ozone, hydrogen peroxide, chloride of nitrogen, nitro-compounds, \&c. Hence they cannot be formed directly from the elements or their simplest compounds, but, on the contrary, decompose into them. In a liquid state chlorine monoxide even explodes on contact with powdery substances, or when rapidly agitated-for instance, if a file be rasped over the vessel in which it is.

34 A solution of chlorine monoxide, or hypochlorous acid, does not explode, owing to the presence of the mass of water. In dissolving, chlorine monoxide evolves about 9000 heat units, so that its store of heat becomes less.

The capacity of hypochlorous acid for entering into combination with the unsaturated hydrocarbons (Carius and others) is very often taken advantage of in organic chemistry. "Thus its solution absorbs ethylene, forming the chlorhydrin $\mathrm{C}_{2} \mathrm{H}_{4} \mathrm{Cl} \cdot \mathrm{OH}$.

The oxidising action of hypochlorous acid and its salts is not only applied to bleaching but also to many reactions of oxidation. Thus it converts the lower oxides of manganese into the peroxide.

35 Chlorous acid, $\mathrm{HClO}_{2}$ (judging from the data given by Millon, Brandau, and others) in many respects resembles hypochlorous acid $\mathrm{HClO}$, whilst they both differ from chloric and perchloric acids in their degree of stability, which is expressed, for instance, in their bleaching properties; the two higher acids do not bleach, but both the lower ones do so (oxidise at the ordinary temperature). On the other hand, chlorous acid is analogous to nitrous acid, $\mathrm{HNO}_{2}$. The anhydride of chlorous acid, $\mathrm{Cl}_{2} \mathrm{O}_{3}$, is not known in a pure state, but it probably occurs in admixture with chlorine dioxide, $\mathrm{ClO}_{2}$, which is obtained by the action of nitric and sulphuric acids on a mixture of potassium chlorate with such reducing substances as nitric oxide, arsenious oxide, sugar, \&c. All that is at present known is that pure chlorine dioxıde $\mathrm{ClO}_{2}$ (see Notes 39-43) is gradually converted into a mixture of hypochlorous and chlorous acids under the action of water (and alkalis) 
thing, yield two fresh salts which are both much more stable; onecontains more oxygen than $\mathrm{MClO}$, the other contains none at all.

$$
\underset{\text { hypochlorite }}{3 \mathrm{MClO}}=\underset{\text { chlorate }}{\mathrm{MClO}_{3}}+\underset{\text { chloride. }}{2 \mathrm{MCl}}
$$

Part of the salt-namely, two-thirds of it - parts with its oxygen in order to oxidise the remaining third. ${ }^{36}$ From an intermediate substance, $\mathrm{RX}$, there proceed two extremes, $\mathrm{R}$ and $\mathrm{RX}_{3}$, just as from nitrous anhydride there proceed nitric oxide and nitric anhydride (or nitric acid): $3 \mathrm{~N}_{2} \mathrm{O}_{3}=\mathrm{N}_{2} \mathrm{O}_{5}+4 \mathrm{NO}$. The resulting salt $\mathrm{MClO}_{3}$ corresponds with chloric acid and potassium chlorate, $\mathrm{KClO}_{3}$. It is evident that a similar salt is obtained directly by the action of chlorine on an alkali if its solution be heated, because $\mathrm{RClO}$ will be first formed, and then $\mathrm{RClO}_{3}$; for example, $6 \mathrm{KHO}+3 \mathrm{Cl}_{2}=\mathrm{KClO}_{3}+5 \mathrm{KCl}+3 \mathrm{H}_{2} \mathrm{O}$. Chlorates are formed thus; for instance, potassium chlorate, which is

that is, it acts like nitric peroxide, $\mathrm{NO}_{2}$ (giving $\mathrm{HNO}_{3}$ and $\mathrm{HNO}_{2}$ ), or as a mixed anhydride, $2 \mathrm{ClO}_{2}+\mathrm{H}_{2} \mathrm{O}=\mathrm{HClO}_{3}+\mathrm{HClO}_{2}$. The silver salt, $\mathrm{AgClO}_{2}$, is sparingly soluble in water. The investigations of Garzarolli-Thurnlackh and others seem to show that the anhydride $\mathrm{Cl}_{2} \mathrm{O}_{3}$ does not exist.

${ }^{36}$ Hydrochloric acid, which forms an example of compounds of this kind, is a saturated substance which does not combine directly with oxygen, but in which, nevertheless, a considerable quantity of oxygen may be inserted between the elements forning it. The same may be observed in a number of other cases. Thus, for instance, oxygen may be added or inserted between the elements, sometimes in considerable quantities, in the saturated hydrocarbons; for instance, in $\mathrm{C}_{3} \mathrm{H}_{8}$, three atoms of oxygen produce an alcohol, glycerin or glycerol $\mathrm{C}_{5} \mathrm{H}_{5}(\mathrm{OH})_{3}$. We shall meet with similar examples hereafter. This is explained generally by regarding oxygen as a bivalent element-that is, as capable of combining with two different elements, such as chlorine, hydrogen, \&c. On the basis of this view, it may be inserted between each pair of combined elements; the oxygen will then be combined with one of the elements by one of its affinities and with the other element by its other affinity. This view does not, however, express the entire substance of the matter, even when applied to the compounds of chlorine. Hypochlorous acid, $\mathrm{HOCl}$-that is, hydrochloric acid in which one atom of oxygen is inserted-is, as we have already seen, a substance of small stability; it would therefore be expected that on the addition of a fresh quantity of oxygen a still less stable substance would be obtained, because, according to the above view, the chlorine and hydrogen, which form such a stable compound together, are then still further removed from each other. But it appears. that chloric and perchloric acid, $\mathrm{HClO}_{3}$ and $\mathrm{HClO}_{4}$, are much more stable substances. Furthermore, the addition of oxygen has also its limit, it can only be added to a certain extent. If the above representation were true and not formal, then a limit to the combination of oxygen could not be looked for, and the more it entered into one uninterrupted chain the more stable would be the resultant compound. But not more than four atoms of oxygen can be added to hydrogen sulphide, nor to hydrochloric acid, nor tohydrogen phosphide. This peculiarity must lie in the properties of oxygen itself; four atoms of oxygen seem to have the power of forming a kind of radicle which retains two. or several atoms of different other substances-for example, chlorine and hydrogen, hydrogen and sulphur, sodium and manganese, phosphorus and metals, \&c., forming comparatively stable compounds, $\mathrm{NaClO}_{4}, \mathrm{Na}_{2} \mathrm{SO}_{4}, \mathrm{NaMnO}_{4}, \mathrm{Na}_{3} \mathrm{PO}_{4}$, \&c. See Chapter X. Note 1. 
easily separated from potassium chloride, because it is sparingly soluble in cold water. ${ }^{37}$.

If dilute sulphuric acid be added to a solution of potassium chlorate, then chloric acid is liberated ; but it cannot be separated by distillation, as it is decomposed in the process. To obtain the free acid sulphuric acid must be added to a solution of barium chlorate. ${ }^{38}$ The sulphuric acid gives a precipitate of barium sulphate with the barium, and free chloric acid remains in solution. The solution may be evaporated under the receiver of an air-pump. This solution is colourless, has no smell, and acts as a powerful acid (it saturates sodium hydroxide, decomposes sodium carbonate, gives hydrogen with zinc, drc.); when

37 If chlorine be passed through a cold solution of potash, then a bleaching compound, potassium chloride and hypochlorite, $\mathrm{HCl}+\mathrm{KClO}$, is formed, but if it be passed through a hot solution potassium chlorate is formed. As this is sparingly soluble in water, it chokes the gas-conducting tube, which should therefore be widened out at the end.

Potassium chlorate is usually prepared on a large scale from calcium chlorate, which is prepared by passing chlorine (as long as it is absorbed) into water containing lime, the mixture being kept warm. A mixture of calcium chlorate and chloride is thus formed in the solution. Potassium chloride is then added to the warm solution, and on cooling a precipitation of potassium chlorate is formed as a substance which is sparingly soluble in cold water, especially in the presence of other salts. The double decomposition taking place is $\mathrm{Ca}\left(\mathrm{ClO}_{5}\right)_{2}+2 \mathrm{KCl}=\mathrm{CaCl}_{2}+2 \mathrm{KClO}_{5}$. On a small scale in the laboratory potassium chlorate is best prepared from a strong solution of bleaching powder by passing chlorine through it and then adding potassium chloride.

Potassium chlorate crystallises easily in large colourless tabular crystals. Its solubility in 100 parts of water at $0^{\circ}=3$ parts, $20^{\circ}=8$ parts, $40^{\circ}=14$ parts, $60^{\circ}=25$ parts, $80^{\circ}=40$ parts. For comparison we will cite the following figures showing the solubility of potassium chloride and perchlorate in 100 parts of water: potassium chloride at $0^{\circ}=28$ parts, $20^{\circ}=35$ parts, $40^{\circ}=40$ parts, $100^{\circ}=57$ parts ; potassium perchlorate at $0^{\circ}$ about 1 part, $20^{\circ}$ about $1 \frac{3}{4}$ parts, $100^{\circ}$ about 18 parts. When heated potassium chlorate melts (the melting point has been given as from $335^{\circ}-376^{\circ}$; according to the latest determination by Carnelley, $359^{\circ}$ ) and decomposes with the evolution of oxygen, potassium perchlorate being at first formed, as will afterwards be described. A mixture of potassium chlorate and nitric and hydrochloric acids brings about oxidation and chlorination in solutions. It deflagrates when thrown upon incandescent carbon, and when mixed with sulphur ( $\frac{1}{3}$ by weight) it sets light to it, even when struck, in which case an explosion takes place. The same occurs with many metallic sulphides and organic substances. Such mixtures are inflamed by a drop of sulphuric acid. All these effects are due to the large amount of oxygen contained in potassium chlorate, and to the ease with which it is evolved. A mixture of two parts of potassium chlorate, one part of sugar, and one part of yellow prussiate of potasls acts like gunpowder, but it burns very rapidly, and therefore bursts the guns, and also it has a very strong oxidising action on their metal. The sodium salt, $\mathrm{NaClO}_{3}$, is much more soluble than the potassium salt, and it is therefore more difficult to free it from sodium chloride, \&c. The barium salt is also more soluble than the potassium salt; $0^{2}=24$ parts, $20^{\circ}=37$ parts, $80^{\circ}=98$ parts of salt per 100 of water.

58 Barium chlorate, $\mathrm{Ba}\left(\mathrm{ClO}_{3}\right)_{2}, \mathrm{H}_{2} \mathrm{O}$, is prepared in the following way : impure chloric acid is first prepared and saturated with baryta, and the barium salt purified by crystallisation. The impure free chloric acid is obtained by converting the potassium in potassium chlorate into an insoluble salt. This is done by adding tartaric or hydrofluosilicie acid to a solution of potassium chlorate, because potassium tartrate and potassium silicofluoride are very sparingly soluble in water. Chloric acid is easily soluble in water. 


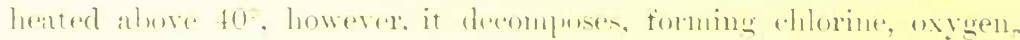

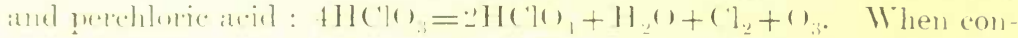

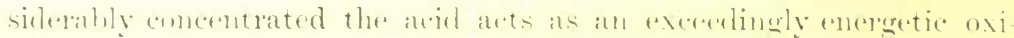

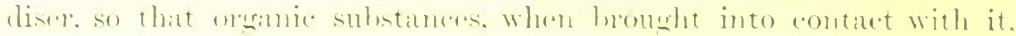

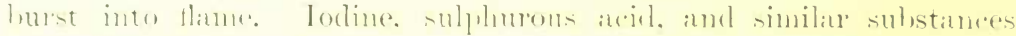
liable to axidition form highere oxidation froducts and reduce the

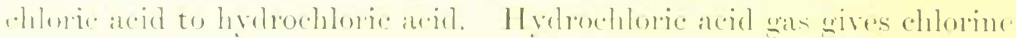

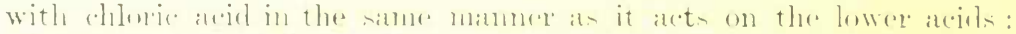
$11(16)+.311(1)=: 311,(1)+: 3(1 \%$

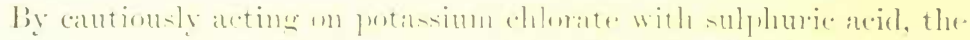

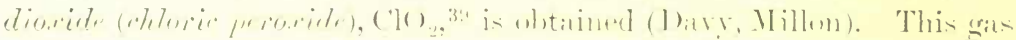
is easily liquetienl in a freering mixture, and the liquin luils at +10 .

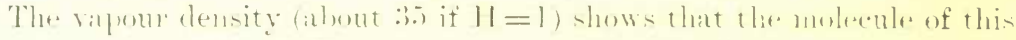

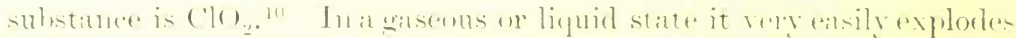

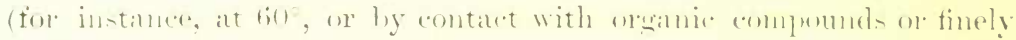

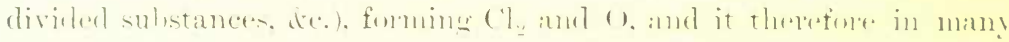

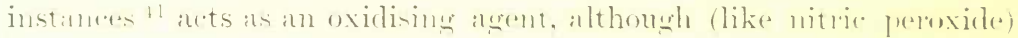
it may be itself further widised. ${ }^{12}$ In dissolving in water or alkalis

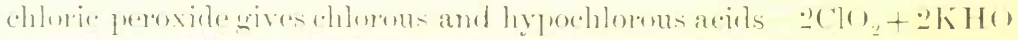

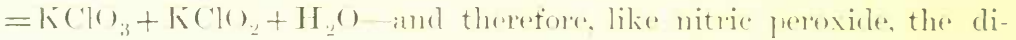

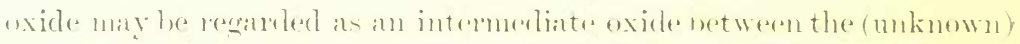

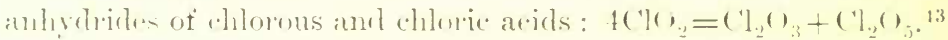

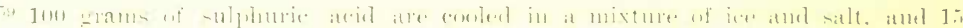

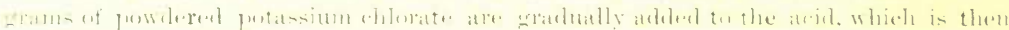

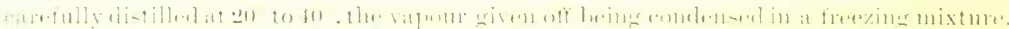

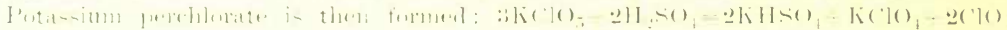

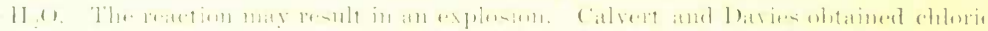

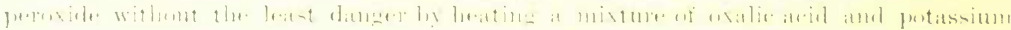

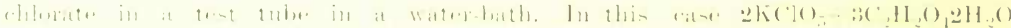

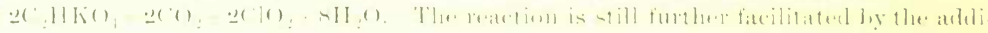

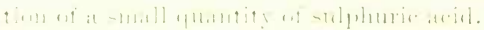

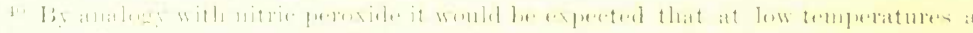

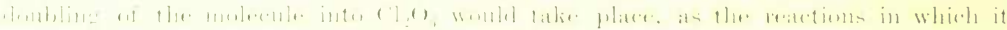

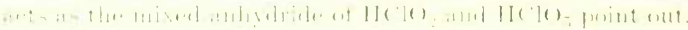

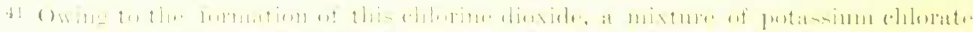

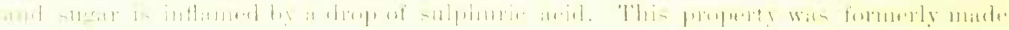

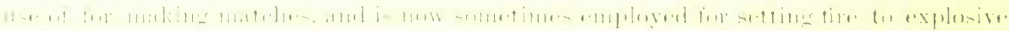

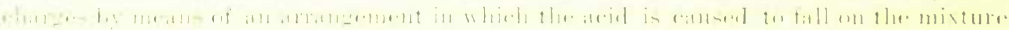

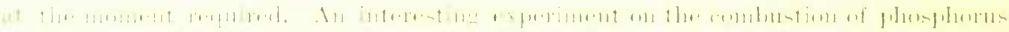

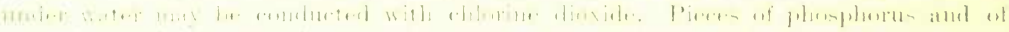

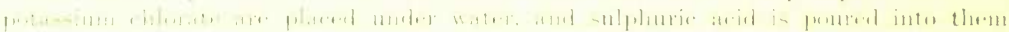

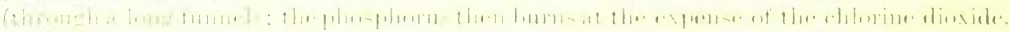

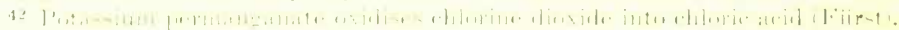

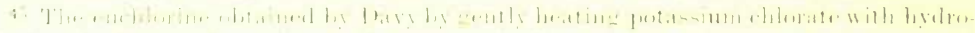

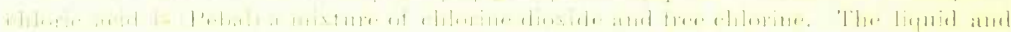

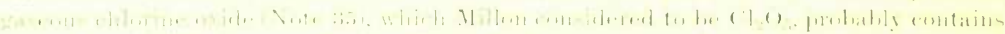

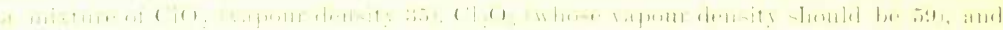

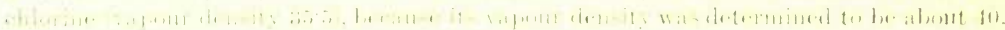


As the salts of chloric acid, $\mathrm{HClO}_{3}$, are produced by the oxidation of the salts of hypochlorous acid, so, in a similar manner, the salts of perchloric acid, $\mathrm{HClO}_{4}$, are produced by the oxidation of the salts of chloricacid, $\mathrm{HClO}_{3}$. But this is the highest form of the oxidation of $\mathrm{HCl}$. Perchloric acid, $\mathrm{HClO}_{4}$, is the most stable of all the acids of chlorine. When fused potassium chlorate begins to swell up and solidify, after having parted with one-third of its oxygen; then potassium chloride and potassium perchlorate are formed, $2 \mathrm{KClO}_{3}=\mathrm{KClO}_{4}+\mathrm{KCl}+\mathrm{O}_{2}$.

The formation of this salt is clearly remarked in the preparation of oxygen from potassium chlorate, owing to the fact that the potassium perchlorate fuses with greater difficulty than the chlorate, and therefore appears in the molten salt as solid grains. (Under the action of certain acids - for instance, sulphuric and nitric-potassium chlorate also gives potassium perchlorate.) It may be easily purified, because it is but sparingly soluble in water, although all the other salts of perchloric acid are very soluble and even effloresce in the air. It is a remarkable fact that the perchlorates, although they contain more oxygen than the chlorates, are decomposed with greater difficulty, and, even when thrown on ignited charcoal, give a much feebler deflagration than the chlorates. Sulphuric acid (at a temperature not below $100^{\circ}$ ) evolves volatile and, to a certain extent, stable perchloric acid from potassium perchlorate. Neither sulphuric nor any other acid will further decompose perchloric acid as it decomposes chloric acid. Of all the acids of chlorine, perchloric acid alone can be distilled. ${ }^{44}$ The pure hydrate $\mathrm{HClO}_{4}{ }^{45}$ is a colourless and exceedingly caustic substance

44 If a solution of chloric acid, $\mathrm{HClO}_{3}$, be first concentrated over sulphuric acid under the receiver of an air-pump and afterwards distilled, then chlorine and oxygen are evolved and perchloric acid formed: $4 \mathrm{HClO}_{3}=2 \mathrm{HClO}_{4}+\mathrm{Cl}_{2}+3 \mathrm{O}+\mathrm{H}_{2} \mathrm{O}$. Roscoe accordingly directly decomposed a solution of potassium chlorate by hydrofluosilicic acid, decanted it from the precipitate of potassium silicofluoride, $\mathrm{K}_{2} \mathrm{SiF}_{6}$, concentrated the solution of chloric acid, and then distilled it, perchloric acid being then obtained (see following footnote). That chloric acid is capable of passing into perchloric acid is also seen from the fact that potassium permanganate is decolorised, although slowly, by the action of a solution of chloric acid. On decomposing a solution of potassium chlorate by the action of an electric current, potassium perchlorate is obtained at the positive electrode (where the oxygen is evolved). Perchloric acid is also formed by the action of an electric current on solutions of chlorine and chlorine monoxide. Perchloric acid was obtained by Count Stadion. and afterwards by Serullas, and was studied by Roscoe and others.

45 Perchloric acid, which is obtained in a free state by the action of sulphuric acid on its salts, may be separated from a solution very easily by distillation, because it is volatile, although it is partially decomposed by distillation. The solution obtained after distillation may be concentrated by evaporation in open vessels. In the distillation the solution reaches a temperature of $200^{\circ}$, and then a very constant liquid hydrate of the composition $\mathrm{HClO}_{4}, 2 \mathrm{H}_{2} \mathrm{O}$ is obtained in the distillate. If this hydrate be mixed with sulphuric acid, it begins to decompose at $100^{\circ}$, but, nevertheless, a portion of the acid passes over into the receiver without decomposing, forming a crystalline hydrate $\mathrm{HClO}_{4}, \mathrm{H}_{2} \mathrm{O}$ which melts at $50^{\circ}$. On carefully heating this hydrate it breaks up into perchloric acid, which 
which fumes in the air and has a sp. gr. 1.78 at $15^{\circ}$ (sometimes, after being kept for some time, it decomposes with a violent explosion). It explodes violently when brought into contact with charcoal, paper, wood, and other organic substances. If a small quantity of water be added to this hydrate, and if it be subjected to cold, then a crystallohydrate, $\mathrm{ClHO}_{4}, \mathrm{H}_{2} \mathrm{O}$, separates out. It is much more stable, but the liquid hydrate $\mathrm{HClO}_{4}, 2 \mathrm{H}_{2} \mathrm{O}$ is still more so. The acid dissolves in water in all proportions, and its solutions are distinguished for their stability. ${ }^{46}$ When ignited both the acid and its salts are decomposed, with the evolution of oxygen. ${ }^{47}$

distils over below $100^{\circ}$, and into the liquid hydrate $\mathrm{HClO}_{4}, 2 \mathrm{H}_{2} \mathrm{O}$. The acid $\mathrm{HClO}_{4}$ may also be obtained by adding one-fourth part of strong sulphuric acid to potassium chlorate, and carefully distilling and subjecting the crystals of the hydrate $\mathrm{HClO}_{4}, \mathrm{H}_{2} \mathrm{O}$ obtained in the distillate to a fresh distillation. A liquid of the composition $\mathrm{HClO}_{4}$ then passes over. Perchloric acid, $\mathrm{HClO}_{4}$, when taken separately, does not distil, and is decomposed in distillation until the more stable hydrate $\mathrm{HClO}_{4}, \mathrm{H}_{2} \mathrm{O}$ is formed; this is decomposed in distillation into $\mathrm{HClO}_{4}$ and $\mathrm{HClO}_{4}, 2 \mathrm{H}_{2} \mathrm{O}$, which latter hydrate distils without alteration. This forms an excellent example of the influence of water on stability, and of the property of chlorine to give compounds of the type $\mathrm{ClX}_{7}$, of which all the above hydrates, $\mathrm{ClO}_{3}(\mathrm{OH}), \mathrm{ClO}_{2}(\mathrm{OH})_{3}$, and $\mathrm{ClO}(\mathrm{OH})_{5}$, are members. Probably further research will lead to the discovery of a hydrate $\mathrm{Cl}(\mathrm{OH})_{7}$.

46 According to Roscoe the sp. gr. of perchloric acid $=1.782$ and of the hydrate $\mathrm{HClO}_{4}, \mathrm{H}_{2} \mathrm{O}$ in a liquid state $\left(50^{\circ}\right) 1 \cdot 811$; hence a considerable contraction takes place in the combination of $\mathrm{HClO}_{4}$ with $\mathrm{H}_{2} \mathrm{O}$.

47 The decomposition of salts analogous to potassium chlorate has been more fully studied in recent years by Potilitzin and P. Frankland. Professor Potilitzin, by decomposing, for example, lithium chlorate $\mathrm{LiClO}_{3}$, found (from the quantity of lithium chloride and oxygen) that at first the decomposition of the fused salt $\left(368^{\circ}\right)$ is accomplished according to the equation, $3 \mathrm{LiClO}_{3}=2 \mathrm{LiCl}+\mathrm{LiClO}_{4}+5 \mathrm{O}$, and that towards the end the remaining salt is decomposed thus: $5 \mathrm{LiClO}_{3}=4 \mathrm{LiCl}+\mathrm{LiClO}_{4}+10 \mathrm{O}$. The phenomena observed by Potilitzin obliged him to admit that lithium perchlorate is capable of decomposing simultaneously with lithium chlorate, with the formation of the latter salt and oxygen; and this was confirmed by direct experiment, which showed that lithium chlorate is always formed in the decomposition of the perchlorate. Potilitzin turned particular attention to the fact that the decomposition of potassium chlorate and of salts analogous to it, although exothermal (Chapter III. Note 12), not only does not proceed by itself, but requires time and a rise of temperature in order to attain completion, which again shows that chemical equilibria are not expressed by the heat effects of reactions only.

P. Frankland and J. Dingwall (1887) showed that at $448^{\circ}$ (in the vapour of sulphur) a mixture of potassium chlorate and pounded glass is decomposed almost in accordance with the equation $2 \mathrm{KClO}_{3}=\mathrm{KClO}_{4}+\mathrm{KCl}+\mathrm{O}_{2}$, whilst the salt by itself evolves about half as much oxygen, in accordance with the equation, $8 \mathrm{KClO}_{3}=5 \mathrm{KClO}_{4}+3 \mathrm{KCl}+2 \mathrm{O}_{2}$. The decomposition of potassium perchlorate in admixture with manganese peroxide proceeds to completion, $\mathrm{KClO}_{4}=\mathrm{KCl}+2 \mathrm{O}_{2}$. But in decomposing by itself the salt at first gives potassium chlorate, approximately according to the equation $7 \mathrm{KClO}_{4}=2 \mathrm{KClO}_{3}+5 \mathrm{KCl}$ $+110_{2}$. Thus there is now no doubt that when potassium chlorate is heated, the perchlorate is formed, and that this salt, in decomposing also with the evolution of oxygen, gives the former salt.

I may further remark that the decomposition of potassium chlorate as a reaction evolving heat from this very reason easily lends itself to the contact action of manganese peroxide and other similar admixtures; for such very feeble influences as those of contact may evince themselves, as is observed either in those cases (for instance, detonating gas, 
On comparing chlorine as an element not only with nitrogen and carbon but with all the other non-metallic elements (chlorine has so little analogy with the metals that a comparison would be superfluous), we find in it the following fundamental properties of the halogens or salt-producers. With metals chlorine gives salts (such as sodium chloride, \&c.); with hydrogen a very energetic and monobasic (containing one $\mathrm{H}$ in its molecule) acid $\mathrm{HCl}$, and the same chlorine is able by metalepsis to replace the hydrogen; with oxygen it forms oxides of an acid character. These properties of chlorine are possessed by three other elements, bromine, iodine, and fluorine. They are members of one natural family. Each representative has its peculiarities, its individual properties, and points of distinction in combination and in the free state-otherwise they would not be independent elements; but the repetition in all of them of the same chief signs of the family enables one to foretell from one element the properties of another, and thus abbrevites an acquaintance with all the differences of their elementary propdrties and a'systemisation of the elements themselves.

In order to have a guiding thread in forming comparisons between the elements, attention must be turned to those of their properties and signs in which they differ most from each other, because it is only under this condition that the comparison ceases to be artificial. And the atomic weights of the elements must be counted as their most elementary property ; it being a quantity which is most undoubtedly established, and which acts in all the manifestations of the element. The halogens are endowed with atomic weights-

$$
\mathrm{F}=19, \quad \mathrm{Cl}=35 \cdot 5, \quad \mathrm{Br}=80, \quad \mathrm{I}=127 .
$$

All the properties, physical and chemical, of the elements and their corresponding compounds must evidently be in a certain dependence on this fundamental point, if the grouping in one family be natural. And we find in reality that, for instance, the properties of bromine, whose atomic weight is almost the mean between those of iodine and chlorine, occupy a mean position between those of these two elements. The second measurable property of the elements is their equivalence or their capacity for forming compounds of definite forms. Thus carbon

hydrogen peroxide, \&c.), when the reaction is accompanied by the evolution of heat, or when (for instance, $\mathrm{H}_{2}+\mathrm{I}_{2}$, \&c.) little heat is absorbed or evolved. In these cases it is evident that the existing equilibrium is not very stable, and that a feeble alteration in it proceeding at the surfaces of contact may suffice to cause its destruction. In order to conceive the modus operandi of contact phenomena, it is enough to imagine, for instance, that at the borders of contact the movement of the atoms in the molecules changes from a circular to an elliptical path. Momentary and transitory compounds may be formed, but their formation cannot alter the explanation of the phenomena.

VOL. I. 
or nitrogen in this respect differs widely from the halogens. Although the form $\mathrm{ClO}_{2}$ corresponds with $\mathrm{NO}_{2}$ and $\mathrm{CO}_{2}$, yet the latter is the highest oxide of carbon, whilst that of nitrogen is $\mathrm{N}_{2} \mathrm{O}_{5}$, and for chlorine, if there was an anhydride of perchloric acid, its composition would be $\mathrm{Cl}_{2} \mathrm{O}_{7}$, which is quite different from that of carbon. In respect to the forms of their compounds the halogens, like all elements of one family or group, are perfectly inalogous to each other, as is seen from their hydrogen compounds :

\section{$\mathrm{HF}, \mathrm{HCl}, \mathrm{HBr}$, $\mathrm{HI}$.}

It is the same with their oxygen compounds. Only fluorine does. not give any oxygen compounds. The iodine and bromine compounds corresponding with $\mathrm{HClO}_{3}$ and $\mathrm{HClO}_{4}$ are $\mathrm{HBrO}_{3}$ and $\mathrm{HBrO}_{4}, \mathrm{HIO}_{3}$ and $\mathrm{HIO}_{4}$. On comparing the properties of these acids we can even foresee the fact that fluorine will not form any oxygen compound. In fact, iodine is easily oxidised - for instance, by nitric acid-whilst chlorine is not directly oxidised. The oxygen acids of iodine are comparatively more stable than the acids of chlorine; and, generally speaking, the affinity of iodine for oxygen is much greater than that of chlorine. Here also bromine occupies an intermediate position. In fluorine we may expect a still smaller affinity for oxygen than in chlorine-and up to now it has not been combined with oxygen. If any oxygen compound of fluorine be obtained, it will be exceedingly unstable. The relation of these elements to hydrogen is the reverse of the above. Fluorine has so great an affinity for hydrogen that it decomposes water at the ordinary temperature; whilst iodine has so little affinity for hydrogen that hydriodic acid, HI, is formed with difficulty, is easily decomposed, and acts as a reducing agent in a number of cases.

From the form of their compounds the halogens are univalent elements with respect to hydrogen and septivalent with respect to oxygen, if $\mathrm{N}$ be trivalent to hydrogen (it gives $\mathrm{NH}_{3}$ ) and quinquivalent to oxygen (it gives $\mathrm{N}_{2} \mathrm{O}_{5}$ ), and if $\mathrm{C}$ be quadrivalent to both $\mathrm{H}$ and $\mathrm{O}$ as it forms $\mathrm{CH}_{4}$ and $\mathrm{CO}_{2}$.

As not only their oxygen compounds, but also their hydrogen compounds, have acid properties, the halogens are elements of an exclusively acid character. Such metals as sodium, potassium, barium only give basic oxides. In the case of nitrogen, although it forms acid oxides, still in ammonia we find that capacity to give an alkali with hydrogen which indicates a less distinctly acid character than in the halogens. In no other elements are the acid characters so strongly developed as in the halogens. 
In describing certain peculiarities characterising the halogens, we shall at every step encounter a confirmation of the above-mentioned general relations.

As fluorine decomposes water with the evolution of oxygen (which forms ozone if the temperature be not high), $\mathrm{F}_{2}+\mathrm{H}_{2} \mathrm{O}=2 \mathrm{HF}+\mathrm{O}$, therefore for a long time all efforts to obtain it in free state by means of methods similar to those for the preparation of chlorine proved fruitless. Thus by the action of hydrofluoric acid on manganese peroxide, or by decomposing a solution of hydrofluoric acid by an electric current, either oxygen or a mixture of oxygen and fluorine were obtained instead of fluorine. Probably a certain quantity of fluorine ${ }^{48}$ was set free by the action of oxygen or an electric current on incandescent and fused calcium fluoride, but it then, at the high temperature, acted even on platinum, and was therefore absorbed, leaving only oxygen. When chlorine acted on silver fluoride, $\mathrm{AgF}$, in a vessel of natural fluor spar, $\mathrm{CaF}_{2}$, fluorine was also liberated; but it was mixed with chlorine, and it was impossible to study the properties of the resultant gas. Brauner also obtained fluorine by igniting cerium fluoride, $2 \mathrm{CeF}_{4}=2 \mathrm{CeF}_{3}+\mathrm{F}_{2}$; but this, like all preceding efforts, only showed fluorine to be a gas which decomposes water, and is capable of acting in a number of instances like chlorine, but gave no possibility of testing its properties. It was evident that it was necessary to avoid as far as possible the presence of water and a rise of temperature; this Moissan succeeded in doing in 1886. He decomposed anhydrous hydrofluoric acid, liquefied at a temperature of $-23^{\circ}$ and contained in a $\mathrm{U}$-shaped tube (to which a small quantity of potassium fluoride had been added to make it a better conductor), by the action of a powerful electric current (twenty Bunsen's elements in series). Hydrogen was then evolved at the negative pole, and fluorine appeared at the positive pole (of iridium platinum) as a colourless gas which decomposed water with the forma tion of ozone and hydrofluoric acid, and combined directly with silicon (forming silicon fluoride, $\mathrm{SiF}_{4}$ ), boron (forming $\mathrm{BF}_{3}$ ), sulphur, \&c. But

48 It is most likely that in this experiment of Fremy's, which corresponds with the action of oxygen on calcium chloride, fluorine was set free, but that a converse reaction also proceeded, $\mathrm{CaO}+\mathrm{F}_{2}=\mathrm{CaF}_{2}+\mathrm{O}$-that is, the calciun distributed itself between the oxygen and fluorine. $\mathrm{MnF}_{4}$, which is capable of splitting up into $\mathrm{MnF}_{2}$ and $\mathrm{F}_{2}$, is without doubt formed by the action of a strong solution of hydrofluoric acid on manganese peroxide, but under the action of water the fluorine gives hydrofluoric acid, and probably this is aided by the affinity of the manganese fluoride and hydrofluoric acid. In all the efforts (Duvy, Knox, Louget, Fremy, Gore, and others) made to decompose fluorides (those of lead, silver, calcium, and others) by chlorine, there were doubtless also cases of distribution, a portion of the metal combined with chlorine and a portion of the fluorine was evolved; but it is improbable that there were pure results. Probably Fremy obtained fluorine, but it was not pure. 
the action of fluorine on metals at the ordinary temperature is comparatively feeble, because the metallic fluoride formed coats the remaining mass of the metals ; it is, however, completely absorbed by iron. Hydrocarbons (for instance, naphtha), alcohol, \&c., immediately absorb fluorine, with the formation of hydrofluoric acid. Fluorine when mixed with hydrogen easily explodes violently, forming hydrofluoric acid. ${ }^{49}$

Among the compounds of fluorine calcium fluoride, $\mathrm{CaF}_{2}$, is rather widely spread in nature as fluor spar, ${ }^{50}$ whilst cryolite, or aluminium sodium fluoride, $\mathrm{Na}_{3} \mathrm{AlF}_{6}$, is found more rarely (in large masses in Greenland). Cryolite, like fluor spar, is also insoluble in water, and gives hydrofluoric acid with sulphuric acid. Small quantities of fluorine have also in a number of cases been found in the bodies of animals, in the blood, urine, and bones. If fluorides occur in the bodies of animals, they must have been introduced by food, and must occur in plants and water. And in reality river, and especially sea, water always contains a certain, although small, quantity of fluorine compounds.

49 According to Moissan, fluorine is disengaged by the action of an electric current on fused hydrogen potassium fluoride, $\mathrm{KHF}_{2}$. The present state of chemical knowledge is such that the notion of an element with its properties is much more general than the notion of that element in the free state. It is profitable and agreeable to learn that even fluorine in the free state has not succeeded in eluding experiment and research, that the efforts to isolate it have been crowned with success, but the sum total of the general chemical data concerning fluorine as an element gain but little by this result. The gain will be augmented, however, if it be now possible to subject fluorine to a comparative study in relation to oxygen and chlorine. There is particular interest in the phenomena of the distribution of fluorine and oxygen, or fluorine and chlorine, competing under different conditions and relations.

so It is called spar because it very frequently occurs as crystals of a clearly laminar structure, and is therefore easily split up into pieces bounded by planes. It is called fluor spar because when used as a flux it renders ores fusible, owing to its reacting with silica, $\mathrm{SiO}_{2}+2 \mathrm{CaF}_{2}=2 \mathrm{CaO}+\mathrm{SiF}_{4}$; the silicon fluoride escapes as a gas and the lime combines with a further quantity of silica, and gives a vitreous slag. Fluor spar occurs in mineral veins and rocks, sometimes in considerable quantities. It always crystallises in the cubic system, sometimes in very large semitransparent cubic crystals, which are colouirless or of different colours. It is insoluble in water. It melts under the action of heat, and crystallises on cooling. The sp. gr. is $3 \cdot 1$. When steam is passed over incandescent fluor spar, lime and hydrofluoric acid are formed: $\mathrm{CaF}_{2}+\mathrm{H}_{2} \mathrm{O}=\mathrm{CaO}+2 \mathrm{HF}$. A double decomposition is also easily produced by fusing ftuor spar with sodium or potassium hydroxides, or potash, or even with their carbonates; the fluorine then passes over to the potassium or sodium, and the oxygen to the calcium. In solutions-for example, $\mathrm{Ca}\left(\mathrm{NO}_{3}\right)_{2}+2 \mathrm{KF}=\mathrm{CaF}_{2}$ (precipitate) $+2 \mathrm{KNO}_{3}$ (in solution)-the formation of calcium fluoride takes place, owing to its very sparing solubility. 26000 parts of water dissolve one part of fluor spar. Fluorine in the form of calcium fluoride also enters into the composition of certain minerals: but in general the amount of fluorine in minerals is inconsiderable. Apatite is a mineral whose chief mass consists of calcium phosphate. Apatites sometimes contain no fluorine whatever, but only chlorine; whilst in other instances a certain amount of fluorine enters into their composition, in which case the atomic proportion of chlorine is proportionately diminished. 
Hydrofluoric acid, HF, cannot be obtained from fluor spar in glass retorts, because glass is acted on by and destroys the hydrofluoric acid. It is prepared in lead vessels, and when it is required pure, in platinum vessels, because lead in reality also acts on hydrofluoric acid, only very feebly at the surface, and when once a coating of fluoride and sulphate of lead is formed there is no further action. Powdered fluor spar and sulphuric acid evolve hydrofluoric acid (which fumes in the air) even at the ordinary temperature, $\mathrm{CaF}_{2}+\mathrm{H}_{2} \mathrm{SO}_{4}=\mathrm{CaSO}_{4}+2 \mathrm{HF}$. At $130^{\circ}$ fluor spar is completely decomposed by sulphuric acid. The acid is then evolved as vapour, which may be condensed by a freezing mixture into an anhydrous acid. The condensation is aided by pouring water into the receiver of the condenser, as the acid is easily soluble in cold water.

In the liquid anhydrous form hydrofluoric acid boils at $+19^{\circ}$, and its sp. gr. at $12 \cdot 8^{\circ}=0.9849 .51$ It dissolves in water with the evolution of a considerable amount of heat, and gives a solution of constant boiling point which distils over at $120^{\circ}$; hence the acid is able to combine with water. The specific gravity of the compound is $1 \cdot 15$, and its composition $\mathrm{HF}, 2 \mathrm{H}_{2} \mathrm{O} .{ }^{52}$ With an excess of water a dilute solution first distils over. The aqueous solution and the acid itself must be kept in platinum vessels, but the dilute acid may be conveniently preserved in vessels made of various organic materials, such as guttapercha, or even in glass vessels having an interior coating of paraffin. Hydrofluoric acid does not act on hydrocarbons and many other substances, but it acts in a highly corrosive manner on metals, glass, porcelain, and the majority of rock substances. ${ }^{53}$ It also corrodes the skin,

51 According to Gore, hydrofluoric acid remains liquid when cooled to $-34^{\circ}$. Fremy obtained anhydrous hydrofluoric acid by decomposing lead fluoride at a red heat, by hydrogen, or by heating the double salt $\mathrm{HKF}_{2}$, which easily crystallises (in cubes) from a solution of hydrofluoric acid, half of which has been saturated with potassium lydroxide, or carbonate.

52 This composition corresponds with the crystallo-hydrate $\mathrm{HCl}, 2 \mathrm{H}_{2} \mathrm{O}$. All the properties of hydrofluoric acid call to mind those of hydrochloric acid, and therefore the comparative ease with which hydrofluoric acid is liquefied (it boils at $+19^{\circ}$, hydrochloric acid at $-35^{\circ}$ ), must be explained by a polymerisation taking place at low temperatures, as will be afterwards explained, $\mathrm{H}_{2} \mathrm{~F}_{2}$, being formed, and therefore in a liquid state it differs from hydrochloric acid, for which a phenomenon of a similar kind has not yet been observed.

53 The corrosive action of hydrofluoric acid on glass and similar siliceous compounds is based upon the fact that it acts on silica, $\mathrm{SiO}_{2}$, as we shall consider more fully in describing that compound, forming gaseous silicon fluoride, $\mathrm{SiO}_{2}+4 \mathrm{HF}=\mathrm{SiF}_{4}+2 \mathrm{H}_{2} \mathrm{O}$. Silica, on the other hand, forms the binding (acid) element of glass and of the mass of mineral substances forming the salts of silica. When it is removed the bond is destroyed. This is made use of in practice, and the laboratory, for etching designs and scales, \&c., on glass. In engraving on glass the surface is covered with a varnish composed of four parts of wax and one part of turpentine. This varnish is not acted on by 
and is distinguished by its poisonous properties, so that in working with the acid a strong draught must be kept up, to prevent the possibility of the fumes being inhaled. The non-metals do not act on hydrofluoric acid, but all metals - with the exception of mercury, silver, gold, and platinum, and, to a certain degree, lead-decompose it with the evolution of gaseous hydrogen. With bases it directly gives metallic fluorides, and behaves in many respects like hydrochloric acid. There are, however, several distinct individual differences, which are furthermore much greater than those between hydrochloric, hydrobromic, and hydriodic acids. Thus the silver compounds of the latter are insoluble in water, whilst silver fluoride is moderately soluble. Calcium fluoride, on the contrary, is insoluble in water, whilst calcium chloride, bromide, and iodide are not only soluble, but attract water with great energy, owing to which gases are frequently dried by passing them over calcium chloride. Neither hydrochloric, nor hydrobromic, nor hydriodic acid acts on sand and glass, whilst hydrofluoric acid corrodes them, forming gaseous silicon fluoride. The other halogen acids only form normal salts, $\mathrm{KCl}, \mathrm{NaCl}$, with $\mathrm{Na}$ or $\mathrm{K}$, whilst hydrofluoric acid gives acid salts, for instance $\mathrm{HKF}_{2}$ (and by dissolving $\mathrm{KF}$ in liquid $\mathrm{HF}, \mathrm{KHF}_{2} 2 \mathrm{HF}$ is obtained). This latter property is in close connection with the fact that at the ordinary temperature the vapour density of hydrofluoric acid is nearly 20, which corresponds with a formula $\mathrm{H}_{2} \mathrm{~F}_{2}$, as Mallet (1881) showed; but a depolymerisation occurs with a rise of temperature, and the density approaches 10 , which answers to the formula HF. ${ }^{54}$

The analogy between chlorine and the other two halogens, bromine

hydrofluoric acid, and it is soft enough to allow designs being drawn upon it whose lines lay bare the glass. The drawing is made with a steel point, and the glass is afterwards laid in a lead trough in which a mixture of fluor spar and sulphuric acid is placed. The sulphuric acid must be taken in considerable excess, as otherwise transparent lines are obtained (owing to the formation of hydrofluosilicic acid). After being exposed for some time, the varnish is removed (melted), and the design drawn by the steel point is found reproduced in dull lines. The drawing may be also made by the direct application of a mixture of a silicofluoride and sulphuric acid, which forms hydrofluoric acid. In the laboratory a solution of hydrofluoric acid or its fumes is employed for decomposing siliceous substances which are insoluble in ordinary acids, or else they are fused with the salts $\mathrm{KHF}_{2}$ or $\mathrm{NH}_{4} \mathrm{~F}$.

54 Mallet (1881) determined the density at $30^{\circ}$ and $100^{\circ}$, previous to which Gore (1869) had determined the vapour density at $100^{\circ}$, whilst Thorpe and Hambly (1888) made fourteen determinations between $26^{\circ}$ and $88^{\circ}$, and showed that within this limit of temperature the density gradually diminishes, just like the vapour of acetic acid. The capacity of $\mathrm{HF}$ to polymerise into $\mathrm{H}_{2} \mathrm{~F}_{2}$ is probably connected with the property of many fluorides of forming acids with $\mathrm{HF}$ - for example, $\mathrm{KHF}_{2}$ and $\mathrm{H}_{2} \mathrm{SiF}_{6}$. We saw above that $\mathrm{HCl}$ has the same property (forming, for instance, $\mathrm{H}_{2} \mathrm{PtCl}_{6}$, \&c., p. 450), and hence this property of hydrofluoric acid does not stand isolated from the properties of the other halogens. 
and iodine, is much more perfect. Not only have their hydrates or halogen acids much in common, but they themselves resemble chlorine in many respects, ${ }^{55}$ and even the properties of the corresponding metallic compounds of bromine and iodine are very much alike. Thus the chlorides, bromides, and iodides of sodium and potassium crystallise in the cubic system, and are soluble in water; the chlorides of calcium, aluminium, magnesium, and barium are soluble in water, like the bromides and iodides of these metals. The iodides and bromides of silver and lead are sparingly soluble in water, like the chlorides of these metals. Besides which even the oxygen compounds of bromine and iodine present a very strong analogy to the corresponding compounds of chlorine. A hypobromous acid is known corresponding with hypochlorous acid. The salts of this acid have the same bleaching property

55 For instance, the experiment with Dutch metal foil (Note 16) may be made with bromine just as well as with chlorine. A very instructive experiment on the direct combination of the halogens with metals may be made by throwing a small piece (a shaving) of aluminium into a vessel containing liquid bromine; the aluminium, being lighter, floats on the bromine, and after a certain time reaction sets in accompanied by the evolution of heat, light, and fumes of bromine. The incandescent piece of metal moves rapidly over the surface of the bromine in which the resultant aluminium bromide is dissolved. It was in this manner that Gustavson prepared that mixture of bromine and aluminium bromide which reacts by metalepsis with the greatest ease in those cases when bromine by itself is not able to bring about metalepsis, or else only acts very slowly, as, for instance, with benzene, $\mathrm{C}_{6} \mathrm{H}_{6}$. When drops of this hydrocarbon are added to bromine containing aluminium bromide, they immediately give a mass of hydrobromic acid and of the product of metalepsis. Gustavson showed that the cause of this facility of reaction must be looked for in the capacity of aluminium bromide to form an unstable compound with the products of reaction which are formed. For the sake of comparison we will proceed to cite several thermochemical data (Thomsen) for analogous actions of (1) chlorine, (2) bromine, and (3) iodine, with respect to metals; the halogen being expressed by the symbol $\mathrm{X}$, and the sign plus connecting the reacting substances. All the figures are given in thousands of calories, and refer to molecular quantities in grams and to the ordinary temperature :-

$\begin{array}{lrrr} & 1 & 2 & 3 \\ \mathrm{~K}_{2}+\mathrm{X}_{2} & 211 & 191 & 160 \\ \mathrm{Na}_{2}+\mathrm{X}_{2} & 195 & 172 & 138 \\ \mathrm{Ag}_{2}+\mathrm{X}_{2} & 59 & 45 & 28 \\ \mathrm{Hg}_{2}+\mathrm{X}_{2} & 83 & 68 & 48 \\ \mathrm{Hg}+\mathrm{X}_{2} & 63 & 51 & 34 \\ \mathrm{Ca}+\mathrm{X}_{2} & 170 & 141 & - \\ \mathrm{Ba}+\mathrm{X}_{2} & 195 & 170 & - \\ \mathrm{Zn}+\mathrm{X}_{2} & 97 & 76 & 49 \\ \mathrm{~Pb}+\mathrm{X}_{2} & 83 & 64 & 40 \\ \mathrm{Al}+\mathrm{X}_{3} & 161 & 120 & 70\end{array}$

We may remark that the latent heat of vaporisation of the molecular weight $\mathrm{Br}_{2}$ is about $7 \cdot 2$, and of iodine 6.0 thousand heat units, whilst the latent heat of fusion of $\mathrm{Br}_{2}$ is about 0.3, and of $\mathrm{I}_{2}$ about 3.0 thousand leat units. From this it is evident that the difference between the amounts of heat evolved does not depend on the difference in physical state. For instance, the vapour of iodine in combining with $\mathrm{Zn}$ to form $\mathrm{ZnI} \mathrm{n}_{2}$ would give $48+8+3$, or about sixty thousand heat units, or $1 \frac{1}{8}$ times less than $\mathrm{Zn}_{n}+\mathrm{Cl}_{2}$. 
as the salts of hypochlorous acid. The higher oxygen acids of iodine, bromine, and chlorine are closely analogous to each other. Iodine was discovered in 1811 by Courtois in kelp, and was soon investigated by Clement, Gay-Lussac, and Dary. Bromine was discovered in 1826 by Balard in the mother liquor of sea water.

Bromine and iodine, like chlorine, occur in sea water in combination with metals. However, the amount of bromides, and especially of iodides, in sea water is so small that their presence can only be discovered by means of sensitive reactions. ${ }^{56}$ In the extraction of salt from sea water the bromides remain in the mother liquor, and can be obtained therefrom; this method is, however, rarely employed on a large scale, as other and richer sources are known for the extraction of this substance. Iodine and bromine also occur combined with silver, in admixture with silver chloride, as a rare ore which is mainly found in America. Certain mineral waters (those of Kreuznach and Schönebeck) contain metallic bromides and iodides, always in admixture with an excess of sodium chloride. Those upper strata of the Stassfurt rock salt (Chapter X.) which are a source of potassium salts also contain metallic bromides, ${ }^{57}$ which collect in the mother liquors left after the crystallisation of the potassium salts; and this now forms the chief source (together with certain American springs) of the bromine in common use. Bromine may be easily liberated from a mixture of bromides and chlorides, owing to the fact that chlorine displaces bromine from its compounds with sodium, magnesium, calcium, dt. A colourless solution of bromides and chlorides turns an orange colour after the passage of chlorine, owing to the disengagement of bromine. ${ }^{58}$. Bromine may be extracted on a large scale by a similar method, but it

56 One litre of sea water contains about 20 grams of chlorine, and about 0.07 grams of bromine. The Dead Sea contains about ten times as much.

${ }^{57}$ But there is no iodine in Stassfurt carnallite.

58 The chlorine must not, however, be in large excess, as otherwise the bromine would contain chlorine. Commercial bromine not unfrequently contains chlorine, as bromine chloride; this is more soluble in water than bromine, which may thus be freed from it. To obtain pure bromine the commercial bromine is washed with water, dried by sulphuric acid, and distilled, the portion coming over at $58^{\circ}$ being collected; the greater part is then converted into potassium bromide and dissolved, and the remainder is added to the solution in order to separate iodine, which is removed by shaking with carbon bisulphide. By heating the potassium bromide thus obtained with manganese peroxide and sulphuric acid, bromine is obtained quite free from iodine, which, however, is not present in certain kinds of commercial bromine (the Stassfurt, for instance). By treatment with potash, the bromine is then converted into a mixture of potassium bromide and bromate, and the mixture (which is in the proportion given in the equation) is distilled with sulphuric acid, bromine being then evolved : $5 \mathrm{KBr}+\mathrm{KBrO}_{3}$ $+6 \mathrm{H}_{2} \mathrm{SO}_{4}=6 \mathrm{KHSO}_{4}+3 \mathrm{H}_{2} \mathrm{O}+3 \mathrm{Br}_{2}$. After dissolving the bromine in a strong solution of calcium bromide and precipitating with an excess of water, it loses all the chlorine it contained, because chlorine forms calcium chloride with $\mathrm{CaBr}_{2}$. 
is simpler to add a small quantity of manganese peroxide and sulphuric acid to the mother liquid direct. This sets free a portion of the chlorine, and this chlorine liberates the bromine. Bromine is a dark brown liquid, giving brown fumes, and having a poisonous suffocating smell, from whence its name (from the Greek $\beta \rho \hat{\omega} \mu o s$, signifying evil smelling). The vapour density of bromine shows that its molecule is $\mathrm{Br}_{2}$. In the cold bromine freezes into brown-grey scales like iodine. The melting. point of pure bromine is $-7.05^{\circ} .59$ The density of liquid bromine at $0^{\circ}$. is $3 \cdot 187$, and at $15^{\circ}$ about $3 \cdot 0$. The boiling point of bromine is about. $58.7^{\circ}$; it is generally purified by distillation. Bromine, like chlorine, is soluble in water; 1 part of bromine at $5^{\circ}$ requires 27 parts of water, and at $15^{\circ} 29$ parts of water. The aqueous solution of bromine is of an orange colour, and when cooled to $-2^{\circ}$ yields crystals containing 10 molecules of water to 1 molecule of bromine. ${ }^{60}$ Alcohol dissolves a

59 There has long existed a difference of opinion as to the melting point of pure bromine. By some (Regnault, Pierre) it was given as between $-7^{\circ}$ and $-8^{\circ}$, and by others (Balard, Liebig, Quincke, Baumhauer) as between $-20^{\circ}$ and $-25^{\circ}$. There is now no doubt, thanks more especially to the researches of Ramsay and Young (1885), that pure bromine melts at about $-7^{\circ}$. This figure is not only established by direct experiment (Van der Plaats confirmed it) but also by means of the determination of the vapour tensions. For solid bromine the vapour tension $p$. in $\mathrm{mm}$. at $t$ was found to be-

$\begin{array}{cccccc}p=20 & 25 & 30 & 35 & 40 & 45 \mathrm{~mm} . \\ t=-16^{\circ} 6^{\circ} & -14^{\circ} & -12^{\circ} & -10^{\circ} & -8.5^{\circ} & -7^{\circ}\end{array}$

For liquid bromine-

$\begin{array}{cccccc}p=50 & 100 & 200 & 400 & 600 & 760 \mathrm{~mm} \\ t=-5 \cdot 0^{\circ}+8 \cdot 2^{\circ} & 23 \cdot 4^{\circ} & 40 \cdot 4^{\circ} & 51 \cdot 9^{\circ} & 58 \cdot 7^{\circ}\end{array}$

These curves intersect at $-7 \cdot 05$. Besides which, in comparing the vapour tension of many liquids (for example, those given in Chapter II. Note 27), Ramsay and Young observed that the ratio of the absolute temperatures $(t+273)$ corresponding with equal tension varies for every pair of substances in rectilinear proportion in dependence upon $t$, and, therefore, for the above pressure $p$, Ramsay and Young determined the ratio of $t+273$ for water and bromine, and found that the straight lines expressing these ratios for liquid and solid bromine intersect also at $7 \cdot 05^{\circ}$; thus, for example, for solid bromine-

$\begin{array}{rllllll}p= & 20 & 25 & 30 & 35 & 40 & 45 \\ 273+t= & 256 \cdot 4 & 259 & 261 & 263 & 264 \cdot 6 & 266 \\ 273+t^{\prime}= & 295 \cdot 3 & 299 & 30 * \cdot 1 & 304 \cdot 8 & 307 \cdot 2 & 309 \cdot 3 \\ c= & 1 \cdot 152 & 1 \cdot 154 & 1 \cdot 157 & 1 \cdot 159 & 1 \cdot 161 & 1 \cdot 163\end{array}$

where $t^{\prime}$ indicates the temperature of water corresponding with a vapour tension $p$, and where $c$ is the ratio of $273+t^{\prime}$ to $273+t$. The magnitude of $c$ is evidently expressed with great accuracy by the straight line $c=1 \cdot 1703+0 \cdot 0011 t$. In exactly the same way we find the ratio for liquid bromine and water to be $c_{1}=1 \cdot 1585+0.00057 t$. The intersection of these straight lines in fact corresponds with $-7 \cdot 06^{\circ}$, which again confirms the melting point given above for bromine. In this manner it is possible with the existing store of data to accurately establish and verify the melting point of substances. Ramsay and Young established the thermal constants of iodine by exactly the same method.

60 The observations made by Paterno and Nasini (by Raoult's method, Chapter-I. Note 49$)$ on the temperature of the formation of ice $\left(-1 \cdot 115^{\circ}\right.$, with $1 \cdot 391$ grams of bromine 


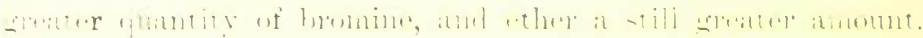

line

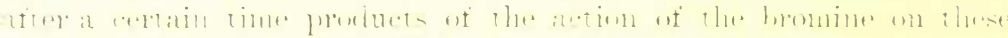

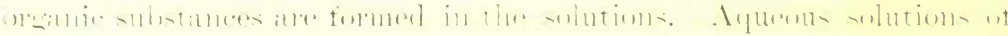

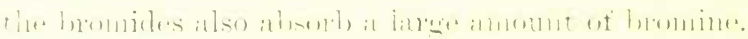

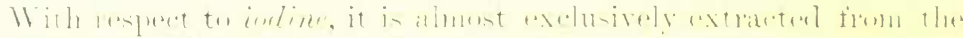

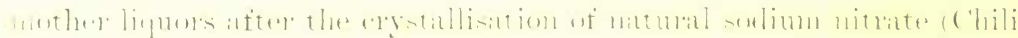

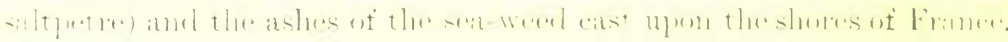

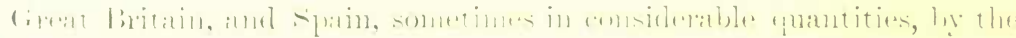

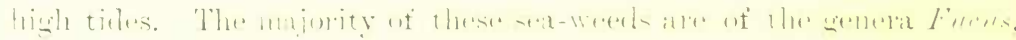

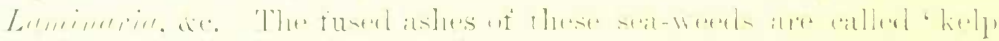

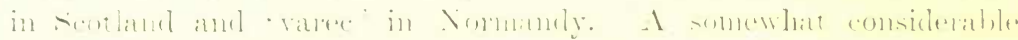

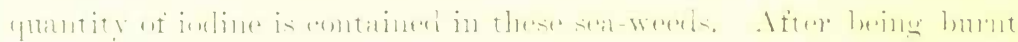

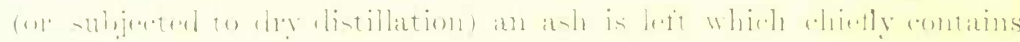

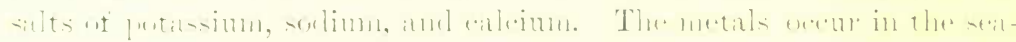

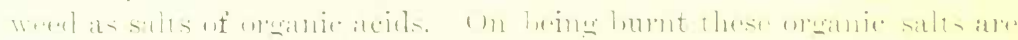

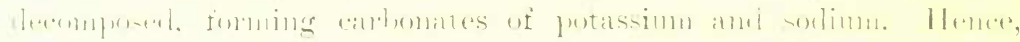

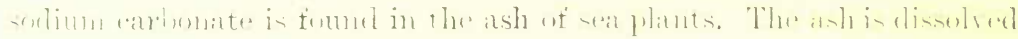

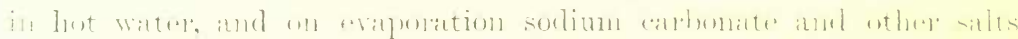

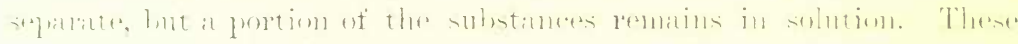

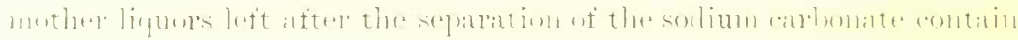

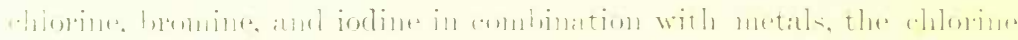

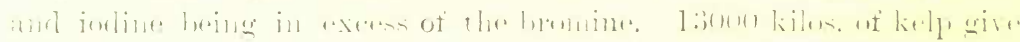

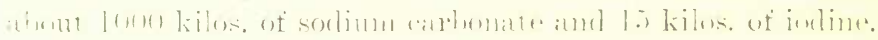

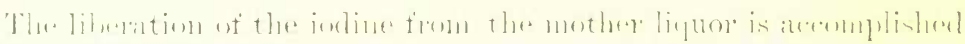

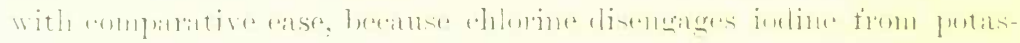

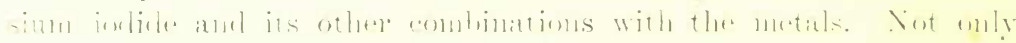

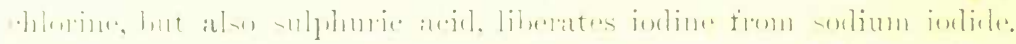

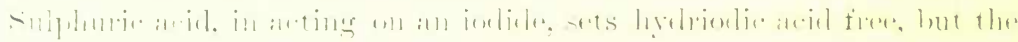

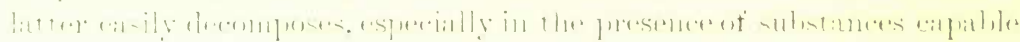

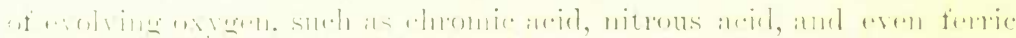

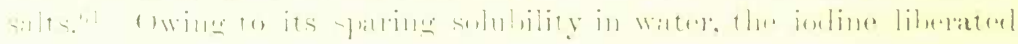

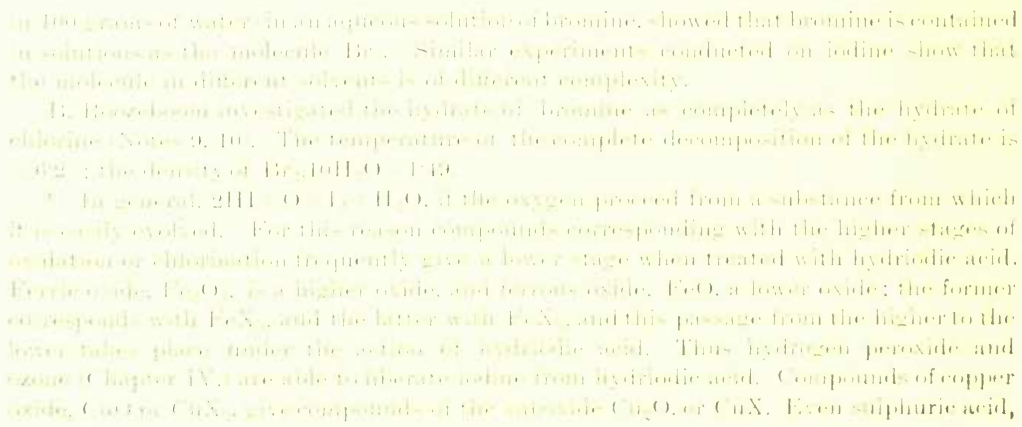


separates as a precipitate. To obtain pure iodine it is enough to distil it, and neglect the first and last portions of the distillate, the middle portion only being collected. Iodine passes directly from a state of vapour into a crystalline form, and settles on the cool portions of the apparatus in tabular crystals, having a black grey colour and metallic lustre. ${ }^{62}$

The specific gravity of the crystals of iodine is 4.95 . It melts at $114^{\circ}$ and boils at $184^{\circ}$. Its vapour is formed at a much lower temperature, and is of a violet colour, from whence iodine receives its name. The smell of iodine calls to mind the characteristic smell of hypochlorous acid; it has a sharp sour taste. It destroys the skin and organs of the body, and is therefore frequently employed for cauterising and as an irritant for the skin. In small quantities it turns the skin brown, but the coloration disappears after a certain time, partly owing to the volatility of the iodine. Water dissolves only $\frac{1}{5000}$ part of iodine. A brown solution is thus obtained, which bleaches, but much more feebly than bromine and chlorine. Water which contains salts, and especially iodides, in solution dissolves iodine in considerable quantities, and the resultant solution is of a dark brown colour. Pure alcohol dissolves a small amount of iodine, and in so doing acquires a brown colour, but the solubility of iodine is considerably increased by the presence of a small quantity of an iodine compound - for instance, ethyl iodide - in the alcohol. ${ }^{63}$ Ether dissolves a larger amount of iodine than alcohol; but

which corresponds to the higher stage $\mathrm{SO}_{5}$, is able to act thus, forming the lower oxide $\mathrm{SO}_{2}$. The liberation of iodine from hydriodic acid proceeds with still greater ease under the action of substances capable of disengaging oxygen. In practice, many methods are employed for liberating iodine from acid liquids containing, for example, sulphuric acid and hydriodic acid. The higher oxides of nitrogen are most commonly used; they then pass into nitric oxide. Iodine may even be disengaged from hydriodic acid by the action of iodic acid, \&c. But there is a limit in these reactions of the oxidation of hydriodic acid because, under certain conditions, especially in dilute solutions, the iodine set free is itself able to act as an oxidising agent-that is, it evinces in itself the character of chlorine, and of the halogens in general, to which we shall again have occasion to refer.

62 For the ultimate purification of iodine, Stas dissolves it in a strong solution of potassium iodide, and precipitates it by the addition of water (see Note 58).

63 The solubility of iodine in solutions containing oxides, and compounds of iodine in general, may serve, on the one hand, as an indication that solution is called forth by analogy (p. 70), and, on the other hand, as an indirect proof of that view as to solutions which was cited in Chapter I., because in many instances unstable highly-iodised compounds, resembling crystallo-hydrates, have been obtained from such solutions. Thus iodide of tetramethylammonium $\mathrm{N}\left(\mathrm{CH}_{3}\right)_{4} \mathrm{I}$, combines with $\mathrm{I}_{2}$ and $\mathrm{I}_{4}$. Even a solution of iodine in a saturated solution of potassium iodide presents traces of the formation of a definite compound $\mathrm{KI}_{3}$. Thus, an alcoholic solution of $\mathrm{KI}_{3}$ does not give up iodine to carbon bisulphide, although this solvent takes up iodine from an alcoholic solution of iodine itself (Girault, Jörgensen, and others). The instability of these compounds resembles the instability of many crystallo-hydrates, for instance of $\mathrm{HCl}, 2 \mathrm{H}_{2} \mathrm{O}$. 
iodine is particularly soluble in liquid hydrocarbons, in carbon bisulphide, and in chloroform. A small quantity of iodine dissolved in carbon bisulphide tints it rose-colour, but in a somewhat larger amount it gives a violet colour. Chloroform (quite free from alcohol) is alsotinted rose colour by a small amount of iodine. This gives an easy means for detecting the presence of free iodine in small quantities. The coloration which free iodine gives with starch may also, as has already been frequently mentioned, serve for the detection of iodine.

If we compare the four elements, fluorine, chlorine, bromine, and iodine, we see in them an example of analogous substances which arrange themselves by their physical properties in the same order as they stand in respect to their atomic and molecular weights. If the weight of the molecule be large, the substance has a higher specific gravity, a higher melting and boiling point, and a whole series of properties depending on this difference in its fundamental properties. A large atomic weight should determine a larger affinity between the molecules, and consequently a greater difficulty in their division and a greater attraction between them. We meet a very evident examplein question in polymeric compounds ; for instance, in the hydrocarbons. expressed by the formula, $\mathrm{C}_{n} \mathrm{H}_{2 n}: \mathrm{C}_{2} \mathrm{H}_{4}, \mathrm{C}_{3} \mathrm{H}_{6}$ are gases, those having a greater molecular weight-for instance, $\mathrm{C}_{5} \mathrm{H}_{10}, \mathrm{C}_{7} \mathrm{H}_{14}$, \&c.-are liquids, and those of a still greater molecular weight are solids. The same relation is found in the above-named four elements. Chlorine in a free state boils at about $-35^{\circ}$, bromine boils at $60^{\circ}$, and iodine only above $180^{\circ}$. According to Avogadro-Gerhardt's law, the vapour densities of these elements in a gaseous state are proportional to their atomic weights, and here, at all events approximately, the densities in a liquid (solid) state are also almost in the ratio of their atomic weights. Dividing the atomic weight of chlorine $(35.5)$ by its sp. gr. in a liquid state $(1 \cdot 3)$, we obtain a volume $=27$, for bromine $(80 / 3 \cdot 1) 26$, as also for iodine $(127 / 4 \cdot 9) 26 .{ }^{64}$

The metallic bromides and iodides are in the majority of cases and

64 The equality of the atomic volumes of the halogens themselves is all the more remarkable because in all the halogen compounds the volume augments with the substitution of fluorine by chlorine, bromine, and iodine. Thus, for example, the volume of sodium fluoride (obtained by dividing the weight expressed by its formula by its specific gravity) is about 15 , of sodium chloride 27 , of sodium bromide 32 , and of sodium iodide 41. The volume of silicon chloroform, $\mathrm{SiHCl}_{3}$, and of the corresponding bromine, and iodine compounds, are 82,108 , and 122 respectively. So in solutions, for example, $\mathrm{NaCl}+200 \mathrm{H}_{2} \mathrm{O}$ has a sp. gr. (at $15^{\circ} / 4^{\circ}$ ) of $1 \cdot 0106$, consequently the volume of the solution $3658^{\circ} 5 / 1 \cdot 0106=3620$, hence the volume of sodium chloride in solution $=3620-3603$ (this is: the vol. of $200 \mathrm{H}_{2} \mathrm{O}$ ) =17. So also in similat solutions, $\mathrm{NaBr}=26$ and $\mathrm{NaI}=35$. 
respects analogous to the corresponding chlorides, ${ }^{65}$ but chlorine displaces the bromine and iodine from them, and bromine liberates iodine from iodides, which is taken advantage of in the preparation of these halogens. However, the researches of Potilitzin showed that a reverse displacement of chlorine by bromine may occur both in solutions of and in ignited metallic chlorides in an atmosphere of bromine vapour - that is, a distribution of the metal (according to Berthollet's doctrine) takes place between the halogens, although, however, the larger portion still unites with the chlorine, which shows its greater affinity for metals as compared with that of bromine and iodine. ${ }^{66}$ The latter, however,

65 But the density (and even volume, Note 64) of a bromine compound is always greater than that of a chlorine compound, whilst that of an iodine compound is still greater. The order is the same in many other respects. For example, an iodine compound has a higher boiling point than a bromine compound, \&c.

66 A. L. Potilitzin showed that in heating various metallic chlorides in a closed tube, with an equivalent quantity of bromine, a distribution of the metal between the halogens always occurs, and that the amounts of chlorine replaced by the bromine in the ultimate product are proportional to the atomic weights of the metals taken and inversely proportional to their equivalency. Thus, if $\mathrm{NaCl}+\mathrm{Br}$ be taken, then out of 100 parts of chlorine, 5.54 are replaced by the bromine, whilst if $\mathrm{AgCl}+\mathrm{Br}$ be taken, the 27.28 parts are replaced. These figures are in the ratio $1: 4.9$, but the atomic weights $\mathrm{Na}: \mathrm{Ag}=1: 4 \cdot 7$. In general terms, if a chloride $\mathrm{MCl}_{n}$ be taken, then it gives with $n \mathrm{Br}$ a percentage substitution $=4 \mathrm{M} / n^{2}$, where $\mathrm{M}$ is the atomic weight of the metal. This law was deduced from observations on the chlorides of $\mathrm{Li}, \mathrm{K}, \mathrm{Na}, \mathrm{Ag}(n=1), \mathrm{Ca}, \mathrm{Sr}, \mathrm{Ba}, \mathrm{Co}, \mathrm{Ni}, \mathrm{Hg}, \mathrm{Pb}(n=2), \mathrm{Bi}(n=3)$, $\mathrm{Sn}(n=4)$, and $\mathrm{Fe}_{2}(n=6)$.

In these determinations of Potilitzin we see not only a brilliant confirmation of Berthollet's doctrine, but also the first effort (there have been no similar determinations since 1879) to directly determine the affinities of elements by means of displacement. The chief object of these researches consisted in proving whether a displacement occurs in those cases where heat is absorbed, and in this instance it should be absorbed, because the formation of all metallic bromides is attended with the evolution of less heat than that of the chlorides, as is seen by the figures given in Note 55. Among the results of other researches made by Potilitzin in the same direction, it is necessary to turn attention to the following.

If the mass of the bromine be increased, then the amount of chlorine displaced also increases. For example, if masses of bromine of 1 and 4 equivalents act on a molecule of sodium chloride, then the percentages of the chlorine displaced will be 6.08 p.c. and 12.46 p.c.; in the action of $1,4,9,16,25$, and 100 molecules of bromine on a molecule of barium chloride, there will be displaced $7 \cdot 8,17 \cdot 6,23 \cdot 5,31 \cdot 0,35^{\circ} 0$, and $45^{\circ} 0$ p.c. of chlorine. If an equivalent quantity of hydrochloric acid act on metallic bromides in closed tubes, and in the absence of water at a temperature of $300^{\circ}$, then the percentages of the substitution -of the bromine by the chlorine in the double decomposition taking place between univalent metals are inversely proportional to their atomic weights. For example, $\mathrm{NaBr}+\mathrm{HCl}$ gives at the limit 21 p.c. of displacement, $\mathrm{KCl} 12$ p.c. and $\mathrm{AgCl} 14 \frac{1}{4}$ p.c. Essentially the same takes place in an aqueous solution, although the phenomena is complicated by the participation of the water. The reactions proceed of themselves in one or the other direction at the ordinary temperature but at different rates. In the action of a dilute solution (1 equivalent per 5 litres) of sodium chloride on silver bromide at the ordinary temperature, 2.07 p.c. of bromine is replaced in six and a half days, with potassium chloride 1.5 p.c. With an excess of the chloride the magnitude of the substitution increases; with four equivalents it is 4.95 p.c. in nine days. In the action of 9 grams of sodium chloride on 1.1 grams of silver bromide, 9.69 p.c. of bromine is replaced in thirteen days. 
sometimes behave with respect to metallic oxides in exactly the samemanner as chlorine. Gay-Lussac, by igniting potassium carbonate in iodine vapour, obtained (as with chlorine) an evolution of oxygen and carbonic anhydride, $\mathrm{K}_{2} \mathrm{CO}_{3}+\mathrm{I}_{2}=2 \mathrm{KI}+\mathrm{CO}_{2}+\mathrm{O}$, only the reactions between the halogens and oxygen are more easily reversible with bromine and iodine than with chlorine. Thus, at a red heat oxygen displaces iodine from barium iodide (besides which the reaction is. here complicated by iodine being more easily oxidised than chlorine). Aluminium iodide burns in a current of oxygen (Deville and Troost), and a similar, although not so highly developed, relation exists for aluminium choride, and shows that the halogens have a distinctly smaller affinity for metals which only form feeble bases. This refers to a still greater extent to non-metals, which form acids and evolve much more heat with oxygen than the halogens. But in all these instances the affinity (and amount of heat evolved) of iodine and bromine is less than that of chlorine, probably because the atomic weights are greater, whilst the properties of the atoms of all the halogens are analogous. The smaller store of energy in iodine and bromine is seen still more clearly in the relation of the halogens to hydrogen. In a gaseous state they all enter, with more or less ease, into direct combination with gaseous hydrogen-for example, in the presence of spongy platinum, forming halogen acids, $\mathrm{HX}$ - but the latter are far from being equally stable ; hydrogen chloride is the most stable, hydrogen iodide the least so, and hydrogen bromide occupies an intermediate position. A very strong heat is required to only partially decompose hydrogen chloride,

These conversions also proceed with the absorption of heat. The reverse reactions evolving heat proceed incomparably more rapidly, but also to a certain limit; for example, in the reaction $\mathrm{AgCl}+\mathrm{RBr}$ the following percentages of silver bromide are formed in different times:

$\begin{array}{lccccc}\text { hours } & 2 & 3 & 22 & 96 & 120 \\ \mathrm{~K} & 79 \cdot 82 & 87 \cdot 4 & 88 \cdot 22 & - & 94 \cdot 21 \\ \mathrm{Na} & 83 \cdot 63 & 90 \cdot 74 & 91 \cdot 70 & 95 \cdot 49 & -\end{array}$

Consequently, the conversions which are accompanied by this evolution of heat proceed with very much greater rapidity than the reverse conversions. If the rates at the commencement of the first reactions be placed side by side with the amounts of heat evolved by them, then a perfect conformity is seen to exist between the data. In the reaction between $\mathrm{AgCl}$ and $\mathrm{KBr} 3.5$ thousand heat units are evolved, and the rate of this reaction in the first two hours is expressed by the formation of 79.8 p.c. of bromide of silver; if $\mathrm{NaBr}$ act on $\mathrm{AgCl}$, then 4.3 thousand heat units are evolved, and the rate during the first two hours is 83.2 p.c. This conformity between the rate of reaction and the thermal figures is also remarked for other compounds. This shows that the thermal figures are not equivalent to the entire work of affinity, but are only proportional to the first rates of reactions. This explains why it is possible upon their basis to foresee the direction of the dominating reaction in a complex medium, but impossible to foretell in what direction the reaction will proceed. 
whilst hydrogen iodide is decomposed by light at even the ordinary temperature, and very easily by a red heat. And therefore the reaction $\mathrm{I}_{2}+\mathrm{H}_{2}=\mathrm{HI}+\mathrm{HI}$ is very easily reversible, and consequently has a limit, and hydrogen iodide easily dissociates. ${ }^{67}$ Judging by the direct measurement of the heat evolved, the conversion of $2 \mathrm{HCl}$ into $\mathrm{H}_{2}+\mathrm{Cl}_{2}$ requires the expenditure of 44000 heat units (because the formation of $\mathrm{HCl}$ evolves 22000 heat units). The decomposition of $2 \mathrm{HBr}$ into $\mathrm{H}_{2}+\mathrm{Br}_{2}$ requires, if the bromine is obtained in a gaseous state, a consumption of only about 24000 units, and in the decomposition of $2 \mathrm{HI}$ into $\mathrm{H}_{2}+\mathrm{I}_{2}$ as vapour about 3000 heat units are evolved, ${ }^{68}$ which, without doubt, stands in causal connection with the great

67 The dissociation of hydriodic acid has been studied in detail by Hautefeuille and Lemoine, from whose researches we will extract the following information. The decomposition of hydriodic acid is distinct, but proceeds slowly at $180^{\circ}$; the rate and limit of decomposition increases with a rise of temperature The reverse action is the same-that is, $\mathrm{I}_{2}+\mathrm{H}_{2}$ forms $2 \mathrm{HI}$, not only under the influence of spongy platinum (Corenwinder), which also accelerates the decomposition of hydriodic acid, but it also proceeds by itself, although slowly. The limit of the reverse reaction remains the same with or without spongy platinum. An increase of pressure lias a very powerful accelerative effect on the rate of the reaction of the formation of hydriodic acid, and therefore spongy platinum by condensing gases has the same effect as increase of pressure. At the atmospheric pressure the decomposition of hydriodic acid reaches the below-mentioned limit at $250^{\circ}$ in several months, and at $446^{\circ}$ in several hours. The limit at $250^{\circ}$ is about 18 p.c. of decomposition - that is, out of 100 parts of hydrogen previously combined in hydriodic acid, about. 10 p.c. may be disengaged at this temperature (this hydrogen may be easily measured, and the measure of dissociation determined), but not more; the limit at $440^{\circ}$ is about 26 p.c. If the pressure under which $2 \mathrm{HI}$ passes into $\mathrm{H}_{2}+\mathrm{I}_{2}$ be $4 \frac{1}{2}$ atmospheres, then the limit is 24 p.c.; under a pressure of $\frac{1}{5}$ atmosphere the limit is 29 p.c. The small influence of pressure on the dissociation of hydriodic acid (compared with $\mathrm{N}_{2} \mathrm{O}_{2}$, Chap. VI. Note 46) is due to the fact that the reaction $2 \mathrm{HI}=\mathrm{I}_{2}+\mathrm{H}_{2}$ is not accompanied by a change of volume, and probably the existing differences are determined by deviations from BoyleMariotte's law. In order to show the influence of time, we will cite the following figures referring to $350^{\circ}$ : 1. Reaction $\mathrm{H}_{2}+\mathrm{I}_{2}, 3$ hours, 88 p.c. of hydrogen remained free; 8 hours, 69 p.c. ; 34 hours, 48 p.c.; 76 hours, 29 p.c.; and 327 hours, 18.5 p.c. of hydrogen remained in 8 free state. 2. The reverse decomposition of $2 \mathrm{HI}, 9$ hours, 3 p.c. of hydrogen set free, and after 250 hours 18.6 p.c. of hydrogen became free-that is, the limit was reached. The addition of extraneous hydrogen diminishes the limit of the reaction of decomposition, or increases the formation of hydriodic acid from iodine and hydrogen, as would be expected from Berthollet's doctrine (Chap. X.). Thus at $440^{\circ} 26$ p.c. of hydriodic acid is decomposed if there be no admixture of lydrogen, while if $\mathrm{H}_{2}$ be added, then at the limit half as small a mass of $\mathrm{HI}$ is decomposed. Therefore, if an infinite mass of hydrogen be taken there will be no decomposition of the hydriodic acid. Light aids the decompo. sition of hydriodic acid very powerfully. At the ordinary temperature 80 p.c. is decomposed under the influence of light, whilst under the influence of heat alone this limit corresponds with a very high temperature. The distinct action of light, spongy platinum, and of imperfect cleanliness in glass (especially of sodium sulphate, which destroys hydriodic acid), not only render the investigations difficult, but also show that in reactions like $2 \mathrm{HI}=\mathrm{I}_{2}+\mathrm{H}_{2}$, which are accompanied by slight heat effects, all foreign and feeble influences may deeply affect the process of the phenomenon (Note 47).

68 The thermal determinations of Thomsen (at $18^{\circ}$ ) gave in thousands of calories, $\mathrm{Cl}+\mathrm{H}=+22, \mathrm{HCl}+\mathrm{Aq}$ (that is, a large amount of water dissolves $\mathrm{HCl}$ ) $=+17 \cdot 3$, and 
stability of hydrogen chloride, the easy decomposability of hydrogen iodide, and the intermediate properties of hydrogen bromide. From this it would be expected that chlorine is capable of decomposing water with the evolution of oxygen, whilst iodine has not the energy to produce this disengagement, ${ }^{69}$ although it is able to liberate the oxygen from the oxides of potassium and sodium, as the affinity of these metals for the halogens is very considerable. For this reason oxygen, especially in compounds from which it can be evolved (for instance, $\mathrm{ClHO}, \mathrm{CrO}_{3}$, dc.), easily decomposes hydrogen iodide. A mixture of hydrogen iodide and oxygen burns in the presence of an ignited substance, forming water and iodine. Drops of nitric acid in an atmosphere of hydrogen iodide produce the disengagement of violet fumes of iodine and brown fumes of nitric peroxide. In the presence of alkalis and an excess of water, however, iodine is able to produce oxidation like chlorine - that is, it decomposes water ; the action is here aided by the affinity of hydrogen iodide for the alkali and water, just as sulphuric acid helps zinc to decompose water. But the relative instability of hydriodic acid is best seen in comparing the acids in a gaseous state. If the halogen acids be dissolved in water, they evolve so much heat that they approach much nearer to each other in properties. This is seen from thermochemical data, because in the formation of $\mathrm{HX}$ in solution (in a large excess of water) from the gaseous elements there is evolved for $\mathrm{HCl} 39000$, for $\mathrm{HBr} 22000$,

therefore $\mathrm{H}+\mathrm{Cl}+\mathrm{Aq}=+39 \cdot 3$. In taking molecules, all these figures must be doubled. $\mathrm{Br}+\mathrm{H}=+8 \cdot 4 ; \mathrm{HBr}+\mathrm{Aq}=19 \cdot 9 ; \mathrm{H}+\mathrm{Br}+\mathrm{Aq}=+28 \cdot 3$. According to Berthelot $7 \cdot 2$ go to the vaporisation of $\mathrm{Br}_{2}$, hence $\mathrm{Br}_{2}+\mathrm{H}_{2}=16 \cdot 8+7 \cdot 2=+24$, if $\mathrm{Br}_{2}$ be taken as vapour for comparison with $\mathrm{Cl}_{2} . \mathrm{H}+\mathrm{I}=-6 \cdot 0, \mathrm{HI}+\mathrm{Aq}=19 \cdot 2 ; \mathrm{H}+\mathrm{I}+\mathrm{Aq}=+13 \cdot 2$, and, according to Berthelot, the heat of fusion of $\mathrm{I}_{2}=3^{*} 0$, and of vaporisation 6.0 thousand heat units, and therefore $\mathrm{I}_{2}+\mathrm{H}_{2}=-2(6 \cdot 0)+3+6=-3 \cdot 0$, if the iodine be taken as vapour. Berthelot, on the basis of his determinations, gives, however, +0.8 thousand heat units. Similar contradictory results are often met with in thermochemistry with the imperfection of the existing methods, and depend on the necessity of obtaining the fundamental figures by an indirect method. Thus Thomsen decomposed a dilute solution of potassium iodide by gaseous chlorine; the reaction gave $+26 \cdot 2$, whence, having first determined the heat effects of the reactions $\mathrm{KHO}+\mathrm{HCl}, \mathrm{KHO}+\mathrm{HI}$ and $\mathrm{Cl}+\mathrm{H}$ in aqueous solutions, it was possible to find $\mathrm{H}+\mathrm{I}+\mathrm{Aq}$; then, knowing $\mathrm{HI}+\mathrm{Aq}$, to find $\mathrm{I}+\mathrm{H}$. It is evident that the unavoidable errors may multiply themselves.

69 One would think, however, that on the basis of Berthollet's doctrine, and the observations of Potilitzin (Note 66), a certain trace of slow decomposition of water by iodine may exist. In this sense the observations of Dossios and Weith on the fact that the solubility of iodine in water increases after lapse of several months, will be comprehensible. Hydriodic acid is then formed, and it increases the solubility. If the iodine be extracted from such a solution by carbon bisulphide, then, as the authors showed, after the action of nitrous anhydride, iodine may be again found in the solution by means of starch. One can easily imagine that a number of like reactions, requiring much time and taking place in small quantities, have up to now eluded the attention of investigators who even still doubt the universal application of Berthollet's doctrine, or only see the thermochemical side of reactions, or else neglect to pay attention to the element of time and the influence of mass. 
and for HI 18000 heat units. $^{70}$ But this is especially evident from the fact that solutions of hydrogen bromide and iodide in water have many points in common with solutions of hydrogen chloride, both in their capacity to form hydrates, fuming solutions of constant boiling point, and in their capacity to form haloid salts, \&c., by reacting on bases.

In consequence of what has been said above, it follows that hydrobromic and hydriodic acids, being substances which are but slightly stable, cannot be evolved in a gaseous state under many of those conditions under which hydrochloric acid is formed. Thus if sulphuric acid in solution acts on sodium iodide, all the same phenomena take place as with sodium chloride (a portion of the sodium iodide gives hydriodic acid, and all remains in solution), but if sodium iodide be mixed with strong sulphuric acid, then its oxygen decomposes the hydriodic acid set free with liberation of iodine, $\mathrm{H}_{2} \mathrm{SO}_{4}+2 \mathrm{HI}=2 \mathrm{H}_{2} \mathrm{O}+\mathrm{SO}_{2}+\mathrm{I}_{2}$. This reaction proceeds in the reverse direction in the presence of a mass of water (2000 parts of water per 1 part of $\mathrm{SO}_{2}$ ), in which not only the affinity of hydriodic acid for water evinces itself, but also the direct participation of water in the direction of chemical reactions accomplished through its medium. ${ }^{71}$ Therefore, having a halogen salt, it is easy to obtain gaseous hydrochloric acid (by the action of sulphuric acid), but neither hydrobromic nor hydriodic acid can be so obtained free (as gases). ${ }^{72}$ Other methods are requisite for their preparation; but above all there must be conditions for the removal of oxygen, which is so easily able to destroy these acids. Therefore hydrogen sulphide, phosphorus, \&c., which themselves easily take up oxygen, are introduced as means for the conversion of bromine and iodine into hydrobromic and hydriodic acids in the presence of water. For example, in the action of phosphorus the essence of the matter is that the oxygen of the water goes to the phosphorus, and the reaction of the remainders leads to the formation of hydrobromic or hydriodic acid; but the matter is complicated by the reversibility of the reaction, the affinity for water, and other circumstances which are understood by following Berthollet's doctrine. Chlorine (also bromine) directly decomposes hydrogen sulphide, forming hydrochloric acid and liberating sulphur, both in a gaseous form and in solutions, whilst iodine only decomposes hydrogen sulphide in weak

70 On the basis of the data in Note 68.

71 A number of like cases confirm what has been said in Notes 26 and 28 of Chapter X.

72 This is prevented by the reducibility of sulphuric acid. If rolatile acids be taken they pass over, together with the hydrobromic and hydriodic acids, when distilled; whilst many non-volatile acids which are not reduced by hydrobromic and hydriodic acids only act feebly (like phosphoric acid), or do not act at all (like boric acid).

VOL. I. 
solutions when its atfinity for hydengen is ateled by the attinity of

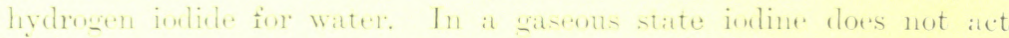
on livdrogen -ulphide. whilst sulphure is ahle to decompore graseous

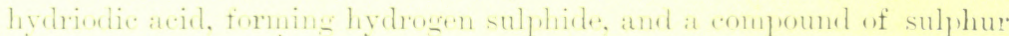

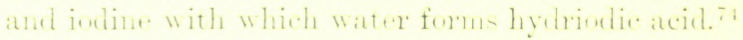

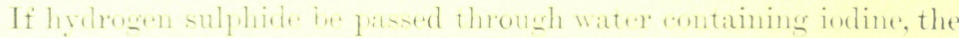

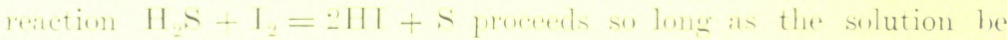

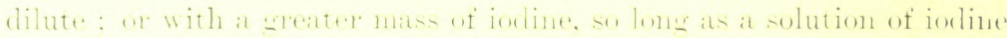

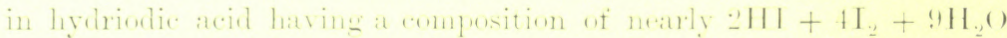
(according to bineatu) he nut formed. Hydrogen sulphicle then mo longer

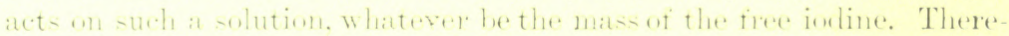
tore. only weak solution of hylriodic acid atu be ohtatined by passing

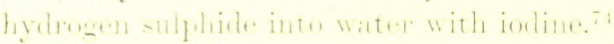

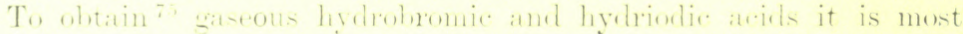

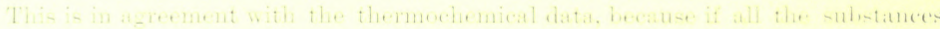

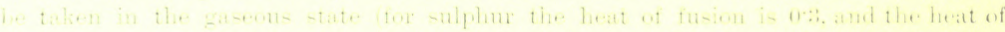

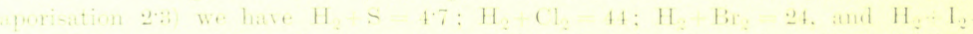

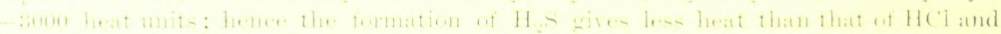

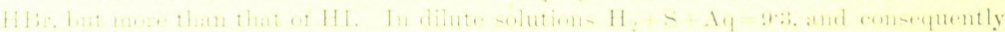

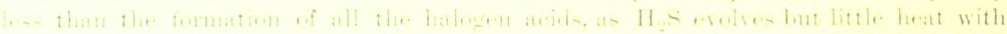

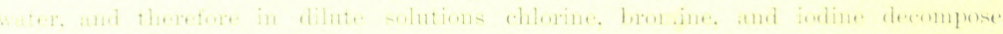

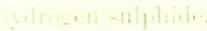

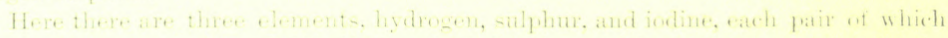

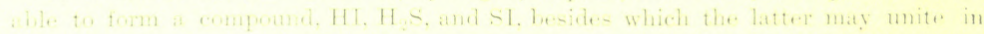

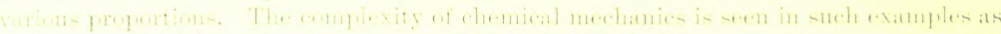

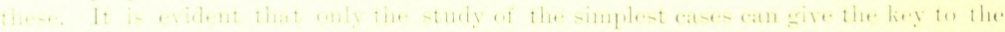

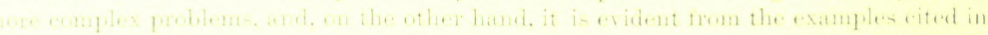

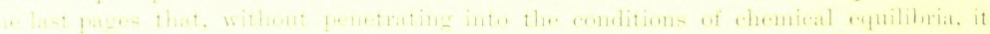

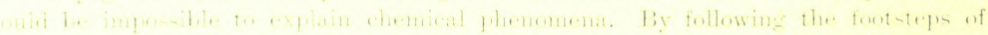

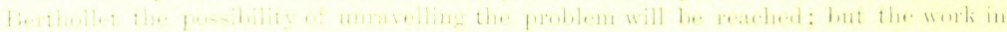

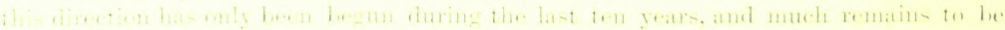

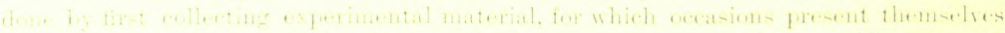

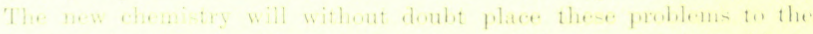

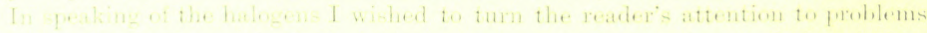

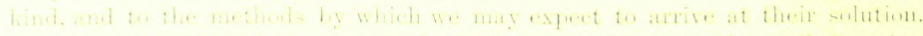

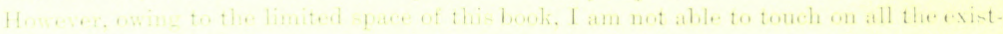

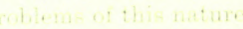

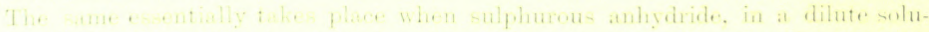

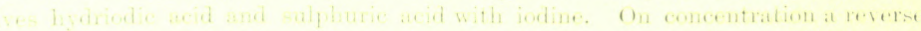

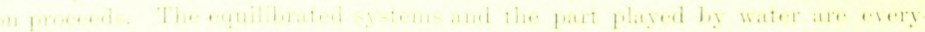

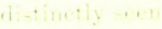

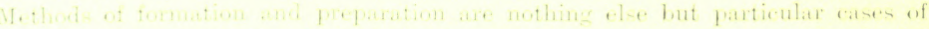

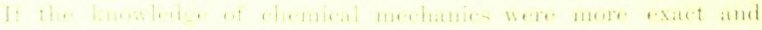

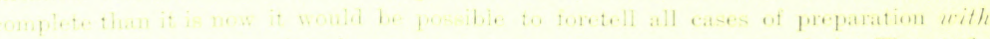

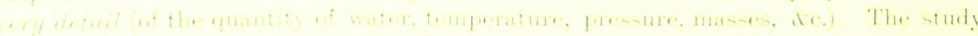

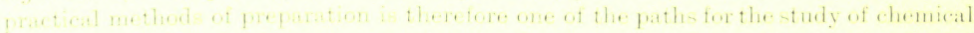

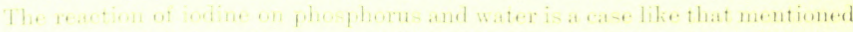

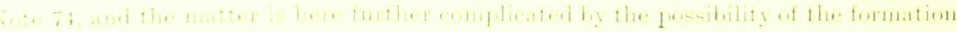

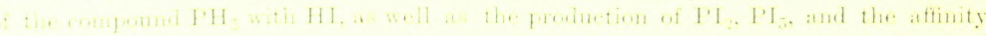

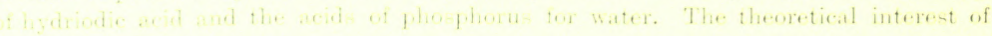


convenient to take advantage of the reactions between phosphorus, the halogens, and water, when the latter is present in small quantity (otherwise the halogen acids formed are dissolved by the water), and the halogen is gradually added to the phosphorus moistened with water. Thus if red phosphorus be placed in a flask and moistened with water, and bromine be added drop by drop (from a closed funnel with a glass tap), hydrobromic acid is abundantly and uniformly disengaged. ${ }^{76}$ Hydrogen iodide is prepared by adding 1 part of common (yellow) dry phosphorus to 10 parts of dry iodine in a glass flask. On revolving the flask, reaction (light and heat are evolved) proceeds quietly between them, and when the mass of the iodide of phosphorus which is formed has cooled, water is added drop by drop (from a funnel with a tap) and hydrogen iodide is evolved even without the aid of heat. These methods of preparation will be at once understood when it is remembered (p. 462) that phosphorus chloride gives hydrogen chloride with water. It is exactly the same here-the oxygen of the water passes over to the phosphorus, and the hydrogen to the iodine; for example, $\mathrm{PI}_{3}+3 \mathrm{H}_{2} \mathrm{O}=\mathrm{PH}_{3} \mathrm{O}_{3}+3 \mathrm{HI} .{ }^{77}$

In a gaseous form hydrobromic and hydriodic acids are closely

equilibria in all their complexity is naturally very great, but it falls in the background before the primary interests of discovering practical methods for the isolation of substances, and the means of employing them for the requirements of man. It is only after the satisfaction of these requirements that interests of the other order arise, which themselves reflect the interests of the primary nature. For these reasons, whilst considering it opportune to point out the theoretical interests of chemical equilibria, the chief attention of the reader is directed in this work to the primary chemical interests.

$76 \mathrm{Hydrobromic}$ acid is obtained by the action of bromine on paraffin heated to $180^{\circ}$. Gustavson proposed preparing it by the action of bromine (it is best if it be added in drops together with traces of aluminium bromide) on anthracene (a solid hydrocarbon from coal tar). Balard prepared it by passing bromine vapour over moist pieces of common phosphorus. The liquid tribromide of phosphorus, directly obtained from phosphorus and bromine, also gives hydrobromic acid when treated with water. Bromide of potassium or sodium, when treated with sulphuric acid in the presence of a piece of phosphorus, also gives hydrobromic acid, but hydriodic acid is decomposed by this method. In order to free hydrobromic acid from bromine vapour it is passed over moist phosphorus and dried either by phosphoric anhydride or calcium bromide (calcium chloride cannot be used, as hydrochloric acid would be formed). Neither hydrobromic nor hydriodic acids can be collected over mercury, on which they act, but they may be directly collected in a dry vessel by letting the gas-conducting tube pass down to the bottom of the ressel, both gases being much heavier than air.

77 But, generally, more phosphorus is taken than is required for the formation of $\mathrm{PI}_{3}$, because, otherwise, a portion of the iodine is distilled over. If less than one-tenth part of iodine be taken, then much phosphonium iodide, $\mathrm{PH}_{4} \mathrm{I}$ is formed. This proportion was established by Gay-Lussac and Kolbe. Hydriodic acid is also prepared in many other ways. Bannoff dissolves two parts of iodine in one part of a previously-prepared strong (sp. gr., 1.67) solution of hydriodic acid, and pours it on to red phosphorus in a retort. Personne takes a mixture of fifteen parts of water, ten of iodine, and one of red phosphorus, which, when heated, disengages hydriodic acid mixed with iodine rapour, which is removed by passing it over moist phosphorus (Note 76). It must be remembered, 


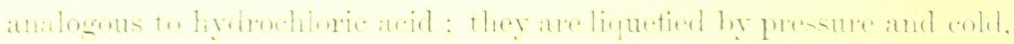

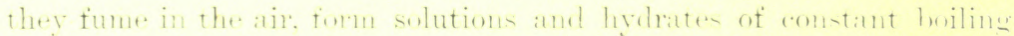

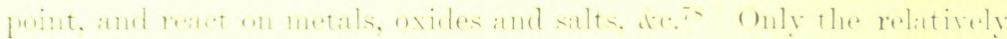

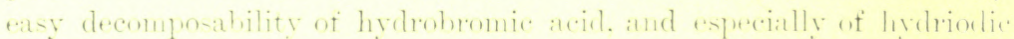

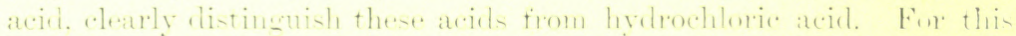

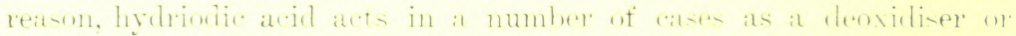

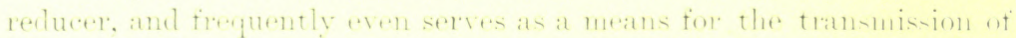

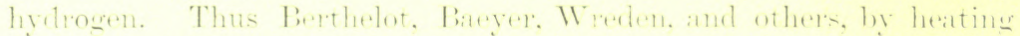

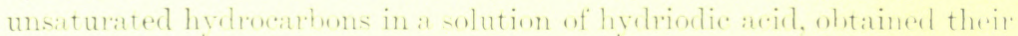

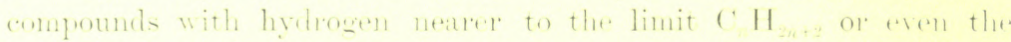

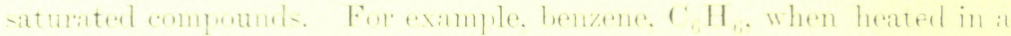

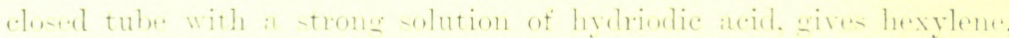

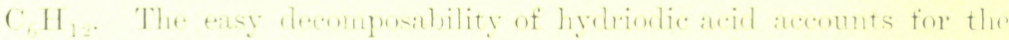

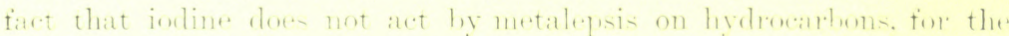

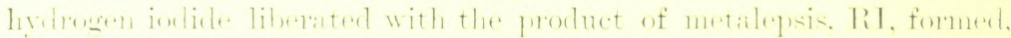

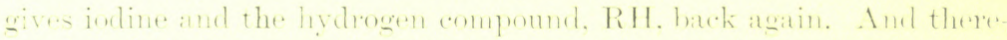

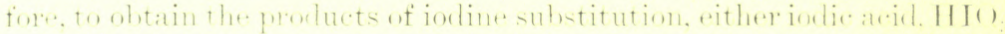

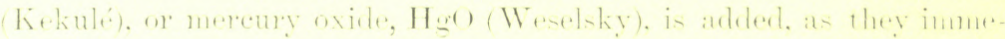

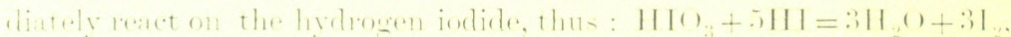

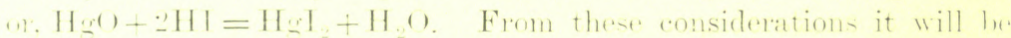

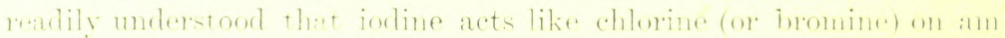
end ia and sodium hydroxide, for in this ease the hydriodie acid produced

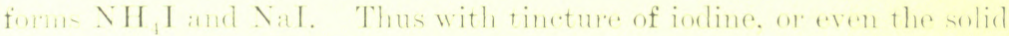
foruent, at -olution of ammoniat immerliately torms a highly-explo-ive

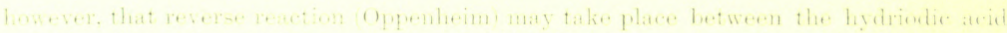

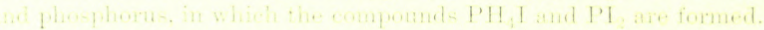

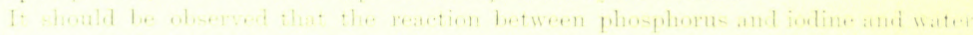

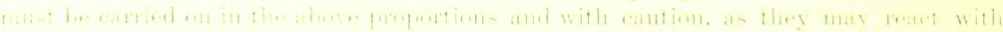

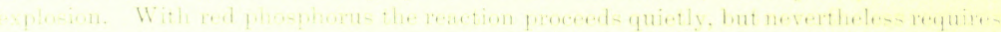

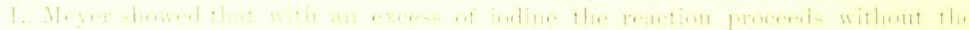

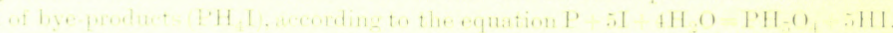

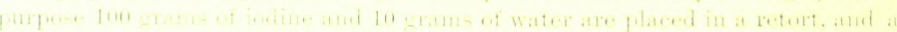

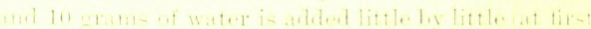

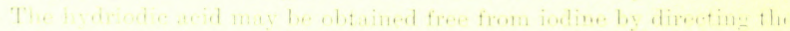

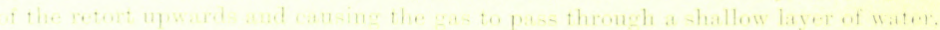

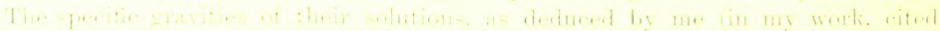

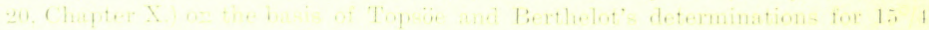

\begin{tabular}{|c|c|c|c|c|c|c|}
\hline & 10 & 211 & $\therefore i 1$ & 111 & int & (ii) $11 .+$. \\
\hline Ifi: & $\mid 10,1$ & 1157 & $1 \ldots+4$ & $1 \cdots 7$ & $1.50,2$ & \\
\hline & $1 \%, \ldots$ & $1 \cdot 1+1$ & 1 wati & $1 \cdots: 2,1$ & 1 stit & $!+$ \\
\hline
\end{tabular}

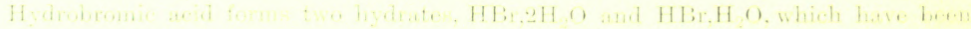

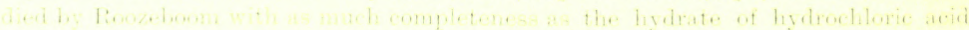


solid black product of metalepsis, generally known as iodide of nitrogen, although it probably contains hydrogen, $3 \mathrm{NH}_{3}+2 \mathrm{I}_{2}=2 \mathrm{NH}_{4} \mathrm{I}+\mathrm{NHI}_{2}$. However, the composition of the product is variable, and with an excess of water $\mathrm{NI}_{3}$ seems to be formed. Iodide of nitrogen is no less explosive than nitrogen chloride. In the action of iodine on sodium hydroxide no bleaching compound is formed (whilst bromine gives one), but a direct reaction is always accomplished with the formation of an iodate $6 \mathrm{NaHO}+3 \mathrm{I}_{2}=5 \mathrm{NaI}+3 \mathrm{H}_{2} \mathrm{O}+\mathrm{NaIO}_{3}$ (Gay-Lussac). Solutions of other alkalis, and even a mixture of water and oxide of mercury, act in the same manner. This direct formation of iodic acid, $\mathrm{HIO}_{3}=\mathrm{IO}_{2}(\mathrm{OH})$, shows the propensity of iodine to give compounds of the type $\mathrm{IX}_{5}$. Indeed, the propensity of iodine to form compounds of a higher type clearly evinces itself in many ways. But it is most important to turn attention to the fact that iodic acid is easily and directly formed by the action of oxidising substances on iodine. Thus, for instance, strong nitric acid directly converts iodine into iodic acid, whilst it has no oxidising action on chlorine. ${ }^{79}$ This shows a greater affinity in iodine for oxygen than in chlorine, and this conclusion is confirmed by the fact that iodine displaces chlorine from its oxygen acids, ${ }^{80}$ and that in the presence of water chlorine oxidises iodine. ${ }^{81}$ Even ozone or a silent discharge passed through a mixture of oxygen and iodine vapour is able to directly oxidise iodine ${ }^{82}$ into iodic acid. It is disengaged from solu-

79 The oxidation of iodine by strong nitric acid was discovered by Connell; Millon showed that it is accomplished, although more slowly, by the action of the hydrates of nitric acid up to $\mathrm{HNO}_{3}, \mathrm{H}_{2} \mathrm{O}$, but that the solution $\mathrm{HNO}_{5}, 2 \mathrm{H}_{2} \mathrm{O}$, and weaker solutions, do not oxidise, but simply dissolve, iodine. The participation of water in reactions is seen in this instance. It is also seen, for example, in the fact that dry ammonia combines directly with iodine-for instance, at $0^{\circ}$ forming the compound $\mathrm{I}_{2}, 4 \mathrm{NH}_{5}$-whilst iodide of nitrogen is only formed under the action of water.

80 Bromine also displaces chlorine-for instance, from chloric acid, directly forming bromic acid. If a solution of potassium chlorate be taken ( 75 parts per 400 parts of water), and iodine be added to it ( 80 parts), and then a small quantity of nitric acid, chlorine is disengaged on boiling, and potassium iodate is formed in the solution. In this instance the nitric acid first evolves a certain portion of the chloric acid, and the latter, with the iodine, evolves chlorine. The iodic acid thus formed acts on a further quantity of the potassium chlorate, sets a portion of the chloric acid free, and in this manner the action is kept up. Potilitzin (1887) remarked, however, that not only do bromine and iodine displace the chlorine from chloric acid and potassium chlorate, but also chlorine displaces bromine from sodium bromate, and, furthermore, the reaction does not proceed as a direct substitution of the halogens, but is accompanied by the formation of free acids; for example, $5 \mathrm{NaClO}_{3}+3 \mathrm{Br}_{2}+3 \mathrm{H}_{2} \mathrm{O}=5 \mathrm{NaBr}+5 \mathrm{HClO}_{3}+\mathrm{HBrO}_{3}$.

81 If iodine be stirred up in water, and chlorine passed through it, then the iodine is dissolved; the liquid becomes colourless, and contains, according to the mass of water and chlorine, either the compounds $\mathrm{IHCl}_{2}$, or $\mathrm{ICl}_{5}$, or $\mathrm{HIO}_{5}$. If there be a small amount of water, then the iodic acid may separate out directly in crystals, but a complete conversion (Bornemann) only occurs when not less than ten parts of water are taken to one part of iodine- $\mathrm{ICl}+3 \mathrm{H}_{2} \mathrm{O}+2 \mathrm{Cl}_{2}=\mathrm{IHO}_{3}+5 \mathrm{HCl}$.

82 Schönebein and Ogier proved this. Ogier found that at $45^{\circ}$ ozone immediately 
tions as a hydrate, $\mathrm{HIO}_{3}$, which loses water at $170^{\circ}$, and gives an anhydride, $\mathrm{I}_{2} \mathrm{O}_{5}$. Both these substances are crystalline (sp. gr. $\mathrm{I}_{2} \mathrm{O}_{5}$ $5 \cdot 037, \mathrm{HIO}_{3} 4 \cdot 869$ at $\left.0^{\circ}\right)$, colourless, soluble in water, ${ }^{83}$ decompose at a red heat into iodine and oxygen, are in many cases powerfully oxidisingfor instance, they oxidise sulphurous anhydride, hydrogen sulphide, carbonic oxide, \&c.- - form chloride of iodine and water with hydrochloric acid, and with bases form salts, not only normal $\mathrm{MIO}_{3}$, but also acid, for example, $\mathrm{KIO}_{3} \mathrm{HIO}_{3}, \mathrm{KIO}_{3} 2 \mathrm{HIO}_{3}$. With hydriodic acid iodic acid immediately reacts, disengaging iodine, $\mathrm{HIO}_{3}+5 \mathrm{HI}=$ $3 \mathrm{H}_{2} \mathrm{O}+3 \mathrm{I}_{2}$.

As with chlorine, so with iodine, a periodic acid, $\mathrm{HIO}_{4}$, is formed. This acid is produced, as its salts, by the action of chlorine on alkaline solutions of iodates, and also by the action of iodine on chloric acid. ${ }^{84}$

oxidises iodine vapour, first forming the oxide $\mathrm{I}_{2} \mathrm{O}_{3}$, which is decomposed by water or on heating into iodic anhydride and iodine. Iodic acid is formed at the positive poles (Riche) when a solution of hydriodic acid is decomposed by a galvanic current. It is also formed in the combustion of hydrogen mixed with a small quantity of hydriodic acid (Salet).

85 Kämmerer showed that a solution of sp. gr. $2 \cdot 127$ at $14^{\circ}$, containing $2 \mathrm{HIO}_{3}, 9 \mathrm{H}_{2} \mathrm{O}$, entirely solidified in the cold. On comparing solutions $\mathrm{HI}+m \mathrm{H}_{2} \mathrm{O}$ with $\mathrm{HIO}_{3}+m \mathrm{H}_{2} \mathrm{O}$, we find that the specific gravity increases but the volume decreases (it is the same in their passage to solutions $\mathrm{HIO}_{3}+m \mathrm{H}_{2} \mathrm{O}$ ), whilst in the passage of solutions $\mathrm{HCl}+m \mathrm{H}_{2} \mathrm{O}$ to $\mathrm{HClO}_{3}+m \mathrm{H}_{2} \mathrm{O}$ the specific gravity augments, and so does the volume, which is remarked also in certain other cases (for example, $\mathrm{H}_{3} \mathrm{PO}_{5}$ and $\mathrm{H}_{5} \mathrm{PO}_{4}$ ). Observations on the specific volume of solution of iodic and periodic acids were made by Thomsen at $17^{\circ} 17^{\circ}$. He expresses for $\mathrm{HIO}_{3}+m \mathrm{H}_{2} \mathrm{O}$ by $18 m+39 \cdot 1-13 \cdot 1 m /(m+18)$, and for $\mathrm{H}_{5} \mathrm{IO}_{6}+m \mathrm{H}_{2} \mathrm{O}$ by $18 m+23 \cdot 8$, which shows the absence of contraction in mixing these acids with water (see Mendeléeff, Investigation of Aqueous Solutions, p. 368).

84 If sodium iodate be mixed with a solution of sodium hydroxide, heated, and chlorine be passed through the solution, then a sparingly soluble salt separates out, which corresponds with periodic acid, and has the composition $\mathrm{Na}_{4} \mathrm{I}_{2} \mathrm{O}_{9}, 3 \mathrm{H}_{2} \mathrm{O}$.

$$
6 \mathrm{NaHO}+2 \mathrm{NaIO}_{3}+4 \mathrm{Cl}=4 \mathrm{NaCl}+\mathrm{Na}_{4} \mathrm{I}_{2} \mathrm{O}_{9}+3 \mathrm{H}_{2} \mathrm{O} \text {. }
$$

This compound is sparingly soluble in water, but easily dissolves in a very dilute solution of nitric acid. If silver nitrate be added to this solution a precipitate is formed which contains the corresponding compound of silver, $\mathrm{Ag}_{4} \mathrm{I}_{2} \mathrm{O}_{9}, 3 \mathrm{H}_{2} \mathrm{O}$. If this sparingly soluble silver compound be dissolved in hot nitric acid, then, on evaporating, orange crystals of a salt having the composition $\mathrm{AgIO}_{4}$ separate. This salt is formed from the preceding by the nitric acid taking up silver oxide. $\mathrm{Ag}_{4} \mathrm{I}_{2} \mathrm{O}_{9}+2 \mathrm{HNO}_{3}=2 \mathrm{AgNO}_{3}$ $+2 \mathrm{AgIO}_{4}+\mathrm{H}_{2} \mathrm{O}$ is decomposed by water, with the re-formation of the preceding salt, while iodic acid remains in solution-

$$
4 \mathrm{AgIO}_{4}+\mathrm{H}_{2} \mathrm{O}=\mathrm{Ag}_{4} \mathrm{I}_{2} \mathrm{O}_{9}+2 \mathrm{HIO}_{4} \text {. }
$$

The structure of the first of these salts, $\mathrm{Na}_{4} \mathrm{I}_{2} \mathrm{O}_{9}, 3 \mathrm{H}_{2} \mathrm{O}$, presents itself in a simpler form if the water of crystallisation is regarded as an integral portion of the salt; the formula is then divided in two, and takes the form of $\mathrm{IO}(\mathrm{OH})_{3}(\mathrm{ONa})_{2}$-that is, it answers to the type $\mathrm{IOX}_{3}$, or $\mathrm{IX}_{7}\left(\right.$ like $\mathrm{AgIO}_{4}$ ) which is $\mathrm{IO}_{5}(\mathrm{OAg})$. The salts of periodic acids are expressed by this type $\mathrm{IX}_{7}$. Kimmins (1889) classes all the salts of periodic acid into four types-the meta-salts of $\mathrm{HIO}_{4}$ (salts of $\mathrm{Ag}, \mathrm{Cu}, \mathrm{Pb}$ ), the meso-salts of $\mathrm{H}_{3} \mathrm{IO}_{3}\left(\mathrm{~Pb}, \mathrm{Ag}_{2}, \mathrm{H}, \mathrm{Cd}, \mathrm{H}\right)$, the para-salts of $\mathrm{H}_{5} \mathrm{IO}_{6}\left(\mathrm{Na}_{2} \mathrm{H}_{5}, \mathrm{Na}_{5} \mathrm{H}_{2}\right)$, and the di-salts of $\mathrm{H}_{4} \mathrm{I}_{2} \mathrm{O}_{3}\left(\mathrm{~K}_{4}, \mathrm{Ag}_{4}, \mathrm{Ni}_{2}\right)$. The three first are direct compounds of the type $\mathrm{IX}_{7}$, namely, $\mathrm{IO}_{3}(\mathrm{OH}), \mathrm{IO}_{2}(\mathrm{OH})_{3}$, and $\mathrm{IO}(\mathrm{OH})_{3}$, and the latter are types of diperiodic 
It crystallises from solutions as a hydrate containing $2 \mathrm{H}_{2} \mathrm{O}$ (corresponding with $\mathrm{HClO}_{4}, 2 \mathrm{H}_{2} \mathrm{O}$ ), but as there are salts containing up to 5 atoms of metals, this water must be counted as water of constitution. Therefore $\mathrm{IO}(\mathrm{OH})_{5}=\mathrm{HIO}_{4}, 2 \mathrm{H}_{2} \mathrm{O}$ corresponds with the higher form of halogen compounds, $\mathrm{IX}_{7} .^{8.5}$ In decomposing (at $200^{\circ}$ ) or acting as an oxidiser, periodic acid first gives iodic acid, but it may also be ultimately decomposed, with the formation of hydriodic acid.

Thus both bromine and iodine present a great analogy to chlorine in their behaviour with various substances, but nevertheless a series of qualitative distinctions characterise each element. The formation of a compound between chlorine and iodine must be classed among these distinctions. ${ }^{86}$ These elements combine directly together with the evolution of heat, and form iodine monochloride, ICl, or iodine trichloride, $\mathrm{ICl}_{3} .{ }^{87} \mathrm{As}$ water reacts on these substances, forming iodic

salts, which correspond with the type of the meso-salts, as pyrophosphoric salts correspond with orthophosphoric salts-i.e. $2 \mathrm{H}_{2} \mathrm{IO}_{5}-\mathrm{H}_{2} \mathrm{O}=\mathrm{H}_{4} \mathrm{I}_{2} \mathrm{O}_{9}$.

85 Periodic acid, discovered by Magnus and Ammermüller, and whose salts were afterwards studied by Langlois, Rammelsberg, and many others, presents an example of hydrates in which it is evident that there is not that distinction between the water of hydration and of crystallisation which was at first considered to be so clear. In $\mathrm{HClO}, 2 \mathrm{H}_{2} \mathrm{O}$ the water, $2 \mathrm{H}_{2} \mathrm{O}$, is not displaced by bases and must be regarded as water of crystallisation, whilst in $\mathrm{HIO}_{4}, 2 \mathrm{H}_{2} \mathrm{O}$ it must be regarded as water of hydration. We shall afterwards see that the system of the elements obliges one to consider the halogens as substances giving a highest saline type, $G X_{7}$, if $G$ signify a halogen, and $X$ oxygen $\left(\mathrm{O}=\mathrm{X}_{2}\right), \mathrm{OH}$, and other like elements. The hydrate $\mathrm{IO}(\mathrm{OH})_{5}$ corresponding with many of the salts of periodic acid (for example, the salts of barium, strontium, mercury) does not exhaust all the possible forms. It is evident that various other pyro-, meta-, \&c., forms are possible by the loss of water, as will be more fully explained in speaking of phosphoric acid, and as was pointed out in the preceding note.

86 With respect to hydrogen, oxygen, chlorine, and other elements, bromine occupies an intermediate position between chlorine and iodine, and therefore there is no particular need for lingering over the compounds of bromine. This is the great advantage of a natural grouping of the elements.

87 They were both obtained by Gay-Iussac and many others. Recent verified data respecting iodine monochloride, $\mathrm{ICl}$, entirely confirm the numerous observations of Trapp (1854), and even confirm his statement as to the existence of two isomeric (liquid and crystalline) forms (Stortenbeker). With a small excess of iodine, iodine monochloride remains liquid, but in the presence of traces of iodine trichloride it easily crystallises. Schiitzenberger amplified the data concerning the action of water on the chlorides (Note 88) and Christomanos gave the fullest data regarding the trichloride.

After being kept for some time, the liquid monochloride of iodine yields red deliquescent octahedra, having the composition $\mathrm{ICl}_{4}$, which are therefore formed from the monochloride with the liberation of free iodine, which dissolves in the remaining quantity of the monochloride. This substance, however, judging by certain observations, is impure iodine trichloride. If 1 part of iodine be stirred up in 20 parts of water, and chlorine be passed through the liquid, then all the iodine is dissolved, and a colourless liquid is ultimately obtained which contains a certain proportion of chlorine, because this compound gives a metallic chloride and iodate with alkalis without evolving any free iodine : $\mathrm{ICl}_{5}+6 \mathrm{KHO}$ $=5 \mathrm{KCl}+\mathrm{KIO}_{3}+3 \mathrm{H}_{2} \mathrm{O}$. The existence of a pentachloride $\mathrm{ICl}_{5}$ is, however, denied, becau se this substance has not been obtained in a free state. 
acid and iodine, they have to be prepared from dry iodine and chlorine. ${ }^{88}$ Both substances are formed in a number of reactions ; for example, by the action of aqua regia on iodine, of chlorine on hydriodic acid, of hydrochloric acid on periodic acid, of iodine on potassium chlorate (with the aid of heat, dc.). Trapp obtained iodine monochloride, in beautiful red crystals, by passing a rapid current of chlorine into molten iodine. The monochloride then distils over and solidifies; it melts at $27^{\circ}$. By passing chlorine over the crystals of the monochloride, it is easy to obtain iodine trichloride in orange crystals, which melt at $34^{\circ}$ and volatilise at $47^{\circ}$, but in so doing decompose (into $\mathrm{Cl}_{2}$ and $\mathrm{ClI}$ ). The chemical properties of these chlorides entirely correspond with the properties of chlorine and iodine, which would be expected, because, in this instance, a combination by similitude took place as in the formation of solutions or alloys. Thus, for instance, the unsaturated hydrocarbons (for example, $\mathrm{C}_{2} \mathrm{H}_{4}$ ), which are capable of directly combining with chlorine and iodine, also directly combine with iodine monochloride.

Stortenbeker (1888) investigated the equilibrium of the system containing the molecules $\mathrm{I}_{2}, \mathrm{ICl}, \mathrm{ICl}_{3}$, and $\mathrm{Cl}_{2}$, in the same way that Roozeboom (Chapter X. Note 38 ) examined the equilibrium of the molecules $\mathrm{HCl}, \mathrm{HCl}, 2 \mathrm{H}_{2} \mathrm{O}$, and $\mathrm{H}_{2} \mathrm{O} . \mathrm{He}$ found that iodine monochloride appears in two states, one (the ordinary) is stable and melts at $27 \cdot 2^{\circ}$, whilst the other is obtained by rapid cooling, and melts at $13.9^{\circ}$, and easily passes into the first form. Iodine trichloride melts at $101^{\circ}$ only in a closed tube under a pressure of 16 , atmospheres.

$88 \mathrm{By}$ the action of water on iodine monochloride and trichloride a compound $\mathrm{IHCl}_{2}$ is obtained, which does not seem to be altered by water. Besides this compound, iodine and iodic acid are always formed, $10 \mathrm{ICl}+3 \mathrm{H}_{2} \mathrm{O}=\mathrm{HIO}_{3}+5 \mathrm{IHCl}_{2}+2 \mathrm{I}_{2}$; and in this respect iodine trichloride may be regarded as a mixture $\mathrm{ICl}+\mathrm{ICl}_{5}=2 \mathrm{ICl}_{5}$, but $\mathrm{ICl}_{5}+3 \mathrm{H}_{2} \mathrm{O}=$ $\mathrm{IHO}_{5}+5 \mathrm{HCl}$; hence iodic acid, iodine, the compound $\mathrm{IHCl}_{2}$, and hydrochloric acid are also formed by the action of water. 


\title{
CHAPTER XII
}

\author{
S O D I U M
}

WHEN common salt is submitted to the action of sulphuric acid, as we saw in Chapter X., hydrochloric acid is disengaged, and a neutral salt, sodium sulphate, $\mathrm{Na}_{2} \mathrm{SO}_{4}$, is formed if the mixture be strongly heated at the end of the reaction. This salt ${ }^{1}$ forms a colourless saline mass consisting of fine crystals, soluble in water. It is the product of many other double decompositions, sometimes produced on a large scale ; for instance, it is formed when ammonium sulphate is heated with common salt, in which case the sal-ammoniac is volatilised, also when sulphuric acid acts on sodium nitrate, \&c. A similar decomposition also takes place when, for instance, a mixture of lead sulphate and common salt is heated ; this mixture easily fuses, and if the temperature be further raised heavy vapours of lead chloride appear. When the disengagement of these vapours ceases, the remaining mass, on being treated with water, yields a solution of sodium sulphate mixed with a solution of undecomposed common salt. A considerable quantity, however, of the lead sulphate remains unchanged during this reaction, $\mathrm{PbSO}_{4}+2 \mathrm{NaCl}$ $=\mathrm{PbCl}_{2}+\mathrm{Na}_{2} \mathrm{SO}_{4}$, the vapours will contain lead chloride, and the residue will contain the mixture of the three remaining salts. Here the decomposition is produced by the lead salt as with sulphuric acid. The cause and nature of the reaction are just the same as were pointed out when considering Berthollet's doctrine. And here it may evidently be shown that the double decomposition is not determined by any other means than the removal of the substance formed from the sphere of the action of the remaining substances. This is seen

1 Whilst describing in some detail the properties of sodium chloride, hydrochloric acid, and sodium sulphate, I wish to impart a conception, by separate examples, of the properties of saline substances, but the dimensions of this treatise and its purpose and aim do not permit the possibility of entering into particulars concerning every salt, acid, or other substance. The fundamental object of this work-an account of the characteristics of the elements and an acquaintance with the forces acting between atoms-has nothing to gain from the multiplication of the number of as yet ungeneralised properties and relations. 


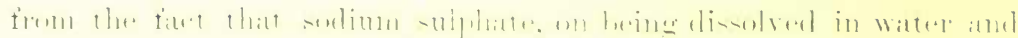

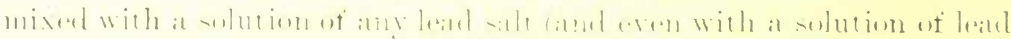

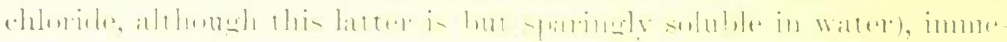

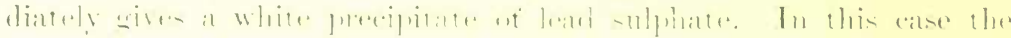

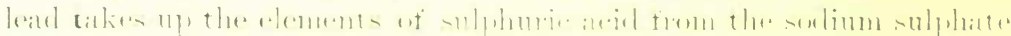

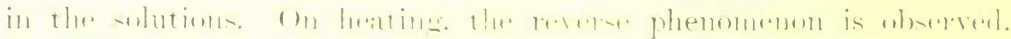

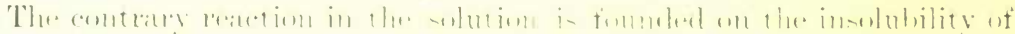

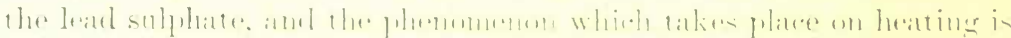

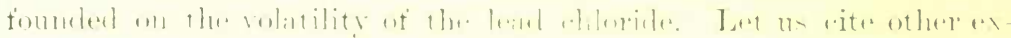

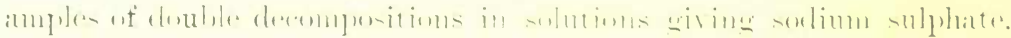

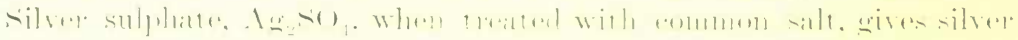

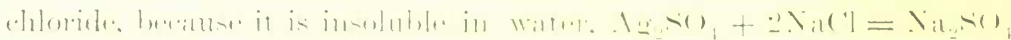

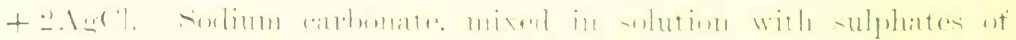

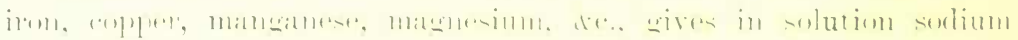

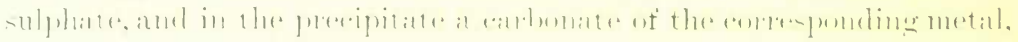

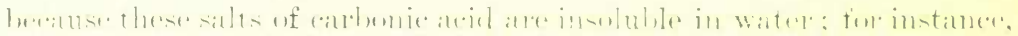

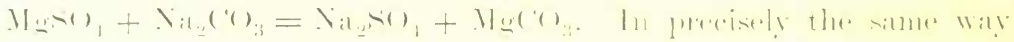

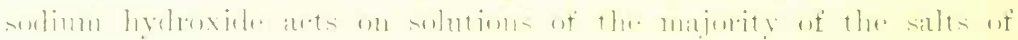

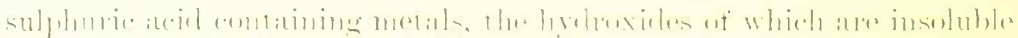

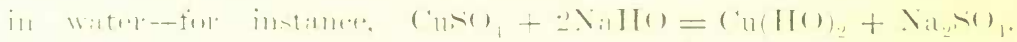

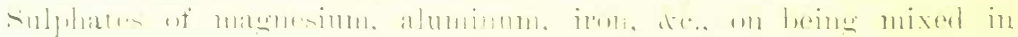

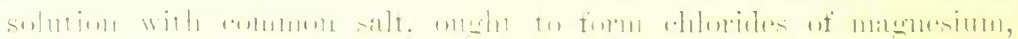

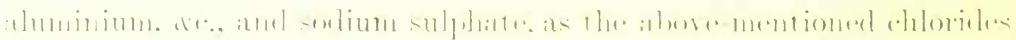

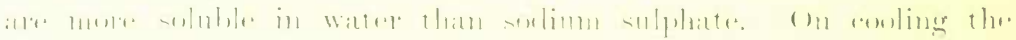

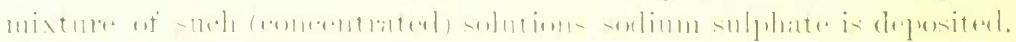

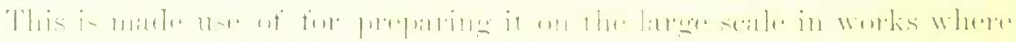

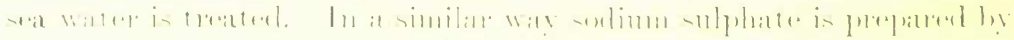

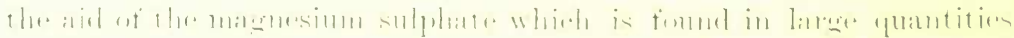

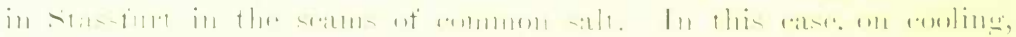

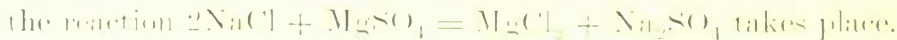

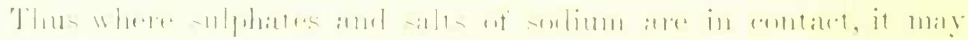

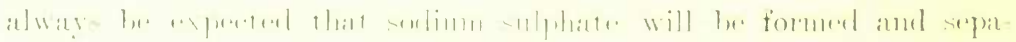

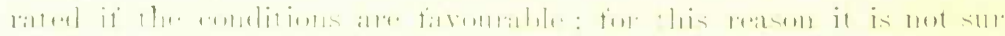

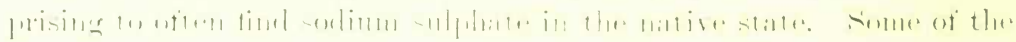

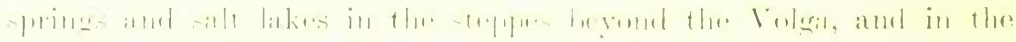

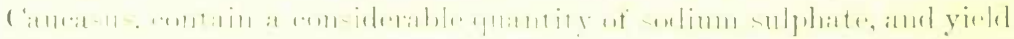

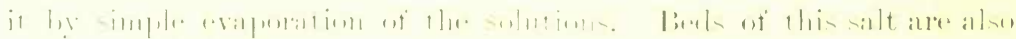

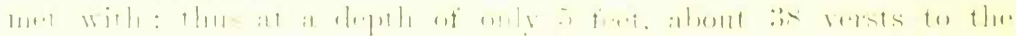

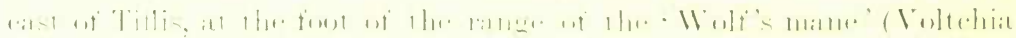

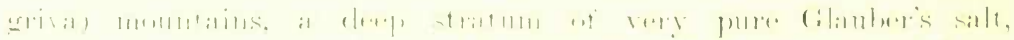

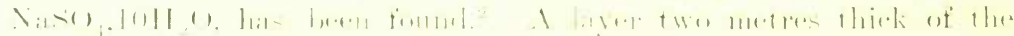


same salt lies at the bottom of several lakes (an area of about 10 square kilometres) in the Kouban district near Batalpaschinsk, and here its working has been commenced (1887). In Spain, near Arangoulz and Madrid, mineral sodium sulphate has likewise been found, and is already being worked.

The methods of obtaining salts by means of double decomposition from others already prepared are so general, that in describing a given salt there is no necessity to enumerate the cases hitherto observed of its being formed through various double decompositions. ${ }^{3}$ The possibility of this occurrence ought to be foreseen according to Berthollet's doctrine from the properties of the salt in question. On this account it is important to know the properties of salts ; all the more so because up to the present time those very properties (solubility, formation of crystallo-hydrates, volatility, \&c.) which may be made use of for separating them from other salts have not been generalised. ${ }^{4}$ These properties as yet remain subjects for investigation, and are rarely to be foreseen.

Sorlium sulphate very easily parts with water, and may be obtained in an anhydrous state if it be carefully heated until the weight remains constant; but if heated further, it partly loses the elements of sulphuric anhydride. It fuses at $861^{\circ}$ (red heat), and volatilises in a small proportion when very strongly heated, in which case it naturally decomposes. At $0^{\circ}$ in 100 parts of water, 5 parts of the anhydrous salt dissolve, at $10^{\circ} 9$ parts, at $20^{\circ} 19 \cdot 4$, at $30^{\circ} 40$, and at $34^{\circ} 55$ parts, the same being the case in the presence of an excess of crystals of $\mathrm{Na}_{2} \mathrm{SO}_{4}, 10 \mathrm{H}_{2} \mathrm{O}^{5}$ At $34^{\circ}$ the latter fuses, and the solubility decreases

salt-cake, in mineralogy thenardite. Crystalline decahydrated salt is termed in mineralogy mirabilite. On fusing it, the monohydrate $\mathrm{Na}_{2} \mathrm{SO}_{4} \mathrm{H}_{2} \mathrm{O}$ is obtained, together with a supersaturated solution.

3 The salts may be obtained not only by methods of substitution of various kinds, but also by many other combinations. Thus sodium sulphate may be formed from sodium oxide, and sulphuric anhydride by oxidising sodium sulphide, $\mathrm{Na}_{2} \mathrm{~S}$, sodium sulphite, $\mathrm{Na}_{2} \mathrm{SO}_{3}$, \&c. When sodium chloride is heated in a mixture of the vapours of water, air, and sulphuric anhydride, sodium sulphate is formed.

4 Many observations have been made, but little general information has been extracted from particular cases. In addition to which, the properties of a given salt are changed by the presence of other salts. This takes place not only in virtue of mutual decomposition or formation of double salts capable of separate existence, but is determined by the influence which some salts exert on others, or by forces similar to those which act during decomposition. Here, nothing has been generalised to that extent which would render it possible to foretell uninvestigated examples. Let us state one of these numerous cases : 100 parts of water at $20^{\circ}$ dissolve 34 parts of potassium nitrate, but on the addition of sodium nitrate the solubility of potassium nitrate increases to 48 parts in 100 of water (Carnelley and Thomson). In general, in all cases of which there are accurate observations, it appears that the presence of foreign salts changes the property of any given salt.

5 The information concerning solubility (Chapter I. p. 72) is given according to the determinations of Gay-Lussac, Lovell, and Mulder. 
at higher temperatures. ${ }^{6}$ A concentrated solution at $34^{\circ}$ has a composition nearly approaching to $\mathrm{Na}_{2} \mathrm{SO}_{4}+14 \mathrm{H}_{2} \mathrm{O}$, and the decahydrated salt, when mixed with 100 parts of water, contains 78.9 of the anhydrous salt, from whence it is seen that the decahydrated salt cannot fuse without decomposing, ${ }^{7}$ similarly to hydrate of chlorine, $\mathrm{Cl}_{2}, 8 \mathrm{H}_{2} \mathrm{O}$, or sulphurous acid, $\mathrm{SO}_{2}, 7 \mathrm{H}_{2} \mathrm{O}$. Not only the fused decahydrated salt, but also the concentrated solution at $34^{\circ}$ (not all at once, but gradually), yields the monohydrated salt $\mathrm{Na}_{2} \mathrm{SO}_{4}, \mathrm{H}_{2} \mathrm{O}$. The heptahydrated salt, $\mathrm{Na}_{2} \mathrm{SO}_{4}, 7 \mathrm{H}_{2} \mathrm{O}$, also splits up, even at low temperatures, with the formation of this monohydrated salt, and therefore from $35^{\circ}$ the solubility can be given only for the latter, and it is as follows for 100 parts of water: at $40^{\circ} 48 \cdot 8$, at $50^{\circ} 46 \cdot 7$, at $80^{\circ} 43 \cdot 7$, at $100^{\circ} 42 \cdot 5$ parts of the anhydrous salt. If the decahydrated salt be fused, and the solution be allowed to cool in the presence of the monohydrated salt, then at $30^{\circ} 50.4$ parts of anhydrous salt are retained in the solution, and at $20^{\circ} 52.8$ parts. Hence, with respect to the anhydrous and monohydrated salts, the solubility is one and the same, and falls with

6 In Chapter I. Note 24, we have already seen that with many other sulphates the solubility also decreases after a certain temperature. Gypsum, $\mathrm{CaSO}_{4}, 2 \mathrm{H}_{2} \mathrm{O}$, lime, and many other compounds present such a phenomenon, nov as yet, however, thoroughly investigated. The observation of Tilden and Shenstone (1884) is most instructive, and shows that on raising the temperature (in closed vessels) above $140^{\circ}$ the solubility of sodium sulplate again begins to increase. At $100^{\circ}, 100$ parts of water dissolve about 43 parts of anhydrous salt, at $140^{\circ} 42$ parts, at $160^{\circ} 43$ parts, at $180^{\circ} 44$ parts, at $230^{\circ}$ 46 parts. It is evident that the phenomenon of saturation, determined by the presence of an excess of the dissolved substance, is very complex, and, therefore, for the theory of solutions considered as liquid indefinite chemical compounds, many useful results can hardly be given ; more especiully as the physico-mechanical side of the transition of the solid into the liquid condition (or the reverse) is up to the present time less clearly understood in theory than the transition of a liquid into vapour.

7 As has been referred to in Chapter I. Note 5 t.

The example of sodium sulphate is historically very important for the theory of solutions. Notwithstunding the mass of investigations which have been made, it is still insufficiently studied, especially from the point of the vapour tension of solutions and crystallo-hydrates, so that those processes camnot be applied to it which Guldberg, Roozeboom, Van't Hoff, and others applied to solutions and crystallo-hydrates. It would also be most important to investigate the influence of pressure on the various phenomena corresponding with the combinations of water and sodium sulphate, because when crystals are separated-for instance, in the decahydrated salt-an increase of volume takes place, as can be seen from the following data:-the sp. gr. of the anlydrous salt is $2 \cdot 66$, that of the decallydrated sult $=1 \cdot 46$, but the sp. gr. of solutions at $15^{\circ} / 4^{\circ}=9992+90 \cdot 2 p+0 \cdot 35 p^{2}$ if $p$ represents the percentage of anhydrous salt in the solution, and if the sp. gr. of water at $4^{\circ}=10000$. Hence, for solutions containing $20^{\circ}$ of anhydrous salt, the sp. gr. $1 \cdot 1936$; therefore the volume of $100 \mathrm{grams}$ of this solution $=83.8 \mathrm{c.c}$., and the volume of unhydrous salt contained in it is equal to $202 \cdot 66$, or $=7.5$ c.c., and the volume of water $=80^{\circ} 1$ c.c. Therefore, the solution, on decomposing into anhydrous salt and water, increases in volume (from 83.8 to 87.6 ) ; but in the same way 83.8 c.c. of 20 p.c. solution are formed from $(45 \cdot 4 \cdot 1 \cdot 46=) 31 \cdot 1$ c.c. of the decahydrated salt, and $54 \cdot 6$ c.c. of water-that is to say, that during the formation of a solution from $85 \cdot 7$ c.c., $83 \cdot 8$ c.c. are formed. 
the temperature, whilst with respect to decahydrated salt, the solubility rises with the temperature. So that if in the presence of a solution of sodium sulphate there be only crystals of that heptahydrated salt (p. 59) $\mathrm{Na}_{2} \mathrm{SO}_{4}, 7 \mathrm{H}_{2} \mathrm{O}$ which is formed from saturated solutions, then saturation sets in when the solution has the following composition per 100 parts of salt: at $0^{\circ} 19 \cdot 6$, at $10^{\circ} 30 \cdot 5$, at $20^{\circ} 44 \cdot 7$, and at $25^{\circ}$ 52.9 parts of anhydrous salt. Above $27^{\circ}$ the heptahydrated salt, like the decahydrated salt at $34^{\circ}$, splits up into the monohydrated salt and a saturated solution. Thus sodium sulphate has three curves of solubility : one for $\mathrm{Na}_{2} \mathrm{SO}_{4}, 7 \mathrm{H}_{2} \mathrm{O}$ (from $0^{\circ}$ to $26^{\circ}$ ), one for $\mathrm{Na}_{2} \mathrm{SO}_{4}, 10 \mathrm{H}_{2} \mathrm{O}$ (from $0^{\circ}$ to $34^{\circ}$ ), and one for $\mathrm{Na}_{2} \mathrm{SO}_{4}, \mathrm{H}_{2} \mathrm{O}$ (a descending curve beginning at $26^{\circ}$ ), because there are three of these crystallo-hydrates, whilst the solubility can only be referred to a definite state of a substance which is present (or separated) in excess. ${ }^{8}$

Thus solutions of sodium sulphate give crystallo-hydrates of three kinds on cooling the saturated solution : the unstable equilibrium of the heptahydrated salt at temperatures below $26^{\circ}$, the decahydrated salt forms under ordinary conditions at temperatures below $34^{\circ}$, and the monohydrated salt at temperatures above $34^{\circ}$. Both the latter crystallo-hydrates present a stable state of equilibrium, and the heptahydrated salt decomposes into them, probably according to the equation $3 \mathrm{Na}_{2} \mathrm{SO}_{4}, 7 \mathrm{H}_{2} \mathrm{O}=2 \mathrm{Na}_{2} \mathrm{SO}_{4}, 10 \mathrm{H}_{2} \mathrm{O}+\mathrm{Na}_{2} \mathrm{SO}_{4}, \mathrm{H}_{2} \mathrm{O}$. The ordinary decahydrated salt is called Glauber's salt. All forms of these crystallo-hydrates entirely lose their water, and give the anhydrous salt when dried over sulphuric acid. ${ }^{9}$

Sodium sulphate, $\mathrm{Na}_{2} \mathrm{SO}_{4}$, enters into only a few reactions of combination with other salts, and chiefly with salts of the same acid, forming double sulphates. Thus, for example, if a solution of sodium

8 From this example it is evident that the phenomena of saturation are not able to contribute much towards the understanding of solutions themselves. The solution remains the same, but from the contact of a solid it becomes either saturated or supersaturated, because crystallisation is determined by the attraction to a solid, as the phenomenon of supersaturation clearly demonstrates.

9 According to the demonstrations of Pickering (1886), the molecular weight in grams (that is, 142 grams) of anhydrous sodium sulphate, on being dissolved in a large mass of water, at $0^{\circ}$ absorbs (therefore the $-\operatorname{sign}$ ) -1100 heat units, at $10^{\circ}-700$, at $15^{\circ}-275$, at $20^{\circ}$ (gives out) $+25^{\circ}$, at $25^{\circ}+300$ calories. For the decahydrated salt $\mathrm{Na}_{2} \mathrm{SO}_{4}, 10 \mathrm{H}_{2} \mathrm{O}$, $5^{\circ}-4225,10^{\circ}-4000,15^{\circ}-3570,20^{\circ}-3160,25^{\circ}-2775$. Hence (just as in Chapter I. Note 56 ) the heat of the combination $\mathrm{Na}_{2} \mathrm{SO}_{4}, 10 \mathrm{H}_{2} \mathrm{O}$ at $5^{\circ}=+3125,10^{\circ}=+3250,20^{\circ}=+3200$ and $25^{\circ}=+3050$.

It is evident that the decahydrated salt dissolving in water gives a decrease of temperature. Solutions in hydrochloric acid give a still greater decrease, because the water of crystallisation is here taken in a solid state-that is, like ice-and on melting absorbs heat. A mixture of 15 parts of $\mathrm{Na}_{2} \mathrm{SO}_{4}, 10 \mathrm{H}_{2} \mathrm{O}$ and 12 parts of strong hydrochloric acid produces sufficient cold to freeze water. During the treatment with hydrochloric acid a certain quantity of sodium chloride is formed. 
sulphate be mixed with a solution of aluminium, magnesium, or ferrous sulphate, it gives crystals of a double salt when evaporated. Sulphuric acid itself forms a compound with sodium sulphate, which is exactly like these double salts. It is formed with great ease if sodium sulphate is dissolved in sulphuric acid and the solution be evaporated. On evaporation, crystals of the acid salt separate, $\mathrm{Na}_{2} \mathrm{SO}_{4}+\mathrm{H}_{2} \mathrm{SO}_{4}$ $=2 \mathrm{NaHSO}_{4}$. This separates from hot solutions, whilst the crystallohydrate $\mathrm{NaHSO}_{4}, \mathrm{H}_{2} \mathrm{O}^{10}$ separates from cold solutions. The crystals, when exposed to damp air, split up into $\mathrm{H}_{2} \mathrm{SO}_{4}$, which deliquesces, and $\mathrm{Na}_{2} \mathrm{SO}_{4}$ (Graham, Rose) ; alcohol also extracts sulphuric acid from the acid salt. This shows the feeble force which holds the sulphuric acid to the sodium sulphate. ${ }^{11}$ Both acid sodium sulphate and all mixtures of the normal salt and sulphuric acid lose water when heated, and are converted into sodium pyrosulphate, $\mathrm{Na}_{2} \mathrm{~S}_{2} \mathrm{O}_{7}$, at a low red heat. This anhydrous salt, at a bright red heat, parts with the elements of sulphuric anhydride, the normal sodium sulphate remaining behind$\mathrm{Na}_{2} \mathrm{~S}_{2} \mathrm{O}_{7}=\mathrm{Na}_{2} \mathrm{SO}_{4}+\mathrm{SO}_{3}$. From this it is seen that the normal salt is able to combine with water, with other sulphates, and with sulphuric anhydride or acid, \&c. ${ }^{11 b}$

Sodium sulphate may, by double decomposition, be converted into a sodium salt of any other acid, by means of heat and taking advantage of the volatility, or by means of solution and taking advantage of the different degree of solubility of the different salts. Thus, for instance, owing to the insolubility of barium sulphate, sodium hydroxide or caustic soda may be prepared from sodium sulphate, if barium hydroxide be added to its solution, $\mathrm{Na}_{2} \mathrm{SO}_{4}+\mathrm{Ba}(\mathrm{HO})_{2}=\mathrm{BaSO}_{4}+2 \mathrm{NaHO}$. And by taking any salt of barium, $\mathrm{BaX}_{2}$, the corresponding salt of sodium

10 The very large and well-formed crystals of this salt resemble the hydrate $\mathrm{H}_{2} \mathrm{SO}_{4}, \mathrm{H}_{2} \mathrm{O}$, or $\mathrm{SO}(\mathrm{OH})_{4}$. In general the replacement of hydrogen by sodium inodifies many of the properties of acids less than its replacement by other metals. This most probably depends on the volumes being nearly equal.

11 In solution (Berthelot) the acid salt in all probability decomposes most in the greatest mass of water. The specific gravity (according to the determinations of Marignac) of solutions at $15^{\circ} / 4^{\circ}=9992+77 \cdot 92 p+0 \cdot 231 p^{2}$ (see Note 7 ). From these figures, and from the specific gravities of sulphuric acid, it is evident that on mixing solutions of this acid and sodium sulphate expansion will always take place; for instance, $\mathrm{H}_{2} \mathrm{SO}_{4}+25 \mathrm{H}_{2} \mathrm{O}$ with $\mathrm{Na}_{2} \mathrm{SO}_{4}+25 \mathrm{H}_{2} \mathrm{O}$ increases from 483 volumes to 486 . In addition to which, in weak solutions heat is absorbed, as shown in Chapter X. Note 27. Nevertheless, even more acid salts may be formed. For instance, on cooling a solution of 1 part of sodium sulphate in 7 parts of sulphuric acid, crystals of the composition $\mathrm{NaHSO}_{4}, \mathrm{H}_{2} \mathrm{SO}_{4}$ are separated (Schultz, 1868). This fuses at about $100^{\circ}$; the ordinary acid salt, $\mathrm{NaHSO}_{4}$, at $149^{\circ}$.

$11 b$ In order to demonstrate the weakness of the bond acting in sodium hydrogen sulphate, $\mathrm{NaHSO}_{4}$, it is useful to remember that on decreasing the pressure this salt dissociates much more easily than at the ordinary pressure; it loses water and forms the pyrosulphate, $\mathrm{Na}_{2} \mathrm{~S}_{2} \mathrm{O}_{7}$; this reaction is utilised in chemical works. 
may be obtained, $\mathrm{Na}_{2} \mathrm{SO}_{4}+\mathrm{BaX}_{2}=\mathrm{BaSO}_{4}+2 \mathrm{NaX}$. Barium sulphate thus formed, being a very sparingly-soluble salt, is obtained as a precipitate, whilst the sodium hydroxide, or salt, $\mathrm{NaX}$, is obtained in solution, because all salts of sodium are soluble. Berthollet's doctrine permits all such cases to be foreseen.

The reactions of decomposition of sodium sulphate are above all noticeable by the separation of oxygen. Sodium sulphate itself is very stable, and it is only at a temperature sufficient to melt iron that it is possible to separate the elements $\mathrm{SO}_{3}$ from it, and then only partially. However, the oxygen may be separated from sodium sulphate, as from all other sulphates, by means of many substances which are able to combine with oxygen, such as charcoal and sulphur, but hydrogen is not able to produce this action. If sodium sulphate be heated with charcoal, then carbonic oxide and anhydride are evolved, and there is produced, according to the circumstances, either the lower oxygen compound, sodium sulphite, $\mathrm{Na}_{2} \mathrm{SO}_{3}$ (for instance, in the formation of glass); or else the decomposition proceeds further and sodium sulphide, $\mathrm{Na}_{2} \mathrm{~S}$, is formed, according to the equation $\mathrm{Na}_{2} \mathrm{SO}_{4}+2 \mathrm{C}$ $=2 \mathrm{CO}_{2}+\mathrm{Na}_{2} \mathrm{~S}$.

On the basis of this reaction the greater part of the sulphate of sodium prepared at chemical works is converted into soda ash - that is, sodium carbonate, $\mathrm{Na}_{2} \mathrm{CO}_{3}$, which is used for many purposes. In the form of carbonates, the metallic oxides behave in many cases just as they do in the state of oxides or hydroxides, owing to the feeble acid properties of carbonic acid. However, the majority of the salts of carbonic acid are insoluble, whilst sodium carbonate is one of the few soluble salts of this acid, and therefore reacts with facility. Therefore sodium carbonate is employed for many purposes, in which it acts owing to its alkaline properties. Thus sodium carbonate, even under the action of feeble organic acids, immediately parts with its carbonic acid, and gives a sodium salt of the acid taken. Furthermore, its solutions alrearly exhibit an alkaline reaction on litmus, and in many cases are able to act as an alkali. Thus, for instance, sodium carbonate, like the alkalis, aids the passage of certain organic substances (tar, acids) into solution, and is therefore used, like alkalis and soap (which also acts by virtue of the alkali it contains), for the removal of certain organic substances, especially in bleaching tissues in cotton and similar works. Besides which, a considerable quantity of sodium carbonate is used for the preparation of sodium hydroxide or caustic soda, which has also a very wide application. In large chemical works where sodium carbonate is manufactured it is usual to first manufacture sulphuric acid, and then by its aid to convert common salt into sodium sulphate, 
and lastly to convert the sodium sulphate thus obtained into carbonate and caustic soda. Hence these works prepare alkaline substances (soda ash and caustic soda) and acid substances (sulphuric and hydrochloric acids), those two forms of chemical products which are distinguished for the greatest energy of their reactions, and which are therefore frequently applied to technical purposes. And therefore the works manufacturing soda are generally called chemical works (alkali works).

The process of the conversion of sodium sulphate into sodium carbonate consists in strongly heating a mixture of the sulphate with charcoal and calcium carbonate. The following reactions then take place : the sodium sulphate is first deoxidised by the charcoal, forming sodium sulphide and carbonic anhydride, $\mathrm{Na}_{2} \mathrm{SO}_{4}+2 \mathrm{C}=\mathrm{Na}_{2} \mathrm{~S}+2 \mathrm{CO}_{2}$. 'The sodium sulphide thus formed then enters into double decomposition with the calcium carbonate taken and gives calcium sulphide and sodium carbonate, $\mathrm{Na}_{2} \mathrm{~S}+\mathrm{CaCO}_{3}=\mathrm{Na}_{2} \mathrm{CO}_{3}+\mathrm{CaS}$.

Besides which, under the action of the heat, a portion of the excess of calcium carbonate is decomposed into lime and carbonic anhydride, $\mathrm{CaCO}_{3}=\mathrm{CaO}+\mathrm{CO}_{2}$, and the carbonic anhydride with the excess of charcoal forms carbon monoxide, which towards the end of the operation shows itself by the appearance of a blue flame. Thus from a mass containing sodium sulphate we obtain a mass which includes sodium carbonate, calcium sulphide, and calcium oxide, but none of the sodium sulphide first formed, or, strictly speaking, only a small portion. The entire process, which proceeds at a high temperature, may be expressed by a combination of the three above-mentioned formulæ, if it be taken into consideration that the product contains one equivalent of calcium oxide to two equivalents of calcium sulphide. ${ }^{12}$ The sum of the reactions may then be expressed thus: $2 \mathrm{Na}_{2} \mathrm{SO}_{4}+3 \mathrm{CaCO}_{3}+9 \mathrm{C}$ $=2 \mathrm{Na}_{2} \mathrm{CO}_{3}+\mathrm{CaO}, 2 \mathrm{CaS}+10 \mathrm{CO}$. Indeed, the quantities in which the substances are mixed together at chemical works approaches to the proportion required by this equation. The entire process of decomposition is carried on in reverberatory furnaces, into which a mixture of 1000 parts of sodium sulphate, 1040 parts of calcium carbonate (as a

12 Calcium sulphide, CaS, like many metallic sulphides which are soluble in water, is decomposed by water (page 426), $\mathrm{CaS}+\mathrm{H}_{2} \mathrm{O}=\mathrm{CaO}+\mathrm{H}_{2} \mathrm{~S}$, because hydrogen sulphide is a very feeble acid. If calcium sulphide be acted on by a large mass of water, lime may be precipitated, a state of equilibrium will be entered on, when the system $\mathrm{CaO}+2 \mathrm{CuS}$ remains unchanged. Lime, being the product of the action of water on CaS, limits this action. Therefore, if in black ash the lime was not in excess, a part of the sulphide compounds would be in solution (actually there is but very little). In this manner in the manufacture of sodium carbonate the conditions of equilibrium which enter into double decompositions have been made use of (see above), and the aim is to form directly the unchangeable product $\mathrm{CaO}, 2 \mathrm{CaS}$. This was first regarded as a special insoluble compound, but nothing points to its independent existence. 
somewhat porous limestone), and 500 parts of small coal is charged from above. This mixture is first heated in the portion of the furnace which is furthest removed from the fire-grate ; it is then brought to the

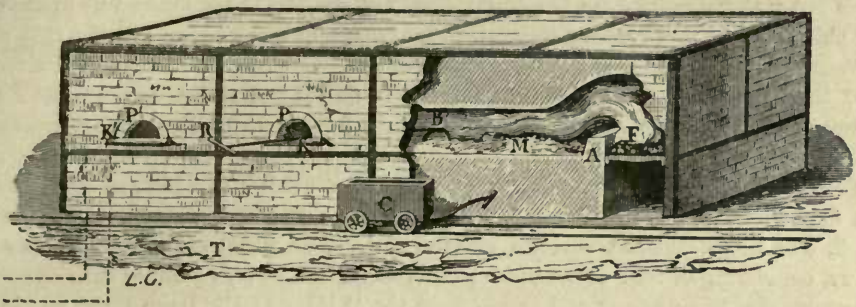

Fig. 69.-Reverberatory furnace for the manufacture of sodium carbonate. F, grate. A, bridge. $\mathrm{M}$, hearth for the ultimate calcination of the mixture of sodium sulphate, coal, and calcium carbonate, which is charged from above into the part of the furnace furthest removed from the fire $\mathrm{F}$. $\mathbf{P}, \mathbf{P}$, doors for stirring and bringing the mass towards the grate $\mathbf{F}$ by means of stirrers $\mathbf{R}$. At the end of the operation the semifused mass is charged into trucks C.

portion nearest to the fire-grate, when it is stirred during heating. The partially-fused mass obtained at the end of the process is cooled, and then subjected to methodical lixiviation ${ }^{13}$ to extract the sodium

15 Methodical lixiviation is the extraction, by means of water, of a soluble substance from the mass containing it. It is carried on so as not to obtain weak aqueous solutions, and in such a way that the residue shall not contain any of the soluble sabstance. This problem is practically of great importance in many industries. It is required to extract from the mass all that is soluble in water. This is easily effected if water be first poured

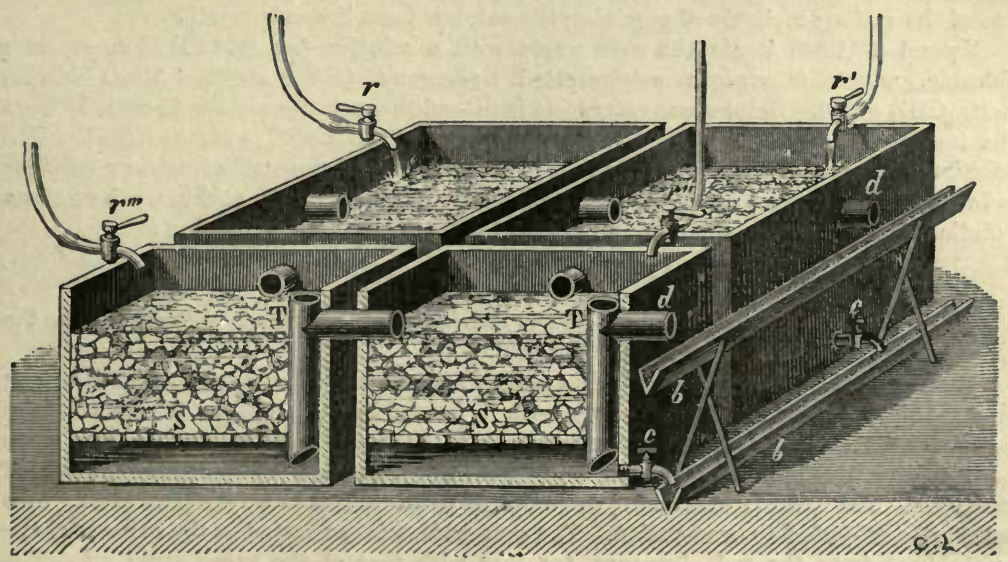

Fig. 70.-Apparatus for the metholical lixiviation of black ash, \&c. Water flows into the tanks from the pipes $r, r$, and the saturated liquid is drawn off from $c, c$.

on the mass; the strong solution thus obtained is then decanted, then water is again poured on, time being allowed for it to act; then again decanted, and so on until the water does not take up anything. But then finally such weak solutions are obtained that it would be very disadvantageous to evaporate them. This is avoided by pouring

VOL. I. 
carbonate, the mixture of calcium oxide and sulphide forming the socalled 'soda waste." '14

the fresh hot water destined for the lixiviation, not on the fresh mass, but upon a mass already subjected to preparatory lixiviation by means of weak solutions. In this way the fresh water gives a weak solution. The solution which is obtained flows to those parts of the apparatus which contain the fresh, as yet unlixiviated, mass, and thus in the latter parts the weak alkali formed in the other parts of the apparatus becomes saturated as far as possible with the soluble substance. Generally several intercommunicating vessels are constructed (standing at the same level) into which in turn the fresh mass is deposited which is intended for lixiviation; the water is poured in, the alkali drawn off, and the lixiviated residue cleared out. The illustration represents such an apparatus, consisting of four communicating vessels. The water poured into one of them flows through the two nearest and issues from the third. If the fresh mass be placed in one of these cases or vessels, the stream of water passing through the apparatus is directed in such a manner as to finally issue from this vessel containing the fresh unlixiviated mass. The fresh water is added to the vessel containing the matter which is almost completely exhausted. The fresh water passing through this vessel is conveyed by the pipe (syphon passing from below the first case to the top of the second) cominunicating with the second; it finally passes (also through a syphon pipe) into the case (the third) containing the fresh stuff. The water will extract all that is soluble in the first vessel, leaving only an insoluble mass. This vessel is then destined to be emptied, and refilled with fresh matter. The level of the liquids in the various vessels will naturally be different, in consequence of the various strengths of the solutions which they contain.

14 The whole of the sulphur used in the production of the sulphuric acid employed in decomposing the common salt enters into this residue. This residue carrying off the sulphur is the great burden and expense of the soda works which use Leblanc's method. As an instructive example from a chemical point of view it is worth while describing here one of the various methods of regaining the sulphur from the soda waste.

Kynaston (1885) treats the soda waste with a solution (sp. gr. 1.21) of magnesium chloride, which disengages sulphuretted hydrogen: $\mathrm{CaS}+\mathrm{MgCl}_{2}+2 \mathrm{H}_{2} \mathrm{O}=\mathrm{CaCl}_{2}$ $+\mathrm{Mg}(\mathrm{OH})_{2}+\mathrm{H}_{2} \mathrm{~S}$. Sulphurous anhydride is passed through the residue in order to form the insoluble calcium sulphite: $\mathrm{CaCl}_{2}+\mathrm{Mg}(\mathrm{OH})_{2}+\mathrm{SO}_{2}=\mathrm{CaSO}_{3}+\mathrm{MgCl}_{2}+\mathrm{H}_{2} \mathrm{O}$. The solution of magnesium chloride obtained is again used, and the washed calcium sulphite is brought into contact at a low temperature with hydrochloric acid (a weak aqueous solution) and hydrogen sulphide, the whole of the sulphur then separating:

$$
\mathrm{CaSO}_{3}+2 \mathrm{H}_{2} \mathrm{~S}+2 \mathrm{HCl}=\mathrm{CaCl}_{2}+3 \mathrm{H}_{2} \mathrm{O}+3 \mathrm{~S} \text {. }
$$

It is necessary to turn once more to the lixiviation of the sodium carbonate from the mass formed in the furnace (see also Note 25).

It must not, however, be thought that sodium carbonate alone passes into the solution ; there is also a good deal of caustic soda with it, formed by the action on the carbonate of sodium of the lime remaining from the first process of the action of the charcoal, and there are also certain sodium sulphur compounds with which we shall partly become acquainted hereafter. The sodium carbonate, therefore, is not obtained in a very pure state. The solution is subjected to evaporation. The evaporation is conducted at the expense of the waste heat from the soda furnaces, together with that of the gases given off. The process in the soda furnaces is only carried on at a high temperature, and therefore the smoke and gases issuing from the furnaces are inevitably very hot. If the heat they contain was not made use of there would be a great waste of fuel; consequently in immediate proximity to the soda furnaces there is generally a series of pans or evaporating boilers, under which the gases from the furnace pass, and into these the alkali solution obtained is poured: On evaporating the solution first of all the undecomposed sodium sulphate separates, then the sodium carbonate or soda crystals. These crystals 
The above-mentioned process for making soda was discovered in the year 1808 by the French doctor Leblanc, and is known as the Leblanc process. The particulars of this discovery are somewhat remarkable. Sodium carbonate, having a considerable application in industry, was for a long time prepared exclusively from the ash of marine plants (Chapter XI. page 490). Even up to the present time this process is carried on in Normandy. In France, where for a long time the manufacture of large quantities of soap (so-called Marseilles soap) and various fabrics required a large amount of soda, the quantity prepared at the coast was insufficient to meet the demand. For this reason during the wars at the beginning of the century, when the import of foreign goods into France was interdicted, the want of sodium carbonate was felt. The French Academy offered a prize for the discovery of a profitable method of preparing it from common salt. Leblanc then proposed the above-mentioned process, which is remarkable for its great simplicity. ${ }^{15}$

which separate are raked out and placed on planks, where the liquid flows off. Caustic soda remains in the residue, and also any sodium chloride which was not decomposed in the foregoing process.

Part of the sodium carbonate is crystallised in order to purify it more thoroughly. In order to do this a saturated solution is left to crystallise at a temperature below $30^{\circ}$ in a current of air, in order to promote the separation of the vapour of water. Then the large transparent crystals (efflorescent in air) of $\mathrm{Na}_{2} \mathrm{CO}_{3}, 10 \mathrm{H}_{2} \mathrm{O}$ are formed which have been spoken of already (Chapter I.).

15 Among the drawbacks of the Leblanc process are the accumulation of 'soda waste,' and the impossibility at the comparatively low price of sulphur (especially in the form of pyrites) of finding a suitable employment for it (although this waste can furnish sulphur and sulphur compounds, for which purposes it is sometimes treated), and also the insufficient purity of the sodium carbonate for many purposes. The advantage of the Leblanc process, besides its simplicity and cheapness, are that almost all acids having a commercial value are obtained as bye-products; chlorine and bleaching powder are produced with the assistance of the large amount of hydrochloric acid which appears as a bye-product, and caustic soda is very easily made, and the demand for it increases every year. In those places where salt, pyrites, charcoal, and limestone (which are the materials required for soda works) are found side by side-as, for instance, in the Ural or Don districts-all conditions are favourable to the development of the manufacture of sodium carbonate on an enormous scale; and where, as in the Caucasus, sodium sulphate occurs naturally, the conditions are still more favourable. A large amount, however, of the latter salt, even from soda works, is used in making glass. The most important soda works, as regards the quantity of products obtained from them, are the English works.

As an example of the other numerous and various methods of manufacturing soda from sodium chloride, the following processes may be mentioned: Sodium chloride is decomposed by oxide of lead, $\mathrm{PbO}$, forming lead chloride and sodium oxide, which, with carbonic anhydride, yields sodium carbonate (Scheele's process). In Cornu's method sodium chloride is treated with lime, and then exposed to the air, when it yields a small quantity of sodium carbonate. In E. Kopp's process sodium sulphate (125 parts) is mixed with oxide of iron ( 80 parts) and charcoal ( 55 parts), and the mixture is heated in reverberatory furnaces. Here a compound, $\mathrm{Na}_{6} \mathrm{Fe}_{4} \mathrm{~S}_{3}$, is formed, which is insoluble in water and absorbs oxygen and carbonic anhydride, and then forms sodium carbonate and ferrous sulphide; this when roasted can give sulphurous anhydride, which is indispensable for the manufacture of sulphuric acid, and ferric oxide, which is again used in the 


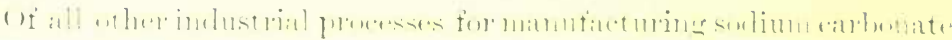

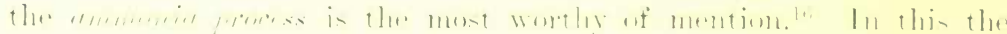

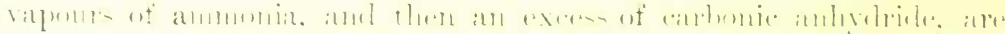

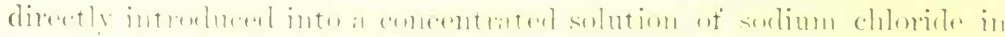

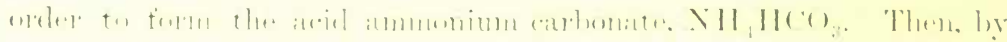

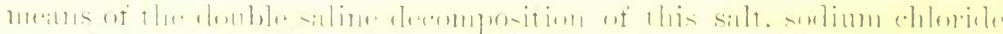

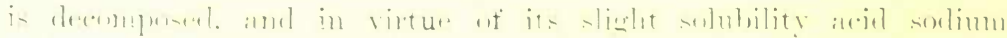

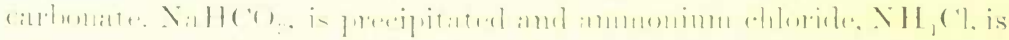

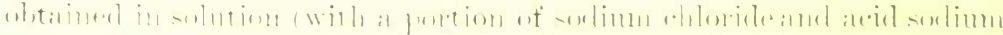

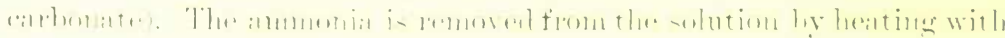

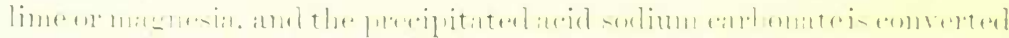

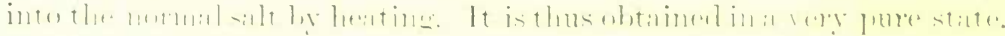

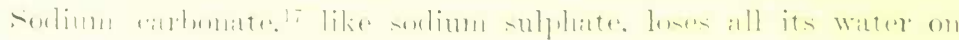

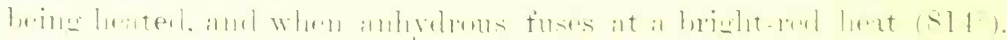

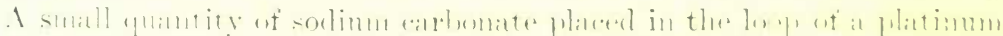

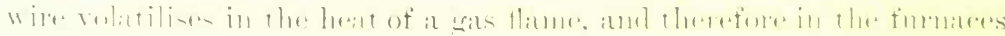

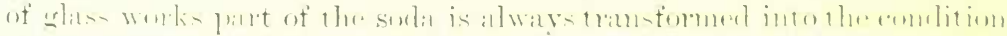

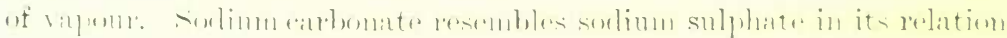

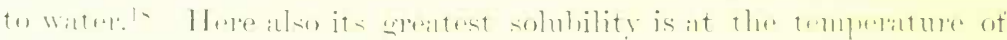

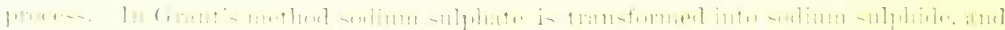

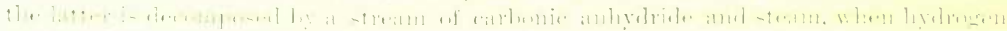

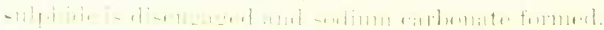

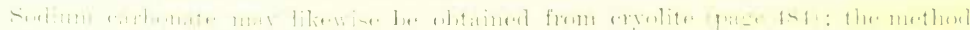

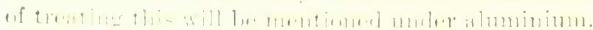

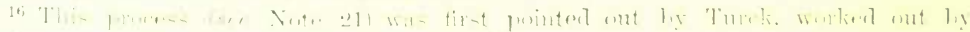

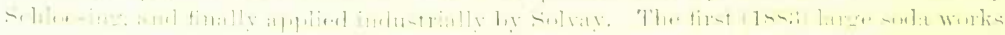

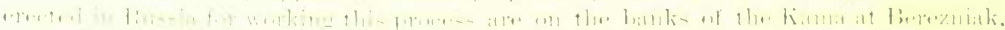

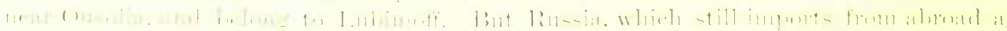

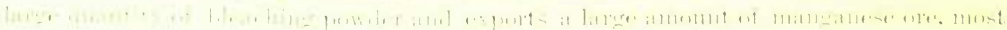

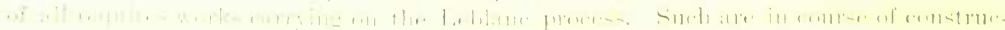

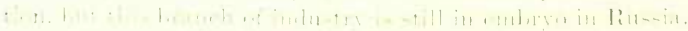

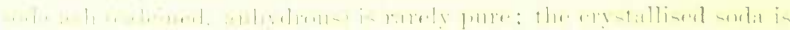

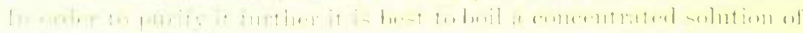

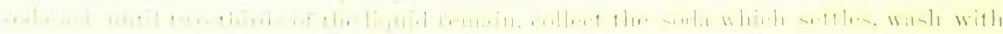

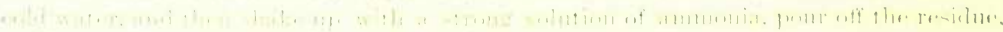

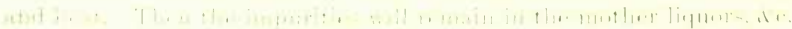

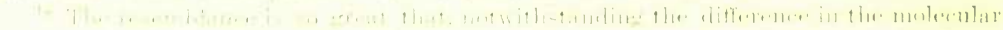

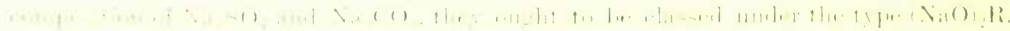

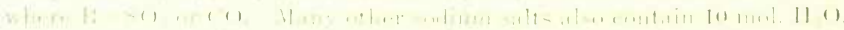

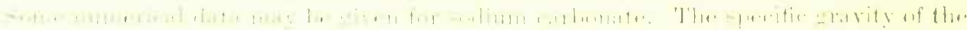

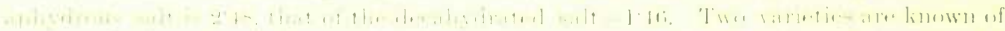

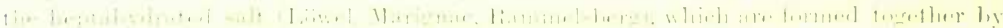

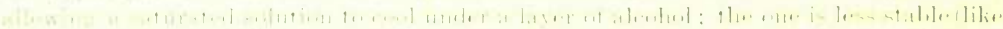

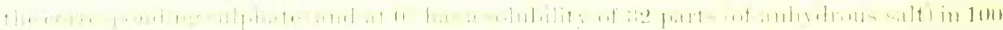

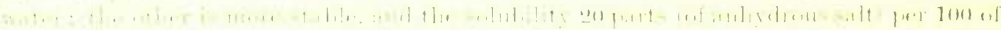

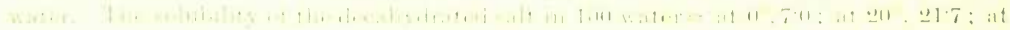

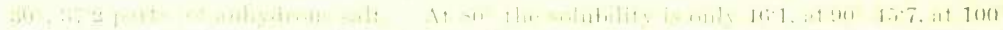

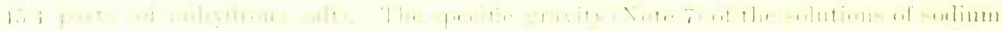

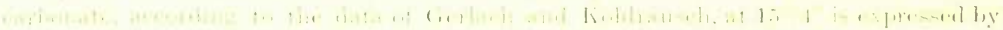


$37^{\circ}$; both salts, on crystallising at the ordinary temperature, combine with ten molecules of water, and such crystals of soda, like crystals of Glauber's salt, fuse at $34^{\circ}$. Sodium carbonate also forms a supersaturated solution, and, according to the conditions, gives various combinations with water of crystallisation (mentioned on page 107), \&c.

At a red heat superheated steam liberates carbonic anhydride from sodium carbonate and forms caustic soda, $\mathrm{Na}_{2} \mathrm{CO}_{3}+\mathrm{H}_{2} \mathrm{O}=2 \mathrm{NaHO}+$ $\mathrm{CO}_{2}$. Here the carbonic anhydride is replaced by water; this depends on the feebly acid character of carbonic anhydride. By direct heating, sodium carbonate is only slightly decomposed into sodium oxide and carbonic anhydride; thus, when sodium carbonate is fused, about 1 per cent. of carbonic anhydride is disengaged. ${ }^{19}$ The carbonates of many other metals - for instance, of calcium, copper, magnesium, iron, \&c.-on being heated lose-all their carbonic anhydride. This shows the considerable basic energy which sodium possesses. With the soluble salts of most metals, sodium carbonate gives precipitates either of insoluble carbonates of the metals, or else of the hydroxides (in this latter case carbonic anhydride is disengaged); for instance, with barium salts it precipitates an insoluble barium carbonate $\left(\mathrm{BaCl}_{2}+\mathrm{Na}_{2} \mathrm{CO}_{3}=2 \mathrm{NaCl}+\mathrm{BaCO}_{3}\right)$ and with the aluminium salts it precipitates aluminium hydroxide, carbonic anhydride being disengaged : $3 \mathrm{Na}_{2} \mathrm{CO}_{3}+\mathrm{Al}_{2}\left(\mathrm{SO}_{4}\right)_{3}+3 \mathrm{H}_{2} \mathrm{O}=3 \mathrm{Na}_{2} \mathrm{SO}_{4}+2 \mathrm{Al}(\mathrm{OH})_{3}+$ $3 \mathrm{CO}_{2}$. Sodium carbonate, like all the salts of carbonic acid, evolves carbonic anhydride on treatment with all acids which are to any extent energetic. But if an acid diluted with water be gradually added to a solution of sodium carbonate, at first such an evolution does not take place, because the excess of the carbonic anhydride forms acid sodium carbonate (sodium bicarbonate), $\mathrm{NaHCO}_{3}$. The composition of this salt, however, may be also represented as a combination of carbonic acid, $\mathrm{H}_{2} \mathrm{CO}_{3}$, with the normal salt, $\mathrm{Na}_{2} \mathrm{CO}_{3}$, just as the latter also combines with water. Such a representation is all the more

the formula, $s=9092+104 \cdot 5 p+0 \cdot 165 p^{2}$. Weak solutions occupy a volume not only less than the sum of the volumes of the anhydrous salt and the water, but even less than the water contained in them. For instance, 1000 grams of 1 p.c. solution occupy $\left(15^{c}\right)$ a volume of 990.4 c.c. (sp. gr. 1.0097), but contain 900 grams of water, occupying at $15^{\circ}$ a volume of 990.8 c.c. A similar case of solubility, which is comparatively rare (it occurs also with sodium hydroxide), occurs in those dilute solutions for which the factor $A$ is greater than 100 if the sp. gr. of water at $4^{\circ}=100000$, and if the sp. gr. of the solution be expressed by the formula $S=S_{o}+A p+B p^{2}$, where $S_{o}$ is the specific gravity of the water (this is more fully discussed in my treatise, The Investigation of Aqueous Solutions, $1887, \S \S 94$ and 95 ). For 5 p.c. the sp. gr. $15^{\circ} / 4^{\mathrm{c}}=1 \cdot 0520$; for 10 p.c. $1 \cdot 1057$; for 15 p.c. 1.1603. The changes in the $\mathrm{sp}$. gr. with the temperature are here almost the same as with solutions of sodinm chloride with an equal value of $p$.

19 According to the observations of Pickering. According to Rose, when solutions of sodium carbonate are boiled a certain amount of carbonic anhydride is disengaged. 
likely because (1) there exists another salt, $\mathrm{Na}_{2} \mathrm{CO}_{3}, 2 \mathrm{NaHCO}_{3}, 2 \mathrm{H}_{2} \mathrm{O}$ (sodium sesquicarbonate), obtained by cooling a boiling solution of sodium bicarbonate, or by mixing this salt with the normal salt; but the formula of this salt cannot be derived from that of normal carbonic acid, as the formula of the bicarbonate can; ${ }^{20}(2)$ water of crystallisation does not enter into the composition of the crystals of the acid salt, so that on its formation (occurring only at low temperatures, as in the formation of crystalline compounds with water) the water of crystallisation of the normal salt separates and the water is, as it were, replaced by the elements of carbonic acid. In any casethe acid sodium carbonate is an unstable salt. Not only when heated alone, but even on being slightly heated in solution, and also at the ordinary temperature in damp air, it loses carbonic anhydride and forms. the normal salt. And at the same time it is easy to obtain it in a pure crystalline form, if a strong solution of sodium carbonate be cooled and a stream of carbonic anhydride gas passed through it. The acid salt is less soluble in water than the normal, ${ }^{21}$ and therefore a strong solution of the latter gives crystals of the acid salt if carbonicanhydride be passed through it. The acid salt may be yet more conveniently formed from effloresced crystals of sodium carbonate,

20 At the same time the sesqui-salt has all the properties of a definite compound; it crystallises in transparent crystals, has a constant composition, its solubility (at $0^{\circ}$ in 100 of water, $12 \cdot 6$ of anhydrous salt) differs from the solubility of the normal and acid salts, and shows changes in composition; it is found in nature, and is known by the names of trona and urao. The observations of Watts and Richards showed (1886) that on pouring a strong solution of the acid salt into a solution of the normal salt saturated by heating, crystals of the salt $\mathrm{NaHCO}_{5}, \mathrm{Na}_{2} \mathrm{CO}_{3}, 2 \mathrm{H}_{2} \mathrm{O}$ may be easily obtained, as long as the temperature is above $35^{\circ}$. The natural urao (Boussingault) has, according to Laurent, the same composition. This salt is very stable in air, and may be used for purifying sodium carbonate on the large scale. From the theoretical side such compounds have been little studied, yet are particularly interesting because, in all probability, they correspond with orthocarbonic acid $\mathrm{C}(\mathrm{OH})_{4}$, and at the same time correspond with double salts like astrachanite (Chapter XIV.).

${ }_{21} 100$ parts of water at $0^{\circ}$ dissolves 7 parts of the acid salt, which corresponds with 4.3 parts of the anhydrous normal salt, but at $0^{\circ} 100$ parts of water dissolves 7 parts of the latter. The solubility of the acid salt varies witl considerable regularity; 100 parts of water dissolves at $15^{\circ} 9$ parts of the salt, at $30^{\circ} 11$ parts.

The ammonium, and more especially the calcium, salt, is much more soluble in water. The ammonia process (see ante, p. 516) is founded upon this. Ammonium bicarbonate (acid carbonate) at $0^{\circ}$ has a solubility of 12 parts in 100 water, at $30^{\circ}$ of 27 parts. The solubility, therefore, increases very rapidly with the temperature. But its saturated solution is more stable than a solution of sodium bicarlonate. In fact, saturated solutions of these salts have the vapour tension of a mixture of carbonic anhydride and water-namely at $15^{\circ}$ and at $50^{\circ}$, for the sodium salt 120 and 750 millimetres, for the ammonium salt 120 and 563 millimetres. These data are of great importance in understanding the phenomena connected with the ammonia process. They demonstrate that with an increased pressure the formation of the sodium salt ought to increase if there be an excess of ammonium salt. 
which, on being considerably heated, very easily absorb carbonic anhydride. ${ }^{22}$ The acid salt crystallises well, but not, however, in such large crystals as the normal salt ; it has a brackish and not an alkaline taste like that of the normal salt, its reaction is feebly alkaline, nearly neutral. At $70^{\circ}$ its solution begins to lose carbonic anhydride, and on boiling the evolution becomes very abundant. From the preceding remarks it is clear that in most reactions this salt, especially when heated, acts similarly to the normal salt, but has, naturally, some distinctions from it. Thus, for example, if a solution of sodium carbonate be added to a normal magnesium salt a turbidity (precipitate) is formed of magnesium carbonate, $\mathrm{MgCO}_{3}$. No such precipitate is formed by the acid salt, because magnesium carbonate is soluble in the presence of an excess of carbonic anhydride.

Sodium carbonate is used for the preparation of caustic soda ${ }^{23}$ that is, the hydrate of sodium oxide, or the alkali which corresponds with sodium. For this purpose the action of lime on a solution of sodium carbonate is generally made use of. The process is as follows : a weak, generally 10 per cent., solution of sodium carbonate is taken, ${ }^{24}$ and boiled in a cast-iron, wrought-iron, or silver boiler (sodium hydroxide does not act on these metals), and lime is added, little by little, during the boiling. This latter is soluble in water, although but very slightly. The clear solution becomes turbid on the addition of the lime because a precipitate is formed; this precipitate consists of calcium carbonate, almost insoluble in water, whilst caustic soda is formed and remains in solution. The decomposition is effected according to the equation: $\mathrm{Na}_{2} \mathrm{CO}_{3}+\mathrm{Ca}(\mathrm{HO})_{2}=\mathrm{CaCO}_{3}+2 \mathrm{NaHO}$. On cooling the solution the calcium carbonate easily settles as a precipitate,

22 Crystalline sodium carbonate (broken into lumps) also absorbs carbonic anhydride, but the water contained in the crystals is then disengaged: $\mathrm{Na}_{2} \mathrm{CO}_{3}, 10 \mathrm{H}_{2} \mathrm{O}+\mathrm{CO}_{2}=$ $\mathrm{Na}_{2} \mathrm{CO}_{3}, \mathrm{H}_{2} \mathrm{CO}_{3}+9 \mathrm{H}_{2} \mathrm{O}$, and dissolves part of the carbonate; therefore part of the sodium carbonate passes into solution together with all the impurities. When it is required to avoid the formation of this solution, a mixture of ignited and crystalline sodium carbonate is taken. Sodium bicarbonate is prepared chiefly for medicinal use, and is then often termed carbonate of soda, also, for instance in the so-called soda powders, for preparing certain artificial mineral waters, for the rapid generation of considerable quantities of carbonic anhydride in the domestic preparation of water charged with carbonic acid, for the manufacture of digestive lozenges like those made at Essentuki, Vichy (pastilles digestives de Vichy), \&c.

${ }_{23}$ In chemistry sodium oxide is termed 'soda,' which word must be carefully distinguished from the word sodium, meaning the metal.

24 With a small quantity of water, the reaction either does not take place, or even proceeds in the reverse way - that is, sodium and potassium hydroxides remove carbonic anhydride from calcium carbonate (Liebig, Watson, Mitscherlich, and others). The influence of the mass of water is evident. According to Gerberts, strong solutions of sodium carbonate are, however, decomposed by lime, which is very interesting if confirmed by further investigation. 
and the clear solution or alkali above it contains the easily-soluble sodium hydroxide formed in the reaction. ${ }^{2.5}$ After the necessary quantity of lime has been added, the solution is allowed to stand, and is then decanted off and evaporated in cast or wrought iron boilers, or in silver pans if a perfectly-pure product is required. ${ }^{26}$ The evaporation cannot be conducted in china, glass, or similar vessels, because caustic soda eats into glass and china, although but slightly. The solution does not crystallise on evaporation, because the solubility of caustic soda when heated is very great, but crystals containing water of crystallisation may be obtained by cooling. If the evaporation of the alkali be conducted until the specific gravity reaches $1 \cdot 38$, and the liquid is then cooled to $0^{\circ}$, transparent crystals appear contain-

25 As long as part of the undecomposed sodium carbonate remains in solution, excess of acid added to the solution disengages carbonic anhydride, and the solution after dilution gives a white precipitate with a barium salt soluble in acids, showing the presence of a carbonate in solution (if there be sulphate present, it also forms a white precipitate, but this is insoluble in acids). For the decomposition of sodium carbonate, milk of lime -that is, slaked lime suspended in water-is employed. Formerly pure sodium hydroxide was prepared (according to Berthollet) by dissolving the impure substance in alcohol (sodium carbonate and sulphate are not soluble), but now that metallic sodium has become cheap and is purified by distillation, pure caustic soda is prepared by acting on a small quantity of water with sodium. By allowing strong solutions to crystallise (in the cold) completely pure sodium hydroxide may also be obtained (Note 27).

In alkali works where the Leblanc process is used, caustic soda is prepared directly from the alkali remaining in the mother liquors after the separation of the sodium carbonate evaporation (Note 14). If excess of lime and charcoal has been used, much sodium hydroxide may be obtained. After the removal as far as possible of the sodium carbonate, a red liquid (from iron oxide) is left, containing sodium hydroxide mixed with compounds of sulphur and of cyanogen (formed in the Leblanc furnaces, see p. 225, and Chapter IX.) and also containing iron. This red alkali is evaporated and air is blown through it, which oxidises the impurities (for this purpose sometimes sodium nitrate is added, or bleaching powder, \&c.) and leaves fused caustic soda. The fused mass is allowed to settle in order to separate the ferruginous precipitate, and poured into iron barrels, where the sodium hydroxide solidifies. Such caustic soda contains about 10 p.c. of water in excess and some saline impurities, but when properly manufactured is almost free from carbonate and from iron.

${ }^{26}$ Löwig gave a method of preparing sodium hydroxide from sodium carbonate by heating it to a dull red heat with an excess of ferric oxide. Carbonic anhydride is given off, and warm water extracts the caustic soda from the remaining mass. This reaction, as experiment shows, proceeds very easily, and is an example of contact action similar to the influence of ferric oxide on the decomposition of potassium chlorate. The reason of this may be that a small quantity of the sodium carbonate enters into double decomposition with the ferric oxide, and the ferric carbonate produced is decomposedinto carbonic anhydride and ferric oxide, the action of which is renewed. Similar explanations expressing the motive of a reaction really add but little to that elementary conception of contact which, according to my opinion, consists in the change of motion of the atoms in the molecules under the influence of the substance in contact. In order to represent this clearly it is sufficient, for instance, to imagine that in the sodium carbonate the elements $\mathrm{CO}_{2}$ move in a circle round the elements $\mathrm{Na}_{2} \mathrm{O}$, but at the points of contact with $\mathrm{Fe}_{2} \mathrm{O}_{3}$ the motion becomes elliptic with a long axis, and at some distance from $\mathrm{Na}_{2} \mathrm{O}$ the elements of $\mathrm{CO}_{2}$ are parted, not having the faculty of attaching themselves to $\mathrm{Fe}_{2} \mathrm{O}_{5}$. 
ing $2 \mathrm{NaHO}, 7 \mathrm{H}_{2} \mathrm{O}$, they fuse at $+6{ }^{\circ} \cdot{ }^{27}$ If the evaporation be conducted as long as water is disengaged, which requires a considerable amount of heat, then, on cooling, the hydroxide, $\mathrm{NaHO}$, solidifies in semi-transparent crystals. ${ }^{28}$

Caustic soda, free from water, forms a colcurless crystalline mass, which eagerly absorbs moisture and carbonic anhydride from the air. ${ }^{29}$ Its specific gravity is $2 \cdot 13 ;^{30}$ it is easily soluble in water, with disengagement of a considerable quantity of heat. ${ }^{31}$ A saturated solution at the ordinary temperature has a specific gravity of about $1 \cdot 5$, contains about 45 per cent. of sodium hydroxide, and boils at $130^{\circ}$; at $55^{\circ}$ water dissolves an equal weight of it. ${ }^{32}$ Caustic soda is not only soluble in water but in alcohol, and even in ether. Solutions of sodium hydroxide produce the sensation of soaping on the skin, because the active base of soap consists of caustic soda. ${ }^{33}$

27 By allowing strong solutions of sodium hydroxide to crystallise in the cold, impurities-such as, for instance, sodium sulphate-may be separated from them. The fused crystallo-hydrate $2 \mathrm{NaHO}, 7 \mathrm{H}_{2} \mathrm{O}$ forms a solution having a specific gravity of 1.405 (Hermes). Let us remark that, according to certain determinations, less water-namely, only $\mathrm{NaHO}, 3 \mathrm{H}_{2} \mathrm{O}$-enters into the composition of the crystallo-hydrate. The crystals on dissolving in water produce cold.

28 In solid caustic soda there is generally an excess of water beyond that required by the formula NaHO. The caustic soda used in laboratories is generally cast in sticks, which are broken into pieces. It must be preserved in carefully closed vessels, because it absorbs water and carbonic anhydride from the air.

29 By the way it changes in air it is easy to distinguish caustic soda from caustic potash, which in general resembles it. Both alkalis absorbs water and carbonic adhydride from the air, but caustic potash forms a deliquescent mass of potassium carbonate, whilst caustic soda forms a dry powder of efflorescent salt.

30 As the molecular weight of $\mathrm{NaHO}=40$, the volume of its molecule $=40 / 2 \cdot 13=18 \cdot 5$, which very nearly approaches the volume of a molecule of water. The same in general refers to the compounds of sodium-for instance, its salts have a molecular volume approaching the volume of the acids from which they are derived.

31 The molecular quantity of sodium hydroxide ( 40 grams), on being dissolved in a large mass (200 gram molecules) of water, develops, according to Berthelot 9780 , and according to Thomsen 9940, heat-units, but at $100^{\circ}$ about 13000 (Berthelot). Solutions of $\mathrm{NaHO}+n \mathrm{H}_{2} \mathrm{O}$, on being mixed with water, evolve heat if they contain less than $6 \mathrm{H}_{2} \mathrm{O}$, but if more they absorb heat.

32 The specific gravity of solutions of sodium hydroxide at $15^{\circ} / 4^{\circ}$ is given in the short table below :-

$$
\begin{array}{lccccccc}
\text { NaHO, p.c. . } & 5 & 5 & 10 & 15 & 20 & 30 & 40 \\
\text { Sp.gr. . . } & 1 \cdot 057 & 1 \cdot 113 & 1 \cdot 169 & 1 \cdot 224 & 1 \cdot 331 & 1 \cdot 436
\end{array}
$$

1000 grams of a 5 p.c. solution occupies a volume of 946 c.c. ; that is, less than the water serving to make the solution (see Note 18).

33 Sodium hydroxide (and other alkalis) is capable of hydrolysing-saponifying, as it is termed - the compounds of acids with alcohols. If $\mathrm{RHO}$ (or $\mathrm{R}(\mathrm{HO}) n$ ) represent the composition of an alcohol - that is, of the hydroxide of a hydrocarbon radicle-and QHO an acid, then the compound of the acid with the alcohol or ethereal salt of the given acid will have the composition RQO. Ethereal salts, therefore, present a similitude to metallic salts, just as alcohols resemble basic hydroxides. Sodium hydroxide acts on 
The chemical reactions of sodium hydroxide serve as a type for thoseof a whole class of alkalis - that is, of soluble basic hydroxides which may be obtained from water and a metallic oxide, and whose composition is expressed as a compound of the metal, M, and hydroxyl, $\mathrm{OH}$; $\mathrm{MOH}$. The solution of sodium hydroxide is a very caustic liquidthat is to say, it acts in a destructive way on most substances, for instance, on most organic tissues - hence caustic soda, like all soluble alkalis, is a poisonous substance; acids, for instance, hydrochloric, forming common salt, serve as antidotes. The action of caustic soda on bones, fat, starch, and similar vegetable and animal substances explains its action on organisms. Thus bones, when plunged into a weak solution of caustic soda, fall to powder, ${ }^{34}$ and evolve a smell of ammonia, which is due to the caustic soda changing the gelatinous. organic substance (it contains carbon, hydrogen, nitrogen, oxygen, and sulphur, like albumin) of the bones, dissolving it and in part destroying it, whence ammonia is disengaged. Fats, tallow, and oils become

ethereal salts in the same way that it acts on the majority of metallic salts-namely, it liberates alcohol, and forms the sodium salt of that acid which was in the ethereal salt. The reaction takes place in the following way :-

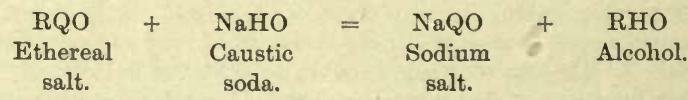

Such a decomposition is termed saponification, because similar reactions were knowm very long ago for the ethereal salts corresponding with glycerin, $\mathrm{C}_{3} \mathrm{H}_{5}(\mathrm{OH})_{3}$ (Chapter IX.), found in animals and plants, and composing what are called fats or oils. Caustic soda, acting on fat and oil, forms glycerin, and sodium salts of those acids which were in union with the glycerin in the fat, as Chevreul showed at the beginning of this century. The sodium salts of the fatty acids are known in practice as soaps. That is to say, that soap is made from fat and caustic soda, glycerin being separated and a sodium salt. formed. As glycerin is usually found in union with certain acids, so also are the sodium salts of certain acids found in soap. The greater part of the acids found in conjunction with glycerin in fats are the solid, palmitic and stearic acids, $\mathrm{C}_{16} \mathrm{H}_{32} \mathrm{O}_{2}$ and $\mathrm{C}_{18} \mathrm{H}_{38} \mathrm{O}_{2}$, and the liquid, oleic acid, $\mathrm{C}_{18} \mathrm{H}_{34} \mathrm{O}_{2}$; hence soap principally contains a mixture of the sodium salts of these acids. In preparing soap the fatty substances are mixed with a solution of caustic soda until an emulsion is formed; the proper quantity of caustic soda is then added in order to produce saponification when heated, the soap being separated from the solution either by means of an excess of caustic soda or else by common salt, which displaces the soap from the aqueous solution (salt water does not dissolve soap, neither does it form a lather). The water, acting on the soap, partly decomposes it (because the acids of the soap are feeble), and the alkali set free acts during the application of soap. Hence it may be replaced by a very feeble alkali. Strong solutions of alkali corrode the skin and tissues. They are not formed from soap, because the reaction is reversible, and the alkali is only set free by the excess of water. Thus we see how the teaching of Berthollet renders it possible to understand many phenomena which occur during everyday experience.

34 On this is founded the process of Henkoff and Engelhardt for treating bones. The bones are mixed with ashes, lime, and water: it is true in this case that more potassium hydroxide than sodium hydroxide is formed, but their action is almost. identical. 
saponified with a solution of caustic soda - that is to say, they form with it soaps soluble in water, or sodium salts of the organic acids contained in the fats..$^{35}$ The clearest reactions of sodium hydroxide are determined by the fact that it saturates all acids forming salts with them, which are almost all soluble in water, and in this respect caustic soda is as characteristic amongst the bases as nitric acid is among the acids. It is impossible to detect sodium by means of the formation of precipitates of insoluble sodium salts, as may be done with other metals, many of whose salts are but slightly soluble. The powerful alkaline properties of caustic soda determine its capacity for combining with even the feeblest acids, its property of disengaging ammonia from ammonium salts, its faculty of acting on salts whose bases are insoluble in water, \&c. If a solution of the salt of almost. any metal be mixed with caustic soda, then a soluble sodium salt will be formed, and an insoluble hydroxide of the metal will be separatedfor instance, copper nitrate yields copper hydroxide, $\mathrm{Cu}\left(\mathrm{NO}_{3}\right)_{2}$ $+2 \mathrm{NaHO}=\mathrm{Cu}(\mathrm{HO})_{2}+2 \mathrm{NaNO}_{3}$. Even many basic oxides - as, for instance, the oxides of zinc and aluminium-precipitated by caustic soda, are capable of combining with it and forming soluble compounds, and therefore caustic soda in the salts of such metals first forms a precipitate of hydroxide, and then, employed in excess, dissolves this precipitate. This phenomenon occurs, for instance, when caustic soda. is added to the salts of aluminium. This shows the property of such an alkali as caustic soda of combining not only with acids, but also with feeble basic oxides. For this reason caustic soda acts on most elements which are capable of forming acids or oxides similar to. them; thus, for instance, the metal aluminium gives hydrogen with caustic soda in consequence of the formation of alumina, which combines with the caustic soda - that is, in this case, the caustic alkali acts on the metal just like an acid. If the substance which is mixed with the caustic soda is capable of combining with the hydrogen evolved (aluminium does not form such a compound), then such a compound will be formed. Thus, for instance, in this way phosphorus acts on caustic soda, yielding hydrogen phosphide. If the hydrogen compound disengaged is capable of combining with the alkali, then, naturally, a salt of the corresponding acid is formed. For instance, chlorine and sulphur act thus on caustic soda. Chlorine, with the hydrogen of the caustic soda, forms hydrochloric acid, and the latter forms common salt with the sodium hydroxide, whilst the other atom in the molecule of chlorine, $\mathrm{Cl}_{2}$, takes the place of the hydrogen, and

33 As explained in Note 33. 
forms the hypochlorite, $\mathrm{NaClO}$. In the same way, by the action of sodium hydroxide on sulphur, hydrogen sulphide is formed, which acts on the soda forming sodium sulphide, in addition to which sodium thiosulphate is formed (see sulphur). In virtue of the possibility of similar reactions, sodium hydroxide acts on many metals and non-metals. Such action is often accelerated by the presence of the oxygen of the air, as by this means the possibility of the formation of acids and oxides rich in oxygen is increased. Thus many metals and their lower oxides, in the presence of an alkali, absorb oxygen and form acids. Even manganese peroxide, when mixed with caustic soda, is capable of absorbing the oxygen of the air, because sodium manganate is formed. Organic acids, when heated with caustic soda, give up to it the elements of carbonic anhydride, forming sodium carbonate, and separating that hydrocarbon group which exists, in combination with carbonic anhydride, in the organic acid.

Thus sodium hydroxide, like the soluble alkalis in general, ranks amongst the most active substances in the chemical sense of the term ; but few substances are capable of resisting it. Even the rocky siliceous substances, as we shall see further on, are transformed by it, forming vitreous slags with it, at all events on fusion. Sodium hydroxide, as a typical example of the basic hydrates, in distinction from many other basic oxides, easily forms acid salts with acids (for instance, $\mathrm{NaHSO}_{4}$, $\mathrm{NaHCO}_{3}$ ), and does not form any basic salts at all; whilst many less energetic bases, such as the oxides of copper and lead, easily form basic salts, but acid salts only with difficulty. This capability of forming acid salts, particularly with polybasic acids, may be explained by the energetic character of the basic properties of sodium hydroxide, and the small development of these properties in the bases which easily form basic salts. These latter bases are even capable of combining with such bases as sodium hydroxide and ammonium hydroxide. An energetic base is capable of retaining a considerable quantity of acid, which a slightly energetic base would not have the power of doing. Certain feeble bases, particularly intermediate ones (like alumina) are not at all capable of retaining such feeble acids as carbonic, or, if they form compounds with them, they are very unstable and basic. The formation of acid salts with such acids as carbonic, oxalic, sulphuric, phosphoric, \&c., which contain two or more atoms of hydrogen, capable of being replaced by metals, is explained by the fact that the normal salt represents the substitution of sodium for all the atoms of hydrogen. Such monobasic acids as nitric, hydrochloric, \&c., do not form any stable acid salts (although they form unstable compounds of the normal salt with the 
acid), because they contain only one atom of hydrogen capable of being replaced by the metal. Also, as will be shown in the subsequent chapters, sodium belongs to the univalent metals, exchangeable for hydrogen atom for atom; amongst metals sodium may, like chlorine amongst the non-metals, serve as a representative of the univalent properties. Most of the other elements which are not. capable of forming acid salts are bivalent, from whence it may be understood that in a bibasic acid-for instance, carbonic, $\mathrm{H}_{2} \mathrm{CO}_{3}$, or sulphuric, $\mathrm{H}_{2} \mathrm{SO}_{4}$ - the hydrogen may be exchanged, atom for atom, for sodium, and yield an acid salt by means of the first substitution and a normal salt by means of the second-for instance, $\mathrm{NaHSO}_{4}$, and $\mathrm{Na}_{2} \mathrm{SO}_{4}$, whilst such bivalent metals as calcium or barium do not form acid salts because one of their atoms at once takes the place of both hydrogen atoms, forming, for instance, $\mathrm{CaCO}_{3}$ and $\mathrm{CaSO}_{4}$. It may be expected, from what has been mentioned above, that bivalent metals easily form acid salts with acids containing more than two atoms of hydrogen - for instance, with tribasic acids, such as phosphoric acid, $\mathrm{H}_{3} \mathrm{PO}_{4}$-and actually such salts do exist; but all such relations are complicated by the fact that the character of the base very often changes and becomes weakened with the increase of valency and the change of atomic weight, and feebler bases (for instance, silver oxide), although corresponding with univalent metals, do not form acid salts, and the feeblest bases (for instance, $\mathrm{CuO}, \mathrm{PbO}$ ) easily form basic salts, and, notwithstanding their valency, do not form any acid salts which are in any degree stable-for instance, which are undecomposable by water. In addition, basic and acid salts ought to be regarded rather as compounds similar to crystallo-hydrates, because such acids as sulphuric form with sodium not only an acid and a normal salt, as might be expected judging from the valency of sodium, but in addition to these also salts containing a greater quantity of acid. In sodium sesquicarbonate we saw an example of such compounds. Taking all this into consideration, we ought to say that the property of more or less easily forming acid salts more readily accords with the energy of the base than with the valency, and it is truest of all to state that the faculty of a base to form acid and basic salts is its characteristic, just as the faculty of forming compounds with hydrogen is a characteristic property of elements. In this respect sodium hydroxide distinguishes itself by the facility with which it forms acid salts and an absence of the faculty of forming basic salts. This property is also shared with sodium by the bases formed from the metals potassium and lithium. The metals lead and copper do not form acid salts, but easily give basic salts. Barium, 
calcium, and silver form acid and basic salts with difficulty, although they easily form normal ones.

We have seen the transformation of common salt into sodium sulphate, of this latter into sodium carbonate, and of sodium carbonate into caustic soda. Lavoisier still regarded sodium hydroxide as an element, because he was unacquainted with its decomposition with the elimination of oxygen and its formation by means of metallic sodium, which separates the hydrogen from water, forming caustic soda. The preparation of metallic sodium was one of the greatest discoveries in chemistry, not only because through it the conception of elements became broader and more regular, but especially because in sodium chemical properties were observed which were but feebly shown in the other metals more familiarly known. This discovery was made in 1807 by the English chemist Davy by means of the galvanic current. By connecting with the positive pole (of copper or charcoal) a piece of moist (in order to obtain electrical conductivity) caustic soda, and boring a hole in it filled with mercury connected with the negative pole of a strong Volta's pile, Davy observed that on passing the current, a peculiar metal dissolved in the mercury, less volatile than mercury, and capable of decomposing water, again forming caustic soda. In this way (by analysis and synthesis) Davy demonstrated the complexity of alkalis, which up to that time had been regarded as undecomposable substances. On being decomposed by the galvanic current, caustic soda disengages hydrogen and sodium at the negative pole and oxygen at the positive pole. Davy showed that the metal formed volatilises at a red heat, which is the most important physical property for extracting sodium, ibecause all further methods are founded on the volatility of sodium. Besides this Davy observed that sodium easily oxidises, that its vapours take fire in air, and the latter circumstance was for a long time an obstacle to the easy preparation of this metal. The properties of sodium were later on more thoroughly investigated by Gay-Lussac and Thenard, who furnished easier means of obtaining sodium, and observed that metallic iron at a high temperature was capable of reducing sodium from caustic soda. ${ }^{36}$ Brunner latterly discovered that not only iron,

36 Deville supposes that such a decomposition of sodium hydroxide by metallic iron depends solely on the dissociation of the alkali at a white heat into sodium, hydrogen, and oxygen. Here the part played by the iron is only that it retains the oxygen formed, otherwise the decomposed elements would again reunite upon cooling, as in other cases of dissociation. If it be supposed that the temperature at the commencement of the dissociation of the iron oxides is higher than that of sodium oxide, then the decomposition may be explained by the hypothesis of Deville. Deville demonstrates his views by the following experiment:-An iron bottle, filled with iron shavings, was heated in such a sway that the upper part became red hot, the lower part remaining cooler; sodium 
but also charcoal, has this property, although hydrogen does not reduce sodium. ${ }^{37}$ But still the methods of extracting sodium were very troublesome, and consequently sodium was a great rarity. The principal obstacle to its production was that they endeavoured to condense the easily-oxidising vapours of sodium in vacuum in complicated apparatus. For this reason, when Donny and Maresca, having thoroughly studied the matter, constructed a specially simple condenser, the production of sodium was much facilitated. Furthermore, in practice the most important epoch in the history of the production of sodium is comprised in the investigation of Sainte-Claire Deville, who avoided the complex methods in vogue up to that time, and furnished those simple means by which the production of sodium is now rendered feasible in chemical works.

For the production of sodium according to the method of Deville, a mixture of sodium carbonate (7 parts) free from water, charcoal (two parts), and lime or chalk ( 7 parts) is heated. This latter ingredient is only added in order that the sodium carbonate, on fusing, shall not separate from the charcoal. ${ }^{38}$ The chalk on being heated loses carbonic anhydride, leaving infusible lime, which is permeated by the sodium carbonate and forms a thick mass, in which the charcoal is situated, in immediate contact with the sodium carbonate. That is to say, the lime only serves as a mechanical admixture. When the charcoal is heated

hydroxide was introduced into the upper part. The decomposition was then effectedthat is, sodium vapours were produced (this experiment was done really with potassium hydroxide). On unfastening the bottle it was found that the iron in the upper part was not oxidised, but only that in the lower part. This may be explained by the decomposition of the alkali into sodium, hydrogen, and oxygen taking place in the upper part, whilst the iron in the lower part absorbed the oxygen set free. If the whole bottle be subjected to the same moderate heat as the lower extremity, no metallic vapours are formed. Then, according to the hypothesis, the temperature would be insufficient for the dissociation of the sodium hydroxide.

37 It has been previously remarked (Chapter II. Note 9) that Beketoff showed the displacement of sodium by hydrogen, not from sodium hydroxide but from the oxide $\mathrm{Na}_{2} \mathrm{O}$; then, however, only one half is displaced, with the formation of $\mathrm{NaHO}$.

38 In latter times in England, where the preparation of sodium is at present carried on on a large commercial scale (from 1860 to 1870 it was only manufactured in a few works in France), they have begun to add to Deville's mixture more iron, or iron oxide, which, with the charcoal, gives metallic and carburetted iron, which still further facilitates the decomposition. At present a kilogram of sodium may be purchased for about the same sum $(2 /-)$ as a gram cost thirty years ago. The industrial preparation of sodium in large quantities ought not only to influence the extraction of such metals as aluminium (metal of clay and alum), but also many other branches of industry. The method of preparation by means of the action of the galvanic current, proposed and tried many times, still requires further improvement. Deville overcame the practical difficulties connected with his process, which is that in use at present, and is distinguished by its simplicity and cheapness; but so far industry has but scantily availed itself of the possibility of obtaining cheap sodium. 
with the sodium carbonate, at a white heat, carbonic oxide and vapours of sodium are disengaged, according to the equation :

$$
\mathrm{Na}_{2} \mathrm{CO}_{3}+2 \mathrm{C}=\mathrm{Na}_{2}+3 \mathrm{CO}
$$

On cooling the vapours and gases disengaged, the vapours condense into molten metal (in this form sodium does not easily oxidise, whilst in vapour it burns) and the carbonic oxide remains as gas.

Here are the simple means by which metallic sodium may be obtained. An iron tube, about a metre long and a decimetre in diameter, is made out of boiler plate. The pipe is cemented into a furnace having a strong

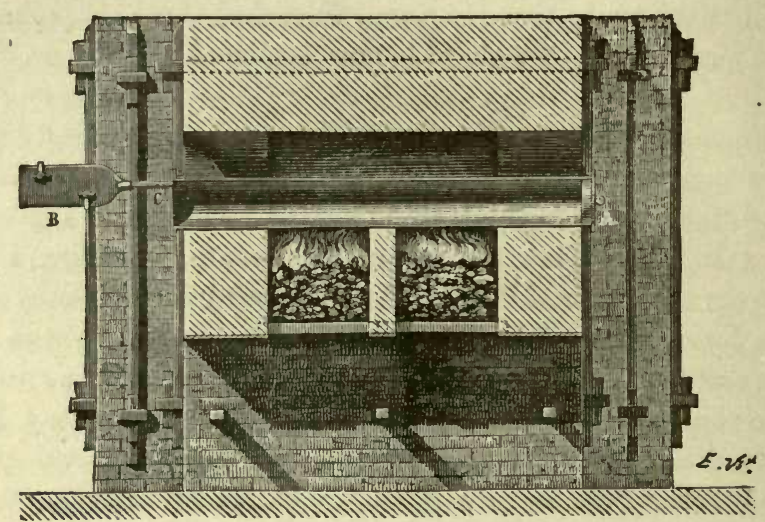

FIG. 71.-Manufacture of sodium by Deville's process. A C, iron tube containing a mixture of soda, charcoal, and chalk. $\mathrm{B}$, condenser.

draught, capable of giving a high temperature, and the tube is charged with the mixture required for the preparation of sodium. One end of the tube is closed with a cast-iron stopper A with clay luting, and the other with the cast-iron stopper $\mathrm{C}$ provided with an aperture. On heating, first of all the moisture contained in the various substances is given off, then carbonic anhydride and the products of the dry distillation of the charcoal, then the latter begins to act on the sodium carbonate, and carbonic oxide and vapours of sodium appear. It is easy to observe the appearance of the latter, because on issuing from the aperture in the stopper $\mathrm{C}$ they take fire spontaneously and burn with a very bright yellow flame. The pipe of the condenser is then introduced into the aperture $\mathrm{C}$, compelling in this way the vapours and gases formed to pass through the condenser B. This condenser consists of two square cast-iron trays, A and $\mathbf{A}^{\prime}$, Fig. 72 , with wide edges firmly screwed together. Between these two trays there is a space in which the condensation of the vapours of sodium is accomplished, because the thin metallic walls of the condenser 
are cooled by the air but remain sufficiently heated to preserve the sodium in a liquid state, and so it does not choke the apparatus, but continually flows from it. The vapours of sodium, condensing in the cooler, flow in the shape of liquid metal into a vessel containing some non-volatile naphtha or hydrocarbon. This is used in order to prevent the sodium oxidising as it issues from the condenser at a somewhat high temperature. During the operation it is necessary (with an iron rod) to occasionally clear the pipe which conducts the vapours to the condenser, because it becomes choked up with solid compounds formed by the sodium. In order to obtain sodium of a pure quality it is necessary to distil

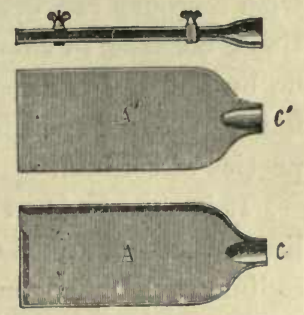

FIG. 72.-Donny and Maresca's sodium ondenser, consisting of two cast-iron plates screwed together. it once more, which may even be done in porcelain retorts, but the distillation must be conducted in a stream of some gas on which sodium does not act, for instance in a stream of nitrogen ; carbonic anhydride is not applicable, because sodium partially decomposes it, absorbing the oxygen from it.

Pure sodium is a lustrous metal, white as silver, soft as wax ; it becomes brittle in the cold. In ordinary moist air it quickly tarnishes and becomes covered with a film of hydroxide, $\mathrm{NaHO}$, formed at the expense of the water in the air. In completely dry air sodium retains its lustre for an indefinite time. Its density at the ordinary temperature is equal to 0.98 , so that it is lighter than water ; it fuses very easily at a temperature of $95^{\circ}$, and distils at a bright red heat (about $900^{\circ}$ ). It forms alloys with most metals, combining with them, heat being sometimes evolved and sometimes absorbed. Thus if sodium (having a clean surface) be thrown into mercury, especially when heated, there is a flash, and such a considerable amount of heat is evolved that part of the mercury is transformed into vapour. ${ }^{39}$ Compounds or solutions of sodium

39 By dissolving sodium amalgams in water and acids, and deducting the heat of solution of the sodium, Berthelot found that for each atom of the sodium in amalgams containing a large amount of mercury (more than 90 p.c.) the amount of heat evolved increases with the quantity of sodium, and also that when a composition is reached which approaches $\mathrm{NaHg}_{5}$ the heat evolved decreases. In the formation of the latter compound, about 18500 calories are evolved; when $\mathrm{NaHg}_{3}$ is formed, about 14000 ; and for $\mathrm{NaHg}$ about 10000 calories. Kraut and Popp regarded the definite crystalline amalgam as having the composition of $\mathrm{NaHg}_{e}$, but at the present time, in accordance with Grimaldi's results, it is thought to be $\mathrm{NaHg}_{5}$. A similar amalgam is very easily obtained if a 3 p.c. amalgam be left several days in a solution of sodium hydroxide until a orystalline mass is formed, from which the mercury may be removed by strongly pressing in chamois leather. This amalgam with a solution of potassium hydroxide forms a potassium amalgam, $\mathrm{KHg}_{10}$ (Crookewitt, Grimaldi). It may be mentioned here that

VOL. I. 
in mercury, or amalgams of sodium, even when containing 2 parts of sodium to 100 parts of mercury, are solids. Only those amalgams which are the very poorest in sodium are liquid. Such alloys of sodium with mercury are often used instead of sodium in chemical investigations, because. in combination with mercury sodium is not easily acted on by air, and is a mass heavier than water, and therefore more convenient to handle, whilst at the same time it retains the principal properties of sodium. ${ }^{40}$

It is easy to form an alloy of mercury and sodium having a crystalline structure, and a definite atomic composition $\mathrm{NaHg}_{5}$. The alloy of sodium with hydrogen or sodium hydride, $\mathrm{Na}_{2} \mathrm{H}$, which has the external appearance of a metal, ${ }^{41}$ is a most instructive example of the characteristics of alloys. At the ordinary temperature sodium does not absorb hydrogen, but from $300^{\circ}$ to $421^{\circ}$ the absorption takes place at the ordinary pressure (and at an increased pressure even at higher temperatures), as shown by Troost and Hautefeuille (1874). One volume of sodium absorbs as much as 238 vols. of hydrogen. The metal increases in volume, and when once formed the alloy can be preserved for some time without change at the ordinary temperature. The appearance of sodium hydride resembles that of sodium itself; it is as soft as this latter, when heated it becomes brittle, and decomposes above $300^{\circ}$, evolving hydrogen. In this decomposition all the phenomena of dissociation are very clearly shown - that is, the hydrogen gas evolved has a definite tension, ${ }^{42}$ which corresponds with each definite temperature. This confirms the fact, that the formation of substances capable of dissociation is only accomplished within the limits of the dissociation. Sodium hydride melts more easily than sodium itself, and

the latent heat of fusion (of atomic quantities) of $\mathrm{Hg}=360$ (Personne), $\mathrm{Na}=730$ (Joannis), and $K=610$ calories (Joannis).

40 Alloys are so similar to solutions (exhibiting such complete parallelism in properties) that they are contained in the same class of so-called indefinite compounds (Chapter I.). But in alloys, as substances passing from the liquid to the solid state, it is easier to discover the formation of definite chemical compounds, and therefore, in order to arrive at a correct understanding of solutions, it is very important to study alloys. In addition to this, they are of themselves of considerable interest. The combination of sodium with hydrogen, where the change of physical properties is so evident and where the conservation of chemical properties and easy dissociation are so apparent, ought in this respect to supply much towards an understanding of alloys and also of solutions. Alloys and solutions are homogeneous; if the decomposition has commenced or increased we may not perceive it, but the products of the splitting up of the alloy $\mathrm{Na}_{2} \mathrm{H}$ are heterogeneous; they are visible.

41 Potassium forms a similar compound, but lithium, under the same circumstances, does not.

42 Namely, the tension of dissociation of hydrogen, $p$, in millimetres of mercury, is :-

$\begin{array}{lccclll}t= & 330^{\circ} & 350^{\circ} & 380^{\circ} & 400^{\circ} & 420^{\circ} & 430^{\circ} \\ p= & 28 & 57 & 150 & 447 & 752 & 900\end{array}$


then does not undergo decomposition if it is in an atmosphere of hydrogen. It oxidises easily in air, but not so easily as potassium hydride. The chemical reactions of sodium are retained in its hydride, and, if we may so express it, they are even increased by the addition of hydrogen. At all events, in the properties of sodium hydride ${ }^{43}$ we see other properties than in such hydrogen compounds as $\mathrm{HCl}, \mathrm{H}_{2} \mathrm{O}, \mathrm{H}_{3} \mathrm{~N}, \mathrm{H}_{4} \mathrm{C}$, or even in the gaseous metallic hydrides $\mathrm{AsH}_{3}, \mathrm{TeH}_{\mathrm{\ell}}$. But platinum, palladium, nickel, and iron, in absorbing hydrogen form compounds in which liydrogen is in a similar state. In them, as in sodium hydride, the hydrogen is compressed, absorbed, occluded (page 141).

The most important property of sodium is its power of easily decomposing water and evolving hydrogen from the majority of the hydrogen compounds, and especially from all acid and hydrate compounds in which hydroxyl ought to be recognised. This depends on the power of sodium of combining with the elements which are in combination with the hydrogen. We already know that sodium disengages hydrogen, not only from water, hydrochloric acid, ${ }^{44}$ and all other acids, but also from ammonia, although it does not displace hydrogen from the hydrocarbons. ${ }^{45}$

43 In general, during the formation of alloys the volumes change very slightly, and therefore from the volume of $\mathrm{Na}_{2} \mathrm{H}$ some idea may be formed of the volume of hydrogen in a solid or liquid state. Archimedes even concluded that there was gold in an alloy of copper and gold by reason of its volume and density. From the fact that the density of $\mathrm{Na}_{2} \mathrm{H}$ is equal to 0.959 , it may be seen that the volume of 47 grams (the gram molecule) of this compound $=49^{\circ} 0$ c.c. The volume of 46 grams of sodium contained in the $\mathrm{Na}_{2} \mathrm{H}$ is equal (the density in the same conditions 0.97 ) to 47.4 c.c. Therefore the volume of 1 gram of hydrogen in $\mathrm{Na}_{2} \mathrm{H}$ is equal to $1 \cdot 6$ c.c., and consequently the density of metallic hydrogen, or the weight of 1 c.c., approaches $0 \cdot 6$ gram. This density is also proper to the hydrogen alloyed with potassium and palladium. Judging from the scanty information which is at present available, liquid hydrogen near its absolute. boiling-point (Chapter II.) has a far less density.

It ought to be mentioned that sodium hydride, according to the usual equivalency of $\mathrm{H}_{2}$ with $\mathrm{O}$, corresponds, not with the oxide $\mathrm{Na}_{2} \mathrm{O}$, but with the suboxide of sodium, $\mathrm{Na}_{4} \mathrm{O}$, and if we judge the atomicity of elements by hydrogen compounds, sodium ought to be counted as semivalent. According to the law of substitution, $\mathrm{Na}$ ought to be taken as univalent in all its ordinary combinations: $\mathrm{Na}_{2} \mathrm{O}, \mathrm{NaCl}, \mathrm{NaHO}, \mathrm{NaHSO}_{4}$, \&c. Therefore sodium hydride belongs to the series $\mathrm{Na}_{2} \mathrm{X}$, and not $\mathrm{NaX}$.

$44 \mathrm{H}$. A. Schmidt remarked that completely dry hydrogen chloride is decomposed with great difficulty by sodium, although the decomposition proceeds easily with potassium and with sodium in moist lyddrogen chloride. Wanklyn also remarked that sodium burns with great difficulty in dry chlorine. Probably in relation to this there is a correspondence with other phenomena observed by Dixon, who found that completely dry carbonic oxide does not explode with oxygen on passing an electric spark.

45 As sodium does not displace hydrogen from the hydrocarbons, it may be preserved in liquid hydrocarbons. Naphtha is generally used for this purpose, as it consists of a mixture of various liquid hydrocarbons. However, in naphtha sodium usually becomes coated with a crust composed of matter produced by the action of the sodium on certain of the substances contained in the mixture composing naphtha. In order that sodium may retain its lustre in naphtha, secondary octyl alcohol is added. (This alcohol is obtained by distilling castor oil with caustic potash.) Potassium and sodium keep well in a mixture of pure benzene and naphthalene. 
Sodium burns both in chlorine and in oxygen, evolving much heat. These properties of sodium are closely connected with its power of taking up oxygen, chlorine, and similar elements from most of their compounds. Just as sodium removes the oxygen from the oxides of nitrogen and from carbonic anhydride, so also does it decompose the majority of oxides at definite temperatures. Here the essence of the matter is the same as in the decomposition of water. Thus, for instance, when acting on magnesium chloride, the sodium displaces the magnesium, or when acting on aluminium chloride sodium displaces metallic aluminium. Sulphur, phosphorus, arsenic, and a whole series of other elements, also combine with sodium. ${ }^{46}$

With oxygen sodium unites in three degrees of combination, forming a suboxide, $\mathrm{Na}_{4} \mathrm{O}$, an oxide, $\mathrm{Na}_{2} \mathrm{O}$, and a peroxide, $\mathrm{NaO}$. They are thus termed because $\mathrm{Na}_{2} \mathrm{O}$ is a basic oxide (with water it forms a basic hydroxide), but $\mathrm{Na}_{4} \mathrm{O}$ and $\mathrm{NaO}$ do not form corresponding saline compounds. The suboxide is a grey inflammable substance which easily decomposes water, disengaging hydrogen; it is formed by the slow oxidation of sodium at the ordinary temperature. The peroxide is a greenish yellow substance, fusing at a bright red heat; it is produced by burning sodium in an excess of oxygen, and it yields oxygen when treated with water :

$$
\begin{aligned}
\text { Suboxide : } & \mathrm{Na}_{4} \mathrm{O}+3 \mathrm{H}_{2} \mathrm{O}=4 \mathrm{NaHO}+\mathrm{H}_{2}{ }^{47} \\
\text { Oxide : } & \mathrm{Na}_{2} \mathrm{O}+\mathrm{H}_{2} \mathrm{O}=2 \mathrm{NaHO}^{48} \\
\text { Peroxide : } & \mathrm{NaO}+\mathrm{H}_{2} \mathrm{O}=2 \mathrm{NaHO}+\mathrm{O}
\end{aligned}
$$

46 If sodium does not directly displace the hydrogen in hydrocarbons, still by indirect means compounds may be obtained which contain sodium and hydrocarbon groups. Some of these compounds have been produced, although not in a pure state. Thus, for instance zinc ethyl $\mathrm{Zn}\left(\mathrm{C}_{2} \mathrm{H}_{5}\right)_{2}$, when treated with sodium, loses zinc and forms sodium ethyl, $\mathrm{C}_{2} \mathrm{H}_{5} \mathrm{Na}$, but this decomposition is not complete, and the compound formed cannot be separated by distillation from the remaining zinc ethyl. In this combination the energy of the sodium clearly appears, for which reason it reacts with substances containing haloids, oxygen, \&c., and directly absorbs carbonic anhydride, forming a salt of a carboxylic acid (propionic).

47 A compound, $\mathrm{Na}_{2} \mathrm{Cl}$, which corresponds with the suboxide, is evidently formed when a galvanic current is passed through fused common salt; the sodium liberated dissolves in the common salt, and does not separate from the compound either on cooling or on treatment with mercury. It is therefore supposed to be $\mathrm{Na}_{2} \mathrm{Cl}$; the more so as the mass obtained gives hydrogen when treated with water: $\mathrm{Na}_{2} \mathrm{Cl}+\mathrm{H}_{2} \mathrm{O}$ $=\mathrm{H}+\mathrm{NaHO}+\mathrm{NaCl}$, that is, it acts like suboxide of sodium. If $\mathrm{Na}_{2} \mathrm{Cl}$ really exists as a salt, then the corresponding base $\mathrm{Na}_{4} \mathrm{O}$, according to the example of other bases of the composition $\mathrm{M}_{4} \mathrm{O}$, ought to be called a quaternary oxide. According to certain evidence, a suboxide is formed when thin sheets or fine drops of sodium slowly oxidise in moist air.

48 According to observations easily made, sodium when fused in air oxidises but does not burn, the combustion only commencing with the formation of vapour - that is, when considerably heated. Davy and Karsten obtained the oxides of potassium, $\mathrm{K}_{2} \mathrm{O}$, and of sodium. 
All three oxides form sodium hydroxide with water, but only the oxide, $\mathrm{Na}_{2} \mathrm{O}$, is directly transformed into a hydrate. The other oxides either liberate hydrogen or oxygen; they also present a similar distinction with reference to many other agents. Thus carbonic anhydride combines directly with the oxide $\mathrm{Na}_{2} \mathrm{O}$, which when heated in the gas burns, forming sodium carbonate, whilst in this case the peroxide ${ }^{49}$ also yields oxygen. When treated with acids, sodium and all its oxides only form the salts corresponding with sodium oxide - that is, of the formula or type $\mathrm{NaX}$. Thus the oxide of sodium $\mathrm{Na}_{2} \mathrm{O}$ is the only salt-forming oxide of this metal, as water is in the case of hydrogen. Although the peroxide $\mathrm{H}_{2} \mathrm{O}_{2}$ is derived from hydrogen, yet there are no corresponding salts known, and if they are formed they are probably as unstable as hydrogen peroxide itself. Although carbon forms carbonic oxide, $\mathrm{CO}$, still it has only one salt-forming oxide-carbonic anhydride, $\mathrm{CO}_{2}$. Nitrogen and chlorine both give several salt-forming oxides and types of salts. But of the oxides of nitrogen, $\mathrm{NO}$ and $\mathrm{NO}_{2}$ do not form salts, as do $\mathrm{N}_{2} \mathrm{O}_{3}, \mathrm{~N}_{2} \mathrm{O}_{4}$, and $\mathrm{N}_{2} \mathrm{O}_{5}$, but $\mathrm{N}_{2} \mathrm{O}_{4}$ does not form special salts, and $\mathrm{N}_{2} \mathrm{O}_{5}$ corresponds with the highest form of the saline compounds of nitrogen. Such distinctions between the elements, according to their power of giving one or several saline forms, is a radical property of no less importance than the basic or acid properties of the oxides produced. Sodium as a typical metal does not form any acid oxides,

$\mathrm{Na}_{2} \mathrm{O}$, by heating the metals with their hydroxides, whence $\mathrm{NaHO}+\mathrm{Na}=\mathrm{Na} \mathrm{O}_{2} \mathrm{O}+\mathrm{H}$, but N. N. Beketoff failed to obtain oxides by this means. He prepared them by directly igniting the metals in dry air, and afterwards heating with the metal in order to destroy any peroxide. The oxide $\mathrm{Na}_{2} \mathrm{O}$ produced, when heated in an atmosphere of hydrogen, gave a mixture of sodium and its hydroxide: $\mathrm{Na}_{2} \mathrm{O}+\mathrm{H}=\mathrm{NaHO}+\mathrm{Na}$ (see Chapter II. Note 9). If both the observations mentioned are accurate, then the reaction is reversible. Sodium oxide ought to be formed during the decomposition of sodium carbonate by oxide of iron (see Note 26), and during the decomposition of sodium nitrite. According to Karsten, its specific gravity is $2 \cdot 8$, according to Beketoff $2 \cdot 3$. The difficulty in obtaining it is owing to an excess of sodium forming the suboxide, and an excess of oxygen the peroxide. The grey colour peculiar to the suboxide and oxide perhaps shows that they contain metallic sodium. In addition to this, in the presence of water it may contain sodium hydride.

49 Of the oxides of sodium, that easiest to form is the peroxide $\mathrm{NaO}$ or $\mathrm{Na}_{2} \mathrm{O}_{2}$; this is obtained when sodium is burnt in an excess of oxygen. When heated with iodine vapour, it loses oxygen: $\mathrm{Na}_{2} \mathrm{O}_{2}+\mathrm{I}_{2}=\mathrm{Na}_{2} \mathrm{OI}_{2}+\mathrm{O}$. The compound $\mathrm{Na}_{2} \mathrm{OI}_{2}$ is akin to the compound $\mathrm{Cu}_{2} \mathrm{OCl}_{2}$ obtained by oxidising $\mathrm{CuCl}$. This reaction is one of the few where iodine directly displaces oxygen. The substance $\mathrm{Na}_{2} \mathrm{OI}_{2}$ is soluble in water, and when acidified gives free iodine and a sodium salt. Carbonic oxide is absorbed by heated sodium peroxide with formation of sodium carbonate: $\mathrm{Na}_{2} \mathrm{CO}_{3}=\mathrm{Na}_{2} \mathrm{O}_{2}+\mathrm{CO}$, whilst carbonic anhydride liberates oxygen from it. With nitrous oxide it reacts thus: $\mathrm{Na}_{2} \mathrm{O}_{2}$ $+2 \mathrm{~N}_{2} \mathrm{O}=2 \mathrm{NaNO}_{2}+\mathrm{N}_{2}$; with nitric oxide it combines directly, forming a nitrite, $\mathrm{NaO}$ $+\mathrm{NO}=\mathrm{NaNO}_{2}$. Sodium peroxide, when treated with water, does not give hydrogen peroxide, because the latter in the presence of the alkali formed $\left(\mathrm{Na}_{2} \mathrm{O}_{2}+2 \mathrm{H}_{2} \mathrm{O}=2 \mathrm{NaHO}\right.$ $+\mathrm{H}_{2} \mathrm{O}_{2}$ ) decomposes into water and oxygen. 
whilst chlorine, being a typical non-metal, does not form bases with oxygen. Therefore sodium as an element may be thus characterised : it forms one very stable salt-forming oxide, $\mathrm{Na}_{2} \mathrm{O}$, having powerful basic properties, its salts are of the general formula, $\mathrm{NaX}$, therefore in saline compounds it is, like hydrogen, a univalent element..$^{50}$

On comparing sodium and its analogues, which will be described later with other metallic elements, it will be seen that those properties, together with the relative lightness of the metal itself and its compounds, and the magnitude of the atomic weight of sodium, comprise the most essential properties of this element, clearly distinguishing it. from others, and enabling us easily to recognise its analogues.

50 By heating sodium in dry ammonia, Gay-Lussac and Thénard obtained an olivegreen, easily-fusible mass, sodamide, $\mathrm{NH}_{2} \mathrm{Na}$, hydrogen being separated. This substance with water forms sodium hydroxide and ammonia; with carbonic oxide, $\mathrm{CO}$, it forms sodium cyanide, $\mathrm{NaCN}$, and water $\mathrm{H}_{2} \mathrm{O}$; and with dry hydrogen chloride it forms sodium and ammonium chlorides. These and other reactions of sodamide show that the metal in it preserves its energetic properties in reaction, and that this compound of sodium is but little more stable than the corresponding chlorine amide, although it does not show its property of spontaneous decomposition, which is evident from the difference between the properties of netallic sodium and gaseous chlorine. When heated, sodamide, $\mathrm{NH}_{2} \mathrm{Na}$, only partially decomposes, with evolution of hydrogen, the principal part of it giving ammonia and sodium nitride, $\mathrm{Na}_{5} \mathrm{~N}$, according to the equation $3 \mathrm{NH}_{2} \mathrm{Na}=2 \mathrm{NH}_{5}$ $+\mathrm{NNa}_{3}$. The latter is an almost black powdery mass, decomposed by water into ammonia. and sodium hydroxide. 


\section{CHAPTER XIII}

POTASSIUM, RUBIDIUM, CASIUM, AND LITHIUN. SPECTRUM ANALYSIS

JusT as the series of halogens, fluorine, bromine, and iodine, correspond with the chlorine contained in common salt, so there also exists a corresponding series of elements: lithium, $\mathrm{Li}=7$, potassium, $\mathrm{K}=39$, rubidium, $\mathrm{Rb}=85$, and crsium, $\mathrm{Cs}=133$, which are analogous to the sodium in common salt. These elements bear as great a resemblance to sodium, $\mathrm{Na}=23$, as fluorine, $\mathrm{F}=19$, bromine, $\mathrm{Br}=80$, and iodine, $\mathrm{I}=127$, have to chlorine, $\mathrm{Cl}=35.5$. Indeed, in a free state, these elements are, like sodium, soft metals, which rapidly oxidise in moist air, and decompose water at the ordinary temperature, forming soluble hydroxides, having cleariy-defined basic properties, and the composition $\mathrm{RHO}$, like that of caustic soda. The resemblance between these metals is sometimes seen with striking clearness, especially in compounds such as salts. The corresponding salts of nitric, sulphuric, carbonic, and nearly all acids with these metals have many points in common. The metals which resemble sodium so much in their reactions are termed the metals of the alkalis.

Among the metals of the alkalis, the most widely distributed in nature, after sodium, is potassium. Like sodium, it does not appear either in a free state or as oxide or hydroxide, but in the form of salts, which present much in common with the salts of sodium in the manner of their occurrence. The compounds of potassium and sodium in the earth's crust occur as mineral compounds of silica. With silica, $\mathrm{SiO}_{2}$, potassium oxide, like sodium oxide, forms saline mineral substances like glass. If different other oxides, such as lime, $\mathrm{CaO}$, and alumina, $\mathrm{Al}_{2} \mathrm{O}_{3}$, combine with these compounds, there is formed glass, or a glassy stony mass, which is distinguished by its great stability. It is such complex silicious compounds as these which contain potash (potassium oxide), $\mathrm{K}_{2} \mathrm{O}$, or soda (sodium oxide), $\mathrm{Na}_{2} \mathrm{O}$, and sometimes both together, silica, $\mathrm{SiO}_{2}$, lime, $\mathrm{CaO}$, alumina, $\mathrm{Al}_{2} \mathrm{O}_{3}$, and other oxides, that form the chief mass of rocks, out of which, judging by the distribution of the strata, the chief mass of the accessible crust (envelope) of the earth is made up. The primary rocks, like granite, 
porphyry, \&c., ${ }^{1}$ are formed of such crystalline silicious rocks as these. The oxides entering into the composition of these rocks do not form a homogeneous amorphous mass like glass, but are distributed in a series of peculiar, and in the majority of cases crystalline, compounds, into which the primary rocks may be divided. Thus, as has been already mentioned, granite contains felspar, quartz, and mica. Potash and soda, \&c., are generally found in these component parts of rocks. Thus a felspar (orthoclase) in granite contains from 8 to 15 per cent. of potassium, whilst another variety (plagioclase) which also occurs in granite contains $1 \cdot 2$ to 6 per cent. of potassium, and 6 to 12 per cent. of sodium. The mica in granite contains 3 to 10 per cent. of potassium. As has already been mentioned, and will be further explained, the friable, crumbling, and stratified formations which in our times cover a large part of the earth's surface have been formed from these primary rocks by the action of the atmosphere, and of water containing carbonic acid. It is evident that in the formation of these friable formations from the primary rocks by the action of water, the compounds of potassium, as well as the compounds of sodium, must have been dissolved by the water (as they are soluble in water), and, therefore, the compounds of potassium must be accumulated together with those of sodium in sea water. And, indeed, compounds of potassium are always, as we have already pointed out (Chaps. I. and X.) found in sea vater. This forms one of the sources from which they are extracted. After the evaporation of sea water, there remains a mother liquor, which contains potassium chloride and a large proportion of magnesium chloride. On cooling this solution crystals separate out, which contain chlorides of magnesium and potassium. A double salt of this kind, called carnallite, $\mathrm{KMgCl}_{3}, 6 \mathrm{H}_{2} \mathrm{O}$, occurs at Stassfurt. This carnallite ${ }^{2}$ is now employed as a material for the extraction of potassium chloride, and of all the compounds of this element. ${ }^{3}$ Besides which, potassium

1 The origin of the primary rocks has been mentioned in Chap. X. Note 2.

2 Carnallite belongs to the number of double salts which are directly decomposed by water, and it only crystallises from solutions which contain an excess of magnesium chloride. It may be prepared artificially by mixing strong solutions of potassium and magnesium chlorides, when colourless crystals of sp. gr. 1.60 separate, whilst the Stassfurt salt is usually of a reddish tint, owing to traces of iron. At the ordinary temperature sixty-five parts of carnallite are soluble in one-hundred parts of water in the presence of an excess of the salt. It deliquesces in the air, forming a solution of magnesium chloride and leaving potassium chloride.

3 The method of separating sodium chloride from potassium chloride has been described on p. 72. On evaporation of a mixture of the saturated solutions, sodium chloride separates; and then, on cooling, potassium chloride separates, owing to the difference of the variation of their solubilities with the temperature. The following are the most trustworthy figures for the solubility of potassium chloride in one hundred parts of water (for sodium chloride, see Chap. X. Note 13) :-

$\begin{array}{lllll}10^{\circ} & 20^{\circ} & 40^{\circ} & 60^{\circ} & 100^{\circ} \\ 32 & 35 & 40 & 40 & 57 .\end{array}$


chloride itself is sometimes found at Stassfurt as sylvine. By a method of double saline decomposition, the chloride of potassium may be converted into all the other potassium salts, ${ }^{4}$ some of which are of practical use. The potassium salts have, however, their greatest importance as an indispensable component of the food of plants. ${ }^{5}$

When mixed with solutions of other salts the solubility of potassium chloride naturally varies, but not to any great extent. The specific gravity of the solid salt is 1.99-that is, less than that of sodium chloride. All the salts of sodium are specifically heavier than the corresponding salts of potassium, as are also their solutions for equal percentage compositions. If the specific gravity of water at $4^{\circ}=10000$, then at $15^{\circ}$ the specific gravity of a solution of $p$ p.c. potassium chloride $=9992+63 \cdot 29 p+0 \cdot 226 p^{2}$, and therefore for 10 p.c. $=10647,20$ p.c. $=11348$, \&c.

Potassium chloride combines with iodine trichloride to form a compound $\mathrm{KCl}+\mathrm{ICl}_{3}=$ $\mathrm{KICl}_{4}$, of a yellow colour, which is fusible, loses the iodine trichloride at a red heat, and gives potassium iodate and hydrochloric acid with water. It is not only formed by direct combination, but also by many other methods; for instance, by passing chlorine into a solution of potassium iodide so long as the gas is absorbed $\mathrm{KI}+2 \mathrm{Cl}_{2}=\mathrm{KCl}, \mathrm{ICl}_{5}$. Potassium iodide, when treated with potassium chlorate and strong hydrochloric acid, also gives this compound; another method for the formation of which is shown by the equation $\mathrm{KClO}_{5}+\mathrm{I}+6 \mathrm{HCl}=\mathrm{KCl}, \mathrm{ICl}_{3}+3 \mathrm{Cl}+3 \mathrm{H}_{2} \mathrm{O}$. This is a kind of a salt corresponding with $\mathrm{KIO}_{2}$ (unknown) in which the oxygen is replaced by chlorine. If atomicity is taken as a starting point in the study of chemical compounds, and if the elements are considered as having a constant atomicity (number of bonds) - that is, if $\mathrm{K}, \mathrm{Cl}$, and I be counted as univalent elements-then it is impossible to explain the formation of such a compound, because, according to this view, univalent elements are only able to form dual compounds with each other; for example, $\mathrm{KCl}, \mathrm{ClI}, \mathrm{KI}$, \&c., whilst here they are grouped together in the molecule $\mathrm{KICl}_{4}$.

4 It is possible to directly extract the compounds of potassium from the primary rocks which are, especially in some localities, so widely distributed over the earth's surface. From a chemical point of view this problem presents no difficulty; for instance, by fusing powdered orthoclase with lime and fluor spar (Ward's method) and then extracting the alkali with water (in the fusion the silica gives an insoluble compound with lime), or by treating the orthoclase with hydrofluoric acid (in which case silicon fluoride is evolved as a gas) it is possible to transfer the alkali of the orthoclase to an aqueous solution, and to separate it in this manner from the other insoluble oxides. Thus after treating with hydrofluoric acid, many fluorides are obtained in solution, chiefly the fluorides of aluminium and potassium. If the solution be evaporated and sulphuric acid then added, hydrofluoric acid is evolved and the metals are obtained as sulphates. On adding ammonia to the solution of these salts, the aluminium is precipitated as hydroxide, and the salts of ammonia and potassium remain in solution. The ammonia salt is then decomposed by igniting, and the potassium sulphate is obtained alone. However, as yet there is no profit in, nor necessity for, having recourse to this treatment, as there still exist abundant sources for the extraction of potassium compounds by cheaper methods. Furthermore, the salts of potassium are now in the majority of chemical reactions replaced by salts of sodium, especially since the preparation of sodium carbonate has been facilitated by Leblanc's method. The replacement of potassium compounds by sodium compounds not only presents the advantage that the salts of sodium are in general cheaper than those of potassium, but also that a less quantity of a sodium salt is needed for a given reaction than of a potassium salt, because the combining weight of sodium (23) is less than that of potassium (39).

s It has been shown by direct experiment on the cultivation of plants in artificial soils and in solutions that under conditions (physical, chemical, and physiological) otherwise identical plants are able to thrive and become fully developed in the entire absence of sodium salts, but that their development is impossible without potassium salts. 
The primary reckis contain an almost equal propertion of potas-ium

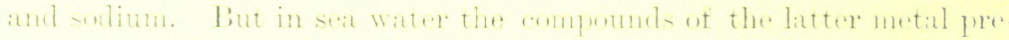

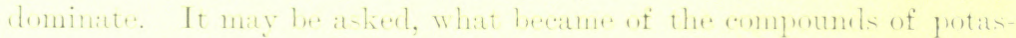

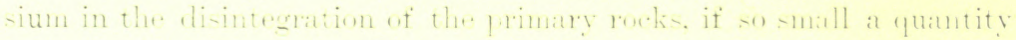

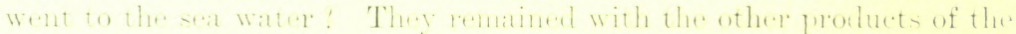

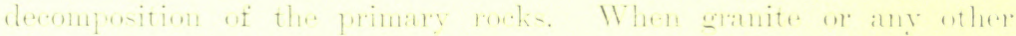

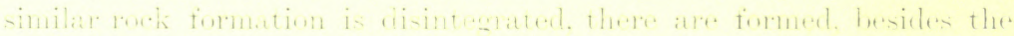

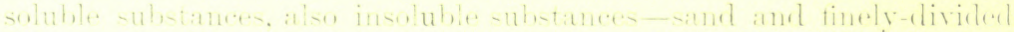

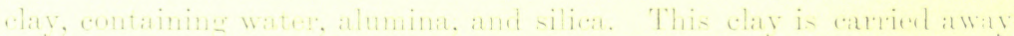

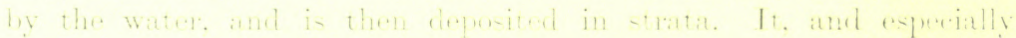

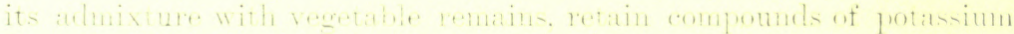

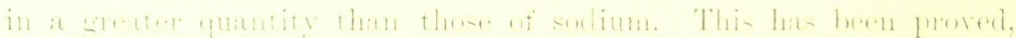

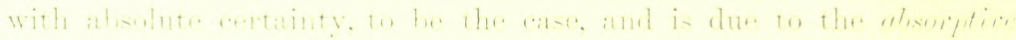

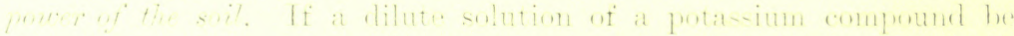

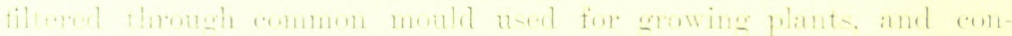

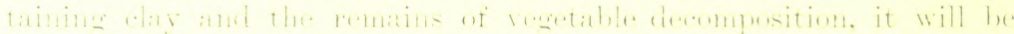

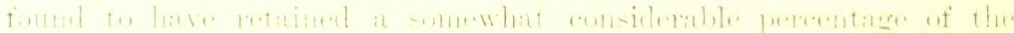

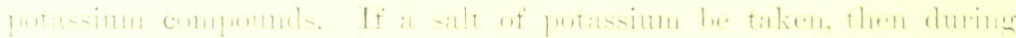

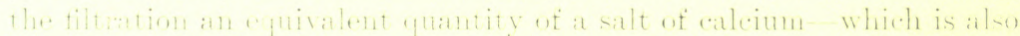

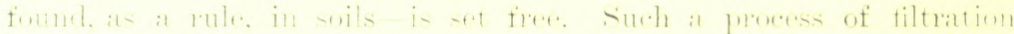

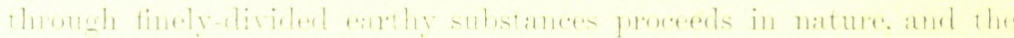

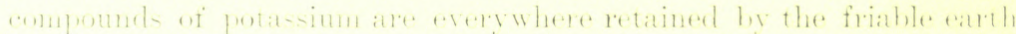

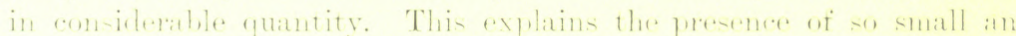

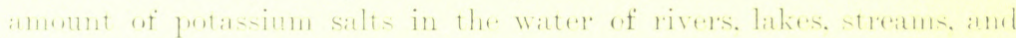

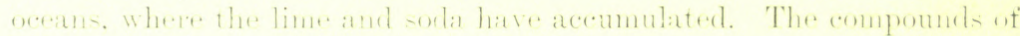

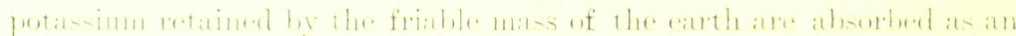

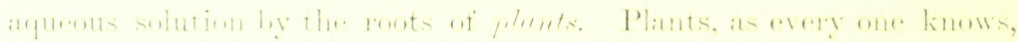

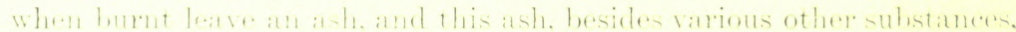

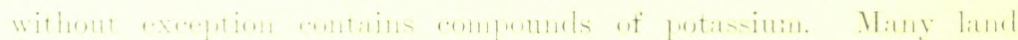

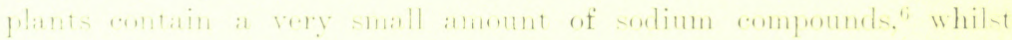

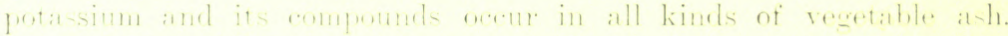

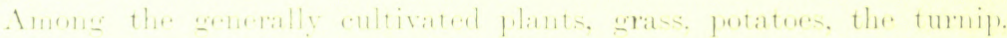

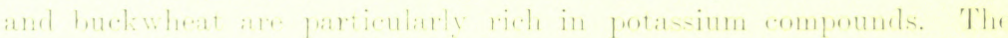

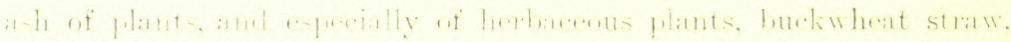

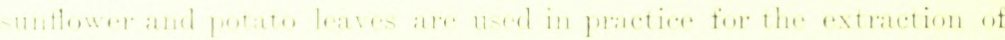

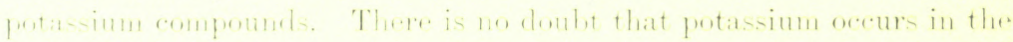

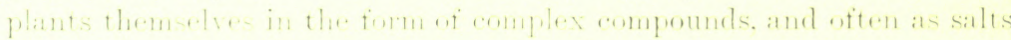

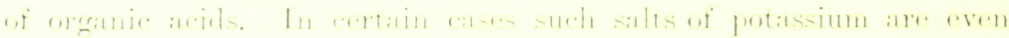

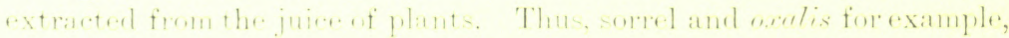

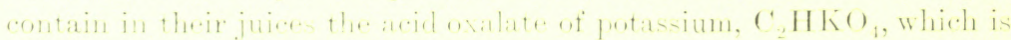


employed for removing ink stains. Grape juice contains the so-called cream of tartar, which is the acid tartrate of potassium, $\mathrm{C}_{4} \mathrm{H}_{5} \mathrm{KO}_{6}{ }^{7}$ This salt also separates as a sediment from wine. When the plants, containing one or more of the salts of potassium, are burnt, then the carbonaceous matter is oxidised, and in consequence the potassium is obtained in the ash as carbonate, $\mathrm{K}_{2} \mathrm{CO}_{3}$, which is generally known as potashes. Hence potashes occur ready prepared in the ash of plants, and therefore the ash of land plants is employed as a source for the extraction of potassium compounds. Potassium carbonate is extracted by lixiviating the ash with water. ${ }^{8}$ Potassium carbonate

7 As plants always contain mineral substances and cannot thrive in a sphere which does not contain them, especially which is free from the salts of the four basic oxides, $\mathrm{K}_{2} \mathrm{O}, \mathrm{CaO}, \mathrm{MgO}$, and $\mathrm{Fe}_{2} \mathrm{O}_{5}$, and of the four acid oxides, $\mathrm{CO}_{2}, \mathrm{~N}_{2} \mathrm{O}_{5}, \mathrm{P}_{2} \mathrm{O}_{5}$, and $\mathrm{SO}_{5}$, the question involuntarily arises as to what part these play in the development of plants. With the present store of chemical data only one answer is possible to this question, and it is still only a hypothesis. This answer was particularly clearly expressed by Professor Gustavson of the Petroffsky Agricultural Academy. Starting from the fact (Chap. XI. Note 55) that a small quantity of aluminium renders possible and facilitates the reaction of bromine on hydrocarbons at the ordinary temperature, it is easy to arrive at the conclusion, which is very probable and in accordance with many data respecting the reactions of organic compounds, that the addition of mineral substances to organic compounds lowers the temperature of reaction and in general facilitates chemical reactions in plants, and thus aids the conversion of the most simple nourishing substances into the complex component parts of the plant organism. The province of chemical reactions proceeding in organic substances in the presence of a small quantity of mineral substances has as yet been but little investigated, although there are already several disconnected data of this kind, and although a great deal is known concerning such reactions among inorganic compounds. The essence of the matter may be expressed thustwo substances, $\mathrm{A}$ and $\mathrm{B}$, do not react on each other of their own accord, but the addition of a small quantity of a third particularly active substance, $\mathrm{C}$, produces the reaction of $\mathrm{A}$ on $\mathrm{B}$, because $\mathrm{A}$ combines with $\mathrm{C}$, forms $\mathrm{AC}$, and $\mathrm{B}$ reacts on this new compound, which has a different store of chemical energy, forming the compound $\mathrm{AB}$ or its products, and setting $\mathrm{C}$ free again.

It may here be remarked that all the mineral substances necessary for plants (those enumerated at the beginning of the note) are the highest saline compounds of their elements, that they enter into the plants as salts, that the lower forms of oxidation of the same elements (for instance, sulphites and phosphites) are harmful to plants (poisonous), and that strong solutions of the salts assimilated by plants (their osmotic pressure is great and contracts the cells, as De Vries showed, see p. 322) not only do not enter into the plants but kill them (poison them).

Besides which, it will be understood from the preceding paragraph that the salts of potassinm may become exhausted from the soil by long cultivation, and that there may therefore be cases when the direct fertilisation by salts of potassium may be profitable. But manure and animal excrements, ashes, and, in general, nearly all refuse which may serve for fertilising the soil, contain a considerable quantity of potassium salts, and therefore, as regards the natural salts of potassium (Stassfurt), and especially potassium sulphate, if they often improve the crops, it is in all probability because they influence the properties of the soil. The agriculturist cannot therefore be advised to add potassium salts, without making special experiments showing the advantage of such a fertiliser on a given kind of soil and plant.

8 The animal body also contains potassium compounds, which is natural, since animals consume plants. For example, milk, and especially woman's milk, contains a somewhat 


\section{may also be obtained from the chloride by a method similar to that by which sodium carbonate is prepared from sodium chloride. There}

.considerable quantity of potassium compounds. However, cow's milk contains but little potassium salts. Sodium compounds generally predominate in animal bodies. The excrement of animals, and especially of herbivorous animals, on the contrary, often contains a large proportion of potassium salts. Thus sheep's dung is rich in them, and in washing sheep's wool salts of potassium pass into the water.

The ash of tree stems, as the already dormant portion of the plant (Chap. VIII. Note 1), contains little potash. For the extraction of potash, which was formerly (before the discovery of the Stassfurt salt) carried on extensively in the east of Russia, the ash of grasses, and the green portions of potatoes, buckwheat, \&c., are taken and treated with water (lixiviated), the solution is evaporated, and the residue ignited in order to destroy the organic matter present in the extract. The residue thus obtained is composed of raw potash. It is refined by a second dissolution in a small quantity of water, because the potash itself is very soluble in water, whilst the impurities are sparingly soluble. The solution thus obtained is again evaporated, and the residue ignited, and this potash is then calle refined potash, pearlasll. This method of treatment cannot give chemically pure potassium carbonate. A certain amount of impurities remain. To obtain chemically pure potassium carbonate, some other salt of potassium is generally taken and purified by crystallisation. Potassium carbonate crystallises with difficulty, and it cannot therefore be purified by this means, whilst other salts, such as the tartrate, acid carbonate, sulphate, or nitrate, \&c., crystallise easily and may thus be directly purified. The tartrate is most frequently taken, all the more as it is prepared in large quantities for medicinal use under the name of crean of tartar. When ignited without the access of air, it leaves a mixture of charcoal and potassium carbonate. The charcoal is then obtained in a finely-divided condition (this mixture is called 'black flux,' and is sometimes used for reducing metals from their oxides, by the aid of heat). A certain quantity of nitre is added to burn the charcoal formed by heating the cream of tartar. Potassium carbonate thus prepared is further purified by converting it into the acid salt, by passing a current of carbonic anhydride through a strong solution. $\mathrm{KHCO}_{3}$ is then formed, which is less soluble than the normal salt (as is the case with the corresponding sodium salts), and therefore crystals of the acid salt separate from the solution on cooling. When ignited, they part with their water and carbonic anhydride, and pure potassium carbonate remains behind. The physical properties of potassium carbonate distinguish it with sufficient distinctness from sodium carbonate; it is obtained from solutions as a powdery white mass, having an alkaline taste and reaction, and, as a rule, shows only traces of crystallisation. It also attracts the moisture of the air with great energy. The crystals do not contain water, but absorb it from the air, deliquescing into a saturated solution. It melts at a red heat $\left(830^{\circ}\right)$, and at a still higher temperature is even converted into vapour, as has been observed at glass works where it is employed. It is very soluble. At the ordinary temperature, water dissolves an equal weight of the salt. Crystals containing two equivalents of water separate from such a saturated solution when strongly cooled. There is no necessity to describe its reactions, because they are entirely analogous to those of sodium carbonate. When manufactured sodium carbonate was but little known, the consumption of potassium carbonate was very considerable, and even now washing soda is frequently replaced for household purposes by 'ley'-i.e. an aqueous solution obtained from ashes. It contains potassium carbonate, which acts like the sodium salt in washing tissues, linen, \&c.

A mixture of potassium and sodium carbonate fuses with much greater ease than the separate salts, and a mixture of their solutions gives salts of a fine crystalline form-for instance (Marguerite's salt), $\mathrm{K}_{2} \mathrm{CO}_{3}, 6 \mathrm{H}_{2} \mathrm{O}, 2 \mathrm{Na}_{2} \mathrm{CO}_{3}, 6 \mathrm{H}_{2} \mathrm{O}$. Crystallisation also proceeds in other multiple proportions of $\mathrm{K}$ and $\mathrm{Na}$ (in the above case $1: 2$, but $1: 1$ and $1: 3$ are known), and always with $6 \mathrm{~mol} . \mathrm{H}_{2} \mathrm{O}$. This is evidently a combination by analogy, as in alloys, solutions, \&c. 


\section{is no difficulty in obtaining any salt of potassium-for example, the sulphate ${ }^{9}$ bromide, and iodide ${ }^{10}$ - by the action of the corresponding .}

${ }^{9}$ Potassium sulphate, $\mathrm{K}_{2} \mathrm{SO}_{4}$, crystallises from its solutions in an anhydrous condition, in which respect it differs from the corresponding sodium salt, just as potassium carbonate differs from sodium carbonate. In general, it must be observed that the majority of sodium salts combine more easily with water of crystallisation than the potassium salts. The solubility of potassium sulphate does not show the same peculiarities as sodium sulphate, because it does not combine with water of crystallisation; at the ordinary temperature 100 parts of water dissolve about 10 parts of the salt, at $0^{\circ} 8.3$ parts, and at $100^{\circ}$ about 26 parts. The acid sulphate, $\mathrm{KHSO}_{4}$, obtained easily by heating crystals of the normal salt with sulphuric acid, is frequently employed in chemical practice. On heating the mixture of acid and salt, fumes of sulphuric acid are at first given off; when they cease to be evolved, the acid salt is contained in the residue. At a higher temperature (of above $600^{\circ}$ ) the acid salt parts with all the acid contained in it, the normal salt being re-formed. The definite composition of this acid salt, and the ease with which it decomposes, render it exceedingly valuable for certain chemical transformations accomplished by means of sulphuric acid at a high temperature, because it is possible to take, in the form of this salt, a strictly definite quantity of sulphuric acid, and to act on a given substance at a high temperature, which it is often necessary to do, more especially in chemical analysis. In this case, the acid salt acts in exactly the same manner as sulphuric acid itself, but the action of the latter is inconvenient at temperatures above $400^{\circ}$, because it would all evaporate, while at that temperature the acid salt still remains in a fused state, and acts with the elements of sulphuric acid on the substance taken. Hence by its means the boiling-point of sulphuric acid is raised. Thus the acid potassium sulphate is employed, for example, where for conversion of certain oxides, such as the oxides of iron, aluminium, and chromium, into salts, a high temperature is required.

Weber, by heating potassium sulphate with an excess of sulphuric acid at $100^{\circ}$, observed the formation of a lower stratum, which was found to contain a definite compound containing eight equivalents of $\mathrm{SO}_{3}$ per equivalent of $\mathrm{K}_{2} \mathrm{O}$. The salts of rubidium, cæsium, and thallium give a similar result, but those of sodium and lithium do not.

10 The bromide and iodide of potassium are used, like the corresponding sodium compounds, in medicine and photography. Potassium iodide is easily obtained in a pure state by saturating a solution of hydriodic acid with caustic potash. In practice, however, this method is rarely had recourse to, other more simple processes being employed although they do not give so pure a product. They aim at the direct formation of hydriodic acid in the liquid in the presence of potassium hydroxide or carbonate. Thus iodine is thrown into a solution of pure potash, and hydrogen sulphide passed through the mixture, the iodine being thus converted into hydriodic acid. Or a solution is prepared from phosphorus, iodine, and water, containing hydriodic and phosphoric acid; lime is then added to this solution, and calcium iodide is obtained in solution, and calcium phosphate as a precipitate. The solution of calcium iodide gives, with potassium carbonate, insoluble calcium carbonate and a solution of potassium iodide. If iodine is added to a slightly-heated solution of caustic potash (free from carbonate-that is, freshly prepared), so long as the solution is not coloured from the presence of an excess of iodine, then there is formed (as in the action of chlorine on a solution of caustic potash) a mixture of potassium iodide and iodate. On evaporating the solution thus obtained and igniting the residue, the iodate is destroyed and converted into iodide, the oxygen being disengaged, and potassium iodide only is left behind. On dissolving the residue in waterand then evaporating, cubical crystals of the anhydrous salt are obtained, which are soluble in water and alcohol, fuse and give an alkaline reaction, owing to the fact that when ignited a portion of the salt decomposes, forming potassium oxide. The neutral salt may be obtained by adding hydriodic acid to this alkaline salt until it gives an acid reaction. It is best to add some finely-divided charcoal to the mixture of iodate- 
acid on the carbonate, whilst the hydroxide, caustic potash, $\mathrm{KHO}$, which is in many respects analogous to caustic soda, is easily obtained by means of lime in exactly the same manner as sodium hydroxide is prepared from sodium carbonate. ${ }^{11}$ Therefore, in order to complete our knowledge of the alkali metals, we will only describe two salts of potassium which are of practical importance, and whose analogues have not been described in the preceding chapter.

Potassium cyanide, which presents in its chemical relations a certain analogy with the halogen salts of potassium, is not only formed accord. ing to the equation, $\mathrm{KHO}+\mathrm{HCN}=\mathrm{H}_{2} \mathrm{O}+\mathrm{KCN}$, but also whenever a nitrogenous carbon compound - for instance, animal matter - is heated in the presence of metallic potassium, or of a compound of potassium, and even when a mixture of potash and carbon is heated in a stream of nitrogen. Potassium cyanide is obtained from yellow prussiate (p. 401), which has been already mentioned and whose preparation on a large scale will be described in speaking of iron. If the yellow prussiate be ground to a powder and dried, so that it loses its water of crystallisation, then it melts at a red heat, and decomposes into carbide of iron, nitrogen, and potassium cyanide, $\mathrm{FeK}_{4} \mathrm{C}_{6} \mathrm{~N}_{6}=$ $4 \mathrm{KCN}+\mathrm{FeC}_{2}+\mathrm{N}_{2}$. After the decomposition it is found that the yellow salt has been converted into a white mass of potassium cyanide. "The carbide of iron formed collects at the bottom of the vessel. If

and iodide before igniting it, as it facilitates the evolution of the oxygen from the iodate. The iodate may also be converted into iodide by the action of certain reducing agents, such as zinc amalgam, which, when boiled with a solution containing an iodate, converts it into iodide. Potassium iodide may also be prepared by mixing a solution of ferrous iodide (it is best if the solution contains an excess of iodine) and potassium carbonate, in which case ferrous carbonate, $\mathrm{FeCO}_{3}$, is precipitated (with an excess of iodine the precipitate is granular, and contains a compound of the suboxide and oxide of iron), while potassium iodide remains in solution. Ferrous iodide, $\mathrm{FeI}_{2}$, is obtained by the direct action of iodine on iron in water. Potassium iodide considerably lowers the temperature (even by $24^{\circ}$ ), when it dissolves in water, 100 parts of the salt dissolve in 73.5 parts of water at $125^{\circ}$, in 70 parts at $18^{\circ}$, whilst the saturated solution which boils at $120^{\circ}$ contains 100 parts of salt per 45 parts of water. Solutions of potassium iodide dissolve a considerable amount of iodine; strong solutions even dissolving as much or more iodine than they contain as potassium iodide (see Chap XI. Note 64).

11 Caustic potash is not only formed by the action of lime on dilute solutions of potassium carbonate (as sodium hydroxide is prepared from sodium carbonate), but also by igniting potassium nitrate with finely-divided copper (see Note 15), and also by mixing solutions of potassium sulphate (or even of alum, $\mathrm{KAlS}_{2} \mathrm{O}_{8}$ ) and barium hydroxide, $\mathrm{BaH}_{2} \mathrm{O}_{2}$. It is sometimes purified by dissolving it in alcohol (the impurities, for example, potassium sulphate and carbonate, are not dissolved) and then evaporating the alcolool.

The specific gravity of potassium hydroxide is 2.04 , but that of its solutions (sec Chap. XII. Note 18) at $15^{\circ} \mathrm{S}=9992+90 \cdot 4 p+0 \cdot 28 p^{2}$ (here $p^{2}$ is + , and for sodium hydroxide it is -). Strong solutions, when cooled, yield a cryst̨llo-hydrate, $\mathrm{KHO}_{2} 4 \mathrm{H}_{2} \mathrm{O}$, which dissolves in water, producing (like $2 \mathrm{NaHO}, 7 \mathrm{H}_{2} \mathrm{O}$ ) cold, whilst potassium hydroxide in solution develops a considerable amount of heat. 
the mass thus obtained be treated with water, the potassium cyanide is partially decomposed by the water, but if it be treated with alcohol, then the cyanide is dissolved, and on cooling separates in a crystalline form. ${ }^{12}$ A solution of potassium cyanide has a powerfully alkaline reaction, a smell like that of bitter almonds, peculiar to prussic acid, and acts as a most powerful poison. Although exceedingly stable in a fused state, potassium cyanide easily changes when in solution. Prussic acid is so very feebly energetic that even water decomposes potassium cyanide. A solution of the salt, even without access of air, easily turns brown and decomposes, and when heated evolves ammonia and forms potassium formate; this is easily comprehensible from the representation of the cyanogen compounds which was developed in Chap. IX., $\mathrm{KCN}+2 \mathrm{H}_{2} \mathrm{O}=\mathrm{CHKO}_{2}+\mathrm{NH}_{3}$. Furthermore, as carbonic anhydride acts on potassium cyanide with evolution of prussic acid, and as potassium cyanate, which is also unstable, is formed by the action of air, it will be easily seen that solutions of potassium cyanide are very unstable. Potassium cyanicle, containing as it does carbon and potassium, is a substance which can act in a very vigorously reducing manner, especially when fused; it is therefore used as a powerful reducing agent at a red heat. A considerable quantity of potassium cyanicle is used in the arts, more particularly for the preparation of metallic solutions which are decomposed by the action of a galvanic current; thus it is very frequently employed in electro-silvering and

12 In this case it is evident that all the cyanogen which was in combination with the iron is decomposed into nitrogen, which is evolved as gas, and into carbon, which combines with the iron. In order to avoid this, potassium carbonate is added to the yellow prussiate while it is being fused. A mixture of 8 parts of anhydrous yellow prussiate and 3 parts of pure potassium carbonate is generally taken. On fusing, double decomposition takes place, resulting in the formation of ferrous carbonate and potassium cyanide. But by this method, as by the first, a pure salt is not obtained, (1) because a portion of the potassium cyanide is oxidised at the expense of the iron carbonate and forms potassium cyanate, $\mathrm{FeCO}_{3}+\mathrm{KCN}=\mathrm{CO}_{2}+\mathrm{Fe}+\mathrm{KCNO}$; (2) a portion of the iron under the action of the water again passes into solution; and (3) the potassium cyanide very easily forms oxide, which acts on the sides of the vessel in which the mixtare is heated (to avoid this iron vessels should be used), \&c. By adding one part of charcoal powder to the mixture of 8 parts of anhydrous yellow prussiate and 3 parts of potassium carbonate a mass is obtained which is free from cyanate, because the carbon absorbs the oxygen, but then it is impossible to obtain a colourless potassium cyanide by simple fusion, although this may be easily done by dissolving it in alcohol. Naturally, pure potassium cyanide may be easily obtained if hydrocyanic acid be saturated with caustic potash, and especially if caustic potash be dissolved in alcohol and hydrocyanic acid gas be passed through this solution; crystals of potassium cyanide then separate directly from the solution. Potassium cyanide is now prepared in large quantities from yellow prussiate for gilding and silvering. When fused in large quantities the action of the oxygen of the air is limited, and the entire operation may be conducted with great care, and therefore, on a large scale very pure salt is sometimes obtained. When slowly cooled, the fused salt separates in cubical crystals like potassium chloride. 
gilding. An alkaline solution is prepared, ${ }^{13}$ which is moderately stable owing to the fact that potassium cyanide in the form of certain double salts - that is, combined with other cyanides-is far more stable than when alone (yellow prussiate, which contains potassium cyanide in combination with ferrous cyanide, is an example of this). The property of potassium cyanide of giving double salts with other cyanides is very clearly shown by the fact that many metals dissolve in a solution of potassium cyanide, with the evolution of hydrogen. For example, iron, copper, and zinc act in this manner. In which case potassium hydroxide is formed ; for example-

$$
4 \mathrm{KCN}+2 \mathrm{H}_{2} \mathrm{O}+\mathrm{Zn}=\mathrm{K}_{2} \mathrm{ZnC}_{4} \mathrm{~N}_{4}+2 \mathrm{KHO}+\mathrm{H}_{2} \text {. }
$$

Gold and silver are soluble in potassium cyanide in the presence of air, in which case the hydrogen, which might be evolved in the reaction, combines with the oxygen of the air, forming water. Platinum, mercury, and tin are not dissolved in a solution of potassium cyanide, even with the access of air.

Potassium nitrate, or common nitre or saltpetre, $\mathrm{KNO}_{3}$, is chiefly used as a component part of gunpowder, in which it cannot be replaced by the sodium salt, because the latter is deliquescent. It is necessary that the nitre in gunpowder should be perfectly pure, as even small traces of sodium, magnesium, and calcium salts, especially chlorides, render the nitre and gunpowder capable of attracting moisture. Nitre may easily be obtained pure, owing to its great disposition to form crystals both large and small, which aids its separation from other salts. The considerable differences between the solubility of nitre at different temperatures aids this crystallisation. A solution of nitre saturated at its boiling point $\left(116^{\circ}\right)$ contains 335 parts of nitre to 100 parts of water, whilst at the ordinary temperature-for instance, $20^{\circ}$-. the solution is only able to retain 32 parts of the salt. Therefore, in the preparation and refining of nitre, its solution, saturated at the boiling point, is cooled, and nearly all the nitre is obtained in the form of crystals. If the solution be quietly and slowly cooled in large quantities then large crystals are formed, but if it be rapidly cooled and agitated then suall crystals are obtained. In this manner, if not all at all events the majority of the impurities present in small quantities. remain in the mother liquor. If an unsaturated solution of nitre be rapidly cooled, so as to prevent the formation of large crystals (in whose crevices the mother liquor, together with the impurities, would remain), the very minute crystals of nitre known as saltpetre flour are obtained.

13 The electro-deposition of metals generally proceeds more uniformly and gives a. purer deposit from alkaline, than from acid, solutions. 
Common nitre occurs in nature, but only in small quantities in admixture with other nitrates, and especially with sodium, magnesium, and calcium nitrates. Such a mixture of salts of nitric acid is formed in nature in fertile earth, and in those localities where, as in the soil, nitrogenous organic remains are decomposed in the presence of alkalis or alkaline bases with free access of air. This method of the formation of nitrates requires moisture, besides the free access of air, and takes place principally during warm weather. Besides which Schloessing and Müntz, by employing similar methods to Pasteur, showed that the formation of nitre in the decomposition of nitrogenous substances is accomplished by the aid of peculiar micro-organisms (ferments) without which the simultaneous action of the other necessary conditions (alkalis, moisture, a somewhat elevated temperature, air, and nitrogenous substances) cannot give nitre. In warm countries, and in temperate climates during the summer months, fertile soils produce a small quantity of nitre. In this respect India is especially known as affording a considerable supply of nitre extracted from the soil. The soil, which contains nitre, after the rainy season sometimes becomes covered during the summer with crystals of nitre, formed by the evaporation of the water in which it was previously dissolved. This soil is collected, subjected to repeated lixiviations, and treated for nitre, as will be presently described. In temperate climates nitrates are obtained from the lime rubbish of demolished buildings which have stood for many years, and especially from those portions which have touched the ground. The conditions are there very propitious to the formation of nitre, because the lime used as a cement in buildings contains the base necessary for the formation of nitrates, while the excrement, urine, and other animal refuse are sources of nitrogen. By the methodical lixiviation of this kind of rubbish a like solution of nitrogenous salts is obtained as by the lixiviation of fertile soil. A similar solution is also obtained by the lixiviation of the so-called nitre plantations. They are composed of manure interlaid with brush-wood, and strewn over with ashes and other alkaline and lime rubbish. These nitre plantations are set up in those localities where the manure is not required for the fertilisation of the soil, as, for example, in the southeastern 'black earth' governments of Russia. The same process of oxidation of nitrogenous matter free to the access of air and moisture during the warm season in the presence of alkalis takes place in nitre plantations as is accomplished in the fertile soil and in the walls of buildings. From all these sources there is obtained a solution containing different salts of nitric acid mixed with soluble organic matter. The simplest method of treating this impure solution of nitre

VOL. I. 
is to add a solution of potassium carbonate, or to simply treat it with ashes containing this substance. The potassium carbonate enters into double decomposition with the calcium and magnesium salts, forming insoluble carbonates of these bases and leaving the nitre in solution. Thus, for instance, $\mathrm{K}_{2} \mathrm{CO}_{3}+\mathrm{Ca}\left(\mathrm{NO}_{3}\right)_{2}=2 \mathrm{KNO}_{3}+\mathrm{CaCO}_{3}$. Both calcium and magnesium carbonates are insoluble, and therefore after treatment with potassium carbonate the solution no longer contains salts of these metals but only the salts of sodium and potassium together with organic matter. The latter partially separates on heating in an insoluble form, and is entirely destroyed by heating the nitre to a low red heat. The nitre thus obtained is easily purified by repeated crystallisation. The greater part of the nitre used for making gunpowder is now obtained from the sodium salt Chili saltpetre or cubic nitre, which occurs, as has been already mentioned, in nature. The conversion of this salt into common nitre is also carried on by means of a double decomposition. This is done either by adding potassium carbonate (when, on mixing the strong and hot solutions, sodium carbonate is directly obtained as a precipitate), or as is now most frequent, potassium chloride. When a mixture of strong solutions of potassium chloride and sodium nitrate is evaporated, sodium chloride first separates, because this salt, which is formed by the double decomposition $\mathrm{KCl}+\mathrm{NaNO}_{3}=\mathrm{KNO}_{3}+\mathrm{NaCl}$, is almost equally soluble in hot and cold water ; on cooling, therefore, a large amount of potassium nitrate separates from the saturated solution, while the sodium chloride remains dissolved. The nitre is ultimately purified by recrystallisation and by washing with a saturated solution of nitre, which cannot dissolve a further quantity of nitre but only the impurities.

Nitre is a colourless salt having a peculiar cool taste. It easily crystallises in long striated six-sided rhombic prisms terminating in similar pyramids. Its crystals (sp. gr. 1.93) do not contain water, but their cavities generally contain a certain quantity of the solution from which they have crystallised. For this reason in refining nitre, larga crystals are not obtained but saltpetre flour is prepared. At a low red heat $\left(339^{\circ}\right)$ nitre melts to a colourless liquid. ${ }^{14}$ Potassium nitrate at the ordinary temperature and in a solid form is inactive and stable,

14 Before fusing, the crystals of potassium nitrate change their form, and take the same form as sodium nitrate-that is, they change into rhombohedra. Nitre crystallises from hot solutions, and in general under the influence of a rise of temperature, in a different form from that given at the ordinary or lower temperatures. Fused nitre solidifies into a radiated crystalline mass; but it does not exhibit this structure if metallic chlorides be present, so that this method may be taken advantage of to determine the degree of purity of nitre. With a small trace of sodium chloride, the mass of nitre solidified after fusion does not present any signs of crystallisation in the middle.

Carnelley and Thomson (1888) determined the fusing point of mixtures of potassium 
but at a high temperature it acts as a powerful oxidising agent, because it gives up a considerable amount of oxygen to substances mixed with it. ${ }^{1.5}$ When thrown on incandescent charcoal it brings about its rapid combustion, and a mechanical mixture of powdered charcoal and nitre ignites when brought into contact with a red-hot substance, and continues to burn by itself. In this action, nitrogen is evolved, and the oxygen goes to oxidise the charcoal, in consequence of which potassium carbonate and carbonic anhydride are formed: $4 \mathrm{KNO}_{3}+5 \mathrm{C}=2 \mathrm{~K}_{2} \mathrm{CO}_{3}+3 \mathrm{CO}_{2}+2 \mathrm{~N}_{2}$. This phenomenon depends on the fact that oxygen in combining with carbon evolves more heat than it does in combining with nitrogen. Hence when once the combustion has been started at the expense of the nitre, it is able to go on without requiring the aid of external heat. A like oxidation or combustion at the expense of the contained oxygen proceeds when nitre is heated with different combustible substances. If a mixture of sulphur and nitre be thrown upon a red-hot surface, then the sulphur burns, forming potassium sulphate and sulphurous anhydride. In this case, also, the nitrogen of the nitre is evolved as gas : $2 \mathrm{KNO}_{3}+2 \mathrm{~S}=\mathrm{K}_{2} \mathrm{SO}_{4}+\mathrm{N}_{2}$ $+\mathrm{SO}_{2}$. A similar phenomenon takes place when nitre is heated with

and sodium nitrates. The first salt fuses at $339^{\circ}$ and the second at $316^{\circ}$, and if $p$ be the percentage amount of potassium nitrate, then the result obtained-

$\begin{array}{ccccccccc}p_{s}=10 & 20 & 30 & 40 & 50 & 60 & 70 & 80 & 90 \\ 298^{\circ} & 283^{\circ} & 268^{\circ} & 242^{\circ} & 231^{\circ} & 231^{\circ} & 242^{\circ} & 284^{\circ} & 306^{\circ}\end{array}$

which confirms Shaffgotsch's observation (1857) that the lowest fusing point (about 231 ${ }^{\circ}$ ) is given by mixing molecular quantities $(p=54 \cdot 3)$ of the salts - that is, in the formation of the alloy, $\mathrm{KNO}_{3}, \mathrm{NaNO}_{3}$.

A somewhat similar result was discovered by the same observers for the solubility of mixtures of these salts at $20^{\circ}$ in 100 parts of water. Thus, if $p$ be the weight of potassium nitrate mixed with $100-p$ parts by weight of sodium nitrate taken for solution, and $c$ be the quantity of the mixed salts which dissolves in 100 , the solubility of sodium nitrate being 85 , and of potassium nitrate 34 , parts in 100 parts of water, then-

$\begin{array}{rrrrrrrrr}p=10 & 20 & 30 & 40 & 50 & 60 & 70 & 80 & 90 \\ c=110 & 136 & 136 & 138 & 106 & 81 & 73 & 54 & 41\end{array}$

The maximum solubility proved not to answer to the most fusible mixture, but to one much richer in sodium nitrate.

Both these phenomena show that in homogeneous liquid mixtures the chemical forces that act between substances are the same as those that determine the molecular weights of substances, even when the mixture consists of such analogous substances as potassium and sodium nitrates, between which there is no direct chemical interchange. It is instructive to note also that the maximum solubility does not correspond with the minimum fusing point, which naturally depends on the fact that a third substance, namely water, participates in solution, although an attraction between the salts, like that which exists between sodium and potassium carbonates (Note 8), also partially acts.

15 Fused nitre, with a further rise of temperature, disengages oxygen and then nitrogen. The nitrite $\mathrm{KNO}_{2}$ is first formed and then potassium oxide. The admixture of certain metals-for example, of finely-divided copper-aids the last decomposition. The oxygen in this case naturally passes over to the metal. 


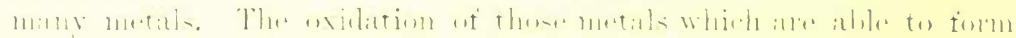

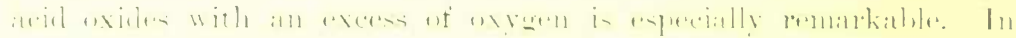

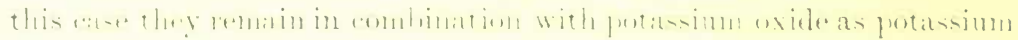

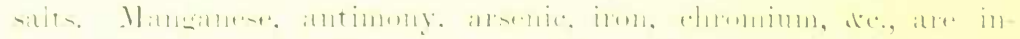

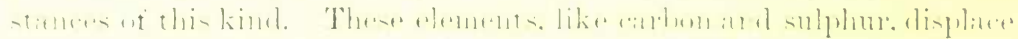

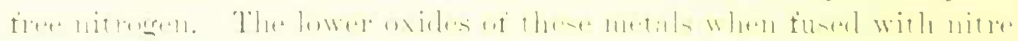

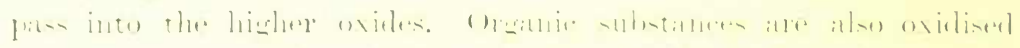

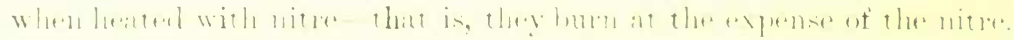

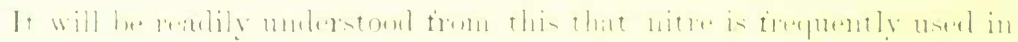

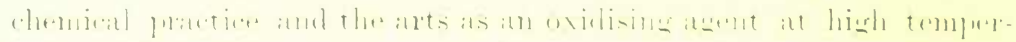

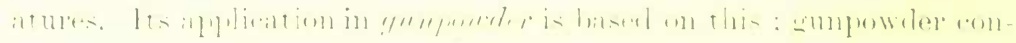

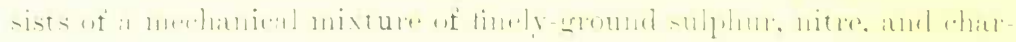

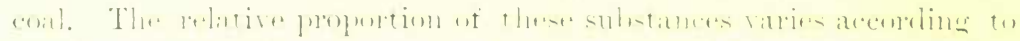

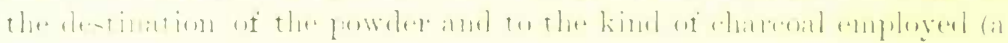

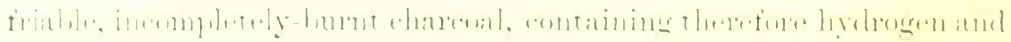

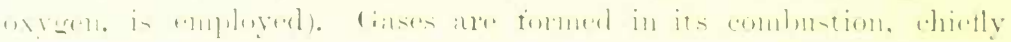

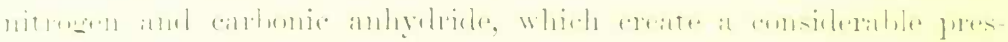

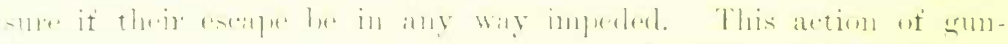

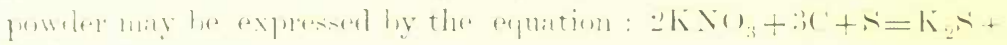
$\therefore(1)+X$.

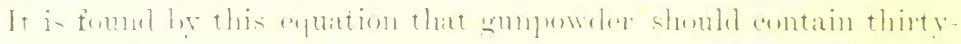

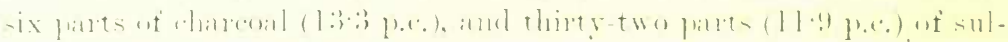

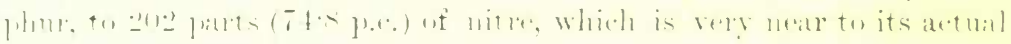

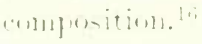

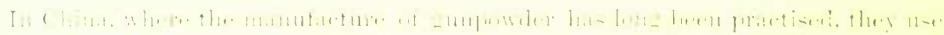

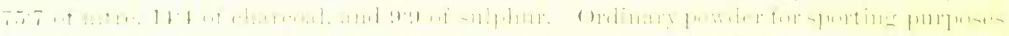

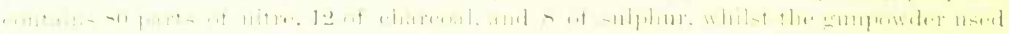

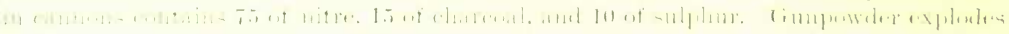

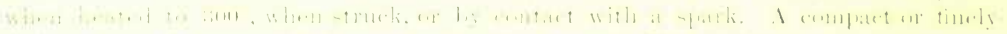

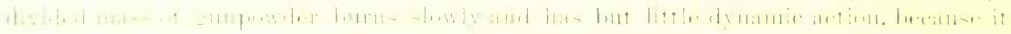

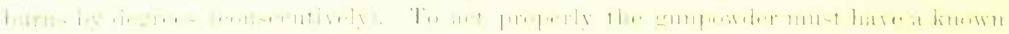

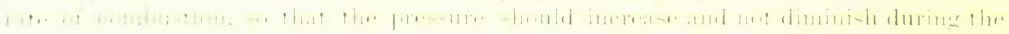

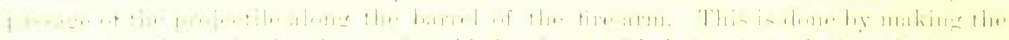

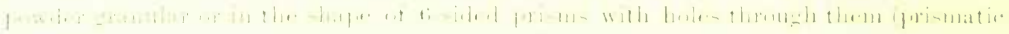
1 w w. in.

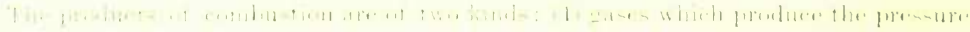

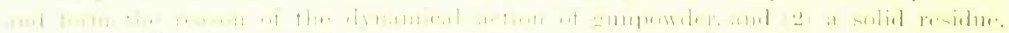

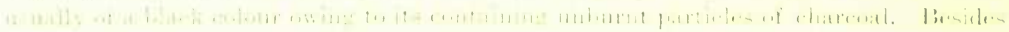

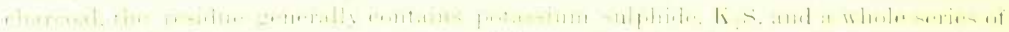

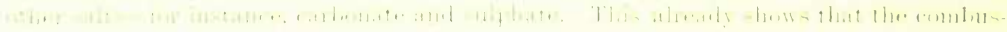

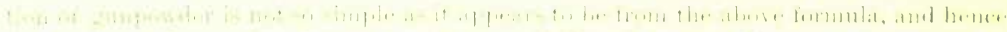

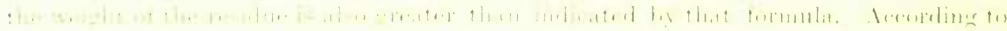

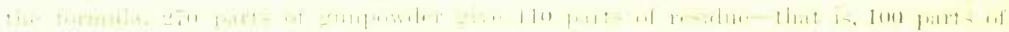

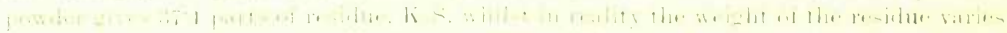

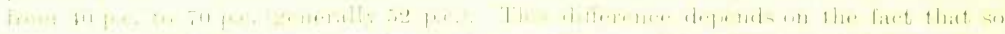

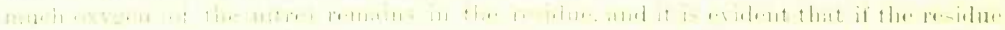

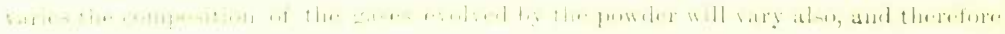


Potassium was obtained like sodium ; first by the action of a galvanic current, then by reduction of the hydroxide by means of metallic iron, and lastly, by the action of charcoal on the carbonate at a high temperature. The preparation of metallic potassium differs, however, from that of sodium in that it easily combines with carbonic oxide, forming an explosive and inflammable mass. ${ }^{17}$

However, in its essential points the method of its extraction is not different from that of sodium, because potassium is quite as volatile as sodium, if not more so. At the ordinary temperature, potassium is even softer than sodium, its freshly-cut surfaces present a whiter colour than sodium, but, like the latter, and with even greater ease, it oxidises in moist air. It is brittle at low temperatures, but is quite soft at $25^{\circ}$, and melts at $58^{\circ}$. At a low red heat $\left(720^{\circ}\right)$ it distils without change, forming a green vapour, whose density, ${ }^{18}$ according to

the entire process will be different in different cases. The difference in the composition of the gases and residue depends, as the researches of Gay-Lussac, Shishkoff and Bunsen, Noble and Abel, Federoff, \&c., show, on the conditions under which the combustion of the powder proceeds. When gunpowder buins in an open space, the gaseous products which are formed do not remain in contact with the residue, and then a considerable portion of the charcoal entering into the composition of the powder remains unburnt because the charcoal burns after the sulphur at the expense of the oxygen of the nitre. In this extreme case the commencement of the combustion of the gunpowder may be expressed by the equation, $2 \mathrm{KNO}_{3}+3 \mathrm{C}+\mathrm{S}=2 \mathrm{C}+\mathrm{K}_{2} \mathrm{SO}_{4}+\mathrm{CO}_{2}+\mathrm{N}_{2}$. The residue in a blank cartridge often consists of a mixture of $\mathrm{C}, \mathrm{K}_{2} \mathrm{SO}_{4}, \mathrm{~K}_{2} \mathrm{CO}_{3}$, and $\mathrm{K}_{2} \mathrm{~S}_{2} \mathrm{O}_{3}$. If the combustion of the gunpowder be impeded-if it take place in a cartridge in the barrel of a gun-the quantity of potassium sulphate will first be diminished, then the amount of sulphite, whilst the amount of carbonic anhydride in the gases and the amount of potassium sulphide in the residue will increase. The quantity of charcoal entering into the action will then be also increased, and hence the amount in the residue will decrease. Under these circumstances the weight of the residue will be less-for example, $4 \mathrm{~K}_{2} \mathrm{CO}_{5}+4 \mathrm{~S}=\mathrm{K}_{2} \mathrm{SO}_{4}+3 \mathrm{~K}_{2} \mathrm{~S}+4 \mathrm{CO}_{2}$. Besides which, carbonic oxide has been found in the gases, and potassium bisulphide, $\mathrm{K}_{2} \mathrm{~S}_{2}$, in the residue of gunpowder. The amount of potassium sulphide, $\mathrm{K}_{2} \mathrm{~S}$, increases with the completeness of the combustion, and is formed in the residue at the expense of the potassium sulphite. In recent times the knowledge of the action of gunpowder and other explosives has made much progress, and has developed into a vast province of artillery science.

17 The substances obtained in this case are mentioned in Chap. IX. Note 31.

$18 \mathrm{~A}$. Scott (1887) determined the vapour densities of many of the compounds of the alkali elements in a platinum vessel heated in a furnace and previously filled with nitrogen. But these, the first data concerning a subject of great importance, have not yet been sufficiently fully described, nor have they received as much attention as could be desired. Taking the density of hydrogen as unity, Scott found the vapour densities of the following substances to be-

$\begin{array}{llllr}\mathrm{Na} & 12 \cdot 75 & (12 \cdot 5) . & \mathrm{KI} & 92(84) . \\ \mathrm{K} & 19 & (19 \cdot 5) . & \mathrm{RbCl} & 70(60) . \\ \mathrm{C} 8 \mathrm{Cl} & 89 \cdot 5 & (84 \cdot 2) . & \mathrm{CsI} & 133(180) . \\ \mathrm{FeCl}_{3} 68 & & \mathrm{AgCl} & 80(71 \cdot 7) .\end{array}$

In brackets are given the densities corresponding with the formulæ, according to Arogadro-Gerhardt's law. This figure is not giren for $\mathrm{FeCl}_{3}$, because in all probability 
A. Scott, is equal to 19 (if that of hydrogen=1). This shows that the molecule of potassium (like that of sodium, mercury, and zinc) contains but one atom. This is also the case with other metals, judging by recent researches. ${ }^{19}$ The specific gravity of potassium at $15^{\circ}$ is 0.87 , and is therefore less than that of sodium, as is also the case with all its compounds. ${ }^{20}$ Potassium decomposes water with great ease at the ordinary temperature, evolving 45000 heat units per atomic weight. The heat evolved is sufficient to inflame the hydrogen, the flame being coloured violet from the presence of particles of potassium. ${ }^{21}$

With regard to the relation of potassium to hydrogen and oxygen, it is closely analogous to sodium in this respect. Thus, with hydrogen it forms potassium hydride, $\mathrm{K}_{2} \mathrm{H}$ (between $200^{\circ}$ and $411^{\circ}$ ), and with oxygen it gives a suboxide $\mathrm{K}_{4} \mathrm{O}$, oxide $\mathrm{K}_{2} \mathrm{O}$, and peroxide, only more oxygen enters into the composition of the latter than in sodium per-

under these conditions (the temperature at which it was determined) a portion of the $\mathrm{FeCl}_{3}$ was decomposed. If it was not decomposed, then a density 81 would correspond with the formula $\mathrm{FeCl}_{3}$, and if the decomposition were $\mathrm{Fe}_{2} \mathrm{Cl}_{6}=2 \mathrm{FeCl}_{2}+\mathrm{Cl}_{2}$, then the deusity should be 54 . With regard to the silver chloride, there is reason to think that the platinum decomposed this salt.

The majority of Scott's results so closely correspond with the formulæ that a better concord cannot be expected in such determinations.

19 The molecules of non-metals are more complex-for instance, $\mathrm{H}_{2}, \mathrm{O}_{3}, \mathrm{Cl}_{2}$, \&c. But arsenic, whose superficial appearance recalls that of metals, but whose chemical properties approach more nearly to the non-metals, has a complex molecule containing $\mathrm{As}_{4}$. With respect to the vapour of iodine, see Chap. VII. p. 313.

20 As the atomic weight of potassium is greater than that of sodium, therefore the volumes of the molecules, or the quotients of the molecular weight by the specific gravity, for potassium compounds are greater than those of sodium compounds, because both the denominator and numerator of the fraction augment. We cite for comparison the volumes of the corresponding compounds-

$\begin{array}{llllll}\mathrm{Na} 24 & \mathrm{NaHO} 18 & \mathrm{NaCl} 28 & \mathrm{NaNO}_{5} 37 & \mathrm{Na}_{2} \mathrm{SO}_{4} 54 \\ \mathrm{~K} & 45 & \mathrm{KHO} 27 & \mathrm{KCl} 39 & \mathrm{KNO}_{3} 48 & \mathrm{~K}_{2} \mathrm{SO}_{4} 66\end{array}$

21 The same precautions must be taken in decomposing water by potassium as have to be observed with sodium (Chap. II. Note 8).

It must be observed that potassium decomposes carbonic anhydride and carbonic oxide when heated, the carbon being liberated and the oxygen taken up by the metal, whilst on the other hand charcoal takes up oxygen from potassium, as is seen from the preparation of potassium by heating potash with charcoal, hence the reaction $\mathrm{K}_{2} \mathrm{O}+\mathrm{C}=$ $\mathrm{K}_{2}+\mathrm{CO}$ is reversible and the relation is the same in this case as between hydrogen and zinc. Nothing of the kind could be expected from a comparison of the quantities of heat evolved in the formation of these compounds, for charcoal in combining with oxygen to form carbonic oxide evolves (for its molecular weight) only about 30000 heat units (Chap. IX. Note 25), whilst potassium in forming the oxide $\mathrm{K}_{2} \mathrm{O}$ gives about 100000 units, and iron in forming ferrous oxide, $\mathrm{FeO}$, evolves about 70000 units. It is evident that the decomposition of potassium oxide by charcoal is accompanied by the absorption of a large amount of heat, and the reverse reaction-which, however, proceeds with greater difficulty-with the development of heat, which again shows the impossibility of judging the direction in which a reaction will proceed from thermal data. 
oxide ; potassium peroxide contains $\mathrm{KO}_{2}$, but it is probable that in the combustion of potassium an oxide $\mathrm{KO}$ is also formed. Potassium, like sodium, is soluble in mercury. ${ }^{22}$ In a word, the relation between sodium and potassium is as close at that between chlorine and bromine, or, better still, between fluorine and chlorine, as the atomic weight of sodium, 23, is as much greater than that of fluorine, 19, as that of potassium, 39 , is greater than that of chlorine, 35.5 .

The resemblance between potassium and sodium is so great that their compounds can only be easily distinguished in the form of certain of their salts. For instance, the acid potassium tartrate, $\mathrm{C}_{4} \mathrm{H}_{5} \mathrm{KO}_{6}$ (cream of tartar), is distinguished by its sparing solubility in water and in alcohol, and in a solution of tartaric acid, whilst the corresponding sodium salt is easily dissolved. Therefore, if a solution of tartaric acid be added in considerable excess to the solutions of the majority of potassium salts, then a precipitate of the sparingly-soluble acid salt is formed, which does not occur with salts of sodium. The chlorides $\mathrm{KCl}$ and $\mathrm{NaCl}$ in solutions easily give double salts $\mathrm{K}_{2} \mathrm{PtCl}_{6}$ and $\mathrm{Na}_{2} \mathrm{PtCl}_{6}$, with platinic chloride, $\mathrm{PtCl}_{4}$, and the solubility of these salts is very different, especially in a mixture of alcohol and ether. The sodium salt is easily soluble, whilst the potassium salt is insoluble or almost so, and therefore the reaction with platinic chloride is that most often used for the separation of potassium from sodium, as is more fully described in works on analytical chemistry.

It is possible to discover the least traces of these metals in admixture together, by means of their property of imparting different colours to a flame. The presence of a salt of sodium in a flame is recognised by a brilliant yellow coloration, and a pure potassium salt colours a colourless flame violet. However, in the presence of a sodium salt the pale violet coloration given by a potassium salt is quite undistinguishable, and it is at first sight impossible in this case to discover the potassium salt in the presence of that of sodium. But by decomposing the light given by a flame coloured by these metals, or a mixture of them, by means of a prism, they are both easily distinguishable, because

22 The definite crystalline amalgam of potassium contains twice as much mercury, $\mathrm{KHg}_{2}$ (as the potassium peroxide contains twice as much oxygen, Chap. XII. Note 39).

Potassium forms alloys with sodium in all proportions. The alloys containing 1 and $: 3$ equivalents of potassium to one equivalent of sodium are liquids, like mercury at the ordinary temperature. Joannis, by determining the amount of heat developed by these alloys in decomposing water, found the evolution for $\mathrm{Na}_{2} \mathrm{~K}, \mathrm{NaK}, \mathrm{NaK}_{2}$, and $\mathrm{NaK}_{3}$ to be $\cdot 44 \cdot 5,44 \cdot 1,43 \cdot 8$ and $44 \cdot 4$ thousand heat units respectively (for $\mathrm{Na} 42 \cdot 6$ and for $\mathrm{K} 45 \cdot 4$ ). The formation of the alloy $\mathrm{NaK}_{2}$ is therefore accompanied by the development of heat, whilst the other alloys may be regarded as solutions of potassium or sodium in this alloy. In any case a fall of the temperature of fusion is evident in this instance as in the alloys of nitre (Note 14). 
the yellow light emitted by the sodium salt depends on a group of light rays having a definite index of refraction which corresponds with the yellow portion of the solar spectrum, having the index of refraction of the Frauenhofer line (strictly speaking, group of lines) D, whilst the salts of potassium give a light in which these rays are entirely wanting, but which contain rays of a red and violet colour. Therefore, if a potassium salt occur in a flame, then, on decomposing the light (from

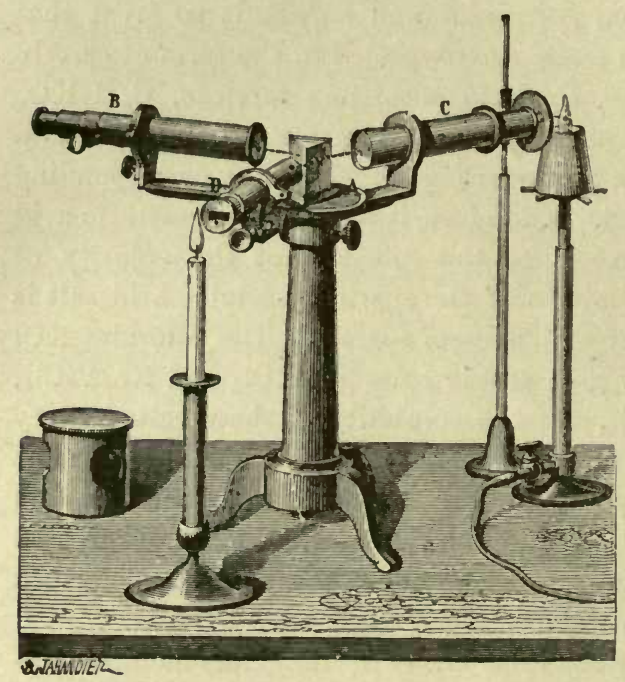

Fig. 72A - Spectroscope. The prism and table are covered with an opaque cover. The spectrum obtained from the flame coloured by a substance introduced on the wire is viewed through $\mathrm{B}$. A light is placed before the scale $\mathrm{D}$ in order to illuminate the image of the scale reflected through $\mathrm{B}$ by the side of the prism. and three tubes directed on the plane of the refracting angle of the prism. One of the tubes, $\mathrm{C}$, has a vertical slit at the end, giving access to the light to be tested, which then passes into the tube (collimator),

23 For accurate measurements and comparative researches more complicated spectroscopes are required which give a greater dispersion, and which are furnished for this: purpose with several prisms-for example, in Browning's spectroscope the light passes through six prisms, and then, having undergone an entire internal reflection, passes through the upper portion of the same six prisms, and again by an entire internal reflection passes into the ocular tube. With such a powerful dispersion the relative position of the spectral lines may be determined with accuracy. For the absolute and exact determination of the wave lengths it is particularly important that the spectroscope should be furnished with diffraction gratings. The construction of spectroscopes destined for special purposes (for example, for investigating the light of stars, or for determining the absorption spectra in microscopic preparations, \&c.) is exceedingly varied Details of the subject must be looked for in works on physics and on spectrum analysis. Among the latter the best kuown for their completeness and merit are those of Roscoe, Kayser, Vogel, and Lecog de Boisbaudran. 
which gives the rays a parallel direction. The rays of light having passed through the slit, and having become parallel, are refracted and dispersed in the prism, and the spectrum formed is observed through the orifice of the other telescope B. The third tube D contains a horizontal transparent scale (at the exterior end) which is divided into equal divisions. The light from a source such as a gas burner or candle placed before this tube, passes through the scale, and its image is thus reflected on that face of the prism which stands before the telescope $B$, so that the image of the scale is seen through this telescope simultaneously with the spectrum given by the rays passing through the slit of the tube C. In this manner the image of the scale and the spectrum given by the source of light under investigation are seen simultaneously. If the sun's rays be directed through the slit of the tube $\mathrm{C}$, then the observer looking through the orifice of $\mathrm{B}$ will see the solar spectrum, and (if the aperture of the slit be narrow and the apparatus truly adjusted) the dark Frauenhofer lines in it.24 Smallsized spectroscopes are usually so adjusted that (looking through B) the violet portion of the spectrum is seen to the right and the red portion to the left, and the Frauenhofer line D (in the bright yellow portion of the spectrum) is situated on the 50th division of the scale. ${ }^{25}$ If the light emitted by an incandescent solid-for example, the Drummond light-be passed through the spectroscope, then all the colours of the solar spectrum are seen, but not the Frauenhofer lines. Let us now consider what will happen if the light from a flame coloured by various salts be passed through the spectroscope. This is done by placing a Bunsen gas burner (or the pale flame of hydrogen gas issuing from a platinum orifice) giving so pale a flame that its spectrum will be invisible before the slit. If any compound of sodium be placed in the flame of

24 The arrangement of all the parts of the apparatus so as to give the clearest possible vision and accuracy of observation must evidently precede every kind of spectroscopic determination. Details concerning the practical use of the spectroscope must be also looked for in special works on the subject. In this treatise the reader is supposed to have a certain knowledge of the physical data respecting the refraction of light, and its dispersion and diffraction, and the theory of light, which allows of the determination of the length of the waves of light in absolute measures on the basis of observations with diffraction gratings, the distance between whose divisions may be easily measured in fractions of a millimetre : by such means it is possible to determine the wave lengths of definite rays of light.

${ }^{25}$ In order to give an idea of the several sizes of the scale, we may observe that the common spectrum extends from the zero of the scale (where the red portion is situated) to the 170th division (where the end of the visible violet portion of the spectrum is situated), and that the Frauenhofer line A (the extreme prominent line in the red) corresponds with the 17 th division of the scale; the Frauenhofer line $F$ (at the beginning of the blue, near the green colour) is situated on the 90th division, and the line $G$, which is clearly seen in the beginning of the violet portion of the spectrum, corresponds with the 127 th division of the scale. 
the gas burner (for which purpose a platinum wire on whose end sodium chloride is fused is fixed to the stand), then the flame is dyed a yellow colour, and on looking through the spectroscope the observer will see a bright yellow line falling upon the 50th division of the scale, which is seen together with the spectrum in the telescope. No yellow lines of other refractive index, nor any rays of any other colour, will be seen, and, therefore, the spectrum corresponding with sodium compounds consists of yellow rays of that index of refraction which belong to the Frauenhofer (black) line D of the solar spectrum. If a potassium salt be introduced into the flame instead of a sodium salt, then two bands will be seen which are much feebler than the bright sodium band -namely, one red line near the Frauenhofer line $\mathrm{A}$ and another violet line. Besides which, a pale, almost continuous, spectrum will be observed in the central portions of the scale. If a mixture of sodium and potassium salts be now introduced into the flame, three lines will be simultaneously seen-namely, the red and pale violet lines of potassium and the yellow line of sodium. In this manner it is possible, by the aid of the spectroscope, to determine the relation between the spectra of metals and known portions of the solar spectrum. The continuity of the latter is interrupted by dark lines (that is, by an absence of light of a definite index of refraction), termed the Frauenhofer lines of the solar spectrum. It has been shown by careful observations (by Frauenhofer, Brewster, Foucault, Ångström, Kirchhoff, Cornu, Lockyer, Dewar, and others), that there exists an exact agreement between the spectra of certain metals and certain of the Frauenhofer lines. Thus the bright yellow sodium line exactly corresponds with the dark Frauenhofer line D of the solar spectrum. A similar agreement is observed in the case of many other metals. This is not an approximate or chance correlation. In fact, if a spectroscope having a large number of refracting prisms and a high magnifying power be used, then it is seen that the dark line $\mathrm{D}$ of the solar spectrum consists of an entire system of closely adjacent but definitely situated fine and wide (sharp, distinct) dark lines, ${ }^{26}$ and an exactly similar group of bright lines is obtained when the yellow sodium line is examined through the same apparatus, so that each bright sodium line exactly corresponds with a dark line in the solar spectrum. In the common spectroscopes which are usually employed in chemical research, one yellow band, which does not split

${ }^{25}$ The two most distinct lines of $\mathrm{D}$, or of sodium, have wave lengths of $589^{\circ} 5$ and $588^{\circ} 0$ millionths of a millimeter, besides which fainter and fainter lines are seen whose wave lengths in millionth parts of a millimeter are $588^{\circ} 7$ and $588 \cdot 1,616.0$ and $615 \cdot 4,515.5$ and $515 \cdot 2,498 \cdot 3$ and $498 \cdot 2, \& c$., according to Liveing and Dewar. Many strive to find a simple relation, subject to a law, in the wave lengths, both of these pairs of lines and those of other elements. 
up into thinner lines, is seen instead of the system of sodium lines, owing to the small dispersive power of the prism and the considerable aperture of the slit of the object tube.

This conformity of the bright lines formed by sodium with the dark lines of the solar spectrum cannot be accidental. This conclusion is further confirmed by the fact that the bright lines of other metals correspond with dark lines of the solar spectrum. Thus, for example, a series of sparks passing between the iron electrodes of a Ruhmkorff coil gives 450 very distinct lines characterising this metal. All these 450 bright lines, or the whole spectrum corresponding with iron, is repeated, as Kirchhoff showed, in the solar spectrum as dark Frauenhofer lines which occur in exactly the same situations as the bright lines occur in the iron spectrum, just as the sodium lines correspond with the band $\mathrm{D}$ in the solar spectrum. Many observers have in this manner simultaneously studied the solar spectrum and the spectra of different metals, and discovered in the former lines which correspond not only with sodium and iron, but also with many other metals. ${ }^{27}$ The spectra of such elements as hydrogen, oxygen, nitrogen, and other gases may be observed in the so-called Geissler's tubes-that is, in glass tubes filled with rarefied gases, through which the discharge of a Ruhmkorff's coil is passed. Thus hydrogen gives a spectrum composed

27 The most accurate investigations made in this respect are carried on with spectra obtained by diffraction, because in this case the position of the dark and bright lines does not depend on the index of refraction of the material of the prism, nor on the dispersive power of the apparatus. The best-that is, the most general and accurate-method of expressing the results of such determinations consists in determining the lengths of the waves corresponding to the rays of a definite index of refraction. We will express this wave length in millionth parts of a millimetre (the ten millionth parts are already doubtful, and fall within the limits of error). In order to illustrate the relation between the wave lengths and the positions of the spectrum, we will cite the wave lengths corresponding with the chief Frauenhofer lines and colours of the spectrum.

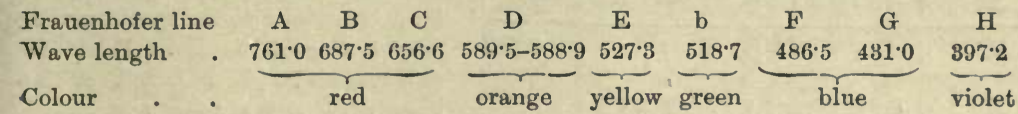

In the following table are given the wave lengths of the light rays (the longest and most distinct, see later) for certain elements, those in black type being the most clearly defined and distinct lines, which are easily obtained either in the flame of a Bunsen's burner, or in Geissler's tubes, or in general, by an electric discharge. These lines refer to the elements (the lines of compounds are different, as will be afterwards explained, but many compounds are decomposed by the flame or by an electric discharge), and furthermore to the elements in an incandescent and rarefied gaseous state, for the spectra sometimes vary considerably with a variation of temperature and pressure.

It may be said that the red colour corresponds with lines having a wavelength of from 780 (with a greater wave length the lines are hardly visible, and are ultra red) to 650 , the orange from 650 to 590 , the yellow from 590 to 520 , the green from 520 to 490 , the 
of three lines-a red line corresponding with the Frauenhofer line C, a green line corresponding with the line F, and a violet line corre-

\begin{tabular}{|c|c|}
\hline 政 & 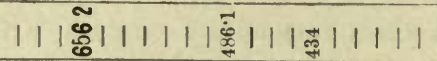 \\
\hline ; & 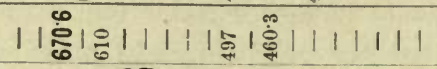 \\
\hline$\stackrel{\pi}{\pi}$ & 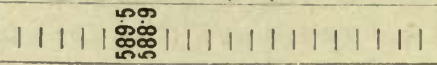 \\
\hline 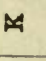 & 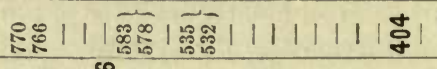 \\
\hline 우 & 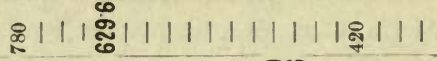 \\
\hline 8 & 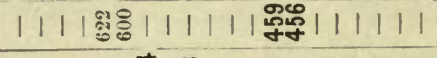 \\
\hline 40 & 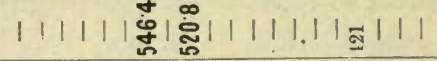 \\
\hline हี & 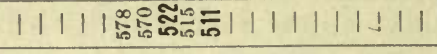 \\
\hline \&̊. & 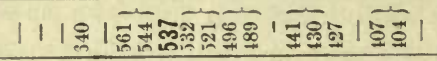 \\
\hline 音 & 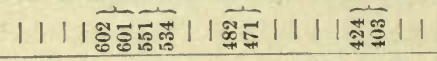 \\
\hline 番 & 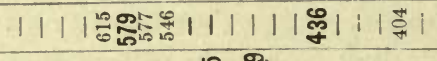 \\
\hline 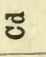 & 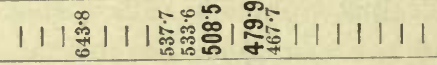 \\
\hline ถี & 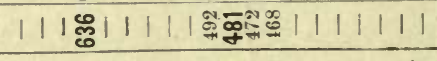 \\
\hline$\stackrel{\infty}{*}$ & 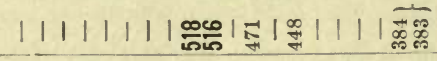 \\
\hline ळ & 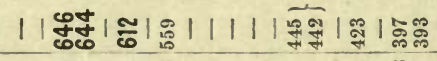 \\
\hline 㟔 & 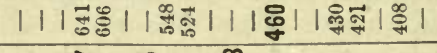 \\
\hline ๓ึ & 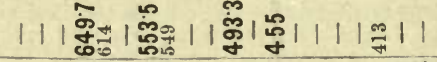 \\
\hline$\overline{4}$ & 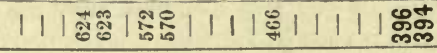 \\
\hline छ็ & 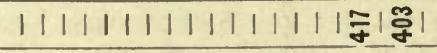 \\
\hline 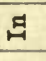 & 1111\%111:8111511111웅 \\
\hline$\vec{F}$ & 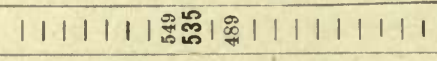 \\
\hline 뽏 & 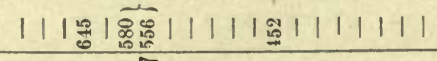 \\
\hline คి & 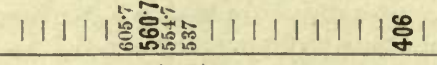 \\
\hline H & 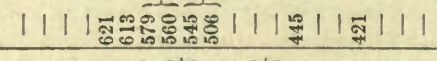 \\
\hline 蛊 & 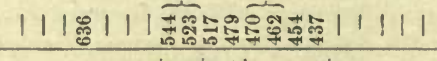 \\
\hline छี & 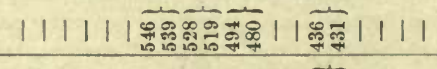 \\
\hline $0^{\circ}$ & 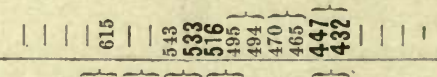 \\
\hline$\dot{4}$ & 11 \& \\
\hline
\end{tabular}

blue from 490 to 420 , and the violet from 420 to 380 millionth parts of a millimetre. Beyond 380 the lines are scarcely visible, and belong to the ultra-violet.

In this table, which is arranged in conformity to the image of the spectra as they are seen (the red lines on the left hand and the violet on the right hand side), the figures in black type correspond with lines which are so bright and distinctly visible that they may easily be made use of, both in determining the relation between the divisions of the scale and the wave lengths, and in determining the admixture of a given element with another. The brackets join those lines between which several other lines are clearly visible if the dispersive power of the spectroscope permits distinguishing the neighbouring lines. In the ordinary laboratory spectroscopes with one prism, even. with all possible precision of arrangement and with a brilliancy of light permitting the observations being made with a very narrow aperture, the lines whose wave lengths only differ by 2-3 millionth parts of a millimetre, are blurred together; and with a wide aperture a series of lines differing by even as much as $20^{\circ}$ millionths of a millimetre appear as one wide line. With a faint light (that is, with a small quantity of light entering into the spectroscope) only the most brilliant lines are clearly visible. The length of the lines does not always correspond with their brilliancy. According to Lockyer this length is determined by placing the carbon electrodes (between which the incandescent vapours of the metals are formed), not. horizontally to the slit (as they are generally placed, to give more light), but vertically to it. Then certain lines appear long and others short. As a rule (Lockyer, Dewar, Cornu), the longest lines are those with which it is easiest to obtain reversed 
sponding with one of the lines between $\mathrm{G}$ and $\mathrm{H}$. Of these rays the red is the brightest, and therefore the general colour of luminous hydrogen (with an electric discharge through a Geissler tube) is reddish.

The correlation of the Frauenhofer lines with the spectra of metals depends on the phenomenon of the so-called reversed spectrum. This phenomenon consists in that instead of the bright spectrum corresponding with a metal, under certain circumstances a similar dark spectrum in the form of Frauenhofer lines may be obtained, as will be directly explained. In order to clearly understand the phenomenon of reversed spectra, it must be known that in the passage of light through certain transparent substances these substances retain rays of a certain refrangibility. The colour of solutions is a proof of this. Light which has passed through a yellow solution of a uranium salt contains no violet rays, and after having passed through a red solution of a permanganate, does not contain many rays in the yellow, blue, and green portions of the spectrum. Solutions of copper salts absorb nearly all red rays. Sometimes colourless solutions also absorb rays of certain definite refractive indexes, and give absorption spectra. Thus solutions of salts of didymium absorb rays of a certain refrangibility ${ }_{1}$ and therefore an impression of black lines is received, ${ }^{28}$ as shown in fig.

spectra (see later). Consequently, these lines are the most characteristic. Only the longest and most brilliant are given in our table, which is composed on the basis of a collection of the data at our disposal for bright spectra of the incandescent and rarefied vapours of the elements. As the spectra change with great variations of temperature and vapour density (the faint lines become brilliant whilst the bright lines sometimes disappear), which is particularly clear from Ciamician's researches on the halogens, therefore, until the method of observation and the theory of the subject are enlarged, particular theoretical importance should not be given to the wave lengths showing the maximum brilliancy, and which only possess any significance in a practical respect for the common methods of spectroscopic observations.

28 The ocular impressions of light (it is essentially the same with all other impressions received by the senses) are all relative; in those portions of a spectrum, received through an absorptive medium, where there appears to be an absence of light, it may be only rendered fainter, and for absorption spectra this is directly proved to be the case both by experiment (by employing solntions of different strengths or strata of different thicknesses), and by direct measurement by the aid of the spectroscope-for instance, by Vierordt's apparatus, which is described in works on physics. The relative distinctness of the dark lines in an absorption spectrum, and of the bright coloured lines in luminous spectra of vapours and gases, which are self-evident in making observations, offer great difficulty with regard to precise measurement, just as is the case, for instance, with the relative brilliancy of the stars.

The method of observing absorption spectra consists in taking a continuous spectrum (one which does not give either dark lines or particularly bright luminous bands in the spectrum) of white light-for instance, the light of a candle, lamp, or other source. The collimator (that is, the tube with the slit) is directed towards this light, and then all the colours of the spectrum are visible in the ocular tube. A transparent absorptive medium - for instance, a solution or tube containing a gas-is then placed between the source of light and the apparatus (or anywhere inside the apparatus itself in the path of the rays). In this case either the entire spectrum is uniformly fainter, or absorption 
73. Many vapours (iodine) and gases (nitric peroxide) give similar spectra. Light which has passed through a deep layer of aqueous vapour, oxygen, or nitrogen also gives its absorption spectrum. For this reason the peculiar (winter) dark lines discovered by Brewster are

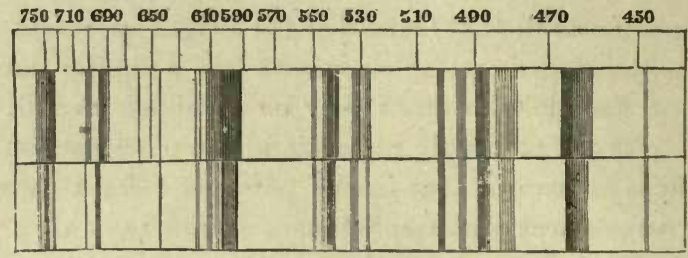

FIG. 73.-Absorption spectrum (Lecoq de Boisbaudran) of salts of didymium in concentrated and dilute solutions.

observed in sunlight, especially in the evening and morning, when the sun's rays pass through the atmosphere (containing these substances) by a longer path than at mid-day. It is evident that the Frauenhofer

bands appear on the bright field of the continuous spectrum in definite positions along it. These bands have different lengths and positions, and distinctness and intensity of absorption, according to the properties of the absorptive medium. Like the luminous spectra given by incandescent gases and vapours, the absorption spectra of a number of substances have already been studied, and some with great precision-as, for example, the spectrum of the brown vapours of nitrogen dioxide by Hasselberg (at Pulkowa), or

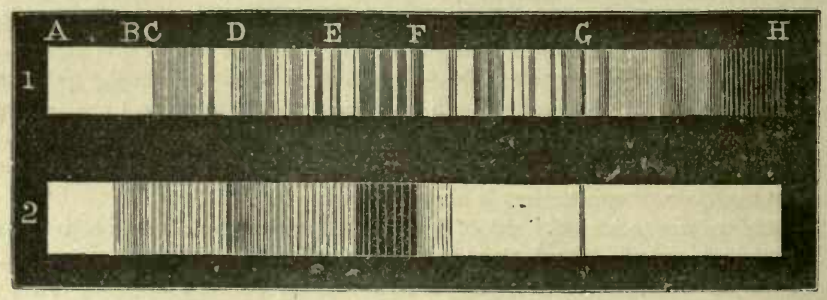

F1G. 74.-Absorption spectra of nitrogen dioxide oxide and iodine.

the spectra of colouring matters (Eder and others), and especially those applied to orthochromatic photography, or the spectra of blood, chlorophyll (the green constituent of leaves), and other similar substances, all the more as by the aid of their spectra tlie presence of these substances may be discovered in small quantities (even in microscopical quantities, by the aid of special appliances or the microscope), and the changes they undergo investigated.

The absorption spectra, obtained at the ordinary temperature and proper to substances in all physical states, offer a most vast but as yet little studied field, both for the theory of all spectroscopy, and for gaining an insight into the structure of substances. The investigation of colouring matters has already shown that in certain cases a definite change of composition and structure entails not only a definite change of the colours but also a displacement of the absorption bands by definite wave lengths. This subject, which has only begun to be worked out, promises great scientific fruition. 
lines may be ascribed to the absorption of certain rays of light in its passage from the luminous mass of the sun to the earth. The remarkable progress made in all spectroscopic research dates from the investigations made by Kirchhoff (1859) on the relation between absorption spectra and the spectra of luminous incandescent gases. It had already been long since observed (by Frauenhofer, Foucault, Angström) that the bright spectrum of the sodium flame gives exactly the same two bright lines which are known as the line $\mathrm{D}$ in the solar spectrum, and expressed by black lines which evidently belong to an absorption spectrum. When Kirchhoff caused moderated sunlight to fall upon the slit of a spectroscope, and placed a sodium flame before it, a perfect superposition was observed-the bright sodium lines completely covered the black lines D of the solar spectrum. When later the continuous spectrum of a Drummond light appeared with the black line $\mathbf{D}$ on placing a sodium flame between it and the slit of the spectroscope-that is, when the Frauenhofer line of the solar spectrum was artificially producedthen there was no doubt that it was seen in the solar spectrum because the light somewhere travelled through incandescent vapours of sodium. Hence a new theory of reversed spectr $a^{29}$ arose - that is, respecting the relation between the waves of light emitted and absorbed by a substance under given conditions of temperature, which is expressed by Kirchhoff's law discovered by a careful analysis of the relations between the luminous rays absorbed and emitted by a substance. This law of the theory of light may be formulated in an elementary form in the following manner: At a given temperature the relation between the intensity of the light emitted (of a definite wave length) and the absorptive capacity with respect to the same colour. (of the same wave length) is a constant quantity. ${ }^{30}$ As a black dull surface emits a considerable

29 A number of methods have been invented to demonstrate the reversibility of spectra; among these methods we will cite two which are most easily carried out. In Bunsen's method sodium chloride is put into an apparatus for evolving hydrogen (the spray of the salt is then carried off by the hydrogen and colours the flame with the yellow sodium colour), and the hydrogen is ignited in two burners - in one large one with a wide flame giving a bright yellow sodium light, and in another with a small fine orifice whose flame is pale: this flame will throw a dark patch on the large bright flame. In Ladoffsky's method the front tube (p. 552) is unscrewed from a spectroscope directed towards the light of a lamp (a continuous spectrum), and the flame of a spirit lamp coloured by a small quantity of $\mathrm{NaCl}$ is placed between the tube and the prism; a black band corresponding to sodium will then be seen on looking through the ocular tube. This experiment is always successful if there be only the requisite relation between the strength of the light of both lamps.

30 The absorptive capacity is the relation between the intensity of the light (of a given wave length) falling upon and retained by a substance. Bunsen and Roscoe showed by direct experiment that this ratio is a constant quantity for every substance. If $A$ stand for this ratio for a given substance at a given temperature-for instance, for a flame coloured by sodium-and $E$ be the intensity of the light of the same wave length 
quantity of heat rays and also absorbs a considerable quantity, whilst a polished metallic surface both absorbs but few and emits but few, so a flame coloured by sodium emits a considerable quantity of yellow rays of a definite refrangibility, and has the property of retaining a considerable quantity of the rays of the same refractive index. In general, the sphere which evolves definite rays also retains them.

Thus the bright spectral rays characteristic of a given metal may be reversed - that is, converted into dark lines - by passing light which gives a continuous spectrum through a space containing the heated vapours of the given metal. A similar phenomenon to that thus artificially produced may be observed naturally in sunlight, which shows dark lines characteristic of known metals-that is, the Frauenhofer lines form an absorption spectrum or depend on a reversed spectrum ; it being presupposed that the sun itself, like all known sources of artificial light, gives a continuous spectrum without Frauenhofer lines. ${ }^{31}$ We must imagine that the sun, owing to the high temperature which is proper to it, emits a brilliant light which gives a continuous spectrum, and that this light, before reaching our eyes, passes through a space full of the vapours of different metals and their compounds. As the earth's atmosphere ${ }^{32}$ contains no, or very little, metallic vapours, and as they cannot be supposed to exist in the heavenly space, therefore the only place in which the existence of such vapours can be admitted is in the atmosphere surrounding the sun itself. As the cause of the sun's luminosity must be looked for in its high temperature, therefore the existence of an atmosphere containing metallic vapours is readily understood, because at its high temperature such metals as sodium, and even iron, are separated from their compounds and converted into vapour. The sun must be imagined as surrounded by an atmosphere of incandescent vaporous emitted at the same temperature by the same substance, then Kirchhoff's law, the explanation and deduction of which must be looked for in text books of physics, states that the fraction $A / E$ is a constant quantity depending on the nature of a substance (as $A$ depends on it) and determined by the temperature and wave length.

31 Heated metals begin to emit light (only visible in the dark) at about $420^{\circ}$ (varying with the metal). On further heating solids first emit red, then yellow, and lastly white light. Compressed or heary gases (see Chap. III. Note 44), when strongly heated, also emit white light. Heated liquids (for example, molten steel or platinum) also give a white compound light. This is readily understood. In a dense mass of matter the collisions of the molecules and atoms are so frequent that waves of only a few definite lengths cannot appear; the reverse is possible in rarefied gases or vapours.

32 Brewster, as is mentioned above, first distinguished the atmospheric, cosmical Frauenhofer lines from the solar lines. Janssen showed that the spectrum of the atmosphere contains lines which depend on the absorption produced by aqueous vapour. Egoreff, Olszewski, Janssen, and Liveing and Dewar showed by a series of experiments that the oxygen of the atmosphere determines certain lines of the solar spectrum, -especially the line A. 
and gaseous matter, ${ }^{33}$ including those elements whose reversed spectra correspond with the Frauenhofer lines-namely, sodium, iron, hydrogen, lithium, calcium, magnesium, \&c. Thus in spectrum analysis we find a means of determining the composition of the inaccessible heavenly luminaries, and much has been done in this respect since Kirchhoff's theory was formulated. By observations on the spectra of many heavenly boclies, changes have been discovered going on in them, ${ }^{34}$ and certain of the elements known to us have been found with certainty in them. ${ }^{3.5}$ From this it must be concluded that the same elements which exist on the earth occur throughout the whole universe, and that at

33 Eruptions, like our volcanic eruptions, but on an incomparably larger scale, are a frequent occurrence on the sun. They are seen as protuberances visible during a total eclipse of the sun, in the form of vaporous masses on the edge of the solar disc and emitting a faint light. These protuberances of the sun are now observed at all times by means of the spectroscope (Lockyer's method), because they contain luminous vapours (giving bright lines) of hydrogen and other elements.

34 The great interest and vastness of astro-physical observations concerning the sun, comets, stars, nebulae, \&c., render this new province of natural science very important, and necessitate referring the reader to special works on the subject. I cannot, however, but caution the reader against those premature conclusions which many form on a first acquaintance with the subject. Just as the astromomer may easily arrive at an arbitrary conclusion about the composition of elements, from observations upon the spectra of the heavenly bodies, so the chemist may easily fall into error as to the nature of the phenomena of the heavens by only judging from spectroscopic observations.

The most important astro-physical data since the time of Kellner are those referring to the displacement of the lines of the spectrum. Just as a musical note changes its pitch with the approach or withdrawal of the resonant object or the ear, so the pitch of the luminous note or wave length of the light varies if the luminous vapour and the earth from which we observe it approach or recede from each other; this expresses itself in a visible displacement of the spectral lines. The solar erruptions even give broken lines in the spectrum, because the rapidly moving eruptive masses of vapour and gases either travel in the direction of the eye or fall back towards the sun. As the earth travels with the solar system among the stars, so it is possible to determine the direction and velocity with which the sun travels in space by the displacement of the spectral lines and light of the stars.

The changes proceeding on the sun in its mass, which must be pronounced as vaporous, and in its atmosphere, are now studied by means of the spectroscope. For this purpose, there now exist many special astro-physical observatories where these investigations are carried on.

35 Spectrum analysis has proved the indubitable existence in the sun and stars of a number of elements known in chemistry. Huggins, Secchi, and others have furnished a large amount of material upon this subject. A compilation of existing information on this subject has been given by Prof. S. A. Kleiber, in the Journal of the Russian Physico-chemical Society for 1885 (vol. xviii. p. 146). Besides which, a peculiar element called helium has been discovered, which is characterised by a line (whose wave length is 587.5 , situated near D), which is seen very brightly in the projections (protuberances) and spots of the sun, but which does not belong to any known element and is not reproducible as a reversed, dark line. This may be a right conclusion-that is to say, it is possible that an element may be discovered to which the spectrum of helium correspondsbut it may be that the helium line belongs to one of the known elements, because spectra vary in the brilliancy and position of their lines with changes of temperature and pressure. Thus, for instance, Lockyer could only see the line 423 , at the very end 


\section{that degree of heat which is proper to the sun those simple matters which we accept as the elements in chemistry are still undecomposed}

of the calcium spectrum, at comparatively low temperatures, whilst the lines 397 and 393 appear at a higher temperature, and at a still higher temperature the line 423 becomes quite invisible.

Lockyer, to whom spectroscopy is indebted for many excellent spectroscopic observations, supposes that the elements existing on the earth are decomposed at the temperature of the sun-for instance, that iron decomposes into two new elements having particular spectra, because an unequal intensity of all the lines of iron is observed in different portions of the sun (spots, prominences, \&c.), and because a displacement (Note 34) of certain of the lines of this metal may be observed in the sun's spots at the same time that other lines remain undisplaced. Lockyer supposes this to depend on the movement of one component part of iron with an immobility of the position (in space) of the other product of the decomposition of iron which remains in a lower stratum. Prof. Kleiber explains these phenomena by the fact that the visible spectrum of the sun is determined by the entire thickness of the atmosphere of the sun, by the inequality of the movement of different strata of the sun's atmosphere, and by the fact that lines of different wave lengths offer a different constant with reference to Kirchhoff's law. It is enough if the thickness, pressure, and temperature of an incandescent vapour be different in a laboratory experiment and in a given stratum of the solar atmosphere to obtain a marked difference in the intensity of the light of different bands of the spectrum of one and the same element. As regards the displacement of only one portion of the iron lines, Kleiber supplements Liveing and Dewar's observation that the displaced lines are those emitted by the most rarefied vapours (Note 27), by pointing out that in the first place one and the same line is sometimes observed on the sun both in a contorted (broken) and normal position (as Lockyer himself observed), and in the second place that. the intensity of the light of different lines depends on the different temperatures and densities of the strata of the solar atmosphere, and therefore the lines determined by the upper strata may, by their movement, be displaced, whilst the other lines determined by the lower strata may remain unchanged. Besides which I may observe, for my part, that if, under ordinary conditions, we see the normal spectrum of iron in the sun, Lockyer's supposed component parts of iron must occur together in the sun, and therefore it is difficult to understand how one component part of iron is able to move whilst the other remains at rest. Furthermore, as the solar spectrum of iron entirely corresponds with that obtained by experiment at the comparatively low temperatures obtainable in the laboratory, it is necessary to admit one of the two following propositions-either the decomposition of iron does not require so high a temperature as that on the sun, and then it would be easy to prove this supposed complexity of iron by a laboratory experiment, or it must be admitted that the two component parts of iron when combined together (forming undecomposed iron) do not alter the positions of their spectral lines, whilst, as we shall see later, the spectra of elements change when they combine together, and therefore that in the case of iron a proposition must be made contradictory to this fact. Other data put forward by Lockyer in favour of the decomposability of certain elements proved when re-investigated (by Liveing and Dewar), by means of spectroscopes of high dispersive power, to be only founded on the confusion of dissimilar lines. The arguments of Lockyer concerning the decomposability of elements, which at one time made a great impression, do not thus appear to support the doctrine of a single common material (p. 20, Note 26), but must be made use of for the further development of spectroscopic science. Besides which I consider it well to turn attention to the facts (1) that the conception of the elements stands in all respects more firmly than any deductions obtained by the spectroscope; (2) this comparatively young doctrine of the spectra of elements is only the fruit of the chemical doctrine of elements; and (3) that as yet no generalisations, besides Kirchhoff's law, have appeared for spectroscopic phenomena, which could allow predictions being made, whilst the conception of elements has already arrived at this stage. When, however, spectroscopic science has been dereloped to the same extent 
and remain unchanged. A high temperature forms one of those conditions under which compounds most easily decompose ; and therefore if sodium or a similar element were a compound, then in all probability it would be decomposed into component parts at the high temperature of the sun. This may already be concluded from the fact that in ordinary spectroscopic experiments the spectra obtained often belong to the metals and not to the compounds taken; this depends on the decomposition of these compounds in the heat of the flame. If common salt be introduced into the flame of a gas-burner, a portion of it is decomposed, first forming, in all probability, with water, hydrochloric acid and sodium hydroxide, and the latter is then partially decomposed by the hydrocarbons, giving metallic sodium, whose incandescent vapour emits light of a definite refrangibility. This conclusion is arrived at from the following experiment :- If hydrochloric acid gas be introduced into a flame coloured by sodium it is observed that the sodium spectrum disappears, owing to the fact that metallic sodium cannot remain in the flame in the presence of an excess of hydrochloric acid. The same thing takes place on the addition of sal-ammoniac, which in the heat of the flame gives hydrochloric acid. If a porcelain tube containing sodium chloride (or sodium hydroxide or carbonate), and closed at both ends by glass plates, be so powerfully heated that the salt volatilises, then the sodium spectrum is not observable; but if the salt be replaced by sodium, then both the bright line and the absorption spectra are obtained, according to whether the light emitted by the incandescent vapour be observer, or only that which passes through the tube. Thus the above spectrum is not given by sodium chloride or other sodium compound, but is proper to the metal sodium itself. It is the same with other analogous metals. The chlorides and other halogen compounds of barium, calcium, copper, \&c., give independent spectra which differ from those of the metals. If barium chloride be introduced into a flame, it gives a mixed spectrum belonging to metallic barium and barium chloride. If besides barium chloride, hydrochloric acid or sal-ammoniac be introduced into the flame, then the spectrum of the metal disappears, and that of the chloride remains, which differs distinctly from the spectrum of barium fluoride, barium bromide, or barium iodide. A certain common resemblance and certain common lines are observed in the spectra of two different compounds of one and the same element obtained in the above-described manner, and also in the spectrum of the metal, but they all have their peculiarities. The approach perfection. As yet spectroscopic science is still, for want of laws, at the epoch of the accumulation of facts and not of their possession. 
independent spectra of the compounds of copper are easily observed (fig. 75). Thus certain compounds which exist and are luminous at a

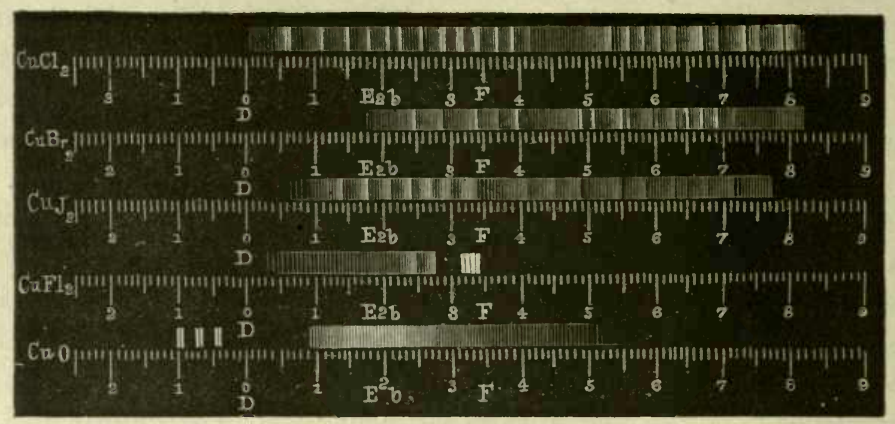

Fig. 75. - Bright Spectra of Copper Compounds.

high temperature give their independent spectra. In the majority of cases the spectra of compounds are composed of indistinct luminous lines and complete bright bands, whilst metallic elements generally give a few clearly-defined spectral lines. ${ }^{36}$ There is no reason for thinking

36 Spectroscopic observations are still further complicated by the fact that one and the same substance gives different spectra at different temperatures. This is especially the case with gases whose spectra are obtained by an electric discharge in tubes. Plïcker; Wüllner, Schuster, and others showed that at different temperatures and pressures the spectra of iodine, sulphur, nitrogen, oxygen, \&c., are quite different from the spectra of the same elements at high temperatures and pressures. This may either depend on the fact that the elements change their molecular structure with a change of temperature, just as ozone is converted into oxygen, or else because at low temperature certain rays have a greater relative intensity than those which appear at higher temperatures. If we suppose that the molecules of a gas are in continual movement, with a velocity dependent on the temperature, then it must be admitted that they often strike against each other and rebound, and thus communicate peculiar movements to each other and the supposed ether, which express themselves in luminiferous phenomena. A rise of the temperature or an increase in the density of a gas must have an influence on the collision of its molecules and luminiferous movements thus produced, and this may be the cause of the difference of the spectra under these circumstances. It has been shown by direct experiment that gases compressed by pressure, when the collision of the molecules must be frequent and varied, exhibit a more complex spectrum on the passage of an electric spark than rarefied gases, and that even a continuous spectrum appears. In order to show the variability of the spectrum according to the circumstances under which it proceeds, it is enough to say that potassium sulphate fused on a platinum wire gives, on the passage of a series of sparks, a distinct system of lines, 583-578, whilst when a series of sparks is passed through a solution of this salt this system of lines is faint, and when Roscoe and Schuster observed the absorption spectrum of the vapour of unetallic potassium (which is green) they remarked a number of lines of the same intensity as the above system in the red, orange, and yellow portions.

The spectra of solutions are best observed by means of Lecoq de Boisbaudran's arrangement, shown in fig. 76. A bent capillary tube, D F, inside which a platinum wire, 
that the spectrum of a compound is equal to the sum of the spectra of its elements - that is, every compound which is not decomposed by heat has its own proper spectrum. This is best proved by absorption spectra, which are essentially only reversed spectra observed at low temperatures. If every salt of sodium, lithium, and potassium gives one and the same spectrum, this must be ascribed to the presence in the flame of the free metals liberated by the decomposition of their salts. Therefore the phenomena of the spectrum are determined by molecules, and not by atomsthat is, the molecules of the metal sodium, and not its atoms, produce those forms of vibrations which are expressed in the spectrum of a sodium salt. Where there is no free metallic sodium there is no sodium spectrum.

Spectrum analysis has not only endowed science with a representation of the composition of the distant heavenly bodies (of the sun, stars, nebulae, comets, \&c.), but has also given a new method for studying the matter of the earth's surface. Bunsen by its means discovered two new elements belonging to the group of the alkali metals, and thallium, inclium, and gallium were afterwards discovered by the same means. The spectroscope is employed in the study of rare metals (which in solution often give distinct absorption spectra), of dyes, and in general of many organic substances, $\& c{ }^{37}$ With respect to the

A $a$ (from 0.3 to $0.5 \mathrm{~mm}$. in diameter) is fused, is immersed in a narrow eylinder, C (in which it is firmly held by a cork). The projecting end, $a$, of the wire is covered by a fine capillary tube, $d$, which extends $1-2 \mathrm{~mm}$. beyond the wire. Another straight capillary tube, E, with a platinum wire, $\mathrm{B} b$, about $1 \mathrm{~mm}$. in diameter (a finer wire soon becomes hot), is held (by a cork or in a stand) above the end of the tube, D. If the wire $A$ be now connected with the positive, and the wire B with the negative, terminal of a Ruhmkorff's coil (if the wires be comnected in the opposite order, the spectrum of air is obtained), a series of sparks rapidy following each other appear between $a$ and $b$, and their light may be examined by placing the apparatus in front of the slit of a spectroscope. The variations to which a spectrum is liable may easily be observed by increasing the distance between the wires, altering the direction of the current or strength of the solution, \& $\mathrm{c}$.

${ }^{37}$ The importance of the spectroscope for the purpose of chemical research was already shown by Gladstone in 1856, but it did not become an accessory to the laboratory until after the discoveries of Kirchhoff and Bunsen. It may be hoped that in time spectroscopic researches will explain certain wants of the theoretical (philosophical) side of chemistry, but as yet all that has been done in this respect can only be regarded as attempts which have not yet led to any trustworthy conclusions. Thus many,

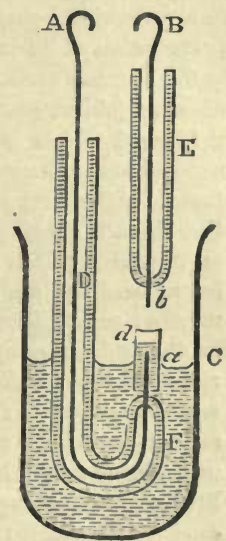

Frg. 76.-Method of showing the spectrum of substances in solution. by collating the wave lengths of all the light vibrations excited by a given element, endeavour to find the law governing their mutual relations; others (especially Hartley and Ciamician), by comparing the spectra of analogous elements (for instance, chlorine, bromine, and iodine), have succeeded in noticing definite features of resemblance in them, whilst others (Griinwald) search for relations between the spectra of compounds 
metals which are analogous to sodium, they all give such very volatile salts and such very characteristic spectra that the least traces of them ${ }^{38}$ are discovered with great ease by means of the spectroscope. For instance, lithium gives a very brilliant red coloration to a flame and a very bright red spectral line (wave length, 670 millionths $\mathrm{mm}$.), which indicates the presence of this metal in admixture with compounds of other alkali metals.

Lithium, $\mathrm{Li}=7$, is, like potassium and sodium, somewhat widely spread in siliceous rocks, but only occurs in small quantities and as mere traces in considerable masses of potassium and sodium salts. Only a very few minerals contain more than traces of it. ${ }^{39}$ The compounds of lithium are in all respects closely analogous to the corresponding compounds of sodium and potassium; but the carbonate is sparingly

and their component elements, \&c.; but-owing to the mutiplicity of the spectral lines proper to many elements, and (especially in the ultra-red and ultra-violet ends of the spectrum) the existence of lines which are undistinguishable owing to their faintness, and also owing to the comparative novelty of spectroscopic research-this subject cannot be considered as in any way perfected in any of its branches.

38 In order to show the degree of sensitiveness of spectroscopic reactions it will be enough to cite the following observation of Dr. Bence Jones. If a solution of 3 grains of a lithium salt be injected under the skin of a guinea-pig, then, after the lapse of four minutes, lithium may already be discoverved in the bile and liquids of the eye, and, after ten minutes, in all parts of the animal.

39 Thus spodumene contains up to 6 p.c. of lithium oxide, and petolite, and lepidolite or lithia mica, about 3 p.c. of lithium oxide. This mica is met with in certain granites in a somewhat considerable quantity, and is therefore most frequently employed for the preparation of lithium compounds. The treatment of lepidolite is carried on on a large scale, because certain salts of lithium are employed in medicine as a remedy for certain diseases (stone, gouty affections), as they have the power of dissolving the insoluble uric acid which is then deposited. Lepidolite, which is unacted on by acids in its natural state, decomposes under the action of strong hydrochloric acid after it has been fused. After being subjected to the action of the hydrochloric acid for several hours all the silica is obtained in an insoluble form, whilst the metallic oxides pass into solution as chlorides. This solution is mixed with nitric acid to convert the ferrous salts into ferric, and sodium carbonate is then added until the liquid becomes neutral, by which means a precipitate is formed of the oxides of iron, alumina, magnesia, \&c., as insoluble oxides and carbonates. The solution (with an excess of water) then contains the chlorides of the alkaline metals $\mathrm{KCl}, \mathrm{NaCl}, \mathrm{LiCl}$, which do not give a precipitate with sodium carbnnate in a dilute solution. It is then evaporated, and a strong solution of sodium carbonate added. This precipitates lithium carbonate, which, although soluble in water, is much less so than sodium carbonate, and therefore the latter precipitates lithium from strong solutions as carbonate $2 \mathrm{LiCl}+\mathrm{Na}_{2} \mathrm{CO}_{3}=2 \mathrm{NaCl}+\mathrm{Li}_{2} \mathrm{CO}_{3}$. Lithium carbonate, which resembles sodium carbonate in many respects, is a substance which is very slightly soluble in cold water and is only somewhat soluble in boiling water. In this respect lithium forms a transition between the metals of the alkalis and other metals, especially the metals of the alkaline earths (magnesium, barium), whose carbonates are only sparingly soluble. Oxide of lithium, $\mathrm{Li}_{2} \mathrm{O}$, may be obtained by heating lithium carbonate with charcoal. Lithium oxide in dissolving gives (per molecule) 26000 heat units; but the combination of $\mathrm{Li}_{2}$ with $\mathrm{O}$ evolves 140000 calories - that is, more than $\mathrm{Na}_{2} \mathrm{O}$ (100000 calories) and $\mathrm{K}_{2} \mathrm{O}$ (97000 calories), as shown by Beketoff (1887). 
soluble in cold water, which fact is taken advantage of for separating lithium from potassium and sodium. This salt, $\mathrm{Li}_{2} \mathrm{CO}_{3}$, is easily converted into the other compounds of lithium. Thus, for instance, the lithium hydroxide, $\mathrm{LiHO}$, is obtained in exactly the same way as caustic soda, by the action of lime on the carbonate, and it is soluble in water and crystallises (from its solution in alcohol) as $\mathrm{LiHO}_{2} \mathrm{H}_{2} \mathrm{O}$. Metallic lithium is obtained by the action of a galvanic current on fused lithium chloride; for this purpose a cast-iron crucible, furnished with a stout cover, is filled with lithium chloride, heated until the latter fuses, and then a strong galvanic current is passed through the molten mass. The positive pole (fig. 77) consists of a dense carbon rod $\mathrm{C}$ (surrounded by a porcelain tube $\mathrm{P}$ fixed in an iron tube B B), and the negative pole of an iron wire, on which the metal is deposited after the current has passed the molten mass for a certain length of

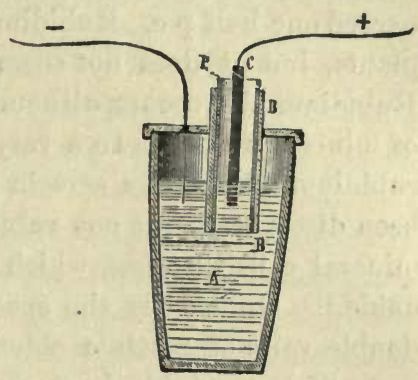

FIG. 77.-Preparation of lithium by the action of a galvanic current on fused lithium chloride.

time. Chlorine is evolved at the positive pole. When a somewhat considerable quantity of the metal has accumulated on the wire it is withdrawn, the metal is collected from it, and the experiment is then carried on as before. Lithium is the lightest of all metals, its specific gravity is 0.59 , owing to which it even floats on naphtha; it melts at $180^{\circ}$, but does not volatilise at a red heat. Its appearance recalls that of sodium, and, like it, it has a yellow tint. At $200^{\circ}$ it burns in air with a very bright flame, forming lithium oxide. In decomposing water it does not ignite the hydrogen. The characteristic test for lithium compounds is the red coloration which they impart to a colourless flame. ${ }^{40}$

Bunsen tried to determine by means of the spectroscope whether any other as yet unknown metals do not occur in different natural products together with lithium, potassium, and sodium, and he soon discovered two new alkali metals showing independent spectra. They are named after the characteristic coloration which they impart to the

40 In determining the presence of lithium in a given compound, it is best to treat the material under investigation with acid (in the case of mineral silicon compounds hydrofluoric acid must be taken), and to treat the residue with sulphuric acid, evaporate to dryness, and extract with alcohol, which dissolves a certain amount of the lithium sulphate. It is easy to discover lithium in such an alcolnolic solution by means of the coloration imparted to the flame on burning it, and in case of doubt by investigating its light in a spectroscope, because lithium gives a red line, which is very characteristic and is found as a dark line in the solar spectrum. Lithium was first discovered in 1817 in petolite by Arfvedson. 
flame. One which gives a red and violet band is named rubidium, from rubidius (dark red), and the other is called caesium, because it colours a pale flame sky blue, which depends on its containing bright blue rays, which appear in the spectrum of caesium as two blue bands. Both metals accompany sodium, potassium, and lithium, but in small quantities; rubidium occurs more frequently than caesium. The amount of the oxides of caesium and rubidium in lepidolite does not generally exceed one-half p.c. Rubidium has also been found in the ashes of many plants, but it does not seem to accompany potassium in sea water. Rubidium also occurs, although in very small quantities, in the majority of mineral waters. In a very few cases caesium is not accompanied by rubidium ; thus, in a certain granite on the Isle of Elba, caesium has been discovered, but not rubidium. This granite contains a very rare mineral called pollux, which contains as much as 34 p.c. of caesium oxide. ${ }^{41}$ Guided by the spectroscope, and aided by the fact that the double salts of platinic chloride and rubidium and caesium chlorides are still less soluble in water than the corresponding potassium salt,

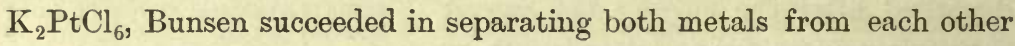
and from potassium, and demonstrated the great resemblance they bear to each other. The isolated metals, ${ }^{42}$ rubidium and caesium, have

41 The salts of the majority of metals are precipitated as carbonates on the addition of ammonium carbonate-for instance, the salts of calcium, iron, \&c. The alkalis whose carbonates are soluble are not, however, precipitated in this case. On evaporating the resultant solution and igniting the residue (to remove the ammonium salts), we obtain salts of the alkali metals. They may be separated by adding hydrochloric acid with a solution of platinic chloride. The chlorides of lithium and sodium give easilysoluble double salts with platinic chloride, whilst the chlorides of potassium, rubidium and caesium form double salts which are sparingly soluble. A hundred parts of water at $0^{\circ}$ dissolve 0.74 part of the potassium platinochloride; the corresponding rubidium platinochloride is only dissolved to the amount of 0.134 part, and the caesium salt, 0.024 part; at $100^{\circ} 5.13$ parts of potassium platinochloride, $\mathrm{K}_{2} \mathrm{PtCl}_{6}$, is dissolved, 0.634 parts of rubidium platinochloride, and 0.177 parts of caesium platinochloride. From this it is clear how the salts of rubidium and caesium may be isolated. The separation of caesium from rubidium by this means is very lengthy. It is better effected by taking advantage of the difference of the solubility of their carbonates in alcohol; caesium carbonate, $\mathrm{Cs}_{2} \mathrm{CO}_{3}$, is soluble in alcohol, whilst the corresponding salts of rubidium and potassium are almost insoluble. Setterberg separated these metals as alums, but the best method, that given by Scharples, is founded on the fact that from a mixture of the chlorides of potassium, sodium, caesium, and rubidium in the pre. sence of hydrochloric acid stannic chloride precipitates a double salt of caesium, which is very slightly soluble. The salts of $\mathrm{Rb}$ and $\mathrm{Cs}$ are closely analogous to those of potassium.

42 Bunsen obtained rubidium by distilling a mixture of the tartrate with soot, and Beketoff (1888) by heating the hydroxide with aluminium $2 \mathrm{RbHO}+\mathrm{Al}=\mathrm{RbAlO}_{2}+$ $\mathrm{H}_{2}+\mathrm{Rb}$. By the action of 85 grams of rubidium on water, 94000 heat units are evolved. Setterberg obtained caesium (1882) by the electrolysis of a fused mixture of cyanide of caesium and of barium. 
specific gravities of 1.52 and 1.88 respectively, and melt at $39^{\circ}$ and $27^{\circ}$.

Judging by the properties of the free metals, and of their corresponding and even very complex compounds, lithium, sodium, potassium, rubidium, and caesium present an indubitable chemical resemblance. The fact that the metals easily decompose water, and that their hydroxides $\mathrm{RHO}$ and carbonates $\mathrm{R}_{2} \mathrm{CO}_{3}$ are soluble in water, whilst the hydroxides and carbonates of nearly all other metals are insoluble, confirms this, and if the resemblance between the corresponding salts be taken into consideration there is no doubt that the resemblance in the chemical character of these metals is very considerable : therefore they form a natural group of alkali metals. The halogens and the alkali metals form, by their character, the two extremes of the elements. Some of the other elements are metals approaching in a certain degree the alkali metals, both in their capacity of forming salts and in not forming acid compounds, but are not so energetic as the alkali metals, and are displaced by the latter from the majority of their compounds; they also evolve less heat in combining with the halogens, and form less energetic bases than the alkali metals. Such are the common metals, silver, iron, copper, \&c. Some other elements, in the character of their compounds, approach the halogens, and, like them, combine with hydrogen, but these compounds do not show the energetic property of the halogen acids; in a free state they easily combine with metals, but they do not then form such saline compounds as the halogens doin a word, the halogen properties are less sharply defined in them than in the halogens themselves. Sulphur, phosphorus, arsenic, \&c., belong to this order of elements. Lastly, there is yet another order of elements, like carbon and nitrogen, in which neither the metallic nor the halogen properties are clearly defined, and which in this respect occupy an intermediate position between the two above-mentioned orders of elements.

The clear distinction of the properties of the halogens and alkali metals is expressed in the fact that the former give acids and do not form bases, whilst the latter, on the contrary, only give bases. The first are true acid elements, the latter clearly-defined basic or metallic elements. On combining together, the halogens form, in a chemical sense, unstable compounds, and the alkali metals alloys in which the character of the metals remains unaltered, just as in the compound ICl the character of the halogens remains undisguised; thus both classes of elements on combining with members of their own class form noncharacteristic compounds, which have the properties of their components. On the other hand, the halogens on combining with the alkali 
metals form compounds which are, in all respects, stable, and in which the original characters of the halogens and alkali metals have entirely disappeared. The formation of such compounds is accompanied by evolution of a large amount of heat, and by an entire change of both the physical and chemical properties of the substances originally taken. The alloy of sodium and potassium, although liquid at the ordinary temperature, is perfectly metallic, like both its components. The compound of sodium and chlorine has neither the appearance nor the properties of the original elements; sodium chloride melts at a higher temperature, and is more difficultly volatile, than either sodium or chlorine.

With all these qualitative differences there is, however, an important quantitative resemblance between the halogens and the alkali metals. This resemblance is clearly expressed by stating that both orders of elements belong to those which are univalent with respect to hydrogen. It is thus possible to express that both the above-named orders of elements replace hydrogen atom for atom. Chlorine is able to take the place of hydrogen by metalepsis, and the alkali metals take the place of hydrogen in water and acids. As it is possible to consecutively replace every equivalent of hydrogen in a hydrocarbon by chlorine, so it is possible in an acid containing several equivalents of hydrogen to replace the hydrogen consecutively equivalent after equivalent by an alkali metal; hence an atom of these elements is analogous to an atom of hydrogen, which is taken, in all respects, as the unit for the comparison of the other elements. In ammonia and in water chlorine and sodium are able to bring about a direct replacement. According to the law of substitution, the formation of sodium chloride, $\mathrm{NaCl}$, already shows the equivalence of the atoms of the alkali metals and the halogens. The halogens and hydrogen and the alkali metals combine with such elements as oxygen, and it is easily seen that in such compounds one atom of oxygen is able to retain two atoms of the halogens, of hydrogen, and of the alkali metals. In this respect it is enough to compare the compounds $\mathrm{KHO}, \mathrm{K}_{2} \mathrm{O}, \mathrm{HClO}$, and $\mathrm{Cl}_{2} \mathrm{O}$, with water. It must not be forgotten, however, that with oxygen the halogens give higher acid grades of oxidation, besides compounds of the type $\mathrm{R}_{2} \mathrm{O}$, which the alkali metals and hydrogen are not capable of forming. We shall soon see that these relations are also subject to a special law, showing the gradual transition of the properties of the elements from the alkali metals to the halogens. ${ }^{43}$

43 We may here observe that the halogens, and especially iodine, may play the part of metals (hence iodine is more easily replaced by metals than the other halogens, and it approaches nearer to the metals in its physical properties than the other halogens). 
The atomic weights of the alkali metals, lithium 7 , sodium 23 , potassium 39, rubidium 85, and caesium 133 , show that here, as in the halogens, the elements may be arranged according to their atomic weights in order to compare the properties of the analogous compounds of the members of this group. Thus, for example, the platinochlorides of lithium and sodium are soluble in water; those of potassium, rubidium, and caesium sparingly soluble, and the greater the atomic weight of the metal the less soluble is the salt. In other cases the reverse is observed-the greater the atomic weight the more soluble are the corresponding salts. The variation of properties with the variation in atomic weights even shows itself in the metals themselves; thus lithium volatilises with difficulty, whilst sodium is obtained by means of distillation, potassium volatilises more easily than sodium, and rubidium and caesium, as we have seen, are still more volatile.

Schützenberger obtained a compound $\mathrm{C}_{2} \mathrm{H}_{3} \mathrm{O}(\mathrm{OCl})$, which he called chlorine acetate, by acting on acetic anhydride, $\left(\mathrm{C}_{2} \mathrm{H}_{5} \mathrm{O}\right)_{2} \mathrm{O}$, with chlorine monoxide, $\mathrm{Cl}_{2} \mathrm{O}$. With iodine this compound disengages chlorine and forms iodine acetate, $\mathrm{C}_{2} \mathrm{H}_{3} \mathrm{O}(\mathrm{OI})$, which also is formed by the action of iodine chloride on sodium acetate, $\mathrm{C}_{2} \mathrm{H}_{5} \mathrm{O}(\mathrm{ONa})$. Such compounds are very unstable, decompose with an explosion when heated, and are changed by the action of water and of many other re-agents, which is in accordance with the fact that they contain very closely-allied elements, as $\mathrm{Cl}_{2} \mathrm{O}$ itself, or $\mathrm{ICl}$ or $\mathrm{KNa}$. By the action of chlorine monoxide on a mixture of iodine and acetic anhydride, Schiitzenberger also obtained the compound $\mathrm{I}\left(\mathrm{C}_{2} \mathrm{H}_{3} \mathrm{O}_{2}\right)$, which is analogous to $\mathrm{ICl}_{3}$, because the group $\mathrm{C}_{2} \mathrm{H}_{3} \mathrm{O}_{2}$ is, like $\mathrm{Cl}$, a halogen, forming salts with the metals. 


\section{CHAPTER XIV}

THF VALENCY AND SPECIFIC HEAT OF THE METALS. MAGNESIUM, CALCIUM, STRONTIUM, BARIUM, AND BERYLLIUM :

IT is easy by investigating the composition of corresponding compounds, to establish the equivalent weights of the metals - that is, the quantity which replaces one part by weight of hydrogen. If a metal directly. decomposes acids, with the evolution of hydrogen, then the equivalent weight of the metal may be determined by taking a definite weight of it and measuring the volume of hydrogen evolved by its action on an excess of acid; then it is easy to calculate the weight of the hydrogen from its volume. ${ }^{1}$ The same result may be arrived at by determining the composition of the normal salts of the metal ; for instance, by finding the weight which combines with 35.5 parts of chlorine or 80 parts of bromine. ${ }^{2}$ The equivalent of a metal may be also learnt by simultaneously (i.e., in one circuit) decomposing an acid and a fused salt of a given metal by an electric current and determining the relation between the amounts of hydrogen and metal separated, because, according to Faraday's law, electrolytes (conductors of the second order) are always decomposed in equivalent quantities. The equivalent of a metal may even be found by simply determining the relation between the weight of a metal and of its salt giving oxide, as by this weight we know the weight of the metal which combines with 8 parts by weight of oxygen, and this will be the weight of an equivalent, because 8 parts of oxygen combine with 1 part by weight of

1 Under favourable circumstances (by taking all the requisite precautions), the weight of the equivalent may be accurately determined by this method. Thus Reynolds and Ramsay (1887) determined the equivalent of zinc to be 32.7 by this method (from the average of 23 experiments), whilst by other methods it has been fixed (by different observers) between $32 \cdot 55$ and 33.95 .

The difference in their equivalents may be demonstrated by taking equal weights of different metals, and by collecting the hydrogen evolved by them (under the action of an acid or alkali).

2 The most accurate determinations of this kind were carried on by Stas, and will bedescribed in speaking of silver. 
hydrogen. One method is verified by another, and all the processes for the accurate determination of the equivalents require the most accurate methods to avoid the absorption of moisture, of further qxidation, volatility, and other like circumstances which influence exact weighings. The description of the methods necessary for the attainment of exact results belongs to the province of analytical chemistry.

For univalent metals, like those of the alkalis, the weight of the equivalent is equal to the weight of the atom. For bivalent metals the atomic weight is equal to the weight of two equivalents, for $n$-valent metals it is equal to the weight of $n$ equivalents. Thus aluminium, $\mathrm{Al}=27$, is trivalent, that is, its equivalent $=9$; magnesium, $\mathrm{Mg}=24$, is bivalent, and its equivalent $=12$. Therefore, if potassium or sodium, or in general a univalent metal, $\mathbf{M}$, gives compounds $\mathbf{M}_{2} \mathrm{O}, \mathbf{M H O}$, $\mathrm{MCl}, \mathrm{MNO}_{3}, \mathrm{M}_{2} \mathrm{SO}_{4}$, \&c., and in general $\mathrm{MX}$, then for bivalent metals like magnesium or calcium the corresponding compounds will be $\mathrm{MgO}, \mathrm{Mg}(\mathrm{HO})_{2}, \mathrm{MgCl}_{2}, \mathrm{Mg}\left(\mathrm{NO}_{3}\right)_{2}, \mathrm{MgSO}_{4}$, drc., or in general $\mathrm{MX}_{2}$.

By what are we to be guided in ascribing to some metals univalency and to others bi-ter-quadri-...n-valency? What obliges us to make this difference? Why are not all metals given the same valency-for instance, why is not magnesium considered as univalent ? If this be done, taking $\mathrm{Mg}=12$ (and not 24 as now used), not only is a simplicity of expression of the composition of all the compounds of magnesium attained, but also we gain the advantage that their composition will be the same as those of the corresponding compounds of sodium and potassium. These compositions were so expressed before, why has this been changed now?

These questions could only be answered after the establishment of the conceptions of multiples of the atomic weights as the minimum quantities of the elements combining together to form compounds-in a word, since the time of the establishment of Avogadro-Gerhardt's law (Chap. VII.). By taking such an element as arsenic, which has many volatile compounds, it is easy to determine the density of these compounds, and therefore to establish their molecular weights, and hence to find the indubitable atomic weight, exactly as for oxygen, nitrogen, chlorine, carbon, dc. It appears that $\mathrm{As}=75$, and its compounds correspond, like the compounds of nitrogen, with the forms $\mathrm{AsX}_{3}$ and $\mathrm{AsX}_{5}$; for example, $\mathrm{AsH}_{3}, \mathrm{AsCl}_{3}, \mathrm{AsFl}_{3}, \mathrm{As}_{2} \mathrm{O}_{5}$, \&c. It is evident that we are here dealing with a metal (or, better, element) of two valencies, and which is furthermore in no way univalent, but tri- or quinqui-valent. This example alone is sufficient for the recognition of the existence of polyvalent atoms among the metals. And as antimony 
and bismuth are closely analogous to arsenic in all their compounds, as potassium is analogous to rubidium and caesium; therefore, although very few volatile compounds of bismuth are known, it was necessary to ascribe to them formulae corresponding with those ascribed to arsenic.

As we shall see in describing them, there are also many analogous metals among the bivalent elements, some of which also give volatile compounds. For example, zinc, which is itself volatile, gives several volatile compounds (for instance, zinc ethyl, $\mathrm{ZnC}_{4} \mathrm{H}_{10}$, which boils at $118^{\circ}$, vapour density $\left.=61 \cdot 3\right)$, and in the molecules of all these compounds there is never less than 65 parts of zinc, which is equivalent to $\mathrm{H}_{2}$, because 65 parts of zinc displace 2 parts by weight of hydrogen ; so that zinc is just such an example of the bivalent metals as oxygen, whose equivalent $=8$ (because $\mathrm{H}_{2}$ is replaced by $\mathrm{O}=16$ ), is a representative of the bivalent elements, or as arsenic is of the tri- and quinquivalent elements. And, as we shall afterwards see, magnesium is in many respects closely analogous to zine, which fact obliges us to regard magnesium as a bivalent metal.

Such metals as mercury and copper, which are able to give not one but two bases, are of particular importance for distinguishing univalent and bivalent metals. Thus copper gives the suboxide $\mathrm{Cu}_{2} \mathrm{O}$ and the oxide $\mathrm{CuO}$ - that is, the compounds $\mathrm{CuX}$ corresponding with the suboxide are analogous (in the quantitative relations, by their composition) to $\mathrm{NaX}$ or $\mathrm{AgX}$, and the compounds of the oxide $\mathrm{CuX}_{2}$ to $\mathrm{MgX}_{2}$, $\mathrm{ZnX}_{2}$, and in general to the bivalent metals. It is clear that in such examples we must distinguish metals of varying atomicity.

In this manner the valency of many metals may be established by means of certain and comparatively few volatile metallic compounds, and by the aid of a search into their analogies (concerning which see Chap. XV.). The law of specific heats discovered by Dulong and Petit has frequently been applied to the same purpose ${ }^{3}$ in the

3 The chief means by which we determine the valency of the elements, or what multiple of the equivalents should be ascribed to the atom, are : (1) The law of AvogadroGerhardt. This method is the most general and trustworthy, and has already been applied to a great number of elements. (2) The different grades of oxidation and their isomorphism or analogy in general; for example, $\mathrm{Fe}=56$ because the suboxide (ferrous oxide) is isomorphous with magnesium oxide, \&c., and the oxide (ferric oxide) contains $1 \frac{1}{2}$ times as much oxygen as the suboxide. Berzelius, Marignac, and others took advantage of this method for determining the composition of the compounds of many elements. (3) The specific heat, according to Dulong and Petit's law. Regnault, and more especially Cannizzaro, used this method to distinguish univalent from bivalent metals. (4) The periodic law (see Chapter XV.) has served as a means for the determination of the atomic weights of cerium, uranium, yttrium, \&c., and more especially of gallium, scandium, and germanium. The correction of the results of one method by those 
history of chemistry, especially since the development given to this law by the researches of Regnault, and since Cannizzaro (1860) showed the agreement between the deductions of this law and the consequences arising from Avogadro-Gerhardt's law.

Dulong and Petit, having determined the specific heat of a number of solid elementary substances, observed that as the atomic weights of the elements increase, their specific heats decrease, and that the product of the specific heat $Q$ into the atomic weight $A$ is an almost constant quantity. This means that to bring different elements into a known thermal state, an equal amount of work is required if atomic quantities. of the elements are taken; that is, the amounts of heat expended in heating equal quantities by weight of the elements are far from equal, but are in inverse proportion to the atomic weights. For thermal changes the atom is a unit ; all atoms, notwithstanding the difference of weight and nature, are equal. This is the simplest expression of the fact discovered by Dulong and Petit. The specific heat measures that quantity of heat which is required to raise the temperature of one unit of weight of a substance by one degree. If the magnitude of the specific heat of elements be multiplied by the atomic weight, then we obtain the atomic heat - that is, the amount of heat required to raise the temperature of the atomic weight of an element by one degree. It. is these products which for the majority of the elements prove to be approximately, if not quite, identical. A complete identity cannot be expected, because the specific heat of one and the same substance varies. with the temperature, with its passage from one state into another, and frequently with even a simple mechanical change of density (for instance by hammering), not to speak of allotropic changes, \&c. We will

of others is generally had recourse to, and is quite necessary, because phenomena of dissociation, polymerisation, \&c., may complicate the individual determinations by each method.

It will be well to observe that a number of other methods, especially from the province of those physical properties which are clearly dependent on the magnitude of the atom (or equivalent) or of the molecule, may lead to the same result. I may point out, for instance, that even the specific gravity of solutions of the metallic chlorides (Chapter VII. p. 322) may serve for this purpose. Thus if beryllium be taken as trivalent-that is, if the composition of its chloride be taken as $\mathrm{BeCl}_{3}$ (or a polymeride of it), then the specific gravity of solutions of beryllium chloride will not fit into the series of the other metallic chlorides. But on ascribing to it an atomic weight $\mathrm{Be}=7$, or taking $\mathrm{Be}$ as bivalent, the composition of its chloride as $\mathrm{BeCl}_{2}$, we arrive at the general rule given on p. 318 . Thus W. G. Burdakoff determined in my laboratory, that the specific gravity at $15^{\circ} / 4^{\circ}$ of the solution $\mathrm{BeCl}_{2}+200 \mathrm{H}_{2} \mathrm{O}=1.0138$-that is, greater than the corresponding solution $\mathrm{KCl}+200 \mathrm{H}_{2} \mathrm{O}(=1 \cdot 0121)$, and less than the solution $\mathrm{MgCl}_{2}+200 \mathrm{H}_{2} \mathrm{O}(=1.0203)$, as would follow from the magnitude of the molecular weight $\mathrm{BeCl}_{2}=80$, because $\mathrm{KCl}=74.5$ and $\mathrm{MgCl}_{2}=95$ (see my work Investigation of Aqueous Solutions, 1887, p. 425). 
cite sereral figures ${ }^{4}$ proving the truth of the conclusions arrived at by Dulong and Petit.

\begin{tabular}{|c|c|c|c|c|}
\hline & $\mathrm{Li}$ & $\mathrm{Na}$ & $\mathrm{Mg}$ & $P$ \\
\hline$A=$ & 7 & 23 & 24 & 31 \\
\hline $\mathrm{Q}=$ & 0.9408 & 0.2934 & $0 \cdot 245$ & $0 \cdot 202$ \\
\hline$A Q=$ & $6 \cdot 59$ & $6 \cdot 75$ & $5 \cdot 88$ & $6 \cdot 26$ \\
\hline & $\mathrm{Fe}$ & $\mathrm{Cu}$ & $\mathrm{Zn}$ & $\mathrm{Br}$ \\
\hline$A=$ & 56 & 63 & 65 & 80 \\
\hline $\mathrm{Q}=$ & 0.112 & 0.093 & 0.093 & 0.0843 \\
\hline $\mathrm{AQ}=$ & $6 \cdot 27$ & $5 \cdot 86$ & $6 \cdot 04$ & $6 \cdot 74$ \\
\hline & $\mathrm{Pd}$ & $\mathrm{Ag}$ & Sn & I \\
\hline$A=$ & 106 & 108 & 118 & 127 \\
\hline$Q=$ & 0.0592 & 0.056 & 0.055 & 0.541 \\
\hline$A Q=$ & $6 \cdot 28$ & $6 \cdot 05$ & $6 \cdot 49$ & $6 \cdot 87$ \\
\hline & $\mathrm{Pt}$ & $A u$ & $\mathrm{Hg}$ & $\mathrm{Pb}$ \\
\hline$A=$ & 196 & 198 & 200 & 206 \\
\hline $\mathrm{Q}=$ & 0.0325 & 0.0324 & 0.0333 & 0.0315 \\
\hline $\mathrm{AQ}=$ & $6 \cdot 37$ & $6 \cdot 41$ & $6 \cdot 66$ & $6 \cdot 49$ \\
\hline
\end{tabular}

It is seen from this that the product of the specific heat of the element into the atomic weight is an almost contant quantity, which is nearly 6. Therefore the possibility arises of judging the valency with a sufficient degree of exactitude, by the specific heats of the

4 The specific heats here given refer to different limits of temperature, but in the majority of cases betwcen $0^{\circ}$ and $100^{\circ}$; only in the case of bromine the specific heat is taken (for the solid state) at a temperature below $-7^{\circ}$, according to Regnault's determination. The variation of the specific heat with a change of temperature forms a very complex phenomenon, the consideration of which I think would here be out of place. I will only cite certain figures as an example. According to Bystrom, the specific heat of iron at $0^{\circ}=0.1116$, at $100^{\circ}=0.1114$, at $200^{\circ}=0.1188$, at $300^{\circ}=0.1267$, and at $1400^{\circ}=$ 0.4031 . Between these last limits of temperature a change takes place in iron (a spontaneous heating, recalescence), as we shall afterwards see. For quartz $\mathrm{SiO}_{2}$, Pionchon gives $\mathrm{Q}=0 \cdot 1737+394 t 10^{-6}-27 t^{2} 10^{-9}$ up to $400^{\circ}$; consequently, as a rule, the specific heat varies with the temperature. Still more remarkable are H. E. Weber's observations on the great variation of the specific heat of charcoal, the diamond and boron:

$\begin{array}{lccccc} & 0^{\circ} & 100^{\circ} & 200^{\circ} & 600^{\circ} & 900^{\circ} \\ \text { Wood charcoal } & 0.15 & 0.23 & 0.29 & 0.44 & 0.46 \\ \text { Diamond } & 0.10 & 0.19 & 0.22 & 0.44 & 0.45 \\ \text { Boron } & 0.22 & 0.29 & 0.35 & - & -\end{array}$

These determinations (they have been verified by Dewar) are of especial importance as confirming the universality of Dulong and Petit's law, because the elements mentioned above form exceptions to the general rule when the mean specific heat for temperatures between $0^{\circ}$ and $100^{\circ}$ is taken. Thus in the case of the diamond the product of $A \times Q$ at $0^{\circ}=1 \cdot 2$, and for boron $=2 \cdot 4$. But if we take the specific heat towards which there is .evidently a tendency with a rise of temperature, then we obtain a product approaching 
metals. Thus, for instance, the specific heats of lithium, sodium, and potassium convince one of the fact that their atomic weights are indeed those which we took, because by multiplying the specific heats found by experiment by the corresponding atomic weights we obtain the following figures: $\mathrm{Li}, 6.59, \mathrm{Na}, 6.75$ and $\mathrm{K}, 6.47$. Of the alkaline earth metals the specific heats have been determined : of magnesium $=0 \cdot 245$ (Regnault and Kopp), of calcium $=0 \cdot 170$ (Bunsen), and of barium $=0.05$ (Mendeléeff). If the same composition be ascribed to the compounds of magnesium as to the corresponding compounds of potassium, then the equivalent of magnesium will be equal to 12 . On multiplying this atomic weight by the specific heat of magnesium, we obtain a figure 2.94 , which is half that which is given by the other elements, and therefore the atomic weight of magnesium must be taken as equal to 24 and not to 12 . Then the atomic heat of magnesium $=$ $24 \times 0 \cdot 245=5.9$; for calcium, giving its compounds a composition $\mathrm{CaX}_{2}$-for example $\mathrm{CaCl}_{2}, \mathrm{CaSO}_{4}, \mathrm{CaO}(\mathrm{Ca}=40)$-we obtain an atomic heat $=40 \times 0.17=6.8$, and for barium it is equal to $137 \times 0.05=6.8$; that is, they must be counted as bivalent, or that their atom replaces $\mathrm{H}_{2}, \mathrm{Na}_{2}$, or $\mathrm{K}_{2}$. This conclusion may be confirmed by a method of analogy, as we shall afterwards see. A strict application of the principle of specific heats to the determination of the magnitudes of the atomic weights of those metals, the magnitude of whose atomic weights

to 6 as with other elements. Thus with the diamond and charcoal, it is evident that the specific heat tends towards $0 \cdot 47$, which multiplied by 12 gives $5 \cdot 6$, the same as for magnesium and aluminium. I may here turn the reader's attention to the fact that for solid elements having a small atomic weight, the specific heat varies considerably if we take the average figures for temperatures $0^{\circ}$ to $100^{\circ}$ :

$\begin{array}{rccc}\mathrm{Li}=7 & \mathrm{Be}=9 & \mathrm{~B}=11 & \mathrm{C}=12 \\ \mathrm{Q}=0.94 & 0.42 & 0.24 & 0.20 \\ \mathrm{AQ}=6.6 & 3.8 & 2.6 & 2.4\end{array}$

It is therefore clear that the specific heat of beryllium determined at a low temperature cannot serve for establighing its atomicity. On the other hand, the low atomic heat of charcoal, graphite, and the diamond, boron, \&c., may perhaps depend on the complexity of the molecules of these elements. The necessity for acknowledging a great complexity of the molecules of carbon was explained in Chapter VIII. In the case of sulphur the molecule contains at least $S_{6}$ and its atomic heat $=32 \times 0 \cdot 163=5 \cdot 22$, which is distinctly below the normal. If a great number of atoms of carbon are gathered together in the molecule of charcoal, this would to a certain extent account for its comparatively small atomic heat. With respect to the specific heat of compounds it will not be out of place to here mention the conclusion arrived at by Kopp, that the molecular heat (that is, the product of MQ) may be looked on as the sum of the atomic heats of its component elements; but as this rule is not a general one, and can only be applied to an approximate judgment of the specific heats of substances, I do not think it necessary to go into the details of the conclusions described in Liebig's 'Annalen Supplement-Band,' 1864; which includes a number of determinations made by Kopp. 
could not be determined according to Avogadro-Gerhardt's law, was made about 1860 by the Italian professor Cannizzaro.

Exactly the same conclusions respecting the bivalence of magnesium and its analogues are obtained by comparing the specific heats of their compounds, especially of the halogen compounds as the most simple, with the specific heats of the corresponding alkali compounds. Thus, for instance, the specific heat of magnesium and calcium chlorides, $\mathrm{MgCl}_{2}$ and $\mathrm{CaCl}_{2}$, equal $0 \cdot 194$ and $0 \cdot 164$, and of sodium and potassium chlorides, $\mathrm{NaCl}$ and $\mathrm{KCl}, 0 \cdot 214$ and $0 \cdot 172$, and therefore their molecular heats (or the products QM, where M is the weight of the molecule) are 18.4 and $18 \cdot 2,12 \cdot 5$ and $12 \cdot 8$, and therefore the atomic heats (or the quotient of QM by the number of atoms) are all nearly 6 , as with the elements. Whilst if, instead of the actual atomic weights $\mathrm{Mg}=24$ and $\mathrm{Ca}=40$, their equivalents 12 and 20 be taken, then the atomic heats of the chlorides of magnesium and calcium would be about $4 \cdot 6$, whilst those of potassium and sodium chlorides are about $6 \cdot 3 .^{5}$

As the specific heat or the amount of heat required to raise the temperature of a unit of weight one degree ${ }^{6}$ is a complex quantity-

$s$ It must be remarked that in the case of oxygen (hydrogen and carbon) compounds the quotient of $\mathrm{MQ} / n$, where $n$ is the number of atoms in the molecule, is always less than 6 for solids; for example, in the case of $\mathrm{MgO}=5 \cdot 0, \mathrm{CuO}=5 \cdot 1, \mathrm{MnO}_{2}=4^{\cdot} \cdot 6$, ice $(\mathrm{Q}=0.504)=3, \mathrm{SiO}_{2}=3.5$, \&c. At present it is impossible to say whether this depends on the smaller specific heat of the atoms of oxygen in its solid compounds (Kopp, Note 4 ) or on some other reason; but, nevertheless, taking into account this decrease, depending on the presence of oxygen, a reflection of the atomicity of the elements may to a certain extent be seen in the specific heat of the oxides. Thus in the case of alumina, $\mathrm{Al}_{2} \mathrm{O}_{3}$ $(Q=0 \cdot 217), M Q=22 \cdot 3$, and therefore the quotient $M Q / n=4 \cdot 5$, which is nearly that given by magnesium oxide, $\mathrm{MgO}$. But if we ascribe the same composition to alumina as. to magnesia - that is, if aluminium were counted as divalent-we should obtain the figure 3.7 , which is much less. In general, in compounds of identical atomic composition and of analogous chemical properties the molecular heats MQ are nearly equal, as many investigators have long remarked. For example, $\mathrm{ZnS}=11 \cdot 7$ and $\mathrm{HgS}=11 \cdot 8$; $\mathrm{MgSO}_{4}=27^{\circ} 0$ and $\mathrm{ZnSO}_{4}=28^{\circ} \cdot 0$, \&c.

6 If $\mathrm{W}$ be the amount of heat contained in a mass $m$ of a substance at a temperature $t$, and $d \mathrm{~W}$ the amount expended in heating it from $t$ to $t+d t$, then the specific heat $\mathrm{Q}=d \mathrm{~W} /(m \times d t)$. The specific heat not only varies with the composition and complexity of the molecules of a substance, but also with the temperature, pressure, and pliysical state of a substance. Even for gases the variation of $Q$ with $t$ is to be observed. Thus it is seen from the experiments of Regnault and Wiedemann that the specific heat of carbonic anhydride at $0^{\circ}=0 \cdot 19$, at $100^{\circ}=0.22$, and at $200^{\circ}=0.24$. But the variation of the specific heat of permanent gases with the temperature is, as far as we know, very inconsiderable. Therefore the specific heat of those permanent gases which contain two atoms in the molecule $\left(\mathrm{H}_{2}, \mathrm{O}_{2}, \mathrm{~N}_{2}, \mathrm{CO}\right.$, and $\left.\mathrm{NO}\right)$ may be, as is shown by experiment, taken as not varying with the temperature. The constancy of the specific heat of perfect gases forms one of the fundamental propositions of the whole theory of heat, and supports the determination of temperatures by means of gas-thermometers containing hydrogen, nitrogen, or air. Le Chatelier (1887), on the basis of existing determinations, concludes that the molecular heat-that is, the product $\mathrm{MQ}$ - of all gases varies in proportion to the temperature, and tends to become equal $(=6.8)$ at the temperature of absolute zero (that is, at $-273^{\circ}$ ); and therefore $M Q=6.8+a(273+t)$, where $a$ is a constant quantity 


\section{including not only the increase of the energy of a substance with its rise in temperature, but also the external work of expansion ${ }^{7}$ and the}

which increases with the complexity of the gaseous molecule. The magnitude $1000 \mathrm{a}$ for ammonia $=6 \cdot 11$, for chloric anhydride $=7 \cdot 42$, for ethylene, $\mathrm{C}_{2} \mathrm{H}_{4}=12 \cdot 7$, for chloroform, $\mathrm{CHCl}_{3}=29.5$, \&c. For permanent gases $a=0$, and $\mathrm{MQ}=6 \cdot 8$ - that is, the atomic heat (if the molecule contains two atoms) $=3 \cdot 4$, as it is in reality. As regards liquids (as well as the vapours formed by them), the specific heat always rises with the temperature: Thus for benzene, it equals $0 \cdot 38+0 \cdot 0014 t$. R. Schiff (1887) showed that the variation of the specific heat of many organic liquids is proportional to the change of temperature (as in the case of gases, according to Le Chatelier), and reduced these variations into dependence with their composition and absolute boiling point. It is very probable that the theory of liquids will make use of these simple relations, which recall the simplicity of the variation of the specific gravity (Chap. II. Note 34), cohesion, and other properties of liquids with the temperature. They are all expressed by the linear function of the temperature, $a+b t$, with the same degree of proximity as the property of gases is expressed by the equation $p v=R t$.

As regards the relation between the specific heats of liquids (of solids) and of their vapours, the specific heat of the vapour (and also of the solid) is always less than that of the liquid. For example, benzene vapour 0.22 , liquid 0.38 ; chloroform vapour 0.13 , liquid 0.23 ; steam 0.475 , liquid water $1 \cdot 0$. But the whole complexity of the relations existing in specific heat is seen from the fact that the specific heat of ice $=0.502$ is less than that of liquid water. According to Regnault, in the case of bromine the specific heat of the vapour $=0.055$ (at $150^{\circ}$ ), of the liquid $=0.107$ (at $30^{\circ}$ ), and of solid bromine $=0.084\left(\right.$ at $-15^{\circ}$ ). The specific heat of solid benzoic acid (according to experiment and calculation, Hess 1888) between $0^{\circ}$ and $100^{\circ}$ is 0.31 , and of liquid benzoic acid 0.50 . One of the problems of the present day is the explanation of those complex relations which exist between the composition and such properties as specific heat, latent heat, expansion by heat, compression, internal friction, cohesion, and other like properties. They can only be connected by a complete theory of liquids, which may now soon be expected, more especially as many sides of the subject have already been partially explained.

7 According to the above reasons the quantity of heat, $Q$, required to raise the temperature of one part by weight of a substance by one degree may be expressed by the sum $Q=K+B+D$, where $K$ is the heat actually expended in heating the substance, or that which is terned the absolute specific heat, B the amount of heat expended in the internal work accomplished with the rise of temperature, and D the amount of heat expended in external work. In the case of gases the last quantity may be easily determined, knowing their coefficient of expansion, which is approximately $=0.00368$. By applying to this case the same argument given at the end of Note 11, Chap I., we find that one cubic metre of a gas heated by $1^{\circ}$ produces an external work of $10333 \times 0.00368$, or 38.02 kilogrammetres, on which $38.02 / 424$ or 0.0897 heat units are expended. This is the heat expended for the external work produced by one cubic metre of a gas, but the specific heat refers to units of weight, and therefore it is necessary in order to know D to 'reduce the above quantity to a unit of weight. One cubic metre of hydrogen at $0^{\circ}$ and $760 \mathrm{~mm}$. pressure weighs $0.0896 \mathrm{kilo}$, a gas of molecular weight $\mathrm{M}$ has a density $\mathrm{M} / 2$, consequently a cubic metre weighs (at $0^{7}$ and $760 \mathrm{~mm}$.) $0.0448 \mathrm{M} \mathrm{kilo,} \mathrm{and} \mathrm{therefore}$ 1 kilogram of the gas occupies a volume $1 / 0.0448 \mathrm{M}$ cubic metres, and hence the external work $\mathrm{D}$ in the heating of $1 \mathrm{kilo}$. of the given gas by $1^{\circ}=0^{\circ} 0896 / 0.0448 \mathrm{M}$, or $\mathrm{D}=2 / \mathrm{M}$.

Taking the magnitude of the internal work $\mathrm{B}$ for gases as minute if permanent gases are taken, and therefore supposing $B=0$, we find the specific heat of gases at a constant pressure $Q=K+2 M$, where $K$ is the specific heat at a constant volume, or the true specific heat, and $M$ the molecular weight. Hence $K=Q-2 / M$. The magnitude of the specific heat $Q$ is given by direct experiment. According to Regnault's experiments, for oxygen it $=0.2175$, for hydrogen $3 \cdot 405$, for nitrogen 0.2438 ; the molecular weights of these gases are 32,2 , and 28 , and therefore for hydrogen $K=0.2438-0.0714=0 \cdot 1724$. These true 


\section{internal work accomplished in the molecules inciting them to decom- position according to the rise of temperature ${ }^{8}$ - therefore it is impos-}

specific heats of elements are in inverse proportion to their atomic weights-that is, their product by the atomic weight is a constant quantity. In reality, for oxygen this product $=0.155 \times 16=2.48$, for hydrogen 2.40 , for nitrogen $0.7724 \times 14=2.414$, and therefore if $\mathrm{A}$ stand for the atomic weight we obtain the expression $\mathrm{K} \times \mathrm{A}=$ a constant, which may be counted as 2.45 ; and this is the true expression of Dulong and Petit's law, because $K$ is the true specific heat and $A$ the weight of the atom. It should be remarked, moreover, that the product of the observed specific heat $\mathrm{Q}$ into $\mathrm{A}$ is also a constant quantity (for oxygen $=3.48$, for hydrogen $=3.40$ ), because the external work $D$ is also inversely proportional to the magnitude of the atomic weight.

In the case of gases we distinguish the specific heat at a constant pressure $c^{\prime}$ (we designated this quantity above by $Q$ ), and at a constant volume $c$. It is evident that the relation between both specific heats, $k$, judging from the above, is the ratio of $Q$ to $K$, or equal to the ratio of $2 \cdot 45 n+2$ to $2 \cdot 45 n$. When $n=1$ this ratio $k=1 \cdot 8$; when $n=2 k=1 \cdot 4$, when $n=3 k=1 \cdot 3$, and with an exceedingly large number, $n$, of atoms in the molecule, $k=1$. That is, the ratio between the specific heats decreases from 1.8 to 1.0 as the number of atoms, $n$, contained in the molecule increases. This deduction is verified to a certain extent by direct experiment. For such gases as hydrogen, oxygen, nitrogen, carbonic oxide, air, and others in which $n=2$, the magnitude of $k$ is determined by methods described in physics (for example, by the change of temperature with an alteration of pressure, by the velocity of sound, \&c.), and is found in reality to be nearly 1.4 , and for such gases as carbonic anhydride, nitric peroxide, and others it is nearly $1 \cdot 3$. Kundt and Warburg (1875), by means of the approximate method mentioned on p. 321 , determined $k$ for mercury vapour when $n=1$, and found it to be $=1 \cdot 67$ - that is, a larger quantity than for air, as would be expected from the above.

It may be admitted that the true atomic heat of gases $=2 \cdot 43$, only under the condition that they are distant from a liquid state, and do not undergo a chemical change when heated-that is, when no internal work is produced in them $(B=0)$. Therefore this work may to a certain extent be judged by the observed specific heat. Thus, forinstance, for chlorine $(\mathrm{Q}=0 \cdot 12$, Regnault; $k=1 \cdot 33$, according to Straker and Martin, and therefore $\mathrm{K}=0^{*} 09$, MK $\left.=6 \cdot 4\right)$, the atomic heat $(3 \cdot 2)$ is much greater than for other gases containing two atoms in a molecule, and one must consider, therefore, that when heated some great internal work is accomplished of whose nature it is at present impossible to form an opinion. And as in the case of such gases as ethylene, $\mathrm{C}_{2} \mathrm{H}_{4}(\mathrm{Q}=0.39)$, according to Wiedemann $k=1 \cdot 2, \mathrm{~K}=0.33, \mathrm{MK}=9 \cdot 2$; hence the true atomic heat is less than for constant gases $=1 \cdot 5$. Therefore the question as to the relation between the specific heats of gases and the number of atoms and composition cannot be counted as sufficiently general if we do not consider Le Chatelier's deduction (Note 6) as proved by the association of data. If the latterl be verified, then it will have to be admitted that Dulong and Petit's law is not applicable to any gases besides those which are permanent and possess a comparatively low molecular weight. The question might be solved by determining the specific heat of mercury vapour at different temperatures, but as yet there are no exact methods of doing this.

All the more remarkable is the adaptability of Dulong and Petit's law to the mass of the common elements in a solid state. In order to generalise the facts concerning the specific heat of gases and solids, it appears to me possible to accept the following general proposition: the atomic heat (that is, $\mathrm{AQ}$ or $\mathrm{QM} / n$, where $\mathrm{M}$ is the molecular weight and $n$ the number of molecules) is less (greatest for solids, 6.8 ; for gases, $3 \cdot 4$ ) the more complex the molecule (that is, the greater the number $(n)$ of the atoms forming it), and to a certain extent (with similar physical states of substances) the less the mean $(\mathrm{M} / n)$ weight of the atom.

8 For an example, it is enough to point out the specific heat of nitrogen tetroxide, $\mathrm{N}_{2} \mathrm{O}_{4}$, which, when heated, gradually passes into $\mathrm{NO}_{2}$-that is, chemical work of decom- 
sible to expect in the magnitude of the specific heat the great simplicity of relation to composition which we see, for instance, in the density of gaseous substances. Therefore, although the specific heat gives one of the important means of judging the atomicity of the elements, still the mainstay for a true judgment of atomicity is only given by Arogadro-Gerhardt's law. All other means can only be accessory or preliminary, until it be possible to have direct recourse to the determination of the vapour density.

Among the bivalent metals the first place, with respect to their distribution in nature, is occupied by magnesium and calcium, just as sodium and potassium stand first amongst the univalent metals. The relation which exists between the atomic weights of these four metals confirms the above comparison. In fact, the combining weight of magnesium is equal to 24 , and of calcium 40 ; whilst the combining weights of sodium and potassium are 23 and 39 -that is, the latter are one unit less than the former. ${ }^{9}$ They all belong to the number of light metals, as they have but a small specific gravity, in which they differ from the ordinary, generally known heavy, or ore, metals (for instance, iron, copper, silver, and lead), which are distinguished by a much greater specific gravity. There is no doubt that their low specific gravity has a significance, not only as a simple point of distinction, but also as a property which determines the fundamental properties of these metals. Indeed, all the light metals have a series of points of resemblance which approximates them to the metals of the alkalis; thus both magnesium and calcium, like the metals of the

position proceeds, which consumes heat. Speaking generally, specific heat is a complex quantity, in which it is clear that thermal data (for instance, the heat of reaction) alone cannot give an idea either of chemical or of physical changes individually, but always depend on an association of the one and the other. If a substance be heated from $t_{0}$ to $t$, it cannot but suffer a chemical change (that is, the state of the atoms in the molecules changes more or less in one way or another) if dissociation sets in at a temperature $t_{1}$. Even in the case of the elements whose molecules contain only one atom, a true chemical change is possible with a rise of temperature, because more heat is evolved in chemical reactions than that quantity which participates in purely physical clianges. One gram of hydrogen (specific heat $=3.4$ at a constant pressure) cooled to the temperature of absolute zero will evolve altogether about one thousand units of heat, 8 grams of oxygen half this amount, whilst in combining together they evolve in the formation of 9 grams of water more than thirty times as much heat. Hence the store of chemical energy (that is, of the movement of the atoms, vortex, or other) is much greater than the physical store proper to the molecules, but it is the change accomplished by this store that is the cause of chemical transformations. Here we evidently touch on those limits of existing knowledge beyond which the discipline of science does not yet allow us to pass. A number of new scientific conquests are necessary before this can become possible.

9 As if $\mathrm{NaH}=\mathrm{Mg}$ and $\mathrm{KH}=\mathrm{Ca}$, which is in accordance with their valency, $\mathrm{KH}$ including two monovalent elements is a bivalent group like $\mathrm{Ca}$. 
alkalis, decompose water (without the addition of acids), although not so easily as the latter metals. The process of the decomposition is essentially one and the same ; for example, $\mathrm{Ca}+2 \mathrm{H}_{2} \mathrm{O}=\mathrm{CaH}_{2} \mathrm{O}_{2}+\mathrm{H}_{2}-$ that is, hydrogen is liberated and a hydroxide of the metal formed. These hydroxides are bases which saturate nearly all acids. However, the hydroxides $\mathrm{RH}_{2} \mathrm{O}_{2}$ of calcium and magnesium are in no respects so energetic as the hydroxides of the true metals of the alkalis ; thus when heated they lose water, are not so soluble, develop less heat with acids, and form various salts, which are less stable and more easily decomposed by heat than the corresponding salts of sodium and potassium. Thus calcium and magnesium carbonates easily part with carbonic anhydride when ignited; the nitrates are also very easily decomposed by heat, calcium and magnesium oxides, $\mathrm{CaO}$ and $\mathrm{MgO}$, being left behind. The chlorides of magnesium and calcium, when heated with water, evolve hydrogen chloride, forming the corresponding hydroxides, and when ignited the oxide itself. All these points already evince a weakening of the alkaline properties.

These metals have been termed the metals of the alkaline earths, because they, like the alkali metals, form energetic bases. They are called alkaline earths because they are met with in nature in a state of combination, forming the insoluble mass of the earth, and because as oxides, RO, they themselves have an earthy appearance. Not a few salts are known of these metals which are insoluble in water, whilst the corresponding salts of the alkali metals are generally soluble-for example, the carbonates, phosphates, borates, and other salts of the alkaline earth metals are nearly insoluble. This serves to distinguish the metals of the alkaline earths from the metals of the alkalis. For this purpose a solution of ammonium carbonate is added to a mixed solution of salts of both metals, when by a double decomposition the insoluble carbonates of the metals of the alkaline earths are formed and pass into a precipitate, whilst the metals of the alkalis remain in solution: $\mathrm{RX}_{2}+\mathrm{Na}_{2} \mathrm{CO}_{3}=\mathrm{RCO}_{3}+2 \mathrm{NaX}$.

We may here remark that the oxides of the metals of the alkaline earths are frequently called by special names: $\mathrm{MgO}$ is called magnesia or bitter earth; $\mathrm{CaO}$, lime; $\mathrm{SrO}$, strontia ; and $\mathrm{BaO}$, baryta.

In the primary rocks the oxides of calcium and magnesium are combined with silica, sometimes in variable quantities, so that in some cases the lime predominates and in other cases the magnesium. Both oxides, being analogous to each other, replace each other in equivalent quantities. The various forms of augite, hornblende or amphibole, and of similar minerals, which enter into the composition of nearly all rocks, 
contain lime and magnesia and silica. The majority of the primary rocks also contain alumina, potash, and soda. These rocks, under the action of water (containing carbonic acid) and air, give up lime and magnesia to the water, and therefore they are contained in all kinds of water, and especially sea water. The carbonates, $\mathrm{CaCO}_{3}$ and $\mathrm{MgCO}_{3}$, frequently met with in nature, are soluble in an excess of water saturated with carbonic antydride, ${ }^{10}$ and therefore many natural waters contain these salts, and are able to yield them when evaporated. However, one kilogram of water saturated with carbonic anhydride does not dissolve more than three grams of calcium carbonate. By gradually expelling the carbonic anhydride from such water, an insoluble precipitate of calcium carbonate separates out. It may confidently be stated that the formation of the very widely distributed strata of calcium and magnesium carbonates was of this nature, because these strata are of a sedimentary character--that is, that which would be exhibited by a gradually accumulating deposit on the bottom of the sea. Furthermore, the remains of sea organisms, plants, ferns, \&c., are frequently found amongst these deposits. These deposits of calcium and magnesium carbonates are the most important sources of these metals. Lime generally predominates, because it is present in rocks and stream water in greater quantity than magnesia, and in this case these sedimentary rocks are termed limestone. Some common flagstones used for paving, \&c., and chalk may be taken as examples of this kind of formation. Those limestones in which a considerable portion of the calcium is replaced by magnesium are termed dolomites. The dolomites are distinguished by their hardness, and by their not parting with the whole of their carbonic anhydride so easily as the limestones under the action of acids. Dolomites ${ }^{11}$ sometimes contain an equal number of molecules of calcium carbonate and magnesium carbonate, and they also sometimes appear in a crystalline form, which is easily understood, because calcium carbonate itself is exceedingly common in this form in nature, and is then known as calc spar, whilst natural

10 Sodium carbonate and other carbonates of the alkalis give acid salts which are less soluble "than the normal ; here, on the contrary, with an excess of carbonic anhydride, a salt is formed which is more soluble than the nornal, but this acid salt is more unstable than sodium hydrogen carbonate, $\mathrm{NaHCO}_{3}$.

11 The formation of dolomite may be explained, if only we imagine that a solution of a magnesium salt acts on calcium carbonate. Magnesium carbonate may be formed by double decomposition, and it must be supposed that this process is able to cease at a certain limit (Cliapter XII.), when we shall obtain a mixture of the carbonates of calcium and magnesium. Haitinger heated a mixture of calcium carbonate, $\mathrm{CaCO}_{3}$, with a solution of an equivalent quantity of magnesium sulphate, $\mathrm{MgSO}_{4}$, in a closed tube at $200^{\circ}$, and then a portion of the magnesia actually passed into the state of magnesium carbonate, $\mathrm{MgCO}_{3}$, and a portion of the lime was converted into gypsum, $\mathrm{CaSO}_{4}$. 
crystalline mannesium carthonate is termed mugnesite. The formation of the crytalline varieties of the insoluble earbonates is explained by the possibility of a slow deposition from solutions containing carbonic acil. Besides which the ealcium and magnesimu sulphates are obtaines from sea wates, and therefore they are met with buth at deposits and in springs. It must be observed that magme-ium is hehl in comsiderahles yuantities in seal water, beraluse tine sulphate and chloride of magnesium are very soluble in water. whils calcejum sulphate is hut little

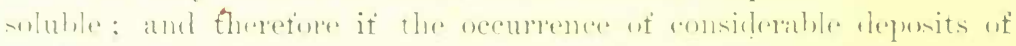

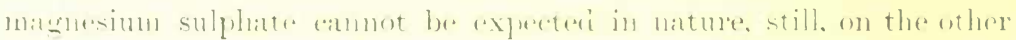

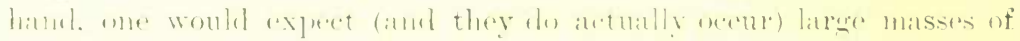

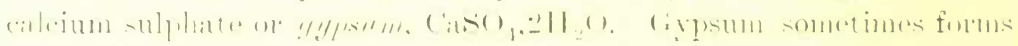

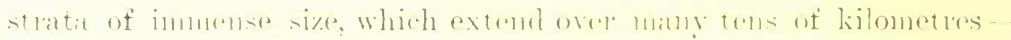

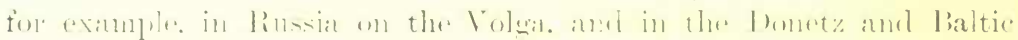
jurince.

Lime and magnesia also, but in much smallere quantities (often to

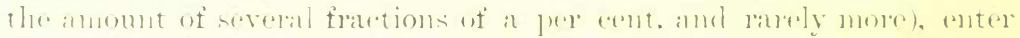

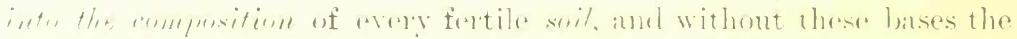

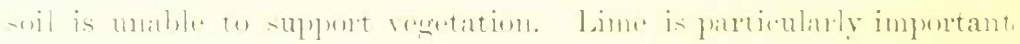

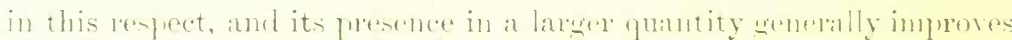

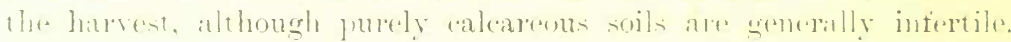
For this masom the soil is fertilised hoth with lime itself and with marl that is, with chay mixed with a certain quantity of calcium carbo

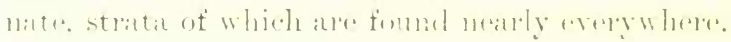

From the wil the lime and magnesia (in at smaller quantity) pass

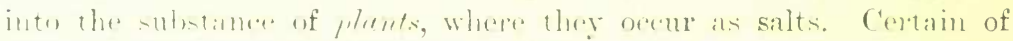

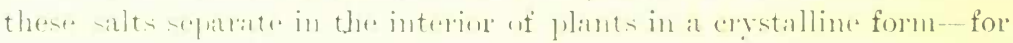

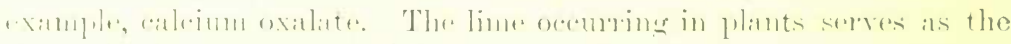

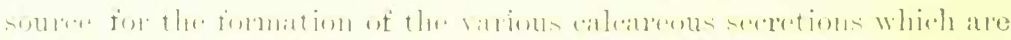

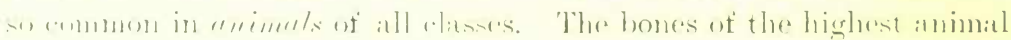

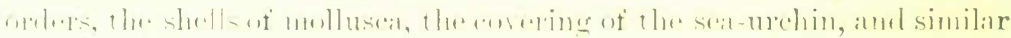

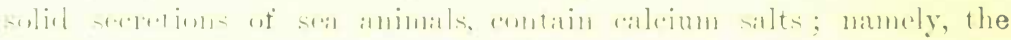

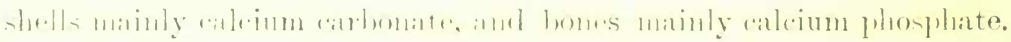

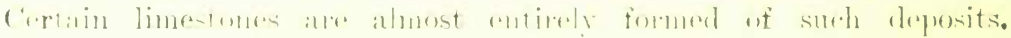

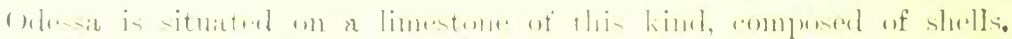

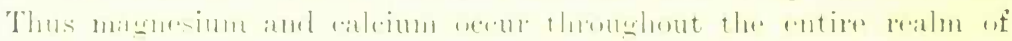
1.ature.

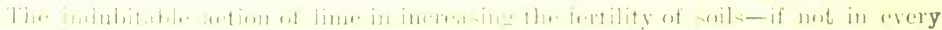

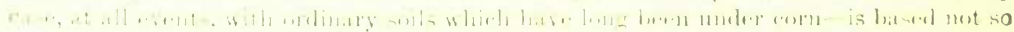

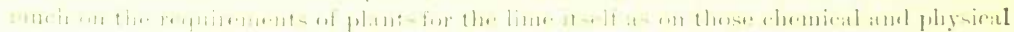

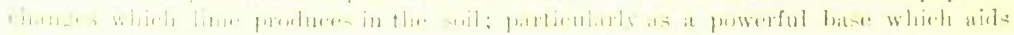

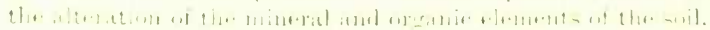


As lime and magnesia form bases which are in many respects. analogous, they were not distinguished from each other for a long time. Magnesia was obtained for the first time in the seventeenth century from Italy, and used as a medicine; and it was only in the last century that Black, Bergmann, and others distinguished magnesia from lime.

Metallic magnesium (and calcium also) is not obtained by heating magnesium oxide or the carbonate with charcoal, as the alkali metals are obtained, ${ }^{13}$ but is liberated by the action of a galvanic current on fused magnesium chloride (best mixed with potassium chloride); but Davy and Bussy first obtained metallic magnesium by acting on magnesium chloride with the vapours of potassium. At the present time (Deville's process) magnesium is prepared in rather considerable quantities by a similar process, only the potassium is replaced by sodium. Anhydrous magnesium chloride, together with sodium chloride and calcium fluoride, is fused in a closed crucible. The latter substances only serve to facilitate the formation of a fusible mass before and after the reaction, which is indispensable in order to prevent the access and action of air. One part of finely-divided sodium to five parts of magnesium chloride is thrown into the strongly-heated molten mass, and after stirring the reaction proceeds very quickly, and magnesium separates, $\mathrm{MgCl}_{2}+\mathrm{Na}_{2}=\mathrm{Mg}+2 \mathrm{NaCl}$. In working on a large scale, the powdery metallic magnesium is then subjected to distillation at a white heat. The distillation of the magnesium is necessary, because the undistilled metal is not homogeneous ${ }^{14}$ and burns unevenly : the metal is prepared for the purpose of illumination. Magnesium

13 Sodium and potassium only decompose magnesium oxide at a white heat and very feebly, probably for two reasons. In the first place, because the reaction $\mathrm{Mg}+\mathrm{O}$ develops more heat (about 140 thousand calories) than $\mathrm{K}_{2}+\mathrm{O}$ or $\mathrm{Na}_{2}+\mathrm{O}$ (about 100 thousand calories); and, in the second place, because magnesia is not fusible at the heat of a furnace and cannot act on the charcoal, sodium, or potassium-that is, it does not pass into that mobile state which is necessary for reaction. The first reason alone is not sufficient to explain the absence of the reaction between charcoal and magnesia, because iron and charcoal in combining with oxygen evolve less heat than sodium or potassium, yet, nevertheless, they can displace them. With respect to magnesium chloride, it acts on sodium and potassium, not only because their combination with chlorine evolves more heat than the combination of chlorine and magnesium $\left(\mathrm{Mg}+\mathrm{Cl}_{2}\right.$ gives 150 and $\mathrm{Na}_{2}+\mathrm{Cl}_{2}$ about 195 thousand calories), but also because a fusion, both of the magnesium chloride and of the double salt, takes place under the action of heat. It is probable, however, that a reverse reaction will take place.

14 Commercial magnesium generally contains a certain amount of magnesium nitride (Deville and Caron), $\mathrm{Mg}_{5} \mathrm{~N}_{2}$ - that is, a product of substitution of ammonia which is directly formed when magnesium is heated in nitrogen. It is yellowisb green powder, which gives ammonia and magnesia with water, and cyanogen when heated with carbonic anhydride. Perfectly pure magnesium may be obtained by the action of a galranic current. 
is a white metal, like silver ; it is not soft like the alkali metals, but is, on the contrary, hard like the majority of the ordinary metals. This is natural from the fact that it melts at a rather high temperature-namely, about $500^{\circ}$-and boils at about $1000^{\circ}$. It is malleable and ductile, like the generality of metals, so that it can be drawn into wires and rolled into ribbon; it is most frequently used for lighting purposes in the latter form. Unlike the alkali metals, magnesium does not decompose the atmospheric moisture at the ordinary temperature, so that it is almost unacted on by air ; it is not even acted on by water at the ordinary temperature, so that it may be washed to free it from sodium chloride. Magnesium only decomposes water with the evolution of hydrogen at the boiling point of water, ${ }^{15}$ and especially at still higher temperatures; but even this is accomplished with difficulty. This is explained by the fact that in decomposing water magnesium forms an insoluble hydroxide, $\mathrm{MgH}_{2} \mathrm{O}_{2}$, which covers the metal and hinders the further action of the water. Magnesium easily displaces hydrogen from acids, forming magnesium salts. When ignited it burns, not only in oxygen but in air (and even in carbonic anhydride), forming a white powder of magnesium oxide, or magnesia ; in burning it emits a white and exceedingly brilliant light. The strength of this light naturally depends on the fact that magnesium (24 parts by weight) in burning evolves about 140 thousand heat units, and that the product of combustion, $\mathrm{MgO}$, is infusible by heat; and therefore the vapour of the burning magnesium will contain an ignited powder of non-volatile and infusible magnesia, and will consequently present all the conditions for the production of a brilliant light. The light emitted by burning magnesium contains many rays which act chemically, and are situated in the violet and ultra-violet parts of the spectrum. For this reason burning magnesium may be employed for taking photographic images. ${ }^{16}$

Owing to its great affinity for oxygen, magnesium reduces many metals (zinc, iron, bismuth, antimony, cadmium, tin, lead, copper, silver, and others) from solutions of their salts at the ordinary temperature, ${ }^{17}$

1) Hydrogen peroxide (Welsing) dissolves magnesium. The reaction has not been investigated.

16 A special form of apparatus is used for burning magnesium. It is a clockwork arrangement in which a cylinder rotates, round which a ribbon or wire of magnesium is wound. The wire is subjected to a uniform unwinding and burning as the cylinder rotates, and in this manner the combustion may continue uniform for a certain time. The same is attained in special lamps, by causing a mixture of sand and finely-divided magnesium to fall from a funnel-shaped reservoir on to the flame. In photography it is best to blow finely-divided magnesium into a colourless (spirit or gas) flame.

17 According to the observations of Maack, Comaille, Böttger, and others. The reduction by heat mentioned further on was pointed out by Geuther, Phipson, Parkinson and Gattermann. 
and at a red heat finely-divided magnesium takes up the oxygen from silica, alumina, boric anhydride, \&c.; so that silicon and similar elements may be obtained by directly heating a mixture of powdered silica and magnesium in an infusible glass tube. ${ }^{18}$ Magnesium acts on fused potassium and sodium hydroxides, with an energetic evolution of hydrogen.

The affinity of magnesium for the halogens is much more feeble than for oxygen, ${ }^{19}$ as is already evident from the fact that a solution of iodine reacts feebly on magnesium; still magnesium burns in the vapours of iodine, bromine, and chlorine. The character of magnesium is also determined by the fact that all its salts, especially in the presence of water, are decomposable at a comparatively moderate temperature, the elements of the acid being evolved, and the magnesium oxide, which is non-volatile and unchangeable by heat, remaining behind. This naturally refers to those acids which are themselves volatilised by heat. Even magnesium sulphate is completely decomposed at the temperature at which iron melts, oxide of magnesium remaining behind. This decomposition of magnesium salts by heat proceeds much more easily than with calcium salts. For example, magnesium carbonate is totally decomposed at $170^{\circ}$, magnesium oxide being left behind. This magnesia, or magnesium oxide, is met with both in an anhydrous and hydrated state in nature (the anhydrous magnesia as the mineral periclase, $\mathrm{MgO}$, and the hydrated magnesia as brucite, $\mathrm{MgH}_{2} \mathrm{O}_{2}$ ). Magnesia is a well-known medicine (calcined magnesia-magnesia usta). It is a white, extremely fine, and very voluminous powder, of specific gravity 3.4 ; it is infusible by heat, and only shrinks or sinters in an oxyhydrogen flame. After long contact the anhydrous magnesia combines with water, although very slowly, forming the hydroxide $\mathrm{Mg}(\mathrm{HO})_{2}$, which, however, parts with its water with great ease when heated even below a red heat, and again yields anhydrous magnesia. This hydroxide is obtained directly as a gelatinous amorphous substance when a soluble alkali is mixed with a solution of any magnesium salt, $\mathrm{MgCl}_{2}+2 \mathrm{KHO}=\mathrm{Mg}(\mathrm{HO})_{2}+2 \mathrm{KCl}$. This decomposition proceeds to the end, and nearly all the magnesium passes into the precipitate; and this clearly shows the almost perfect insolubility of magnesia in water. Water dissolves a scarcely per-

18 This action of metallic magnesium in all probability depends, although only partially (see Note 13), on its volatility, and on the fact that, in combining with a given quantity of oxygen, it evolves more heat than aluminiuun, silicon, potassium, and other elements.

${ }^{19}$ Davy, on heating magnesia in chlorine, observed a complete substitution, because the volume of oxygen was half the volume of chlorine ; it is probable, however, that owing to the formation of chlorine oxide (Chapter XI. Note 30) the decomposition is not complete and is limited by a reverse reaction if the mass of the oxygen have no effect. 


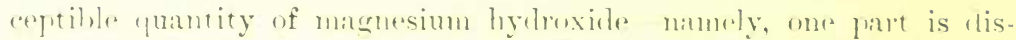

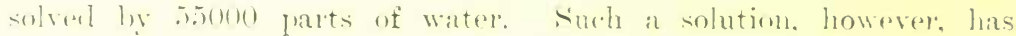
an alkaline reaction, and erives, with a salt of phosphorie arde a

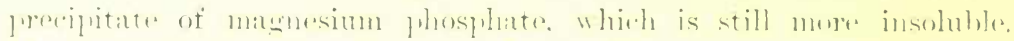

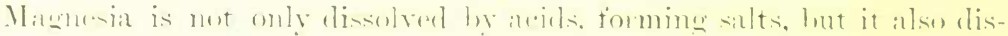

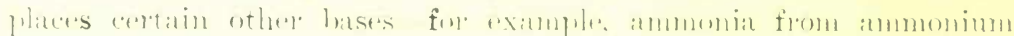

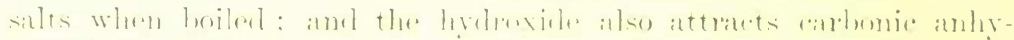

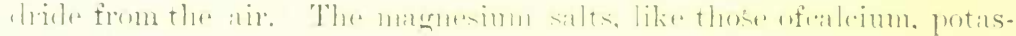

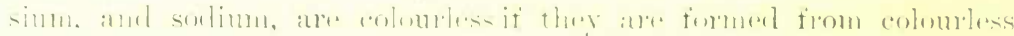

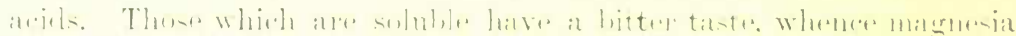

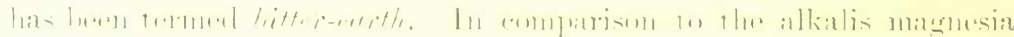

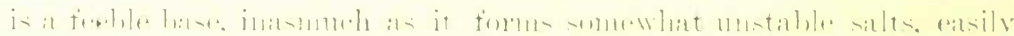

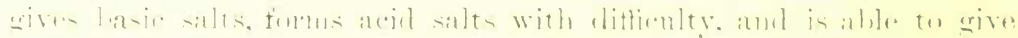

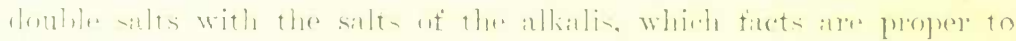

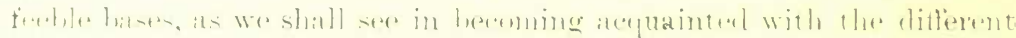
$|1+1+1 a|<$.

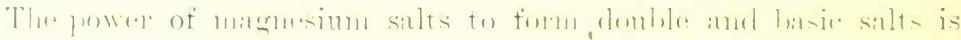

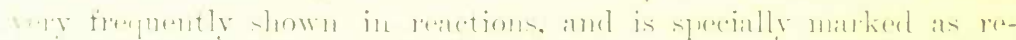

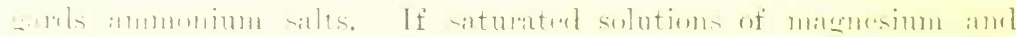

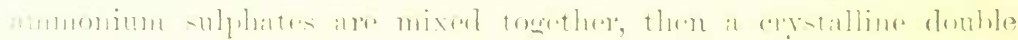

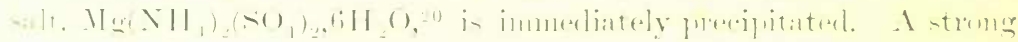

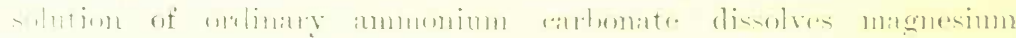

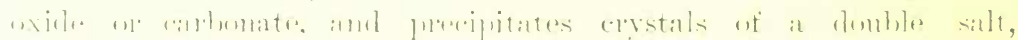

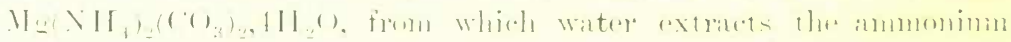

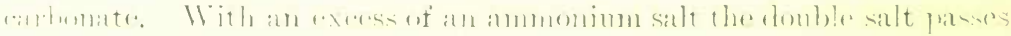

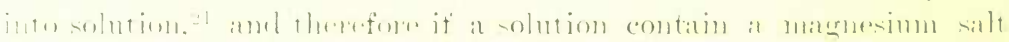

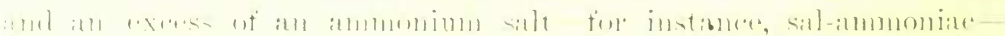

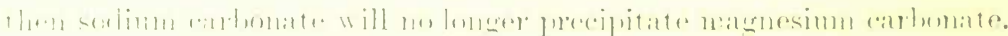

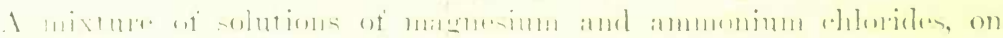

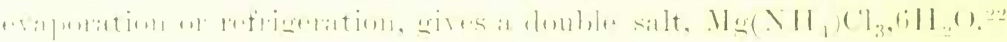

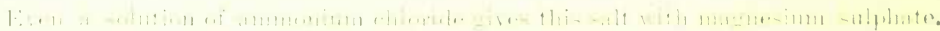

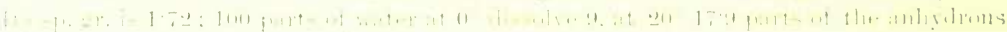

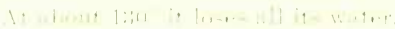

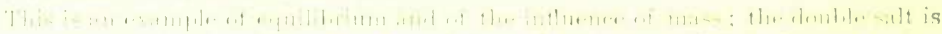

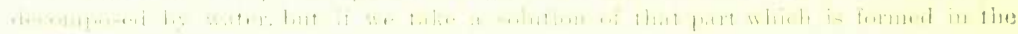

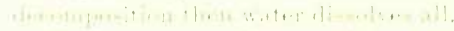

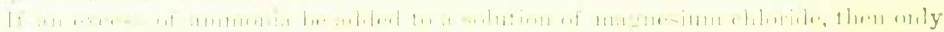

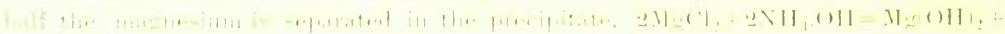

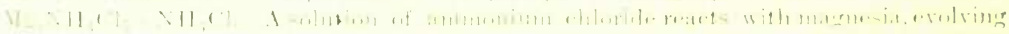

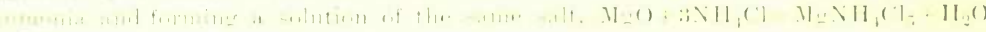
$2 \div 11$

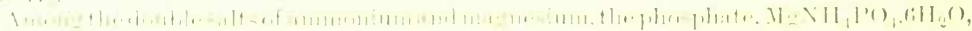

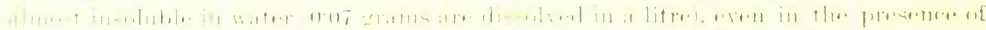

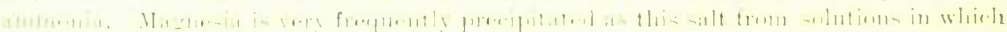

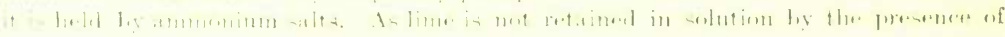


The salts of potassium, like those of ammonium, are able to enter into combination with the magnesium salts. ${ }^{23}$ For instance, the double salt, $\mathrm{MgKCl}_{3}, 6 \mathrm{H}_{2} \mathrm{O}$, which is known as carnallite, ${ }^{24}$ and occurs in the salt mines of Stassfurt, may be formed by freezing a saturated solution of potassium chloride with an excess of magnesium chloride. A saturated solution of magnesium sulphate dissolves potassium sulphate, and solid magnesium sulphate is soluble in a saturated solution of potassium sulphate. A double salt, $\mathrm{K}_{2} \mathrm{Mg}\left(\mathrm{SO}_{4}\right)_{2}, 6 \mathrm{H}_{2} \mathrm{O}$, which closely resembles the above-mentioned ammonium salt, crystallises from these solutions. ${ }^{25}$

ammonia salts, but is then precipitated by sodium carbonate, \&c., it is therefore very easy to separate calcium from magnesium on the basis of these properties.

25 In order to see the nature and cause of the formation of double salts, it is sufficient to regard them from the aspect (although this does not embrace the whole essence of the matter) that one of the metals of such salts (for instance, potassium) easily gives acid salts, and the other (in this instance, magnesium) basic salts; the properties of distinctly basic elements predominate in the former, whilst in the latter these properties are enfeebled, and the salts formed by them bear the character of acids-for instance, the salts of aluminium or magnesium act in many cases like acids. By their mutual combination both these properties of the salts are satisfied.

24 Carnallite has been mentioned in Chapter X. (Note 4) and in Chapter XIII. These deposits also contain much kainite, $\mathrm{KMgCl}\left(\mathrm{SO}_{4}\right), 3 \mathrm{H}_{2} \mathrm{O}$ (sp. gr., $2 \cdot 13 ; 100$ parts of water dissolve 79.6 parts at $18^{\circ}$ ). This double salt contains two metals and two haloids.

25. The component parts of certain double salts diffuse at different rates, and as the diffused solution contains a different proportion of the component salts than the solution taken of the double salt, it shows that such salts are decomposed by water. According to Riidorff, the double salts, like carnallite, $\mathrm{MgK}_{2}\left(\mathrm{SO}_{4}\right)_{2}, 6 \mathrm{H}_{2} \mathrm{O}$, and the alums, all belong to this order (1888). But such salts as tartar emetic, the double oxalates, and double cyanides are not separated by diffusion, which in all probability depends both on the relative rate of the diffusion of the component salts and on the measure of affinity acting between them. Those complex states of equilibrium which exist between water, the individual salts MX and NY, and the double salt MNXY, have been already partially analysed (as will be shown hereafter) in that case when the system is heterogeneous (that is, when something separates out in a solid state from the liquid solution), but in the case of equilibria in a homogeneous liquid medium (in a solution) the phenomenon is not so clear, because it concerns that very theory of solution which cannot yet be considered as established (Chapter I. Note 9, and others). As regards the heterogeneous decomposition of double salts, it has long been known that such salts as carnallite and $\mathrm{K}_{2} \mathrm{Mg}\left(\mathrm{SO}_{4}\right)_{2}$ give up the more soluble salt of magnesium if an insufficient quantity of water for their complete solution be taken. The complete saturation of 100 parts of water requires at $0^{\circ} 14 \cdot 1$, at $20^{\circ} 25$, and at $60^{\circ} 50^{\circ} 2$ parts of the latter double salt (anhydrous), while 100 parts of water dissolve 27 parts of magnesium sulphate at $0^{\circ}, 36$ parts at $20^{\circ}$, and 55 parts at $60^{\circ}$, of the anhydrous salt taken.

Of all the states of equilibrium exhibited by double salts the most fully investigated as yet is the system containing water, sodium sulphate, magnesium sulphate, and their double salt, $\mathrm{Na}_{2} \mathrm{Mg}\left(\mathrm{SO}_{4}\right)_{2}$, which crystallises with 4 and $6 \mathrm{~mol} . \mathrm{OH}_{2}$. The first crystallohydrate, $\mathrm{MgNa}_{2}\left(\mathrm{SO}_{4}\right)_{2}, 4 \mathrm{H}_{2} \mathrm{O}$, occurs at Stassfurt, and as a sedimentary deposit in many of the salt lakes near Astrakhan, and is therefore called astrakhanite. The specific gravity of the monoclinic prisms of this salt is $2 \cdot 22$. If this salt, in a finely-divided state, be mixed with the necessary (according to the equation) quantity of water, then the mixture solidifies like plaster of Paris into a lomogeneous mass if the temperature be below $22^{\circ}$ (Van't Hoff und Van Deventer, 1886; Bakhius Roozeboom, 1887); but if the temperature be above this transition-point then the water and double salt do not react 
The nearest analogues of magnesium are able to give exactly similar double salts, both in crystalline form (monoclinic system) and composition ; they, like this salt (see Chapter XV.), are easily able to $\left(140^{\circ}\right)$ part with all their water of crystallisation, and correspond with the salts of sulphuric acid, whose type may be taken as magnesium sulphate, $\mathrm{MgSO}_{4} \cdot{ }^{26}$ It occurs at Stassfurt as kieserite, $\mathrm{MgSO}_{4}, \mathrm{H}_{2} \mathrm{O}$,

on each other: $\mathrm{MgNa}_{2}\left(\mathrm{SO}_{4}\right)_{2}, 4 \mathrm{H}_{2} \mathrm{O}+13 \mathrm{H}_{2} \mathrm{O}=\mathrm{Na}_{2} \mathrm{SO}_{4}, 10 \mathrm{H}_{2} \mathrm{O}+\mathrm{MgSO}_{4}, 7 \mathrm{H}_{2} \mathrm{O}$; that is, they do not solidify or give a mixture of sodium and magnesium sulphates. If a mixture (in. equivalent quantities) of solutions of these salts be evaporated, and crystals of astrakhanite and of the individual salts capable of proceeding from it be added to the concentrated solution to avoid the possibility of a supersaturated solution, then at temperatures above $22^{\circ}$ astrakhanite is exclusively formed (this is the method of its production), but at lower temperatures the individual salts are alone produced. If equivalent amounts. of Glauber's salt and magnesium sulphate be mixed together in a solid state, then there is no change at temperatures below $22^{\circ}$, but at higher temperatures astrakhanite and water are formed. The volume corresponding with $\mathrm{Na}_{2} \mathrm{SO}_{4}, 10 \mathrm{H}_{2} \mathrm{O}$ in grams $=322 / 1 \cdot 46=220^{\circ}$. cubic centimetres, and of $\mathrm{MgSO}_{4}, 7 \mathrm{H}_{2} \mathrm{O}=246 / 1 \cdot 68=146.4$; hence their mixture in equivalent. quantities occupies a volume of $366^{\circ} 9$ c.c. The volume of astrakhanite $=334 / 2 \cdot 22=150 \cdot 5$, and the volume of $13 \mathrm{H}_{2} \mathrm{O}=234$, hence their sum $=380.5$ c.c., and therefore it is easy to follow the formation of the astrakhanite in a suitable apparatus (a kind of thermometer. containing oil and a powdered mixture of sodium and magnesium sulphates), and to see by the variation in volume that before $22^{\circ}$ it remains unchanged, and at higher temperatures proceeds more quickly the higher the temperature. At the transition temperature the solubility of astrakhanite and of the mixture of the component salts is one and the same, whilst at higher temperatures a solution which is saturated for a mixture of the individual salts would be supersaturated for astrakhanite, and at lower temperatures the solution of astrakhanite will be supersaturated for the component salts, as has been shown with especial detail by Karsten, Diacon, and others. Roozeboom showed that there are two limits to the composition of the solutions which can exist for a double salt; these limits are respectively obtained by dissolving a mixture of the double salt with each of its component simple salts. Van't Hoff demonstrated, besides this, that the tendency towards the formation of double salts has a distinct influence on the progress of double decomposition, because at temperatures above $31^{\circ}$ the mixture $2 \mathrm{MgSO}_{4}, 7 \mathrm{H}_{2} \mathrm{O}$ $+2 \mathrm{NaCl}$ passes into $\mathrm{MgNa}_{2}\left(\mathrm{SO}_{4}\right)_{2}, 4 \mathrm{H}_{2} \mathrm{O}+\mathrm{MgCl}_{2}, 6 \mathrm{H}_{2} \mathrm{O}+4 \mathrm{H}_{2} \mathrm{O}$, whilst below $31^{\circ}$ there is not this double decomposition, but it proceeds in the opposite direction, as may be demonstrated by the above-described methods.

From these examples on double salts we see that there is as close a dependence between the temperature and the formation of substances as there is between the temperature and a change of state. It is a case of the conceptions of Deville concerning dissociation, but extended in the direction of the passage of a solid into a liquid. On the other hand, we here see how essential a rôle water plays in the formation of compounds, and how the affinity for water of crystallisation is essentially analogous to the affinity between salts, and hence also to the affinity of acids for bases, because the formation of double salts does not in any essential point (except the measure of affinity-that is, from a quantitative aspect) differ from the formation of salts themselves. When sodium hydroxide with nitric acid gives sodium nitrate and water the phenomenon is essentially the same as in the fornation of astrakhanite from the salts $\mathrm{Na}_{2} \mathrm{SO}_{4}, 10 \mathrm{H}_{2} \mathrm{O}$ and $\mathrm{MgSO}_{4}, 7 \mathrm{H}_{2} \mathrm{O}$. Water is disengaged in both cases, and hence the volumes are altered.

26 This salt, and especially its erystallo-hydrate with $7 \mathrm{H}_{2} \mathrm{O}$, is generally known as. Epsom salts. It has long been used as a purgative. It is easily obtained from magnesia. and sulphuric acid, and it separates on the evaporation of sea water and of many saline springs. When carbonic anhydride is obtained by the action of sulphuric acid on 
and generally separates from solutions as a heptahydrated salt, $\mathrm{MgSO}_{4}, 7 \mathrm{H}_{2} \mathrm{O}$, and from supersaturated solutions as a hexahydrated salt, $\mathrm{MgSO}_{4}, 6 \mathrm{H}_{2} \mathrm{O}$; at temperatures below $0^{\circ}$ it crystallises out as a dodecahydrated salt, $\mathrm{MgSO}_{4}, 12 \mathrm{H}_{2} \mathrm{O}$, and a solution of the composition $\mathrm{MgSO}_{4}, 2 \mathrm{H}_{2} \mathrm{O}$ completely solidifies at $5^{\circ} .{ }^{27}$ Thus between water and magnesium sulphate there may exist several definite and more or less stable degrees of equilibrium, and the double salt $\mathrm{MgSO}_{4} \mathrm{~K}_{2} \mathrm{SO}_{4}, 6 \mathrm{H}_{2} \mathrm{O}$ may be regarded as one of these equilibrated systems, all the more as it contains $6 \mathrm{H}_{2} \mathrm{O}$, whilst $\mathrm{MgSO}_{4}$ is able to

magnesite, magnesium sulphate remains in solution. When dolomite-that is, a mixture of magnesium and calcium carbonates-is subjected to the action of a solution of hydrochloric acid until about half of the salt remains, then the calcium carbonate is chiefly dissolved and magnesium carbonate is left, and, by treatment with sulphuric acid, gives a solution of magnesium sulphate.

27 The anhydrous salt, $\mathrm{MgSO}_{4}$ (sp. gr., $2 \cdot 61$ ), attracts moisture $\left(7 \mathrm{~mol} . \mathrm{H}_{2} \mathrm{O}\right.$ ) from moist air; when heated in steam or hydrogen chloride it gives sulphuric acid, and when heated with carbon is decomposed, according to the equation $2 \mathrm{MgSO}_{4}+\mathrm{C}=2 \mathrm{SO}_{2}$ $+\mathrm{CO}_{2}+2 \mathrm{MgO}$. The monohydrated salt (kieserite), $\mathrm{MgSO}_{4}, \mathrm{H}_{2} \mathrm{O}$ (sp. gr., 2.56), dissolves in water with difficulty, and remains when the other crystallo-hydrates are heated to $135^{\circ}$. The hexahydrated salt is dimorphous. If a solution, saturated at the boiling-point, be prepared, and cooled without access of crystals of the heptahydrated salt, then $\mathrm{MgSO}_{4}, 6 \mathrm{H}_{2} \mathrm{O}$ crystallises out in monoclinic prisms (Loewel, Marignac), which are quite as unstable as the salt, $\mathrm{NaSO}_{4}, 7 \mathrm{H}_{2} \mathrm{O}$ (p. 509); but if prismatic crystals of the cubic system of the copper-nickel salts of the composition $\mathrm{MSO}_{4}, 6 \mathrm{H}_{2} \mathrm{O}$ be added, then crystals of $\mathrm{MgSO}_{4}, 6 \mathrm{H}_{2} \mathrm{O}$ are deposited on them as prisms of the cubic system (Lecoq de Boisbaudran). The common crystallo-hydrate, $\mathrm{MgSO}_{4}, 7 \mathrm{H}_{2} \mathrm{O}$, Epsom salts, belongs to the rhombic system, and is obtained by crystallisation below $30^{\circ}$. Its specific gravity is 1.69 . In a vacuum, or at $100^{\circ}$, it loses $5 \mathrm{H}_{2} \mathrm{O}$, at $132^{\circ} 6 \mathrm{H}_{2} \mathrm{O}$, and at $210^{\circ}$ all the $7 \mathrm{H}_{2} \mathrm{O}$ (Graham). If crystals of ferrous or cobaltic sulphate be placed in a saturated solution, then hexagonal crystals of the heptahydrated salt are formed (Lecoq de Boisbaudran); they present an unstable state of equilibrium, and soon become cloudy, probably owing to their transformation into the more stable common form. Fritzsche, by cooling saturated solutions below $0^{\circ}$, obtained a mixture of crystals of ice and of a dodecahydrated salt, which easily split up at temperatures above $0^{\circ}$. Guthrie showed that dilute solutions of magnesium sulphate, when refrigerated, separate ice until the solution attains a composition $\mathrm{MgSO}_{4}, 24 \mathrm{H}_{2} \mathrm{O}$, which will completely freeze into a crystallo-hydrate at $-5 \cdot 3^{\circ}$ (p. 97). According to Coppet and Riidorff, the temperature of the formation of ice falls by $0.073^{\circ}$ for every part by weight of the heptahydrated salt per 100 of water. This figure gives (Chapter I. Note 49) $i=1$ for both the heptahydrated and the anhydrous salt, from which it is clearly seen that it is impossible to judge the state of combination in which a dissolved substance occurs by the temperature of the formation of ice.

The solubility of the different crystallo-hydrates of magnesium sulphate, according to Loewel, also varies, like those of sodium sulphate or carbonate (see Chapter XII. Notes 7 and 18). At $0^{\circ} 100$ parts of water dissolves $40.75 \mathrm{MgSO}_{4}$ in the presence of the hexahydrated salt, $34.67 \mathrm{MgSO}_{4}$ in the presence of the hexagonal heptaliydrated salt, and only 26 parts of $\mathrm{MgSO}_{4}$ in the presence of the ordinary heptahydrated salt-that is, solutions giving the remaining crystallo-hydrates will be supersaturated for the ordinary heptahydrated salt.

All this shows how many diverse aspects of more or less stable equilibria may exist between water and a substance dissolved in it; this has already been enlarged on in Chapter I.

Carefully purified magnesium sulphate in its aqueous solution gives, according to. 


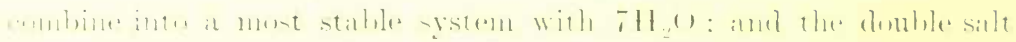

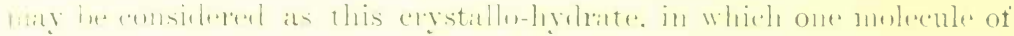

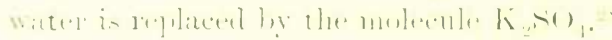

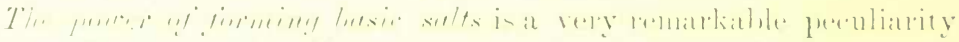

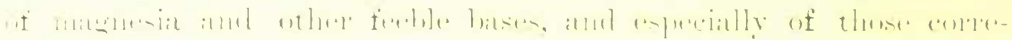

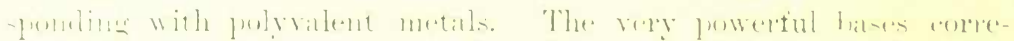

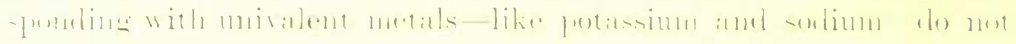

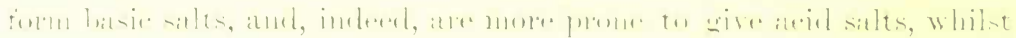

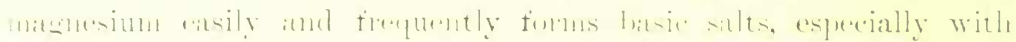

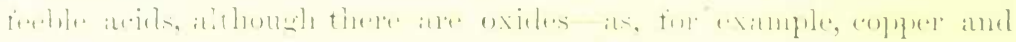

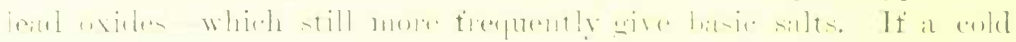

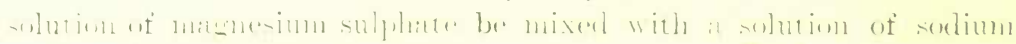

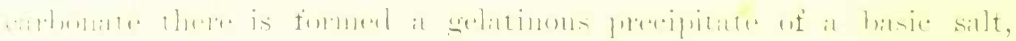

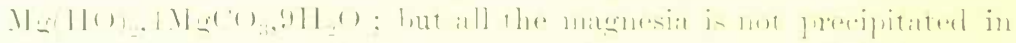

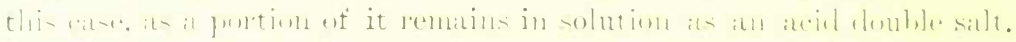

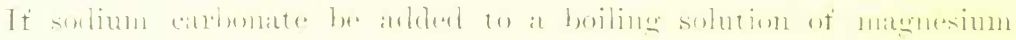

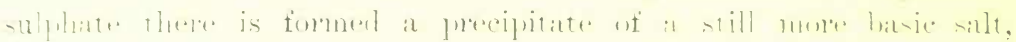

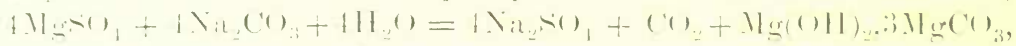

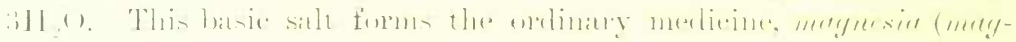

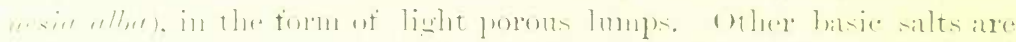

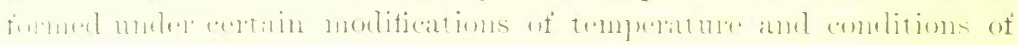

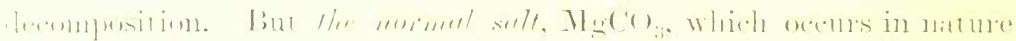

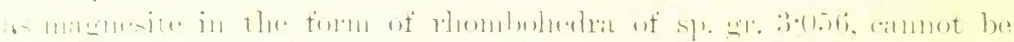

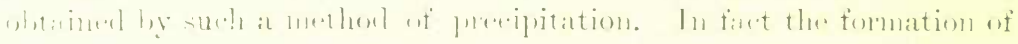

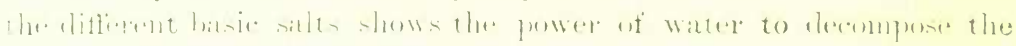

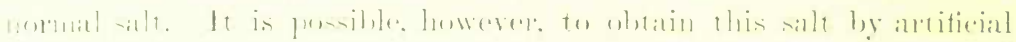

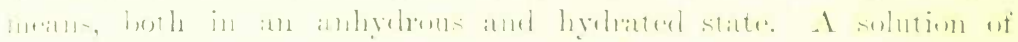

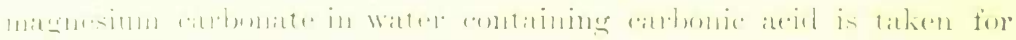

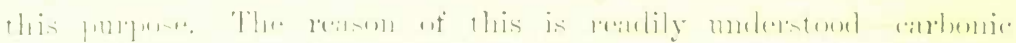

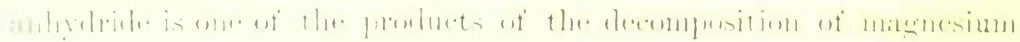

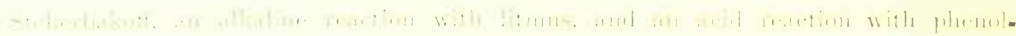

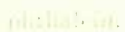

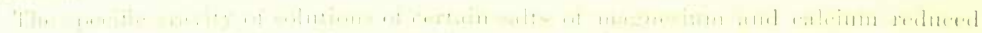

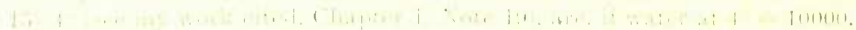

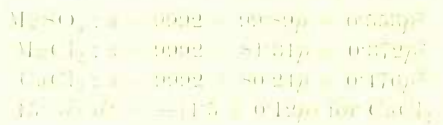

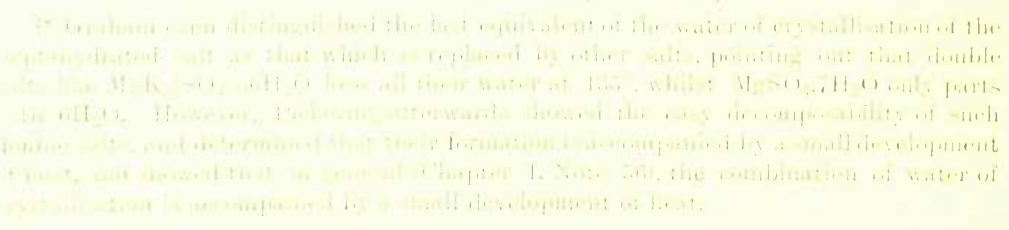


carbonate in the presence of water. If this solution be left to evaporate spontaneously the normal salt separates in a hydrated form, but in the evaporation of a heated solution, through which a stream of carbonic anhydride is passed, the anhydrous salt is formed as a crystalline mass, which remains unaltered in the air, like the natural mineral. ${ }^{29}$ The decomposing influence of water on the salts of magnesium, which is directly dependent on the feeble basic properties of magnesia, ${ }^{30}$ is most clearly seen in magnesium chloride, $\mathrm{MgCl}_{2}$. This salt is contained, ${ }^{31}$ as we have already seen (Chapter X.), in sea water, and remains in the last mother liquors of its evaporation. On cooling a sufficiently concentrated solution, the crystallo-hydrate, $\mathrm{MgCl}_{2}, 6 \mathrm{H}_{2} \mathrm{O}$, separates $;^{32}$ but if it be further heated (above $106^{\circ}$ ) to remove the water, then hydrochloric acid passes off together with the latter, so that there ultimately remains magnesia with a small quantity of magnesium chloride. ${ }^{33}$ From what has been said it is evident that anhydrous magnesium chloride cannot be obtained by simple evaporation. But if sal-ammoniac or sodium chloride be added to a solution of magnesium chloride, then the evolution of hydrochloric acid does not take place, and after complete evaporation the remaining mass is entirely soluble in water. This renders it possible to obtain anhydrous

29 The crystalline form of the anhydrous salt obtained in this manner is not the same as that of the natural salt. The former gives rhombohedra, like those in which calcium carbonate appears as calc spar, whilst the natural salt appears as rhombic prisms, like those sometimes presented by the same carbonate, as aragonite, which will soon be described.

30 Magnesium sulphate enters into certain reactions which are proper to sulphuric acid itself. Thus, for instance, if a carefully-prepared mixture of equivalent quantities of hydrous magnesium sulphate and sodium chloride be heated to a red heat, the evolution of hydrochloric acid is observed just as in the action of sulphuric acid on common salt, $\mathrm{MgSO}_{4}+2 \mathrm{NaCl}+\mathrm{H}_{2} \mathrm{O}=\mathrm{Na}_{2} \mathrm{SO}_{4}+\mathrm{MgO}+2 \mathrm{HCl}$. Magnesium sulphate acts in a similar manner on nitrates, with the evolution of nitric acid. A mixture of it with common salt and manganese peroxide gives chlorine. Sulphuric acid is sometimes replaced by magnesium sulphate in galvanic batteries-for example, in the well-known Meidinger battery. In the above-mentioned reactions we see a striking example of how alike the reactions of acids and salts are, especially of salts which are formed by such feeble bases as magnesia.

31 As sea water contains many salts, $\mathrm{MCl}$ and $\mathrm{MgX}_{2}$, it follows, according to Berthollet's teaching, that $\mathrm{MgCl}_{2}$ is also present.

32 As the crystallo-hydrates of the salts of sodium often contain $10 \mathrm{H}_{2} \mathrm{O}$, so many of the salts of magnesium contain $6 \mathrm{H}_{2} \mathrm{O}$.

$5 \overline{3}$ This decomposition is most simply defined as the result of the two reverse reactionf, $\mathrm{MgCl}_{2}+\mathrm{H}_{2} \mathrm{O}=\mathrm{MgO}+2 \mathrm{HCl}$ and $\mathrm{MgO}+2 \mathrm{HCl}=\mathrm{MgCl}_{2}+\mathrm{H}_{2} \mathrm{O}$, or as a distribution between $\mathrm{O}$ and $\mathrm{Cl}_{2}$ on the one hand and $\mathrm{H}_{2}$ and $\mathrm{Mg}$ on the other. It is then clear that according to Berthollet's doctrine, the mass of the hydrochloric acid converts the magnesium oxide into chloride, and the mass of the water converts the magnesium chloride into oxide. The crystallo-hydrate, $\mathrm{MgCl}_{2}, 6 \mathrm{H}_{2} \mathrm{O}$, forms the limit of the reversibility. But an intermediate state of equilibrium may exist in the form of basic salts. On mixing ignited magnesia with a solution of magnesium chloride of specific gravity about $1 \cdot 2$, a solid mass is obtrined which is scarcely decomposed by water at the ordinary temperature (see Zinc).

voL. 1. 


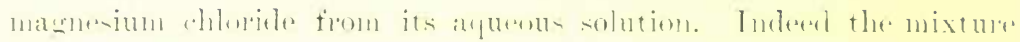

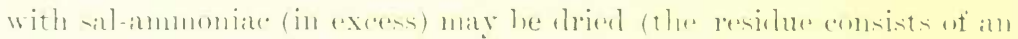

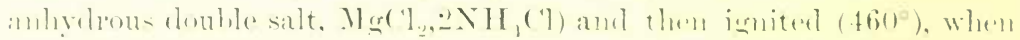

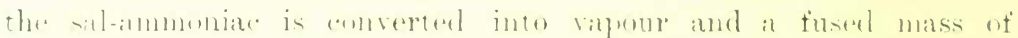

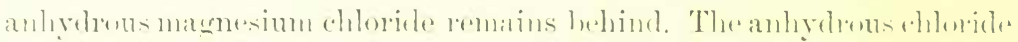
evelves at very eonsiderable andunt of heat on the aldition of water, which shews the ereat attinity the salt has for water. ${ }^{3}$ Anhedrous magnesiun chloride is not only ohtained ly the ahove methed. hut is

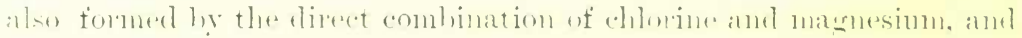

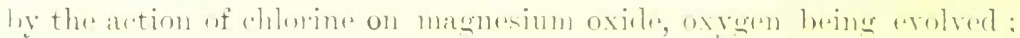

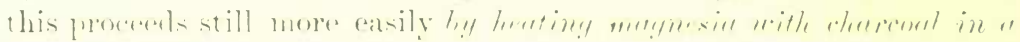

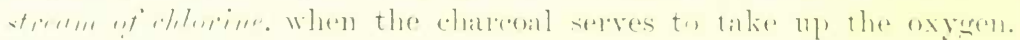

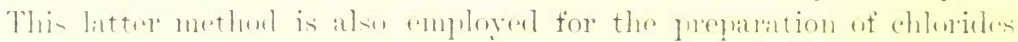
which are formed in an anhyllous form with -till eneater dittienly

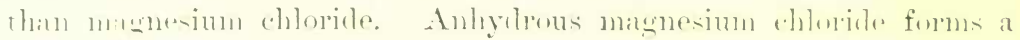
coldourlene, transparent mass, composel of flexible erystalline plates

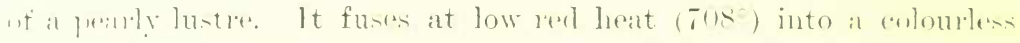

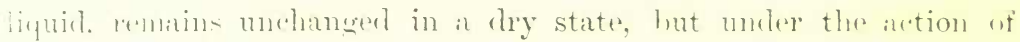

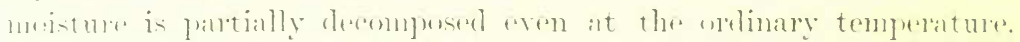
with formation of hriluehloric acid.

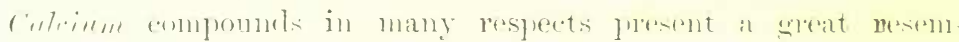

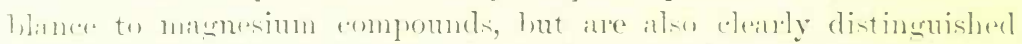

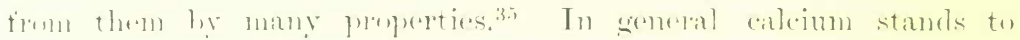

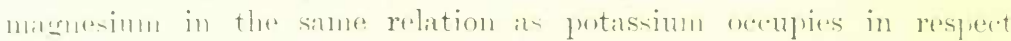

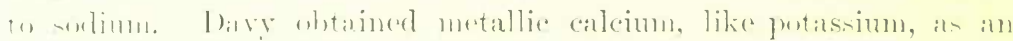

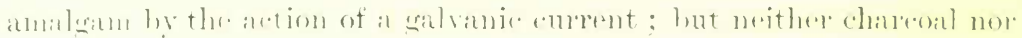

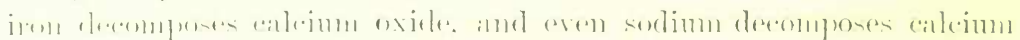

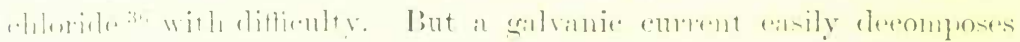

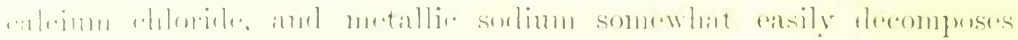

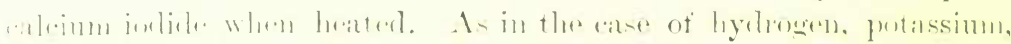

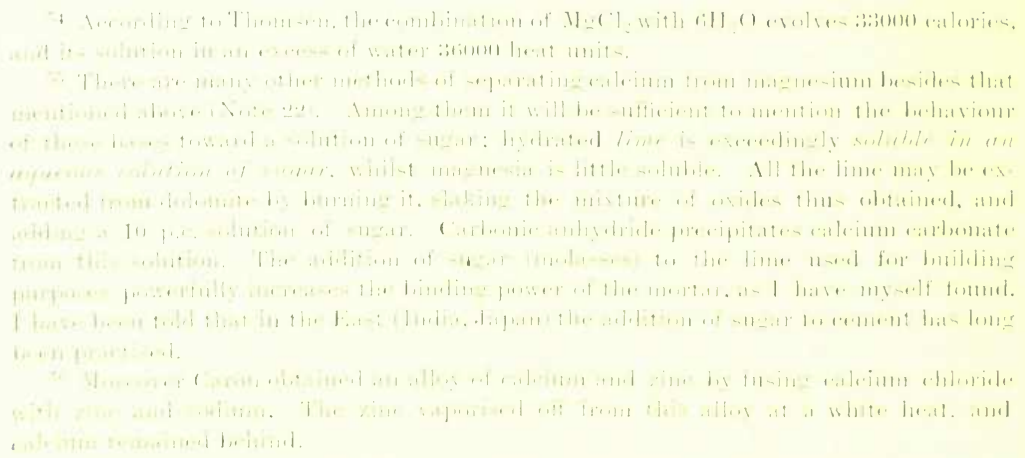


and magnesium, the affinity of iodine for calcium is feebler than that of chlorine (and oxygen), and therefore it is not astonishing that calcium iodide may be subjected to that decomposition, into which the chloride and oxide enter with difficulty. ${ }^{37}$ Metallic calcium is of a yellow colour, and has a considerable lustre, which it preserves in dry air. Its specific gravity is $1 \cdot 58$. Calcium is distinguished by its great ductility ; it melts at a red heat and then burns in the air with a very brilliant flame, which is easily understood from the fact that finely-divided infusible calcium oxide is formed. Judging from the fact that in burning calcium gives a very large flame, it is probable that this metal is volatile. Calcium decomposes water at the ordinary temperature, and is oxidised in moist air, but not so rapidly as sodium. In burning, calcium gives its oxide or lime, $\mathrm{CaO}$, a substance which is familiar to -every one, and of which we have already frequently had occasion to speak. This oxide is not met with in nature in a free state, because it is an energetic base which everywhere encounters acid substances, with which it forms salts. Generally it is combined with silica, or occurs as calcium carbonate or sulphate. The calcium carbonate and nitrate are decomposed, at a red heat, with the formation of lime. As a rule, the carbonate, which is so frequently met with in nature, serves as the source of the calcium oxide, both commercial and pure. When heated calcium carbonate dissociates: $\mathrm{CaCO}_{3}=\mathrm{CaO}+\mathrm{CO}_{2}$. In practice the decomposition is conducted at a bright red heat, in the presence of steam, or a current of a foreign gas, in heaps or in special kilns. ${ }^{38}$

37 Calcium iodide may be prepared by saturating lime with hydriodic acid. It is a very soluble salt (at $20^{\circ}$ one part of the salt requires 0.49 parts and at $43^{\circ} 0.35$ parts of water for solution), is deliquescent in the air, and resembles calcium clloride in many respects. It changes but little when evaporated, and, like calcium chloride, fuses when heated, and therefore all the water may be driven off by heat. If anhydrous calcium iodide be heated with an equivalent quantity of sodium in a closely-covered iron crucible, sodium iodide and metallic calcium are formed (Liés-Bodart). Dumas advises carrying on this reaction in a closed space under pressure.

38 Kilns which act either intermittently or continuously are built for this purpose. The kilns of the first kind are filled with alternate layers of fuel and limestone; the fuel is lighted, and the heat developer by its combustion serves for decomposing the limestone. When the process is completed the kiln is allowed to cool somewhat, the lime raked out, and the same process repeated. In the continuously-acting furnaces, constructed like that shown in fig. 78 , the kiln itself only contains limestone, and there are lateral hearths for burning the fuel, whose flame passes through the limestone and serves for its decomposition. Such furnaces are able to work continuously because the unburnt linestone may be charged from above, and the burnt lime raked out from below.

It is not every limestone that is suitable for the preparation of lime, becruse many contain impurities, especially clay, dolomite, and sand. Such limestones when burnt either partially fuse or give an impure lime, called poor lime in distinction from that pure lime which is obtained from purer limestone, and which is called rich lime. The latter quality is characterised by its disintegrating into a fine powder when treated with water, and is suitable for the majority of uses to which lime is applied, and for which 
Calcium oxide that is, quicklime is a sub-tance (spe gr. $3 \cdot 15)$

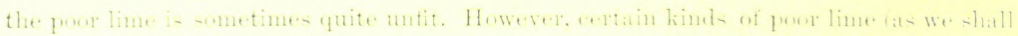

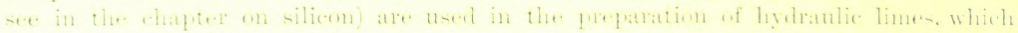

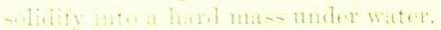

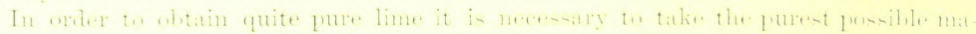

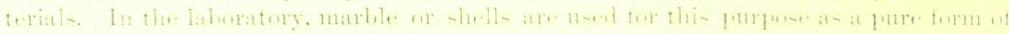

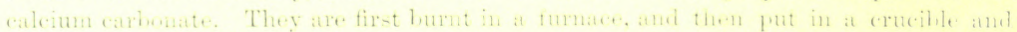
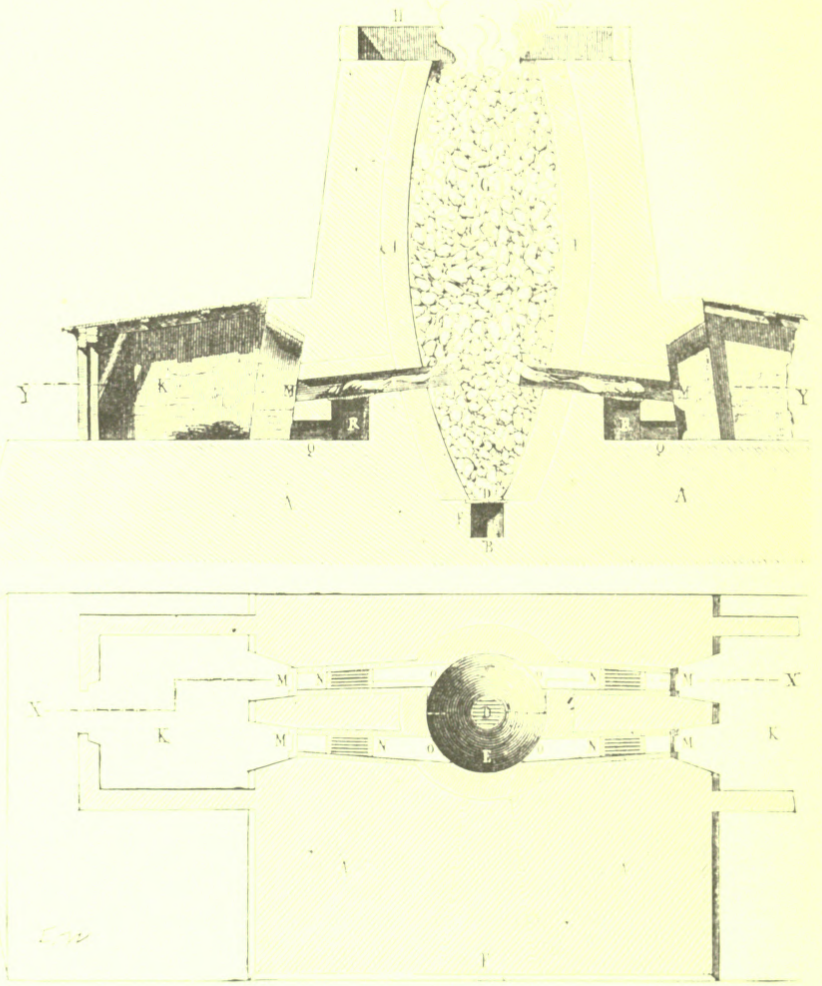

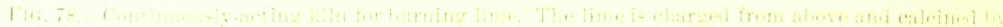

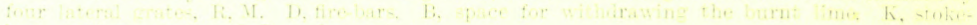

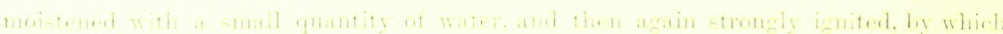

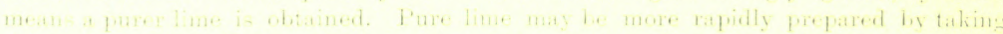

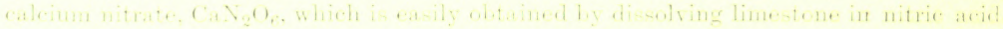

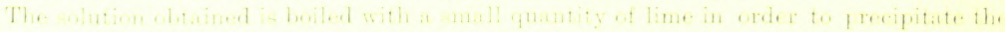

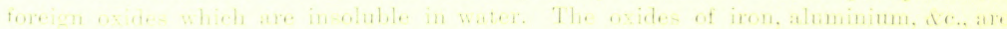

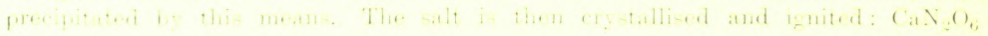

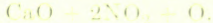

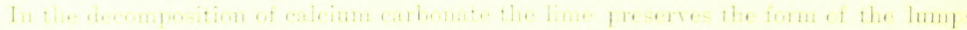

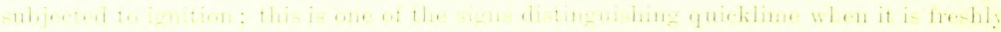


which is unaffected by heat, ${ }^{39}$ and may therefore serve as a fireresisting material, and was employed by Deville for the construction of furnaces in which platinum was melted, and silver volatilised by the action of the heat evolved by the combustion of detonating gas. The hydrated lime, slaked lime, or calcium hydroxide, $\mathrm{CaH}_{2} \mathrm{O}_{2}$ (sp. gr. 2.07 ) is a most common alkaline substance, which is employed largely in building for making mortars or cements, in which case its binding property is mainly due to the absorption of carbonic anhydride. ${ }^{40}$ Lime, like other alkalis, acts on many animal and vegetable substances, and for this reason has many practical uses-for example, for removing fats, and in agriculture for accelerating the decomposition of organic substances in the so-called composts or accumulations of vegetable and animal remains used for fertilising land. Calcium hydroxide easily loses its water at a moderate heat $\left(530^{\circ}\right)$, but it does not part with water at $100^{\circ}$. When mixed with water lime forms a pasty mass, which is known as slaked lime, and in a more dilute form as milk of lime, because when shaken up in water

burnt and unaltered by air. It attracts moisture from the air and then disintegrates into a powder; if left long exposed in the air it also attracts carbonic anliydride and increases in volume; it does not entirely pass into carbonate, but forms a compound of the latter with caustic lime.

${ }^{39}$ Lime, when heated to a white heat in the vapour of potassium, gives calcium, and in chlorine it disengages oxygen. Sulphur, phosphorus, \&c., when heated with lime, are absorbed by it.

40 The greater quantity of lime is used in making mortar for binding bricks or stones together, in the form of lime or cement, or the so-called slaked lime. For this purpose the lime is mixed with water and sand, which serves to separate the particles of lime from each other. If only lime paste were put between two bricks they would not hold firmly together, because after the water had evaporated the lime would occupy a smaller space than before, and therefore cracks and powder would form in its mass, so that it would not at all produce that complete cementation of the bricks which it is desired to attain. Pieces of stone-that is, sand-mixed with the lime hinder this process of disintegration, because the lime binds together the individual grains of sand mixed with it, and forms one concrete mass, in consequence of a process which proceeds after the desiccation or removal of the water. The process of the solidification of lime, taken as slaked lime, consists first in the direct evaporation of the water and crystallisation of the hydrate, so that the lime binds the stones and sand mixed with it, just as glue binds two pieces of wood. But this preliminary binding action of lime is feeble (as is seen by direct experiment) unless there be further alteration of the lime leading to the formation of carbonates, silicates, and other salts of calcium which are distinguished by their great cohesion. With the progress of time the cement is partially subjected to the action of the carbonic anhydride in the air, owing to which calcium carbonate is formed, but not more than half the lime is thus converted into carbonate. Besides which, the lime partially acts on the silica of the bricks, and it is owing to these new combinations simultaneously forming in the cement that it gradually becomes stronger and stronger. Hence the binding action of the lime becomes stronger with the progress of time. This is the reason (and not, as is sometimes said, because the ancients knew how to build stronger than we) why buildings which have stood for centuries possess a very strongly binding cement. Hydraulic cements will be described in the chapter on silicon. 


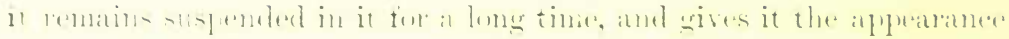

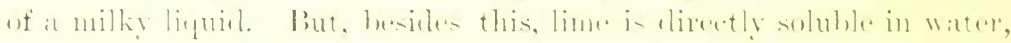

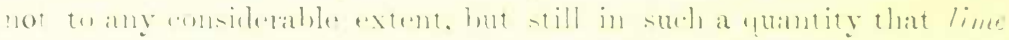

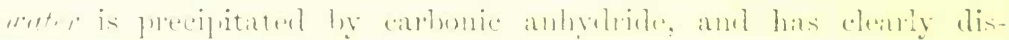

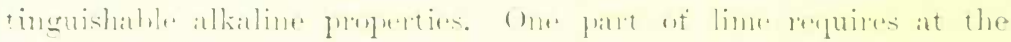

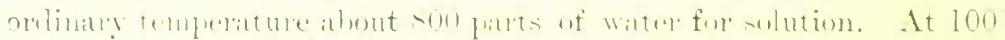

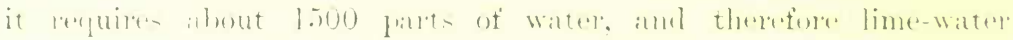

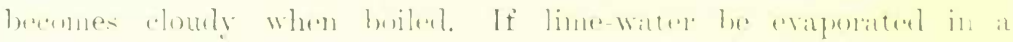

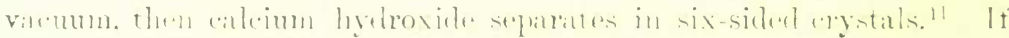

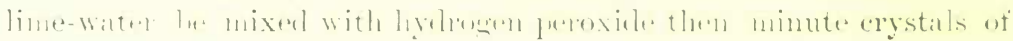

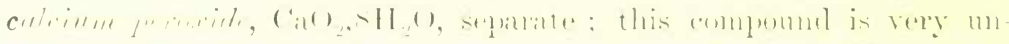

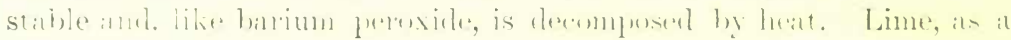

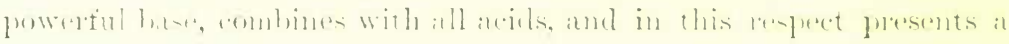

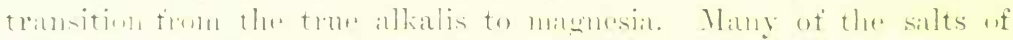

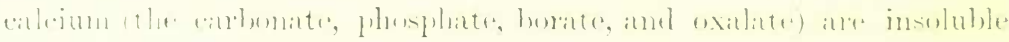
in watel : lesiles which the sulphate is only spatringly soluhle in

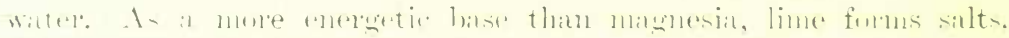

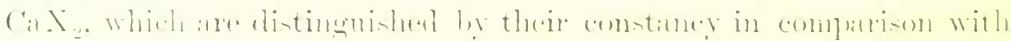

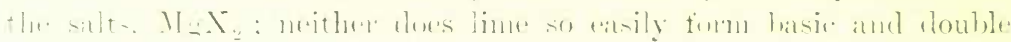
sillts it mitultesite

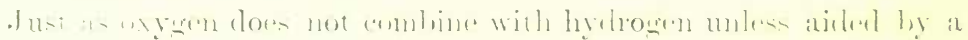

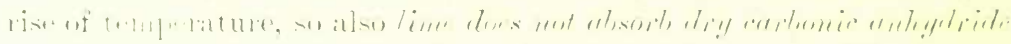

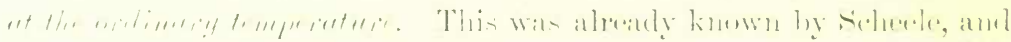

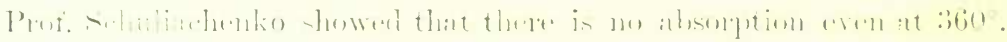

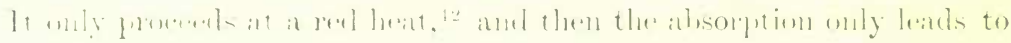

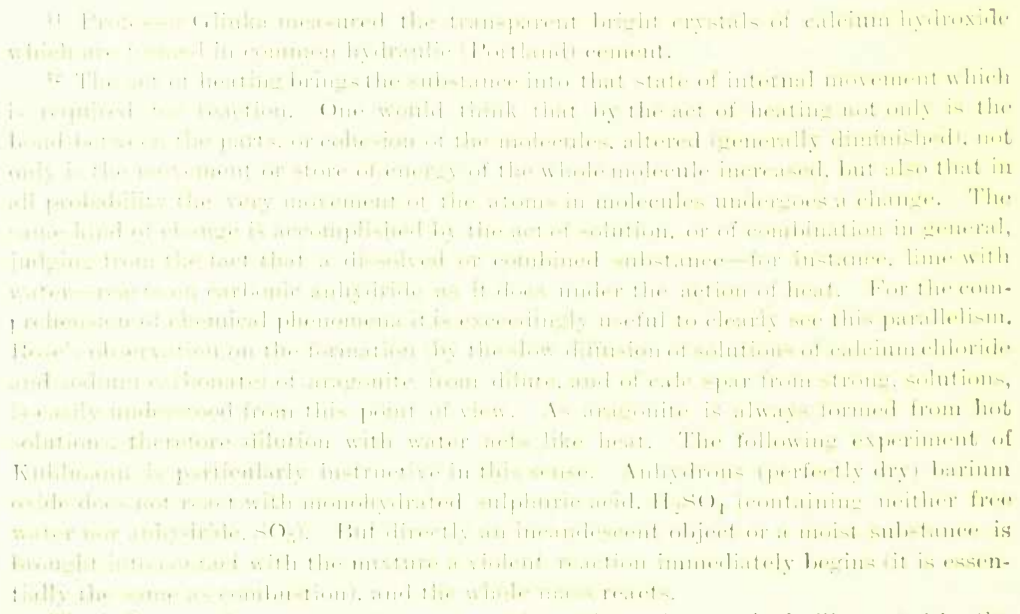

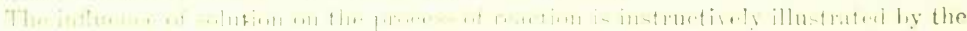


the formation of a mixture of calcium oxide and carbonate (Rose). But if the lime be slaked or taken in solution, then the absorption of earbonic anhydride proceeds rapidly and to the end. These phenomena are connected with the dissociation of calcium carbonate, studied by Debray (1867) under the influence of the conceptions of dissociation introduced into science by Henri Sainte-Claire Deville. Just as there is no vapour tension for non-volatile substances, so there is no dissociation tension of carbonic anhydride for calcium carbonate at the ordinary temperature. Just as every volatile substance has a maximum possible vapour tension for every temperature, so also calcium carbonate has its corresponding dissociation tension; thus at $770^{\circ}$ (the boiling point of cadmium) about $85 \mathrm{~mm}$. (of the mercury column), and at $930^{\circ}$ (the boiling point of $\mathrm{Zn}$ ) it is about $520 \mathrm{~mm}$. As, if the tension be greater, there will be no evaporation, so also there will be no decomposition. Debray took crystals of calc spar, and could not observe the least change in them at the boiling point of zinc $\left(930^{\circ}\right)$ in an atmosphere of carbonic anhydride taken at the atmospheric pressure $(760 \mathrm{~mm}$.), whilst, on the other hand, calcium carbonate may be completely decomposed at a much lower temperature if the tension of the carbonic anhydride be less than the dissociation tension, which may be arrived at either by directly pumping away the gas with an air-pump, or by mixing it with some other gas - that is, by diminishing the partial pressure of the carbonic anhydride, ${ }^{43}$ just as an object may be dried at the ordinary temperature by removing the aqueous vapour or by carrying it off in a stream of another gas. Thus it is possible to obtain calcium carbonate from lime and carbonic anhydride at a certain temperature above that at which dissociation begins, and conversely to decompose calcium carbonate at the same temperature into lime and carbonic anhydride. ${ }^{44}$

following experiment. Lime, or barium oxide, is placed in a flask or retort having an upper orifice and connected with a tube immersed in mercury. A funnel furnished with a stopcock and filled with water is fixed into the upper orifice of the retort, which is then filled with dry carbonic anhydride. There is no absorption. When the temperature of equilibrium is arrived at, the unslaked oxide is made to absorb all the carbonic anhydride by carefully letting in water. A vacuum is formed, as is seen by the mercury rising up the neck of the retort. With water the absorption goes on to the end, whilst under the action of heat there remains the dissociating tension of the carbonic anhydride. Furthermore, we here see that, with a certain resemblance, there is also a distinction, depending on the fact that at low temperatures calcium carbonate does not dissociate; this determines the complete absorption of the carbonic anhydride in the aqueous solution.

43 Experience has long shown that by moistening partially-burnt lime with water and re-heating it, it is easy to drive off the last traces of carbonic anhydride from it, and that, in general, by blowing air or steam through the lime, and even by using moist fuel, it is possible to accelerate the decomposition of the calcium carbonate. The partial pressure is decreased by these means.

44 Before Deville's conception of dissociation, the modus operandi of decompo. 


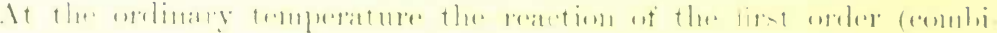

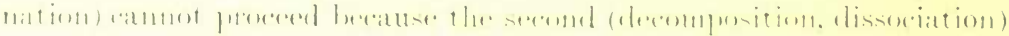

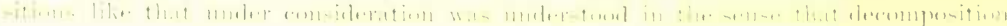

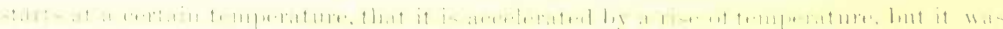

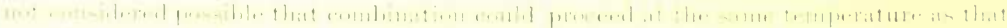

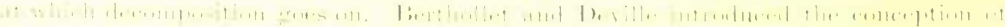

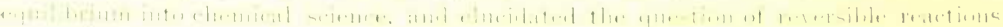

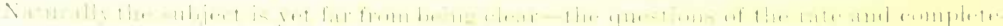

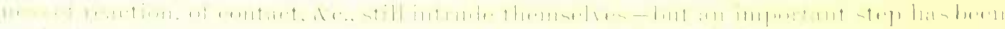

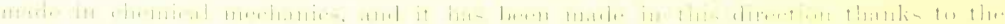

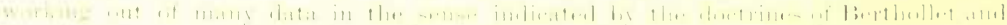

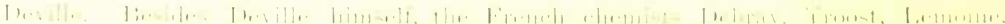

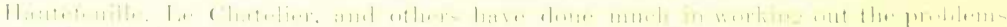

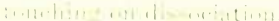

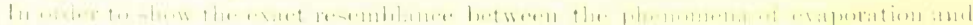

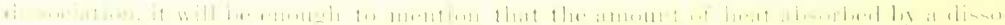

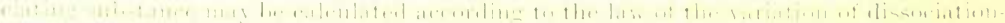

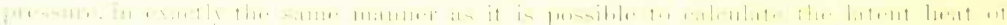

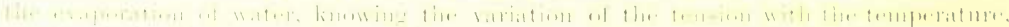

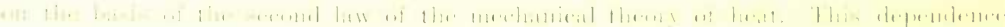

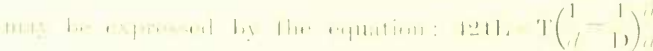

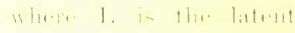

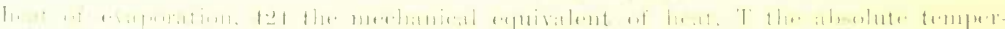
ant

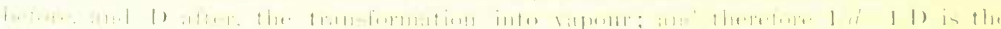

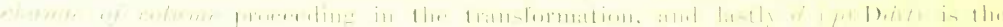

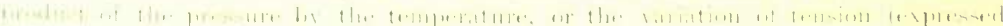

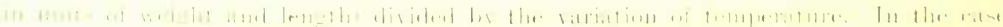

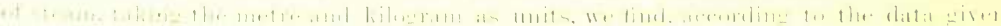

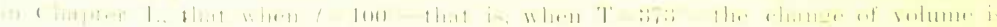

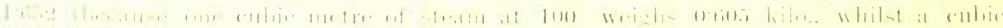

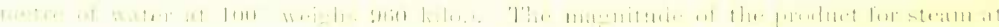

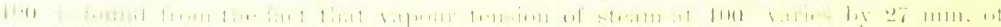

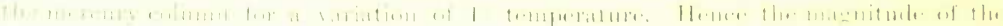

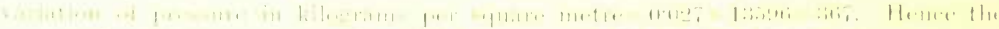

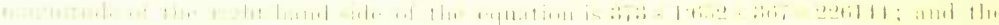

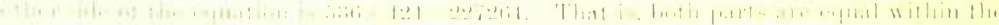

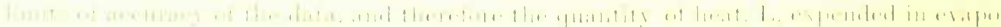

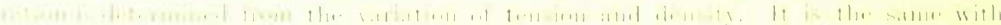

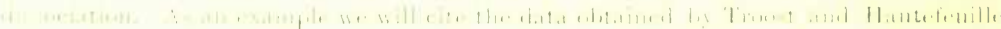

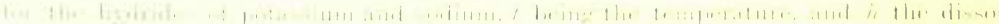

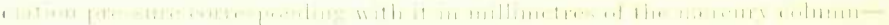

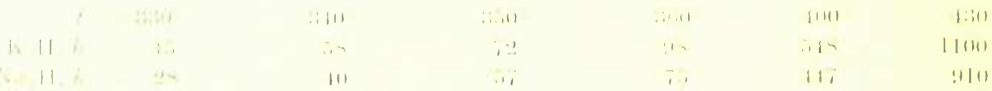

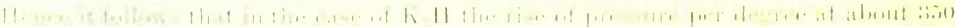

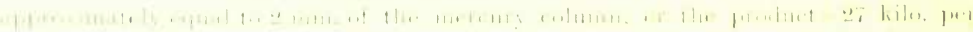

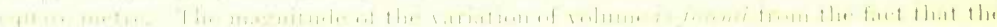

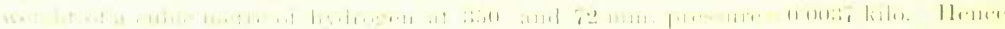

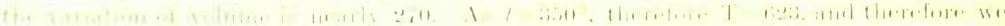

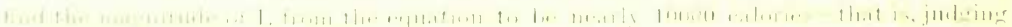

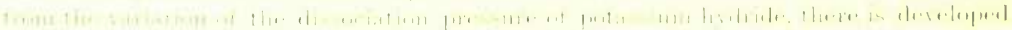

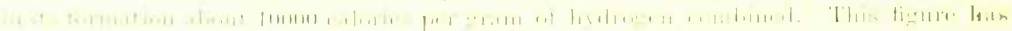


cannot take place, and thus all the most important phenomena with respect to the behaviour of lime towards carbonic anhydride are explained by starting from one common basis. ${ }^{45}$

Calcium carbonate, $\mathrm{CaCO}_{3}$-which occurs, as has already been mentioned, in limestone, marble, dr.-is sometimes met with in nature in a crystalline form, and it forms an example of the phenomenon termed dimorphism - that is, it appears in two crystalline forms. When it exhibits combinations of forms belonging to the hexagonal system (six-sided prisms, rhombohedra, dc.) it is called calc spar. Calc spar has a specific gravity of $2 \cdot 7$, and is further characterised by a distinct cleavage along the planes of the fundamental rhombohedron having an angle of $105^{\circ}$. Perfectly transparent Iceland spar presents a clear example of double refraction (for which reason it is frequently

not been verified by experiment, nor has the calculated figure of 13 calories for the formation of sodium hydride, $\mathrm{Na}_{2} \mathrm{H}$, at 300 been verified; but the figure of $4 \cdot 1$ calories calculated $\left(20^{\circ}\right)$ by Moutier from Troost's data for the dissociation pressure of palladium hydride, entirely agrees with the direct results of Favre, which showed that each gram of hydrogen which combines with palladium at the ordinary temperature develops $4 \cdot 174$ calories, and therefore one and the same conception of the mechanical theory of heat is applicable to dissociation and evaporation. The heat evolved in the absorption of hydrogen depends, naturally, not only on the physical process of the condensation of the gas, but also on the formation of a new chemical compound, as is seen from the fact that different metals in absorbing a gram of hydrogen develop a different amount of heatnamely, palladium 4 calories, potassium 10 calories, sodium 13 calories, platinum 20 calories-and therefore in the concordance of calculation with fact we should see, not an identity, but only a resemblance between the phenomena of dissociation and evaporation.

45 But still the question as to the formation of a basic calcium carbonate remains undecided. According to certain data one would think that, with a rise of temperature, not only calcium carbonate but also a basic salt may be formed, which, however, is denied by certain investigators. Probably the presence of water complicates all the relations between lime and carbonic anhydride, all the more as the existence of an attraction between calcium carbonate and water is seen from its being able to give a crystallohydrate, $\mathrm{CaCO}_{3}, 5 \mathrm{H}_{2} \mathrm{O}$ (Pelouze, Salm-Horstmar), which crystallises in rhombic prisms of sp. gr. about 1.77 and loses its water at $20^{\circ}$. These crystals are obtained when a solution of lime in sugar and water is left long exposed to the air and slowly attracts carbonic anhydride from it, and also by the evaporation of such a solution at a temperature of about $3^{\circ}$. On the other hand, it is probable that an acid salt $\mathrm{CaH}_{2}\left(\mathrm{CO}_{3}\right)_{2}$ is formed in an aqueous solution, not only because water containing carbonic acid dissolves calcium carbonate, but more especially judging by the researches of Schloesing (1872), which showed that at $16^{\circ}$ a litre of water in an atmosphere of earbonic anhydride (pressure 0.984 atmosphere) dissolves 1.086 grams of calcium carbonate and 1.778 grams of carbonic anliydride, which corresponds with the formation of calcium hydrogen carbonate, and the solution of carbonic anhydride in the remaining water. Caro showed that a litre of water is able to dissolve as mueh as 3 grams of calcium carbonate if the pressure be increased to 4 and more atmospheres. The calcium carbonate is precipitated when the carbonic anhydride passes off in the air or in a current of another gas; this also takes place in many uatural springs. Tufa, stalactites, and other like formations from waters containing calcium carbonate and carbonic acid in solution, are formed in this manner. The solubility of calcium carbonate itself at the ordinary temperature does not exceed 13 milligrams per litre of water. 
employed in physical apparatus). The other form of calcium carbonate occurs in crystals belonging to the rhombic system, and it is then called aragonite; its specific gravity is 3.0 . If calcium carbonate is artificially produced by slow crystallisation at the ordinary temperature, then it appears in the rhombohedral form, but if the crystallisation be aided by heat then it appears as aragonite. One may suppose, therefore, that calc spar presents the form corresponding with a low temperature, and aragonite with a higher temperature during crystallisation. ${ }^{46}$

Calcium sulphate in combination with two equivalents of water, $\mathrm{CaSO}_{4}, 2 \mathrm{H}_{2} \mathrm{O}$, is very widely distributed in nature, and is known as gypsum. Gypsum loses its two equivalents of water at a moderate temperature, ${ }^{47}$ and anhydrous or burnt gypsum is then obtained, which is also known as plaster of Paris, and is employed in large quantities. for modelling. ${ }^{48}$ This use depends on the fact that burnt and finelydivided and sifted gypsum forms a paste when mixed with water; after a certain time this paste becomes slightly heated and solidifies, owing to the fact that the anhydrous calcium sulphate, $\mathrm{CaSO}_{4}$, again combines with water. When the plaster of Paris and water are first made into a paste they form a mechanical mixture, but when the mass

46 When calcium carbonate separates out from solutions, it at first has a gelatinous. appearance, which leads one to think that this salt appears in a colloidal state. It only crystallises with the progress of time. The colloidal state of calcium carbonate is particularly clear from the following observations made by Prof. Famintsin, who showed that when it separates from solutions it is obtained under certain conditions in the form of grains having the peculiar paste-like structure proper to starch, which fact has not only an independent interest, but presents an example of a mineral substance being obtained in a form until then only known in the organic substances elaborated in plants. This shows that the forms (cells, vessels, \&c.) in which vegetable and animal substances occur in organisms do not present in themselves anything peculiar to organisms, but are only the result of those particular conditions in which these substances are formed. Traube and afterwards Monnier and Vogt(1882) obtained formations which, under the microscope, were in every respect identical in appearance witl vegetable cells, by means of a similar slow formation of precipitates (by reacting on sulphates of different metals with sodium silicate or carbonate). Owing to its insolubility in water, calcium carbonate may be easily obtained from any other soluble calcium salt by the addition of a solution of an alkali carbonate; for example, ammonium carbonate.

${ }_{47}$ According to Le Chatelier (1888), $1 \frac{1}{2} \mathrm{H}_{2} \mathrm{O}$ is lost at $120^{\circ}$-that is, $\mathrm{H}_{2} \mathrm{O}, 2 \mathrm{CaSO}_{4}$ is formed, but at $194^{\circ}$ all the water is expelled. According to Shenstone and Cundall (1888) gypsum begins to lose water at $70^{\circ}$ in dry air. The semi-hydrated compcund, $\mathrm{H}_{2} \mathrm{O}, 2 \mathrm{CaSO}_{4}$, is also formed when gypsum is heated with water in a closed vessel at $150^{\circ}$ (Hoppe-Seyler).

48 For stucco-work it is usual to add lime and sand, as the mass is then harder and does not solidify so quickly. For imitating marble, glue is added to the plaster, and the mass is polished when thoroughly dry. Reburnt gypsum cannot be used over again, as that which has once solidified is, like the natural anhydride, not able to re-combine with water. It is evident that the structure of the molecules in the crystallised mass, or in general in any dense mass, exerts an influence on the chemical action, as is particularly clearly seen in metals in their different forms (powder, crystalline, rolled, \&c.). 
solidifies, then a compound of the calcium sulphate with two molecules of water is produced; and this may be regarded as derived from $\mathrm{S}(\mathrm{OH})_{6}$ by the substitution of two atoms of hydrogen by one atom of bivalent calcium. Natural gypsum sometimes appears as perfectly colourless, or variegated marble-like, masses, and sometimes in perfectly colourless crystals, selenite, of sp. gr. $2 \cdot 33$. The semi-transparent gypsum, or alabaster, is often carved into small statues. Besides which an anhydrous calcium sulphate, $\mathrm{CaSO}_{4}$, called anhydrite (sp. gr. 2.97), occurs in nature. It sometimes occurs along with gypsum. It is no longer capable of combining directly with water, and differs in this respect from the anhydrous salt obtained by gently igniting gypsum. If gypsum be very strongly heated it shrinks and loses its power of combining with water. Gypsum also passes into anhydrite if it be heated in water, if the temperature be as high as $150^{\circ}$. One part of calcium sulphate requires at $0^{\circ} 525$ parts of water for solution, at $38^{\circ} 466$ parts, and at $100^{\circ} 517$ parts of water. The maximum solubility, which is at about $36^{\circ}$, is nearly the same as that of sodium sulphate. ${ }^{49}$

As lime is a more energetic base than magnesia, so calcium chloride, $\mathrm{CaCl}_{2}$, is not so easily decomposed by water, and its solutions only disengage a small quantity of hydrochloric acid when evaporated, and when the evaporation is conducted in a stream of hydrochloric acid it. easily gives an anhydrous salt which fuses at $719^{\circ}$; otherwise an aqueous solution yields a crystallo-hydrate, $\mathrm{CaCl}_{2}, 6 \mathrm{H}_{2} \mathrm{O}$, which inelts at $28^{\circ} .50$

49 As Marignac showed, gypsum especially desiccated at $120^{\circ}$ easily gives supersaturated solutions with respect to $\mathrm{CaSO}_{4}, 2 \mathrm{H}_{2} \mathrm{O}$, which contain as much as 1 part of $\mathrm{CaSO}_{4}$ to 110 parts of water. Boiling dilute hydrochloric acid dissolves gypsum, forming calcium chloride. The behaviour of gypsum towards the alkali carbonates has been described in Chap. X. Alcohol precipitates gypsum from its aqueous solutions, because, like the sulphates in general, it is sparingly soluble in alcohol. Gypsum, like all the sulphates, when heated with charcoal, gives up its oxygen, forming the sulphide, CaS.

Calcium sulphate, like magnesium sulphate, is capable of forming double salts, but with difficulty, and they are chemically less stable. They contain, as is always the case with double salts, less water of crystallisation than the component salts. Rose, Phillips, Schott, Stchefarovitch, Struvé, Ditte, and otkers obtained the salt $\mathrm{CaK}_{2}\left(\mathrm{SO}_{4}\right)_{2}, \mathrm{H}_{2} \mathrm{O}$; a mixture of gypsum with an equivalent amount of potassium sulphate and water solidifies into a homogeneous mass. Fritzsche obtained the corresponding sodium salt in a hydrated and anhydrous state, by heating a mixture of gypsum with a saturated solution of sodium sulphate. The anhydrous salt occurs in nature as glauberite. Fritzsche also obtained gaylussite, $\mathrm{Na}_{2} \mathrm{Ca}\left(\mathrm{CO}_{3}\right)_{2}, 5 \mathrm{H}_{2} \mathrm{O}$, by pouring a saturated solution of sodium carbonate over freshly-precipitated calcium carbonate.

so Calcium chloride has a specific gravity $2 \cdot 20$, or, when fused, $2 \cdot 12$, and the sp. gr. of the crystallised salt $\mathrm{CaCl}_{2}, 6 \mathrm{H}_{2} \mathrm{O}$ is $1^{\circ} 69$. If the volume of the crystals at $0^{\circ}=1$, then at $29^{\circ}$ it is 1.020 , and the volume of the fused mass at the same temperature is 1.118 (Kopp) (specific gravity of solutions, see Note 27). The solution containing 50 p.c. $\mathrm{CaCl}_{2}$ boils at 130,70 p.c. at $158^{\circ}$. Superheated steam decomposes calcium chloride with more diffi- 
As for potassium, $\mathrm{K}=39$ (and sodium, $\mathrm{Na}=23$ ), there are the near analogues, $\mathrm{Rb}=85$ and $\mathrm{Cs}=133$, and also another, $\mathrm{Li}=7$, so in exactly the same manner for calcium, $\mathrm{Ca}=40$ (and magnesium, $\mathbf{M g}=24)$, there is another analogue of lighter atomic weight, or beryllium, $\mathrm{Be}=9$, besides the near analogues, strontium, $\mathrm{Sr}=87$, and barium, $\mathrm{Ba}=137$. As rubidium and caesium are more rarely met with in nature than potassium, so also strontium and barium are rarer

culty than magnesium chloride and with greater ease than barium chloride (Kuhnheim). Sodium does not decompose fused calcium chloride even with further heating (LiésBodart), but an alloy of sodium with zinc, lead, or bismuth decomposes it, forming an alloy of calcium with one of the above-named metals (Caron). The zinc alloy may be obtained with as much as 15 p.c. of calcium. Calcium chloride is soluble in alcohol and absorbs ammonia.

A molecular gram weight of calcium chloride in dissolving in an excess of water evolves 18723 calories, and in dissolving in alcohol 17555 units of heat, according to Pickering.

Roozeboom made detailed researches on the crystallo-hydrates of calcium chloride (1889), and found that $\mathrm{CaCl}_{2}, 6 \mathrm{H}_{2} \mathrm{O}$ melts at $30^{\circ} 2^{\circ}$, and is formed at low temperatures from solutions containing not more than 103 parts of calcium chloride per 100 parts of water; if the amount of salt (always to 100 parts of water) reaches 120 parts, then tabular crystals of $\mathrm{CaCl}_{2}, 4 \mathrm{H}_{2} \mathrm{O} \beta$ are formed, which at temperatures above $38.4^{\circ}$ are converted into the crystallo-hydrates $\mathrm{CaCl}_{2}, 2 \mathrm{H}_{2} \mathrm{O}$, whilst at temperatures below $18^{\circ}$ the variety $\beta$ passes into the more stable $\mathrm{CaCl}_{2}, 4 \mathrm{H}_{2} \mathrm{Oa}$, which process is aided by mechanical friction. Hence, as is the case with magnesium sulphate (Note 27), one and the same crystallo-hydrate appears in two forms-the $\beta$, which easily forms, but does not keep and is unstable, and the other, $\alpha$, which is stable. The solubility of the above-mentioned hydrates of chloride of calcium, or amount of calcium chloride per 100 parts of water, is as follows :-

\begin{tabular}{lcrccr} 
& $0^{\circ}$ & $20^{\circ}$ & $30^{\circ}$ & $40^{\circ}$ & \multicolumn{2}{c}{$60^{\circ}$} \\
$\mathrm{CaCl}_{2}, 6 \mathrm{H}_{2} \mathrm{O}$ & 60 & 75 & 100 & \multicolumn{2}{c}{$(102 \cdot 8)$} \\
$\mathrm{CaCl}_{2}, 4 \mathrm{H}_{2} \mathrm{O} \alpha$ & - & 90 & 101 & 117 \\
$\mathrm{CaCl}_{2}, 4 \mathrm{H}_{2} \mathrm{O} \beta$ & - & 104 & 114 & - & $(154 \cdot 2)$ \\
$\mathrm{CaCl}_{2}, 2 \mathrm{H}_{2} \mathrm{O}$ & - & - & $(308 \cdot 3)$ & 128 & 137
\end{tabular}

The amount of calcium chloride to 100 parts of water in the crystallo-hydrate given in brackets. The point of intersection of the curves of solubility lies at about $30^{\circ}$ for the first two salts and about $45^{\circ}$ for the salts with $4 \mathrm{H}_{2} \mathrm{O}$ and $2 \mathrm{H}_{2} \mathrm{O}$. The crystals $\mathrm{CaCl}_{2}, 2 \mathrm{H}_{2} \mathrm{O}$ may, however, be obtained (Ditte) at the ordinary temperature from solutions containing hydrochloric acid. The vapour tension of this crystallo-hydrate equals the atmospheric at $165^{\circ}$, and therefore the crystals may be dried in an atmosphere of steam and obtained without a mother liquor, whose vapour tension is greater. This crystallo-hydrate decomposes at about $175^{\circ}$ into $\mathrm{CaCl}_{2}, \mathrm{H}_{2} \mathrm{O}$ and a solution; this is easily brought about in a closed ressel when the pressure is greater than the atmosphere. This crystallo-hydrate is destroyed at temperatures above $260^{\circ}$, anhydrous calcium chloride being formed.

On the other hand, Hammerl showed that solutions of calcium chloride, when frozen, deposit ice if they contain less than 48 parts of salt per 100 of water, and if more the crystallo-hydrate $\mathrm{CaCl}_{2}, 6 \mathrm{H}_{2} \mathrm{O}$ separates, and that a solution of the above composition $\left(\mathrm{CaCl}_{2}, 14 \mathrm{H}_{2} \mathrm{O}\right.$ requires 44.0 parts calcium chloride per 100 of water) solidifies as a cryohydrate at about $-55^{\circ}$. Thus the solubility of calcium chloride is better known than that of any other salt.

Neglecting the unstable equilibrium $\mathrm{CaCl}_{2}, 4 \mathrm{H}_{2} \mathrm{O} \beta$, we will cite the temperatures $t$ at which the passage of one hydrate into another takes place and at which the solution $\mathrm{CaCl}_{2}+n \mathrm{H}_{2} \mathrm{O}$, the two solids $\mathrm{A}$ and $\mathrm{B}$ and aqueous vapour, whose tension is given an $p$ 
than calcium (as also bromine and iodine are rarer than chlorine). Whilst exhibiting many points of resemblance with calcium, strontium and barium may be characterised after a very short acquaintance with their chief compounds; this shows the important advantages gained by distributing the elements according to their natural groups, to which matter we shall turn our attention in the next chapter.

Among the compounds of barium met with in nature the commonest is the sulphate, $\mathrm{BaSO}_{4}$, which forms anhydrous crystals of the rhombic system, which are identical in their crystalline form with anhydrite, and which generally occur as transparent and semi-transparent masses of tabular crystals having a high specific gravity, namely $4 \cdot 45$, for which reason this salt bears the name of heavy spar or barytes. Analogous to it is celestine, $\mathrm{SrSO}_{4}$, which is, however, more rarely met with; heavy spar frequently forms the gangue separated on dressing metallic ores from the vein stuff. This mineral is the source of all other barium compounds, because the carbonate, although more easily transformed into the other compounds (because acids act directly on it, evolving carbonic anhydride), is, however, a comparatively rare mineral $\left(\mathrm{BaCO}_{3}\right.$ forms the mineral witherite; $\mathrm{SrCO}_{3}$, strontianite; both are rare, the latter is found at Etna). The treatment of barium sulphate is rendered difficult from the fact that it is insoluble both in water and acids, and has therefore to be conducted by a method of reduction. ${ }^{51}$ Like sodium sulphate and calcium sulphate, heavy spar when heated with charcoal parts with its oxygen and forms barium sulphide, BaS. For this purpose a pasty mixture of powdered heavy spar, charcoal, and tar is subjected to the action of a strong heat, in which case $\mathrm{BaSO}_{4}+4 \mathrm{C}=\mathrm{BaS}+4 \mathrm{CO}$. The residue is then treated with water, in which the barium sulphide is soluble. ${ }^{52}$ When boiled with hydrochloric

in millimetres, are able to exist together in stable equilibrium, according to Roozeboom's determinations-

\begin{tabular}{ccclc}
$t$ & $n$ & \multicolumn{1}{c}{$\mathrm{A}$} & \multicolumn{1}{c}{$\mathrm{B}$} & $p$ \\
$-55^{\circ}$ & $14 \cdot 5$ & ice & $\mathrm{CaCl}_{2}, 6 \mathrm{H}_{2} \mathrm{O}$ & 0 \\
$+29 \cdot 8^{\circ}$ & $6 \cdot 1$ & $\mathrm{CaCl}_{2}, 6 \mathrm{H}_{2} \mathrm{O}$ & $\mathrm{CaCl}_{2}, 4 \mathrm{H}_{2} \mathrm{O}$ & $6 \cdot 8$ \\
$45 \cdot 3^{\circ}$ & $4 \cdot 7$ & $\mathrm{CaCl}_{2}, 4 \mathrm{H}_{2} \mathrm{O}$ & $\mathrm{CaCl}_{2}, 2 \mathrm{H}_{2} \mathrm{O}$ & $11 \cdot 8$ \\
$175 \cdot 5^{\circ}$ & $2 \cdot 1$ & $\mathrm{CaCl}_{2}, 2 \mathrm{H}_{2} \mathrm{O}$ & $\mathrm{CaCl}_{2}, \mathrm{H}_{2} \mathrm{O}$ & 842 \\
$260^{\circ}$ & $1 \cdot 8$ & $\mathrm{CaCl}_{2}, \mathrm{H}_{2} \mathrm{O}$ & $\mathrm{CaCl}_{2}$ & Several atmospheres.
\end{tabular}

Solutions of calcium chloride may serve as a convenient example for the study of the supersaturated state, which easily comes about in this case, becanse different hydrates are formed. Thus at $25^{\circ}$ solutions containing more than 83 parts of anhydrous calcium chloride per 100 of water will be supersaturated for the hydrate $\mathrm{CaCl}_{2}, 6 \mathrm{H}_{2} \mathrm{O}$. p. 427.

The action of barium sulphate on sodium and potassium carbonates is given on

32 Barium sulphide is decomposed by water, $\mathrm{BaS}+2 \mathrm{H}_{2} \mathrm{O}=\mathrm{H}_{2} \mathrm{~S}+\mathrm{Ba}(\mathrm{OH})_{2}$ (the reaction is reversible), but both substances are soluble in water, and their separation is complicated by the fact that barium sulphide absorbs oxygen and gives insoluble barium 


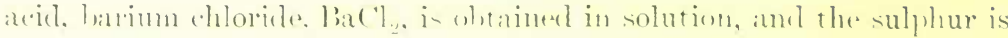

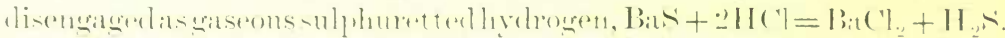

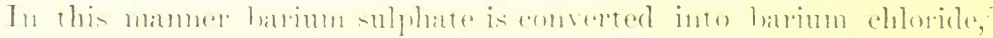

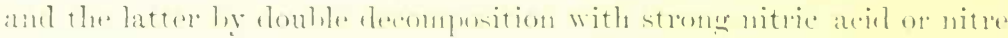

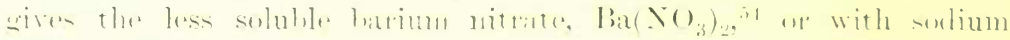

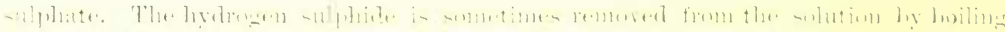

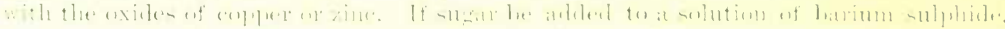

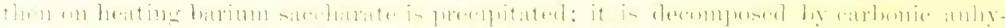

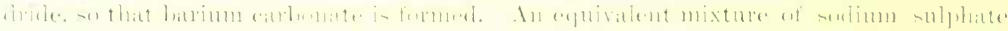

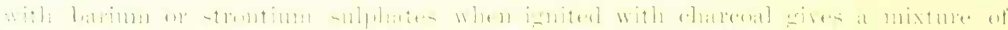

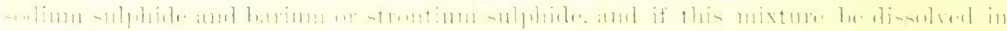

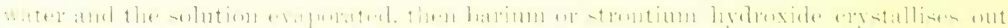

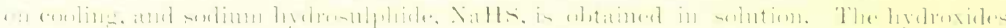

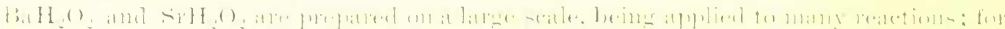

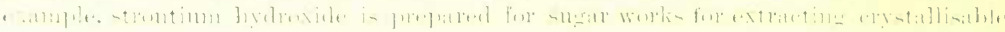

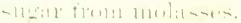

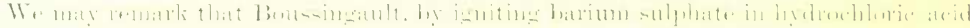

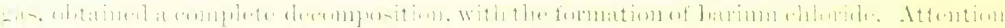

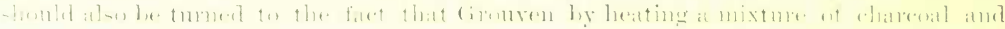

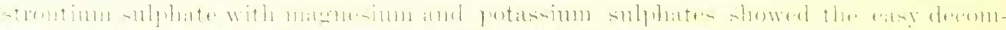

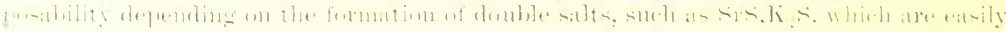

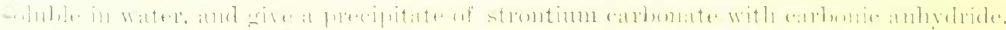

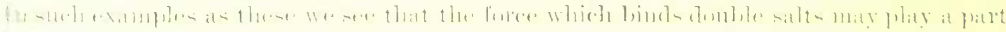

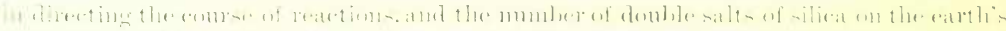

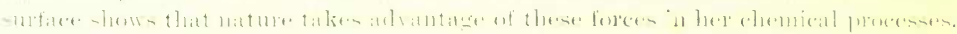

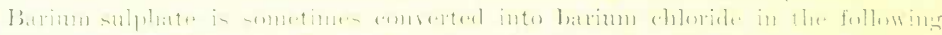

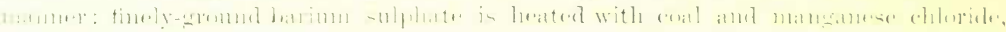

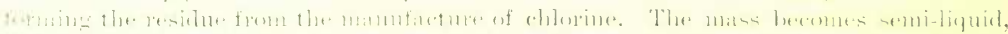

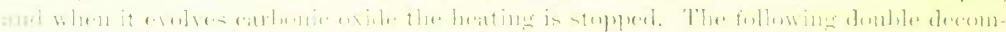

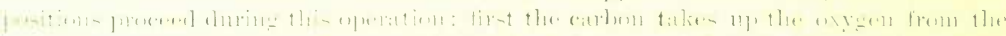

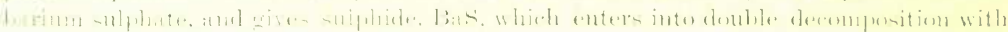

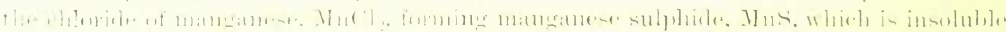

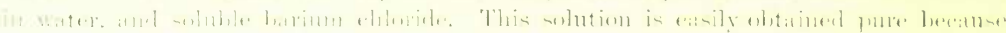

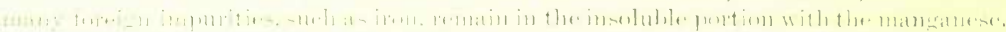

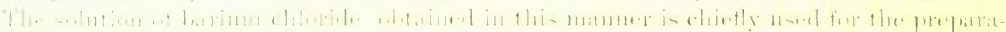

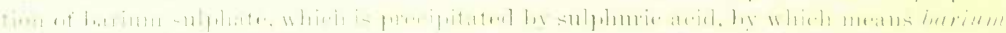

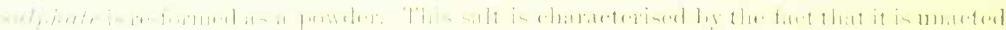

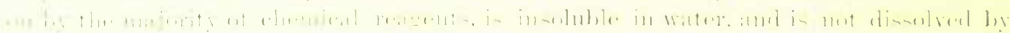

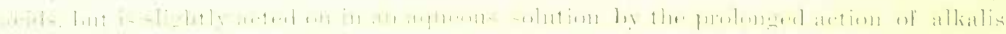

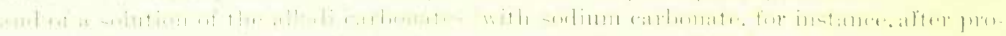

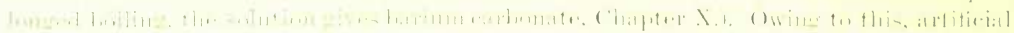

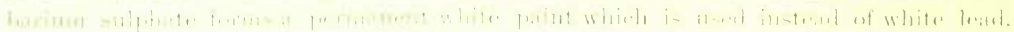

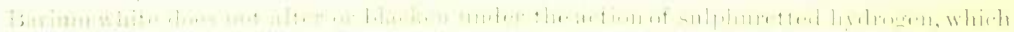

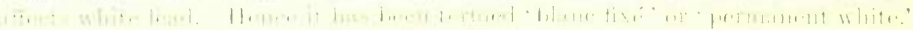

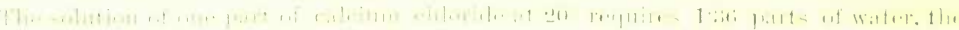

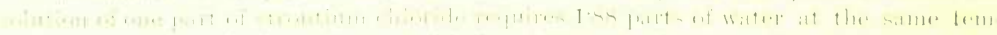

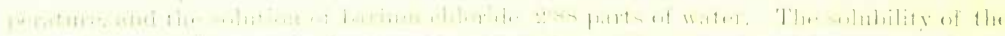

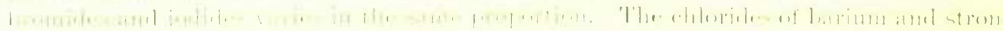

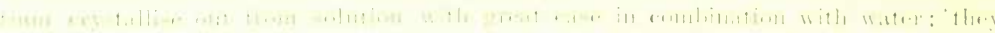

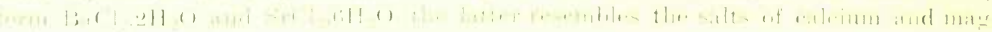


carbonate a precipitate of barium earbonate, $\mathrm{BaCO}_{3}$. Both these salts are able to give barium oxide, or baryta, $\mathrm{BaO}$, and the hydroxide, $\mathrm{Ba}(\mathrm{HO})_{2}$, which differs from lime by its great solubility in water, ${ }^{5.5}$ and by the ease with which it forms a crystallo-hydrate, $\mathrm{BaH}_{2} \mathrm{O}_{2}, 8 \mathrm{H}_{2} \mathrm{O}$, from its solutions. Owing to its solubility, baryta is frequently employed in the manufactures and in chemical practice as an alkali which has the very important property that it may be always entirely removed from solution by the addition of sulphuric acid, which entirely separates the baryta as the insoluble sulphate, $\mathrm{BaSO}_{4}$. It may also be removed so long as it remains in an alkaline state (for example, the excess which may remain when it is taken for saturating acids) by means of carbonic anhydride, which also entirely precipitates barium oxide as a sparingly soluble, colourless, and powdery carbonate. Both these reactions show that baryta has such properties as would render its use in practice most widely extended were its compounds as widely distributed as those of sodium and calcium, and if its soluble compounds were not poisonous. Barium nitrate is directly decomposed by the action of heat, barium oxide being left behind. The same takes place with barium carbonate, especially that precipitated from solutions and when mixed with charcoal or ignited in an atmosphere of steam.

Barium oxide combines with water with the development of a large amount of heat, and the resultant hydroxide is so stable in its retention of the water that it will not part with it under the action of heat alone, although it entirely dissociates when strongly ignited in a current of hydrogen or other gas, and especially of air. With oxygen the

or strontium chlorides. They are obtained by the action of nitric acid on the carbonates or oxides. They may also be obtained by the action of nitric acid on solutions of the chlorides, all the more as tiney are comparatively little soluble, especially in water containing nitric acid-100 parts of water at $15^{\circ}$ dissolve 6.5 parts strontium nitrate and 8.2 parts of barium nitrate, whilst more than 300 parts of calcium nitrate are soluble at the same temperature. Strontium nitrate communicates a crimson coloration to the flame of burning substances, and is therefore frequently used for Bengal fire, fireworks, and signal lights, for which purpose the salts of lithium are still better fitted. Calcium nitrate is exceedingly hygroscopic. The barium nitrate, on the contrary, does not show this property in the least degree, and in this respect it resembles potassium nitrate, and is therefore used instead of the latter for the preparation of a gunpowder which is called 'saxifragin powder' (76 parts of barium nitrate, 2 parts of nitre, and 22 parts of charcoal).

55 The dissociation of the crystallo-hydrate of baryta is given in Chapter I. Note 65 . 100 parts of water dissolve

$\begin{array}{cccccc} & 0^{\circ} & 20^{\circ} & 40^{\circ} & 60^{\circ} & 80^{\circ} \\ \mathrm{BaO} & 1.5 & 3.5 & 7 \cdot 4 & 18.8 & 90.8 \\ \mathrm{SrO} & 0.3 & 0.7 & 1.4 & 3 & 9\end{array}$

Supersaturated solutions are easily formed.

The anhydrous oxide $\mathrm{BaO}$ fuses in the oxyhydrogen flame. When ignited in the vapour of potassium, the latter takes up the oxygen; whilst in chlorine the oxygen is separated and barium chloride formed. 
anhydrous oxide gives, as has already been mentioned in Chapters III. and IV., a peroxide, $\mathrm{BaO}_{2}{ }^{56}$ Neither calcium nor strontium oxides are able to give such a peroxide directly, but they form peroxides under the action of hydrogen peroxide.

Barium oxide is decomposed when heated with potassium ; and fused barium chloride is decomposed, as Davy showed, by the action of a galvanic current, forming metallic barium ; and Crookes (1862) obtained an amalgam of barium from which the mercury could easily be driven off, by heating sodium amalgam in a saturated solution of barium chloride. The same occurs with strontium. Both metals are soluble in mercury, and seem to be non-volatile or at least very slightly volatile. They are both heavier than water ; the sp. gr. of barium is $3 \cdot 6$, and of strontium $2 \cdot 5$. They both decompose water at the ordinary temperature, like the metals of the alkalis.

Barium and strontium as saline elements are characterised by their powerful basic properties, so that they form acid salts with difficulty, and scarcely form basic salts. On comparing them together and with calcium, it is evident that the alkaline properties in this group (as in the group potassium, rubidium, caesium) increase with the atomic weight, and this succession clearly shows itself in many of their corresponding compounds. Thus, for instance, the solubility of the hydroxides $\mathrm{RH}_{2} \mathrm{O}_{2}$ and the specific gravity ${ }^{57}$ rise in passing from calcium to strontium and barium, while the solubility of the sulphates decreases,, 58 and therefore in the case of magnesium and beryllium as metals whose atomic weights are still less, we should expect the solubility of the sulphates to be greater, and this is the case in reality.

${ }^{5 i}$ The property of barium oxide of absorbing oxygen when heated, and giving the peroxide $\mathrm{BaO}_{2}$, is very characteristic for this oxide. It is only proper to the anhydrous oxide. The lyydroxide does not absorb oxygen. Peroxides of calcium and strontium may be obtained by means of hydrogen peroxide. Barium peroxide is insoluble in water, but is able to form a hydrate with it, and also to combine with liydrogen peroxide, forming a very unstable compound having the composition $\mathrm{BaH}_{2} \mathrm{O}_{4}$ (obtained by Professor Schöne), and which in the course of time evolves oxygen (Chapter IV. Note 21).

57 Even in solutions a gradual progression in the increase of the specific gravity shows itself, not only for equivalent solutions (for instance, $\mathrm{RCl}_{2}+200 \mathrm{H}_{2} \mathrm{O}$ ), but even with an equal percentage composition, as is seen from the curves giving the specific gravity (water $4^{\circ}=10000$ ) at $15^{\circ}$ (for barium chloride, according to Bourdiakoff's determinations) :

$$
\begin{aligned}
& \mathrm{BeCl}_{2}: \mathrm{S}=9992+67 \cdot 21 p+0 \cdot 111 p^{2} \\
& \mathrm{CaCl}_{2}: \mathrm{S}=9992+80 \cdot 24 p+0 \cdot 476 p^{3} \\
& \mathrm{SrCl}_{2}: \mathrm{S}=9992+85 \cdot 57 p+0 \cdot 733 p^{2} \\
& \mathrm{BaCl}_{2}: \mathrm{S}=9992+86 \cdot 56 p+0 \cdot 813 p^{3}
\end{aligned}
$$

38 One part of calcium sulphate at the ordinary temperature requires about 500 parts of water for solution, strontium sulphate abont 7000 parts, barium sulphate about 400000 parts, whilst beryllium sulphate is easily soluble in water. 
As in the series of the alkali metals we saw the metals potassium, rubidium, and caesium approaching near to each other in their properties, and besides them two metals having smaller combining weights-namely, sodium, and the lightest of all, lithium, which already exhibited certain particular characteristic properties - so also in the case of the metals of the alkaline earths we find, besides calcium, barium, and strontium, the metal magnesium and also beryllium or glucinum. In respect to the magnitude of its atomic weight, it occupies the same position in the series of the metals of the alkaline earths as lithium does in the series of the alkali metals, because the combining weight of beryllium, $\mathrm{Be}$ or $\mathrm{Gl}=9$. This combining weight is greater than the combining weight of lithium (7), as the combining weight of magnesium (24) is greater than that of sodium (23), or of calcium (40) is greater than that of potassium (39), \&c. ${ }^{59}$ Beryllium was so named because it occurs in the mineral beryl. The metal is also called glucinum (from the Greek word $\gamma \lambda v \kappa v$ s, 'sweet'), because its salts have a sweet taste. It occurs in beryl, aquamarine, the emerald, and other minerals, which are generally of a green colour, and sometimes occur in considerable masses, but which are as a rule comparatively rare and, as transparent crystals, form precious stones. The composition of beryl, the emerald, and smaragd is as follows: $\mathrm{Al}_{2} \mathrm{O}_{3}, 3 \mathrm{BeO}, 6 \mathrm{SiO}_{2}$. The Siberian and Brazilian beryls are the best known. The sp. gr. of beryl is about $2 \cdot 7$. Beryllium oxide, from the feebleness of its basic properties, presents an analogy to aluminium oxide in the same degree as lithium oxide is analogous to magnesium oxide. ${ }^{60}$ Owing to its rare occur-

59 We refer beryllium to the class of the bivalent metals of the alkaline earths-that is, we ascribe to its oxide the formula $\mathrm{BeO}$, and do not count it as trivalent ( $\mathrm{Be}=13 \cdot 5$, p. 318), as has been proposed and argued by many. The true atomic composition of beryllium oxide was first given by the Rassian chemist, Avdéeff (1819), in his researches on the compounds of this metal. He compared the compounds of beryllium to those of magnesium, and set aside the then reigning opinion of the resemblance between the oxides of beryllinm and alurninium, by proving that beryllium sulphate presents a greater resemblance to magnesinm sulphate than to aluminium sulphate. It was especially noticed that the analogues of alumina give alams, whilst beryllium oxide, although it is a feeble base, easily giving, like magnesia, basic and double salts, does not form true alums. The establishment of the periodic system of the elements (1869), which is considered in the following chapter, immediately showed that Ardéeff's view corresponded with the trath -that is, that beryllium is bivalent, and therefore necessitated the refutation of the trivalency of beryllium. This scientific controversy resulted in a vast series of researches (1870-80) concerning this element, and ended in Nilson and Pettersson-two of the chief advocates of the trivalency of beryllium-determining the vapour density of $\mathrm{BeCl}_{2}(=40$, p. 318), which gare an undonbted proof of the bivalency of beryllium.

${ }_{60}$ Beryllinm oxide, like aluminium oxide, is precipitated from solutions of its salts by alkalis as a gelatinous hydroxide $\mathrm{BeH}_{2} \mathrm{O}_{2}$, which, like alnmina, is soluble in an excess of canstic potash or soda. This reaction may be taken advantage of for distinguishing and separating beryllium from aluminium, because when the alkaline solution is diluted with water and boiled beryllium hydroxide is precipitated, whilst the alumina remains

voL. 1 . 
rence in nature, to the absence of any especially distinct individual properties, and to the possibility of foretelling them to a certain extent on the basis of the periodic system of the elements given in the following chapter, and owing to the brevity of this treatise, we will not linger long over the compounds of beryllium, and will only observe that the individuality of the compounds of beryllium was pointed out in 1798 by Vauquelin, and that metallic beryllium was obtained by Wöhler and Bussy. Wöhler obtained metallic beryllium (like magnesium) by acting on beryllium chloride, $\mathrm{BeCl}_{2}$, with potassium. Metallic beryllium has a sp. gr. $1 \cdot 64$ (Nillson and Pettersson). It is very infusible, melting at nearly the same temperature as silver, which it resembles in its white colour and lustre. It is characterised by the fact that it is very difficultly oxidised, and even in the oxidising flame of a blow-pipe is only superficially covered by a coating of oxide; it does not burn in pure oxygen, does not decompose water at the ordinary temperature or at a red heat, but gaseous hydrochloric acid is decomposed by it when slightly heated, with evolution of hydrogen and development of a considerable amount of heat. Even dilute hydrochloric acid acts in the same manner at the ordinary temperature. Beryllium also acts easily on sulphuric acid, but it is remarkable that neither dilute nor strong nitric acid acts on beryllium, which seems to

in solution. The solubility of the beryllium oxide already clearly indicates its feeble basic properties, and, as it were, separates this oxide from the class of the alkaline earths. But on arranging the oxides of the above-described metals of the alkaline earths according to their decreasing atomic weights we have the series

$$
\mathrm{BaO}, \mathrm{SrO}, \mathrm{CaO}, \mathrm{MgO}, \mathrm{BeO} \text {, }
$$

in which the basic properties and solubility of the oxides consecutively and distinctly decrease until we reach a point when, had we not known of the existence of the beryllium oxide, we should expect to find in its place an oxide insoluble in water and of feeble basic properties. So also in the series of the metals of the alkalis the basicity of lithium oxide is distinctly more feeble than sodium and potassium oxides, and lithium carbonate is insoluble in water.

Another characteristic of the salts of beryllium is that they give a gelatinous precipitate with aqueous ammonia, which is soluble in an excess of ammonium carbonate, like the precipitate of magnesia; in this beryllium oxide differs from the oxide of aluminium. Beryllium oxide easily forms a carbonate which is insoluble in water, and resembles magnesium carbonate in many respects. Beryllium sulphate is distinguished by its considerable solubility in water-thus, at the ordinary temperature it dissolves in an equal weight of water; it crystallises out from its solutions in well-formed crystals, which do not change in the air, and contain $\mathrm{BeSO}_{4}, 4 \mathrm{H}_{2} \mathrm{O}$. When ignited it leaves beryllium oxide, but this oxide, after prolonged ignition, is re-dissolved by sulphuric acid, whilst aluminium sulphate, after a similar treatment, leaves aluminium oxide, which is no longer soluble in acids. With a few exceptions, the salts of beryllium crystallise with great difficulty, and to a considerable extent resemble the salts of magnesium; thus, for instance, beryllium chloride is analogous to magnesium chloride. It is volatile in an anhydrous state, and in a hydrated state it decomposes, with the evolution of bydrochloric acid. 
resist oxidising agents with particular ease. Potassium hydroxide acts on beryllium as on aluminium, hydrogen being disengaged and the metal dissolved, but anmonia has no action on it. These properties of metallic beryllium seem to isolate it from the series of the other metals described in this chapter, but if we compare the properties of calcium, magnesium, and beryllium we shall see that magnesium occupies an intermediate position between the other two. Whilst calcium decomposes water with great ease, magnesium does so with difficulty, and beryllium not at all. The peculiarities of beryllium among the metals of the alkaline earths recalls the fact that in the series of the halogens we saw that fluorine differed from the other halogens in many of its properties and has the smallest atomic weight. Just the same is the case with beryllium among the other metals of the alkaline earths.

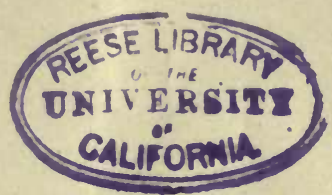

END OF THE FIRST VOLUME 





\section{RETURN TO the circulation desk of any}

University of California Library or to the

NORTHERN REGIONAL LIBRARY FACILITY Bldg. 400, Richmond Field Station University of California Richmond, CA 94804-4698

ALL BOOKS MAY BE RECALLED AFTER 7 DAYS

- 2-month loans may be renewed by calling (510) 642-6753

- 1 -year loans may be recharged by bringing books to NRLF

- Renewals and recharges may be made 4 days prior to due date.

DUE AS STAMPED BELOW

\section{JAN 042001}

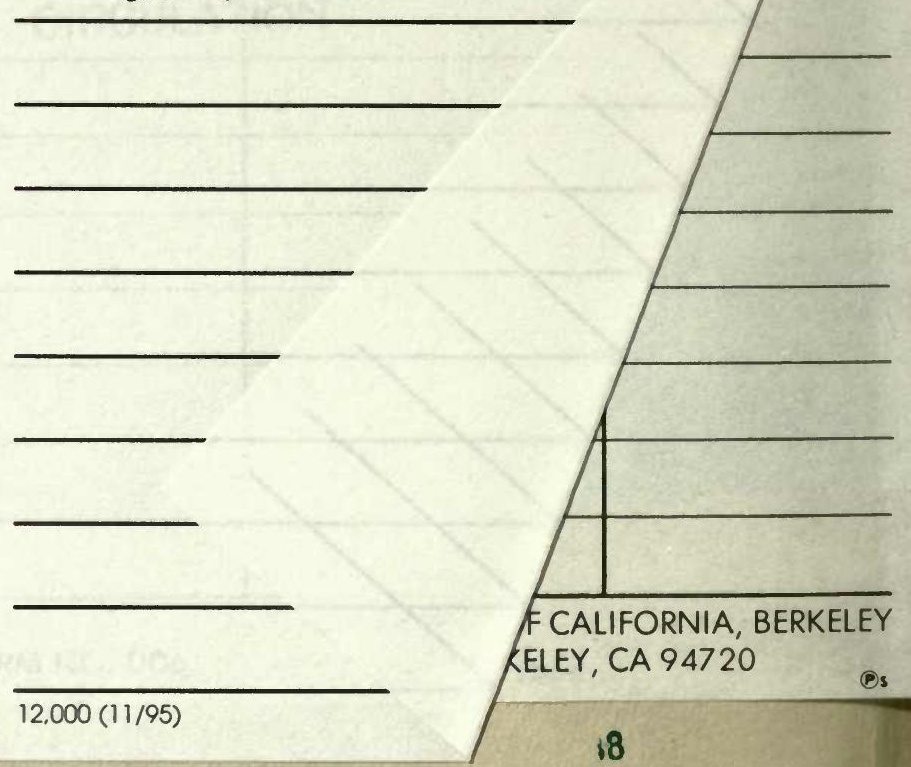




\section{U.C. BERKELEY LIBRARIES}

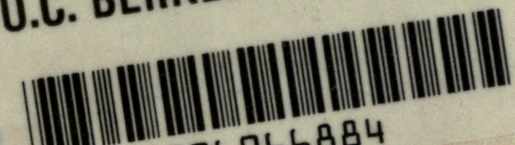

C006066884 
10.

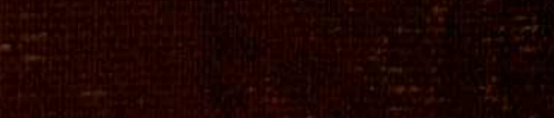

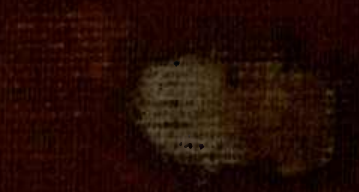

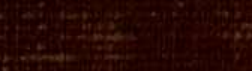
sing

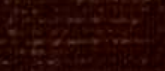

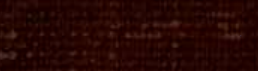

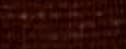
ats

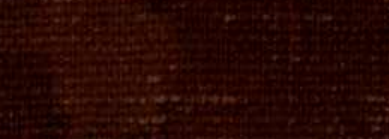

